This document was prepared in conjunction with work accomplished under Contract No. DE-AC09-96SR18500 with the U. S. Department of Energy.

\title{
DISCLAIMER
}

This report was prepared as an account of work sponsored by an agency of the United States Government. Neither the United States Government nor any agency thereof, nor any of their employees, nor any of their contractors, subcontractors or their employees, makes any warranty, express or implied, or assumes any legal liability or responsibility for the accuracy, completeness, or any third party's use or the results of such use of any information, apparatus, product, or process disclosed, or represents that its use would not infringe privately owned rights. Reference herein to any specific commercial product, process, or service by trade name, trademark, manufacturer, or otherwise, does not necessarily constitute or imply its endorsement, recommendation, or favoring by the United States Government or any agency thereof or its contractors or subcontractors. The views and opinions of authors expressed herein do not necessarily state or reflect those of the United States Government or any agency thereof. 


\section{A STATISTICAL REVIEW OF DWPF LABORATORY DATA INCLUDING MEASURMENTS OF THE ARG-1 STANDARD FOR BATCHES 94 - 263}

T. B. Edwards

November 2004

Statistical Consulting Section

Savannah River National Laboratory

Aiken, SC 29808

Prepared for the U.S. Department of Energy Under Contract Number DEAC09-96SR18500

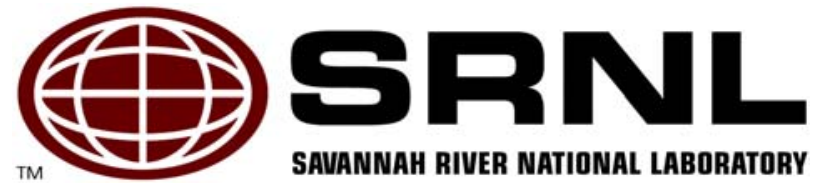


This page was intentionally left blank 
Key Words: statistics, fusion, mixed acid, cold chemical

Retention: Permanent

\section{A STATISTICAL REVIEW OF DWPF LABORATORY DATA INCLUDING MEASURMENTS OF THE ARG-1 STANDARD FOR BATCHES 94 - 263}

T. B. Edwards

November 2004

Statistical Consulting Section

Savannah River National Laboratory

Aiken, SC 29808

Prepared for the U.S. Department of Energy Under Contract Number DEAC09-96SR18500

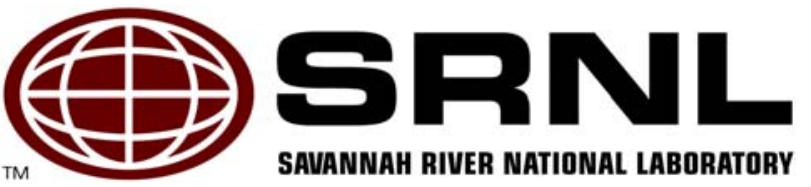




\section{EXECUTIVE SUMMARY}

Measurements of calibration and bench standards as well as samples of ARG-1 that were performed by the DWPF Lab during the processing of batches 94 through 263 were provided to SCS for review. Three datasets, one associated with each of three preparation methods (Cold Chem, Mixed Acid, and Fusion) were included in the review.

The review conducted covered several areas of investigation. Biases in these measurements relative to the reference values for the standards including ARG-1 were estimated and found to be of no practical concern. Percent relative standard deviations for these data also were determined. Sources of variation in the measurements (i.e., batch-to-batch and within process batch) were estimated and compared. An investigation into evidence of instrument drift during a group of measurements representing a prototypical block was conducted for each preparation method using pairs of calibration and bench standards. No evidence of instrument drift at levels of practical concern was seen in these data. Also, the replicates of calibration standards at the beginning of each analytical block were found to be unnecessary.

The uncertainties of the measurements, which incorporated biases and precision errors, were computed for the calibration standards, bench standards, and ARG-1. The limits for these uncertainties were compared to the current, LIMS operating limits for the errors in the measurements of these standards. These comparisons may provide opportunities for revising the LIMS limits.

Comparisons between MFT and SME results were made for both the Mixed Acid and the Fusion prep methods. There was no evidence of differences (in either bias or precision) between the results for these two tanks for either of the two prep methods. 


\section{TABLE OF CONTENTS}

EXECUTIVE SUMMARY iii

LIST OF TABLES $\quad \mathrm{V}$

LIST OF ACRONYMS vi

1.0 INTRODUCTION AND BACKGROUND 1

1.1 Overview of Data 1

1.1.1 Cold Chem (SRAT) Data 2

1.1.2 Mixed Acid (SME and MFT) Data 3

1.1.3 Fusion (SME and MFT) Data 4

2.0 Results $\quad 5$

2.1 Cold Chem Results $\quad 5$

2.1.1 Cold Chem Calibration and Bench Standards 5

2.1.2 Cold Chem ARG-1 Results 13

2.2 Mixed Acid Results $\quad 14$

2.2.1 Mixed Acid Calibration and Bench Standards 14

2.2.2 Mixed Acid ARG-1 Results $\quad 22$

2.3 Fusion Results 23

2.3.1 Fusion Calibration and Bench Standards 23

2.3.2 Fusion ARG-1 Results $\quad 29$

2.4 LIMS Limits 31

2.4.1 Measurement Uncertainties of Calibration and Bench Standards 31

2.4.2 Measurement Uncertainties of ARG-1 32

2.5 Sum of oxides $\quad 33$

2.6 MFT versus SME Results

3.0 CONCLUSIONS $\quad 36$

4.0 REFERENCES 37

APPENDIX A. 39 


\section{LIST OF TABLES}

Table 1-1 Reference Composition of the ARG-1 Standard with LIMS Limits for Errors ....... 2

Table 1-2 Protocol for SRAT Samples Prepared Using Cold Chem Method.......................... 2

Table 1-3 Reference Values (mg/L) for Cold Chem Calibration and Bench Standards......... 3

Table 1-4 Protocol for SME and MFT Samples Prepared Using Mixed Acid Method........... 3

Table 1-5 Reference Values (mg/L) for Mixed Acid Calibration and Bench Standards........ 4

Table 1-6 Protocol for SME and MFT Samples Prepared Using the Fusion Method ............. 4

Table 1-7 Reference Values (mg/L) for Fusion Calibration and Bench Standards ................ 4

Table 2-1 All Measurements (mg/L) for Cold Chem Calibration \& Bench Standards Means

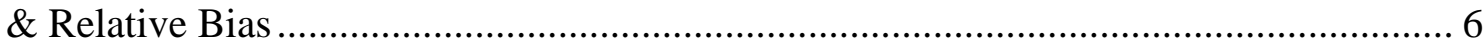

Table 2-2 All Measurements (mg/L) for Cold Chem Calibration and Bench Standards........ 7

Table 2-3 Measurements (mg/L) for Cold Chem Calibration and Bench Standards Reported

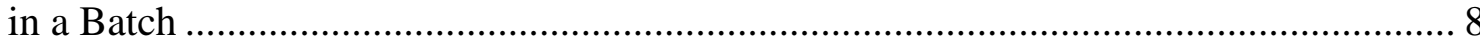

Table 2-4 Variance Components and Percent Contribution by Standard for Cold Chem ........ 9

Table 2-5 Insights into Instrument Drift by Comparisons of IN35 vs IN33 for Cold Chem. 10

Table 2-6 Insights into Instrument Drift by Comparisons of IN36 vs IN34 for Cold Chem. 11

Table 2-7 Insights into Instrument Drift by Comparisons of IN39 vs IN38 for Cold Chem.. 12

Table 2-8 ARG-1 Results for Cold Chem Samples ........................................................... 13

Table 2-9 Components of Variation for ARG-1 Results for Cold Chem Samples................ 14

Table 2-10 Means \& Relative Biases for All Measurements (mg/L) for Mixed Acid

Calibration \& Bench Standards .................................................................................. 15

Table 2-11 Descriptive Statistics for All Mixed Acid Calibration and Bench Standard

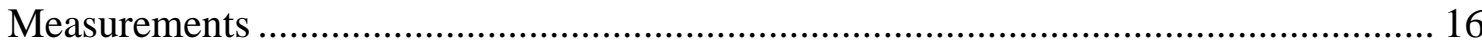

Table 2-12 Descriptive Statistics for Mixed Acid Calibration and Bench Standards in a

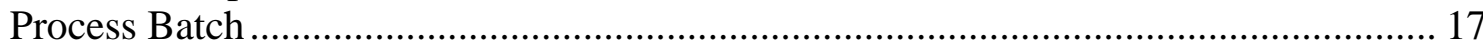

Table 2-13 Components of Variance for Mixed Acid Calibration and Bench Standards in a

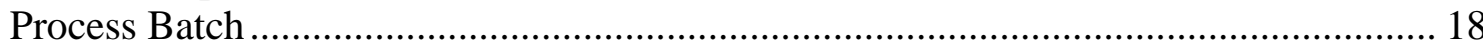

Table 2-14 Insights into Mixed Acid Instrument Drift by Comparisons of SM35 vs SM33 . 19

Table 2-15 Insights into Instrument Drift by Comparisons of SM36 vs SM34 for Mixed Acid

Table 2-16 Insights into Instrument Drift by Comparisons of SM38 vs SM37 for Mixed Acid

Table 2-17 ARG-1 Results for Mixed Acid Samples ........................................................ 22

Table 2-18 Components of Variation for ARG-1 Results for Mixed Acid Samples.............. 23

Table 2-19 Averages and Relative Biases for All Calibration and Bench Standard

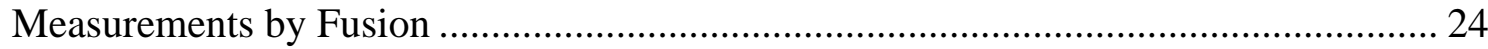

Table 2-20 Descriptive Statistics for All Fusion Calibration and Bench Standard

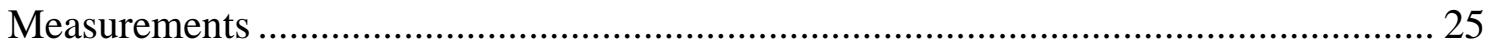

Table 2-21 Descriptive Statistics for Fusion Calibration and Bench Standards in a Process

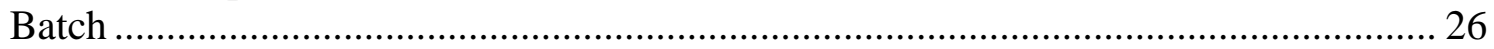

Table 2-22 Components of Variance for Fusion Calibration and Bench Standards in a Process

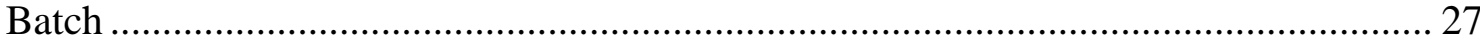

Table 2-23 Insights into Instrument Drift for Fusion Measurements of SM53 vs SM51...... 28 
Table 2-24 Insights into Instrument Drift by Comparisons of SM52 vs SM54 for Fusion .... 29

Table 2-25 Summary Statistics for ARG-1 Fusion Measurements ..................................... 30

Table 2-26 Components of Variation for ARG-1 Results for Mixed Acid Samples.............. 30

Table 2-27 Uncertainties for ARG-1 Measurements ............................................................ 33

Table 2-28 Descriptive Statistics for ARG-1 Sums of Oxides by Preparation Method ......... 34

Table 2-29 Descriptive Statistics for ARG-1 Mixed Acid Measurements by Tank .............. 35

Table 2-30 Descriptive Statistics for ARG-1 Fusion Measurements by Tank ...................... 35

\section{LIST OF ACRONYMS}

DWPF Defense Waste Processing Facility

MFT Melter Feed Tank

RSD Relative Standard Deviation

SME Slurry Mix Evaporator

SRAT Sludge Receipt and Adjustment Tank

Std Dev Standard Deviation

Prep preparation

SRS Savannah River Site

SCS Statistical Consulting Section 


\subsection{INTRODUCTION AND BACKGROUND}

The Defense Waste Processing Facility (DWPF) at the Savannah River Site (SRS) in Aiken, South Carolina, has been immobilizing high level radioactive waste (HLW) in borosilicate glass since 1996. Chemical composition analyses of the material being processed at the facility are among the measurements used by the DWPF to manage and control their operations and to ensure that they meet product specifications. These analyses, which include measurements of samples from the Sludge Receipt and Adjustment Tank (SRAT), the Slurry Mix Evaporator (SME), and (in the past) the Melter Feed Tank (MFT), are provided by the DWPF Laboratory (DWPF Lab) of Waste Laboratory Services. The DWPF Lab employs several standards to establish and monitor the comprehensive quality control program under which the process sample analyses are conducted.

Statistical reviews of data generated by this program have been conducted in the past. Measurements of process samples have been reviewed by Edwards ([1] and [2]). The most recent review of DWPF Lab measurements of the standards was conducted by Harris in 2000 [3], and that review covered data through process batch 93. The purpose of this report, which was initiated as part of the response to a Technical Task Request (TTR) issued by DWPF Process Engineering [4], is to conduct a statistical review of the measurements of the standards generated by the DWPF Lab during the processing of batches 94 through 263. The information in this review is intended to provide a basis for assessing the performance of the laboratory and for initiating potential improvement activities.

\subsection{OVERVIEW OF DATA}

The DWPF Lab, through their WG17 server on the SRS computer network, provided the measurement data for this analysis to the Statistical Consulting Section (SCS) of the Savannah River National Laboratory (SRNL). The measurements were grouped into three datasets, each related to the preparation method utilized for the samples being measured. The preparation/dissolution method(s) used was determined by the vessel being sampled, and samples from the SRAT, the SME, and the MFT were included in the data for this study. For the SRAT samples, there was only one preparation/dissolution method used and it was denoted as "Cold Chem." For the SME and MFT, two different dissolution methods were used. These were denoted as "Mixed Acid" and "Fusion." Thus, the data were organized into three datasets corresponding to these three preparation or prep methods: Cold Chem, Mixed Acid, and Fusion. For each of these datasets, measurements were provided in analytical sequence. The measurements related to a process batch, whether measurements of process samples or standards, were identified by the number of the process batch involved. Other measurements also were included in the datasets that were not part of the information reported for a process batch. The analyses of this study were initiated using all of the available data.

One of the standards utilized in each of the three datasets was the Analytical Reference Glass One (ARG-1). This was the standard of primary interest in this study. It is a powered glass that was prepared by Corning Glass and characterized by the Material Characterization Center (MCC) at Pacific Northwest National Laboratory (PNNL). Its reference composition is provided in Table 1-1 in both elemental and oxide weight percents (wt\%'s). Also in Table 1-1 are the errors (as percentages) that the DWPF Lab utilizes as part of its quality control in the measurement of the ARG-1 standard. The limits for these errors are incorporated in the Laboratory Information Management System (LIMS) used by the DWPF Lab. If the measurements for one of the elements of this standard fall outside the permitted errors for the element, the measurements for that element are repeated for the block of samples being processed. 
Table 1-1 Reference Composition of the ARG-1 Standard with LIMS Limits for Errors

\begin{tabular}{|c|c|c|c|c|c|c|c|}
\hline Element & $\begin{array}{c}\begin{array}{c}\text { Weight Percent } \\
\text { (wt\%) }\end{array} \\
\end{array}$ & Oxide & $\begin{array}{c}\text { Gravimetric } \\
\text { Factor }\end{array}$ & $\begin{array}{c}\begin{array}{c}\text { Weight Percent } \\
\text { (wt\%) }\end{array} \\
\end{array}$ & $\begin{array}{l}\begin{array}{l}\text { Cold Chem } \\
\text { +/- Error }\end{array} \\
\end{array}$ & $\begin{array}{l}\text { Mixed Acid } \\
+/ \text { - Error }\end{array}$ & $\begin{array}{c}\text { Fusion } \\
\text { +/- Error }\end{array}$ \\
\hline Aluminum (Al) & 2.5 & $\mathrm{Al}_{2} \mathrm{O}_{3}$ & 1.8895 & 4.7238 & 12 & 9 & 8 \\
\hline Boron (B) & 2.69 & $\mathrm{~B}_{2} \mathrm{O}_{3}$ & 3.2199 & 8.6615 & - & - & 11 \\
\hline Calcium $(\mathrm{Ca})$ & 1.02 & $\mathrm{CaO}$ & 1.3992 & 1.4272 & 22 & 13 & 20 \\
\hline Chromium (Cr) & 0.06 & $\mathrm{Cr}_{2} \mathrm{O}_{3}$ & 1.4616 & 0.0877 & 35 & 35 & 35 \\
\hline Copper (Cu) & 0.0001 & $\mathrm{CuO}$ & 1.2518 & 0.0001 & - & - & - \\
\hline Iron $(\mathrm{Fe})$ & 9.79 & $\mathrm{Fe}_{2} \mathrm{O}_{3}$ & 1.4297 & 13.9968 & 15 & 8 & 8 \\
\hline Potassium (K) & 2.25 & $\mathrm{~K}_{2} \mathrm{O}$ & 1.2046 & 2.7104 & 20 & 11 & 10 \\
\hline Lithium (Li) & 1.49 & $\mathrm{Li}_{2} \mathrm{O}$ & 2.1529 & 3.2078 & 15 & 8 & 8 \\
\hline Magnesium (Mg) & 0.52 & $\mathrm{MgO}$ & 1.6581 & 0.8622 & 17 & 10 & 9 \\
\hline Manganese (Mn) & 1.46 & $\mathrm{MnO}$ & 1.2912 & 1.8852 & 14 & 8 & 8 \\
\hline Sodium (Na) & 8.53 & $\mathrm{Na}_{2} \mathrm{O}$ & 1.3480 & 11.4984 & 15 & 13 & - \\
\hline Nickel (Ni) & 0.83 & $\mathrm{NiO}$ & 1.2725 & 1.0562 & 15 & 9 & 10 \\
\hline Silicon (Si) & 22.39 & $\mathrm{SiO}_{2}$ & 2.1393 & 47.8989 & 18 & 10 & 9 \\
\hline Titanium (Ti) & 0.69 & $\mathrm{TiO}_{2}$ & 1.6680 & 1.1509 & 17 & 9 & 8 \\
\hline Uranium (U) & 0 & $\mathrm{U}_{3} \mathrm{O}_{8}$ & 1.1792 & 0.0000 & - & - & - \\
\hline Zirconium (ZrO) & 0.1 & $\mathrm{ZrO}_{2}$ & 1.3508 & 0.1351 & 21 & 12 & - \\
\hline
\end{tabular}

Other standards are also included in each of the three datasets. These include blanks, calibration standards, and bench standards. The standards in each datasets are identified during the discussion of the analytical protocols provided below.

\subsubsection{Cold Chem (SRAT) Data}

The Cold Chem method is used in the preparation of SRAT samples, and the typical protocol used to process a set of 6 SRAT samples is provided in Table 1-2. As indicated in this table, Calibration Standard C and Bench Standard C were not used for every process batch.

Table 1-2 Protocol for SRAT Samples Prepared Using Cold Chem Method

\begin{tabular}{||c|c|}
\hline Analytical Sequence & Description \\
\hline IN35 & Calibration Std A \\
\hline IN35 & Calibration Std A \\
\hline IN36 & Calibration Std B \\
\hline IN36 & Calibration Std B \\
\hline IN38 & Calibration Std C - used for some batches \\
\hline IN38 & Calibration Std C - used for some batches \\
\hline IN32 & Blank \\
\hline ARGC & ARAT sample \# 1 \\
\hline SRAT-1 & SRAT sample \# 2 \\
\hline SRAT-2 & SRAT sample \# 3 \\
\hline SRAT-3 & SRAT sample \# 4 \\
\hline SRAT-4 & SRAT sample \# 5 \\
\hline SRAT-5 & SRAT sample \# 6 \\
\hline SRAT-6 & ARG-1 std \\
\hline ARGC & Blank \\
\hline IN32 & Bench A Std \\
\hline IN33 & Bench B Std \\
\hline IN34 & Bench C Std - used for some batches \\
\hline IN39 & \\
\hline & \\
\hline
\end{tabular}

The reference values for each of the standards used to support the Cold Chem analyses are provided in Table 1-3. As the DWPF Lab processes a set of SRAT samples using this protocol, the measurements of the standards are assessed against an acceptance criterion: the measurements generated for the standards are expected to be no more that $+/-7 \%$ (the LIMS limits) from the reference values in this table. 
Otherwise, the block of samples is considered to be in question, and the measurements of each element failing to meet this criterion are repeated.

Table 1-3 Reference Values (mg/L) for Cold Chem Calibration and Bench Standards

\begin{tabular}{|c|c|c|c|c|c|c|c|c|c|c|c|c|c|c|c|c|}
\hline Seq & $\overline{\mathrm{Al}}$ & 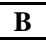 & $\overline{C a}$ & $\mathrm{Cr}$ & $\overline{c \mathbf{C u}}$ & 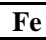 & $\bar{K}$ & $\overline{\mathbf{L i}}$ & Mg & Mn & $\mathrm{Na}$ & $\mathrm{Ni}$ & $\mathrm{Si}$ & $\mathbf{T i}$ & $\mathbf{U}$ & $\mathrm{Zr}$ \\
\hline IN32 & 0 & 0 & 0 & 0 & 0 & 0 & 0 & 0 & 0 & 0 & 0 & 0 & 0 & 0 & 0 & 0 \\
\hline IN33 & 10 & 0 & 2 & 2 & 1 & 20 & 0 & 0 & 1 & 1 & 20 & 2 & 0 & 2 & 0 & 2 \\
\hline IN34 & 0 & 10 & 0 & 0 & 0 & 0 & 10 & 10 & 0 & 0 & 65 & 0 & 40 & 0 & 0 & 0 \\
\hline IN35 & 10 & 0 & 2 & 2 & 1 & 20 & 0 & 0 & 1 & 1 & 20 & 2 & 0 & 2 & 0 & 2 \\
\hline IN36 & 0 & 10 & 0 & 0 & 0 & 0 & 10 & 10 & 0 & 0 & 65 & 0 & 40 & 0 & 0 & 0 \\
\hline IN38 & 0 & 0 & 0 & 0 & 0 & 0 & 0 & 0 & 0 & 0 & 0 & 0 & 0 & 0 & 10 & 0 \\
\hline IN39 & 0 & 0 & 0 & 0 & 0 & 0 & 0 & 0 & 0 & 0 & 0 & 0 & 0 & 0 & 10 & 0 \\
\hline
\end{tabular}

Also, note that the pairs of standards: IN33 and IN35, IN34 and IN36, and IN38 and IN39, where one is a calibration standard and the other is a bench standard, provide opportunities to look for drift in the measurements over the course of an analytical block. More will be said on instrument drift in the sections that follow.

\subsubsection{Mixed Acid (SME and MFT) Data}

The Mixed Acid (MA) method is used in the preparation of SME and MFT samples, and the typical protocol used to process a set of 6 samples from either of these tanks is provided in Table 1-4.

Table 1-4 Protocol for SME and MFT Samples Prepared Using Mixed Acid Method

\begin{tabular}{||c|c|}
\hline Analytical Sequence & Description \\
\hline SM35 & Calibration Std A \\
\hline SM35 & Calibration Std A \\
\hline SM36 & Calibration Std B \\
\hline SM36 & Calibration Std B \\
\hline SM38 & Calibration Std C \\
\hline SM38 & Calibration Std C \\
\hline SM32 & Blank \\
\hline ARGB & ARG-1 std \\
\hline SME or MFT-1 & SME or MFT sample \# 1 \\
\hline SME or MFT-2 & SME or MFT sample \# 2 \\
\hline SME or MFT--3 & SME or MFT sample \# 3 \\
\hline SME or MFT--4 & SME or MFT sample \# 4 \\
\hline SME or MFT--5 & SME or MFT sample \# 5 \\
\hline SME or MFT--6 & SME or MFT sample \# 6 \\
\hline ARGB & ARG-1 std \\
\hline SM32 & Blank \\
\hline SM33 & Bench A Std \\
\hline SM34 & Bench B Std \\
\hline SM37 & Bench C Std \\
\hline
\end{tabular}

The reference values for each of the standards used to support the Mixed Acid analyses are provided in Table 1-5. As the DWPF Lab processes a set of SME or MFT samples using this protocol, the measurements of the standards are assessed against an acceptance criterion: the measurements generated for the standards are expected to be no more that $+/-7 \%$ (the LIMS limits) from the reference values in this table. Otherwise, the block of samples is considered questionable, and the measurements of each element failing to meet this criterion are repeated. Note that the pairs SM33 and SM35, SM34 and SM36, and SM37 and SM38 may be used to investigate for potential instrument drift in the measurement of samples prepared using the Mixed Acid method. 
WSRC-TR-2004-00576

Revision 0

Table 1-5 Reference Values (mg/L) for Mixed Acid Calibration and Bench Standards

\begin{tabular}{|c|c|c|c|c|c|c|c|c|c|c|c|c|c|c|c|c|}
\hline Seq & Al & B & $\mathbf{C a}$ & $\mathrm{Cr}$ & $\overline{\mathrm{Cu}}$ & $\mathbf{F e}$ & K & $\mathbf{L i}$ & Mg & Mn & $\mathrm{Na}$ & $\mathrm{Ni}$ & Si & $\mathbf{T i}$ & $\mathbf{U}$ & $\mathrm{Zr}$ \\
\hline SM32 & 0 & 0 & 0 & 0 & 0 & 0 & 0 & 0 & 0 & 0 & 0 & 0 & 0 & 0 & 0 & 0 \\
\hline SM33 & 0 & 0 & 1 & 1 & 1 & 0 & 5 & 2 & 1 & 0 & 32 & 1 & 20 & 0 & 0 & 0 \\
\hline SM34 & 2 & 0 & 0 & 0 & 0 & 10 & 0 & 0 & 0 & 2 & 5 & 0 & 0 & 1 & 0 & 1 \\
\hline SM35 & 0 & 0 & 1 & 1 & 1 & 0 & 5 & 2 & 1 & 0 & 32 & 1 & 20 & 0 & 0 & 0 \\
\hline SM36 & 2 & 0 & 0 & 0 & 0 & 10 & 0 & 0 & 0 & 2 & 5 & 0 & 0 & 1 & 0 & 1 \\
\hline SM37 & 0 & 0 & 0 & 0 & 0 & 0 & 0 & 0 & 0 & 0 & 0 & 0 & 0 & 0 & 10 & 0 \\
\hline SM38 & 0 & 0 & 0 & 0 & 0 & 0 & 0 & 0 & 0 & 0 & 0 & 0 & 0 & 0 & 10 & 0 \\
\hline
\end{tabular}

\subsubsection{Fusion (SME and MFT) Data}

The Fusion (F) method is used in the preparation of SME and MFT samples, and the typical protocol used to process a set of 6 samples from either of these tanks is provided in Table 1-6.

Table 1-6 Protocol for SME and MFT Samples Prepared Using the Fusion Method

\begin{tabular}{|c|c|}
\hline Analytical Sequence & Description \\
\hline SM53 & Calibration Std A \\
\hline SM53 & Calibration Std A \\
\hline SM54 & Calibration Std B \\
\hline SM54 & Calibration Std B \\
\hline SM55 & Blank \\
\hline ARGB & ARG-1 std \\
\hline SME or MFT-1 & SME or MFT sample \# 1 \\
\hline SME or MFT-2 & SME or MFT sample \# 2 \\
\hline SME or MFT--3 & SME or MFT sample \# 3 \\
\hline SME or MFT--4 & SME or MFT sample \# 4 \\
\hline SME or MFT--5 & SME or MFT sample \# 5 \\
\hline SME or MFT--6 & SME or MFT sample \# 6 \\
\hline ARGB & ARG-1 std \\
\hline SM55 & Blank \\
\hline SM51 & Bench A Std \\
\hline SM52 & Bench B Std \\
\hline
\end{tabular}

The reference values for each of the standards used to support the Fusion analyses are provided in Table 1-7. As the DWPF Lab processes a set of SME or MFT samples using this protocol, the measurements of the standards are assessed against an acceptance criterion: the measurements generated for the standards are expected to be no more that $+/-7 \%$ (the LIMS limits) from the reference values in this table.

Otherwise, the block of samples is considered questionable, and the measurements of each element failing to meet the criterion are repeated. Note that the pairs SM53 and SM51 and SM52 and SM54 may be used to investigate potential drift in the measurement of samples prepared using the Fusion method.

Table 1-7 Reference Values (mg/L) for Fusion Calibration and Bench Standards

\begin{tabular}{||c|c|c|c|c|c|c|c|c|c|c|c|c|c||}
\hline \hline Seq & $\mathrm{Al}$ & $\mathrm{B}$ & $\mathrm{Ca}$ & $\mathrm{Cr}$ & $\mathrm{Cu}$ & $\mathrm{Fe}$ & $\mathrm{K}$ & $\mathrm{Li}$ & $\mathrm{Mg}$ & $\mathrm{Mn}$ & $\mathrm{Ni}$ & $\mathrm{Si}$ & $\mathrm{Ti}$ \\
\hline SM51 & 0 & 4 & 1 & 1 & 1 & 0 & 5 & 2 & 1 & 0 & 1 & 20 & 0 \\
\hline SM52 & 2 & 2 & 0 & 0 & 0 & 10 & 0 & 0 & 0 & 2 & 0 & 0 & 1 \\
\hline SM53 & 0 & 4 & 1 & 1 & 1 & 0 & 5 & 2 & 1 & 0 & 1 & 20 & 0 \\
\hline SM54 & 2 & 2 & 0 & 0 & 0 & 10 & 0 & 0 & 0 & 2 & 0 & 0 & 1 \\
\hline SM55 & 0 & 0 & 0 & 0 & 0 & 0 & 0 & 0 & 0 & 0 & 0 & 0 & 0 \\
\hline
\end{tabular}




\subsection{RESULTS}

The information in Section 1 provides the framework for the data that are reviewed in this report. Most, but not all, of the measurement data were generated along with measurements that were reported for SRAT, SME, and MFT samples. A review of the measurements of these process samples is not in the scope of the analyses conducted in this report. However, the grouping of the standards data by process batch provides an opportunity to investigate and estimate the components or sources of variation in these data as well as to assess possible instrument drift over the block of measurements making up the batch. Drift in the measurements might be seen as a shift in the mean elemental value between a calibration standard and its corresponding bench standard, or it might be seen as less precision in a bench standard's measurements versus its corresponding calibration standard for an element. There also are opportunities to estimate bias in the elemental measurements of the standards. All of these aspects of the comparisons of the measurement data are discussed in this section. Each of the three major datasets, the Cold Chem, Mixed Acid, and Fusion measurements, is discussed in turn with the calibration and bench standard measurements being considered initially followed by an investigation of the ARG-1 data.

The statistical analyses were conducted using JMP Version 5.0 [5].

\subsection{COLD CHEM RESULTS}

Recall the analytical protocol (provided in Table 1-1) for SRAT samples analyzed by the Cold Chem prep method. In Section 2.1.1, the calibration and bench standards are investigated. All of the available data are initially explored with the measurements of the standards associated with the SRAT batches subsequently investigated. The elemental measurements for these standards are expressed as mg/L. Section 2.1.2 provides a discussion of the ARG-1 measurements generated under the Cold Chem prep. The ARG-1 measurements are expressed in elemental wt\%.

\subsubsection{Cold Chem Calibration and Bench Standards}

Exhibit A1 in the Appendix provides time plots in the form of control charts of the elemental measurements of each of the calibration and bench standards used as part of the Cold Chem protocol. These measurement are in $\mathrm{mg} / \mathrm{L}$, the displays are in analytical sequence by element for each of the standards. The plotted values represented by an " $x$ " are measurements that were not associated with a particular process batch. There are obvious outliers indicated in some of these plots as well as mean shifts, trends, and inconsistencies in precision.

Exhibit A2 provides histograms and descriptive statistics for all of these data. The means and reference values for the standards are given in Table 2-1 as are values of the \% relative difference (\% Rel Diff) for the active elements (i.e., those present in the standard). Note that all of the $\%$ relative differences (or \% biases) of the measured versus reference values are less than $1.5 \%$ except for the $U$ measurements for IN39, which show a $2.2 \%$ relative bias. 
WSRC-TR-2004-00576

Revision 0

Table 2-1 All Measurements (mg/L) for Cold Chem Calibration \& Bench Standards Means \& Relative Bias

\begin{tabular}{|c|c|c|c|c|c|c|c|c|}
\hline & & IN32 & IN33 & IN34 & IN35 & IN36 & IN38 & IN39 \\
\hline Element & N Rows & 484 & 432 & 420 & 932 & 894 & 302 & 142 \\
\hline \multirow[t]{3}{*}{$\mathrm{Al}(\mathrm{mg} / \mathrm{L})$} & Mean & 0.02 & 9.90 & 0.01 & 9.97 & 0.02 & 0.02 & 0.02 \\
\hline & Ref Value & 0 & 10 & 0 & 10 & 0 & 0 & 0 \\
\hline & \% Rel Diff & & $-1.0 \%$ & & $-0.3 \%$ & & & \\
\hline \multirow[t]{3}{*}{$\mathrm{B}(\mathrm{mg} / \mathrm{L})$} & Mean & 0.00 & -0.01 & 10.01 & 0.00 & 10.05 & 0.00 & 0.00 \\
\hline & Ref Value & 0 & 0 & 10 & 0 & 10 & 0 & 0 \\
\hline & \% Rel Diff & & & $0.1 \%$ & & $0.5 \%$ & & \\
\hline \multirow[t]{3}{*}{$\mathrm{Ca}(\mathrm{mg} / \mathrm{L})$} & Mean & 0.11 & 1.99 & 0.08 & 1.99 & 0.08 & 0.29 & 0.29 \\
\hline & Ref Value & 0 & 2 & 0 & 2 & 0 & 0 & 0 \\
\hline & \% Rel Diff & & $-0.5 \%$ & & $-0.3 \%$ & & & \\
\hline \multirow[t]{3}{*}{$\mathrm{Cr}(\mathrm{mg} / \mathrm{L})$} & Mean & 0.00 & 1.98 & 0.00 & 2.00 & 0.00 & 0.03 & 0.03 \\
\hline & Ref Value & 0 & 2 & 0 & 2 & 0 & 0 & 0 \\
\hline & \% Rel Diff & & $-0.8 \%$ & & $0.0 \%$ & & & \\
\hline \multirow[t]{3}{*}{$\mathrm{Cu}(\mathrm{mg} / \mathrm{L})$} & Mean & 0.00 & 0.99 & 0.00 & 1.00 & 0.00 & 0.02 & 0.02 \\
\hline & Ref Value & 0 & 1 & 0 & 1 & 0 & 0 & 0 \\
\hline & \% Rel Diff & & $-0.5 \%$ & & $0.5 \%$ & & & \\
\hline \multirow[t]{3}{*}{$\mathrm{Fe}(\mathrm{mg} / \mathrm{L})$} & Mean & 0.00 & 19.84 & 0.03 & 19.97 & 0.01 & 0.00 & 0.01 \\
\hline & Ref Value & 0 & 20 & 0 & 20 & 0 & 0 & 0 \\
\hline & \% Rel Diff & & $-0.8 \%$ & & $-0.1 \%$ & & & \\
\hline \multirow[t]{3}{*}{$\mathrm{K}(\mathrm{mg} / \mathrm{L})$} & Mean & 0.04 & 0.00 & 9.90 & 0.02 & 9.93 & -0.02 & -0.02 \\
\hline & Ref Value & 0 & 0 & 10 & 0 & 10 & 0 & 0 \\
\hline & \% Rel Diff & & & $-1.0 \%$ & & $-0.7 \%$ & & \\
\hline \multirow[t]{3}{*}{$\mathrm{Li}(\mathrm{mg} / \mathrm{L})$} & Mean & 0.00 & 0.00 & 9.94 & 0.00 & 9.97 & 0.00 & 0.00 \\
\hline & Ref Value & 0 & 0 & 10 & 0 & 10 & 0 & 0 \\
\hline & \% Rel Diff & & & $-0.6 \%$ & & $-0.3 \%$ & & \\
\hline \multirow[t]{3}{*}{$\mathrm{Mg}(\mathrm{mg} / \mathrm{L})$} & Mean & 0.00 & 1.00 & 0.01 & 1.01 & 0.01 & 0.00 & 0.00 \\
\hline & Ref Value & 0 & 1 & 0 & 1 & 0 & 0 & 0 \\
\hline & \% Rel Diff & & $0.3 \%$ & & $0.9 \%$ & & & \\
\hline \multirow[t]{3}{*}{ Mn (mg/L) } & Mean & 0.00 & 0.99 & 0.01 & 1.00 & 0.01 & 0.00 & 0.00 \\
\hline & Ref Value & 0 & 1 & 0 & 1 & 0 & 0 & 0 \\
\hline & \% Rel Diff & & $-0.7 \%$ & & $0.0 \%$ & & & \\
\hline \multirow[t]{3}{*}{$\mathrm{Na}(\mathrm{mg} / \mathrm{L})$} & Mean & 0.03 & 19.83 & 65.70 & 19.94 & 65.82 & 0.40 & 0.40 \\
\hline & Ref Value & 0 & 20 & 65 & 20 & 65 & 0 & 0 \\
\hline & \% Rel Diff & & $-0.8 \%$ & $1.1 \%$ & $-0.3 \%$ & $1.3 \%$ & & \\
\hline \multirow[t]{3}{*}{$\mathrm{Ni}(\mathrm{mg} / \mathrm{L})$} & Mean & 0.00 & 1.97 & 0.00 & 1.99 & 0.00 & 0.00 & 0.00 \\
\hline & Ref Value & 0 & 2 & 0 & 2 & 0 & 0 & 0 \\
\hline & \% Rel Diff & & $-1.5 \%$ & & $-0.7 \%$ & & & \\
\hline \multirow[t]{3}{*}{ Si (mg/L) } & Mean & -0.09 & -0.37 & 39.95 & -0.70 & 40.23 & -0.04 & 0.19 \\
\hline & Ref Value & 0 & 0 & 40 & 0 & 40 & 0 & 0 \\
\hline & \% Rel Diff & & & $-0.1 \%$ & & $0.6 \%$ & & \\
\hline \multirow[t]{3}{*}{$\mathrm{Ti}(\mathrm{mg} / \mathrm{L})$} & Mean & 0.00 & 1.98 & 0.00 & 1.99 & 0.00 & 0.00 & 0.00 \\
\hline & Ref Value & 0 & 2 & 0 & 2 & 0 & 0 & 0 \\
\hline & \% Rel Diff & & $-1.1 \%$ & & $-0.4 \%$ & & & \\
\hline \multirow[t]{3}{*}{$\mathrm{U}(\mathrm{mg} / \mathrm{L})$} & Mean & 0.13 & 0.08 & 0.05 & 0.14 & 0.06 & 9.84 & 9.78 \\
\hline & Ref Value & 0 & 0 & 0 & 0 & 0 & 10 & 10 \\
\hline & \% Rel Diff & & & & & & $-1.6 \%$ & $-2.2 \%$ \\
\hline \multirow[t]{3}{*}{$\mathrm{Zr}(\mathrm{mg} / \mathrm{L})$} & Mean & 0.00 & 2.00 & 0.01 & 2.02 & 0.01 & 0.00 & 0.00 \\
\hline & Ref Value & 0 & 2 & 0 & 2 & 0 & 0 & 0 \\
\hline & \%RSD & & $0.1 \%$ & & $0.8 \%$ & & & \\
\hline
\end{tabular}

The means and standard deviations for these data appear in Table 2-2. Also, in this table are values for the percent relative standard deviation (\%RSD), which is the ratio of the standard deviation to the mean expressed as a percentage, for elements present in the standard. RSD is also called the coefficient of 
WSRC-TR-2004-00576

Revision 0

variation (CV). Even with no screening of the data for outliers, note that the \%RSDs are all less than or equal to $2.4 \%$.

Table 2-2 All Measurements (mg/L) for Cold Chem Calibration and Bench Standards

\begin{tabular}{|c|c|c|c|c|c|c|c|c|}
\hline & & IN32 & IN33 & IN34 & IN35 & IN36 & IN38 & IN39 \\
\hline Element & N Rows & 484 & 432 & 420 & 932 & 894 & 302 & 142 \\
\hline \multirow[t]{3}{*}{$\mathrm{Al}(\mathrm{mg} / \mathrm{L})$} & Mean & 0.02 & 9.90 & 0.01 & 9.97 & 0.02 & 0.02 & 0.02 \\
\hline & Std Dev & 0.042 & 0.148 & 0.068 & 0.130 & 0.199 & 0.029 & 0.031 \\
\hline & \%RSD & & $1.5 \%$ & & $1.3 \%$ & & & \\
\hline \multirow[t]{3}{*}{$\mathrm{B}(\mathrm{mg} / \mathrm{L})$} & Mean & 0.00 & -0.01 & 10.01 & 0.00 & 10.05 & 0.00 & 0.00 \\
\hline & Std Dev & 0.031 & 0.032 & 0.142 & 0.032 & 0.120 & 0.031 & 0.031 \\
\hline & \%RSD & & & $1.4 \%$ & & $1.2 \%$ & & \\
\hline \multirow[t]{3}{*}{$\mathrm{Ca}(\mathrm{mg} / \mathrm{L})$} & Mean & 0.11 & 1.99 & 0.08 & 1.99 & 0.08 & 0.29 & 0.29 \\
\hline & Std Dev & 0.108 & 0.047 & 0.082 & 0.043 & 0.097 & 0.354 & 0.385 \\
\hline & \%RSD & & $2.4 \%$ & & $2.2 \%$ & & & \\
\hline \multirow[t]{3}{*}{$\mathrm{Cr}(\mathrm{mg} / \mathrm{L})$} & Mean & 0.00 & 1.98 & 0.00 & 2.00 & 0.00 & 0.03 & 0.03 \\
\hline & Std Dev & 0.007 & 0.032 & 0.003 & 0.029 & 0.003 & 0.006 & 0.006 \\
\hline & \%RSD & & $1.6 \%$ & & $1.4 \%$ & & & \\
\hline \multirow[t]{3}{*}{$\mathrm{Cu}(\mathrm{mg} / \mathrm{L})$} & Mean & 0.00 & 0.99 & 0.00 & 1.00 & 0.00 & 0.02 & 0.02 \\
\hline & Std Dev & 0.004 & 0.017 & 0.005 & 0.014 & 0.004 & 0.010 & 0.010 \\
\hline & \%RSD & & $1.7 \%$ & & $1.4 \%$ & & & \\
\hline \multirow[t]{3}{*}{$\mathrm{Fe}(\mathrm{mg} / \mathrm{L})$} & Mean & 0.00 & 19.84 & 0.03 & 19.97 & 0.01 & 0.00 & 0.01 \\
\hline & Std Dev & 0.073 & 0.303 & 0.298 & 0.260 & 0.058 & 0.035 & 0.031 \\
\hline & \%RSD & & $1.5 \%$ & & $1.3 \%$ & & & \\
\hline \multirow[t]{3}{*}{ K (mg/L) } & Mean & 0.04 & 0.00 & 9.90 & 0.02 & 9.93 & -0.02 & -0.02 \\
\hline & Std Dev & 0.062 & 0.064 & 0.137 & 0.066 & 0.125 & 0.072 & 0.078 \\
\hline & \%RSD & & & $1.4 \%$ & & $1.3 \%$ & & \\
\hline \multirow[t]{3}{*}{$\mathrm{Li}(\mathrm{mg} / \mathrm{L})$} & Mean & 0.00 & 0.00 & 9.94 & 0.00 & 9.97 & 0.00 & 0.00 \\
\hline & Std Dev & 0.002 & 0.003 & 0.125 & 0.003 & 0.113 & 0.005 & 0.006 \\
\hline & \%RSD & & & $1.3 \%$ & & $1.1 \%$ & & \\
\hline \multirow[t]{3}{*}{$\mathrm{Mg}(\mathrm{mg} / \mathrm{L})$} & Mean & 0.00 & 1.00 & 0.01 & 1.01 & 0.01 & 0.00 & 0.00 \\
\hline & Std Dev & 0.005 & 0.024 & 0.021 & 0.023 & 0.020 & 0.003 & 0.003 \\
\hline & $\%$ RSD & & $2.4 \%$ & & $2.3 \%$ & & & \\
\hline \multirow[t]{3}{*}{$\mathrm{Mn}(\mathrm{mg} / \mathrm{L})$} & Mean & 0.00 & 0.99 & 0.01 & 1.00 & 0.01 & 0.00 & 0.00 \\
\hline & Std Dev & 0.010 & 0.017 & 0.028 & 0.016 & 0.027 & 0.002 & 0.002 \\
\hline & \%RSD & & $1.7 \%$ & & $1.6 \%$ & & & \\
\hline \multirow[t]{3}{*}{$\mathrm{Na}(\mathrm{mg} / \mathrm{L})$} & Mean & 0.03 & 19.83 & 65.70 & 19.94 & 65.82 & 0.40 & 0.40 \\
\hline & Std Dev & 0.103 & 0.364 & 1.560 & 0.346 & 2.484 & 0.953 & 0.927 \\
\hline & \%RSD & & $1.8 \%$ & $2.4 \%$ & $1.7 \%$ & $3.8 \%$ & & \\
\hline \multirow[t]{3}{*}{$\mathrm{Ni}(\mathrm{mg} / \mathrm{L})$} & Mean & 0.00 & 1.97 & 0.00 & 1.99 & 0.00 & 0.00 & 0.00 \\
\hline & Std Dev & 0.009 & 0.036 & 0.008 & 0.034 & 0.008 & 0.010 & 0.010 \\
\hline & \%RSD & & $1.8 \%$ & & $1.7 \%$ & & & \\
\hline \multirow[t]{3}{*}{ Si (mg/L) } & Mean & -0.09 & -0.37 & 39.95 & -0.70 & 40.23 & -0.04 & 0.19 \\
\hline & Std Dev & 0.505 & 0.742 & 0.846 & 1.009 & 0.792 & 1.155 & 1.271 \\
\hline & \%RSD & & & $2.1 \%$ & & $2.0 \%$ & & \\
\hline \multirow[t]{3}{*}{$\mathrm{Ti}(\mathrm{mg} / \mathrm{L})$} & Mean & 0.00 & 1.98 & 0.00 & 1.99 & 0.00 & 0.00 & 0.00 \\
\hline & Std Dev & 0.002 & 0.031 & 0.004 & 0.027 & 0.004 & 0.002 & 0.002 \\
\hline & \%RSD & & $1.6 \%$ & & $1.3 \%$ & & & \\
\hline \multirow[t]{3}{*}{$\mathrm{U}(\mathrm{mg} / \mathrm{L})$} & Mean & 0.13 & 0.08 & 0.05 & 0.14 & 0.06 & 9.84 & 9.78 \\
\hline & Std Dev & 0.177 & 0.186 & 0.178 & 0.719 & 0.166 & 0.170 & 0.195 \\
\hline & \%RSD & & & & & & $1.7 \%$ & $2.0 \%$ \\
\hline \multirow[t]{3}{*}{$\mathrm{Zr}(\mathrm{mg} / \mathrm{L})$} & Mean & 0.00 & 2.00 & 0.01 & 2.02 & 0.01 & 0.00 & 0.00 \\
\hline & Std Dev & 0.004 & 0.040 & 0.023 & 0.037 & 0.023 & 0.003 & 0.003 \\
\hline & \%RSD & & $2.0 \%$ & & $1.8 \%$ & & & \\
\hline
\end{tabular}


WSRC-TR-2004-00576

Revision 0

Table 2-3 provides the same type of summary information, but this time for only those measurements of the standards that were reported as part of the information for a process batch. For these data, the largest \%RSD is that for $\mathrm{Na}(2.6 \%)$ for the IN34 standard.

Table 2-3 Measurements (mg/L) for Cold Chem Calibration and Bench Standards Reported in a Batch

\begin{tabular}{|c|c|c|c|c|c|c|c|c|}
\hline & & IN32 & IN33 & IN34 & IN35 & IN36 & IN38 & IN39 \\
\hline Element & N Rows & 344 & 180 & 177 & 370 & 366 & 120 & 60 \\
\hline \multirow[t]{3}{*}{$\mathrm{Al}(\mathrm{mg} / \mathrm{L})$} & Mean & 0.02 & 9.89 & 0.00 & 9.98 & 0.00 & 0.02 & 0.02 \\
\hline & Std Dev & 0.031 & 0.150 & 0.030 & 0.141 & 0.023 & 0.025 & 0.025 \\
\hline & \%RSD & & $1.5 \%$ & & $1.4 \%$ & & & \\
\hline \multirow[t]{3}{*}{$\mathrm{B}(\mathrm{mg} / \mathrm{L})$} & Mean & 0.00 & -0.01 & 10.01 & 0.00 & 10.05 & 0.00 & 0.00 \\
\hline & Std Dev & 0.035 & 0.038 & 0.136 & 0.038 & 0.110 & 0.037 & 0.036 \\
\hline & \%RSD & & & $1.4 \%$ & & $1.1 \%$ & & \\
\hline \multirow[t]{3}{*}{ Ca (mg/L) } & Mean & 0.12 & 1.99 & 0.09 & 2.00 & 0.09 & 0.24 & 0.26 \\
\hline & Std Dev & 0.111 & 0.043 & 0.069 & 0.046 & 0.092 & 0.215 & 0.238 \\
\hline & \%RSD & & $2.2 \%$ & & $2.3 \%$ & & & \\
\hline \multirow[t]{3}{*}{$\mathrm{Cr}(\mathrm{mg} / \mathrm{L})$} & Mean & 0.00 & 1.98 & 0.00 & 2.00 & 0.00 & 0.03 & 0.03 \\
\hline & Std Dev & 0.006 & 0.032 & 0.003 & 0.030 & 0.003 & 0.004 & 0.005 \\
\hline & \%RSD & & $1.6 \%$ & & $1.5 \%$ & & & \\
\hline \multirow[t]{3}{*}{$\mathrm{Cu}(\mathrm{mg} / \mathrm{L})$} & Mean & 0.00 & 0.99 & 0.00 & 1.00 & 0.00 & 0.02 & 0.02 \\
\hline & Std Dev & 0.004 & 0.018 & 0.005 & 0.016 & 0.004 & 0.012 & 0.013 \\
\hline & \%RSD & & $1.8 \%$ & & $1.6 \%$ & & & \\
\hline \multirow[t]{3}{*}{$\mathrm{Fe}(\mathrm{mg} / \mathrm{L})$} & Mean & 0.00 & 19.82 & 0.01 & 19.98 & 0.01 & 0.00 & 0.01 \\
\hline & Std Dev & 0.080 & 0.305 & 0.050 & 0.277 & 0.048 & 0.029 & 0.020 \\
\hline & \%RSD & & $1.5 \%$ & & $1.4 \%$ & & & \\
\hline \multirow[t]{3}{*}{$\mathrm{K}(\mathrm{mg} / \mathrm{L})$} & Mean & 0.04 & 0.00 & 9.90 & 0.02 & 9.94 & -0.02 & -0.03 \\
\hline & Std Dev & 0.066 & 0.060 & 0.144 & 0.075 & 0.120 & 0.064 & 0.067 \\
\hline & \%RSD & & & $1.5 \%$ & & $1.2 \%$ & & \\
\hline \multirow[t]{3}{*}{$\mathrm{Li}(\mathrm{mg} / \mathrm{L})$} & Mean & 0.00 & 0.00 & 9.94 & 0.00 & 9.97 & 0.00 & 0.00 \\
\hline & Std Dev & 0.002 & 0.002 & 0.126 & 0.004 & 0.109 & 0.005 & 0.005 \\
\hline & \%RSD & & & $1.3 \%$ & & $1.1 \%$ & & \\
\hline \multirow[t]{3}{*}{$\mathrm{Mg}(\mathrm{mg} / \mathrm{L})$} & Mean & 0.00 & 1.00 & 0.01 & 1.01 & 0.01 & 0.00 & 0.00 \\
\hline & Std Dev & 0.005 & 0.024 & 0.016 & 0.023 & 0.016 & 0.002 & 0.003 \\
\hline & \%RSD & & $2.4 \%$ & & $2.2 \%$ & & & \\
\hline \multirow[t]{3}{*}{$\mathrm{Mn}(\mathrm{mg} / \mathrm{L})$} & Mean & 0.00 & 0.99 & 0.01 & 1.00 & 0.01 & 0.00 & 0.00 \\
\hline & Std Dev & 0.011 & 0.017 & 0.021 & 0.016 & 0.021 & 0.001 & 0.001 \\
\hline & \%RSD & & $1.7 \%$ & & $1.6 \%$ & & & \\
\hline \multirow[t]{3}{*}{$\mathrm{Na}(\mathrm{mg} / \mathrm{L})$} & Mean & 0.02 & 19.86 & 65.93 & 19.98 & 66.13 & 0.49 & 0.50 \\
\hline & Std Dev & 0.087 & 0.366 & 1.731 & 0.365 & 1.619 & 1.045 & 1.048 \\
\hline & \%RSD & & $1.8 \%$ & $2.6 \%$ & $1.8 \%$ & $2.4 \%$ & & \\
\hline \multirow[t]{3}{*}{$\mathrm{Ni}(\mathrm{mg} / \mathrm{L})$} & Mean & 0.00 & 1.97 & 0.00 & 1.99 & 0.00 & 0.00 & 0.00 \\
\hline & Std Dev & 0.008 & 0.037 & 0.007 & 0.034 & 0.007 & 0.007 & 0.008 \\
\hline & \%RSD & & $1.9 \%$ & & $1.7 \%$ & & & \\
\hline \multirow[t]{3}{*}{ Si (mg/L) } & Mean & -0.08 & -0.27 & 40.01 & -0.60 & 40.32 & 0.02 & 0.28 \\
\hline & Std Dev & 0.526 & 0.514 & 0.747 & 0.480 & 0.724 & 0.913 & 0.920 \\
\hline & \%RSD & & & $1.9 \%$ & & $1.8 \%$ & & \\
\hline \multirow[t]{3}{*}{$\mathrm{Ti}$ (mg/L) } & Mean & 0.00 & 1.97 & 0.00 & 1.99 & 0.00 & 0.00 & 0.00 \\
\hline & Std Dev & 0.002 & 0.033 & 0.004 & 0.029 & 0.004 & 0.002 & 0.002 \\
\hline & \%RSD & & $1.7 \%$ & & $1.5 \%$ & & & \\
\hline \multirow[t]{3}{*}{$\mathrm{U}(\mathrm{mg} / \mathrm{L})$} & Mean & 0.11 & 0.06 & 0.03 & 0.12 & 0.06 & 9.86 & 9.81 \\
\hline & Std Dev & 0.186 & 0.179 & 0.179 & 0.277 & 0.150 & 0.165 & 0.206 \\
\hline & \%RSD & & & & & & $1.7 \%$ & $2.1 \%$ \\
\hline \multirow[t]{3}{*}{$\mathrm{Zr}(\mathrm{mg} / \mathrm{L})$} & Mean & 0.00 & 2.00 & 0.01 & 2.02 & 0.01 & 0.00 & 0.00 \\
\hline & Std Dev & 0.005 & 0.040 & 0.030 & 0.039 & 0.030 & 0.003 & 0.003 \\
\hline & \%RSD & & $2.0 \%$ & & $1.9 \%$ & & & \\
\hline
\end{tabular}


Exhibit A3 in the Appendix provides an investigation into sources of variation for the standards with replicate measurements for each batch of SRAT analyses. The results are summarized in Table 2-4, which shows that the batch-to-batch contribution to the variation is usually larger than the within batch contribution for all of the standards except IN32.

Table 2-4 Variance Components and Percent Contribution by Standard for Cold Chem

\begin{tabular}{|c|c|c|c|c|c|c|c|c|c|}
\hline & & \multicolumn{4}{|c|}{ Components of Variance } & \multicolumn{4}{|c|}{ \% of Variance } \\
\hline Element & & IN32 & IN35 & IN36 & IN38 & IN32 & IN35 & IN36 & IN38 \\
\hline \multirow[t]{3}{*}{$\mathrm{Al}(\mathrm{mg} / \mathrm{L})$} & Batch-to-batch & 0.000299 & 0.016965 & 0.000493 & 0.000633 & $31.2 \%$ & $85.2 \%$ & $95.2 \%$ & $98.6 \%$ \\
\hline & Residual & 0.000659 & 0.002947 & 0.000025 & 0.000009 & $68.8 \%$ & $14.8 \%$ & $4.8 \%$ & $1.4 \%$ \\
\hline & Total & 0.000958 & 0.019912 & 0.000518 & 0.000642 & & & & \\
\hline \multirow[t]{3}{*}{$\mathrm{B}(\mathrm{mg} / \mathrm{L})$} & Batch-to-batch & 0.001074 & 0.001393 & 0.009086 & 0.001342 & $89.6 \%$ & $94.1 \%$ & $75.1 \%$ & $98.2 \%$ \\
\hline & Residual & 0.000125 & 0.000088 & 0.003010 & 0.000025 & $10.4 \%$ & $5.9 \%$ & $24.9 \%$ & $1.8 \%$ \\
\hline & Total & 0.001199 & 0.001481 & 0.012096 & 0.001367 & & & & \\
\hline \multirow[t]{3}{*}{ Ca (mg/L) } & Batch-to-batch & 0.010481 & 0.001878 & 0.007691 & 0.046479 & $84.6 \%$ & $87.2 \%$ & $91.2 \%$ & $100.0 \%$ \\
\hline & Residual & 0.001902 & 0.000275 & 0.000741 & 0.000019 & $15.4 \%$ & $12.8 \%$ & $8.8 \%$ & $0.0 \%$ \\
\hline & Total & 0.012383 & 0.002153 & 0.008432 & 0.046498 & & & & \\
\hline \multirow[t]{3}{*}{$\mathrm{Cr}(\mathrm{mg} / \mathrm{L})$} & Batch-to-batch & 0.000010 & 0.000747 & 0.000006 & 0.000019 & $24.4 \%$ & $84.1 \%$ & $75.0 \%$ & $96.4 \%$ \\
\hline & Residual & 0.000031 & 0.000141 & 0.000002 & 0.000001 & $75.6 \%$ & $15.9 \%$ & $25.0 \%$ & $3.6 \%$ \\
\hline & Total & 0.000041 & 0.000888 & 0.000008 & 0.000020 & & & & \\
\hline \multirow[t]{3}{*}{$\mathrm{Cu}(\mathrm{mg} / \mathrm{L})$} & Batch-to-batch & 0.000013 & 0.000208 & 0.000012 & 0.000148 & $76.5 \%$ & $83.5 \%$ & $92.3 \%$ & $99.5 \%$ \\
\hline & Residual & \begin{tabular}{|l|}
0.000004 \\
\end{tabular} & 0.000041 & 0.000001 & 0.000001 & $23.5 \%$ & $16.5 \%$ & $7.7 \%$ & $0.5 \%$ \\
\hline & Total & 0.000017 & 0.000249 & 0.000013 & 0.000149 & & & & \\
\hline \multirow[t]{3}{*}{$\mathrm{Fe}(\mathrm{mg} / \mathrm{L})$} & Batch-to-b & 0.001963 & 0.063345 & 0.002277 & 0.000875 & $30.5 \%$ & $82.6 \%$ & $99.6 \%$ & $99.9 \%$ \\
\hline & Residual & 0.004475 & 0.013354 & 0.000009 & 0.000001 & $69.5 \%$ & $17.4 \%$ & $0.4 \%$ & $0.1 \%$ \\
\hline & Total & 0.006438 & 0.076699 & 0.002286 & 0.000876 & & & & \\
\hline \multirow[t]{3}{*}{$\mathrm{K}(\mathrm{mg} / \mathrm{L})$} & Batch-to-batch & 0.002230 & 0.002630 & 0.011662 & 0.003819 & $51.5 \%$ & $46.9 \%$ & $80.2 \%$ & $93.2 \%$ \\
\hline & Residual & 0.002100 & 0.002982 & 0.002877 & 0.000280 & $48.5 \%$ & $53.1 \%$ & $19.8 \%$ & $6.8 \%$ \\
\hline & Total & 0.004330 & 0.005612 & 0.014539 & 0.004099 & & & & \\
\hline \multirow[t]{3}{*}{$\mathrm{Li}(\mathrm{mg} / \mathrm{L})$} & Batch-to-batch & 0.000003 & 0.000006 & 0.009523 & 0.000019 & $60.0 \%$ & $42.9 \%$ & $80.3 \%$ & $95.0 \%$ \\
\hline & Residual & \begin{tabular}{|l|}
0.000002 \\
\end{tabular} & 0.000008 & 0.002334 & 0.000001 & $40.0 \%$ & $57.1 \%$ & $19.7 \%$ & $5.0 \%$ \\
\hline & Total & 0.000005 & 0.000014 & \begin{tabular}{|l|}
0.011857 \\
\end{tabular} & 0.000020 & & & & \\
\hline \multirow[t]{3}{*}{$\mathrm{Mg}(\mathrm{mg} / \mathrm{L})$} & Batch & 0.000006 & 0.000442 & 0.000257 & 0.000006 & $27.3 \%$ & $86.0 \%$ & $99.9 \%$ & $99.9 \%$ \\
\hline & Residual & 0.000016 & 0.000072 & 0.000000 & 0.000000 & $72.7 \%$ & $14.0 \%$ & $0.1 \%$ & $0.1 \%$ \\
\hline & Total & 0.000022 & 0.000514 & 0.000257 & 0.000006 & & & & \\
\hline \multirow[t]{3}{*}{ Mn (mg/L) } & Batch-to-batch & 0.000001 & 0.000230 & 0.000461 & 0.000001 & $0.7 \%$ & $85.5 \%$ & $99.9 \%$ & $97.6 \%$ \\
\hline & Residual & 0.000126 & 0.000039 & 0.000000 & 0.000000 & $99.3 \%$ & $14.5 \%$ & $0.1 \%$ & $2.4 \%$ \\
\hline & Total & \begin{tabular}{|l|}
0.000127 \\
\end{tabular} & 0.000269 & \begin{tabular}{|l|}
0.000461 \\
\end{tabular} & 0.000001 & & & & \\
\hline \multirow[t]{3}{*}{$\mathrm{Na}(\mathrm{mg} / \mathrm{L})$} & Batch-to-batch & 0.001589 & 0.120675 & 2.491725 & 1.101650 & $20.9 \%$ & $90.1 \%$ & $94.7 \%$ & $100.0 \%$ \\
\hline & Residual & 0.006006 & 0.013223 & 0.138330 & 0.000060 & $79.1 \%$ & $9.9 \%$ & $5.3 \%$ & $0.0 \%$ \\
\hline & Total & 0.007595 & 0.133898 & 2.630055 & 1.101710 & & & & \\
\hline \multirow[t]{3}{*}{$\mathrm{Ni}(\mathrm{mg} / \mathrm{L})$} & Batch-to-batch & \begin{tabular}{|l|}
0.000031 \\
\end{tabular} & 0.000870 & 0.000033 & \begin{tabular}{|l|}
0.000039 \\
\end{tabular} & $44.9 \%$ & $73.5 \%$ & $58.9 \%$ & $72.2 \%$ \\
\hline & Residual & 0.000038 & 0.000313 & 0.000023 & 0.000015 & $55.1 \%$ & $26.5 \%$ & $41.1 \%$ & $27.8 \%$ \\
\hline & Total & \begin{tabular}{|l|}
0.000069 \\
\end{tabular} & 0.001183 & 0.000056 & 0.000054 & & & & \\
\hline \multirow[t]{3}{*}{ Si (mg/L) } & Batch-to-batch & 0.246320 & 0.225288 & 0.482651 & 0.816861 & $88.9 \%$ & $97.4 \%$ & $91.8 \%$ & $97.3 \%$ \\
\hline & Residual & 0.030616 & 0.006131 & 0.043309 & 0.022892 & $11.1 \%$ & $2.6 \%$ & $8.2 \%$ & $2.7 \%$ \\
\hline & Total & 0.276936 & 0.231419 & 0.525960 & 0.839753 & & & & \\
\hline \multirow[t]{3}{*}{$\mathrm{Ti}(\mathrm{mg} / \mathrm{L})$} & Batch-to-batch & 0.000002 & 0.000710 & 0.000018 & 0.000003 & $69.7 \%$ & $84.4 \%$ & $97.8 \%$ & $94.0 \%$ \\
\hline & Residual & 0.000001 & 0.000131 & 0.000000 & 0.000000 & $30.3 \%$ & $15.6 \%$ & $2.2 \%$ & $6.0 \%$ \\
\hline & Total & \begin{tabular}{|l|}
0.000003 \\
\end{tabular} & 0.000841 & 0.000018 & 0.000003 & & & & \\
\hline \multirow[t]{3}{*}{$\mathrm{U}(\mathrm{mg} / \mathrm{L})$} & Batch-to-b & \begin{tabular}{|l|}
0.025843 \\
\end{tabular} & 0.025468 & 0.019956 & \begin{tabular}{|l|}
0.024381 \\
\end{tabular} & $74.6 \%$ & $33.1 \%$ & $88.3 \%$ & $88.5 \%$ \\
\hline & Residual & 0.008816 & 0.051482 & 0.002644 & 0.003165 & $25.4 \%$ & $66.9 \%$ & $11.7 \%$ & $11.5 \%$ \\
\hline & Total & \begin{tabular}{|l|}
0.034659 \\
\end{tabular} & 0.076950 & 0.022600 & 0.027546 & & & & \\
\hline \multirow[t]{3}{*}{$\mathrm{Zr}(\mathrm{mg} / \mathrm{L})$} & Batch-to-batch & 0.000020 & 0.001389 & \begin{tabular}{|l|}
0.000917 \\
\end{tabular} & 0.000006 & $95.2 \%$ & $90.8 \%$ & $99.9 \%$ & $96.9 \%$ \\
\hline & Residual & 0.000001 & 0.000140 & 0.000001 & 0.000000 & $4.8 \%$ & $9.2 \%$ & $0.1 \%$ & $3.1 \%$ \\
\hline & Total & 0.000021 & 0.001529 & 0.000918 & 0.000006 & & & & \\
\hline
\end{tabular}


As mentioned earlier, attempts were made during this data analysis to investigate for possible instrument drift. Comparisons between IN35 (Calibration Standard A) and IN33 (Bench Standard A) provide some insight into this possible problem for the Cold Chem data. Exhibit A4 in the Appendix presents a paired comparison between the measurements for these standards across the SRAT batches. The average of the two IN35 measurements was compared to the IN33 measurement for each batch. The mean difference between the measurements of this pair of standards was found to be statistically significant for all of the active elements (i.e., $\mathrm{Al}, \mathrm{Cr}, \mathrm{Cu}, \mathrm{Fe}, \mathrm{K}, \mathrm{Mg}, \mathrm{Mn}, \mathrm{Na}, \mathrm{Ni}$, Ti, and $\mathrm{Zr}$ ) except Ca. Table 2-5 provides summary information for these measurements. This table reveals that although statistically significant differences are seen, all of these differences are less than $1.25 \%$. So no dramatic drift, in the form of a consistent mean shift, is evident from these data. The standard deviations of these measurements across the SRAT batches are also provided in this table, and a review of these statistics does not suggest that the measurements of the bench standard are dramatically less precise than the measurements of the corresponding calibration standard.

Table 2-5 Insights into Instrument Drift by Comparisons of IN35 vs IN33 for Cold Chem

\begin{tabular}{|c|c|c|c|}
\hline & Bench A & Cal A & \\
\hline & IN33 & IN35 & $\%$ \\
\hline N Rows & 173 & 173 & Diff \\
\hline Mean(Al (mg/L)) & 9.8813 & 9.9783 & $-0.97 \%$ \\
\hline $\operatorname{Mean}(\mathrm{B}(\mathrm{mg} / \mathrm{L}))$ & -0.0119 & -0.0035 & \\
\hline Mean(Ca (mg/L)) & 1.9938 & 1.9985 & $-0.23 \%$ \\
\hline Mean(Cr (mg/L)) & 1.9820 & 2.0023 & $-1.01 \%$ \\
\hline $\operatorname{Mean}(\mathrm{Cu}(\mathrm{mg} / \mathrm{L}))$ & 0.9926 & 1.0049 & $-1.23 \%$ \\
\hline Mean(Fe (mg/L)) & 19.8142 & 19.9818 & $-0.84 \%$ \\
\hline $\operatorname{Mean}(\mathrm{K}(\mathrm{mg} / \mathrm{L}))$ & 0.0027 & 0.0195 & \\
\hline Mean(Li (mg/L)) & 0.0007 & 0.0015 & \\
\hline Mean(Mg (mg/L)) & 1.0019 & 1.0099 & $-0.79 \%$ \\
\hline Mean(Mn (mg/L)) & 0.9910 & 1.0013 & $-1.03 \%$ \\
\hline Mean(Na (mg/L)) & 19.8535 & 19.9922 & $-0.69 \%$ \\
\hline Mean(Ni (mg/L)) & 1.9680 & 1.9869 & $-0.95 \%$ \\
\hline $\operatorname{Mean}(\mathrm{Si}(\mathrm{mg} / \mathrm{L}))$ & -0.2732 & -0.6047 & \\
\hline Mean(Ti (mg/L)) & 1.9722 & 1.9894 & $-0.87 \%$ \\
\hline Mean(U (mg/L)) & 0.0646 & 0.1213 & \\
\hline Mean(Zr (mg/L)) & 1.9991 & 2.0171 & $-0.89 \%$ \\
\hline Std Dev(Al (mg/L)) & 0.1464 & 0.1335 & \\
\hline Std Dev(B (mg/L)) & 0.0381 & 0.0385 & \\
\hline Std Dev(Ca (mg/L)) & 0.0426 & 0.0444 & \\
\hline Std Dev(Cr (mg/L)) & 0.0315 & 0.0286 & \\
\hline Std Dev(Cu (mg/L)) & 0.0177 & 0.0150 & \\
\hline Std Dev(Fe (mg/L)) & 0.2973 & 0.2617 & \\
\hline Std Dev(K (mg/L)) & 0.0580 & 0.0627 & \\
\hline Std Dev(Li (mg/L)) & 0.0023 & 0.0032 & \\
\hline Std Dev(Mg (mg/L)) & 0.0241 & 0.0217 & \\
\hline Std Dev(Mn (mg/L)) & 0.0164 & 0.0158 & \\
\hline Std Dev(Na (mg/L)) & 0.3634 & 0.3534 & \\
\hline Std Dev(Ni (mg/L)) & 0.0352 & 0.0321 & \\
\hline Std Dev(Si (mg/L)) & 0.5221 & 0.4882 & \\
\hline Std Dev(Ti (mg/L)) & 0.0326 & 0.0279 & \\
\hline Std Dev(U (mg/L)) & 0.1707 & 0.2245 & \\
\hline Std Dev(Zr (mg/L)) & 0.0401 & 0.0379 & \\
\hline
\end{tabular}

Comparisons between IN34 (Bench Standard B) and IN36 (Calibration Standard B) also provide some insight into the possible problem of instrument drift for the Cold Chem data. Exhibit A5 in the Appendix presents a paired comparison between the measurements for these standards across the SRAT batches. 
The average of the two IN36 measurements was compared to the IN34 measurement for each batch. The mean difference between the measurements of this pair of standards was found to be statistically significant for all of the active elements (i.e., B, K, Li, Na, and $\mathrm{Si}$ ). Table 2-6 provides summary information for these measurements. This table reveals that although statistically significant differences are seen, all of these differences are less than $1.0 \%$. So no dramatic drift, in the form of a consistent mean shift, is evident from these data. The standard deviations of these measurements across the SRAT batches are also provided in this table, and a review of these statistics does not suggest that the measurements of the bench standard are dramatically less precise than the measurements of the corresponding calibration standard.

Table 2-6 Insights into Instrument Drift by Comparisons of IN36 vs IN34 for Cold Chem

\begin{tabular}{|c|c|c|c|}
\hline & Bench B & Cal B & \\
\hline & IN34 & IN36 & $\%$ \\
\hline N Rows & 172 & 172 & Diff \\
\hline $\operatorname{Mean}(\mathrm{Al}(\mathrm{mg} / \mathrm{L}))$ & 0.0006 & 0.0051 & \\
\hline Mean(B (mg/L)) & 10.0074 & 10.0553 & $-0.48 \%$ \\
\hline Mean(Ca (mg/L)) & 0.0877 & 0.0873 & \\
\hline Mean(Cr (mg/L)) & 0.0006 & 0.0009 & \\
\hline $\operatorname{Mean}(\mathrm{Cu}(\mathrm{mg} / \mathrm{L}))$ & -0.0002 & 0.0003 & \\
\hline Mean(Fe (mg/L)) & 0.0114 & 0.0086 & \\
\hline $\operatorname{Mean}(\mathrm{K}(\mathrm{mg} / \mathrm{L}))$ & 9.8971 & 9.9359 & $-0.39 \%$ \\
\hline Mean(Li (mg/L)) & 9.9393 & 9.9740 & $-0.35 \%$ \\
\hline Mean(Mg (mg/L)) & 0.0054 & 0.0050 & \\
\hline Mean(Mn (mg/L)) & 0.0066 & 0.0064 & \\
\hline Mean(Na (mg/L)) & 65.9269 & 66.1319 & $-0.31 \%$ \\
\hline Mean(Ni (mg/L)) & 0.0008 & 0.0006 & \\
\hline Mean(Si (mg/L)) & 40.0109 & 40.3450 & $-0.83 \%$ \\
\hline Mean(Ti (mg/L)) & 0.0025 & 0.0027 & \\
\hline Mean(U (mg/L)) & 0.0347 & 0.0659 & \\
\hline $\operatorname{Mean}(\mathrm{Zr}(\mathrm{mg} / \mathrm{L}))$ & 0.0096 & 0.0096 & \\
\hline Std Dev(Al (mg/L)) & 0.0299 & 0.0230 & \\
\hline Std Dev(B (mg/L)) & 0.1375 & 0.1034 & \\
\hline Std Dev(Ca (mg/L)) & 0.0686 & 0.0913 & \\
\hline Std Dev(Cr (mg/L)) & 0.0031 & 0.0027 & \\
\hline Std Dev(Cu (mg/L)) & 0.0046 & 0.0037 & \\
\hline Std Dev(Fe (mg/L)) & 0.0508 & 0.0485 & \\
\hline Std Dev(K (mg/L)) & 0.1439 & 0.1159 & \\
\hline Std Dev(Li (mg/L)) & 0.1260 & 0.1053 & \\
\hline Std Dev(Mg (mg/L)) & 0.0162 & 0.0160 & \\
\hline Std Dev(Mn (mg/L)) & 0.0214 & 0.0214 & \\
\hline Std Dev(Na (mg/L)) & 1.7407 & 1.6068 & \\
\hline Std Dev(Ni (mg/L)) & 0.0071 & 0.0066 & \\
\hline Std Dev(Si (mg/L)) & 0.7504 & 0.6954 & \\
\hline Std Dev(Ti (mg/L)) & 0.0044 & 0.0043 & \\
\hline Std Dev(U (mg/L)) & 0.1734 & 0.1477 & \\
\hline Std Dev(Zr (mg/L)) & 0.0302 & 0.0303 & \\
\hline
\end{tabular}

Finally, comparisons between IN38 (Bench Standard C) and IN39 (Calibration Standard C) provide some insight into the possible problem of instrument drift for the Cold Chem data. Exhibit A6 in the Appendix presents a paired comparison between the measurements for these standards across the SRAT batches. The average of the two IN39 measurements was compared to the IN38 measurement for each batch. U is the only active element for this pair of standards, and the mean difference between the two standards was found to be statistically significant at the $5 \%$ level. Table 2-7 provides summary information for these measurements. This table reveals that although statistically significant, the $\%$ difference for $U$ was only 
$0.53 \%$. So no dramatic drift, in the form of a consistent mean shift, is evident from these data. The standard deviations of these measurements across the SRAT batches are also provided in this table, and a review of these statistics shows that the standard deviation for $U$ for the bench standard is actually smaller than the U standard deviation for the corresponding calibration standard.

Table 2-7 Insights into Instrument Drift by Comparisons of IN39 vs IN38 for Cold Chem

\begin{tabular}{|c|c|c|c|}
\hline & IN38 (Bench C) & IN39 (Cal C) & $\%$ \\
\hline N Rows & 59 & 59 & Diff \\
\hline Mean(Al (mg/L)) & 0.0204 & 0.0207 & \\
\hline Mean(B (mg/L)) & 0.0013 & -0.0025 & \\
\hline Mean(Ca (mg/L)) & 0.2387 & 0.2551 & \\
\hline $\operatorname{Mean}(\mathrm{Cr}(\mathrm{mg} / \mathrm{L}))$ & 0.0280 & 0.0272 & \\
\hline $\operatorname{Mean}(\mathrm{Cu}(\mathrm{mg} / \mathrm{L}))$ & 0.0237 & 0.0234 & \\
\hline Mean(Fe (mg/L)) & 0.0044 & 0.0098 & \\
\hline Mean(K (mg/L)) & -0.0227 & -0.0317 & \\
\hline Mean(Li (mg/L)) & 0.0016 & 0.0019 & \\
\hline Mean $(\mathrm{Mg}(\mathrm{mg} / \mathrm{L}))$ & 0.0017 & 0.0022 & \\
\hline Mean(Mn (mg/L)) & 0.0021 & 0.0025 & \\
\hline Mean(Na (mg/L)) & 0.4935 & 0.5075 & \\
\hline Mean(Ni (mg/L)) & 0.0038 & 0.0033 & \\
\hline Mean(Si (mg/L)) & 0.0420 & 0.2809 & \\
\hline Mean(Ti (mg/L)) & 0.0003 & 0.0001 & \\
\hline Mean(U (mg/L)) & 9.8575 & 9.8052 & $0.53 \%$ \\
\hline $\operatorname{Mean}(\mathrm{Zr}(\mathrm{mg} / \mathrm{L}))$ & 0.0012 & 0.0010 & \\
\hline Std Dev(Al (mg/L)) & 0.0250 & 0.0252 & \\
\hline Std Dev(B (mg/L)) & 0.0371 & 0.0357 & \\
\hline Std Dev(Ca (mg/L)) & 0.2167 & 0.2398 & \\
\hline Std Dev(Cr (mg/L)) & 0.0044 & 0.0045 & \\
\hline Std Dev(Cu (mg/L)) & 0.0123 & 0.0132 & \\
\hline Std Dev(Fe (mg/L)) & 0.0181 & 0.0205 & \\
\hline Std Dev(K (mg/L)) & 0.0634 & 0.0678 & \\
\hline Std Dev(Li (mg/L)) & 0.0045 & 0.0049 & \\
\hline Std Dev(Mg (mg/L)) & 0.0024 & 0.0027 & \\
\hline Std Dev(Mn (mg/L)) & 0.0007 & 0.0015 & \\
\hline Std Dev(Na (mg/L)) & 1.0566 & 1.0552 & \\
\hline Std Dev(Ni (mg/L)) & 0.0069 & 0.0080 & \\
\hline Std Dev(Si (mg/L)) & 0.8987 & 0.9276 & \\
\hline Std Dev(Ti (mg/L)) & 0.0017 & 0.0019 & \\
\hline Std Dev(U (mg/L)) & 0.1625 & 0.2077 & \\
\hline Std Dev(Zr (mg/L)) & 0.0026 & 0.0027 & \\
\hline
\end{tabular}

Thus, the review of the Cold Chem bench and calibration standard measurements gives no indication of any instrument drift of practical concern over the course of the block of Cold Chem measurements needed to process a SRAT batch. The results presented in Table 2-1 through Table 2-7 also provide an opportunity to address one additional question regarding the calibration standards: Are the replicate measurements of IN35, IN36, and IN38 at the beginning of each Cold Chem block worthwhile as a feature of the quality assurance of this analytical process? In Table 2-4 note that the within batch (residual) variances for these calibrations standards are typically smaller than the between batch (batchto-batch) variances, while the standard deviations of Table 2-5 through Table 2-7 suggest that the batch to batch variances of the calibration standards are comparable to the batch-to-batch variances of the bench standards. Thus, these results indicate that there is no practical drift in the precision of these measurements over a Cold Chem block and that the replicate measurements of the calibration standards at the beginning of the Cold Chem block are not needed. 


\subsubsection{Cold Chem ARG-1 Results}

Exhibit A7 in the Appendix provides time plots in the form of control charts of the elemental measurements of the ARG-1 standard glass under the Cold Chem protocol. These measurements are in elemental weight percent (wt\%), and the displays are in analytical sequence. The plotted values represented by an " $x$ " are measurements that were not associated with a particular process batch. There are obvious outliers indicated in some of these plots as well as mean shifts, trends, and inconsistencies in precision.

Exhibit A8 in the Appendix provides histograms and descriptive statistics for all of the ARG-1 data while Exhibit A9 in the Appendix provides the same information for the ARG-1 results associated with a SRAT batch. The reference values and the means for both groups of ARG-1 measurements are given in Table 28. The $\%$ relative difference (\% Rel Diff) for each element for each group is also provided. Note that all of the \% relative differences (or \% biases) of the measured versus reference values for all of the major oxides (those in concentrations greater than $0.5 \mathrm{wt} \%$ ) are negative except for Ca. Also, the largest bias for these major oxides is that of Al. Also, the standard deviations and percent relative standard deviations (\% Rel Std Dev's) are presented in this table. All of these \%RSD's are less than 5\% (for the major elements) except for the $8.5 \%$ RSD for Ca.

Table 2-8 ARG-1 Results for Cold Chem Samples

\begin{tabular}{|c|c|c|c|c|c|c|c|c|c|}
\hline & & \multicolumn{4}{|c|}{$\begin{array}{l}\text { All of the Available Data } \\
(\mathrm{n}=480)\end{array}$} & \multicolumn{4}{|c|}{$\begin{array}{l}\text { Data Associated with SRAT Batches } \\
\qquad(\mathrm{n}=323)\end{array}$} \\
\hline & Ref & Mean & \% Rel & Std & \% Rel & Mean & \% Rel & Std & \% Rel \\
\hline Element & Value & $\mathrm{wt} \%$ & Diff & Dev & Std Dev & $\mathrm{wt} \%$ & Diff & Dev & Std Dev \\
\hline $\mathrm{Al}(\mathrm{wt} \%)$ & 2.5 & 2.347 & $-6.11 \%$ & 0.126 & $5.37 \%$ & 2.357 & $-5.72 \%$ & 0.083 & $3.54 \%$ \\
\hline $\mathrm{B}(\mathrm{wt} \%)$ & 2.69 & 2.629 & $-2.25 \%$ & 0.089 & $3.38 \%$ & 2.635 & $-2.03 \%$ & 0.094 & $3.58 \%$ \\
\hline Ca (wt\%) & 1.02 & 1.030 & $0.97 \%$ & 0.109 & $10.54 \%$ & 1.040 & $1.93 \%$ & 0.088 & $8.51 \%$ \\
\hline Cr (wt\%) & 0.06 & 0.068 & $13.28 \%$ & 0.003 & $4.96 \%$ & 0.068 & $12.84 \%$ & 0.003 & $4.70 \%$ \\
\hline $\mathrm{Cu}(\mathrm{wt} \%)$ & 0.0001 & 0.005 & & 0.003 & & 0.004 & & 0.003 & \\
\hline $\mathrm{Fe}(\mathrm{wt} \%)$ & 9.79 & 9.675 & $-1.18 \%$ & 0.286 & $2.96 \%$ & 9.667 & $-1.26 \%$ & 0.286 & $2.96 \%$ \\
\hline $\mathrm{K}(\mathrm{wt} \%)$ & 2.25 & 2.242 & $-0.35 \%$ & 0.074 & $3.30 \%$ & 2.238 & $-0.53 \%$ & 0.075 & $3.34 \%$ \\
\hline $\mathrm{Li}(\mathrm{wt} \%)$ & 1.49 & 1.478 & $-0.80 \%$ & 0.052 & $3.53 \%$ & 1.483 & $-0.47 \%$ & 0.047 & $3.20 \%$ \\
\hline $\mathrm{Mg}(\mathrm{wt} \%)$ & 0.52 & 0.492 & $-5.47 \%$ & 0.045 & $9.13 \%$ & 0.497 & $-4.46 \%$ & 0.024 & $4.86 \%$ \\
\hline $\mathrm{Mn}(\mathrm{wt} \%)$ & 1.46 & 1.430 & $-2.06 \%$ & 0.044 & $3.06 \%$ & 1.428 & $-2.17 \%$ & 0.042 & $2.94 \%$ \\
\hline $\mathrm{Na}(\mathrm{wt} \%)$ & 8.53 & 8.419 & $-1.30 \%$ & 0.292 & $3.47 \%$ & 8.438 & $-1.08 \%$ & 0.288 & $3.41 \%$ \\
\hline $\mathrm{Ni}(\mathrm{wt} \%)$ & 0.83 & 0.817 & $-1.61 \%$ & 0.025 & $3.09 \%$ & 0.815 & $-1.78 \%$ & 0.026 & $3.13 \%$ \\
\hline Si (wt\%) & 22.39 & 22.088 & $-1.35 \%$ & 0.793 & $3.59 \%$ & 22.162 & $-1.02 \%$ & 0.816 & $3.68 \%$ \\
\hline $\mathrm{Ti}(\mathrm{wt} \%)$ & 0.69 & 0.683 & $-1.07 \%$ & 0.020 & $2.95 \%$ & 0.681 & $-1.27 \%$ & 0.020 & $2.97 \%$ \\
\hline $\mathrm{U}(\mathrm{wt} \%)$ & 0 & 0.111 & & 0.122 & & 0.096 & & 0.132 & \\
\hline $\mathrm{Zr}(\mathrm{wt} \%)$ & 0.1 & 0.105 & $4.62 \%$ & 0.005 & $4.84 \%$ & 0.104 & $4.39 \%$ & 0.005 & $4.78 \%$ \\
\hline
\end{tabular}

Exhibit A10 in the Appendix provides an investigation into sources of variation for the replicated ARG-1 measurements within each block of SRAT analyses. The results are summarized in Table 2-9. For most of the major elements, the variation within a batch is comparable to the variation between the batches for these data. 
WSRC-TR-2004-00576

Revision 0

Table 2-9 Components of Variation for ARG-1 Results for Cold Chem Samples

\begin{tabular}{|c|c|c|c|c|c|c|c|c|c|}
\hline & Mean & \multicolumn{3}{|c|}{ Variance } & \multicolumn{2}{|c|}{ \% Variance } & \%RSD & \%RSD & \%RSD \\
\hline Element & $\mathrm{wt} \%$ & Batch & Residual & Total & \% Batch & \% Residual & Batch & Residual & Total \\
\hline $\mathrm{Al}$ (wt\%) & 2.356 & 0.001801 & 0.005295 & 0.007096 & $25.38 \%$ & $74.62 \%$ & $1.80 \%$ & $3.09 \%$ & $3.58 \%$ \\
\hline B (wt\%) & 2.634 & 0.003657 & .005411 & 0.009068 & $40.33 \%$ & $59.67 \%$ & $2.30 \%$ & $2.79 \%$ & $3.61 \%$ \\
\hline Ca (wt\%) & 1.039 & 04858 & 002965 & 0.007823 & 62.10\% & $37.90 \%$ & $6.71 \%$ & $5.24 \%$ & $8.52 \%$ \\
\hline Cr (wt\%) & 0.068 & .000004 & 0.000006 & 0.000010 & $40.00 \%$ & $60.00 \%$ & $2.96 \%$ & $3.62 \%$ & $4.67 \%$ \\
\hline $\mathrm{Cu}(\mathrm{wt} \%)$ & 0.004 & 0.000009 & 0.000001 & 0.000010 & $90.00 \%$ & $10.00 \%$ & $69.17 \%$ & $23.06 \%$ & 72.91\% \\
\hline Fe (wt\%) & 9.661 & 0.013305 & 0.067327 & 0.080632 & $16.50 \%$ & $83.50 \%$ & $1.19 \%$ & $2.69 \%$ & $2.94 \%$ \\
\hline K (wt\%) & 2.238 & 0.001781 & 0.003892 & 0.005673 & $31.39 \%$ & $68.61 \%$ & $1.89 \%$ & $2.79 \%$ & $3.37 \%$ \\
\hline Li (wt\%) & 1.483 & 0.000684 & 0.001619 & 0.002303 & $29.70 \%$ & $70.30 \%$ & $1.76 \%$ & $2.71 \%$ & $3.24 \%$ \\
\hline$\overline{M g}$ (wt\%) & 0.497 & 0.000250 & 0.000351 & 0.000601 & $41.60 \%$ & $58.40 \%$ & $3.18 \%$ & $3.77 \%$ & $4.94 \%$ \\
\hline Mn (wt\%) & 1.427 & \begin{tabular}{|l|}
0.000271 \\
\end{tabular} & \begin{tabular}{|l|l|}
0.001474 \\
\end{tabular} & 0.001745 & $15.53 \%$ & $84.47 \%$ & $1.15 \%$ & $2.69 \%$ & $2.93 \%$ \\
\hline $\mathrm{Na}(\mathrm{wt} \%)$ & 8.435 & 0.017106 & 0.066766 & 0.083872 & $20.40 \%$ & $79.60 \%$ & $1.55 \%$ & $3.06 \%$ & $3.43 \%$ \\
\hline $\mathrm{Ni}(\mathrm{wt} \%)$ & 0.814 & 0.001210 & 0.000514 & 0.001724 & $70.19 \%$ & $29.81 \%$ & $4.27 \%$ & $2.78 \%$ & $5.10 \%$ \\
\hline Si (wt\%) & 22.160 & 0.274867 & 0.404762 & 0.679629 & $40.44 \%$ & $59.56 \%$ & $2.37 \%$ & $2.87 \%$ & $3.72 \%$ \\
\hline Ti (wt\%) & 0.681 & 0.000073 & 0.000327 & 0.000400 & $18.25 \%$ & $81.75 \%$ & $1.26 \%$ & $2.66 \%$ & $2.94 \%$ \\
\hline $\mathrm{U}(\mathrm{wt} \%)$ & 0.098 & 0.015337 & 0.001946 & 0.017283 & $88.74 \%$ & $11.26 \%$ & $126.13 \%$ & $44.93 \%$ & $133.89 \%$ \\
\hline Zr (wt\%) & 0.104 & 0.000014 & 0.000010 & 0.000024 & $58.33 \%$ & $41.67 \%$ & $3.59 \%$ & $3.03 \%$ & $4.69 \%$ \\
\hline
\end{tabular}

\subsection{MIXED ACID RESULTS}

Recall the analytical protocol (provided in Table 1-4) for SME and MFT samples analyzed by the Mixed Acid prep method. In Section 2.2.1, the calibration and bench standards are investigated. All of the available data are initially explored with the measurements of the standards associated with the SME and MFT batches subsequently investigated. The elemental measurements for these standards are expressed as mg/L. Section 2.2.2 provides a discussion of the ARG-1 measurements generated under the Mixed Acid prep. The ARG-1 measurements are expressed in elemental wt\%.

\subsubsection{Mixed Acid Calibration and Bench Standards}

Exhibit A11 in the Appendix provides time plots in the form of control charts of the elemental measurements of each of the calibration and bench standards used as part of the Mixed Acid protocol for both SME and MFT samples. These measurement are in $\mathrm{mg} / \mathrm{L}$, the displays are in analytical sequence by element for each of the standards. The plotted values represented by an " $x$ " are measurements that were not associated with a particular process batch. There are obvious outliers indicated in some of these plots as well as mean shifts, trends, and inconsistency precision.

Exhibit A12 provides histograms and descriptive statistics for all of these data. The means and reference values for the standards are given in Table $2-10$. The \% relative differences (\% Rel Diff) of these measurements are also in this table; note that even without any screening of the data the largest $\%$ Rel Diff is only $3.4 \%$. 
WSRC-TR-2004-00576

Revision 0

Table 2-10 Means \& Relative Biases for All Measurements (mg/L) for Mixed Acid Calibration \& Bench Standards

\begin{tabular}{|c|c|c|c|c|c|c|c|c|}
\hline & & SM32 & SM33 & SM34 & SM35 & SM36 & SM37 & SM38 \\
\hline Element & N Rows & 687 & 508 & 509 & 1104 & 1098 & 702 & 1176 \\
\hline \multirow[t]{3}{*}{$\mathrm{Al}(\mathrm{mg} / \mathrm{L})$} & Mean & 0.01 & 0.01 & 1.97 & 0.01 & 1.99 & 0.03 & 0.05 \\
\hline & Ref Value & 0 & 0 & 2 & 0 & 2 & 0 & 0 \\
\hline & \% Rel Diff & & & $-1.3 \%$ & & $-0.7 \%$ & & \\
\hline \multirow[t]{3}{*}{$\mathrm{Ca}(\mathrm{mg} / \mathrm{L})$} & Mean & 0.01 & 1.00 & 0.02 & 1.01 & 0.01 & 0.26 & 0.26 \\
\hline & Ref Value & 0 & 1 & 0 & 1 & 0 & 0 & 0 \\
\hline & $\%$ Rel Diff & & $-0.2 \%$ & & $0.8 \%$ & & & \\
\hline \multirow[t]{3}{*}{$\mathrm{Cr}(\mathrm{mg} / \mathrm{L})$} & Mean & 0.00 & 0.99 & 0.00 & 1.00 & 0.00 & 0.03 & 0.03 \\
\hline & Ref Value & 0 & 1 & 0 & 1 & 0 & 0 & 0 \\
\hline & $\%$ Rel Diff & & $-0.6 \%$ & & $0.5 \%$ & & & \\
\hline \multirow[t]{3}{*}{$\mathrm{Cu}(\mathrm{mg} / \mathrm{L})$} & Mean & 0.00 & 1.00 & 0.00 & 1.00 & 0.00 & 0.02 & 0.02 \\
\hline & Ref Value & 0 & 1 & 0 & 1 & 0 & 0 & 0 \\
\hline & \% Rel Diff & & $-0.4 \%$ & & $0.4 \%$ & & & \\
\hline \multirow[t]{3}{*}{$\mathrm{Fe}(\mathrm{mg} / \mathrm{L})$} & Mean & 0.01 & -0.01 & 9.90 & -0.01 & 9.94 & 0.01 & 0.00 \\
\hline & Ref Value & 0 & 0 & 10 & 0 & 10 & 0 & 0 \\
\hline & \% Rel Diff & & & $-1.0 \%$ & & $-0.6 \%$ & & \\
\hline \multirow[t]{3}{*}{$\mathrm{K}(\mathrm{mg} / \mathrm{L})$} & Mean & 0.06 & 4.92 & 0.01 & 4.98 & 0.01 & -0.01 & 0.00 \\
\hline & Ref Value & 0 & 5 & 0 & 5 & 0 & 0 & 0 \\
\hline & \% Rel Diff & & $-1.6 \%$ & & $-0.4 \%$ & & & \\
\hline \multirow[t]{3}{*}{$\mathrm{Li}(\mathrm{mg} / \mathrm{L})$} & Mean & 0.00 & 1.98 & 0.01 & 1.99 & 0.01 & 0.00 & 0.00 \\
\hline & Ref Value & 0 & 2 & 0 & 2 & 0 & 0 & 0 \\
\hline & \% Rel Diff & & $-1.2 \%$ & & $-0.4 \%$ & & & \\
\hline \multirow[t]{3}{*}{$\mathrm{Mg}(\mathrm{mg} / \mathrm{L})$} & Mean & 0.00 & 1.01 & 0.00 & 1.02 & 0.00 & 0.00 & 0.00 \\
\hline & Ref Value & 0 & 1 & 0 & 1 & 0 & 0 & 0 \\
\hline & \% Rel Diff & & $0.7 \%$ & & $1.6 \%$ & & & \\
\hline \multirow[t]{3}{*}{$\mathrm{Mn}(\mathrm{mg} / \mathrm{L})$} & Mean & 0.00 & 0.00 & 1.97 & 0.00 & 1.98 & 0.00 & 0.00 \\
\hline & Ref Value & 0 & 0 & 2 & 0 & 2 & 0 & 0 \\
\hline & \% Rel Diff & & & $-1.5 \%$ & & $-0.9 \%$ & & \\
\hline \multirow[t]{3}{*}{$\mathrm{Na}(\mathrm{mg} / \mathrm{L})$} & Mean & 0.01 & 32.80 & 4.90 & 33.10 & 4.92 & 0.08 & 0.09 \\
\hline & Ref Value & 0 & 32 & 5 & 32 & 5 & 0 & 0 \\
\hline & $\%$ Rel Diff & & $2.5 \%$ & $-1.9 \%$ & $3.4 \%$ & $-1.6 \%$ & & \\
\hline \multirow[t]{3}{*}{$\mathrm{Ni}(\mathrm{mg} / \mathrm{L})$} & Mean & 0.01 & 1.00 & 0.01 & 1.01 & 0.01 & 0.00 & 0.00 \\
\hline & Ref Value & 0 & 1 & 0 & 1 & 0 & 0 & 0 \\
\hline & \% Rel Diff & & $-0.4 \%$ & & $0.6 \%$ & & & \\
\hline \multirow[t]{3}{*}{ Si (mg/L) } & Mean & 0.09 & 20.27 & 0.09 & 20.43 & 0.09 & 0.30 & 0.13 \\
\hline & Ref Value & 0 & 20 & 0 & 20 & 0 & 0 & 0 \\
\hline & \% Rel Diff & & $1.3 \%$ & & $2.1 \%$ & & & \\
\hline \multirow[t]{3}{*}{$\mathrm{Ti}(\mathrm{mg} / \mathrm{L})$} & Mean & 0.01 & 0.00 & 0.99 & 0.00 & 1.00 & 0.00 & 0.00 \\
\hline & Ref Value & 0 & 0 & 1 & 0 & 1 & 0 & 0 \\
\hline & \% Rel Diff & & & $-0.7 \%$ & & $-0.3 \%$ & & \\
\hline \multirow[t]{3}{*}{$\mathrm{U}(\mathrm{mg} / \mathrm{L})$} & Mean & 0.18 & 0.04 & 0.06 & 0.11 & 0.09 & 9.86 & 9.93 \\
\hline & Ref Value & 0 & 0 & 0 & 0 & 0 & 10 & 10 \\
\hline & \% Rel Diff & & & & & & $-1.4 \%$ & $-0.7 \%$ \\
\hline \multirow[t]{3}{*}{$\mathrm{Zr}(\mathrm{mg} / \mathrm{L})$} & Mean & -0.01 & -0.01 & 0.98 & -0.01 & 0.98 & 0.00 & 0.00 \\
\hline & Ref Value & 0 & 0 & 1 & 0 & 1 & 0 & 0 \\
\hline & $\%$ Rel Diff & & & $-2.2 \%$ & & $-1.8 \%$ & & \\
\hline
\end{tabular}

The \% relative standard deviations (\% RSDs) were computed from the means and standard deviations of Exhibit A12 and these data are provided in Table 2-11. Note that all of the \%RSDs are less than or equal to $3.1 \%$ even without any screening of the data. 
WSRC-TR-2004-00576

Revision 0

Table 2-11 Descriptive Statistics for All Mixed Acid Calibration and Bench Standard Measurements

\begin{tabular}{|c|c|c|c|c|c|c|c|c|}
\hline & & SM32 & SM33 & SM34 & SM35 & SM36 & SM37 & SM38 \\
\hline Element & N Rows & 687 & 508 & 509 & 1104 & 1098 & 702 & 1176 \\
\hline \multirow[t]{3}{*}{$\mathrm{Al}(\mathrm{mg} / \mathrm{L})$} & Mean & 0.01 & 0.01 & 1.97 & 0.01 & 1.99 & 0.03 & 0.05 \\
\hline & Std Dev & 0.050 & 0.232 & 0.032 & 0.225 & 0.031 & 0.042 & 0.107 \\
\hline & $\%$ RSD & & & $1.6 \%$ & & $1.6 \%$ & & \\
\hline \multirow[t]{3}{*}{$\mathrm{Ca}(\mathrm{mg} / \mathrm{L})$} & Mean & 0.01 & 1.00 & 0.02 & 1.01 & 0.01 & 0.26 & 0.26 \\
\hline & Std Dev & 0.053 & 0.026 & 0.065 & 0.025 & 0.045 & 0.339 & 0.320 \\
\hline & $\%$ RSD & & $2.6 \%$ & & $2.5 \%$ & & & \\
\hline \multirow[t]{3}{*}{$\mathrm{Cr}(\mathrm{mg} / \mathrm{L})$} & Mean & 0.00 & 0.99 & 0.00 & 1.00 & 0.00 & 0.03 & 0.03 \\
\hline & Std Dev & 0.007 & 0.019 & 0.003 & 0.019 & 0.003 & 0.014 & 0.010 \\
\hline & $\%$ RSD & & $1.9 \%$ & & $1.9 \%$ & & & \\
\hline \multirow[t]{3}{*}{$\mathrm{Cu}(\mathrm{mg} / \mathrm{L})$} & Mean & 0.00 & 1.00 & 0.00 & 1.00 & 0.00 & 0.02 & 0.02 \\
\hline & Std Dev & 0.004 & 0.015 & 0.007 & 0.015 & 0.011 & 0.027 & 0.027 \\
\hline & $\%$ RSD & & $1.5 \%$ & & $1.5 \%$ & & & \\
\hline \multirow[t]{3}{*}{$\mathrm{Fe}(\mathrm{mg} / \mathrm{L})$} & Mean & 0.01 & -0.01 & 9.90 & -0.01 & 9.94 & 0.01 & 0.00 \\
\hline & Std Dev & 0.078 & 0.023 & 0.159 & 0.024 & 0.147 & 0.341 & 0.037 \\
\hline & $\%$ RSD & & & $1.6 \%$ & & $1.5 \%$ & & \\
\hline \multirow[t]{3}{*}{$\mathrm{K}(\mathrm{mg} / \mathrm{L})$} & Mean & 0.06 & 4.92 & 0.01 & 4.98 & 0.01 & -0.01 & 0.00 \\
\hline & Std Dev & 0.083 & 0.088 & 0.062 & 0.091 & 0.061 & 0.057 & 0.058 \\
\hline & $\%$ RSD & & $1.8 \%$ & & $1.8 \%$ & & & \\
\hline \multirow[t]{3}{*}{$\mathrm{Li}(\mathrm{mg} / \mathrm{L})$} & Mean & 0.00 & 1.98 & 0.01 & 1.99 & 0.01 & 0.00 & 0.00 \\
\hline & Std Dev & 0.003 & 0.031 & 0.019 & 0.031 & 0.092 & 0.003 & 0.002 \\
\hline & $\%$ RSD & & $1.6 \%$ & & $1.6 \%$ & & & \\
\hline \multirow[t]{3}{*}{$\mathrm{Mg}(\mathrm{mg} / \mathrm{L})$} & Mean & 0.00 & 1.01 & 0.00 & 1.02 & 0.00 & 0.00 & 0.00 \\
\hline & Std Dev & 0.005 & 0.025 & 0.010 & 0.022 & 0.008 & 0.007 & 0.005 \\
\hline & $\%$ RSD & & $2.5 \%$ & & $2.2 \%$ & & & \\
\hline \multirow[t]{3}{*}{$\mathrm{Mn}(\mathrm{mg} / \mathrm{L})$} & Mean & 0.00 & 0.00 & 1.97 & 0.00 & 1.98 & 0.00 & 0.00 \\
\hline & Std Dev & 0.010 & 0.002 & 0.035 & 0.002 & 0.033 & 0.006 & 0.001 \\
\hline & $\%$ RSD & & & $1.8 \%$ & & $1.7 \%$ & & \\
\hline \multirow[t]{3}{*}{$\mathrm{Na}(\mathrm{mg} / \mathrm{L})$} & Mean & 0.01 & 32.80 & 4.90 & 33.10 & 4.92 & 0.08 & 0.09 \\
\hline & Std Dev & 0.184 & 1.023 & 0.107 & 0.994 & 0.103 & 0.450 & 0.493 \\
\hline & $\%$ RSD & & $3.1 \%$ & $2.2 \%$ & $3.0 \%$ & $2.1 \%$ & & \\
\hline \multirow[t]{3}{*}{$\mathrm{Ni}(\mathrm{mg} / \mathrm{L})$} & Mean & 0.01 & 1.00 & 0.01 & 1.01 & 0.01 & 0.00 & 0.00 \\
\hline & Std Dev & 0.012 & 0.018 & 0.008 & 0.018 & 0.008 & 0.008 & 0.008 \\
\hline & $\%$ RSD & & $1.9 \%$ & & $1.8 \%$ & & & \\
\hline \multirow[t]{3}{*}{$\mathrm{Si}(\mathrm{mg} / \mathrm{L})$} & Mean & 0.09 & 20.27 & 0.09 & 20.43 & 0.09 & 0.30 & 0.13 \\
\hline & Std Dev & 0.589 & 0.476 & 0.434 & 0.432 & 0.373 & 0.607 & 0.525 \\
\hline & $\%$ RSD & & $2.3 \%$ & & $2.1 \%$ & & & \\
\hline \multirow[t]{3}{*}{$\mathrm{Ti}(\mathrm{mg} / \mathrm{L})$} & Mean & 0.01 & 0.00 & 0.99 & 0.00 & 1.00 & 0.00 & 0.00 \\
\hline & Std Dev & 0.025 & 0.001 & 0.018 & 0.001 & 0.018 & 0.002 & 0.002 \\
\hline & $\%$ RSD & & & $1.8 \%$ & & $1.8 \%$ & & \\
\hline \multirow[t]{3}{*}{$\mathrm{U}(\mathrm{mg} / \mathrm{L})$} & Mean & 0.18 & 0.04 & 0.06 & 0.11 & 0.09 & 9.86 & 9.93 \\
\hline & Std Dev & 0.187 & 0.149 & 0.148 & 0.337 & 0.338 & 0.216 & 0.202 \\
\hline & $\%$ RSD & & & & & & $2.2 \%$ & $2.0 \%$ \\
\hline \multirow[t]{3}{*}{$\mathrm{Zr}(\mathrm{mg} / \mathrm{L})$} & Mean & -0.01 & -0.01 & 0.98 & -0.01 & 0.98 & 0.00 & 0.00 \\
\hline & Std Dev & 0.016 & 0.015 & 0.014 & 0.016 & 0.014 & 0.014 & 0.015 \\
\hline & $\%$ RSD & & & $1.5 \%$ & & $1.4 \%$ & & \\
\hline
\end{tabular}

Table 2-12 provides the same type of summary information, but this time for only those measurements of the calibration and bench standards that were reported as part of the information for a process (SME or MFT) batch. Note that the largest \%RSD is only 3.1\% for these data. 
WSRC-TR-2004-00576

Revision 0

Table 2-12 Descriptive Statistics for Mixed Acid Calibration and Bench Standards in a Process Batch

\begin{tabular}{|c|c|c|c|c|c|c|c|c|}
\hline & & "SM32 & "SM33 & "SM34 & "SM35 & "SM36 & "SM37 & SM38 \\
\hline Element & N Rows & 619 & 319 & 324 & 640 & 642 & 334 & 640 \\
\hline \multirow[t]{3}{*}{$\mathrm{Al}(\mathrm{mg} / \mathrm{L})$} & Mean & 0.011 & 0.015 & 1.972 & 0.021 & 1.986 & 0.044 & 0.054 \\
\hline & Std Dev & 0.050 & 0.292 & 0.030 & 0.295 & 0.028 & 0.019 & 0.136 \\
\hline & $\%$ RSD & & & $1.5 \%$ & & $1.4 \%$ & & \\
\hline \multirow[t]{3}{*}{ Ca (mg/L) } & Mean & 0.013 & 0.996 & 0.020 & 1.010 & 0.014 & 0.281 & 0.277 \\
\hline & Std Dev & 0.052 & 0.026 & 0.071 & 0.024 & 0.050 & 0.360 & 0.350 \\
\hline & $\%$ RSD & & $2.6 \%$ & & $2.4 \%$ & & & \\
\hline \multirow[t]{3}{*}{$\mathrm{Cr}(\mathrm{mg} / \mathrm{L})$} & Mean & 0.004 & 0.994 & 0.002 & 1.006 & 0.002 & 0.028 & 0.029 \\
\hline & Std Dev & 0.007 & 0.019 & 0.003 & 0.017 & 0.002 & 0.005 & 0.005 \\
\hline & $\%$ RSD & & $1.9 \%$ & & $1.7 \%$ & & & \\
\hline \multirow[t]{3}{*}{$\mathrm{Cu}(\mathrm{mg} / \mathrm{L})$} & Mean & 0.002 & 0.996 & 0.002 & 1.004 & 0.002 & 0.020 & 0.021 \\
\hline & Std Dev & 0.004 & 0.014 & 0.003 & 0.014 & 0.003 & 0.037 & 0.033 \\
\hline & $\%$ RSD & & $1.4 \%$ & & $1.4 \%$ & & & \\
\hline \multirow[t]{3}{*}{ Fe (mg/L) } & Mean & 0.004 & -0.007 & 9.897 & -0.009 & 9.940 & -0.005 & -0.006 \\
\hline & Std Dev & 0.056 & 0.022 & 0.143 & 0.024 & 0.126 & 0.024 & 0.025 \\
\hline & $\%$ RSD & & & $1.4 \%$ & & $1.3 \%$ & & \\
\hline \multirow[t]{3}{*}{$\mathrm{K}(\mathrm{mg} / \mathrm{L})$} & Mean & 0.059 & 4.912 & 0.008 & 4.986 & 0.015 & -0.006 & 0.003 \\
\hline & Std Dev & 0.078 & 0.083 & 0.052 & 0.084 & 0.053 & 0.051 & 0.048 \\
\hline & $\%$ RSD & & $1.7 \%$ & & $1.7 \%$ & & & \\
\hline \multirow[t]{3}{*}{$\mathrm{Li}(\mathrm{mg} / \mathrm{L})$} & Mean & 0.003 & 1.971 & 0.007 & 1.993 & 0.011 & 0.001 & 0.001 \\
\hline & Std Dev & 0.003 & 0.028 & 0.020 & 0.031 & 0.120 & 0.002 & 0.002 \\
\hline & $\%$ RSD & & $1.4 \%$ & & $1.6 \%$ & & & \\
\hline \multirow[t]{3}{*}{$\mathrm{Mg}(\mathrm{mg} / \mathrm{L})$} & Mean & 0.001 & 1.007 & 0.003 & 1.017 & 0.003 & 0.005 & 0.005 \\
\hline & Std Dev & 0.005 & 0.023 & 0.012 & 0.021 & 0.008 & 0.005 & 0.005 \\
\hline & $\%$ RSD & & $2.3 \%$ & & $2.1 \%$ & & & \\
\hline \multirow[t]{3}{*}{$\mathrm{Mn}(\mathrm{mg} / \mathrm{L})$} & Mean & 0.001 & 0.000 & 1.969 & 0.000 & 1.980 & 0.002 & 0.001 \\
\hline & Std Dev & 0.010 & 0.002 & 0.033 & 0.002 & 0.030 & 0.001 & 0.001 \\
\hline & $\%$ RSD & & & $1.7 \%$ & & $1.5 \%$ & & \\
\hline \multirow[t]{3}{*}{$\mathrm{Na}(\mathrm{mg} / \mathrm{L})$} & Mean & 0.013 & 32.702 & 4.906 & 33.050 & 4.921 & 0.115 & 0.117 \\
\hline & Std Dev & 0.191 & 0.999 & 0.103 & 0.916 & 0.097 & 0.544 & 0.556 \\
\hline & $\%$ RSD & & $3.1 \%$ & $2.1 \%$ & $2.8 \%$ & $2.0 \%$ & & \\
\hline \multirow[t]{3}{*}{$\mathrm{Ni}(\mathrm{mg} / \mathrm{L})$} & Mean & 0.005 & 0.995 & 0.009 & 1.007 & 0.009 & 0.002 & 0.002 \\
\hline & Std Dev & 0.012 & 0.017 & 0.008 & 0.018 & 0.008 & 0.008 & 0.008 \\
\hline & $\%$ RSD & & $1.7 \%$ & & $1.8 \%$ & & & \\
\hline \multirow[t]{3}{*}{ Si (mg/L) } & Mean & 0.088 & 20.257 & 0.121 & 20.458 & 0.105 & 0.427 & 0.172 \\
\hline & Std Dev & 0.567 & 0.481 & 0.469 & 0.411 & 0.377 & 0.646 & 0.577 \\
\hline & $\%$ RSD & & $2.4 \%$ & & $2.0 \%$ & & & \\
\hline \multirow[t]{3}{*}{$\mathrm{Ti}(\mathrm{mg} / \mathrm{L})$} & Mean & 0.014 & 0.000 & 0.993 & 0.001 & 0.997 & 0.001 & 0.001 \\
\hline & Std Dev & 0.026 & 0.001 & 0.018 & 0.001 & 0.018 & 0.002 & 0.001 \\
\hline & $\%$ RSD & & & $1.8 \%$ & & $1.8 \%$ & & \\
\hline \multirow[t]{3}{*}{$\mathrm{U}(\mathrm{mg} / \mathrm{L})$} & Mean & 0.172 & 0.031 & 0.056 & 0.100 & 0.080 & 9.846 & 9.913 \\
\hline & Std Dev & 0.187 & 0.145 & 0.144 & 0.105 & 0.107 & 0.180 & 0.179 \\
\hline & $\%$ RSD & & & & & & $1.8 \%$ & $1.8 \%$ \\
\hline \multirow[t]{3}{*}{$\mathrm{Zr}(\mathrm{mg} / \mathrm{L})$} & Mean & -0.007 & -0.007 & 0.977 & -0.006 & 0.982 & -0.005 & -0.005 \\
\hline & Std Dev & 0.016 & 0.015 & 0.013 & 0.015 & 0.012 & 0.016 & 0.015 \\
\hline & $\%$ RSD & & & $1.3 \%$ & & $1.2 \%$ & & \\
\hline
\end{tabular}

Exhibit A13 in the Appendix provides an investigation into sources of variation for the calibration and bench standards with replicate measurements within each block of SME or MFT analyses. The analysis combines the SME and MFT blocks into a single dataset with the intention of estimating between batch and within batch (residual) variation. A summary of the results is provided in Table 2-13, which shows, in general, that the larger contributor to the variation in these data was the batch-to-batch variation. 
WSRC-TR-2004-00576

Revision 0

Table 2-13 Components of Variance for Mixed Acid Calibration and Bench Standards in a Process Batch

\begin{tabular}{|c|c|c|c|c|c|c|c|c|c|}
\hline & & \multicolumn{4}{|c|}{ Components of Variance } & \multicolumn{4}{|c|}{ \% of Variance } \\
\hline Element & & SM32 & SM35 & SM36 & SM38 & SM32 & SM35 & SM36 & SM38 \\
\hline \multirow[t]{3}{*}{$\mathrm{Al}(\mathrm{mg} / \mathrm{L})$} & Batch-to-batch & 0.001169 & 0.087404 & 0.000745 & 0.018593 & $46.0 \%$ & $100.0 \%$ & $91.7 \%$ & $99.9 \%$ \\
\hline & Residual & 0.001373 & 0.000016 & 0.000067 & 0.000023 & $54.0 \%$ & $0.0 \%$ & $8.3 \%$ & $0.1 \%$ \\
\hline & Total & 0.002542 & 0.087420 & 0.000812 & 0.018616 & & & & \\
\hline \multirow[t]{3}{*}{$\mathrm{Ca}(\mathrm{mg} / \mathrm{L})$} & Batch-to-batch & 0.000775 & 0.000508 & 0.002482 & 0.117891 & $28.6 \%$ & $86.7 \%$ & $97.7 \%$ & $96.2 \%$ \\
\hline & Residual & 0.001934 & 0.000078 & 0.000058 & 0.004636 & $71.4 \%$ & $13.3 \%$ & $2.3 \%$ & $3.8 \%$ \\
\hline & Total & 0.002709 & 0.000586 & 0.002540 & 0.122527 & & & & \\
\hline \multirow[t]{3}{*}{$\mathrm{Cr}(\mathrm{mg} / \mathrm{L})$} & Batch-to-batch & 0.000012 & 0.000272 & 0.000004 & 0.000023 & $26.7 \%$ & $91.9 \%$ & $89.3 \%$ & $88.5 \%$ \\
\hline & Residual & 0.000033 & 0.000024 & 0.000000 & 0.000003 & $73.3 \%$ & $8.1 \%$ & $10.7 \%$ & $11.5 \%$ \\
\hline & Total & 0.000045 & 0.000296 & 0.000004 & 0.000026 & & & & \\
\hline \multirow[t]{3}{*}{$\mathrm{Cu}(\mathrm{mg} / \mathrm{L})$} & Batch-to-batch & 0.000012 & 0.000175 & 0.000007 & 0.001116 & $63.2 \%$ & $89.3 \%$ & $94.4 \%$ & $99.7 \%$ \\
\hline & Residual & 0.000007 & 0.000021 & 0.000000 & 0.000003 & $36.8 \%$ & $10.7 \%$ & $5.6 \%$ & $0.3 \%$ \\
\hline & Total & 0.000019 & 0.000196 & 0.000007 & 0.001119 & & & & \\
\hline \multirow[t]{3}{*}{$\mathrm{Fe}(\mathrm{mg} / \mathrm{L})$} & Batch-to-batch & 0.000896 & 0.000550 & 0.014124 & 0.000612 & $28.2 \%$ & $98.9 \%$ & $89.4 \%$ & $96.8 \%$ \\
\hline & Residual & 0.002278 & 0.000006 & 0.001668 & 0.000020 & $71.8 \%$ & $1.1 \%$ & $10.6 \%$ & $3.2 \%$ \\
\hline & Total & 0.003174 & 0.000556 & 0.015792 & 0.000632 & & & & \\
\hline \multirow[t]{3}{*}{$\mathrm{K}(\mathrm{mg} / \mathrm{L})$} & Batch-to-batch & 0.003043 & 0.005879 & 0.001766 & 0.001624 & $49.6 \%$ & $82.6 \%$ & $62.8 \%$ & $69.6 \%$ \\
\hline & Residual & 0.003091 & 0.001235 & 0.001048 & 0.000708 & $50.4 \%$ & $17.4 \%$ & $37.2 \%$ & $30.4 \%$ \\
\hline & Total & 0.006134 & 0.007114 & 0.002814 & 0.002332 & & & & \\
\hline \multirow[t]{3}{*}{$\mathrm{Li}(\mathrm{mg} / \mathrm{L})$} & Batch-to-batch & 0.000005 & 0.000913 & 0.000704 & 0.000003 & $62.5 \%$ & $91.9 \%$ & $4.9 \%$ & $85.7 \%$ \\
\hline & Residual & 0.000003 & 0.000080 & 0.013696 & 0.000001 & $37.5 \%$ & $8.1 \%$ & $95.1 \%$ & $14.3 \%$ \\
\hline & Total & 0.000008 & 0.000993 & 0.014400 & 0.000004 & & & & \\
\hline \multirow[t]{3}{*}{$\mathrm{Mg}(\mathrm{mg} / \mathrm{L})$} & Batch-to-batch & 0.000011 & 0.000395 & 0.000071 & 0.000027 & $42.3 \%$ & $86.4 \%$ & $99.5 \%$ & $97.4 \%$ \\
\hline & Residual & 0.000015 & 0.000062 & 0.000000 & 0.000001 & $57.7 \%$ & $13.6 \%$ & $0.5 \%$ & $2.6 \%$ \\
\hline & Total & 0.000026 & 0.000457 & 0.000071 & 0.000028 & & & & \\
\hline \multirow[t]{3}{*}{ Mn (mg/L) } & Batch-to-batch & 0.000002 & 0.000004 & 0.000831 & 0.000001 & $2.0 \%$ & $96.5 \%$ & $93.5 \%$ & $83.1 \%$ \\
\hline & Residual & 0.000099 & 0.000000 & 0.000058 & 0.000000 & $98.0 \%$ & $3.5 \%$ & $6.5 \%$ & $16.9 \%$ \\
\hline & Total & 0.000101 & 0.000004 & 0.000889 & 0.000001 & & & & \\
\hline \multirow[t]{3}{*}{$\mathrm{Na}(\mathrm{mg} / \mathrm{L})$} & Batch-to-batch & 0.008084 & \begin{tabular}{|l|}
0.819909 \\
\end{tabular} & 0.008932 & \begin{tabular}{|l|}
0.309782 \\
\end{tabular} & $22.0 \%$ & $97.7 \%$ & $95.3 \%$ & $100.0 \%$ \\
\hline & Residual & 0.028598 & 0.019729 & 0.000439 & 0.000041 & $78.0 \%$ & $2.3 \%$ & $4.7 \%$ & $0.0 \%$ \\
\hline & Total & 0.036682 & 0.839638 & 0.009371 & 0.309823 & & & & \\
\hline \multirow[t]{3}{*}{$\mathrm{Ni}(\mathrm{mg} / \mathrm{L})$} & Batch-to-batch & 0.000055 & 0.000213 & 0.000033 & 0.000035 & $39.9 \%$ & $68.5 \%$ & $56.9 \%$ & $61.4 \%$ \\
\hline & Residual & 0.000083 & 0.000098 & 0.000025 & 0.000022 & $60.1 \%$ & $31.5 \%$ & $43.1 \%$ & $38.6 \%$ \\
\hline & Total & 0.000138 & 0.000311 & 0.000058 & 0.000057 & & & & \\
\hline \multirow[t]{3}{*}{$\mathrm{Si}(\mathrm{mg} / \mathrm{L})$} & Batch-to-batch & 0.258251 & 0.142166 & 0.126453 & 0.268065 & $80.2 \%$ & $84.1 \%$ & $88.8 \%$ & $80.3 \%$ \\
\hline & Residual & 0.063628 & \begin{tabular}{|l}
0.026969 \\
\end{tabular} & 0.015925 & 0.065902 & $19.8 \%$ & $15.9 \%$ & $11.2 \%$ & $19.7 \%$ \\
\hline & Total & 0.321879 & 0.169135 & 0.142378 & 0.333967 & & & & \\
\hline \multirow[t]{3}{*}{$\mathrm{Ti}(\mathrm{mg} / \mathrm{L})$} & Batch-to-batch & 0.000432 & \begin{tabular}{|l|}
0.000001 \\
\end{tabular} & 0.000299 & 0.000002 & $66.3 \%$ & $82.7 \%$ & $95.5 \%$ & $89.2 \%$ \\
\hline & Residual & 0.000220 & 0.000000 & 0.000014 & 0.000000 & $33.7 \%$ & $17.3 \%$ & $4.5 \%$ & $10.8 \%$ \\
\hline & Total & 0.000652 & 0.000001 & 0.000313 & 0.000002 & & & & \\
\hline \multirow[t]{3}{*}{$\mathrm{U}(\mathrm{mg} / \mathrm{L})$} & Batch-to-batch & 0.021752 & 0.009920 & 0.010613 & 0.027521 & $61.9 \%$ & $89.4 \%$ & $93.0 \%$ & $85.3 \%$ \\
\hline & Residual & 0.013400 & 0.001179 & 0.000796 & 0.004741 & $38.1 \%$ & $10.6 \%$ & $7.0 \%$ & $14.7 \%$ \\
\hline & Total & 0.035152 & 0.011099 & 0.011409 & 0.032262 & & & & \\
\hline \multirow[t]{3}{*}{$\mathrm{Zr}(\mathrm{mg} / \mathrm{L})$} & Batch-to-batch & 0.000243 & 0.000228 & 0.000123 & 0.000235 & $99.2 \%$ & $99.7 \%$ & $89.8 \%$ & $99.6 \%$ \\
\hline & Residual & 0.000002 & 0.000001 & 0.000014 & 0.000001 & $0.8 \%$ & $0.3 \%$ & $10.2 \%$ & $0.4 \%$ \\
\hline & Total & 0.000245 & 0.000229 & 0.000137 & 0.000236 & & & & \\
\hline
\end{tabular}

As for the Cold Chem measurements, attempts were made during this data analysis to investigate for possible instrument drift in the Mixed Acid data. Comparisons between SM35 (Calibration Standard A) and SM33 (Bench Standard A) provide some insight into this possible problem for the Mixed Acid data. Exhibit A14 in the Appendix presents a paired comparison between the measurements for these standards across the SME and MFT batches. The average of the two SM35 measurements was compared to the 
SM33 measurement for each batch. The mean difference between the measurements of this pair of standards was found to be statistically significant for all of the active elements (i.e., $\mathrm{Ca}, \mathrm{Cr}, \mathrm{Cu}, \mathrm{K}, \mathrm{Li}$, $\mathrm{Mg}, \mathrm{Na}, \mathrm{Ni}$, and $\mathrm{Si}$ ). Table 2-14 provides summary information for these measurements. This table reveals that although statistically significant differences are seen, all of these differences are less than $1.50 \%$. So no dramatic drift, in the form of a consistent mean shift, is evident from these data. The standard deviations of these Mixed Acid measurements across the SME and MFT batches are also provided in this table, and a review of these statistics does not suggest that the measurements of the bench standard are dramatically less precise than the measurements of the corresponding calibration standard.

Table 2-14 Insights into Mixed Acid Instrument Drift by Comparisons of SM35 vs SM33

\begin{tabular}{||c|c|c|c||}
\hline & Bench A & Cal A & \\
\hline & SM33 & SM35 & $\%$ \\
\hline N Rows & 305 & 305 & Diff \\
\hline Mean(Al (mg/L)) & 0.0162 & 0.0217 & \\
\hline Mean(Ca (mg/L)) & 0.9953 & 1.0103 & $-1.49 \%$ \\
\hline Mean(Cr (mg/L)) & 0.9944 & 1.0066 & $-1.21 \%$ \\
\hline Mean(Cu (mg/L)) & 0.9942 & 1.0054 & $-1.11 \%$ \\
\hline Mean(Fe (mg/L)) & -0.0070 & -0.0081 & \\
\hline Mean(K (mg/L)) & 4.9121 & 4.9847 & $-1.46 \%$ \\
\hline Mean(Li (mg/L)) & 1.9706 & 1.9930 & $-1.12 \%$ \\
\hline Mean(Mg (mg/L)) & 1.0064 & 1.0176 & $-1.10 \%$ \\
\hline Mean(Mn (mg/L)) & 0.0002 & 0.0001 & \\
\hline Mean(Na (mg/L)) & 32.7116 & 33.0419 & $-1.00 \%$ \\
\hline Mean(Ni (mg/L)) & 0.9947 & 1.0072 & $-1.24 \%$ \\
\hline Mean(Si (mg/L)) & 20.2611 & 20.4596 & $-0.97 \%$ \\
\hline Mean(Ti (mg/L)) & 0.0001 & 0.0007 & \\
\hline Mean(U (mg/L)) & 0.0298 & 0.0980 & \\
\hline Mean(Zr (mg/L)) & -0.0069 & -0.0058 & \\
\hline Std Dev(Al (mg/L)) & 0.2989 & 0.3028 & \\
\hline Std Dev(Ca (mg/L)) & 0.0258 & 0.0235 & \\
\hline Std Dev(Cr (mg/L)) & 0.0185 & 0.0168 & \\
\hline Std Dev(Cu (mg/L)) & 0.0139 & 0.0136 & \\
\hline Std Dev(Fe (mg/L)) & 0.0227 & 0.0229 & \\
\hline Std Dev(K (mg/L)) & 0.0832 & 0.0804 & \\
\hline Std Dev(Li (mg/L)) & 0.0279 & 0.0309 & \\
\hline Std Dev(Mg (mg/L)) & 0.0228 & 0.0201 & \\
\hline Std Dev(Mn (mg/L)) & 0.0019 & 0.0020 & \\
\hline Std Dev(Na (mg/L)) & 1.0046 & 0.8880 & \\
\hline Std Dev(Ni (mg/L)) & 0.0172 & 0.0164 & \\
\hline Std Dev(Si (mg/L)) & 0.4827 & 0.3965 & \\
\hline Std Dev(Ti (mg/L)) & 0.0014 & 0.0011 & \\
\hline Std Dev(U (mg/L)) & 0.1468 & 0.1031 & \\
\hline Std Dev(Zr (mg/L)) & 0.0155 & 0.0154 & \\
\hline \hline
\end{tabular}

Comparisons between SM36 (Calibration Standard B) and SM34 (Bench Standard B) also provide some insight into the possible problem of instrument drift for the Mixed Acid data. Exhibit A15 in the Appendix presents a paired comparison between the measurements for these standards across the SME and MFT batches for this prep method. The average of the two SM36 measurements was compared to the SM34 measurement for each batch and vessel combination. The mean difference between the measurements of this pair of standards was found to be statistically significant for all of the active elements (i.e., Al, Fe, Mn, Na, Ti, and Zr). Table 2-15 provides summary information for these measurements. This table reveals that although statistically significant differences are seen, all of these 
differences are less than $1.0 \%$. So no dramatic drift, in the form of a consistent mean shift, is evident from these data. The standard deviations of these measurements across the SME and MFT batches are also provided in this table, and a review of these statistics does not suggest that the measurements of the bench standard are dramatically less precise than the measurements of the corresponding calibration standard.

Table 2-15 Insights into Instrument Drift by Comparisons of SM36 vs SM34 for Mixed Acid

\begin{tabular}{||c|c|c|c||}
\hline & Bench B & Cal B & \\
\hline & SM34 & SM36 & $\%$ \\
\hline N Rows & 314 & 313 & Diff \\
\hline Mean(Al (mg/L)) & 1.9725 & 1.9857 & $-0.67 \%$ \\
\hline Mean(Ca (mg/L)) & 0.0186 & 0.0142 & \\
\hline Mean(Cr (mg/L)) & 0.0019 & 0.0023 & \\
\hline Mean(Cu (mg/L)) & 0.0007 & 0.0012 & \\
\hline Mean(Fe (mg/L)) & 9.8966 & 9.9390 & $-0.43 \%$ \\
\hline Mean(K (mg/L)) & 0.0075 & 0.0143 & \\
\hline Mean(Li (mg/L)) & 0.0069 & 0.0116 & \\
\hline Mean(Mg (mg/L)) & 0.0034 & 0.0027 & \\
\hline Mean(Mn (mg/L)) & 1.9694 & 1.9795 & $-0.51 \%$ \\
\hline Mean(Na (mg/L)) & 4.9059 & 4.9208 & $-0.30 \%$ \\
\hline Mean(Ni (mg/L)) & 0.0087 & 0.0091 & \\
\hline Mean(Si (mg/L)) & 0.1246 & 0.1131 & \\
\hline Mean(Ti (mg/L)) & 0.9929 & 0.9974 & $-0.45 \%$ \\
\hline Mean(U (mg/L)) & 0.0554 & 0.0802 & \\
\hline Mean(Zr (mg/L)) & 0.9769 & 0.9815 & $-0.47 \%$ \\
\hline Std Dev(Al (mg/L)) & 0.0302 & 0.0281 & \\
\hline Std Dev(Ca (mg/L)) & 0.0699 & 0.0506 & \\
\hline Std Dev(Cr (mg/L)) & 0.0027 & 0.0021 & \\
\hline Std Dev(Cu (mg/L)) & 0.0033 & 0.0027 & \\
\hline Std Dev(Fe (mg/L)) & 0.1424 & 0.1225 & \\
\hline Std Dev(K (mg/L)) & 0.0524 & 0.0482 & \\
\hline Std Dev(Li (mg/L)) & 0.0205 & 0.0870 & \\
\hline Std Dev(Mg (mg/L)) & 0.0118 & 0.0086 & \\
\hline Std Dev(Mn (mg/L)) & 0.0329 & 0.0295 & \\
\hline Std Dev(Na (mg/L)) & 0.1036 & 0.0964 & \\
\hline Std Dev(Ni (mg/L)) & 0.0079 & 0.0068 & \\
\hline Std Dev(Si (mg/L)) & 0.4747 & 0.3604 & \\
\hline Std Dev(Ti (mg/L)) & 0.0182 & 0.0176 & \\
\hline Std Dev(U (mg/L)) & 0.1449 & 0.1058 & \\
\hline Std Dev(Zr (mg/L)) & 0.0127 & 0.0114 & \\
\hline \hline
\end{tabular}

Finally, comparisons between SM38 (Bench Standard C) and SM37 (Calibration Standard C) provide some insight into the possible problem of instrument drift for the Mixed Acid data. Exhibit A16 in the Appendix presents a paired comparison between the measurements for these standards across the SME and MFT batches that used the Mixed Acid prep. The average of the two SM38 measurements was compared to the SM37 measurement for each batch. U is the only active element for this pair of standards, and the mean difference between the two standards was found to be statistically significant at the 5\% level. Table 2-16 provides summary information for these measurements. This table reveals that although statistically significant, the \% difference for $U$ was only $0.68 \%$. So no dramatic drift, in the form of a consistent mean shift, is evident from these data. The standard deviations of these measurements across the SRAT batches are also provided in this table, and a review of these statistics 
WSRC-TR-2004-00576

Revision 0

shows that the standard deviation for $\mathrm{U}$ for the bench standard is actually smaller than the $\mathrm{U}$ standard deviation for the corresponding calibration standard.

Table 2-16 Insights into Instrument Drift by Comparisons of SM38 vs SM37 for Mixed Acid

\begin{tabular}{||c|c|c|c||}
\hline & Bench C & Cal C & \\
\hline & SM37 & SM38 & $\%$ \\
\hline N Rows & 312 & 311 & Diff \\
\hline Mean(Al (mg/L)) & 0.0444 & 0.0540 & \\
\hline Mean(Ca (mg/L)) & 0.2877 & 0.2778 & \\
\hline Mean(Cr (mg/L)) & 0.0286 & 0.0291 & \\
\hline Mean(Cu (mg/L)) & 0.0251 & 0.0251 & \\
\hline Mean(Fe (mg/L)) & -0.0052 & -0.0060 & \\
\hline Mean(K (mg/L)) & -0.0069 & 0.0032 & \\
\hline Mean(Li (mg/L)) & 0.0005 & 0.0006 & \\
\hline Mean(Mg (mg/L)) & 0.0052 & 0.0048 & \\
\hline Mean(Mn (mg/L)) & 0.0016 & 0.0015 & \\
\hline Mean(Na (mg/L)) & 0.1181 & 0.1184 & \\
\hline Mean(Ni (mg/L)) & 0.0019 & 0.0025 & \\
\hline Mean(Si (mg/L)) & 0.4320 & 0.1827 & \\
\hline Mean(Ti (mg/L)) & 0.0009 & 0.0011 & \\
\hline Mean(U (mg/L)) & 9.8445 & 9.9120 & $-0.68 \%$ \\
\hline Mean(Zr (mg/L)) & -0.0052 & -0.0048 & \\
\hline Std Dev(Al (mg/L)) & 0.0182 & 0.1383 & \\
\hline Std Dev(Ca (mg/L)) & 0.3688 & 0.3501 & \\
\hline Std Dev(Cr (mg/L)) & 0.0047 & 0.0049 & \\
\hline Std Dev(Cu (mg/L)) & 0.0380 & 0.0339 & \\
\hline Std Dev(Fe (mg/L)) & 0.0246 & 0.0250 & \\
\hline Std Dev(K (mg/L)) & 0.0515 & 0.0446 & \\
\hline Std Dev(Li (mg/L)) & 0.0022 & 0.0018 & \\
\hline Std Dev(Mg (mg/L)) & 0.0051 & 0.0052 & \\
\hline Std Dev(Mn (mg/L)) & 0.0012 & 0.0012 & \\
\hline Std Dev(Na (mg/L)) & 0.5612 & 0.5639 & \\
\hline Std Dev(Ni (mg/L)) & 0.0077 & 0.0068 & \\
\hline Std Dev(Si (mg/L)) & 0.6324 & 0.5342 & \\
\hline Std Dev(Ti (mg/L)) & 0.0016 & 0.0014 & \\
\hline Std Dev(U (mg/L)) & 0.1773 & 0.1728 & \\
\hline Std Dev(Zr (mg/L)) & 0.0158 & 0.0155 & \\
\hline
\end{tabular}

Thus, overall for the Mixed Acid data there is no indication of any instrument drift of practical concern over the course of a block of measurements needed to process a SME or MFT batch. The results presented in Table 2-10 through Table 2-16 also provide an opportunity to address one additional question regarding the calibration standards: Are the replicate measurements of SM36, SM37, and SM38 at the beginning of each Mixed Acid block worthwhile as a feature of the quality assurance of this analytical process? In Table 2-13 note that the within batch (residual) variances for these calibration standards are typically smaller than the between batch (batch-to-batch) variances, while the standard deviations of Table 2-14 through Table 2-16 suggest that the batch to batch variances of the calibration standards are comparable to the batch-to-batch variances of the bench standards. Thus, these results indicate that there is no practical drift in the precision of these measurements over a Mixed Acid block and that the replicate measurements of the calibration standards at the beginning of the Mixed Acid block are not needed. 


\subsubsection{Mixed Acid ARG-1 Results}

Exhibit A17 in the Appendix provides time plots in the form of control charts of the elemental measurements of the ARG-1 standard glass under the Mixed Acid protocol. These measurements are in elemental weight percent (wt\%), and the displays are in analytical sequence. The plotted values represented by an " $x$ " are measurements that were not associated with a particular process (SME or MFT) batch. There are obvious outliers indicated in some of these plots as well as mean shifts, trends, and inconsistencies in precision.

Exhibit A18 in the Appendix provides histograms and descriptive statistics for all of the ARG-1 data while Exhibit A19 in the Appendix provides the same information for the ARG-1 results associated with a SME or MFT batch. The means and reference values for both groups of ARG-1 measurements are given in Table 2-17. The \% relative difference (\% Rel Diff) for each element for each group is also provided. Note that all of the \% relative differences (or \% biases) of the measured versus reference values are less than 5\% for all of the elements present in ARG-1 at concentrations of at least $0.1 \mathrm{wt} \%$. The table also presents the standard deviations and \% RSDs for these data. For the major elements, the \%RSDs are all less than 5\%. Also, note that the bias in the Si values generated using the Mixed Acid prep is negative (i.e., the Si measurements are slightly less than the ARG-1 reference value for this element).

Table 2-17 ARG-1 Results for Mixed Acid Samples

\begin{tabular}{|c|c|c|c|c|c|c|c|c|c|}
\hline & & \multicolumn{4}{|c|}{ A All of the Available Data $(\mathrm{N}=649)$} & \multicolumn{4}{|c|}{ Data for SME \& MFT Batches $(\mathrm{N}=597)$} \\
\hline & Ref & Mean & \% Rel & Std & \% Rel & Mean & $\%$ Rel & Std & \% Rel \\
\hline Element & Value & $\mathrm{wt} \%$ & Diff & Dev & Std Dev & wt $\%$ & Diff & Dev & Std Dev \\
\hline $\mathrm{Al}(\mathrm{wt} \%)$ & 2.5 & 2.386 & $-4.54 \%$ & 0.073 & $3.06 \%$ & 2.388 & $-4.49 \%$ & 0.071 & $2.97 \%$ \\
\hline Ca (wt\%) & 1.02 & 1.028 & $0.74 \%$ & 0.045 & $4.38 \%$ & 1.027 & $0.73 \%$ & 0.043 & $4.21 \%$ \\
\hline $\mathrm{Cr}(\mathrm{wt} \%)$ & 0.06 & 0.071 & $18.35 \%$ & 0.006 & $8.42 \%$ & 0.071 & $18.29 \%$ & 0.006 & $8.32 \%$ \\
\hline $\mathrm{Cu}$ (wt\%) & 0.0001 & 0.006 & & 0.005 & & 0.006 & & 0.004 & \\
\hline $\mathrm{Fe}(\mathrm{wt} \%)$ & 9.79 & 9.592 & $-2.02 \%$ & 0.263 & $2.74 \%$ & 9.597 & $-1.97 \%$ & 0.254 & $2.65 \%$ \\
\hline $\mathrm{K}(\mathrm{wt} \%)$ & 2.25 & 2.282 & $1.41 \%$ & 0.087 & $3.79 \%$ & 2.284 & $1.53 \%$ & 0.085 & $3.71 \%$ \\
\hline $\mathrm{Li}(\mathrm{wt} \%)$ & 1.49 & 1.486 & $-0.29 \%$ & 0.042 & $2.80 \%$ & 1.488 & $-0.16 \%$ & 0.039 & $2.62 \%$ \\
\hline $\mathrm{Mg}(\mathrm{wt} \%)$ & 0.52 & 0.518 & $-0.34 \%$ & 0.017 & $3.28 \%$ & 0.519 & $-0.26 \%$ & 0.016 & $3.15 \%$ \\
\hline $\mathrm{Mn}(\mathrm{wt} \%)$ & 1.46 & 1.421 & $-2.64 \%$ & 0.039 & $2.76 \%$ & 1.422 & $-2.62 \%$ & 0.038 & $2.66 \%$ \\
\hline $\mathrm{Na}(\mathrm{wt} \%)$ & 8.53 & 8.347 & $-2.15 \%$ & 0.259 & $3.10 \%$ & 8.352 & $-2.09 \%$ & 0.249 & $2.98 \%$ \\
\hline $\mathrm{Ni}(\mathrm{wt} \%)$ & 0.83 & 0.832 & $0.23 \%$ & 0.026 & $3.17 \%$ & 0.832 & $0.27 \%$ & 0.026 & $3.10 \%$ \\
\hline Si (wt\%) & 22.39 & 21.506 & $-3.95 \%$ & 1.190 & $5.53 \%$ & 21.488 & $-4.03 \%$ & 0.863 & $4.02 \%$ \\
\hline $\mathrm{Ti}(\mathrm{wt} \%)$ & 0.69 & 0.706 & $2.26 \%$ & 0.033 & $4.68 \%$ & 0.706 & $2.31 \%$ & 0.033 & $4.69 \%$ \\
\hline $\mathrm{U}(\mathrm{wt} \%)$ & 0 & 0.194 & & 0.162 & & 0.194 & & 0.161 & \\
\hline $\mathrm{Zr}(\mathrm{wt} \%)$ & 0.1 & 0.098 & $-2.47 \%$ & 0.017 & $17.92 \%$ & 0.098 & $-2.41 \%$ & 0.017 & $17.72 \%$ \\
\hline
\end{tabular}

Exhibit A20 in the Appendix provides an investigation into sources of variation for the replicated ARG-1 measurements within each block of SME and MFT analyses. The results are summarized in Table 2-18, which shows that the batch-to-batch variation is the smaller source of variation as compared to the within batch variation for most of the major elements. 
WSRC-TR-2004-00576

Revision 0

Table 2-18 Components of Variation for ARG-1 Results for Mixed Acid Samples

\begin{tabular}{|c|c|c|c|c|c|c|c|c|c|}
\hline & Tean & & $\bar{e}$ & & $\%$ & $\mathrm{e}$ & \%RSD & \%RSD & \%RSD \\
\hline Element & $\mathrm{wt} \%$ & Batch & ial & Total & o Batch & \% Residua & Batch & Residual & Total \\
\hline$\%)$ & 387 & 22 & & 4917 & $0<70$ & & $0 \%$ & $8 \%$ & $2.94 \%$ \\
\hline $\mathrm{Ca}(\mathrm{v}$ & 027 & 338 & 24 & 01862 & $\%$ & $\%$ & $82 \%$ & $1 \%$ & $4.20 \%$ \\
\hline Cr (wt\%) & 0.071 & 000018 & 0.000017 & 0.000035 & $51.43 \%$ & $48.57 \%$ & $5.97 \%$ & $5.81 \%$ & $8.33 \%$ \\
\hline $\mathrm{Cu}(\mathrm{v}$ & 0.006 & 0.000010 & 0.000005 & 0.000015 & $66.67 \%$ & $33.33 \%$ & $51.70 \%$ & $36.55 \%$ & $63.32 \%$ \\
\hline $\mathrm{Fe}(\mathrm{v}$ & 9.590 & 0.001959 & 0.058366 & 0.060325 & $3.25 \%$ & $96.75 \%$ & $0.46 \%$ & $2.52 \%$ & $2.56 \%$ \\
\hline $\mathrm{K}$ & 2.284 & 51 & 0.00 & 0.007178 & $42.55 \%$ & $\%$ & $2.42 \%$ & $2.81 \%$ & $3.71 \%$ \\
\hline $\mathrm{Li} \mathrm{(v}$ & 37 & 0.000108 & 3 & 1 & $7.44 \%$ & $92.56 \%$ & 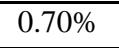 & 2 . & $\%$ \\
\hline$\overline{M g}($ & 8 & 000054 & 0.000210 & 4 & 0 & $79.55 \%$ & $1.42 \%$ & 2. & $\%$ \\
\hline$\overline{M n}($ & 1.421 & 0 & 1 & 1 & $8.08 \%$ & $91.92 \%$ & $\%$ & 2. & $2.60 \%$ \\
\hline $\mathrm{Na}($ & 8.349 & 0.003927 & 0.056634 & 0.060561 & $6.48 \%$ & $93.52 \%$ & $0.75 \%$ & $2.85 \%$ & $2.95 \%$ \\
\hline $\mathrm{Ni}(\mathrm{y}$ & 0.832 & 0.000101 & 0.000544 & 0.000645 & $15.66 \%$ & $84.34 \%$ & $1.21 \%$ & $2.80 \%$ & $3.05 \%$ \\
\hline Si (w & 21.500 & 311759 & 0.413924 & 0.725683 & $42.96 \%$ & $57.04 \%$ & $2.60 \%$ & $2.99 \%$ & $3.96 \%$ \\
\hline Ti (wt\%) & 0.706 & 0.000573 & 0.000544 & 0.001117 & $51.30 \%$ & $48.70 \%$ & $3.39 \%$ & $3.30 \%$ & $4.74 \%$ \\
\hline $\mathrm{U}(\mathrm{wt} \%)$ & 0.197 & 0.020960 & 0.003742 & 0.024702 & $84.85 \%$ & $15.15 \%$ & $73.48 \%$ & $31.05 \%$ & $79.77 \%$ \\
\hline $\mathrm{Zr}(\mathrm{wt} \%)$ & 0.098 & 0.000261 & 0.000010 & 0.000271 & $96.31 \%$ & $3.69 \%$ & $16.48 \%$ & $3.23 \%$ & $16.80 \%$ \\
\hline
\end{tabular}

\subsection{FUSION RESULTS}

Recall the analytical protocol (provided in Table 1-6) for SME and MFT samples analyzed by the Fusion prep method. In Section 2.3.1, the calibration and bench standards are investigated. All of the available data are initially explored with the measurements of the standards associated with the SME and MFT batches subsequently investigated. The elemental measurements for these standards are expressed as $\mathrm{mg} / \mathrm{L}$. Section 2.3.2 provides a discussion of the ARG-1 measurements generated under the Fusion prep. The ARG-1 measurements are expressed in elemental wt\%.

\subsubsection{Fusion Calibration and Bench Standards}

Exhibit A21 in the Appendix provides time plots in the form of control charts of the elemental measurements of each of the calibration and bench standards used as part of the Fusion protocol for both SME and MFT samples. These measurement are in $\mathrm{mg} / \mathrm{L}$, the displays are in analytical sequence by element for each of the standards. The plotted values represented by an " $x$ " are measurements that were not associated with a particular process batch. There are obvious outliers indicated in some of these plots as well as mean shifts, trends, and inconsistencies in precision.

Exhibit A22 provides histograms and descriptive statistics for all of these data. The means and reference values for the standards are given in Table 2-19. The \% relative differences (\% Rel Diffs) of these measurements are also in this table; note that even without any screening of the data the largest \% Rel Diff is only $2.6 \%$. 
WSRC-TR-2004-00576

Revision 0

Table 2-19 Averages and Relative Biases for All Calibration and Bench Standard Measurements by Fusion

\begin{tabular}{|c|c|c|c|c|c|c|}
\hline & & SM51 & SM52 & SM53 & SM54 & SM55 \\
\hline Element & N Rows & 687 & 508 & 509 & 1104 & 1098 \\
\hline \multirow[t]{3}{*}{$\mathrm{Al}(\mathrm{mg} / \mathrm{L})$} & Mean & 0.006 & 1.972 & 0.010 & 1.979 & 0.024 \\
\hline & Ref Value & 0 & 2 & 0 & 2 & 0 \\
\hline & \% Rel Diff & & $-1.4 \%$ & & $-1.1 \%$ & \\
\hline \multirow[t]{3}{*}{$\mathrm{B}(\mathrm{mg} / \mathrm{L})$} & Mean & 3.897 & 1.986 & 3.916 & 1.990 & 0.007 \\
\hline & Ref Value & 4 & 2 & 4 & 2 & 0 \\
\hline & \% Rel Diff & $-2.6 \%$ & $-0.7 \%$ & $-2.1 \%$ & $-0.5 \%$ & \\
\hline \multirow[t]{3}{*}{ Ca (mg/L) } & Mean & 0.999 & 0.012 & 1.002 & 0.007 & -0.002 \\
\hline & Ref Value & 1 & 0 & 1 & 0 & 0 \\
\hline & \% Rel Diff & $-0.1 \%$ & & $0.2 \%$ & & \\
\hline \multirow[t]{3}{*}{$\mathrm{Cr}(\mathrm{mg} / \mathrm{L})$} & Mean & 0.990 & 0.001 & 0.993 & 0.002 & 0.009 \\
\hline & Ref Value & 1 & 0 & 1 & 0 & 0 \\
\hline & \% Rel Diff & $-1.0 \%$ & & $-0.7 \%$ & & \\
\hline \multirow[t]{3}{*}{$\mathrm{Cu}(\mathrm{mg} / \mathrm{L})$} & Mean & 0.990 & 0.000 & 0.992 & 0.001 & 0.005 \\
\hline & Ref Value & 1 & 0 & 1 & 0 & 0 \\
\hline & \% Rel Diff & $-1.0 \%$ & & $-0.8 \%$ & & \\
\hline \multirow[t]{3}{*}{$\mathrm{Fe}(\mathrm{mg} / \mathrm{L})$} & Mean & 0.006 & 9.874 & 0.006 & 9.889 & 0.047 \\
\hline & Ref Value & 0 & 10 & 0 & 10 & 0 \\
\hline & \% Rel Diff & & $-1.3 \%$ & & $-1.1 \%$ & \\
\hline \multirow[t]{3}{*}{$\mathrm{K}(\mathrm{mg} / \mathrm{L})$} & Mean & 4.929 & 0.016 & 4.944 & 0.018 & 0.051 \\
\hline & Ref Value & 5 & 0 & 5 & 0 & 0 \\
\hline & \% Rel Diff & $-1.4 \%$ & & $-1.1 \%$ & & \\
\hline \multirow[t]{3}{*}{$\mathrm{Li}(\mathrm{mg} / \mathrm{L})$} & Mean & 1.974 & 0.001 & 1.978 & 0.001 & 0.005 \\
\hline & Ref Value & 2 & 0 & 2 & 0 & 0 \\
\hline & \% Rel Diff & $-1.3 \%$ & & $-1.1 \%$ & & \\
\hline \multirow[t]{3}{*}{$\mathrm{Mg}(\mathrm{mg} / \mathrm{L})$} & Mean & 1.004 & 0.002 & 1.004 & 0.001 & 0.004 \\
\hline & Ref Value & 1 & 0 & 1 & 0 & 0 \\
\hline & \% Rel Diff & $0.4 \%$ & & $0.4 \%$ & & \\
\hline \multirow[t]{3}{*}{$\mathrm{Mn}(\mathrm{mg} / \mathrm{L})$} & Mean & 0.001 & 1.971 & 0.001 & 1.975 & 0.005 \\
\hline & Ref Value & 0 & 2 & 0 & 2 & 0 \\
\hline & \% Rel Diff & & $-1.5 \%$ & & $-1.2 \%$ & \\
\hline \multirow[t]{3}{*}{$\mathrm{Ni}(\mathrm{mg} / \mathrm{L})$} & Mean & 0.987 & 0.008 & 0.990 & 0.008 & 0.009 \\
\hline & Ref Value & 1 & 0 & 1 & 0 & 0 \\
\hline & $\%$ Rel Diff & $-1.3 \%$ & & $-1.0 \%$ & & \\
\hline \multirow[t]{3}{*}{$\mathrm{Si}(\mathrm{mg} / \mathrm{L})$} & Mean & 20.124 & 0.006 & 20.050 & 0.011 & 0.103 \\
\hline & Ref Value & 20 & 0 & 20 & 0 & 0 \\
\hline & \% Rel Diff & $0.6 \%$ & & $0.2 \%$ & & \\
\hline \multirow[t]{3}{*}{$\mathrm{Ti}(\mathrm{mg} / \mathrm{L})$} & Mean & 0.000 & 0.993 & 0.001 & 0.995 & 0.004 \\
\hline & Ref Value & 0 & 1 & 0 & 1 & 0 \\
\hline & \% Rel Diff & & $-0.7 \%$ & & $-0.5 \%$ & \\
\hline
\end{tabular}

The \% relative standard deviations (\% RSDs) were computed from the means and standard deviations of Exhibit A22 and these data are provided in Table 2-20. Note that all of the \%RSDs are no larger than $6.0 \%$ even without any screening of the data. 
WSRC-TR-2004-00576

Revision 0

Table 2-20 Descriptive Statistics for All Fusion Calibration and Bench Standard Measurements

\begin{tabular}{|c|c|c|c|c|c|c|}
\hline & & " SM51 & " SM52 & " SM53 & " SM54 & "SM55 \\
\hline Element & N Rows & 687 & 508 & 509 & 1104 & 1098 \\
\hline \multirow[t]{3}{*}{$\mathrm{Al}(\mathrm{mg} / \mathrm{L})$} & Mean & 0.006 & 1.972 & 0.010 & 1.979 & 0.024 \\
\hline & Std Dev & 0.016 & 0.033 & 0.012 & 0.030 & 0.042 \\
\hline & $\%$ RSD & & $1.7 \%$ & & $1.5 \%$ & \\
\hline \multirow[t]{3}{*}{$\mathrm{B}(\mathrm{mg} / \mathrm{L})$} & Mean & 3.897 & 1.986 & 3.916 & 1.990 & 0.007 \\
\hline & Std Dev & 0.231 & 0.034 & 0.234 & 0.033 & 0.023 \\
\hline & $\%$ RSD & $5.9 \%$ & $1.7 \%$ & $6.0 \%$ & $1.7 \%$ & \\
\hline \multirow[t]{3}{*}{$\mathrm{Ca}(\mathrm{mg} / \mathrm{L})$} & Mean & 0.999 & 0.012 & 1.002 & 0.007 & -0.002 \\
\hline & Std Dev & 0.028 & 0.065 & 0.025 & 0.044 & 0.048 \\
\hline & $\%$ RSD & $2.8 \%$ & & $2.5 \%$ & & \\
\hline \multirow[t]{3}{*}{$\mathrm{Cr}(\mathrm{mg} / \mathrm{L})$} & Mean & 0.990 & 0.001 & 0.993 & 0.002 & 0.009 \\
\hline & Std Dev & 0.016 & 0.003 & 0.015 & 0.002 & 0.017 \\
\hline & $\%$ RSD & $1.6 \%$ & & $1.5 \%$ & & \\
\hline \multirow[t]{3}{*}{$\mathrm{Cu}(\mathrm{mg} / \mathrm{L})$} & Mean & 0.990 & 0.000 & 0.992 & 0.001 & 0.005 \\
\hline & Std Dev & 0.014 & 0.004 & 0.013 & 0.004 & 0.009 \\
\hline & $\%$ RSD & $1.4 \%$ & & $1.3 \%$ & & \\
\hline \multirow[t]{3}{*}{$\mathrm{Fe}(\mathrm{mg} / \mathrm{L})$} & Mean & 0.006 & 9.874 & 0.006 & 9.889 & 0.047 \\
\hline & Std Dev & 0.075 & 0.157 & 0.061 & 0.150 & 0.108 \\
\hline & $\%$ RSD & & $1.6 \%$ & & $1.5 \%$ & \\
\hline \multirow[t]{3}{*}{$\mathrm{K}(\mathrm{mg} / \mathrm{L})$} & Mean & 4.929 & 0.016 & 4.944 & 0.018 & 0.051 \\
\hline & Std Dev & 0.079 & 0.058 & 0.072 & 0.049 & 0.349 \\
\hline & $\%$ RSD & $1.6 \%$ & & $1.4 \%$ & & \\
\hline \multirow[t]{3}{*}{$\mathrm{Li}(\mathrm{mg} / \mathrm{L})$} & Mean & 1.974 & 0.001 & 1.978 & 0.001 & 0.005 \\
\hline & Std Dev & 0.029 & 0.002 & 0.025 & 0.002 & 0.007 \\
\hline & $\%$ RSD & $1.5 \%$ & & $1.3 \%$ & & \\
\hline \multirow[t]{3}{*}{$\mathrm{Mg}(\mathrm{mg} / \mathrm{L})$} & Mean & 1.004 & 0.002 & 1.004 & 0.001 & 0.004 \\
\hline & Std Dev & 0.024 & 0.020 & 0.020 & 0.003 & 0.008 \\
\hline & $\%$ RSD & $2.4 \%$ & & $2.0 \%$ & & \\
\hline \multirow[t]{3}{*}{ Mn (mg/L) } & Mean & 0.001 & 1.971 & 0.001 & 1.975 & 0.005 \\
\hline & Std Dev & 0.002 & 0.034 & 0.002 & 0.031 & 0.012 \\
\hline & $\%$ RSD & & $1.7 \%$ & & $1.6 \%$ & \\
\hline \multirow[t]{3}{*}{$\mathrm{Ni}(\mathrm{mg} / \mathrm{L})$} & Mean & 0.987 & 0.008 & 0.990 & 0.008 & 0.009 \\
\hline & Std Dev & 0.019 & 0.008 & 0.017 & 0.008 & 0.020 \\
\hline & $\%$ RSD & $1.9 \%$ & & $1.7 \%$ & & \\
\hline \multirow[t]{3}{*}{ Si (mg/L) } & Mean & 20.124 & 0.006 & 20.050 & 0.011 & 0.103 \\
\hline & Std Dev & 0.396 & 0.046 & 0.340 & 0.032 & 0.206 \\
\hline & $\%$ RSD & $2.0 \%$ & & $1.7 \%$ & & \\
\hline \multirow[t]{3}{*}{$\mathrm{Ti}(\mathrm{mg} / \mathrm{L})$} & Mean & 0.000 & 0.993 & 0.001 & 0.995 & 0.004 \\
\hline & Std Dev & 0.002 & 0.020 & 0.002 & 0.019 & 0.009 \\
\hline & $\%$ RSD & & $2.0 \%$ & & $1.9 \%$ & \\
\hline
\end{tabular}

Table 2-21 provides the same type of summary information for the fusion measurements, but this time for only those measurements of the calibration and bench standards that were reported as part of the information for a process (SME or MFT) batch. Note that the largest \%RSD for these data is that for boron, with a value of $6.2 \%$. All other \%RSDs are less than $3 \%$. 
WSRC-TR-2004-00576

Revision 0

Table 2-21 Descriptive Statistics for Fusion Calibration and Bench Standards in a Process Batch

\begin{tabular}{|c|c|c|c|c|c|c|}
\hline & & SM51 & "SM52 & SM53 & "SM54 & "SM55 \\
\hline Element & N Rows & 325 & 321 & 648 & 646 & 635 \\
\hline \multirow[t]{3}{*}{$\mathrm{Al}(\mathrm{mg} / \mathrm{L})$} & Mean & 0.007 & 1.973 & 0.010 & 1.980 & 0.026 \\
\hline & \begin{tabular}{|l} 
Std Dev \\
\end{tabular} & 0.014 & 0.032 & 0.011 & 0.028 & 0.042 \\
\hline & $\%$ RSD & & $1.6 \%$ & & $1.4 \%$ & \\
\hline \multirow[t]{3}{*}{$\mathrm{B}(\mathrm{mg} / \mathrm{L})$} & Mean & 3.882 & 1.986 & 3.902 & 1.988 & 0.009 \\
\hline & Std Dev & 0.241 & 0.032 & 0.240 & 0.032 & 0.023 \\
\hline & $\%$ RSD & $6.2 \%$ & $1.6 \%$ & $6.2 \%$ & $1.6 \%$ & \\
\hline \multirow[t]{3}{*}{$\mathrm{Ca}(\mathrm{mg} / \mathrm{L})$} & Mean & 0.998 & 0.014 & 1.002 & 0.006 & 0.002 \\
\hline & Std Dev & 0.028 & 0.071 & 0.025 & 0.038 & 0.049 \\
\hline & \%RSD & $2.8 \%$ & & $2.5 \%$ & & \\
\hline \multirow[t]{3}{*}{$\mathrm{Cr}(\mathrm{mg} / \mathrm{L})$} & Mean & 0.989 & 0.001 & 0.992 & 0.002 & 0.010 \\
\hline & Std Dev & 0.015 & 0.003 & 0.014 & 0.002 & 0.019 \\
\hline & $\%$ RSD & $1.5 \%$ & & $1.4 \%$ & & \\
\hline \multirow[t]{3}{*}{$\mathrm{Cu}(\mathrm{mg} / \mathrm{L})$} & Mean & 0.989 & 0.000 & 0.992 & 0.001 & 0.005 \\
\hline & Std Dev & 0.014 & 0.003 & 0.013 & 0.002 & 0.010 \\
\hline & $\%$ RSD & $1.4 \%$ & & $1.3 \%$ & & \\
\hline \multirow[t]{3}{*}{$\mathrm{Fe}(\mathrm{mg} / \mathrm{L})$} & Mean & 0.010 & 9.881 & 0.008 & 9.895 & 0.054 \\
\hline & \begin{tabular}{|l} 
Std Dev \\
\end{tabular} & 0.093 & 0.145 & 0.080 & 0.132 & 0.111 \\
\hline & $\%$ RSD & & $1.5 \%$ & & $1.3 \%$ & \\
\hline \multirow[t]{3}{*}{$\mathrm{K}(\mathrm{mg} / \mathrm{L})$} & Mean & 4.921 & 0.013 & 4.938 & 0.016 & 0.055 \\
\hline & Std Dev & 0.080 & 0.051 & 0.069 & 0.040 & 0.388 \\
\hline & $\%$ RSD & $1.6 \%$ & & $1.4 \%$ & & \\
\hline \multirow[t]{3}{*}{$\mathrm{Li}(\mathrm{mg} / \mathrm{L})$} & Mean & 1.974 & 0.001 & 1.979 & 0.001 & 0.005 \\
\hline & \begin{tabular}{|l} 
Std Dev \\
\end{tabular} & 0.028 & 0.002 & 0.025 & 0.002 & 0.008 \\
\hline & $\%$ RSD & $1.4 \%$ & & $1.2 \%$ & & \\
\hline \multirow[t]{3}{*}{$\mathrm{Mg}(\mathrm{mg} / \mathrm{L})$} & Mean & 1.003 & 0.003 & 1.003 & 0.001 & 0.004 \\
\hline & Std Dev & 0.024 & 0.027 & 0.020 & 0.002 & 0.008 \\
\hline & $\%$ RSD & $2.4 \%$ & & $2.0 \%$ & & \\
\hline \multirow[t]{3}{*}{$\mathrm{Mn}(\mathrm{mg} / \mathrm{L})$} & Mean & 0.001 & 1.972 & 0.001 & 1.976 & 0.005 \\
\hline & Std Dev & 0.002 & 0.031 & 0.002 & 0.029 & 0.013 \\
\hline & $\%$ RSD & & $1.6 \%$ & & $1.5 \%$ & \\
\hline \multirow[t]{3}{*}{$\mathrm{Ni}(\mathrm{mg} / \mathrm{L})$} & Mean & 0.986 & 0.008 & 0.990 & 0.008 & 0.009 \\
\hline & \begin{tabular}{|l} 
Std Dev \\
\end{tabular} & 0.018 & 0.008 & 0.016 & 0.007 & 0.021 \\
\hline & $\%$ RSD & $1.8 \%$ & & $1.6 \%$ & & \\
\hline \multirow[t]{3}{*}{ Si (mg/L) } & Mean & 20.151 & 0.006 & 20.064 & 0.011 & 0.111 \\
\hline & \begin{tabular}{|l|} 
Std Dev \\
\end{tabular} & 0.406 & 0.047 & 0.326 & 0.029 & 0.210 \\
\hline & $\%$ RSD & $2.0 \%$ & & $1.6 \%$ & & \\
\hline \multirow[t]{3}{*}{$\mathrm{Ti}(\mathrm{mg} / \mathrm{L})$} & Mean & 0.000 & 0.993 & 0.001 & 0.994 & 0.005 \\
\hline & Std Dev & 0.001 & 0.019 & 0.001 & 0.019 & 0.010 \\
\hline & $\%$ RSD & & $1.9 \%$ & & $1.9 \%$ & \\
\hline
\end{tabular}

Exhibit A23 in the Appendix provides an investigation into sources of variation for the calibration and bench standards with replicate measurements within each block of SME or MFT analyses. The analysis combines the SME and MFT blocks into a single dataset with the intention of estimating between batch and within batch (residual) variation. A summary of the results is provided in Table 2-22, which shows that the batch-to-batch variation is, in general, larger than the within-batch (or residual) variation for these data. 
WSRC-TR-2004-00576

Revision 0

Table 2-22 Components of Variance for Fusion Calibration and Bench Standards in a Process Batch

\begin{tabular}{|c|c|c|c|c|c|c|c|}
\hline & & \multicolumn{3}{|c|}{ Components of Variance } & \multicolumn{3}{|c|}{ \% of Variance } \\
\hline Element & & SM53 & SM54 & SM55 & SM53 & SM54 & SM55 \\
\hline \multirow[t]{3}{*}{$\mathrm{Al}(\mathrm{mg} / \mathrm{L})$} & Batch-to-batch & 0.000098 & 0.000716 & 0.000329 & $87.5 \%$ & $88.4 \%$ & $18.9 \%$ \\
\hline & Residual & 0.000014 & 0.000094 & 0.001408 & $12.5 \%$ & $11.6 \%$ & $81.1 \%$ \\
\hline & Total & 0.000112 & 0.000810 & 0.001737 & & & \\
\hline \multirow[t]{3}{*}{$\mathrm{B}(\mathrm{mg} / \mathrm{L})$} & Batch-to-batch & 0.056529 & 0.000785 & 0.000242 & $97.9 \%$ & $78.5 \%$ & $47.2 \%$ \\
\hline & Residual & 0.001202 & 0.000215 & 0.000271 & $2.1 \%$ & $21.5 \%$ & $52.8 \%$ \\
\hline & Total & 0.057731 & 0.001000 & 0.000513 & & & \\
\hline \multirow[t]{3}{*}{ Ca (mg/L) } & Batch-to-batch & 0.000549 & 0.001353 & 0.000938 & $88.3 \%$ & $92.4 \%$ & $38.9 \%$ \\
\hline & Residual & 0.000073 & 0.000111 & 0.001474 & $11.7 \%$ & $7.6 \%$ & $61.1 \%$ \\
\hline & Total & 0.000622 & 0.001464 & 0.002412 & & & \\
\hline \multirow[t]{3}{*}{$\mathrm{Cr}(\mathrm{mg} / \mathrm{L})$} & Batch-to-batch & 0.000183 & 0.000004 & 0.000047 & $89.3 \%$ & $80.9 \%$ & $13.2 \%$ \\
\hline & Residual & 0.000022 & 0.000001 & 0.000308 & $10.7 \%$ & $19.1 \%$ & $86.8 \%$ \\
\hline & Total & 0.000205 & 0.000005 & 0.000355 & & & \\
\hline \multirow[t]{3}{*}{$\mathrm{Cu}(\mathrm{mg} / \mathrm{L})$} & Batch-to-batch & 0.000151 & 0.000004 & 0.000039 & $89.9 \%$ & $87.1 \%$ & $39.0 \%$ \\
\hline & Residual & 0.000017 & 0.000001 & 0.000061 & $10.1 \%$ & $12.9 \%$ & $61.0 \%$ \\
\hline & Total & 0.000168 & 0.000005 & 0.000100 & & & \\
\hline \multirow[t]{3}{*}{$\mathrm{Fe}(\mathrm{mg} / \mathrm{L})$} & Batch-to-batch & 0.004083 & 0.015135 & 0.001558 & $64.1 \%$ & $86.8 \%$ & $12.7 \%$ \\
\hline & Residual & 0.002282 & 0.002292 & 0.010691 & $35.9 \%$ & $13.2 \%$ & $87.3 \%$ \\
\hline & Total & 0.006365 & 0.017427 & 0.012249 & & & \\
\hline \multirow[t]{3}{*}{$\mathrm{K}(\mathrm{mg} / \mathrm{L})$} & Batch-to-batch & 0.003658 & 0.000998 & 0.148998 & $76.3 \%$ & $63.4 \%$ & $98.8 \%$ \\
\hline & Residual & 0.001137 & 0.000575 & 0.001856 & $23.7 \%$ & $36.6 \%$ & $1.2 \%$ \\
\hline & Total & 0.004795 & 0.001573 & 0.150854 & & & \\
\hline \multirow[t]{3}{*}{$\mathrm{Li}(\mathrm{mg} / \mathrm{L})$} & Batch-to-batch & 0.000533 & 0.000002 & 0.000011 & $88.0 \%$ & $73.5 \%$ & $17.2 \%$ \\
\hline & Residual & 0.000073 & 0.000001 & 0.000053 & $12.0 \%$ & $26.5 \%$ & $82.8 \%$ \\
\hline & Total & 0.000606 & 0.000003 & 0.000064 & & & \\
\hline \multirow[t]{3}{*}{$\mathrm{Mg}(\mathrm{mg} / \mathrm{L})$} & Batch-to-batch & 0.000337 & 0.000001 & 0.000028 & $85.1 \%$ & $24.0 \%$ & $40.0 \%$ \\
\hline & Residual & 0.000059 & 0.000003 & 0.000042 & $14.9 \%$ & $76.0 \%$ & $60.0 \%$ \\
\hline & Total & 0.000396 & 0.000004 & 0.000070 & & & \\
\hline \multirow[t]{3}{*}{$\mathrm{Mn}(\mathrm{mg} / \mathrm{L})$} & Batch-to-batch & 0.000003 & 0.000767 & 0.000014 & $90.3 \%$ & $90.4 \%$ & $7.8 \%$ \\
\hline & Residual & 0.000000 & 0.000081 & 0.000165 & $9.7 \%$ & $9.6 \%$ & $92.2 \%$ \\
\hline & Total & 0.000003 & 0.000848 & 0.000179 & & & \\
\hline \multirow[t]{3}{*}{$\mathrm{Ni}(\mathrm{mg} / \mathrm{L})$} & Batch-to-batch & 0.000181 & 0.000033 & 0.000057 & $68.3 \%$ & $60.0 \%$ & $12.9 \%$ \\
\hline & Residual & 0.000084 & 0.000022 & 0.000386 & $31.7 \%$ & $40.0 \%$ & $87.1 \%$ \\
\hline & Total & 0.000265 & 0.000055 & 0.000443 & & & \\
\hline \multirow[t]{3}{*}{$\mathrm{Si}(\mathrm{mg} / \mathrm{L})$} & Batch-to-batch & 0.095160 & 0.000752 & 0.005051 & $89.2 \%$ & $91.5 \%$ & $11.4 \%$ \\
\hline & Residual & 0.011507 & 0.000070 & 0.039115 & $10.8 \%$ & $8.5 \%$ & $88.6 \%$ \\
\hline & Total & 0.106667 & 0.000822 & 0.044166 & & & \\
\hline \multirow[t]{3}{*}{$\mathrm{Ti}(\mathrm{mg} / \mathrm{L})$} & Batch-to-batch & 0.000001 & 0.000347 & 0.000015 & $79.8 \%$ & $95.3 \%$ & $15.8 \%$ \\
\hline & Residual & 0.000000 & 0.000017 & 0.000080 & $20.2 \%$ & $4.7 \%$ & $84.2 \%$ \\
\hline & Total & 0.000001 & 0.000364 & 0.000095 & & & \\
\hline
\end{tabular}

As for the measurements for the other two prep methods, attempts were made during this data analysis to investigate for possible instrument drift in the Fusion data. Comparisons between SM53 (Calibration Standard A) and SM51 (Bench Standard A) provide some insight into this possible problem for the Fusion data. Exhibit A24 in the Appendix presents a paired comparison between the Fusion measurements for these standards across the SME and MFT batches. The average of the two SM53 measurements was compared to the SM51 measurement for each batch. The mean difference between the measurements of this pair of standards was found to be statistically significant for all of the active elements (i.e., B, Ca, Cr, Cu, K, Li, Ni, and $\mathrm{Si}$ ) except $\mathrm{Mg}$. Table 2-23 provides summary information for these measurements. This table reveals that although statistically significant differences are seen, all of these differences are less than $1.00 \%$. So no dramatic drift, in the form of a consistent mean shift, is 
evident from these data. The standard deviations of these Fusion measurements across the SME and MFT batches are also provided in this table, and a review of these statistics does not suggest that the measurements of the bench standard are dramatically less precise than the measurements of the corresponding calibration standard.

Table 2-23 Insights into Instrument Drift for Fusion Measurements of SM53 vs SM51

\begin{tabular}{|c|c|c|c|}
\hline & Bench A & Cal A & \\
\hline & SM51 & SM53 & $\%$ \\
\hline N Rows & 317 & 317 & Diff \\
\hline Mean(Al (mg/L)) & 0.0067 & 0.0099 & \\
\hline $\operatorname{Mean}(\mathrm{B}(\mathrm{mg} / \mathrm{L}))$ & 3.8806 & 3.9013 & $-0.53 \%$ \\
\hline Mean(Ca (mg/L)) & 0.9980 & 1.0017 & $-0.37 \%$ \\
\hline Mean(Cr (mg/L)) & 0.9891 & 0.9921 & $-0.30 \%$ \\
\hline Mean $(\mathrm{Cu}(\mathrm{mg} / \mathrm{L}))$ & 0.9892 & 0.9918 & $-0.27 \%$ \\
\hline Mean(Fe (mg/L)) & 0.0100 & 0.0080 & \\
\hline $\operatorname{Mean}(\mathrm{K}(\mathrm{mg} / \mathrm{L}))$ & 4.9217 & 4.9381 & $-0.33 \%$ \\
\hline $\operatorname{Mean}(\mathrm{Li}(\mathrm{mg} / \mathrm{L}))$ & 1.9743 & 1.9792 & $-0.25 \%$ \\
\hline $\operatorname{Mean}(\mathrm{Mg}(\mathrm{mg} / \mathrm{L}))$ & 1.0032 & 1.0032 & $0.00 \%$ \\
\hline $\operatorname{Mean}(\mathrm{Mn}(\mathrm{mg} / \mathrm{L}))$ & 0.0009 & 0.0008 & \\
\hline Mean(Ni (mg/L)) & 0.9866 & 0.9901 & $-0.36 \%$ \\
\hline Mean(Si (mg/L)) & 20.1574 & 20.0630 & $0.47 \%$ \\
\hline Mean(Ti (mg/L)) & 0.0005 & 0.0009 & \\
\hline Std Dev(Al (mg/L)) & 0.0138 & 0.0102 & \\
\hline Std Dev(B (mg/L)) & 0.2396 & 0.2380 & \\
\hline Std Dev(Ca (mg/L)) & 0.0278 & 0.0244 & \\
\hline Std Dev(Cr (mg/L)) & 0.0152 & 0.0139 & \\
\hline Std Dev(Cu (mg/L)) & 0.0140 & 0.0126 & \\
\hline Std Dev(Fe (mg/L)) & 0.0945 & 0.0729 & \\
\hline $\operatorname{Std} \operatorname{Dev}(\mathrm{K}(\mathrm{mg} / \mathrm{L}))$ & 0.0807 & 0.0651 & \\
\hline Std Dev(Li (mg/L)) & 0.0275 & 0.0240 & \\
\hline Std Dev(Mg (mg/L)) & 0.0239 & 0.0192 & \\
\hline Std Dev(Mn (mg/L)) & 0.0018 & 0.0017 & \\
\hline Std Dev(Ni (mg/L)) & 0.0178 & 0.0149 & \\
\hline Std Dev(Si (mg/L)) & 0.4014 & 0.3182 & \\
\hline Std Dev(Ti (mg/L)) & 0.0014 & 0.0011 & \\
\hline
\end{tabular}

Comparisons between SM52 (Bench Standard B) and SM54 (Calibration Standard B) also provide some insight into the possible problem of instrument drift for the Fusion data. Exhibit A25 in the Appendix presents a paired comparison between the measurements for these standards across the SME and MFT batches for this prep method. The average of the two SM54 measurements was compared to the SM52 measurement for each batch and vessel combination. The mean difference between the measurements of this pair of standards was found to be statistically significant for all of the active elements (i.e., Al, Fe, $\mathrm{Mn}$, and Ti) except B. Table 2-24 provides summary information for these measurements. This table reveals that although statistically significant differences are seen, all of these differences are less than $0.50 \%$. So no dramatic drift, in the form of a consistent mean shift, is evident from these data. The standard deviations of these measurements across the SME and MFT batches are also provided in this table, and a review of these statistics does not suggest that the measurements of the bench standard are dramatically less precise than the measurements of the corresponding calibration standard. 
WSRC-TR-2004-00576

Revision 0

Table 2-24 Insights into Instrument Drift by Comparisons of SM52 vs SM54 for Fusion

\begin{tabular}{|c|c|c|c|}
\hline & "Bench B & Cal B & \\
\hline & SM52 & SM54 & $\%$ \\
\hline N Rows & 312 & 317 & Diff \\
\hline Mean(Al (mg/L)) & 1.9733 & 1.9799 & $-0.33 \%$ \\
\hline $\operatorname{Mean}(\mathrm{B}(\mathrm{mg} / \mathrm{L}))$ & 1.9863 & 1.9884 & $-0.11 \%$ \\
\hline Mean(Ca (mg/L)) & 0.0145 & 0.0058 & \\
\hline Mean(Cr (mg/L)) & 0.0012 & 0.0018 & \\
\hline Mean $(\mathrm{Cu}(\mathrm{mg} / \mathrm{L}))$ & 0.0002 & 0.0008 & \\
\hline Mean(Fe (mg/L)) & 9.8842 & 9.8951 & $-0.11 \%$ \\
\hline $\operatorname{Mean}(\mathrm{K}(\mathrm{mg} / \mathrm{L}))$ & 0.0134 & 0.0153 & \\
\hline $\operatorname{Mean}(\mathrm{Li}(\mathrm{mg} / \mathrm{L}))$ & 0.0012 & 0.0013 & \\
\hline $\operatorname{Mean}(\mathrm{Mg}(\mathrm{mg} / \mathrm{L}))$ & 0.0032 & 0.0008 & \\
\hline $\operatorname{Mean}(\mathrm{Mn}(\mathrm{mg} / \mathrm{L}))$ & 1.9722 & 1.9761 & $-0.19 \%$ \\
\hline Mean(Ni (mg/L)) & 0.0077 & 0.0080 & \\
\hline Mean(Si (mg/L)) & 0.0061 & 0.0109 & \\
\hline Mean(Ti (mg/L)) & 0.9928 & 0.9942 & $-0.14 \%$ \\
\hline Std Dev(Al (mg/L)) & 0.0321 & 0.0277 & \\
\hline Std Dev(B (mg/L)) & 0.0321 & 0.0297 & \\
\hline Std Dev(Ca (mg/L)) & 0.0716 & 0.0378 & \\
\hline Std Dev(Cr (mg/L)) & 0.0028 & 0.0021 & \\
\hline Std Dev(Cu (mg/L)) & 0.0031 & 0.0022 & \\
\hline Std Dev(Fe (mg/L)) & 0.1441 & 0.1279 & \\
\hline Std Dev(K (mg/L)) & 0.0499 & 0.0358 & \\
\hline Std Dev(Li (mg/L)) & 0.0021 & 0.0017 & \\
\hline Std Dev(Mg (mg/L)) & 0.0271 & 0.0016 & \\
\hline Std Dev(Mn (mg/L)) & 0.0313 & 0.0284 & \\
\hline Std Dev(Ni (mg/L)) & 0.0080 & 0.0066 & \\
\hline Std Dev(Si (mg/L)) & 0.0464 & 0.0281 & \\
\hline Std Dev(Ti (mg/L)) & 0.0194 & 0.0189 & \\
\hline
\end{tabular}

Thus, overall for the Fusion data there is no indication of any instrument drift of practical concern over the course of a block of measurements needed to process a SME or MFT batch. The results presented in Table 2-19 through Table 2-24 also provide an opportunity to address one additional question regarding the calibration standards: Are the replicate measurements of SM53 and SM54 at the beginning of each Fusion block worthwhile as a feature of the quality assurance of this analytical process? In Table 2-22 note that the within batch (residual) variances for these calibration standards are typically smaller than the between batch (batch-to-batch) variances, while the standard deviations of Table 2-23 and Table 2-24 suggest that the batch to batch variances of the calibration standards are comparable to the batch-to-batch variances of the bench standards. Thus, these results indicate that there is no practical drift in the precision of these measurements over a Fusion block and that the replicate measurements of the calibration standards at the beginning of the Fusion block are not needed.

\subsubsection{Fusion ARG-1 Results}

Exhibit A26 in the Appendix provides time plots in the form of control charts of the elemental measurements of the ARG-1 standard glass under the Fusion protocol. These measurements are in elemental weight percent (wt\%), and the displays are in analytical sequence. The plotted values represented by an " $x$ " are measurements that were not associated with a particular process (SME or MFT) batch. There are obvious outliers indicated in some of these plots as well as mean shifts, trends, and inconsistencies in precision. 
Exhibit A27 in the Appendix provides histograms and descriptive statistics for all of the ARG-1 data while Exhibit A28 in the Appendix provides the same information for the ARG-1 results associated with a SME or MFT batch. The means and reference values for both groups of ARG-1 measurements are given in Table 2-25. The \% relative difference (\% Rel Diff) for each element for each group is also provided. Note that all of the \% relative differences (or \% biases) of the measured versus reference values are less than or equal to 3.12\% for all of the elements present in ARG-1 at concentrations of at least $0.1 \mathrm{wt} \%$. Also, note that the bias in the Si values generated using the Fusion prep is positive (i.e., the Si measurements are slightly greater than the ARG-1 reference value for this element). Recall that the Si bias for the Mixed Acid prep was negative. These results support the current DWPF Engineering approach to estimating the Si content in process samples by averaging the Si measurements from the two preparation methods.

Table 2-25 Summary Statistics for ARG-1 Fusion Measurements

\begin{tabular}{|c|c|c|c|c|c|c|c|c|c|}
\hline & & \multicolumn{4}{|c|}{ All of the Available Data $(\mathrm{N}=712)$} & \multicolumn{4}{|c|}{ "Data for SME \& MFT Batches $(\mathrm{N}=596)$} \\
\hline & Ref & Mean & \% Rel & Std & \% Rel & Mean & \% Rel & Std & \% Rel \\
\hline Element & Value & $\mathrm{wt} \%$ & Diff & Dev & Std Dev & $\mathrm{wt} \%$ & Diff & Dev & Std Dev \\
\hline $\mathrm{Al}(\mathrm{wt} \%)$ & 2.5 & 2.462 & $-1.51 \%$ & 0.118 & $4.79 \%$ & 2.465 & $-1.41 \%$ & 0.077 & $3.13 \%$ \\
\hline $\mathrm{B}(\mathrm{wt} \%)$ & 2.69 & 2.634 & $-2.09 \%$ & 0.125 & $4.74 \%$ & 2.635 & $-2.05 \%$ & 0.075 & $2.83 \%$ \\
\hline $\mathrm{Ca}(\mathrm{wt} \%)$ & 1.02 & 0.989 & $-3.02 \%$ & 0.068 & $6.89 \%$ & 0.989 & $-2.99 \%$ & 0.055 & $5.57 \%$ \\
\hline $\mathrm{Cr}(\mathrm{wt} \%)$ & 0.06 & 0.074 & $22.67 \%$ & 0.011 & $15.35 \%$ & 0.073 & $22.03 \%$ & 0.009 & $12.33 \%$ \\
\hline $\mathrm{Cu}(\mathrm{wt} \%)$ & 0.0001 & 0.010 & & 0.055 & & 0.010 & & 0.060 & \\
\hline $\mathrm{Fe}(\mathrm{wt} \%)$ & 9.79 & 9.567 & $-2.28 \%$ & 0.436 & $4.56 \%$ & 9.557 & $-2.38 \%$ & 0.261 & $2.73 \%$ \\
\hline $\mathrm{K}(\mathrm{wt} \%)$ & 2.25 & 2.226 & $-1.08 \%$ & 0.123 & $5.55 \%$ & 2.222 & $-1.24 \%$ & 0.095 & $4.30 \%$ \\
\hline $\mathrm{Li}(\mathrm{wt} \%)$ & 1.49 & 1.460 & $-2.03 \%$ & 0.067 & $4.56 \%$ & 1.459 & $-2.08 \%$ & 0.039 & $2.65 \%$ \\
\hline $\mathrm{Mg}(\mathrm{wt} \%)$ & 0.52 & 0.508 & $-2.33 \%$ & 0.025 & $4.91 \%$ & 0.507 & $-2.42 \%$ & 0.017 & $3.26 \%$ \\
\hline Mn (wt\%) & 1.46 & 1.416 & $-3.04 \%$ & 0.066 & $4.68 \%$ & 1.414 & $-3.12 \%$ & 0.041 & $2.90 \%$ \\
\hline $\mathrm{Ni}(\mathrm{wt} \%)$ & 0.83 & 0.808 & $-2.66 \%$ & 0.041 & $5.12 \%$ & 0.807 & $-2.76 \%$ & 0.029 & $3.63 \%$ \\
\hline Si (wt\%) & 22.39 & 22.965 & $2.57 \%$ & 1.173 & $5.11 \%$ & 22.978 & $2.63 \%$ & 0.770 & $3.35 \%$ \\
\hline $\mathrm{Ti}(\mathrm{wt} \%)$ & 0.69 & 0.675 & $-2.15 \%$ & 0.032 & $4.68 \%$ & 0.674 & $-2.25 \%$ & 0.019 & $2.88 \%$ \\
\hline
\end{tabular}

Exhibit A29 in the Appendix provides an investigation into sources of variation for the replicated ARG-1 measurements generated from samples prepared using the Fusion method within each block of SME and MFT analyses. The results are summarized in Table 2-26.

Table 2-26 Components of Variation for ARG-1 Results for Fusion Samples

\begin{tabular}{|c|c|c|c|c|c|c|c|c|c|}
\hline & Mean & & & & & & \%RSD & \%RSD & \%RSD \\
\hline Element & wt $\%$ & Batch & Residual & Total & \% Batch & \% Residual & Batch & Residual & Total \\
\hline $\mathrm{Al}(\mathrm{wt} \%)$ & 2.467 & 0.001728 & 0.004295 & 0.006023 & $28.69 \%$ & $71.31 \%$ & $1.69 \%$ & $2.66 \%$ & $3.15 \%$ \\
\hline $\mathrm{B}(\mathrm{wt} \%)$ & 2.635 & 0.000741 & 0.004753 & 0.005494 & $13.49 \%$ & $86.51 \%$ & $1.03 \%$ & $2.62 \%$ & $2.81 \%$ \\
\hline Ca (wt\%) & 0.991 & 0.001182 & 0.001822 & 0.003004 & $39.35 \%$ & $60.65 \%$ & $3.47 \%$ & $4.31 \%$ & $5.53 \%$ \\
\hline $\mathrm{Cr}(\mathrm{wt} \%)$ & 0.073 & 0.000028 & 0.000050 & 0.000078 & $35.90 \%$ & $64.10 \%$ & $7.23 \%$ & $9.67 \%$ & $12.07 \%$ \\
\hline $\mathrm{Cu}(\mathrm{wt} \%)$ & 0.0105 & 0.000002 & 0.003989 & 0.003991 & $0.05 \%$ & $99.95 \%$ & & & \\
\hline $\mathrm{Fe}(\mathrm{wt} \%)$ & 9.563 & 0.005424 & 0.060836 & 0.066260 & $8.19 \%$ & $91.81 \%$ & $0.77 \%$ & $2.58 \%$ & $2.69 \%$ \\
\hline $\mathrm{K}(\mathrm{wt} \%)$ & 2.223 & 0.005712 & 0.003838 & 0.009550 & $59.81 \%$ & $40.19 \%$ & $3.40 \%$ & $2.79 \%$ & $4.40 \%$ \\
\hline $\mathrm{Li}$ (wt\%) & 1.461 & 0.000163 & 0.001309 & 0.001472 & $11.07 \%$ & $88.93 \%$ & $0.87 \%$ & $2.48 \%$ & $2.63 \%$ \\
\hline $\mathrm{Mg}(\mathrm{wt} \%)$ & 0.508 & 0.000063 & 0.000202 & 0.000265 & $23.77 \%$ & $76.23 \%$ & $1.56 \%$ & $2.80 \%$ & $3.21 \%$ \\
\hline Mn (wt\%) & 1.415 & 0.000171 & 0.001492 & 0.001663 & $10.28 \%$ & $89.72 \%$ & $0.92 \%$ & $2.73 \%$ & $2.88 \%$ \\
\hline $\mathrm{Ni}$ (wt\%) & 0.808 & 0.000086 & 0.000784 & 0.000870 & $9.89 \%$ & $90.11 \%$ & $1.15 \%$ & $3.47 \%$ & $3.65 \%$ \\
\hline $\mathrm{Si}(\mathrm{wt} \%)$ & 22.983 & 0.239982 & 0.348987 & 0.588969 & $40.75 \%$ & $59.25 \%$ & $2.13 \%$ & $2.57 \%$ & $3.34 \%$ \\
\hline $\mathrm{Ti}(\mathrm{wt} \%)$ & 0.675 & 0.000028 & 0.000347 & 0.000375 & $7.47 \%$ & $92.53 \%$ & $0.78 \%$ & $2.76 \%$ & $2.87 \%$ \\
\hline
\end{tabular}




\subsection{LIMS LIMITS}

Section 1 provided a discussion of the current LIMS (Laboratory Information Management System) limits utilized by the DWPF Lab to screen for errors in the measurements of the standards discussed in this report. For the calibration and bench standards, the relative error was $+/-7 \%$. For ARG-1 the limits, which depend on the preparation method used, are provided in Table 1-1.

In this section, the results of the previous sections are revisited with an emphasis on comparisons between the LIMS limits and the uncertainties in the measurements of the standards as estimated in this study. The uncertainties are estimated by combining the biases and standard deviations presented earlier in one of two ways as outlined in [6]: (1) using a root sum square (RSS) approach to estimate the uncertainty at approximately a 95\% confidence level and (2) using a additive approach to estimate the uncertainty at approximately a $99 \%$ confidence level.

The approaches may best be explained with the help of an example. Consider the \% relative bias, $-1.0 \%$, of the Al measurements of the IN33 standard for the Cold Chem results of Table 2-1. Since this is a negative bias, a lower bound on the true $\mathrm{Al}$ bias for this standard can be computed using the sample size of $n=432$ from Table 2-1 and the corresponding \% relative standard deviation, $\% \mathrm{RSD}=1.5 \%$, from Table 2-2. Representing the $-1.0 \%$ relative bias as the $\%$ sample mean bias, $\% \overline{\mathrm{x}}$, the equation for the lower bound, \%B, (at the $95 \%$ confidence level) for the bias is given by

$$
\% \mathrm{~B}=\% \overline{\mathrm{x}}-\mathrm{t}_{0.05,431} \cdot \frac{(\% \mathrm{RSD})}{\sqrt{432}}=-1.0-1.645 \cdot \frac{1.5}{20.7}=-1.0-0.12=-1.12 \%
$$

Using the RSS approach as outlined in [6], the uncertainty in the Al measurement of the IN33 standard under the Cold Chem protocol at a 95\% confidence level is given by:

$$
\text { Uncertainty }_{95 \%}=\sqrt{\% \mathrm{~B}^{2}+(2 \cdot \% \mathrm{RSD})^{2}}=\sqrt{(-1.12 \%)^{2}+(2 \cdot 1.5 \%)^{2}}=\sqrt{10.2544}=3.20 \%
$$

Note that for this situation, the estimated bias is negative, so the uncertainty is asymmetrical and this estimates bounds the errors on the low side with $95 \%$ confidence. When the uncertainty is expressed as $+/-3.2 \%$, it conservatively bounds the potential positive errors as well.

Using the additive approach as outlined in [6], the uncertainty of negative errors in the $\mathrm{Al}$ measurement of the IN33 standard under the Cold Chem protocol at a 99\% confidence level is given by:

$$
\text { Uncertainty }_{99 \%}=\% \mathrm{~B}-(2 \cdot \% \mathrm{RSD})=-1.0-(2 \cdot 1.5)=-4.0 \%
$$

Thus, the uncertainty may be stated as $+/-4.0 \%$ with $99 \%$ confidence.

\subsubsection{Measurement Uncertainties of Calibration and Bench Standards}

This approach was used to determine uncertainties for the measurements of the active elements (i.e., those at non-zero concentrations) of the calibration and bench standards discussed in this report. Situations where the bias is positive are handled in a similar manner. For the elements at zero concentrations in the standards (i.e., not present in the standard), the measurements are in the noise associated with the detection limits of the instrumentation. The uncertainties for these measurements were determined in a manner similar to that just described except instead of expressing the uncertainty in percent relative error, 
the uncertainties are expressed in the same units as the measurements (i.e., mg/L). One other modification in the method of determining uncertainty at a 99\% confidence level was introduced for these measurements at a nominal zero concentration. The uncertainty at this confidence level was determined as the maximum of the upper sample mean bias plus 2 times the sample standard deviation and 3 times the sample standard deviation. Thus,

$$
\text { Uncertainty }_{99 \%}=\operatorname{MAXIMUM}(\mathrm{B}+2 \cdot \mathrm{s}, 3 \cdot \mathrm{s})
$$

where $B$ is the upper bound on the sample mean bias in $\mathrm{mg} / \mathrm{L}$ and $\mathrm{s}$ represents the sample standard deviation in $\mathrm{mg} / \mathrm{L}$.

The uncertainties for the calibration and bench standards were computed using the methods described in this section, and the resulting uncertainties are presented in Table A1 in the Appendix. The measurements summarized in this table are those associated with a process batch. The summary information includes the number of measurements available (n), the reference value for each element of each standard, the sample mean and the sample standard deviation. From these statistics, the average bias and uncertainties are determined and provided in absolute units $(\mathrm{mg} / \mathrm{L})$ if the reference value is 0 or as a percentage relative to the reference value if the value is non-zero. Also, note that the relative standard deviation (RSD) in this table is computed relative to the reference value.

Some highlights from the table follow. The uncertainties of the Na values for the SM33 bench standard (8.4\%) and for the SM35 calibration standard (9.0\%) under the Mixed Acid prep are estimated to be larger than the 7\% LIMS limit as is the uncertainty for B for the SM51 bench standard (15.0\%) and the SM53 calibration standard (14.5\%) under the Fusion prep. The uncertainties estimated for all of the other active elements in these bench and calibration standards are less than the $7 \%$ limit in LIMS.

The uncertainties in the measurements of elements at nominal zero concentrations in these standards may be useful as threshold levels in identifying possible contamination during the analytical process.

\subsubsection{Measurement Uncertainties of ARG-1}

The same approach as that just described was used to determine uncertainties for the measurements of the primary components of the ARG-1 standard glass. The resulting uncertainties are provided in Table 2-27. For the Cold Chem prep, only the 99\% uncertainty for Al exceeds the LIMS limit (13.1\% versus 12\%). For the Mixed Acid prep, the LIMS limits are exceeded by the $99 \%$ uncertainties for $\mathrm{Al}(10.6 \%$ versus $9 \%)$, $\mathrm{Cr}$ (35.7\% versus 35\%), Mn (8.1\% versus $8 \%$ ), Si (12.3\% versus $10 \%)$, Ti (12.1\% versus 9\%), and Zr (39.0\% versus $12 \%)$. 
WSRC-TR-2004-00576

Revision 0

Table 2-27 Uncertainties for ARG-1 Measurements

\begin{tabular}{|c|c|c|c|c|c|c|c|c|c|}
\hline & \multicolumn{3}{|c|}{ Cold Chem Method } & \multicolumn{3}{|c|}{ Mixed Acid Method } & \multicolumn{3}{|c|}{ Fusion Method } \\
\hline & uncertainty & uncertainty & & uncertainty & uncertainty & & uncertainty & uncertainty & \\
\hline & @ 95\% & (a) 99\% & LIMS & @ 95\% & @99\% & LIMS & @ 95\% & @99\% & LIMS \\
\hline Element & Confidence & Confidence & Limits & Confidence & Confidence & Limits & Confidence & Confidence & Limits \\
\hline $\mathrm{Al}$ & $9.31 \%$ & $13.13 \%$ & $12 \%$ & $7.57 \%$ & $10.63 \%$ & $9 \%$ & $6.47 \%$ & $7.89 \%$ & $8 \%$ \\
\hline $\mathrm{B}$ & $7.54 \%$ & $9.52 \%$ & - & - & - & - & $6.09 \%$ & $7.91 \%$ & $11 \%$ \\
\hline $\mathrm{Ca}$ & $17.24 \%$ & $19.74 \%$ & $22 \%$ & $8.49 \%$ & $9.53 \%$ & $13 \%$ & $11.64 \%$ & $14.51 \%$ & $20 \%$ \\
\hline $\mathrm{Cr}$ & $16.26 \%$ & $22.67 \%$ & $35 \%$ & $25.29 \%$ & $35.68 \%$ & $35 \%$ & $33.62 \%$ & $47.51 \%$ & $35 \%$ \\
\hline $\mathrm{Cu}$ & - & - & - & - & - & - & & & - \\
\hline $\mathrm{Fe}$ & $6.11 \%$ & $7.45 \%$ & $15 \%$ & $5.72 \%$ & $7.45 \%$ & $8 \%$ & $6.03 \%$ & $8.03 \%$ & $8 \%$ \\
\hline $\mathrm{K}$ & $6.74 \%$ & $7.53 \%$ & $20 \%$ & $7.65 \%$ & $9.29 \%$ & $11 \%$ & $8.73 \%$ & $10.12 \%$ & $10 \%$ \\
\hline $\mathrm{Li}$ & $6.44 \%$ & $7.15 \%$ & $15 \%$ & $5.25 \%$ & $5.58 \%$ & $8 \%$ & $5.75 \%$ & $7.54 \%$ & $8 \%$ \\
\hline $\mathrm{Mg}$ & $10.89 \%$ & $14.63 \%$ & $17 \%$ & $6.33 \%$ & $6.78 \%$ & $10 \%$ & $7.03 \%$ & $9.16 \%$ & $9 \%$ \\
\hline $\mathrm{Mn}$ & $6.37 \%$ & $8.32 \%$ & $14 \%$ & $6.00 \%$ & $8.11 \%$ & $8 \%$ & $6.68 \%$ & $9.11 \%$ & $8 \%$ \\
\hline $\mathrm{Na}$ & $6.97 \%$ & $8.22 \%$ & $15 \%$ & $6.39 \%$ & $8.26 \%$ & $13 \%$ & & & \\
\hline $\mathrm{Ni}$ & $6.59 \%$ & $8.33 \%$ & $15 \%$ & $6.22 \%$ & $6.74 \%$ & $9 \%$ & $7.85 \%$ & $10.26 \%$ & $10 \%$ \\
\hline $\mathrm{Si}$ & $7.49 \%$ & $8.72 \%$ & $18 \%$ & $9.11 \%$ & $12.33 \%$ & $10 \%$ & $7.28 \%$ & $9.55 \%$ & $9 \%$ \\
\hline $\mathrm{Ti}$ & $6.14 \%$ & $7.49 \%$ & $17 \%$ & $9.76 \%$ & $12.11 \%$ & $9 \%$ & $6.26 \%$ & $8.20 \%$ & $8 \%$ \\
\hline $\mathrm{U}$ & - & - & - & - & - & - & - & - & - \\
\hline $\mathrm{Zr}$ & $10.72 \%$ & $14.40 \%$ & $21 \%$ & $35.61 \%$ & $39.03 \%$ & $12 \%$ & & & \\
\hline
\end{tabular}

The ARG-1 uncertainties from this study may provide an opportunity to update the LIMS limits to reflect the historical results for this standard glass.

\subsection{SUM OF OXIDES}

As a final area of investigation, the measured ARG-1 sums of oxides for each of the three preparation methods are compared to the theoretical values, which are based on the reference concentrations for the standard glass. Exhibit A30 in the Appendix provides time series plots of these data (wt\% oxides) in analytical sequence by preparation method for all of the ARG-1 measurements provided in the datasets. Once again, the plotted values represented by an " $x$ " are measurements that were not associated with a particular process batch. Exhibit A31 provides time series plots of the sum of oxides for ARG-1 measurements associated with a batch of process samples. And, finally, Exhibit A31 in the Appendix provides histograms and descriptive statistics for these sums of oxides. Table 2-28 provides summaries of the information from these exhibits in the Appendix. The reference sum is also provided. The results show that the Cold Chem and Mixed Acid preps sums of oxides, on average, are a little more than $1 \mathrm{wt} \%$ below their reference values while the sum of oxides for the Fusion prep is, on average, a little less than $0.5 \mathrm{wt} \%$ above its reference value. This may be due to the positive Si bias for the Fusion prep and the negative Si bias for the other two preps. 
WSRC-TR-2004-00576

Revision 0

Table 2-28 Descriptive Statistics for ARG-1 Sums of Oxides by Preparation Method

\begin{tabular}{|r|c|c|c|c|c||}
\hline \hline & $\begin{array}{c}\text { Cold Chem- } \\
\text { SRAT-With } \\
\text { Batch }\end{array}$ & $\begin{array}{c}\text { Fusion-MFT- } \\
\text { With Batch }\end{array}$ & $\begin{array}{c}\text { Fusion-SME- } \\
\text { With Batch }\end{array}$ & $\begin{array}{c}\text { Mixed Acid- } \\
\text { MFT-With } \\
\text { Batch }\end{array}$ & $\begin{array}{c}\text { Mixed Acid- } \\
\text { SME-With } \\
\text { Batch }\end{array}$ \\
\hline N Rows & 323 & 278 & 318 & 278 & 319 \\
\hline Average Sum of Oxides & 98.1 & 88.1 & 88.1 & 88.2 & 88.3 \\
\hline Reference Value of Sum of Oxides & 99.3 & 87.7 & 87.7 & 90.6 & 90.6 \\
\hline Std Dev of Sum of Oxides & 3.0 & 2.3 & 2.4 & 2.2 & 2.7 \\
\hline Minimum Sum of Oxides & 86.8 & 80.1 & 79.3 & 79.5 & 71.6 \\
\hline Maximum Sum of Oxides & 114.3 & 98.2 & 99.9 & 96.8 & 105.3 \\
\hline
\end{tabular}

\subsection{MFT VERSUS SME RESULTS}

In the discussion of the sum of oxides, a comparison was offered between the MFT and SME results for both the Mixed Acid and the Fusion preps. Even though the analytical protocol for measuring samples from the MFT is the same as that used for SME samples, measurements of the SME samples are tied to the production schedule while those for the MFT samples are not. Thus, comparisons of measurements of the standards between the two vessels are of interest. This section makes such comparisons for the calibration and bench standards and for ARG-1 for the Mixed Acid and the Fusion preps.

Table A2 in the Appendix provides a summary of the measurements of the calibration and bench standards associated with the Mixed Acid protocol by tank. The means and standard deviations (both expressed as $\mathrm{mg} / \mathrm{L}$ values) of all of the elements are provided in the table. For the active elements, values for the percent relative difference of the MFT average as compared to the SME average are given as are values of the ratio of the MFT standard deviation to that of the SME. The percent relative standard deviations (\%RSDs) for both tanks are also provided for the active elements. The largest \% relative difference is that for Si for the SM35 standard at $0.21 \%$, and ratios of standard deviations show comparable precision between the two tanks in the measurements of the active elements of these standards.

Table A3 in the Appendix provides a summary of the measurements of the calibration and bench standards associated with the Fusion protocol by tank. The means and standard deviations (both expressed as $\mathrm{mg} / \mathrm{L}$ values) of all of the elements are provided in the table. For the active elements, values for the percent relative difference of the MFT average as compared to the SME average are given as are values of the ratio of the MFT standard deviation to that of the SME. The percent relative standard deviations (\%RSDs) for both tanks are also provided for the active elements. The largest \% relative difference is that for B for the SM51 standard at $0.40 \%$, and ratios of standard deviations show comparable precision between the two tanks in the measurements of the active elements of these standards.

Table 2-29 provides similar information for the ARG-1 measurements (as wt\% oxides) for the Mixed Acid prep method by tank. For those oxides at reference concentrations of at least $0.1 \mathrm{wt} \%$ in the ARG-1 standard, the \% differences of the MFT means versus those of the SME are less than $0.25 \%$ except for $\mathrm{ZrO}_{2}$. The \% difference for $\mathrm{ZrO}_{2}$ is $1.28 \%$. For most of the oxides, the precision of the MFT measurements appears to be slightly better than their SME counterparts. 
WSRC-TR-2004-00576

Revision 0

Table 2-29 Descriptive Statistics for ARG-1 Mixed Acid Measurements by Tank

\begin{tabular}{|c|c|c|c|c|c|c|c|c|}
\hline Oxide & $\begin{array}{l}\text { MFT } \\
\text { Mean }\end{array}$ & $\begin{array}{l}\text { SME } \\
\text { Mean }\end{array}$ & \begin{tabular}{|c|}
$\%$ \\
Difference
\end{tabular} & $\begin{array}{c}\text { MFT } \\
\text { Std Dev }\end{array}$ & $\begin{array}{c}\text { SME } \\
\text { Std Dev }\end{array}$ & \begin{tabular}{|l} 
MFT Std Dev/ \\
SME Std Dev
\end{tabular} & $\begin{array}{l}\text { \%RSD } \\
\text { MFT }\end{array}$ & $\begin{array}{l}\text { \%RSD } \\
\text { SME }\end{array}$ \\
\hline $\mathrm{Al}_{2} \mathrm{O}_{3}(\mathrm{wt} \%)$ & 4.511 & 4.513 & $-0.05 \%$ & 0.1173 & 0.1472 & 0.80 & $2.60 \%$ & $3.26 \%$ \\
\hline $\mathrm{CaO}(\mathrm{wt} \%)$ & 1.437 & 1.438 & $-0.06 \%$ & 0.0566 & 0.0638 & 0.89 & $3.94 \%$ & $4.44 \%$ \\
\hline $\mathrm{Cr}_{2} \mathrm{O}_{3}(\mathrm{wt} \%)$ & 0.104 & 0.104 & $-0.23 \%$ & 0.0084 & 0.0088 & 0.96 & $8.13 \%$ & $8.49 \%$ \\
\hline $\mathrm{CuO}(\mathrm{wt} \%)$ & 0.008 & 0.008 & & 0.0051 & 0.0050 & & & \\
\hline $\mathrm{Fe}_{2} \mathrm{O}_{3}(\mathrm{wt} \%)$ & 13.705 & 13.734 & $-0.21 \%$ & 0.3026 & 0.4088 & 0.74 & $2.21 \%$ & $2.98 \%$ \\
\hline $\mathrm{K}_{2} \mathrm{O}(\mathrm{wt} \%)$ & 2.754 & 2.750 & $0.12 \%$ & 0.1007 & 0.1034 & 0.97 & $3.66 \%$ & $3.76 \%$ \\
\hline $\mathrm{Li}_{2} \mathrm{O}(\mathrm{wt} \%)$ & 3.200 & 3.205 & $-0.14 \%$ & 0.0667 & 0.0964 & 0.69 & $2.08 \%$ & $3.01 \%$ \\
\hline $\mathrm{MgO}(\mathrm{wt} \%)$ & 0.860 & 0.860 & $-0.06 \%$ & 0.0241 & 0.0296 & 0.81 & $2.80 \%$ & $3.44 \%$ \\
\hline $\mathrm{MnO}(\mathrm{wt} \%)$ & 1.834 & 1.837 & $-0.14 \%$ & 0.0406 & 0.0549 & 0.74 & $2.21 \%$ & $2.99 \%$ \\
\hline $\mathrm{Na}_{2} \mathrm{O}$ (wt\%) & 11.248 & 11.267 & $-0.16 \%$ & 0.2697 & 0.3848 & 0.70 & $2.40 \%$ & $3.42 \%$ \\
\hline $\mathrm{NiO}$ (wt\%) & 1.059 & 1.059 & $-0.07 \%$ & 0.0278 & 0.0366 & 0.76 & $2.63 \%$ & $3.46 \%$ \\
\hline $\mathrm{SiO}_{2}(\mathrm{wt} \%)$ & 45.947 & 45.990 & $-0.09 \%$ & 1.7495 & 1.9293 & 0.91 & $3.81 \%$ & $4.20 \%$ \\
\hline $\mathrm{Ti}_{2} \mathrm{O}$ (wt\%) & 1.177 & 1.178 & $-0.14 \%$ & 0.0557 & 0.0548 & 1.02 & $4.73 \%$ & $4.65 \%$ \\
\hline $\mathrm{U}_{3} \mathrm{O}_{8}(\mathrm{wt} \%)$ & 0.226 & 0.231 & $-2.05 \%$ & 0.2058 & 0.1759 & 1.17 & $91.00 \%$ & $76.20 \%$ \\
\hline $\mathrm{ZrO}_{2}(\mathrm{wt} \%)$ & 0.133 & 0.131 & $1.28 \%$ & 0.0204 & 0.0256 & 0.80 & $15.39 \%$ & $19.57 \%$ \\
\hline
\end{tabular}

Table 2-30 provides similar information for the ARG-1 measurements (as wt\% oxides) for the Fusion prep method by tank. For those oxides at reference concentrations of at least $0.1 \mathrm{wt} \%$ in the ARG-1 standard, the \% differences of the MFT means versus those of the SME are less than $0.50 \%$ except for $\mathrm{Cr}_{2} \mathrm{O}_{3}$. The \% difference for $\mathrm{Cr}_{2} \mathrm{O}_{3}$ is $1.78 \%$. The precision of the measurements of the standards for the two vessels appears to be comparable.

Table 2-30 Descriptive Statistics for ARG-1 Fusion Measurements by Tank

\begin{tabular}{|c|c|c|c|c|c|c|c|c|}
\hline Oxide & $\begin{array}{l}\text { MFT } \\
\text { Mean }\end{array}$ & $\begin{array}{l}\text { SME } \\
\text { Mean }\end{array}$ & \begin{tabular}{c|}
$\%$ \\
Difference
\end{tabular} & $\begin{array}{c}\text { MFT } \\
\text { Std Dev }\end{array}$ & $\begin{array}{c}\text { SME } \\
\text { Std Dev }\end{array}$ & \begin{tabular}{|c|} 
MFT Std Dev/ \\
SME Std Dev
\end{tabular} & $\begin{array}{l}\text { \%RSD } \\
\text { MFT }\end{array}$ & $\begin{array}{c}\text { \%RSD } \\
\text { SME }\end{array}$ \\
\hline $\mathrm{Al} 2 \mathrm{O} 3$ (wt\%) & 4.657 & 4.657 & $0.01 \%$ & 0.1435 & 0.1483 & 0.97 & $3.08 \%$ & $3.19 \%$ \\
\hline B2O3 (wt\%) & 8.483 & 8.485 & $-0.02 \%$ & 0.2296 & 0.2495 & 0.92 & $2.71 \%$ & $2.94 \%$ \\
\hline $\mathrm{CaO}(\mathrm{wt} \%)$ & 1.381 & 1.387 & $-0.40 \%$ & 0.0779 & 0.0765 & 1.02 & $5.64 \%$ & $5.52 \%$ \\
\hline Cr2O3 (wt\%) & 0.108 & 0.106 & $1.78 \%$ & 0.0150 & 0.0114 & 1.32 & $13.86 \%$ & $10.70 \%$ \\
\hline $\mathrm{CuO}(\mathrm{wt} \%)$ & 0.010 & 0.015 & & 0.0265 & 0.0997 & & & \\
\hline Fe2O3 (wt\%) & 13.651 & 13.675 & $-0.18 \%$ & 0.3730 & 0.3737 & 1.00 & $2.73 \%$ & $2.73 \%$ \\
\hline K2O (wt\%) & 2.672 & 2.681 & $-0.36 \%$ & 0.0912 & 0.1324 & 0.69 & $3.41 \%$ & $4.94 \%$ \\
\hline Li2O (wt\%) & 3.143 & 3.140 & $0.12 \%$ & 0.0831 & 0.0832 & 1.00 & $2.64 \%$ & $2.65 \%$ \\
\hline MgO (wt\%) & 0.842 & 0.841 & $0.16 \%$ & 0.0273 & 0.0276 & 0.99 & $3.24 \%$ & $3.28 \%$ \\
\hline $\mathrm{MnO}(\mathrm{wt} \%)$ & 1.824 & 1.828 & $-0.25 \%$ & 0.0533 & 0.0526 & 1.01 & $2.92 \%$ & $2.87 \%$ \\
\hline $\mathrm{NiO}$ (wt\%) & 1.030 & 1.025 & $0.50 \%$ & 0.0398 & 0.0348 & 1.14 & $3.86 \%$ & $3.40 \%$ \\
\hline $\mathrm{SiO} 2(\mathrm{wt} \%)$ & 49.196 & 49.122 & $0.15 \%$ & 1.5856 & 1.7005 & 0.93 & $3.22 \%$ & $3.46 \%$ \\
\hline Ti2O (wt\%) & 1.125 & 1.125 & $-0.01 \%$ & 0.0339 & 0.0311 & 1.09 & $3.01 \%$ & $2.77 \%$ \\
\hline
\end{tabular}




\subsection{CONCLUSIONS}

Measurements of calibration and bench standards as well as samples of ARG-1 that were performed by the DWPF Lab during the processing of batches 94 through 263 were provided to SCS for review. Three datasets, one associated with each of three preparation methods (Cold Chem, Mixed Acid, and Fusion), were included in the review.

The review conducted covered several areas of investigation. Biases in these measurements relative to the reference values for the standards including ARG-1 were estimated and found to be of no practical concern. Percent relative standard deviations for these data also were determined. Sources of variation in the measurements (i.e., batch-to-batch and within process batch) were estimated and compared. An investigation into evidence of instrument drift during a group of measurements representing a prototypical block was conducted for each preparation method using pairs of calibration and bench standards. No evidence of instrument drift at levels of practical concern was seen in these data. Also, the replicates of calibration standards at the beginning of each analytical block were found to be unnecessary.

The uncertainties of the measurements, which incorporated biases and precision errors, were computed for the calibration standards, bench standards, and ARG-1. The limits for these uncertainties were compared to the current, LIMS operating limits for the errors in the measurements of these standards. These comparisons may provide opportunities for revising these LIMS limits.

Comparisons between MFT and SME results were made for both the Mixed Acid and the Fusion prep methods. There was no evidence of differences (in either bias or precision) between the results for these two tanks for either of the two prep methods. 


\subsection{REFERENCES}

[1] Edwards, T.B., "Statistical Review of Composition Data from DWPF’s Process Samples for Macro-Batch 1 (U),” WSRC-RP-2000-00174, Revision 0, February 15, 2000.

[2] Edwards, T.B., “A Statistical Review of Analytical Laboratory Measurements from DWPF’s Macrobatch 1and Macrobatch 2 (U),” WSRC-TR-2003-00045, Revision 0, January 22, 2003.

[3] Harris, S.P., "Statistical Analysis of DWPF ARG-1 Data (U),” WSRC-TR-2000-00070, May 24, 2000.

[4] Patel, P.M., “Technical Task Request: Statistical Analysis of DWPF Process Data and Lab Analytical Data (U),” HLW/DWPF/TTR-03-0020, Revision 0, December 9, 2003.

[5] SAS Institute, JMP®: Statistics and Graphics Guide, Version 5, SAS Institute, Inc., Cary, NC, 2002.

[6] Coleman, H.W. and W. G. Steele, Jr., Experimental and Uncertainty Analysis for Engineers, John Wiley \& Sons, Inc., New York, 1989. 
WSRC-TR-2004-00576

Revision 0

This page intentionally left blank. 
WSRC-TR-2004-00576

Revision 0

APPENDIX A. 
WSRC-TR-2004-00576

Revision 0

This page intentionally left blank. 
WSRC-TR-2004-00576

Revision 0

Table A1. Measurement Uncertainties for Calibration and Bench Standards

\begin{tabular}{|c|c|c|c|c|c|c|c|c|c|c|c|c|c|c|c|}
\hline & & & & & & & & & 95\% Abs. & & & & & & \\
\hline & & & & & Abs. & & Std & & Bound & $95 \%$ & $99 \%$ & 95\% Rel. & $95 \%$ & $99 \%$ & \\
\hline & & & Ref. & Sample & Bias & & Dev & & for Bias & Uncert. & Uncert. & Bound & Rel. & Rel. & LIMS \\
\hline Std. & Elem. & $\mathbf{n}$ & Value & Mean & $\mathrm{mg} / \mathrm{L}$ & \%Bias & $\mathrm{mg} / \mathrm{L}$ & \% RSD & $\mathrm{mg} / \mathrm{L}$ & $\mathrm{mg} / \mathrm{L}$ & $\mathrm{mg} / \mathrm{L}$ & for Bias & \begin{tabular}{|l} 
Uncert. \\
\end{tabular} & Uncert. & Limits \\
\hline IN32 & $\mathrm{Al}$ & 344 & 0 & 0.017 & 0.017 & & 0.031 & & 0.020 & 0.065 & 0.093 & & & & \\
\hline IN32 & $B$ & 344 & 0 & 0.000 & 0.000 & & 0.035 & & 0.003 & 0.069 & 0.104 & & & & \\
\hline IN32 & $\mathrm{Ca}$ & 344 & 0 & 0.116 & 0.116 & & 0.111 & & 0.126 & 0.255 & 0.348 & & & & \\
\hline IN32 & $\mathrm{Cr}$ & 344 & 0 & 0.002 & 0.002 & & 0.006 & & 0.003 & 0.013 & 0.019 & & & & \\
\hline IN32 & $\mathrm{Cu}$ & 344 & 0 & 0.001 & 0.001 & & 0.004 & & 0.001 & 0.009 & 0.013 & & & & \\
\hline IN32 & $\mathrm{Fe}$ & 344 & 0 & 0.004 & 0.004 & & 0.080 & & 0.011 & 0.161 & 0.241 & & & & \\
\hline IN32 & $\mathrm{K}$ & 344 & 0 & 0.038 & 0.038 & & 0.066 & & 0.044 & 0.139 & 0.197 & & & & \\
\hline IN32 & $\mathrm{Li}$ & 344 & 0 & 0.002 & 0.002 & & 0.002 & & 0.002 & 0.005 & 0.007 & & & & \\
\hline IN32 & $\mathrm{Mg}$ & 344 & 0 & 0.001 & 0.001 & & 0.005 & & 0.002 & 0.010 & 0.014 & & & & \\
\hline IN32 & Mn & 344 & 0 & 0.001 & 0.001 & & 0.011 & & 0.002 & 0.023 & 0.034 & & & & \\
\hline IN32 & $\mathrm{Na}$ & 344 & 0 & 0.020 & 0.020 & & 0.087 & & 0.028 & 0.176 & 0.261 & & & & \\
\hline IN32 & $\mathrm{Ni}$ & 344 & 0 & 0.002 & 0.002 & & 0.008 & & 0.003 & 0.017 & 0.025 & & & & \\
\hline IN32 & $\mathrm{Si}$ & 344 & 0 & -0.085 & -0.085 & & 0.526 & & 0.047 & 1.052 & 1.577 & & & & \\
\hline IN32 & $\mathrm{Ti}$ & 344 & 0 & 0.001 & 0.001 & & 0.002 & & 0.001 & 0.004 & 0.005 & & & & \\
\hline IN32 & $\mathrm{U}$ & 344 & 0 & 0.109 & 0.109 & & 0.186 & & 0.126 & 0.393 & 0.558 & & & & \\
\hline IN32 & $\mathrm{Zr}$ & 344 & 0 & $\begin{array}{l}-0.002 \\
\end{array}$ & -0.002 & & 0.005 & & 0.000 & 0.009 & 0.014 & & & & \\
\hline IN33 & $\mathrm{Al}$ & 180 & 10 & 9.885 & -0.115 & $-1.15 \%$ & 0.150 & $1.50 \%$ & & & & $-1.33 \%$ & $2.78 \%$ & $4.15 \%$ & $7 \%$ \\
\hline IN33 & $\mathrm{B}$ & 180 & 0 & -0.012 & -0.012 & & 0.038 & & 0.005 & 0.076 & 0.113 & & & & \\
\hline IN33 & $\mathrm{Ca}$ & 180 & 2 & 1.994 & -0.006 & $-0.32 \%$ & 0.043 & $2.17 \%$ & & & & $-0.59 \%$ & $4.34 \%$ & $4.67 \%$ & $7 \%$ \\
\hline IN33 & $\mathrm{Cr}$ & 180 & 2 & 1.983 & -0.017 & $-0.87 \%$ & 0.032 & $1.59 \%$ & & & & $-1.07 \%$ & $3.06 \%$ & $4.05 \%$ & $7 \%$ \\
\hline IN33 & $\mathrm{Cu}$ & 180 & 1 & 0.993 & -0.007 & $-0.70 \%$ & 0.018 & $1.78 \%$ & & & & $-0.92 \%$ & $3.49 \%$ & $4.26 \%$ & $7 \%$ \\
\hline IN33 & $\mathrm{Fe}$ & 180 & 20 & 19.821 & -0.179 & $-0.90 \%$ & 0.305 & $1.52 \%$ & & & & $-1.08 \%$ & $2.91 \%$ & $3.94 \%$ & $7 \%$ \\
\hline IN33 & $\mathrm{K}$ & 180 & 0 & 0.003 & 0.003 & & 0.060 & & 0.010 & 0.120 & 0.180 & & & & \\
\hline IN33 & $\mathrm{Li}$ & 180 & 0 & 0.001 & 0.001 & & 0.002 & & 0.001 & 0.005 & 0.007 & & & & \\
\hline IN33 & $\mathrm{Mg}$ & 180 & 1 & 1.002 & 0.002 & $0.23 \%$ & 0.024 & $2.43 \%$ & & & & $0.53 \%$ & $4.87 \%$ & $5.09 \%$ & $7 \%$ \\
\hline IN33 & $\mathrm{Mn}$ & 180 & 1 & 0.991 & -0.009 & $-0.87 \%$ & 0.017 & $1.66 \%$ & & & & $-1.08 \%$ & $3.21 \%$ & $4.20 \%$ & $7 \%$ \\
\hline IN33 & $\mathrm{Na}$ & 180 & 20 & 19.857 & -0.143 & $-0.72 \%$ & 0.366 & $1.83 \%$ & & & & $-0.94 \%$ & $3.59 \%$ & $4.37 \%$ & $7 \%$ \\
\hline IN33 & $\mathrm{Ni}$ & 180 & 2 & 1.968 & -0.032 & $-1.61 \%$ & 0.037 & $1.83 \%$ & & & & $-1.84 \%$ & $3.29 \%$ & $5.27 \%$ & $7 \%$ \\
\hline IN33 & $\mathrm{Si}$ & 180 & 0 & -0.269 & -0.269 & & 0.514 & & 0.063 & 1.031 & 1.543 & & & & \\
\hline IN33 & $\mathrm{Ti}$ & 180 & 2 & 1.973 & -0.027 & $-1.35 \%$ & 0.033 & $1.65 \%$ & & & & $-1.55 \%$ & $3.02 \%$ & $4.65 \%$ & $7 \%$ \\
\hline IN33 & $\mathrm{U}$ & 180 & 0 & 0.064 & 0.064 & & 0.179 & & 0.086 & 0.368 & 0.537 & & & & \\
\hline IN33 & $\mathrm{Zr}$ & 180 & 2 & 2.000 & 0.000 & $-0.01 \%$ & 0.040 & $2.02 \%$ & & & & $-0.26 \%$ & $4.05 \%$ & $4.05 \%$ & $7 \%$ \\
\hline IN34 & $\mathrm{Al}$ & 177 & 0 & 0.001 & 0.001 & & 0.030 & & 0.004 & 0.060 & 0.089 & & & & \\
\hline IN34 & $\mathrm{B}$ & 177 & 10 & 10.007 & 0.007 & $0.07 \%$ & 0.136 & $1.36 \%$ & & & & $0.24 \%$ & $2.72 \%$ & $2.79 \%$ & $7 \%$ \\
\hline IN34 & $\mathrm{Ca}$ & 177 & 0 & 0.087 & 0.087 & & 0.069 & & 0.095 & 0.168 & 0.234 & & & & \\
\hline IN34 & $\mathrm{Cr}$ & 177 & 0 & 0.001 & 0.001 & & 0.003 & & 0.001 & 0.007 & 0.010 & & & & \\
\hline IN34 & $\mathrm{Cu}$ & 177 & 0 & 0.000 & 0.000 & & 0.005 & & 0.001 & 0.009 & 0.014 & & & & \\
\hline IN34 & $\mathrm{Fe}$ & 177 & 0 & 0.011 & 0.011 & & 0.050 & & 0.017 & 0.102 & 0.150 & & & & \\
\hline IN34 & $\mathrm{K}$ & 177 & 10 & 9.897 & -0.103 & $-1.03 \%$ & 0.144 & $1.44 \%$ & & & & $-1.20 \%$ & $2.70 \%$ & $3.91 \%$ & $7 \%$ \\
\hline IN34 & $\mathrm{Li}$ & 177 & 10 & 9.938 & -0.062 & $-0.62 \%$ & 0.126 & $1.26 \%$ & & & & $-0.78 \%$ & $2.44 \%$ & $3.14 \%$ & $7 \%$ \\
\hline IN34 & $\mathrm{Mg}$ & 177 & 0 & 0.005 & 0.005 & & 0.016 & & 0.007 & 0.033 & 0.048 & & & & \\
\hline IN34 & $\mathrm{Mn}$ & 177 & 0 & 0.006 & 0.006 & & 0.021 & & 0.009 & 0.043 & 0.063 & & & & \\
\hline IN34 & $\mathrm{Na}$ & 177 & 65 & 65.930 & 0.930 & $1.43 \%$ & 1.731 & $2.66 \%$ & & & & $1.76 \%$ & $5.51 \%$ & $6.76 \%$ & $7 \%$ \\
\hline IN34 & $\mathrm{Ni}$ & 177 & 0 & 0.001 & 0.001 & & 0.007 & & 0.002 & 0.014 & 0.021 & & & & \\
\hline IN34 & $\mathrm{Si}$ & 177 & 40 & 40.008 & 0.008 & $0.02 \%$ & 0.747 & $1.87 \%$ & & & & $0.25 \%$ & $3.74 \%$ & $3.76 \%$ & $7 \%$ \\
\hline IN34 & $\mathrm{Ti}$ & 177 & 0 & 0.003 & 0.003 & & 0.004 & & 0.003 & 0.009 & 0.013 & & & & \\
\hline IN34 & $\mathrm{U}$ & 177 & 0 & 0.034 & 0.034 & & 0.179 & & 0.056 & 0.362 & 0.537 & & & & \\
\hline IN34 & $\mathrm{Zr}$ & 177 & 0 & 0.009 & 0.009 & & 0.030 & & 0.013 & 0.061 & 0.089 & & & & \\
\hline IN35 & $\mathrm{Al}$ & 370 & 10 & 9.978 & -0.022 & $-0.22 \%$ & 0.141 & $1.41 \%$ & & & & $-0.34 \%$ & $2.81 \%$ & $3.03 \%$ & $7 \%$ \\
\hline IN35 & B & 370 & 0 & -0.004 & -0.004 & & 0.038 & & 0.003 & 0.077 & 0.115 & & & & \\
\hline IN35 & $\mathrm{Ca}$ & 370 & 2 & 1.996 & -0.004 & $-0.20 \%$ & 0.046 & $2.32 \%$ & & & & $-0.40 \%$ & $4.63 \%$ & $4.84 \%$ & $7 \%$ \\
\hline IN35 & $\mathrm{Cr}$ & 370 & 2 & 2.002 & 0.002 & $0.09 \%$ & 0.030 & $1.49 \%$ & & & & $0.22 \%$ & $2.98 \%$ & $3.06 \%$ & $7 \%$ \\
\hline IN35 & $\mathrm{Cu}$ & 370 & 1 & 1.005 & 0.005 & $0.49 \%$ & 0.016 & $1.57 \%$ & & & & $0.63 \%$ & $3.19 \%$ & $3.64 \%$ & $7 \%$ \\
\hline IN35 & $\mathrm{Fe}$ & 370 & 20 & 19.980 & -0.020 & $-0.10 \%$ & 0.277 & $1.38 \%$ & & & & $-0.22 \%$ & $2.76 \%$ & $2.86 \%$ & $7 \%$ \\
\hline IN35 & $\mathrm{K}$ & 370 & 0 & 0.018 & 0.018 & & 0.075 & & 0.024 & 0.152 & 0.225 & & & & \\
\hline IN35 & $\mathrm{Li}$ & 370 & 0 & 0.001 & 0.001 & & 0.004 & & 0.002 & 0.008 & 0.011 & & & & \\
\hline IN35 & $\mathrm{Mg}$ & 370 & 1 & 1.009 & 0.009 & $0.91 \%$ & 0.023 & $2.26 \%$ & & & & $1.11 \%$ & $4.62 \%$ & $5.44 \%$ & $7 \%$ \\
\hline IN35 & Mn & 370 & 1 & 1.001 & 0.001 & $0.12 \%$ & 0.016 & $1.64 \%$ & & & & $0.26 \%$ & $3.28 \%$ & $3.39 \%$ & $7 \%$ \\
\hline IN35 & $\mathrm{Na}$ & 370 & 20 & 19.976 & -0.024 & $-0.12 \%$ & 0.365 & $1.83 \%$ & & & & $-0.28 \%$ & $3.65 \%$ & $3.77 \%$ & $7 \%$ \\
\hline IN35 & $\mathrm{Ni}$ & 370 & 2 & 1.986 & -0.014 & $-0.69 \%$ & 0.034 & $1.72 \%$ & & & & $-0.84 \%$ & $3.36 \%$ & $4.13 \%$ & $7 \%$ \\
\hline IN35 & $\mathrm{Si}$ & 370 & 0 & -0.600 & -0.600 & & 0.480 & & 0.041 & 0.961 & 1.441 & & & & \\
\hline
\end{tabular}


WSRC-TR-2004-00576

Revision 0

Table A1. Measurement Uncertainties for Calibration and Bench Standards

\begin{tabular}{|c|c|c|c|c|c|c|c|c|c|c|c|c|c|c|c|}
\hline & & & & & & & & & 95\% Abs. & & & & & & \\
\hline & & & & & Abs. & & Std & & Bound & $95 \%$ & $99 \%$ & \begin{tabular}{|l} 
95\% Rel. \\
\end{tabular} & $95 \%$ & $99 \%$ & \\
\hline & & & Ref. & Sample & Bias & & Dev & & for Bias & Uncert. & Uncert. & Bound & Rel. & Rel. & LIMS \\
\hline Std. & Elem. & $\mathrm{n}$ & Value & Mean & $\mathrm{mg} / \mathrm{L}$ & \%Bias & $\mathrm{mg} / \mathrm{L}$ & $\%$ RSD & $\mathrm{mg} / \mathrm{L}$ & $\mathrm{mg} / \mathrm{L}$ & $\mathrm{mg} / \mathrm{L}$ & \begin{tabular}{|l|} 
for Bias \\
\end{tabular} & Uncert. & Uncert. & Limits \\
\hline IN35 & $\mathrm{Ti}$ & 370 & 2 & 1.990 & -0.010 & \begin{tabular}{|l|}
$-0.52 \%$ \\
\end{tabular} & 0.029 & $1.45 \%$ & & & & $-0.64 \%$ & \begin{tabular}{|l}
$2.85 \%$ \\
\end{tabular} & $3.42 \%$ & $7 \%$ \\
\hline IN35 & $\mathrm{U}$ & 370 & 0 & 0.117 & 0.117 & & 0.277 & & 0.140 & 0.572 & 0.832 & & & & \\
\hline IN35 & $\mathrm{Zr}$ & 370 & 2 & 2.015 & 0.015 & \begin{tabular}{|l|}
$0.77 \%$ \\
\end{tabular} & 0.039 & $1.95 \%$ & & & & $0.93 \%$ & $3.98 \%$ & $4.67 \%$ & $7 \%$ \\
\hline IN36 & $\mathrm{Al}$ & 366 & 0 & 0.005 & 0.005 & & 0.023 & & 0.007 & 0.046 & 0.068 & & & & \\
\hline IN36 & $\mathrm{B}$ & 366 & 10 & 10.052 & 0.052 & \begin{tabular}{|l|}
$0.52 \%$ \\
\end{tabular} & 0.110 & $1.10 \%$ & & & & $0.61 \%$ & $2.26 \%$ & $2.71 \%$ & $7 \%$ \\
\hline IN36 & $\mathrm{Ca}$ & 366 & 0 & 0.086 & 0.086 & & 0.092 & & 0.094 & 0.206 & 0.277 & & & & \\
\hline IN36 & $\mathrm{Cr}$ & 366 & 0 & 0.001 & 0.001 & & 0.003 & & 0.001 & 0.006 & 0.009 & & & & \\
\hline IN36 & $\mathrm{Cu}$ & 366 & 0 & 0.000 & 0.000 & & 0.004 & & 0.001 & 0.007 & 0.011 & & & & \\
\hline IN36 & $\mathrm{Fe}$ & 366 & 0 & 0.009 & 0.009 & & 0.048 & & 0.013 & 0.096 & 0.143 & & & & \\
\hline IN36 & $\mathrm{K}$ & 366 & 10 & 9.937 & -0.063 & $-0.63 \%$ & 0.120 & $1.20 \%$ & & & & $-0.74 \%$ & $2.32 \%$ & $3.04 \%$ & $7 \%$ \\
\hline IN36 & $\mathrm{Li}$ & 366 & 10 & 9.971 & -0.029 & \begin{tabular}{|l|}
$-0.29 \%$ \\
\end{tabular} & 0.109 & $1.09 \%$ & & & & $-0.38 \%$ & $2.16 \%$ & $2.46 \%$ & $7 \%$ \\
\hline IN36 & $\mathrm{Mg}$ & 366 & 0 & 0.005 & 0.005 & & 0.016 & & 0.006 & 0.033 & 0.048 & & & & \\
\hline IN36 & Mn & 366 & 0 & 0.006 & 0.006 & & 0.021 & & 0.008 & 0.044 & 0.064 & & & & \\
\hline IN36 & $\mathrm{Na}$ & 366 & 65 & 66.133 & 1.133 & \begin{tabular}{|l|}
$1.74 \%$ \\
\end{tabular} & 1.619 & $2.49 \%$ & & & & $1.96 \%$ & $5.28 \%$ & $6.72 \%$ & $7 \%$ \\
\hline IN36 & $\mathrm{Ni}$ & 366 & 0 & 0.001 & 0.001 & & 0.007 & & 0.001 & 0.015 & 0.022 & & & & \\
\hline IN36 & $\mathrm{Si}$ & 366 & 40 & 40.316 & 0.316 & \begin{tabular}{|l|}
$0.79 \%$ \\
\end{tabular} & 0.724 & $1.81 \%$ & & & & $0.95 \%$ & $3.71 \%$ & $4.41 \%$ & $7 \%$ \\
\hline IN36 & $\mathrm{Ti}$ & 366 & 0 & 0.003 & 0.003 & & 0.004 & & 0.003 & 0.009 & 0.013 & & & & \\
\hline IN36 & $\mathrm{U}$ & 366 & 0 & 0.064 & 0.064 & & 0.150 & & 0.077 & 0.310 & 0.450 & & & & \\
\hline IN36 & $\mathrm{Zr}$ & 366 & 0 & 0.010 & 0.010 & & 0.030 & & 0.012 & 0.062 & 0.091 & & & & \\
\hline IN38 & $\mathrm{Al}$ & 120 & 0 & 0.021 & 0.021 & & 0.025 & & 0.025 & 0.056 & 0.076 & & & & \\
\hline IN38 & $\mathrm{B}$ & 120 & 0 & 0.002 & 0.002 & & 0.037 & & 0.007 & 0.074 & 0.110 & & & & \\
\hline IN38 & $\mathrm{Ca}$ & 120 & 0 & 0.241 & 0.241 & & 0.215 & & 0.274 & 0.509 & 0.703 & & & & \\
\hline IN38 & $\mathrm{Cr}$ & 120 & 0 & 0.028 & 0.028 & & 0.004 & & 0.029 & 0.030 & 0.038 & & & & \\
\hline IN38 & $\mathrm{Cu}$ & 120 & 0 & 0.024 & 0.024 & & 0.012 & & 0.026 & 0.035 & 0.050 & & & & \\
\hline IN38 & $\mathrm{Fe}$ & 120 & 0 & 0.001 & 0.001 & & 0.029 & & 0.006 & 0.059 & 0.088 & & & & \\
\hline IN38 & $\mathrm{K}$ & 120 & 0 & -0.022 & -0.022 & & 0.064 & & 0.010 & 0.128 & 0.191 & & & & \\
\hline IN38 & $\mathrm{Li}$ & 120 & 0 & 0.002 & 0.002 & & 0.005 & & 0.002 & 0.009 & 0.014 & & & & \\
\hline IN38 & $\mathrm{Mg}$ & 120 & 0 & 0.002 & 0.002 & & 0.002 & & 0.002 & 0.005 & 0.007 & & & & \\
\hline IN38 & $\mathrm{Mn}$ & 120 & 0 & 0.002 & 0.002 & & 0.001 & & 0.002 & 0.003 & 0.004 & & & & \\
\hline IN38 & $\mathrm{Na}$ & 120 & 0 & 0.485 & 0.485 & & 1.045 & & 0.643 & 2.187 & 3.136 & & & & \\
\hline IN38 & $\mathrm{Ni}$ & 120 & 0 & 0.004 & 0.004 & & 0.007 & & 0.005 & 0.015 & 0.022 & & & & \\
\hline IN38 & $\mathrm{Si}$ & 120 & 0 & 0.018 & 0.018 & & 0.913 & & 0.156 & 1.832 & 2.738 & & & & \\
\hline IN38 & $\mathrm{Ti}$ & 120 & 0 & 0.000 & 0.000 & & 0.002 & & 0.001 & 0.003 & 0.005 & & & & \\
\hline IN38 & $\mathrm{U}$ & 120 & 10 & 9.858 & -0.142 & \begin{tabular}{|l|}
$-1.42 \%$ \\
\end{tabular} & 0.165 & $1.65 \%$ & & & & $-1.67 \%$ & $2.99 \%$ & $4.73 \%$ & $7 \%$ \\
\hline IN38 & $\mathrm{Zr}$ & 120 & 0 & 0.001 & 0.001 & & 0.003 & & 0.002 & 0.005 & 0.008 & & & & \\
\hline IN39 & $\mathrm{Al}$ & 60 & 0 & 0.020 & 0.020 & & 0.025 & & 0.026 & 0.056 & 0.076 & & & & \\
\hline IN39 & $\mathrm{B}$ & 60 & 0 & -0.002 & -0.002 & & 0.036 & & 0.008 & 0.072 & 0.107 & & & & \\
\hline IN39 & $\mathrm{Ca}$ & 60 & 0 & 0.255 & 0.255 & & 0.238 & & 0.306 & 0.566 & 0.782 & & & & \\
\hline IN39 & $\mathrm{Cr}$ & 60 & 0 & 0.027 & 0.027 & & 0.005 & & 0.028 & 0.030 & 0.037 & & & & \\
\hline IN39 & $\mathrm{Cu}$ & 60 & 0 & 0.023 & 0.023 & & 0.013 & & 0.026 & 0.037 & 0.052 & & & & \\
\hline IN39 & $\mathrm{Fe}$ & 60 & 0 & 0.010 & 0.010 & & 0.020 & & 0.014 & 0.043 & 0.061 & & & & \\
\hline IN39 & $\mathrm{K}$ & 60 & 0 & -0.032 & -0.032 & & 0.067 & & 0.014 & 0.135 & 0.202 & & & & \\
\hline IN39 & $\mathrm{Li}$ & 60 & 0 & 0.002 & 0.002 & & 0.005 & & 0.003 & 0.010 & 0.015 & & & & \\
\hline IN39 & $\mathrm{Mg}$ & 60 & 0 & 0.002 & 0.002 & & 0.003 & & 0.003 & 0.006 & 0.008 & & & & \\
\hline IN39 & $\mathrm{Mn}$ & 60 & 0 & 0.002 & 0.002 & & 0.001 & & 0.003 & 0.004 & 0.006 & & & & \\
\hline IN39 & $\mathrm{Na}$ & 60 & 0 & 0.499 & 0.499 & & 1.048 & & 0.725 & 2.218 & 3.144 & & & & \\
\hline IN39 & $\mathrm{Ni}$ & 60 & 0 & 0.003 & 0.003 & & 0.008 & & 0.005 & 0.017 & 0.024 & & & & \\
\hline IN39 & $\mathrm{Si}$ & 60 & 0 & 0.281 & 0.281 & & 0.920 & & 0.479 & 1.901 & 2.759 & & & & \\
\hline IN39 & $\mathrm{Ti}$ & 60 & 0 & 0.000 & 0.000 & & 0.002 & & 0.001 & 0.004 & 0.006 & & & & \\
\hline IN39 & $\mathrm{U}$ & 60 & 10 & 9.807 & -0.193 & \begin{tabular}{|l|}
$-1.93 \%$ \\
\end{tabular} & 0.206 & $2.06 \%$ & & & & $-2.38 \%$ & $3.65 \%$ & $6.06 \%$ & $7 \%$ \\
\hline IN39 & $\mathrm{Zr}$ & 60 & 0 & 0.001 & 0.001 & & 0.003 & & 0.002 & 0.006 & 0.008 & & & & \\
\hline SM32 & $\mathrm{Al}$ & 619 & 0 & 0.011 & 0.011 & & 0.050 & & 0.014 & 0.102 & 0.151 & & & & \\
\hline SM32 & $\mathrm{Ca}$ & 619 & 0 & 0.013 & 0.013 & & 0.052 & & 0.017 & 0.105 & 0.156 & & & & \\
\hline SM32 & $\mathrm{Cr}$ & 619 & 0 & 0.004 & 0.004 & & 0.007 & & 0.005 & 0.014 & 0.020 & & & & \\
\hline SM32 & $\mathrm{Cu}$ & 619 & 0 & 0.002 & 0.002 & & 0.004 & & 0.002 & 0.009 & 0.013 & & & & \\
\hline SM32 & $\mathrm{Fe}$ & 619 & 0 & 0.004 & 0.004 & & 0.056 & & 0.008 & 0.113 & 0.169 & & & & \\
\hline SM32 & $\mathrm{K}$ & 619 & 0 & 0.059 & 0.059 & & 0.078 & & 0.065 & 0.169 & 0.235 & & & & \\
\hline SM32 & $\mathrm{Li}$ & 619 & 0 & 0.003 & 0.003 & & 0.003 & & 0.003 & 0.006 & 0.009 & & & & \\
\hline SM32 & $\mathrm{Mg}$ & 619 & 0 & 0.001 & 0.001 & & 0.005 & & 0.001 & 0.010 & 0.015 & & & & \\
\hline SM32 & Mn & 619 & 0 & 0.001 & 0.001 & & 0.010 & & 0.002 & 0.020 & 0.030 & & & & \\
\hline SM32 & $\mathrm{Na}$ & 619 & 0 & 0.013 & 0.013 & & 0.191 & & 0.026 & 0.384 & 0.574 & & & & \\
\hline SM32 & $\mathrm{Ni}$ & 619 & 0 & 0.005 & 0.005 & & 0.012 & & 0.006 & 0.024 & 0.035 & & & & \\
\hline
\end{tabular}


WSRC-TR-2004-00576

Revision 0

Table A1. Measurement Uncertainties for Calibration and Bench Standards

\begin{tabular}{|c|c|c|c|c|c|c|c|c|c|c|c|c|c|c|c|}
\hline & & & & & & & & & 95\% Abs. & & & & & & \\
\hline & & & & & Abs. & & Std & & Bound & $95 \%$ & $99 \%$ & 95\% Rel. & $95 \%$ & $99 \%$ & \\
\hline & & & Ref. & Sample & Bias & & Dev & & for Bias & \begin{tabular}{|l|} 
Uncert. \\
\end{tabular} & Uncert. & Bound & Rel. & Rel. & LIMS \\
\hline Std. & Elem. & n & Value & Mean & $\mathrm{mg} / \mathrm{L}$ & \%Bias & $\mathrm{mg} / \mathrm{L}$ & \% RSD & $\mathrm{mg} / \mathrm{L}$ & $\mathrm{mg} / \mathrm{L}$ & $\mathrm{mg} / \mathrm{L}$ & for Bias & Uncert. & Uncert. & Limits \\
\hline SM32 & $\mathrm{Si}$ & 619 & 0 & 0.088 & 0.088 & & 0.567 & & 0.126 & 1.141 & 1.701 & & & & \\
\hline SM32 & $\mathrm{Ti}$ & 619 & 0 & 0.014 & 0.014 & & 0.026 & & 0.016 & 0.053 & 0.077 & & & & \\
\hline SM32 & $\mathrm{U}$ & 619 & 0 & 0.172 & 0.172 & & 0.187 & & 0.184 & 0.418 & 0.562 & & & & \\
\hline SM32 & $\mathrm{Zr}$ & 619 & 0 & -0.007 & $\begin{array}{l}-0.007 \\
\end{array}$ & & 0.016 & & 0.001 & 0.031 & 0.047 & & & & \\
\hline SM33 & $\mathrm{Al}$ & 319 & 0 & 0.015 & 0.015 & & 0.292 & & 0.042 & 0.586 & 0.877 & & & & \\
\hline SM33 & $\mathrm{Ca}$ & 319 & 1 & 0.996 & -0.004 & $-0.39 \%$ & 0.026 & $2.61 \%$ & & & & $-0.63 \%$ & $5.20 \%$ & $5.61 \%$ & $7 \%$ \\
\hline SM33 & $\mathrm{Cr}$ & 319 & 1 & 0.994 & -0.006 & $-0.57 \%$ & 0.019 & $1.86 \%$ & & & & $-0.75 \%$ & $3.67 \%$ & $4.29 \%$ & $7 \%$ \\
\hline SM33 & $\mathrm{Cu}$ & 319 & 1 & 0.994 & -0.006 & $-0.58 \%$ & 0.014 & $1.39 \%$ & & & & $-0.71 \%$ & $2.71 \%$ & $3.35 \%$ & $7 \%$ \\
\hline SM33 & $\mathrm{Fe}$ & 319 & 0 & -0.007 & -0.007 & & 0.022 & & 0.002 & 0.045 & 0.067 & & & & \\
\hline SM33 & $\mathrm{K}$ & 319 & 5 & 4.912 & -0.088 & $-1.77 \%$ & 0.083 & $1.66 \%$ & & & & $-1.92 \%$ & $2.81 \%$ & $5.09 \%$ & $7 \%$ \\
\hline SM33 & $\mathrm{Li}$ & 319 & 2 & 1.971 & -0.029 & $-1.46 \%$ & 0.028 & $1.40 \%$ & & & & $-1.59 \%$ & $2.39 \%$ & $4.26 \%$ & $7 \%$ \\
\hline SM33 & $\mathrm{Mg}$ & 319 & 1 & 1.007 & 0.007 & $0.67 \%$ & 0.023 & $2.31 \%$ & & & & $0.88 \%$ & $4.68 \%$ & $5.30 \%$ & $7 \%$ \\
\hline SM33 & $\mathrm{Mn}$ & 319 & 0 & 0.000 & 0.000 & & 0.002 & & 0.000 & 0.004 & 0.006 & & & & \\
\hline SM33 & $\mathrm{Na}$ & 319 & 32 & 32.702 & 0.702 & $2.20 \%$ & 0.999 & $3.12 \%$ & & & & $2.48 \%$ & $6.62 \%$ & $8.44 \%$ & $7 \%$ \\
\hline SM33 & $\mathrm{Ni}$ & 319 & 1 & 0.995 & -0.005 & $-0.53 \%$ & 0.017 & $1.73 \%$ & & & & $-0.69 \%$ & $3.42 \%$ & $3.99 \%$ & $7 \%$ \\
\hline SM33 & $\mathrm{Si}$ & 319 & 20 & 20.257 & 0.257 & $1.28 \%$ & 0.481 & $2.40 \%$ & & & & $1.51 \%$ & $4.98 \%$ & $6.09 \%$ & $7 \%$ \\
\hline SM33 & $\mathrm{Ti}$ & 319 & 0 & 0.000 & 0.000 & & 0.001 & & 0.000 & 0.003 & 0.004 & & & & \\
\hline SM33 & $\mathrm{U}$ & 319 & 0 & 0.031 & 0.031 & & 0.145 & & 0.044 & 0.294 & 0.435 & & & & \\
\hline SM33 & $\mathrm{Zr}$ & 319 & 0 & -0.007 & $\begin{array}{l}-0.007 \\
\end{array}$ & & 0.015 & & 0.001 & 0.030 & 0.046 & & & & \\
\hline SM34 & $\mathrm{Al}$ & 324 & 2 & 1.972 & -0.028 & $-1.38 \%$ & 0.030 & $1.52 \%$ & & & & $-1.52 \%$ & $2.71 \%$ & $4.43 \%$ & $7 \%$ \\
\hline SM34 & $\mathrm{Ca}$ & 324 & 0 & 0.020 & 0.020 & & 0.071 & & 0.026 & 0.144 & 0.212 & & & & \\
\hline SM34 & $\mathrm{Cr}$ & 324 & 0 & 0.002 & 0.002 & & 0.003 & & 0.002 & 0.006 & 0.008 & & & & \\
\hline SM34 & $\mathrm{Cu}$ & 324 & 0 & 0.001 & 0.001 & & 0.003 & & 0.001 & 0.007 & 0.010 & & & & \\
\hline SM34 & $\mathrm{Fe}$ & 324 & 10 & 9.897 & -0.103 & $-1.03 \%$ & 0.143 & $1.43 \%$ & & & & $-1.16 \%$ & $2.67 \%$ & $3.89 \%$ & $7 \%$ \\
\hline SM34 & $\mathrm{K}$ & 324 & 0 & 0.008 & 0.008 & & 0.052 & & 0.013 & 0.105 & 0.156 & & & & \\
\hline SM34 & $\mathrm{Li}$ & 324 & 0 & 0.007 & 0.007 & & 0.020 & & 0.009 & 0.041 & 0.061 & & & & \\
\hline SM34 & $\mathrm{Mg}$ & 324 & 0 & 0.003 & 0.003 & & 0.012 & & 0.004 & 0.024 & 0.035 & & & & \\
\hline SM34 & $\mathrm{Mn}$ & 324 & 2 & 1.969 & -0.031 & $-1.53 \%$ & 0.033 & $1.64 \%$ & & & & $-1.68 \%$ & $2.90 \%$ & $4.81 \%$ & $7 \%$ \\
\hline SM34 & $\mathrm{Na}$ & 324 & 5 & 4.906 & -0.094 & $-1.87 \%$ & 0.103 & $2.05 \%$ & & & & $-2.06 \%$ & $3.66 \%$ & $5.98 \%$ & $7 \%$ \\
\hline SM34 & $\mathrm{Ni}$ & 324 & 0 & 0.009 & 0.009 & & 0.008 & & 0.009 & 0.018 & 0.025 & & & & \\
\hline SM34 & $\mathrm{Si}$ & 324 & 0 & 0.121 & 0.121 & & 0.469 & & 0.164 & 0.952 & 1.407 & & & & \\
\hline SM34 & $\mathrm{Ti}$ & 324 & 1 & 0.993 & -0.007 & $-0.72 \%$ & 0.018 & $1.82 \%$ & & & & $-0.88 \%$ & $3.56 \%$ & $4.35 \%$ & $7 \%$ \\
\hline SM34 & $\mathrm{U}$ & 324 & 0 & 0.056 & 0.056 & & 0.144 & & 0.069 & 0.296 & 0.432 & & & & \\
\hline SM34 & $\mathrm{Zr}$ & 324 & 1 & 0.977 & -0.023 & $-2.30 \%$ & 0.013 & $1.27 \%$ & & & & $-2.42 \%$ & $1.07 \%$ & $4.84 \%$ & $7 \%$ \\
\hline SM35 & $\mathrm{Al}$ & 640 & 0 & 0.021 & 0.021 & & 0.295 & & 0.040 & 0.592 & 0.886 & & & & \\
\hline SM35 & $\mathrm{Ca}$ & 640 & 1 & 1.010 & 0.010 & $1.02 \%$ & 0.024 & $2.42 \%$ & & & & $1.18 \%$ & $4.94 \%$ & $5.86 \%$ & $7 \%$ \\
\hline SM35 & $\mathrm{Cr}$ & 640 & 1 & 1.006 & 0.006 & $0.64 \%$ & 0.017 & $1.72 \%$ & & & & $0.75 \%$ & $3.49 \%$ & $4.07 \%$ & $7 \%$ \\
\hline SM35 & $\mathrm{Cu}$ & 640 & 1 & 1.005 & 0.005 & $0.55 \%$ & 0.014 & $1.40 \%$ & & & & $0.64 \%$ & $2.85 \%$ & $3.34 \%$ & $7 \%$ \\
\hline SM35 & $\mathrm{Fe}$ & 640 & 0 & $\begin{array}{l}-0.009 \\
\end{array}$ & -0.009 & & 0.024 & & 0.002 & 0.047 & 0.071 & & & & \\
\hline SM35 & $\mathrm{K}$ & 640 & 5 & 4.986 & -0.014 & $-0.29 \%$ & 0.084 & $1.69 \%$ & & & & $-0.40 \%$ & $3.36 \%$ & $3.66 \%$ & $7 \%$ \\
\hline SM35 & $\mathrm{Li}$ & 640 & 2 & 1.993 & -0.007 & $-0.34 \%$ & 0.031 & $1.57 \%$ & & & & $-0.44 \%$ & $3.13 \%$ & $3.49 \%$ & $7 \%$ \\
\hline SM35 & $\mathrm{Mg}$ & 640 & 1 & 1.017 & 0.017 & $1.73 \%$ & 0.021 & $2.13 \%$ & & & & $1.86 \%$ & $4.60 \%$ & $5.99 \%$ & $7 \%$ \\
\hline SM35 & $\mathrm{Mn}$ & 640 & 0 & 0.000 & 0.000 & & 0.002 & & 0.000 & 0.004 & 0.006 & & & & \\
\hline SM35 & $\mathrm{Na}$ & 640 & 32 & 33.050 & 1.050 & $3.28 \%$ & 0.916 & $2.86 \%$ & & & & $3.47 \%$ & $6.60 \%$ & $9.00 \%$ & $7 \%$ \\
\hline SM35 & $\mathrm{Ni}$ & 640 & 1 & 1.007 & 0.007 & $0.72 \%$ & 0.018 & $1.76 \%$ & & & & $0.84 \%$ & $3.60 \%$ & $4.25 \%$ & $7 \%$ \\
\hline SM35 & $\mathrm{Si}$ & 640 & 20 & 20.458 & 0.458 & $2.29 \%$ & 0.411 & $2.05 \%$ & & & & $2.42 \%$ & $4.70 \%$ & $6.40 \%$ & $7 \%$ \\
\hline SM35 & $\mathrm{Ti}$ & 640 & 0 & 0.001 & 0.001 & & 0.001 & & 0.001 & 0.002 & 0.003 & & & & \\
\hline SM35 & $\mathrm{U}$ & 640 & 0 & 0.100 & 0.100 & & 0.105 & & 0.107 & 0.236 & 0.317 & & & & \\
\hline SM35 & $\mathrm{Zr}$ & 640 & 0 & -0.006 & -0.006 & & 0.015 & & 0.001 & 0.030 & 0.045 & & & & \\
\hline SM36 & $\mathrm{Al}$ & 642 & 2 & 1.986 & -0.014 & $-0.71 \%$ & 0.028 & $1.42 \%$ & & & & $-0.81 \%$ & $2.76 \%$ & $3.56 \%$ & $7 \%$ \\
\hline SM36 & $\mathrm{Ca}$ & 642 & 0 & 0.014 & 0.014 & & 0.050 & & 0.017 & 0.102 & 0.151 & & & & \\
\hline SM36 & $\mathrm{Cr}$ & 642 & 0 & 0.002 & 0.002 & & 0.002 & & 0.002 & 0.005 & 0.007 & & & & \\
\hline SM36 & $\mathrm{Cu}$ & 642 & 0 & 0.001 & 0.001 & & 0.003 & & 0.001 & 0.006 & 0.008 & & & & \\
\hline SM36 & $\mathrm{Fe}$ & 642 & 10 & 9.940 & -0.060 & $-0.60 \%$ & 0.126 & $1.26 \%$ & & & & $-0.68 \%$ & $2.44 \%$ & $3.11 \%$ & $7 \%$ \\
\hline SM36 & $\mathrm{K}$ & 642 & 0 & 0.015 & 0.015 & & 0.053 & & 0.018 & 0.108 & 0.159 & & & & \\
\hline SM36 & $\mathrm{Li}$ & 642 & 0 & 0.011 & 0.011 & & 0.120 & & 0.019 & 0.241 & 0.360 & & & & \\
\hline SM36 & $\mathrm{Mg}$ & 642 & 0 & 0.003 & 0.003 & & 0.008 & & 0.003 & 0.017 & 0.025 & & & & \\
\hline SM36 & $\mathrm{Mn}$ & 642 & 2 & 1.980 & -0.020 & $-1.02 \%$ & 0.030 & $1.49 \%$ & & & & $-1.11 \%$ & $2.80 \%$ & $4.00 \%$ & $7 \%$ \\
\hline SM36 & $\mathrm{Na}$ & 642 & 5 & 4.921 & \begin{tabular}{l|l}
-0.079 \\
\end{tabular} & $-1.57 \%$ & 0.097 & $1.93 \%$ & & & & $-1.70 \%$ & $3.54 \%$ & $5.44 \%$ & $7 \%$ \\
\hline SM36 & $\mathrm{Ni}$ & 642 & 0 & 0.009 & 0.009 & & 0.008 & & 0.010 & 0.018 & 0.025 & & & & \\
\hline SM36 & $\mathrm{Si}$ & 642 & 0 & 0.105 & 0.105 & & 0.377 & & 0.130 & 0.765 & 1.131 & & & & \\
\hline SM36 & $\mathrm{Ti}$ & 642 & 1 & 0.997 & -0.003 & $-0.27 \%$ & 0.018 & $1.77 \%$ & & & & $-0.39 \%$ & $3.53 \%$ & $3.81 \%$ & $7 \%$ \\
\hline
\end{tabular}


WSRC-TR-2004-00576

Revision 0

Table A1. Measurement Uncertainties for Calibration and Bench Standards

\begin{tabular}{|c|c|c|c|c|c|c|c|c|c|c|c|c|c|c|c|}
\hline & & & & & & & & & 95\% Abs. & & & & & & \\
\hline & & & & & Abs. & & Std & & Bound & $95 \%$ & $99 \%$ & 95\% Rel. & $95 \%$ & $99 \%$ & \\
\hline & & & Ref. & Sample & Bias & & Dev & & for Bias & \begin{tabular}{|l|} 
Uncert. \\
\end{tabular} & Uncert. & Bound & Rel. & Rel. & LIMS \\
\hline Std. & Elem. & n & Value & Mean & $\mathrm{mg} / \mathrm{L}$ & \%Bias & $\mathrm{mg} / \mathrm{L}$ & \% RSD & $\mathrm{mg} / \mathrm{L}$ & $\mathrm{mg} / \mathrm{L}$ & $\mathrm{mg} / \mathrm{L}$ & for Bias & Uncert. & Uncert. & Limits \\
\hline SM36 & $\mathrm{U}$ & 642 & 0 & 0.080 & 0.080 & & 0.107 & & 0.087 & 0.231 & 0.320 & & & & \\
\hline SM36 & $\mathrm{Zr}$ & 642 & 1 & 0.982 & -0.018 & $-1.85 \%$ & 0.012 & $1.17 \%$ & & & & $-1.92 \%$ & $1.43 \%$ & $4.18 \%$ & $7 \%$ \\
\hline SM37 & $\mathrm{Al}$ & 334 & 0 & 0.044 & 0.044 & & 0.019 & & 0.045 & 0.059 & 0.084 & & & & \\
\hline SM37 & $\mathrm{Ca}$ & 334 & 0 & 0.281 & 0.281 & & 0.360 & & 0.313 & 0.785 & 1.079 & & & & \\
\hline SM37 & $\mathrm{Cr}$ & 334 & 0 & 0.028 & 0.028 & & 0.005 & & 0.029 & 0.031 & 0.039 & & & & \\
\hline SM37 & $\mathrm{Cu}$ & 334 & 0 & 0.025 & 0.025 & & 0.037 & & 0.028 & 0.079 & 0.110 & & & & \\
\hline SM37 & $\mathrm{Fe}$ & 334 & 0 & -0.005 & -0.005 & & 0.024 & & 0.002 & 0.049 & 0.073 & & & & \\
\hline SM37 & $\mathrm{K}$ & 334 & 0 & -0.006 & -0.006 & & 0.051 & & 0.005 & 0.103 & 0.154 & & & & \\
\hline SM37 & $\mathrm{Li}$ & 334 & 0 & 0.001 & 0.001 & & 0.002 & & 0.001 & 0.005 & 0.007 & & & & \\
\hline SM37 & $\mathrm{Mg}$ & 334 & 0 & 0.005 & 0.005 & & 0.005 & & 0.006 & 0.012 & 0.016 & & & & \\
\hline SM37 & $\mathrm{Mn}$ & 334 & 0 & 0.002 & 0.002 & & 0.001 & & 0.002 & 0.003 & 0.004 & & & & \\
\hline SM37 & $\mathrm{Na}$ & 334 & 0 & 0.115 & 0.115 & & 0.544 & & 0.164 & 1.101 & 1.633 & & & & \\
\hline SM37 & $\mathrm{Ni}$ & 334 & 0 & 0.002 & 0.002 & & 0.008 & & 0.003 & 0.016 & 0.023 & & & & \\
\hline SM37 & $\mathrm{Si}$ & 334 & 0 & 0.427 & 0.427 & & 0.646 & & 0.486 & 1.380 & 1.938 & & & & \\
\hline SM37 & $\mathrm{Ti}$ & 334 & 0 & 0.001 & 0.001 & & 0.002 & & 0.001 & 0.003 & 0.005 & & & & \\
\hline SM37 & $\mathrm{U}$ & 334 & 10 & 9.846 & -0.154 & $-1.54 \%$ & 0.180 & $1.80 \%$ & & & & $-1.71 \%$ & $3.25 \%$ & $5.14 \%$ & $7 \%$ \\
\hline SM37 & $\mathrm{Zr}$ & 334 & 0 & -0.005 & -0.005 & & 0.016 & & 0.001 & 0.032 & 0.048 & & & & \\
\hline SM38 & $\mathrm{Al}$ & 640 & 0 & 0.054 & 0.054 & & 0.136 & & 0.062 & 0.280 & 0.409 & & & & \\
\hline SM38 & $\mathrm{Ca}$ & 640 & 0 & 0.277 & 0.277 & & 0.350 & & 0.299 & 0.761 & 1.049 & & & & \\
\hline SM38 & $\mathrm{Cr}$ & 640 & 0 & 0.029 & 0.029 & & 0.005 & & 0.029 & 0.031 & 0.040 & & & & \\
\hline SM38 & $\mathrm{Cu}$ & 640 & 0 & 0.025 & 0.025 & & 0.033 & & 0.027 & 0.072 & 0.100 & & & & \\
\hline SM38 & $\mathrm{Fe}$ & 640 & 0 & -0.006 & -0.006 & & 0.025 & & 0.002 & 0.050 & 0.075 & & & & \\
\hline SM38 & $\mathrm{K}$ & 640 & 0 & 0.003 & 0.003 & & 0.048 & & 0.006 & 0.097 & 0.145 & & & & \\
\hline SM38 & $\mathrm{Li}$ & 640 & 0 & 0.001 & 0.001 & & 0.002 & & 0.001 & 0.004 & 0.006 & & & & \\
\hline SM38 & $\mathrm{Mg}$ & 640 & 0 & 0.005 & 0.005 & & 0.005 & & 0.005 & 0.012 & 0.016 & & & & \\
\hline SM38 & $\mathrm{Mn}$ & 640 & 0 & 0.001 & 0.001 & & 0.001 & & 0.002 & 0.003 & 0.004 & & & & \\
\hline SM38 & $\mathrm{Na}$ & 640 & 0 & 0.117 & 0.117 & & 0.556 & & 0.153 & 1.123 & 1.668 & & & & \\
\hline SM38 & $\mathrm{Ni}$ & 640 & 0 & 0.002 & 0.002 & & 0.008 & & 0.003 & 0.015 & 0.023 & & & & \\
\hline SM38 & $\mathrm{Si}$ & 640 & 0 & 0.172 & 0.172 & & 0.577 & & 0.209 & 1.174 & 1.732 & & & & \\
\hline SM38 & $\mathrm{Ti}$ & 640 & 0 & 0.001 & 0.001 & & 0.001 & & 0.001 & 0.003 & 0.004 & & & & \\
\hline SM38 & $\mathrm{U}$ & 640 & 10 & 9.913 & -0.087 & $-0.87 \%$ & 0.179 & $1.79 \%$ & & & & $-0.98 \%$ & $3.48 \%$ & $4.46 \%$ & $7 \%$ \\
\hline SM38 & $\mathrm{Zr}$ & 640 & 0 & -0.005 & -0.005 & & 0.015 & & 0.001 & 0.031 & 0.046 & & & & \\
\hline SM51 & $\mathrm{Al}$ & 325 & 0 & 0.007 & 0.007 & & 0.014 & & 0.008 & 0.029 & 0.042 & & & & \\
\hline SM51 & $\mathrm{B}$ & 325 & 4 & 3.882 & -0.118 & $-2.94 \%$ & 0.241 & $6.03 \%$ & & & & $-3.49 \%$ & $11.70 \%$ & $15.00 \%$ & $7 \%$ \\
\hline SM51 & $\mathrm{Ca}$ & 325 & 1 & 0.998 & -0.002 & $-0.24 \%$ & 0.028 & $2.78 \%$ & & & & $-0.49 \%$ & $5.56 \%$ & $5.81 \%$ & $7 \%$ \\
\hline SM51 & $\mathrm{Cr}$ & 325 & 1 & 0.989 & -0.011 & $-1.09 \%$ & 0.015 & $1.51 \%$ & & & & $-1.23 \%$ & $2.83 \%$ & $4.12 \%$ & $7 \%$ \\
\hline SM51 & $\mathrm{Cu}$ & 325 & 1 & 0.989 & -0.011 & $-1.10 \%$ & 0.014 & $1.41 \%$ & & & & $-1.23 \%$ & $2.59 \%$ & $3.91 \%$ & $7 \%$ \\
\hline SM51 & $\mathrm{Fe}$ & 325 & 0 & 0.010 & 0.010 & & 0.093 & & 0.018 & 0.188 & 0.280 & & & & \\
\hline SM51 & $\mathrm{K}$ & 325 & 5 & 4.921 & -0.079 & $-1.58 \%$ & 0.080 & $1.61 \%$ & & & & $-1.73 \%$ & $2.81 \%$ & $4.80 \%$ & $7 \%$ \\
\hline SM51 & $\mathrm{Li}$ & 325 & 2 & 1.974 & -0.026 & $-1.31 \%$ & 0.028 & $1.40 \%$ & & & & $-1.44 \%$ & $2.47 \%$ & $4.11 \%$ & $7 \%$ \\
\hline SM51 & $\mathrm{Mg}$ & 325 & 1 & 1.003 & 0.003 & $0.31 \%$ & 0.024 & $2.38 \%$ & & & & $0.53 \%$ & $4.77 \%$ & $5.07 \%$ & $7 \%$ \\
\hline SM51 & $\mathrm{Mn}$ & 325 & 0 & 0.001 & 0.001 & & 0.002 & & 0.001 & 0.004 & 0.005 & & & & \\
\hline SM51 & $\mathrm{Ni}$ & 325 & 1 & 0.986 & -0.014 & $-1.37 \%$ & 0.018 & $1.80 \%$ & & & & $-1.53 \%$ & $3.32 \%$ & $4.96 \%$ & $7 \%$ \\
\hline SM51 & $\mathrm{Si}$ & 325 & 20 & 20.151 & 0.151 & $\begin{array}{l}0.75 \% \\
\end{array}$ & 0.406 & $2.03 \%$ & & & & $0.94 \%$ & $4.13 \%$ & $4.81 \%$ & $7 \%$ \\
\hline SM51 & $\mathrm{Ti}$ & 325 & 0 & 0.000 & 0.000 & & 0.001 & & 0.001 & 0.003 & 0.004 & & & & \\
\hline SM52 & $\mathrm{Al}$ & 321 & 2 & 1.973 & -0.027 & $-1.36 \%$ & 0.032 & $1.62 \%$ & & & & $-1.51 \%$ & $2.94 \%$ & $4.60 \%$ & $7 \%$ \\
\hline SM52 & $\mathrm{B}$ & 321 & 2 & 1.986 & -0.014 & $-0.70 \%$ & 0.032 & $1.60 \%$ & & & & $-0.85 \%$ & $3.12 \%$ & $3.90 \%$ & $7 \%$ \\
\hline SM52 & $\mathrm{Ca}$ & 321 & 0 & 0.014 & 0.014 & & 0.071 & & 0.021 & 0.143 & 0.212 & & & & \\
\hline SM52 & $\mathrm{Cr}$ & 321 & 0 & 0.001 & 0.001 & & 0.003 & & 0.001 & 0.006 & 0.009 & & & & \\
\hline SM52 & $\mathrm{Cu}$ & 321 & 0 & 0.000 & 0.000 & & 0.003 & & 0.001 & 0.006 & 0.009 & & & & \\
\hline SM52 & $\mathrm{Fe}$ & 321 & 10 & 9.881 & -0.119 & $-1.19 \%$ & 0.145 & $1.45 \%$ & & & & $-1.32 \%$ & $2.65 \%$ & $4.09 \%$ & $7 \%$ \\
\hline SM52 & $\mathrm{K}$ & 321 & 0 & 0.013 & 0.013 & & 0.051 & & 0.018 & 0.103 & 0.152 & & & & \\
\hline SM52 & $\mathrm{Li}$ & 321 & 0 & 0.001 & 0.001 & & 0.002 & & 0.001 & 0.004 & 0.006 & & & & \\
\hline SM52 & $\mathrm{Mg}$ & 321 & 0 & 0.003 & 0.003 & & 0.027 & & 0.006 & 0.054 & 0.080 & & & & \\
\hline SM52 & $\mathrm{Mn}$ & 321 & 2 & 1.972 & -0.028 & $-1.41 \%$ & 0.031 & $1.57 \%$ & & & & $-1.56 \%$ & $2.81 \%$ & $4.56 \%$ & $7 \%$ \\
\hline SM52 & $\mathrm{Ni}$ & 321 & 0 & 0.008 & 0.008 & & 0.008 & & 0.008 & 0.018 & 0.024 & & & & \\
\hline SM52 & $\mathrm{Si}$ & 321 & 0 & 0.006 & 0.006 & & 0.047 & & 0.011 & 0.095 & 0.142 & & & & \\
\hline SM52 & $\mathrm{Ti}$ & 321 & 1 & 0.993 & -0.007 & $-0.74 \%$ & 0.019 & $1.92 \%$ & & & & $-0.91 \%$ & $3.77 \%$ & $4.58 \%$ & $7 \%$ \\
\hline SM53 & $\mathrm{Al}$ & 648 & 0 & 0.010 & 0.010 & & 0.011 & & 0.011 & 0.024 & 0.032 & & & & \\
\hline SM53 & $\mathrm{B}$ & 648 & 4 & 3.902 & -0.098 & $-2.45 \%$ & 0.240 & $6.00 \%$ & & & & $-2.83 \%$ & $11.75 \%$ & $14.45 \%$ & $7 \%$ \\
\hline SM53 & $\mathrm{Ca}$ & 648 & 1 & 1.002 & 0.002 & $0.18 \%$ & 0.025 & $2.49 \%$ & & & & $0.34 \%$ & $4.99 \%$ & $5.17 \%$ & $7 \%$ \\
\hline SM53 & $\mathrm{Cr}$ & 648 & 1 & 0.992 & -0.008 & $-0.78 \%$ & 0.014 & $1.43 \%$ & & & & $-0.87 \%$ & $2.75 \%$ & $3.64 \%$ & $7 \%$ \\
\hline
\end{tabular}


WSRC-TR-2004-00576

Revision 0

Table A1. Measurement Uncertainties for Calibration and Bench Standards

\begin{tabular}{|c|c|c|c|c|c|c|c|c|c|c|c|c|c|c|c|}
\hline & & & & & & & & & 95\% Abs. & & & & & & \\
\hline & & & & & Abs. & & Std & & Bound & $95 \%$ & $99 \%$ & 95\% Rel. & $95 \%$ & $99 \%$ & \\
\hline & & & Ref. & Sample & Bias & & Dev & & for Bias & Uncert. & Uncert. & Bound & Rel. & Rel. & LIMS \\
\hline Std. & Elem. & n & Value & Mean & $\mathrm{mg} / \mathrm{L}$ & \%Bias & $\mathrm{mg} / \mathrm{L}$ & \% RSD & $\mathrm{mg} / \mathrm{L}$ & $\mathrm{mg} / \mathrm{L}$ & $\mathrm{mg} / \mathrm{L}$ & for Bias & Uncert. & Uncert. & Limits \\
\hline SM53 & $\mathrm{Cu}$ & 648 & 1 & 0.992 & -0.008 & $-0.82 \%$ & 0.013 & $1.29 \%$ & & & & $-0.90 \%$ & $2.45 \%$ & $3.40 \%$ & $7 \%$ \\
\hline SM53 & $\mathrm{Fe}$ & 648 & 0 & 0.008 & 0.008 & & 0.080 & & 0.013 & 0.160 & 0.239 & & & & \\
\hline SM53 & $\mathrm{K}$ & 648 & 5 & 4.938 & -0.062 & $-1.23 \%$ & 0.069 & $1.38 \%$ & & & & $-1.32 \%$ & $2.48 \%$ & $4.00 \%$ & $7 \%$ \\
\hline SM53 & $\mathrm{Li}$ & 648 & 2 & 1.979 & -0.021 & $-1.05 \%$ & 0.025 & $1.23 \%$ & & & & $-1.13 \%$ & $2.22 \%$ & $3.51 \%$ & $7 \%$ \\
\hline SM53 & $\mathrm{Mg}$ & 648 & 1 & 1.003 & 0.003 & $0.32 \%$ & 0.020 & $1.99 \%$ & & & & $0.45 \%$ & $3.99 \%$ & $4.30 \%$ & $7 \%$ \\
\hline SM53 & Mn & 648 & 0 & 0.001 & 0.001 & & 0.002 & & 0.001 & 0.004 & 0.005 & & & & \\
\hline SM53 & $\mathrm{Ni}$ & 648 & 1 & 0.990 & -0.010 & $-0.98 \%$ & 0.016 & $1.63 \%$ & & & & $-1.08 \%$ & $3.11 \%$ & $4.24 \%$ & $7 \%$ \\
\hline SM53 & $\mathrm{Si}$ & 648 & 20 & 20.064 & 0.064 & $0.32 \%$ & 0.326 & $1.63 \%$ & & & & $0.43 \%$ & $3.28 \%$ & $3.58 \%$ & $7 \%$ \\
\hline SM53 & $\mathrm{Ti}$ & 648 & 0 & 0.001 & 0.001 & & 0.001 & & 0.001 & 0.002 & 0.003 & & & & \\
\hline SM54 & $\mathrm{Al}$ & 646 & 2 & 1.980 & -0.020 & $-1.01 \%$ & 0.028 & $1.42 \%$ & & & & $-1.10 \%$ & $2.66 \%$ & $3.86 \%$ & $7 \%$ \\
\hline SM54 & B & 646 & 2 & 1.988 & -0.012 & $-0.58 \%$ & 0.032 & $1.58 \%$ & & & & $-0.68 \%$ & $3.11 \%$ & $3.74 \%$ & $7 \%$ \\
\hline SM54 & $\mathrm{Ca}$ & 646 & 0 & 0.006 & 0.006 & & 0.038 & & 0.008 & 0.077 & 0.115 & & & & \\
\hline SM54 & $\mathrm{Cr}$ & 646 & 0 & 0.002 & 0.002 & & 0.002 & & 0.002 & 0.005 & 0.007 & & & & \\
\hline SM54 & $\mathrm{Cu}$ & 646 & 0 & 0.001 & 0.001 & & 0.002 & & 0.001 & 0.005 & 0.007 & & & & \\
\hline SM54 & $\mathrm{Fe}$ & 646 & 10 & 9.895 & -0.105 & $-1.05 \%$ & 0.132 & $1.32 \%$ & & & & $-1.14 \%$ & $2.42 \%$ & $3.69 \%$ & $7 \%$ \\
\hline SM54 & $\mathrm{K}$ & 646 & 0 & 0.016 & 0.016 & & 0.040 & & 0.018 & 0.081 & 0.119 & & & & \\
\hline SM54 & $\mathrm{Li}$ & 646 & 0 & 0.001 & 0.001 & & 0.002 & & 0.001 & 0.004 & 0.005 & & & & \\
\hline SM54 & $\mathrm{Mg}$ & 646 & 0 & 0.001 & 0.001 & & 0.002 & & 0.001 & 0.004 & 0.006 & & & & \\
\hline SM54 & Mn & 646 & 2 & 1.976 & -0.024 & $-1.19 \%$ & 0.029 & $1.46 \%$ & & & & $-1.29 \%$ & $2.66 \%$ & $4.10 \%$ & $7 \%$ \\
\hline SM54 & $\mathrm{Ni}$ & 646 & 0 & 0.008 & 0.008 & & 0.007 & & 0.008 & 0.017 & 0.023 & & & & \\
\hline SM54 & $\mathrm{Si}$ & 646 & 0 & 0.011 & 0.011 & & 0.029 & & 0.013 & 0.059 & 0.086 & & & & \\
\hline SM54 & $\mathrm{Ti}$ & 646 & 1 & 0.994 & -0.006 & $-0.58 \%$ & 0.019 & $1.91 \%$ & & & & $-0.71 \%$ & $3.77 \%$ & $4.40 \%$ & $7 \%$ \\
\hline SM55 & $\mathrm{Al}$ & 635 & 0 & 0.026 & 0.026 & & 0.042 & & 0.028 & 0.088 & 0.125 & & & & \\
\hline SM55 & B & 635 & 0 & 0.009 & 0.009 & & 0.023 & & 0.011 & 0.046 & 0.068 & & & & \\
\hline SM55 & $\mathrm{Ca}$ & 635 & 0 & 0.002 & 0.002 & & 0.049 & & 0.005 & 0.098 & 0.147 & & & & \\
\hline SM55 & $\mathrm{Cr}$ & 635 & 0 & 0.010 & 0.010 & & 0.019 & & 0.011 & 0.039 & 0.056 & & & & \\
\hline SM55 & $\mathrm{Cu}$ & 635 & 0 & 0.005 & 0.005 & & 0.010 & & 0.006 & 0.021 & 0.030 & & & & \\
\hline SM55 & $\mathrm{Fe}$ & 635 & 0 & 0.054 & 0.054 & & 0.111 & & 0.061 & 0.230 & 0.332 & & & & \\
\hline SM55 & $\mathrm{K}$ & 635 & 0 & 0.055 & 0.055 & & 0.388 & & 0.080 & 0.780 & 1.164 & & & & \\
\hline SM55 & $\mathrm{Li}$ & 635 & 0 & 0.005 & 0.005 & & 0.008 & & 0.006 & 0.017 & 0.024 & & & & \\
\hline SM55 & $\mathrm{Mg}$ & 635 & 0 & 0.004 & 0.004 & & 0.008 & & 0.005 & 0.017 & 0.025 & & & & \\
\hline SM55 & $\mathrm{Mn}$ & 635 & 0 & 0.005 & 0.005 & & 0.013 & & 0.006 & 0.027 & 0.040 & & & & \\
\hline SM55 & $\mathrm{Ni}$ & 635 & 0 & 0.009 & 0.009 & & 0.021 & & 0.011 & 0.043 & 0.063 & & & & \\
\hline SM55 & $\mathrm{Si}$ & 635 & 0 & 0.111 & 0.111 & & 0.210 & & 0.125 & 0.438 & 0.630 & & & & \\
\hline SM55 & $\mathrm{Ti}$ & 635 & 0 & 0.005 & 0.005 & & 0.010 & & 0.005 & 0.020 & 0.029 & & & & \\
\hline
\end{tabular}


WSRC-TR-2004-00576

Revision 0

\section{Table A2. Comparisons of SME and MFT Measurements for Mixed Acid Calibration and Bench Standards}

\begin{tabular}{|c|c|c|c|c|c|c|c|c|c|c|}
\hline & & & & & & & & "MFT Std Dev/ & & \\
\hline Standard & $\mathbf{n}$ & Element & MFT Mean & SME Mean & \% Difference & MFT Std Dev & SME Std Dev & SME Std Dev & $\%$ RSD MFT & \%RSD SME \\
\hline SM32 & 294 & $\mathrm{Al}(\mathrm{mg} / \mathrm{L})$ & 0.011 & 0.010 & & 0.0405 & 0.0580 & & & \\
\hline SM32 & 294 & $\mathrm{Ca}(\mathrm{mg} / \mathrm{L})$ & 0.013 & 0.013 & & 0.0436 & 0.0587 & & & \\
\hline SM32 & 294 & $\mathrm{Cr}(\mathrm{mg} / \mathrm{L})$ & 0.004 & 0.004 & & 0.0064 & 0.0071 & & & \\
\hline SM32 & 294 & $\mathrm{Cu}(\mathrm{mg} / \mathrm{L})$ & 0.002 & 0.002 & & 0.0040 & 0.0045 & & & \\
\hline SM32 & 294 & $\mathrm{Fe}(\mathrm{mg} / \mathrm{L})$ & 0.008 & 0.001 & & 0.0566 & 0.0559 & & & \\
\hline SM32 & 294 & $\mathrm{~K}(\mathrm{mg} / \mathrm{L})$ & 0.058 & 0.060 & & 0.0628 & 0.0901 & & & \\
\hline SM32 & 294 & $\mathrm{Li}(\mathrm{mg} / \mathrm{L})$ & 0.002 & 0.003 & & 0.0022 & 0.0033 & & & \\
\hline SM32 & 294 & $\mathrm{Mg}(\mathrm{mg} / \mathrm{L})$ & 0.001 & 0.001 & & 0.0056 & 0.0044 & & & \\
\hline SM32 & 294 & $\mathrm{Mn}(\mathrm{mg} / \mathrm{L})$ & 0.001 & 0.002 & & 0.0023 & 0.0137 & & & \\
\hline SM32 & 294 & $\mathrm{Na}(\mathrm{mg} / \mathrm{L})$ & 0.009 & 0.016 & & 0.1909 & 0.1923 & & & \\
\hline SM32 & 294 & $\mathrm{Ni}(\mathrm{mg} / \mathrm{L})$ & 0.006 & 0.005 & & 0.0113 & 0.0122 & & & \\
\hline SM32 & 294 & $\mathrm{Si}(\mathrm{mg} / \mathrm{L})$ & 0.101 & 0.077 & & 0.4823 & 0.6344 & & & \\
\hline SM32 & 294 & $\mathrm{Ti}(\mathrm{mg} / \mathrm{L})$ & 0.014 & 0.014 & & 0.0245 & 0.0264 & & & \\
\hline SM32 & 294 & $\mathrm{U}(\mathrm{mg} / \mathrm{L})$ & 0.169 & 0.175 & & 0.1710 & 0.2013 & & & \\
\hline SM32 & 294 & $\mathrm{Zr}(\mathrm{mg} / \mathrm{L})$ & -0.006 & -0.007 & & 0.0148 & 0.0163 & & & \\
\hline SM33 & 153 & $\mathrm{Al}(\mathrm{mg} / \mathrm{L})$ & 0.000 & 0.030 & & 0.0148 & 0.4050 & & & \\
\hline SM33 & 153 & $\mathrm{Ca}(\mathrm{mg} / \mathrm{L})$ & 0.996 & 0.996 & $0.01 \%$ & 0.0263 & 0.0260 & 1.01 & $2.64 \%$ & $2.61 \%$ \\
\hline SM33 & 153 & $\mathrm{Cr}(\mathrm{mg} / \mathrm{L})$ & 0.994 & 0.995 & $-0.09 \%$ & 0.0188 & 0.0184 & 1.02 & $1.89 \%$ & $1.85 \%$ \\
\hline SM33 & 153 & $\mathrm{Cu}(\mathrm{mg} / \mathrm{L})$ & 0.994 & 0.994 & $-0.07 \%$ & 0.0137 & 0.0140 & 0.98 & $1.38 \%$ & $1.41 \%$ \\
\hline SM33 & 153 & $\mathrm{Fe}(\mathrm{mg} / \mathrm{L})$ & -0.004 & -0.010 & & 0.0140 & 0.0279 & & & \\
\hline SM33 & 153 & $\mathrm{~K}(\mathrm{mg} / \mathrm{L})$ & 4.914 & 4.909 & $0.10 \%$ & 0.0848 & 0.0814 & 1.04 & $1.73 \%$ & $1.66 \%$ \\
\hline SM33 & 153 & $\mathrm{Li}(\mathrm{mg} / \mathrm{L})$ & 1.971 & 1.971 & $-0.01 \%$ & 0.0293 & 0.0269 & 1.09 & $1.49 \%$ & $1.37 \%$ \\
\hline SM33 & 153 & $\mathrm{Mg}(\mathrm{mg} / \mathrm{L})$ & 1.007 & 1.007 & $0.02 \%$ & 0.0227 & 0.0236 & 0.96 & $2.26 \%$ & $2.35 \%$ \\
\hline SM33 & 153 & $\mathrm{Mn}(\mathrm{mg} / \mathrm{L})$ & 0.000 & 0.000 & & 0.0012 & 0.0025 & & & \\
\hline SM33 & 153 & $\mathrm{Na}(\mathrm{mg} / \mathrm{L})$ & 32.724 & 32.683 & $0.13 \%$ & 0.9660 & 1.0306 & 0.94 & $2.95 \%$ & $3.15 \%$ \\
\hline SM33 & 153 & $\mathrm{Ni}(\mathrm{mg} / \mathrm{L})$ & 0.994 & 0.996 & $-0.19 \%$ & 0.0171 & 0.0175 & 0.97 & $1.72 \%$ & $1.76 \%$ \\
\hline SM33 & 153 & $\mathrm{Si}(\mathrm{mg} / \mathrm{L})$ & 20.264 & 20.251 & $0.06 \%$ & 0.4672 & 0.4945 & 0.94 & $2.31 \%$ & $2.44 \%$ \\
\hline SM33 & 153 & $\mathrm{Ti}(\mathrm{mg} / \mathrm{L})$ & 0.000 & 0.000 & & 0.0014 & 0.0014 & & & \\
\hline SM33 & 153 & $\mathrm{U}(\mathrm{mg} / \mathrm{L})$ & 0.028 & 0.033 & & 0.1556 & 0.1351 & & & \\
\hline SM33 & 153 & $\mathrm{Zr}(\mathrm{mg} / \mathrm{L})$ & -0.006 & -0.007 & & 0.0144 & 0.0160 & & & \\
\hline SM34 & 154 & $\mathrm{Al}(\mathrm{mg} / \mathrm{L})$ & 1.971 & 1.974 & $-0.13 \%$ & 0.0315 & 0.0295 & 1.07 & $1.60 \%$ & $1.50 \%$ \\
\hline SM34 & 154 & $\mathrm{Ca}(\mathrm{mg} / \mathrm{L})$ & 0.021 & 0.019 & & 0.0705 & 0.0712 & & & \\
\hline SM34 & 154 & $\mathrm{Cr}(\mathrm{mg} / \mathrm{L})$ & 0.002 & 0.002 & & 0.0027 & 0.0026 & & & \\
\hline SM34 & 154 & $\mathrm{Cu}(\mathrm{mg} / \mathrm{L})$ & 0.001 & 0.001 & & 0.0035 & 0.0031 & & & \\
\hline SM34 & 154 & $\mathrm{Fe}(\mathrm{mg} / \mathrm{L})$ & 9.889 & 9.904 & $-0.14 \%$ & 0.1413 & 0.1445 & 0.98 & $1.43 \%$ & $1.46 \%$ \\
\hline SM34 & 154 & $\mathrm{~K}(\mathrm{mg} / \mathrm{L})$ & 0.009 & 0.007 & & 0.0539 & 0.0506 & & & \\
\hline SM34 & 154 & $\mathrm{Li}(\mathrm{mg} / \mathrm{L})$ & 0.007 & 0.006 & & 0.0218 & 0.0187 & & & \\
\hline SM34 & 154 & $\mathrm{Mg}(\mathrm{mg} / \mathrm{L})$ & 0.004 & 0.003 & & 0.0146 & 0.0080 & & & \\
\hline SM34 & 154 & $\mathrm{Mn}(\mathrm{mg} / \mathrm{L})$ & 1.968 & 1.971 & $-0.18 \%$ & 0.0301 & 0.0350 & 0.86 & $1.53 \%$ & $1.77 \%$ \\
\hline SM34 & 154 & $\mathrm{Na}(\mathrm{mg} / \mathrm{L})$ & 4.907 & 4.905 & $0.04 \%$ & 0.1056 & 0.1003 & 1.05 & $2.15 \%$ & $2.05 \%$ \\
\hline SM34 & 154 & $\mathrm{Ni}(\mathrm{mg} / \mathrm{L})$ & 0.009 & 0.009 & & 0.0076 & 0.0082 & & & \\
\hline SM34 & 154 & $\mathrm{Si}(\mathrm{mg} / \mathrm{L})$ & 0.169 & 0.077 & & 0.5105 & 0.4245 & & & \\
\hline SM34 & 154 & $\mathrm{Ti}(\mathrm{mg} / \mathrm{L})$ & 0.993 & 0.993 & $-0.02 \%$ & 0.0185 & 0.0180 & 1.03 & $1.86 \%$ & $1.81 \%$ \\
\hline SM34 & 154 & $\mathrm{U}(\mathrm{mg} / \mathrm{L})$ & 0.052 & 0.060 & & 0.1573 & 0.1309 & & & \\
\hline SM34 & 154 & $\mathrm{Zr}(\mathrm{mg} / \mathrm{L})$ & 0.977 & 0.977 & $0.05 \%$ & 0.0116 & 0.0137 & 0.85 & $1.19 \%$ & $1.40 \%$ \\
\hline SM35 & 300 & $\mathrm{Al}(\mathrm{mg} / \mathrm{L})$ & 0.005 & 0.035 & & 0.0106 & 0.4049 & & & \\
\hline SM35 & 300 & $\mathrm{Ca}(\mathrm{mg} / \mathrm{L})$ & 1.010 & 1.010 & $0.04 \%$ & 0.0248 & 0.0236 & 1.05 & $2.46 \%$ & $2.34 \%$ \\
\hline SM35 & 300 & $\mathrm{Cr}(\mathrm{mg} / \mathrm{L})$ & 1.006 & 1.007 & $-0.02 \%$ & 0.0183 & 0.0161 & 1.14 & $1.82 \%$ & $1.60 \%$ \\
\hline SM35 & 300 & $\mathrm{Cu}(\mathrm{mg} / \mathrm{L})$ & 1.005 & 1.006 & $-0.01 \%$ & 0.0144 & 0.0136 & 1.06 & $1.44 \%$ & $1.35 \%$ \\
\hline SM35 & 300 & $\mathrm{Fe}(\mathrm{mg} / \mathrm{L})$ & -0.005 & -0.012 & & 0.0133 & 0.0295 & & & \\
\hline SM35 & 300 & $\mathrm{~K}(\mathrm{mg} / \mathrm{L})$ & 4.990 & 4.982 & $0.17 \%$ & 0.0886 & 0.0802 & 1.11 & $1.78 \%$ & $1.61 \%$ \\
\hline SM35 & 300 & Li (mg/L) & 1.995 & 1.992 & $0.13 \%$ & 0.0321 & 0.0310 & 1.04 & $1.61 \%$ & $1.55 \%$ \\
\hline SM35 & 300 & $\mathrm{Mg}(\mathrm{mg} / \mathrm{L})$ & 1.018 & 1.016 & $0.19 \%$ & 0.0218 & 0.0209 & 1.05 & $2.15 \%$ & $2.06 \%$ \\
\hline SM35 & 300 & $\mathrm{Mn}(\mathrm{mg} / \mathrm{L})$ & 0.000 & 0.000 & & 0.0010 & 0.0025 & & & \\
\hline SM35 & 300 & $\mathrm{Na}(\mathrm{mg} / \mathrm{L})$ & 33.078 & 33.026 & $0.16 \%$ & 0.8707 & 0.9539 & 0.91 & $2.63 \%$ & $2.89 \%$ \\
\hline SM35 & 300 & $\mathrm{Ni}(\mathrm{mg} / \mathrm{L})$ & 1.008 & 1.006 & $0.16 \%$ & 0.0183 & 0.0170 & 1.08 & $1.82 \%$ & $1.69 \%$ \\
\hline SM35 & 300 & $\mathrm{Si}(\mathrm{mg} / \mathrm{L})$ & 20.481 & 20.438 & $0.21 \%$ & 0.4119 & 0.4097 & 1.01 & $2.01 \%$ & $2.00 \%$ \\
\hline SM35 & 300 & $\mathrm{Ti}(\mathrm{mg} / \mathrm{L})$ & 0.001 & 0.001 & & 0.0010 & 0.0013 & & & \\
\hline SM35 & 300 & $\mathrm{U}(\mathrm{mg} / \mathrm{L})$ & 0.094 & 0.106 & & 0.0984 & 0.1108 & & & \\
\hline SM35 & 300 & $\mathrm{Zr}(\mathrm{mg} / \mathrm{L})$ & -0.005 & -0.006 & & 0.0142 & 0.0158 & & & \\
\hline SM36 & 300 & $\mathrm{Al}(\mathrm{mg} / \mathrm{L})$ & 1.986 & 1.986 & $-0.01 \%$ & 0.0310 & 0.0261 & 1.19 & $1.56 \%$ & $1.31 \%$ \\
\hline SM36 & 300 & $\mathrm{Ca}(\mathrm{mg} / \mathrm{L})$ & 0.012 & 0.016 & & 0.0312 & 0.0625 & & & \\
\hline SM36 & 300 & $\mathrm{Cr}(\mathrm{mg} / \mathrm{L})$ & 0.002 & 0.002 & & 0.0019 & 0.0023 & & & \\
\hline SM36 & 300 & $\mathrm{Cu}(\mathrm{mg} / \mathrm{L})$ & 0.001 & 0.001 & & 0.0026 & 0.0028 & & & \\
\hline SM36 & 300 & $\mathrm{Fe}(\mathrm{mg} / \mathrm{L})$ & 9.940 & 9.940 & $0.00 \%$ & 0.1316 & 0.1202 & 1.09 & $1.32 \%$ & $1.21 \%$ \\
\hline SM36 & 300 & $\mathrm{~K}(\mathrm{mg} / \mathrm{L})$ & 0.016 & 0.013 & & 0.0620 & 0.0437 & & & \\
\hline
\end{tabular}


WSRC-TR-2004-00576

Revision 0

Table A2. Comparisons of SME and MFT Measurements for Mixed Acid Calibration and Bench Standards

\begin{tabular}{|c|c|c|c|c|c|c|c|c|c|c|}
\hline & & & & & & & & "MFT Std Dev/ & & \\
\hline Standard & n & Element & MFT Mean & SME Mean & \% Difference & MFT Std Dev & SME Std Dev & SME Std Dev & \%RSD MFT & \%RSD SME \\
\hline SM36 & 300 & $\mathrm{Li}(\mathrm{mg} / \mathrm{L})$ & 0.008 & 0.015 & & 0.0221 & 0.1631 & & & \\
\hline SM36 & 300 & $\mathrm{Mg}(\mathrm{mg} / \mathrm{L})$ & 0.003 & 0.002 & & 0.0092 & 0.0077 & & & \\
\hline SM36 & 300 & $\mathrm{Mn}(\mathrm{mg} / \mathrm{L})$ & 1.979 & 1.980 & $-0.06 \%$ & 0.0294 & 0.0301 & 0.98 & $1.49 \%$ & $1.52 \%$ \\
\hline SM36 & 300 & $\mathrm{Na}(\mathrm{mg} / \mathrm{L})$ & 4.926 & 4.917 & $0.18 \%$ & 0.1059 & 0.0878 & 1.21 & $2.15 \%$ & $1.79 \%$ \\
\hline SM36 & 300 & $\mathrm{Ni}(\mathrm{mg} / \mathrm{L})$ & 0.009 & 0.009 & & 0.0072 & 0.0079 & & & \\
\hline SM36 & 300 & $\mathrm{Si} \mathrm{(mg/L)}$ & 0.152 & 0.064 & & 0.3591 & 0.3880 & & & \\
\hline SM36 & 300 & $\mathrm{Ti}(\mathrm{mg} / \mathrm{L})$ & 0.998 & 0.997 & $0.12 \%$ & 0.0187 & 0.0167 & 1.12 & $1.88 \%$ & $1.68 \%$ \\
\hline SM36 & 300 & $\mathrm{U}(\mathrm{mg} / \mathrm{L})$ & 0.074 & 0.086 & & 0.1042 & 0.1088 & & & \\
\hline SM36 & 300 & $\mathrm{Zr}(\mathrm{mg} / \mathrm{L})$ & 0.982 & 0.981 & $0.18 \%$ & 0.0116 & 0.0118 & 0.98 & $1.18 \%$ & $1.20 \%$ \\
\hline SM37 & 161 & $\mathrm{Al}(\mathrm{mg} / \mathrm{L})$ & 0.043 & 0.044 & & 0.0194 & 0.0189 & & & \\
\hline SM37 & 161 & $\mathrm{Ca}(\mathrm{mg} / \mathrm{L})$ & 0.290 & 0.272 & & 0.3927 & 0.3270 & & & \\
\hline SM37 & 161 & $\mathrm{Cr}(\mathrm{mg} / \mathrm{L})$ & 0.029 & 0.028 & & 0.0043 & 0.0056 & & & \\
\hline SM37 & 161 & $\mathrm{Cu}(\mathrm{mg} / \mathrm{L})$ & 0.024 & 0.026 & & 0.0249 & 0.0452 & & & \\
\hline SM37 & 161 & $\mathrm{Fe}(\mathrm{mg} / \mathrm{L})$ & -0.003 & -0.008 & & 0.0161 & 0.0298 & & & \\
\hline SM37 & 161 & $\mathrm{~K}(\mathrm{mg} / \mathrm{L})$ & -0.006 & -0.007 & & 0.0529 & 0.0501 & & & \\
\hline SM37 & 161 & $\mathrm{Li}(\mathrm{mg} / \mathrm{L})$ & 0.000 & 0.001 & & 0.0024 & 0.0021 & & & \\
\hline SM37 & 161 & $\mathrm{Mg}(\mathrm{mg} / \mathrm{L})$ & 0.005 & 0.005 & & 0.0052 & 0.0051 & & & \\
\hline SM37 & 161 & $\mathrm{Mn}(\mathrm{mg} / \mathrm{L})$ & 0.002 & 0.002 & & 0.0012 & 0.0013 & & & \\
\hline SM37 & 161 & $\mathrm{Na}(\mathrm{mg} / \mathrm{L})$ & 0.057 & 0.170 & & 0.3498 & 0.6734 & & & \\
\hline SM37 & 161 & $\mathrm{Ni}(\mathrm{mg} / \mathrm{L})$ & 0.002 & 0.002 & & 0.0077 & 0.0079 & & & \\
\hline SM37 & 161 & $\mathrm{Si}(\mathrm{mg} / \mathrm{L})$ & 0.471 & 0.387 & & 0.6063 & 0.6802 & & & \\
\hline SM37 & 161 & $\mathrm{Ti}(\mathrm{mg} / \mathrm{L})$ & 0.001 & 0.001 & & 0.0015 & 0.0017 & & & \\
\hline SM37 & 161 & $\mathrm{U}(\mathrm{mg} / \mathrm{L})$ & 9.846 & 9.845 & $0.01 \%$ & 0.1958 & 0.1641 & 1.19 & $1.99 \%$ & $1.67 \%$ \\
\hline SM37 & 161 & $\mathrm{Zr}(\mathrm{mg} / \mathrm{L})$ & -0.005 & -0.005 & & 0.0163 & 0.0158 & & & \\
\hline SM38 & 300 & $\mathrm{Al}(\mathrm{mg} / \mathrm{L})$ & 0.046 & 0.060 & & 0.0163 & 0.1863 & & & \\
\hline SM38 & 300 & $\mathrm{Ca}(\mathrm{mg} / \mathrm{L})$ & 0.292 & 0.263 & & 0.3754 & 0.3254 & & & \\
\hline SM38 & 300 & $\mathrm{Cr}(\mathrm{mg} / \mathrm{L})$ & 0.029 & 0.029 & & 0.0040 & 0.0059 & & & \\
\hline SM38 & 300 & $\mathrm{Cu}(\mathrm{mg} / \mathrm{L})$ & 0.024 & 0.026 & & 0.0162 & 0.0433 & & & \\
\hline SM38 & 300 & $\mathrm{Fe}(\mathrm{mg} / \mathrm{L})$ & -0.003 & -0.008 & & 0.0162 & 0.0307 & & & \\
\hline SM38 & 300 & $\mathrm{~K}(\mathrm{mg} / \mathrm{L})$ & 0.002 & 0.004 & & 0.0490 & 0.0476 & & & \\
\hline SM38 & 300 & $\mathrm{Li}(\mathrm{mg} / \mathrm{L})$ & 0.000 & 0.001 & & 0.0017 & 0.0021 & & & \\
\hline SM38 & 300 & $\mathrm{Mg}(\mathrm{mg} / \mathrm{L})$ & 0.005 & 0.005 & & 0.0053 & 0.0051 & & & \\
\hline SM38 & 300 & $\mathrm{Mn}(\mathrm{mg} / \mathrm{L})$ & 0.001 & 0.002 & & 0.0010 & 0.0015 & & & \\
\hline SM38 & 300 & $\mathrm{Na}(\mathrm{mg} / \mathrm{L})$ & 0.053 & 0.174 & & 0.3600 & 0.6796 & & & \\
\hline SM38 & 300 & $\mathrm{Ni}(\mathrm{mg} / \mathrm{L})$ & 0.003 & 0.002 & & 0.0077 & 0.0074 & & & \\
\hline SM38 & 300 & $\mathrm{Si}(\mathrm{mg} / \mathrm{L})$ & 0.210 & 0.138 & & 0.4877 & 0.6453 & & & \\
\hline SM38 & 300 & $\mathrm{Ti}(\mathrm{mg} / \mathrm{L})$ & 0.001 & 0.001 & & 0.0010 & 0.0017 & & & \\
\hline SM38 & 300 & $\mathrm{U}(\mathrm{mg} / \mathrm{L})$ & 9.914 & 9.912 & $0.02 \%$ & 0.1904 & 0.1696 & 1.12 & $1.92 \%$ & $1.71 \%$ \\
\hline SM38 & 300 & $\mathrm{Zr}(\mathrm{mg} / \mathrm{L})$ & -0.005 & -0.005 & & 0.0147 & 0.0159 & & & \\
\hline
\end{tabular}


WSRC-TR-2004-00576

Revision 0

Table A3. Comparisons of SME and MFT Measurements for Fusion Calibration and Bench Standards

\begin{tabular}{|c|c|c|c|c|c|c|c|c|c|c|}
\hline & & & & & & & & "MFT Std Dev/ & & \\
\hline Standard & n & Element & MFT Mean & SME Mean & \% Difference & MFT Std Dev & SME Std Dev & SME Std Dev & \%RSD MFT & \%RSD SME \\
\hline SM51 & 153 & AL (mg/L) & 0.006 & 0.008 & & 0.0144 & 0.0133 & & & \\
\hline SM51 & 153 & $\mathrm{~B}(\mathrm{mg} / \mathrm{L})$ & 3.874 & 3.890 & $-0.40 \%$ & 0.2420 & 0.2410 & 1.00 & $6.25 \%$ & $6.20 \%$ \\
\hline SM51 & 153 & $\mathrm{Cr}(\mathrm{mg} / \mathrm{L})$ & 0.989 & 0.989 & $-0.08 \%$ & 0.0166 & 0.0138 & 1.20 & $1.68 \%$ & $1.39 \%$ \\
\hline SM51 & 153 & $\mathrm{Cu}(\mathrm{mg} / \mathrm{L})$ & 0.989 & 0.989 & $0.02 \%$ & 0.0148 & 0.0134 & 1.11 & $1.50 \%$ & $1.36 \%$ \\
\hline SM51 & 153 & $\mathrm{Fe}(\mathrm{mg} / \mathrm{L})$ & 0.016 & 0.004 & & 0.1354 & 0.0128 & & & \\
\hline SM51 & 153 & $\mathrm{~K}(\mathrm{mg} / \mathrm{L})$ & 4.922 & 4.920 & $0.06 \%$ & 0.0841 & 0.0774 & 1.09 & $1.71 \%$ & $1.57 \%$ \\
\hline SM51 & 153 & $\mathrm{Li}(\mathrm{mg} / \mathrm{L})$ & 1.975 & 1.973 & $0.08 \%$ & 0.0273 & 0.0286 & 0.96 & $1.38 \%$ & $1.45 \%$ \\
\hline SM51 & 153 & $\mathrm{Mg}(\mathrm{mg} / \mathrm{L})$ & 1.004 & 1.002 & $0.25 \%$ & 0.0241 & 0.0236 & 1.02 & $2.40 \%$ & $2.35 \%$ \\
\hline SM51 & 153 & $\mathrm{Mn}(\mathrm{mg} / \mathrm{L})$ & 0.001 & 0.001 & & 0.0018 & 0.0018 & & & \\
\hline SM51 & 153 & $\mathrm{Ni}(\mathrm{mg} / \mathrm{L})$ & 0.986 & 0.987 & $-0.12 \%$ & 0.0193 & 0.0167 & 1.16 & $1.96 \%$ & $1.69 \%$ \\
\hline SM51 & 153 & Si (mg/L) & 20.159 & 20.144 & $0.08 \%$ & 0.4072 & 0.4053 & 1.00 & $2.02 \%$ & $2.01 \%$ \\
\hline SM51 & 153 & $\mathrm{Ti}(\mathrm{mg} / \mathrm{L})$ & 0.000 & 0.001 & & 0.0015 & 0.0015 & & & \\
\hline SM52 & 151 & AL (mg/L) & 1.971 & 1.975 & $-0.20 \%$ & 0.0328 & 0.0320 & 1.03 & $1.67 \%$ & $1.62 \%$ \\
\hline SM52 & 151 & $\mathrm{~B}(\mathrm{mg} / \mathrm{L})$ & 1.984 & 1.988 & $-0.18 \%$ & 0.0302 & 0.0335 & 0.90 & $1.52 \%$ & $1.69 \%$ \\
\hline SM52 & 151 & $\mathrm{Ca}(\mathrm{mg} / \mathrm{L})$ & 0.018 & 0.011 & & 0.0820 & 0.0590 & & & \\
\hline SM52 & 151 & $\mathrm{Cu}(\mathrm{mg} / \mathrm{L})$ & 0.000 & 0.000 & & 0.0032 & 0.0030 & & & \\
\hline SM52 & 151 & $\mathrm{Fe}(\mathrm{mg} / \mathrm{L})$ & 9.872 & 9.890 & $-0.18 \%$ & 0.1421 & 0.1479 & 0.96 & $1.44 \%$ & $1.50 \%$ \\
\hline SM52 & 151 & $\mathrm{~K}(\mathrm{mg} / \mathrm{L})$ & 0.011 & 0.014 & & 0.0523 & 0.0490 & & & \\
\hline SM52 & 151 & $\mathrm{Li}(\mathrm{mg} / \mathrm{L})$ & 0.001 & 0.001 & & 0.0019 & 0.0023 & & & \\
\hline SM52 & 151 & $\mathrm{Mg}(\mathrm{mg} / \mathrm{L})$ & 0.004 & 0.003 & & 0.0280 & 0.0255 & & & \\
\hline SM52 & 151 & $\mathrm{Mn}(\mathrm{mg} / \mathrm{L})$ & 1.970 & 1.974 & $-0.21 \%$ & 0.0302 & 0.0325 & 0.93 & $1.54 \%$ & $1.65 \%$ \\
\hline SM52 & 151 & $\mathrm{Ni}(\mathrm{mg} / \mathrm{L})$ & 0.008 & 0.008 & & 0.0077 & 0.0083 & & & \\
\hline SM52 & 151 & Si (mg/L) & 0.005 & 0.007 & & 0.0355 & 0.0558 & & & \\
\hline SM52 & 151 & $\mathrm{Ti}(\mathrm{mg} / \mathrm{L})$ & 0.992 & 0.993 & $-0.09 \%$ & 0.0195 & 0.0190 & 1.02 & $1.96 \%$ & $1.92 \%$ \\
\hline SM53 & 305 & AL (mg/L) & 0.009 & 0.011 & & 0.0106 & 0.0104 & & & \\
\hline SM53 & 305 & $\mathrm{~B}(\mathrm{mg} / \mathrm{L})$ & 3.897 & 3.907 & $-0.23 \%$ & 0.2411 & 0.2395 & 1.01 & $6.19 \%$ & $6.13 \%$ \\
\hline SM53 & 305 & $\mathrm{Ca}(\mathrm{mg} / \mathrm{L})$ & 1.003 & 1.001 & $0.14 \%$ & 0.0251 & 0.0248 & 1.01 & $2.50 \%$ & $2.48 \%$ \\
\hline SM53 & 305 & $\mathrm{Cr}(\mathrm{mg} / \mathrm{L})$ & 0.991 & 0.993 & $-0.17 \%$ & 0.0145 & 0.0141 & 1.03 & $1.46 \%$ & $1.42 \%$ \\
\hline SM53 & 305 & $\mathrm{Cu}(\mathrm{mg} / \mathrm{L})$ & 0.991 & 0.992 & $-0.08 \%$ & 0.0129 & 0.0129 & 1.00 & $1.30 \%$ & $1.30 \%$ \\
\hline SM53 & 305 & $\mathrm{Fe}(\mathrm{mg} / \mathrm{L})$ & 0.012 & 0.004 & & 0.1153 & 0.0130 & & & \\
\hline SM53 & 305 & $\mathrm{~K}(\mathrm{mg} / \mathrm{L})$ & 4.935 & 4.941 & $-0.12 \%$ & 0.0669 & 0.0712 & 0.94 & $1.36 \%$ & $1.44 \%$ \\
\hline SM53 & 305 & $\mathrm{Li}(\mathrm{mg} / \mathrm{L})$ & 1.980 & 1.978 & $0.06 \%$ & 0.0232 & 0.0257 & 0.90 & $1.17 \%$ & $1.30 \%$ \\
\hline SM53 & 305 & $\mathrm{Ni}(\mathrm{mg} / \mathrm{L})$ & 0.989 & 0.991 & $-0.19 \%$ & 0.0171 & 0.0155 & 1.11 & $1.73 \%$ & $1.56 \%$ \\
\hline SM53 & 305 & Si (mg/L) & 20.067 & 20.062 & $0.03 \%$ & 0.3073 & 0.3428 & 0.90 & $1.53 \%$ & $1.71 \%$ \\
\hline SM53 & 305 & $\mathrm{Ti}(\mathrm{mg} / \mathrm{L})$ & 0.001 & 0.001 & & 0.0010 & 0.0012 & & & \\
\hline SM54 & 304 & AL (mg/L) & 1.979 & 1.981 & $-0.12 \%$ & 0.0295 & 0.0275 & 1.07 & $1.49 \%$ & $1.39 \%$ \\
\hline SM54 & 304 & $\mathrm{~B}(\mathrm{mg} / \mathrm{L})$ & 1.988 & 1.989 & $-0.06 \%$ & 0.0321 & 0.0311 & 1.03 & $1.62 \%$ & $1.57 \%$ \\
\hline SM54 & 304 & $\mathrm{Ca}(\mathrm{mg} / \mathrm{L})$ & 0.008 & 0.004 & & 0.0511 & 0.0208 & & & \\
\hline SM54 & 304 & $\mathrm{Cr}(\mathrm{mg} / \mathrm{L})$ & 0.002 & 0.002 & & 0.0021 & 0.0022 & & & \\
\hline SM54 & 304 & $\mathrm{Cu}(\mathrm{mg} / \mathrm{L})$ & 0.001 & 0.001 & & 0.0026 & 0.0019 & & & \\
\hline SM54 & 304 & $\mathrm{Fe}(\mathrm{mg} / \mathrm{L})$ & 9.889 & 9.900 & $-0.11 \%$ & 0.1380 & 0.1262 & 1.09 & $1.40 \%$ & $1.27 \%$ \\
\hline SM54 & 304 & $\mathrm{~K}(\mathrm{mg} / \mathrm{L})$ & 0.015 & 0.017 & & 0.0429 & 0.0365 & & & \\
\hline SM54 & 304 & $\mathrm{Li}(\mathrm{mg} / \mathrm{L})$ & 0.001 & 0.001 & & 0.0018 & 0.0017 & & & \\
\hline SM54 & 304 & $\mathrm{Mg}(\mathrm{mg} / \mathrm{L})$ & 0.001 & 0.001 & & 0.0018 & 0.0023 & & & \\
\hline SM54 & 304 & $\mathrm{Mn}(\mathrm{mg} / \mathrm{L})$ & 1.974 & 1.978 & $-0.17 \%$ & 0.0295 & 0.0287 & 1.03 & $1.50 \%$ & $1.45 \%$ \\
\hline SM54 & 304 & $\mathrm{Ni}(\mathrm{mg} / \mathrm{L})$ & 0.008 & 0.008 & & 0.0075 & 0.0073 & & & \\
\hline SM54 & 304 & $\mathrm{Si}(\mathrm{mg} / \mathrm{L})$ & 0.013 & 0.009 & & 0.0237 & 0.0324 & & & \\
\hline SM54 & 304 & Ti (mg/L) & 0.994 & 0.994 & $-0.07 \%$ & 0.0193 & 0.0189 & 1.02 & $1.94 \%$ & $1.90 \%$ \\
\hline SM55 & 299 & AL (mg/L) & 0.029 & 0.023 & & 0.0494 & 0.0331 & & & \\
\hline SM55 & 299 & $\mathrm{~B}(\mathrm{mg} / \mathrm{L})$ & 0.009 & 0.009 & & 0.0247 & 0.0207 & & & \\
\hline SM55 & 299 & $\mathrm{Ca}(\mathrm{mg} / \mathrm{L})$ & 0.001 & 0.003 & & 0.0527 & 0.0457 & & & \\
\hline SM55 & 299 & $\mathrm{Cr}(\mathrm{mg} / \mathrm{L})$ & 0.011 & 0.008 & & 0.0261 & 0.0078 & & & \\
\hline SM55 & 299 & $\mathrm{Cu}(\mathrm{mg} / \mathrm{L})$ & 0.005 & 0.005 & & 0.0128 & 0.0066 & & & \\
\hline SM55 & 299 & $\mathrm{Fe}(\mathrm{mg} / \mathrm{L})$ & 0.063 & 0.046 & & 0.1497 & 0.0556 & & & \\
\hline SM55 & 299 & $\mathrm{~K}(\mathrm{mg} / \mathrm{L})$ & 0.027 & 0.079 & & 0.0533 & 0.5303 & & & \\
\hline SM55 & 299 & $\mathrm{Li}(\mathrm{mg} / \mathrm{L})$ & 0.005 & 0.005 & & 0.0096 & 0.0062 & & & \\
\hline SM55 & 299 & $\mathrm{Mg}(\mathrm{mg} / \mathrm{L})$ & 0.004 & 0.004 & & 0.0084 & 0.0083 & & & \\
\hline SM55 & 299 & $\mathrm{Mn}(\mathrm{mg} / \mathrm{L})$ & 0.006 & 0.005 & & 0.0178 & 0.0075 & & & \\
\hline SM55 & 299 & $\mathrm{Ni}(\mathrm{mg} / \mathrm{L})$ & 0.011 & 0.008 & & 0.0282 & 0.0113 & & & \\
\hline SM55 & 299 & $\mathrm{Si}(\mathrm{mg} / \mathrm{L})$ & 0.115 & 0.108 & & 0.2081 & 0.2122 & & & \\
\hline SM55 & 299 & $\mathrm{Ti}(\mathrm{mg} / \mathrm{L})$ & 0.005 & 0.004 & & 0.0138 & 0.0032 & & & \\
\hline
\end{tabular}


WSRC-TR-2004-00576

\section{Exhibit A1. Cold Chem Standards in Analytical Sequence}

\section{Revision 0}

STCd=IN32

Control Chart

Individual Measurement of $\mathrm{Al}$ (mg/L)

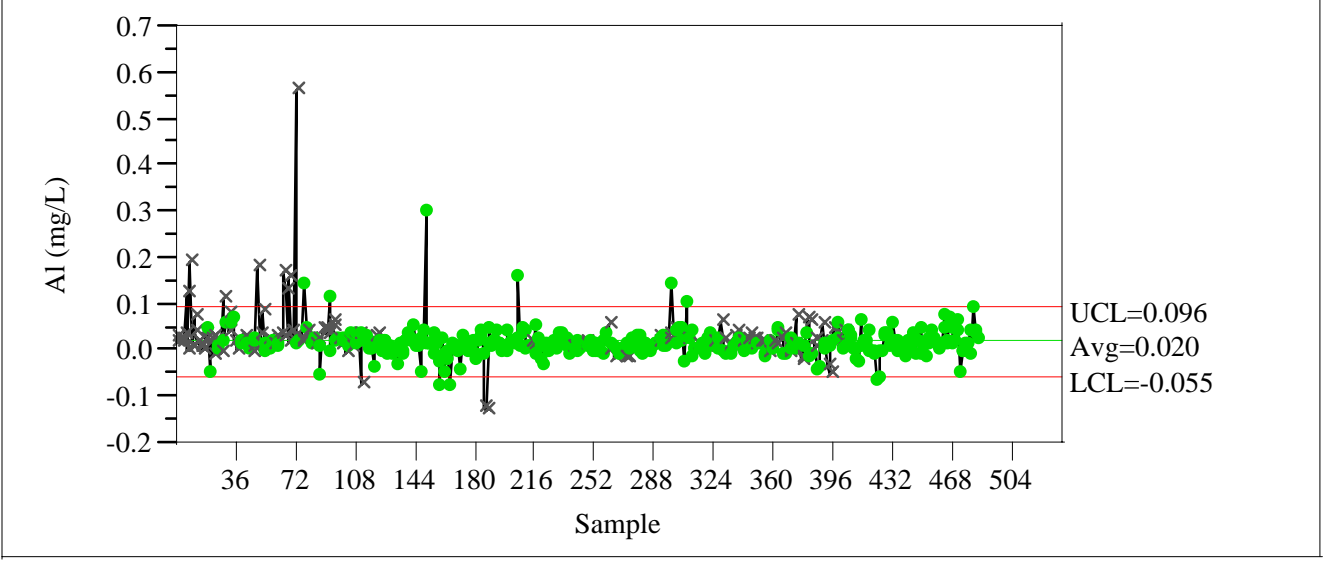

Individual Measurement of B (mg/L)

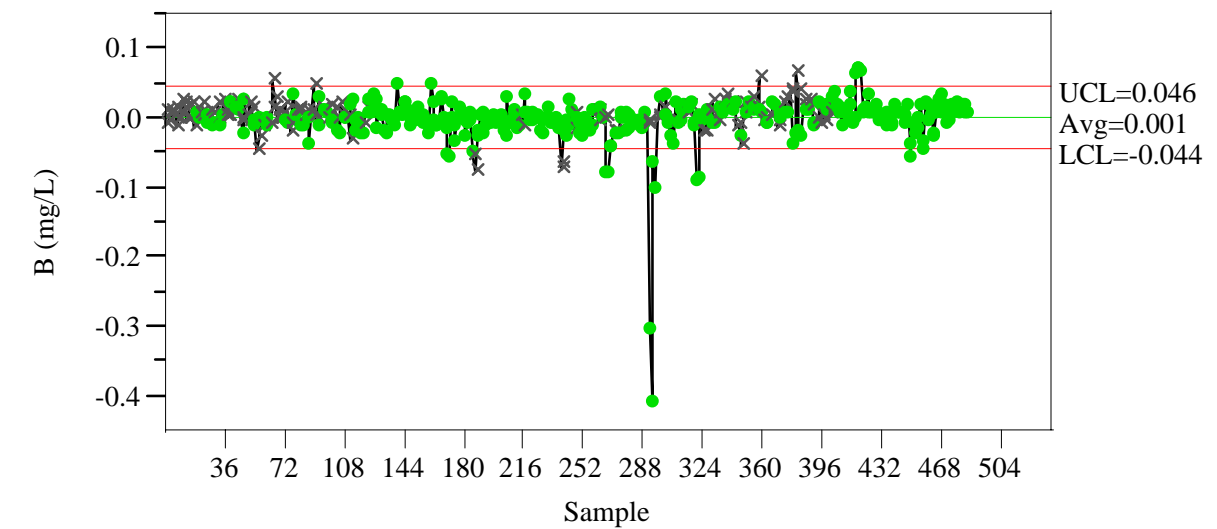

Individual Measurement of Ca (mg/L)

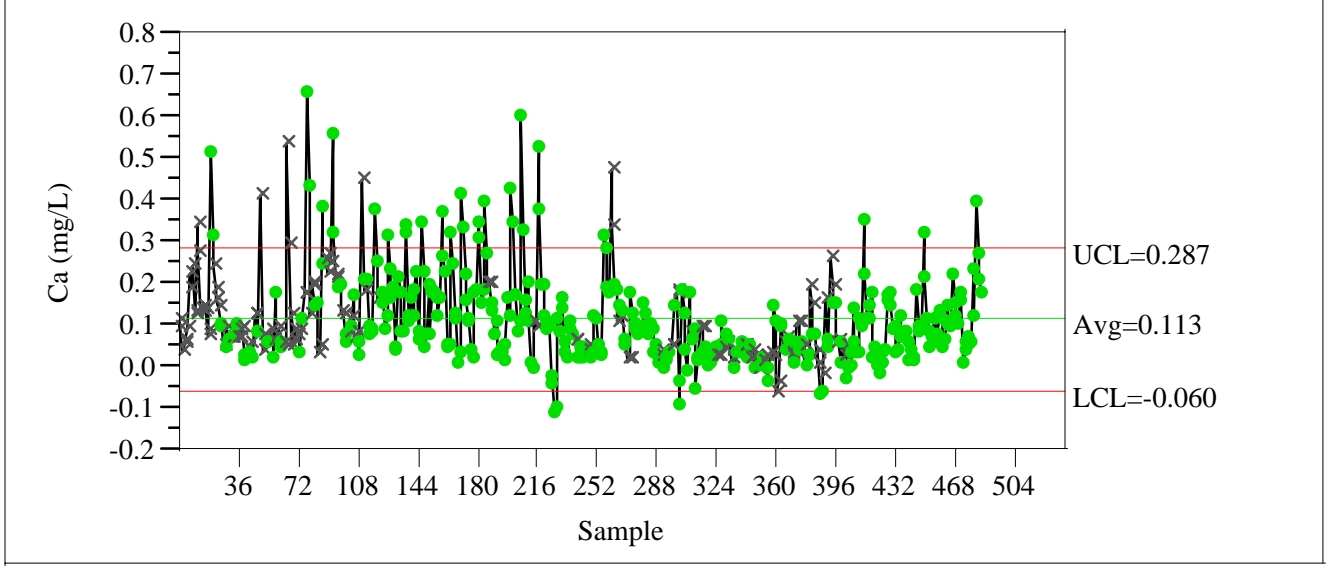


WSRC-TR-2004-00576

\section{Exhibit A1. Cold Chem Standards in Analytical Sequence}

\section{Revision 0}

Individual Measurement of $\mathrm{Cr}$ (mg/L)

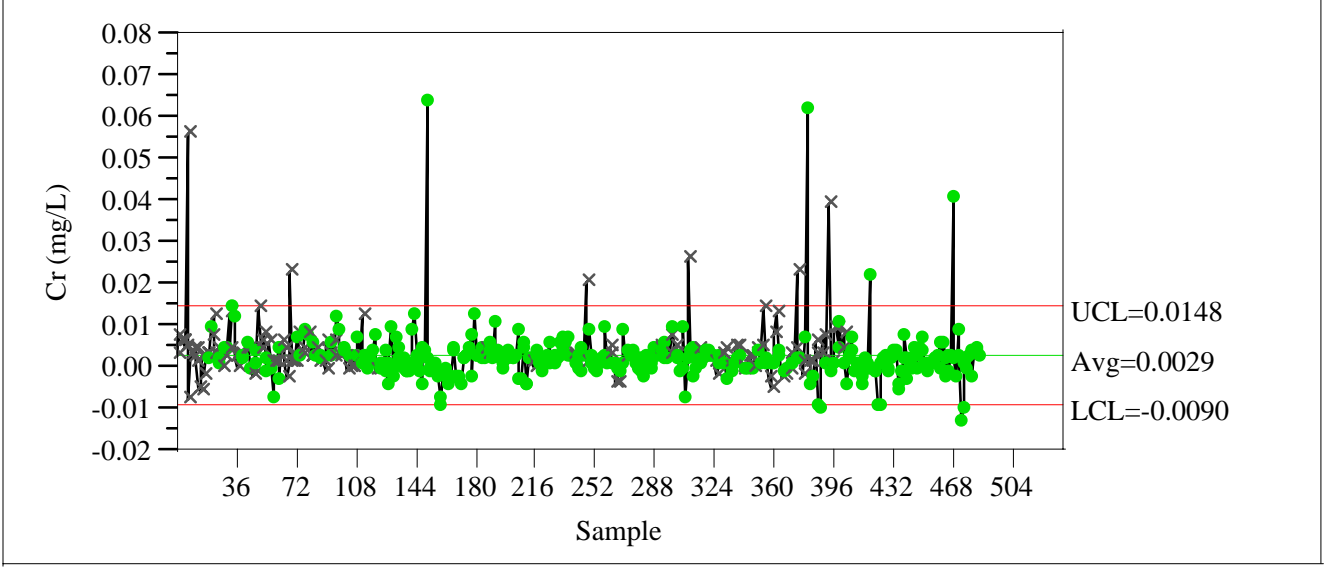

Individual Measurement of $\mathrm{Cu}$ (mg/L)

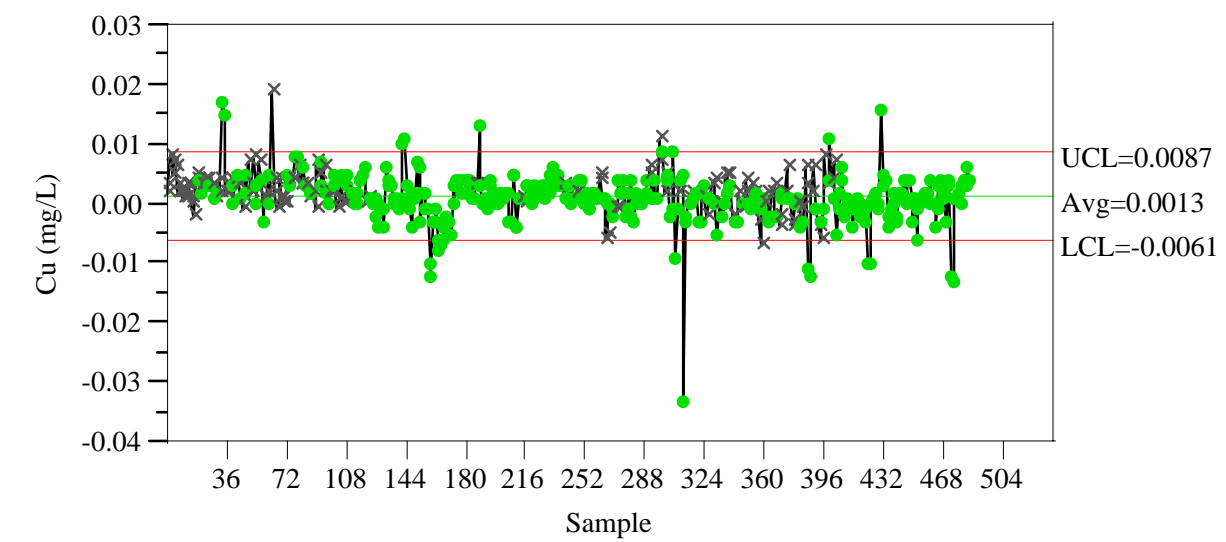

Individual Measurement of Fe (mg/L)

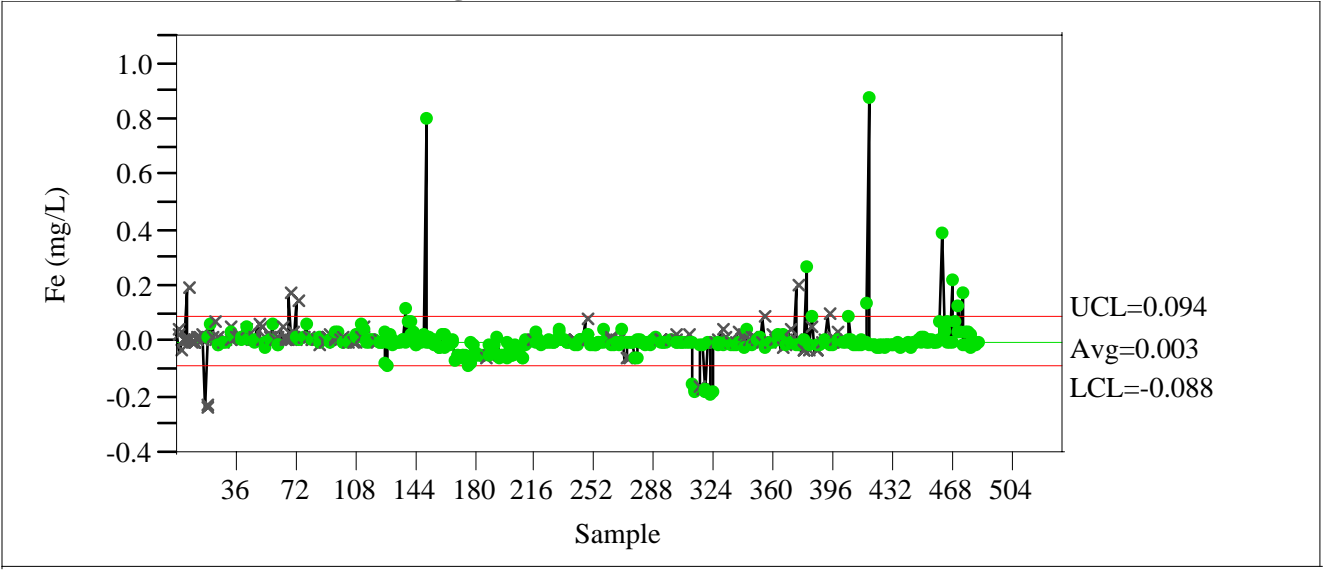




\section{Exhibit A1. Cold Chem Standards in Analytical Sequence}

\section{Revision 0}

Individual Measurement of K (mg/L)

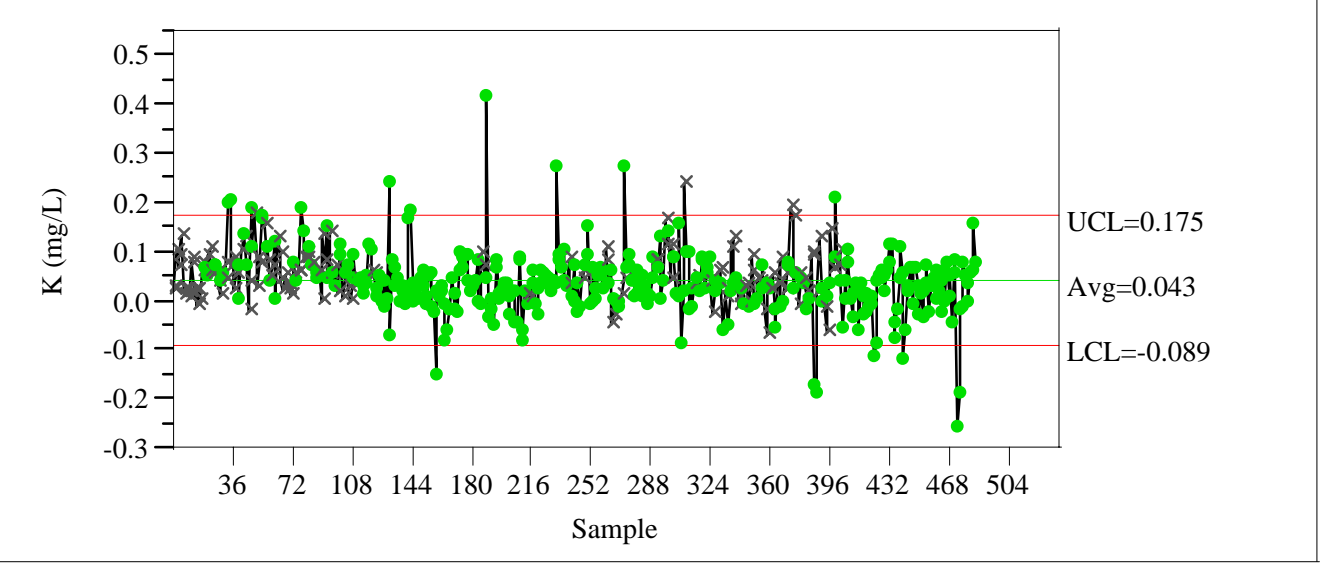

Individual Measurement of $\mathrm{Li}(\mathrm{mg} / \mathrm{L})$

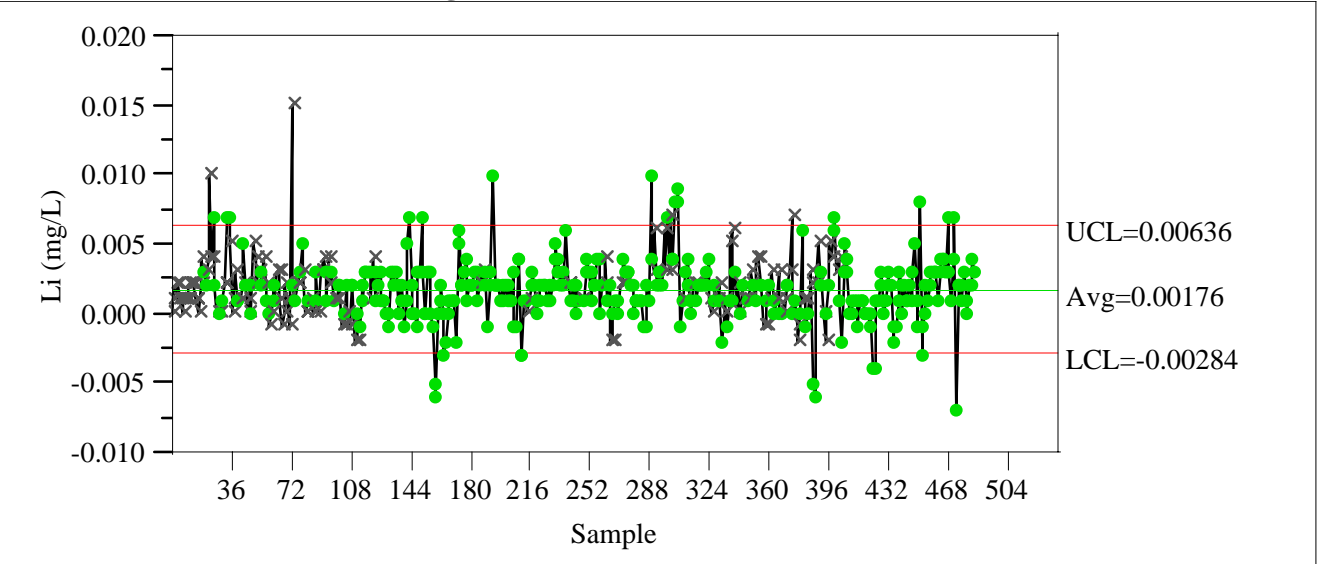

Individual Measurement of $\mathrm{Mg}(\mathrm{mg} / \mathrm{L})$

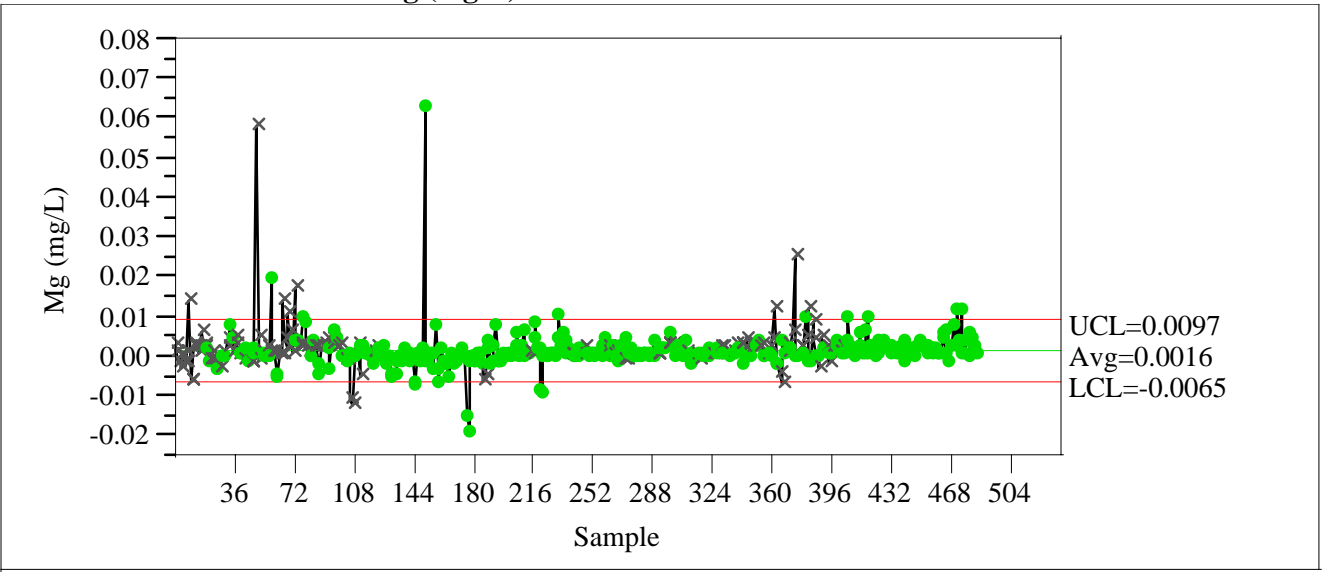


WSRC-TR-2004-00576

\section{Exhibit A1. Cold Chem Standards in Analytical Sequence}

\section{Revision 0}

Individual Measurement of Mn (mg/L)

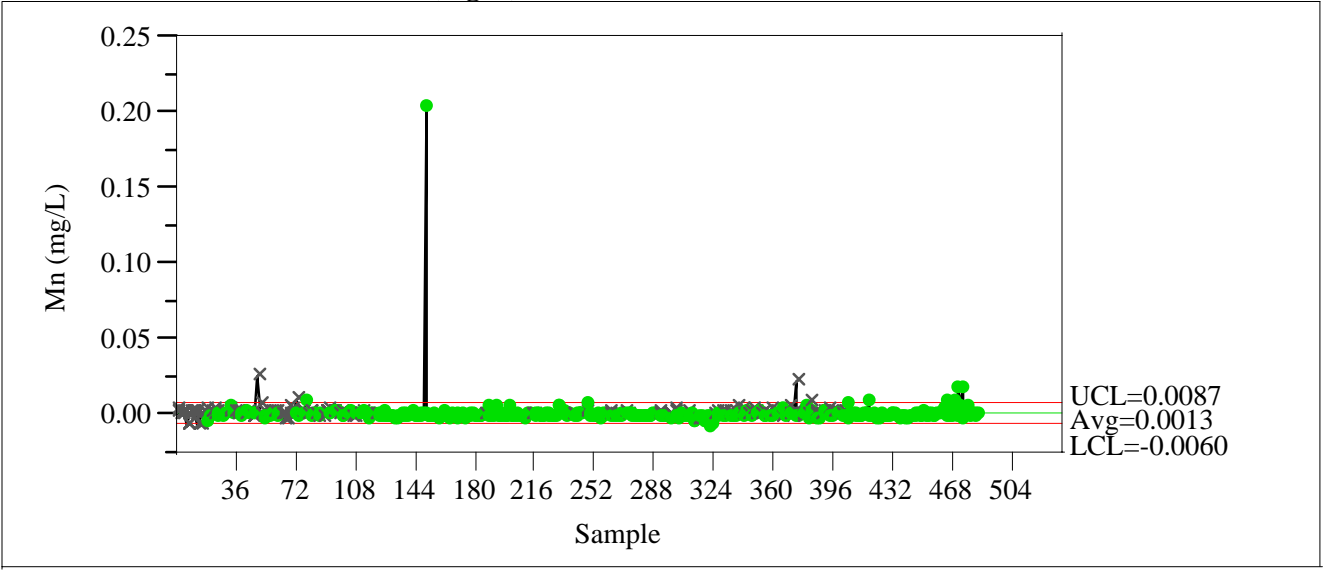

Individual Measurement of $\mathrm{Na}$ (mg/L)

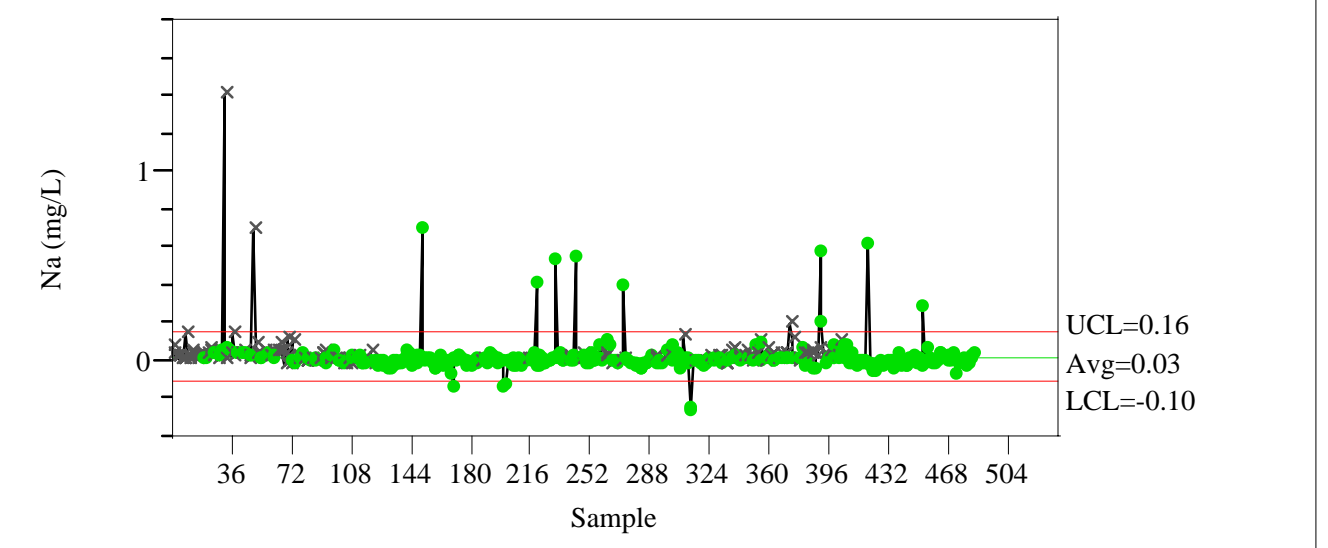

Individual Measurement of $\mathrm{Ni}(\mathrm{mg} / \mathrm{L})$

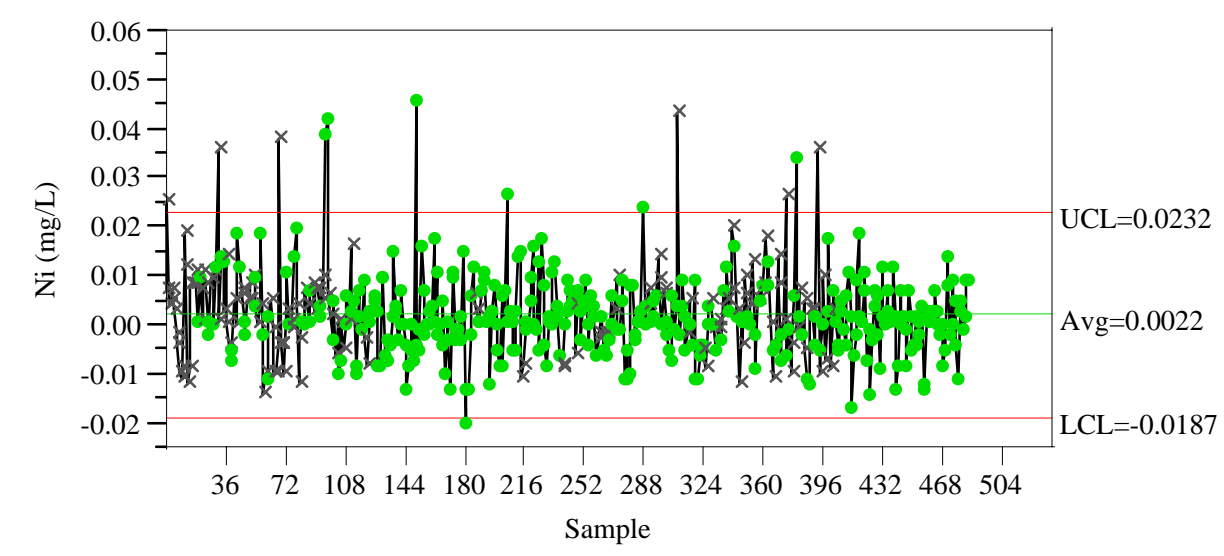




\section{Exhibit A1. Cold Chem Standards in Analytical Sequence}

Individual Measurement of Si (mg/L)

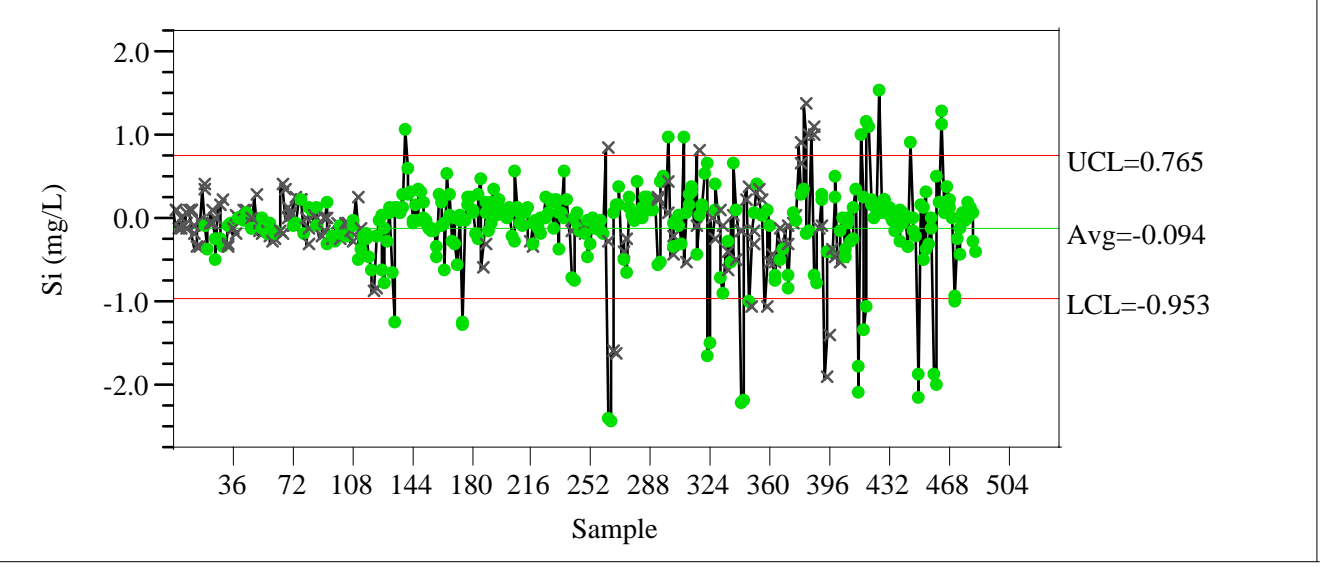

Individual Measurement of Ti (mg/L)

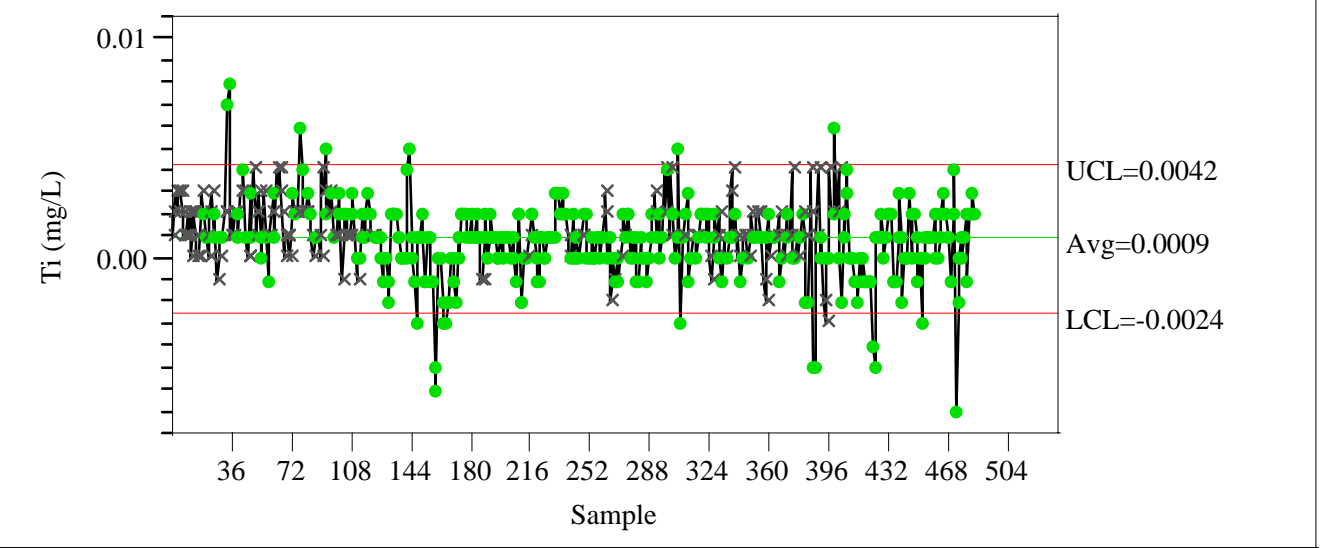

Individual Measurement of Zr (mg/L)

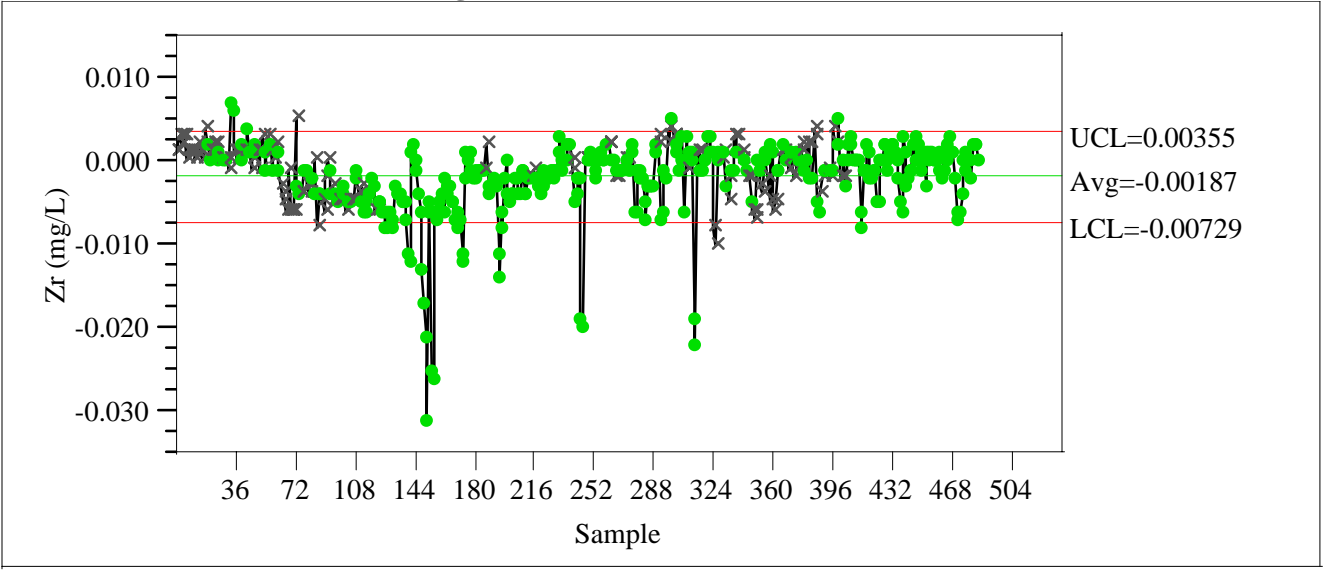




\section{Exhibit A1. Cold Chem Standards in Analytical Sequence}

Individual Measurement of U (mg/L)

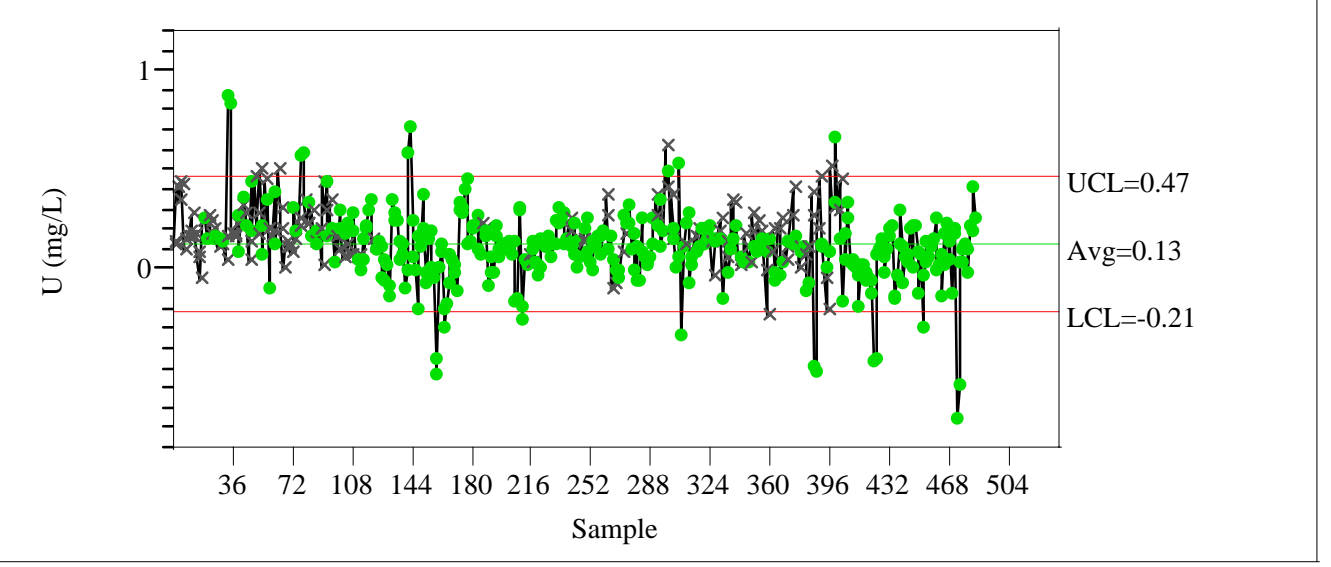

STCd=IN33

Control Chart

Individual Measurement of $\mathrm{Al}(\mathrm{mg} / \mathrm{L})$

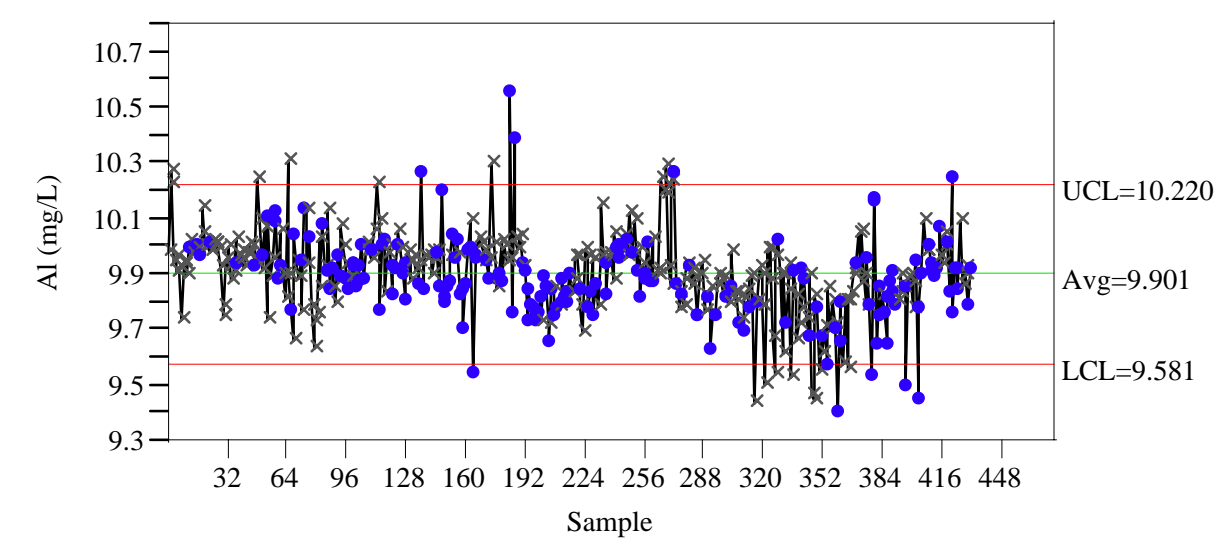

Individual Measurement of B (mg/L)

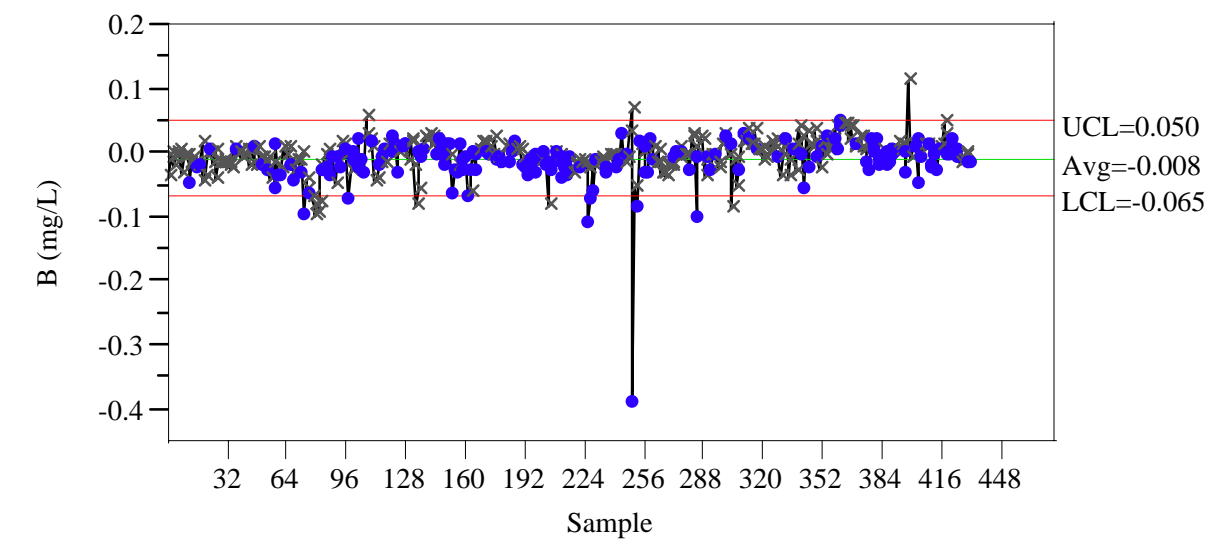




\section{Exhibit A1. Cold Chem Standards in Analytical Sequence}

Individual Measurement of Ca (mg/L)

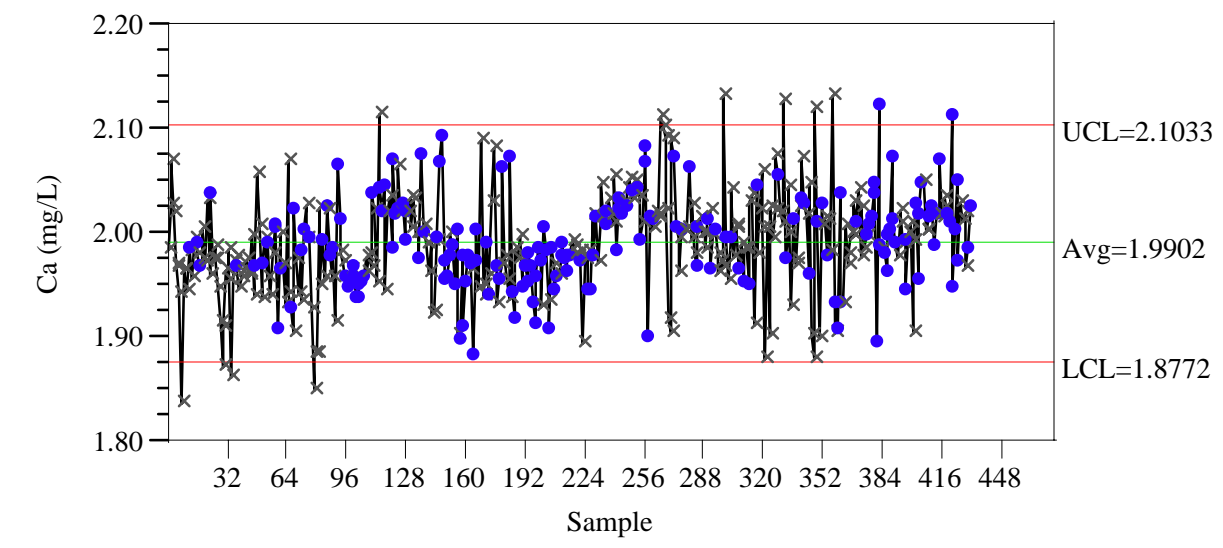

Individual Measurement of $\mathrm{Cr}$ (mg/L)

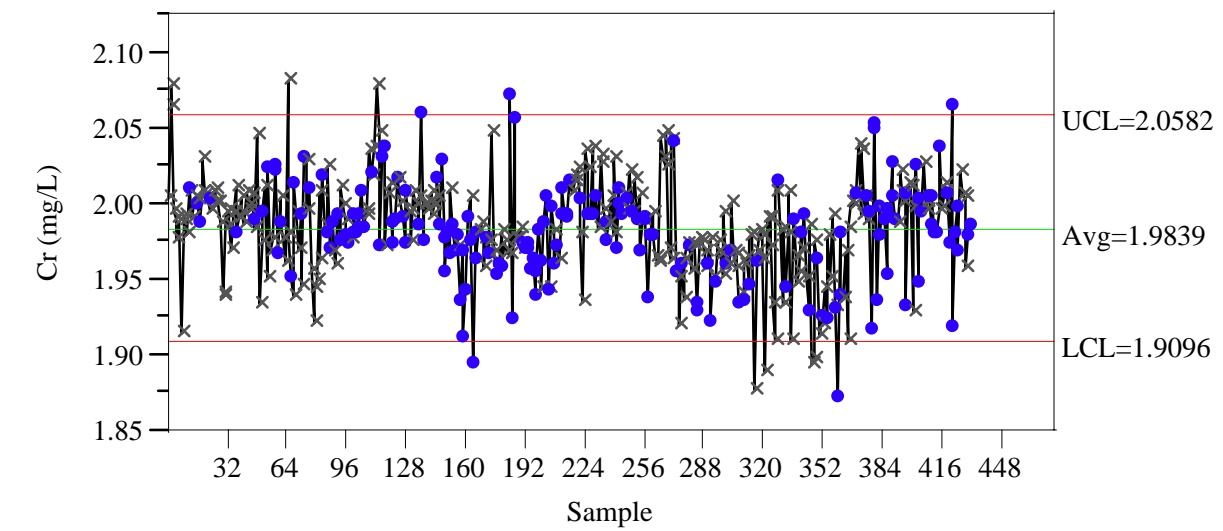

Individual Measurement of $\mathrm{Cu}(\mathrm{mg} / \mathrm{L})$

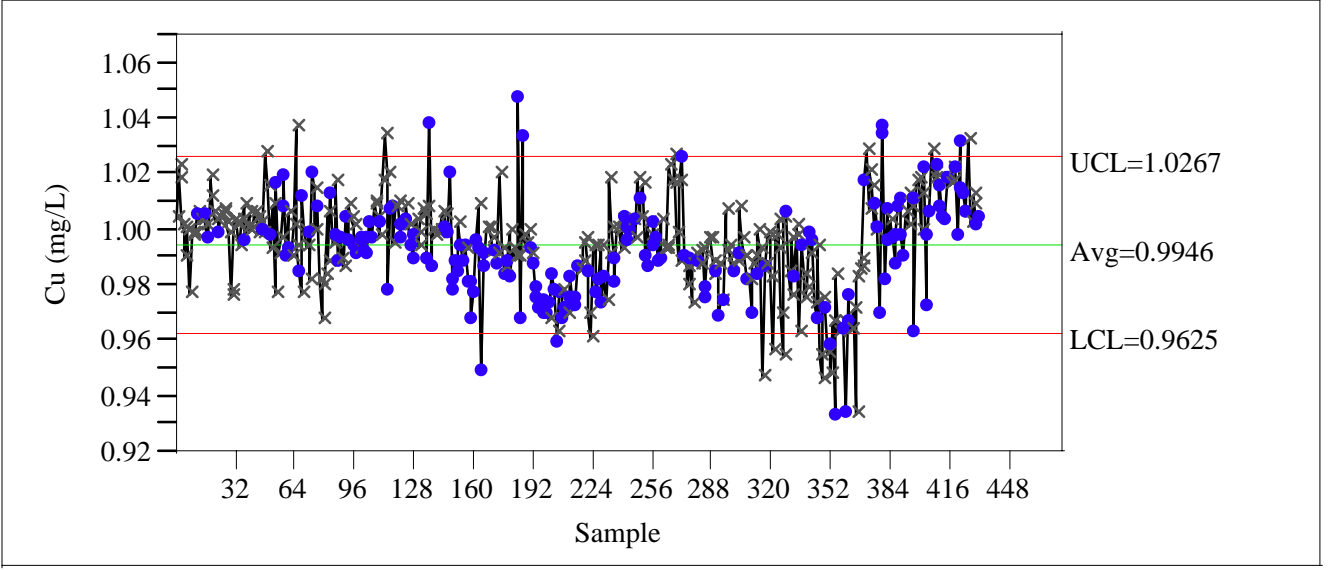




\section{Exhibit A1. Cold Chem Standards in Analytical Sequence}

Individual Measurement of Fe (mg/L)

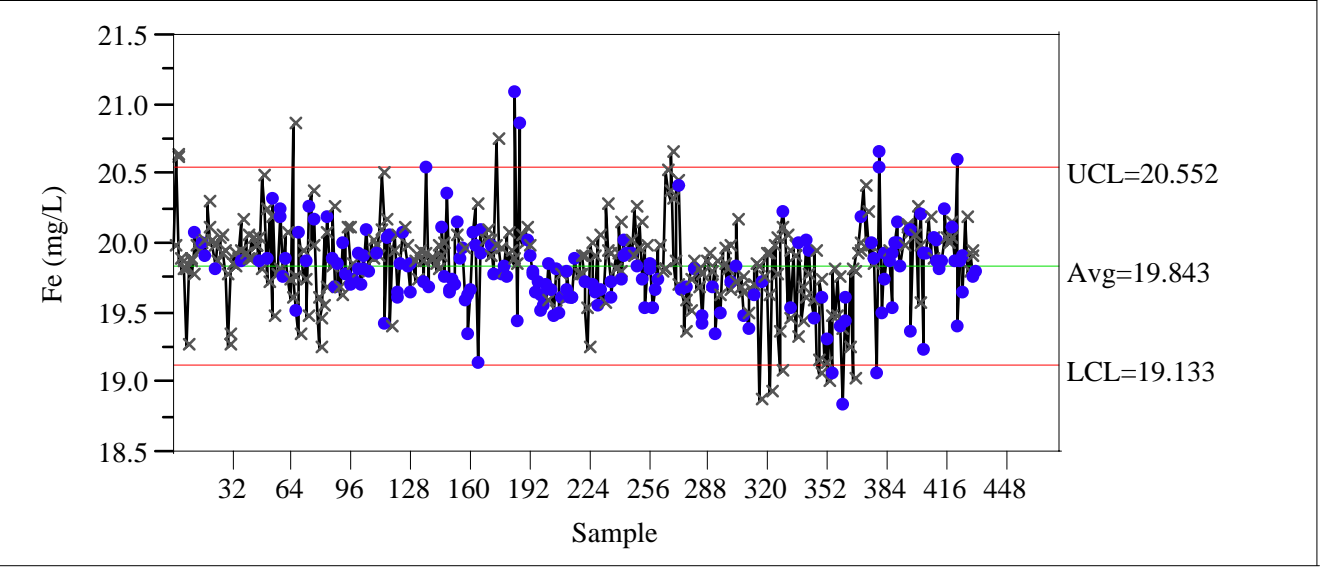

Individual Measurement of K (mg/L)

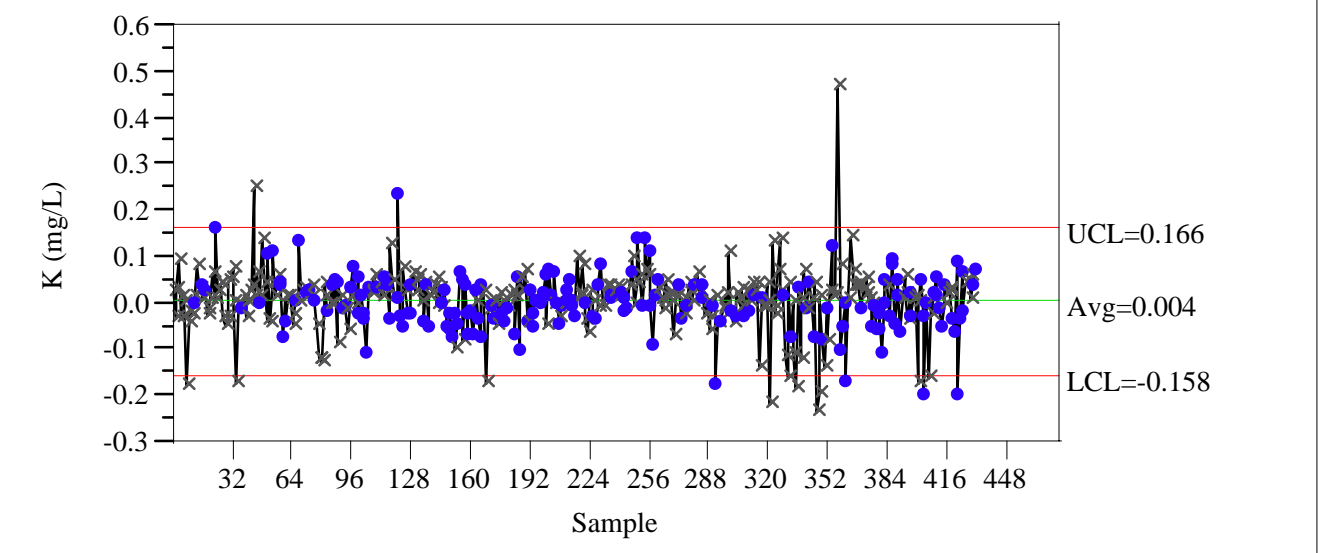

Individual Measurement of $\mathrm{Li}(\mathrm{mg} / \mathrm{L})$

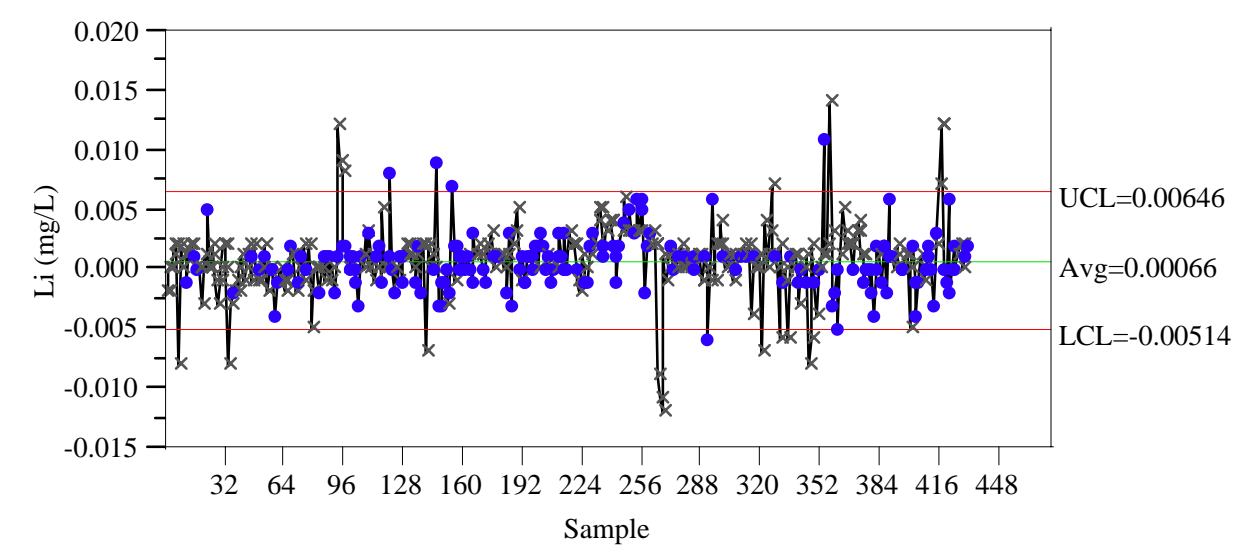




\section{Exhibit A1. Cold Chem Standards in Analytical Sequence}

Individual Measurement of $\mathrm{Mg}(\mathrm{mg} / \mathrm{L})$

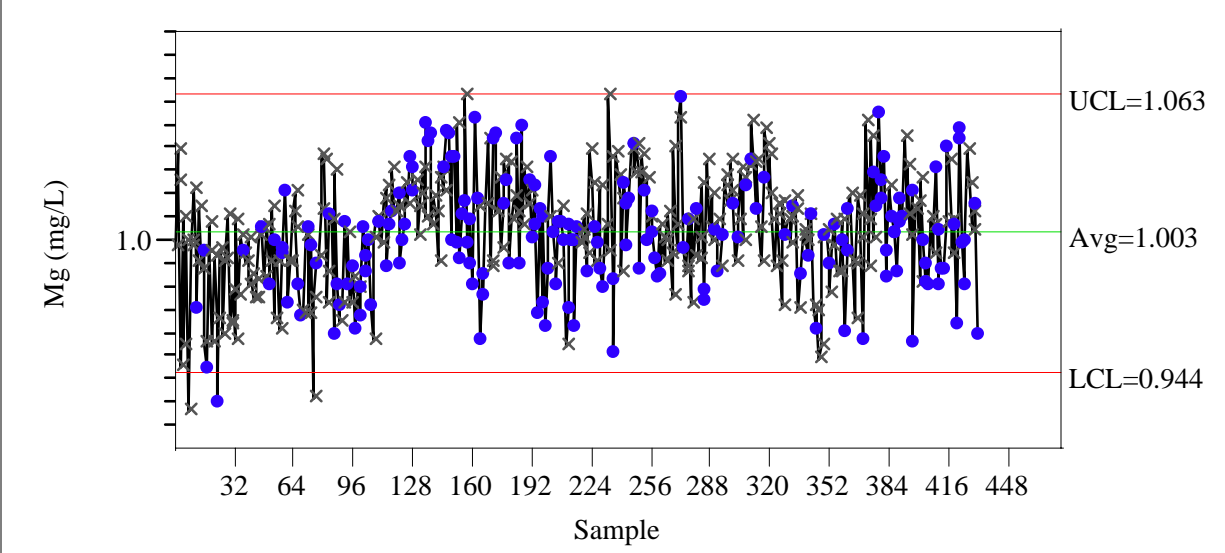

Individual Measurement of Mn (mg/L)

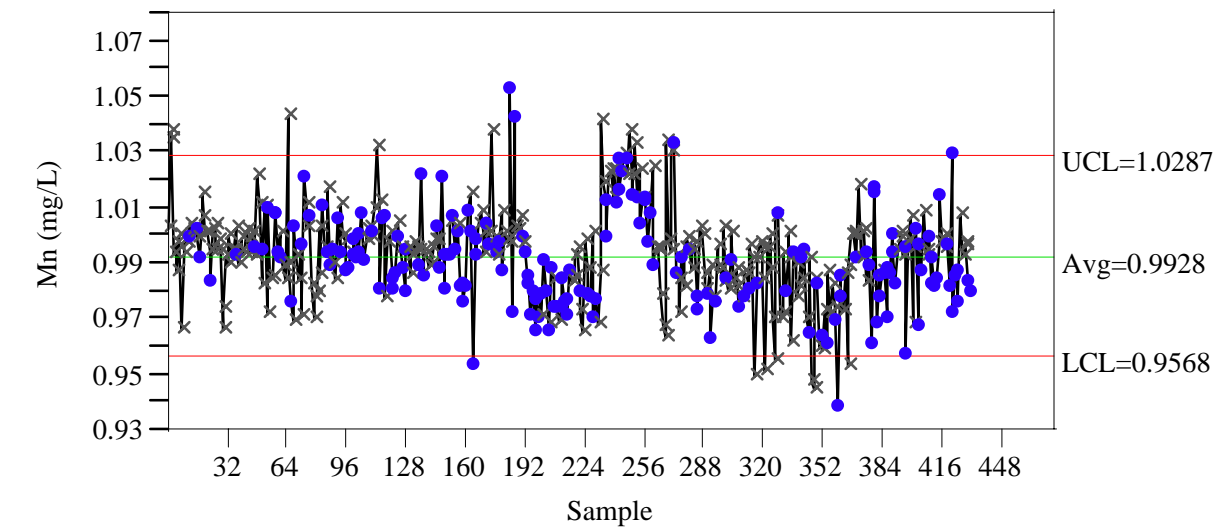

Individual Measurement of $\mathrm{Na}(\mathrm{mg} / \mathrm{L})$

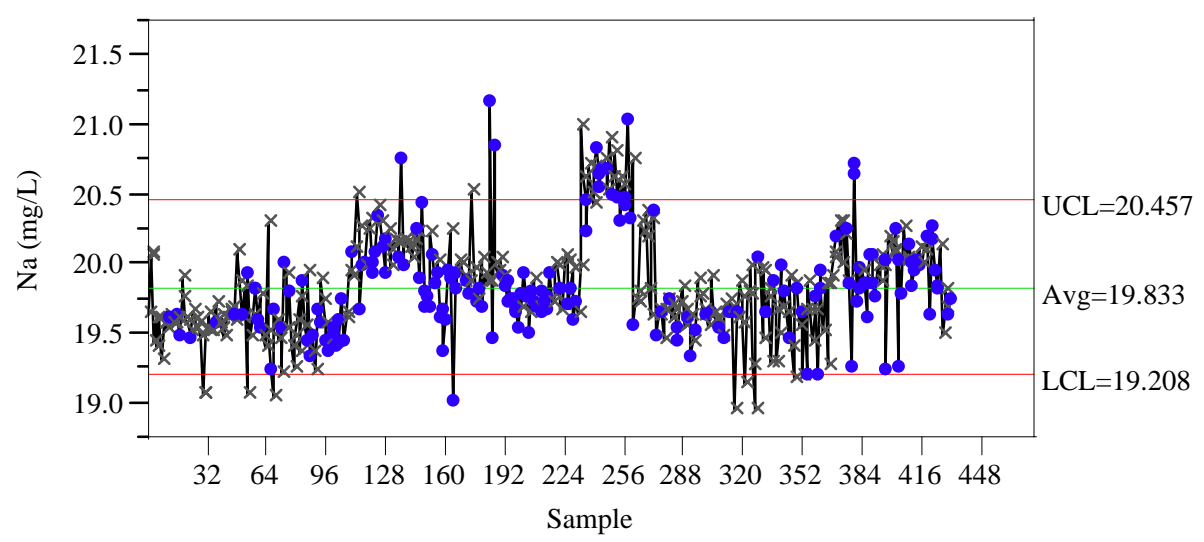




\section{Exhibit A1. Cold Chem Standards in Analytical Sequence}

Individual Measurement of Ni (mg/L)

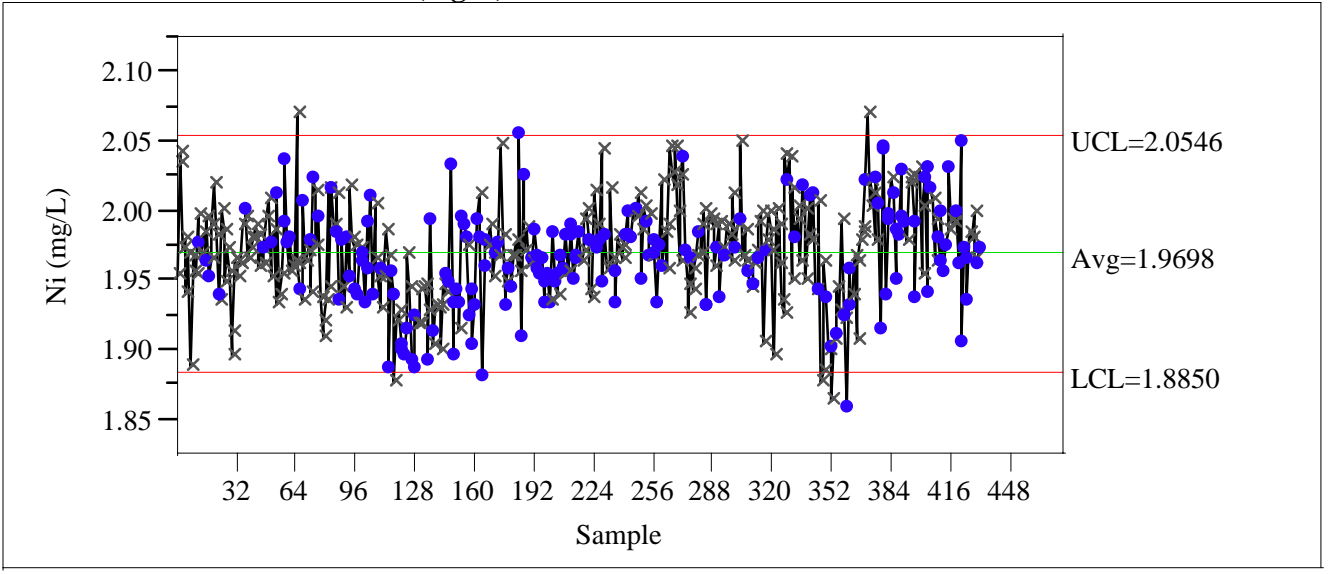

Individual Measurement of Si (mg/L)

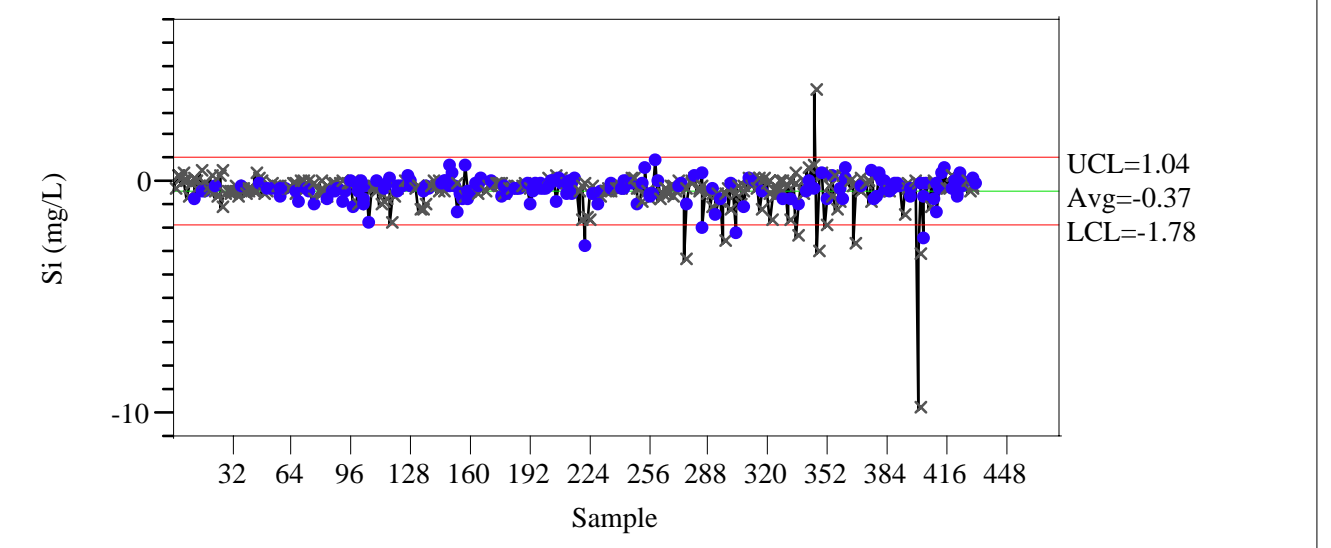

Individual Measurement of $\mathrm{Ti}(\mathrm{mg} / \mathrm{L})$

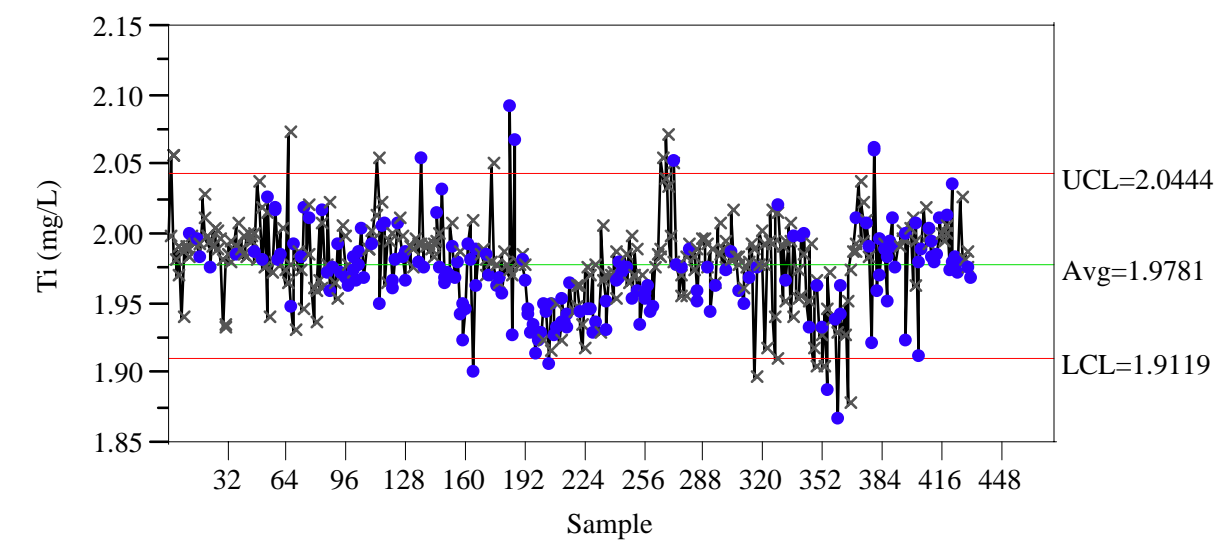




\section{Exhibit A1. Cold Chem Standards in Analytical Sequence}

Individual Measurement of $\mathrm{Zr}$ (mg/L)

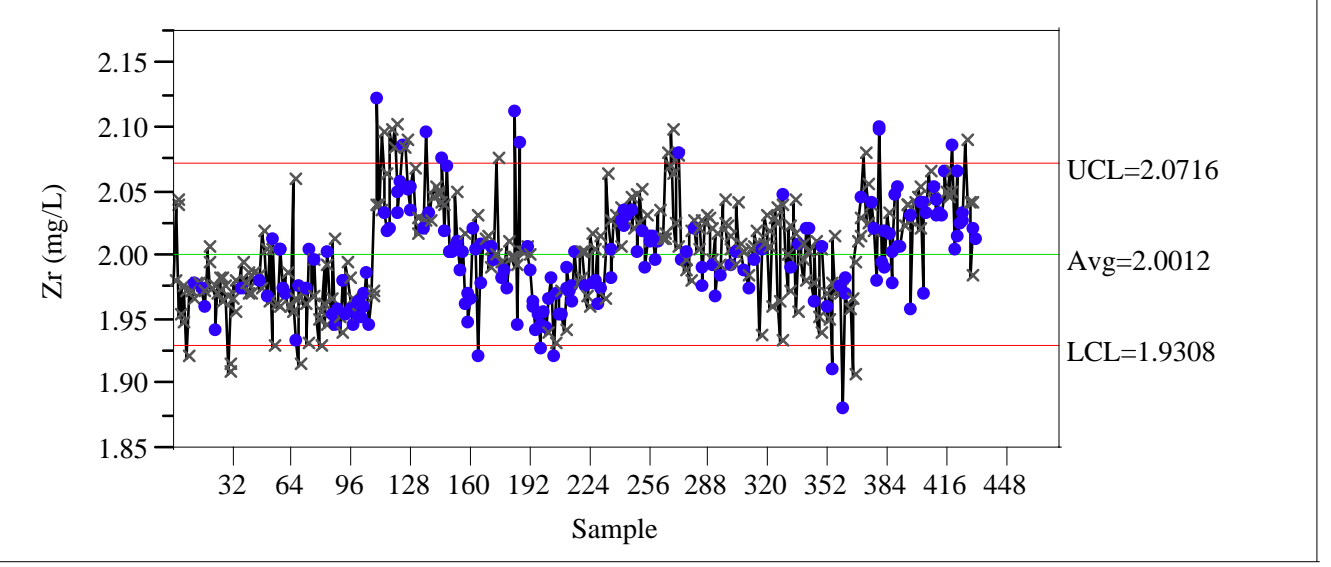

Individual Measurement of U (mg/L)

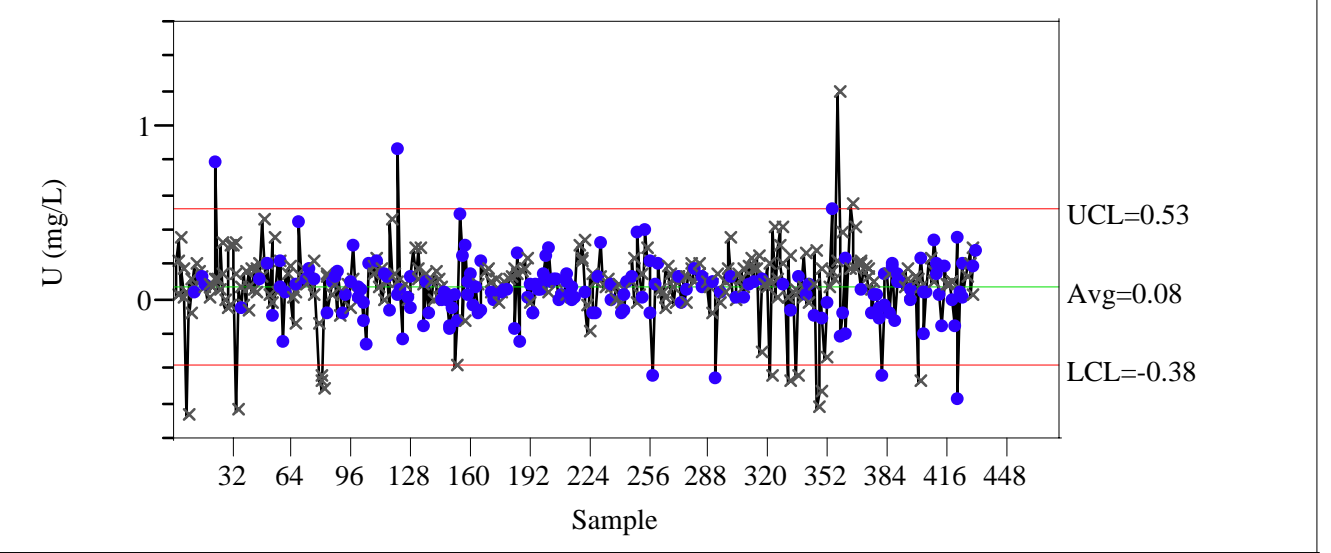

\section{STCd=IN34}

Control Chart

Individual Measurement of $\mathrm{Al}(\mathrm{mg} / \mathrm{L})$

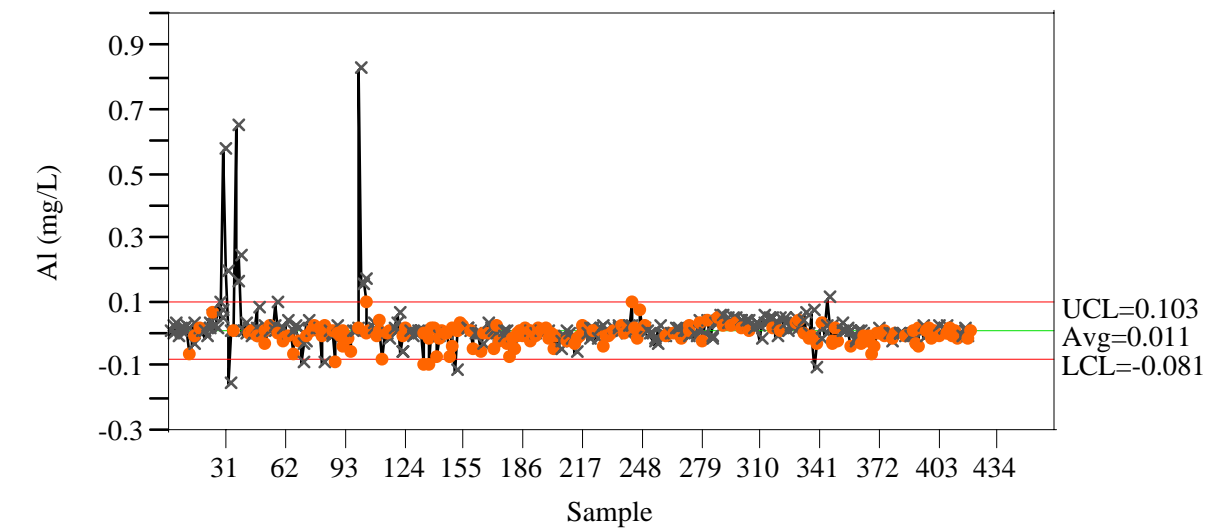




\section{Exhibit A1. Cold Chem Standards in Analytical Sequence}

\section{Revision 0}

Individual Measurement of B (mg/L)

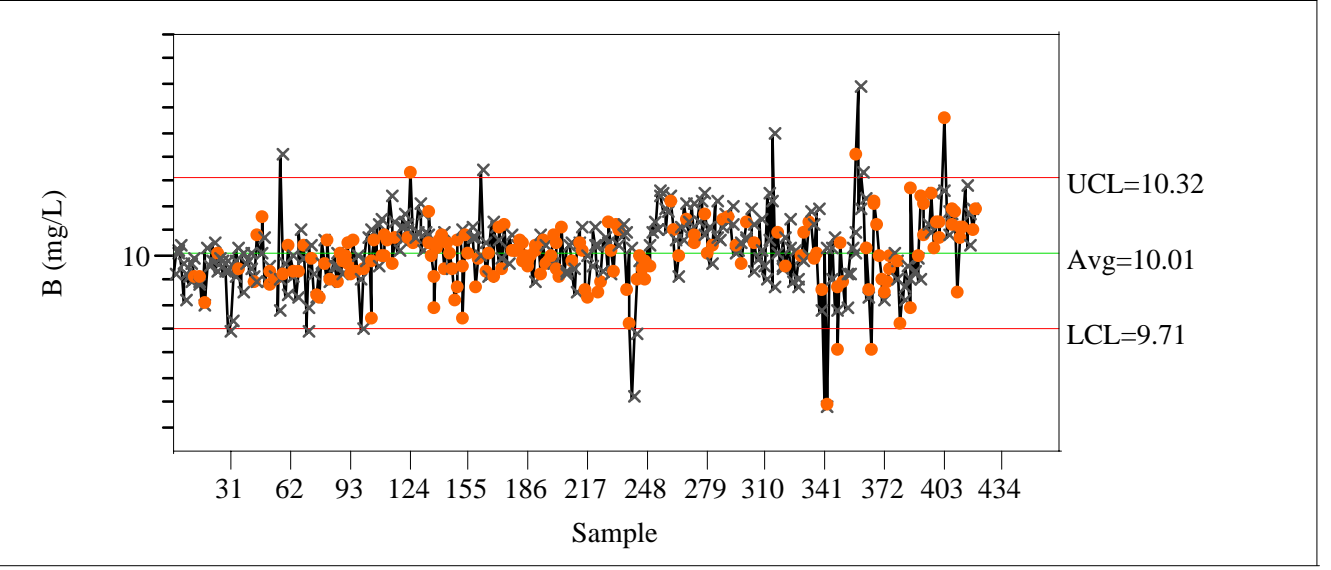

Individual Measurement of Ca (mg/L)

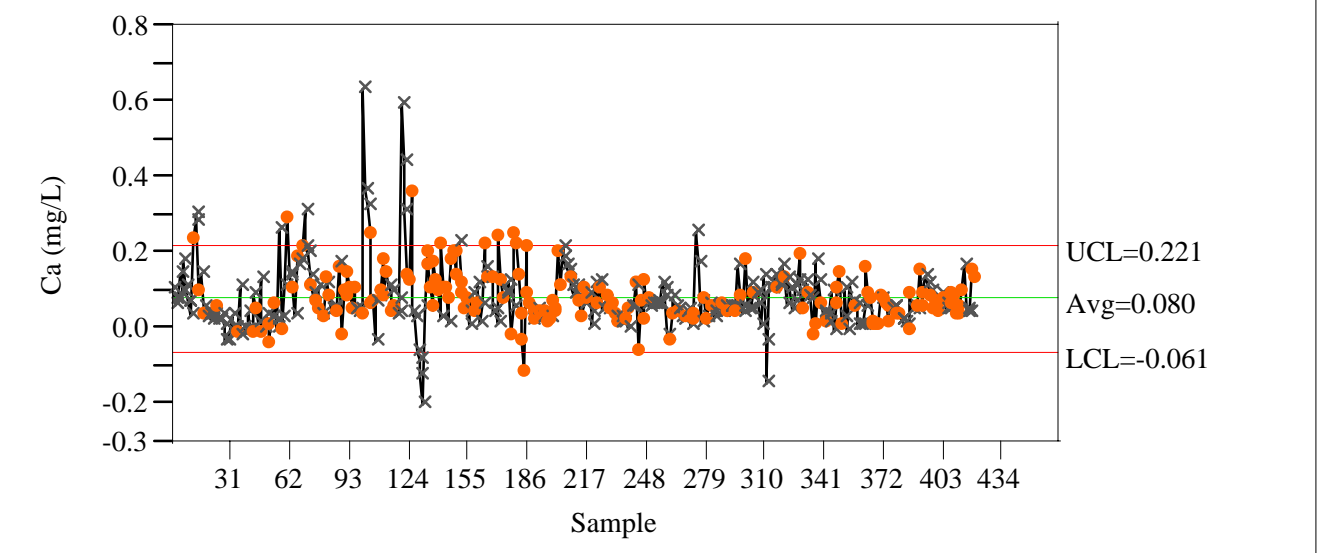

Individual Measurement of $\mathrm{Cr}(\mathrm{mg} / \mathrm{L})$

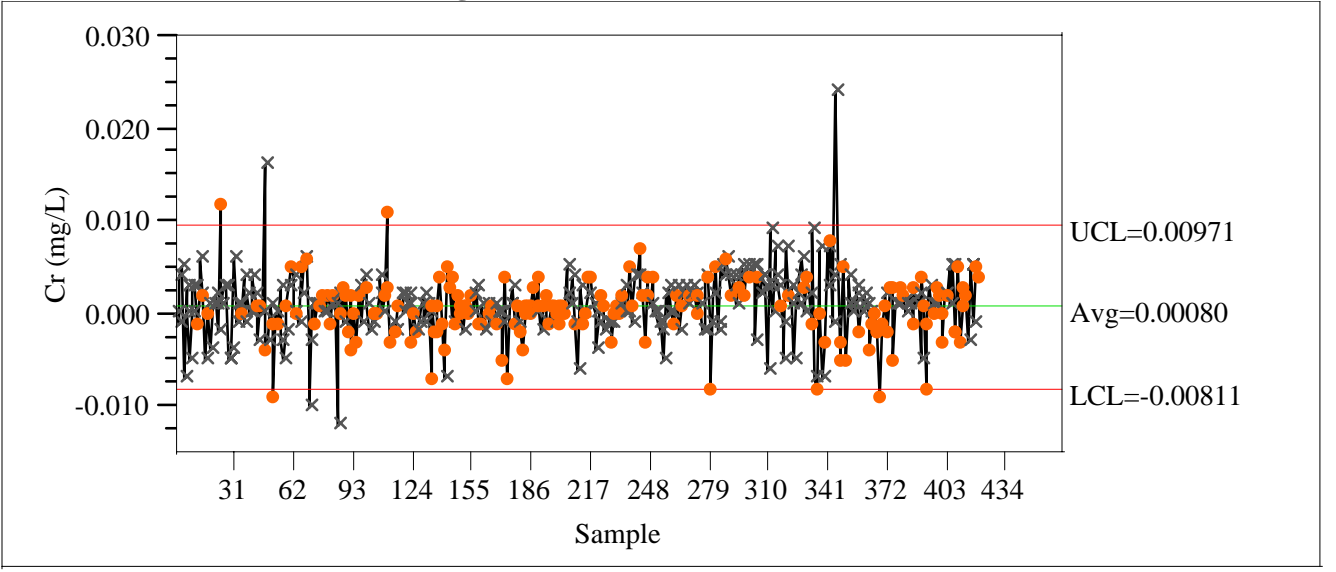


WSRC-TR-2004-00576

\section{Exhibit A1. Cold Chem Standards in Analytical Sequence}

\section{Revision 0}

Individual Measurement of $\mathrm{Cu}(\mathrm{mg} / \mathrm{L})$

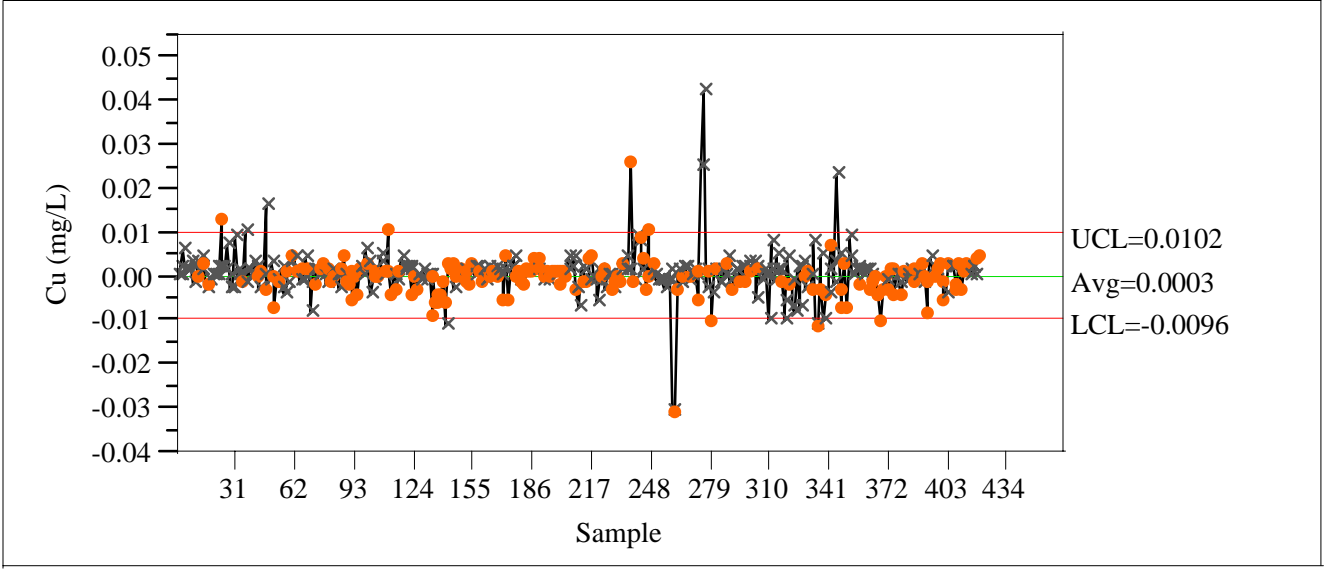

Individual Measurement of Fe (mg/L)

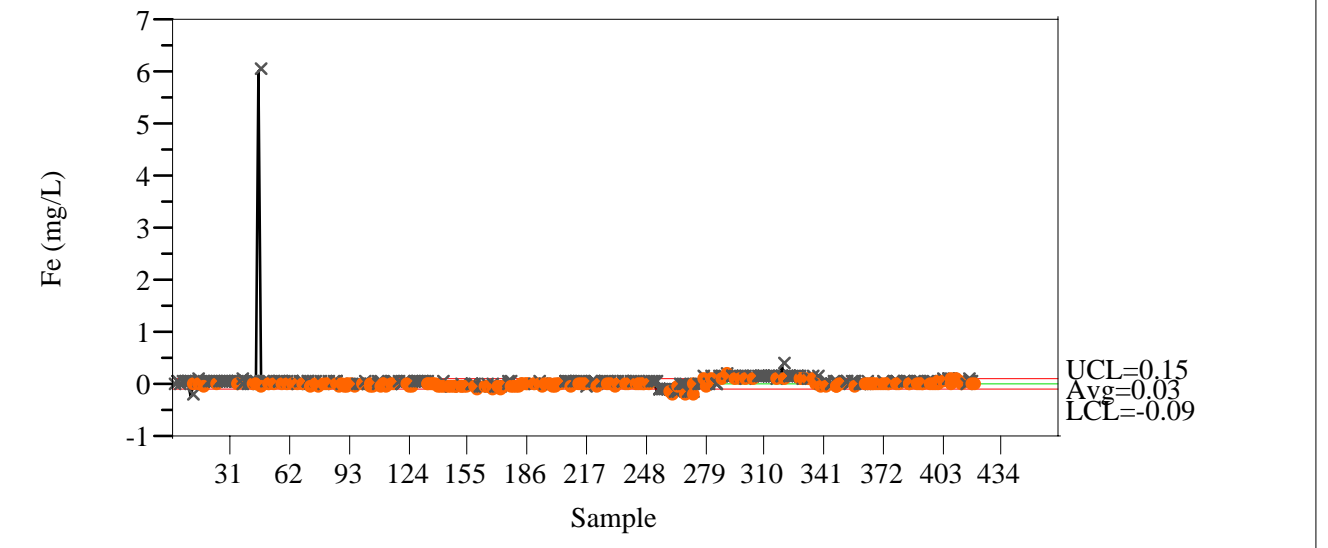

Individual Measurement of K (mg/L)

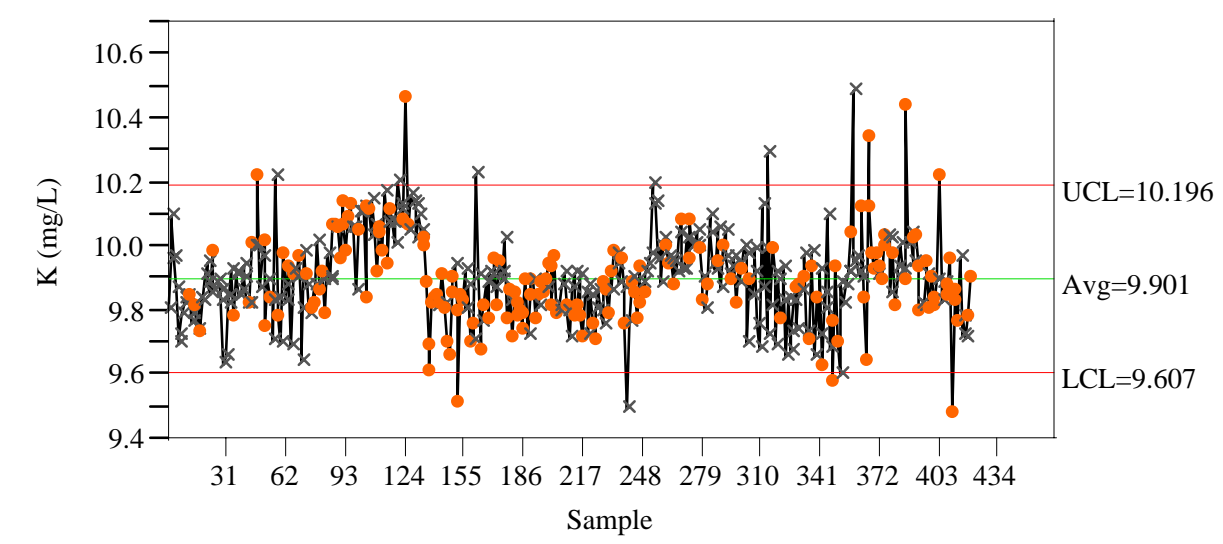


WSRC-TR-2004-00576

\section{Exhibit A1. Cold Chem Standards in Analytical Sequence}

\section{Revision 0}

Individual Measurement of $\mathrm{Li}(\mathrm{mg} / \mathrm{L})$

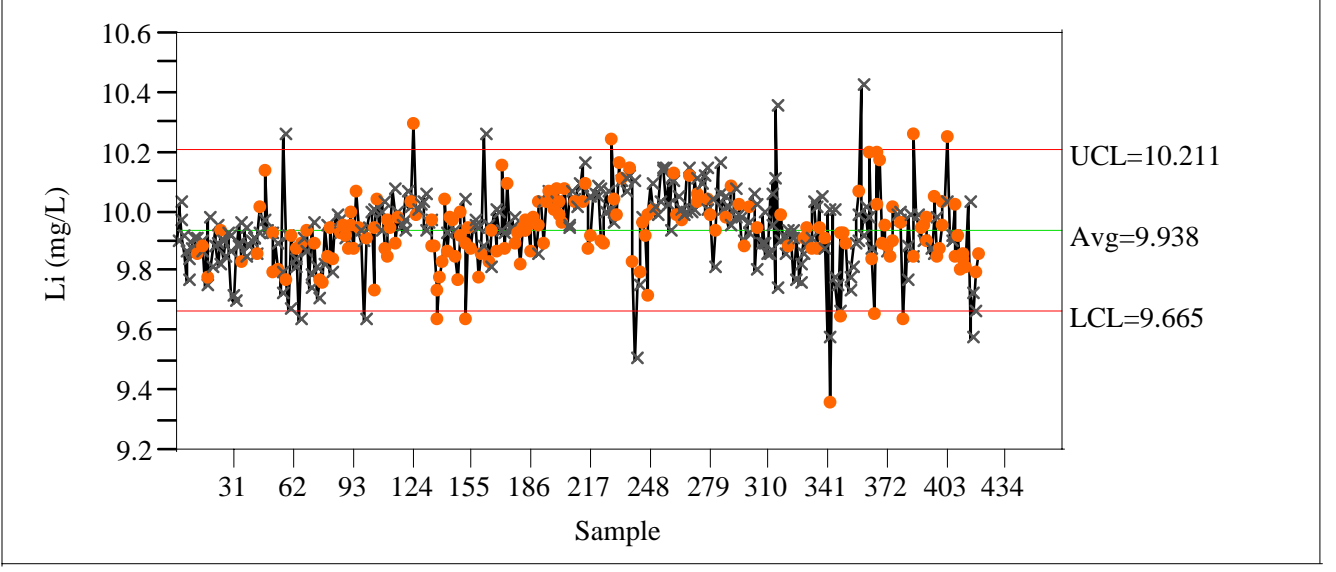

Individual Measurement of Mg (mg/L)

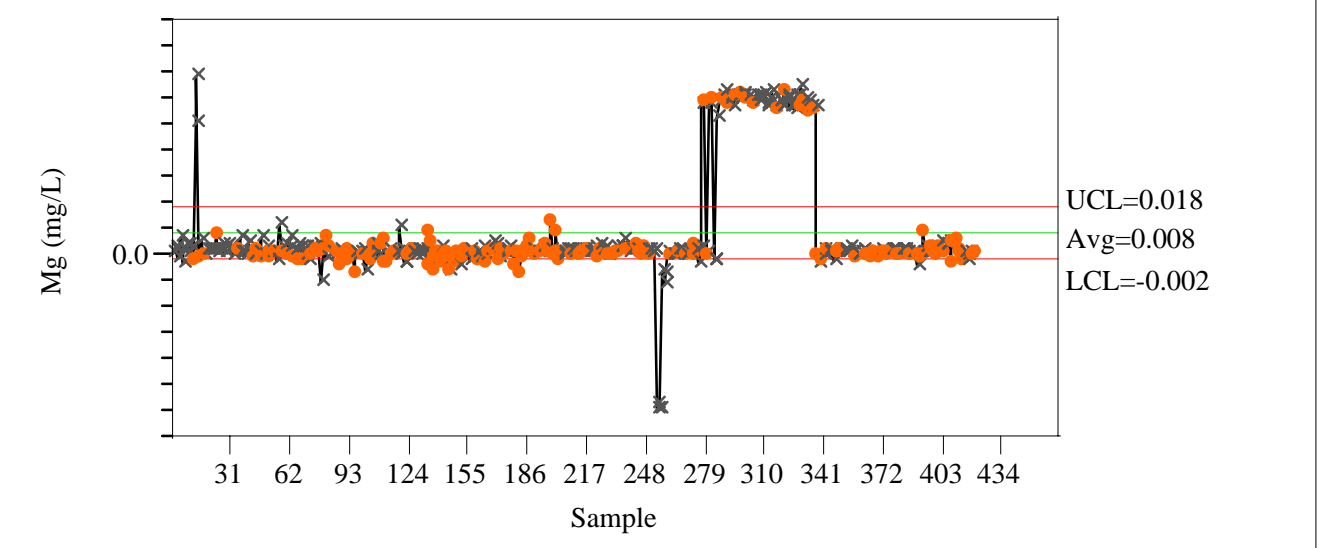

Individual Measurement of Mn (mg/L)

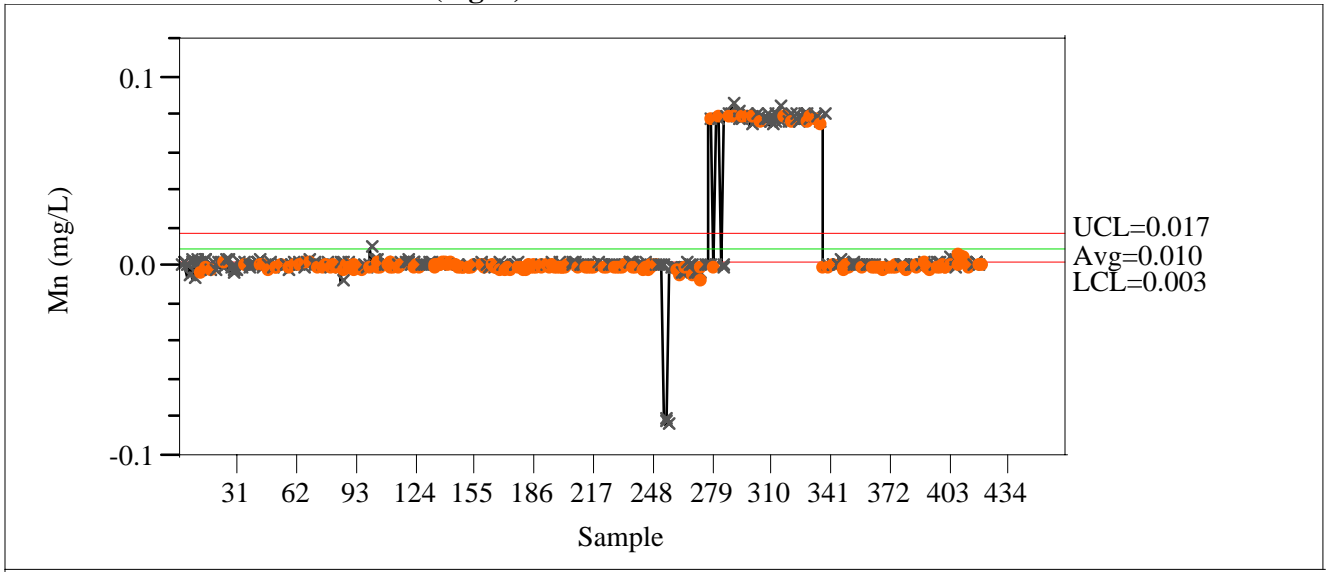




\section{Exhibit A1. Cold Chem Standards in Analytical Sequence}

\section{Revision 0}

Individual Measurement of Na (mg/L)

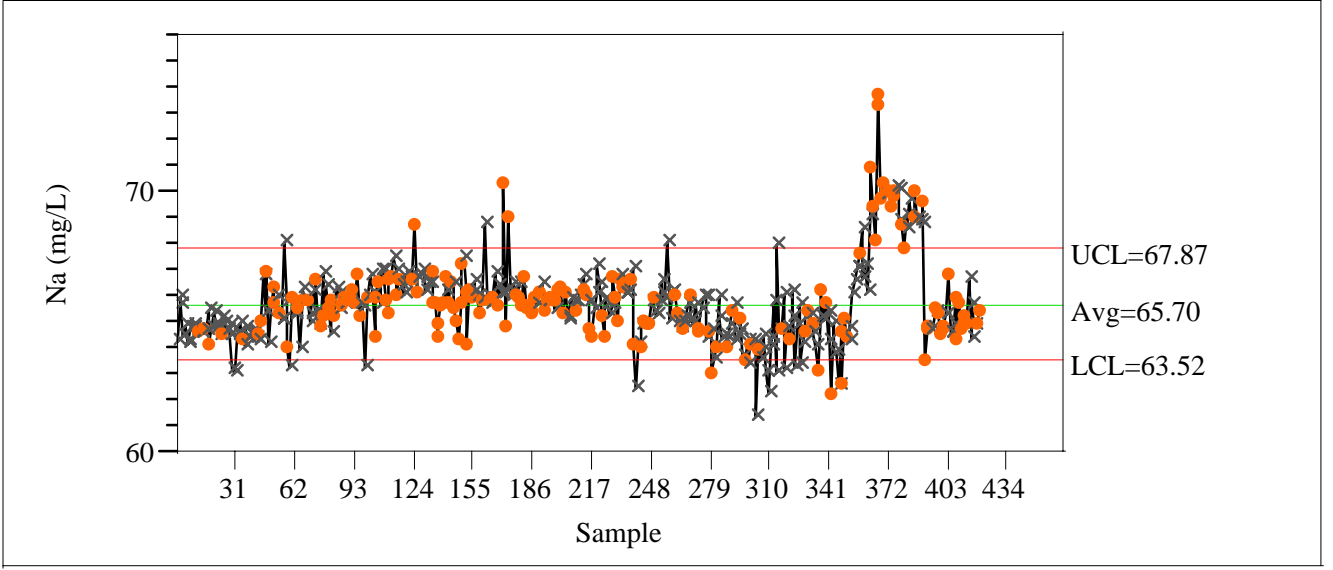

Individual Measurement of $\mathrm{Ni}(\mathrm{mg} / \mathrm{L})$

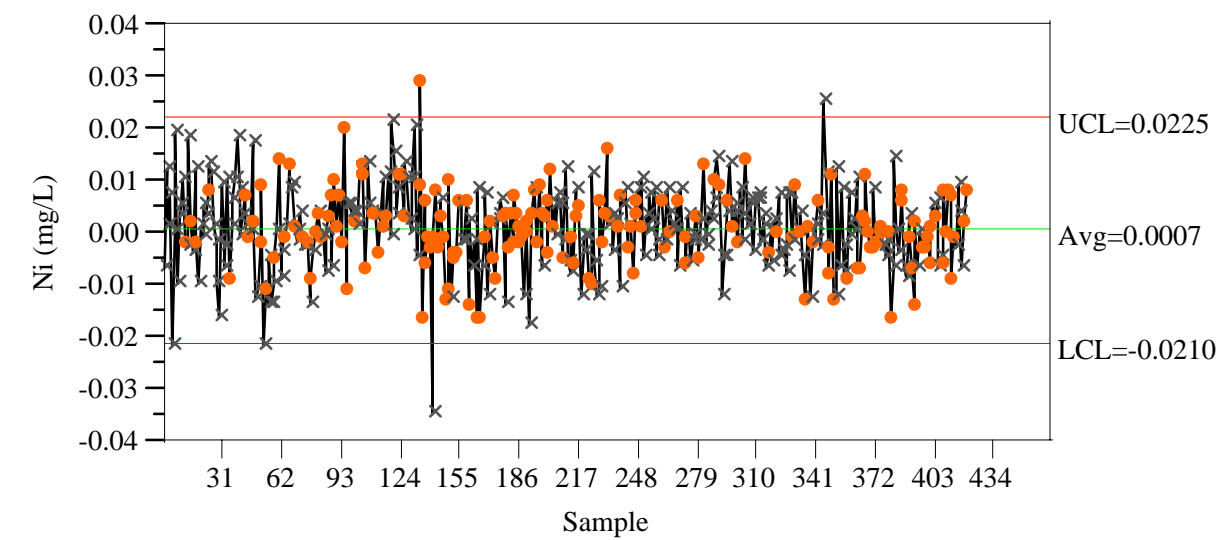

Individual Measurement of Si (mg/L)

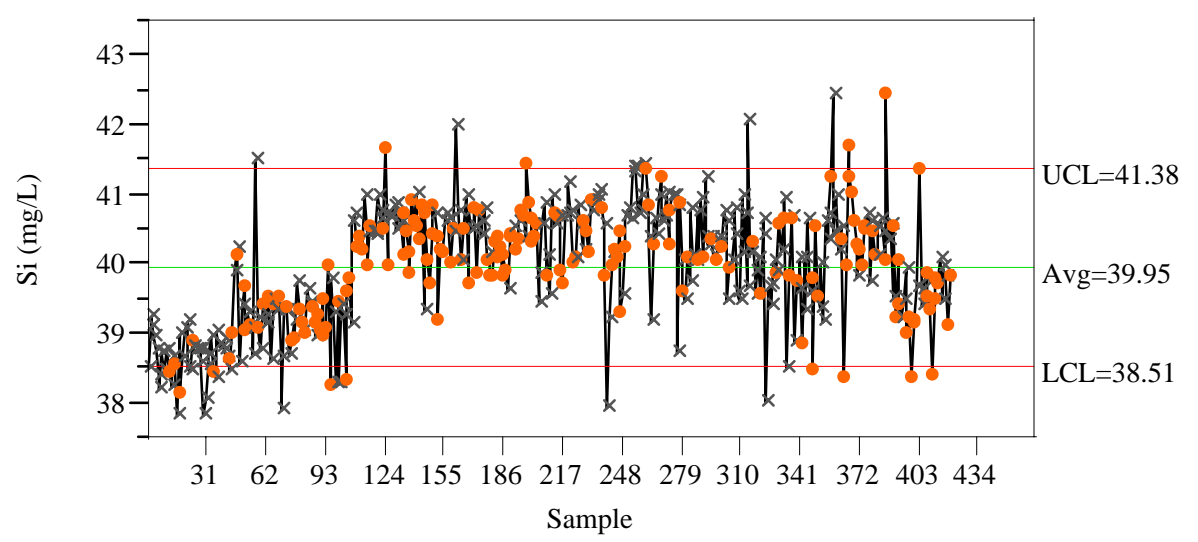


WSRC-TR-2004-00576

\section{Exhibit A1. Cold Chem Standards in Analytical Sequence}

\section{Revision 0}

Individual Measurement of $\mathrm{Ti}(\mathrm{mg} / \mathrm{L})$

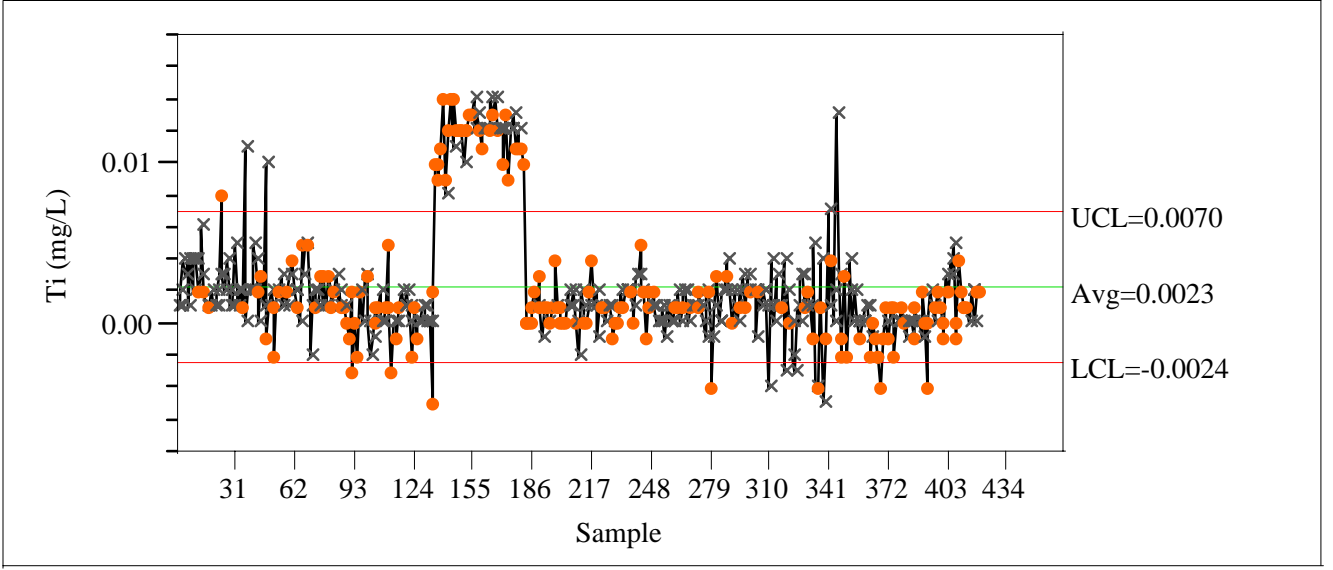

Individual Measurement of $\mathrm{Zr}$ (mg/L)

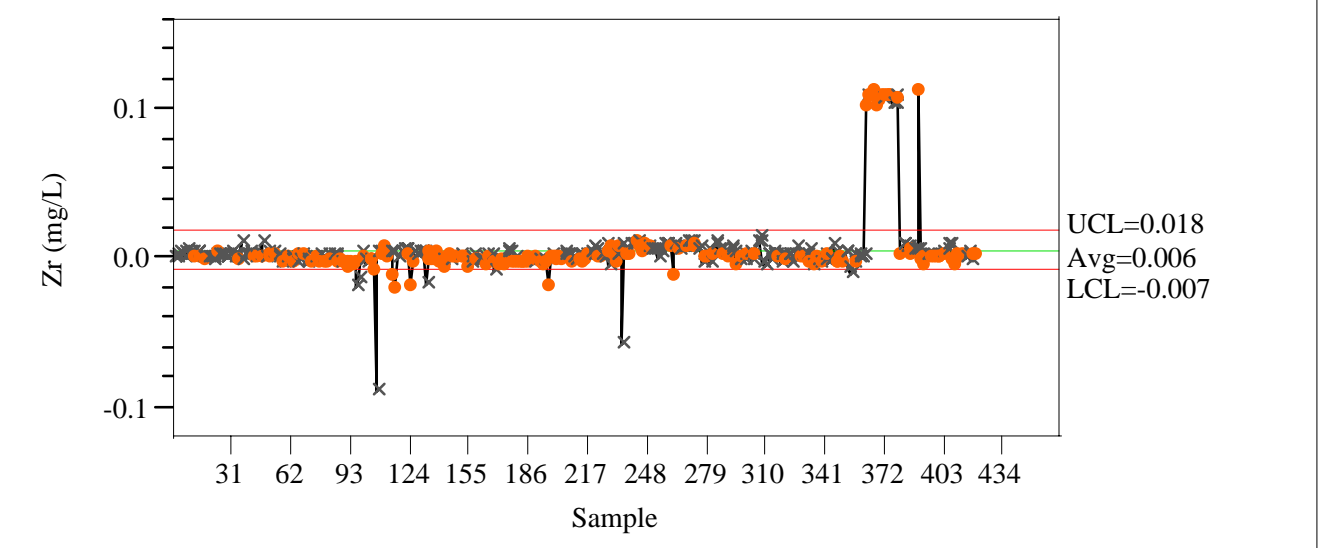

Individual Measurement of U (mg/L)

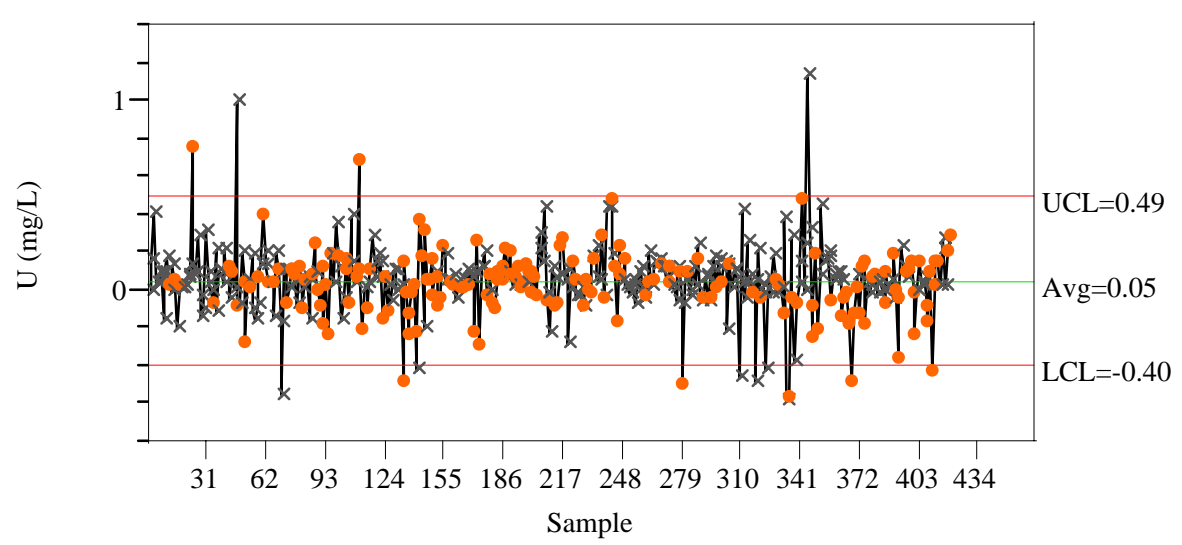




\section{Exhibit A1. Cold Chem Standards in Analytical Sequence}

\section{Revision 0}

STCd=IN35

Control Chart

Individual Measurement of $\mathrm{Al}$ (mg/L)

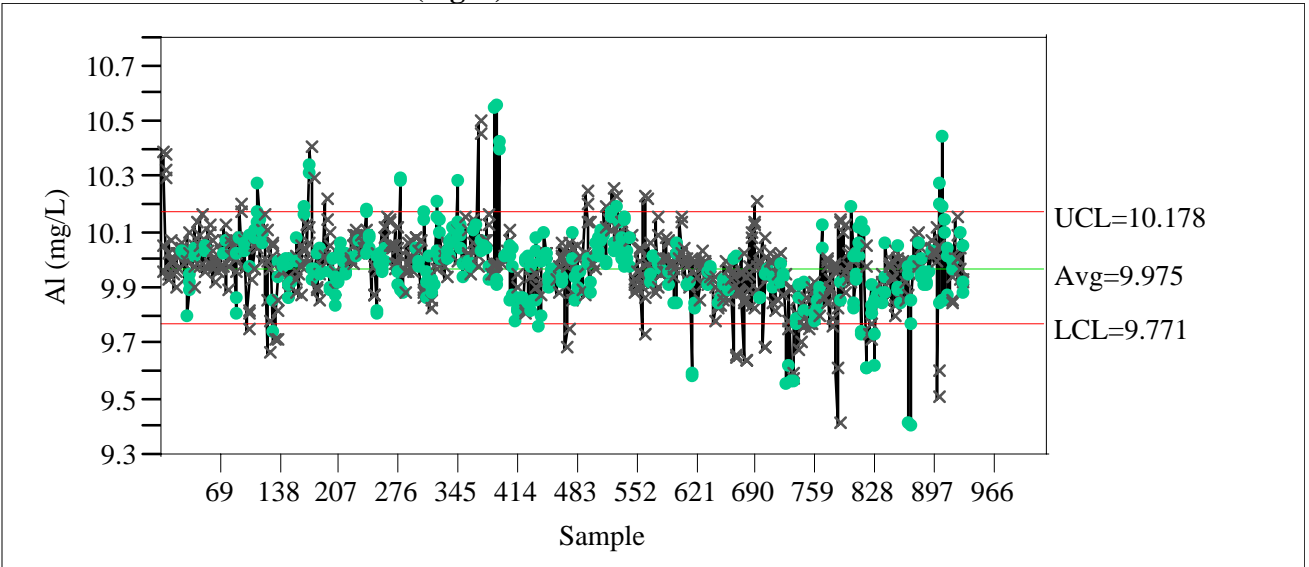

Individual Measurement of B (mg/L)

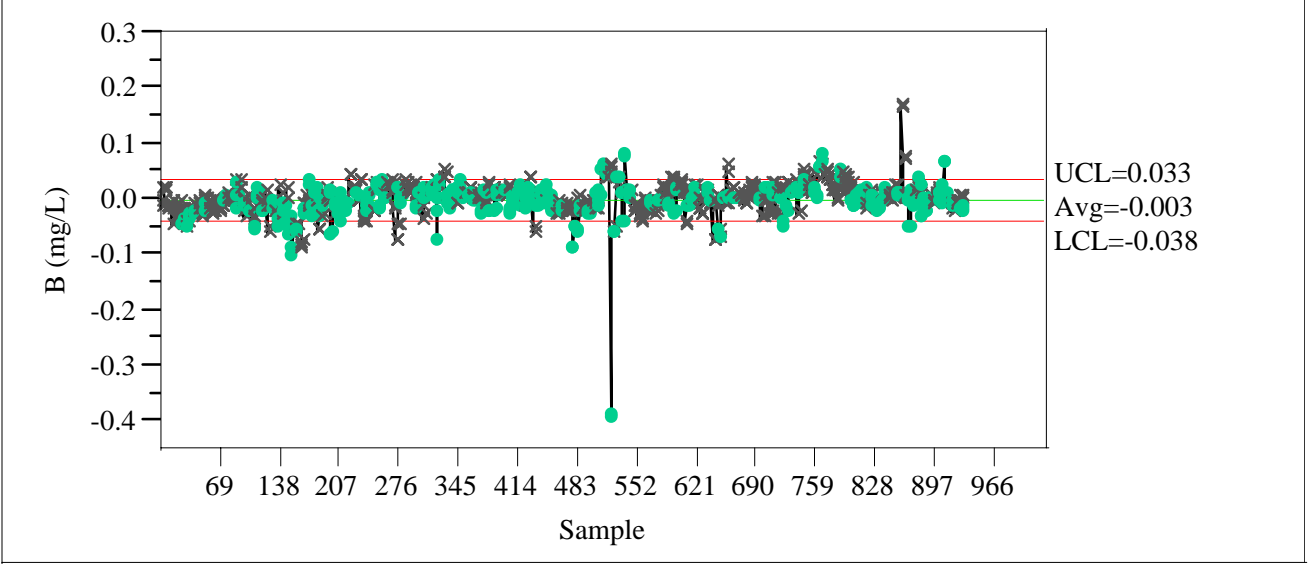

Individual Measurement of Ca (mg/L)

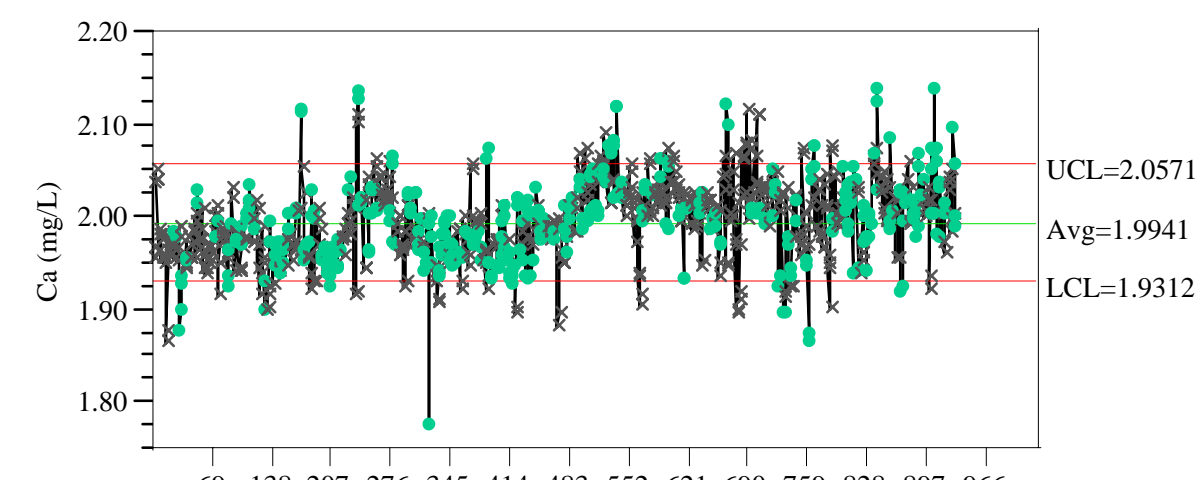

$\begin{array}{llllllllllllll}69 & 138 & 207 & 276 & 345 & 414 & 483 & 552 & 621 & 690 & 759 & 828 & 897 & 966\end{array}$

Sample 


\section{Exhibit A1. Cold Chem Standards in Analytical Sequence}

Revision 0

Individual Measurement of $\mathrm{Cr}(\mathrm{mg} / \mathrm{L})$

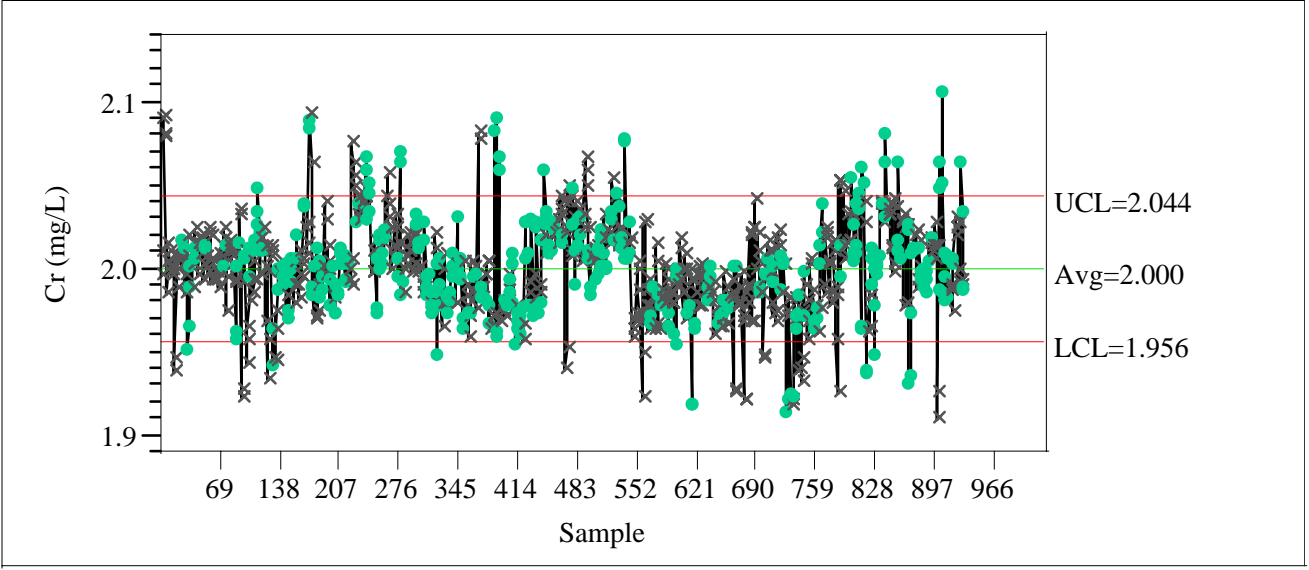

Individual Measurement of $\mathrm{Cu}$ (mg/L)

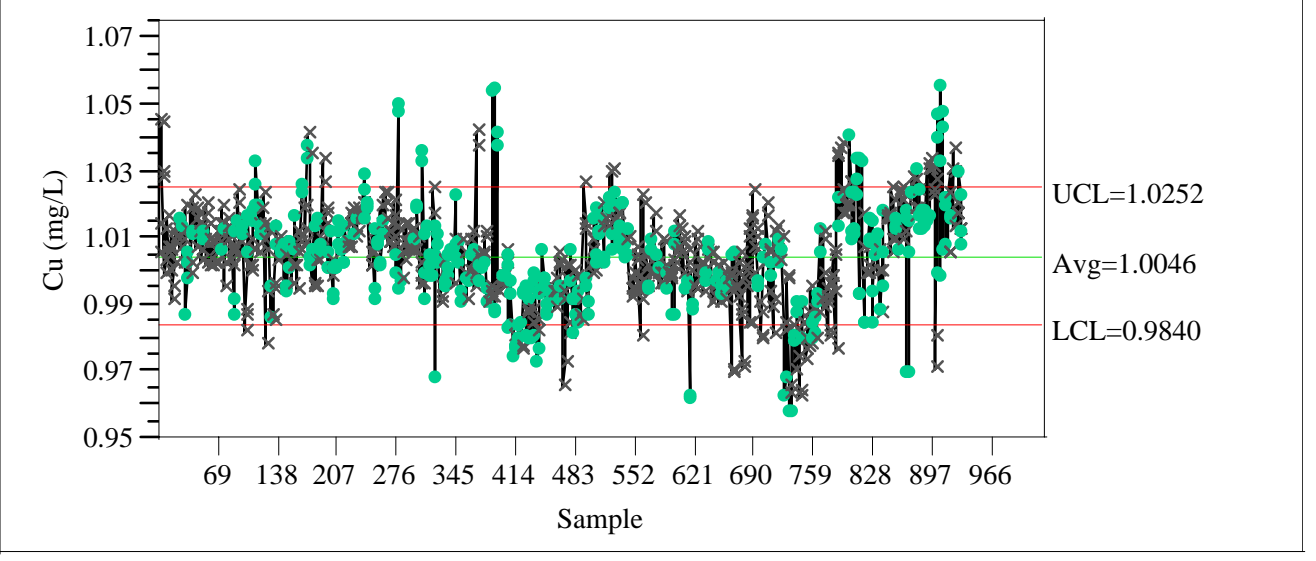

Individual Measurement of Fe (mg/L)

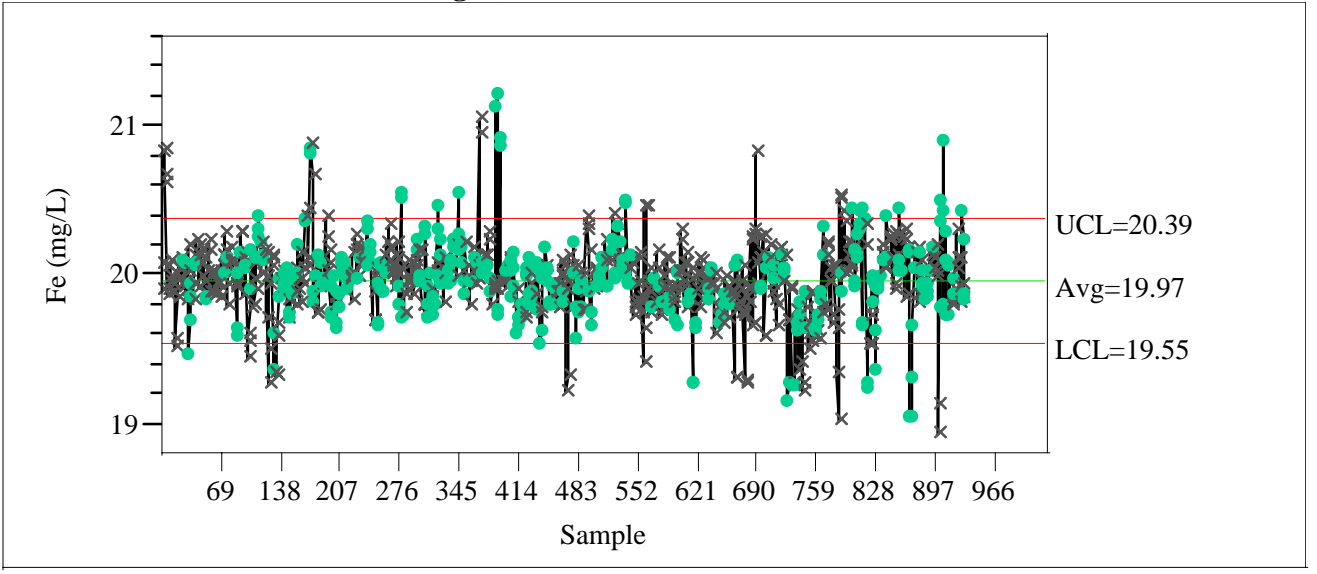


WSRC-TR-2004-00576

\section{Exhibit A1. Cold Chem Standards in Analytical Sequence}

\section{Revision 0}

Individual Measurement of K (mg/L)

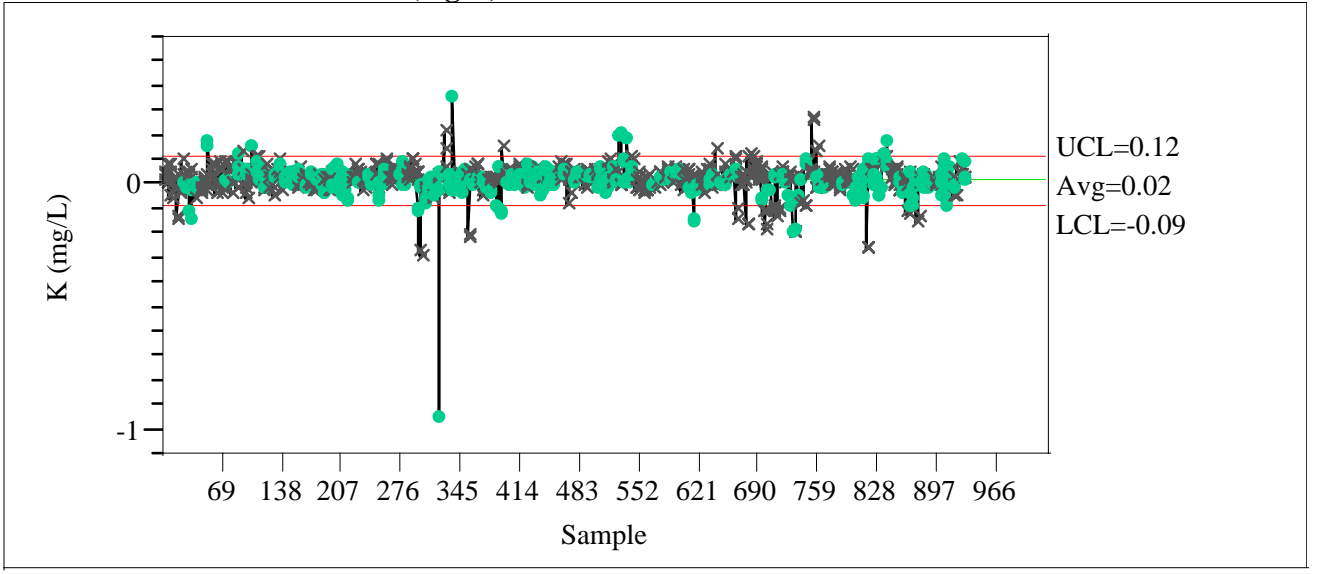

Individual Measurement of $\mathrm{Li}(\mathrm{mg} / \mathrm{L})$

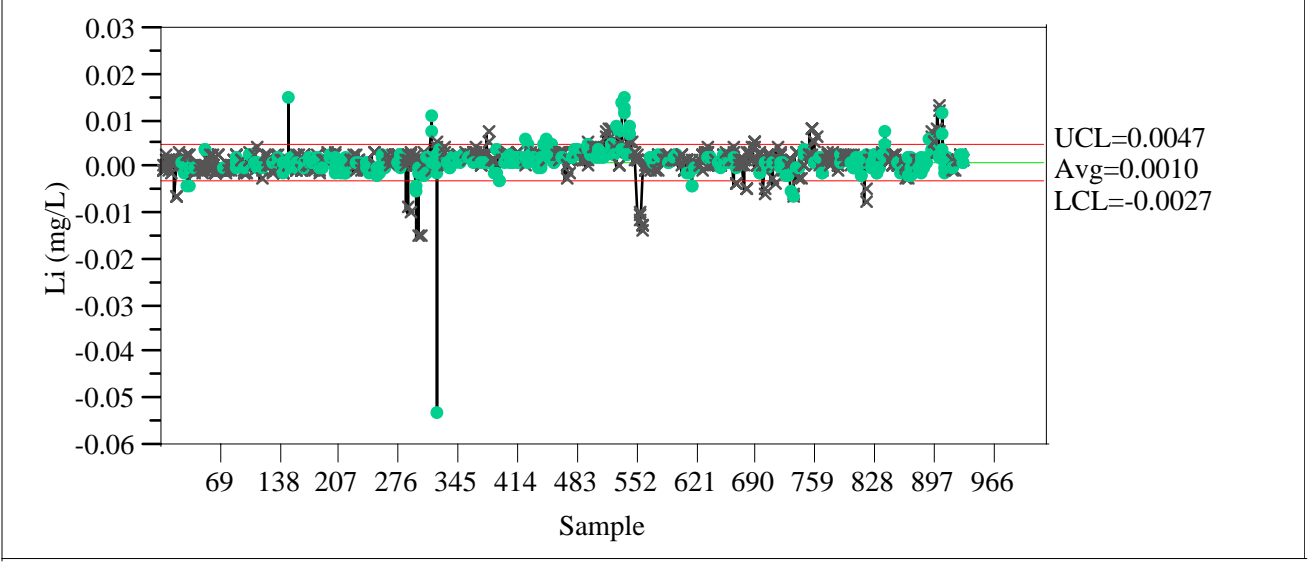

Individual Measurement of $\mathrm{Mg}(\mathrm{mg} / \mathrm{L})$

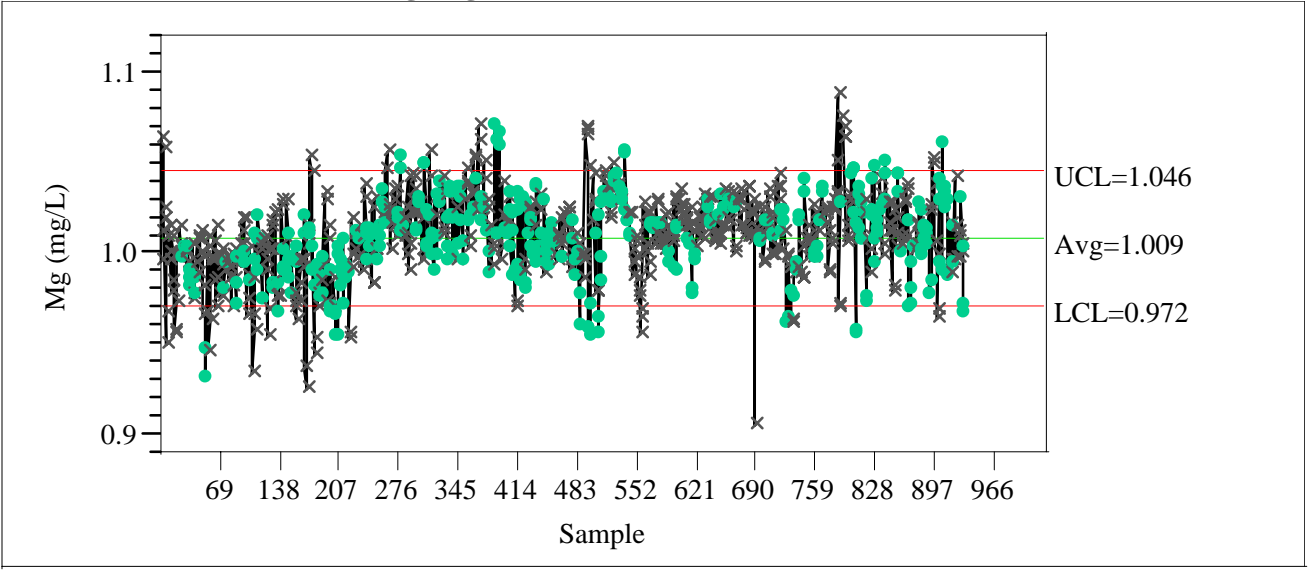




\section{Exhibit A1. Cold Chem Standards in Analytical Sequence}

\section{Revision 0}

Individual Measurement of Mn (mg/L)

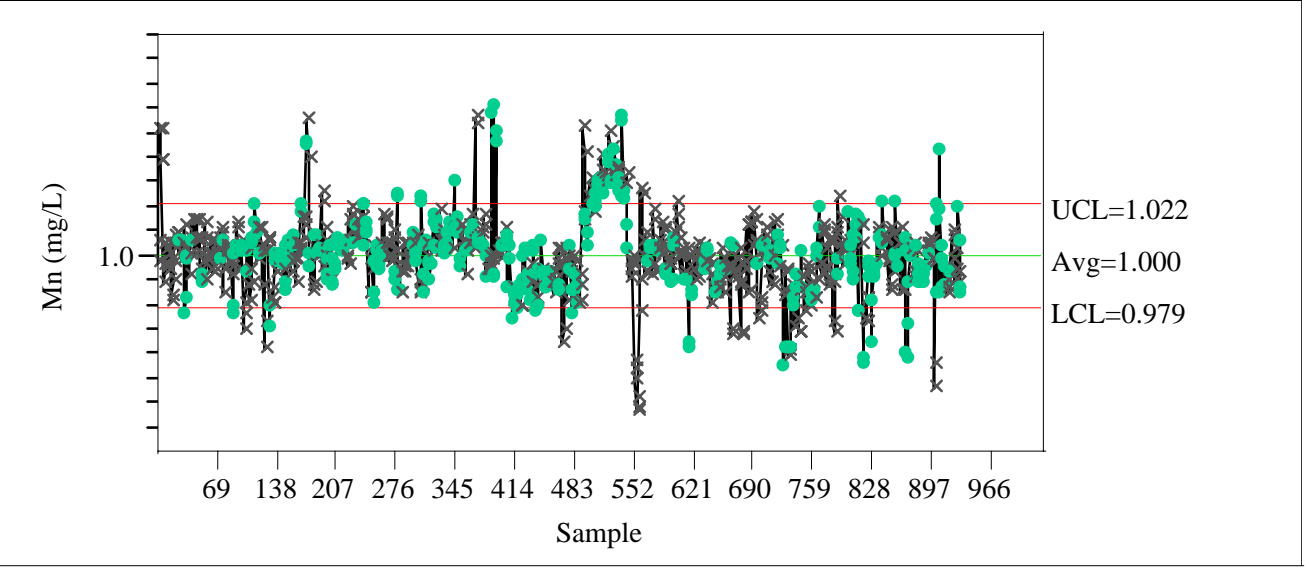

Individual Measurement of $\mathrm{Na}(\mathrm{mg} / \mathrm{L})$

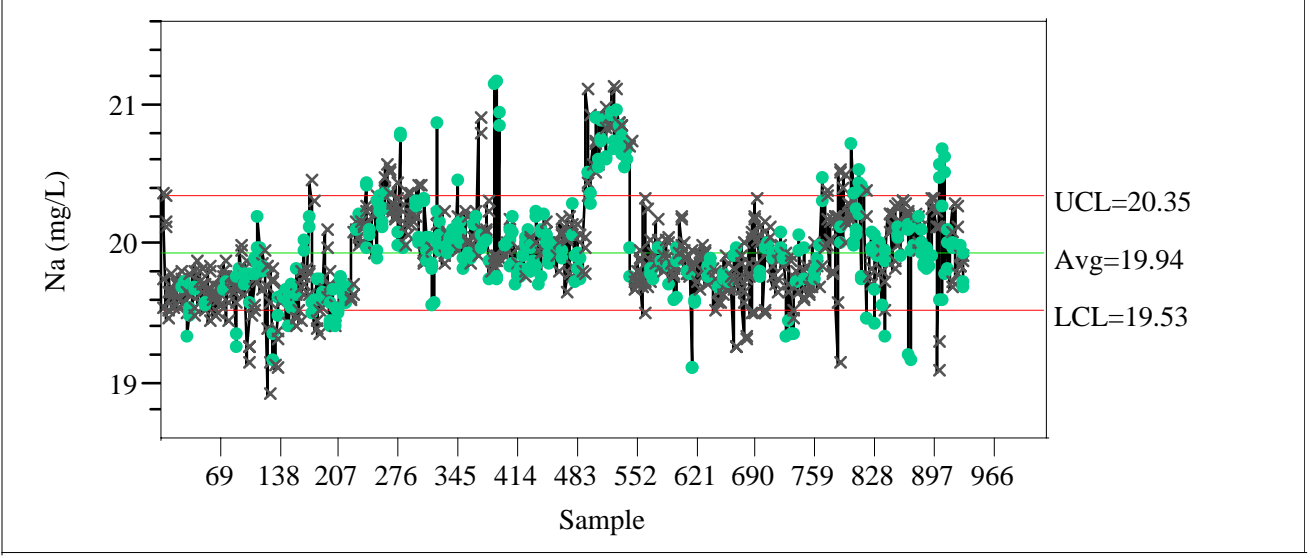

Individual Measurement of $\mathrm{Ni}$ (mg/L)

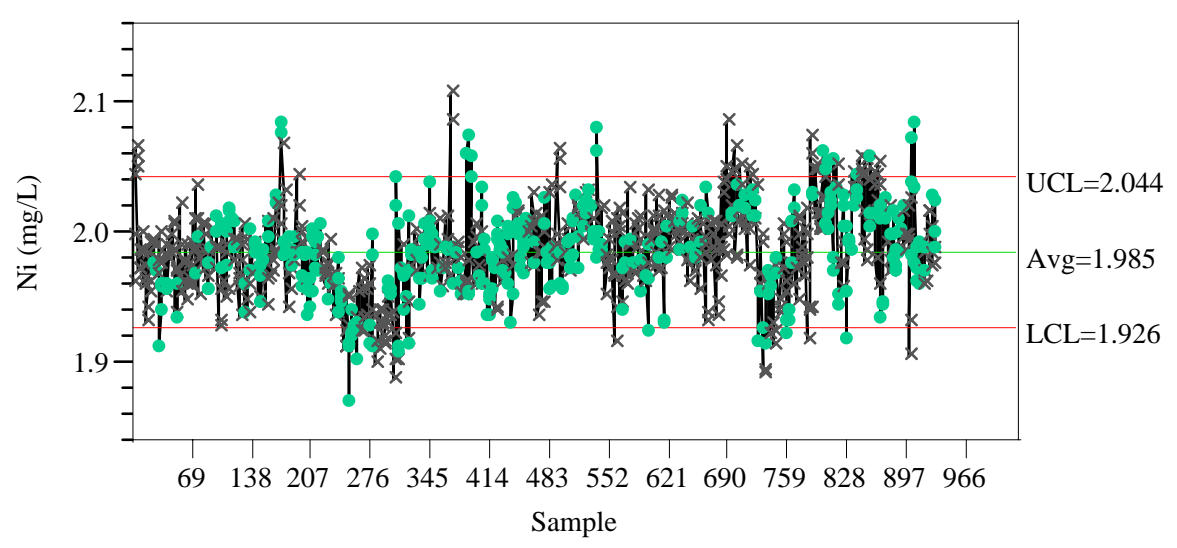




\section{Exhibit A1. Cold Chem Standards in Analytical Sequence}

\section{Revision 0}

Individual Measurement of Si (mg/L)

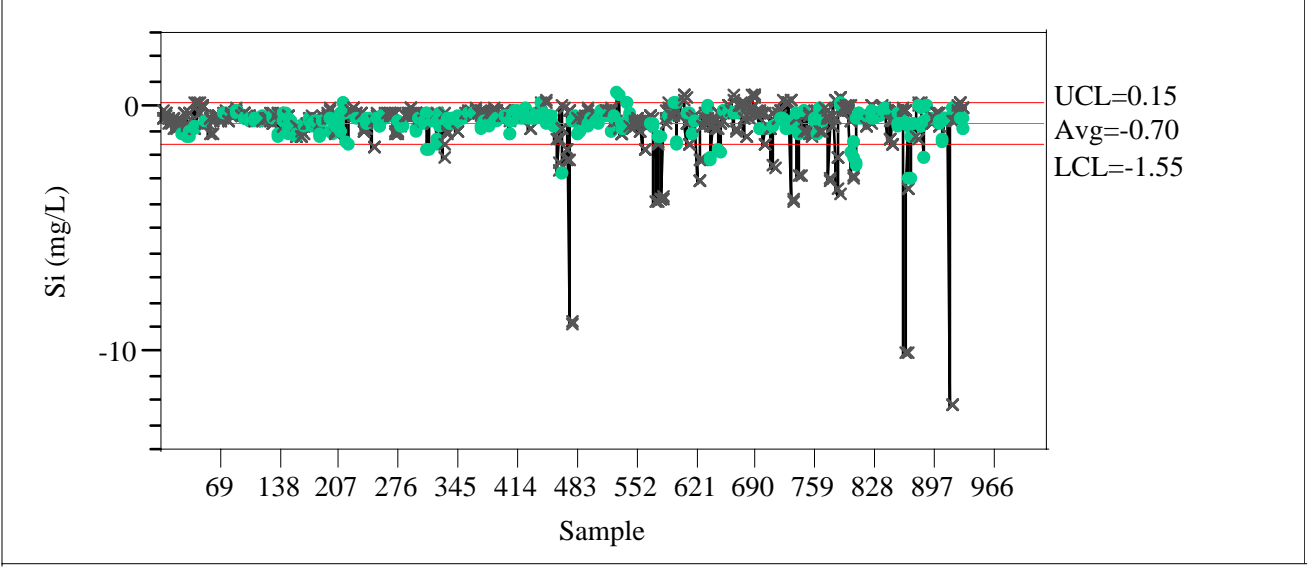

Individual Measurement of Ti (mg/L)

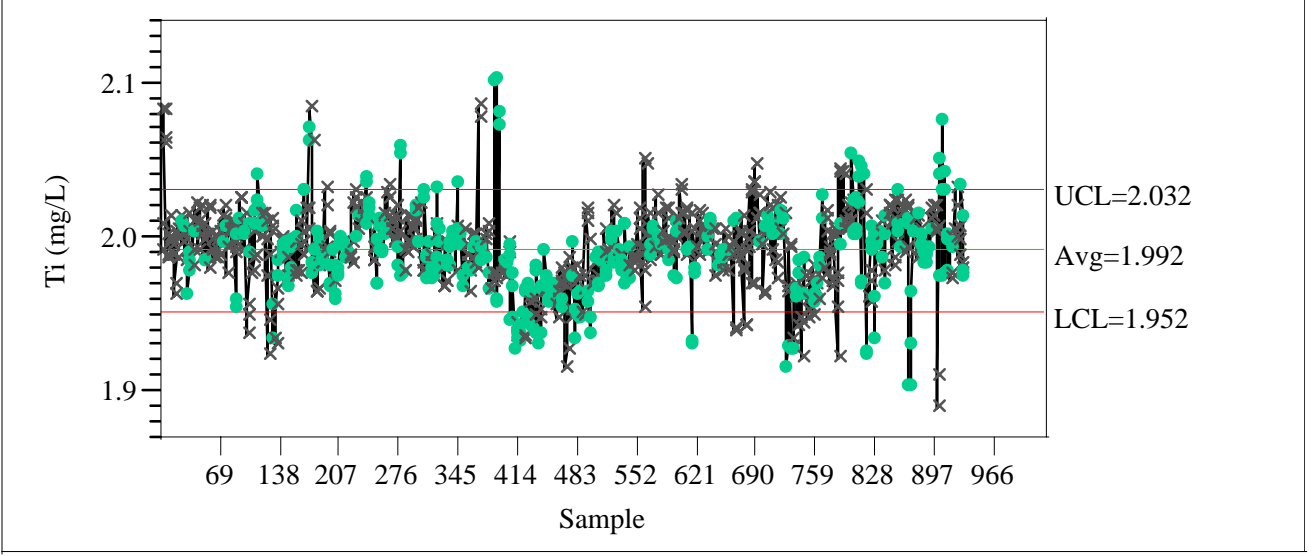

Individual Measurement of $\mathrm{Zr}(\mathrm{mg} / \mathrm{L})$

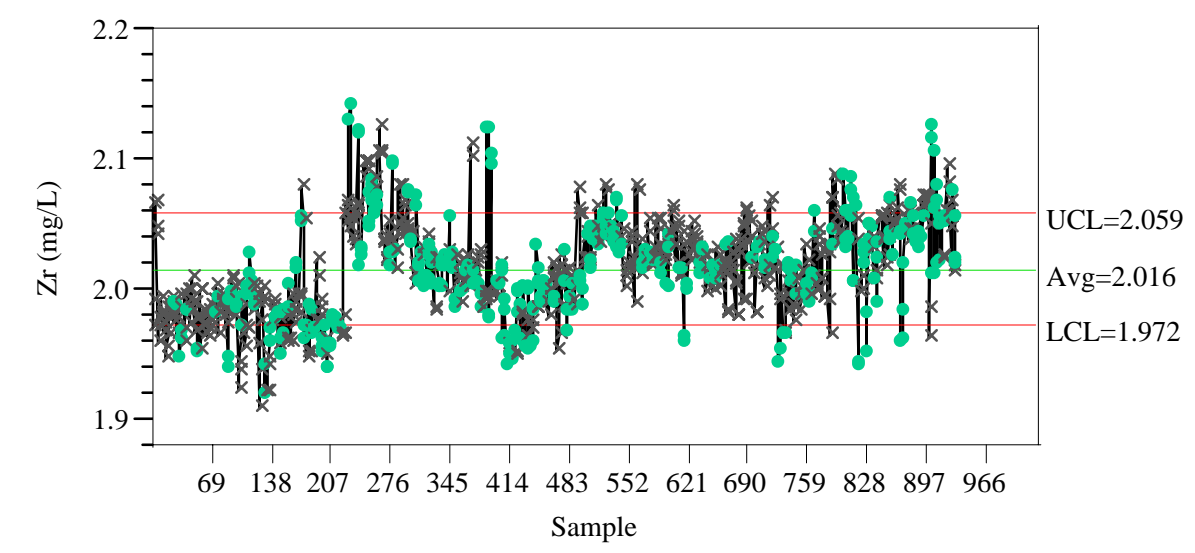


WSRC-TR-2004-00576

\section{Exhibit A1. Cold Chem Standards in Analytical Sequence}

\section{Revision 0}

Individual Measurement of U (mg/L)

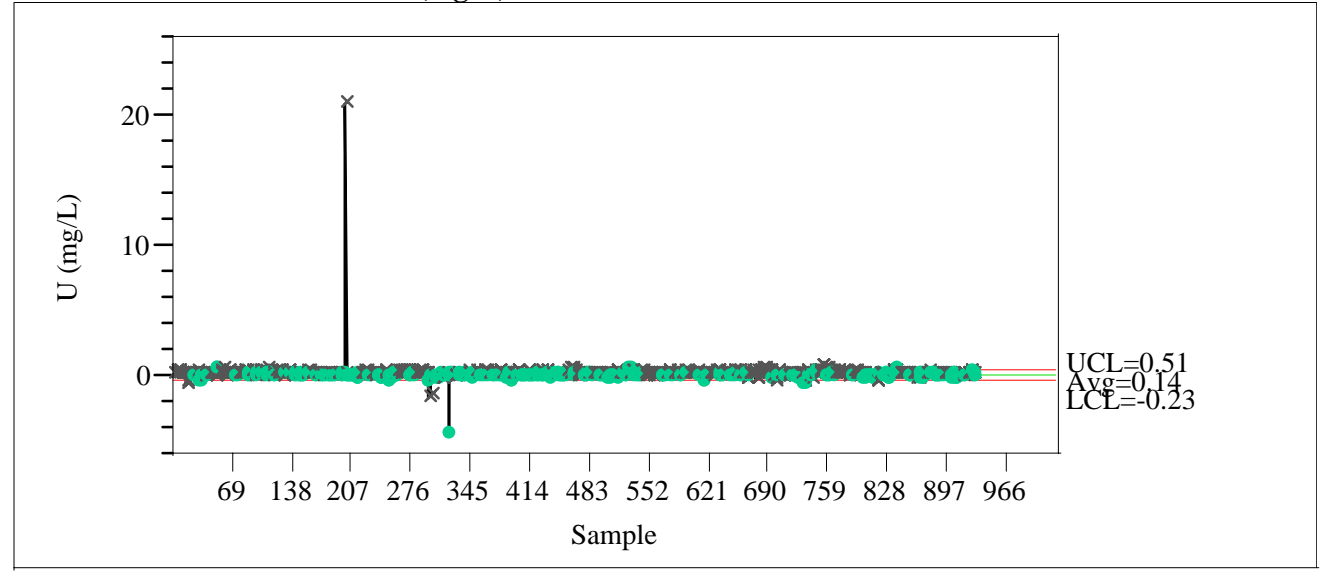

STCd=IN36

Control Chart

Individual Measurement of $\mathrm{Al}$ (mg/L)

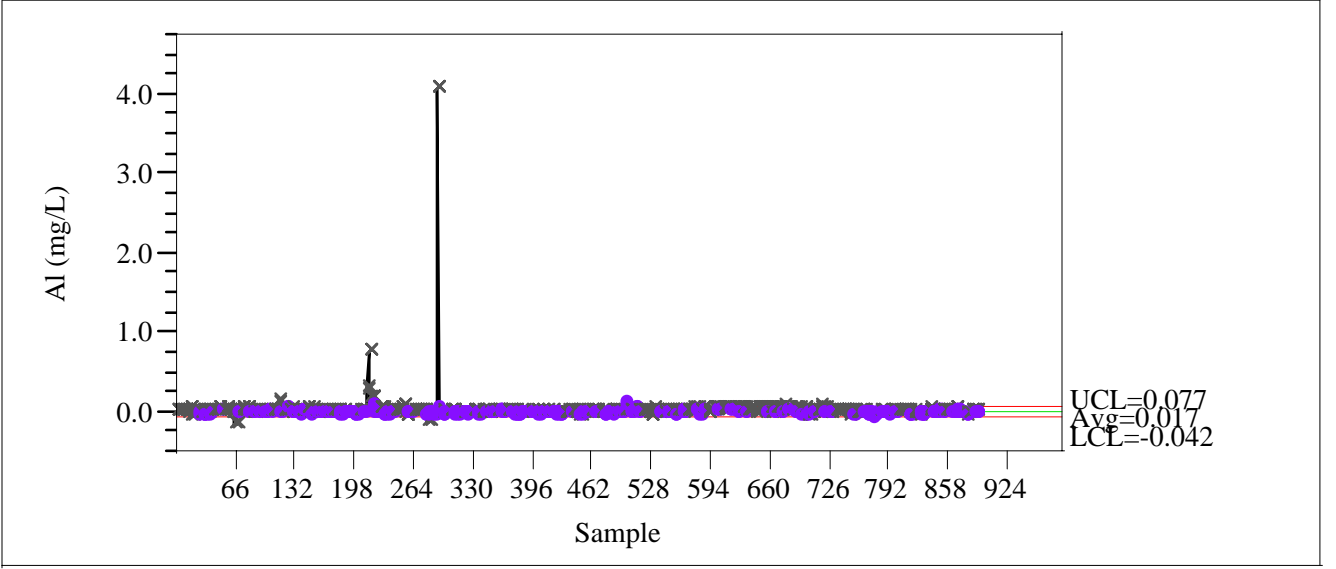

Individual Measurement of B (mg/L)

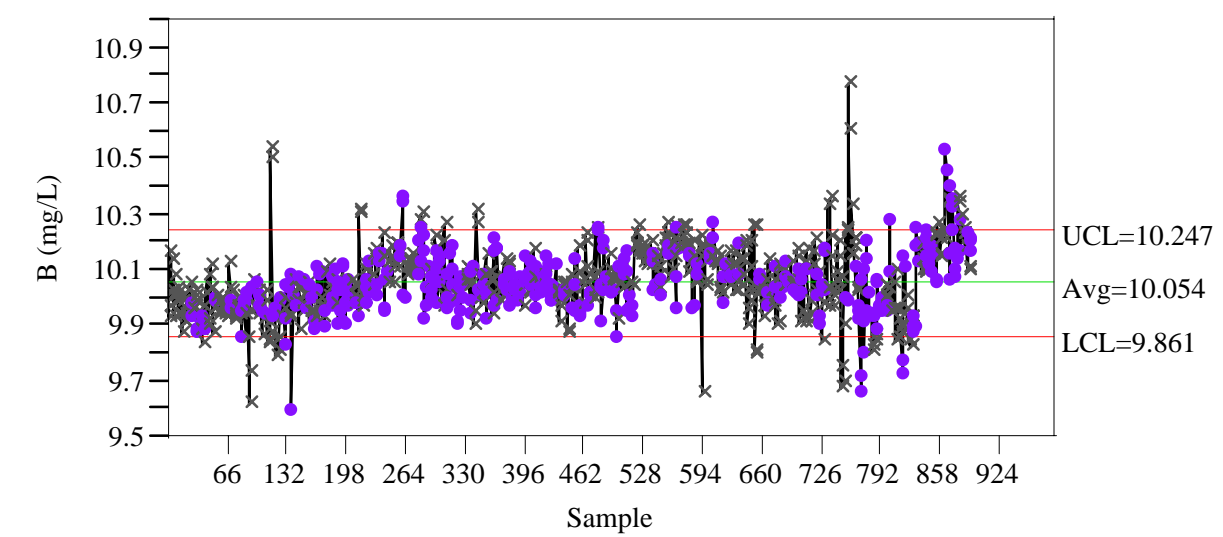




\section{Exhibit A1. Cold Chem Standards in Analytical Sequence}

\section{Revision 0}

Individual Measurement of Ca (mg/L)

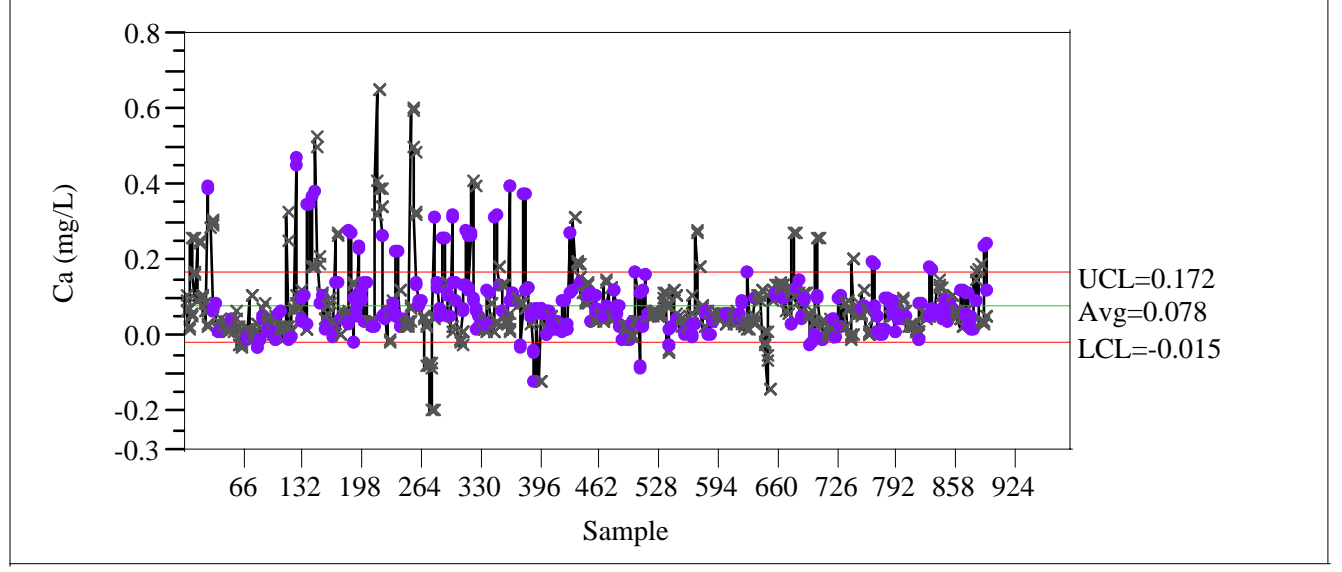

Individual Measurement of $\mathrm{Cr}$ (mg/L)

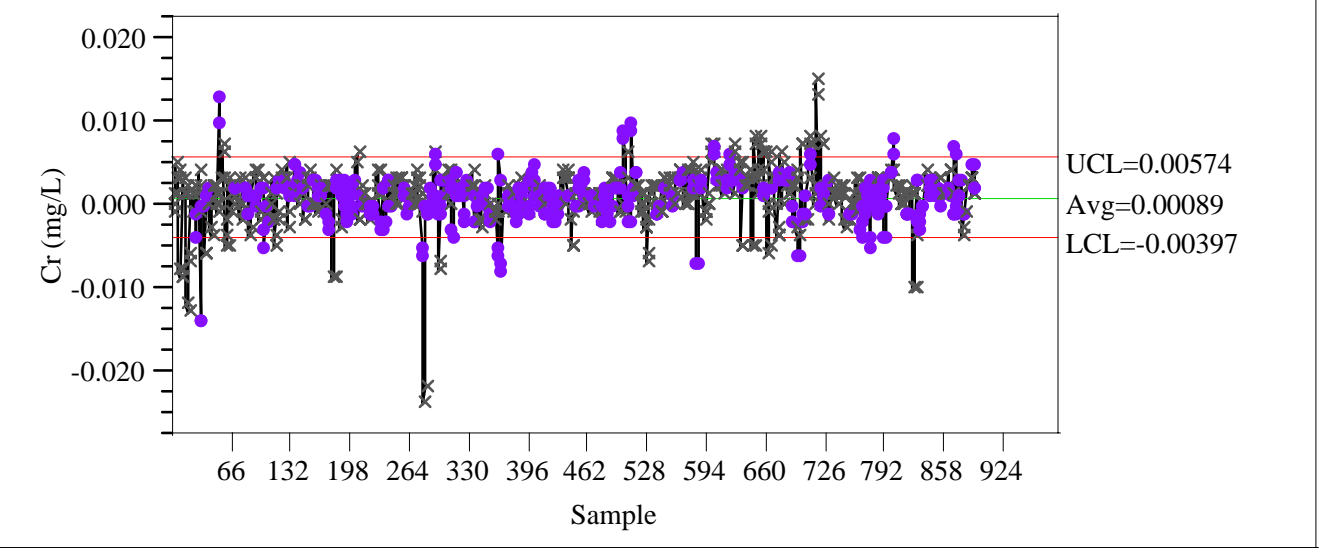

Individual Measurement of $\mathrm{Cu}(\mathrm{mg} / \mathrm{L})$

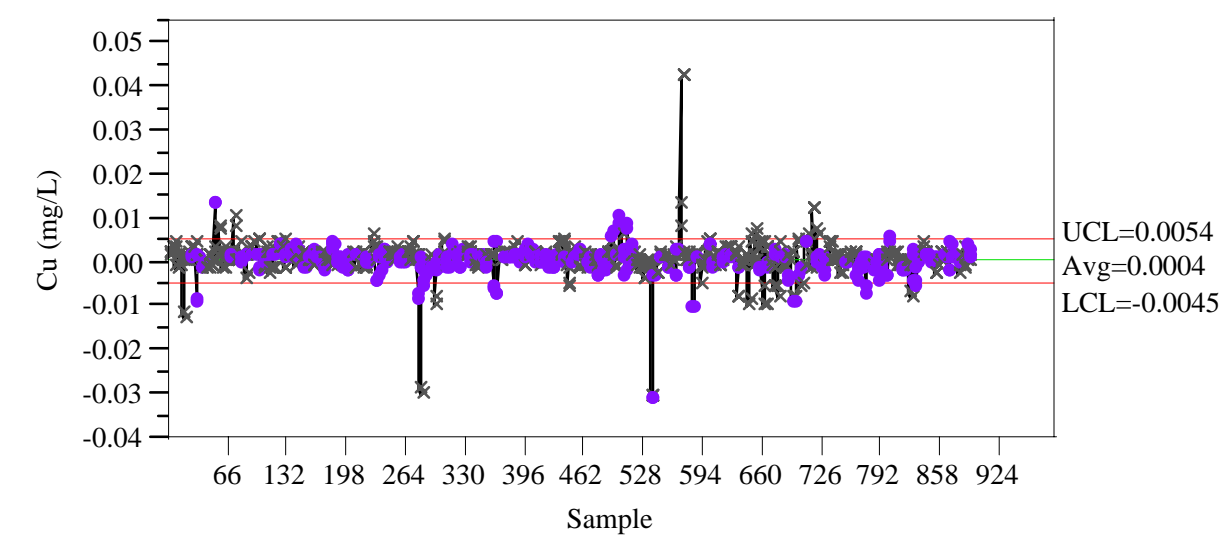




\section{Exhibit A1. Cold Chem Standards in Analytical Sequence}

Revision 0

Individual Measurement of Fe (mg/L)

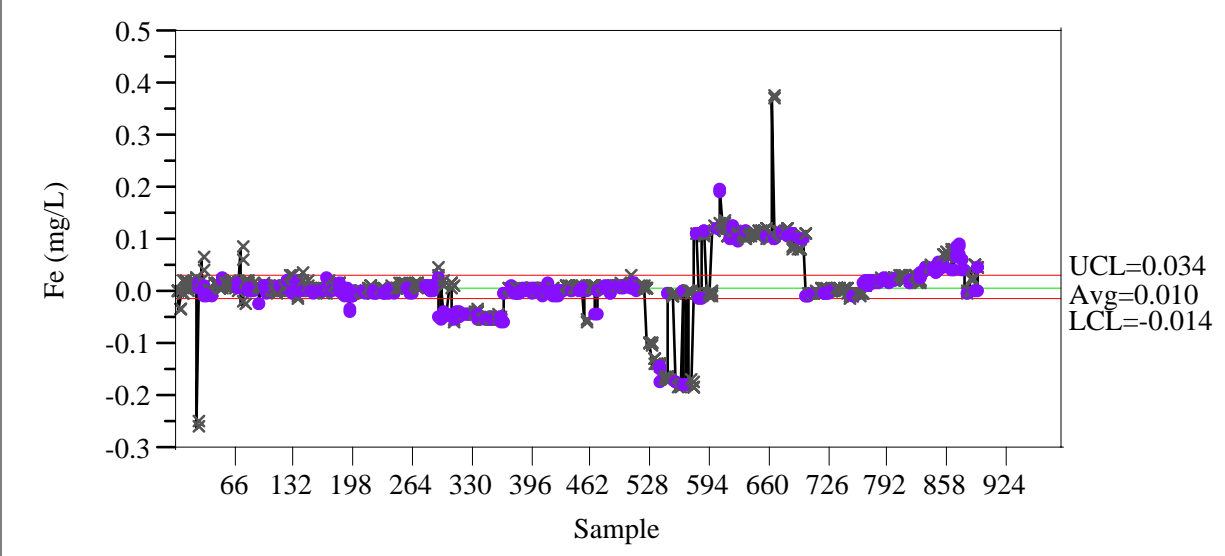

Individual Measurement of K (mg/L)

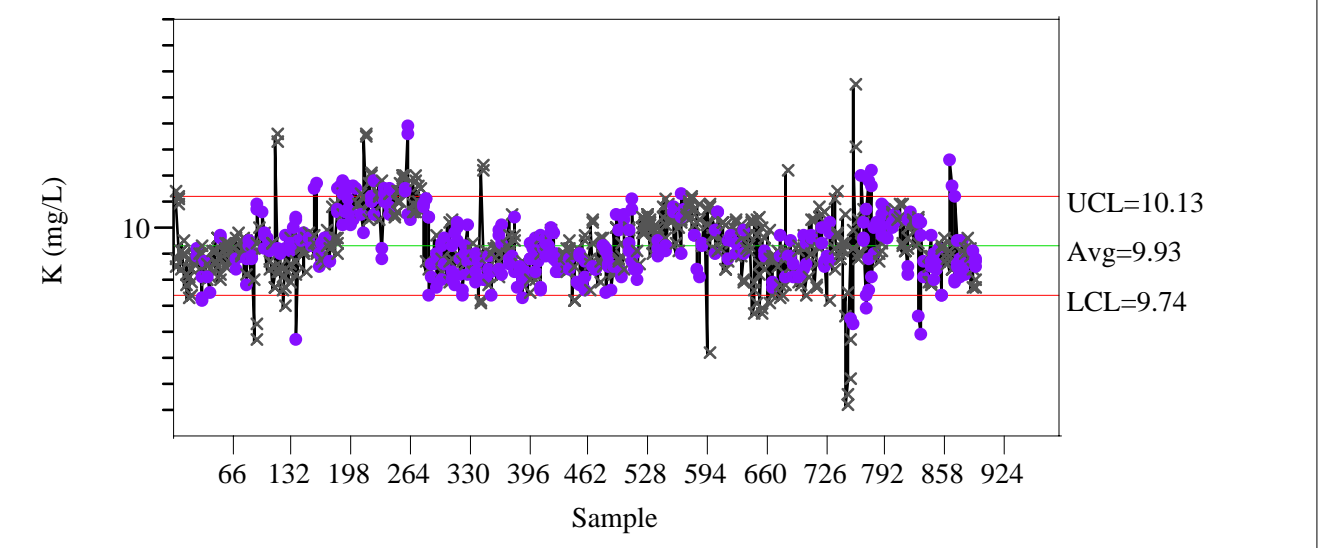

Individual Measurement of $\mathrm{Li}(\mathrm{mg} / \mathrm{L})$

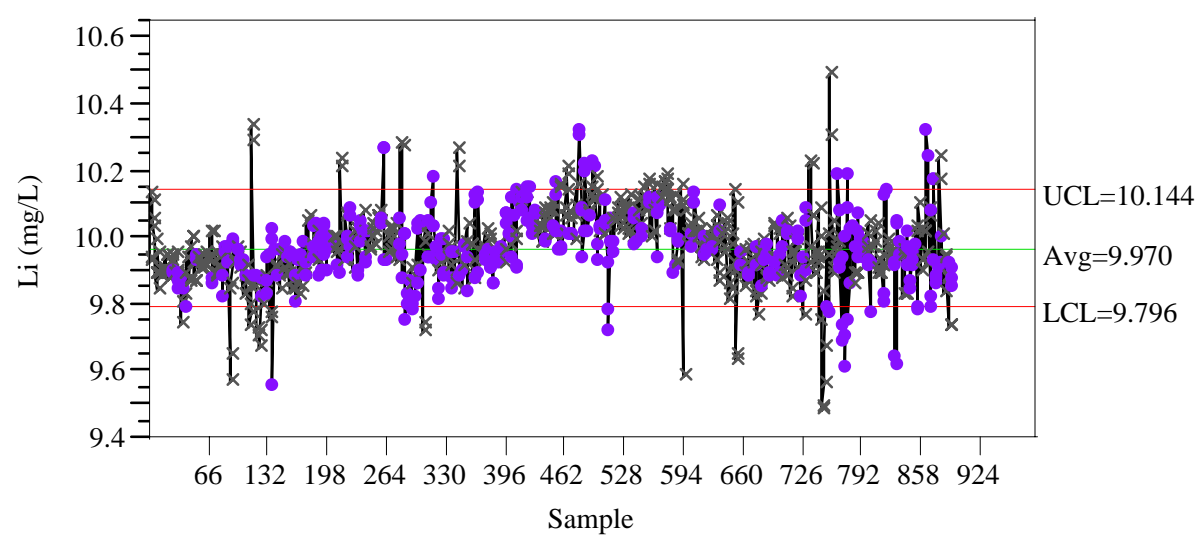


WSRC-TR-2004-00576

\section{Exhibit A1. Cold Chem Standards in Analytical Sequence}

\section{Revision 0}

Individual Measurement of $\mathrm{Mg}(\mathrm{mg} / \mathrm{L})$

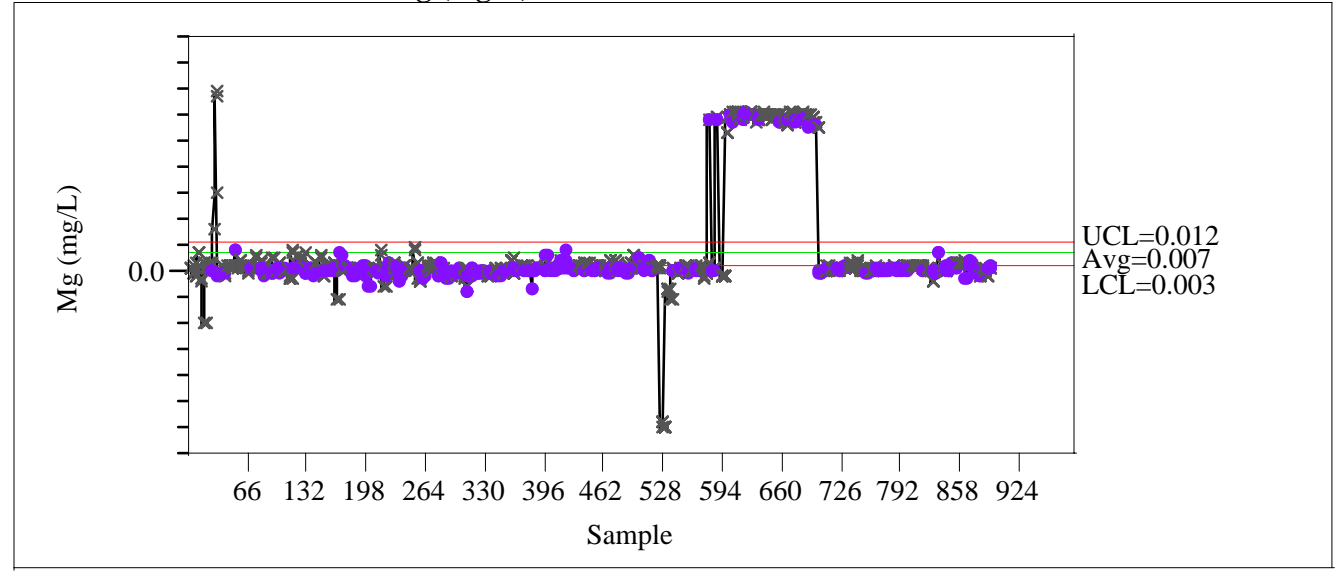

Individual Measurement of Mn (mg/L)

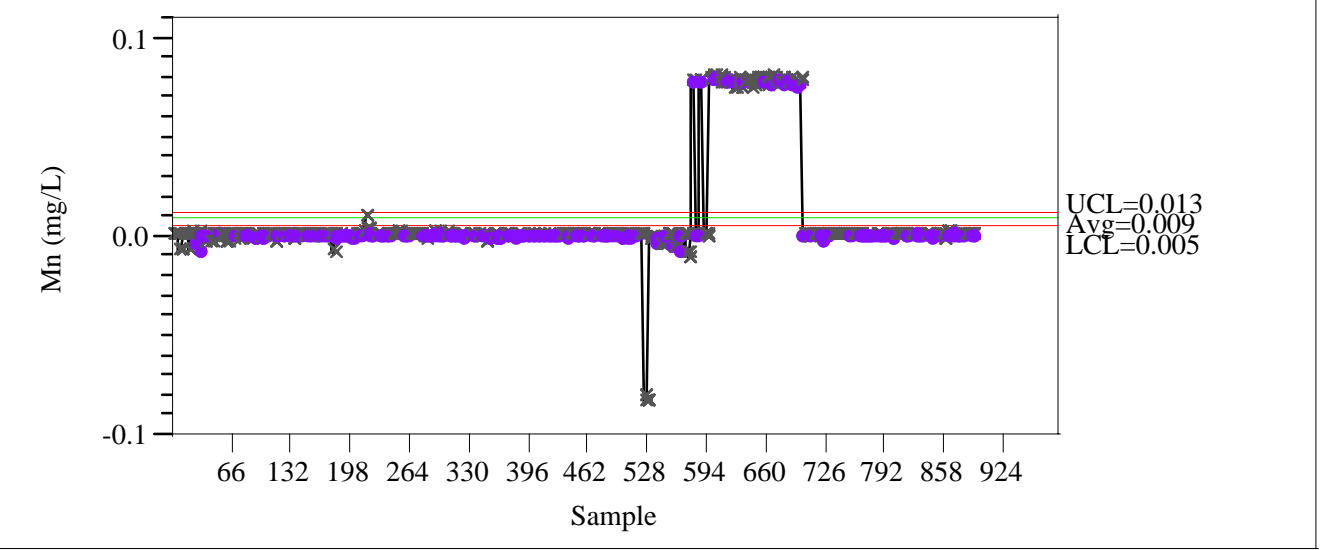

Individual Measurement of $\mathrm{Na}(\mathrm{mg} / \mathrm{L})$

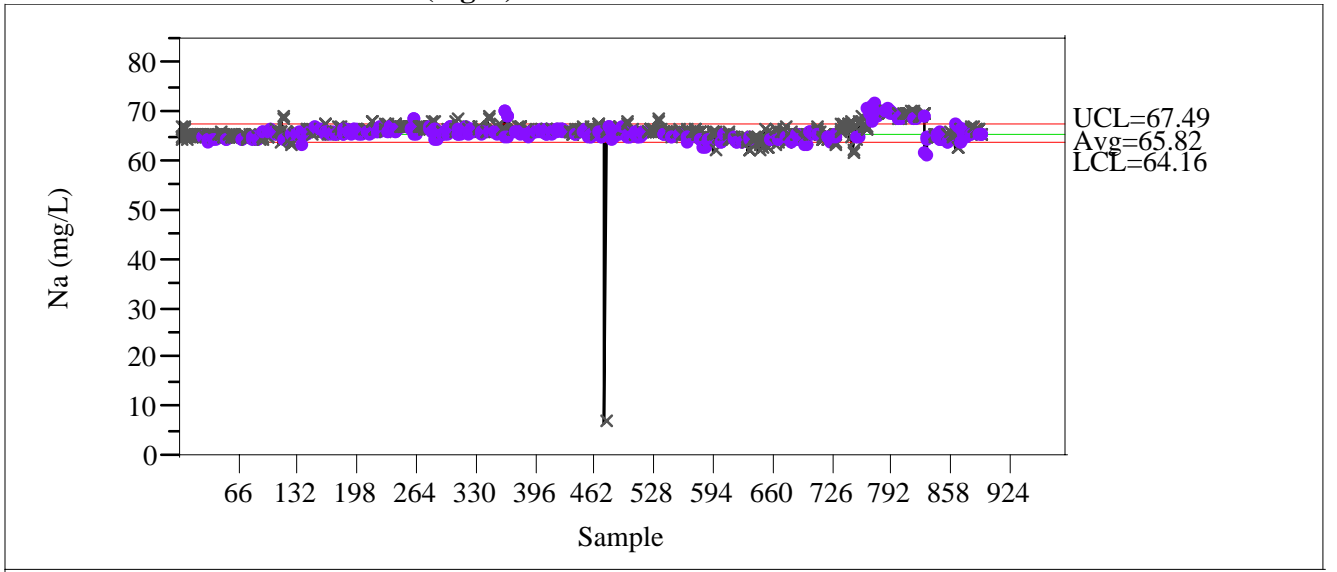




\section{Exhibit A1. Cold Chem Standards in Analytical Sequence}

\section{Revision 0}

Individual Measurement of Ni (mg/L)

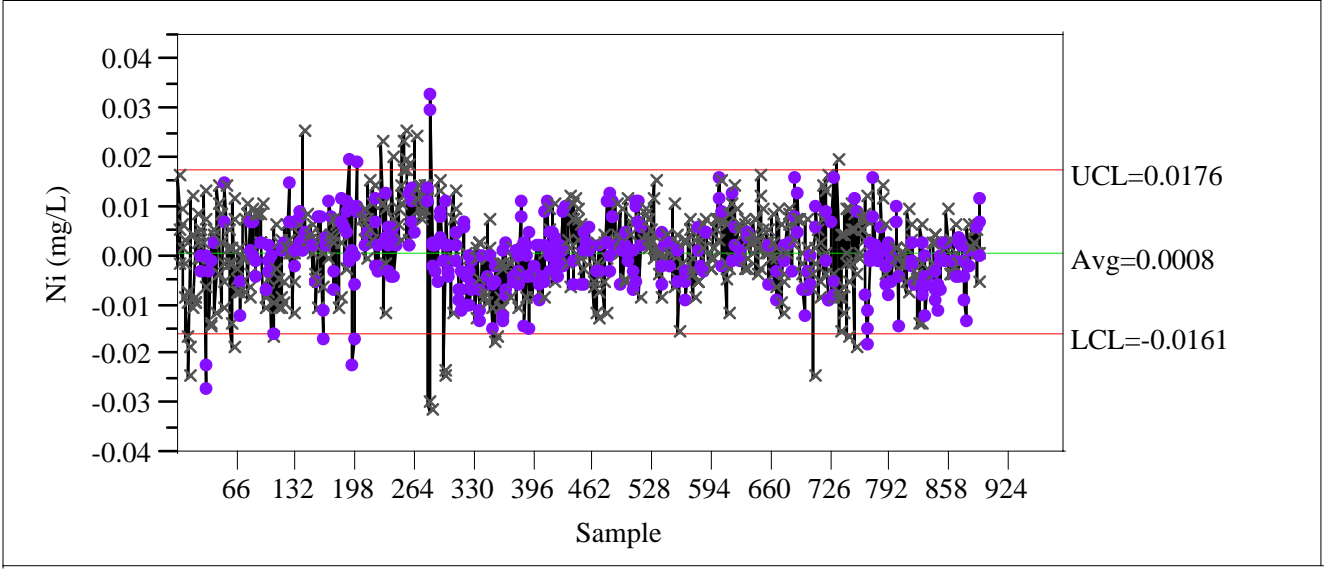

Individual Measurement of Si (mg/L)

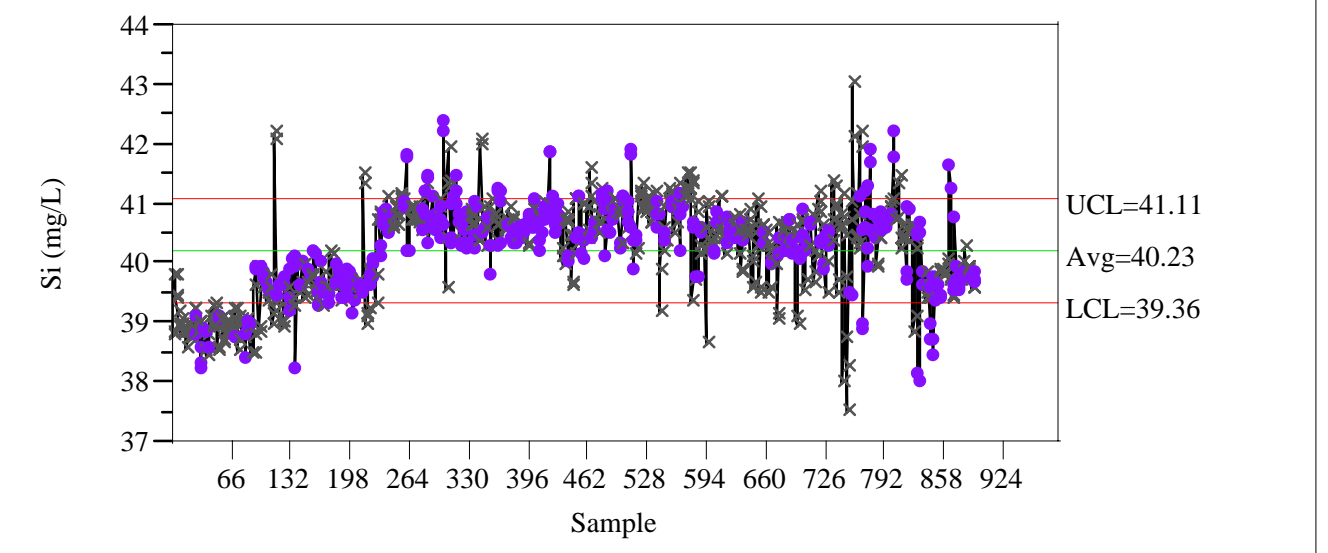

Individual Measurement of $\mathrm{Ti}(\mathrm{mg} / \mathrm{L})$

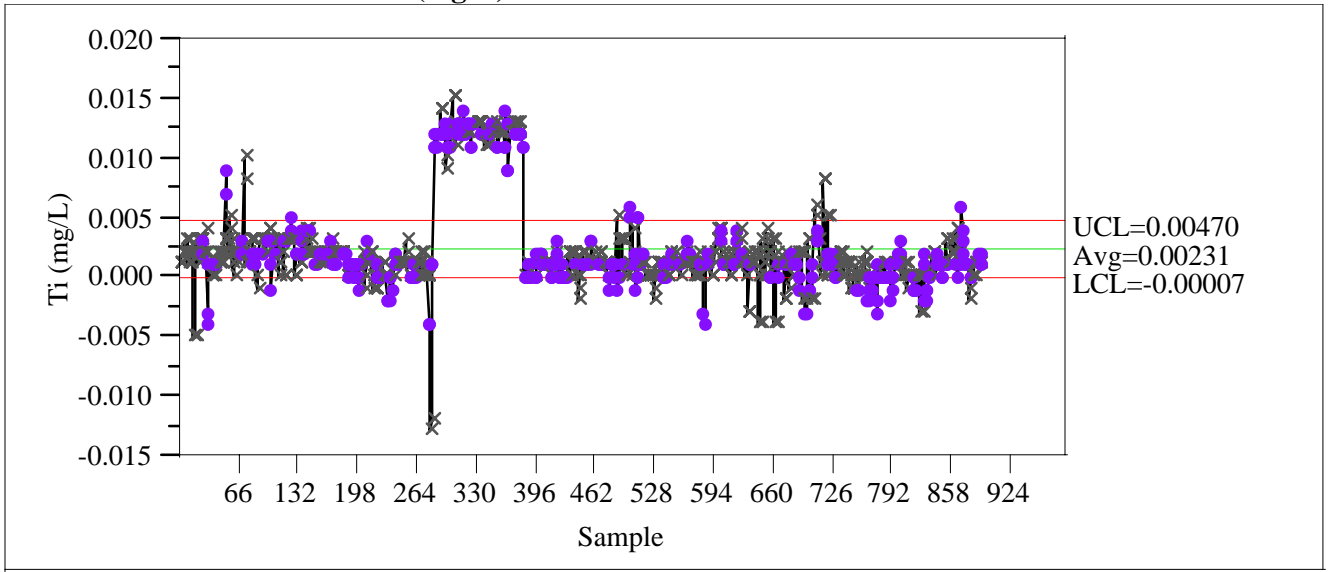


WSRC-TR-2004-00576

\section{Exhibit A1. Cold Chem Standards in Analytical Sequence}

\section{Revision 0}

Individual Measurement of $\mathrm{Zr}(\mathrm{mg} / \mathrm{L})$

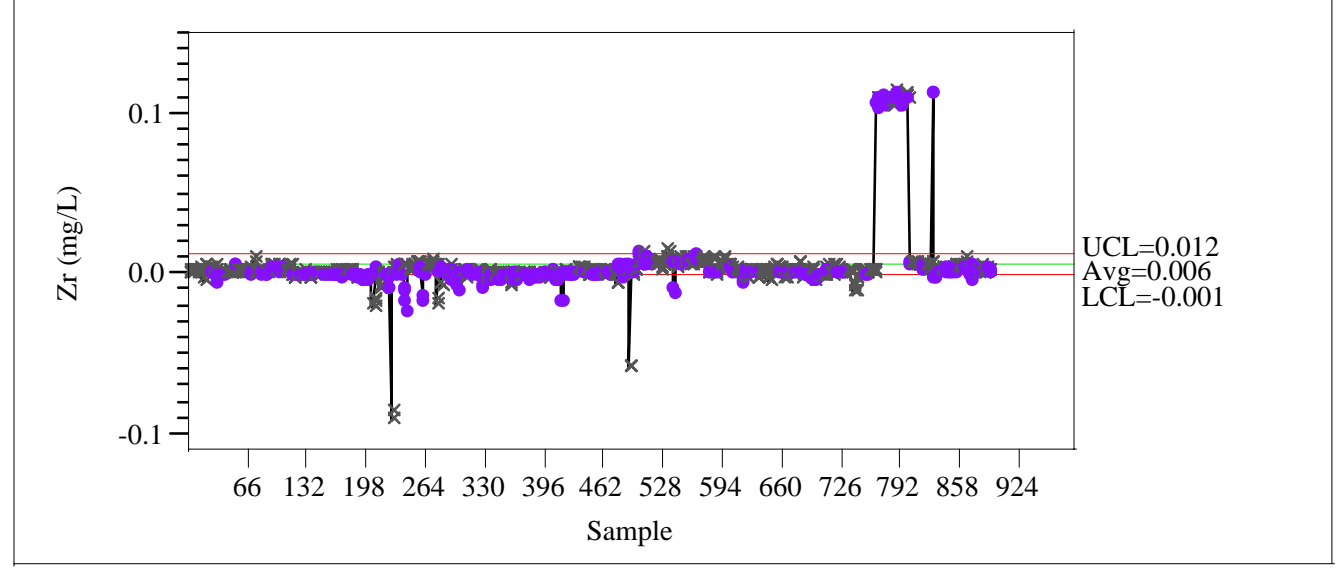

Individual Measurement of U (mg/L)

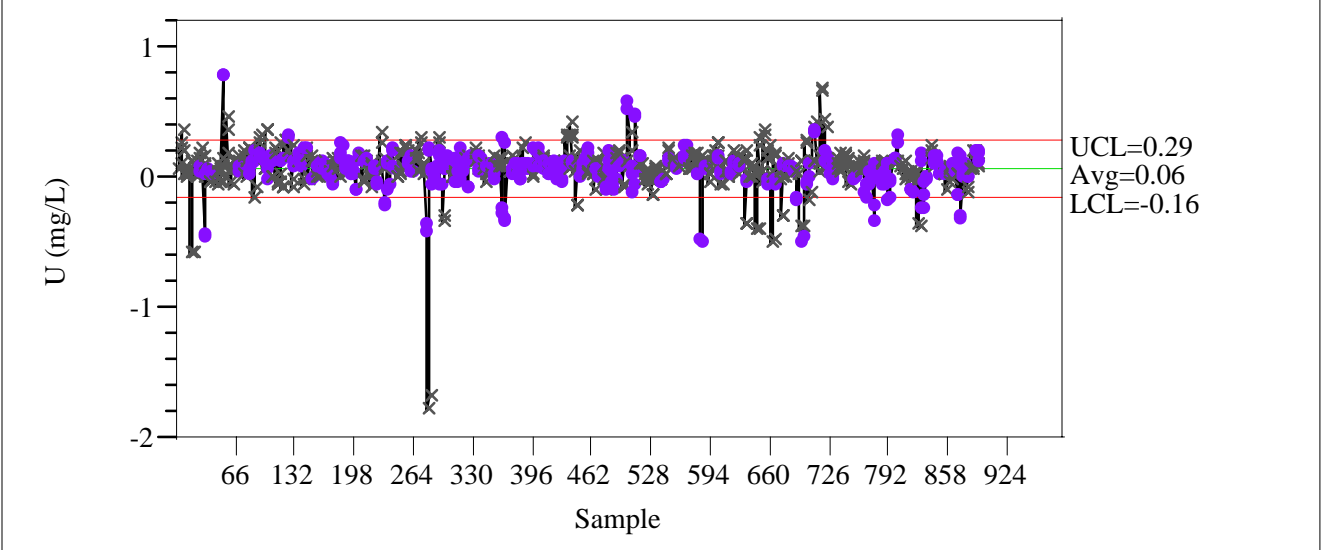

\section{STCd=IN38}

Control Chart

Individual Measurement of Al (mg/L)

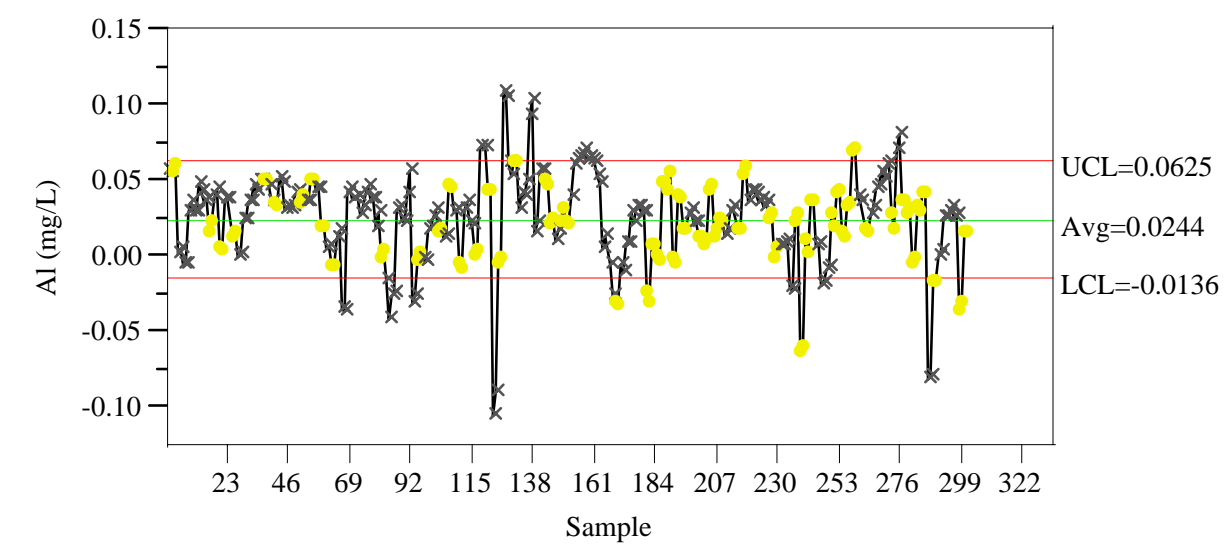


WSRC-TR-2004-00576

\section{Exhibit A1. Cold Chem Standards in Analytical Sequence}

\section{Revision 0}

Individual Measurement of B (mg/L)

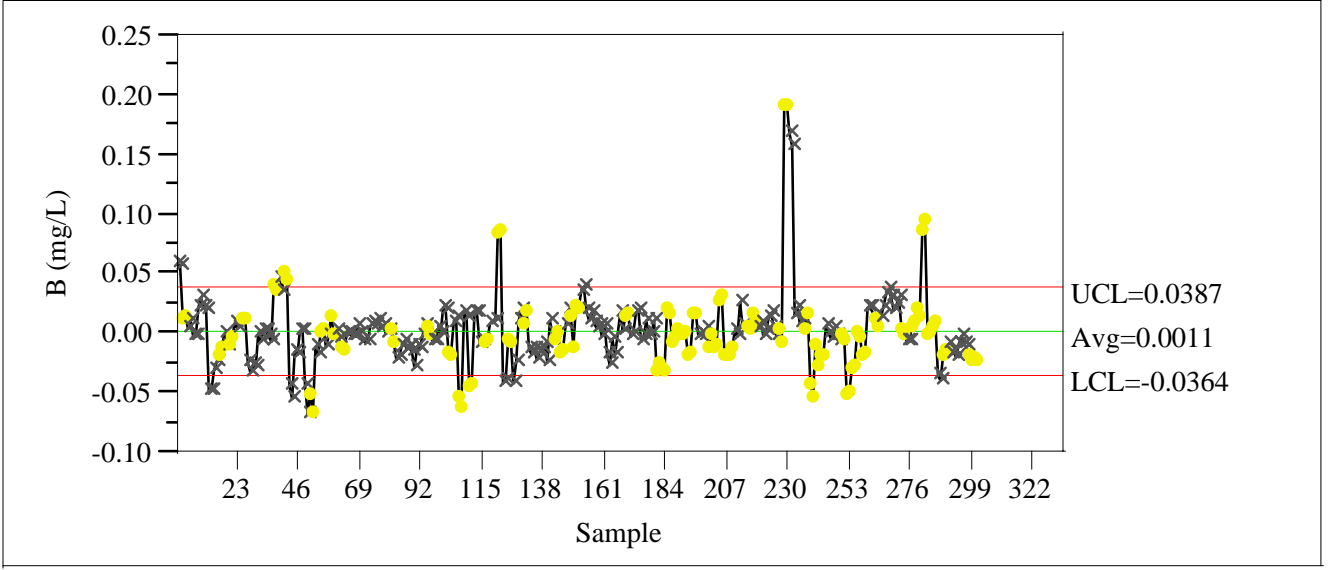

Individual Measurement of Ca (mg/L)

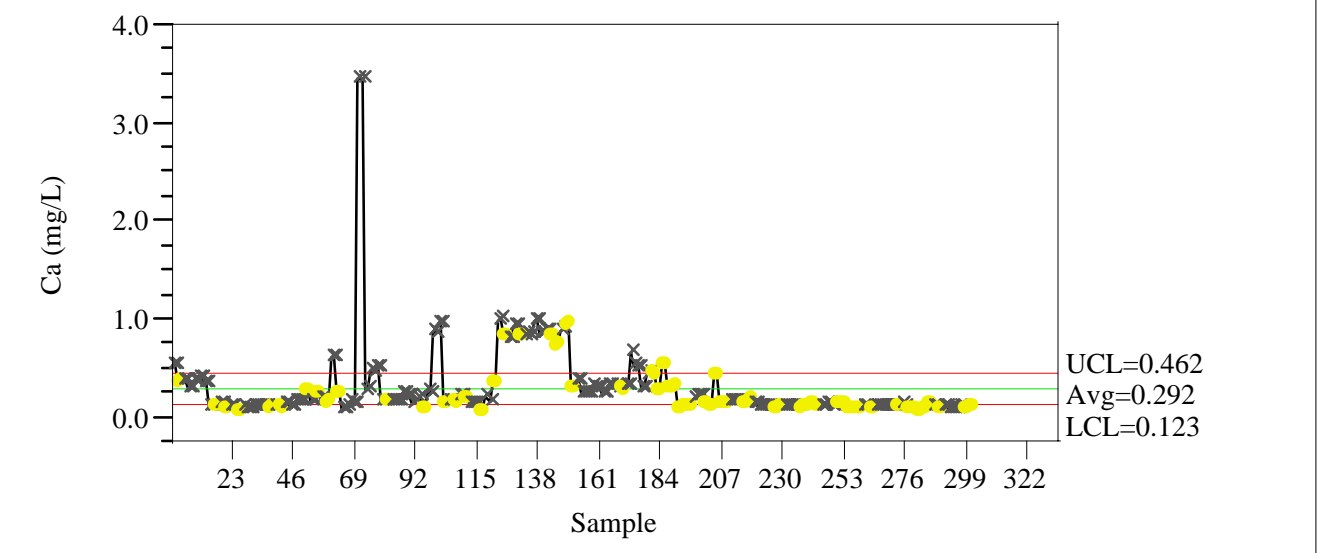

Individual Measurement of $\mathrm{Cr}(\mathrm{mg} / \mathrm{L})$

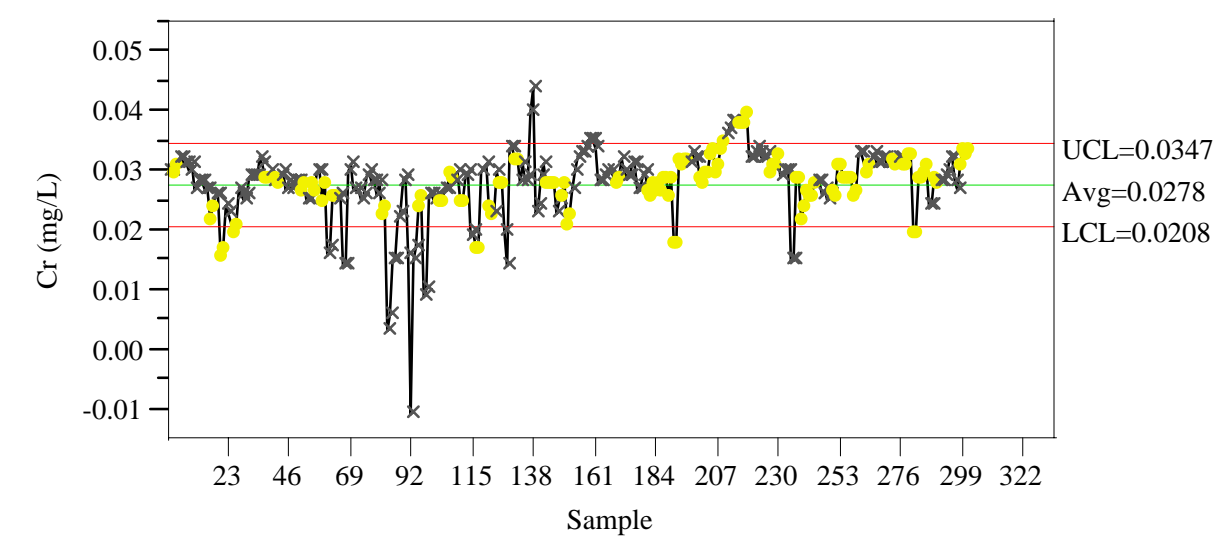


WSRC-TR-2004-00576

\section{Exhibit A1. Cold Chem Standards in Analytical Sequence}

\section{Revision 0}

Individual Measurement of $\mathrm{Cu}(\mathrm{mg} / \mathrm{L})$

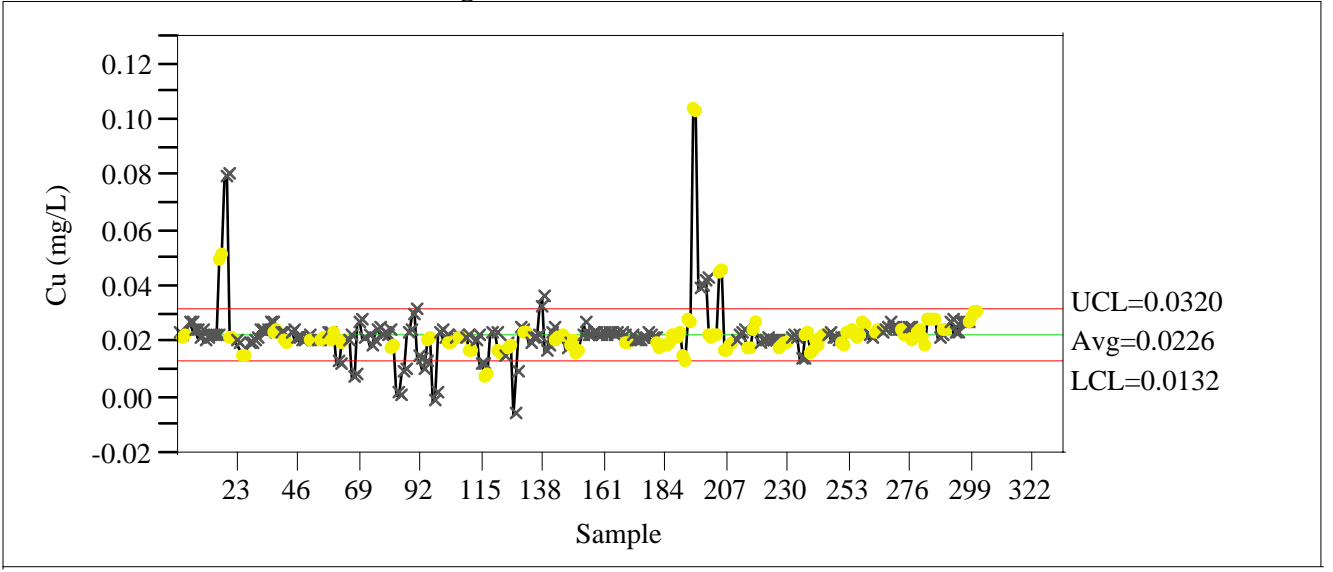

Individual Measurement of Fe (mg/L)

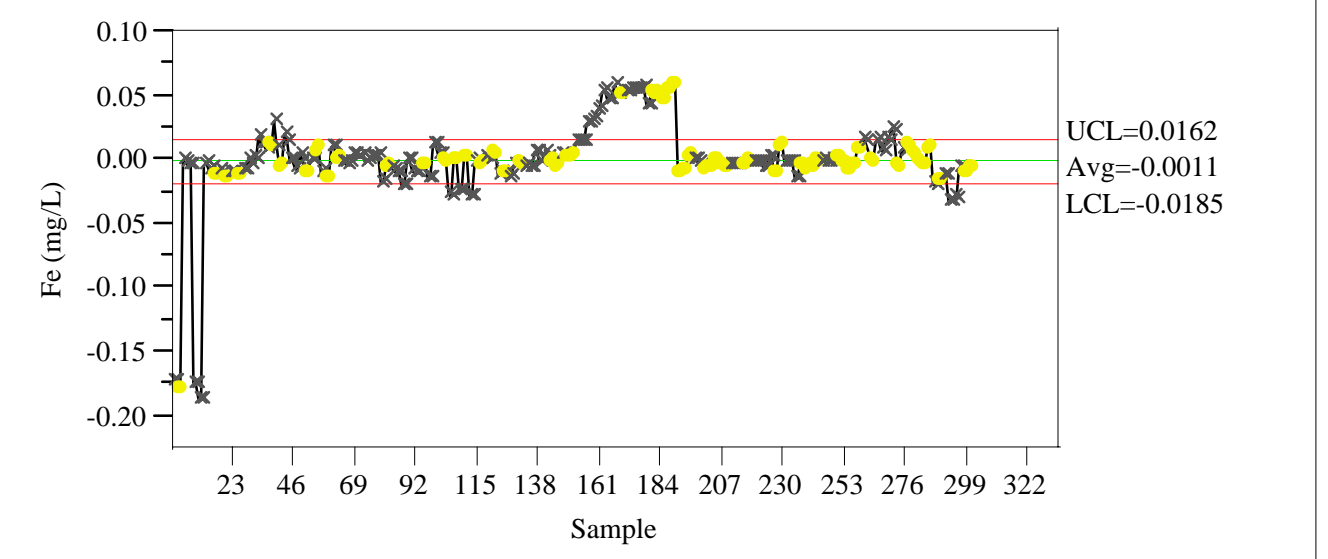

Individual Measurement of K (mg/L)

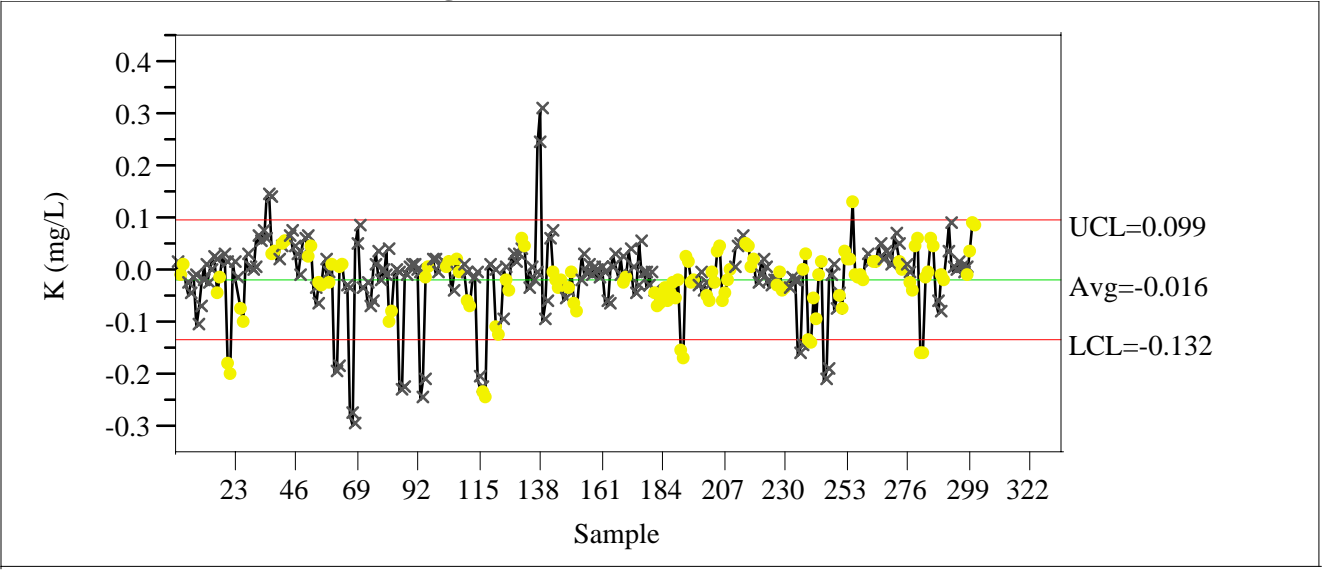


WSRC-TR-2004-00576

\section{Exhibit A1. Cold Chem Standards in Analytical Sequence}

\section{Revision 0}

Individual Measurement of $\mathrm{Li}(\mathrm{mg} / \mathrm{L})$

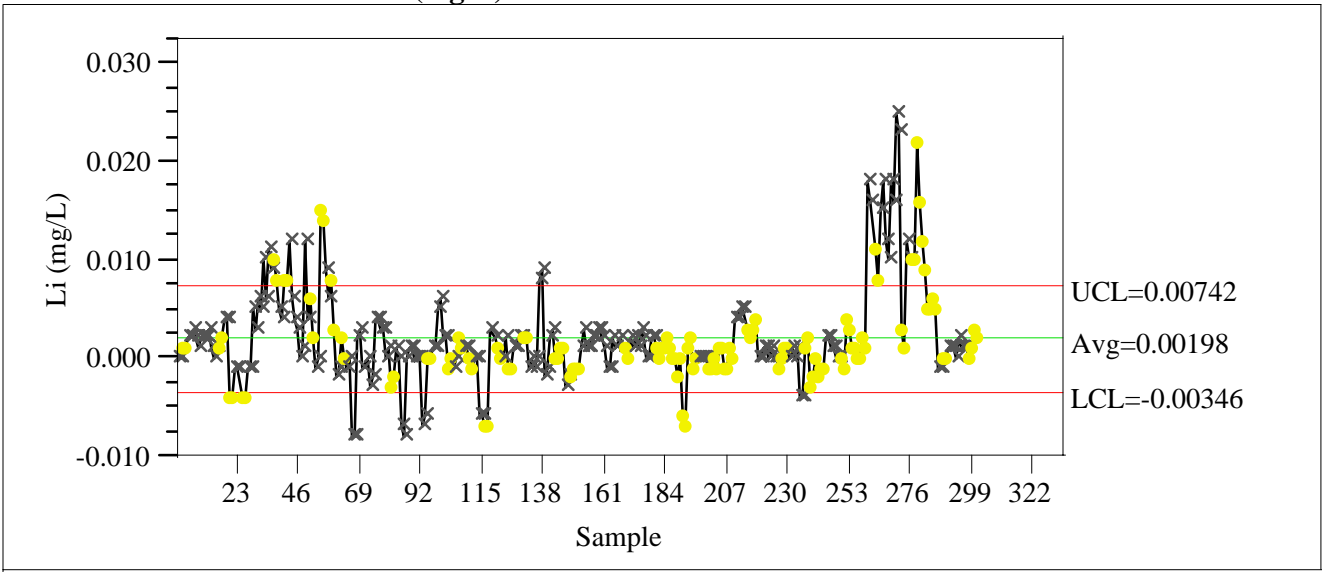

Individual Measurement of Mg (mg/L)

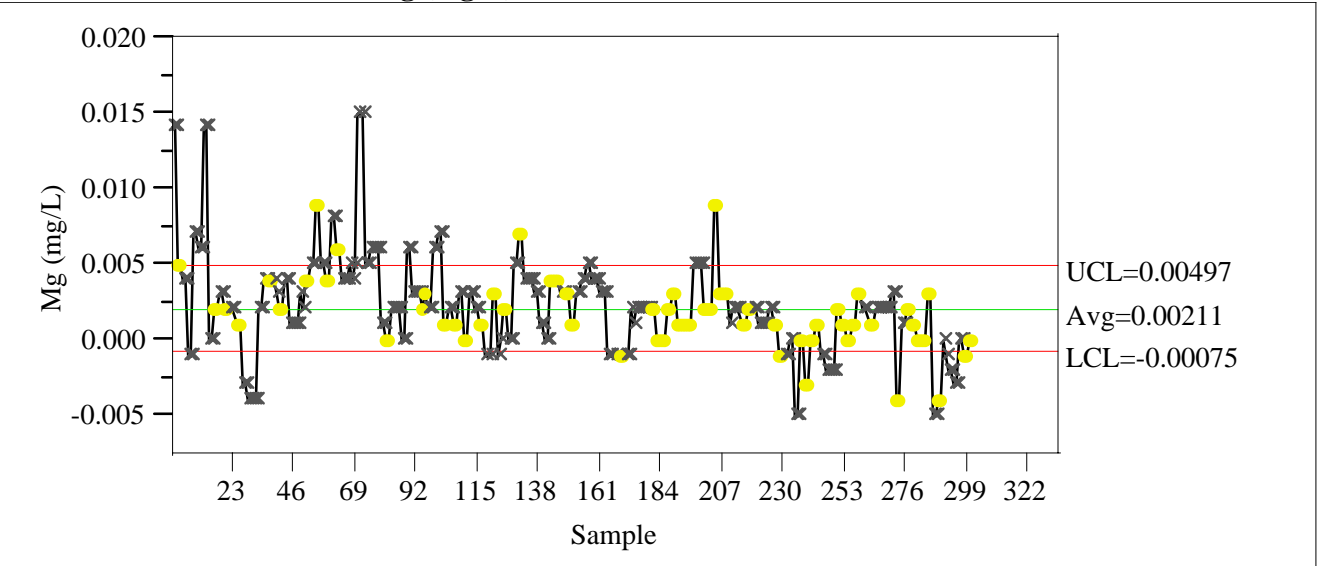

Individual Measurement of Mn (mg/L)

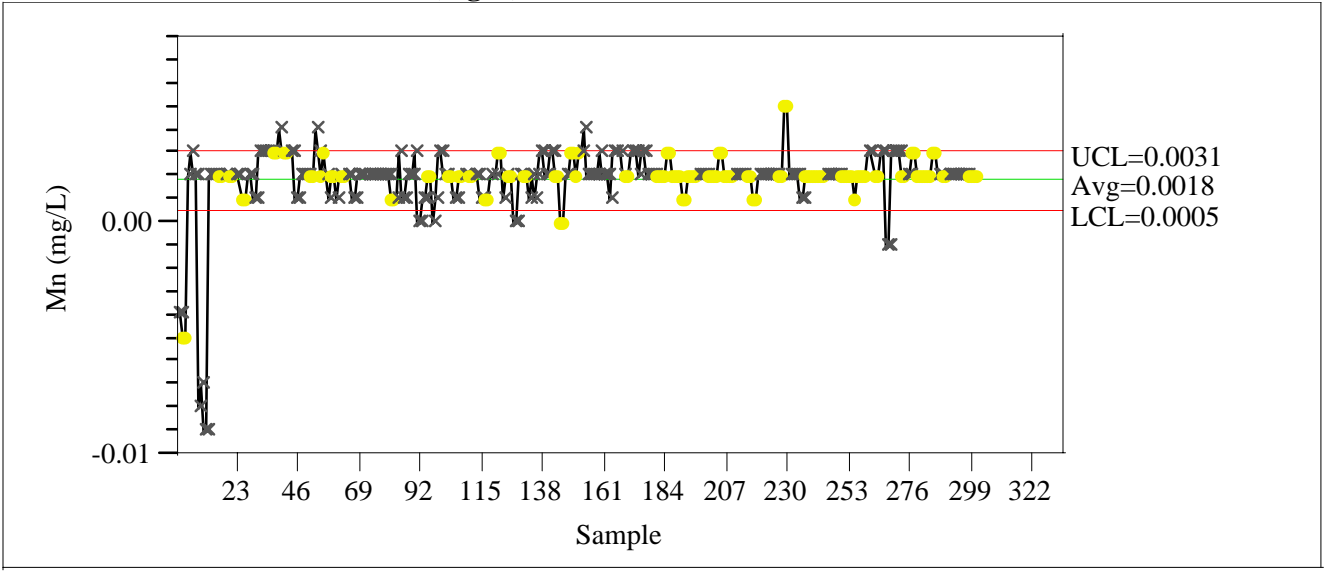


WSRC-TR-2004-00576

\section{Exhibit A1. Cold Chem Standards in Analytical Sequence}

\section{Revision 0}

Individual Measurement of Na (mg/L)

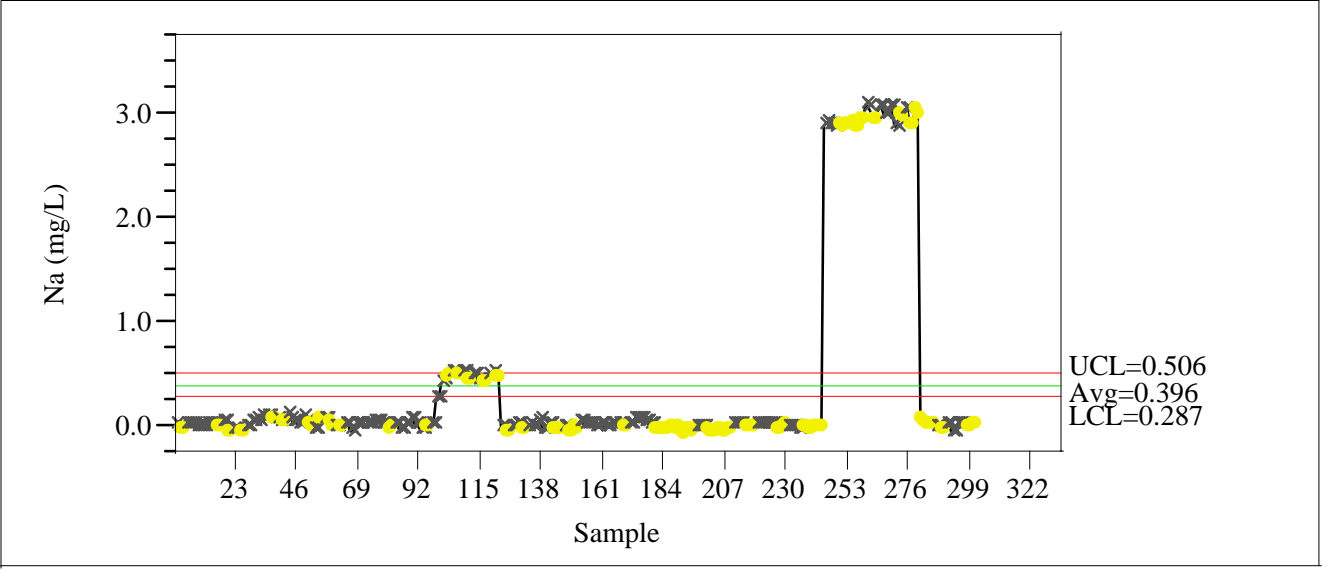

Individual Measurement of Ni (mg/L)

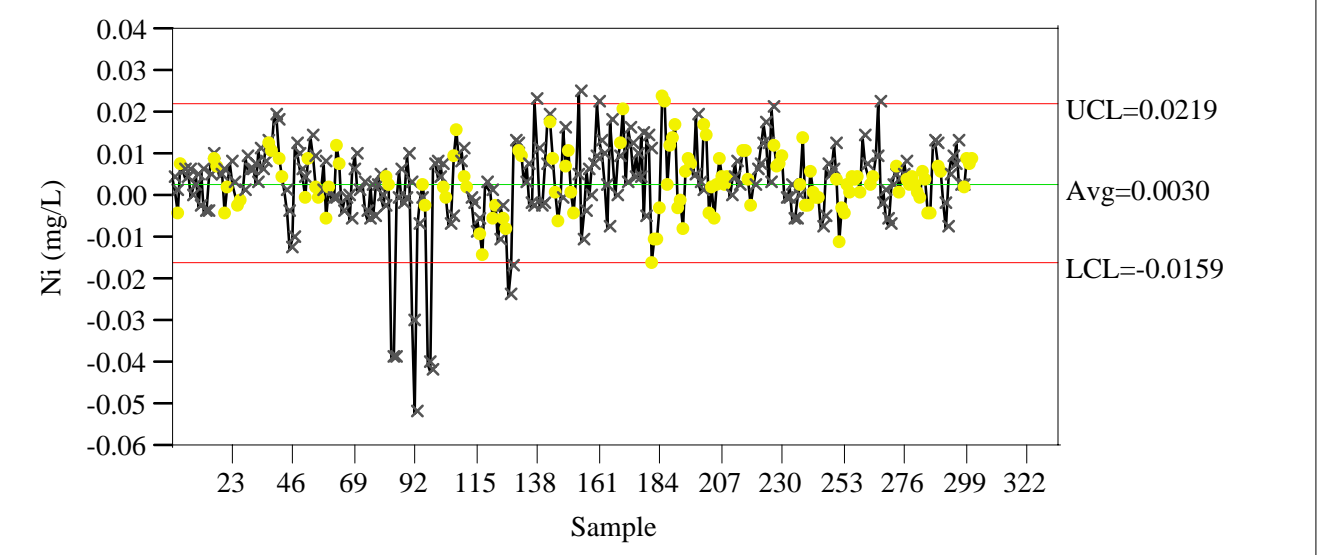

Individual Measurement of Si (mg/L)

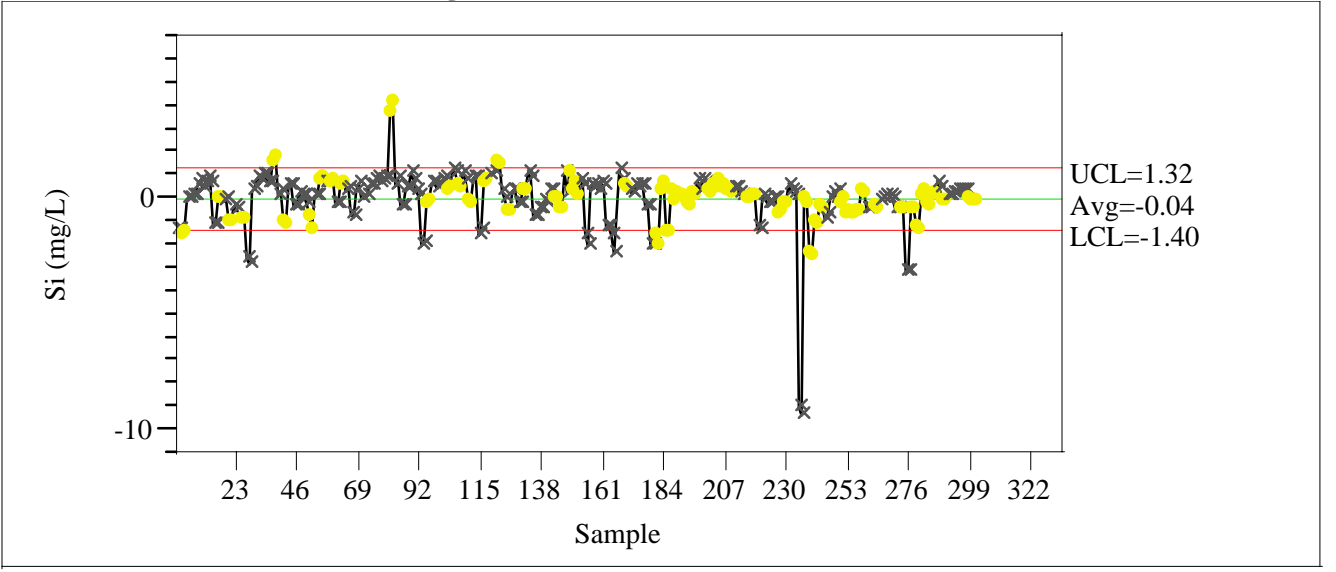




\section{Exhibit A1. Cold Chem Standards in Analytical Sequence}

\section{Revision 0}

Individual Measurement of Ti (mg/L)

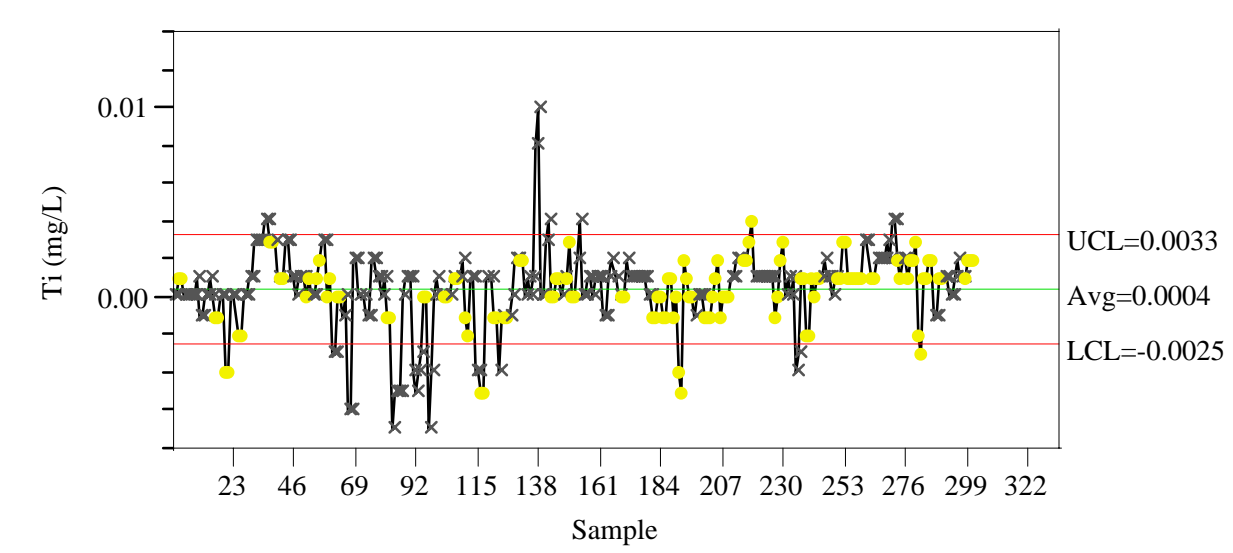

Individual Measurement of $\mathrm{Zr}$ (mg/L)

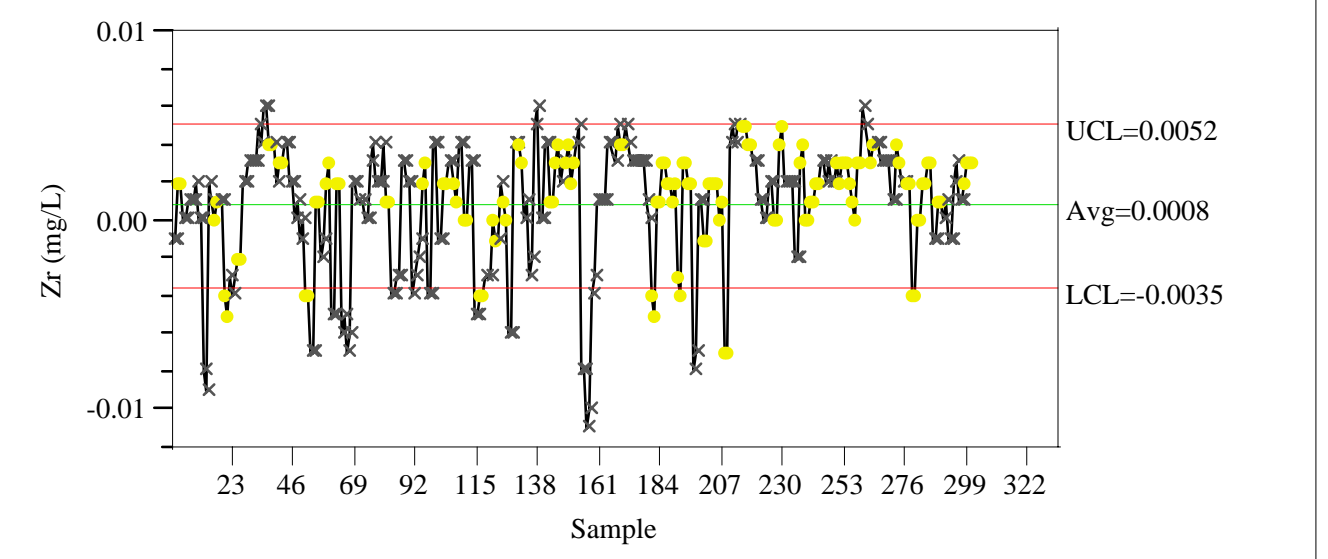

Individual Measurement of U (mg/L)

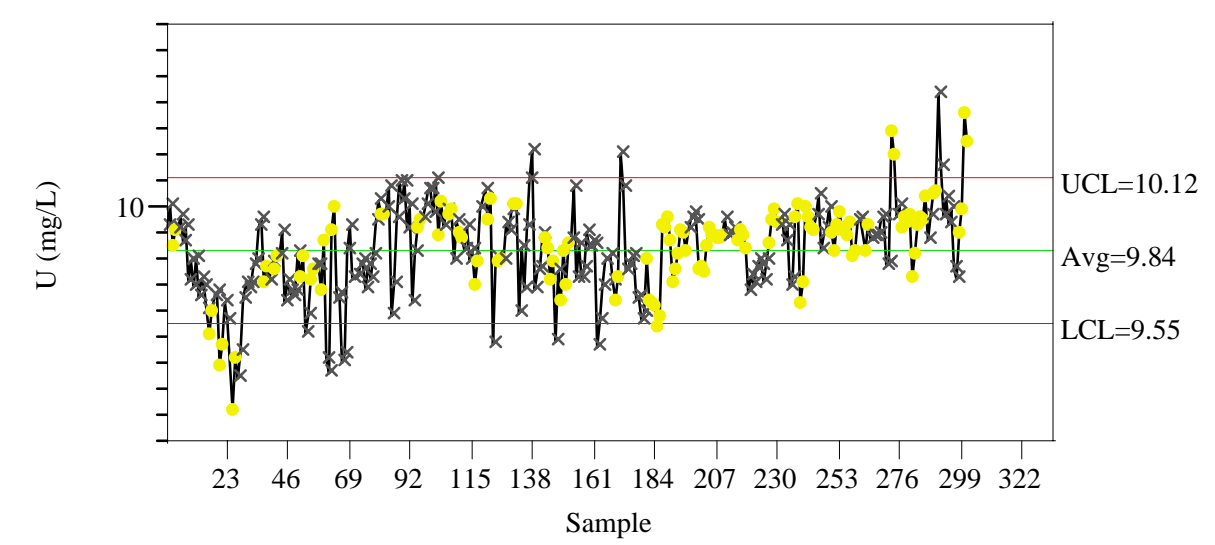


WSRC-TR-2004-00576

\section{Exhibit A1. Cold Chem Standards in Analytical Sequence}

\section{Revision 0}

STCd=IN39

Control Chart

Individual Measurement of $\mathrm{Al}$ (mg/L)

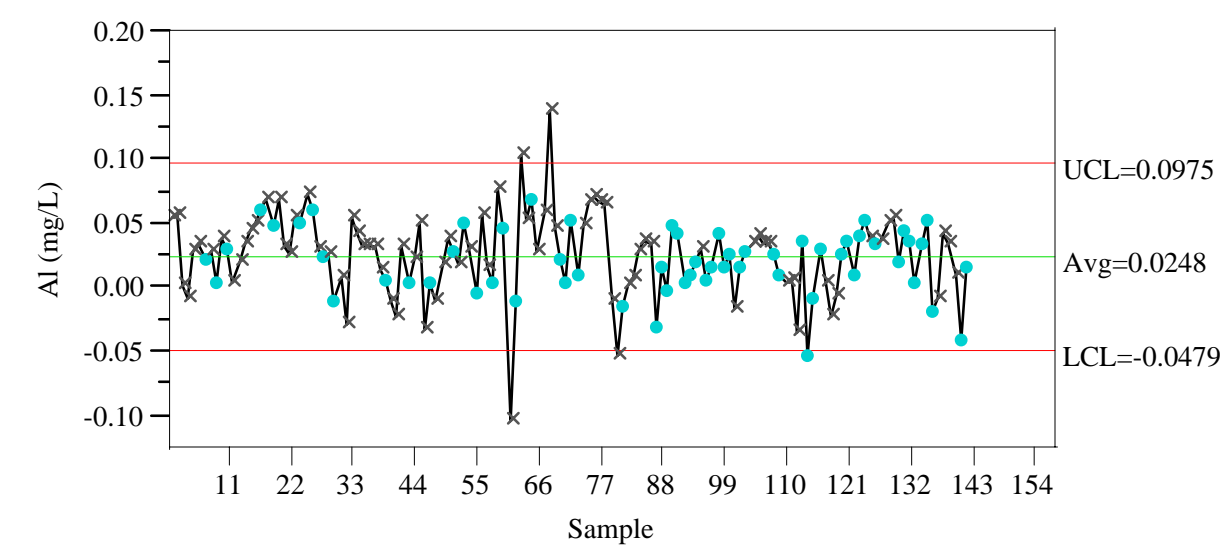

Individual Measurement of B (mg/L)

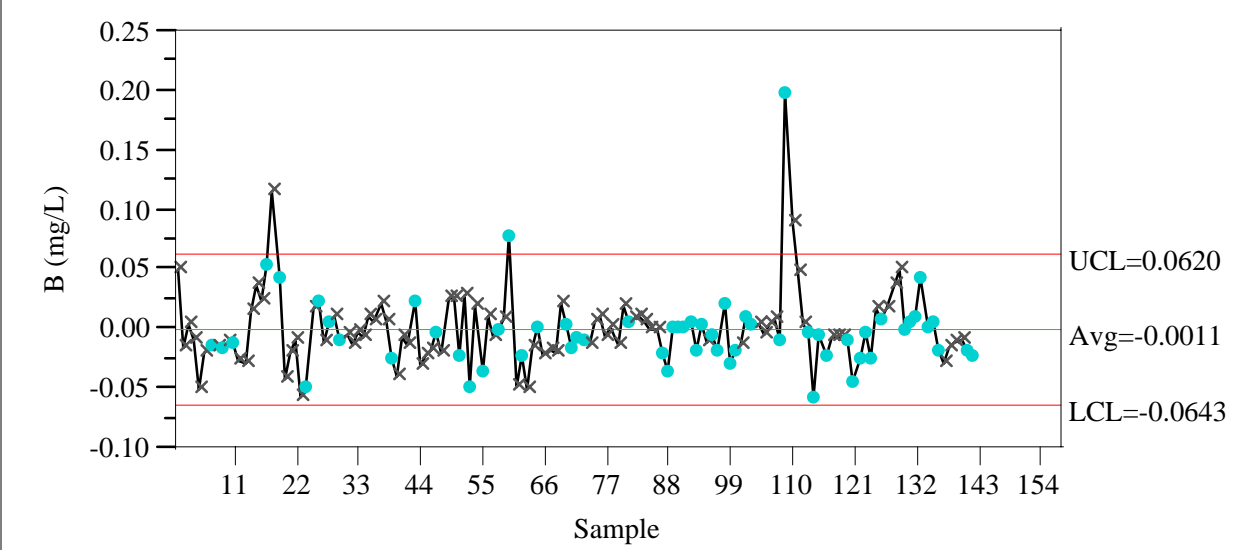

Individual Measurement of Ca (mg/L)

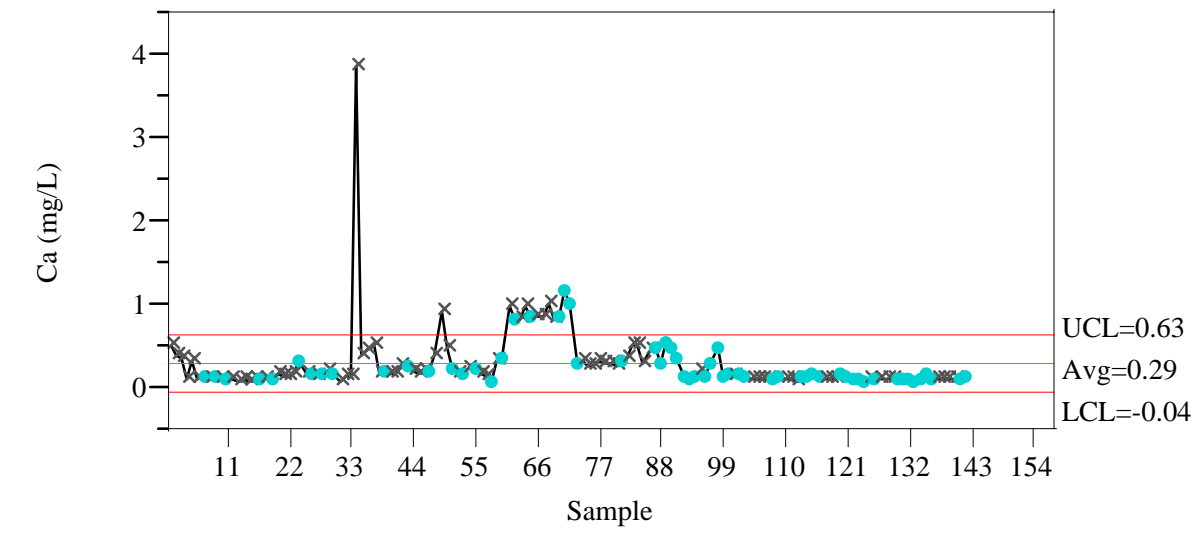


WSRC-TR-2004-00576

\section{Exhibit A1. Cold Chem Standards in Analytical Sequence}

\section{Revision 0}

Individual Measurement of $\mathrm{Cr}$ (mg/L)

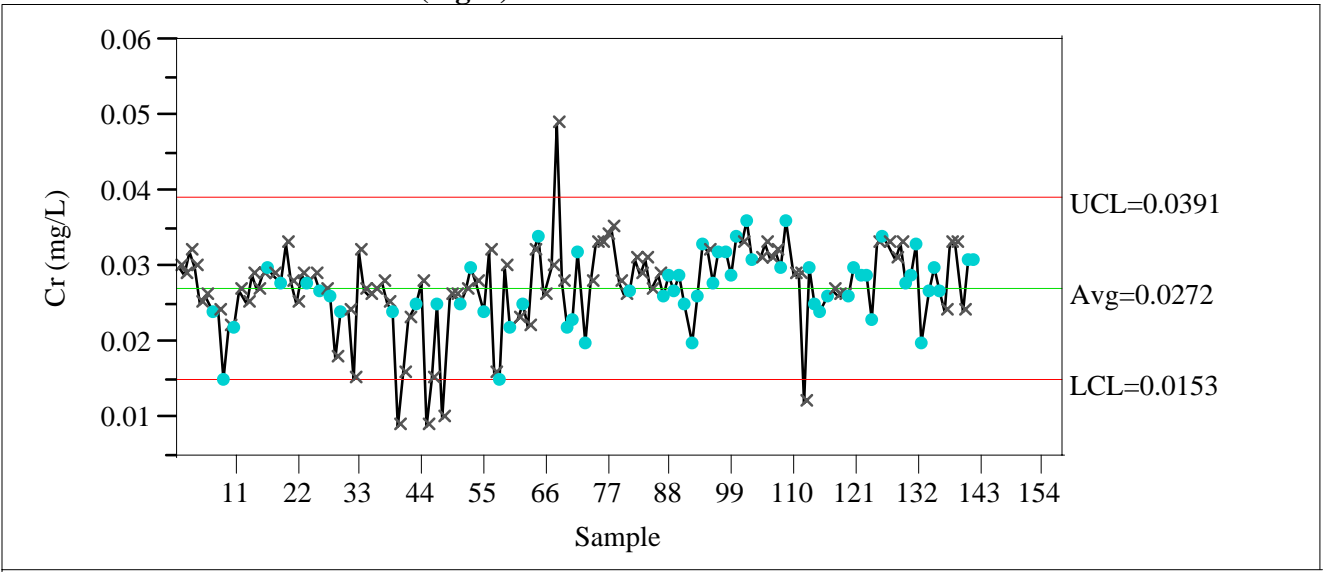

Individual Measurement of $\mathrm{Cu}$ (mg/L)

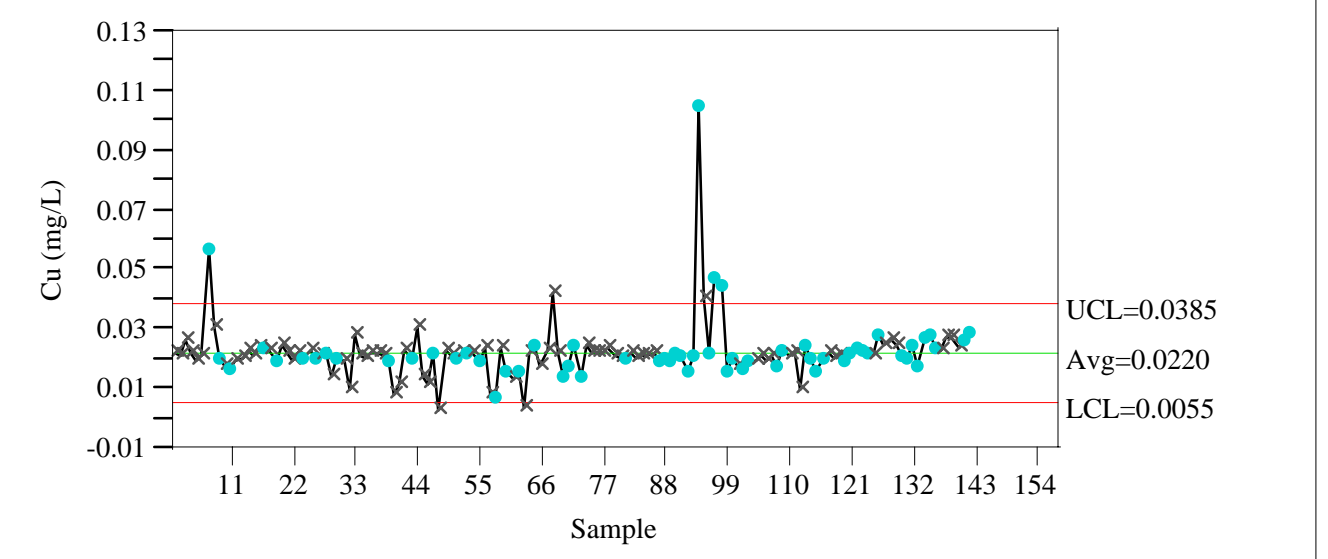

Individual Measurement of Fe (mg/L)

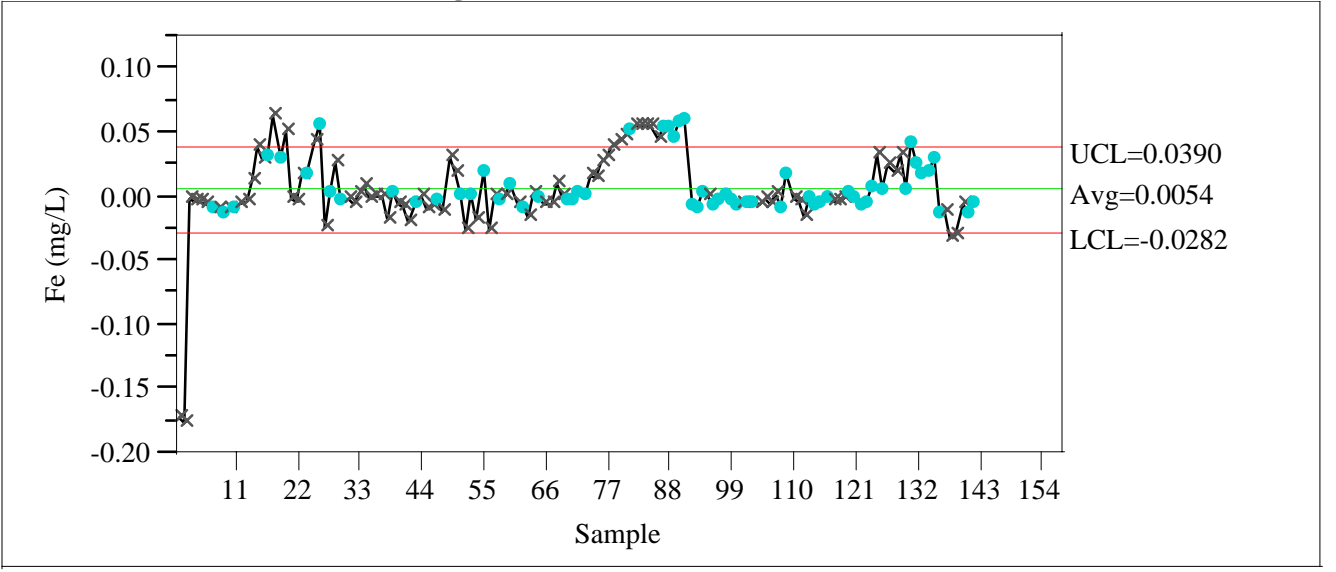




\section{Exhibit A1. Cold Chem Standards in Analytical Sequence}

\section{Revision 0}

Individual Measurement of K (mg/L)

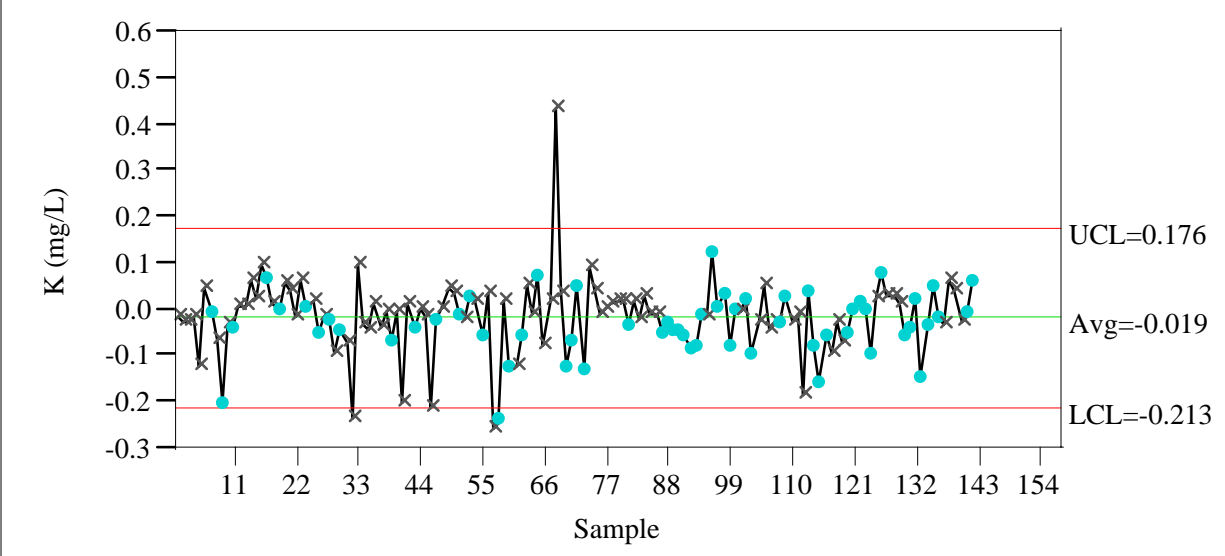

Individual Measurement of $\mathrm{Li}(\mathrm{mg} / \mathrm{L})$

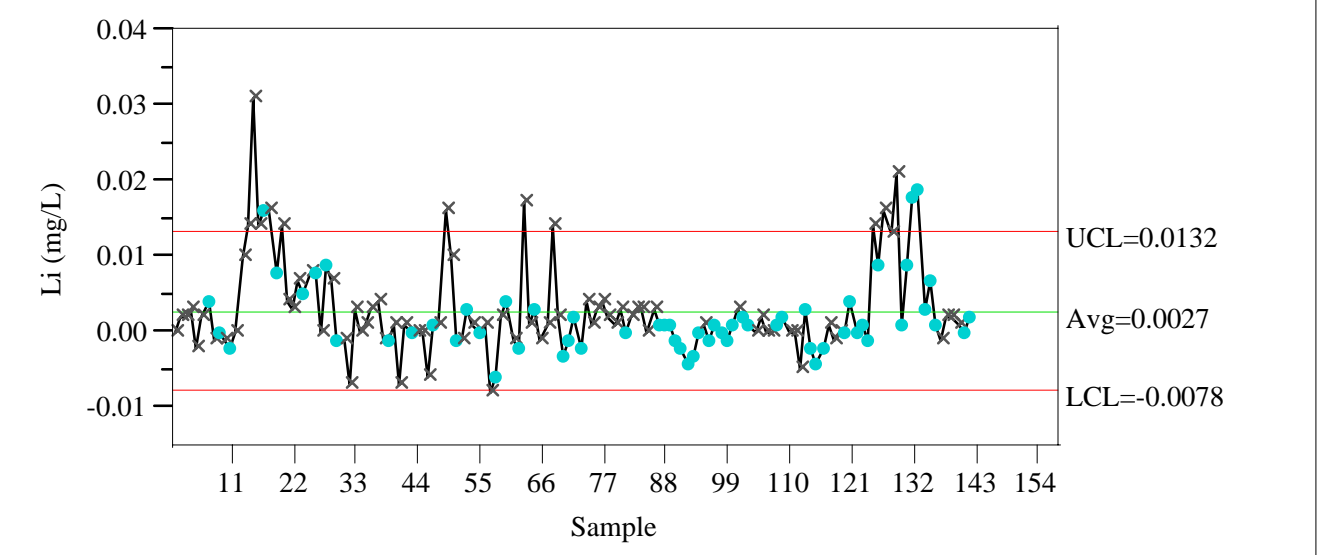

Individual Measurement of $\mathrm{Mg}(\mathrm{mg} / \mathrm{L})$

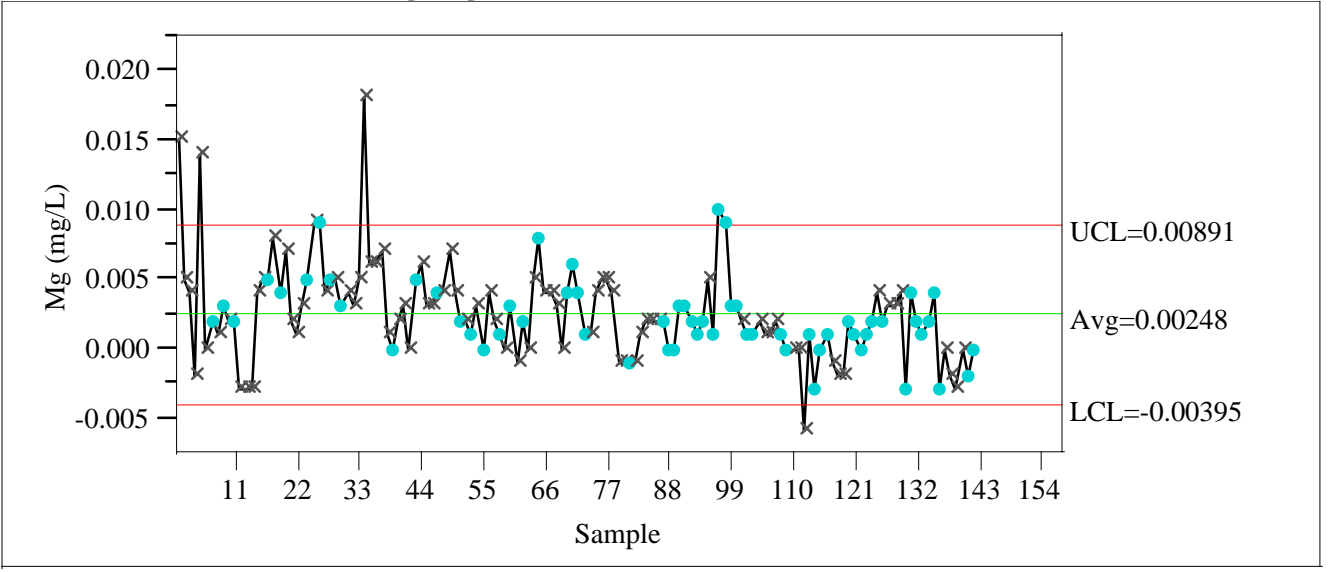


WSRC-TR-2004-00576

\section{Exhibit A1. Cold Chem Standards in Analytical Sequence}

\section{Revision 0}

Individual Measurement of Mn (mg/L)

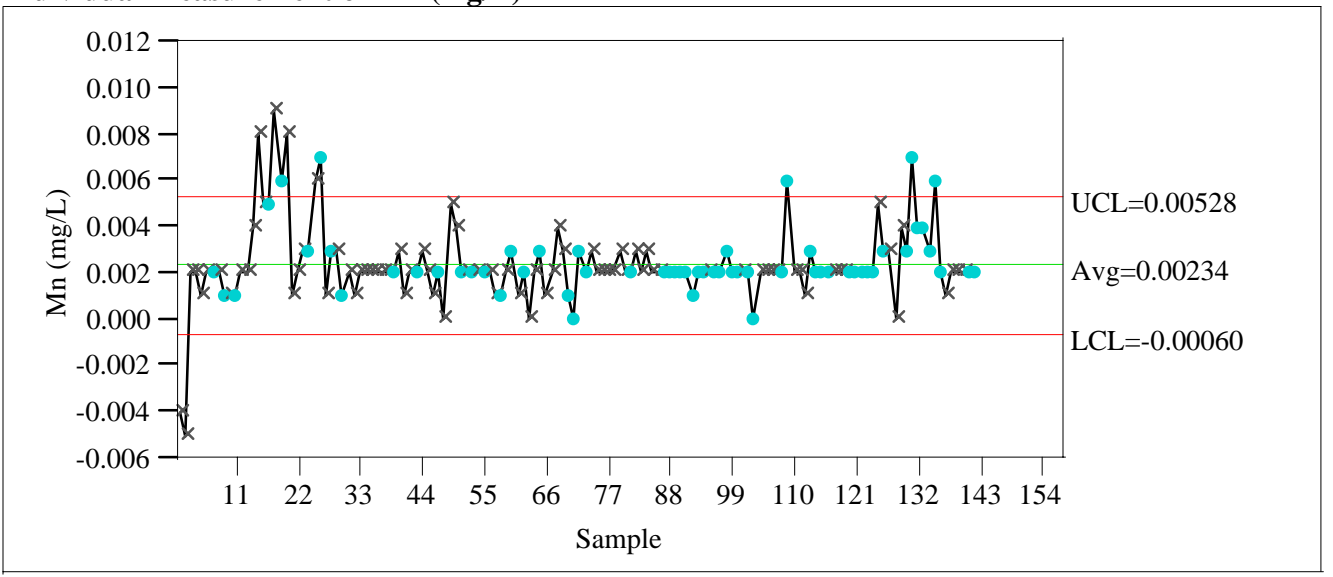

Individual Measurement of $\mathrm{Na}$ (mg/L)

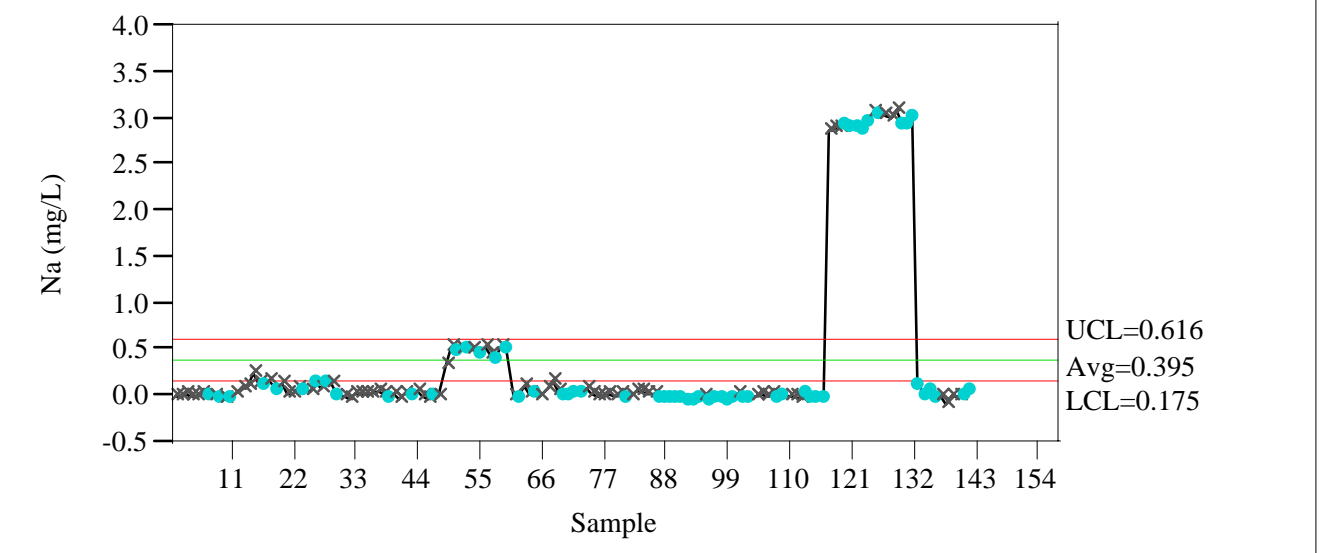

Individual Measurement of $\mathrm{Ni}(\mathrm{mg} / \mathrm{L})$

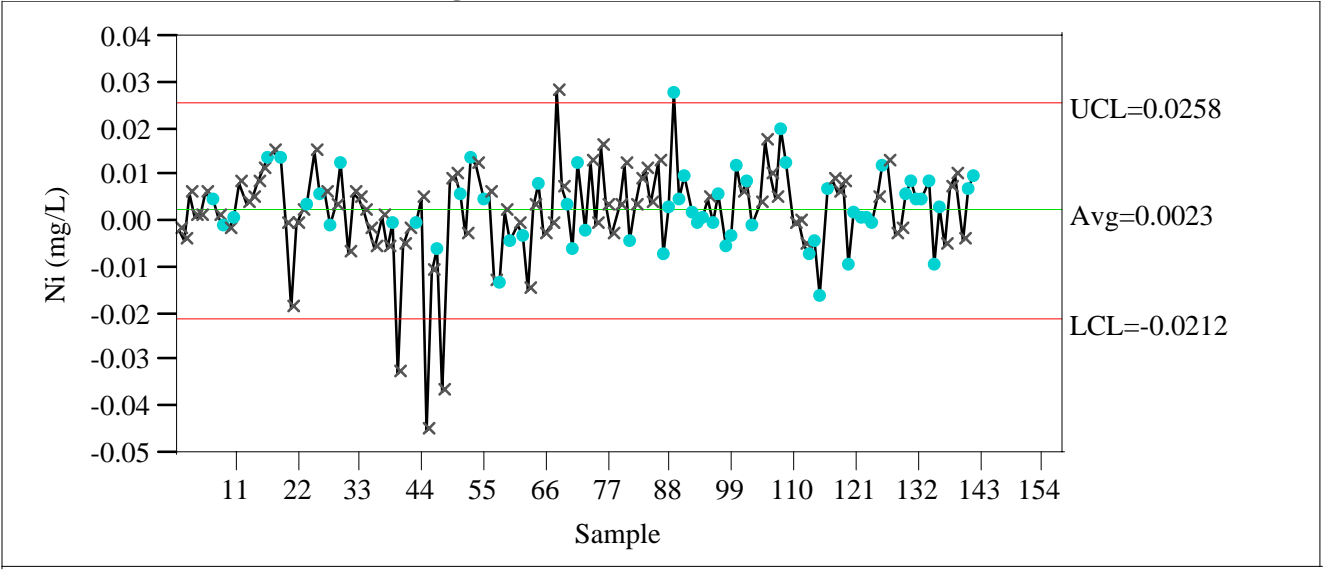




\section{Exhibit A1. Cold Chem Standards in Analytical Sequence}

\section{Revision 0}

Individual Measurement of Si (mg/L)

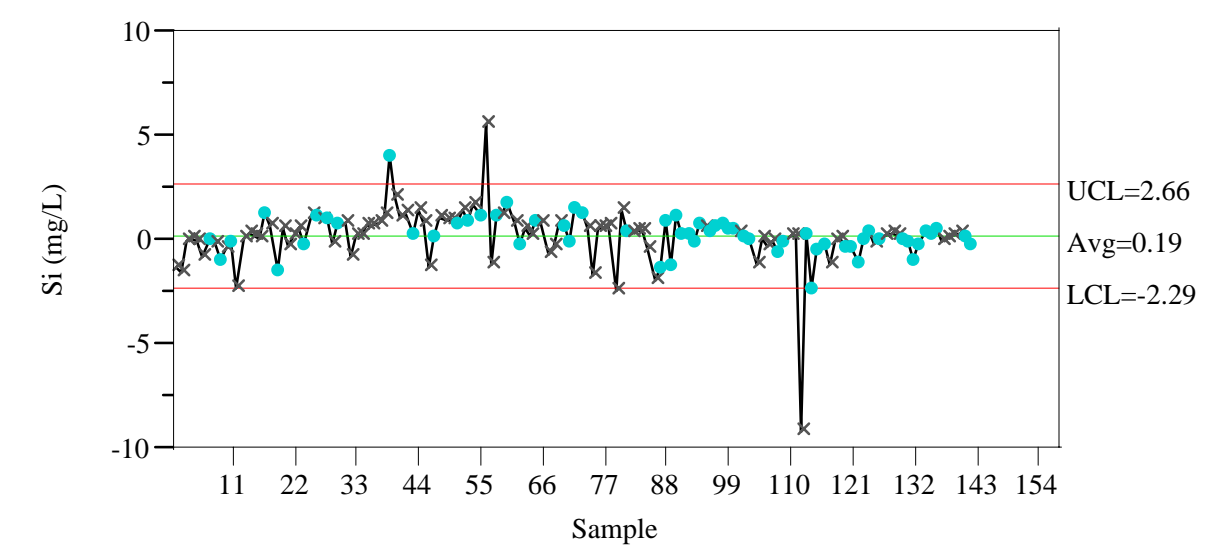

Individual Measurement of Ti (mg/L)

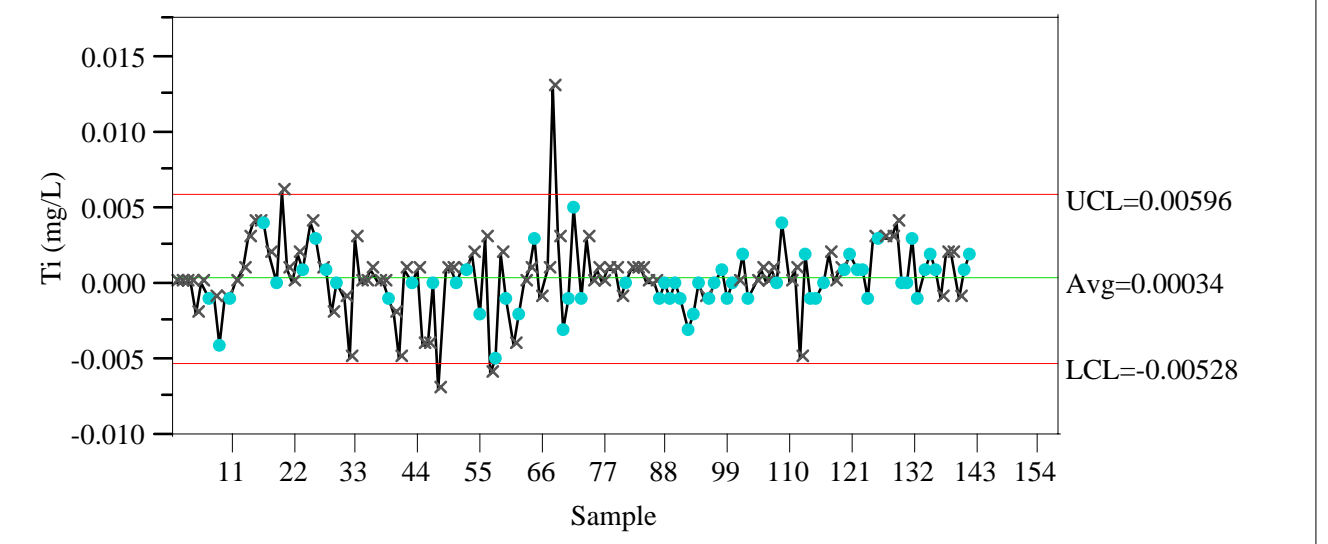

Individual Measurement of $\mathrm{Zr}(\mathrm{mg} / \mathrm{L})$

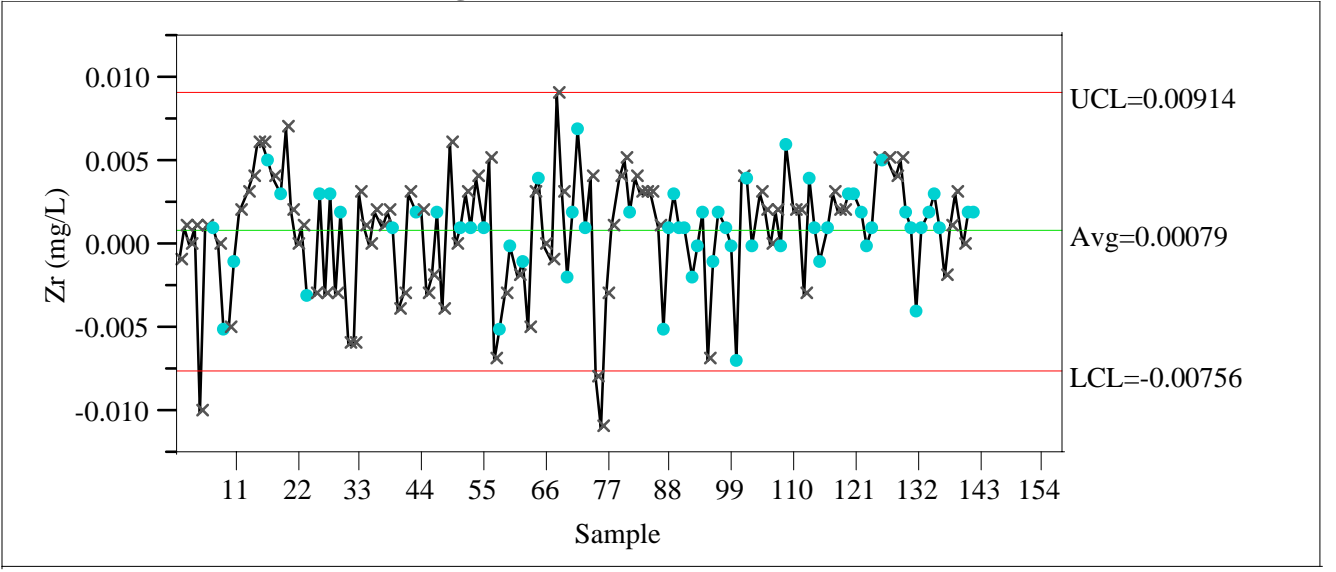


WSRC-TR-2004-00576

\section{Exhibit A1. Cold Chem Standards in Analytical Sequence}

Revision 0

Individual Measurement of U (mg/L)

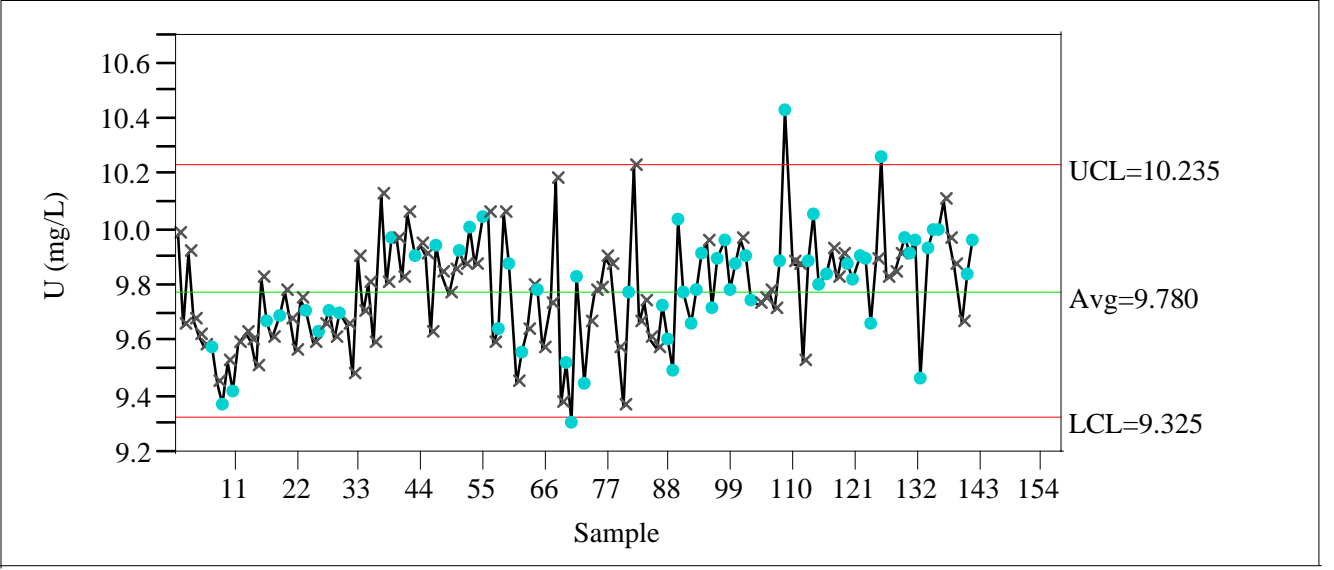


WSRC-TR-2004-00576

Revision 0

\section{Exhibit A2. Histograms and Descriptive Statistics for Cold Chem Measurements of Calibration and Bench Standards}

STCd=IN32

Distributions

Al (mg/L)

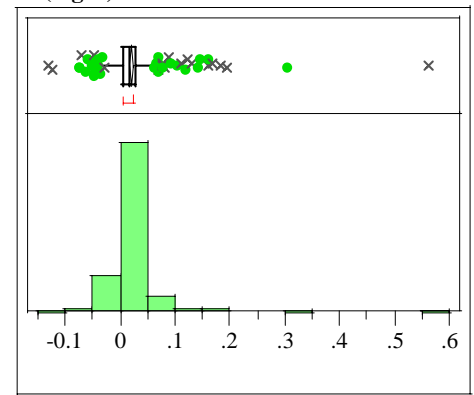

Quantiles

$100.0 \%$ maximum

$99.5 \%$

$97.5 \%$

$90.0 \%$

$75.0 \%$

$50.0 \%$

$25.0 \%$

$10.0 \%$

$2.5 \%$

$0.5 \%$

$0.0 \%$

Moments

Mean

Std Dev

Std Err Mean

upper 95\% Mean

lower 95\% Mean

$\mathrm{N}$

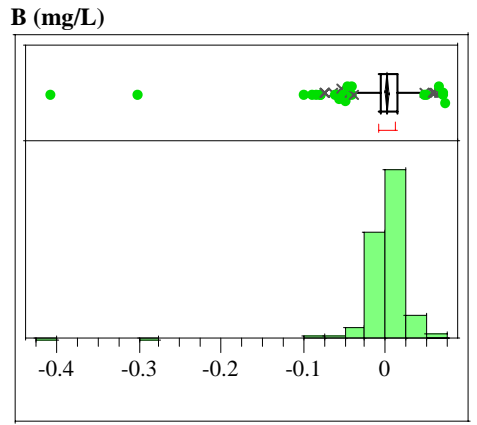

Quantiles

$\begin{array}{ll}100.0 \% & \text { maximum } \\ 99.5 \% & \\ 97.5 \% & \\ 90.0 \% & \\ 75.0 \% & \text { quartile } \\ 50.0 \% & \text { median } \\ 25.0 \% & \text { quartile } \\ 10.0 \% & \\ 2.5 \% & \\ 0.5 \% & \\ 0.0 \% & \text { minimum } \\ \text { Moments } & \\ & \\ \text { Mean } & \\ \text { Std Dev } & \\ \text { Std Err Mean } & \\ \text { upper 95\% Mean } \\ \text { lower 95\% Mean } \\ \text { N }\end{array}$

0.0014231

$-0.0016$
Ca (mg/L)

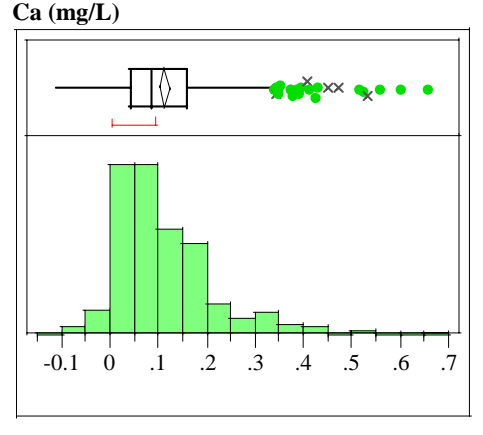

Quantiles

$100.0 \%$ maximum 0.6590

$99.5 \% \quad 0.5850$

$97.5 \%$

$90.0 \%$

$75.0 \%$

$50.0 \%$

$25.0 \%$

$10.0 \%$

$2.5 \%$

$0.5 \%$

$0.0 \% \quad$ minimum

Moments

Mean

Std Dev

Std Err Mean

upper 95\% Mean

lower 95\% Mean

$\mathrm{N}$

Cr (mg/L)

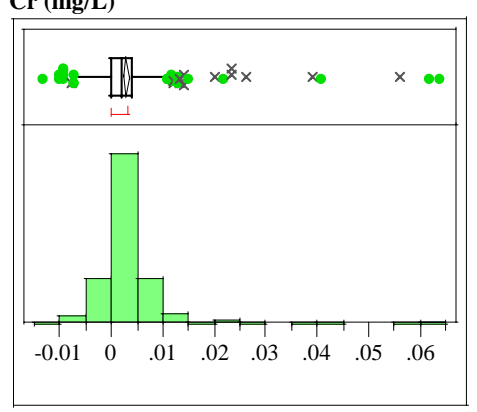

Quantiles

$100.0 \%$

$99.5 \%$

$97.5 \%$

$90.0 \%$

$75.0 \%$

$50.0 \%$

$25.0 \%$

$10.0 \%$

$2.5 \%$

$0.5 \%$

$0.0 \%$

Moments

minimum

Mean

Std Dev

Std Err Mean

upper 95\% Mean

lower 95\% Mean

$\mathrm{N}$

$-0.0254$

$-0.0956$

0.1075139

0.004887

0.1229846

0.1037798

0.0000

-0.0020
-0.0059

$-0.0100$

$-0.0130$
0.0003052

0.0034923

0.0022928
Cu (mg/L)

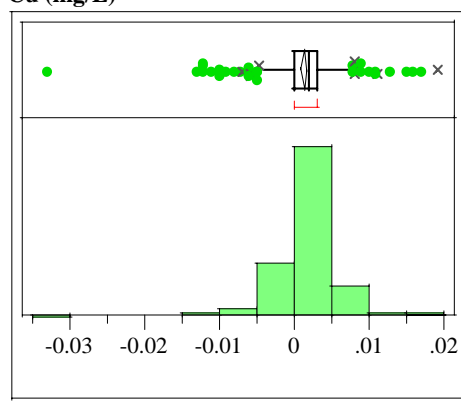

Quantiles

maximum

0.0190

$99.5 \%$

$97.5 \%$

$90.0 \%$

$75.0 \%$

$50.0 \%$

$25.0 \%$

$10.0 \%$

$2.5 \%$

$0.5 \%$

$0.0 \%$

Moments

quartile

median

quartile

Mean

Std Dev

Std Err Mean

upper 95\% Mean

lower 95\% Mean

484

Fe (mg/L)

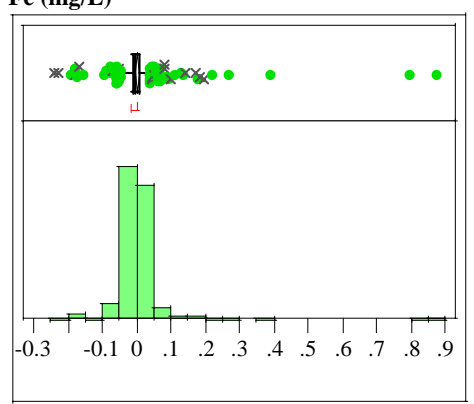

Quantiles

$100.0 \%$

maximum

0.8790

$99.5 \%$

$97.5 \%$

$90.0 \%$

$75.0 \%$

$50.0 \%$

$25.0 \%$

$10.0 \%$

$2.5 \%$

$0.5 \%$

$0.0 \%$

Moments

Mean

Std Dev

Std Err Mean

upper 95\% Mean

lower 95\% Mean

$\mathrm{N}$
0.0166

0.0080

0.0050

0.0030

0.0020

0.0000

$-0.0030$

$-0.0070$

$-0.0126$

$-0.0330$

0.0012934 0.0040104 0.0001823 0.0016516 0.0009352

484 quartile

median

quartile

0.6273

0.1293

0.0360

0.0090

0.0090
-0.0020

$-0.0108$

$-0.0400$

$-0.0872$

$-0.2130$

$-0.2430$

minimum

0.0030909

0.0730918

0.0033224

0.009619

$-0.003437$

484 
WSRC-TR-2004-00576

Revision 0

\section{Exhibit A2. Histograms and Descriptive Statistics for Cold Chem Measurements of Calibration and Bench Standards}

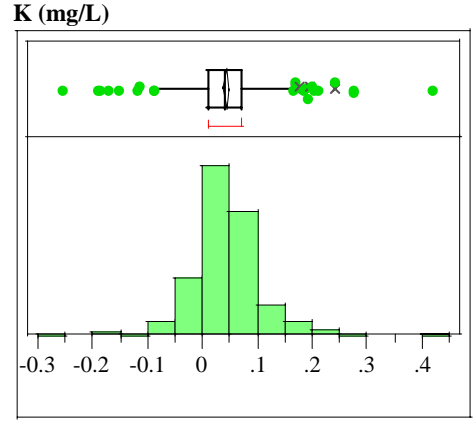

Quantiles

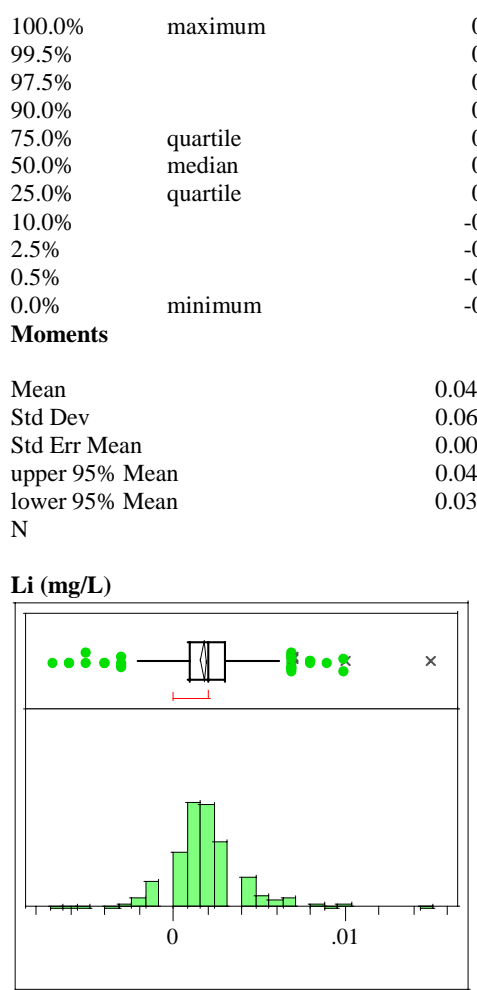

Quantiles

$100.0 \% \quad$ maximum

$99.5 \%$

$97.5 \%$

$90.0 \%$

$75.0 \%$

$50.0 \%$

$25.0 \%$

$10.0 \%$

$2.5 \%$

$0.5 \%$

$0.0 \%$
Moments

minimum

Mean

Std Dev

Std Err Mean

upper 95\% Mean

lower 95\% Mean

$\mathrm{N}$
$\operatorname{Mg}(\mathrm{mg} / \mathrm{L})$

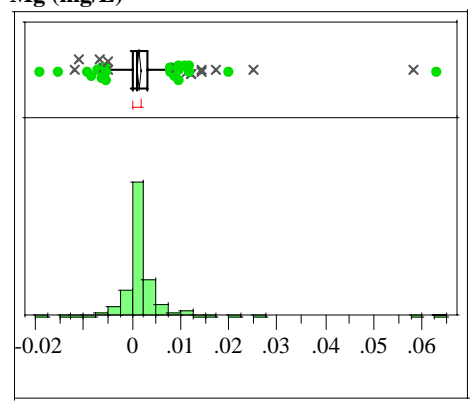

Quantiles

$100.0 \%$

$99.5 \%$

$97.5 \%$

$90.0 \%$

$75.0 \%$

$50.0 \%$

$25.0 \%$

$10.0 \%$

$2.5 \%$

$0.5 \%$

$0.0 \%$

Moments

maximum

Mean

Std Dev

Std Err Mean

upper 95\% Mean

lower 95\% Mean

$\mathrm{N}$

Mn (mg/L)

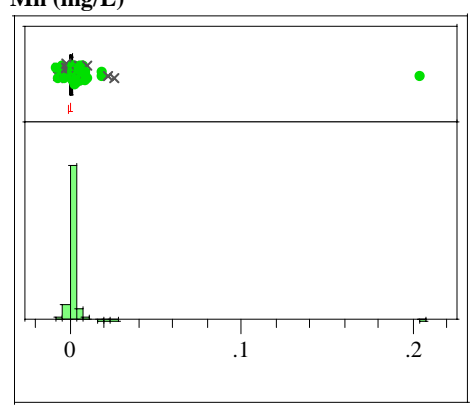

Quantiles

$100.0 \%$ maximum

$99.5 \%$

$97.5 \%$

$90.0 \%$

$75.0 \%$

$50.0 \%$

$25.0 \%$

$10.0 \%$

$2.5 \%$

$0.5 \%$

$0.0 \%$ minimum

Moments

quartile

median
quartile

Mean

Std Dev

Std Err Mean

upper 95\% Mean

lower 95\% Mean

$\mathrm{N}$

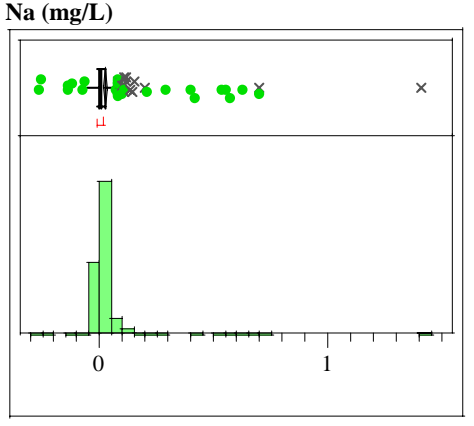

Quantiles

$100.0 \%$

maximum

1.404

$99.5 \%$

$97.5 \%$

$90.0 \%$

$75.0 \%$

$50.0 \%$

$25.0 \%$

$10.0 \%$

$2.5 \%$

$0.5 \%$

$0.0 \%$

Moments

quartile

median

quartile

$-0.0137$

$-0.0190$

Mean

Std Dev

Std Err Mean

upper 95\% Mean

lower 95\% Mean

0.0002384

0.0020594

0.0011224

$\mathrm{N}$

Ni (mg/L)

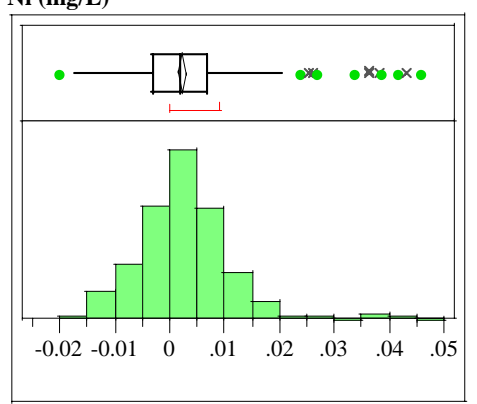

Quantiles

$100.0 \%$

0.2050

0.0237

0.0090

0.0030

0.0010

0.0000

0.0000

0.0000

$-0.0039$

$-0.0070$

$99.5 \%$

$97.5 \%$

$90.0 \%$

$75.0 \%$

$50.0 \%$

$25.0 \%$

$10.0 \%$

$2.5 \%$

$0.5 \%$

$0.0 \%$

quartile

median

quartile

Moments

minimum

Mean

Std Dev

Std Err Mean

upper 95\% Mean

lower 95\% Mean

$\mathrm{N}$
0.0460

0.0426

0.0235

0.0115

0.0070

0.0020

$-0.0030$

$-0.0080$

$-0.0120$

$-0.0157$

$-0.0200$

0.00225

0.0087749

0.0003989

0.0030337

0.0014663 
WSRC-TR-2004-00576

Revision 0

\section{Exhibit A2. Histograms and Descriptive Statistics for Cold Chem Measurements of Calibration and Bench Standards}

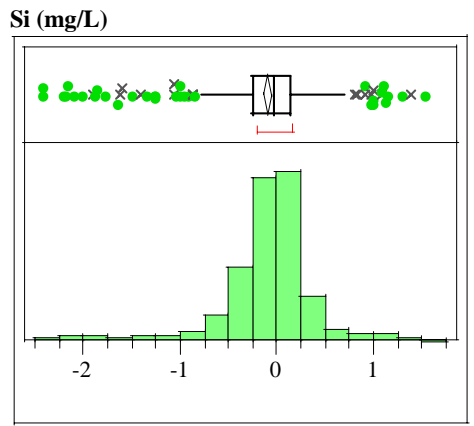

Quantiles

$99.5 \%$

$97.5 \%$

$90.0 \%$

$75.0 \%$

$50.0 \%$

$25.0 \%$

$10.0 \%$

$2.5 \%$

$0.5 \%$

$0.0 \%$

Moments

Mean

Std Dev

Std Err Mean

upper 95\% Mean

lower 95\% Mean

$\mathrm{N}$

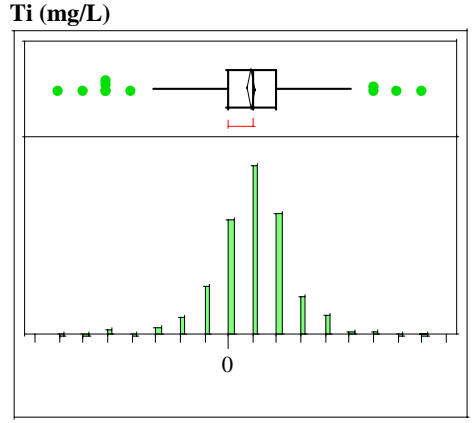

Quantiles

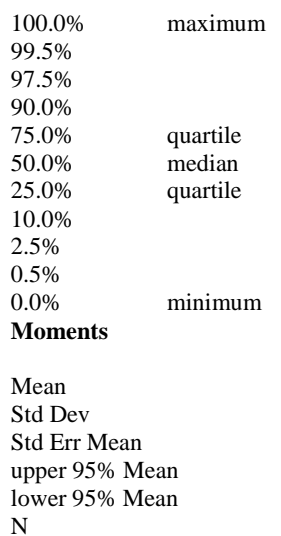

Zr (mg/L)

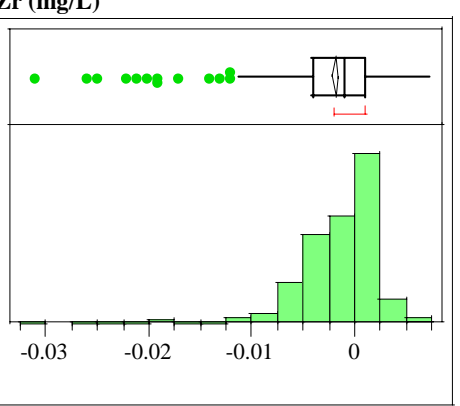

Quantiles

$100.0 \%$

$99.5 \%$

$97.5 \%$

$90.0 \%$

$75.0 \%$

$50.0 \%$

$25.0 \%$

$10.0 \%$

$2.5 \%$

$0.5 \%$

$0.0 \%$

Moments

maximum

quartile

median

quartile

minimum

Mean

Std Dev

Std Err Mean

upper 95\% Mean

lower 95\% Mean

$\mathrm{N}$

$\mathbf{U}(\mathrm{mg} / \mathrm{L})$

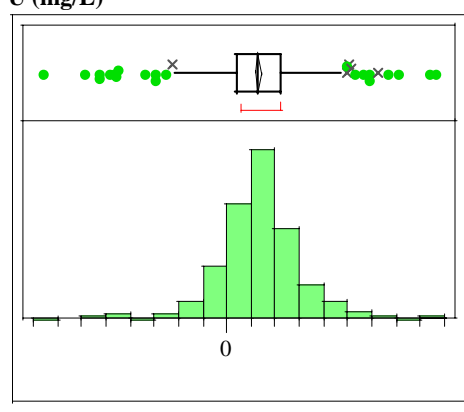

Quantiles

$\begin{array}{ll}100.0 \% & \text { maximum } \\ 99.5 \% & \\ 97.5 \% & \\ 90.0 \% & \\ 75.0 \% & \text { quartile } \\ 50.0 \% & \text { median } \\ 25.0 \% & \text { quartile } \\ 10.0 \% & \\ 2.5 \% & \\ 0.5 \% & \\ 0.0 \% & \text { minimum } \\ \text { Moments } & \\ & \\ \text { Mean } & \\ \text { Std Dev } & \\ \text { Std Err Mean } & \\ \text { upper 95\% Mean } \\ \text { lower 95\% Mean } \\ \text { N }\end{array}$

STCd=IN33

Distributions

Al (mg/L)

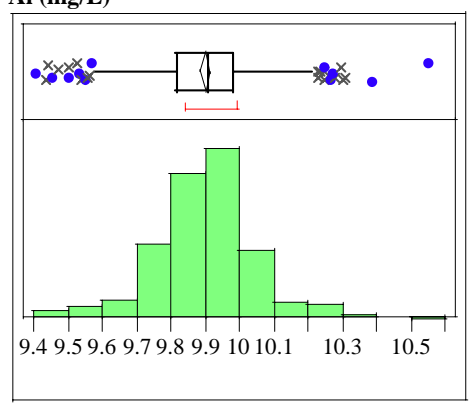

Quantiles

$100.0 \%$ maximum $\quad 10.557$

$99.5 \% \quad 10.376$

$97.5 \%$

$90.0 \%$

$75.0 \%$

$50.0 \%$

$25.0 \%$

$10.0 \%$

$2.5 \%$

$0.5 \%$

$0.0 \%$

Moments

quartile

median

quartile

9.981

9.819

9.734

9.550

9.437

9.409

Mean

Std Dev

Std Err Mean

upper $95 \%$ Mean

lower $95 \%$ Mean

minimum

9.9006157

0.1478098

0.0071115

9.9145933

$-0.001491 \quad$

$484 \quad \mathrm{~N}$

432

B (mg/L)

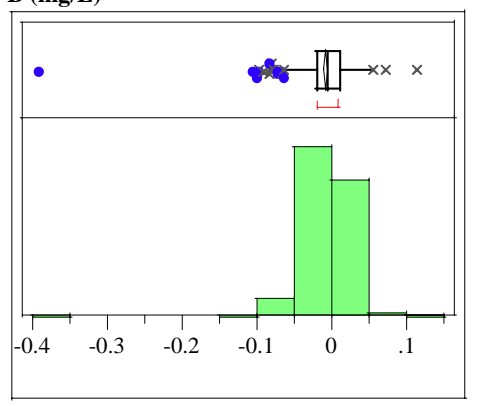

Quantiles

$\begin{array}{llr}100.0 \% & \text { maximum } & 0.1120 \\ 99.5 \% & & 0.0674 \\ 97.5 \% & & 0.0392 \\ 90.0 \% & & 0.0220 \\ 75.0 \% & \text { quartile } & 0.0100 \\ 50.0 \% & \text { median } & -0.0050 \\ 25.0 \% & \text { quartile } & -0.0190 \\ 10.0 \% & & -0.0357 \\ 2.5 \% & & -0.0820 \\ 0.5 \% & & -0.1042 \\ 0.0 \% & \text { minimum } & -0.3890 \\ \text { Moments } & & \\ & & -0.00762 \\ \text { Mean } & & 0.0317911 \\ \text { Std Dev } & & 0.0015295 \\ \text { Std Err Mean } & & -0.004614 \\ \text { upper 95\% Mean } & -0.010627 \\ \text { lower 95\% Mean } & 432 \\ \text { N } & \end{array}$


WSRC-TR-2004-00576

Revision 0

\section{Exhibit A2. Histograms and Descriptive Statistics for Cold Chem Measurements of} Calibration and Bench Standards

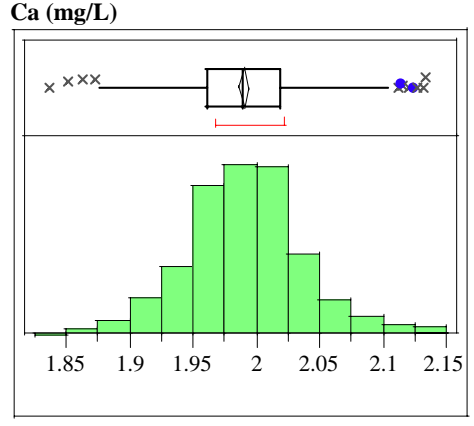

Quantiles

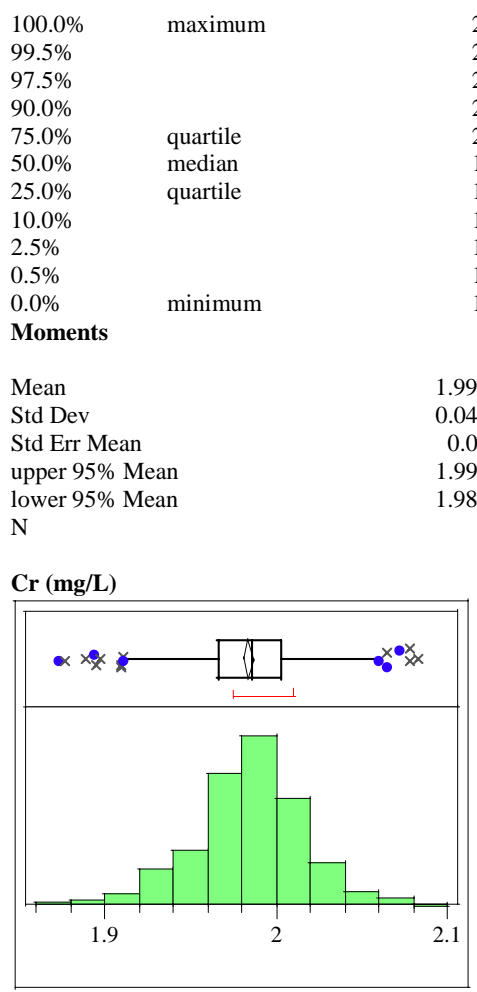

Quantiles

$100.0 \%$

$99.5 \%$

$97.5 \%$

$90.0 \%$

$75.0 \%$

$50.0 \%$

$25.0 \%$

$10.0 \%$

$2.5 \%$

$0.5 \%$

$0.0 \%$

minimum

Mean

Std Dev

Std Err Mean

upper $95 \%$ Mean

lower 95\% Mean

$\mathrm{N}$
Cu (mg/L)

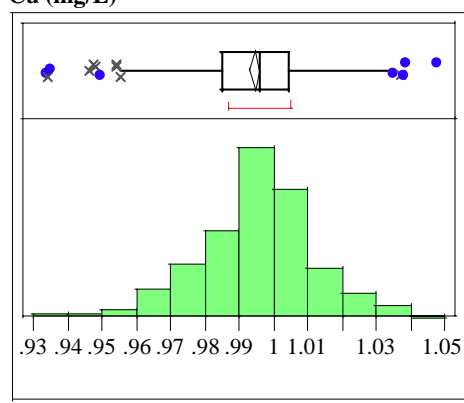

Quantiles

$99.5 \%$

$97.5 \%$

$90.0 \%$

$75.0 \%$

$50.0 \%$

$25.0 \%$

$10.0 \%$

$2.5 \%$

$0.5 \%$

$0.0 \%$

Moments

maximum

Mean

Std Dev

Std Err Mean

upper 95\% Mean

lower 95\% Mean

$\mathrm{N}$

Fe (mg/L)

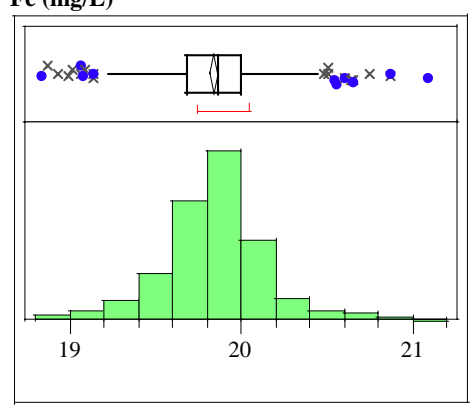

Quantiles

$100.0 \%$ maximum

$99.5 \%$

$97.5 \%$

$90.0 \%$

$75.0 \%$

$50.0 \%$

$25.0 \%$

$10.0 \%$

$2.5 \%$

$0.5 \%$

$0.0 \%$ minimum

Moments

quartile

median

quartile

1.9128

1.8780

1.9839051

0.0323171

0.0015549

1.9869611

1.9808491

432
Mean

Std Dev

Std Err Mean

upper 95\% Mean

lower 95\% Mean

$\mathrm{N}$
K (mg/L)

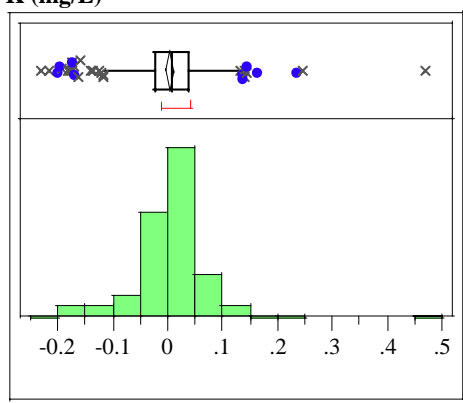

Quantiles

$100.0 \%$

maximum

0.4660

$99.5 \%$

$97.5 \%$

$90.0 \%$

$75.0 \%$

$50.0 \%$

$25.0 \%$

$10.0 \%$

$2.5 \%$

$0.5 \%$

$0.0 \%$

Moments

quartile

median

quartile

Mean

Std Dev

Std Err Mean

upper 95\% Mean

lower 95\% Mean

0.0008226

0.9962372

0.9930036

$\mathrm{N}$

Li (mg/L)

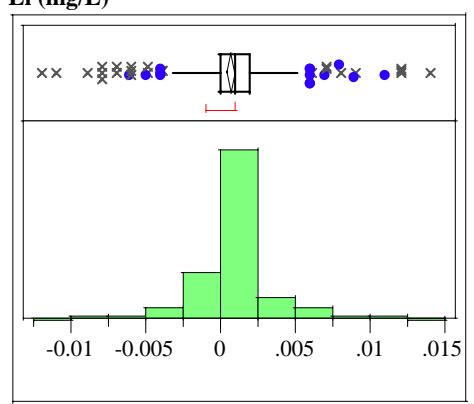

Quantiles

$100.0 \%$ maximum 0.0140

$99.5 \% \quad 0.0120$

$97.5 \%$

$75.0 \%$

$50.0 \%$

$25.0 \%$

$10.0 \%$

$2.5 \%$

$0.5 \%$

$0.0 \%$

minimum

0.0640

0.0367

0.0090

$-0.0240$

$-0.0617$

$-0.1722$

$-0.2129$

$-0.2340$

0.0040926

0.0644453

0.0031006

0.0101868

$-0.002002$

432

Moments

quartile

median

quartile

0.0070

0.0030

0.0020

0.0020
0.0010

0.0010
0.0000

$-0.0020$

$-0.0060$

$-0.0107$

Mean

minimum

$-0.0120$

19.842933

0.3027379

0.0145655

19.871561

Std Dev

0.0006597

Std Err Mean

upper 95\% Mean

lower 95\% Mean

0.0027783

0.0001337

0.0009225

$\mathrm{N}$

432 
WSRC-TR-2004-00576

Revision 0

\section{Exhibit A2. Histograms and Descriptive Statistics for Cold Chem Measurements of Calibration and Bench Standards}

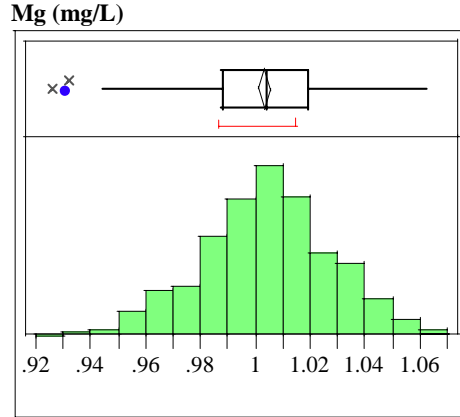

Quantiles

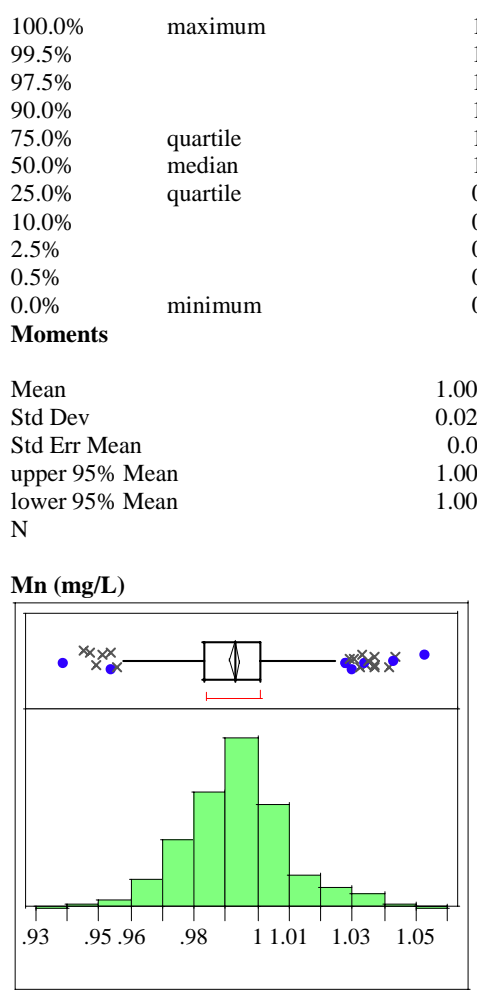

Quantiles

$100.0 \%$

$99.5 \%$

$97.5 \%$

$90.0 \%$

$75.0 \%$

$50.0 \%$

$25.0 \%$

$10.0 \%$

$2.5 \%$

$0.5 \%$

$0.0 \%$

maximum

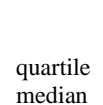

median

quartile

minimum

Mean

Std Dev

Std Err Mean

upper $95 \%$ Mean

lower 95\% Mea

$\mathrm{N}$
Na (mg/L)

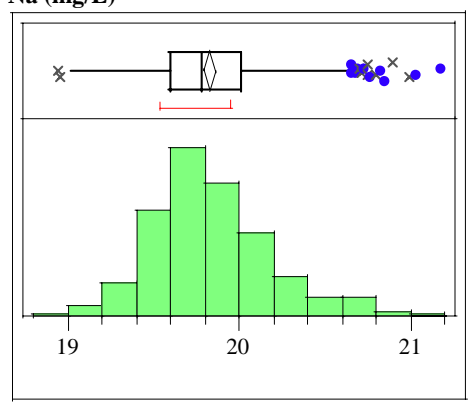

Quantiles

$100.0 \%$

$99.5 \%$

$97.5 \%$

$90.0 \%$

$75.0 \%$

$50.0 \%$

$25.0 \%$

$10.0 \%$

$2.5 \%$

$0.5 \%$

$0.0 \%$

Moments

maximum

Mean

Std Dev

Std Err Mean

upper 95\% Mean

lower 95\% Mean

$\mathrm{N}$

$\mathrm{Ni}$ (mg/L)

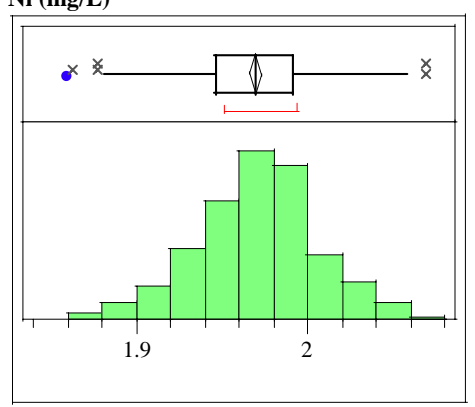

Quantiles

$100.0 \%$ maximum

$99.5 \%$

$97.5 \%$

$90.0 \%$

$75.0 \%$

$50.0 \%$

$25.0 \%$

$10.0 \%$

$2.5 \%$

$0.5 \%$

$0.0 \%$ minimum

Moments

quartile

median

quartile

Mean

Std Dev

Std Err Mean

upper 95\% Mean

lower 95\% Mea

$\mathrm{N}$
Si (mg/L)

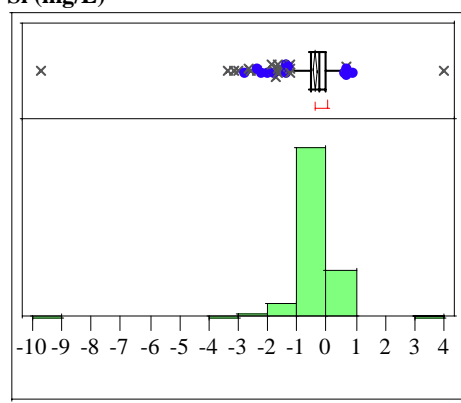

Quantiles

$100.0 \%$

maximum

3.960

$99.5 \%$

$97.5 \%$

$90.0 \%$

$75.0 \%$

$50.0 \%$

$25.0 \%$

$10.0 \%$

$2.5 \%$

$0.5 \%$

$0.0 \%$

Moments

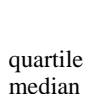

median

quartile

minimum

0.462

0.137

$-0.056$

$-0.274$

$-0.527$

$-0.937$

$-2.017$

$-3.354$

$-9.764$

Mean

Std Dev

Std Err Mean

upper 95\% Mean

lower 95\% Mean

0.3638111

0.0175039

19.866961

19.798154

432

$\mathrm{N}$

$-0.301402$

$-0.441714$

Ti (mg/L)

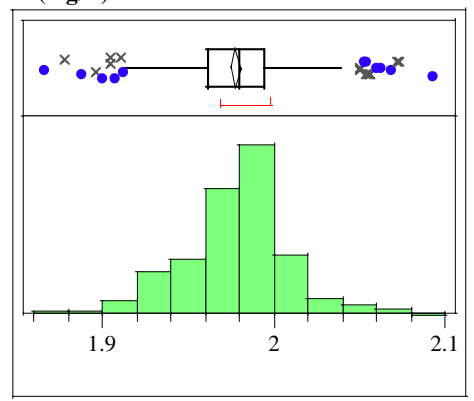

Quantiles

$100.0 \%$ maximum 2.0930

$99.5 \% \quad 2.0727$

$97.5 \%$

$75.0 \%$

$50.0 \%$

$25.0 \%$

$10.0 \%$

$2.5 \%$

$0.5 \%$

$0.0 \%$

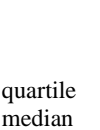

2.0532

2.0120

1.9940

1.9940
1.9800

1.9800
1.9623

1.9363

1.9147

1.8798

Moments

minimum

1.8670

\section{Mean}

Std Dev

Std Err Mean

upper 95\% Mean

lower 95\% Mean

$\mathrm{N}$

1.9781412

0.0312213

0.0015021

1.9810936

1.9751888

432 
WSRC-TR-2004-00576

Revision 0

\section{Exhibit A2. Histograms and Descriptive Statistics for Cold Chem Measurements of Calibration and Bench Standards}

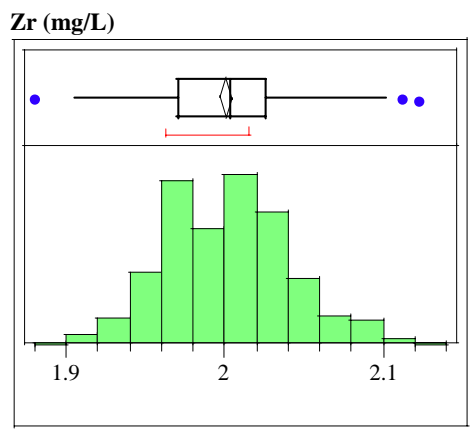

Quantiles

$100.0 \%$

$99.5 \%$

$97.5 \%$

$90.0 \%$

$75.0 \%$

$50.0 \%$

$25.0 \%$

$10.0 \%$

$2.5 \%$

$0.5 \%$

$0.0 \%$

Moments

Mean

Std Dev

Std Err Mean

upper 95\% Mean

lower 95\% Mean

$\mathrm{N}$

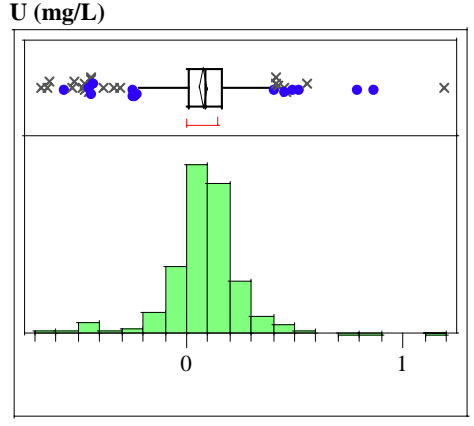

Quantiles

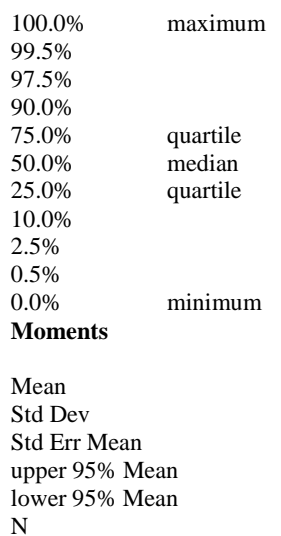

STCd=IN34

Distributions

Al (mg/L)

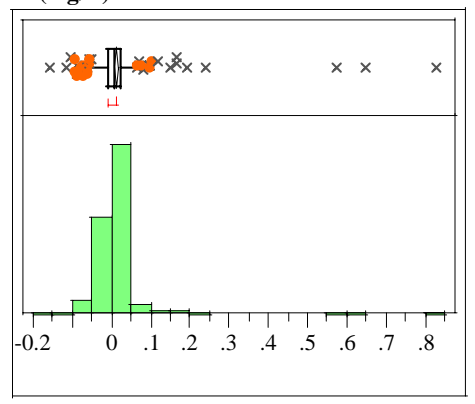

Quantiles

$100.0 \%$ maximum

$99.5 \%$

$97.5 \%$

$90.0 \%$

$75.0 \%$

$50.0 \%$

$25.0 \%$

$10.0 \%$

$2.5 \%$

$0.5 \%$

$0.0 \%$

Moments

minimum

Mean

Std Dev

Std Err Mean

upper $95 \%$ Mean

lower 95\% Mean

$\mathrm{N}$

B (mg/L)

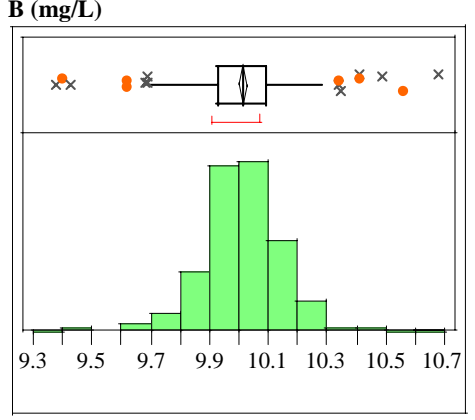

Quantiles

$100.0 \%$

$99.5 \%$

$97.5 \%$

$90.0 \%$

$75.0 \%$

$50.0 \%$

$25.0 \%$

$10.0 \%$

$2.5 \%$

$0.5 \%$

$0.0 \%$

Moments

maximum

Mean

Std Dev

Std Err Mean

upper 95\% Mean

lower 95\% Mean

$\mathrm{N}$
Ca (mg/L)

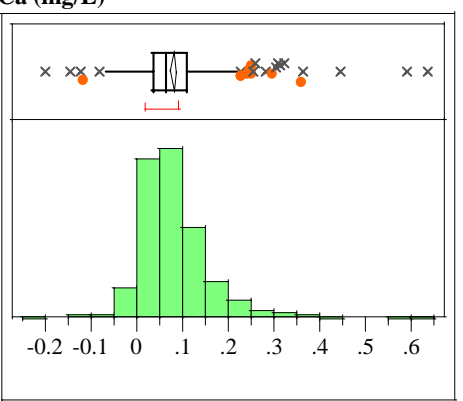

Quantiles

$100.0 \%$ maximum 0.6340

$99.5 \% \quad 0.5735$

$97.5 \% \quad 0.2891$

0.1717

$75.0 \%$ quartile 0.1108

$50.0 \%$ median 0.0640

$25.0 \%$ quartile 0.0343

$10.0 \% \quad 0.0080$

$2.5 \% \quad-0.0354$

$-0.1438$

$0.0 \%$ minimum -0.2020

Moments

Mean

Std Dev

Std Err Mean

upper $95 \%$ Mean

lower $95 \%$ Mean

0.0176927

0.0046978

$\mathrm{N}$

0.0818628

0.0039945

0.0878517

0.0721483

420

$\operatorname{Cr}(\mathrm{mg} / \mathrm{L})$

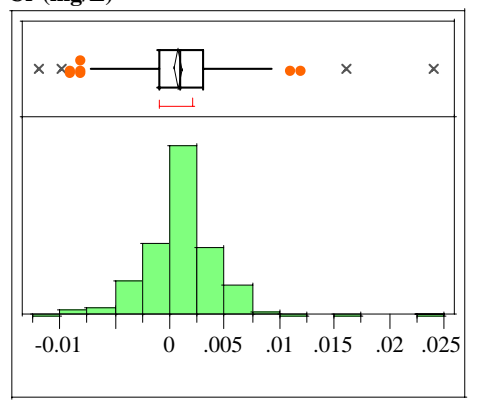

Quantiles

$\begin{array}{llr}100.0 \% & \text { maximum } & 0.0240 \\ 99.5 \% & & 0.0156 \\ 97.5 \% & & 0.0070 \\ 90.0 \% & & 0.0040 \\ 75.0 \% & \text { quartile } & 0.0030 \\ 50.0 \% & \text { median } & 0.0010 \\ 25.0 \% & \text { quartile } & -0.0010 \\ 10.0 \% & & -0.0030 \\ 2.5 \% & & -0.0070 \\ 0.5 \% & & -0.0099 \\ 0.0 \% & \text { minimum } & -0.0120 \\ \text { Moments } & & \\ & & 0.0008 \\ \text { Mean } & & 0.0034334 \\ \text { Std Dev } & & 0.0001675 \\ \text { Std Err Mean } & & 0.0011293 \\ \text { upper 95\% Mean } & 0.0004707 \\ \text { lower 95\% Mean } & 420 \\ \text { N } & \end{array}$


WSRC-TR-2004-00576

Revision 0

\section{Exhibit A2. Histograms and Descriptive Statistics for Cold Chem Measurements of} Calibration and Bench Standards

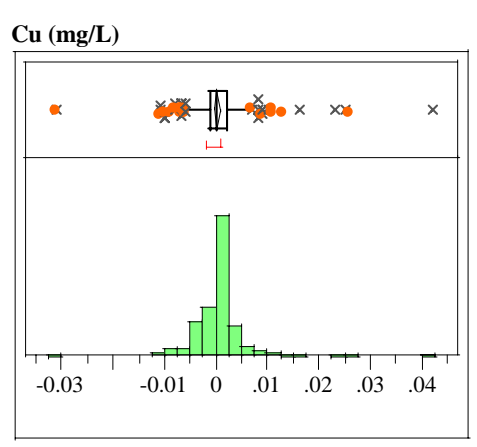

Quantiles

$\begin{array}{llr}100.0 \% & \text { maximum } & 0.0420 \\ 99.5 \% & & 0.0259 \\ 97.5 \% & & 0.0090 \\ 90.0 \% & & 0.0040 \\ 75.0 \% & \text { quartile } & 0.0020 \\ 50.0 \% & \text { median } & 0.0000 \\ 25.0 \% & \text { quartile } & -0.0010 \\ 10.0 \% & & -0.0040 \\ 2.5 \% & & -0.0095 \\ 0.5 \% & & -0.0289 \\ 0.0 \% & \text { minimum } & -0.0310 \\ \text { Moments } & & \\ & & \\ \text { Mean } & & 0.0002833 \\ \text { Std Dev } & & 0.0049694 \\ \text { Std Err Mean } & & 0.0002425 \\ \text { upper 95\% Mean } & 0.00076 \\ \text { lower 95\% Mean } & -0.000193 \\ \text { N } & 420\end{array}$

Fe (mg/L)

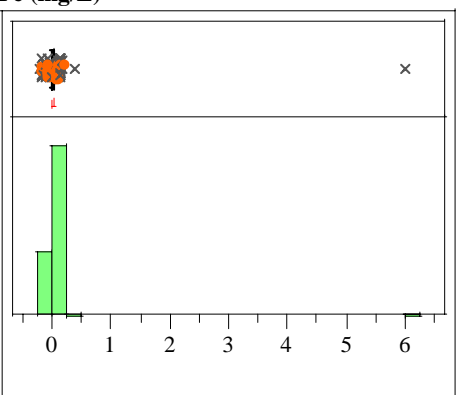

Quantiles

$100.0 \%$

$99.5 \%$

$97.5 \%$

$90.0 \%$

$75.0 \%$

$50.0 \%$

$25.0 \%$

$10.0 \%$

$2.5 \%$

$0.5 \%$

$0.0 \%$

Moments

minimum

quartile

median

quartile

Mean

Std Dev

Std Err Mean

upper 95\% Mean

lower 95\% Mean

$\mathrm{N}$
K (mg/L)

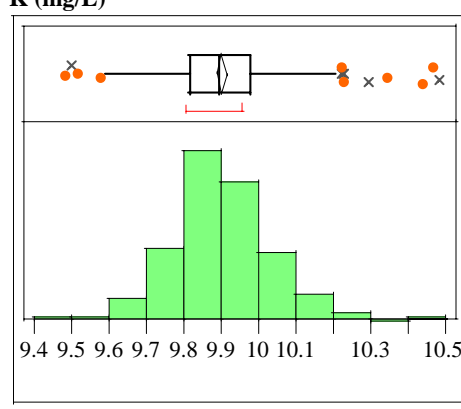

Quantiles

$99.5 \%$

$97.5 \%$

$90.0 \%$

$75.0 \%$

$50.0 \%$

$25.0 \%$

$10.0 \%$

$2.5 \%$

$0.5 \%$

$0.0 \%$

Moments

Mean

Std Dev

Std Err Mean

upper 95\% Mean

lower 95\% Mean

N

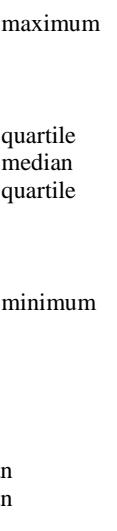

Li (mg/L)

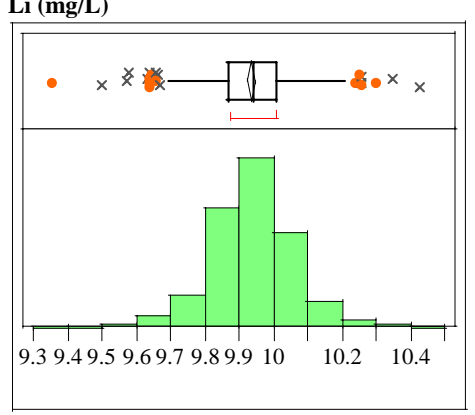

Quantiles

$100.0 \%$ maximum

$99.5 \%$

$97.5 \%$

$90.0 \%$

$75.0 \%$

$50.0 \%$

$25.0 \%$

$10.0 \%$

$2.5 \%$

$0.5 \%$

$0.0 \%$ minimum

Moments

Mean

Std Dev

Std Err Mean

upper 95\% Mean

lower 95\% Mean

$\mathrm{N}$
$\operatorname{Mg}(\mathrm{mg} / \mathrm{L})$

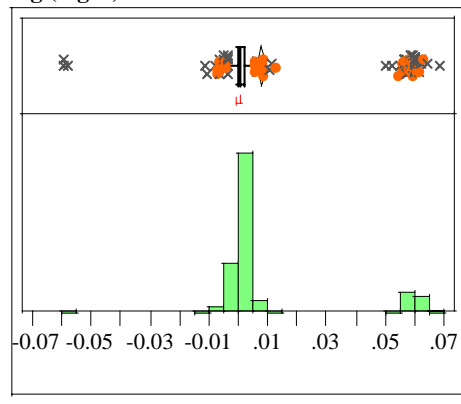

Quantiles

$100.0 \%$

$99.5 \%$

$97.5 \%$

$90.0 \%$

$75.0 \%$

$50.0 \%$

$25.0 \%$

$10.0 \%$

$2.5 \%$

$0.5 \%$

$0.0 \%$

Moments

maximum

0.0680

Mean

Std Dev

Std Err Mean

upper $95 \%$ Mean

lower 95\% Mean

9.9144707

9.8881436

$\mathrm{N}$

Mn (mg/L)

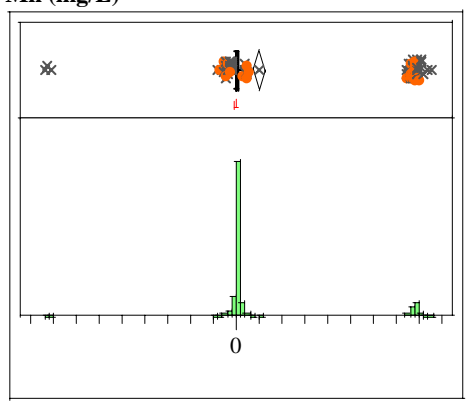

Quantiles

$100.0 \%$ maximum 0.0850

$99.5 \% \quad 0.0828$

$90.0 \%$

$75.0 \%$ quartile

$50.0 \%$ median

$25.0 \%$

$10.0 \%$

$2.5 \%$

$0.5 \%$

$0.0 \%$

quartile

median

quartile

minimum

0.0080881

0.021038

0.0010265

0.0101059

420

Moments

minimum

0.0790

0.0770

0.0010

0.0010
0.0000

0.0000
0.0000

$-0.0010$

$-0.0050$

$-0.0828$

$-0.0840$

Mean

Std Dev

Std Err Mean

upper 95\% Mean

lower 95\% Mean

$\mathrm{N}$
0.0099238

0.0276642

0.0013499

0.0125772

0.0072704 
WSRC-TR-2004-00576

Revision 0

\section{Exhibit A2. Histograms and Descriptive Statistics for Cold Chem Measurements of} Calibration and Bench Standards

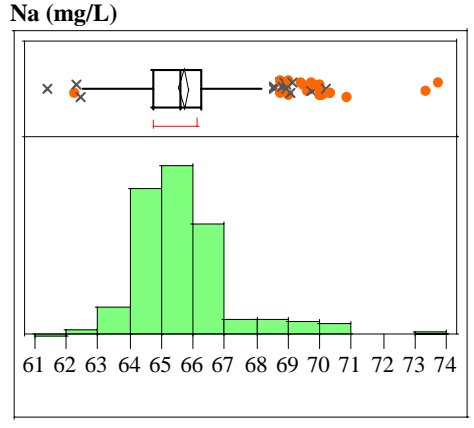

Quantiles

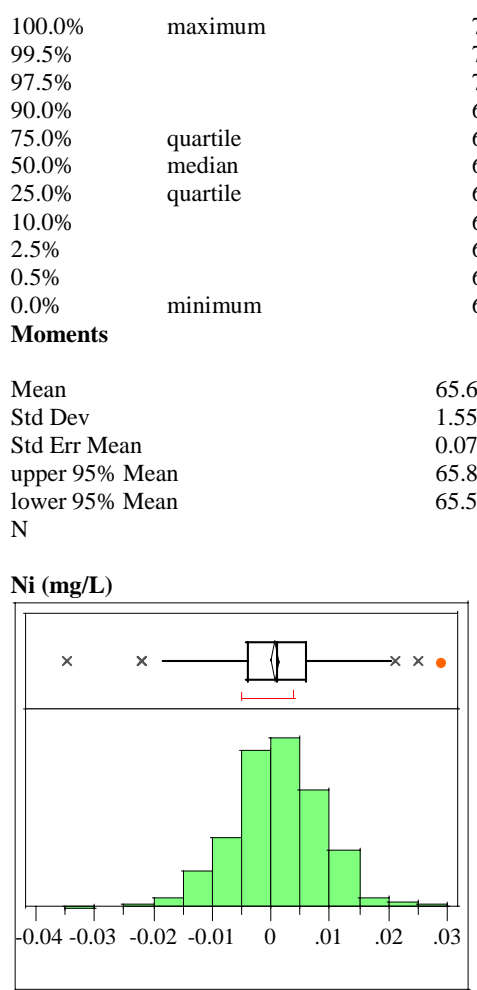

Quantiles

$100.0 \%$

$99.5 \%$

$97.5 \%$

$90.0 \%$

$75.0 \%$

$50.0 \%$

$25.0 \%$

$10.0 \%$

$2.5 \%$

$0.5 \%$

$0.0 \%$
Moments

maximum

quartile

median

quartile

minimum

Mean

Std Dev

Std Err Mean

upper 95\% Mean

lower 95\% Mean

$\mathrm{N}$
Si (mg/L)

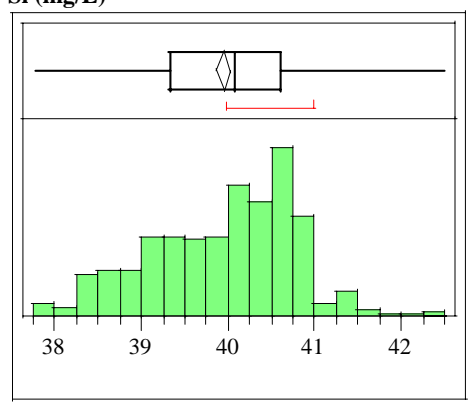

Quantiles

$100.0 \%$

$99.5 \%$

$97.5 \%$

$90.0 \%$

$75.0 \%$

$50.0 \%$

$25.0 \%$

$10.0 \%$

$2.5 \%$

$0.5 \%$

$0.0 \%$

Moments

Mean

Std Dev

Std Err Mean

upper 95\% Mean

lower 95\% Mean

$\mathrm{N}$

Ti (mg/L)

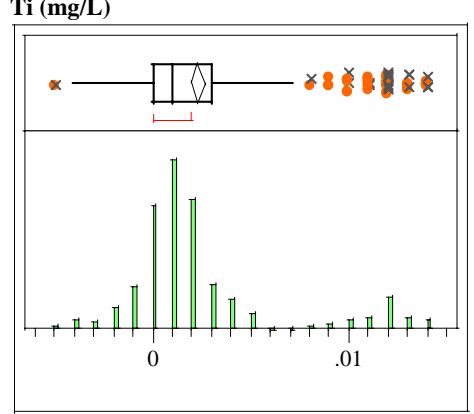

Quantiles

$100.0 \%$ maximum

$99.5 \%$

$97.5 \%$

$90.0 \%$

$75.0 \%$

$50.0 \%$

$25.0 \%$

$10.0 \%$

$2.5 \%$

$0.5 \%$

$0.0 \%$ minimum

Moments

quartile

median

quartile

Mean

Std Dev

Std Err Mean

upper 95\% Mean

lower 95\% Mean

$\mathrm{N}$
Zr (mg/L)

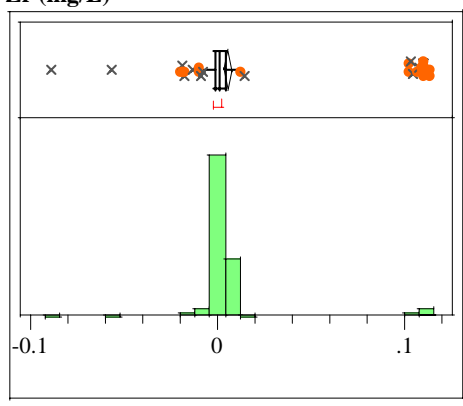

Quantiles

$100.0 \%$

maximum

0.1140

$99.5 \%$

$97.5 \%$

$90.0 \%$

$75.0 \%$

$50.0 \%$

$25.0 \%$

$10.0 \%$

$2.5 \%$

$0.5 \%$

$0.0 \%$

Moments

quartile

median

quartile

Mean

Std Dev

Std Err Mean

upper $95 \%$ Mean

lower 95\% Mean

40.029723

39.867382

420

$\mathbf{U}(\mathbf{m g} / \mathrm{L})$

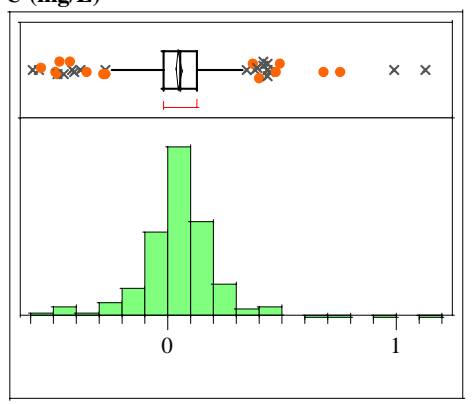

Quantiles

$100.0 \%$ maximum $\quad 1.126$

$99.5 \% \quad 0.966$

$97.5 \%$

$75.0 \%$

$50.0 \%$

$25.0 \%$

$10.0 \%$

$2.5 \%$

$0.5 \%$

$0.0 \%$

minimum

0.1095

0.0090
0.0040

0.0010

$-0.0008$

-0.0008
-0.0030

$-0.0100$

$-0.0531$

$-0.0900$

0.0059548 0.0234382 0.0011437 0.0082028 0.0037067

420
0.0023238

0.003885

0.0001896

0.0026964

0.0019512

\section{Mean}

Std Dev

Std Err Mean

upper 95\% Mean

N
0.966
0.423

0.214

0.124

0.050

$-0.022$

$-0.142$

$-0.418$

$-0.564$

$-0.593$

0.049494

0.1781261

0.008702

0.0665992

0.0323888 
WSRC-TR-2004-00576

Revision 0

\section{Exhibit A2. Histograms and Descriptive Statistics for Cold Chem Measurements of} Calibration and Bench Standards

STCd=IN35

Distributions

Al (mg/L)

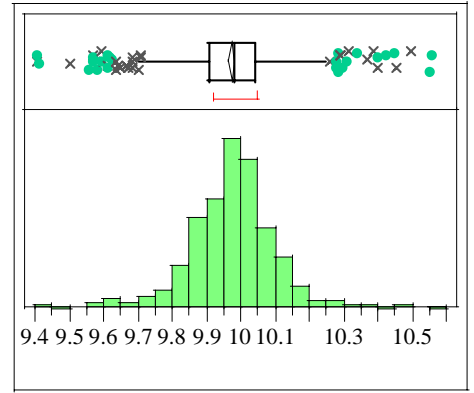

Quantiles

$99.5 \%$

$97.5 \%$

$90.0 \%$

$75.0 \%$

$50.0 \%$

$25.0 \%$

$10.0 \%$

$2.5 \%$

$0.5 \%$

$0.0 \%$

Moments

Mean

Std Dev

Std Err Mean

upper 95\% Mean

lower 95\% Mean

$\mathrm{N}$

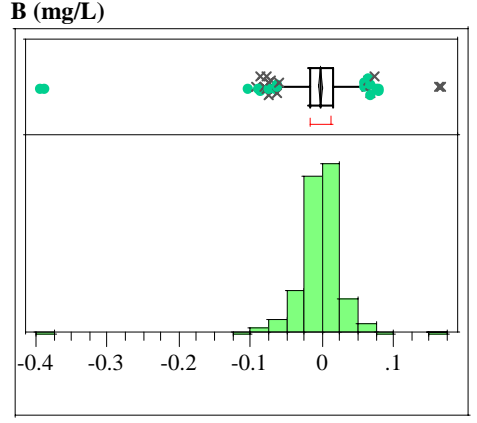

Quantiles

$\begin{array}{ll}100.0 \% & \text { maximum } \\ 99.5 \% & \\ 97.5 \% & \\ 90.0 \% & \\ 75.0 \% & \text { quartile } \\ 50.0 \% & \text { median } \\ 25.0 \% & \text { quartile } \\ 10.0 \% & \\ 2.5 \% & \\ 0.5 \% & \\ 0.0 \% & \text { minimum } \\ \text { Moments } & \\ & \\ \text { Mean } & \\ \text { Std Dev } & \\ \text { Std Err Mean } & \\ \text { upper 95\% Mean } \\ \text { lower 95\% Mean } \\ \text { N }\end{array}$

Ca (mg/L)

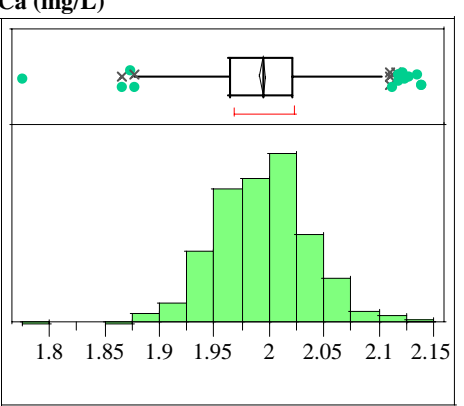

Quantiles

$100.0 \%$ maximum

$99.5 \%$

$97.5 \%$

$90.0 \%$

$75.0 \%$

$50.0 \%$

$25.0 \%$

$10.0 \%$

$2.5 \%$

$0.5 \%$

$0.0 \%$ minimum

Moments

Mean

Std Dev

Std Err Mean

upper 95\% Mean

lower 95\% Mean

$\mathrm{N}$

Cr (mg/L)

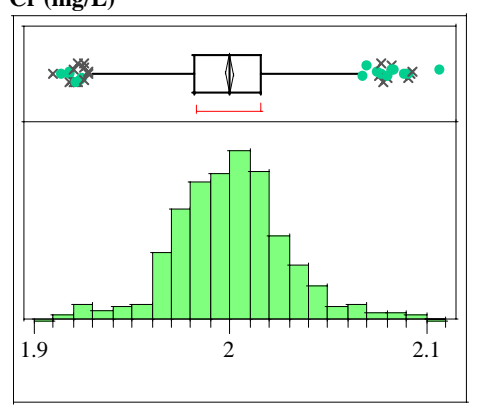

Quantiles

$\begin{array}{ll}100.0 \% & \text { maximum } \\ 99.5 \% & \\ 97.5 \% & \\ 90.0 \% & \\ 75.0 \% & \text { quartile } \\ 50.0 \% & \text { median } \\ 25.0 \% & \text { quartile } \\ 10.0 \% & \\ 2.5 \% & \\ 0.5 \% & \\ 0.0 \% & \text { minimum } \\ \text { Moments } & \\ & \\ \text { Mean } & \\ \text { Std Dev } & \\ \text { Std Err Mean } & \\ \text { upper 95\% Mean } \\ \text { lower 95\% Mean } \\ \text { N }\end{array}$

Cu (mg/L)

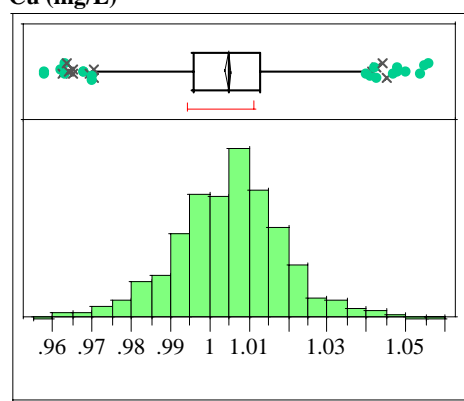

Quantiles

$100.0 \%$ maximum 1.0560

$\begin{array}{ll}99.5 \% & 1.0487\end{array}$

$97.5 \%$

$90.0 \%$

$75.0 \%$ quartile

$50.0 \%$ median

$25.0 \%$ quartile

$10.0 \%$

$2.5 \%$

$0.5 \%$

$0.0 \%$

Moments

minimum

1.0130

1.0050

0.9960

0.9870

0.9733

0.9627

0.9580

Mean

Std Dev

Std Err Mean

upper $95 \%$ Mean

lower 95\% Mean

1.0045912

0.0144782

0.0004742

1.0055219

1.9969254

1.9913492

$\mathrm{N}$

932

Fe (mg/L)

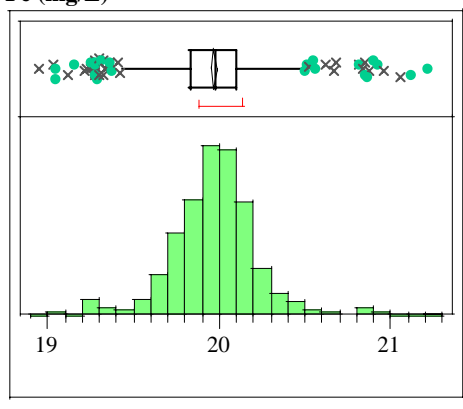

Quantiles

$\begin{array}{llr}100.0 \% & \text { maximum } & 21.226 \\ 99.5 \% & & 20.937 \\ 97.5 \% & & 20.496 \\ 90.0 \% & & 20.233 \\ 75.0 \% & \text { quartile } & 20.103 \\ 50.0 \% & \text { median } & 19.977 \\ 25.0 \% & \text { quartile } & 19.836 \\ 10.0 \% & & 19.690 \\ 2.5 \% & & 19.302 \\ 0.5 \% & & 19.094 \\ 0.0 \% & \text { minimum } & 18.940 \\ \text { Moments } & & \\ & & 19.97019 \\ \text { Mean } & & 0.259994 \\ \text { Std Dev } & & 0.0085164 \\ \text { Std Err Mean } & & 19.986903 \\ \text { upper 95\% Mean } & 19.953476 \\ \text { lower 95\% Mean } & 932 \\ \text { N } & \end{array}$


WSRC-TR-2004-00576

Revision 0

\section{Exhibit A2. Histograms and Descriptive Statistics for Cold Chem Measurements of} Calibration and Bench Standards

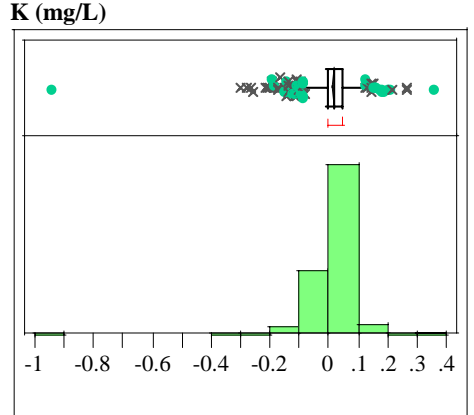

Quantiles

$\begin{array}{ll}100.0 \% & \text { maximum } \\ 99.5 \% & \\ 97.5 \% & \\ 90.0 \% & \\ 75.0 \% & \text { quartile } \\ 50.0 \% & \text { median } \\ 25.0 \% & \text { quartile } \\ 10.0 \% & \\ 2.5 \% & \\ 0.5 \% & \\ 0.0 \% & \text { minimum } \\ \text { Moments } & \\ & \\ \text { Mean } & \\ \text { Std Dev } & \\ \text { Std Err Mean } & \\ \text { upper 95\% Mean } \\ \text { lower 95\% Mean } \\ \text { N }\end{array}$

Li (mg/L)

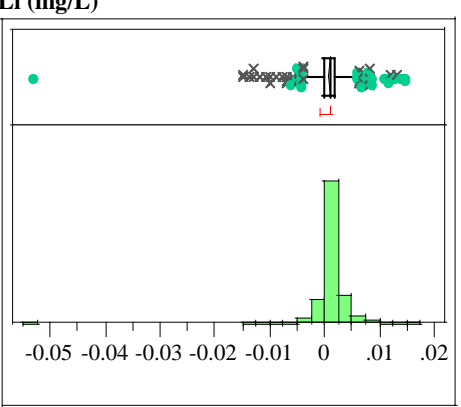

Quantiles

$100.0 \%$

$99.5 \%$

$97.5 \%$

$90.0 \%$

$75.0 \%$

$50.0 \%$

$25.0 \%$

$10.0 \%$

$2.5 \%$

$0.5 \%$

$0.0 \%$

minimum

Mean

Std Dev

Std Err Mean

upper $95 \%$ Mean

lower 95\% Mean

$\mathrm{N}$
$\mathrm{Mg}(\mathrm{mg} / \mathrm{L})$

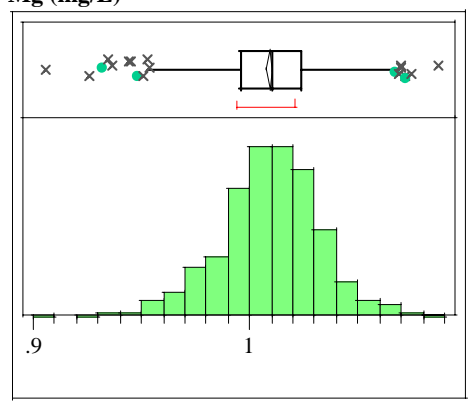

Quantiles

$99.5 \%$

$97.5 \%$

$90.0 \%$

$75.0 \%$

$50.0 \%$

$25.0 \%$

$10.0 \%$

$2.5 \%$

$0.5 \%$

$0.0 \%$

Moments

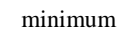

Mean

Std Dev

Std Err Mean

upper 95\% Mean

lower 95\% Mean

$\mathrm{N}$

Mn (mg/L)

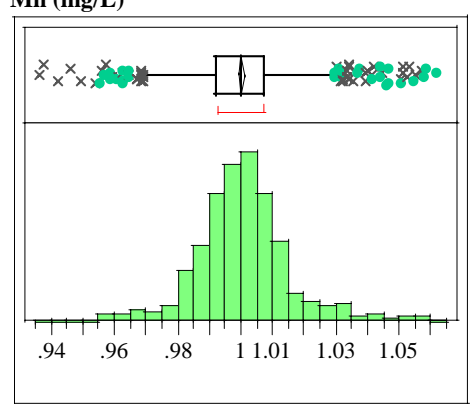

Quantiles

$100.0 \%$ maximum

$99.5 \%$

$97.5 \%$

$90.0 \%$

$75.0 \%$

$50.0 \%$

$25.0 \%$

$10.0 \%$

$2.5 \%$

$0.5 \%$

$0.0 \%$ minimum

Moments

quartile

median

Mean

Std Dev

Std Err Mean

upper 95\% Mean

lower 95\% Mean

$\mathrm{N}$

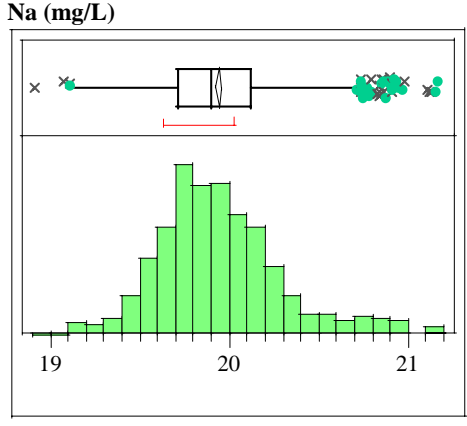

Quantiles

$100.0 \%$

maximum

21.174

$99.5 \%$

$97.5 \%$

$90.0 \%$

$75.0 \%$

$50.0 \%$

$25.0 \%$

$10.0 \%$

$2.5 \%$

$0.5 \%$

$0.0 \%$

Moments

quartile

median

quartile

Mean

Std Dev

Std Err Mean

upper 95\% Mean

lower 95\% Mean

0.0007492

1.0106162

1.0076756

932

$\mathrm{N}$

Ni (mg/L)

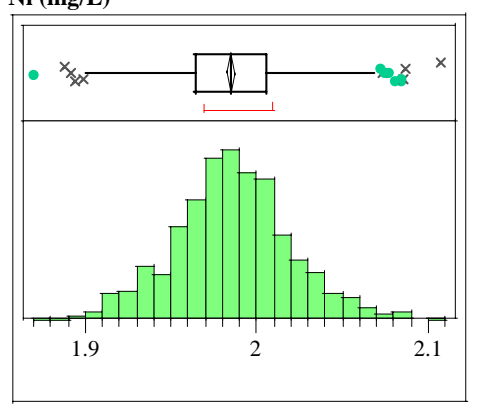

Quantiles

$100.0 \%$ maximum 2.1070

$99.5 \% \quad 2.0850$

$97.5 \%$

$75.0 \%$

$50.0 \%$

$25.0 \%$

$10.0 \%$

$2.5 \%$

$0.5 \%$

$0.0 \%$

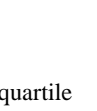

2.0850
2.0567

2.0287

2.0060

1.00000
0.9920

0.9840

0.9480

0.9360

Moments

median

quartile

1.9850

1.9642

1.9403

1.9153

1.8973

Mean

Std Dev

Std Err Mean

upper $95 \%$ Mean

lower 95\% Mean

minimum

1.8710

1.0001685

0.000518

1.001185

$\mathrm{N}$

1.9851373

0.0339141

0.0011109

1.9873175

1.9829572 
WSRC-TR-2004-00576

Revision 0

\section{Exhibit A2. Histograms and Descriptive Statistics for Cold Chem Measurements of} Calibration and Bench Standards

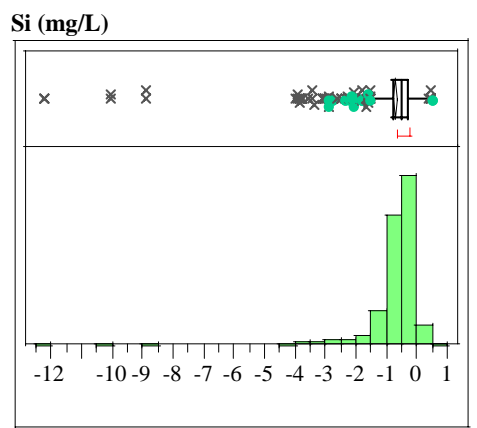

Quantiles

$97.5 \%$

$90.0 \%$

$75.0 \%$

$50.0 \%$

$25.0 \%$

$10.0 \%$

$2.5 \%$

$0.5 \%$

$0.0 \%$

Moments

Mean

Std Dev

Std Err Mean

upper 95\% Mean

lower 95\% Mean

$\mathrm{N}$

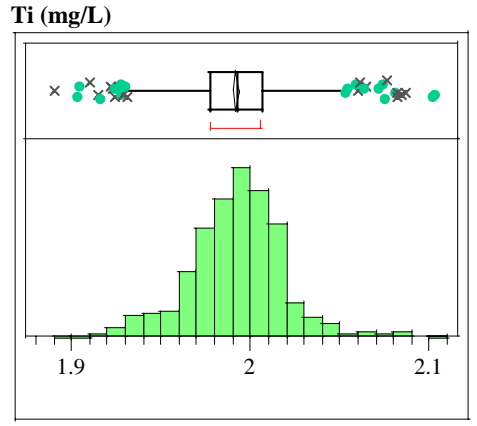

Quantiles

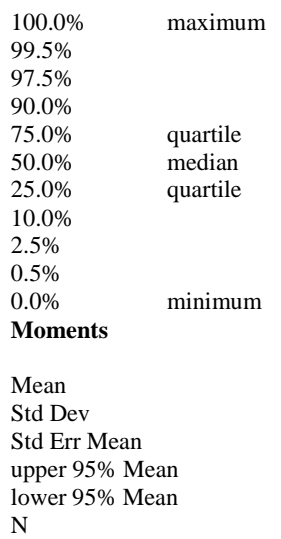

$\mathrm{N}$

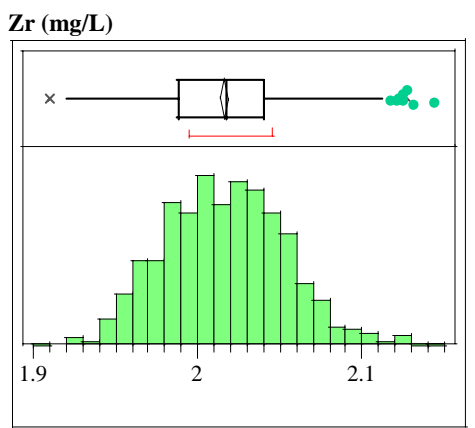

Quantiles

$100.0 \% \quad$ maximum

$99.5 \%$

$97.5 \%$

$90.0 \%$

$75.0 \%$

$50.0 \%$

$25.0 \%$

$10.0 \%$

$2.5 \%$

$0.5 \%$

$0.0 \%$

Moments

minimum

quartile

median

quartile

Mean

Std Dev

Std Err Mean

upper 95\% Mean

lower 95\% Mean

$\mathrm{N}$

$\mathbf{U}(\mathbf{m g} / \mathbf{L})$

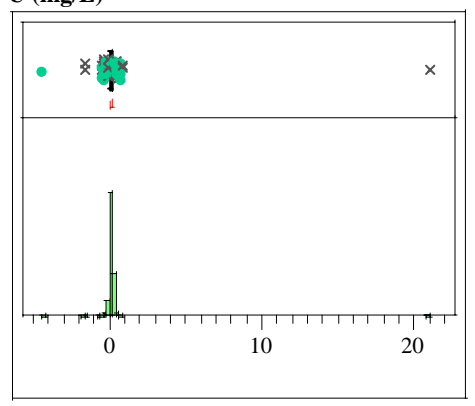

Quantiles

$\begin{array}{ll}100.0 \% & \text { maximum } \\ 99.5 \% & \\ 97.5 \% & \\ 90.0 \% & \\ 75.0 \% & \text { quartile } \\ 50.0 \% & \text { median } \\ 25.0 \% & \text { quartile } \\ 10.0 \% & \\ 2.5 \% & \\ 0.5 \% & \\ 0.0 \% & \text { minimum } \\ \text { Moments } & \\ & \\ \text { Mean } & \\ \text { Std Dev } & \\ \text { Std Err Mean } & \\ \text { upper 95\% Mean } \\ \text { lower 95\% Mean } \\ \text { N }\end{array}$

STCd=IN36

Distributions

Al (mg/L)

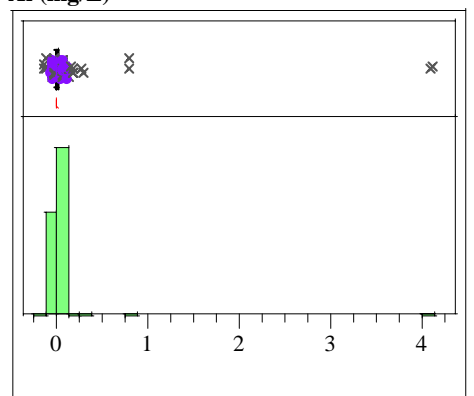

Quantiles

$99.5 \%$

$97.5 \%$

$90.0 \%$

$75.0 \%$

$50.0 \%$

$25.0 \%$

$10.0 \%$

$2.5 \%$

$0.5 \%$

$0.0 \%$

Moments

maximum

quartile

median

quartile

minimum

Mean

Std Dev

Std Err Mean

upper 95\% Mean

lower 95\% Mean

2.0181566

2.0134185

$\mathrm{N}$

B (mg/L)

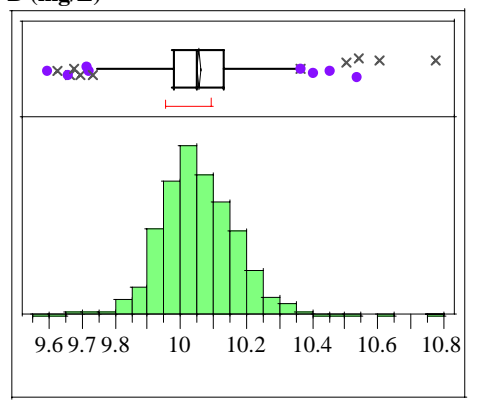

Quantiles

$100.0 \%$

maximum

10.773

$99.5 \%$

$97.5 \%$

$90.0 \%$

$75.0 \%$

$50.0 \%$

$25.0 \%$

$10.0 \%$

$2.5 \%$

$0.5 \%$

$0.0 \%$

Moments

quartile

median

quartile

quartile

minimum

Mean

Std Dev

Std Err Mean

upper 95\% Mean

lower 95\% Mean

$\mathrm{N}$
4.091 0.546

0.063

0.035

0.015

$-0.005$

$-0.017$

$-0.040$

0.017387 0.1985419 0.0066402 0.0304193 0.0043547 894 
WSRC-TR-2004-00576

Revision 0

\section{Exhibit A2. Histograms and Descriptive Statistics for Cold Chem Measurements of} Calibration and Bench Standards

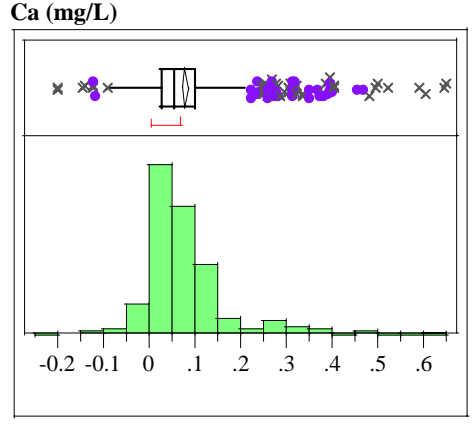

Quantiles

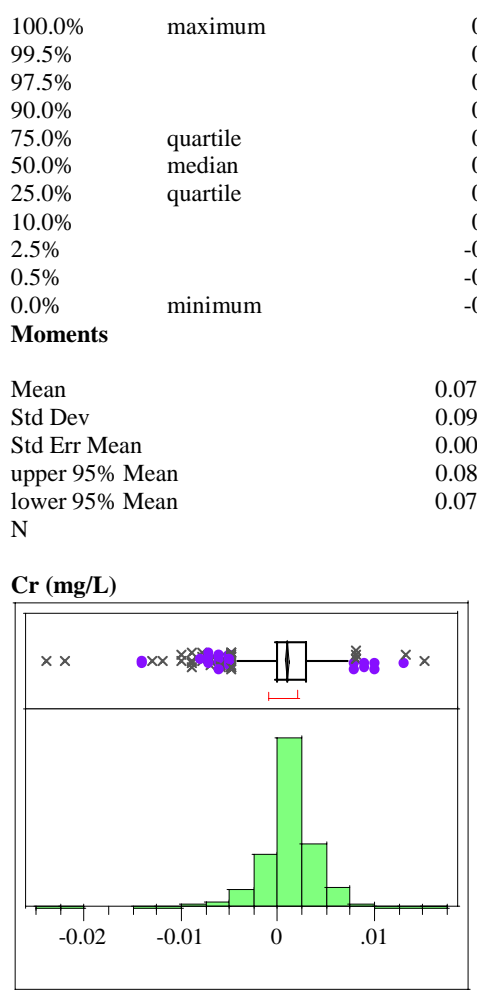

Quantiles

$100.0 \% \quad$ maximum

$99.5 \%$

$97.5 \%$

$90.0 \%$

$75.0 \%$

$50.0 \%$

$25.0 \%$

$10.0 \%$

$2.5 \%$

$0.5 \%$

$0.0 \%$

minimum

Mean

Std Dev

Std Err Mean

upper 95\% Mean

lower 95\% Mean

$\mathrm{N}$
Cu (mg/L)

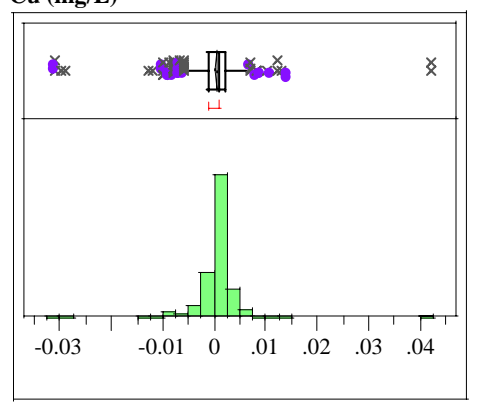

Quantiles

$99.5 \%$

$97.5 \%$

$90.0 \%$

$75.0 \%$

$50.0 \%$

$25.0 \%$

$10.0 \%$

$2.5 \%$

$0.5 \%$

$0.0 \%$

Moments

Mean

Std Dev

Std Err Mean

upper 95\% Mean

lower 95\% Mean

$\mathrm{N}$

Fe (mg/L)

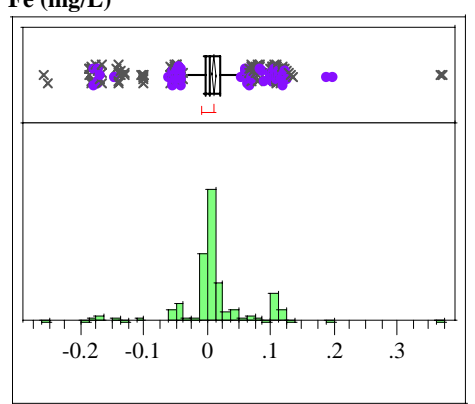

Quantiles

$100.0 \%$ maximum

$99.5 \%$

$97.5 \%$

$90.0 \%$

$75.0 \%$

$50.0 \%$

$25.0 \%$

$10.0 \%$

$2.5 \%$

$0.5 \%$

$0.0 \%$ minimum

Moments

quartile

median

quartile

Mean

Std Dev

Std Err Mean

upper 95\% Mean

lower 95\% Mean

$\mathrm{N}$
K (mg/L)

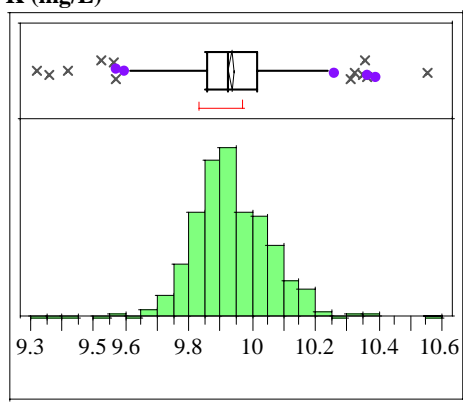

Quantiles

$100.0 \%$

maximum

10.550

$99.5 \%$

$97.5 \%$

$90.0 \%$

$75.0 \%$

$50.0 \%$

$25.0 \%$

$10.0 \%$

$2.5 \%$

$0.5 \%$

$0.0 \%$

Moments

quartile

median

quartile

$-0.0020$

$-0.0305$

$-0.0310$

0.0004362

0.0043039

0.0001439

Mean

Std Dev

Std Err Mean

upper $95 \%$ Mean

lower 95\% Mean

0.0001537

894

$\mathrm{N}$

Li (mg/L)

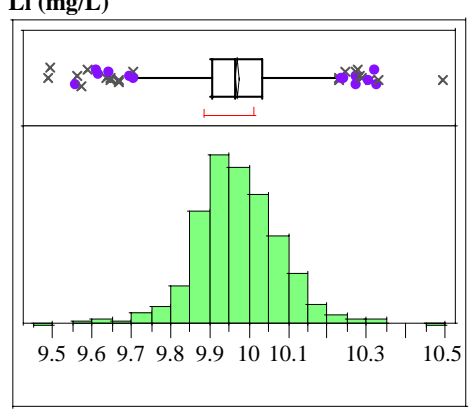

Quantiles

$100.0 \%$ maximum 10.493

$\begin{array}{ll}99.5 \% & 10.315\end{array}$

$97.5 \%$

$75.0 \%$

$50.0 \%$

$25.0 \%$

$10.0 \%$

$2.5 \%$

$0.5 \%$

$0.0 \%$

Moments

\section{quartile}

median

quartile

9.9349989

0.1252223

9.9432185

9.9267793

894
0.0001077

0.0010984

894
0.0099396

Mean

Std Dev

Std Err Mean

upper 95\% Mean

lower 95\% Mean

$\mathrm{N}$ 
WSRC-TR-2004-00576

Revision 0

\section{Exhibit A2. Histograms and Descriptive Statistics for Cold Chem Measurements of} Calibration and Bench Standards

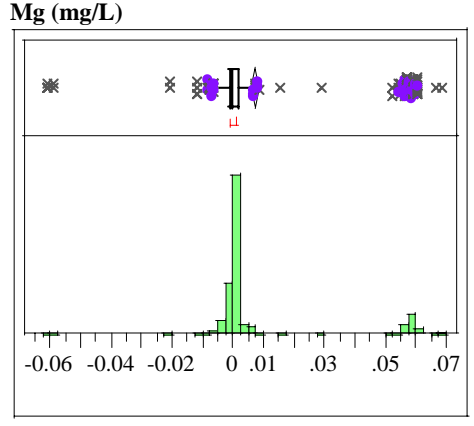

Quantiles

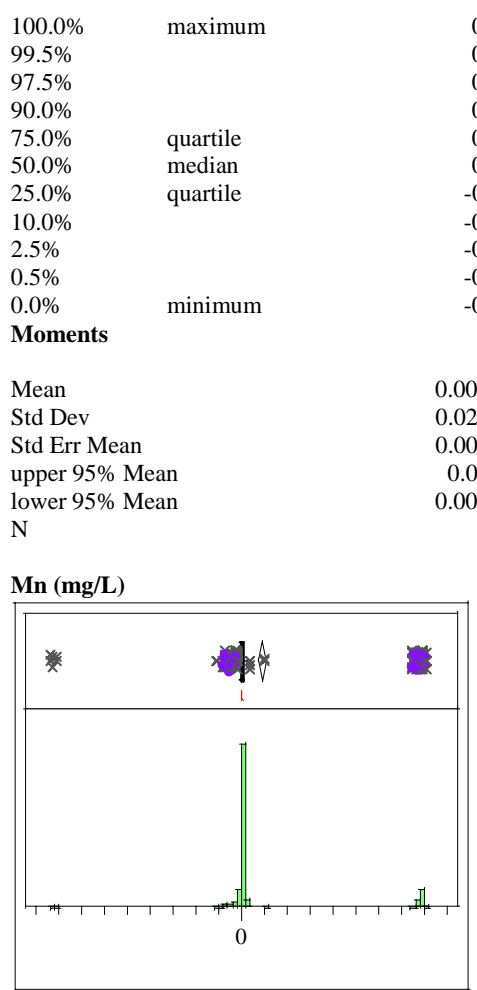

Quantiles

$100.0 \%$

$99.5 \%$

$97.5 \%$

$90.0 \%$

$75.0 \%$

$50.0 \%$

$25.0 \%$

$10.0 \%$

$2.5 \%$

$0.5 \%$

$0.0 \%$
Moments

maximum

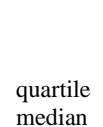

median

quartile

minimum

Mean

Std Dev

Std Err Mean

upper 95\% Mean

lower 95\% Mean

$\mathrm{N}$
Na (mg/L)

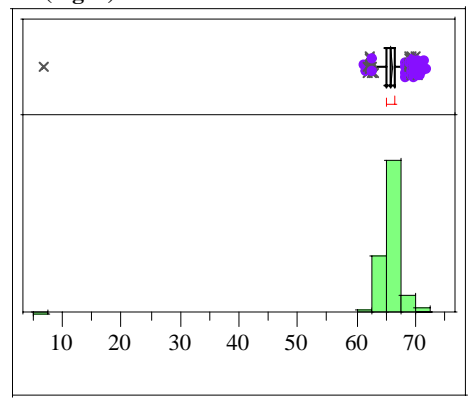

Quantiles

$100.0 \% \quad$ maximum

$99.5 \%$

$97.5 \%$

$90.0 \%$

$75.0 \%$

$50.0 \%$

$25.0 \%$

$10.0 \%$

$2.5 \%$

$0.5 \%$

$0.0 \%$ minimum

Moments

quartile

median

quartile

Mean

Std Dev

Std Err Mean

upper 95\% Mean

lower 95\% Mean

$\mathrm{N}$

$\mathrm{Ni}(\mathrm{mg} / \mathrm{L})$

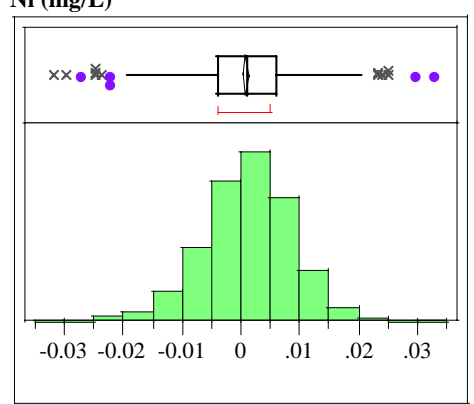

Quantiles

$100.0 \%$ maximum

$99.5 \%$

$97.5 \%$

$90.0 \%$

$75.0 \%$

$50.0 \%$

$25.0 \%$

$10.0 \%$

$2.5 \%$

$0.5 \%$

$0.0 \%$ minimum

Moments

quartile

median

quartile

$-0.0066$

$-0.0840$

0.0090705

0.0269138

0.0009001

0.0108371

0.0073039

894
Mean

Std Dev

Std Err Mean

upper 95\% Mean

$\mathrm{N}$

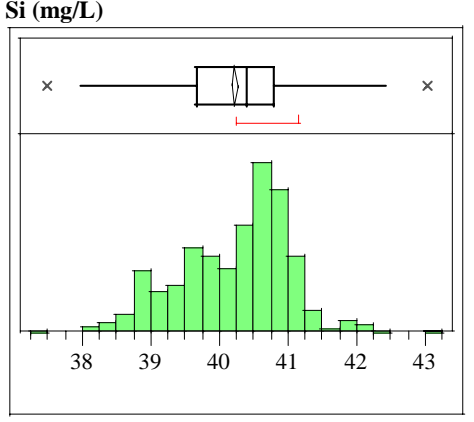

Quantiles

$100.0 \%$

maximum

43.033

$99.5 \%$

$97.5 \%$

$90.0 \%$

$75.0 \%$

$50.0 \%$

$25.0 \%$

$10.0 \%$

$2.5 \%$

$0.5 \%$

$0.0 \%$

Moments

quartile

median

quartile

61.667

6.674

Mean

Std Dev

Std Err Mean

upper 95\% Mean

lower 95\% Mean

0.0830714

65.986532

65.660456

894

Ti (mg/L)

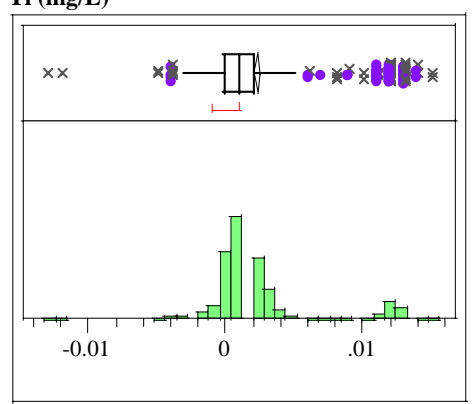

Quantiles

$100.0 \%$ maximum 0.0150

$99.5 \%$

$97.5 \%$

$90.0 \%$

$75.0 \%$

$50.0 \%$

$25.0 \%$

$10.0 \%$

$2.5 \%$

$0.5 \%$

$0.0 \%$

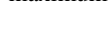

quartile

median

quartile

$-0.0160$

$-0.0250$

Moments

minimum

40.232102

0.791628

0.026476

40.284064

40.180139

894

\section{Mean}

0.0007528

0.0078838

0.0002637

0.0012703

Std Dev

Std Err Mean

upper 95\% Mean

lower 95\% Mean

894

0.0140
0.0130

0.0110

0.0020

0.0010

0.0000

0.0000

$-0.0020$

$-0.0045$

$-0.0130$

0.0023143

0.0038865

0.00013

0.0025694

0.0020592 
WSRC-TR-2004-00576

Revision 0

Exhibit A2. Histograms and Descriptive Statistics for Cold Chem Measurements of Calibration and Bench Standards

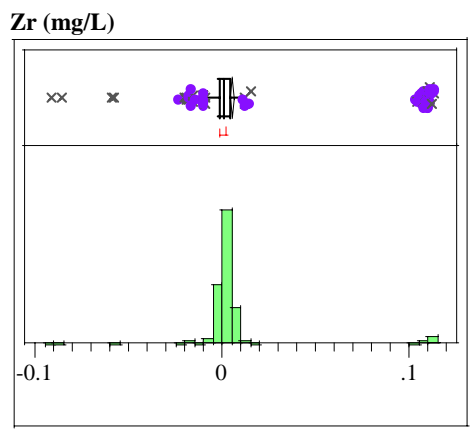

Quantiles

$97.5 \%$

$90.0 \%$

$75.0 \%$

$50.0 \%$

$25.0 \%$

$10.0 \%$

$2.5 \%$

$0.5 \%$

$0.0 \%$

Moments

Mean

Std Dev

Std Err Mean

upper 95\% Mean

lower 95\% Mean

$\mathrm{N}$

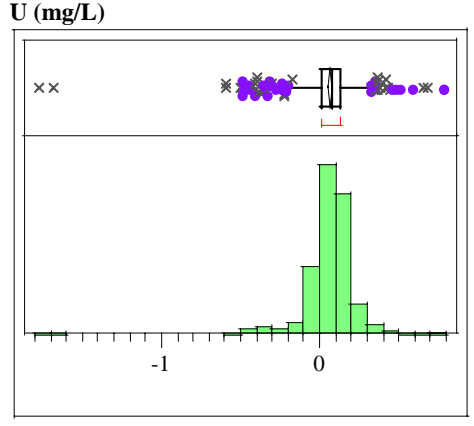

Quantiles

$\begin{array}{ll}100.0 \% & \text { maximum } \\ 99.5 \% & \\ 97.5 \% & \\ 90.0 \% & \\ 75.0 \% & \text { quartile } \\ 50.0 \% & \text { median } \\ 25.0 \% & \text { quartile } \\ 10.0 \% & \\ 2.5 \% & \\ 0.5 \% & \\ 0.0 \% & \text { minimum } \\ \text { Moments } & \\ & \\ \text { Mean } & \\ \text { Std Dev } & \\ \text { Std Err Mean } & \\ \text { upper 95\% Mean } \\ \text { lower 95\% Mean } \\ \text { N }\end{array}$

STCd=IN38

Distributions

Al (mg/L)

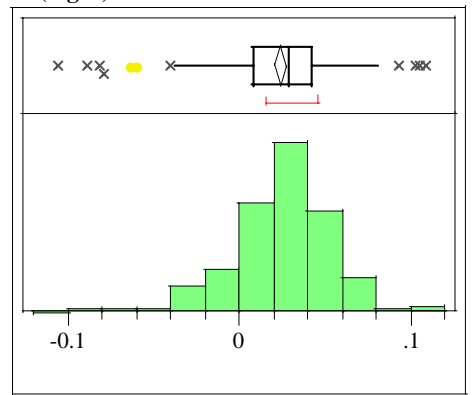

Quantiles

$100.0 \%$ maximum

$99.5 \%$

$97.5 \%$

$90.0 \%$

$75.0 \%$

$50.0 \%$

$25.0 \%$

$10.0 \%$

$2.5 \%$

$0.5 \%$

$0.0 \%$

Moments

minimum

Mean

Std Dev

Std Err Mean

upper $95 \%$ Mean

lower 95\% Mean

$\mathrm{N}$

B (mg/L)

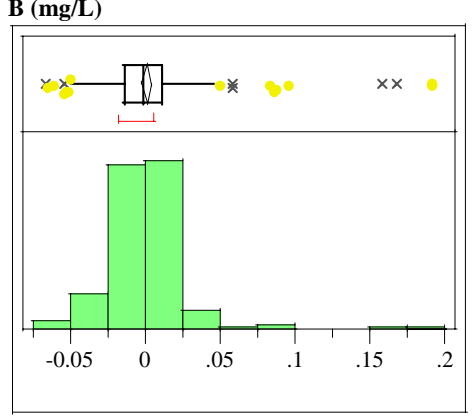

Quantiles

$\begin{array}{ll}100.0 \% & \text { maximum } \\ 99.5 \% & \\ 97.5 \% & \\ 90.0 \% & \\ 75.0 \% & \text { quartile } \\ 50.0 \% & \text { median } \\ 25.0 \% & \text { quartile } \\ 10.0 \% & \\ 2.5 \% & \\ 0.5 \% & \\ 0.0 \% & \text { minimum } \\ \text { Moments } & \\ & \\ \text { Mean } & \\ \text { Std Dev } & \\ \text { Std Err Mean } & \\ \text { upper 95\% Mean } \\ \text { lower 95\% Mean } \\ \text { N }\end{array}$

Ca (mg/L)

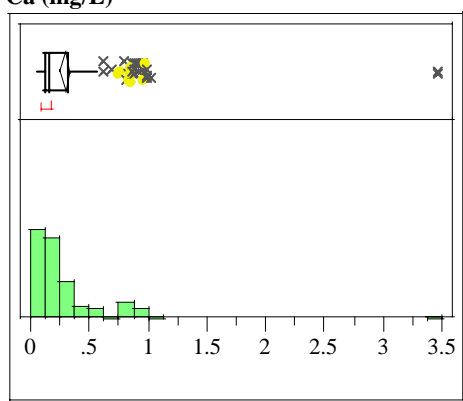

Quantiles

$100.0 \%$ maximum $\quad 3.4580$

$99.5 \% \quad 3.4549$

$97.5 \%$

$90.0 \%$

$75.0 \%$ quartile

$50.0 \%$ median

$25.0 \%$ quartile

$10.0 \%$

$2.5 \%$

$0.5 \%$

$0.0 \%$

Moments

minimum

0.9652

0.8198

0.3142

0.1620

0.1150

0.1000

0.0807

0.0690

0.0690

Mean

Std Dev

Std Err Mean

upper 95\% Mean

lower 95\% Mean

0.2920364

0.3540215

0.0203717

0.3321253

0.0277514

0.0211426

$\mathrm{N}$

302

Cr (mg/L)

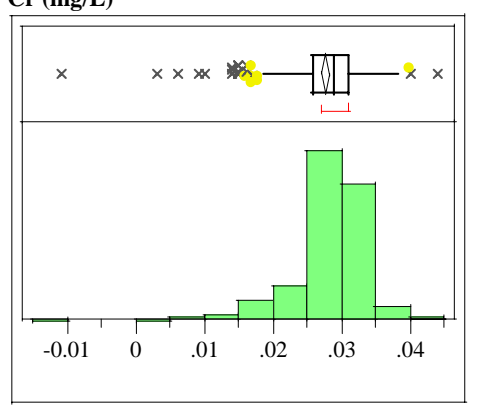

Quantiles

$\begin{array}{llr}100.0 \% & \text { maximum } & 0.0440 \\ 99.5 \% & & 0.0419 \\ 97.5 \% & & 0.0380 \\ 90.0 \% & & 0.0330 \\ 75.0 \% & \text { quartile } & 0.0310 \\ 50.0 \% & \text { median } & 0.0290 \\ 25.0 \% & \text { quartile } & 0.0260 \\ 10.0 \% & & 0.0210 \\ 2.5 \% & & 0.0140 \\ 0.5 \% & & -0.0038 \\ 0.0 \% & \text { minimum } & -0.0110 \\ \text { Moments } & & \\ & & 0.0277682 \\ \text { Mean } & & 0.0057633 \\ \text { Std Dev } & & 0.0003316 \\ \text { Std Err Mean } & & 0.0284208 \\ \text { upper 95\% Mean } & 0.0271156 \\ \text { lower 95\% Mean } & 302 \\ \text { N } & & \end{array}$


WSRC-TR-2004-00576

Revision 0

\section{Exhibit A2. Histograms and Descriptive Statistics for Cold Chem Measurements of Calibration and Bench Standards}

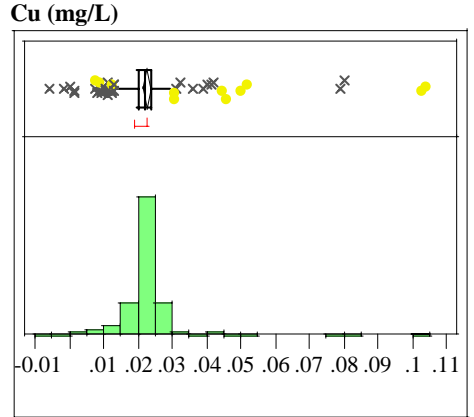

Quantiles

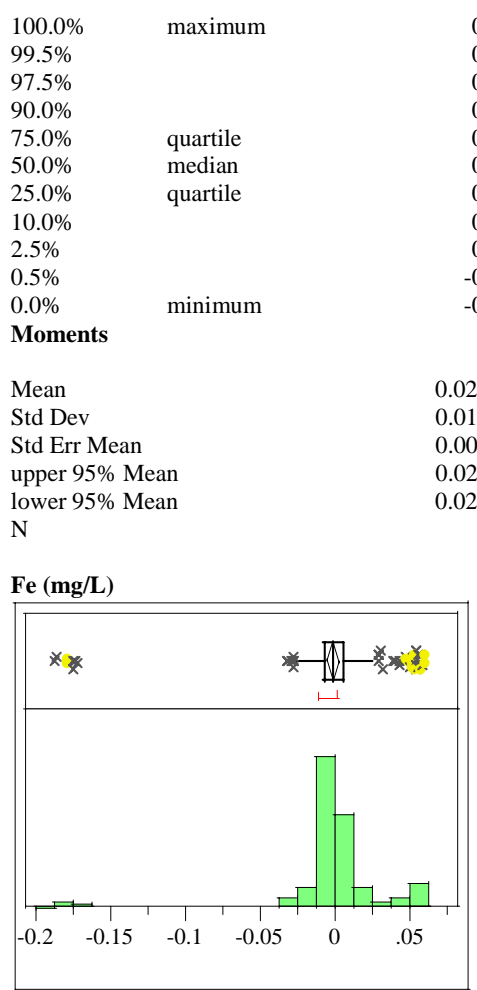

Quantiles

$100.0 \%$

$99.5 \%$

$97.5 \%$

$90.0 \%$

$75.0 \%$

$50.0 \%$

$25.0 \%$

$10.0 \%$

$2.5 \%$

$0.5 \%$

$0.0 \%$

maximum

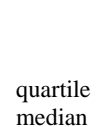

median

quartile

minimum

Mean

Std Dev

Std Err Mean

upper 95\% Mean

lower 95\% Mean

$\mathrm{N}$
K (mg/L)

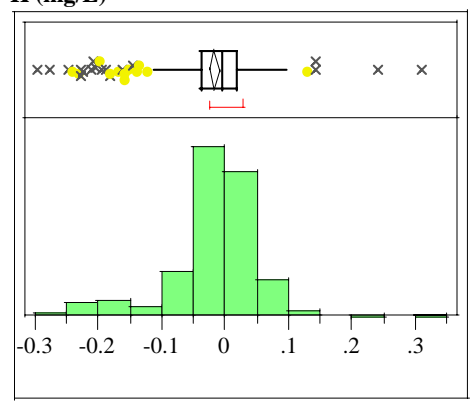

Quantiles

$\begin{array}{ll}100.0 \% & \text { maximum } \\ 99.5 \% & \\ 97.5 \% & \\ 90.0 \% & \\ 75.0 \% & \text { quartile } \\ 50.0 \% & \text { median } \\ 25.0 \% & \text { quartile } \\ 10.0 \% & \\ 2.5 \% & \\ 0.5 \% & \\ 0.0 \% & \text { minimum } \\ \text { Moments } & \\ & \\ \text { Mean } & \\ \text { Std Dev } & \\ \text { Std Err Mean } & \\ \text { upper 95\% Mean } \\ \text { lower 95\% Mean }\end{array}$

$\mathrm{N}$

Li (mg/L)

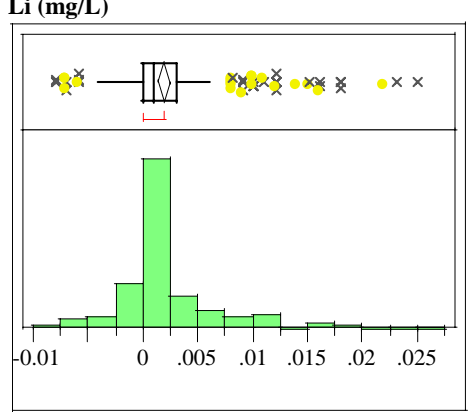

Quantiles

$100.0 \%$ maximum

$99.5 \%$

$97.5 \%$

$90.0 \%$

$75.0 \%$

$50.0 \%$

$25.0 \%$

$10.0 \%$

$2.5 \%$

$0.5 \%$

$0.0 \%$ minimum

Moments

quartile

median

quartile

-0.1734
-0.1875

$-0.1880$

$-0.001126$

0.034847

0.0020052

0.0028202

$-0.005072$

302
Mean

Std Dev

Std Err Mean

upper 95\% Mean

lower 95\% Mean

$\mathrm{N}$
Mg (mg/L)

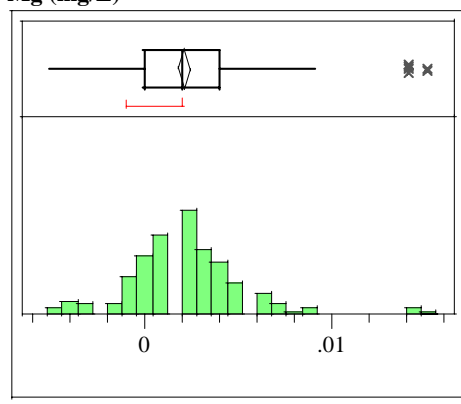

Quantiles

$100.0 \%$

maximum

0.0150

$99.5 \%$

$97.5 \%$

$90.0 \%$

$75.0 \%$

$50.0 \%$

$25.0 \%$

$10.0 \%$

$2.5 \%$

$0.5 \%$

$0.0 \%$

Moments

quartile

median

quartile

$-0.2273$

$-0.2882$

$-0.2980$

$-0.016417$

0.0717532

0.0041289

$-0.008292$

Mean

Std Dev

Std Err Mean

upper $95 \%$ Mean

lower 95\% Mean

$\mathrm{N}$

0.0090

0.0057

0.0040

0.0020

0.0000

$-0.0010$

$-0.0040$

$-0.0050$

minimum

$-0.0050$

0.0021126

0.0031307

0.0024671

0.0017581

302

Mn (mg/L)

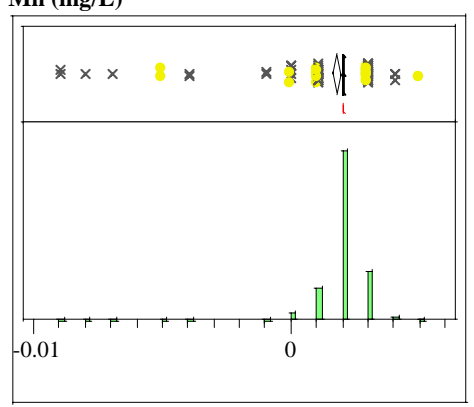

Quantiles

$100.0 \%$ maximum 0.0050

$99.5 \% \quad 0.0050$

$90.0 \%$

$75.0 \%$

$50.0 \%$ median

$25.0 \%$

$10.0 \%$

$2.5 \%$

$0.5 \%$

Moments

0.0019768

Mean

Std Dev

Std Err Mean

upper 95\% Mean

lower 95\% Mean

$\mathrm{N}$ 
WSRC-TR-2004-00576

Revision 0

\section{Exhibit A2. Histograms and Descriptive Statistics for Cold Chem Measurements of} Calibration and Bench Standards

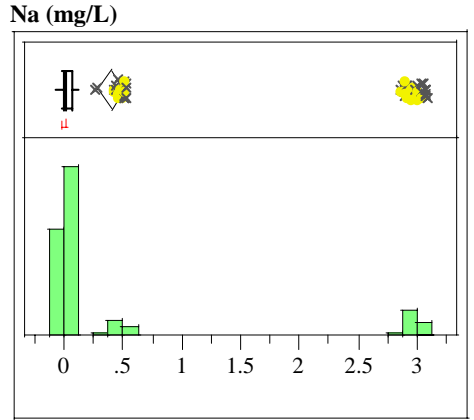

Quantiles

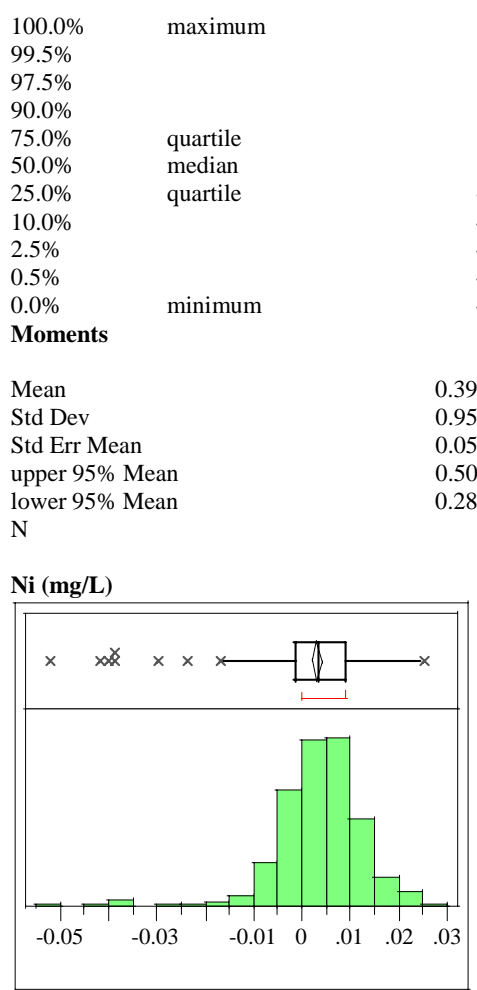

Quantiles

$100.0 \% \quad$ maximum

$99.5 \%$

$97.5 \%$

$90.0 \%$

$75.0 \%$

$50.0 \%$

$25.0 \%$

$10.0 \%$

$2.5 \%$

$0.5 \%$

$0.0 \% \quad$ minimum

Moments

Mean

Std Dev

Std Err Mean

upper 95\% Mean

lower 95\% Mean

$\mathrm{N}$
Si (mg/L)

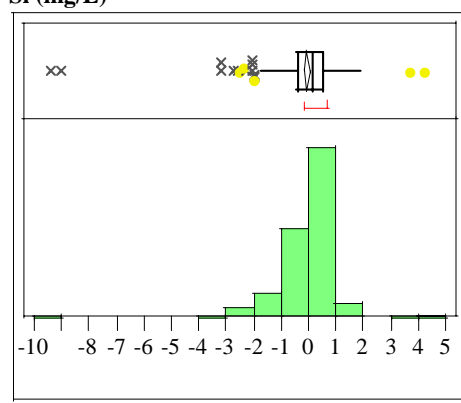

Quantiles

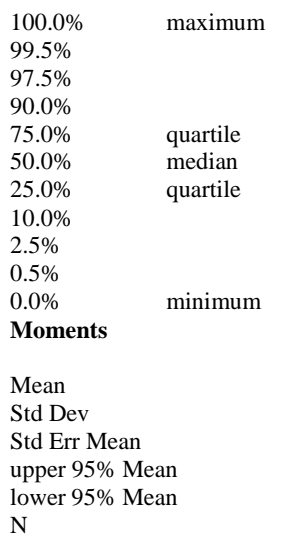

$\mathrm{N}$

Ti (mg/L)

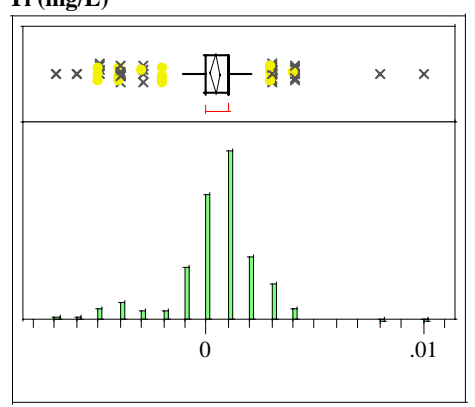

Quantiles

$100.0 \%$ maximum

$99.5 \%$

$97.5 \%$

$90.0 \%$

$75.0 \%$

$50.0 \%$

$25.0 \%$

$10.0 \%$

$2.5 \%$

$0.5 \%$

$0.0 \%$ minimum

Moments

quartile

median
quartile

Mean

Std Dev

Std Err Mean

upper 95\% Mean

lower 95\% Mean

$\mathrm{N}$
$\operatorname{Zr}$ (mg/L)

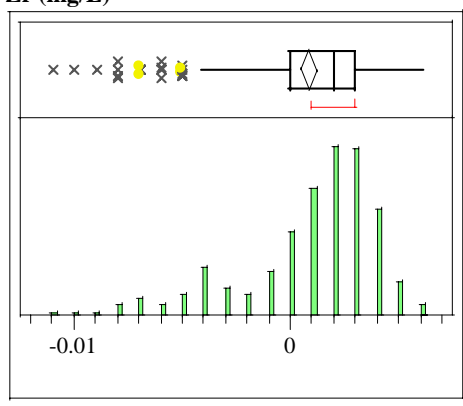

Quantiles

$100.0 \%$

maximum

0.0060

$99.5 \%$

$97.5 \%$

$90.0 \%$

$75.0 \%$

$50.0 \%$

$25.0 \%$

$10.0 \%$

$2.5 \%$

$0.5 \%$

$0.0 \%$

Moments

quartile

median

quartile

minimum

Mean

Std Dev

Std Err Mean

upper 95\% Mean

lower 95\% Mean

$\mathrm{N}$

0.0050

0.0040

0.0030

0.0020

0.0000

$-0.0040$

$-0.0074$

$-0.0105$

$-0.0110$

$-0.172735$

302

U (mg/L)

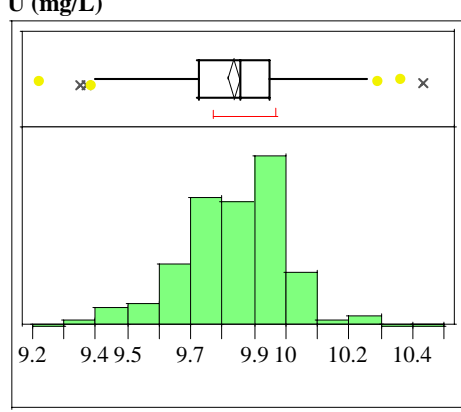

Quantiles

$100.0 \%$ maximum 10.434

$\begin{array}{ll}99.5 \% & 10.397\end{array}$

$90.0 \%$

$75.0 \%$

$50.0 \%$

$25.0 \%$

$10.0 \%$

$2.5 \%$

$0.5 \%$

$0.0 \%$

Moments

quartile

median

quartile

0.0008444 0.0031966 0.0001839 0.0012063 0.0004824 302

\section{Mean}

Std Dev

Std Err Mean

upper 95\% Mean

lower 95\% Mean

$\mathrm{N}$ 
WSRC-TR-2004-00576

Revision 0

\section{Exhibit A2. Histograms and Descriptive Statistics for Cold Chem Measurements of Calibration and Bench Standards}

STCd=IN39

Distributions

Al (mg/L)

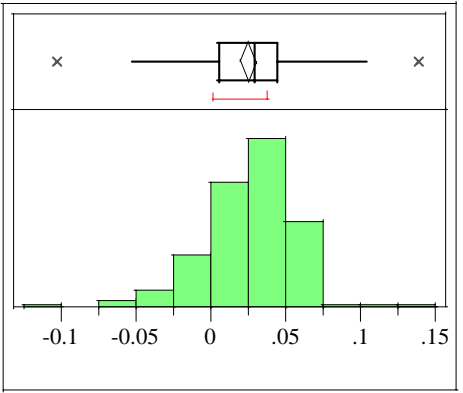

Quantiles

$99.5 \%$

$97.5 \%$

$90.0 \%$

$75.0 \%$

$50.0 \%$

$25.0 \%$

$10.0 \%$

$2.5 \%$

$0.5 \%$

$0.0 \%$

Moments

Mean

Std Dev

Std Err Mean

upper 95\% Mean

lower 95\% Mean

N

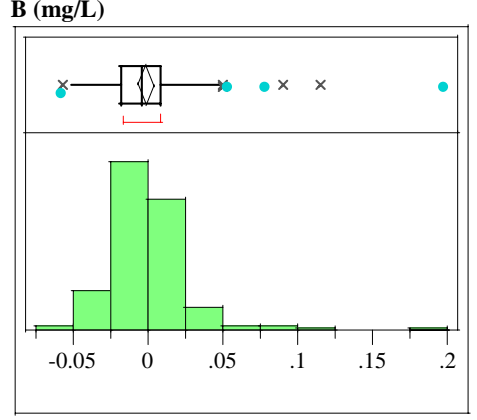

Quantiles

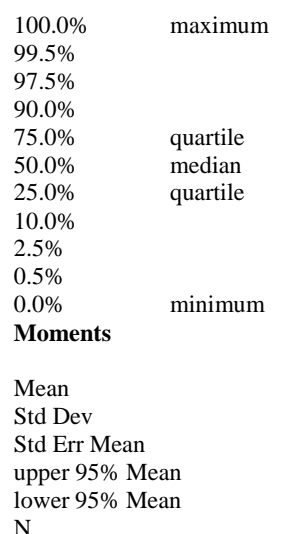

$\mathrm{N}$

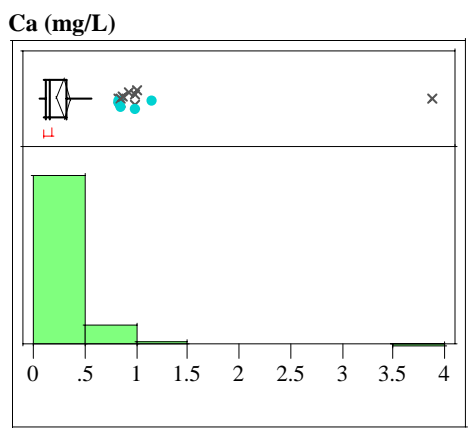

Quantiles

$100.0 \% \quad$ maximum $\quad 3.8590$

$99.5 \%$

$97.5 \%$

$90.0 \%$

$75.0 \%$

$50.0 \%$

$25.0 \%$

$10.0 \%$

$2.5 \%$

$0.5 \%$

$0.0 \%$

Moments

quartile

median

quartile

$-0.0451$

minimum

0.024838

0.0310336

0.0026043

0.0299865

0.0196895

142

0.3576061

0.2300136
Mean

Std Dev

Std Err Mean

upper 95\% Mean

lower 95\% Mean

N

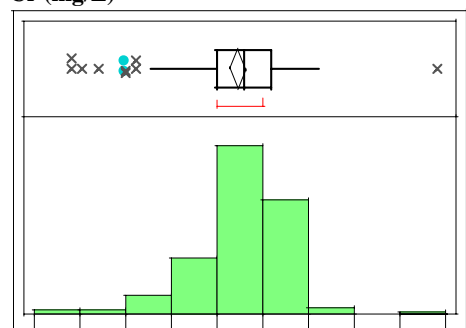

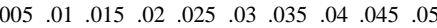

Quantiles

$100.0 \%$

$99.5 \%$

$97.5 \%$

$90.0 \%$

$75.0 \%$

$50.0 \%$

$25.0 \%$

$10.0 \%$

$2.5 \%$

$0.5 \%$

$0.0 \%$

Moments

Mean

Std Dev

Std Err Mean

upper 95\% Mean

lower 95\% Mean

$\mathrm{N}$

quartile

median

quartile

minimum
Cu (mg/L)

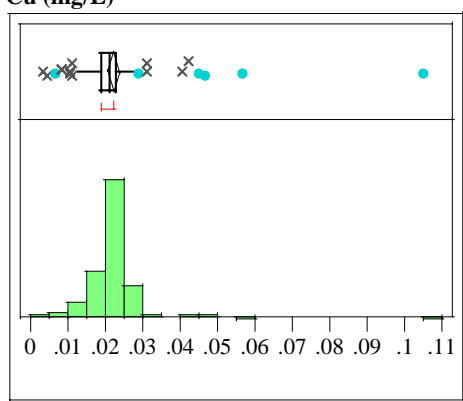

Quantiles

$100.0 \%$ maximum $\quad 0.10500$

$99.5 \% \quad 0.10500$

$97.5 \%$

$90.0 \%$

$75.0 \%$ quartile

$50.0 \%$ median

$25.0 \%$ quartile

$10.0 \%$

$2.5 \%$

$0.5 \%$

$0.0 \%$

Moments

minimum

0.04585

0.02700

0.02300

0.02300
0.02100

0.01900

0.01460

0.00758

0.00300

0.00300

Mean

Std Dev

Std Err Mean

upper 95\% Mean

lower 95\% Mean

$\mathrm{N}$

0.022007

0.0096921

0.0008133

0.023615

0.0203991

142

$\mathrm{Fe}(\mathrm{mg} / \mathrm{L})$

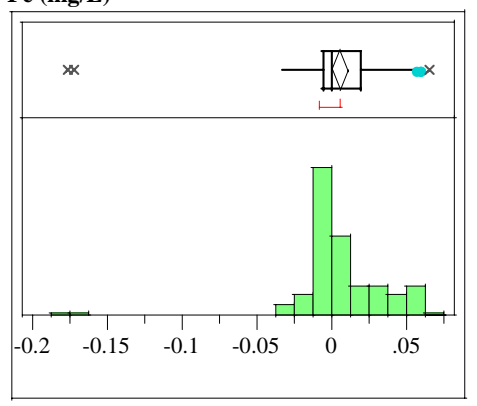

Quantiles

$100.0 \%$

maximum

0.0640

$99.5 \%$

0.04900

0.03542

0.03300

0.03100

0.02800

0.0310199

0.0026031

0.0040265

$-0.006266$

0.02500

0.02060

0.01115

0.00900

$97.5 \%$

$90.0 \%$

$75.0 \%$

$50.0 \%$

$25.0 \%$

$10.0 \%$

$2.5 \%$

0.00900

$0.5 \%$

$0.0 \%$

Moments

quartile

median

quartile

Mean

0.0272042

0.0055742

Std Dev

Std Err Mean

0.028129

upper 95\% Mean

0.0262795

$\mathrm{N}$ 
WSRC-TR-2004-00576

Revision 0

\section{Exhibit A2. Histograms and Descriptive Statistics for Cold Chem Measurements of Calibration and Bench Standards}

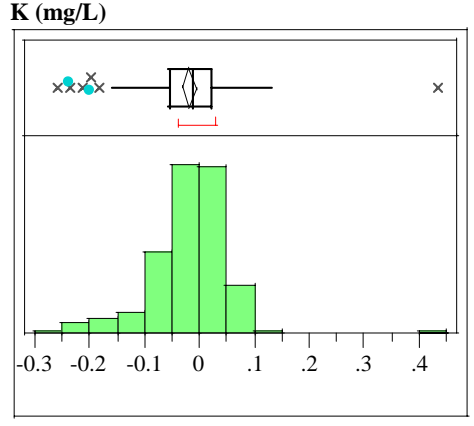

Quantiles

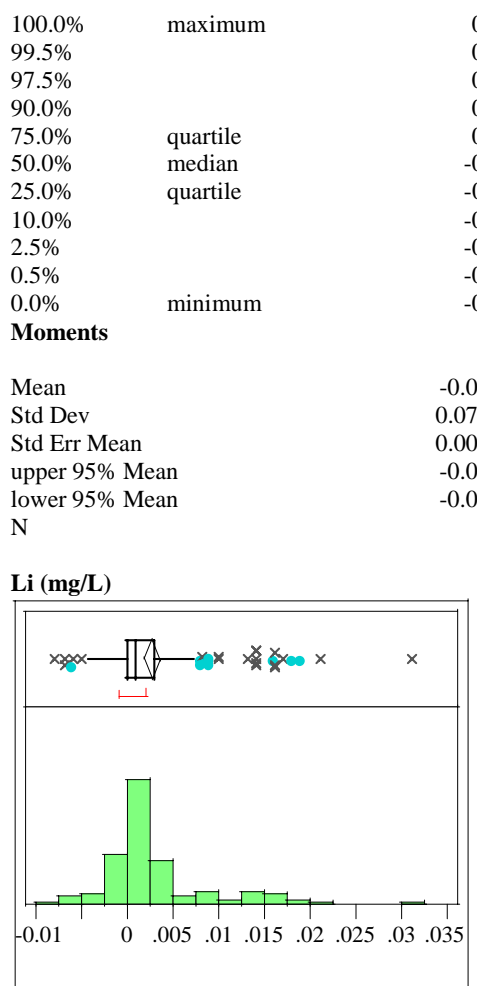

Quantiles

$100.0 \%$

$99.5 \%$

$97.5 \%$

$90.0 \%$

$75.0 \%$

$50.0 \%$

$25.0 \%$

$10.0 \%$

$2.5 \%$

$0.5 \%$

$0.0 \%$
Moments

maximum

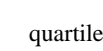

median

quartile

minimum

Mean

Std Dev

Std Err Mean

upper $95 \%$ Mean

lower 95\% Mean

$\mathrm{N}$
$\operatorname{Mg}(\mathrm{mg} / \mathrm{L})$

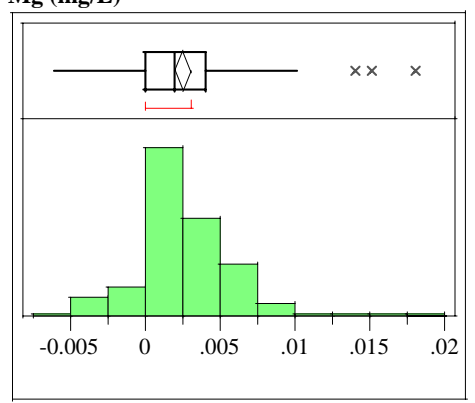

Quantiles

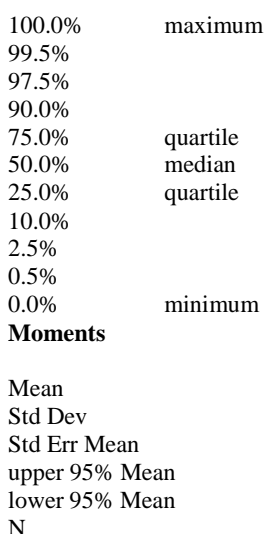

$\mathrm{N}$

Mn (mg/L)

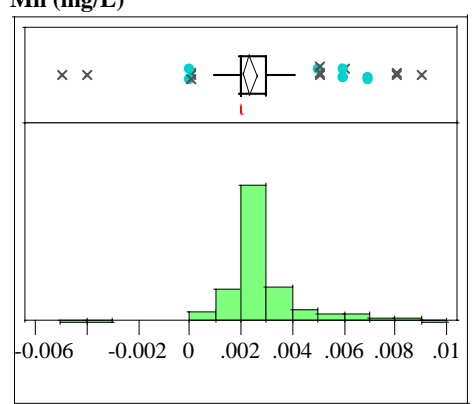

Quantiles

$100.0 \% \quad$ maximum

$99.5 \%$

$97.5 \%$

$90.0 \%$

$75.0 \%$

$50.0 \%$

$25.0 \%$

$10.0 \%$

$2.5 \%$

$0.5 \%$

$0.0 \%$ minimum

Moments

quartile

median

Mean

Std Dev

Std Err Mean

upper 95\% Mean

lower 95\% Mean

$\mathrm{N}$

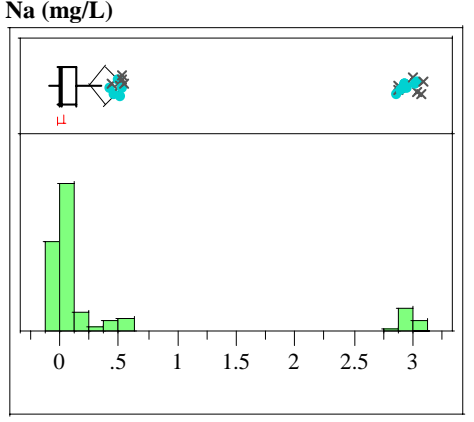

Quantiles

$\begin{array}{llr}100.0 \% & \text { maximum } & 3.083 \\ 99.5 \% & & 3.083 \\ 97.5 \% & & 3.035 \\ 90.0 \% & & 2.878 \\ 75.0 \% & \text { quartile } & 0.140 \\ 50.0 \% & \text { median } & 0.022 \\ 25.0 \% & \text { quartile } & -0.002 \\ 10.0 \% & & -0.016 \\ 2.5 \% & & -0.044 \\ 0.5 \% & & -0.079 \\ 0.0 \% & \text { minimum } & -0.079 \\ \text { Moments } & & \\ & & 0.3951761 \\ \text { Mean } & & 0.9274652 \\ \text { Std Dev } & & 0.0778311 \\ \text { Std Err Mean } & & 0.5490429 \\ \text { upper 95\% Mean } & 0.2413092 \\ \text { lower 95\% Mean } & 142 \\ \text { N } & \end{array}$

Ni (mg/L)

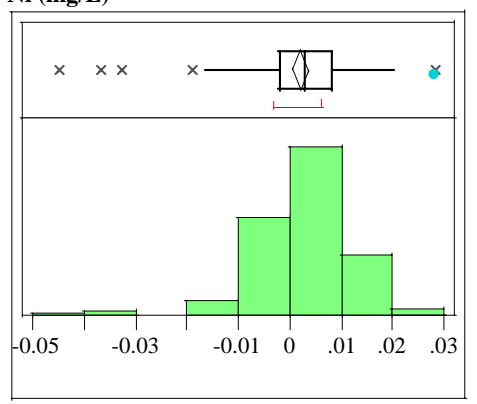

Quantiles

$100.0 \% \quad$ maximum 0.0280

$99.5 \% \quad 0.0280$

$97.5 \%$

$75.0 \%$

$50.0 \%$

$25.0 \%$

$10.0 \%$

$2.5 \%$

$0.5 \%$

$0.0 \%$

quartile

median

quartile

0.0010

0.0000
-0.0050

$-0.0050$

Moments

minimum

0.0183

0.0130

0.0080

0.0030

0.0030
-0.0020

$-0.0067$

$-0.0249$

$-0.0450$

$-0.0450$

0.002338

Mean

Std Dev

Std Err Mean

upper 95\% Mean

0.002625

0.0020511

N

0.0023169

0.0097503

0.0008182

0.0039345

0.0006993 
WSRC-TR-2004-00576

Revision 0

\section{Exhibit A2. Histograms and Descriptive Statistics for Cold Chem Measurements of} Calibration and Bench Standards

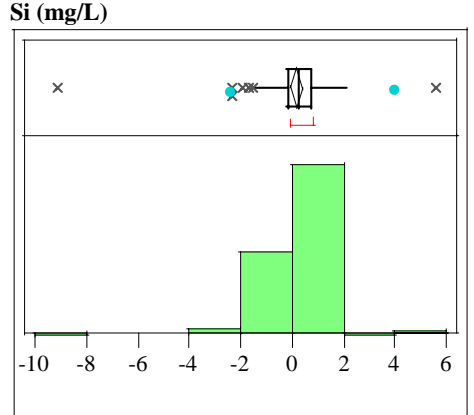

Quantiles

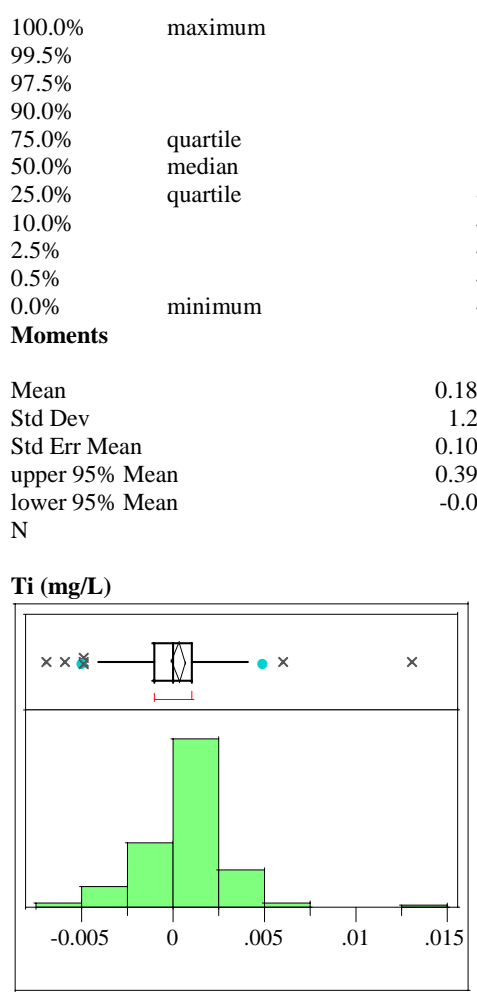

Quantiles

$100.0 \%$

$99.5 \%$

$97.5 \%$

$90.0 \%$

$75.0 \%$

$50.0 \%$

$25.0 \%$

$10.0 \%$

$2.5 \%$

$0.5 \%$

$0.0 \%$

Moments

maximum

quartile

median
quartile

minimum

Mean

Std Dev

Std Err Mean

upper $95 \%$ Mean

lower 95\% Mean

$\mathrm{N}$

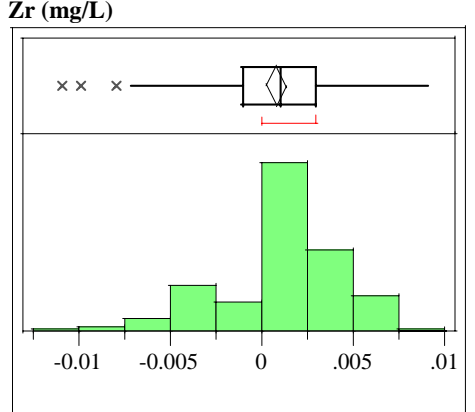

Quantiles

$\begin{array}{lcr}100.0 \% & \text { maximum } & 0.0090 \\ 99.5 \% & & 0.0090 \\ 97.5 \% & & 0.0064 \\ 90.0 \% & & 0.0047 \\ 75.0 \% & \text { quartile } & 0.0030 \\ 50.0 \% & \text { median } & 0.0010 \\ 25.0 \% & \text { quartile } & -0.0010 \\ 10.0 \% & & -0.0040 \\ 2.5 \% & & -0.0074 \\ 0.5 \% & & -0.0110 \\ 0.0 \% & \text { minimum } & -0.0110 \\ \text { Moments } & & \\ & & \\ \text { Mean } & & 0.0007887 \\ \text { Std Dev } & & 0.0034288 \\ \text { Std Err Mean } & & 0.0002877 \\ \text { upper 95\% Mean } & 0.0013576 \\ \text { lower 95\% Mean } & 0.0002199 \\ \text { N } & \end{array}$

U (mg/L)

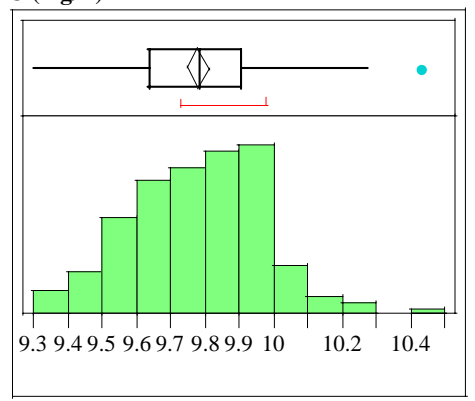

Quantiles

$100.0 \% \quad$ maximum $\quad 10.435$

$\begin{array}{ll}99.5 \% & 10.435\end{array}$

$\begin{array}{ll}97.5 \% & 10.203\end{array}$

10.002

$\begin{array}{lll}75.0 \% & \text { quartile } & 9.908\end{array}$

$50.0 \%$ median $\quad 9.787$

$\begin{array}{ll}25.0 \% & \text { quartile }\end{array}$

$10.0 \% \quad 9.523$

$2.5 \% \quad 9.376$

$0.5 \% \quad 9.306$

$\begin{array}{lll}0.0 \% & \text { minimum } & 9.306\end{array}$

9.7802887

Std Dev $\quad 0.1946235$

Std Err Mean $\quad 0.0163324$

upper 95\% Mean 9.8125769

lower 95\% Mean 9.7480006 
WSRC-TR-2004-00576

Revision 0

Exhibit A3. Components of Variance for Cold Chem Measurements of Calibration and Bench Standards

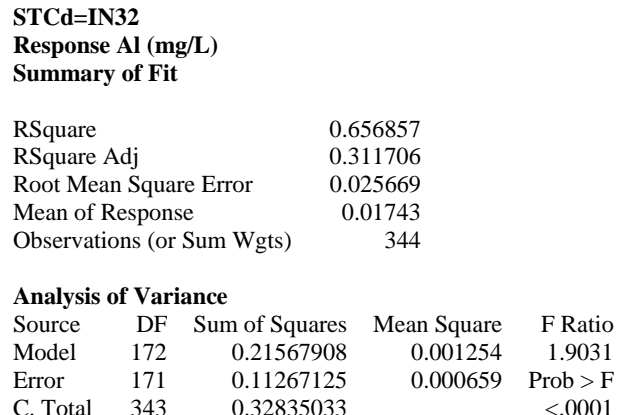

\begin{tabular}{lrr}
\multicolumn{3}{l}{ Variance Component Estimates } \\
Component & Var Comp Est & Percent of Total \\
Batch\&Random & 0.000299 & 31.234 \\
Residual & 0.000659 & 68.766 \\
Total & 0.000958 & 100.000
\end{tabular}

These estimates based on equating Mean Squares to Expected Value.

Response B (mg/L)

Summary of Fi

\begin{tabular}{|c|c|c|c|c|}
\hline \multicolumn{3}{|l|}{ RSquare } & \multicolumn{2}{|c|}{0.947822} \\
\hline \multicolumn{3}{|c|}{ RSquare Adj } & \multicolumn{2}{|c|}{0.895339} \\
\hline \multicolumn{3}{|c|}{ Root Mean Square Error } & \multicolumn{2}{|c|}{0.011189} \\
\hline \multicolumn{3}{|c|}{ Mean of Response } & \multicolumn{2}{|c|}{-0.00017} \\
\hline \multicolumn{3}{|c|}{ Observations (or Sum Wgts) } & 344 & \\
\hline \multicolumn{5}{|c|}{ Analysis of Variance } \\
\hline Source & $\mathrm{DF}$ & Sum of Squares & Mean Square & F Ratio \\
\hline Model & 172 & 0.38887206 & 0.002261 & 18.0596 \\
\hline Error & 171 & 0.02140750 & 0.000125 & Prob $>F$ \\
\hline C. Total & 343 & 0.41027956 & & $<.0001$ \\
\hline
\end{tabular}

\begin{tabular}{lrr}
\multicolumn{3}{c}{ Variance Component Estimates } \\
Component & Var Comp Est & Percent of Total \\
Batch\&Random & 0.001074 & 89.562 \\
Residual & 0.000125 & 10.438 \\
Total & 0.001199 & 100.000
\end{tabular}

These estimates based on equating Mean Squares to Expected Value.

Response Ca (mg/L)

Summary of Fit

$\begin{array}{lr}\text { RSquare } & 0.923231 \\ \text { RSquare Adj } & 0.846012 \\ \text { Root Mean Square Error } & 0.043614 \\ \text { Mean of Response } & 0.115875 \\ \text { Observations (or Sum Wgts) } & 344\end{array}$

Analysis of Variance

Source DF Sum of Squares Mean Square F Ratio

$\begin{array}{lllll}\text { Model } & 172 & 3.9116654 & 0.022742 & 11.9561\end{array}$

$\begin{array}{lllll}\text { Error } & 171 & 0.3252663 & 0.001902 & \text { Prob }>\text { F }\end{array}$

$\begin{array}{lllll}\text { C. Total } & 343 & 4.2369316 & \end{array}$

Variance Component Estimates

Component Var Comp Est Percent of Total

Batch\&Random $\quad 0.010481 \quad 84.640$

$\begin{array}{lll}\text { Residual } & 0.001902 & 15.360\end{array}$

Total $\quad 0.012383 \quad 100.000$

These estimates based on equating Mean Squares to Expected Value.

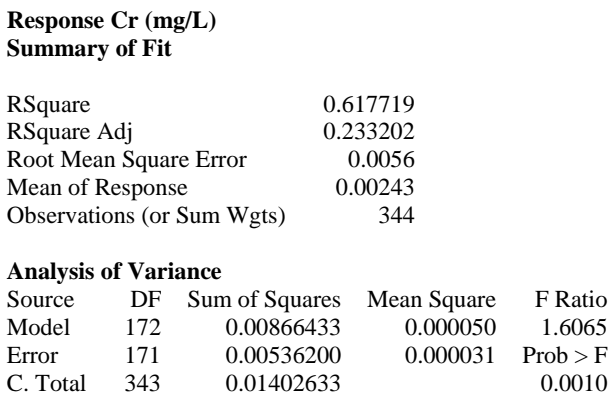

RSquare

0.617719

RSquare Adj

0.233202

Root Mean Square Error $\quad 0.0056$

Mean of Response

Observations (or Sum Wgts) $\quad 344$

Analysis of Variance

$\begin{array}{lrrrr}\text { Source } & \text { DF } & \text { Sum of Squares } & \text { Mean Square } & \text { F Ratio } \\ \text { Model } & 172 & 0.00866433 & 0.000050 & 1.6065 \\ \text { Error } & 171 & 0.00536200 & 0.000031 & \text { Prob > F } \\ \text { C. Total } & 343 & 0.01402633 & & 0.0010\end{array}$

C. Total $343 \quad 0.01402633$

\begin{tabular}{lrr}
\multicolumn{3}{l}{ Variance Component Estimates } \\
Component & Var Comp Est & Percent of Total \\
Batch\&Random & 0.00001 & 23.373 \\
Residual & 0.000031 & 76.627 \\
Total & 0.000041 & 100.000
\end{tabular}

These estimates based on equating Mean Squares to Expected Value.

Response Cu (mg/L)

Summary of Fit

RSquare

0.878763

0.756817

Root Mean Square Error $\quad 0.002074$

Mean of Response 0.000901

Observations (or Sum Wgts) 344

Analysis of Variance

Source DF Sum of Squares Mean Square F Ratio

$\begin{array}{lrrrr}\text { Model } & 172 & 0.00533114 & 0.000031 & 7.2062\end{array}$

$\begin{array}{lllll}\text { Error } & 171 & 0.00073550 & 0.000004 & \text { Prob }>\text { F }\end{array}$

$\begin{array}{llll}\text { C. Total } 343 \quad 0.00606664 & <.0001\end{array}$

\begin{tabular}{lrr}
\multicolumn{3}{l}{ Variance Component Estimates } \\
Component & Var Comp Est & Percent of Total \\
Batch\&Random & 0.000013 & 75.736 \\
Residual & 0.000004 & 24.264 \\
Total & 0.000018 & 100.000
\end{tabular}

These estimates based on equating Mean Squares to Expected Value.

Response Fe (mg/L)

Summary of Fit

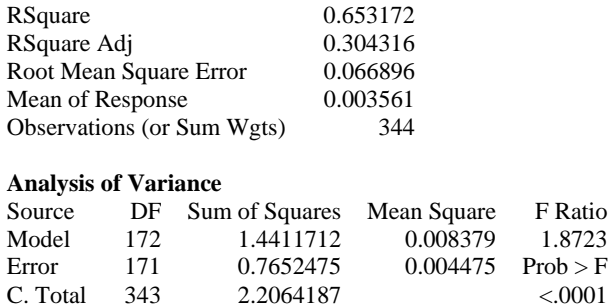

Variance Component Estimates

Component Var Comp Est Percent of Total

Batch\&Random $\quad 0.001963 \quad 30.494$

$\begin{array}{lll}\text { Residual } & 0.004475 & 69.506\end{array}$

Total $0.006438 \quad 100.000$

These estimates based on equating Mean Squares to Expected Value. 
WSRC-TR-2004-00576

Revision 0

Exhibit A3. Components of Variance for Cold Chem Measurements of Calibration and Bench Standards

Response K (mg/L)

Summary of Fit

$\begin{array}{lr}\text { RSquare } & 0.757873 \\ \text { RSquare Adj } & 0.514331 \\ \text { Root Mean Square Error } & 0.045823 \\ \text { Mean of Response } & 0.038078 \\ \text { Observations (or Sum Wgts) } & 344\end{array}$

Analysis of Variance

Source DF Sum of Squares Mean Square F Ratio

$\begin{array}{lllll}\text { Model } & 172 & 1.1238631 & 0.006534 & 3.1119\end{array}$

$\begin{array}{lllll}\text { Error } & 171 & 0.3590537 & 0.002100 & \text { Prob }>\text { F }\end{array}$

$\begin{array}{llll}\text { C. Total } & 343 & 1.4829169 & <.0001\end{array}$

Variance Component Estimates

$\begin{array}{lrr}\text { Component } & \text { Var Comp Est } & \text { Percent of Total } \\ \text { Batch\&Random } & 0.00223 & 51.507 \\ \text { Residual } & 0.0021 & 48.493 \\ \text { Total } & 0.00433 & 100.000\end{array}$

These estimates based on equating Mean Squares to Expected Value.

Response Li (mg/L)

Summary of Fi

RSquare $\quad 0.77327$

RSquare Adj $\quad 0.545214$

Root Mean Square Error $\quad 0.001511$

Mean of Response

Observations (or Sum Wgts) 344

Analysis of Variance

Source DF Sum of Squares Mean Square F Ratio

$\begin{array}{lllll}\text { Model } & 172 & 0.00133181 & 0.0000077 & 3.3907\end{array}$

$\begin{array}{lllll}\text { Error } & 171 & 0.00039050 & 0.0000023 & \text { Prob }>\text { F }\end{array}$

C. Total $343 \quad 0.00172231$
Response Mn (mg/L)

Summary of Fit

RSquare

0.505088

RSquare Adj $\quad 0.007283$

Root Mean Square Error $\quad 0.011235$

Mean of Response $\quad 0.00148$

Observations (or Sum Wgts) 344

Analysis of Variance

Source DF Sum of Squares Mean Square F Ratio

$\begin{array}{lllll}\text { Model } & 172 & 0.02202886 & 0.000128 & 1.0146\end{array}$

$\begin{array}{lllll}\text { Error } & 171 & 0.02158500 & 0.000126 & \text { Prob }>\text { F }\end{array}$

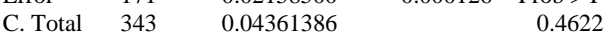

\begin{tabular}{lrr}
\multicolumn{3}{l}{ Variance Component Estimates } \\
Component & Var Comp Est & Percent of Total \\
Batch\&Random & $9.288 \mathrm{e}-7$ & 0.730 \\
Residual & 0.000126 & 99.270 \\
Total & 0.000127 & 100.000
\end{tabular}

These estimates based on equating Mean Squares to Expected Value.

Response Na (mg/L)

Summary of Fit

RSquare

0.605544

RSquare Adj

0.208782

Root Mean Square Error $\quad 0.077498$

Mean of Response

0.019855

Observations (or Sum Wgts) 344

Analysis of Variance

Source DF Sum of Squares Mean Square F Ratio

$\begin{array}{lrrrr}\text { Model } & 172 & 1.5766100 & 0.009166 & 1.5262\end{array}$

$\begin{array}{lllll}\text { Error } & 171 & 1.0270148 & 0.006006 & \text { Prob }>\text { F }\end{array}$

$\begin{array}{llll}\text { C. Total } & 343 & 2.6036247 & 0.0029\end{array}$ \begin{tabular}{lrr}
\multicolumn{3}{c}{ Variance Component Estimates } \\
Component & Var Comp Est & Percent of Total \\
Batch\&Random & 0.000003 & 54.595 \\
Residual & 0.000002 & 45.405 \\
Total & 0.000005 & 100.000
\end{tabular}

These estimates based on equating Mean Squares to Expected Value.

Response Mg (mg/L)

Summary of Fit

RSquare

Root Mean Square Error $\quad 0.00403$

Mean of Response

Observations (or Sum Wgts)

0.001398

344

Analysis of Variance

Source DF Sum of Squares Mean Square F Ratio

$\begin{array}{lrrrr}\text { Model } & 172 & 0.00470669 & 0.000027 & 1.6846\end{array}$

$\begin{array}{lllll}\text { Error } & 171 & 0.00277775 & 0.000016 & \text { Prob }>\text { F }\end{array}$

$\begin{array}{llll}\text { C. Total } & 343 & 0.00748444 & 0.0003\end{array}$

Variance Component Estimates

$\begin{array}{lrr}\text { Component } & \text { Var Comp Est } & \text { Percent of Total } \\ \text { Batch\&Random } & 0.000006 & 25.612 \\ \text { Residual } & 0.000016 & 74.388 \\ \text { Total } & 0.000022 & 100.000\end{array}$

These estimates based on equating Mean Squares to Expected Value.
Variance Component Estimates

$\begin{array}{lrc}\text { Component } & \text { Var Comp Est } & \text { Percent of Total } \\ \text { Batch\&Random } & 0.001589 & 20.927 \\ \text { Residual } & 0.006006 & 79.073 \\ \text { Total } & 0.007595 & 100.000 \\ \text { These estimates based on equating } & \text { Mean Squares to Expected Value. }\end{array}$

Response Ni (mg/L)

Summary of Fit

RSquare

0.723613

RSquare Adj

0.44561

Root Mean Square Error $\quad 0.006168$

Mean of Response

0.001887

Observations (or Sum Wgts) $\quad 344$

$\begin{array}{lrrrr}\text { Analysis of Variance } & & & \\ \text { Source } & \text { DF } & \text { Sum of Squares } & \text { Mean Square } & \text { F Ratio } \\ \text { Model } & 172 & 0.01703283 & 0.000099 & 2.6029 \\ \text { Error } & 171 & 0.00650575 & 0.000038 & \text { Prob }>\text { F } \\ \text { C. Total } & 343 & 0.02353858 & & <.0001\end{array}$

Variance Component Estimates

$\begin{array}{lrc}\text { Component } & \text { Var Comp Est } & \text { Percent of Total } \\ \text { Batch\&Random } & 0.000031 & 44.634 \\ \text { Residual } & 0.000038 & 55.366 \\ \text { Total } & 0.000069 & 100.000 \\ \text { These estimates based on equating } & \text { Mean Squares to Expected Value. }\end{array}$


WSRC-TR-2004-00576

Revision 0

Exhibit A3. Components of Variance for Cold Chem Measurements of Calibration and Bench Standards

Response Si (mg/L)

Summary of Fit

$\begin{array}{lr}\text { RSquare } & 0.944741 \\ \text { RSquare Adj } & 0.889158 \\ \text { Root Mean Square Error } & 0.174973 \\ \text { Mean of Response } & -0.0845 \\ \text { Observations (or Sum Wgts) } & 344\end{array}$

Analysis of Variance

Source DF Sum of Squares Mean Square F Ratio

$\begin{array}{lllll}\text { Model } & 172 & 89.504563 & 0.520375 & 16.9971\end{array}$

$\begin{array}{lllll}\text { Error } & 171 & 5.235263 & 0.030616 & \text { Prob }>\text { F }\end{array}$

$\begin{array}{llll}\text { C. Total } & 343 & 94.739826 & <.0001\end{array}$

Variance Component Estimates

$\begin{array}{lrr}\text { Component } & \text { Var Comp Est } & \text { Percent of Total } \\ \text { Batch\&Random } & 0.24632 & 88.945 \\ \text { Residual } & 0.030616 & 11.055 \\ \text { Total } & 0.276936 & 100.000\end{array}$

These estimates based on equating Mean Squares to Expected Value.

Response Ti (mg/L)

Summary of Fit

RSquare

RSquare Adj 0.717354

Root Mean Square Error $\quad 0.000932$

Mean of Response $\quad 0.00073$

Observations (or Sum Wgts) $\quad 344$

Analysis of Variance

Source DF Sum of Squares Mean Square F Ratio

$\begin{array}{lllll}\text { Model } & 172 & 0.00090536 & 0.0000053 & 6.0612\end{array}$

$\begin{array}{lllll}\text { Error } & 171 & 0.00014850 & 8.6842 \mathrm{e}-7 & \text { Prob }>\text { F }\end{array}$

$\begin{array}{llll}\text { C. Total } & 343 & 0.00105386 & <.0001\end{array}$

Variance Component Estimates

Component Var Comp Est Percent of Total

Batch\&Random $\quad 0.000002 \quad 71.795$

Residual $\quad 8.684 \mathrm{e}-7 \quad 28.205$

$\begin{array}{lll}\text { Total } & 0.000003 & 100.000\end{array}$

These estimates based on equating Mean Squares to Expected Value.

Response Zr (mg/L)

Summary of Fit

RSquare

RSquare Adj

Root Mean Square Erro
Mean of Response

0.932491

0.001188

Observations (or Sum Wgts)

344

Analysis of Variance

$\begin{array}{lrrrr}\text { Source } & \text { DF } & \text { Sum of Squares } & \text { Mean Square } & \text { F Ratio } \\ \text { Model } & 172 & 0.00693399 & 0.000040 & 28.5452 \\ \text { Error } & 171 & 0.00024150 & 0.000001 & \text { Prob }>\text { F } \\ \text { C. Total } & 343 & 0.00717549 & & <.0001\end{array}$

Variance Component Estimates

Component Var Comp Est Percent of Total

Batch\&Random $\quad 0.00002 \quad 93.268$

$\begin{array}{lrr}\text { Residual } & 0.000001 & 6.732\end{array}$

Total

0.00002 100.000

These estimates based on equating Mean Squares to Expected Value.

\begin{tabular}{|c|c|c|c|c|c|}
\hline \multicolumn{6}{|c|}{$\begin{array}{l}\text { Response U (mg/L) } \\
\text { Summary of Fit }\end{array}$} \\
\hline \multicolumn{3}{|l|}{ RSquare } & \multicolumn{3}{|c|}{0.872907} \\
\hline \multicolumn{3}{|c|}{ RSquare Adj } & \multicolumn{3}{|c|}{0.745071} \\
\hline \multicolumn{3}{|c|}{ Root Mean Square Error } & \multicolumn{3}{|c|}{0.093895} \\
\hline \multicolumn{3}{|c|}{ Mean of Response } & \multicolumn{3}{|c|}{0.109384} \\
\hline \multicolumn{3}{|c|}{ Observations (or Sum Wgts) } & \multicolumn{3}{|c|}{344} \\
\hline \multicolumn{6}{|c|}{ Analysis of Variance } \\
\hline Source & DF & Sum of Squ & & Mean Square & F Ratio \\
\hline Model & 172 & 10.35 & & 0.060201 & 6.8283 \\
\hline Error & 171 & 1.50 & 588 & 0.008816 & Prob $>$ F \\
\hline C. Total & 343 & 11.86 & 2075 & & $<.0001$ \\
\hline
\end{tabular}

\begin{tabular}{lrr}
\multicolumn{3}{l}{ Variance Component Estimates } \\
Component & Var Comp Est & Percent of Total \\
Batch\&Random & 0.025843 & 74.563 \\
Residual & 0.008816 & 25.437 \\
Total & 0.03466 & 100.000
\end{tabular}

These estimates based on equating Mean Squares to Expected Value.

STCd=IN35

Response Al (mg/L)

Summary of Fit

RSquare

0.921157

RSquare A

0.851565

Root Mean Square Error $\quad 0.054285$

Mean of Response $\quad 9.978497$

Observations (or Sum Wgts) $\quad 370$

Analysis of Variance

$\begin{array}{lrrrr}\text { Source } & \text { DF } & \text { Sum of Squares } & \text { Mean Square } & \text { F Ratio } \\ \text { Model } & 173 & 6.7481716 & 0.039007 & 13.2367 \\ \text { Error } & 196 & 0.5775869 & 0.002947 & \text { Prob }>\text { F } \\ \text { C. Total } & 369 & 7.3257585 & & <.0001\end{array}$

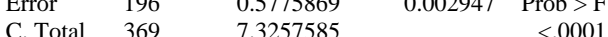

Variance Component Estimates

$\begin{array}{lrr}\text { Component } & \text { Var Comp Est } & \text { Percent of Total } \\ \text { Batch\&Random } & 0.016965 & 85.200 \\ \text { Residual } & 0.002947 & 14.800 \\ \text { Total } & 0.019912 & 100.000 \\ \text { These estimates based on equating } & \text { Mean Squares to Expected Value. }\end{array}$

Response B (mg/L)

Summary of Fit

0.968199

RSquare Adj

0.940129

Root Mean Square Error $\quad 0.009402$

Mean of Response $\quad-0.00419$

Observations (or Sum Wgts) $\quad 370$

Analysis of Variance

$\begin{array}{lrrrr}\text { Source } & \text { DF } & \text { Sum of Squares } & \text { Mean Square } & \text { F Ratio } \\ \text { Model } & 173 & 0.52752767 & 0.003049 & 34.4929 \\ \text { Error } & 196 & 0.01732708 & 0.000088 & \text { Prob }>\text { F } \\ \text { C. Total } & 369 & 0.54485476 & & <.0001\end{array}$

Variance Component Estimates

Component Var Comp Est Percent of Total

Batch\&Random $\quad 0.001393 \quad 94.032$

$\begin{array}{lrr}\text { Residual } & 0.000088 & 5.968\end{array}$

Total $\quad 0.001481 \quad 100.000$

These estimates based on equating Mean Squares to Expected Value. 
WSRC-TR-2004-00576

Revision 0

Exhibit A3. Components of Variance for Cold Chem Measurements of Calibration and Bench Standards

Response Ca (mg/L) Summary of Fit

$\begin{array}{lr}\text { RSquare } & 0.931883 \\ \text { RSquare Adj } & 0.871759 \\ \text { Root Mean Square Error } & 0.016593 \\ \text { Mean of Response } & 1.995949 \\ \text { Observations (or Sum Wgts) } & 370\end{array}$

Analysis of Variance

Source DF Sum of Squares Mean Square F Ratio

$\begin{array}{lllll}\text { Model } & 173 & 0.73821894 & 0.004267 & 15.4994\end{array}$

$\begin{array}{lllll}\text { Error } & 196 & 0.05396108 & 0.000275 & \text { Prob }>\text { F }\end{array}$

$\begin{array}{llll}\text { C. Total } & 369 & 0.79218002 & <.0001\end{array}$

Variance Component Estimates

$\begin{array}{lrr}\text { Component } & \text { Var Comp Est } & \text { Percent of Total } \\ \text { Batch\&Random } & 0.001878 & 87.215 \\ \text { Residual } & 0.000275 & 12.785 \\ \text { Total } & 0.002153 & 100.000\end{array}$

These estimates based on equating Mean Squares to Expected Value.

Response Cr (mg/L)

Summary of Fit

RSquare

RSquare Adj

Root Mean Square Error

Mean of Response

Observations (or Sum Wgts)

$$
\begin{array}{r}
0.91563 \\
0.841161 \\
0.01186 \\
2.001754 \\
370
\end{array}
$$

Analysis of Variance

Source DF Sum of Squares Mean Square F Ratio

$\begin{array}{llrrr}\text { Model } & 173 & 0.29919187 & 0.001729 & 12.2954\end{array}$

$\begin{array}{lllll}\text { Error } & 196 & 0.02756875 & 0.000141 & \text { Prob }>\text { F }\end{array}$

C. Total $369 \quad 0.32676062$ \begin{tabular}{lrr}
\multicolumn{3}{c}{ Variance Component Estimates } \\
Component & Var Comp Est & Percent of Total \\
Batch\&Random & 0.000747 & 84.162 \\
Residual & 0.000141 & 15.838 \\
Total & 0.000888 & 100.000
\end{tabular}

$\begin{array}{lcc}\text { Total } & 0.000888 & 100.000 \\ \text { These estimates based on equating Mean Squares to Expected Value. }\end{array}$

Response Cu (mg/L)

Summary of Fit

RSquare

Root Mean Square Error $\quad 0.006369$

Mean of Response $\quad 1.004914$

Observations (or Sum Wgts) 370

Analysis of Variance

$\begin{array}{lrrrr}\text { Source } & \text { DF } & \text { Sum of Squares } & \text { Mean Square } & \text { F Ratio } \\ \text { Model } & 173 & 0.08354415 & 0.000483 & 11.9042\end{array}$

$\begin{array}{lllll}\text { Model } & 173 & 0.08354415 & 0.000483 & 11.9042 \\ \text { Error } & 196 & 0.00795108 & 0.000041 & \text { Prob }>\text { F }\end{array}$

\begin{tabular}{|c|c|c|c|c|c|}
\hline \multicolumn{6}{|c|}{$\begin{array}{l}\text { Response Fe }(\mathrm{mg} / \mathrm{L}) \\
\text { Summary of Fit }\end{array}$} \\
\hline \multicolumn{3}{|l|}{ RSquare } & \multicolumn{3}{|c|}{0.907255} \\
\hline \multicolumn{3}{|c|}{ RSquare Adj } & \multicolumn{3}{|c|}{0.825393} \\
\hline \multicolumn{3}{|c|}{ Root Mean Square Error } & \multicolumn{3}{|c|}{0.115559} \\
\hline \multicolumn{3}{|c|}{ Mean of Response } & \multicolumn{3}{|c|}{19.98021} \\
\hline \multicolumn{3}{|c|}{ Observations (or Sum Wgts) } & \multicolumn{3}{|c|}{370} \\
\hline \multicolumn{6}{|c|}{ Analysis of Variance } \\
\hline Source & DF & Sum of Sq & & Mean Square & F Ratio \\
\hline Model & 173 & 25.60 & & 0.147997 & 11.0827 \\
\hline Error & 196 & 2.61 & & 0.013354 & Prob $>F$ \\
\hline C. Total & 369 & 28.22 & 836 & & $<.0001$ \\
\hline
\end{tabular}

$\begin{array}{llll}\text { C. Total } & 369 & 0.09149523 & <.0001\end{array}$

Variance Component Estimates

$\begin{array}{lrr}\text { Component } & \text { Var Comp Est } & \text { Percent of Total } \\ \text { Batch\&Random } & 0.000208 & 83.687 \\ \text { Residual } & 0.000041 & 16.313 \\ \text { Total } & 0.000249 & 100.000\end{array}$

These estimates based on equating Mean Squares to Expected Value.

\begin{tabular}{lrr}
\multicolumn{3}{l}{ Variance Component Estimates } \\
Component & Var Comp Est & Percent of Total \\
Batch\&Random & 0.063345 & 82.589 \\
Residual & 0.013354 & 17.411 \\
Total & 0.076699 & 100.000
\end{tabular}

These estimates based on equating Mean Squares to Expected Value.

Response K (mg/L)

Summary of Fit

RSquare

0.717264

RSquare Adj

0.467707

Root Mean Square Error $\quad 0.054612$

Mean of Response

0.017919

Observations (or Sum Wgts)

370

Analysis of Variance

Source DF Sum of Squares Mean Square F Ratio

$\begin{array}{lrrrr}\text { Model } & 173 & 1.4829492 & 0.008572 & 2.8741\end{array}$

$\begin{array}{lllll}\text { Error } & 196 & 0.5845584 & 0.002982 & \text { Prob }>\text { F }\end{array}$

$\begin{array}{llll}\text { C. Total } 369 & 2.0675076 & <.0001\end{array}$

\begin{tabular}{lrr}
\multicolumn{3}{l}{ Variance Component Estimates } \\
Component & Var Comp Est & Percent of Total \\
Batch\&Random & 0.00263 & 46.857 \\
Residual & 0.002982 & 53.143 \\
Total & 0.005612 & 100.000 \\
These estimates based on equating & Mean Squares to Expected Value.
\end{tabular}

Response Li (mg/L)

Summary of Fit

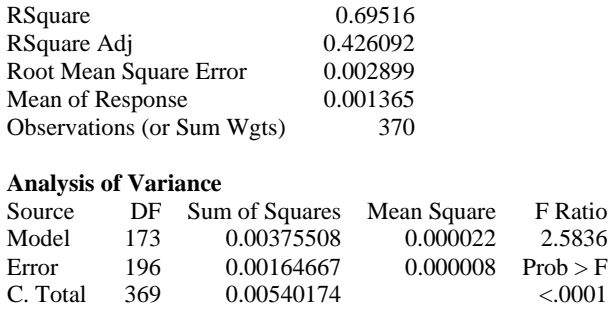

Variance Component Estimates

$\begin{array}{lrc}\text { Component } & \text { Var Comp Est } & \text { Percent of Total } \\ \text { Batch\&Random } & 0.000006 & 42.694 \\ \text { Residual } & 0.000008 & 57.306 \\ \text { Total } & 0.000015 & 100.000 \\ \text { These estimates based on equating } & \text { Mean Squares to Expected Value. }\end{array}$


WSRC-TR-2004-00576

Revision 0

Exhibit A3. Components of Variance for Cold Chem Measurements of Calibration and Bench Standards

Response Mg (mg/L) Summary of Fit

$\begin{array}{lr}\text { RSquare } & 0.925357 \\ \text { RSquare Adj } & 0.859474 \\ \text { Root Mean Square Error } & 0.008486 \\ \text { Mean of Response } & 1.009135 \\ \text { Observations (or Sum Wgts) } & 370\end{array}$

Analysis of Variance

Source DF Sum of Squares Mean Square F Ratio

$\begin{array}{lllll}\text { Model } & 173 & 0.17498808 & 0.001011 & 14.0453\end{array}$

$\begin{array}{lllll}\text { Error } & 196 & 0.01411517 & 0.000072 & \text { Prob }>\text { F }\end{array}$

$\begin{array}{llll}\text { C. Total } & 369 & 0.18910324 & <.0001\end{array}$

Variance Component Estimates

$\begin{array}{lrr}\text { Component } & \text { Var Comp Est } & \text { Percent of Total } \\ \text { Batch\&Random } & 0.000442 & 85.989 \\ \text { Residual } & 0.000072 & 14.011 \\ \text { Total } & 0.000514 & 100.000\end{array}$

These estimates based on equating Mean Squares to Expected Value.

Response Mn (mg/L)

Summary of Fit

RSquare $\quad 0.922755$

RSquare Adj $\quad 0.854574$

Root Mean Square Error $\quad 0.006251$

Mean of Response

Observations (or Sum Wgts) 370

Analysis of Variance

Source DF Sum of Squares Mean Square F Ratio

$\begin{array}{lllll}\text { Model } & 173 & 0.09148764 & 0.000529 & 13.5339\end{array}$

$\begin{array}{lllll}\text { Error } & 196 & 0.00765858 & 0.000039 & \text { Prob }>\text { F }\end{array}$

C. Total $369 \quad 0.09914622$

$<.0001$

Variance Component Estimates

$\begin{array}{lrr}\text { Component } & \text { Var Comp Est } & \text { Percent of Total } \\ \text { Batch\&Random } & 0.00023 & 85.501 \\ \text { Residual } & 0.000039 & 14.499 \\ \text { Total } & 0.000269 & 100.000\end{array}$

Total $0.000269 \quad 100.000$

These estimates based on equating Mean Squares to Expected Value.

Response Na (mg/L)

Summary of Fit

RSquare

0.947381

RSquare Adj 0.900937

Root Mean Square Error $\quad 0.114991$

Mean of Response 19.97589

Observations (or Sum Wgts) 370

Analysis of Variance

$\begin{array}{lrrrr}\text { Source } & \text { DF } & \text { Sum of Squares } & \text { Mean Square } & \text { F Ratio } \\ \text { Model } & 173 & 46.661920 & 0.269722 & 20.3982 \\ \text { Error } & 196 & 2.591672 & 0.013223 & \text { Prob }>\text { F } \\ \text { C. Total } & 369 & 49.253593 & & <.0001\end{array}$

C. Total $369-49.253593$

Variance Component Estimates

$\begin{array}{lrr}\text { Component } & \text { Var Comp Est } & \text { Percent of Total } \\ \text { Batch\&Random } & 0.120675 & 90.125 \\ \text { Residual } & 0.013223 & 9.875 \\ \text { Total } & 0.133898 & 100.000\end{array}$

These estimates based on equating Mean Squares to Expected Value.

\begin{tabular}{|c|c|c|c|c|}
\hline \multicolumn{5}{|c|}{$\begin{array}{l}\text { Response Ni (mg/L) } \\
\text { Summary of Fit }\end{array}$} \\
\hline \multicolumn{3}{|l|}{ RSquare } & \multicolumn{2}{|c|}{0.859103} \\
\hline \multicolumn{3}{|c|}{ RSquare Adj } & \multicolumn{2}{|c|}{0.73474} \\
\hline \multicolumn{3}{|c|}{ Root Mean Square Error } & \multicolumn{2}{|c|}{0.017688} \\
\hline \multicolumn{3}{|c|}{ Mean of Response } & \multicolumn{2}{|c|}{1.986154} \\
\hline \multicolumn{3}{|c|}{ Observations (or Sum Wgts) } & \multicolumn{2}{|c|}{370} \\
\hline \multicolumn{5}{|c|}{ Analysis of Variance } \\
\hline Source & DF & Sum of Squares & Mean Square & F Ratio \\
\hline Model & 173 & 0.37389730 & 0.002161 & 6.9080 \\
\hline Error & 196 & 0.06132092 & 0.000313 & Prob $>$ F \\
\hline C. Total & 369 & 0.43521822 & & $<.0001$ \\
\hline
\end{tabular}

\begin{tabular}{lrr}
\multicolumn{3}{l}{ Variance Component Estimates } \\
Component & Var Comp Est & Percent of Total \\
Batch\&Random & 0.00087 & 73.542 \\
Residual & 0.000313 & 26.458 \\
Total & 0.001182 & 100.000
\end{tabular}

These estimates based on equating Mean Squares to Expected Value.

Response Si (mg/L)

Summary of Fit

RSquare

0.98588

RSquare Adj $\quad 0.973416$

Root Mean Square Error $\quad 0.078302$

Mean of Response $\quad-0.60047$

Observations (or Sum Wgts) $\quad 370$

Analysis of Variance

Source DF Sum of Squares Mean Square F Ratio

$\begin{array}{lrrrr}\text { Model } & 173 & 83.903511 & 0.484991 & 79.1017\end{array}$

$\begin{array}{lllll}\text { Error } & 196 & 1.201723 & 0.006131 & \text { Prob }>\text { F }\end{array}$

$\begin{array}{llll}\text { C. Total } 369<.0001 & 85.105234\end{array}$ \begin{tabular}{lrr}
\multicolumn{3}{l}{ Variance Component Estimates } \\
Component & Var Comp Est & Percent of Total \\
Batch\&Random & 0.225288 & 97.351 \\
Residual & 0.006131 & 2.649 \\
Total & 0.23142 & 100.000 \\
These estimates based on equating & Mean Squares to Expected Value.
\end{tabular}

Response Ti (mg/L)

Summary of Fit

RSquare

0.916756

RSquare Adj

0.843281

Root Mean Square Error $\quad 0.011467$

Mean of Response 1.989592

Observations (or Sum Wgts) $\quad 370$

$\begin{array}{lrrrr}\text { Analysis of Variance } & & & \\ \text { Source } & \text { DF } & \text { Sum of Squares } & \text { Mean Square } & \text { F Ratio } \\ \text { Model } & 173 & 0.28381796 & 0.001641 & 12.4770 \\ \text { Error } & 196 & 0.02577142 & 0.000131 & \text { Prob }>\text { F } \\ \text { C. Total } & 369 & 0.30958938 & & <.0001\end{array}$

Variance Component Estimates

$\begin{array}{lrc}\text { Component } & \text { Var Comp Est } & \text { Percent of Total } \\ \text { Batch\&Random } & 0.00071 & 84.374 \\ \text { Residual } & 0.000131 & 15.626 \\ \text { Total } & 0.000841 & 100.000 \\ \text { These estimates based on equating } & \text { Mean Squares to Expected Value. }\end{array}$


WSRC-TR-2004-00576

Revision 0

Exhibit A3. Components of Variance for Cold Chem Measurements of Calibration and Bench Standards

Response Zr (mg/L)

Summary of Fit

$\begin{array}{lr}\text { RSquare } & 0.951275 \\ \text { RSquare Adj } & 0.908268 \\ \text { Root Mean Square Error } & 0.011823 \\ \text { Mean of Response } & 2.015308 \\ \text { Observations (or Sum Wgts) } & 370\end{array}$

Analysis of Variance

Source DF Sum of Squares Mean Square F Ratio

$\begin{array}{lllll}\text { Model } & 173 & 0.53486871 & 0.003092 & 22.1191\end{array}$

$\begin{array}{lllll}\text { Error } & 196 & 0.02739617 & 0.000140 & \text { Prob }>\text { F }\end{array}$

$\begin{array}{llll}\text { C. Total } & 369 & 0.56226488 & <.0001\end{array}$

Variance Component Estimates

$\begin{array}{lrr}\text { Component } & \text { Var Comp Est } & \text { Percent of Total } \\ \text { Batch\&Random } & 0.001389 & 90.856 \\ \text { Residual } & 0.00014 & 9.144 \\ \text { Total } & 0.001529 & 100.000\end{array}$

These estimates based on equating Mean Squares to Expected Value.

Response U (mg/L)

Summary of Fit

\begin{tabular}{|c|c|c|c|c|}
\hline \multicolumn{3}{|l|}{ RSquare } & \multicolumn{2}{|c|}{0.644226} \\
\hline \multicolumn{3}{|c|}{ RSquare Adj } & \multicolumn{2}{|c|}{0.330201} \\
\hline \multicolumn{3}{|c|}{ Root Mean Square Error } & \multicolumn{2}{|c|}{0.226897} \\
\hline \multicolumn{3}{|c|}{ Mean of Response } & \multicolumn{2}{|c|}{0.116586} \\
\hline \multicolumn{3}{|c|}{ Observations (or Sum Wgts) } & \multicolumn{2}{|c|}{370} \\
\hline \multicolumn{5}{|c|}{ Analysis of Variance } \\
\hline Source & $\mathrm{DF}$ & Sum of Squares & Mean Square & F Ratio \\
\hline Model & 173 & 18.271676 & 0.105617 & 2.0515 \\
\hline Error & 196 & 10.090542 & 0.051482 & Prob $>$ F \\
\hline C. Total & 369 & 28.362218 & & $<.0001$ \\
\hline
\end{tabular}

Variance Component Estimates

$\begin{array}{lrr}\text { Component } & \text { Var Comp Est } & \text { Percent of Total } \\ \text { Batch\&Random } & 0.025468 & 33.097 \\ \text { Residual } & 0.051482 & 66.903 \\ \text { Total } & 0.076951 & 100.000\end{array}$

These estimates based on equating Mean Squares to Expected Value.

STCd=IN36

Response Al (mg/L)

Summary of Fit

RSquare $\quad 0.97468$

RSquare Adj 0.951868

Root Mean Square Error $\quad 0.004985$

Mean of Response 0.004836

Observations (or Sum Wgts) 366

Analysis of Variance

Source DF Sum of Squares Mean Square F Ratio

$\begin{array}{lllll}\text { Model } & 173 & 0.18364566 & 0.001062 & 42.7240\end{array}$

$\begin{array}{lllll}\text { Error } & 192 & 0.00477050 & 0.000025 & \text { Prob }>\text { F }\end{array}$

C. Total $365 \quad 0.18841616$

$<.0001$

Variance Component Estimates

$\begin{array}{lrr}\text { Component } & \text { Var Comp Est } & \text { Percent of Total } \\ \text { Batch\&Random } & 0.000493 & 95.202 \\ \text { Residual } & 0.000025 & 4.798 \\ \text { Total } & 0.000518 & 100.000\end{array}$

These estimates based on equating Mean Squares to Expected Value.
Response B (mg/L)

Summary of Fit

RSquare $\quad 0.868769$

RSquare Adj $\quad 0.750524$

Root Mean Square Error $\quad 0.054865$

Mean of Response $\quad 10.0517$

Observations (or Sum Wgts) 366

Analysis of Variance

$\begin{array}{lrrrr}\text { Source } & \text { DF } & \text { Sum of Squares } & \text { Mean Square } & \text { F Ratio } \\ \text { Model } & 173 & 3.8261756 & 0.022117 & 7.3472 \\ \text { Error } & 192 & 0.5779605 & 0.003010 & \text { Prob }>\text { F } \\ \text { C. Total } & 365 & 4.4041361 & & <.0001\end{array}$

Variance Component Estimates

Component Var Comp Est Percent of Total

Batch\&Random $\quad 0.009086 \quad 75.114$

Residual $\quad 0.00301 \quad 24.886$

Total $\quad 0.012096 \quad 100.000$

These estimates based on equating Mean Squares to Expected Value.

Response Ca (mg/L)

Summary of Fit

RSquare

0.953611

RSquare A

0.911812

Root Mean Square Error $\quad 0.027229$

Mean of Response $\quad 0.085713$

Observations (or Sum Wgts) $\quad 366$

Analysis of Variance

$\begin{array}{lrrrr}\text { Source } & \text { DF } & \text { Sum of Squares } & \text { Mean Square } & \text { F Ratio } \\ \text { Model } & 173 & 2.9263594 & 0.016915 & 22.8144 \\ \text { Error } & 192 & 0.1423555 & 0.000741 & \text { Prob }>\text { F } \\ \text { C. Total } & 365 & 3.0687149 & & <.0001\end{array}$

$\begin{array}{llll}\text { C. Total } 365 & 3.0687149 & 0.000741< & \text { Prob }>\text { F }\end{array}$

Variance Component Estimates

$\begin{array}{lrr}\text { Component } & \text { Var Comp Est } & \text { Percent of Total } \\ \text { Batch\&Random } & 0.007691 & 91.208 \\ \text { Residual } & 0.000741 & 8.792 \\ \text { Total } & 0.008433 & 100.000 \\ \text { These estimates based on equating } & \text { Mean Squares to Expected Value. }\end{array}$

These estimates based on equating Mean Squares to Expected Value.

Response Cr (mg/L)

Summary of Fit

RSquare Adj $\quad 0.736173$

Root Mean Square Error $\quad 0.001482$

Mean of Response $\quad 0.000885$

Observations (or Sum Wgts) 366

Analysis of Variance

Source DF Sum of Squares Mean Square F Ratio

$\begin{array}{lllll}\text { Model } & 173 & 0.00261568 & 0.000015 & 6.8872\end{array}$

$\begin{array}{lllll}\text { Error } & 192 & 0.00042150 & 0.000002 & \text { Prob }>\text { F }\end{array}$

$\begin{array}{llll}\text { C. Total } 365 & 0.00303718 & <.0001\end{array}$

\begin{tabular}{lrr}
\multicolumn{3}{l}{ Variance Component Estimates } \\
Component & Var Comp Est & Percent of Total \\
Batch\&Random & 0.000006 & 73.681 \\
Residual & 0.000002 & 26.319 \\
Total & 0.000008 & 100.000
\end{tabular}

These estimates based on equating Mean Squares to Expected Value. 
WSRC-TR-2004-00576

Revision 0

Exhibit A3. Components of Variance for Cold Chem Measurements of Calibration and Bench Standards

Response Cu (mg/L)

Summary of Fit

$\begin{array}{lr}\text { RSquare } & 0.948701 \\ \text { RSquare Adj } & 0.902478 \\ \text { Root Mean Square Error } & 0.001152 \\ \text { Mean of Response } & 0.000262 \\ \text { Observations (or Sum Wgts) } & 366\end{array}$

Analysis of Variance

Source DF Sum of Squares Mean Square F Ratio

$\begin{array}{lllll}\text { Model } & 173 & 0.00471582 & 0.000027 & 20.5245\end{array}$

$\begin{array}{lllll}\text { Error } & 192 & 0.00025500 & 0.000001 & \text { Prob }>\text { F }\end{array}$

$\begin{array}{llll}\text { C. Total } & 365 & 0.00497082 & <.0001\end{array}$

Variance Component Estimates

$\begin{array}{lrr}\text { Component } & \text { Var Comp Est } & \text { Percent of Total } \\ \text { Batch\&Random } & 0.000012 & 90.277 \\ \text { Residual } & 0.000001 & 9.723 \\ \text { Total } & 0.000014 & 100.000\end{array}$

These estimates based on equating Mean Squares to Expected Value.

Response Fe (mg/L)

Summary of Fit

RSquare $\quad 0.997913$

RSquare Adj 0.996032

Root Mean Square Error $\quad 0.003007$

Mean of Response

Observations (or Sum Wgts) 366

Analysis of Variance

Source DF Sum of Squares Mean Square F Ratio

$\begin{array}{lrrrr}\text { Model } & 173 & 0.82998386 & 0.004798 & 530.6095\end{array}$

$\begin{array}{lllll}\text { Error } & 192 & 0.00173600 & 0.000009 & \text { Prob }>\text { F }\end{array}$

C. Total $365 \quad 0.83171986$

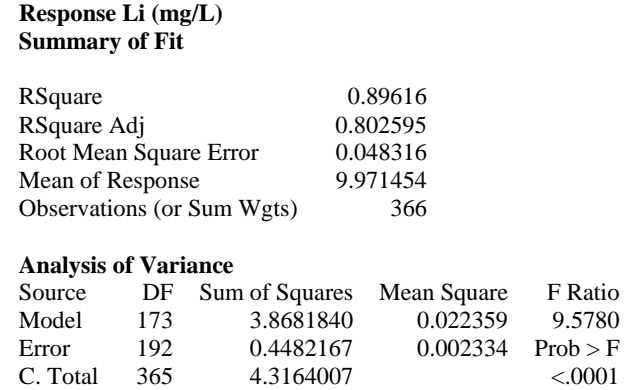

\begin{tabular}{lrr}
\multicolumn{3}{c}{ Variance Component Estimates } \\
Component & Var Comp Est & Percent of Total \\
Batch\&Random & 0.009523 & 80.311 \\
Residual & 0.002334 & 19.689 \\
Total & 0.011857 & 100.000
\end{tabular}

These estimates based on equating Mean Squares to Expected Value.

Response Mg (mg/L)

Summary of Fit

RSquare

0.999421

RSquare Adj

0.998899

Root Mean Square Error $\quad 0.000532$

Mean of Response

Observations (or Sum Wgts)

0.005

Analysis of Variance

Source DF Sum of Squares Mean Square F Ratio

Model $\quad \begin{array}{lrrr}173 & 0.09358575 & 0.000541 & 1914.543\end{array}$

$\begin{array}{lllll}\text { Error } & 192 & 0.00005425 & 0.000000 & \text { Prob }>\text { F }\end{array}$

$\begin{array}{llll}\text { C. Total } 365 & 0.09364000 & <.0001\end{array}$ \begin{tabular}{lrr}
\multicolumn{3}{c}{ Variance Component Estimates } \\
Component & Var Comp Est & Percent of Total \\
Batch\&Random & 0.002277 & 99.605 \\
Residual & 0.000009 & 0.395 \\
Total & 0.002286 & 100.000
\end{tabular}

These estimates based on equating Mean Squares to Expected Value.

Response K (mg/L)

Summary of Fi

RSquare

Root Mean Square Error $\quad 0.053634$

Mean of Response

Observations (or Sum Wgts) $\quad 366$

Analysis of Variance

$\begin{array}{lrrrr}\text { Source } & \text { DF } & \text { Sum of Squares } & \text { Mean Square } & \text { F Ratio } \\ \text { Model } & 173 & 4.7404665 & 0.027402 & 9.5256 \\ \text { Error } & 192 & 0.5523120 & 0.002877 & \text { Prob > F } \\ \text { C. Total } & 365 & 5.2927785 & & <.0001\end{array}$

Variance Component Estimates

$\begin{array}{lrr}\text { Component } & \text { Var Comp Est } & \text { Percent of Total } \\ \text { Batch\&Random } & 0.011662 & 80.214 \\ \text { Residual } & 0.002877 & 19.786 \\ \text { Total } & 0.014539 & 100.000\end{array}$

These estimates based on equating Mean Squares to Expected Value.
Variance Component Estimates

$\begin{array}{lrr}\text { Component } & \text { Var Comp Est } & \text { Percent of Total } \\ \text { Batch\&Random } & 0.000257 & 99.890 \\ \text { Residual } & 2.826 \mathrm{e}-7 & 0.110 \\ \text { Total } & 0.000257 & 100.000\end{array}$

These estimates based on equating Mean Squares to Expected Value.

Response Mn (mg/L)

Summary of Fit

RSquare

0.999521

RSquare Adj

0.999089

Root Mean Square Error $\quad 0.000648$

Mean of Response $\quad 0.006426$

Observations (or Sum Wgts) $\quad 366$

$\begin{array}{lrrrr}\text { Analysis of Variance } & & \\ \text { Source } & \text { DF } & \text { Sum of Squares } & \text { Mean Square } & \text { F Ratio } \\ \text { Model } & 173 & 0.16782301 & 0.000970 & 2313.72 \\ \text { Error } & 192 & 0.00008050 & 0.000000 & \text { Prob }>\text { F } \\ \text { C. Total } & 365 & 0.16790351 & & <.0001\end{array}$

Variance Component Estimates

$\begin{array}{lrc}\text { Component } & \text { Var Comp Est } & \text { Percent of Total } \\ \text { Batch\&Random } & 0.000461 & 99.909 \\ \text { Residual } & 4.193 \mathrm{e}-7 & 0.091 \\ \text { Total } & 0.000462 & 100.000 \\ \text { These estimates based on equating } & \text { Mean Squares to Expected Value. }\end{array}$


WSRC-TR-2004-00576

Revision 0

Exhibit A3. Components of Variance for Cold Chem Measurements of Calibration and Bench Standards

Response Na (mg/L) Summary of Fit

$\begin{array}{lr}\text { RSquare } & 0.972247 \\ \text { RSquare Adj } & 0.94724 \\ \text { Root Mean Square Error } & 0.371927 \\ \text { Mean of Response } & 66.13271 \\ \text { Observations (or Sum Wgts) } & 366\end{array}$

Analysis of Variance

Source DF Sum of Squares Mean Square F Ratio

$\begin{array}{lllll}\text { Model } & 173 & 930.42887 & 5.37820 & 38.8796\end{array}$

$\begin{array}{llll}\text { Error } & 192 & 26.55929 & 0.13833 \quad \text { Prob }>\text { F }\end{array}$

C. Total $365 \quad 956.98816 \quad<.0001$

Variance Component Estimates

$\begin{array}{lrr}\text { Component } & \text { Var Comp Est } & \text { Percent of Total } \\ \text { Batch\&Random } & 2.491725 & 94.740 \\ \text { Residual } & 0.13833 & 5.260 \\ \text { Total } & 2.630055 & 100.000\end{array}$

These estimates based on equating Mean Squares to Expected Value.

Response Ni (mg/L)

Summary of Fit

RSquare $\quad 0.782118$

RSquare Adj $\quad 0.585798$

Root Mean Square Error $\quad 0.004805$

Mean of Response

Observations (or Sum Wgts) $\quad 366$

Analysis of Variance

Source DF Sum of Squares Mean Square F Ratio

$\begin{array}{lllll}\text { Model } & 173 & 0.01590933 & 0.000092 & 3.9839\end{array}$

$\begin{array}{lllll}\text { Error } & 192 & 0.00443200 & 0.000023 & \text { Prob }>\text { F }\end{array}$

C. Total $365 \quad 0.02034133$

\begin{tabular}{|c|c|c|c|c|c|}
\hline \multicolumn{6}{|c|}{$\begin{array}{l}\text { Response Ti }(\mathrm{mg} / \mathrm{L}) \\
\text { Summary of Fit }\end{array}$} \\
\hline \multicolumn{3}{|l|}{ RSquare } & \multicolumn{3}{|c|}{0.988755} \\
\hline \multicolumn{3}{|c|}{ RSquare Adj } & \multicolumn{3}{|c|}{0.978623} \\
\hline \multicolumn{3}{|c|}{ Root Mean Square Error } & \multicolumn{3}{|c|}{0.000634} \\
\hline \multicolumn{3}{|c|}{ Mean of Response } & \multicolumn{3}{|c|}{0.002694} \\
\hline \multicolumn{3}{|c|}{ Observations (or Sum Wgts) } & \multicolumn{3}{|c|}{366} \\
\hline \multicolumn{6}{|c|}{ Analysis of Variance } \\
\hline Source & DF & Sum of Squa & & Mean Square & F Ratio \\
\hline Model & 173 & 0.006792 & & 0.000039 & 97.5854 \\
\hline Error & 192 & 0.000077 & & 0.000000 & Prob $>F$ \\
\hline C. Total & 365 & 0.006869 & & & $<.0001$ \\
\hline
\end{tabular}

\begin{tabular}{lrr}
\multicolumn{3}{l}{ Variance Component Estimates } \\
Component & Var Comp Est & Percent of Total \\
Batch\&Random & 0.000018 & 97.869 \\
Residual & $4.023 \mathrm{e}-7$ & 2.131 \\
Total & 0.000019 & 100.000
\end{tabular}

These estimates based on equating Mean Squares to Expected Value.

Response Zr (mg/L)

Summary of Fit

RSquare

0.99955

RSquare Adj

0.999145

Root Mean Square Error $\quad 0.000885$

Mean of Response

Observations (or Sum Wgts)

366

Analysis of Variance

$\begin{array}{lrrrr}\text { Source } & \text { DF } & \text { Sum of Squares } & \text { Mean Square } & \text { F Ratio } \\ \text { Model } & 173 & 0.33380530 & 0.001930 & 2465.664 \\ \text { Error } & 192 & 0.00015025 & 0.000001 & \text { Prob }>\text { F } \\ \text { C. Total } & 365 & 0.33395555 & & <.0001\end{array}$

Variance Component Estimates

$\begin{array}{lrr}\text { Component } & \text { Var Comp Est } & \text { Percent of Total } \\ \text { Batch\&Random } & 0.000033 & 58.659 \\ \text { Residual } & 0.000023 & 41.341 \\ \text { Total } & 0.000056 & 100.000\end{array}$

Total $0.000056 \quad 100.000$

These estimates based on equating Mean Squares to Expected Value.

Response Si (mg/L)

Summary of Fit

\begin{tabular}{|c|c|c|c|c|}
\hline \multicolumn{3}{|l|}{ RSquare } & \multicolumn{2}{|c|}{0.956555} \\
\hline \multicolumn{3}{|c|}{ RSquare Adj } & \multicolumn{2}{|c|}{0.917409} \\
\hline \multicolumn{3}{|c|}{ Root Mean Square Error } & \multicolumn{2}{|c|}{0.208107} \\
\hline \multicolumn{3}{|c|}{ Mean of Response } & \multicolumn{2}{|c|}{40.31627} \\
\hline \multicolumn{3}{|c|}{ Observations (or Sum Wgts) } & \multicolumn{2}{|l|}{366} \\
\hline \multicolumn{5}{|c|}{ Analysis of Variance } \\
\hline Source & $\mathrm{DF}$ & Sum of Squares & Mean Square & F Ratio \\
\hline Model & 173 & 183.08250 & 1.05828 & 24.4358 \\
\hline Error & 192 & 8.31526 & 0.04331 & Prob $>$ F \\
\hline C. Total & 365 & 191.39776 & & $<.0001$ \\
\hline
\end{tabular}

Variance Component Estimates

$\begin{array}{lrr}\text { Component } & \text { Var Comp Est } & \text { Percent of Total } \\ \text { Batch\&Random } & 0.482651 & 91.766 \\ \text { Residual } & 0.043309 & 8.234 \\ \text { Total } & 0.52596 & 100.000\end{array}$

Total 0.52596

These estimates based on equating Mean Squares to Expected Value.
Variance Component Estimates

$\begin{array}{lrr}\text { Component } & \text { Var Comp Est } & \text { Percent of Total } \\ \text { Batch\&Random } & 0.000917 & 99.915 \\ \text { Residual } & 7.826 \mathrm{e}-7 & 0.085 \\ \text { Total } & 0.000918 & 100.000 \\ \text { These estimates based on equating } & \text { Mean Squares to Expected Value. }\end{array}$

Response U (mg/L)

Summary of Fit

RSquare

0.938278

RSquare Adj

0.882664

Root Mean Square Error $\quad 0.051421$

Mean of Response $\quad 0.06382$

Observations (or Sum Wgts) $\quad 366$

$\begin{array}{lrrrr}\text { Analysis of Variance } & & \\ \text { Source } & \text { DF } & \text { Sum of Squares } & \text { Mean Square } & \text { F Ratio } \\ \text { Model } & 173 & 7.7175661 & 0.044610 & 16.8712 \\ \text { Error } & 192 & 0.5076780 & 0.002644 & \text { Prob }>\text { F } \\ \text { C. Total } & 365 & 8.2252441 & & <.0001\end{array}$

Variance Component Estimates

$\begin{array}{lrc}\text { Component } & \text { Var Comp Est } & \text { Percent of Total } \\ \text { Batch\&Random } & 0.019956 & 88.300 \\ \text { Residual } & 0.002644 & 11.700 \\ \text { Total } & 0.0226 & 100.000 \\ \text { These estimates based on equating } & \text { Mean Squares to Expected Value. }\end{array}$


WSRC-TR-2004-00576

Revision 0

Exhibit A3. Components of Variance for Cold Chem Measurements of Calibration and Bench Standards

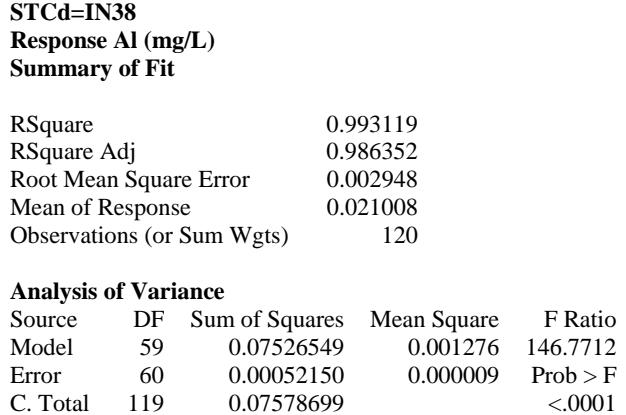

Variance Component Estimates

$\begin{array}{lrr}\text { Component } & \text { Var Comp Est } & \text { Percent of Total } \\ \text { Batch\&Random } & 0.000633 & 98.647 \\ \text { Residual } & 0.000009 & 1.353 \\ \text { Total } & 0.000642 & 100.000\end{array}$

These estimates based on equating Mean Squares to Expected Value.

Response B (mg/L)

Summary of Fit

\begin{tabular}{|c|c|c|c|c|}
\hline \multicolumn{3}{|l|}{ RSquare } & \multicolumn{2}{|c|}{0.990775} \\
\hline \multicolumn{3}{|c|}{ RSquare Adj } & \multicolumn{2}{|c|}{0.981703} \\
\hline \multicolumn{3}{|c|}{ Root Mean Square Error } & \multicolumn{2}{|c|}{0.00498} \\
\hline \multicolumn{3}{|c|}{ Mean of Response } & \multicolumn{2}{|c|}{0.001533} \\
\hline \multicolumn{3}{|c|}{ Observations (or Sum Wgts) } & \multicolumn{2}{|l|}{120} \\
\hline \multicolumn{5}{|c|}{ Analysis of Variance } \\
\hline Source & DF & Sum of Squares & Mean Square & F Ratio \\
\hline Model & 59 & 0.15980987 & 0.002709 & 109.2194 \\
\hline Error & 60 & 0.00148800 & 0.000025 & Prob $>$ F \\
\hline C. Total & 119 & 0.16129787 & & $<.0001$ \\
\hline
\end{tabular}

Variance Component Estimates

$\begin{array}{lrr}\text { Component } & \text { Var Comp Est } & \text { Percent of Total } \\ \text { Batch\&Random } & 0.001342 & 98.185 \\ \text { Residual } & 0.000025 & 1.815 \\ \text { Total } & 0.001367 & 100.000\end{array}$

Total $\quad 0.001367 \quad 100.000$

These estimates based on equating Mean Squares to Expected Value.

Response Ca (mg/L)

Summary of Fit

RSquare

RSquare Adj

Root Mean Square Error

0.999792

Root $\mathrm{Me}$

Observations (or Sum Wgts)

0.999587

0.241108

120

Analysis of Variance

$\begin{array}{lrrrr}\text { Source } & \text { DF } & \text { Sum of Squares } & \text { Mean Square } & \text { F Ratio } \\ \text { Model } & 59 & 5.4855981 & 0.092976 & 4878.508 \\ \text { Error } & 60 & 0.0011435 & 0.000019 & \text { Prob }>\text { F } \\ \text { C. Total } & 119 & 5.4867416 & & <.0001\end{array}$

Variance Component Estimates

$\begin{array}{lrr}\text { Component } & \text { Var Comp Est } & \text { Percent of Total } \\ \text { Batch\&Random } & 0.046479 & 99.959 \\ \text { Residual } & 0.000019 & 0.041 \\ \text { Total } & 0.046498 & 100.000\end{array}$

These estimates based on equating Mean Squares to Expected Value.

\begin{tabular}{|c|c|c|c|c|c|}
\hline \multicolumn{5}{|c|}{ Response Cr (mg/L) } & Summary of Fit \\
\hline \multicolumn{3}{|c|}{ RSquare } & \multicolumn{3}{|c|}{0.981639} \\
\hline \multicolumn{3}{|c|}{ RSquare Adj } & \multicolumn{3}{|c|}{0.963585} \\
\hline \multicolumn{3}{|c|}{ Root Mean Square Error } & \multicolumn{3}{|c|}{0.000847} \\
\hline \multicolumn{3}{|c|}{ Mean of Response } & \multicolumn{3}{|c|}{0.028017} \\
\hline \multicolumn{3}{|c|}{ Observations (or Sum Wgts) } & \multicolumn{3}{|c|}{120} \\
\hline \multicolumn{6}{|c|}{ Analysis of Variance } \\
\hline Source & DF & Sum of Squ & & Mean Square & F Ratio \\
\hline Model & 59 & 0.0022 & 397 & 0.000039 & 54.3705 \\
\hline Error & 60 & 0.0000 & 300 & 0.000001 & Prob $>$ F \\
\hline C. Total & 119 & 0.0023 & 197 & & $<.0001$ \\
\hline
\end{tabular}

\begin{tabular}{lrr}
\multicolumn{3}{l}{ Variance Component Estimates } \\
Component & Var Comp Est & Percent of Total \\
Batch\&Random & 0.000019 & 96.388 \\
Residual & $7.167 \mathrm{e}-7$ & 3.612 \\
Total & 0.00002 & 100.000 \\
These estimates based on equating Mean Squares to Expected Value.
\end{tabular}

Response Cu (mg/L)

Summary of Fit

RSquare

0.9976

RSquare Adj

0.995239

Root Mean Square Error $\quad 0.000837$

Mean of Response $\quad 0.023683$

Observations (or Sum Wgts) $\quad 120$

$\begin{array}{lrrrr}\text { Analysis of Variance } & & & \\ \text { Source } & \text { DF } & \text { Sum of Squares } & \text { Mean Square } & \text { F Ratio } \\ \text { Model } & 59 & 0.01745597 & 0.000296 & 422.6626 \\ \text { Error } & 60 & 0.00004200 & 0.000001 & \text { Prob }>\text { F } \\ \text { C. Total } & 119 & 0.01749797 & & <.0001\end{array}$

Variance Component Estimates

$\begin{array}{lrr}\text { Component } & \text { Var Comp Est } & \text { Percent of Total } \\ \text { Batch\&Random } & 0.000148 & 99.528 \\ \text { Residual } & 7 \mathrm{e}-7 & 0.472 \\ \text { Total } & 0.000148 & 100.000 \\ \text { These estimates based on equating } & \text { Mean Squares to Expected Value. }\end{array}$

Response Fe (mg/L)

Summary of Fit

\begin{tabular}{|c|c|c|c|c|c|}
\hline \multicolumn{3}{|l|}{ RSquare } & \multicolumn{3}{|c|}{0.999492} \\
\hline \multicolumn{3}{|c|}{ RSquare Adj } & \multicolumn{3}{|c|}{0.998992} \\
\hline \multicolumn{3}{|c|}{ Root Mean Square Error } & \multicolumn{3}{|c|}{0.000935} \\
\hline \multicolumn{3}{|c|}{ Mean of Response } & \multicolumn{3}{|c|}{0.001325} \\
\hline \multicolumn{3}{|c|}{ Observations (or Sum Wgts) } & \multicolumn{3}{|c|}{120} \\
\hline \multicolumn{6}{|c|}{ Analysis of Variance } \\
\hline Source & DF & Sum of Sq & & Mean Square & F Ratio \\
\hline Model & 59 & 0.1032 & 783 & 0.001750 & 2000.539 \\
\hline Error & 60 & 0.0000 & & 0.000001 & Prob $>$ F \\
\hline C. Total & 119 & 0.1033 & 033 & & $<.0001$ \\
\hline
\end{tabular}

Variance Component Estimates

$\begin{array}{lrr}\text { Component } & \text { Var Comp Est } & \text { Percent of Total } \\ \text { Batch\&Random } & 0.000875 & 99.900 \\ \text { Residual } & 8.75 \mathrm{e}-7 & 0.100 \\ \text { Total } & 0.000876 & 100.000 \\ \text { These estimates based on equating Mean Squares to Expected Value. }\end{array}$ 
WSRC-TR-2004-00576

Revision 0

Exhibit A3. Components of Variance for Cold Chem Measurements of Calibration and Bench Standards

Response K (mg/L)

Summary of Fit

$\begin{array}{lr}\text { RSquare } & 0.965343 \\ \text { RSquare Adj } & 0.931263 \\ \text { Root Mean Square Error } & 0.016718 \\ \text { Mean of Response } & -0.02228 \\ \text { Observations (or Sum Wgts) } & 120\end{array}$

Analysis of Variance

Source DF Sum of Squares Mean Square F Ratio

$\begin{array}{lllll}\text { Model } & 59 & 0.46711237 & 0.007917 & 28.3261\end{array}$

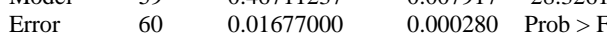

C. Total $119 \quad 0.48388237$

$<.0001$

Variance Component Estimates

$\begin{array}{lrr}\text { Component } & \text { Var Comp Est } & \text { Percent of Total } \\ \text { Batch\&Random } & 0.003819 & 93.180 \\ \text { Residual } & 0.00028 & 6.820 \\ \text { Total } & 0.004098 & 100.000\end{array}$

These estimates based on equating Mean Squares to Expected Value.

Response Li (mg/L)

Summary of Fit

RSquare $\quad 0.970666$

RSquare Adj $\quad 0.94182$

Root Mean Square Error $\quad 0.001088$

Mean of Response

Observations (or Sum Wgts) 120

Analysis of Variance

Source DF Sum of Squares Mean Square F Ratio

$\begin{array}{llrrr}\text { Model } & 59 & 0.00234937 & 0.000040 & 33.6505\end{array}$

$\begin{array}{lllll}\text { Error } & 60 & 0.00007100 & 0.000001 & \text { Prob }>\text { F }\end{array}$

C. Total $119 \quad 0.00242037$

$<.0001$

Variance Component Estimates

$\begin{array}{lrr}\text { Component } & \text { Var Comp Est } & \text { Percent of Total } \\ \text { Batch\&Random } & 0.000019 & 94.228 \\ \text { Residual } & 0.000001 & 5.772 \\ \text { Total } & 0.000021 & 100.000\end{array}$

These estimates based on equating Mean Squares to Expected Value.

Response Mg (mg/L)

Summary of Fit

RSquare

RSquare Adj

Root Mean Square Error

Mean of Response

0.001708

Observations (or Sum Wgts)

120

Analysis of Variance

$\begin{array}{lrrrr}\text { Source } & \text { DF } & \text { Sum of Squares } & \text { Mean Square } & \text { F Ratio } \\ \text { Model } & 59 & 0.00069829 & 0.000012 & 1420.254 \\ \text { Error } & 60 & 0.00000050 & 0.000000 & \text { Prob }>\text { F } \\ \text { C. Total } & 119 & 0.00069879 & & <.0001\end{array}$

C. Total $119 \quad 0.00069879<001$

Variance Component Estimates

$\begin{array}{lrr}\text { Component } & \text { Var Comp Est } & \text { Percent of Total } \\ \text { Batch\&Random } & 0.000006 & 99.859 \\ \text { Residual } & 8.333 \mathrm{e}-9 & 0.141 \\ \text { Total } & 0.000006 & 100.000\end{array}$

These estimates based on equating Mean Squares to Expected Value.

\begin{tabular}{|c|c|c|c|c|c|}
\hline \multicolumn{6}{|c|}{$\begin{array}{l}\text { Response Mn (mg/L) } \\
\text { Summary of Fit }\end{array}$} \\
\hline \multicolumn{3}{|l|}{ RSquare } & \multicolumn{3}{|c|}{0.990183} \\
\hline \multicolumn{3}{|c|}{ RSquare Adj } & \multicolumn{3}{|c|}{0.980529} \\
\hline \multicolumn{3}{|c|}{ Root Mean Square Error } & \multicolumn{3}{|c|}{0.000158} \\
\hline \multicolumn{3}{|c|}{ Mean of Response } & \multicolumn{3}{|c|}{0.001958} \\
\hline \multicolumn{3}{|c|}{ Observations (or Sum Wgts) } & \multicolumn{3}{|c|}{120} \\
\hline \multicolumn{6}{|c|}{ Analysis of Variance } \\
\hline Source & DF & Sum of Squ & & Mean Square & F Ratio \\
\hline Model & 59 & 0.0001 & & 0.0000026 & 102.5706 \\
\hline Error & 60 & 0.0000 & 150 & $2.5 e-8$ & Prob $>F$ \\
\hline C. Total & 119 & 0.0001 & & & $<.0001$ \\
\hline
\end{tabular}

\begin{tabular}{lrr}
\multicolumn{3}{l}{ Variance Component Estimates } \\
Component & Var Comp Est & Percent of Total \\
Batch\&Random & 0.000001 & 98.069 \\
Residual & $2.5 \mathrm{e}-8$ & 1.931 \\
Total & 0.000001 & 100.000
\end{tabular}

These estimates based on equating Mean Squares to Expected Value.

Response Na (mg/L)

Summary of Fit

RSquare

0.999972

RSquare Adj $\quad 0.999945$

Root Mean Square Error $\quad 0.007739$

Mean of Response $\quad 0.485142$

Observations (or Sum Wgts) 120

Analysis of Variance

$\begin{array}{lrrrr}\text { Source } & \text { DF } & \text { Sum of Squares } & \text { Mean Square } & \text { F Ratio } \\ \text { Model } & 59 & 129.99820 & 2.20336 & 36789.08 \\ \text { Error } & 60 & 0.00359 & 0.00006 & \text { Prob }>\text { F } \\ \text { C. Total } & 119 & 130.00179 & & <.0001\end{array}$ \begin{tabular}{lrr}
\multicolumn{3}{l}{ Variance Component Estimates } \\
Component & Var Comp Est & Percent of Total \\
Batch\&Random & 1.10165 & 99.995 \\
Residual & 0.00006 & 0.005 \\
Total & 1.10171 & 100.000 \\
These estimates based on equating Mean Squares to Expected Value.
\end{tabular}

Response Ni (mg/L)

Summary of Fit

RSquare

0.861096

RSquare Adj

0.724507

Root Mean Square Error $\quad 0.003843$

Mean of Response $\quad 0.00375$

Observations (or Sum Wgts) 120

$\begin{array}{lrrrr}\text { Analysis of Variance } & & \\ \text { Source } & \text { DF } & \text { Sum of Squares } & \text { Mean Square } & \text { F Ratio } \\ \text { Model } & 59 & 0.00549250 & 0.000093 & 6.3043 \\ \text { Error } & 60 & 0.00088600 & 0.000015 & \text { Prob }>\text { F } \\ \text { C. Total } & 119 & 0.00637850 & & <.0001\end{array}$

Variance Component Estimates

$\begin{array}{lrc}\text { Component } & \text { Var Comp Est } & \text { Percent of Total } \\ \text { Batch\&Random } & 0.000039 & 72.619 \\ \text { Residual } & 0.000015 & 27.381 \\ \text { Total } & 0.000054 & 100.000 \\ \text { These estimates based on equating } & \text { Mean Squares to Expected Value. }\end{array}$


WSRC-TR-2004-00576

Revision 0

Exhibit A3. Components of Variance for Cold Chem Measurements of Calibration and Bench Standards

Response Si (mg/L)

Summary of Fit

$\begin{array}{lr}\text { RSquare } & 0.986142 \\ \text { RSquare Adj } & 0.972515 \\ \text { Root Mean Square Error } & 0.1513 \\ \text { Mean of Response } & 0.018117 \\ \text { Observations (or Sum Wgts) } & 120\end{array}$

Analysis of Variance

Source DF Sum of Squares Mean Square F Ratio

$\begin{array}{lllll}\text { Model } & 59 & 97.740267 & 1.65661 & 72.3672\end{array}$

$\begin{array}{lllll}\text { Error } & 60 & 1.373507 & 0.02289 & \text { Prob }>\text { F }\end{array}$

$\begin{array}{llll}\text { C. Total } & 119 & 99.113774 & <.0001\end{array}$

Variance Component Estimates

$\begin{array}{lrr}\text { Component } & \text { Var Comp Est } & \text { Percent of Total } \\ \text { Batch\&Random } & 0.816861 & 97.274 \\ \text { Residual } & 0.022892 & 2.726 \\ \text { Total } & 0.839753 & 100.000\end{array}$

These estimates based on equating Mean Squares to Expected Value.

Response Ti (mg/L)

Summary of Fit

RSquare $\quad 0.967477$

RSquare Adj 0.935495

Root Mean Square Error $\quad 0.000438$

Mean of Response

Observations (or Sum Wgts)

120

Analysis of Variance

Source DF Sum of Squares Mean Square F Ratio

$\begin{array}{lrrrr}\text { Model } & 59 & 0.00034209 & 0.0000058 & 30.2513\end{array}$

$\begin{array}{lllll}\text { Error } & 60 & 0.00001150 & 1.9167 \mathrm{e}-7 & \text { Prob }>\text { F }\end{array}$

$\begin{array}{llll}\text { C. Total } & 119 & 0.00035359 & <.0001\end{array}$

Variance Component Estimates

$\begin{array}{lrr}\text { Component } & \text { Var Comp Est } & \text { Percent of Total } \\ \text { Batch\&Random } & 0.000003 & 93.600 \\ \text { Residual } & 1.917 \mathrm{e}-7 & 6.400 \\ \text { Total } & 0.000003 & 100.000\end{array}$

Total $\quad 0.000003 \quad 100.000$

These estimates based on equating Mean Squares to Expected Value.

Response U (mg/L)

Summary of Fit

RSquare $\quad 0.941637$

RSquare Adj 0.884246

Root Mean Square Error $\quad 0.056257$

Mean of Response 9.857983

Observations (or Sum Wgts) 120

Analysis of Variance

Source DF Sum of Squares Mean Square F Ratio

$\begin{array}{lllll}\text { Model } & 59 & 3.0637070 & 0.051927 & 16.4075\end{array}$

$\begin{array}{lllll}\text { Error } & 60 & 0.1898910 & 0.003165 & \text { Prob }>\text { F }\end{array}$

$\begin{array}{llll}\text { C. Total } & 119 & 3.2535980 & <.0001\end{array}$

Variance Component Estimates

$\begin{array}{lrr}\text { Component } & \text { Var Comp Est } & \text { Percent of Total } \\ \text { Batch\&Random } & 0.024381 & 88.511 \\ \text { Residual } & 0.003165 & 11.489 \\ \text { Total } & 0.027546 & 100.000\end{array}$

100.000

These estimates based on equating Mean Squares to Expected Value.

\begin{tabular}{|c|c|c|c|c|}
\hline \multicolumn{4}{|c|}{ Response Zr (mg/L) } & Summary of Fit \\
\hline \multicolumn{3}{|l|}{ RSquare } & \multicolumn{2}{|c|}{0.985142} \\
\hline \multicolumn{3}{|c|}{ RSquare Adj } & \multicolumn{2}{|c|}{0.970532} \\
\hline \multicolumn{3}{|c|}{ Root Mean Square Error } & \multicolumn{2}{|c|}{0.000438} \\
\hline \multicolumn{3}{|c|}{ Mean of Response } & \multicolumn{2}{|c|}{0.001242} \\
\hline \multicolumn{3}{|c|}{ Observations (or Sum Wgts) } & \multicolumn{2}{|c|}{120} \\
\hline \multicolumn{5}{|c|}{ Analysis of Variance } \\
\hline Source & DF & Sum of Squares & Mean Square & F Ratio \\
\hline Model & 59 & 0.00076249 & 0.000013 & 67.4274 \\
\hline Error & 60 & 0.00001150 & 0.000000 & Prob $>$ F \\
\hline C. Total & 119 & 0.00077399 & & $<.0001$ \\
\hline
\end{tabular}

\begin{tabular}{lrr}
\multicolumn{3}{l}{ Variance Component Estimates } \\
Component & Var Comp Est & Percent of Total \\
Batch\&Random & 0.000006 & 97.077 \\
Residual & $1.917 \mathrm{e}-7$ & 2.923 \\
Total & 0.000007 & 100.000
\end{tabular}

These estimates based on equating Mean Squares to Expected Value. 
WSRC-TR-2004-00576

Exhibit A4. Paired Differences for IN33 and IN35

Matched Pairs

Difference: Mean(Al (mg/L)) IN35-Mean(Al (mg/L)) IN33

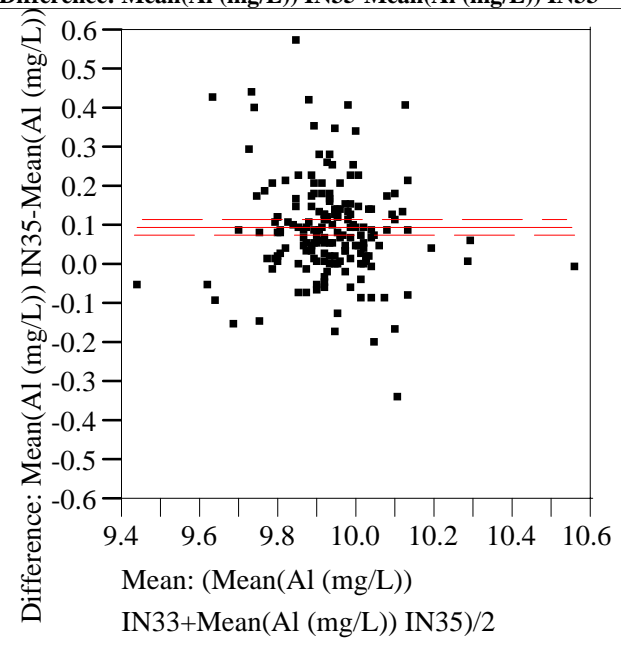

$\begin{array}{lrlr}\text { Mean(Al (mg/L)) IN35 } & 9.97829 & \text { t-Ratio } & 10.04499 \\ \text { Mean(Al (mg/L)) IN33 } & 9.88129 & \text { DF } & 172 \\ \text { Mean Difference } & 0.097 & \text { Prob }>|t| & <.0001 \\ \text { Std Error } & 0.00966 & \text { Prob }>\text { t } & <.0001 \\ \text { Upper95\% } & 0.11606 & \text { Prob }<\mathrm{t} & 1.0000 \\ \text { Lower95\% } & 0.07794 & & \\ \text { N } & 173 & & \\ \text { Correlation } & 0.59167 & & \end{array}$

Difference: Mean(B (mg/L)) IN35-Mean(B (mg/L)) IN33

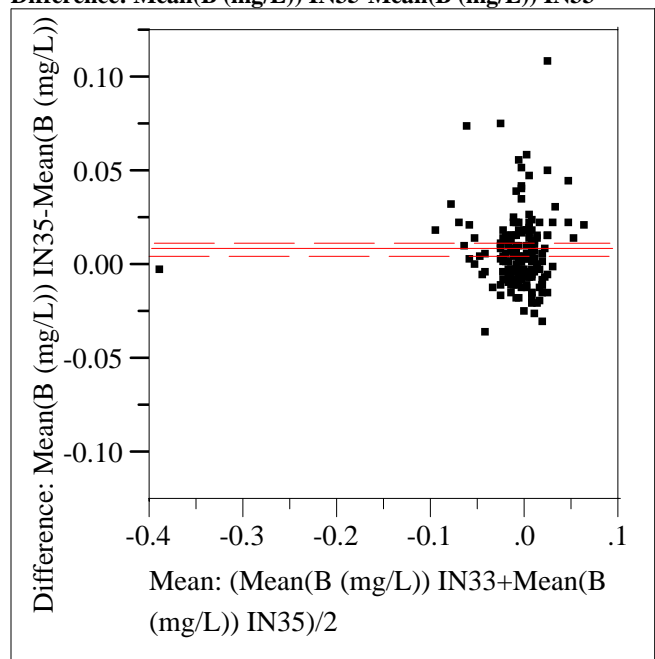

Mean(B (mg/L)) IN35

Mean(B (mg/L)) IN33

Mean Difference

Std Error

Upper95\%

Lower95\%

Correlation

$\begin{array}{rlr}-0.0035 & \text { t-Ratio } & 5.650437 \\ -0.0119 & \text { DF } & 172 \\ 0.00837 & \text { Prob }>|t| & <.0001 \\ 0.00148 & \text { Prob }>t & <.0001 \\ 0.01129 & \text { Prob }<\text { t } & 1.0000 \\ 0.00544 & & \\ 173 & & \\ 0.87068 & & \end{array}$

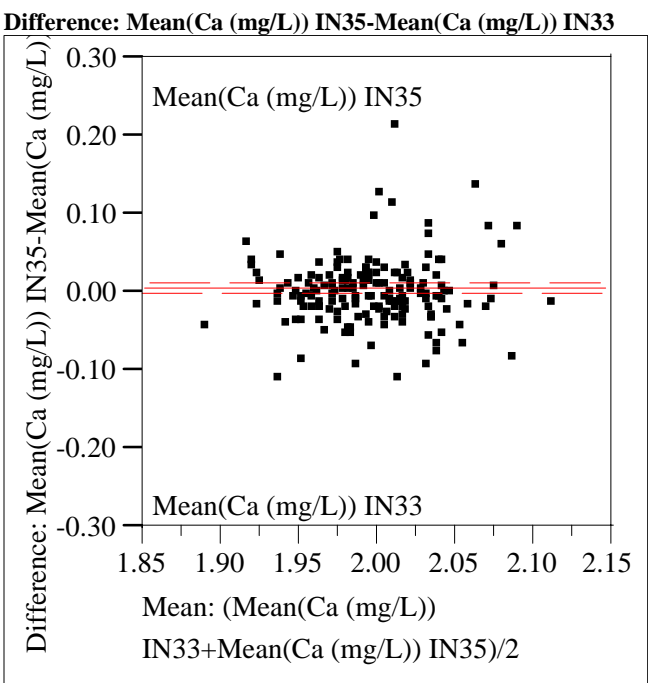

$\begin{array}{lrlr}\text { Mean(Ca (mg/L)) IN35 } & 1.99846 & \mathrm{t} \text {-Ratio } & 1.454665 \\ \text { Mean(Ca (mg/L)) IN33 } & 1.99382 & \text { DF } & 172 \\ \text { Mean Difference } & 0.00464 & \text { Prob }>|t| & 0.1476 \\ \text { Std Error } & 0.00319 & \text { Prob }>\mathrm{t} & 0.0738 \\ \text { Upper95\% } & 0.01094 & \text { Prob }<\mathrm{t} & 0.9262 \\ \text { Lower95\% } & -0.0017 & & \\ \text { N } & 173 & & \\ \text { Correlation } & 0.53464 & & \end{array}$

Difference: Mean(Cr (mg/L)) IN35-Mean(Cr (mg/L)) IN33

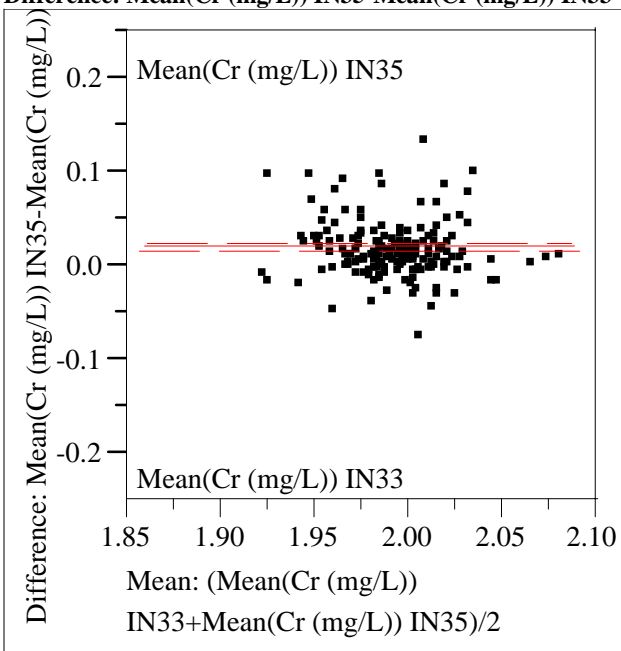

$\begin{array}{lrlr}\text { Mean(Cr (mg/L)) IN35 } & 2.00226 & \mathrm{t}-\text { Ratio } & 9.10471 \\ \text { Mean(Cr (mg/L)) IN33 } & 1.98201 & \text { DF } & 172 \\ \text { Mean Difference } & 0.02025 & \text { Prob }>|t| & <.0001 \\ \text { Std Error } & 0.00222 & \text { Prob }>t & <.0001 \\ \text { Upper95\% } & 0.02464 & \text { Prob }<\mathrm{t} & 1.0000 \\ \text { Lower95\% } & 0.01586 & & \\ \text { N } & 173 & & \\ \text { Correlation } & 0.52972 & & \end{array}$


WSRC-TR-2004-00576

Exhibit A4. Paired Differences for IN33 and IN35

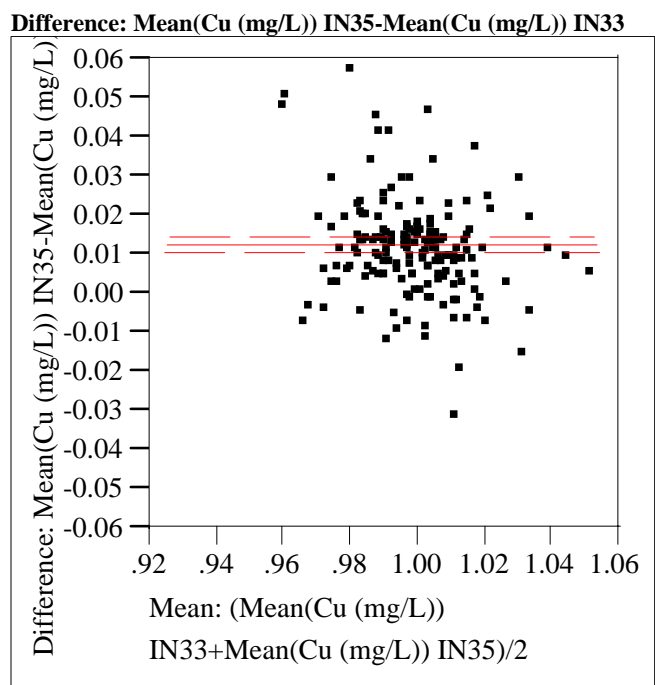

$\begin{array}{lrlr}\text { Mean }(\mathrm{Cu}(\mathrm{mg} / \mathrm{L})) \text { IN35 } & 1.00495 & \mathrm{t}-\text { Ratio } & 12.98091 \\ \text { Mean(Cu (mg/L)) IN33 } & 0.99263 & \text { DF } & 172 \\ \text { Mean Difference } & 0.01232 & \text { Prob }>|\mathrm{t}| & <.0001 \\ \text { Std Error } & 0.00095 & \text { Prob }>\mathrm{t} & <.0001 \\ \text { Upper95\% } & 0.01419 & \text { Prob }<\mathrm{t} & 1.0000 \\ \text { Lower95\% } & 0.01045 & & \\ \mathrm{~N} & 173 & & \\ \text { Correlation } & 0.7187 & & \end{array}$

Difference: Mean(Fe (mg/L)) IN35-Mean(Fe (mg/L)) IN33

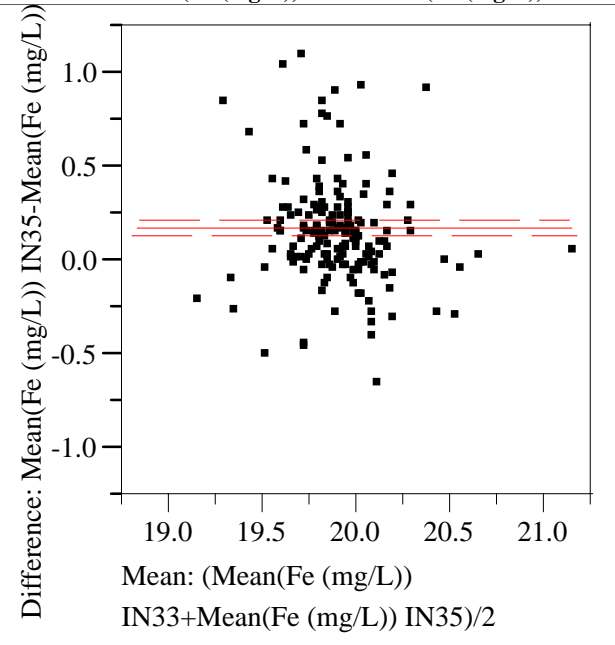

$\begin{array}{lrlr}\text { Mean(Fe (mg/L)) IN35 } & 19.9818 & \text { t-Ratio } & 7.884254 \\ \text { Mean(Fe (mg/L)) IN33 } & 19.8142 & \text { DF } & 172 \\ \text { Mean Difference } & 0.16759 & \text { Prob }>|t| & <.0001 \\ \text { Std Error } & 0.02126 & \text { Prob }>\text { t } & <.0001 \\ \text { Upper95\% } & 0.20955 & \text { Prob }<\mathrm{t} & 1.0000 \\ \text { Lower95\% } & 0.12563 & & \\ \text { N } & 173 & & \\ \text { Correlation } & 0.50585 & & \end{array}$

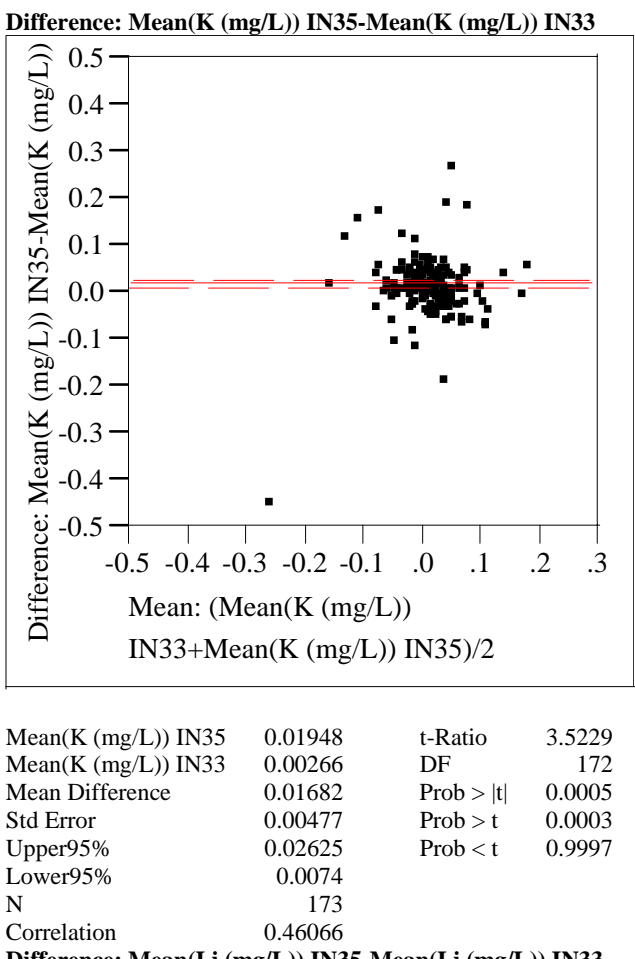

Difference: Mean(Li (mg/L)) IN35-Mean(Li (mg/L)) IN33

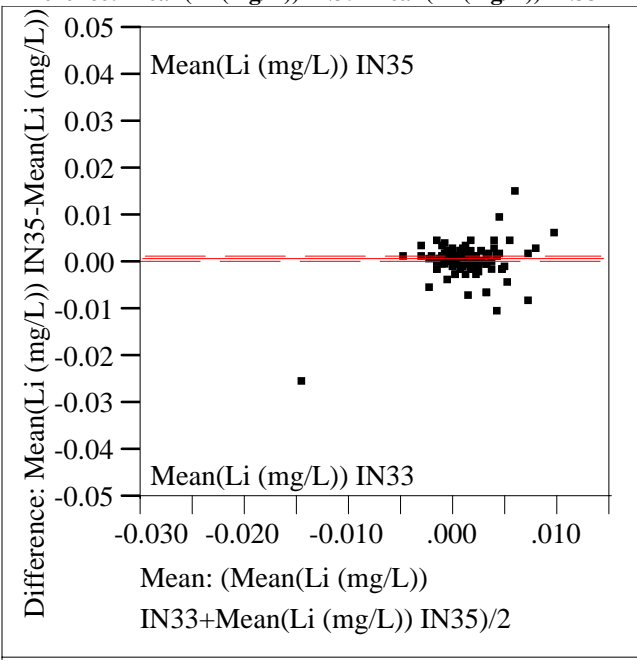

$\begin{array}{lrlr}\text { Mean(Li (mg/L)) IN35 } & 0.00146 & \text { t-Ratio } & 3.244913 \\ \text { Mean(Li (mg/L)) IN33 } & 0.00067 & \text { DF } & 172 \\ \text { Mean Difference } & 0.00079 & \text { Prob }>|t| & 0.0014 \\ \text { Std Error } & 0.00024 & \text { Prob }>\text { t } & 0.0007 \\ \text { Upper95\% } & 0.00127 & \text { Prob }<\mathrm{t} & 0.9993 \\ \text { Lower95\% } & 0.00031 & & \\ \text { N } & 173 & & \\ \text { Correlation } & 0.36487 & & \end{array}$


WSRC-TR-2004-00576

Exhibit A4. Paired Differences for IN33 and IN35

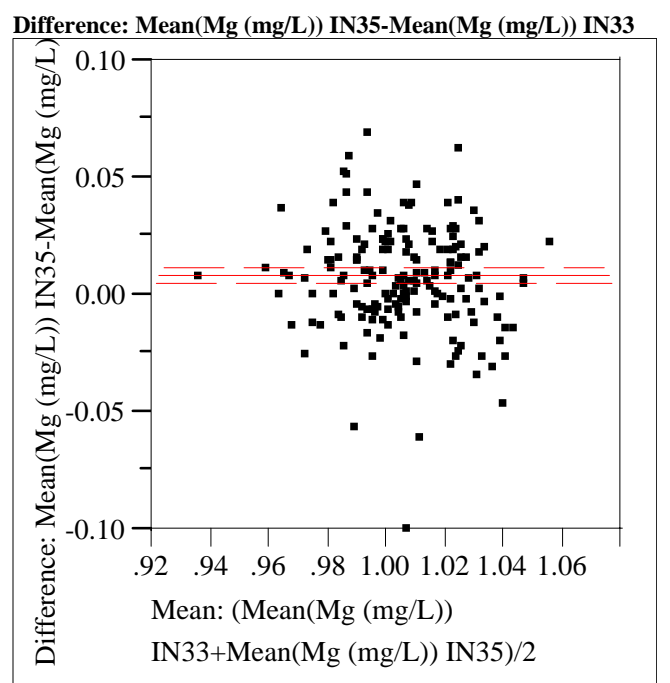

$\begin{array}{lrlr}\text { Mean }(\mathrm{Mg}(\mathrm{mg} / \mathrm{L})) \mathrm{IN} 35 & 1.00989 & \mathrm{t}-\text { Ratio } & 4.678915 \\ \text { Mean(Mg (mg/L)) IN33 } & 1.00188 & \mathrm{DF} & 172 \\ \text { Mean Difference } & 0.008 & \text { Prob }>|\mathrm{t}| & <.0001 \\ \text { Std Error } & 0.00171 & \text { Prob }>\mathrm{t} & <.0001 \\ \text { Upper95\% } & 0.01138 & \text { Prob }<\mathrm{t} & 1.0000 \\ \text { Lower95\% } & 0.00463 & & \\ \mathrm{~N} & 173 & & \\ \text { Correlation } & 0.5213 & & \end{array}$

Difference: Mean(Mn (mg/L)) IN35-Mean(Mn (mg/L)) IN33

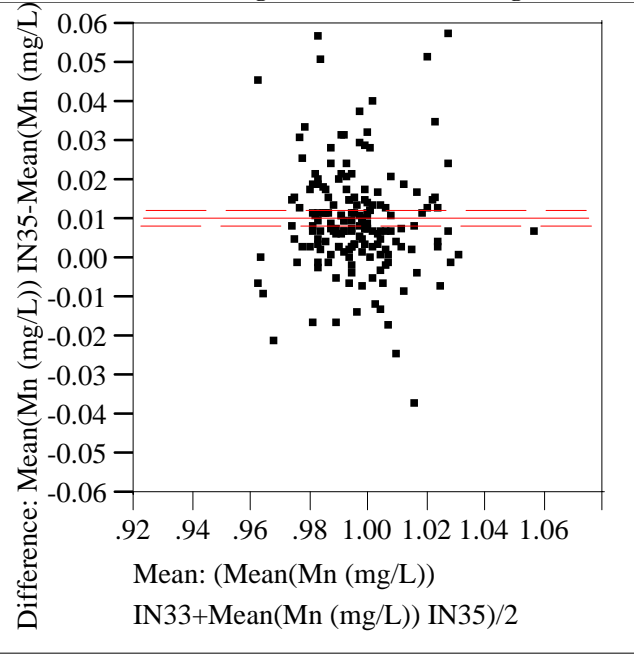

$\begin{array}{lrlr}\text { Mean }(\mathrm{Mn}(\mathrm{mg} / \mathrm{L})) \text { IN35 } & 1.00128 & \mathrm{t}-\text { Ratio } & 9.949129 \\ \text { Mean(Mn (mg/L)) IN33 } & 0.99097 & \text { DF } & 172 \\ \text { Mean Difference } & 0.01032 & \text { Prob }>|\mathrm{t}| & <.0001 \\ \text { Std Error } & 0.00104 & \text { Prob }>\mathrm{t} & <.0001 \\ \text { Upper95\% } & 0.01236 & \text { Prob }<\mathrm{t} & 1.0000 \\ \text { Lower95\% } & 0.00827 & & \\ \text { N } & 173 & & \\ \text { Correlation } & 0.64171 & & \end{array}$

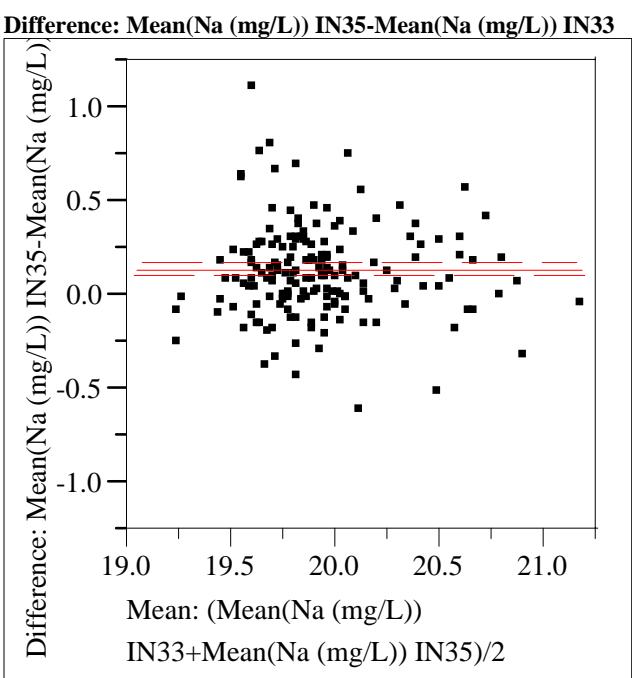

$\begin{array}{lrlr}\text { Mean(Na (mg/L)) IN35 } & 19.9922 & \text { t-Ratio } & 7.454294 \\ \text { Mean(Na (mg/L)) IN33 } & 19.8535 & \text { DF } & 172 \\ \text { Mean Difference } & 0.13865 & \text { Prob }>|t| & <.0001 \\ \text { Std Error } & 0.0186 & \text { Prob }>\text { t } & <.0001 \\ \text { Upper95\% } & 0.17536 & \text { Prob }<\mathrm{t} & 1.0000 \\ \text { Lower95\% } & 0.10193 & & \\ \text { N } & 173 & & \\ \text { Correlation } & 0.7674 & & \end{array}$

Difference: Mean(Ni (mg/L)) IN35-Mean(Ni (mg/L)) IN33

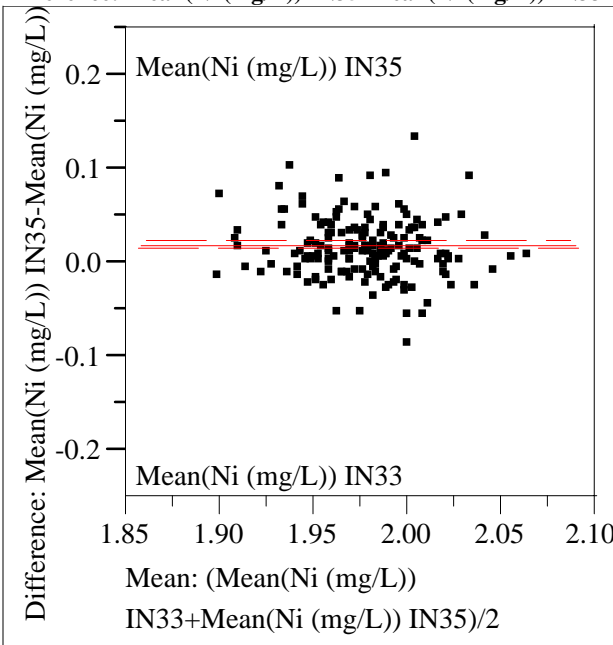

$\begin{array}{lrlr}\text { Mean(Ni (mg/L)) IN35 } & 1.98689 & \text { t-Ratio } & 7.848886 \\ \text { Mean(Ni (mg/L)) IN33 } & 1.96802 & \text { DF } & 172 \\ \text { Mean Difference } & 0.01887 & \text { Prob }>|t| & <.0001 \\ \text { Std Error } & 0.0024 & \text { Prob }>\mathrm{t} & <.0001 \\ \text { Upper95\% } & 0.02362 & \text { Prob }<\mathrm{t} & 1.0000 \\ \text { Lower95\% } & 0.01413 & & \\ \mathrm{~N} & 173 & & \\ \text { Correlation } & 0.56247 & & \end{array}$


WSRC-TR-2004-00576

Exhibit A4. Paired Differences for IN33 and IN35

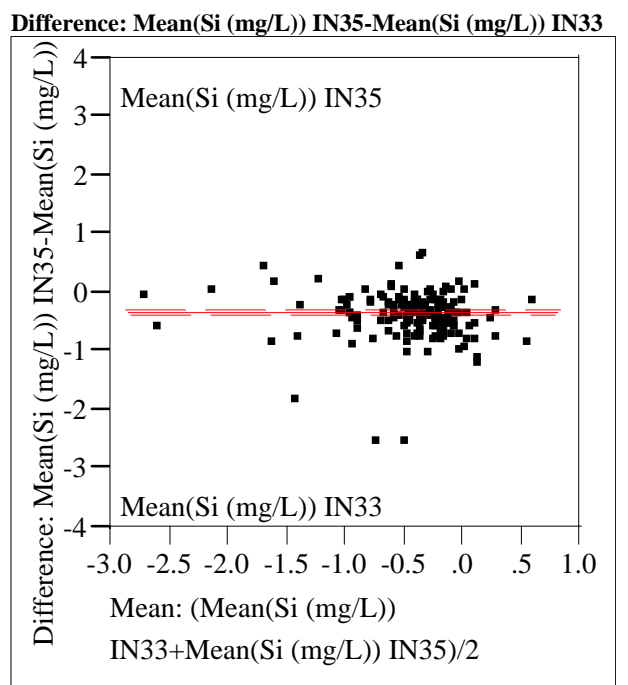

Mean(Si (mg/L)) IN35 $\quad-0.6047 \quad$ t-Ratio $\quad-10.8793$

Mean(Si (mg/L)) IN33

Mean Difference

Std Error

Upper95\%

Lower95\%

$\mathrm{N}$

Correlation

Difference: Mean(Ti (mg/L)) IN35-Mean(Ti (mg/L)) IN33

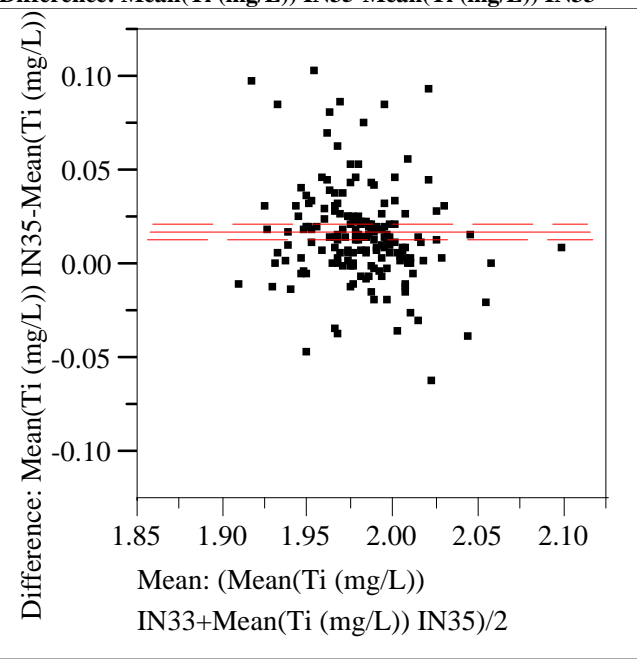

Mean(Ti (mg/L)) IN35 1.98943

Mean(Ti (mg/L)) IN33 1.9722

Mean Difference $\quad 0.01723$

Std Error

Upper95\%

Lower95\%

$\mathrm{N}$

Correlation
t-Ratio $\quad 8.872537$

DF 172

Prob $>\mathrm{t} \quad<.0001$

Prob $<\mathrm{t} \quad 1.0000$
Prob $>|\mathrm{t}| \quad<.0001$

Revision 0

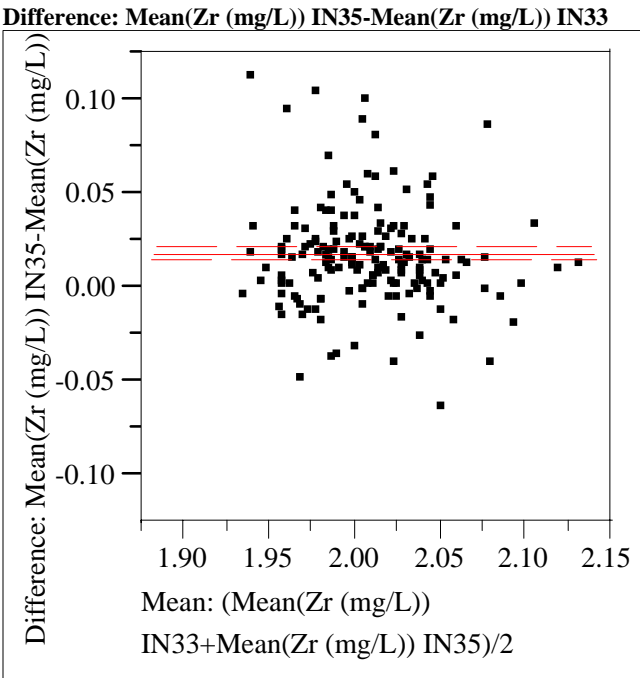

$\begin{array}{lrlr}\text { Mean }(\mathrm{Zr}(\mathrm{mg} / \mathrm{L})) \text { IN35 } & 2.01713 & \mathrm{t}-\text { Ratio } & 8.902987 \\ \text { Mean }(\mathrm{Zr}(\mathrm{mg} / \mathrm{L})) \text { IN33 } & 1.99913 & \text { DF } & 172 \\ \text { Mean Difference } & 0.01799 & \text { Prob }>|\mathrm{t}| & <.0001 \\ \text { Std Error } & 0.00202 & \text { Prob }>\mathrm{t} & <.0001 \\ \text { Upper95\% } & 0.02198 & \text { Prob }<\mathrm{t} & 1.0000 \\ \text { Lower95\% } & 0.014 & & \\ \text { N } & 173 & & \\ \text { Correlation } & 0.76931 & & \end{array}$

Correlation

0.76931

(mg/L)) IN35-Mean(U (mg/L)) IN33

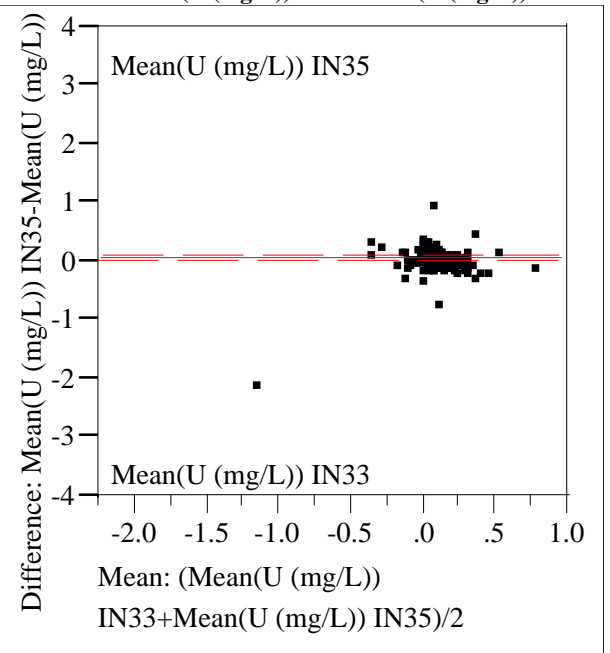

$\begin{array}{lrlr}\text { Mean(U (mg/L)) IN35 } & 0.12129 & \text { t-Ratio } & 3.345885 \\ \text { Mean(U (mg/L)) IN33 } & 0.06457 & \text { DF } & 172 \\ \text { Mean Difference } & 0.05673 & \text { Prob > }|\mathrm{t}| & 0.0010 \\ \text { Std Error } & 0.01695 & \text { Prob }>\text { t } & 0.0005 \\ \text { Upper95\% } & 0.09019 & \text { Prob }<\mathrm{t} & 0.9995 \\ \text { Lower95\% } & 0.02326 & & \\ \text { N } & 173 & & \\ \text { Correlation } & 0.38918 & & \end{array}$


WSRC-TR-2004-00576

Exhibit A5. Paired Differences for IN34 and IN36
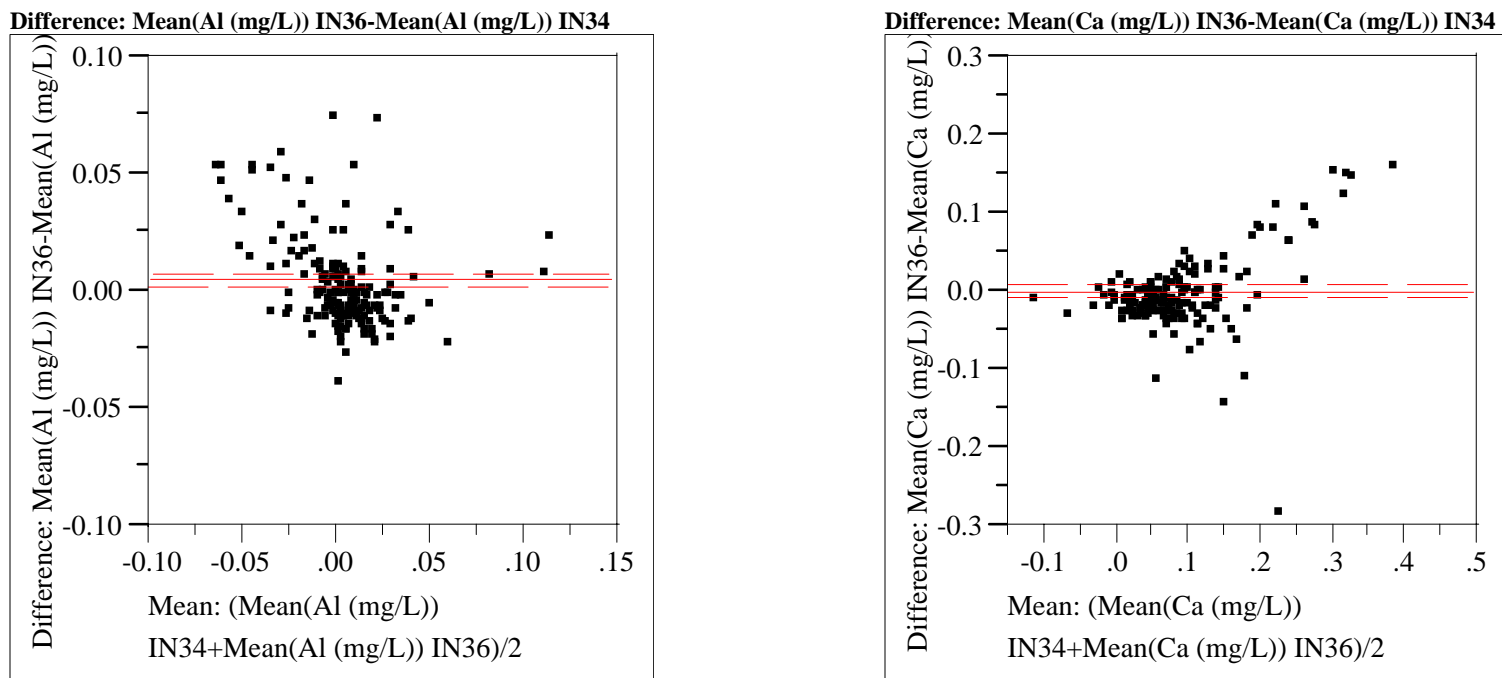

$\begin{array}{lrlr}\text { Mean(Al (mg/L)) IN36 } & 0.00508 & \text { t-Ratio } & 2.971443 \\ \text { Mean(Al (mg/L)) IN34 } & 0.00062 & \text { DF } & 171 \\ \text { Mean Difference } & 0.00446 & \text { Prob }>|t| & 0.0034 \\ \text { Std Error } & 0.0015 & \text { Prob }>t & 0.0017 \\ \text { Upper95\% } & 0.00743 & \text { Prob }<\mathrm{t} & 0.9983 \\ \text { Lower95\% } & 0.0015 & & \\ \text { N } & 172 & & \\ \text { Correlation } & 0.75306 & & \end{array}$

Difference: Mean(B (mg/L)) IN36-Mean(B (mg/L)) IN34

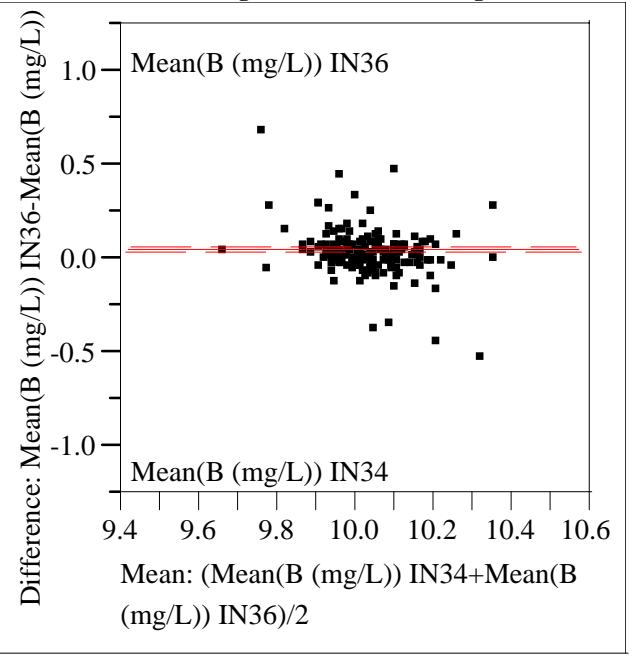

$\begin{array}{lrlr}\text { Mean(B (mg/L)) IN36 } & 10.0553 & \text { t-Ratio } & 4.868207 \\ \text { Mean(B (mg/L)) IN34 } & 10.0074 & \text { DF } & 171 \\ \text { Mean Difference } & 0.04795 & \text { Prob }>|t| & <.0001 \\ \text { Std Error } & 0.00985 & \text { Prob }>t & <.0001 \\ \text { Upper95\% } & 0.06739 & \text { Prob }<\mathrm{t} & 1.0000 \\ \text { Lower95\% } & 0.02851 & & \\ \text { N } & 172 & & \\ \text { Correlation } & 0.45439 & & \end{array}$

$\begin{array}{lrlr}\text { Mean(Ca (mg/L)) IN36 } & 0.08731 & \text { t-Ratio } & -0.09392 \\ \text { Mean(Ca (mg/L)) IN34 } & 0.08766 & \text { DF } & 171 \\ \text { Mean Difference } & -0.0003 & \text { Prob }>|t| & 0.9253 \\ \text { Std Error } & 0.00368 & \text { Prob }>\mathrm{t} & 0.5374 \\ \text { Upper95\% } & 0.00692 & \text { Prob }<\mathrm{t} & 0.4626 \\ \text { Lower95\% } & -0.0076 & & \\ \text { N } & 172 & & \\ \text { Correlation } & 0.8546 & & \end{array}$

Difference: Mean(Cr (mg/L)) IN36-Mean(Cr (mg/L)) IN34

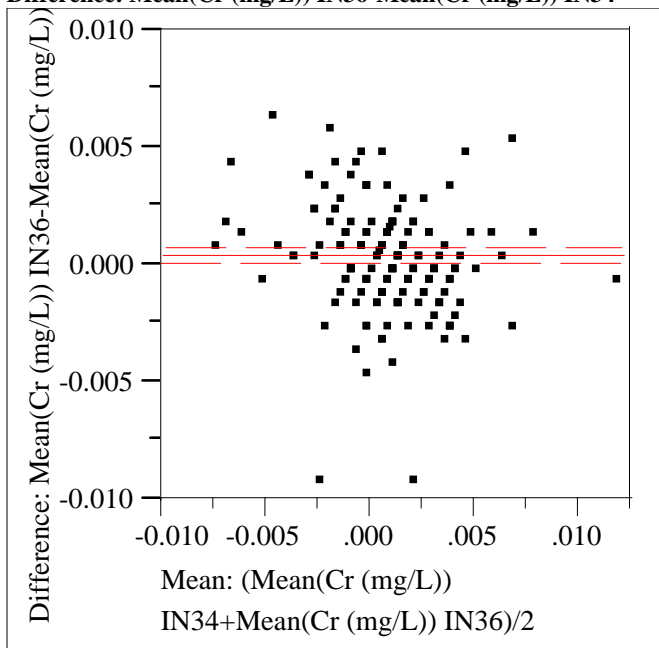

$\begin{array}{lrlr}\text { Mean }(\mathrm{Cr}(\mathrm{mg} / \mathrm{L})) \mathrm{IN} 36 & 0.00094 & \mathrm{t}-\text { Ratio } & 2.065946 \\ \text { Mean(Cr (mg/L)) IN34 } & 0.00058 & \text { DF } & 171 \\ \text { Mean Difference } & 0.00035 & \text { Prob }>|\mathrm{t}| & 0.0403 \\ \text { Std Error } & 0.00017 & \text { Prob }>\mathrm{t} & 0.0202 \\ \text { Upper95\% } & 0.00069 & \text { Prob }<\mathrm{t} & 0.9798 \\ \text { Lower95\% } & 0.00002 & & \\ \mathrm{~N} & 172 & & \\ \text { Correlation } & 0.70603 & & \end{array}$


WSRC-TR-2004-00576

\section{Exhibit A5. Paired Differences for IN34 and IN36}

Revision 0
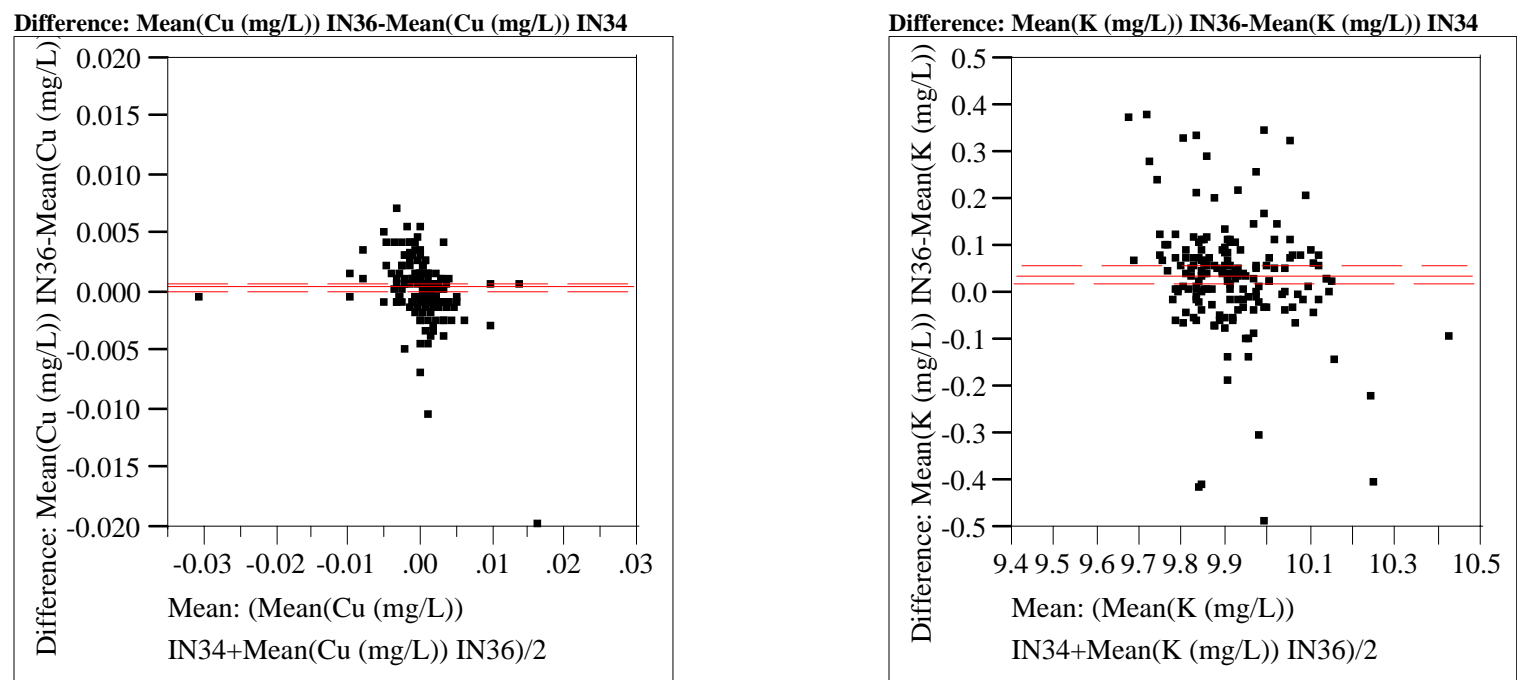

$\begin{array}{lrlr}\text { Mean(Cu (mg/L)) IN36 } & 0.00029 & \text { t-Ratio } & 2.197645 \\ \text { Mean(Cu (mg/L)) IN34 } & -0.0002 & \text { DF } & 171 \\ \text { Mean Difference } & 0.00046 & \text { Prob }>|t| & 0.0293 \\ \text { Std Error } & 0.00021 & \text { Prob }>\mathrm{t} & 0.0147 \\ \text { Upper95\% } & 0.00087 & \text { Prob }<\mathrm{t} & 0.9853 \\ \text { Lower95\% } & 0.00005 & & \\ \text { N } & 172 & & \\ \text { Correlation } & 0.80463 & & \end{array}$

Difference: Mean(Fe (mg/L)) IN36-Mean(Fe (mg/L)) IN34

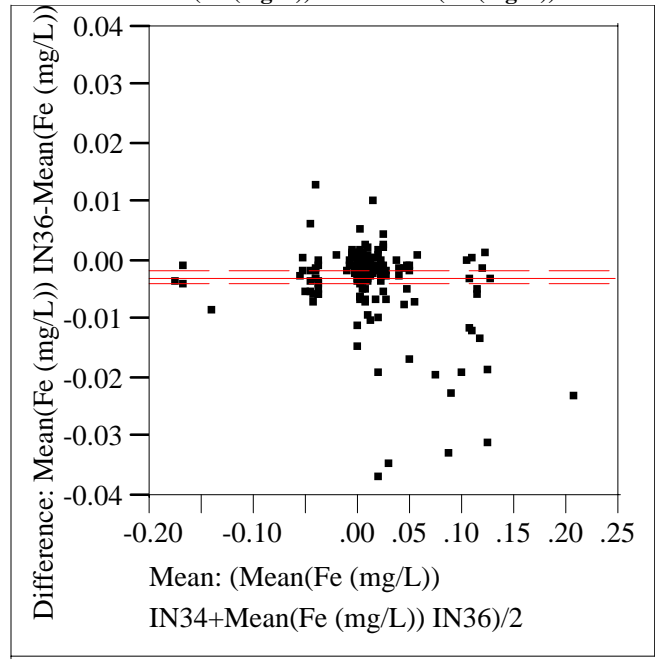

$\begin{array}{lrlr}\text { Mean(Fe (mg/L)) IN36 } & 0.0086 & \text { t-Ratio } & -5.34193 \\ \text { Mean(Fe (mg/L)) IN34 } & 0.01141 & \text { DF } & 171 \\ \text { Mean Difference } & -0.0028 & \text { Prob }>|t| & <.0001 \\ \text { Std Error } & 0.00052 & \text { Prob }>\text { t } & 1.0000 \\ \text { Upper95\% } & -0.0018 & \text { Prob }<\mathrm{t} & <.0001 \\ \text { Lower95\% } & -0.0038 & & \\ \text { N } & 172 & & \\ \text { Correlation } & 0.99148 & & \end{array}$

$\begin{array}{lrlr}\text { Mean }(\mathrm{K}(\mathrm{mg} / \mathrm{L})) \text { IN36 } & 9.93594 & \mathrm{t}-\text { Ratio } & 4.122717 \\ \text { Mean(K (mg/L)) IN34 } & 9.89708 & \text { DF } & 171 \\ \text { Mean Difference } & 0.03886 & \text { Prob }>|\mathrm{t}| & <.0001 \\ \text { Std Error } & 0.00943 & \text { Prob }>\mathrm{t} & <.0001 \\ \text { Upper95\% } & 0.05747 & \text { Prob }<\mathrm{t} & 1.0000 \\ \text { Lower95\% } & 0.02026 & & \\ \mathrm{~N} & 172 & & \\ \text { Correlation } & 0.56512 & & \end{array}$

Difference: Mean(Li (mg/L)) IN36-Mean(Li (mg/L)) IN34

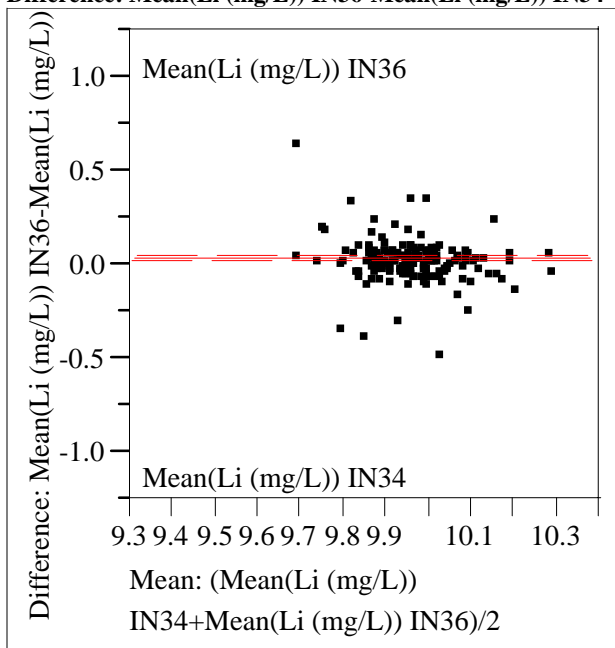

$\begin{array}{lrlr}\text { Mean(Li (mg/L)) IN36 } & 9.97395 & \text { t-Ratio } & 3.962486 \\ \text { Mean(Li (mg/L)) IN34 } & 9.93931 & \text { DF } & 171 \\ \text { Mean Difference } & 0.03464 & \text { Prob }>|t| & 0.0001 \\ \text { Std Error } & 0.00874 & \text { Prob }>\text { t } & <.0001 \\ \text { Upper95\% } & 0.0519 & \text { Prob }<\mathrm{t} & 0.9999 \\ \text { Lower95\% } & 0.01738 & & \\ \text { N } & 172 & & \\ \text { Correlation } & 0.52087 & & \end{array}$


WSRC-TR-2004-00576

Exhibit A5. Paired Differences for IN34 and IN36
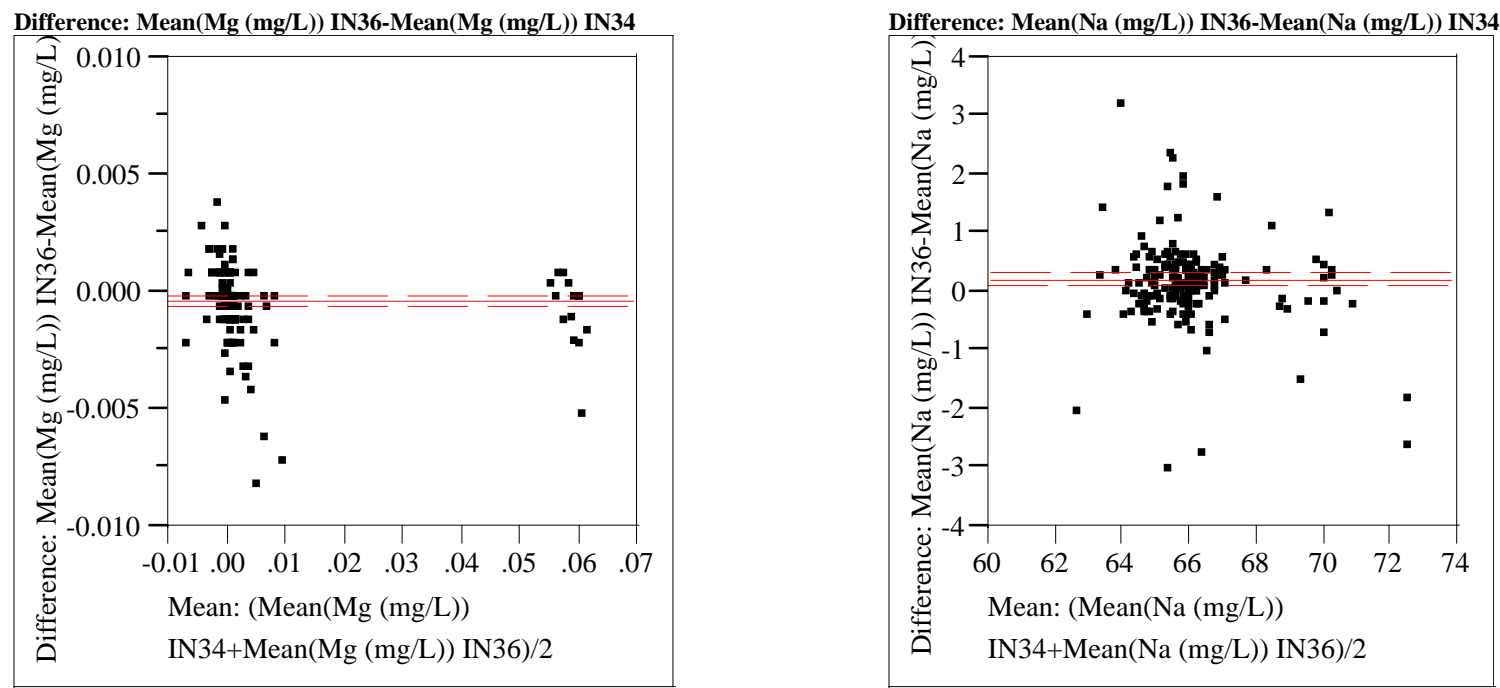

$\begin{array}{lrlr}\text { Mean }(\mathrm{Mg}(\mathrm{mg} / \mathrm{L})) \text { IN36 } & 0.00501 & \text { t-Ratio } & -3.16373 \\ \text { Mean(Mg (mg/L)) IN34 } & 0.00539 & \text { DF } & 171 \\ \text { Mean Difference } & -0.0004 & \text { Prob }>|t| & 0.0018 \\ \text { Std Error } & 0.00012 & \text { Prob }>\text { t } & 0.9991 \\ \text { Upper95\% } & -0.0001 & \text { Prob }<\mathrm{t} & 0.0009 \\ \text { Lower95\% } & -0.0006 & & \\ \text { N } & 172 & & \\ \text { Correlation } & 0.99537 & & \end{array}$

$\begin{array}{lrlr}\text { Mean(Na (mg/L)) IN36 } & 66.1319 & \text { t-Ratio } & 3.654829 \\ \text { Mean(Na (mg/L)) IN34 } & 65.9269 & \text { DF } & 171 \\ \text { Mean Difference } & 0.20496 & \text { Prob }>|t| & 0.0003 \\ \text { Std Error } & 0.05608 & \text { Prob }>\text { t } & 0.0002 \\ \text { Upper95\% } & 0.31566 & \text { Prob }<\mathrm{t} & 0.9998 \\ \text { Lower95\% } & 0.09426 & & \\ \text { N } & 172 & & \\ \text { Correlation } & 0.90651 & & \end{array}$

Difference: Mean(Mn (mg/L)) IN36-Mean(Mn (mg/L)) IN34

Difference: Mean(Ni (mg/L)) IN36-Mean(Ni (mg/L)) IN34
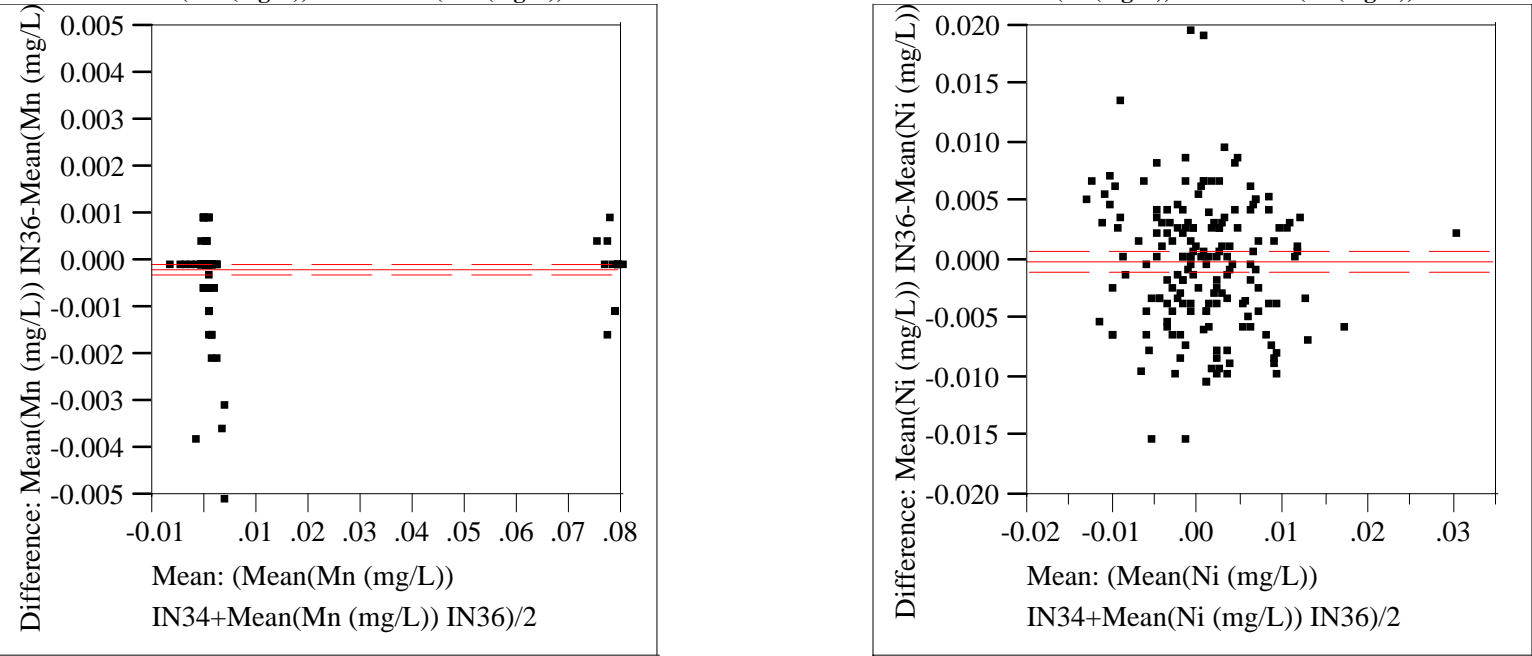

$\begin{array}{lrlr}\text { Mean(Mn (mg/L)) IN36 } & 0.00639 & \text { t-Ratio } & -3.51619 \\ \text { Mean(Mn (mg/L)) IN34 } & 0.0066 & \text { DF } & 171 \\ \text { Mean Difference } & -0.0002 & \text { Prob }>|t| & 0.0006 \\ \text { Std Error } & 0.00006 & \text { Prob }>\text { t } & 0.9997 \\ \text { Upper95\% } & -9.3 e-5 & \text { Prob }<\mathrm{t} & 0.0003 \\ \text { Lower95\% } & -0.0003 & & \\ \text { N } & 172 & & \\ \text { Correlation } & 0.99932 & & \end{array}$

$\begin{array}{lrlr}\text { Mean(Ni (mg/L)) IN36 } & 0.00062 & \text { t-Ratio } & -0.33968 \\ \text { Mean(Ni (mg/L)) IN34 } & 0.00077 & \text { DF } & 171 \\ \text { Mean Difference } & -0.0001 & \text { Prob }>|t| & 0.7345 \\ \text { Std Error } & 0.00043 & \text { Prob }>\text { t } & 0.6327 \\ \text { Upper95\% } & 0.0007 & \text { Prob }<\mathrm{t} & 0.3673 \\ \text { Lower95\% } & -0.001 & & \\ \text { N } & 172 & & \\ \text { Correlation } & 0.66338 & & \end{array}$


WSRC-TR-2004-00576

\section{Exhibit A5. Paired Differences for IN34 and IN36}

Revision 0
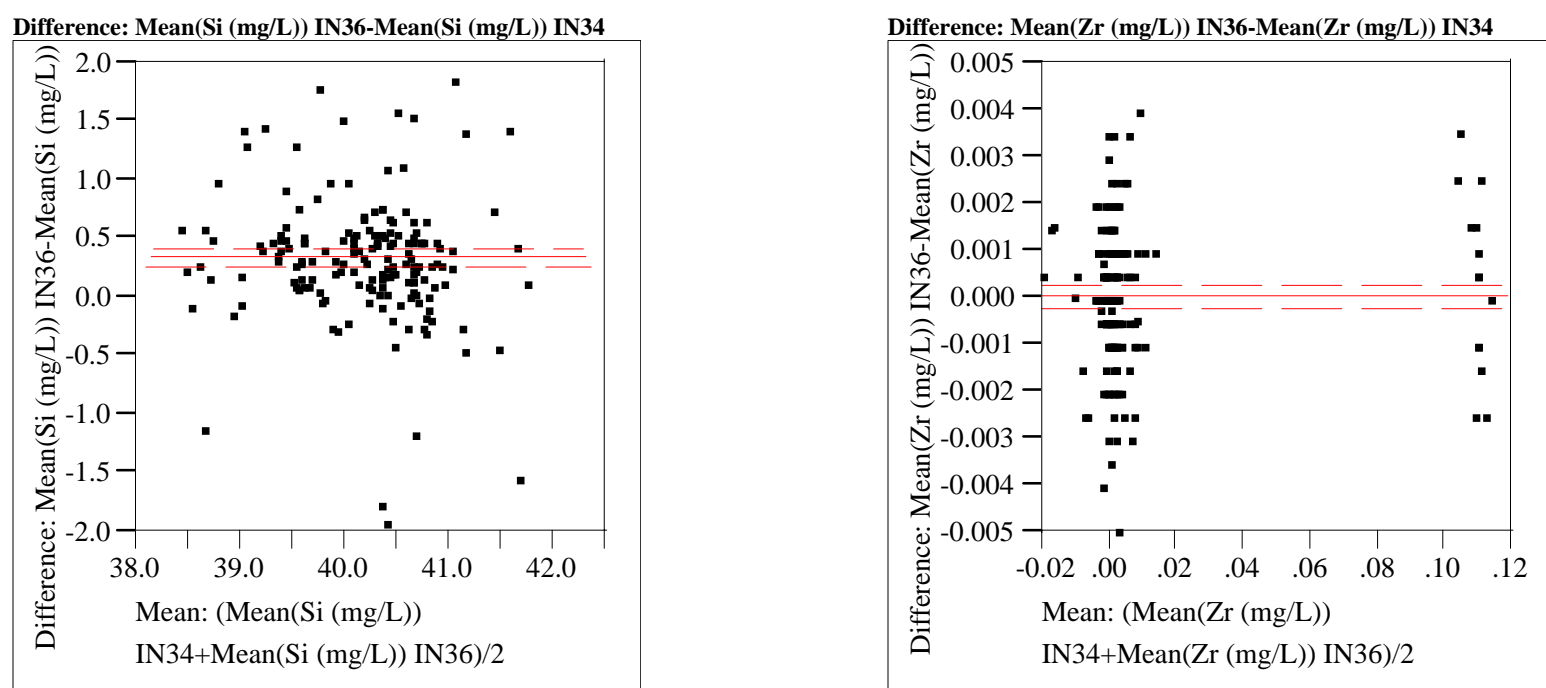

$\begin{array}{lrlr}\text { Mean(Si (mg/L)) IN36 } & 40.345 & \text { t-Ratio } & 8.287566 \\ \text { Mean(Si (mg/L)) IN34 } & 40.0109 & \text { DF } & 171 \\ \text { Mean Difference } & 0.33409 & \text { Prob }>|t| & <.0001 \\ \text { Std Error } & 0.04031 & \text { Prob }>\text { t } & <.0001 \\ \text { Upper95\% } & 0.41367 & \text { Prob }<\mathrm{t} & 1.0000 \\ \text { Lower95\% } & 0.25452 & & \\ \text { N } & 172 & & \\ \text { Correlation } & 0.73509 & & \end{array}$

Difference: Mean(Ti (mg/L)) IN36-Mean(Ti (mg/L)) IN34

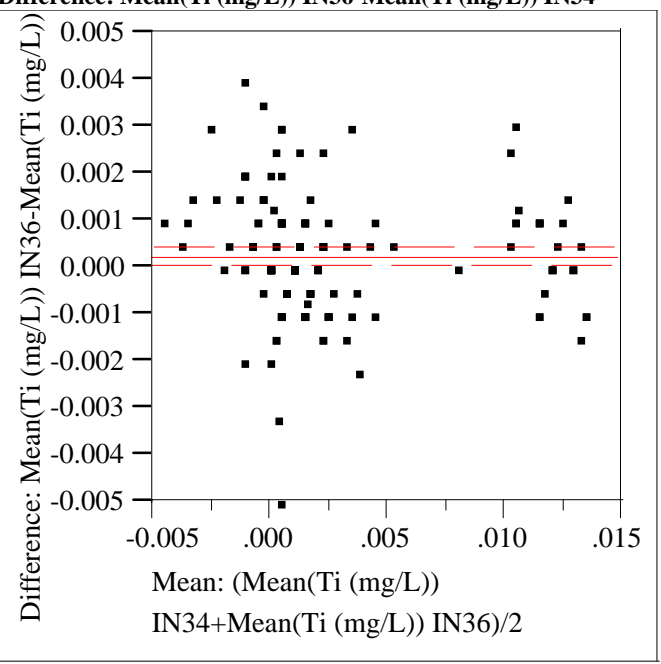

$\begin{array}{lrlr}\text { Mean(Zr (mg/L)) IN36 } & 0.0096 & \text { t-Ratio } & 0.037726 \\ \text { Mean(Zr (mg/L)) IN34 } & 0.00959 & \text { DF } & 171 \\ \text { Mean Difference } & 4.36 \mathrm{e}-6 & \text { Prob }>|t| & 0.9700 \\ \text { Std Error } & 0.00012 & \text { Prob }>\text { t } & 0.4850 \\ \text { Upper95\% } & 0.00023 & \text { Prob }<\mathrm{t} & 0.5150 \\ \text { Lower95\% } & -0.0002 & & \\ \text { N } & 172 & & \\ \text { Correlation } & 0.99875 & & \end{array}$

Difference: Mean(U (mg/L)) IN36-Mean(U (mg/L)) IN34

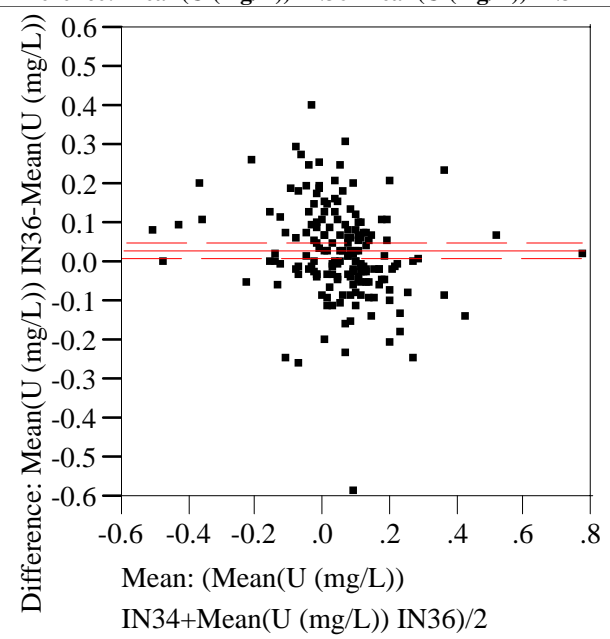

$\begin{array}{lrlr}\text { Mean(Ti (mg/L)) IN36 } & 0.00269 & \text { t-Ratio } & 2.262904 \\ \text { Mean(Ti (mg/L)) IN34 } & 0.00248 & \text { DF } & 171 \\ \text { Mean Difference } & 0.00021 & \text { Prob }>|t| & 0.0249 \\ \text { Std Error } & 0.00009 & \text { Prob }>\text { t } & 0.0124 \\ \text { Upper95\% } & 0.0004 & \text { Prob }<\mathrm{t} & 0.9876 \\ \text { Lower95\% } & 0.00003 & & \\ \text { N } & 172 & & \\ \text { Correlation } & 0.95992 & & \end{array}$

$\begin{array}{lrlr}\text { Mean(U (mg/L)) IN36 } & 0.06586 & \text { t-Ratio } & 3.357473 \\ \text { Mean(U (mg/L)) IN34 } & 0.03474 & \text { DF } & 171 \\ \text { Mean Difference } & 0.03112 & \text { Prob }>|t| & 0.0010 \\ \text { Std Error } & 0.00927 & \text { Prob }>\text { t } & 0.0005 \\ \text { Upper95\% } & 0.04941 & \text { Prob }<\mathrm{t} & 0.9995 \\ \text { Lower95\% } & 0.01282 & & \\ \text { N } & 172 & & \\ \text { Correlation } & 0.72433 & & \end{array}$


WSRC-TR-2004-00576

Exhibit A6. Paired Differences for IN38 and IN39
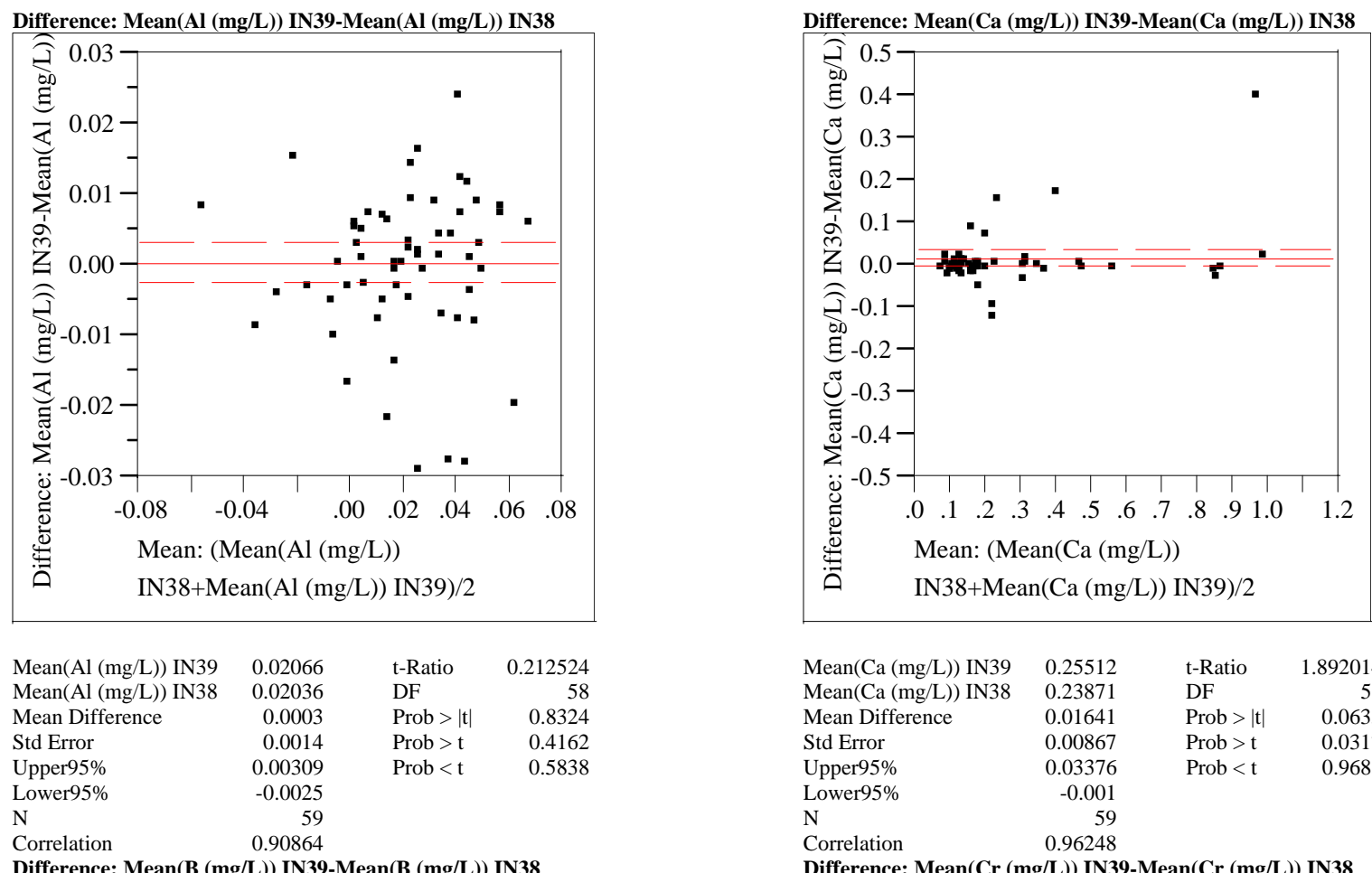

Difference: Mean(B (mg/L)) IN39-Mean(B (mg/L)) IN38

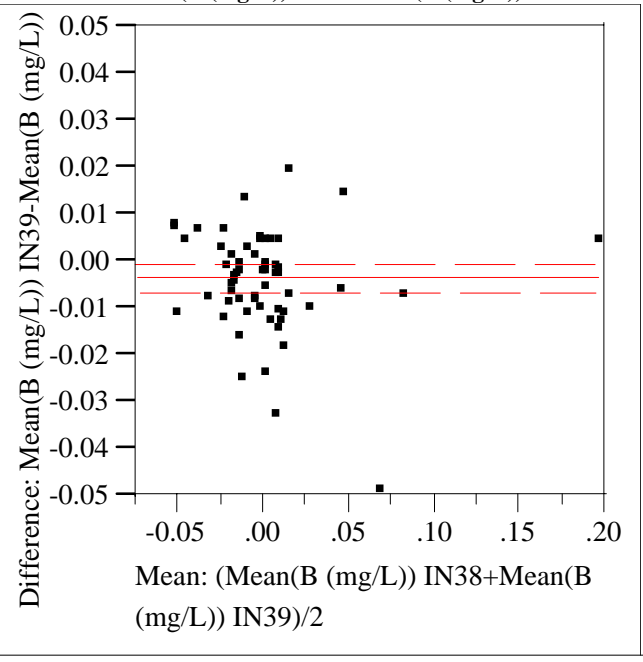

$\begin{array}{lrlr}\text { Mean(B (mg/L)) IN39 } & -0.0025 & \mathrm{t}-\text { Ratio } & -2.64729 \\ \text { Mean(B (mg/L)) IN38 } & 0.00133 & \text { DF } & 58 \\ \text { Mean Difference } & -0.0038 & \text { Prob }>|\mathrm{t}| & 0.0104 \\ \text { Std Error } & 0.00144 & \text { Prob }>\mathrm{t} & 0.9948 \\ \text { Upper95\% } & -0.0009 & \text { Prob }<\mathrm{t} & 0.0052 \\ \text { Lower95\% } & -0.0067 & & \\ \text { N } & 59 & & \\ \text { Correlation } & 0.95428 & & \end{array}$

$\begin{array}{lrlr}\text { Mean(Ca (mg/L)) IN39 } & 0.25512 & \text { t-Ratio } & 1.892014 \\ \text { Mean(Ca (mg/L)) IN38 } & 0.23871 & \text { DF } & 58 \\ \text { Mean Difference } & 0.01641 & \text { Prob }>|t| & 0.0635 \\ \text { Std Error } & 0.00867 & \text { Prob }>\text { t } & 0.0317 \\ \text { Upper95\% } & 0.03376 & \text { Prob }<\mathrm{t} & 0.9683 \\ \text { Lower95\% } & -0.001 & & \\ \text { N } & 59 & & \\ \text { Correlation } & 0.96248 & & \end{array}$

Difference: Mean(Cr (mg/L)) IN39-Mean(Cr (mg/L)) IN38

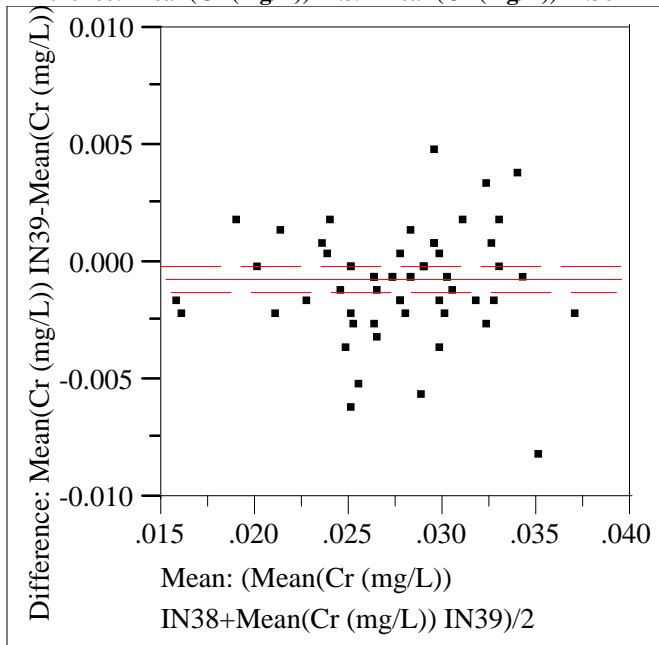

$\begin{array}{lrlr}\text { Mean }(\mathrm{Cr}(\mathrm{mg} / \mathrm{L})) \mathrm{IN} 39 & 0.02724 & \mathrm{t}-\text { Ratio } & -2.49352 \\ \text { Mean }(\mathrm{Cr}(\mathrm{mg} / \mathrm{L})) \text { IN38 } & 0.02797 & \text { DF } & 58 \\ \text { Mean Difference } & -0.0007 & \text { Prob }>|\mathrm{t}| & 0.0155 \\ \text { Std Error } & 0.0003 & \text { Prob }>\mathrm{t} & 0.9922 \\ \text { Upper95\% } & -0.0001 & \text { Prob }<\mathrm{t} & 0.0078 \\ \text { Lower95\% } & -0.0013 & & \\ \text { N } & 59 & & \\ \text { Correlation } & 0.87224 & & \end{array}$


WSRC-TR-2004-00576

Exhibit A6. Paired Differences for IN38 and IN39

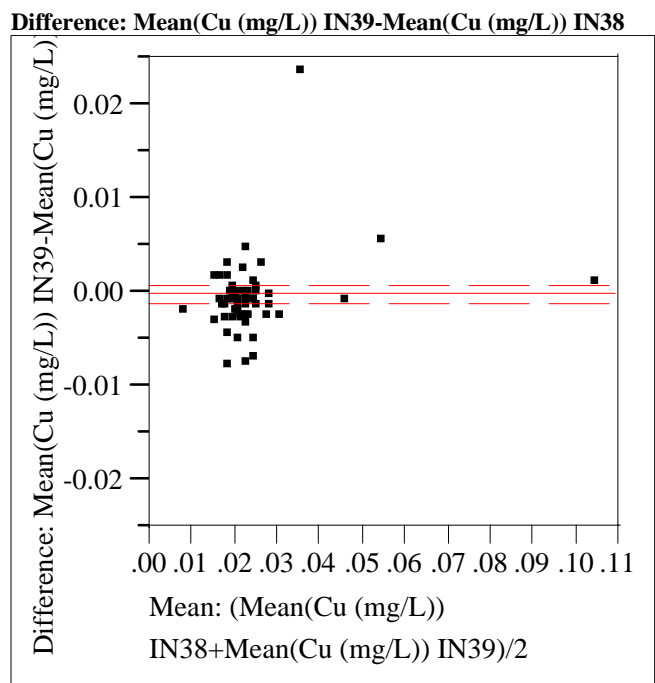

$\begin{array}{lrlr}\text { Mean(Cu (mg/L)) IN39 } & 0.02344 & \text { t-Ratio } & -0.49557 \\ \text { Mean(Cu (mg/L)) IN38 } & 0.0237 & \text { DF } & 58 \\ \text { Mean Difference } & -0.0003 & \text { Prob }>|t| & 0.6221 \\ \text { Std Error } & 0.00053 & \text { Prob }>\mathrm{t} & 0.6890 \\ \text { Upper95\% } & 0.0008 & \text { Prob }<\mathrm{t} & 0.3110 \\ \text { Lower95\% } & -0.0013 & & \\ \text { N } & 59 & & \\ \text { Correlation } & 0.95145 & & \end{array}$

Difference: Mean(Fe (mg/L)) IN39-Mean(Fe (mg/L)) IN38

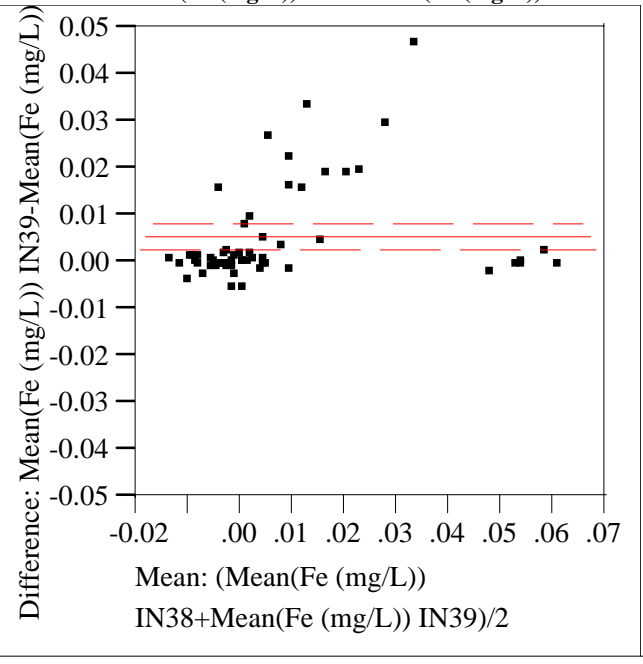

$\begin{array}{lrlr}\text { Mean(Fe (mg/L)) IN39 } & 0.00981 & \text { t-Ratio } & 4.032934 \\ \text { Mean(Fe (mg/L)) IN38 } & 0.00436 & \text { DF } & 58 \\ \text { Mean Difference } & 0.00545 & \text { Prob }>|t| & 0.0002 \\ \text { Std Error } & 0.00135 & \text { Prob }>\text { t } & <.0001 \\ \text { Upper95\% } & 0.00815 & \text { Prob }<\mathrm{t} & 0.9999 \\ \text { Lower95\% } & 0.00274 & & \\ \text { N } & 59 & & \\ \text { Correlation } & 0.86304 & & \end{array}$

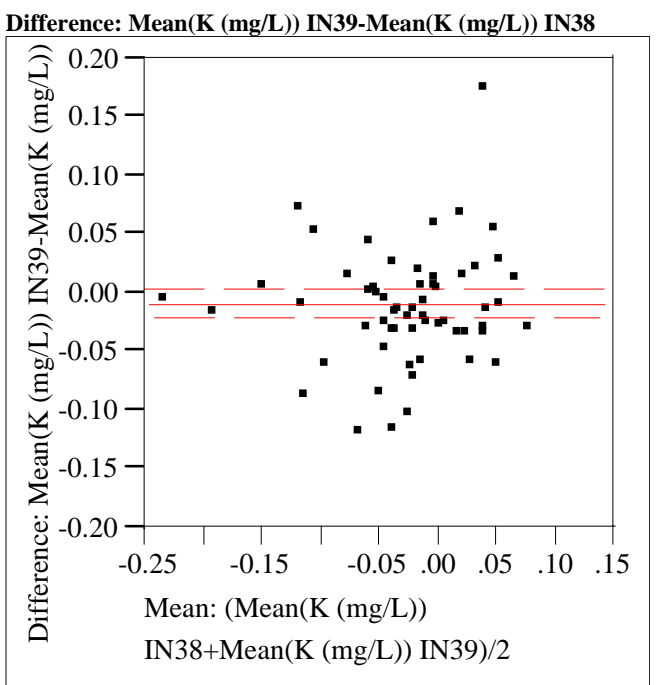

$\begin{array}{lrlr}\text { Mean }(\mathrm{K}(\mathrm{mg} / \mathrm{L})) \text { IN39 } & -0.0317 & \mathrm{t}-\text { Ratio } & -1.417 \\ \text { Mean(K (mg/L)) IN38 } & -0.0227 & \text { DF } & 58 \\ \text { Mean Difference } & -0.009 & \text { Prob }>|\mathrm{t}| & 0.1618 \\ \text { Std Error } & 0.00634 & \text { Prob }>\mathrm{t} & 0.9191 \\ \text { Upper95\% } & 0.00371 & \text { Prob }<\mathrm{t} & 0.0809 \\ \text { Lower95\% } & -0.0217 & & \\ \mathrm{~N} & 59 & & \\ \text { Correlation } & 0.72612 & & \end{array}$

Difference: Mean(Li (mg/L)) IN39-Mean(Li (mg/L)) IN38

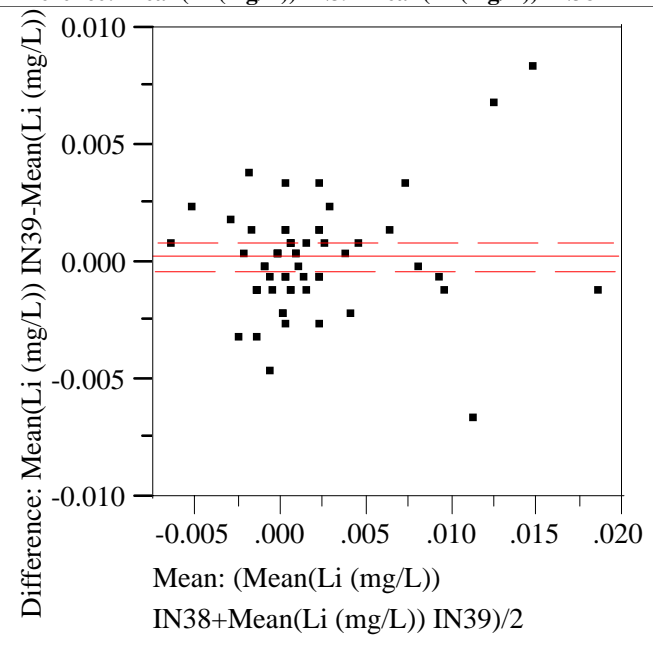

$\begin{array}{lrlr}\text { Mean(Li (mg/L)) IN39 } & 0.00188 & \text { t-Ratio } & 0.824099 \\ \text { Mean(Li (mg/L)) IN38 } & 0.00163 & \text { DF } & 58 \\ \text { Mean Difference } & 0.00025 & \text { Prob }>|t| & 0.4133 \\ \text { Std Error } & 0.00031 & \text { Prob }>\text { t } & 0.2066 \\ \text { Upper95\% } & 0.00087 & \text { Prob }<\mathrm{t} & 0.7934 \\ \text { Lower95\% } & -0.0004 & & \\ \text { N } & 59 & & \\ \text { Correlation } & 0.87734 & & \end{array}$


WSRC-TR-2004-00576

\section{Exhibit A6. Paired Differences for IN38 and IN39}

Revision 0
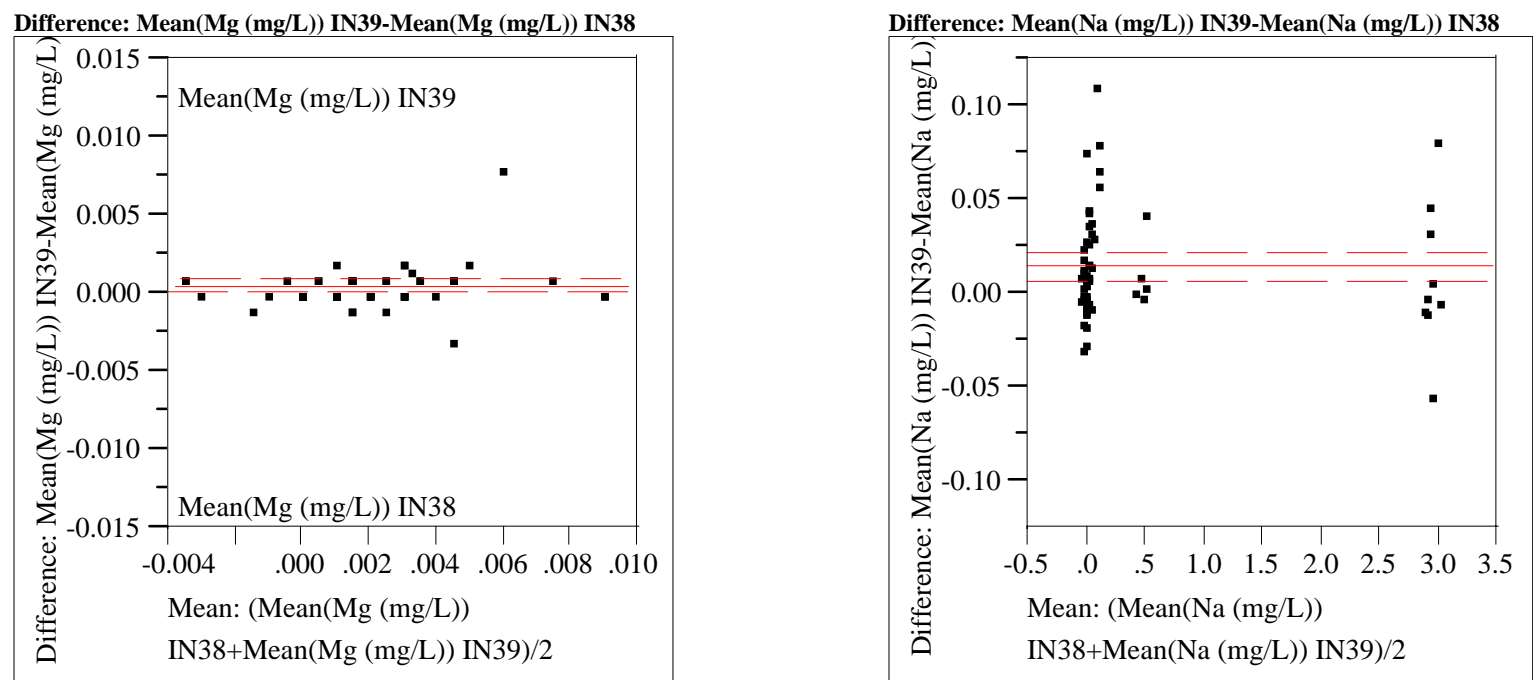

$\begin{array}{lrlr}\text { Mean }(\mathrm{Mg}(\mathrm{mg} / \mathrm{L})) \text { IN39 } & 0.00215 & \mathrm{t}-\text { Ratio } & 2.932233 \\ \text { Mean(Mg (mg/L)) IN38 } & 0.00165 & \text { DF } & 58 \\ \text { Mean Difference } & 0.0005 & \text { Prob }>|\mathrm{t}| & 0.0048 \\ \text { Std Error } & 0.00017 & \text { Prob }>\mathrm{t} & 0.0024 \\ \text { Upper95\% } & 0.00084 & \text { Prob }<\mathrm{t} & 0.9976 \\ \text { Lower95\% } & 0.00016 & & \\ \text { N } & 59 & & \\ \text { Correlation } & 0.87262 & & \end{array}$

Difference: Mean(Mn (mg/L)) IN39-Mean(Mn (mg/L)) IN38

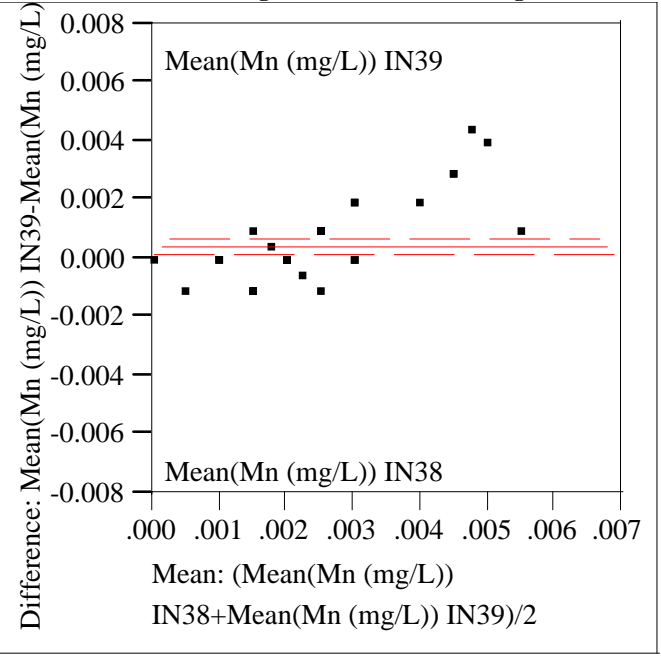

$\begin{array}{lrlr}\text { Mean(Mn (mg/L)) IN39 } & 0.00249 & \text { t-Ratio } & 2.89151 \\ \text { Mean(Mn (mg/L)) IN38 } & 0.00208 & \text { DF } & 58 \\ \text { Mean Difference } & 0.00042 & \text { Prob > |t } & 0.0054 \\ \text { Std Error } & 0.00014 & \text { Prob }>t & 0.0027 \\ \text { Upper95\% } & 0.0007 & \text { Prob }<\mathrm{t} & 0.9973 \\ \text { Lower95\% } & 0.00013 & & \\ \text { N } & 59 & & \\ \text { Correlation } & 0.70195 & & \end{array}$

$\begin{array}{lrlr}\text { Mean(Na (mg/L)) IN39 } & 0.50751 & \text { t-Ratio } & 3.635412 \\ \text { Mean(Na (mg/L)) IN38 } & 0.49352 & \text { DF } & 58 \\ \text { Mean Difference } & 0.01399 & \text { Prob }>|t| & 0.0006 \\ \text { Std Error } & 0.00385 & \text { Prob }>t & 0.0003 \\ \text { Upper95\% } & 0.0217 & \text { Prob }<t & 0.9997 \\ \text { Lower95\% } & 0.00629 & & \\ \text { N } & 59 & & \\ \text { Correlation } & 0.99961 & & \end{array}$

Difference: Mean(Ni (mg/L)) IN39-Mean(Ni (mg/L)) IN38

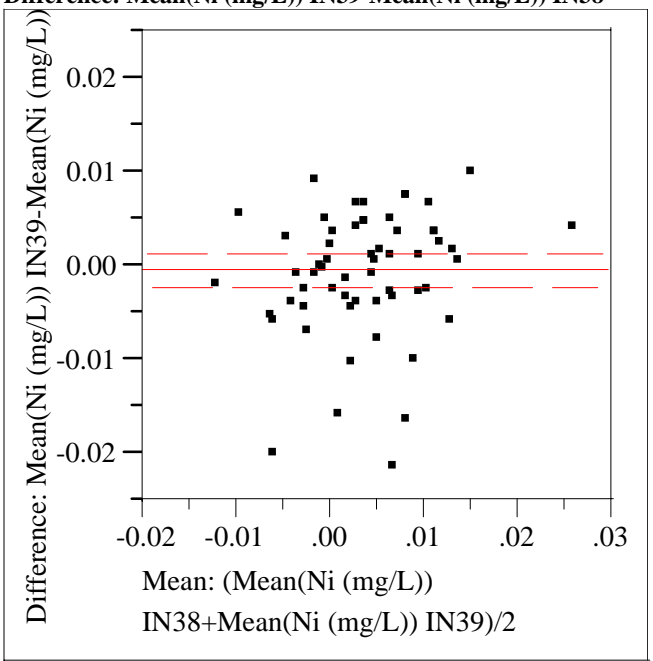

$\begin{array}{lrlr}\text { Mean(Ni (mg/L)) IN39 } & 0.00325 & \mathrm{t}-\text { Ratio } & -0.60716 \\ \text { Mean(Ni (mg/L)) IN38 } & 0.00378 & \text { DF } & 58 \\ \text { Mean Difference } & -0.0005 & \text { Prob }>|t| & 0.5461 \\ \text { Std Error } & 0.00087 & \text { Prob }>\mathrm{t} & 0.7269 \\ \text { Upper95\% } & 0.00121 & \text { Prob }<\mathrm{t} & 0.2731 \\ \text { Lower95\% } & -0.0023 & & \\ \text { N } & 59 & & \\ \text { Correlation } & 0.61148 & & \end{array}$


WSRC-TR-2004-00576

Exhibit A6. Paired Differences for IN38 and IN39
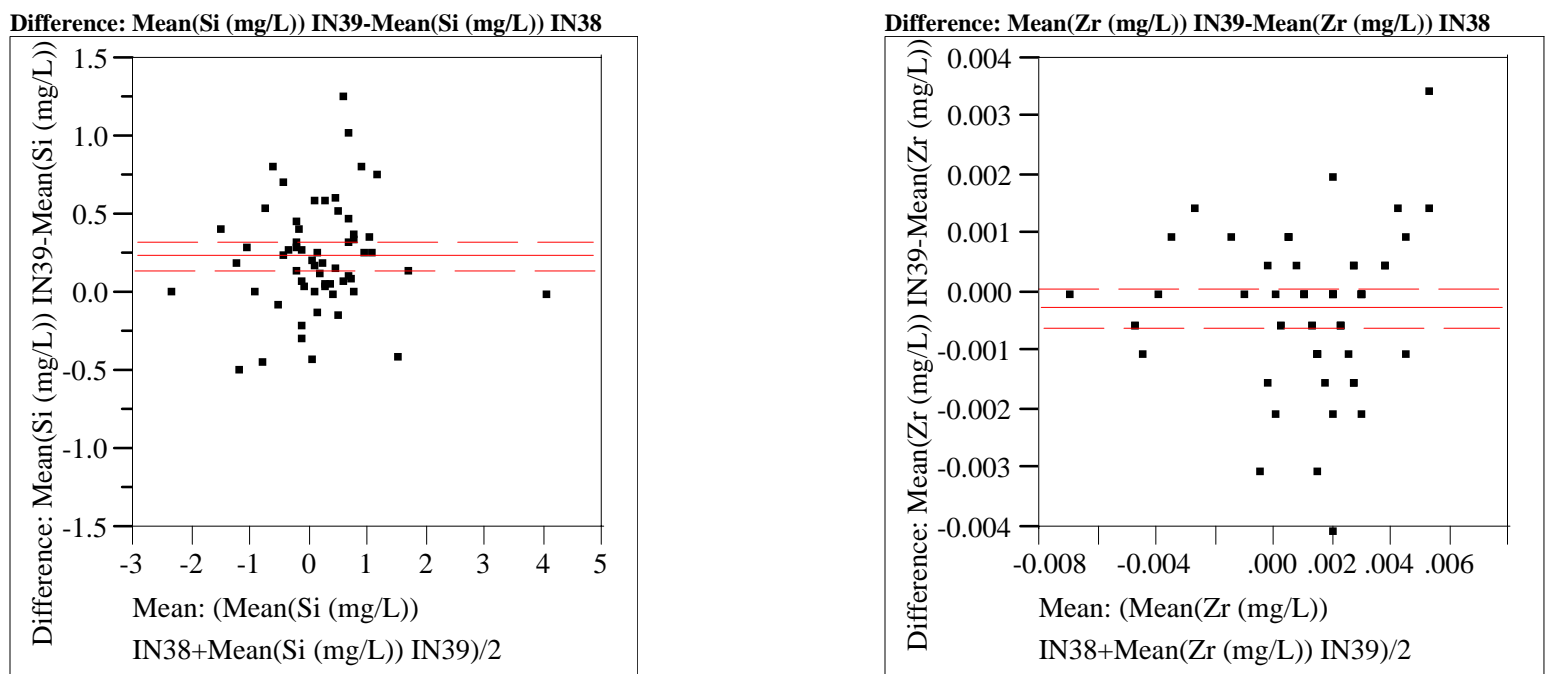

$\begin{array}{lrlr}\text { Mean(Si (mg/L)) IN39 } & 0.28093 & \text { t-Ratio } & 5.314948 \\ \text { Mean(Si (mg/L)) IN38 } & 0.04201 & \text { DF } & 58 \\ \text { Mean Difference } & 0.23892 & \text { Prob }>|t| & <.0001 \\ \text { Std Error } & 0.04495 & \text { Prob }>\text { t } & <.0001 \\ \text { Upper95\% } & 0.32891 & \text { Prob }<\mathrm{t} & 1.0000 \\ \text { Lower95\% } & 0.14894 & & \\ \text { N } & 59 & & \\ \text { Correlation } & 0.929 & & \end{array}$

Difference: Mean(Ti (mg/L)) IN39-Mean(Ti (mg/L)) IN38

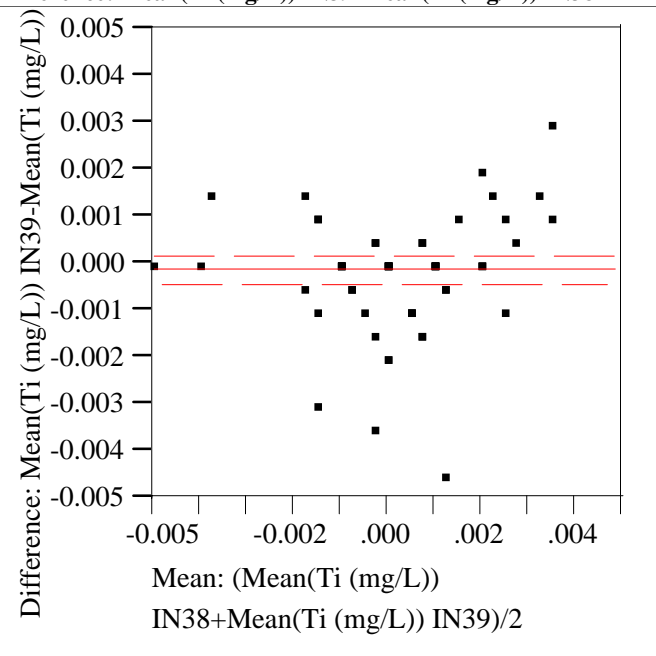

$\begin{array}{lrlr}\text { Mean(Ti (mg/L)) IN39 } & 0.00014 & \text { t-Ratio } & -0.99165 \\ \text { Mean(Ti (mg/L)) IN38 } & 0.0003 & \text { DF } & 58 \\ \text { Mean Difference } & -0.0002 & \text { Prob }>|t| & 0.3255 \\ \text { Std Error } & 0.00016 & \text { Prob }>\text { t } & 0.8373 \\ \text { Upper95\% } & 0.00016 & \text { Prob }<\mathrm{t} & 0.1627 \\ \text { Lower95\% } & -0.0005 & & \\ \text { N } & 59 & & \\ \text { Correlation } & 0.76617 & & \end{array}$

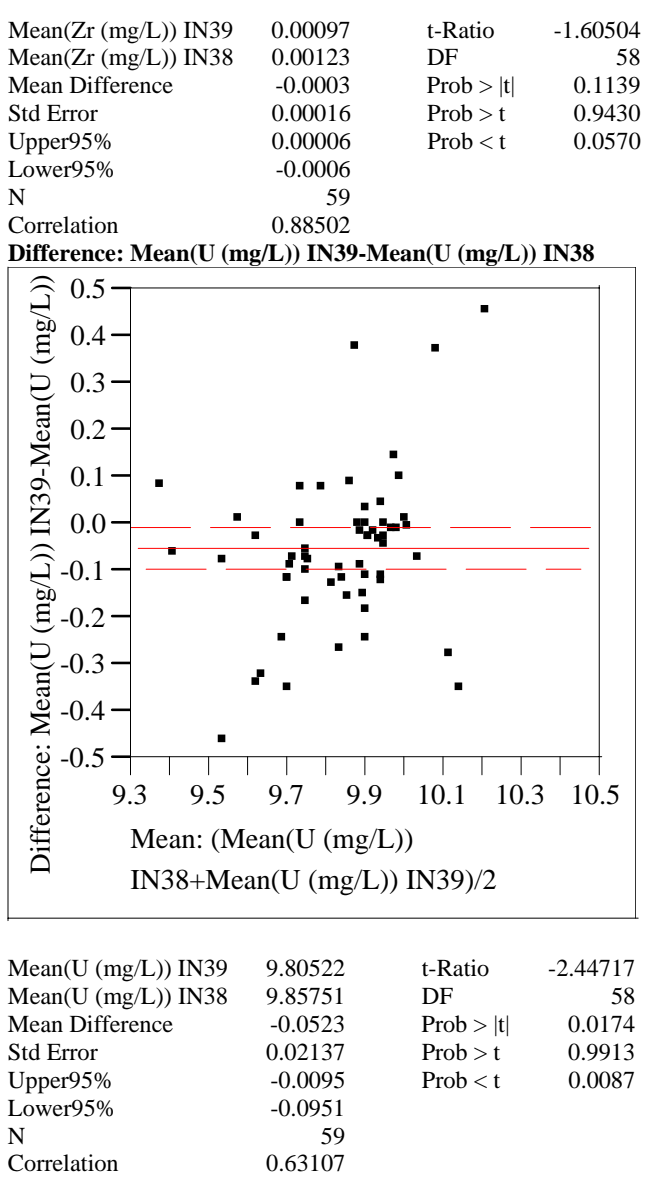




\section{Exhibit A7. Control Charts for Cold Chem ARG-1 Measurements}

Individual Measurement of $\mathrm{Al}$ wt\%

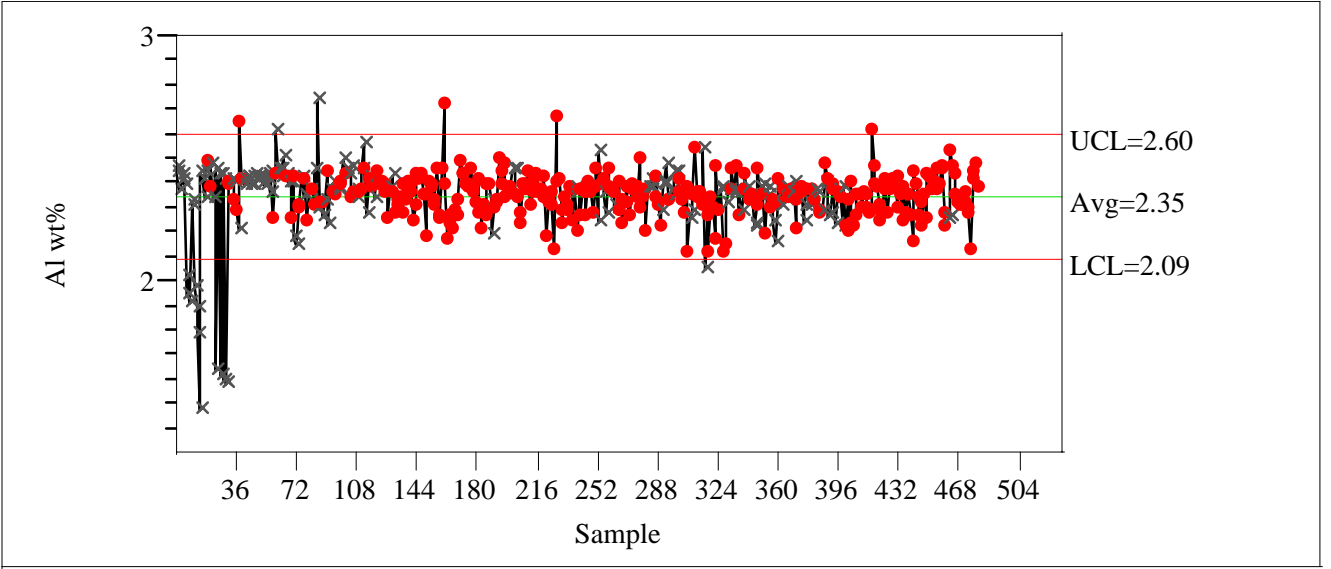

Individual Measurement of B wt \%

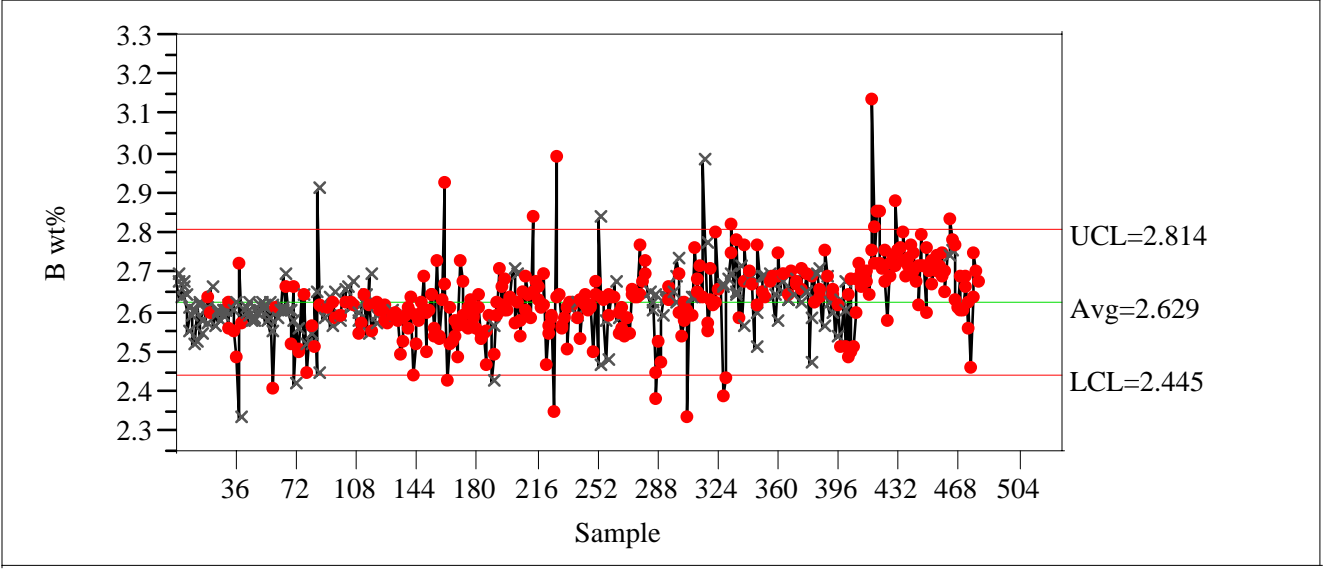

Individual Measurement of Ca wt \%

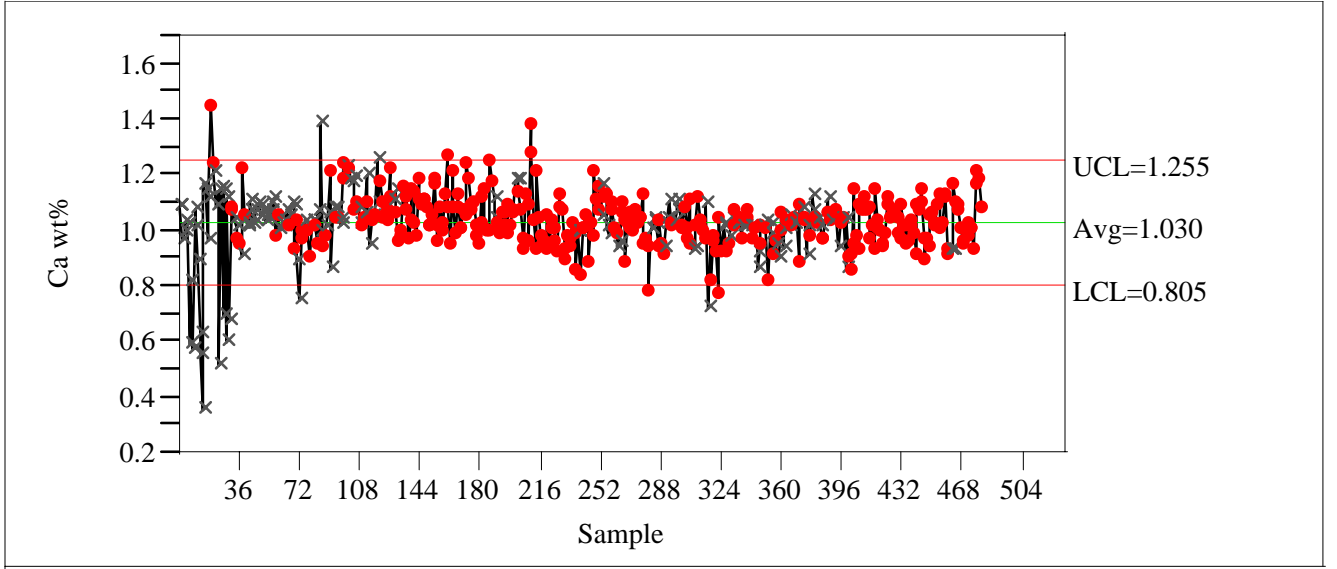


WSRC-TR-2004-00576

Revision 0

\section{Exhibit A7. Control Charts for Cold Chem ARG-1 Measurements}

Individual Measurement of $\mathrm{Cr}$ wt\%

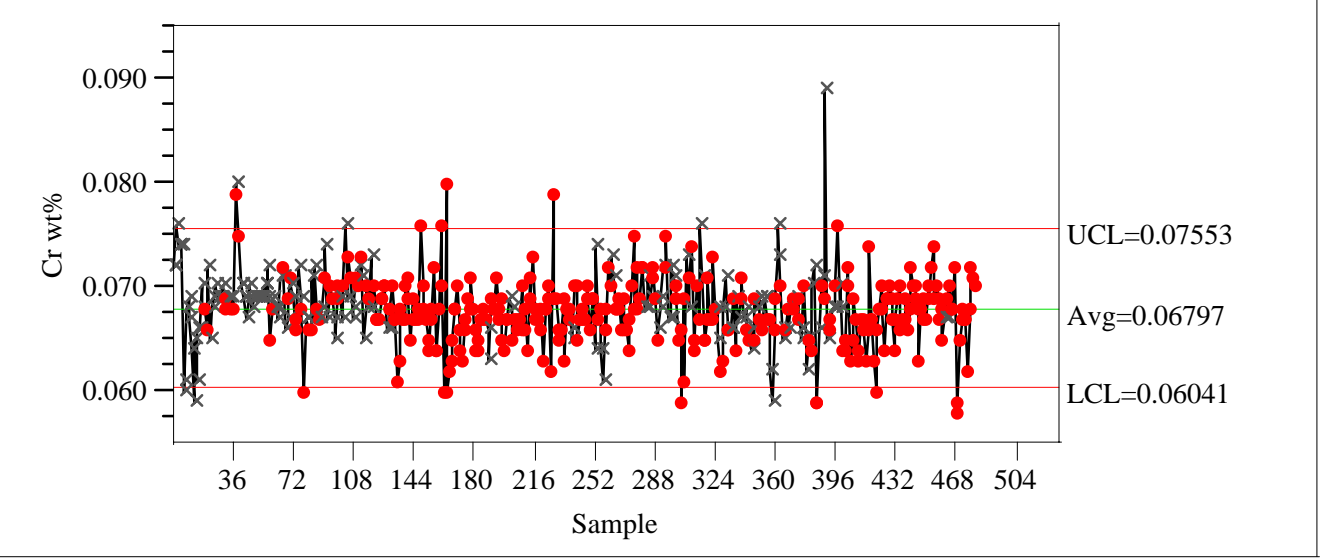

Individual Measurement of $\mathrm{Cu}$ wt \%

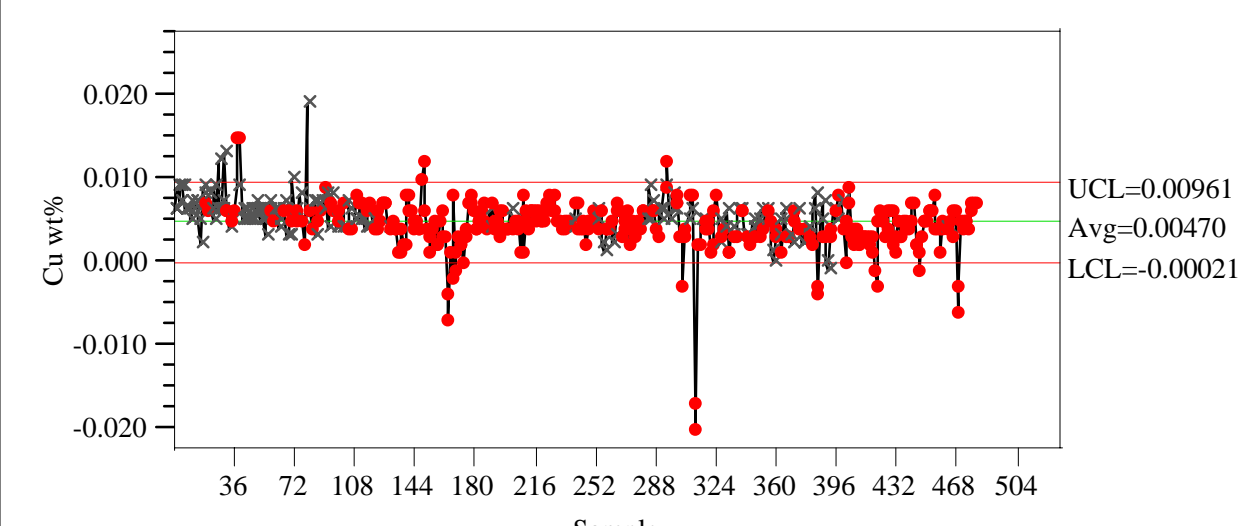

Sample

Individual Measurement of Fe wt \%

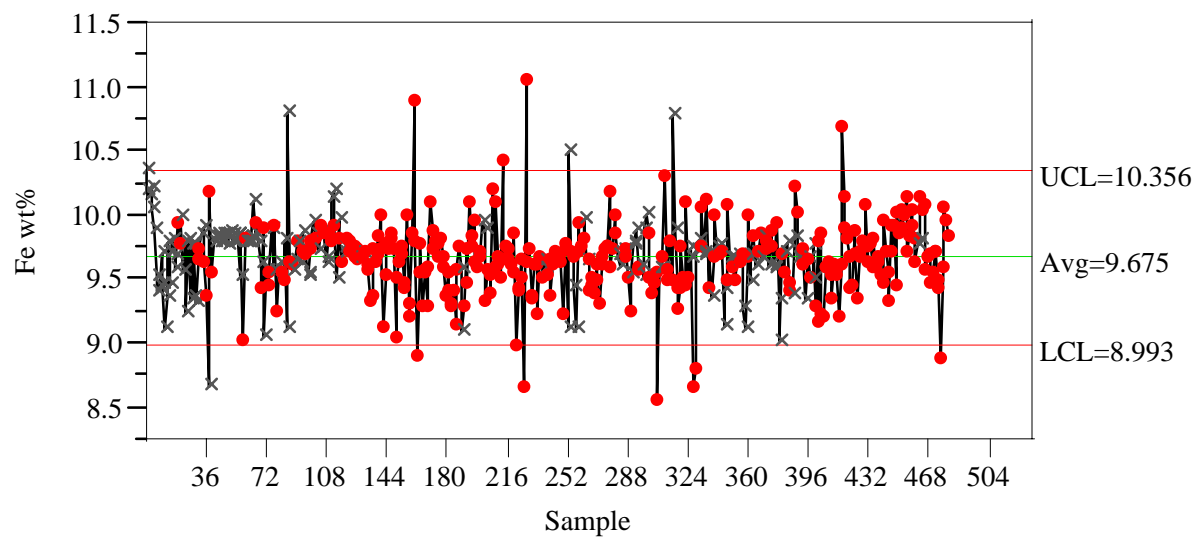




\section{Exhibit A7. Control Charts for Cold Chem ARG-1 Measurements}

Individual Measurement of K wt \%

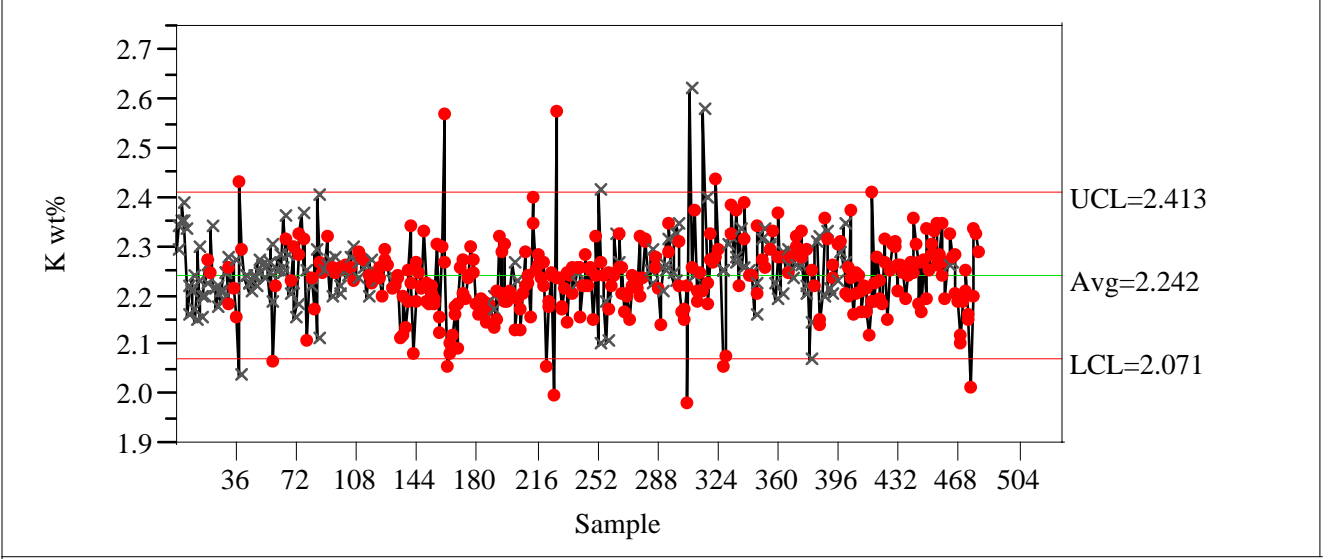

Individual Measurement of $\mathrm{Li}$ wt \%

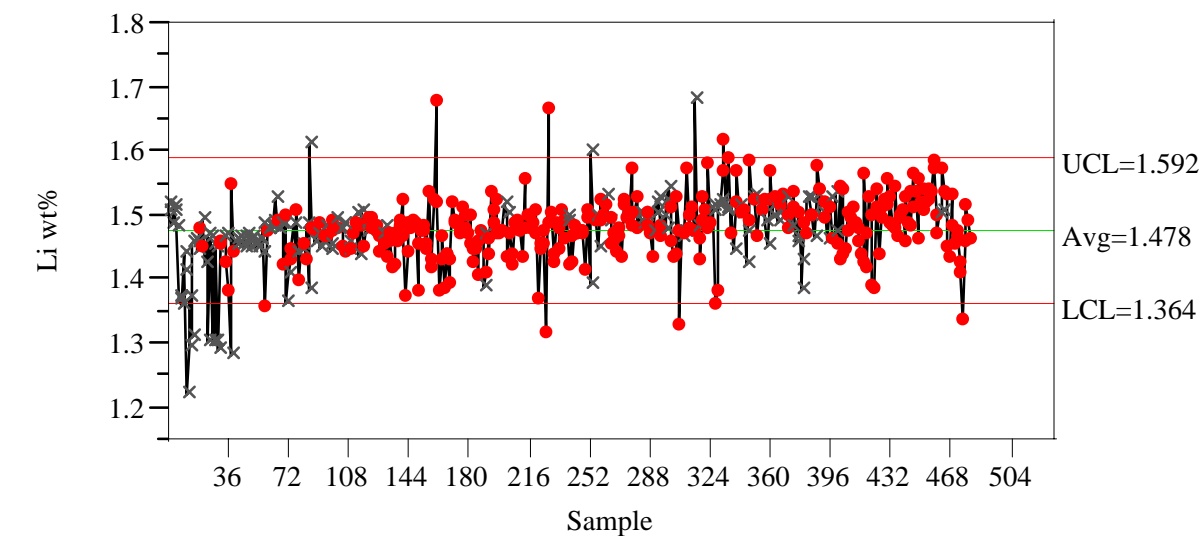

Individual Measurement of Mg wt\%

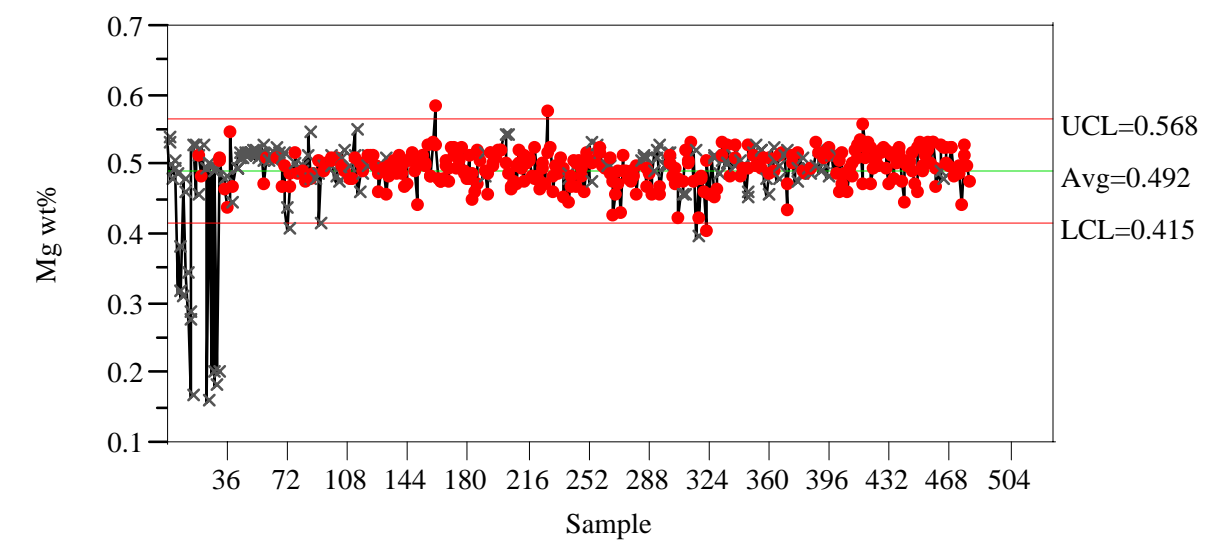




\section{Exhibit A7. Control Charts for Cold Chem ARG-1 Measurements}

Individual Measurement of Mn wt\%

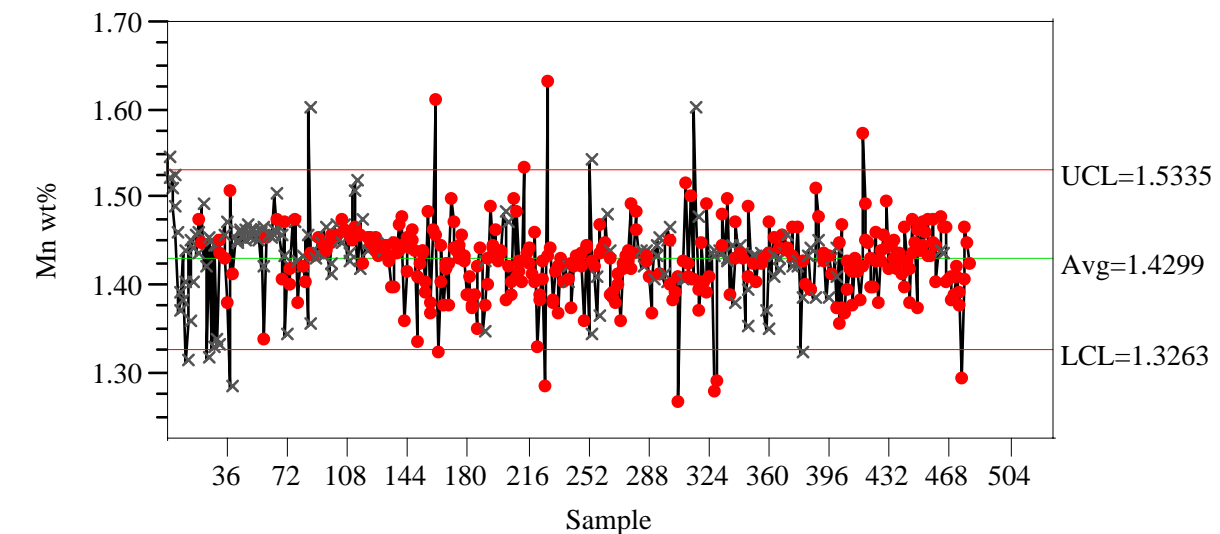

Individual Measurement of Na wt \%

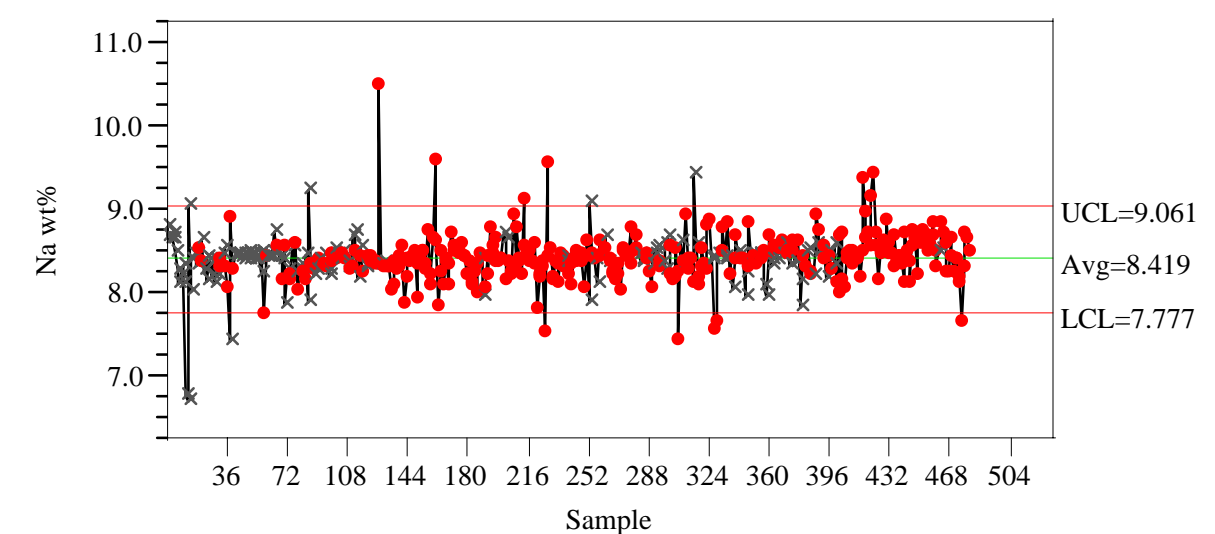

Individual Measurement of $\mathrm{Ni}$ wt\%

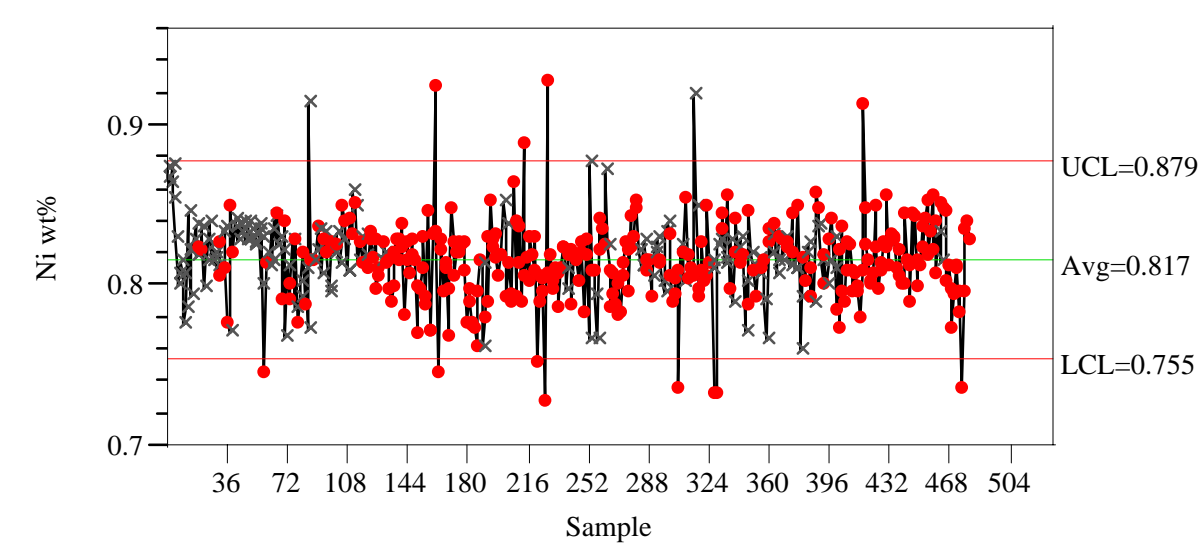




\section{Exhibit A7. Control Charts for Cold Chem ARG-1 Measurements}

Individual Measurement of Si wt $\%$

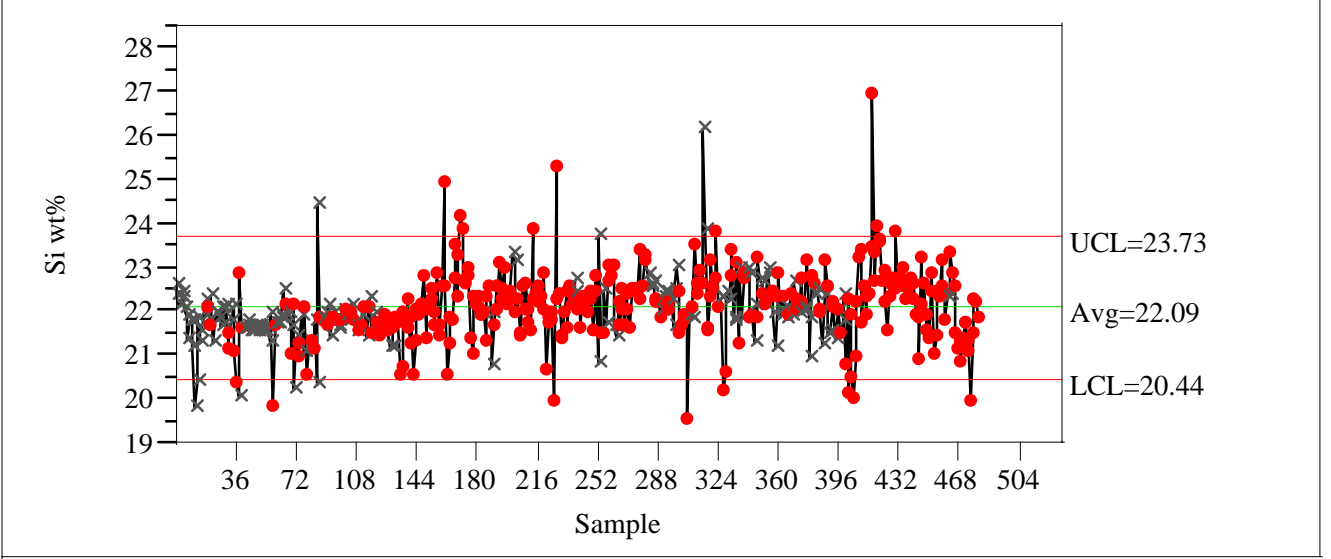

Individual Measurement of Ti wt \%

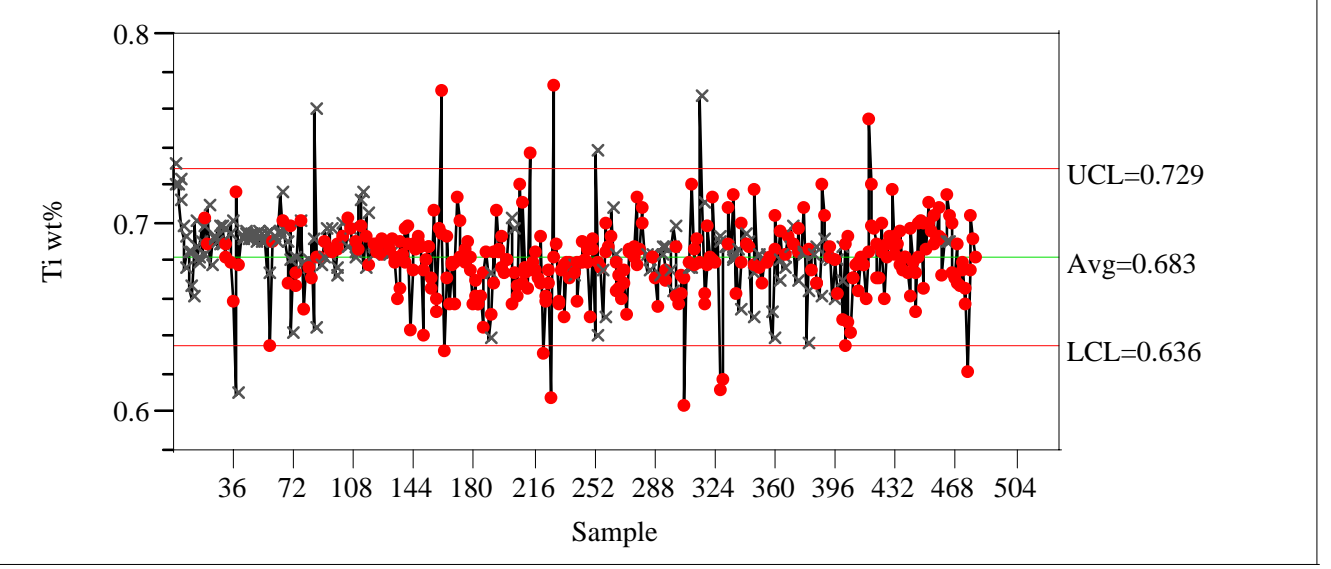

Individual Measurement of $\mathrm{U}$ wt \%

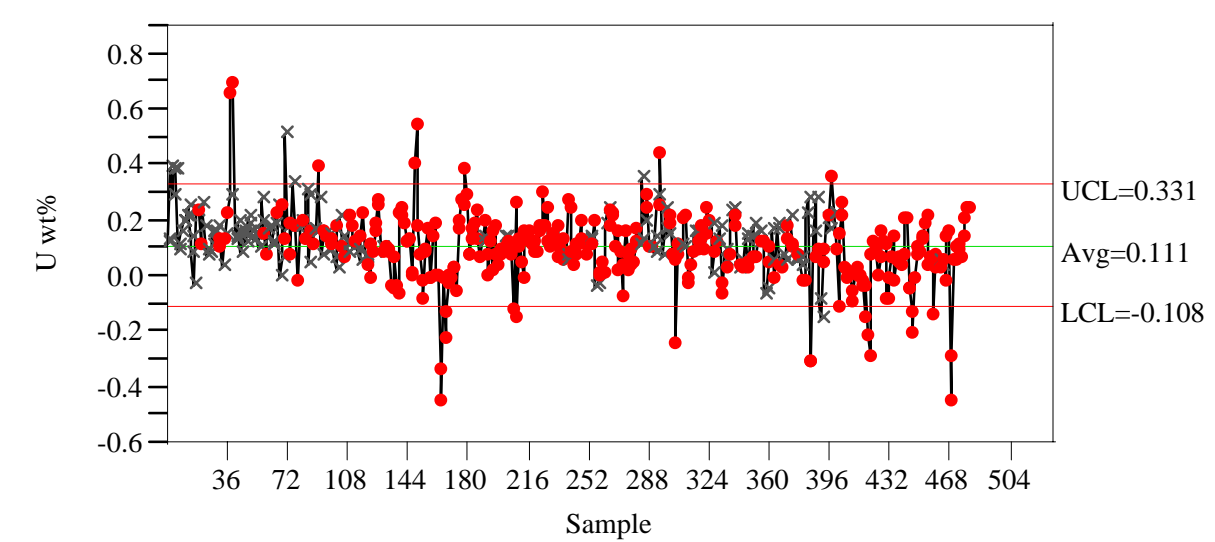


WSRC-TR-2004-00576

Revision 0

\section{Exhibit A7. Control Charts for Cold Chem ARG-1 Measurements}

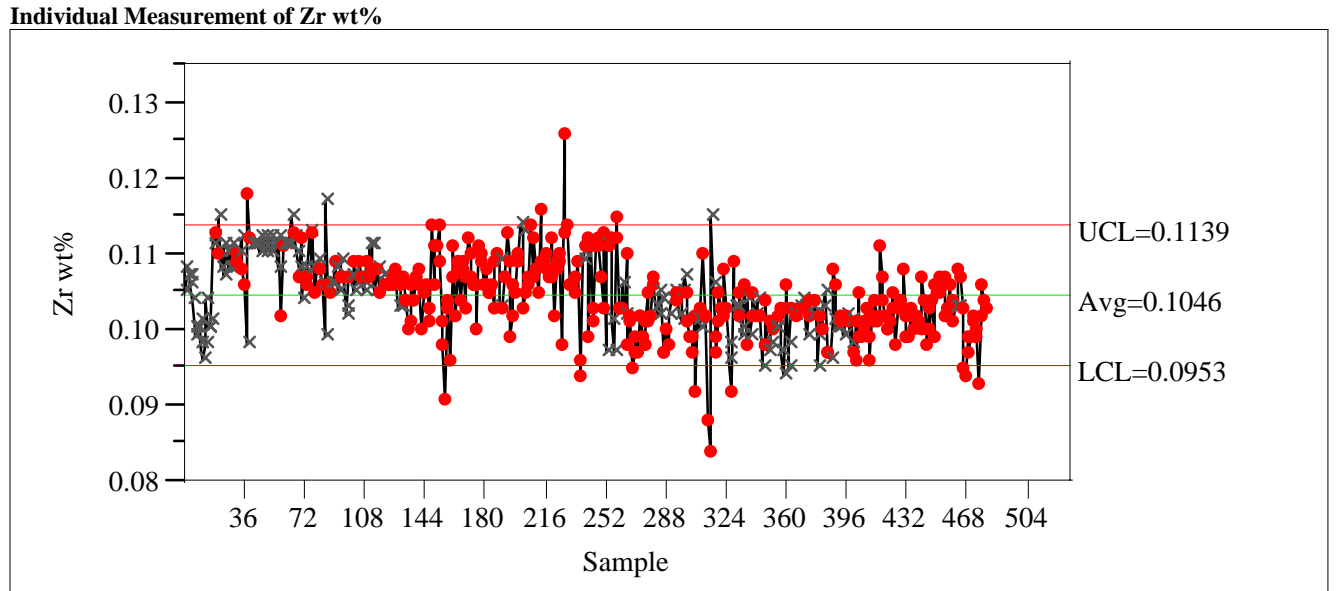


WSRC-TR-2004-00576

Revision 0

Exhibit A8. Histograms and Descriptive Statistics for All ARG-1 Cold Chem Data
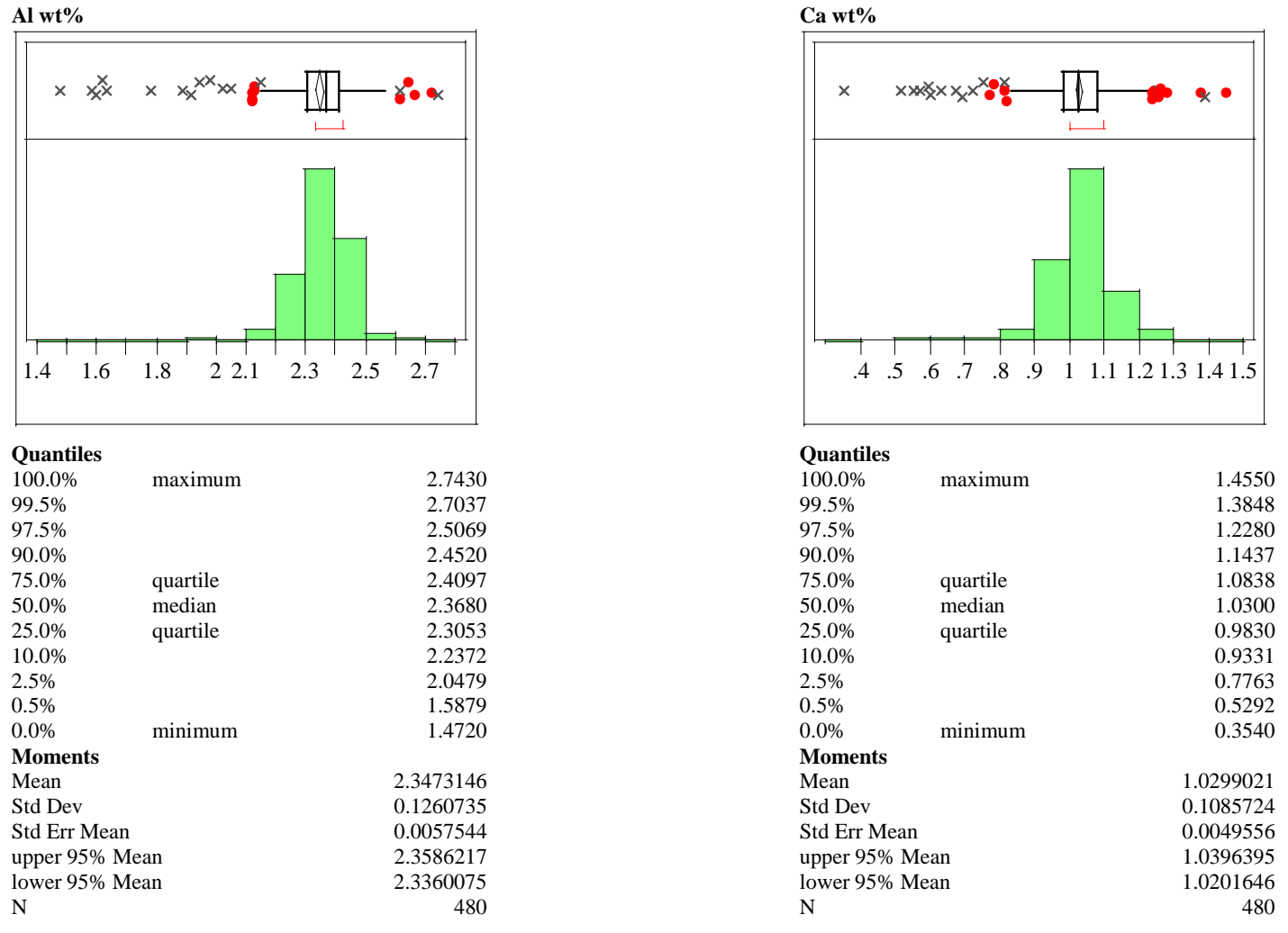

B wt $\%$

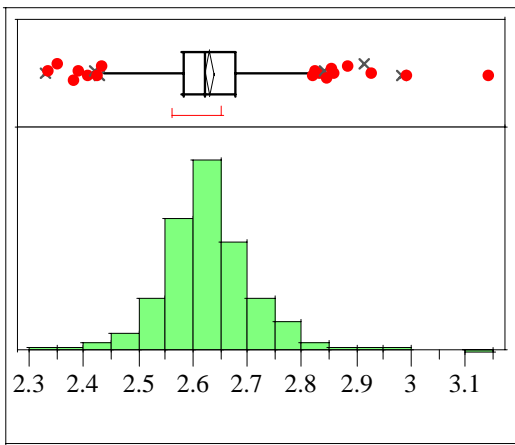

Crwt\%

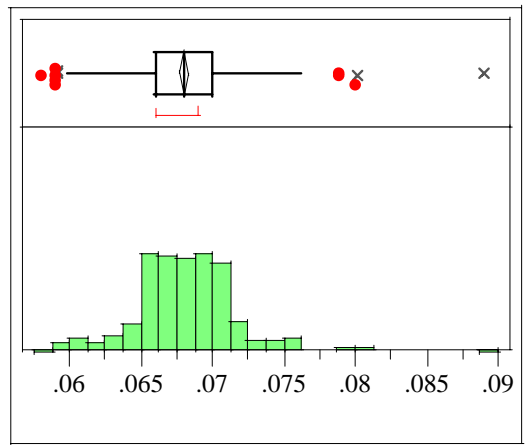

\begin{tabular}{|c|c|c|}
\hline \multicolumn{3}{|c|}{ Quantiles } \\
\hline $100.0 \%$ & maximum & 3.1420 \\
\hline $99.5 \%$ & & 2.9885 \\
\hline $97.5 \%$ & & 2.8259 \\
\hline $90.0 \%$ & & 2.7298 \\
\hline $75.0 \%$ & quartile & 2.6777 \\
\hline $50.0 \%$ & median & 2.6230 \\
\hline $25.0 \%$ & quartile & 2.5833 \\
\hline $10.0 \%$ & & 2.5330 \\
\hline $2.5 \%$ & & 2.4432 \\
\hline $0.5 \%$ & & 2.3441 \\
\hline $0.0 \%$ & minimum & 2.3300 \\
\hline \multicolumn{3}{|c|}{ Moments } \\
\hline \multicolumn{3}{|r|}{2.6293729} \\
\hline \multicolumn{2}{|c|}{ Std Dev } & 0.0889848 \\
\hline \multirow{2}{*}{\multicolumn{2}{|c|}{ Std Err Mean }} & 0.0040616 \\
\hline & & 2.6373536 \\
\hline \multicolumn{2}{|c|}{$\begin{array}{l}\text { upper 95\% Mean } \\
\text { lower 95\% Mean }\end{array}$} & 2.6213922 \\
\hline
\end{tabular}

$\begin{array}{llr}\text { Quantiles } & & \\ 100.0 \% & \text { maximum } & 0.08900 \\ 99.5 \% & & 0.08000 \\ 97.5 \% & & 0.07597 \\ 90.0 \% & & 0.07200 \\ 75.0 \% & \text { quartile } & 0.07000 \\ 50.0 \% & \text { median } & 0.06800 \\ 25.0 \% & \text { quartile } & 0.06600 \\ 10.0 \% & & 0.06400 \\ 2.5 \% & 0.06002 \\ 0.5 \% & & 0.05900 \\ 0.0 \% & \text { minimum } & 0.05800 \\ \text { Moments } & & \\ \text { Mean } & & 0.0679708 \\ \text { Std Dev } & & 0.003368 \\ \text { Std Err Mean } & & 0.0001537 \\ \text { upper 95\% Mean } & 0.0682729 \\ \text { lower 95\% Mean } & 0.0676688 \\ \text { N } & 480\end{array}$


WSRC-TR-2004-00576

Revision 0

Exhibit A8. Histograms and Descriptive Statistics for All ARG-1 Cold Chem Data

Cuwt \%

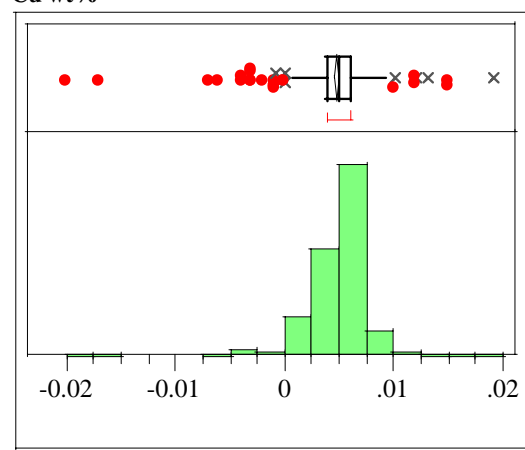

Quantiles

$100.0 \%$

$99.5 \%$

$97.5 \%$

$90.0 \%$

$75.0 \%$

$50.0 \%$

$25.0 \%$

$10.0 \%$

$2.5 \%$

$0.5 \%$

$0.0 \%$

Momen

Mean
Std Dev

Std Err Mean

upper 95\% Mean

lower 95\% Mean

$\mathrm{N}$

Fe wt \%

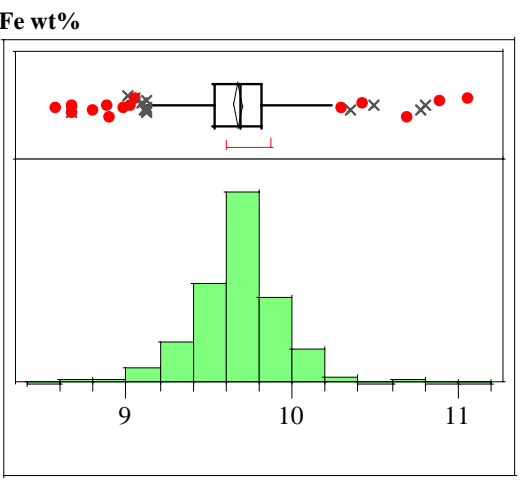

\section{Quantiles}

$100.0 \%$

$99.5 \%$

$97.5 \%$

$90.0 \%$

$75.0 \%$

$50.0 \%$

$25.0 \%$

$10.0 \%$

$2.5 \%$

$0.5 \%$

$0.0 \%$

Moments

Mean

Std Dev

Std Err Mean

upper 95\% Mean

lower 95\% Mean

$\mathrm{N}$

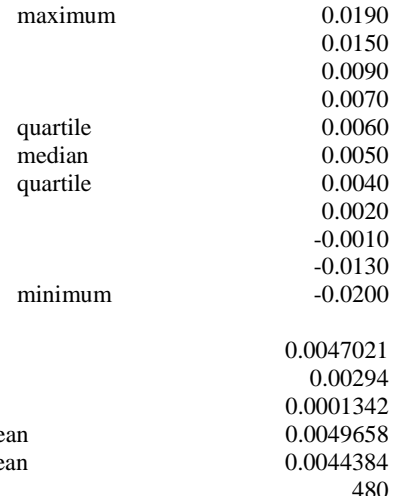

480

$\begin{array}{lr} & \\ \text { maximum } & 11.064 \\ & 10.858 \\ & 10.214 \\ & 9.970 \\ \text { quartile } & 9.815 \\ \text { median } & 9.686 \\ \text { quartile } & 9.537 \\ & 9.346 \\ & 9.059 \\ \text { minimum } & 8.667 \\ & 8.575 \\ & \\ & 9.6745917 \\ & 0.286491 \\ & 0.0130765 \\ \text { Mean } & 9.700286 \\ & 9.6488973\end{array}$

480
K wt \%

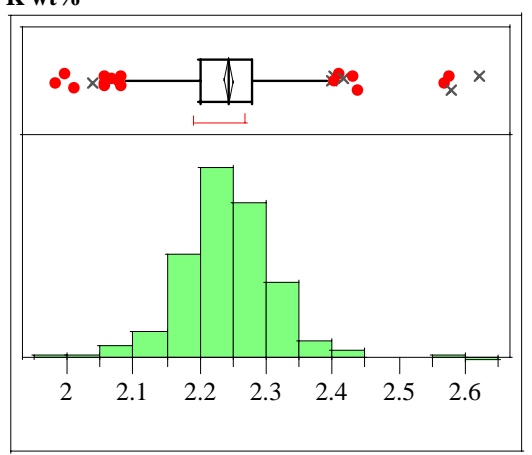

Quantiles

$\begin{array}{llr}100.0 \% & \text { maximum } & 2.6200 \\ 99.5 \% & & 2.5776 \\ 97.5 \% & 2.3889 \\ 90.0 \% & & 2.3268 \\ 75.0 \% & \text { quartile } & 2.2780 \\ 50.0 \% & \text { median } & 2.2430 \\ 25.0 \% & \text { quartile } & 2.2003 \\ 10.0 \% & & 2.1590 \\ 2.5 \% & 2.0823 \\ 0.5 \% & & 2.0041 \\ 0.0 \% & \text { minimum } & 1.9820 \\ \text { Moments } & & \\ \text { Mean } & & 2.2422167 \\ \text { Std Dev } & & 0.0740392 \\ \text { Std Err Mean } & & 0.0033794 \\ \text { upper 95\% Mean } & 2.248857 \\ \text { lower 95\% Mean } & 2.2355764 \\ \text { N } & \end{array}$

Li wt \%

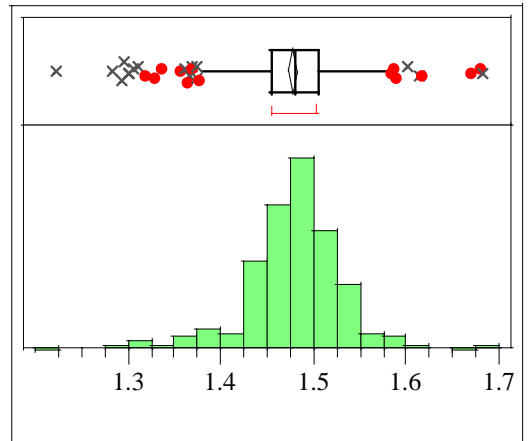

$\begin{array}{llr}\begin{array}{l}\text { Quantiles } \\ 100.0 \%\end{array} & \text { maximum } & \\ 99.5 \% & & 1.6810 \\ 97.5 \% & 1.6759 \\ 90.0 \% & & 1.5760 \\ 75.0 \% & \text { quartile } & 1.5290 \\ 50.0 \% & \text { median } & 1.5057 \\ 25.0 \% & \text { quartile } & 1.4810 \\ 10.0 \% & & 1.4550 \\ 2.5 \% & 1.4261 \\ 0.5 \% & & 1.3590 \\ 0.0 \% & & 1.2851 \\ \text { Moments } & & 1.2210 \\ \text { Mean } & & \\ \text { Std Dev } & & 1.4780812 \\ \text { Std Err Mean } & & 0.0521977 \\ \text { upper 95\% Mean } & 0.0023825 \\ \text { lower 95\% Mean } & 1.4827627 \\ \text { N } & & 1.4733998 \\ \end{array}$


WSRC-TR-2004-00576

Revision 0

Exhibit A8. Histograms and Descriptive Statistics for All ARG-1 Cold Chem Data

Mg wt \%

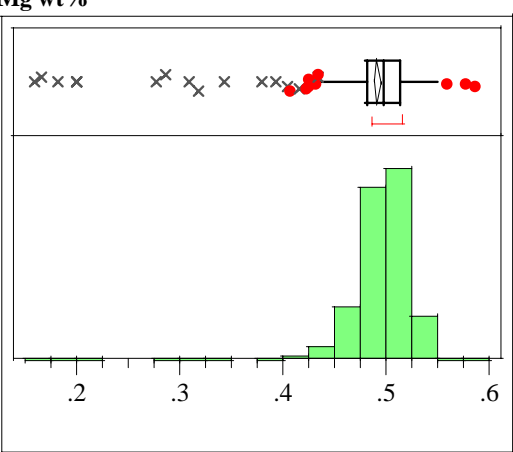

Quantiles

$100.0 \%$

$99.5 \%$

$97.5 \%$

$90.0 \%$

$75.0 \%$

$50.0 \%$

$25.0 \%$

$10.0 \%$

$2.5 \%$

$0.5 \%$

$0.0 \%$

Moments

Mean

Std Dev

Std Err Mean

upper 95\% Mean

lower 95\% Mean

$\mathrm{N}$

$\begin{array}{lr}\text { maximum } & 0.58700 \\ & 0.57111 \\ & 0.53497 \\ \text { quartile } & 0.52400 \\ \text { median } & 0.51300 \\ \text { quartile } & 0.49800 \\ & 0.48200 \\ & 0.46110 \\ \text { minimum } & 0.39328 \\ & 0.17108 \\ & 0.15700 \\ & \\ & 0.4915313 \\ & 0.0448749 \\ & 0.0020483 \\ \text { Mean } & 0.4955559 \\ & 0.4875066 \\ & \end{array}$

Mn wt\%

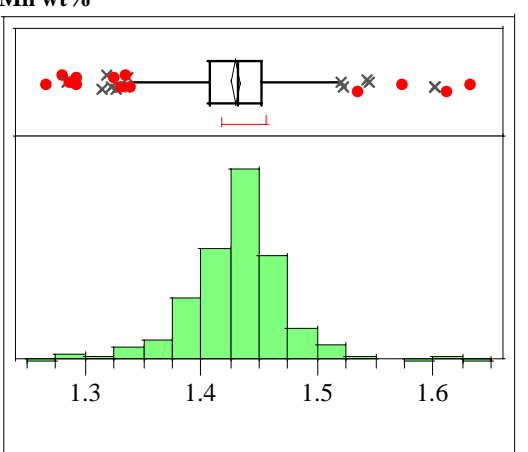

\section{Quantiles}

$100.0 \%$

$99.5 \%$

$97.5 \%$

$90.0 \%$

$75.0 \%$

$50.0 \%$

$25.0 \%$

$10.0 \%$

$2.5 \%$

$0.5 \%$

$0.0 \%$

Moments

Mean

Std Dev

Std Err Mean

upper 95\% Mean

lower 95\% Mean

$\mathrm{N}$

$\begin{array}{lr}\text { maximum } & 1.6330 \\ & 1.6075 \\ & 1.5159 \\ \text { quartile } & 1.4730 \\ \text { median } & 1.4518 \\ \text { quartile } & 1.4325 \\ & 1.4083 \\ & 1.3791 \\ \text { minimum } & 1.3300 \\ & 1.2822 \\ & 1.2670 \\ & \\ & 1.4298833 \\ \text { Mean } & 0.0437338 \\ \text { ean } & 0.0019962 \\ & 1.4338057 \\ & 1.425961 \\ & \end{array}$

Na wt \%

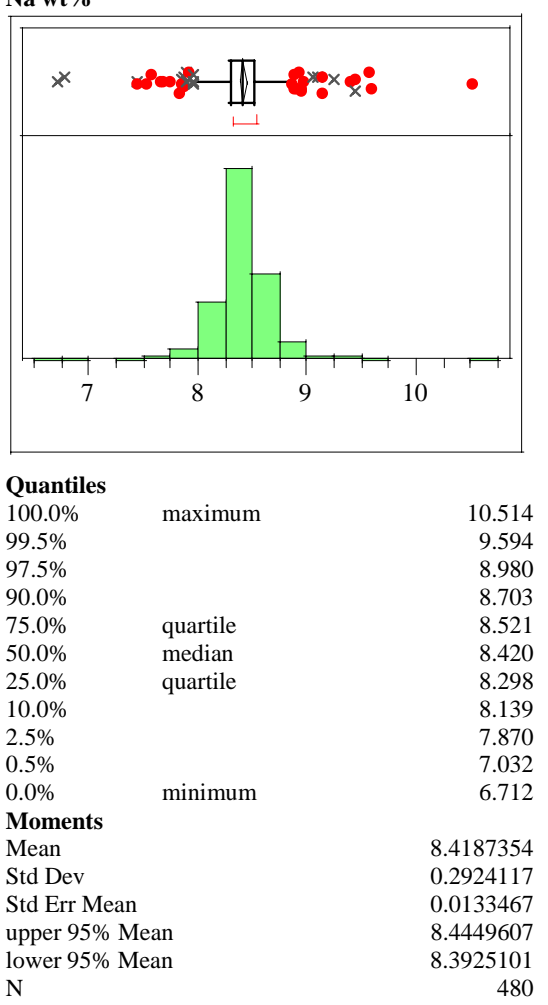

Ni wt \%

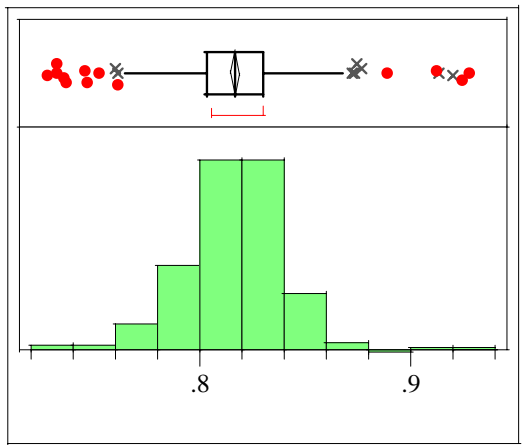

$\begin{array}{llr}\text { Quantiles } & & \\ 100.0 \% & \text { maximum } & 0.92800 \\ 99.5 \% & & 0.92257 \\ 97.5 \% & & 0.86495 \\ 90.0 \% & & 0.84400 \\ 75.0 \% & \text { quartile } & 0.83000 \\ 50.0 \% & \text { median } & 0.81700 \\ 25.0 \% & \text { quartile } & 0.80300 \\ 10.0 \% & & 0.78900 \\ 2.5 \% & & 0.76600 \\ 0.5 \% & & 0.73300 \\ 0.0 \% & \text { minimum } & 0.72800 \\ \text { Moments } & & \\ \text { Mean } & & 0.8166562 \\ \text { Std Dev } & & 0.0252176 \\ \text { Std Err Mean } & & 0.001151 \\ \text { upper 95\% Mean } & 0.8189179 \\ \text { lower 95\% Mean } & 0.8143946 \\ \text { N } & & \end{array}$

$\mathrm{N}$ 
WSRC-TR-2004-00576

Revision 0

Exhibit A8. Histograms and Descriptive Statistics for All ARG-1 Cold Chem Data

Si wt\%

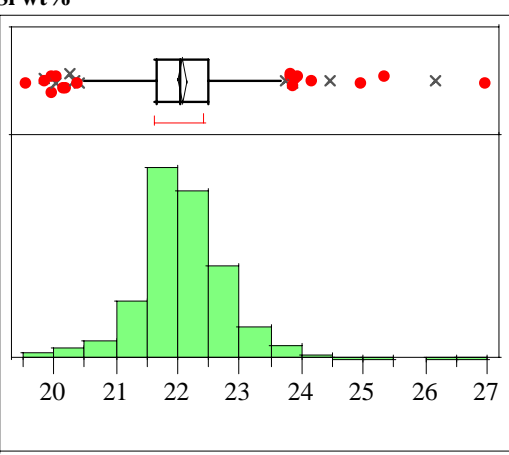

Quantiles

$100.0 \%$

$99.5 \%$

$97.5 \%$

$90.0 \%$

$75.0 \%$

$50.0 \%$

$25.0 \%$

$10.0 \%$

$2.5 \%$

$0.5 \%$

$0.0 \%$

Momen

Mean

Std Dev

Std Err Mean

upper 95\% Mean

lower 95\% Mean

$\mathrm{N}$

Ti wt \%

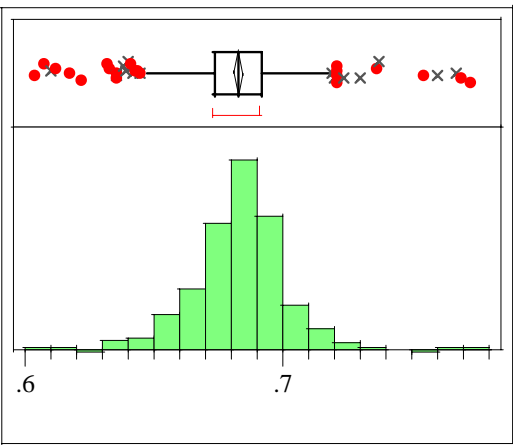

\section{Quantiles}

$100.0 \%$

$99.5 \%$

$97.5 \%$

$90.0 \%$

$75.0 \%$

$50.0 \%$

$25.0 \%$

$10.0 \%$

$2.5 \%$

$0.5 \%$

$0.0 \%$

Moments

Mean

Std Dev

Std Err Mean

upper 95\% Mean

lower 95\% Mean

$\mathrm{N}$

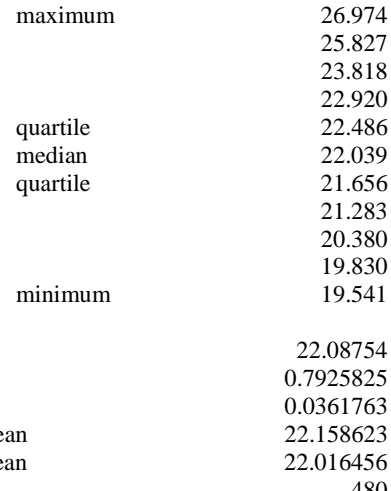

480

$\begin{array}{lr} & \\ \text { maximum } & 0.77300 \\ & 0.76878 \\ & 0.72100 \\ & 0.70200 \\ \text { quartile } & 0.69200 \\ \text { median } & 0.68300 \\ \text { quartile } & 0.67400 \\ & 0.65900 \\ & 0.63802 \\ \text { minimum } & 0.60881 \\ & 0.60400 \\ & \\ & 0.6825833 \\ & 0.0201457 \\ & 0.0009195 \\ \text { ean } & 0.6843901 \\ & 0.6807765 \\ & \end{array}$

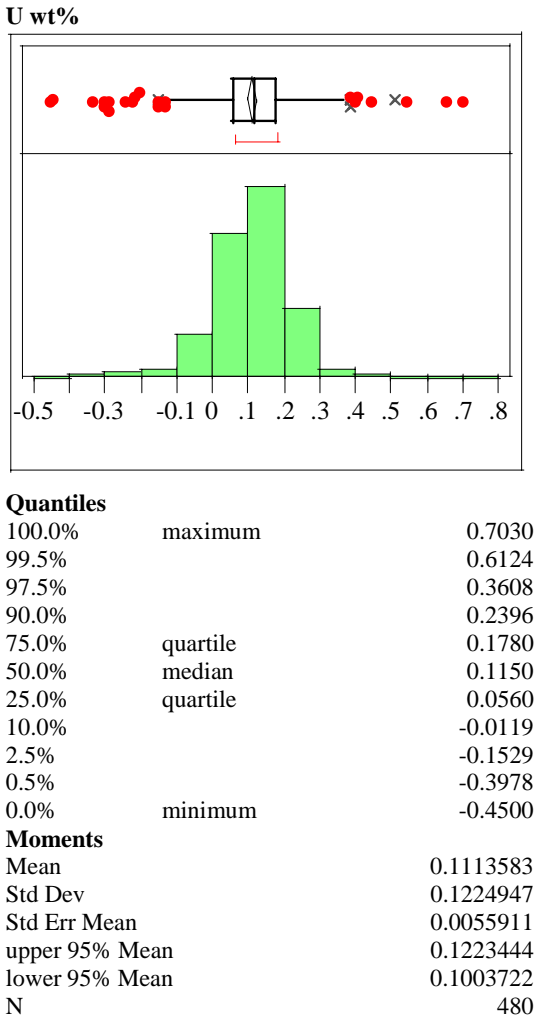

Zr wt\%

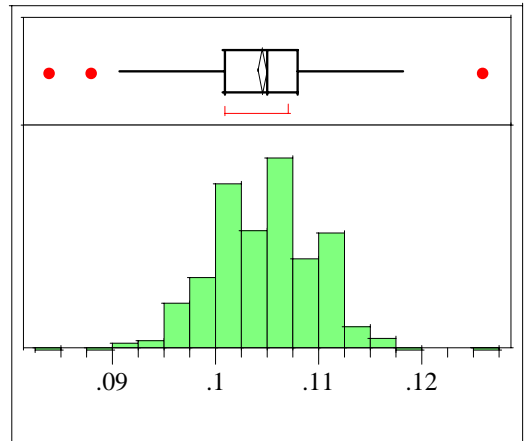

$\begin{array}{llr}\text { Quantiles } & & \\ 100.0 \% & \text { maximum } & 0.12600 \\ 99.5 \% & & 0.11759 \\ 97.5 \% & & 0.11400 \\ 90.0 \% & & 0.11100 \\ 75.0 \% & \text { quartile } & 0.10800 \\ 50.0 \% & \text { median } & 0.10500 \\ 25.0 \% & \text { quartile } & 0.10100 \\ 10.0 \% & & 0.09800 \\ 2.5 \% & & 0.09500 \\ 0.5 \% & 0.08921 \\ 0.0 \% & \text { minimum } & 0.08400 \\ \text { Moments } & & \\ \text { Mean } & & 0.1046187 \\ \text { Std Dev } & & 0.0050598 \\ \text { Std Err Mean } & & 0.0002309 \\ \text { upper 95\% Mean } & 0.1050725 \\ \text { lower 95\% Mean } & 0.104165 \\ \text { N } & & 480\end{array}$

$\mathrm{N}$ 
WSRC-TR-2004-00576

Revision 0

\section{Exhibit A9. Histograms and Descriptive Statistics for ARG-1 Cold Chem Data Associated with a SRAT Batch}
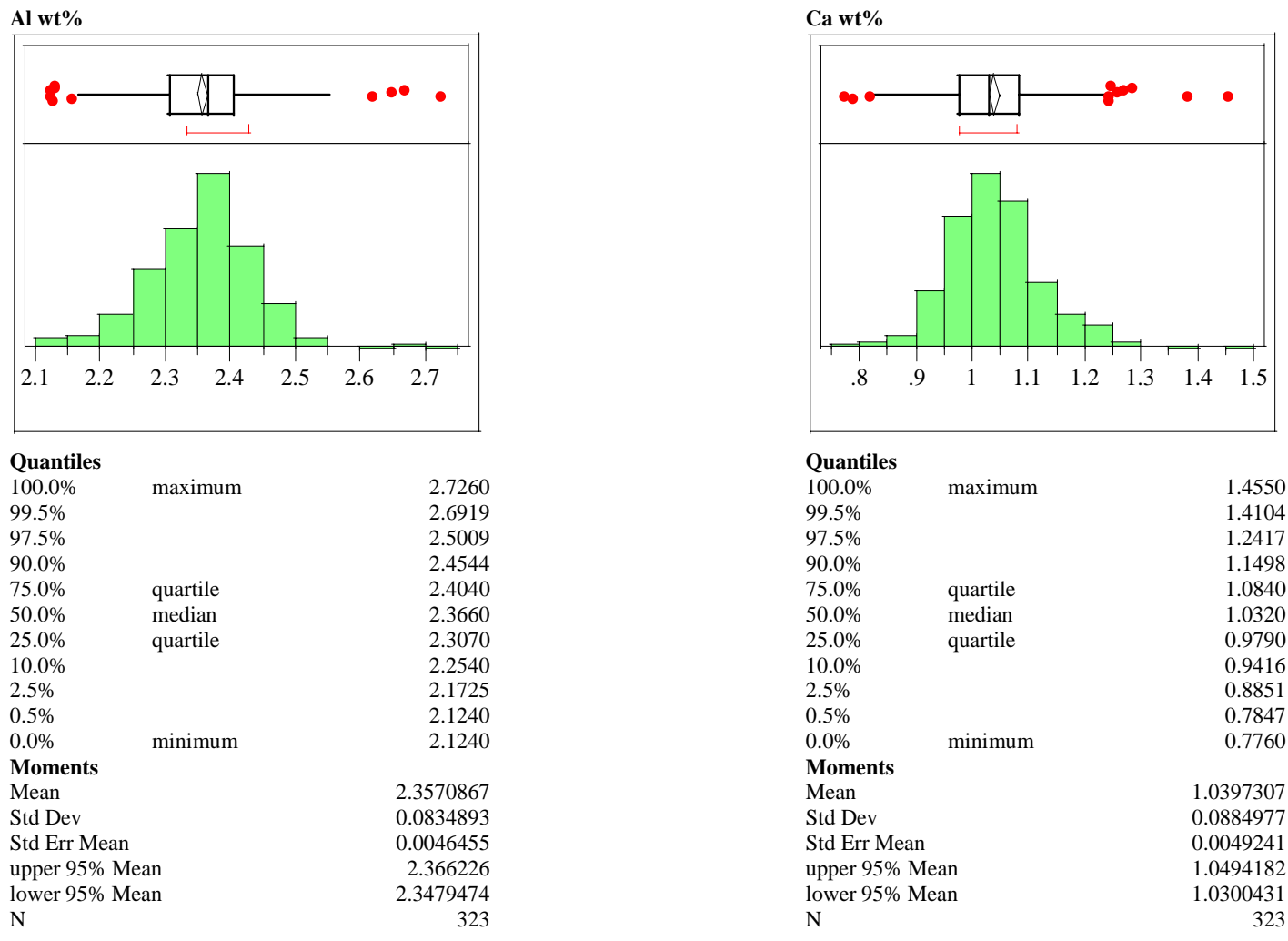

B wt \%
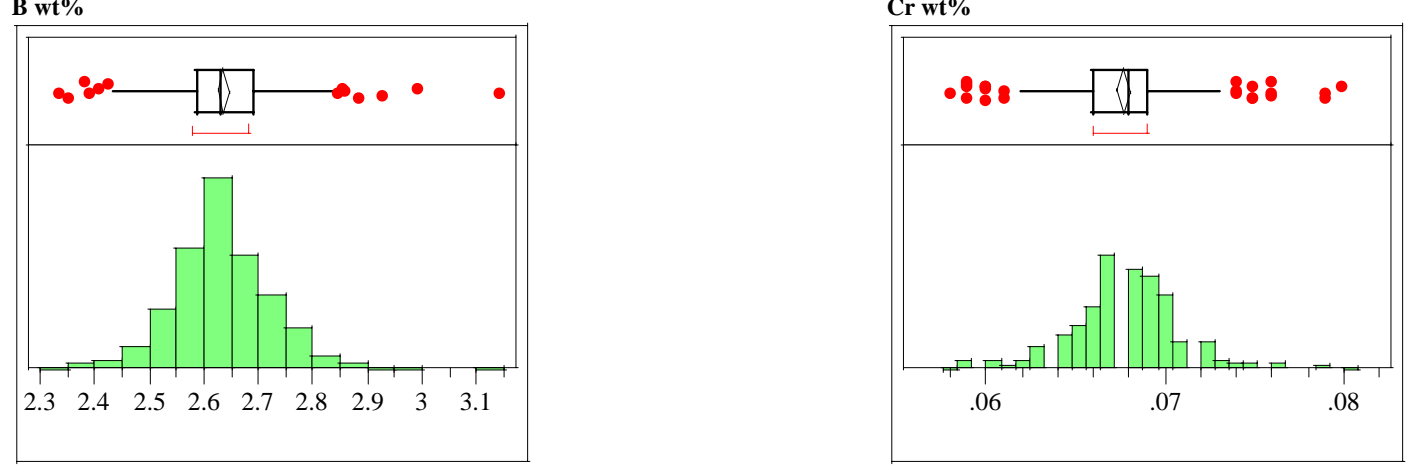

Quantiles

$100.0 \% \quad$ maximum 3.1420

2.8350

$90.0 \%$

$75.0 \%$

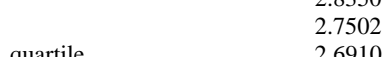

2.7502

$50.0 \%$ median 2.6300

$25.0 \%$ quartile 2.5870

$10.0 \% \quad 2.5254$

$2.5 \%$

$0.5 \% \quad 2.3473$

$0.0 \%$ minimum 2.3380

Moments

2.6354427
0.0943969

Mean

0.0052524

2.645776

upper 95\% Mean

2.6251094

lower 95\% Mean

323

$\begin{array}{llr}\text { Quantiles } & & \\ 100.0 \% & \text { maximum } & 0.08000 \\ 99.5 \% & & 0.07938 \\ 97.5 \% & & 0.07500 \\ 90.0 \% & & 0.07100 \\ 75.0 \% & \text { quartile } & 0.06900 \\ 50.0 \% & \text { median } & 0.06800 \\ 25.0 \% & \text { quartile } & 0.06600 \\ 10.0 \% & & 0.06400 \\ 2.5 \% & & 0.06000 \\ 0.5 \% & & 0.05862 \\ 0.0 \% & 0.05800 \\ \text { Moments } & & \\ \text { Mean } & & 0.0677059 \\ \text { Std Dev } & & 0.0031804 \\ \text { Std Err Mean } & & 0.000177 \\ \text { upper 95\% Mean } & 0.068054 \\ \text { lower 95\% Mean } & 0.0673577 \\ \text { N } & & 323\end{array}$


WSRC-TR-2004-00576

Revision 0

\section{Exhibit A9. Histograms and Descriptive Statistics for ARG-1 Cold Chem Data Associated with a SRAT Batch}
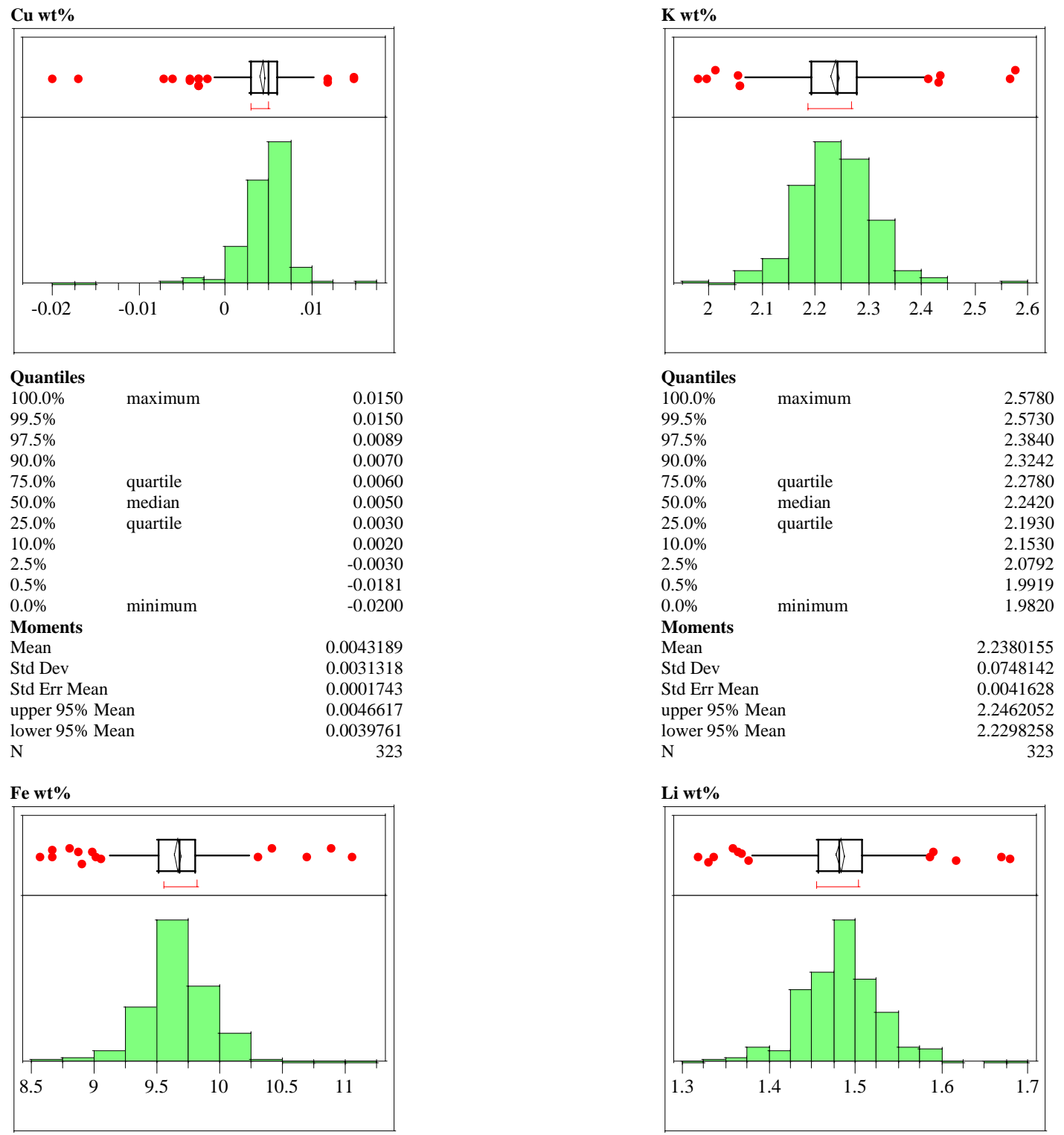

Quantiles

$100.0 \%$ maximum 11.064

10.194

$90.0 \%$

$75.0 \%$

quartile

10.002

$50.0 \%$

median

9.809

$25.0 \%$

quartile

9.676

9.347

$2.5 \%$

$0.5 \%$

$0.0 \%$

minimum

9.028

Moments

Mean

8.633
8.575

Std Dev

9.6671331

Std Err Mean

upper 95\% Mean

0.2861554

0.0159221

ower 95\% Mean

9.6984576

$\mathrm{N}$

9.6358086
323

$\begin{array}{lcr}\text { Quantiles } & & \\ 100.0 \% & \text { maximum } & 1.6800 \\ 99.5 \% & & 1.6738 \\ 97.5 \% & 1.5787 \\ 90.0 \% & & 1.5380 \\ 75.0 \% & \text { quartile } & 1.5090 \\ 50.0 \% & \text { median } & 1.4820 \\ 25.0 \% & \text { quartile } & 1.4570 \\ 10.0 \% & & 1.4300 \\ 2.5 \% & 1.3821 \\ 0.5 \% & 1.3268 \\ 0.0 \% & 1.3200 \\ \text { Moments } & & \\ \text { Mean } & & 1.4830712 \\ \text { Std Dev } & & 0.0474279 \\ \text { Std Err Mean } & & 0.002639 \\ \text { upper 95\% Mean } & 1.488263 \\ \text { lower 95\% Mean } & 1.4778794 \\ \text { N } & & 323\end{array}$


WSRC-TR-2004-00576

Revision 0

\section{Exhibit A9. Histograms and Descriptive Statistics for ARG-1 Cold Chem Data Associated with a SRAT Batch}
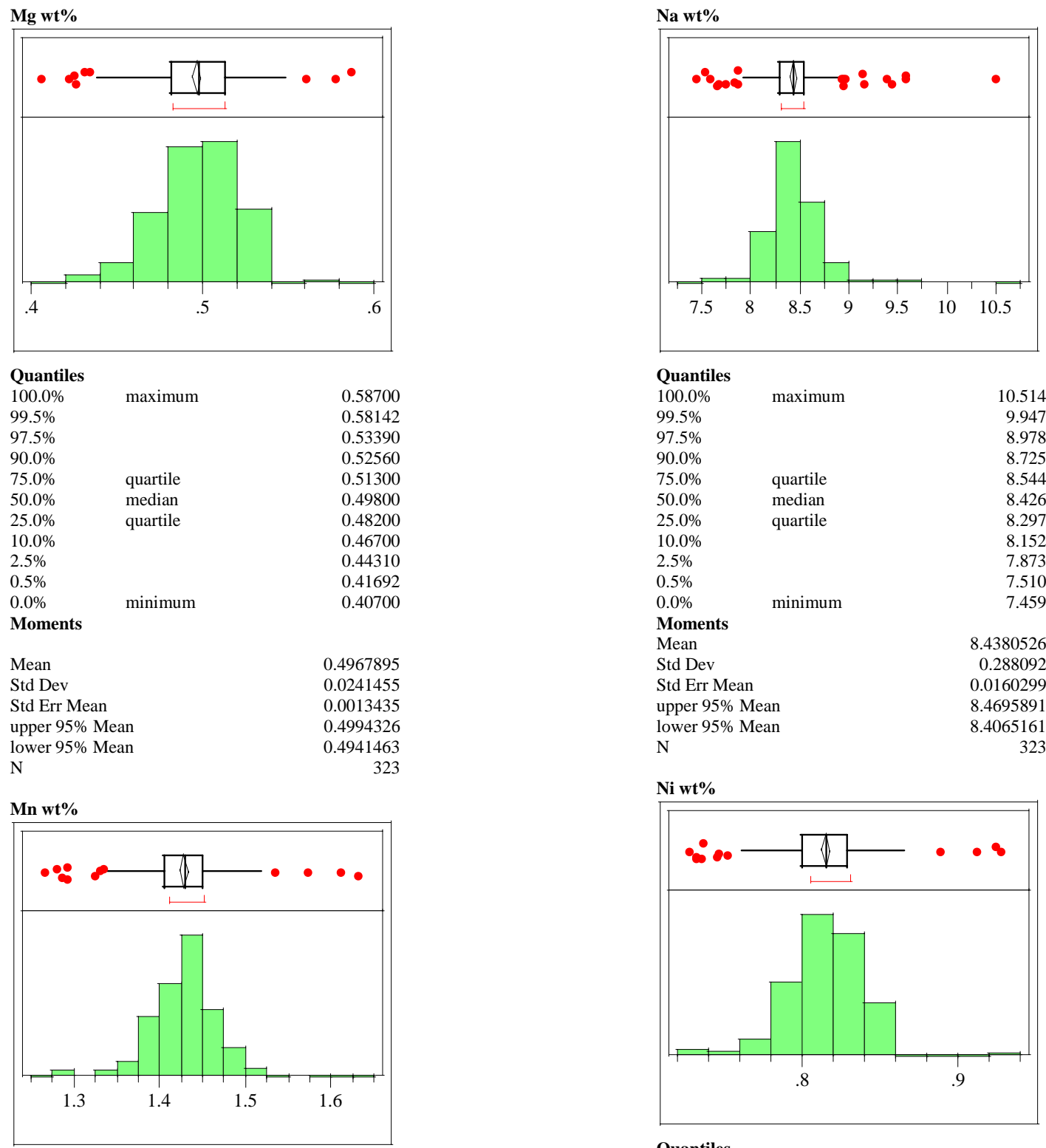

$\begin{array}{llr}\begin{array}{ll}\text { Quantiles } \\ 100.0 \%\end{array} & \text { maximum } & \\ 99.5 \% & & 1.6330 \\ 97.5 \% & 1.6200 \\ 90.0 \% & 1.5037 \\ 75.0 \% & \text { quartile } & 1.4736 \\ 50.0 \% & \text { median } & 1.4500 \\ 25.0 \% & \text { quartile } & 1.4300 \\ 10.0 \% & & 1.4060 \\ 2.5 \% & 1.3804 \\ 0.5 \% & & 1.3364 \\ 0.0 \% & & 1.2757 \\ \text { Moments } & & 1.2670 \\ \text { Mean } & & \\ \text { Std Dev } & & 1.4282972 \\ \text { Std Err Mean } & & 0.041983 \\ \text { upper 95\% Mean } & 0.002336 \\ \text { lower 95\% Mean } & 1.432893 \\ \text { N } & & 1.4237015 \\ \end{array}$

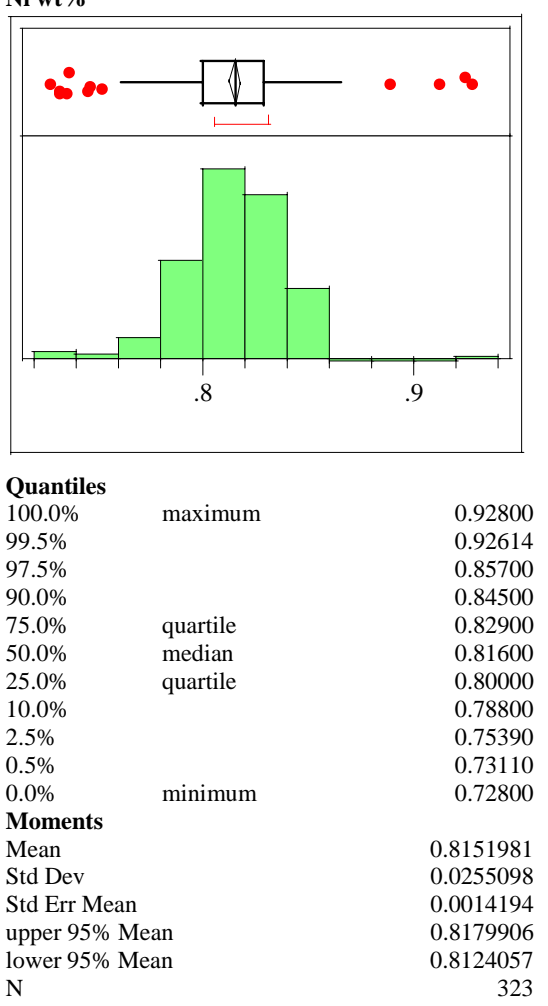


WSRC-TR-2004-00576

Revision 0

\section{Exhibit A9. Histograms and Descriptive Statistics for ARG-1 Cold Chem Data Associated with a SRAT Batch}
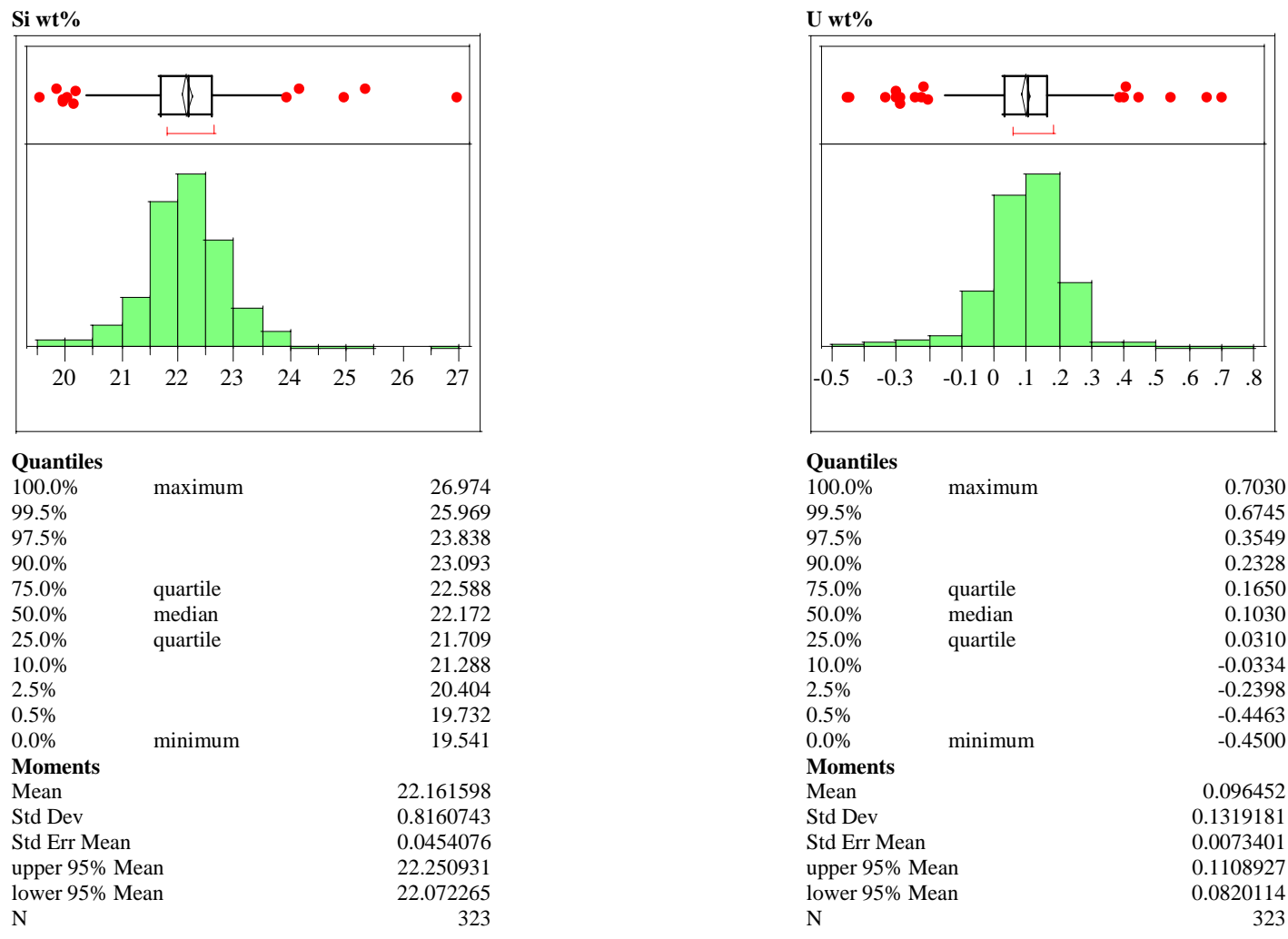

Ti wt \%
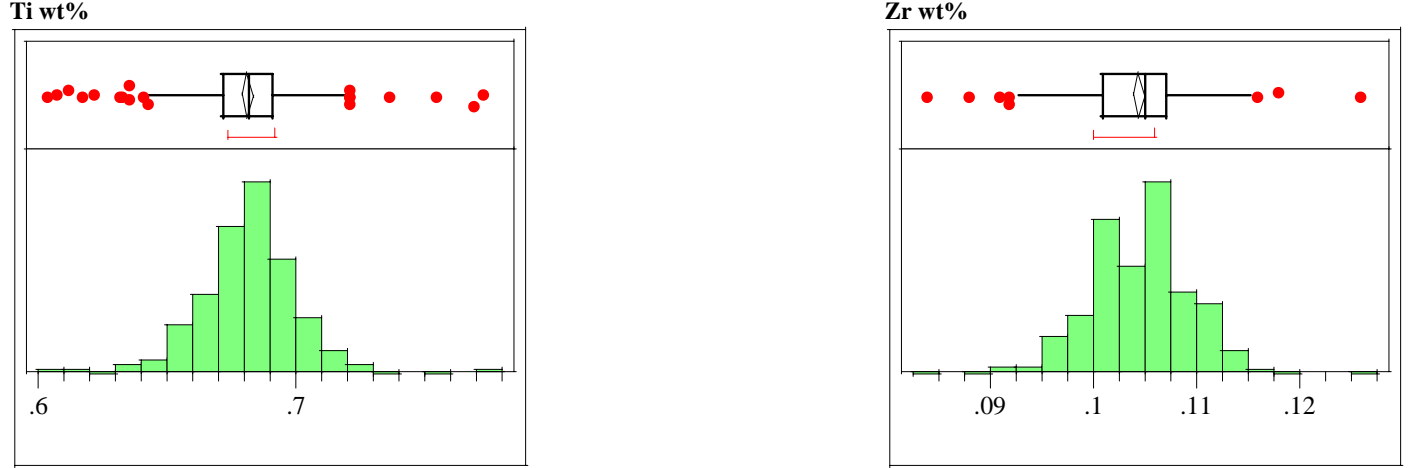

Quantiles

$100.0 \%$ maximum 0.77300

$99.5 \%$

0.72070

0.70200

$75.0 \% \quad$ quartile

$50.0 \%$ median

$25.0 \%$ quartile

$10.0 \%$

0.68200

0.67200

0.65800

$2.5 \%$

0.63600

$0.5 \% \quad 0.60648$

$0.0 \% \quad$ minimum $\quad 0.60400$

Moments

0.681226

Mean

0.0202557

0.0011271

Std Err Mean

upper 95\% Mean

0.6834433

lower 95\% Mean

0.6790087

$\mathrm{N}$

323 
WSRC-TR-2004-00576

Revision 0

\section{Exhibit A10. Components of Variance for ARG-1 Cold Chem Data Associated with a SRAT Batch}

Response Al wt\%

Summary of Fit

RSquare

RSquare Adj

Root Mean Square Error

Mean of Response

Observations (or Sum Wgts)

0.625316

0.253129

0.07277

2.35624

300

Analysis of Variance

$\begin{array}{lrrrr}\text { Source } & \text { DF } & \text { Sum of Squares } & \text { Mean Square } & \text { F Ratio } \\ \text { Model } & 149 & 1.3256557 & 0.008897 & 1.6801 \\ \text { Error } & 150 & 0.7943230 & 0.005295 & \text { Prob }>\text { F } \\ \text { C. Total } & 299 & 2.1199787 & & 0.0008\end{array}$

Variance Component Estimates

Component Var Comp Est Percent of Total

Batch\&Random $\quad 0.001801 \quad 25.376$

Residual $\quad 0.005295 \quad 74.624$

Total $\quad 0.007096 \quad 100.000$

These estimates based on equating Mean Squares to Expected Value.

Response B wt \%

Summary of Fit

RSquare 0.70023

RSquare Adj $\quad 0.402459$

Root Mean Square Error $\quad 0.07356$

Mean of Response

Observations (or Sum Wgts) 300

Analysis of Variance

Source DF Sum of Squares Mean Square F Ratio

$\begin{array}{lllll}\text { Model } & 149 & 1.8960237 & 0.012725 & 2.3516\end{array}$

$\begin{array}{lllll}\text { Error } & 150 & 0.8116910 & 0.005411 \quad \text { Prob }>\text { F }\end{array}$

$\begin{array}{llll}\text { C. Total } & 299 & 2.7077147 & <.0001\end{array}$

Variance Component Estimates

Component Var Comp Est Percent of Total

Batch\&Random $\quad 0.003657 \quad 40.326$

$\begin{array}{lll}\text { Residual } & 0.005411 & 59.674\end{array}$

Total $0.009068 \quad 100.000$

These estimates based on equating Mean Squares to Expected Value.

Response Ca wt \%

Summary of Fit

$\begin{array}{lr}\text { RSquare } & 0.809481 \\ \text { RSquare Adj } & 0.620232 \\ \text { Root Mean Square Error } & 0.054449 \\ \text { Mean of Response } & 1.038653 \\ \text { Observations (or Sum Wgts) } & 300\end{array}$

Analysis of Variance

Source DF Sum of Squares Mean Square F Ratio

$\begin{array}{lllll}\text { Model } & 149 & 1.8894989 & 0.012681 & 4.2773\end{array}$

$\begin{array}{lllll}\text { Error } & 150 & 0.4447110 & 0.002965 & \text { Prob }>\text { F }\end{array}$

$\begin{array}{llll}\text { C. Total } 299 & 2.3342099 & <.0001\end{array}$

Variance Component Estimates

Component Var Comp Est Percent of Total

Batch\&Random $\quad 0.004858 \quad 62.102$

Residual $\quad 0.002965 \quad 37.898$

Total $\quad 0.007823 \quad 100.000$

These estimates based on equating Mean Squares to Expected Value.
Response Cr wt\%

Summary of Fit

\begin{tabular}{|c|c|c|c|c|}
\hline \multicolumn{3}{|l|}{ RSquare } & \multicolumn{2}{|c|}{.703876} \\
\hline \multicolumn{3}{|c|}{ RSquare Adj } & \multicolumn{2}{|c|}{0.409726} \\
\hline \multicolumn{3}{|c|}{ Root Mean Square Error } & \multicolumn{2}{|c|}{0.00243} \\
\hline \multicolumn{3}{|c|}{ Mean of Response } & \multicolumn{2}{|c|}{0.067673} \\
\hline \multicolumn{3}{|c|}{ Observations (or Sum Wgts) } & 300 & \\
\hline \multicolumn{5}{|c|}{ Analysis of Variance } \\
\hline Source & DF & Sum of Squares & Mean Square & F Rati \\
\hline Model & 149 & 0.00210599 & 0.000014 & 2.3929 \\
\hline Error & 150 & 0.00088600 & 0.000006 & Prob $>$ F \\
\hline C. Total & 299 & 0.00299199 & & $<.0001$ \\
\hline
\end{tabular}

Variance Component Estimates

$\begin{array}{lrr}\text { Component } & \text { Var Comp Est } & \text { Percent of Total } \\ \text { Batch\&Random } & 0.000004 & 41.054\end{array}$

$\begin{array}{lll}\text { Batch\&Random } & 0.000004 & 41.054\end{array}$

Total $\quad 0.00001 \quad 100.000$

These estimates based on equating Mean Squares to Expected Value.

Response Cu wt\%

Summary of Fit

RSquare

0.945743

RSquare Ad

0.891847

Root Mean Square Error $\quad 0.001041$

Mean of Response

0.004337

Observations (or Sum Wgts)

300

Analysis of Variance

$\begin{array}{lrrrr}\text { Source } & \text { DF } & \text { Sum of Squares } & \text { Mean Square } & \text { F Ratio } \\ \text { Model } & 149 & 0.00283250 & 0.000019 & 17.5477 \\ \text { Error } & 150 & 0.00016250 & 0.000001 & \text { Prob }>\text { F } \\ \text { C. Total } & 299 & 0.00299500 & & <.0001\end{array}$

Variance Component Estimates

Component Var Comp Est Percent of Total

Batch\&Random $\quad 0.000009 \quad 89.217$

$\begin{array}{lll}\text { Residual } & 0.000001 & 10.783\end{array}$

Total $\quad 0.00001 \quad 100.000$

These estimates based on equating Mean Squares to Expected Value.

Response Fe wt\%

Summary of Fit

RSquare

0.580876

RSquare Adj $\quad 0.164546$

Root Mean Square Error $\quad 0.259474$

Mean of Response 9.660673

Observations (or Sum Wgts) 300

Analysis of Variance

$\begin{array}{lrrrr}\text { Source } & \text { DF } & \text { Sum of Squares } & \text { Mean Square } & \text { F Ratio } \\ \text { Model } & 149 & 13.996515 & 0.093936 & 1.3952 \\ \text { Error } & 150 & 10.099011 & 0.067327 & \text { Prob }>\text { F } \\ \text { C. Total } & 299 & 24.095526 & & 0.0212\end{array}$

Variance Component Estimates

Component Var Comp Est Percent of Total

Batch\&Random $\quad 0.013305 \quad 16.501$

Residual $\quad 0.067327 \quad 83.499$

Total $\quad 0.080632 \quad 100.000$

These estimates based on equating Mean Squares to Expected Value. 
WSRC-TR-2004-00576

Revision 0

\section{Exhibit A10. Components of Variance for ARG-1 Cold Chem Data Associated with a SRAT Batch}

Response K wt \%
Summary of Fit

RSquare

RSquare Adj

Root Mean Square Error

Mean of Response

Observations (or Sum Wgts)

0.655459

0.313215

0.062387

2.23771

300

Analysis of Variance

$\begin{array}{lrrrr}\text { Source } & \text { DF } & \text { Sum of Squares } & \text { Mean Square } & \text { F Ratio } \\ \text { Model } & 149 & 1.1106713 & 0.007454 & 1.9152 \\ \text { Error } & 150 & 0.5838225 & 0.003892 & \text { Prob }>\text { F } \\ \text { C. Total } & 299 & 1.6944938 & & <.0001\end{array}$

Variance Component Estimates

$\begin{array}{lrr}\text { Component } & \text { Var Comp Est } & \text { Percent of Total } \\ \text { Batch\&Random } & 0.001781 & 31.394 \\ \text { Residual } & 0.003892 & 68.606 \\ \text { Total } & 0.005673 & 100.000\end{array}$

$\begin{array}{lcc}\text { Total } & 0.005673 & 100.000 \\ \text { These estimates based on equating Mean Squares to Expected Value. }\end{array}$

Response Li wt\%

Summary of Fit

RSquare 0.646925

RSquare Adj $\quad 0.296203$

Root Mean Square Error $\quad 0.040234$

Mean of Response

Observations (or Sum Wgts) 300

Analysis of Variance

$\begin{array}{lrrrr}\text { Source } & \text { DF } & \text { Sum of Squares } & \text { Mean Square } & \text { F Ratio } \\ \text { Model } & 149 & 0.44490930 & 0.002986 & 1.8446 \\ \text { Error } & 150 & 0.24282050 & 0.001619 & \text { Prob }>\text { F }\end{array}$

0.0001

Variance Component Estimates

Component Var Comp Est Percent of Total

Batch\&Random $\quad 0.000684 \quad 29.690$

$\begin{array}{lll}\text { Residual } & 0.001619 & 70.310\end{array}$

Total $0.002302 \quad 100.000$

These estimates based on equating Mean Squares to Expected Value.

Response Mg wt\%

Summary of Fit

$\begin{array}{lr}\text { RSquare } & 0.706454 \\ \text { RSquare Adj } & 0.414866 \\ \text { Root Mean Square Error } & 0.018748 \\ \text { Mean of Response } & 0.496703 \\ \text { Observations (or Sum Wgts) } & 300\end{array}$

Analysis of Variance

Source DF Sum of Squares Mean Square F Ratio

$\begin{array}{lllll}\text { Model } & 149 & 0.12688810 & 0.000852 & 2.4228\end{array}$

$\begin{array}{lllll}\text { Error } & 150 & 0.05272450 & 0.000351 & \text { Prob }>\text { F }\end{array}$

$\begin{array}{llllr}\text { C. Total } & 299 & 0.17961260 & & <.0001\end{array}$

Variance Component Estimates

Component Var Comp Est Percent of Total

Batch\&Random $\quad 0.00025 \quad 41.568$

Residual $\quad 0.000351 \quad 58.432$

Total $\quad 0.000602 \quad 100.000$

These estimates based on equating Mean Squares to Expected Value.

\section{Response Mn wt $\%$}

Summary of Fit

\begin{tabular}{|c|c|c|c|c|}
\hline \multicolumn{3}{|l|}{ RSquare } & \multicolumn{2}{|c|}{575902} \\
\hline \multicolumn{3}{|c|}{ RSquare Adj } & \multicolumn{2}{|c|}{0.154631} \\
\hline \multicolumn{3}{|c|}{ Root Mean Square Error } & \multicolumn{2}{|c|}{0.038398} \\
\hline \multicolumn{3}{|c|}{ Mean of Response } & \multicolumn{2}{|c|}{1.427333} \\
\hline \multicolumn{3}{|c|}{ Observations (or Sum Wgts) } & \multicolumn{2}{|l|}{300} \\
\hline \multicolumn{5}{|c|}{ Analysis of Variance } \\
\hline Source & DF & Sum of Square & Mean Square & F Ratio \\
\hline Model & 149 & 0.3003296 & 0.002016 & 1.3671 \\
\hline Error & 150 & 0.2211650 & 0.001474 & Prob $>$ F \\
\hline C. Total & 299 & 0.5214946 & & 0.0284 \\
\hline
\end{tabular}

Variance Component Estimates

$\begin{array}{lrr}\text { Variance Component Estimates } & \\ \text { Component } & \text { Var Comp Est } & \text { Percent of Total } \\ \text { Batch\&Random } & 0.000271 & 15.507 \\ \text { Residual } & 0.001474 & 84.493 \\ \text { Total } & 0.001745 & 100.000\end{array}$

Total $\quad 0.001745 \quad 100.000$

These estimates based on equating Mean Squares to Expected Value.

Response Na wt \%

Summary of Fit

RSquare

0.600372

RSquare Adj

0.203409

Root Mean Square Error $\quad 0.258392$

Mean of Response

8.434523

Observations (or Sum Wgts)

300

Analysis of Variance

$\begin{array}{lrrrr}\text { Source } & \text { DF } & \text { Sum of Squares } & \text { Mean Square } & \text { F Ratio } \\ \text { Model } & 149 & 15.045749 & 0.100978 & 1.5124 \\ \text { Error } & 150 & 10.014949 & 0.066766 & \text { Prob }>\text { F } \\ \text { C. Total } & 299 & 25.060699 & & 0.0059\end{array}$

C. Total $299 \quad 25.060699$

Variance Component Estimates

Component Var Comp Est Percent of Total

Batch\&Random $\quad 0.017106 \quad 20.395$

$\begin{array}{lll}\text { Residual } & 0.066766 & 79.605\end{array}$

Total $0.083872 \quad 100.000$

These estimates based on equating Mean Squares to Expected Value.

Response Ni wt \%

Summary of Fit

RSquare

0.59347

RSquare Adj $\quad 0.189651$

Root Mean Square Error $\quad 0.022666$

Mean of Response

0.814423

Observations (or Sum Wgts)

300

Analysis of Variance

$\begin{array}{lrrrr}\text { Source } & \text { DF } & \text { Sum of Squares } & \text { Mean Square } & \text { F Ratio } \\ \text { Model } & 149 & 0.11250374 & 0.000755 & 1.4696 \\ \text { Error } & 150 & 0.07706550 & 0.000514 & \text { Prob }>\text { F } \\ \text { C. Total } & 299 & 0.18956924 & & 0.0096\end{array}$

Variance Component Estimates

Component Var Comp Est Percent of Total

Batch\&Random $\quad 0.000121$

Residual $\quad 0.000514 \quad 80.983$

Total $\quad 0.000634 \quad 100.000$

These estimates based on equating Mean Squares to Expected Value. 
WSRC-TR-2004-00576

Revision 0

\section{Exhibit A10. Components of Variance for ARG-1 Cold Chem Data Associated with a SRAT Batch}

Response Si wt\%

Summary of Fit

RSquare

RSquare Adj

Root Mean Square Error $\quad 0.636209$

Mean of Response 22.15984

Observations (or Sum Wgts) 300

Analysis of Variance

$\begin{array}{lrrrr}\text { Source } & \text { DF } & \text { Sum of Squares } & \text { Mean Square } & \text { F Ratio } \\ \text { Model } & 149 & 142.22005 & 0.954497 & 2.3582 \\ \text { Error } & 150 & 60.71431 & 0.404762 & \text { Prob > F } \\ \text { C. Total } & 299 & 202.93435 & & <.0001\end{array}$

Variance Component Estimates

$\begin{array}{lll}\text { Component } & \text { Var Comp Est } & \text { Percent of Total }\end{array}$

$\begin{array}{lll}\text { Batch\&Random } & 0.274867 & 40.444\end{array}$

Residual $\quad 0.404762 \quad 59.556$

Total $\quad 0.679629 \quad 100.000$

These estimates based on equating Mean Squares to Expected Value.

Response Ti wt \%

Summary of Fit

RSquare

Root Mean Square Error

Mean of Response

Observations (or Sum Wgts)

Analysis of Variance

$\begin{array}{lrrrr}\text { Source } & \text { DF } & \text { Sum of Squares } & \text { Mean Square } & \text { F Ratio } \\ \text { Model } & 149 & 0.07054375 & 0.000473 & 1.4476 \\ \text { Error } & 150 & 0.04905850 & 0.000327 & \text { Prob }>\text { F } \\ \text { C. Total } & 299 & 0.11960225 & & 0.0122\end{array}$

C. Total $299 \quad 0.11960225$

Variance Component Estimates

Component Var Comp Est Percent of Total

$\begin{array}{lll}\text { Batch\&Random } & 0.000073 & 18.287\end{array}$

$\begin{array}{lll}\text { Residual } & 0.000327 & 81.713\end{array}$

Total $\quad 0.0004 \quad 100.000$

These estimates based on equating Mean Squares to Expected Value.

Response U wt\%

Summary of Fit

RSquare $\quad 0.943342$

RSquare Adj 0.887062

Root Mean Square Error $\quad 0.044115$

Mean of Response 0.09819

Observations (or Sum Wgts) 300

Analysis of Variance

Source DF Sum of Squares Mean Square F Ratio

$\begin{array}{lllll}\text { Model } & 149 & 4.8604357 & 0.032620 & 16.7615\end{array}$

$\begin{array}{lllll}\text { Error } & 150 & 0.2919225 & 0.001946 & \text { Prob }>\text { F }\end{array}$

$\begin{array}{llll}\text { C. Total } & 299 & 5.1523582 & <.0001\end{array}$

Variance Component Estimates

Component Var Comp Est Percent of Total

Batch\&Random $\quad 0.015337 \quad 88.740$

Residual $\quad 0.001946 \quad 11.260$

Total $\quad 0.017283 \quad 100.000$

These estimates based on equating Mean Squares to Expected Value.

\section{Response Zr wt \%}

Summary of Fit

$\begin{array}{lr}\text { RSquare } & 0.782691 \\ \text { RSquare Adj } & 0.566831 \\ \text { Root Mean Square Error } & 0.003237 \\ \text { Mean of Response } & 0.104347 \\ \text { Observations (or Sum Wgts) } & 300\end{array}$

Analysis of Variance

$\begin{array}{lrrrr}\text { Source } & \text { DF } & \text { Sum of Squares } & \text { Mean Square } & \text { F Ratio } \\ \text { Model } & 149 & 0.00566195 & 0.000038 & 3.6259 \\ \text { Error } & 150 & 0.00157200 & 0.000010 & \text { Prob }>\text { F } \\ \text { C. Total } & 299 & 0.00723395 & & <.0001\end{array}$

Variance Component Estimates

Component Var Comp Est Percent of Total

$\begin{array}{lrr}\text { Batch\&Random } \quad 0.000014 & 56.765\end{array}$

Residual $\quad 0.00001 \quad 43.235$

Total $\quad 0.000024 \quad 100.000$

These estimates based on equating Mean Squares to Expected Value. 
WSRC-TR-2004-00576

Exhibit A11. MA Standards in Analytical Sequence

Revision 0

STCd=SM32

Control Chart

Individual Measurement of Al (mg/L)

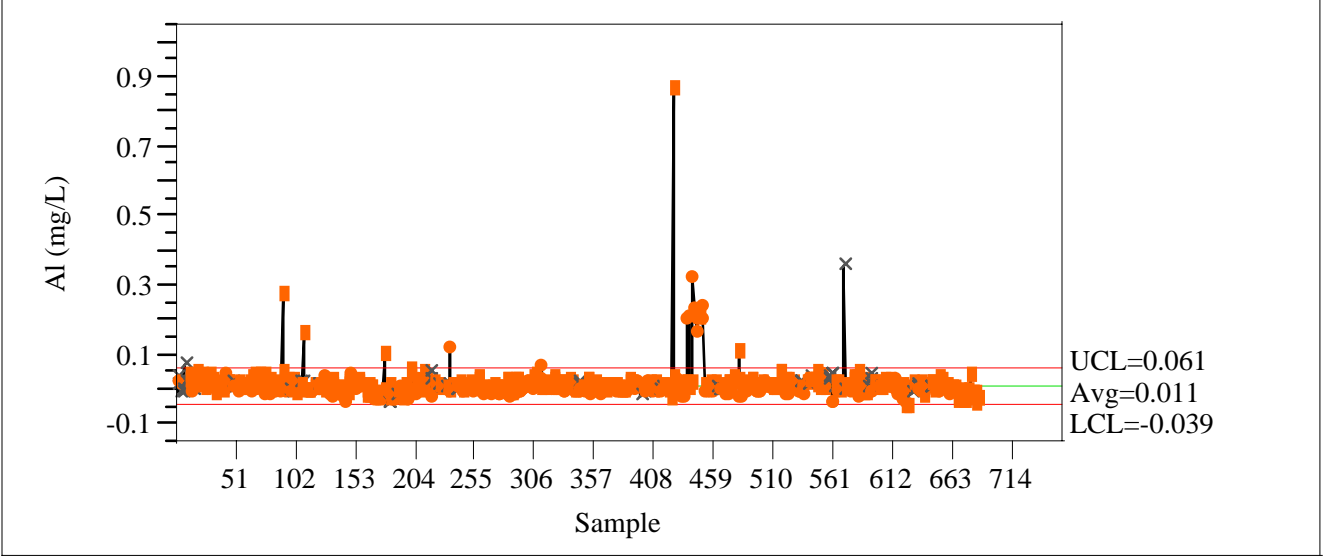

Individual Measurement of Ca (mg/L)

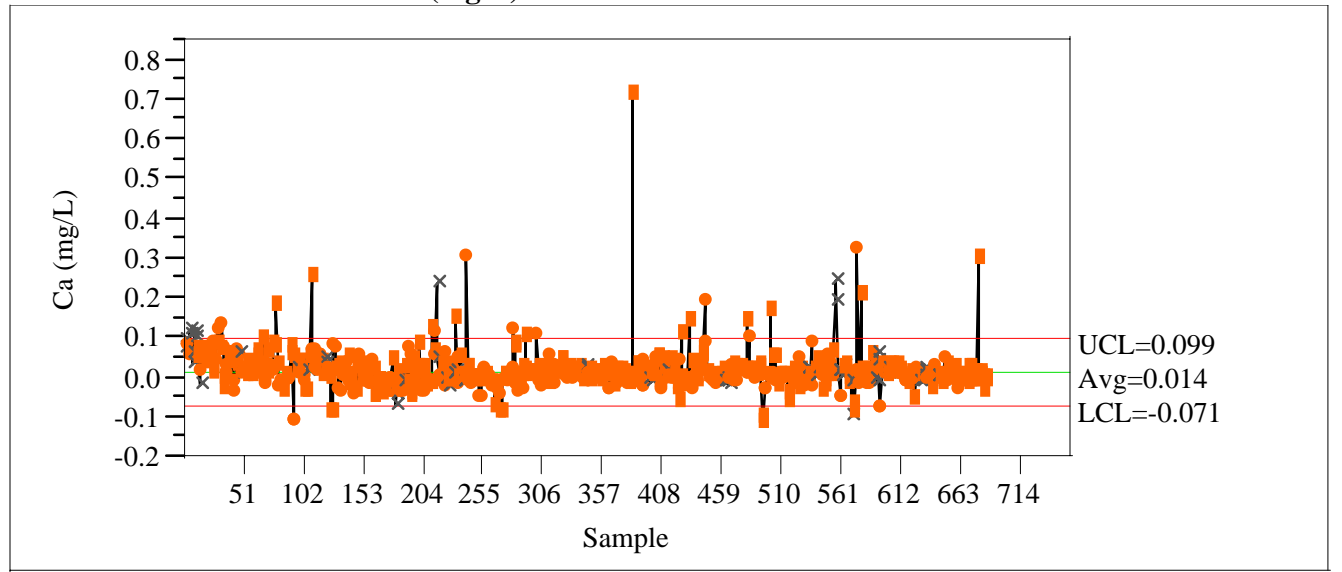

Individual Measurement of $\mathrm{Cr}$ (mg/L)

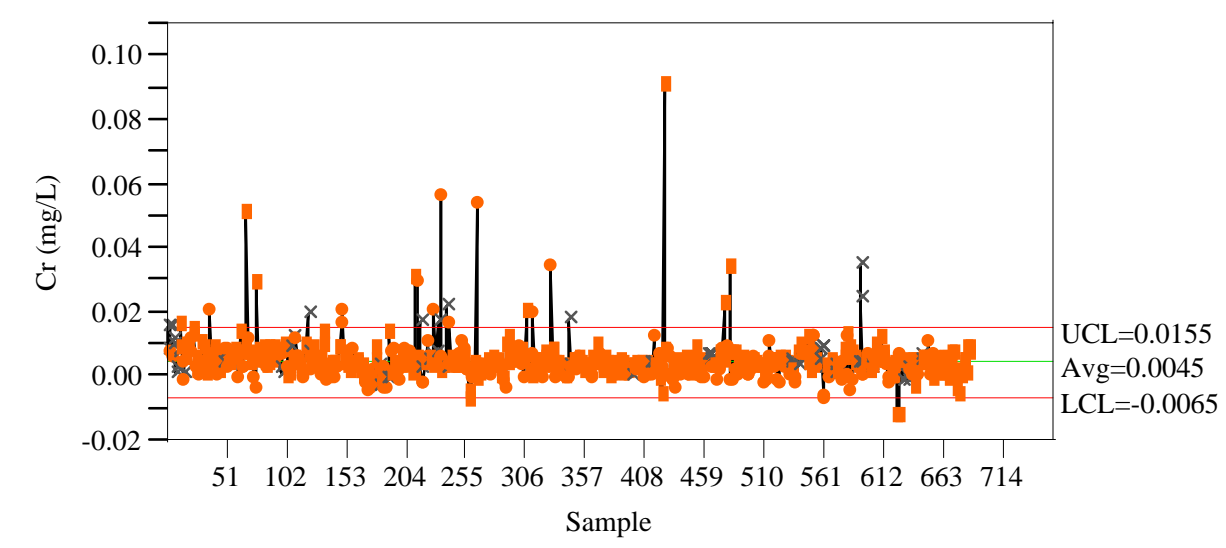


WSRC-TR-2004-00576

Exhibit A11. MA Standards in Analytical Sequence

Revision 0

Individual Measurement of $\mathrm{Cu}(\mathrm{mg} / \mathrm{L})$

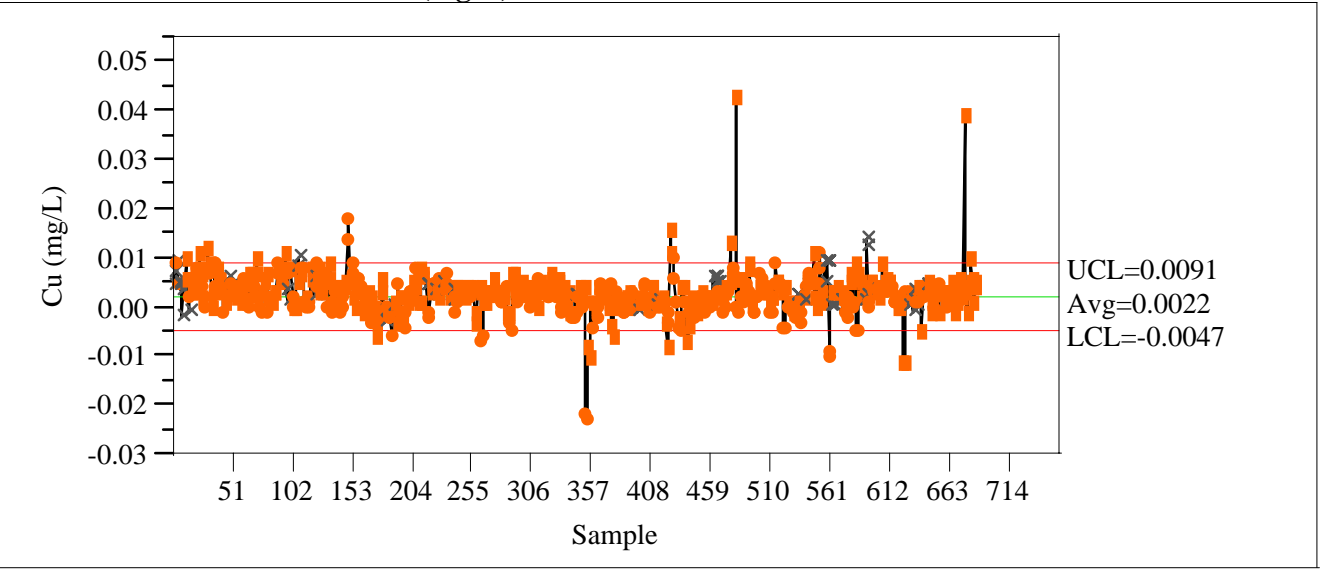

Individual Measurement of Fe (mg/L)

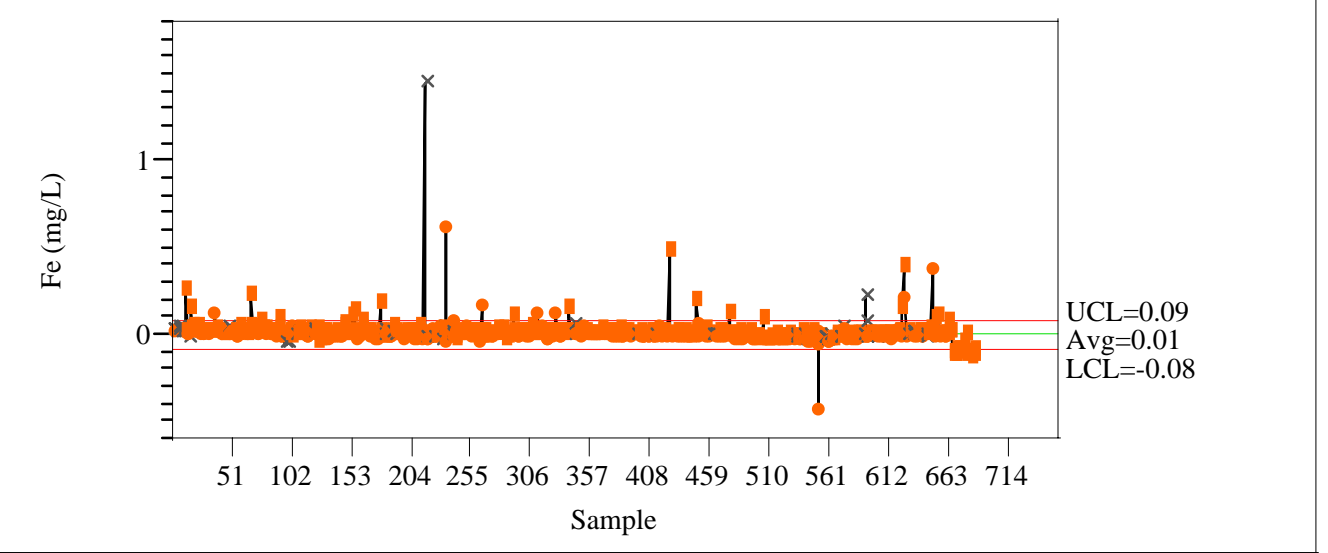

Individual Measurement of K (mg/L)

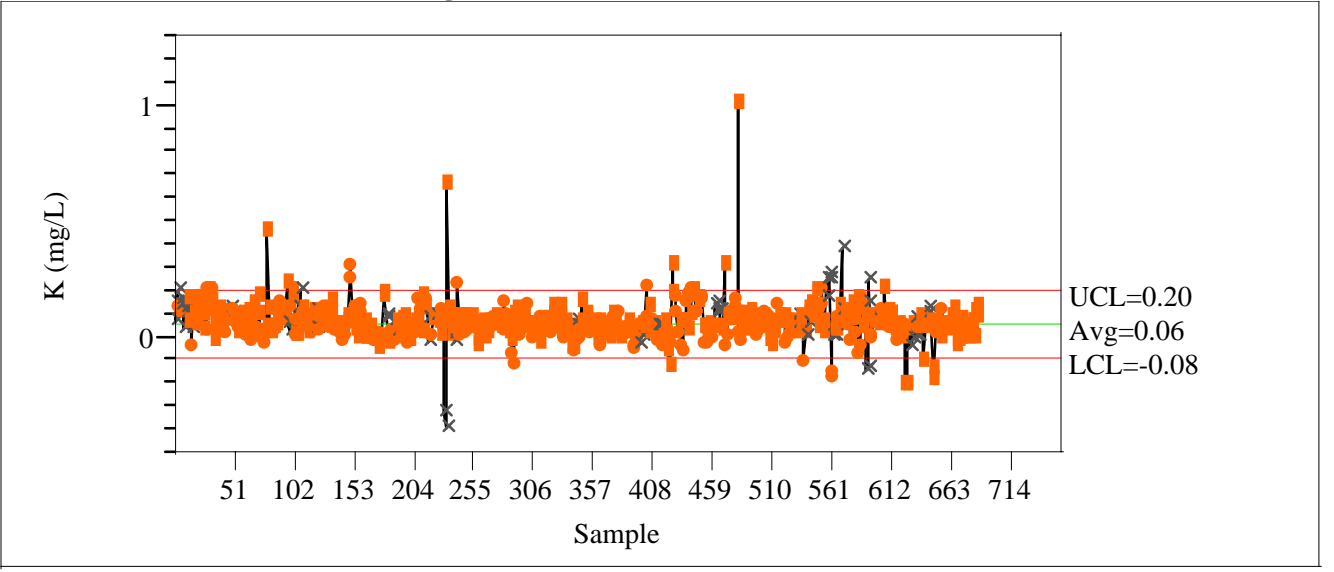


WSRC-TR-2004-00576

\section{Exhibit A11. MA Standards in Analytical Sequence}

\section{Revision 0}

Individual Measurement of $\mathrm{Li}(\mathrm{mg} / \mathrm{L})$

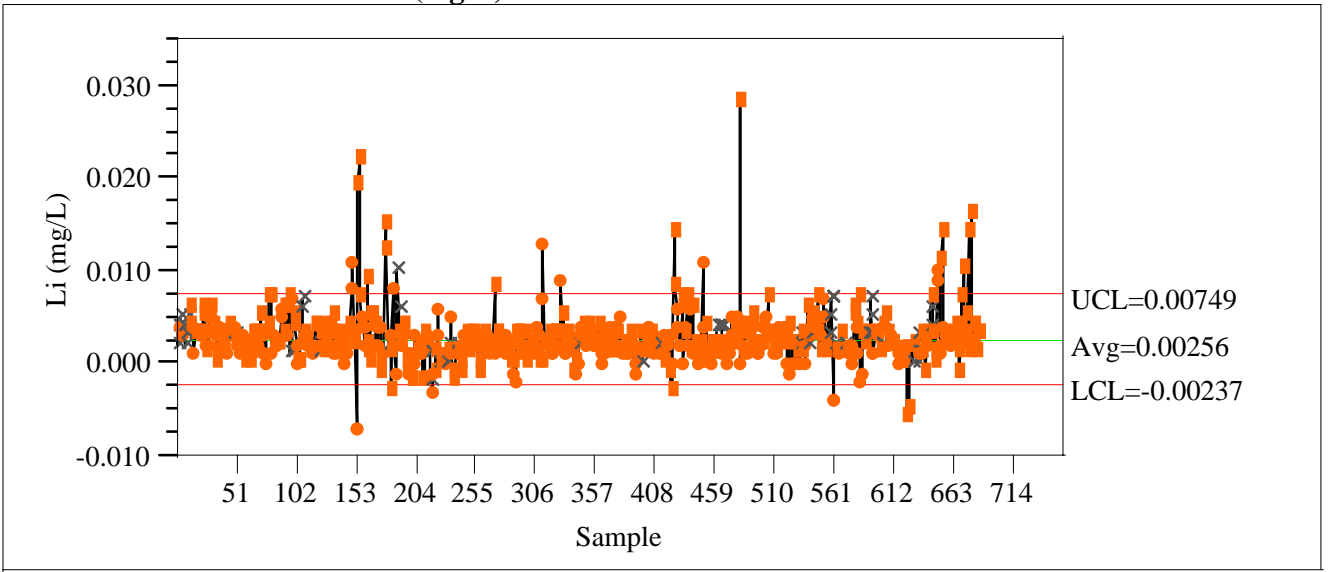

Individual Measurement of Mg (mg/L)

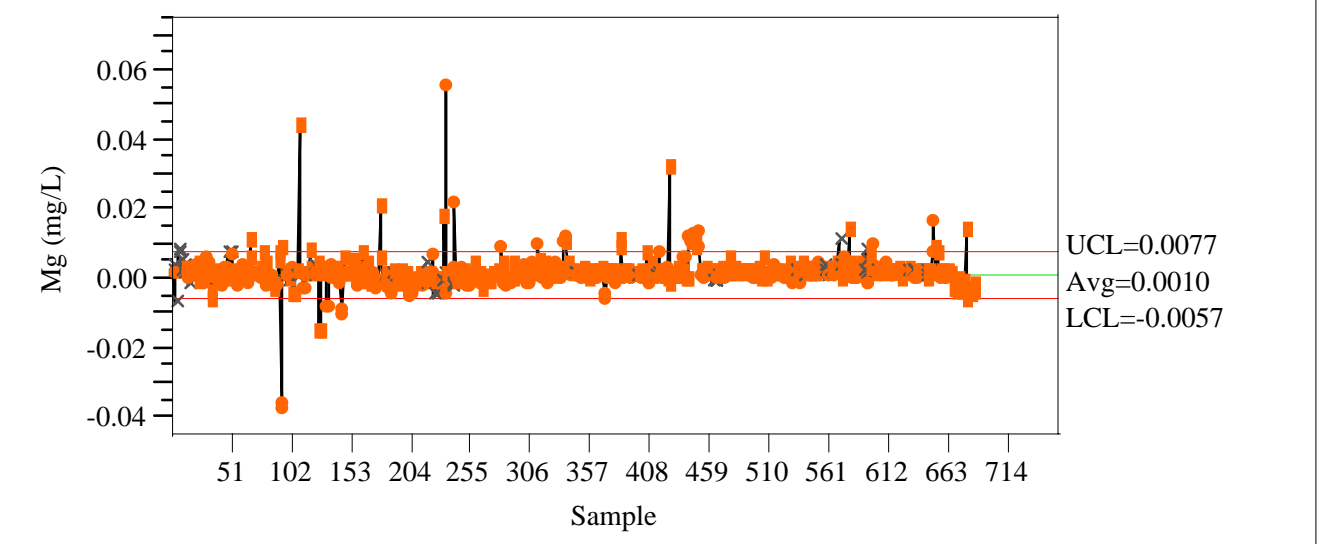

Individual Measurement of Mn (mg/L)

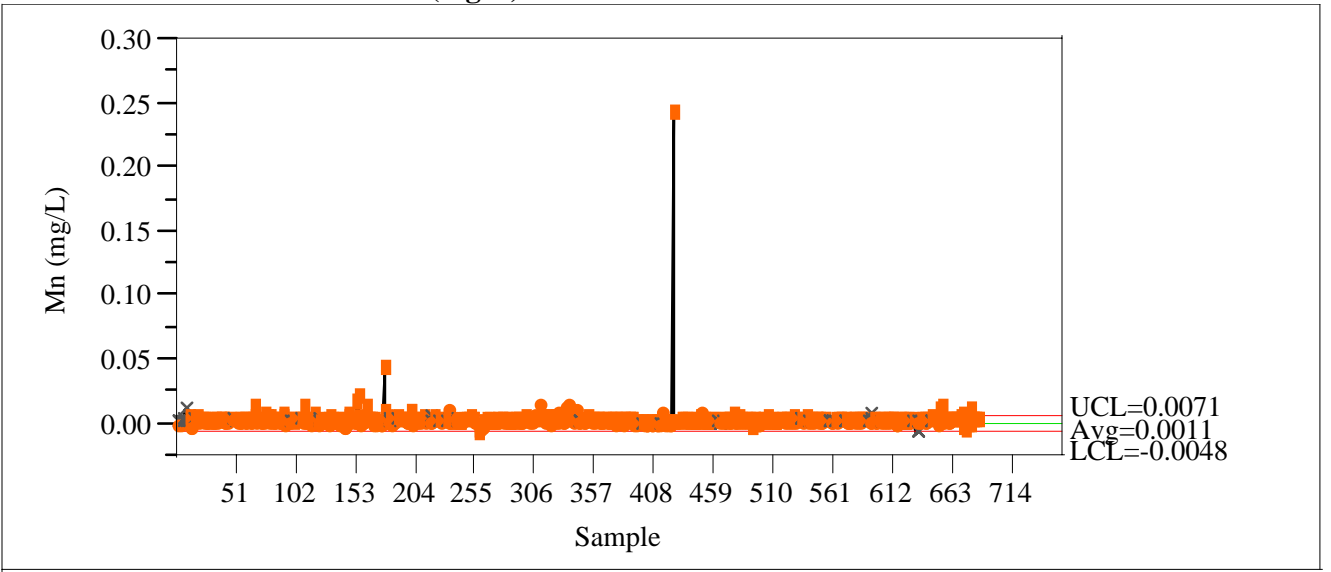


WSRC-TR-2004-00576

Exhibit A11. MA Standards in Analytical Sequence

Revision 0

Individual Measurement of $\mathrm{Na}(\mathrm{mg} / \mathrm{L})$

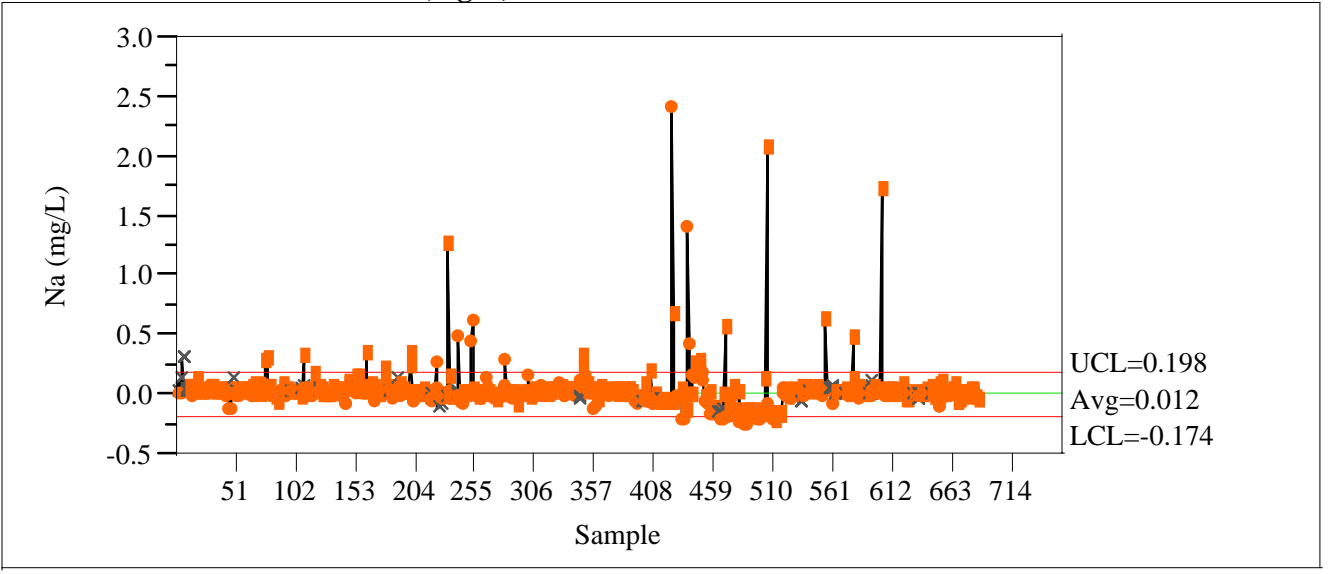

Individual Measurement of Ni (mg/L)

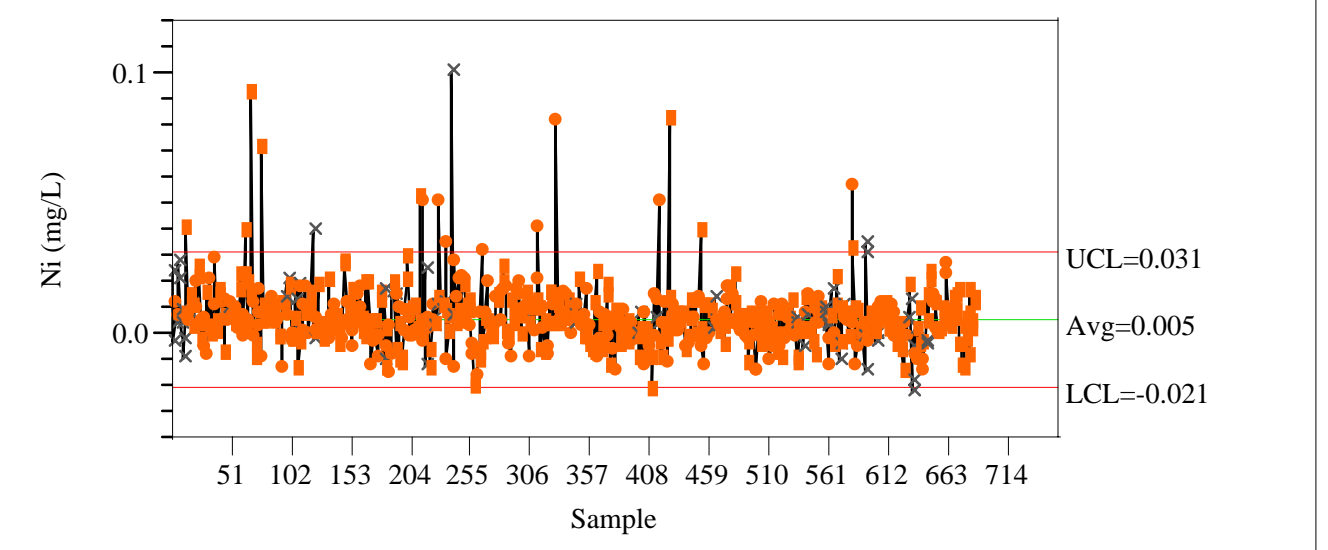

Individual Measurement of Si (mg/L)

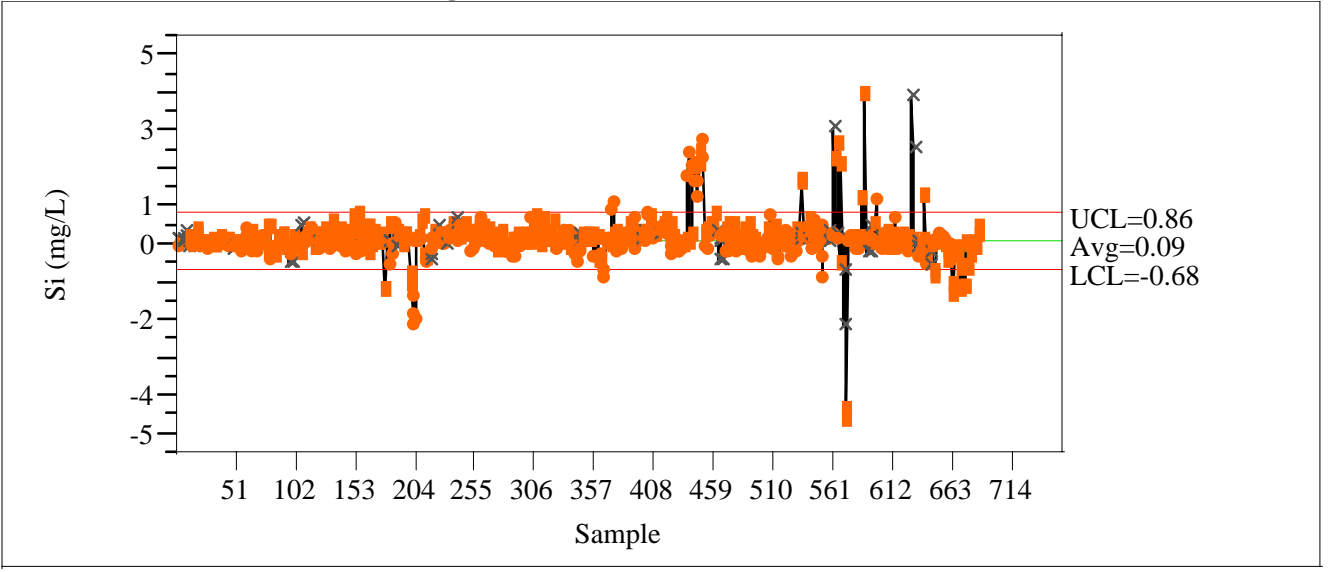


WSRC-TR-2004-00576

\section{Exhibit A11. MA Standards in Analytical Sequence}

Revision 0

Individual Measurement of Ti (mg/L)

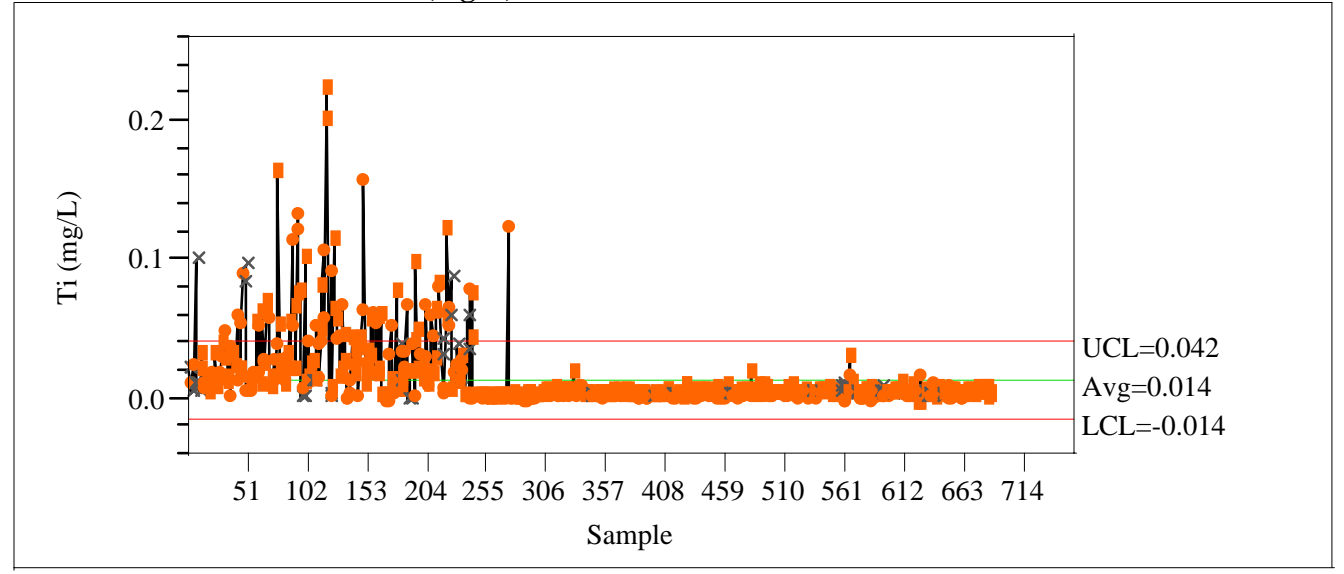

Individual Measurement of U (mg/L)

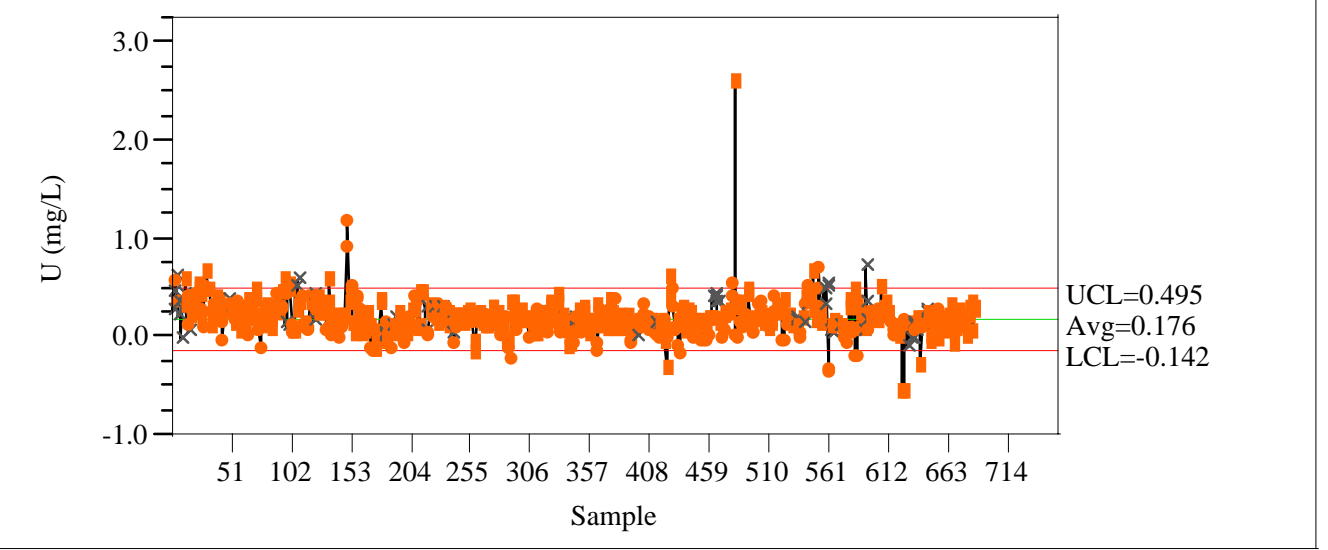

STCd=SM33

Control Chart

Individual Measurement of $\mathrm{Al}$ (mg/L)

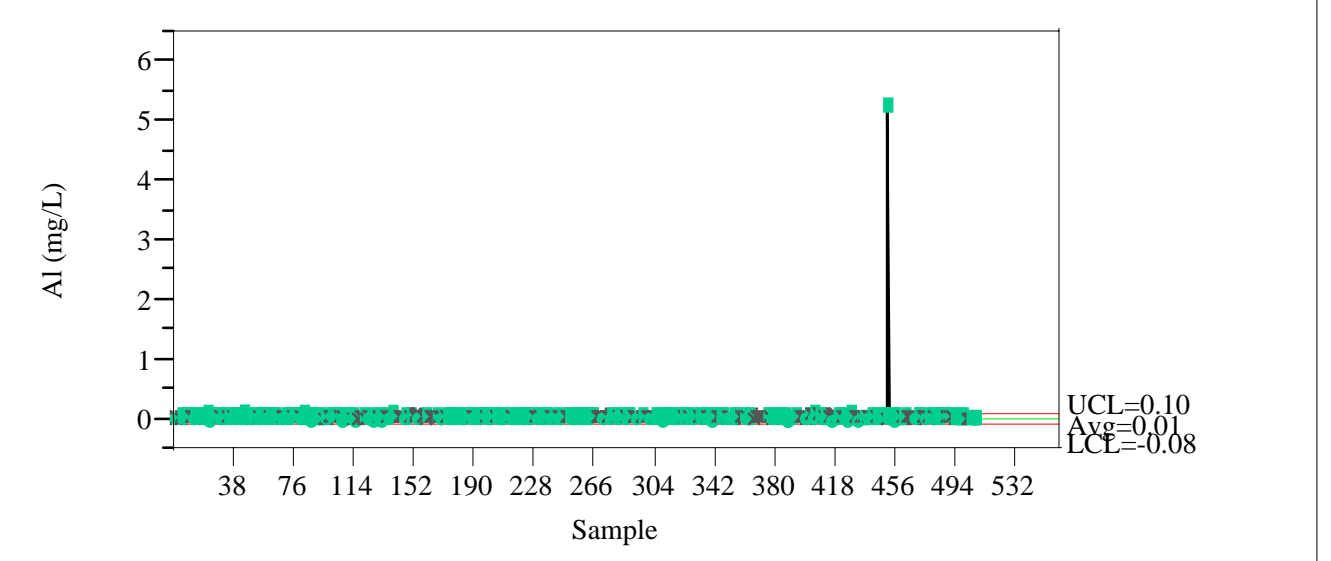


WSRC-TR-2004-00576

\section{Exhibit A11. MA Standards in Analytical Sequence}

Revision 0

Individual Measurement of Ca (mg/L)

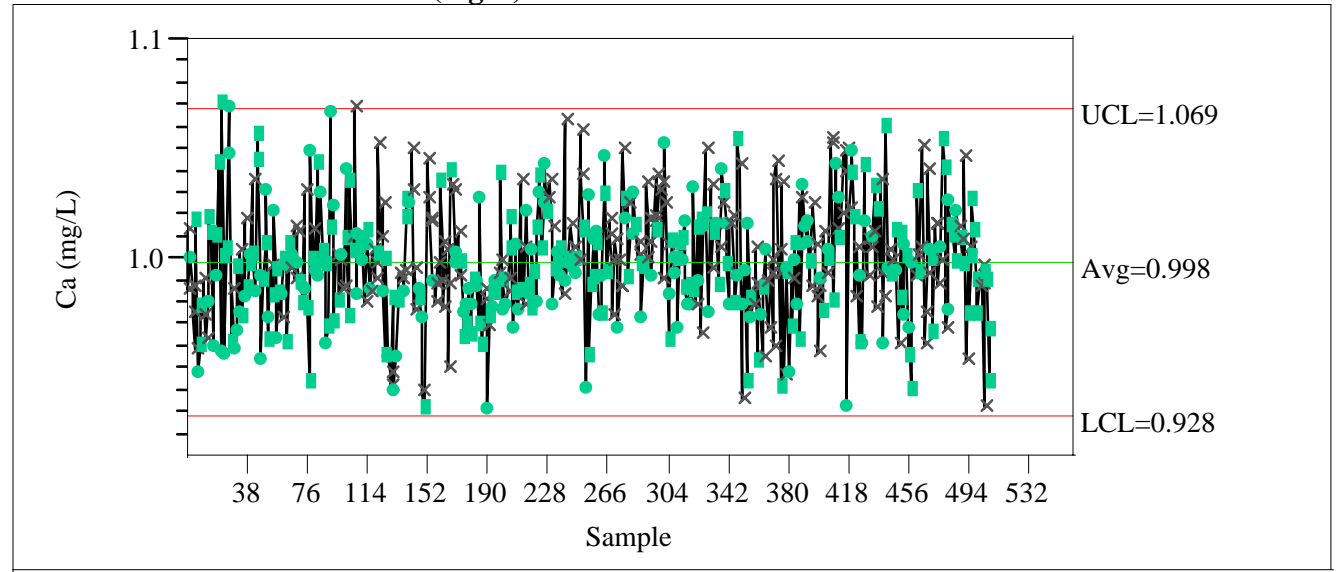

Individual Measurement of $\mathrm{Cr}$ (mg/L)

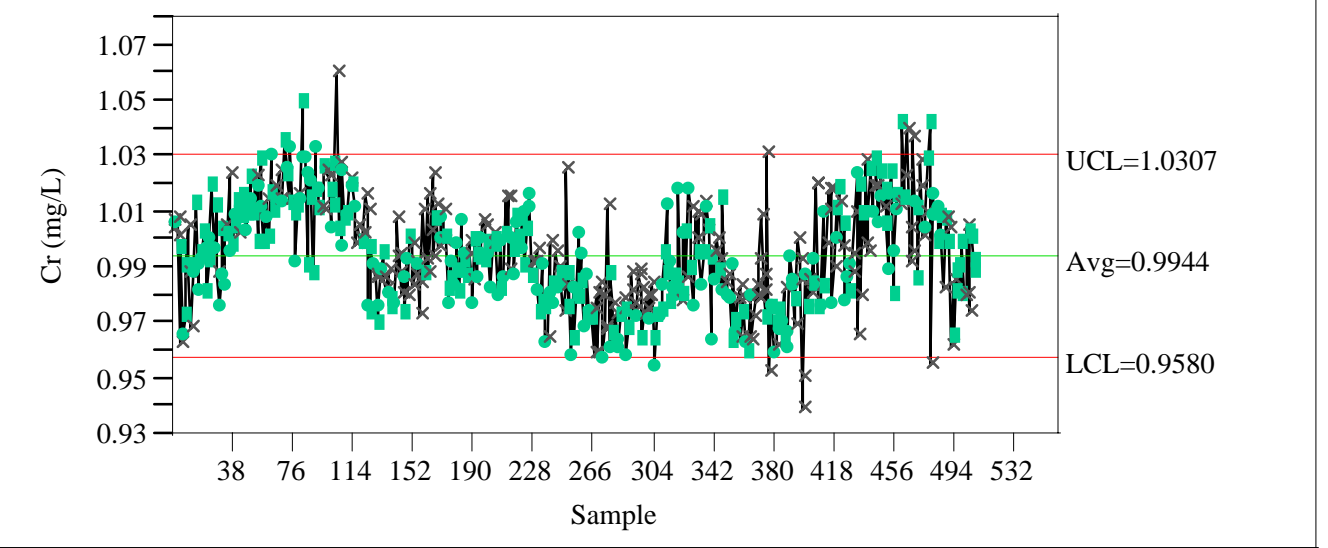

Individual Measurement of $\mathrm{Cu}(\mathrm{mg} / \mathrm{L})$

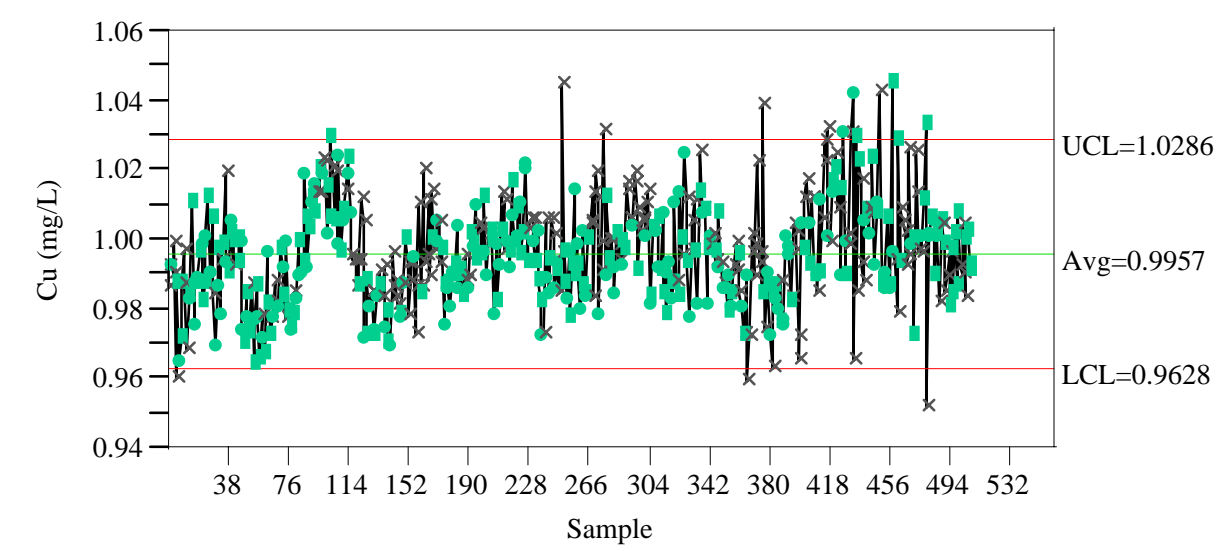


WSRC-TR-2004-00576

\section{Exhibit A11. MA Standards in Analytical Sequence}

Revision 0

Individual Measurement of Fe (mg/L)

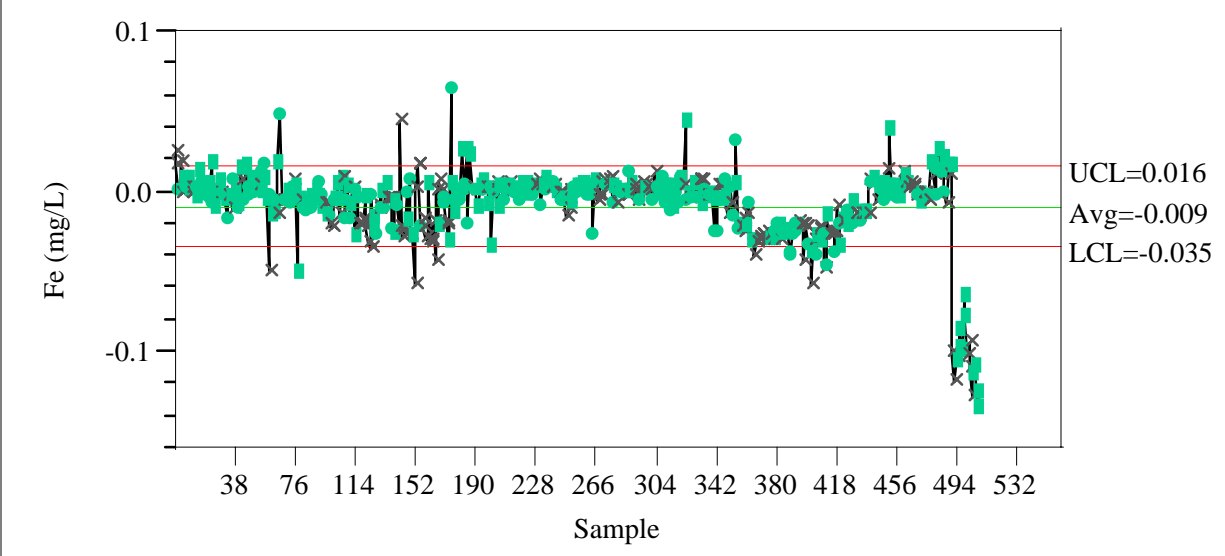

Individual Measurement of K (mg/L)

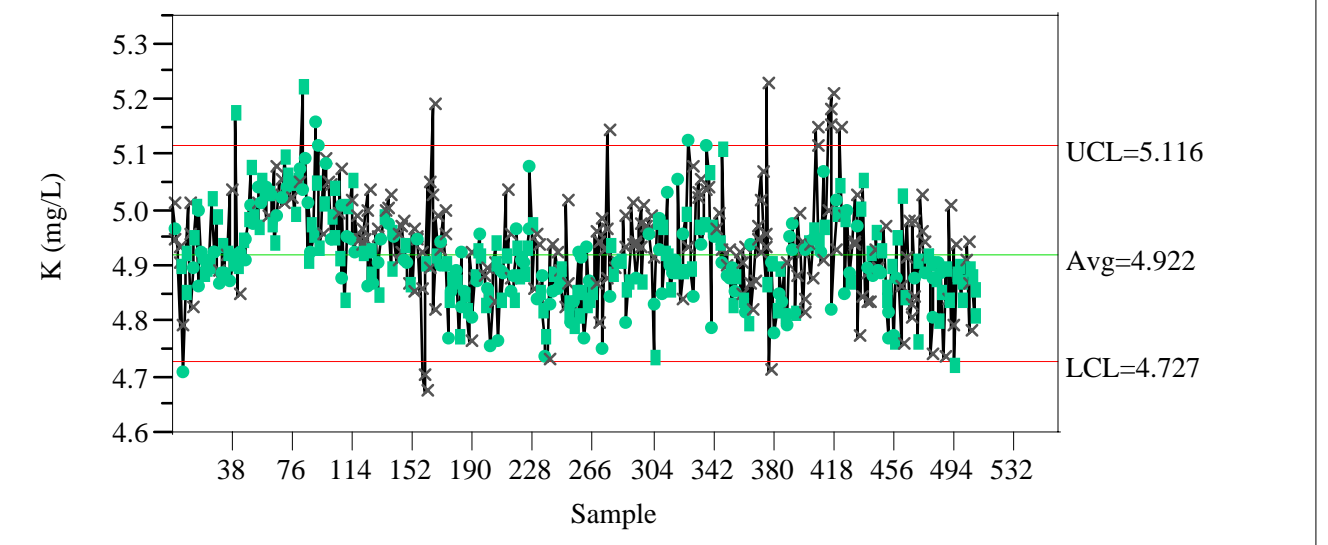

Individual Measurement of $\mathrm{Li}(\mathrm{mg} / \mathrm{L})$

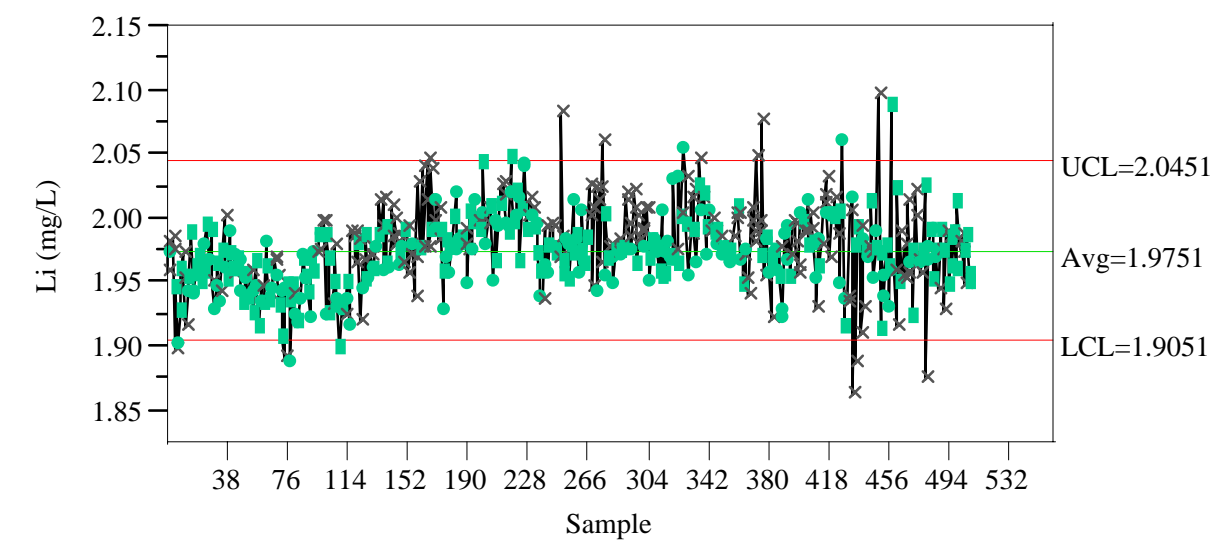


WSRC-TR-2004-00576

\section{Exhibit A11. MA Standards in Analytical Sequence}

\section{Revision 0}

Individual Measurement of $\mathrm{Mg}(\mathrm{mg} / \mathrm{L})$

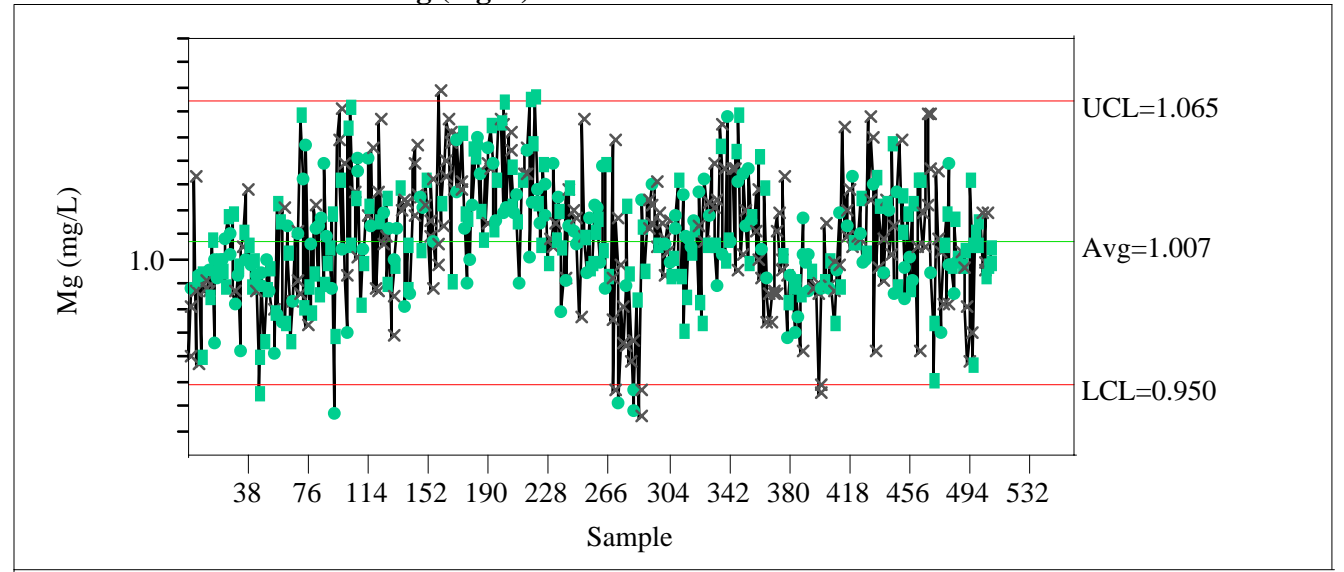

Individual Measurement of Mn (mg/L)

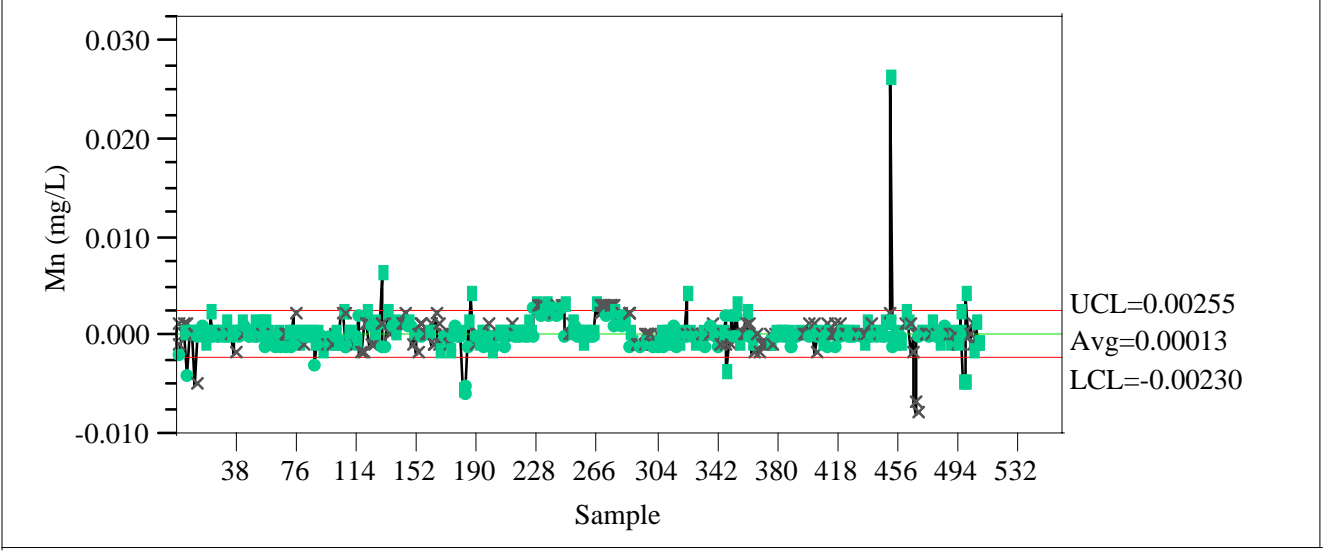

Individual Measurement of $\mathrm{Na}(\mathrm{mg} / \mathrm{L})$

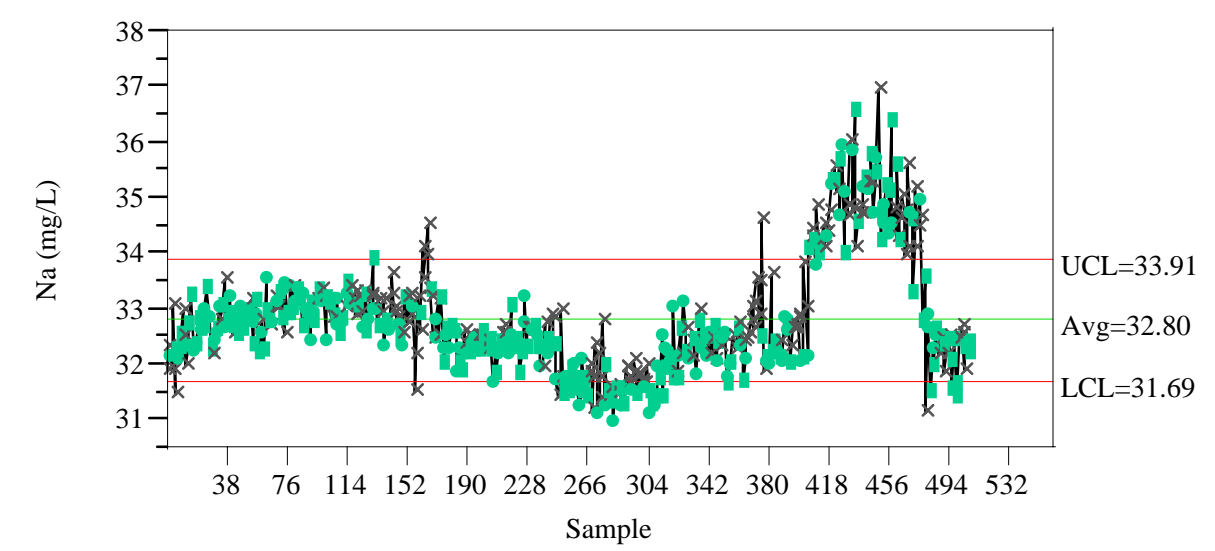


WSRC-TR-2004-00576

\section{Exhibit A11. MA Standards in Analytical Sequence}

Revision 0

Individual Measurement of Ni (mg/L)

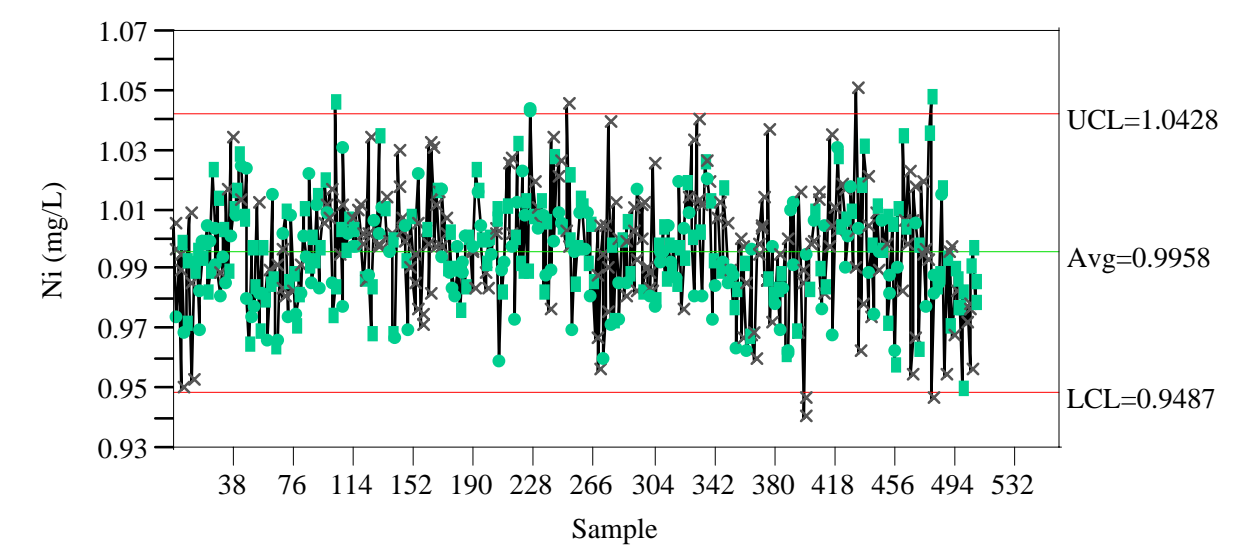

Individual Measurement of Si (mg/L)

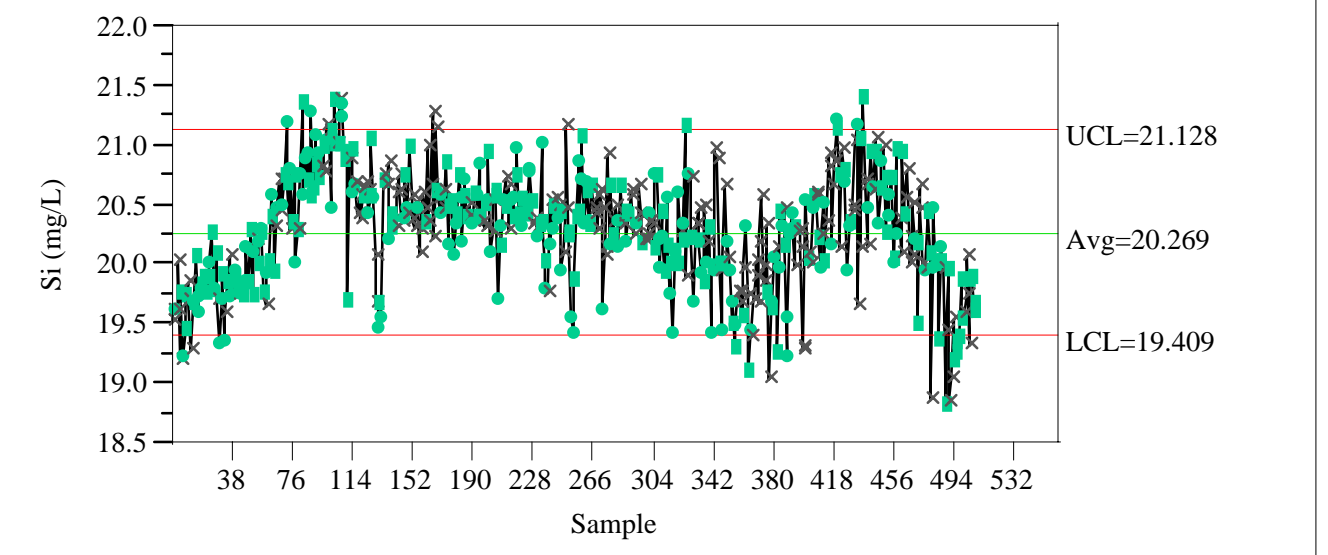

Individual Measurement of $\mathrm{Ti}(\mathrm{mg} / \mathrm{L})$

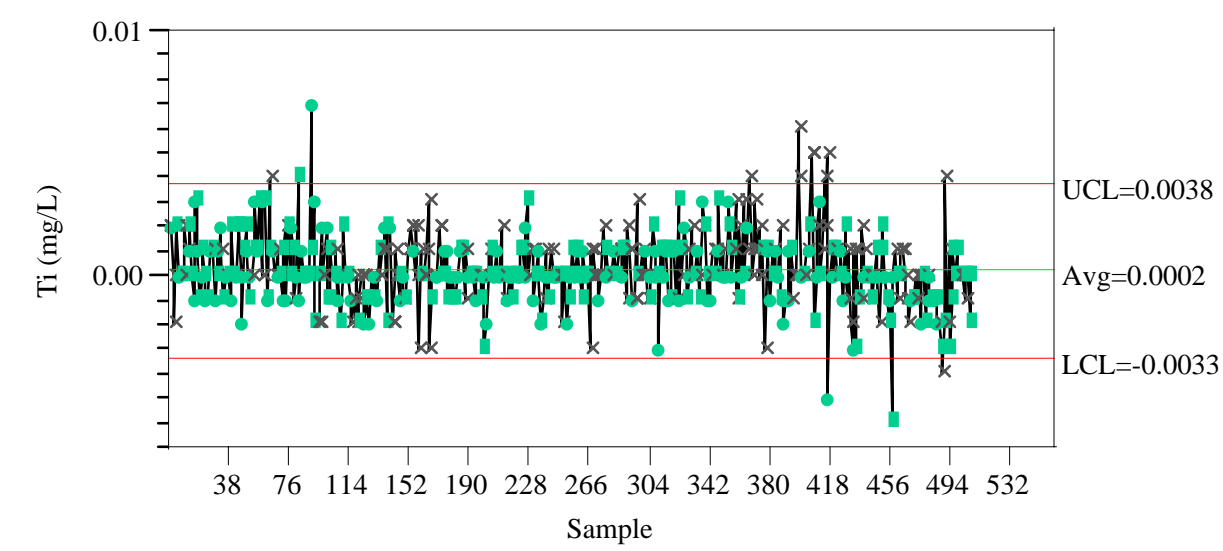


WSRC-TR-2004-00576

\section{Exhibit A11. MA Standards in Analytical Sequence}

Revision 0

Individual Measurement of U (mg/L)

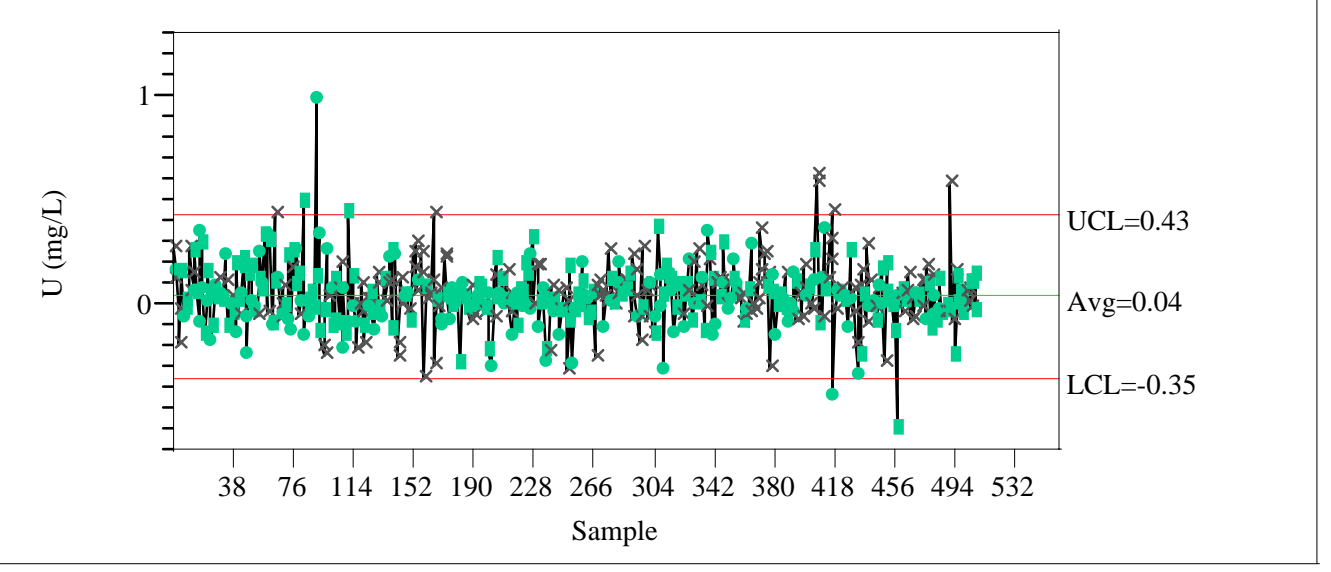

STCd=SM34

Control Chart

Individual Measurement of $\mathrm{Al}$ (mg/L)

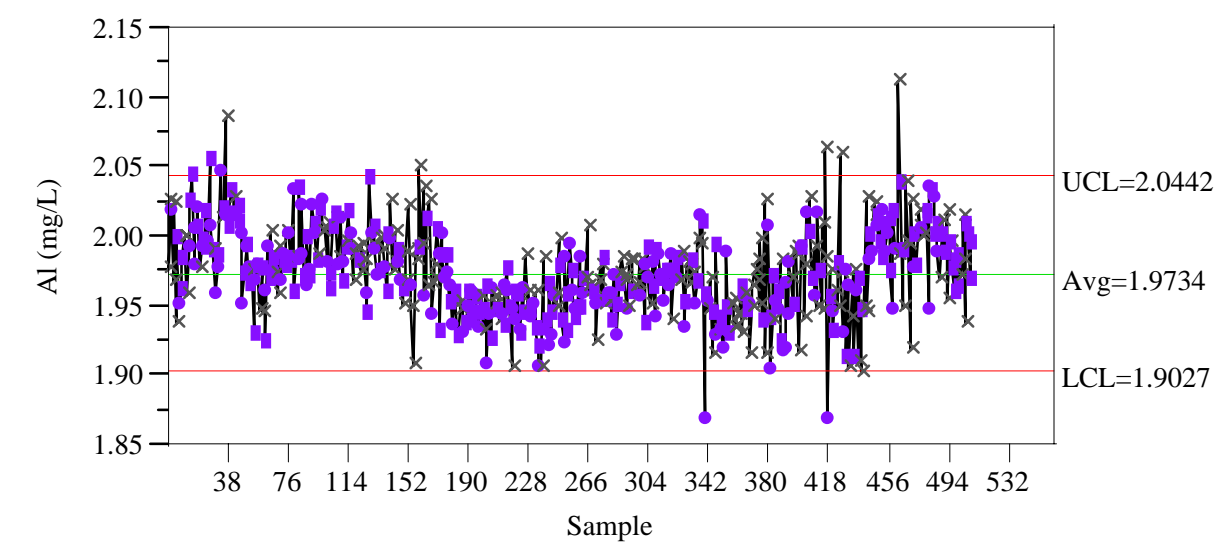

Individual Measurement of $\mathrm{Ca}(\mathrm{mg} / \mathrm{L})$

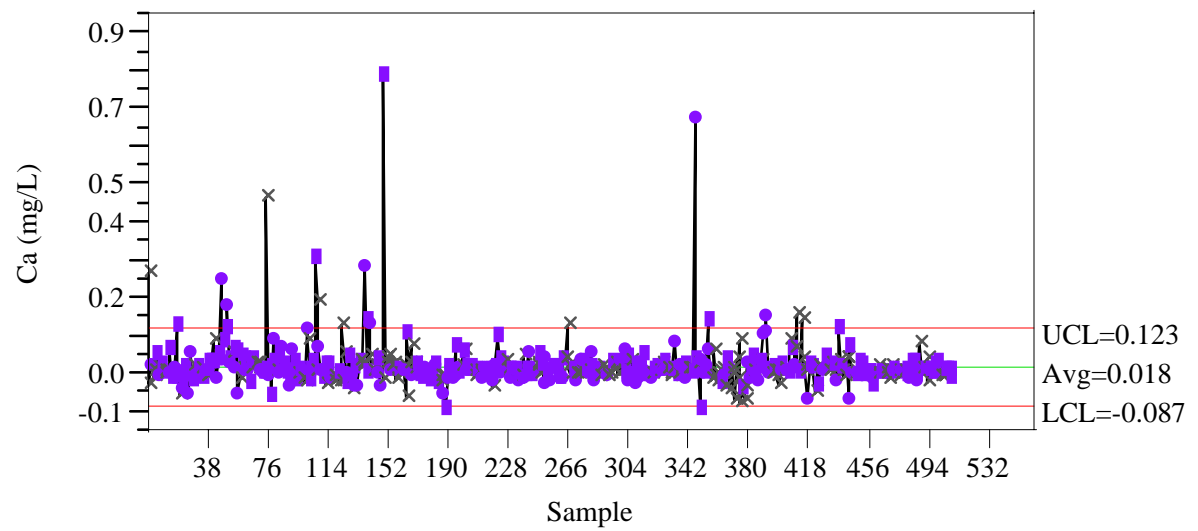


WSRC-TR-2004-00576

\section{Exhibit A11. MA Standards in Analytical Sequence}

Revision 0

Individual Measurement of $\mathrm{Cr}$ (mg/L)

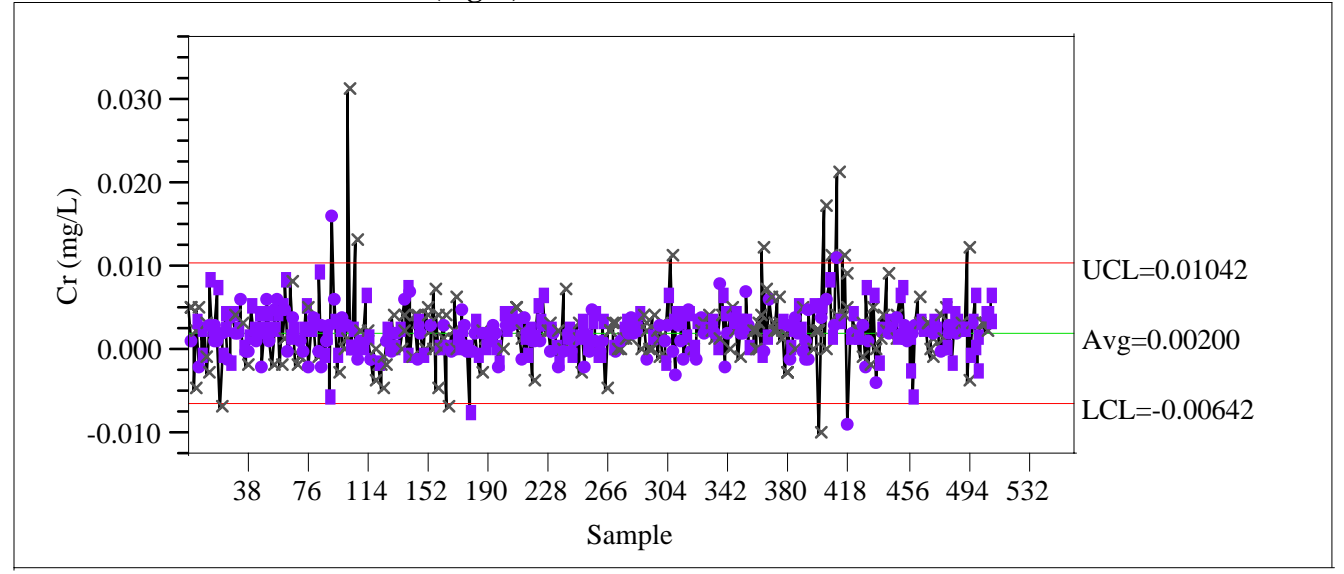

Individual Measurement of $\mathrm{Cu}$ (mg/L)

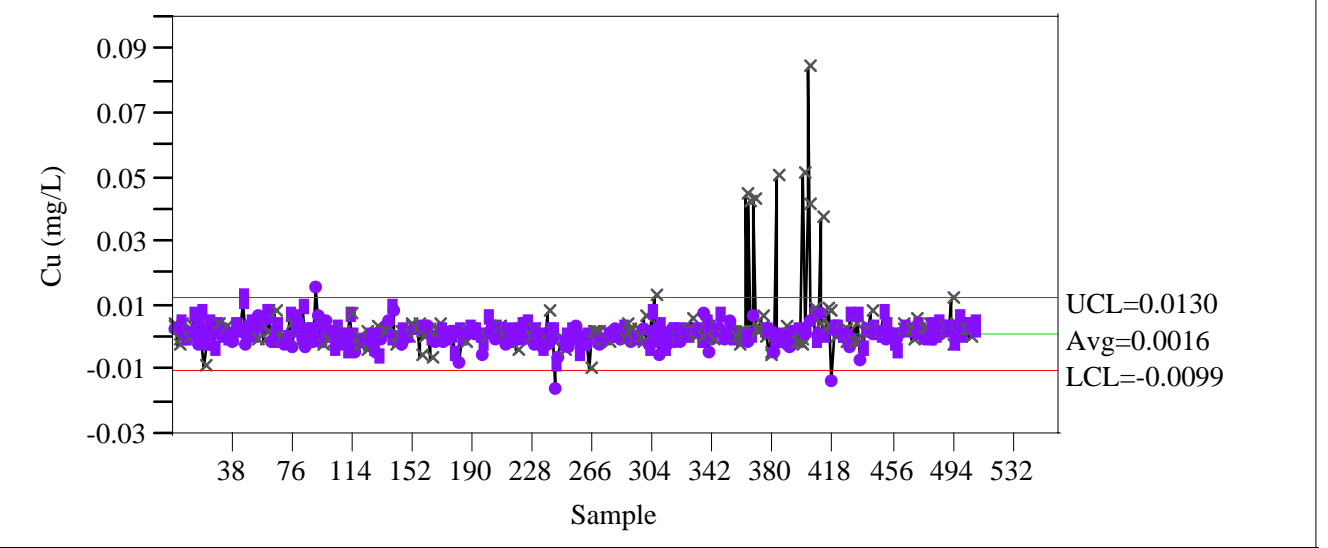

Individual Measurement of Fe (mg/L)

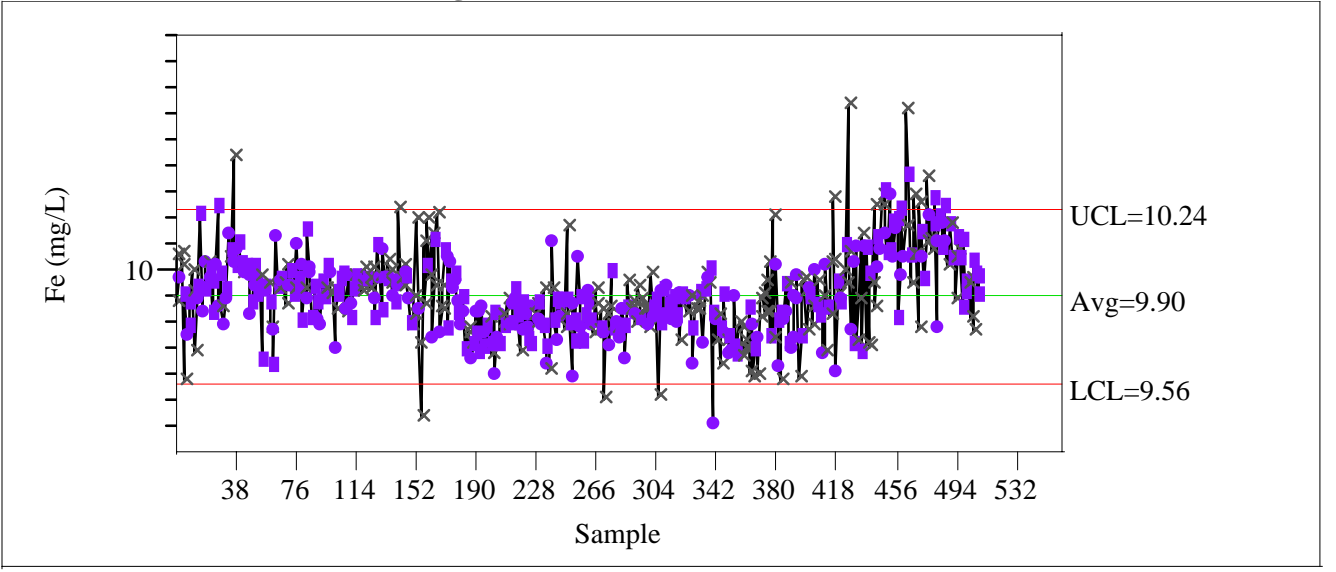


WSRC-TR-2004-00576

\section{Exhibit A11. MA Standards in Analytical Sequence}

\section{Revision 0}

Individual Measurement of K (mg/L)

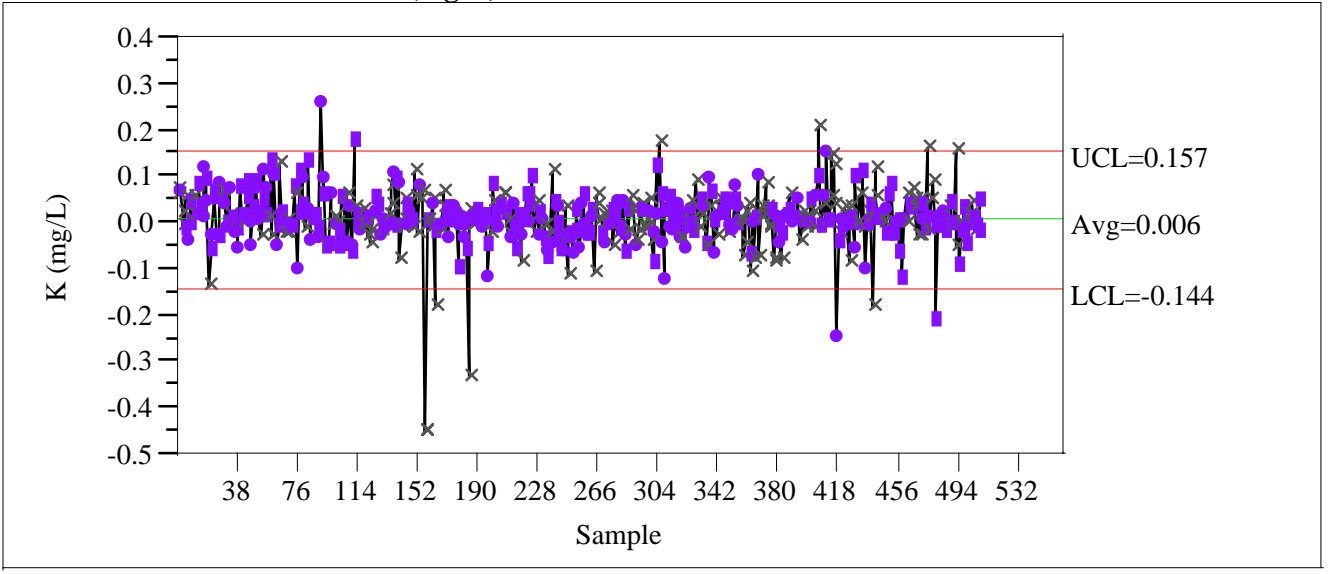

Individual Measurement of $\mathrm{Li}(\mathrm{mg} / \mathrm{L})$

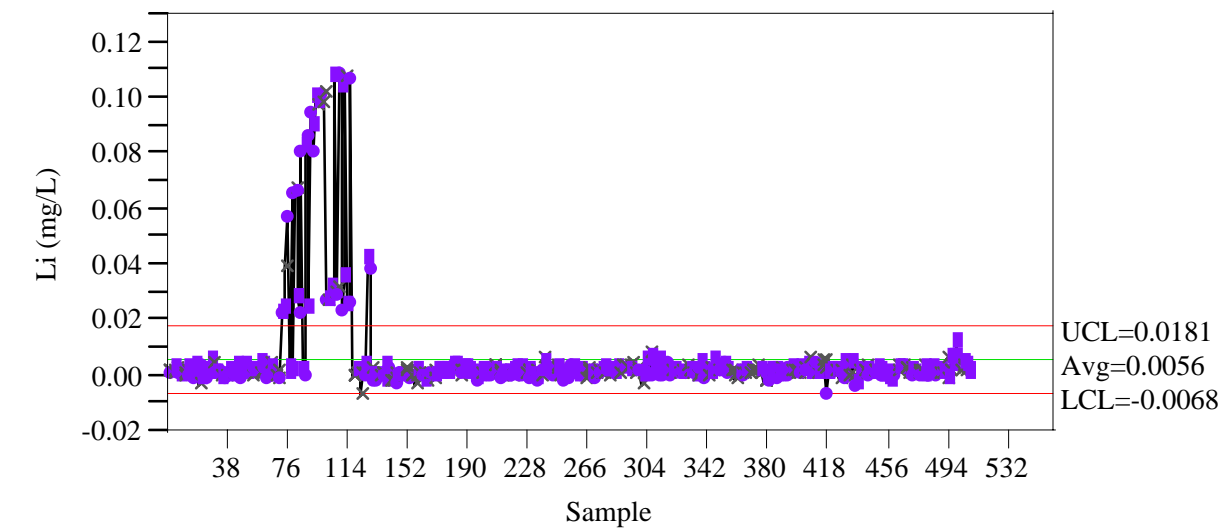

Individual Measurement of $\mathrm{Mg}(\mathrm{mg} / \mathrm{L})$

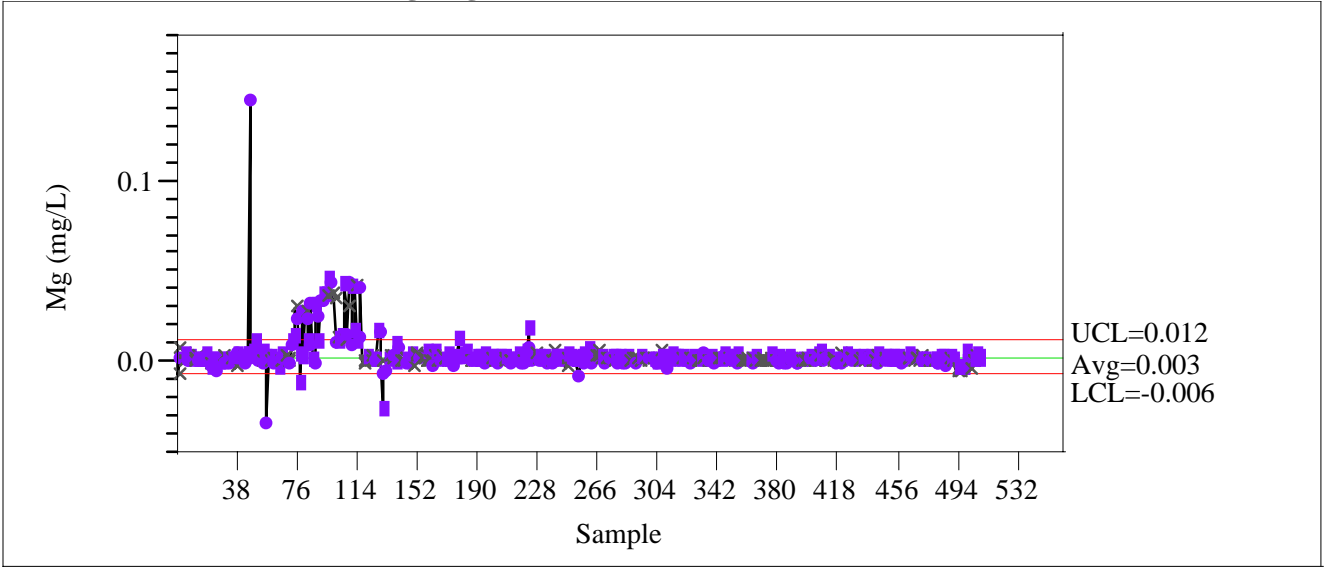


WSRC-TR-2004-00576

\section{Exhibit A11. MA Standards in Analytical Sequence}

Revision 0

Individual Measurement of Mn (mg/L)

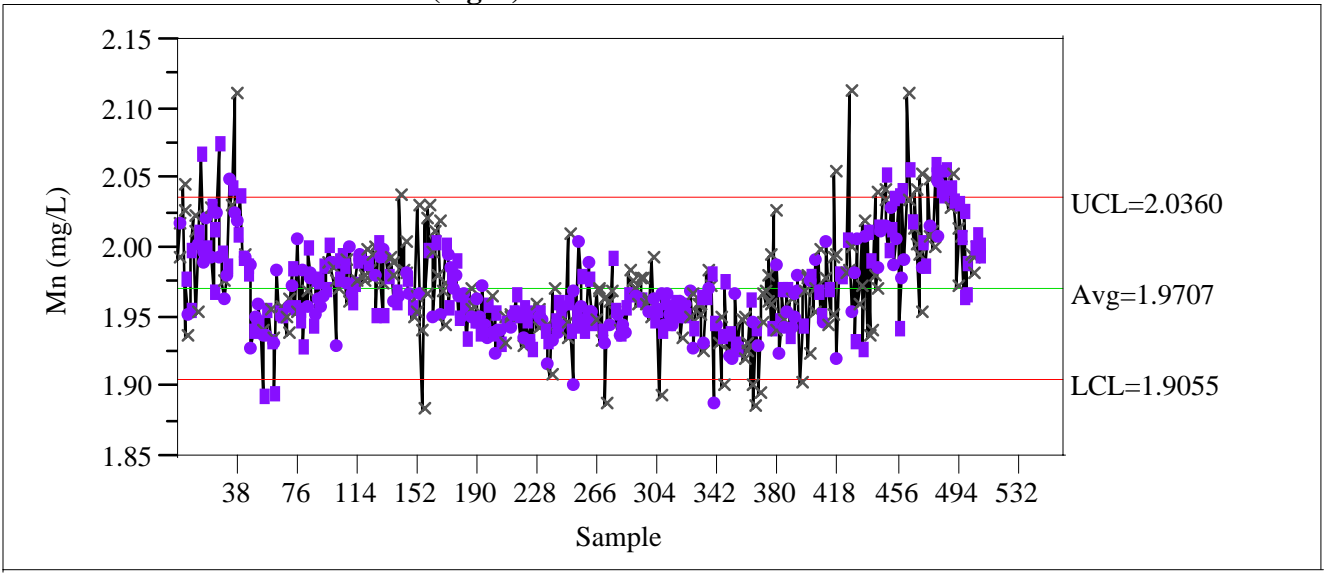

Individual Measurement of $\mathrm{Na}$ (mg/L)

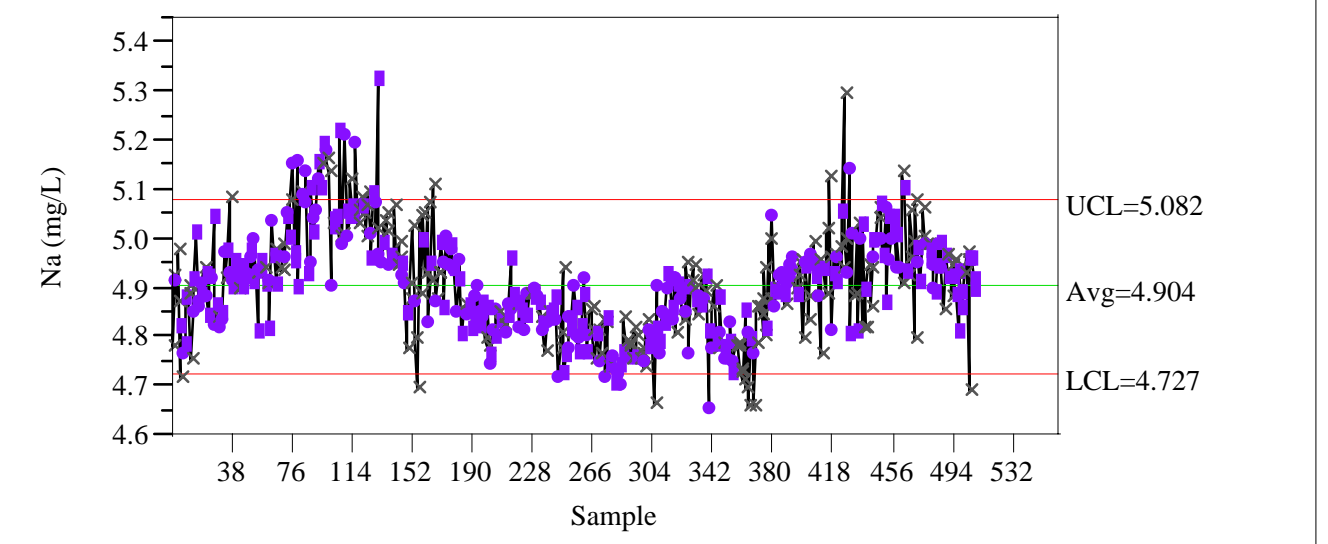

Individual Measurement of $\mathrm{Ni}(\mathrm{mg} / \mathrm{L})$

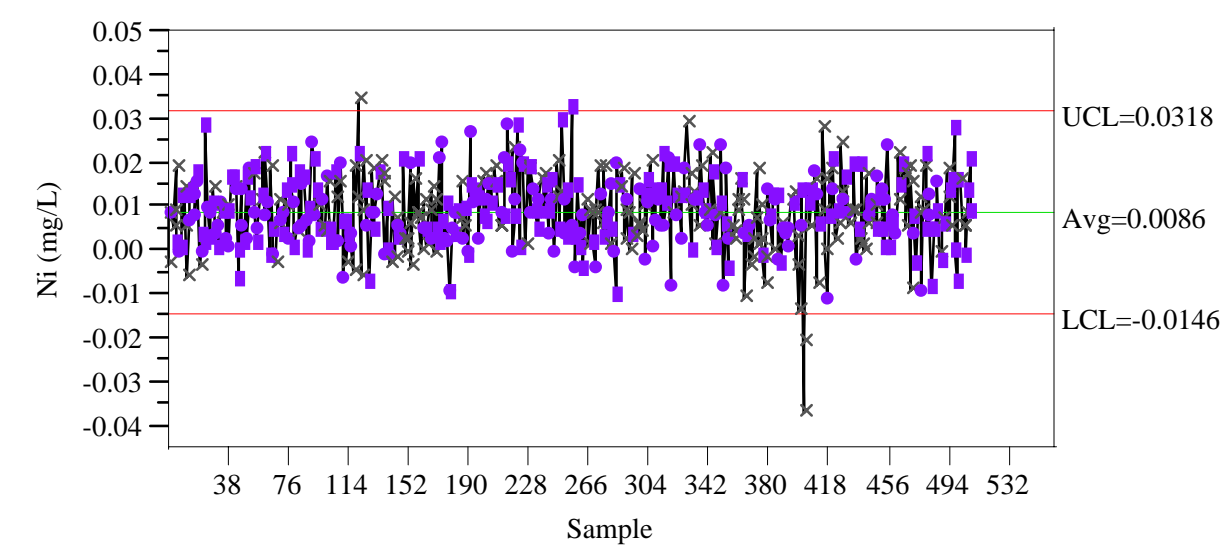




\section{Exhibit A11. MA Standards in Analytical Sequence}

Revision 0

Individual Measurement of Si (mg/L)

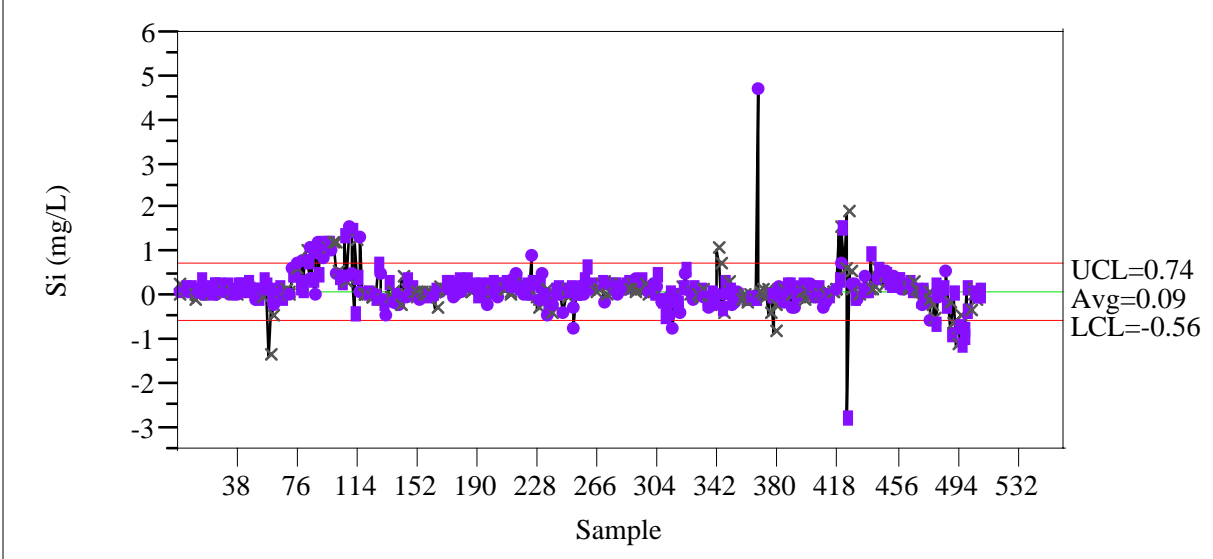

Individual Measurement of Ti (mg/L)

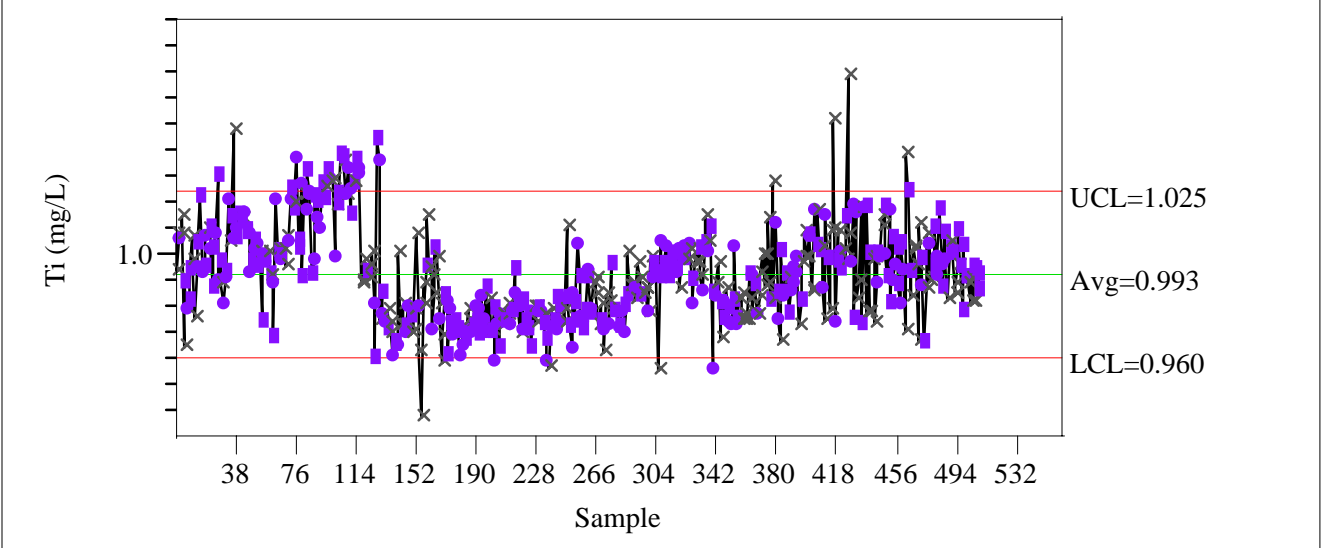

Individual Measurement of U (mg/L)

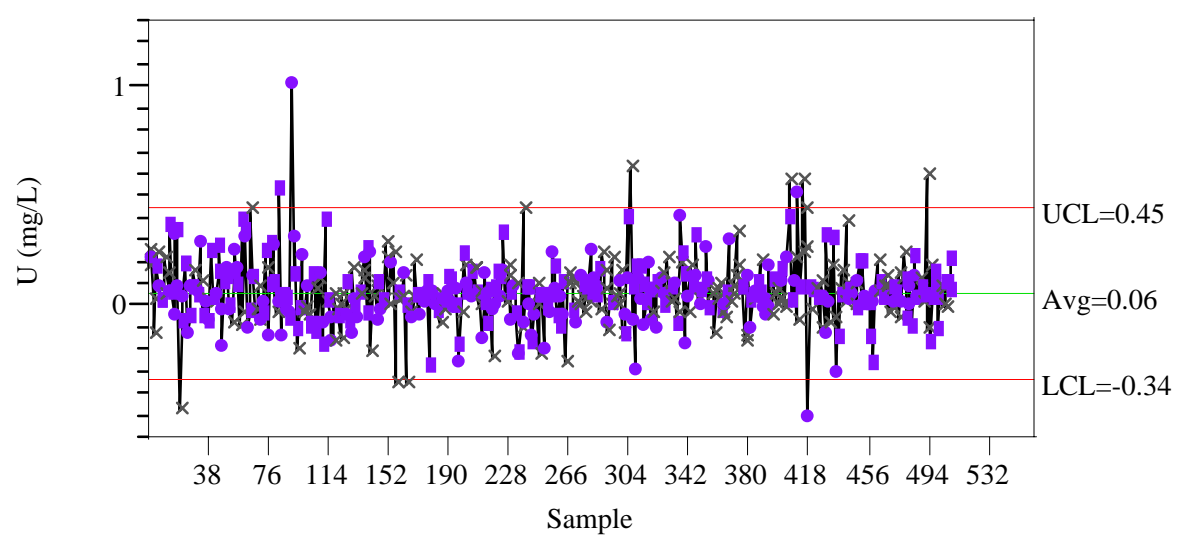


WSRC-TR-2004-00576

Exhibit A11. MA Standards in Analytical Sequence

Revision 0

STCd=SM35

Control Chart

Individual Measurement of $\mathrm{Al}$ (mg/L)

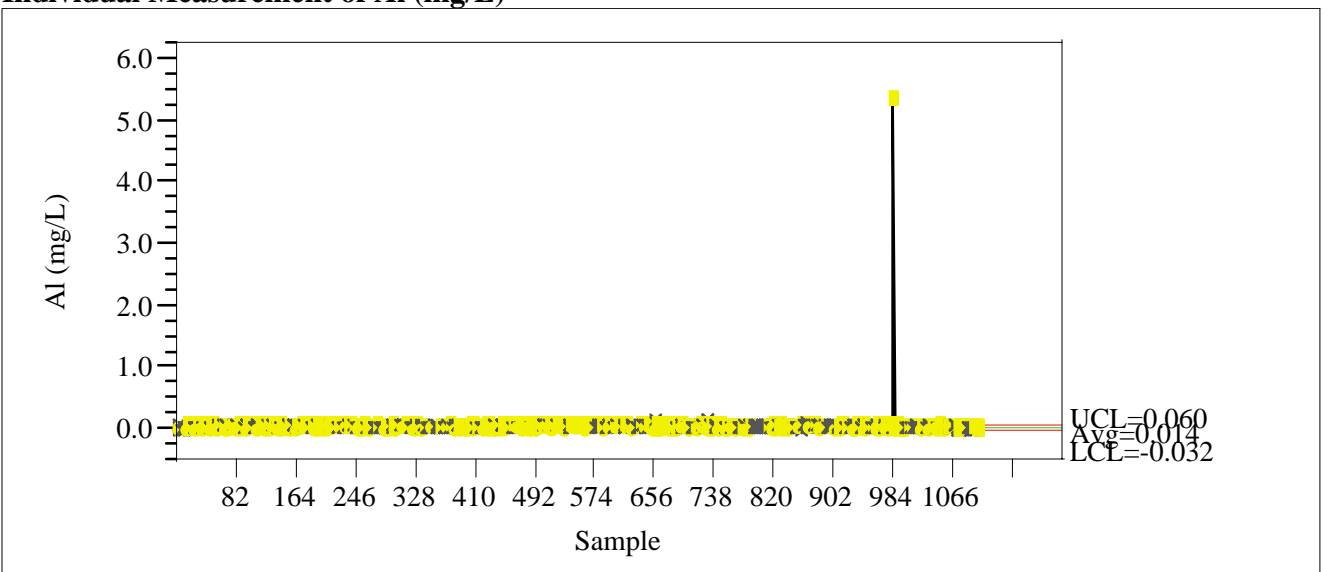

Individual Measurement of Ca (mg/L)

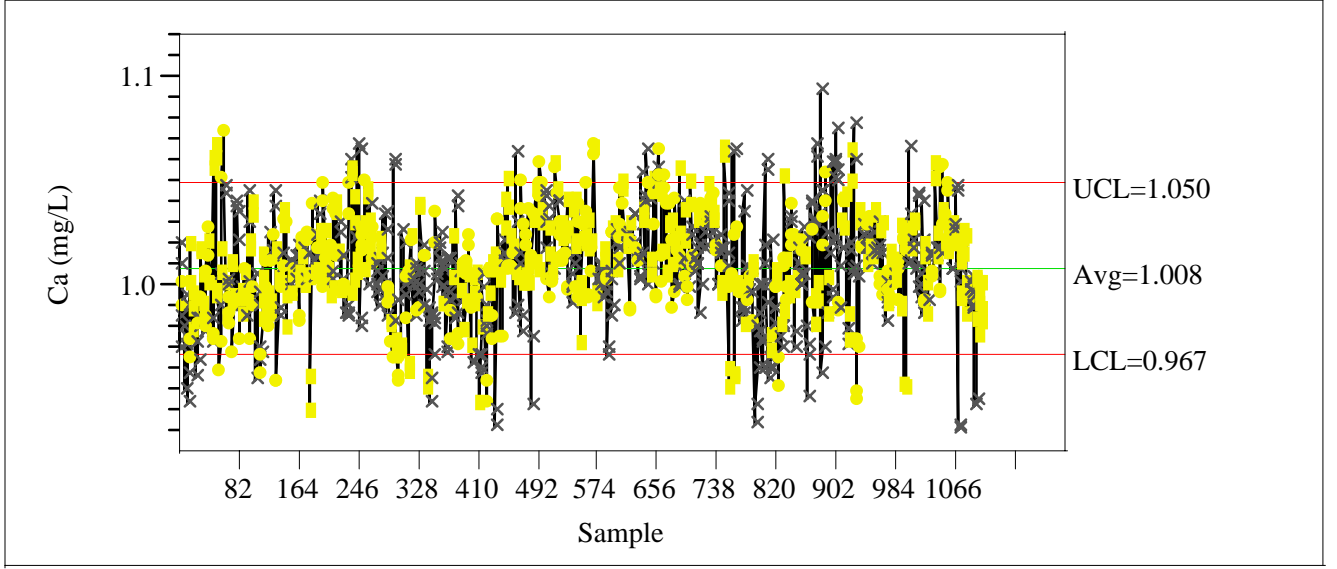

Individual Measurement of $\mathrm{Cr}$ (mg/L)

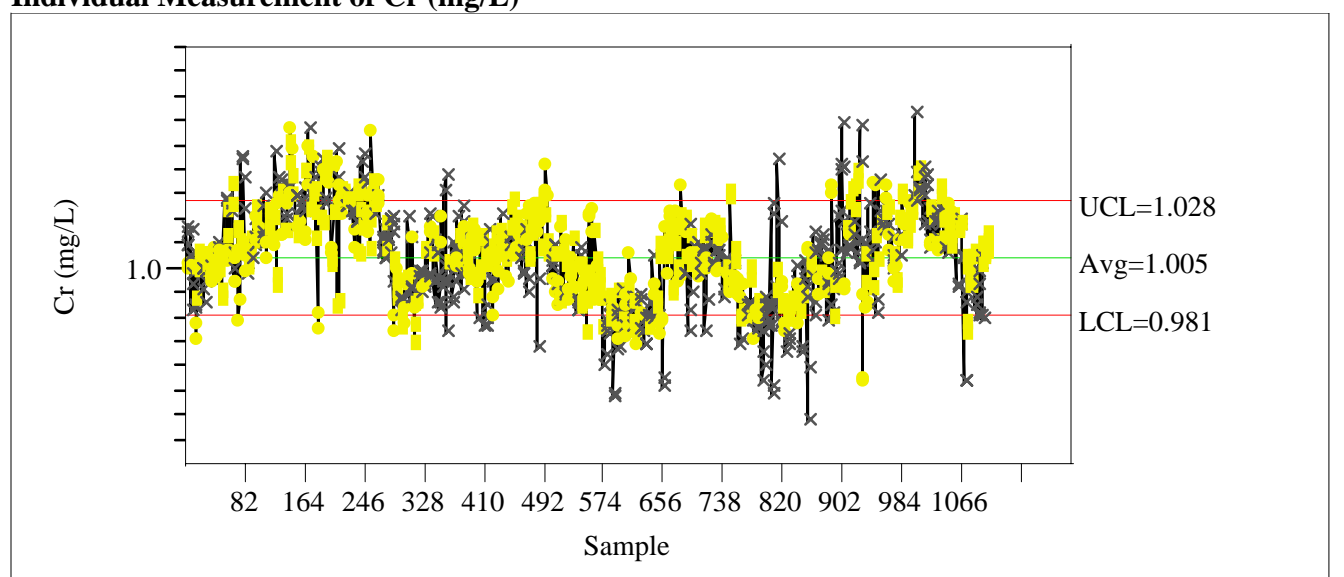


WSRC-TR-2004-00576

\section{Exhibit A11. MA Standards in Analytical Sequence}

Revision 0

Individual Measurement of $\mathrm{Cu}$ (mg/L)

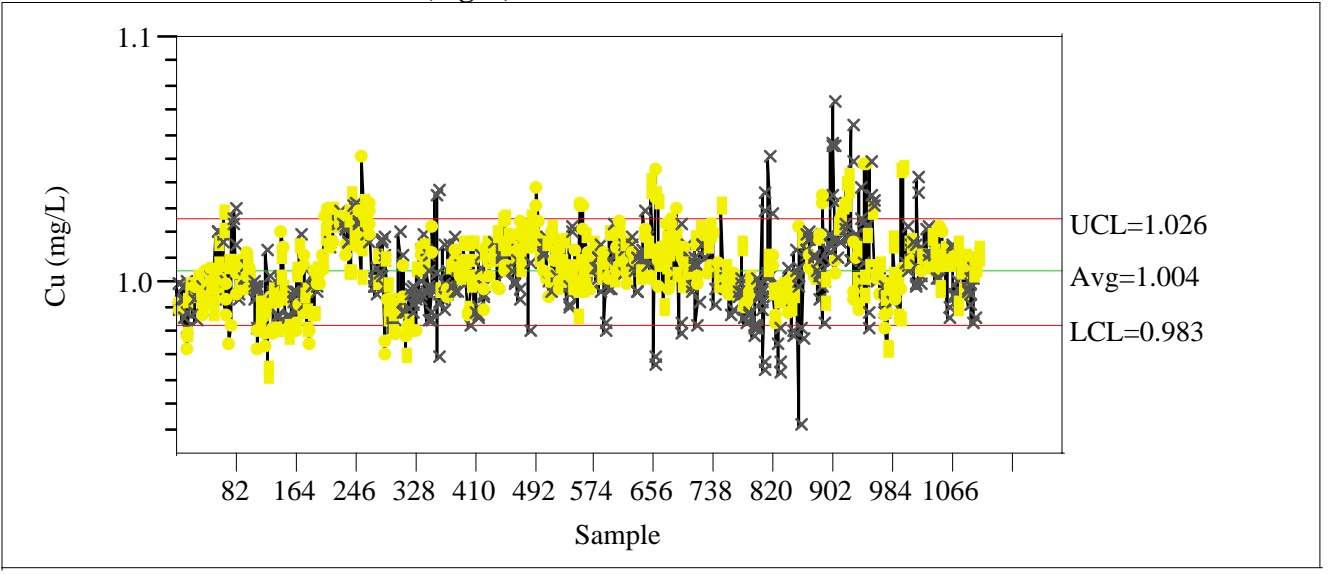

Individual Measurement of Fe (mg/L)

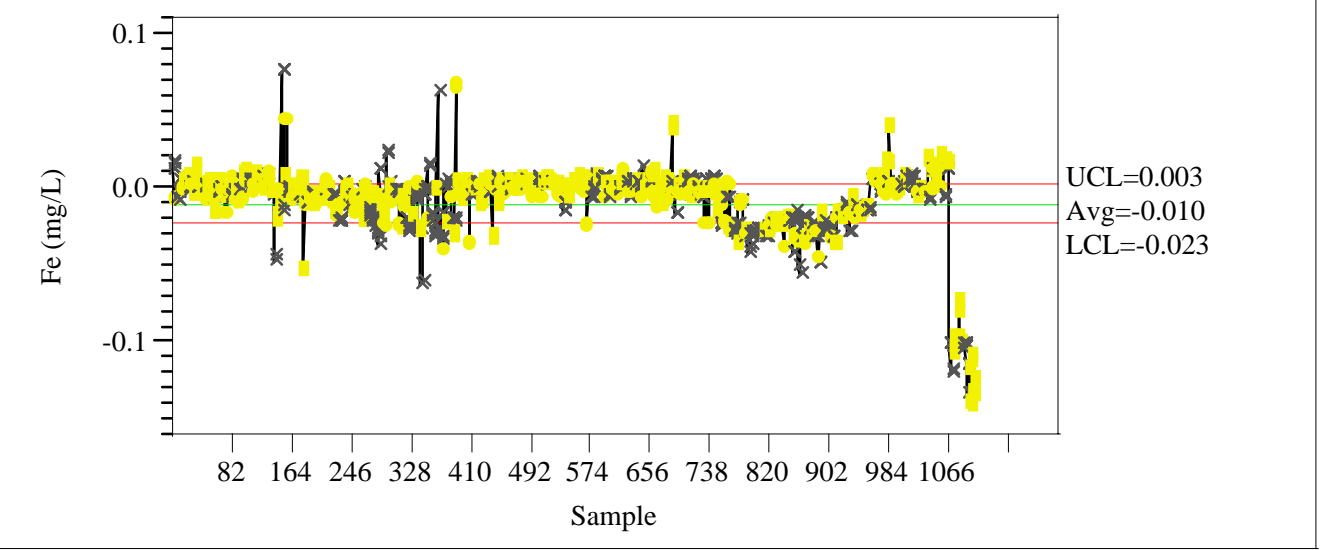

Individual Measurement of K (mg/L)

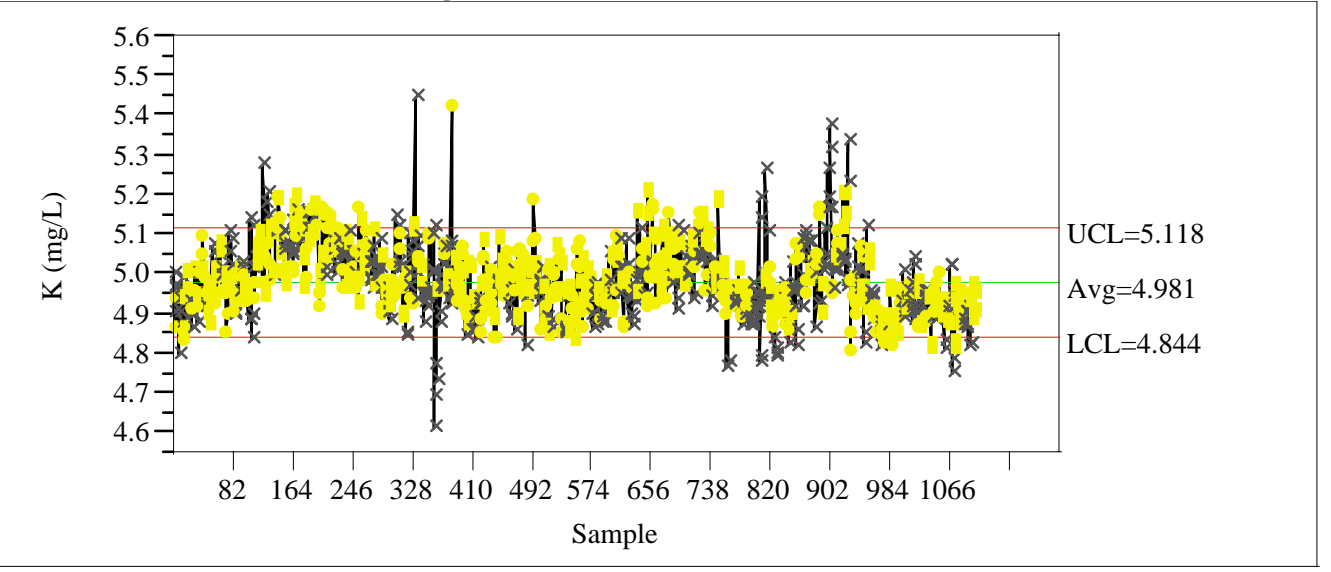


WSRC-TR-2004-00576

\section{Exhibit A11. MA Standards in Analytical Sequence}

Revision 0

Individual Measurement of $\mathrm{Li}(\mathrm{mg} / \mathrm{L})$

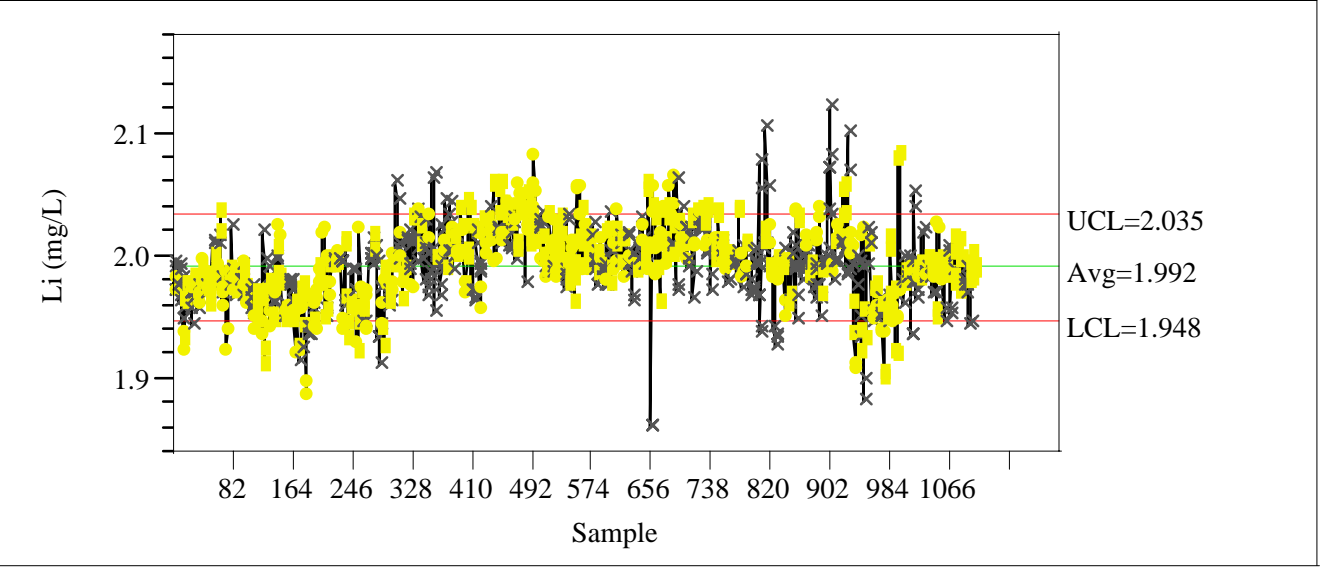

Individual Measurement of Mg (mg/L)

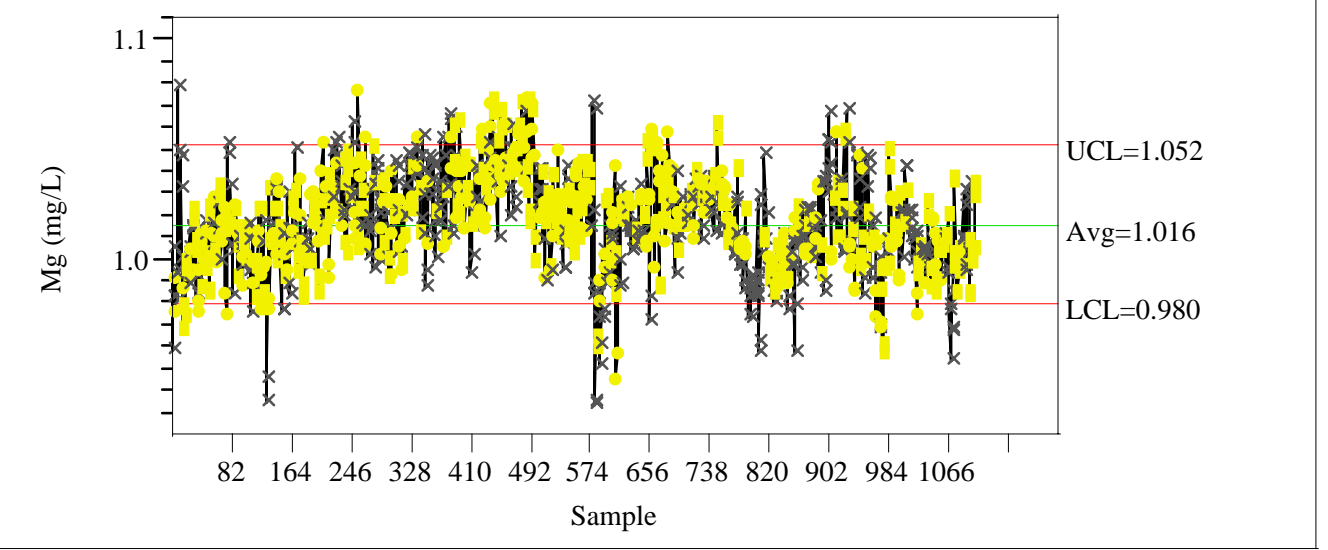

Individual Measurement of Mn (mg/L)

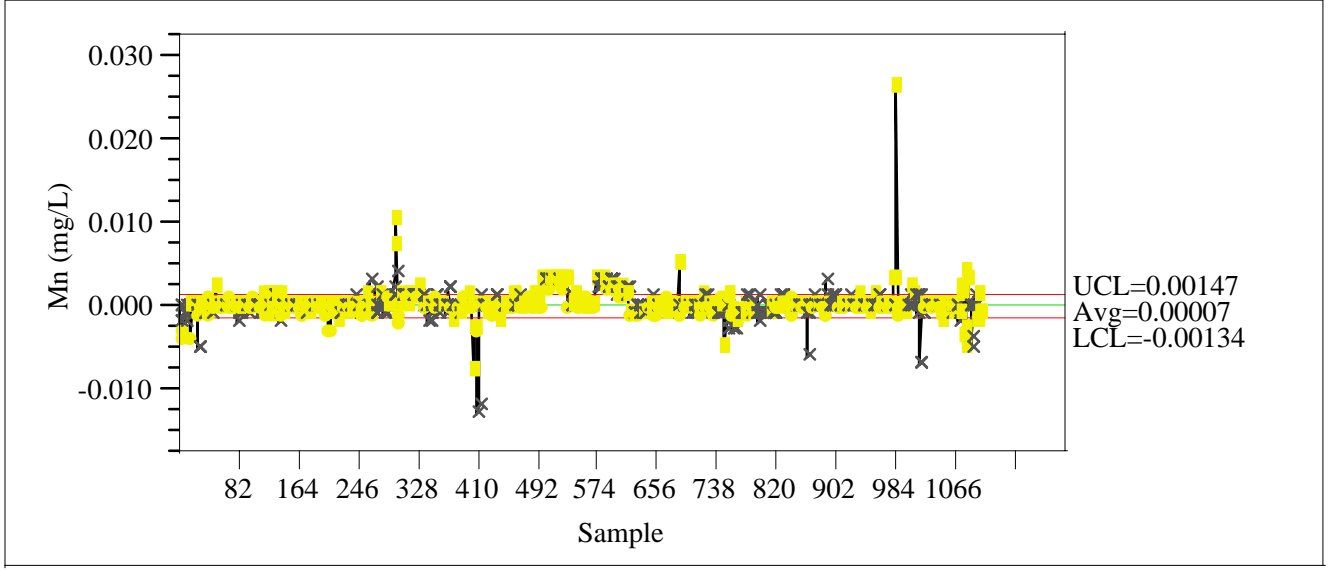


WSRC-TR-2004-00576

\section{Exhibit A11. MA Standards in Analytical Sequence}

Revision 0

Individual Measurement of Na (mg/L)

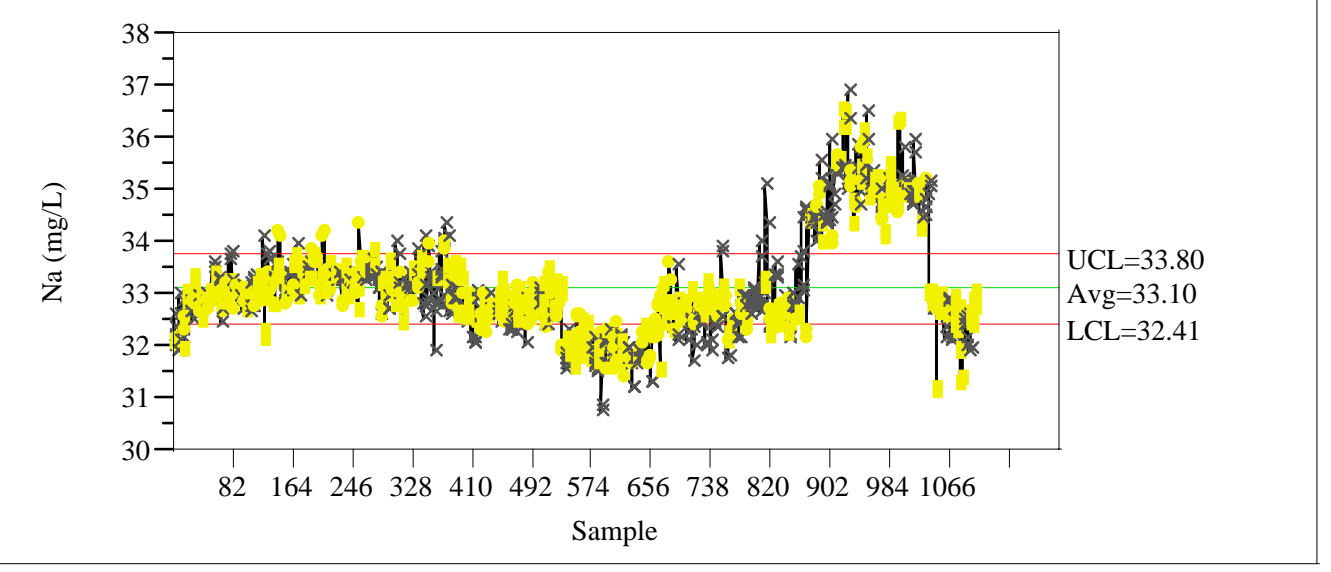

Individual Measurement of Ni (mg/L)

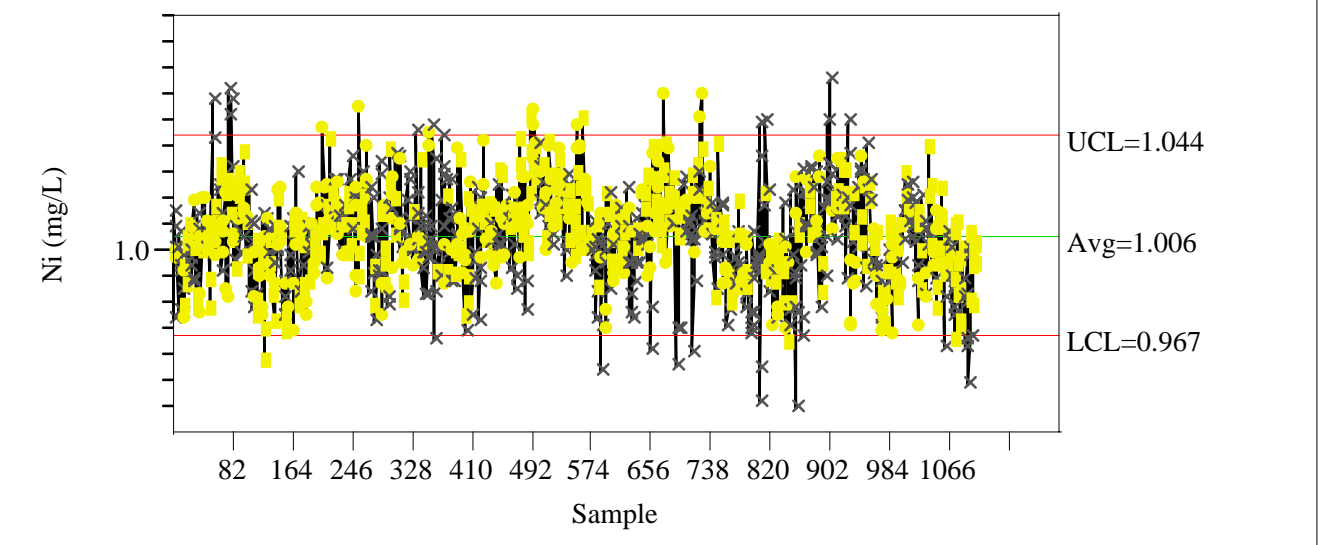

Individual Measurement of Si (mg/L)

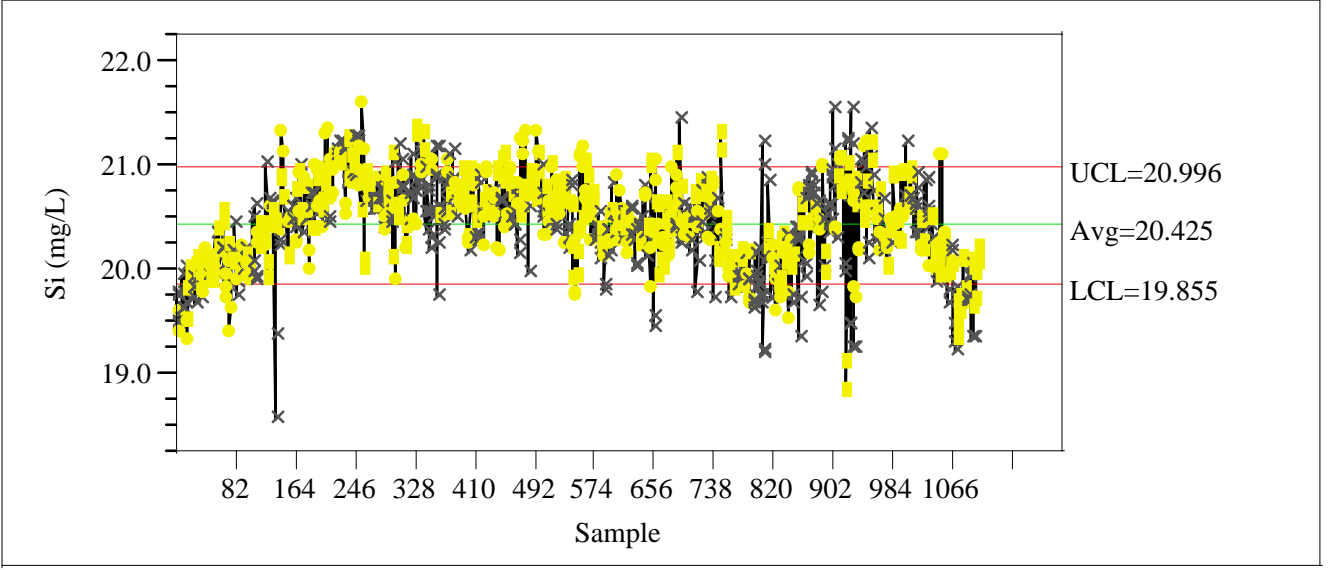


WSRC-TR-2004-00576

\section{Exhibit A11. MA Standards in Analytical Sequence}

\section{Revision 0}

Individual Measurement of Ti (mg/L)

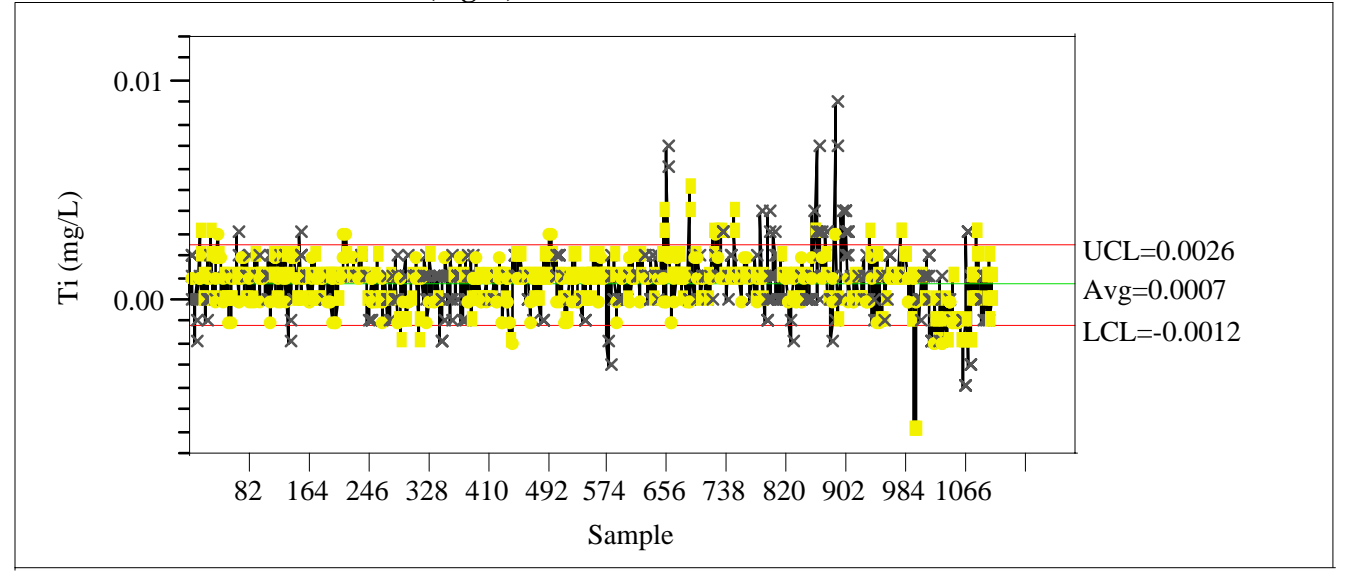

Individual Measurement of U (mg/L)

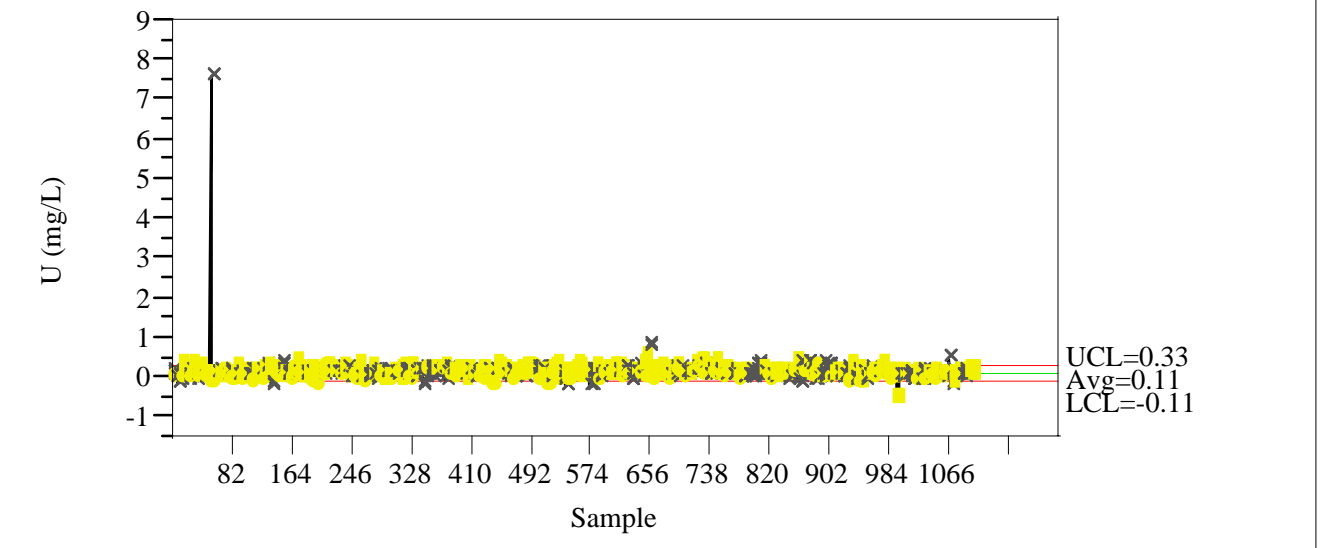

\section{STCd=SM36}

Control Chart

Individual Measurement of $\mathrm{Al}$ (mg/L)

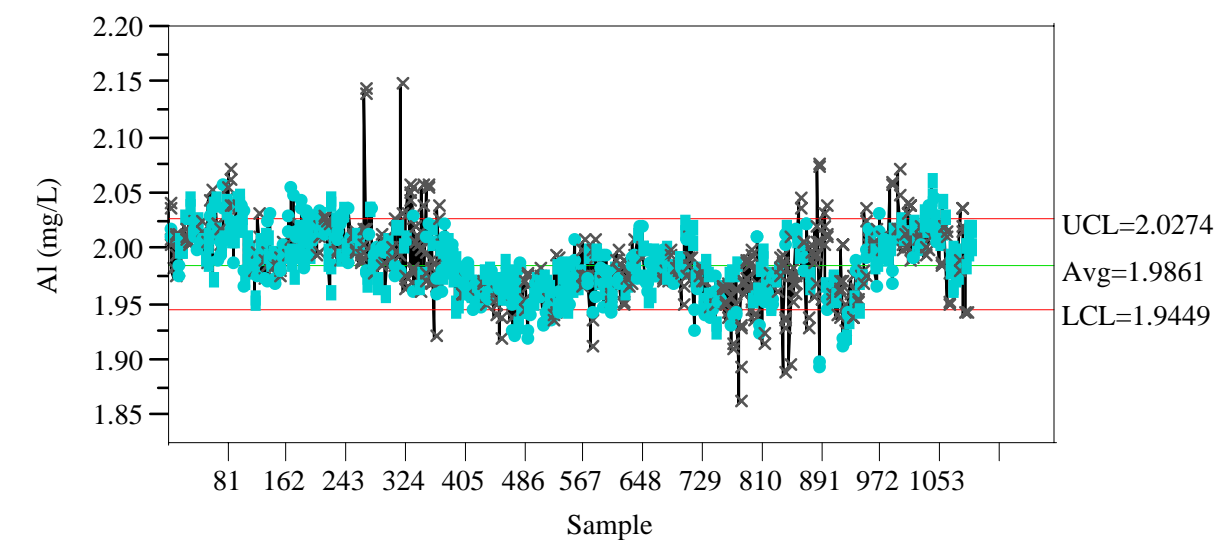


WSRC-TR-2004-00576

Exhibit A11. MA Standards in Analytical Sequence

Revision 0

Individual Measurement of Ca (mg/L)

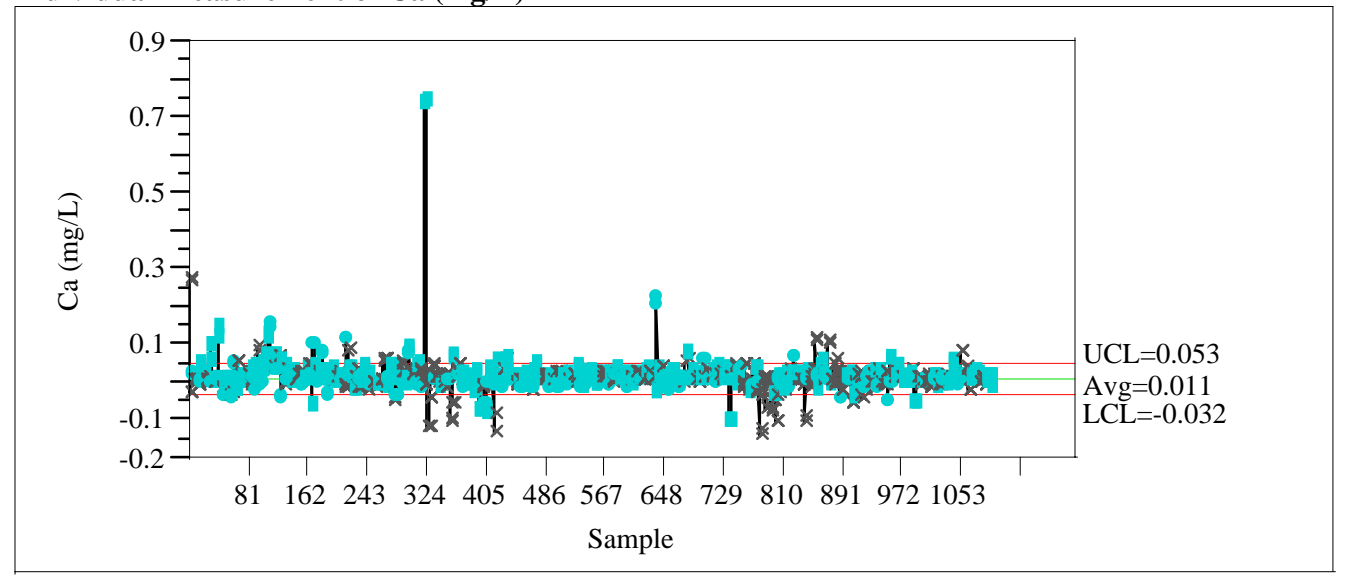

Individual Measurement of $\mathrm{Cr}(\mathrm{mg} / \mathrm{L})$

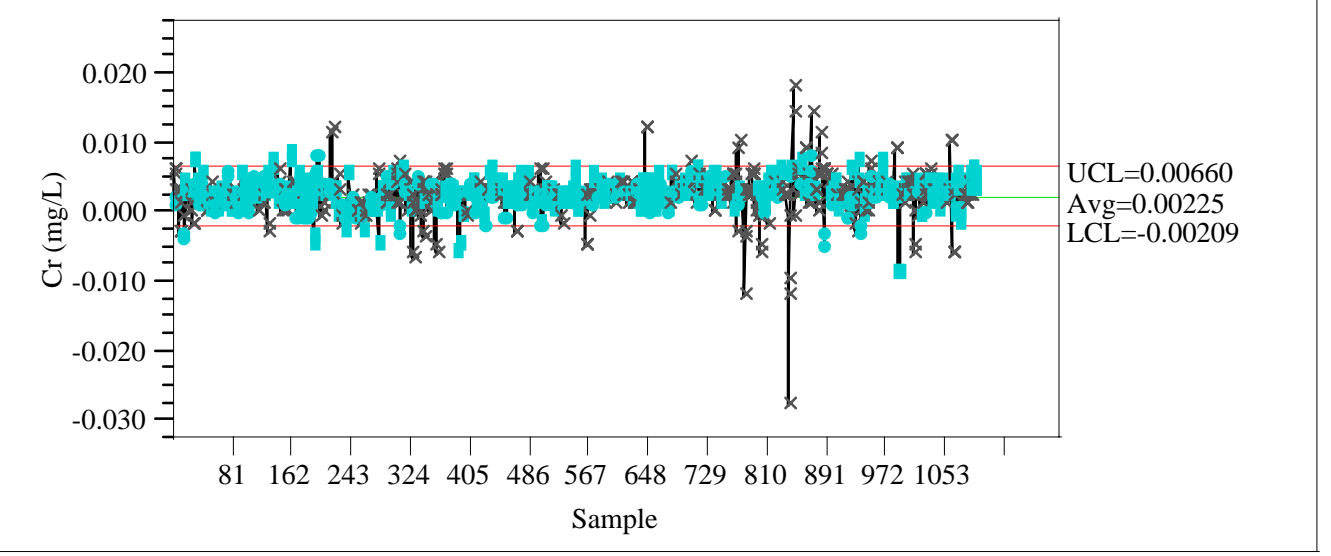

Individual Measurement of $\mathrm{Cu}(\mathrm{mg} / \mathrm{L})$

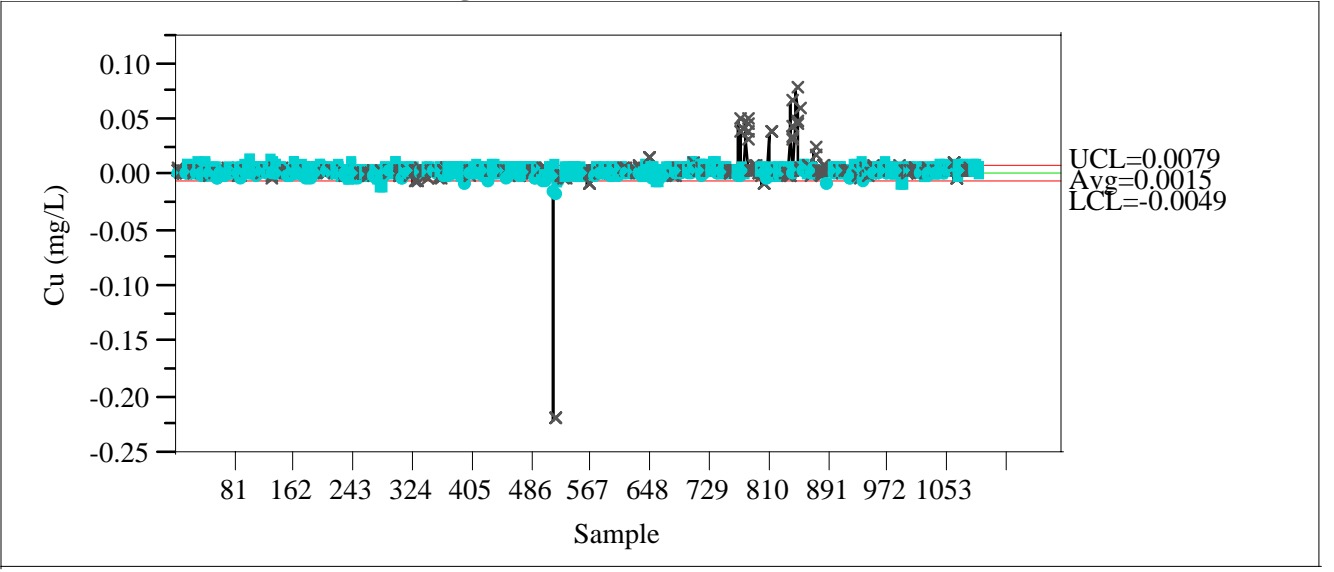


WSRC-TR-2004-00576

\section{Exhibit A11. MA Standards in Analytical Sequence}

\section{Revision 0}

Individual Measurement of Fe (mg/L)

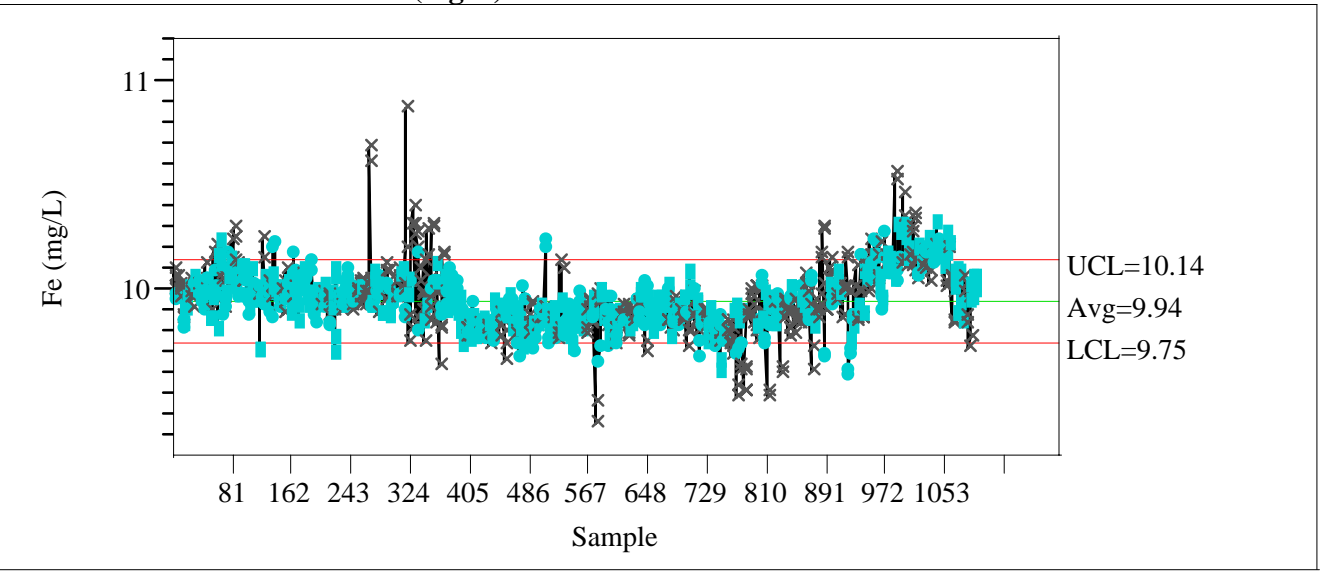

Individual Measurement of K (mg/L)

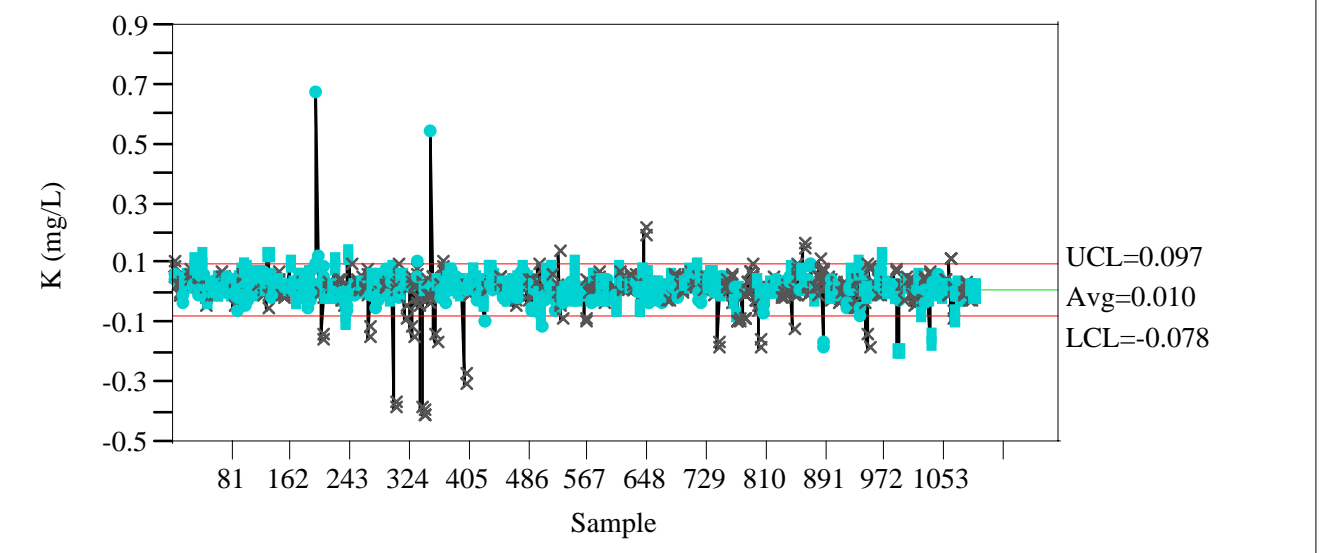

Individual Measurement of $\mathrm{Li}(\mathrm{mg} / \mathrm{L})$

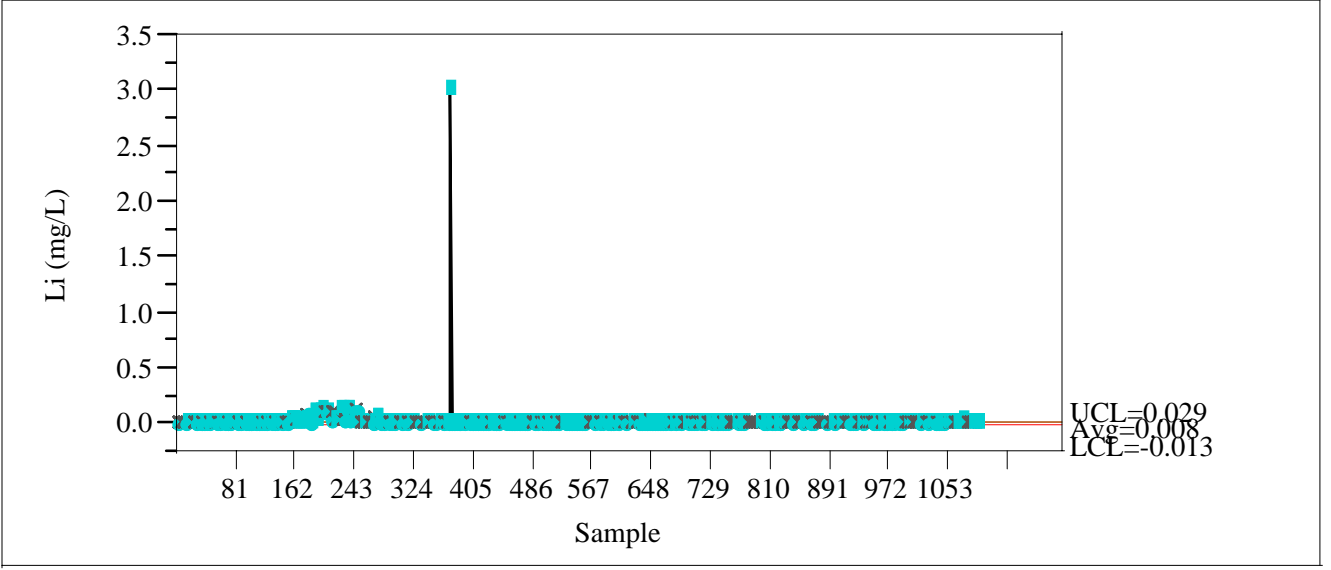


WSRC-TR-2004-00576

\section{Exhibit A11. MA Standards in Analytical Sequence}

\section{Revision 0}

Individual Measurement of $\mathrm{Mg}(\mathrm{mg} / \mathrm{L})$

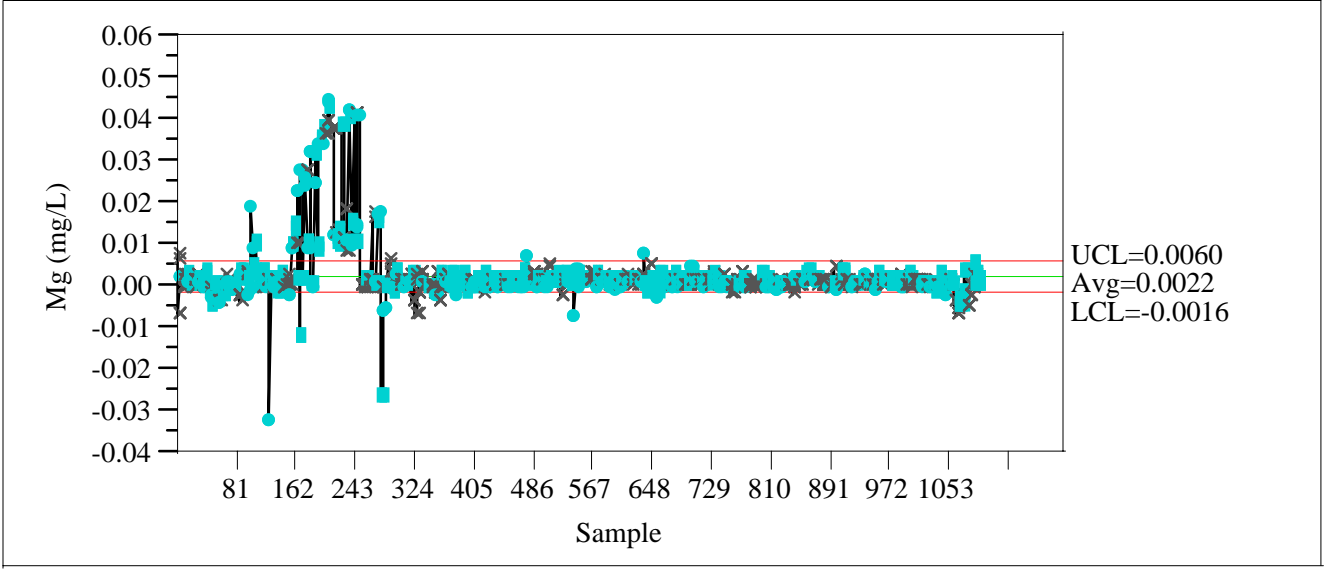

Individual Measurement of Mn (mg/L)

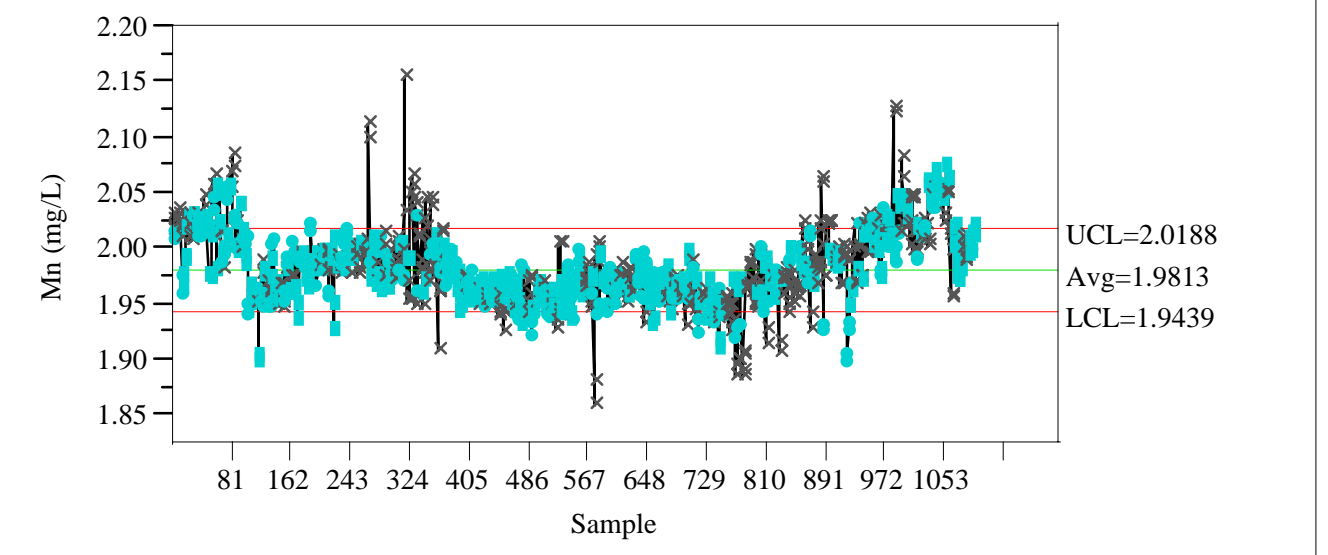

Individual Measurement of $\mathrm{Na}(\mathrm{mg} / \mathrm{L})$

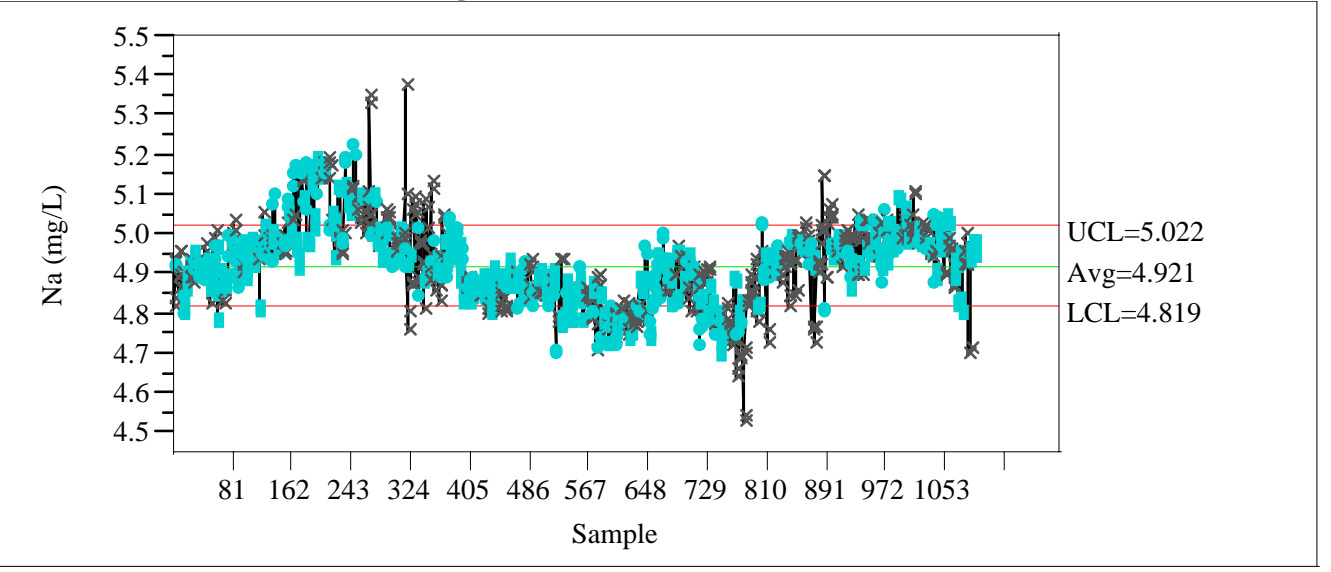


WSRC-TR-2004-00576

\section{Exhibit A11. MA Standards in Analytical Sequence}

\section{Revision 0}

Individual Measurement of Ni (mg/L)

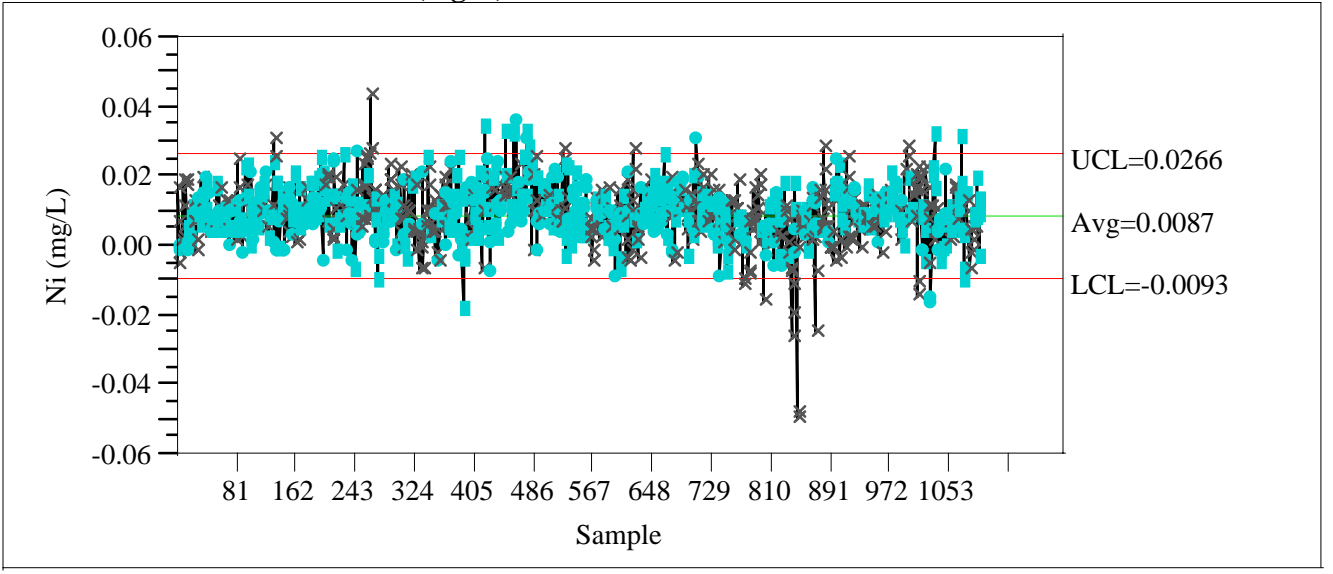

Individual Measurement of Si (mg/L)

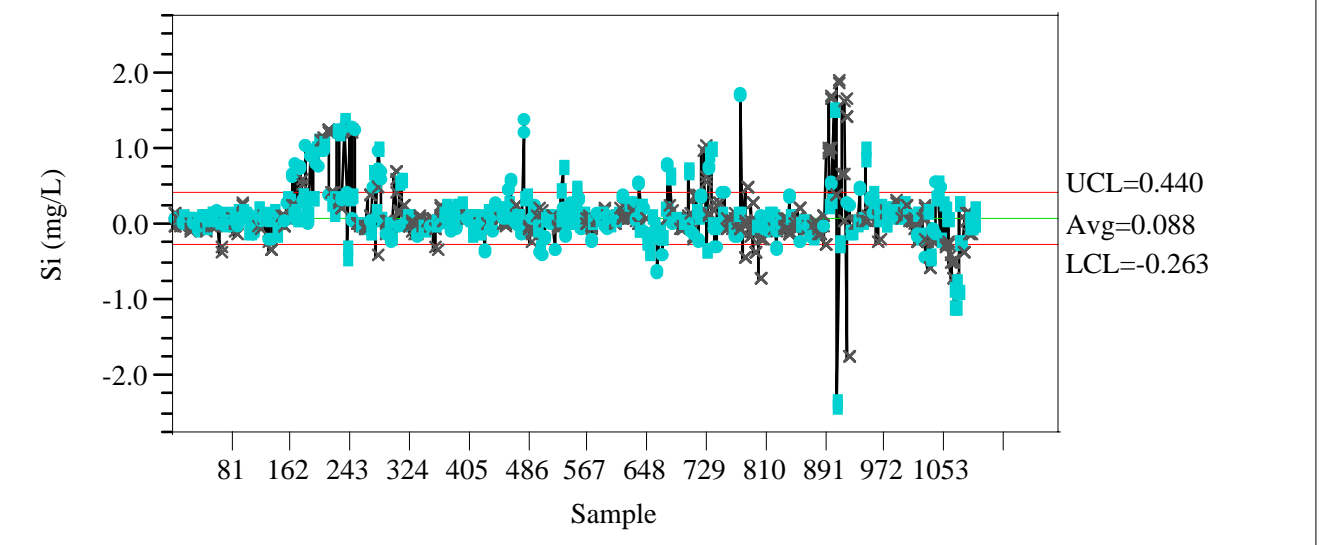

Individual Measurement of $\mathrm{Ti}(\mathrm{mg} / \mathrm{L})$

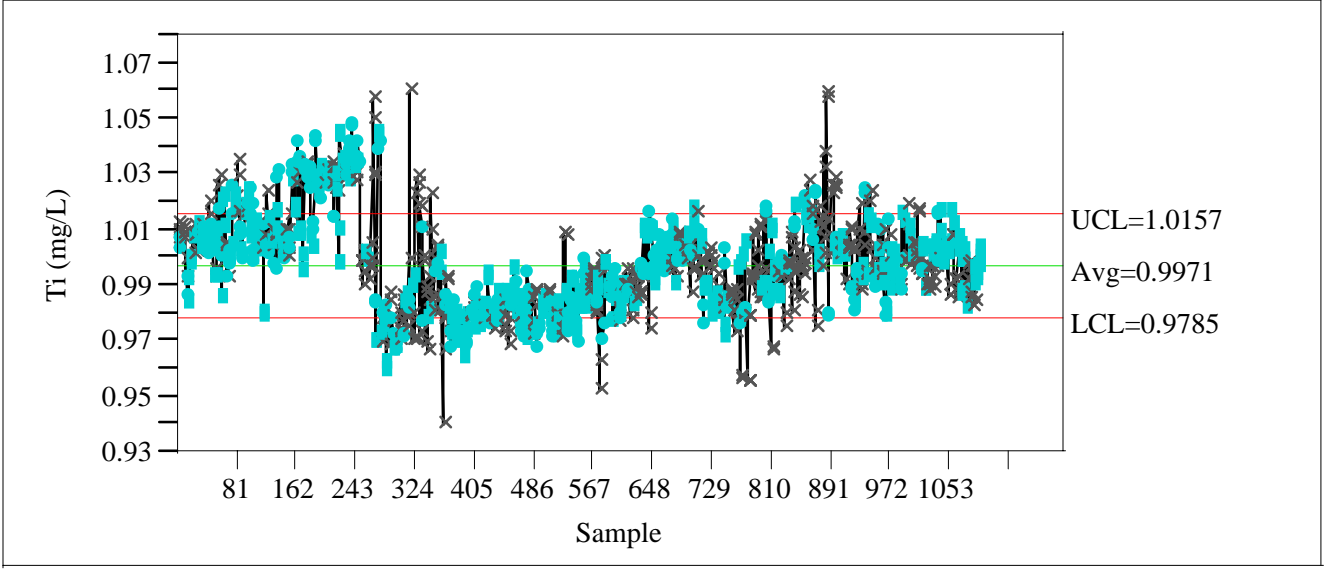


WSRC-TR-2004-00576

Exhibit A11. MA Standards in Analytical Sequence

Revision 0

Individual Measurement of U (mg/L)

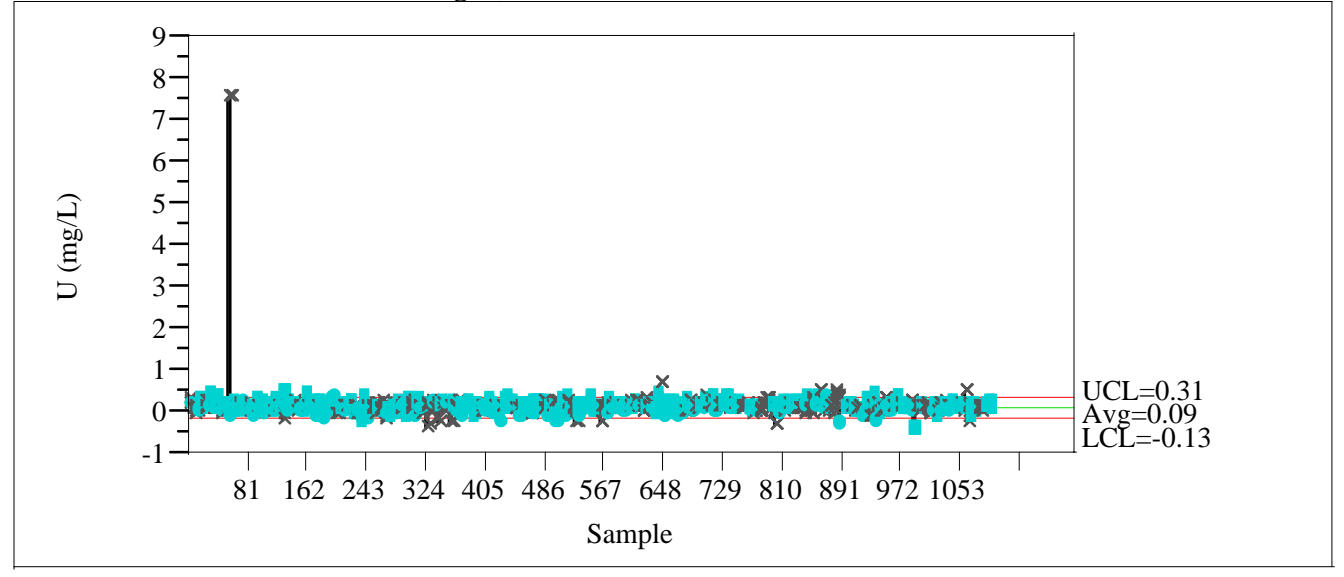

STCd=SM37

Control Chart

Individual Measurement of $\mathrm{Al}$ (mg/L)

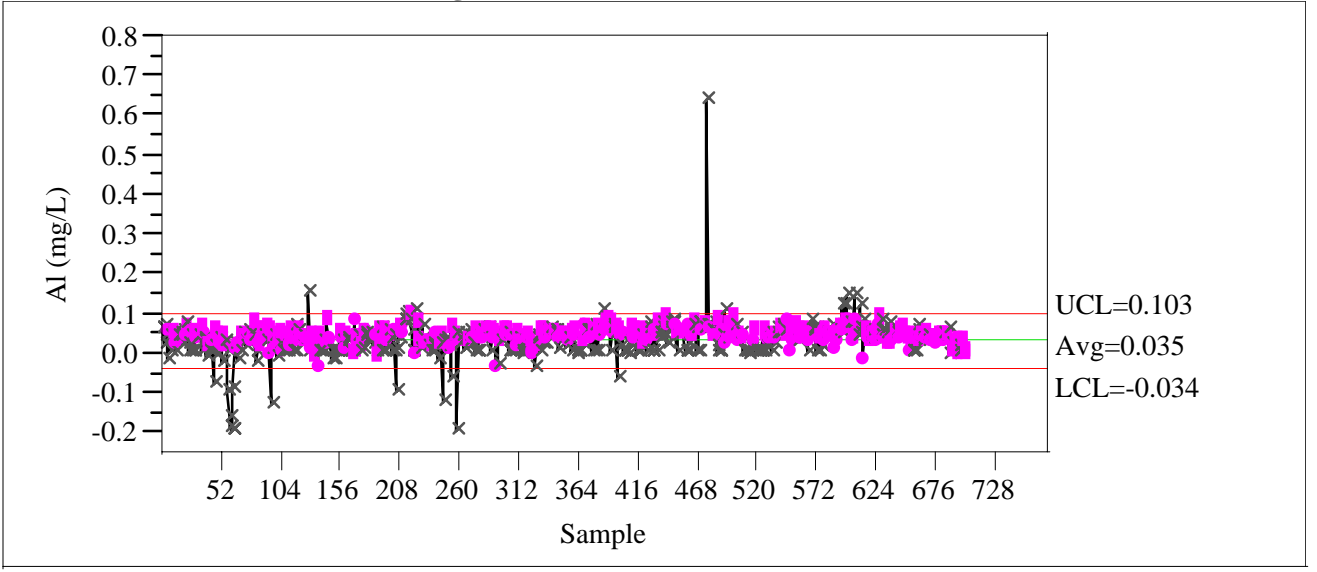

Individual Measurement of Ca (mg/L)

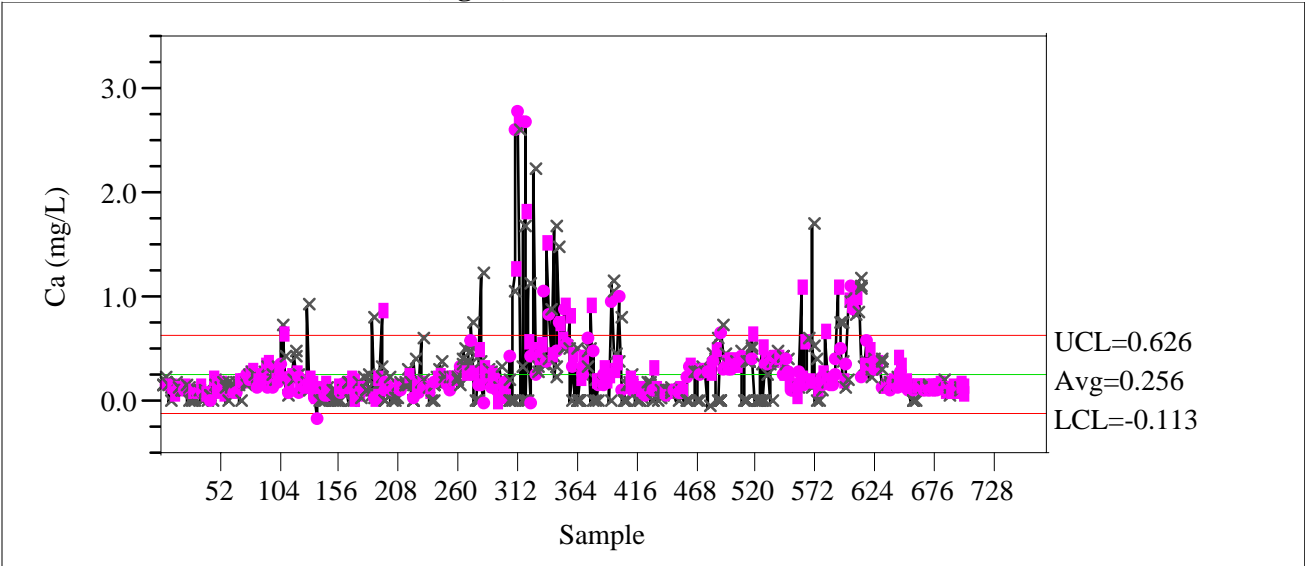


WSRC-TR-2004-00576

Exhibit A11. MA Standards in Analytical Sequence

Revision 0

Individual Measurement of $\mathrm{Cr}$ (mg/L)

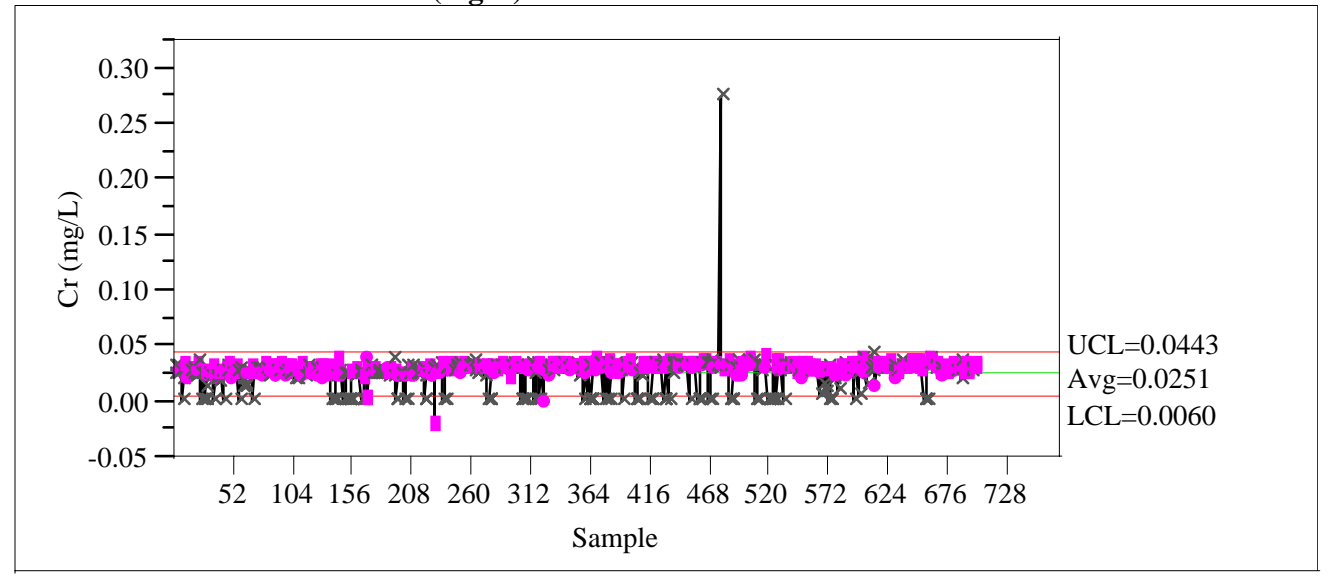

Individual Measurement of $\mathrm{Cu}(\mathrm{mg} / \mathrm{L})$

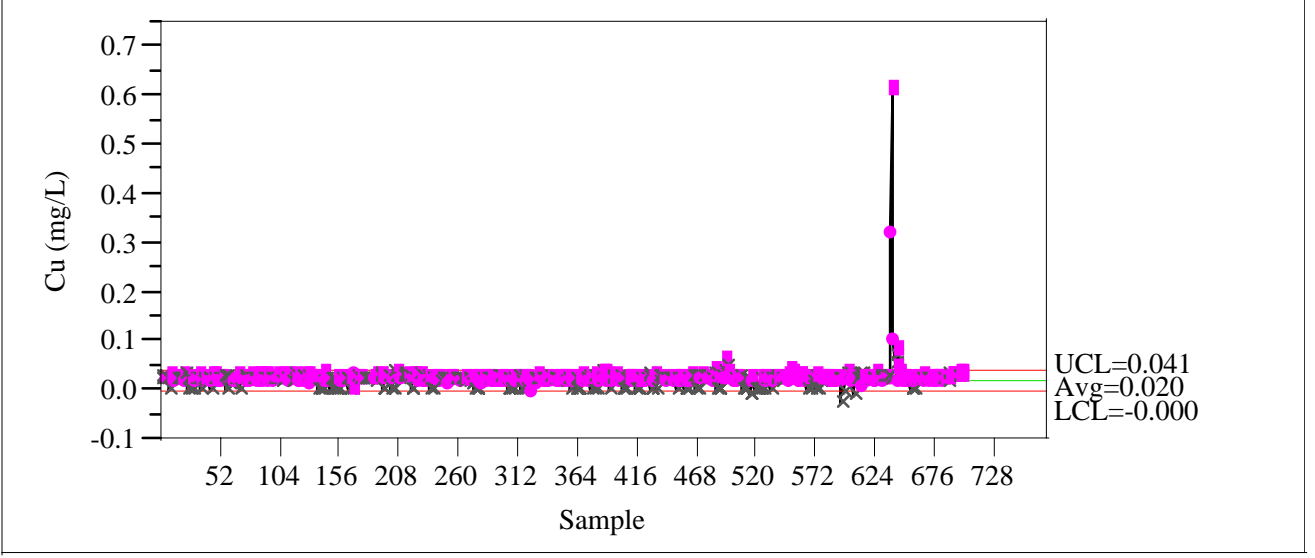

Individual Measurement of Fe (mg/L)

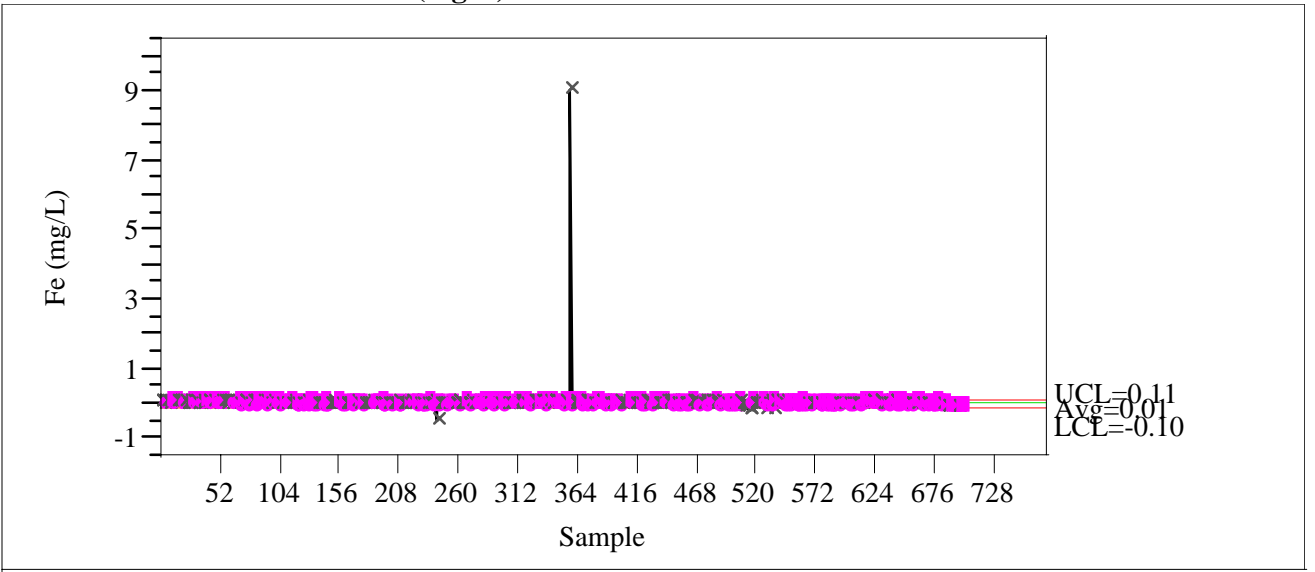




\section{Exhibit A11. MA Standards in Analytical Sequence}

\section{Revision 0}

Individual Measurement of K (mg/L)

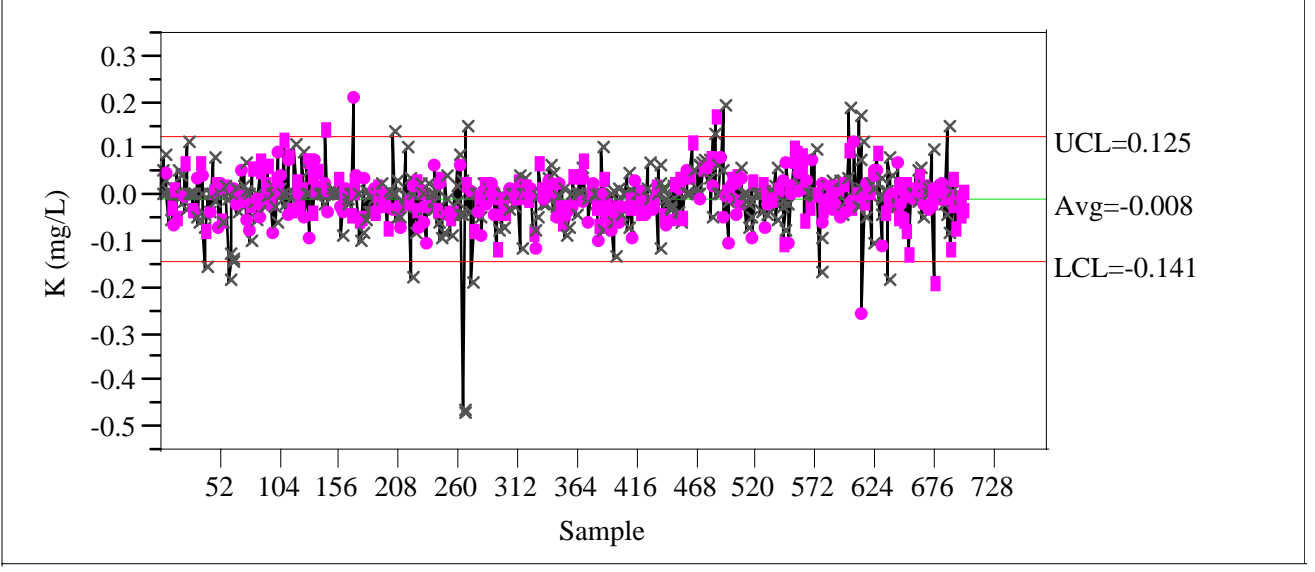

Individual Measurement of $\mathrm{Li}(\mathrm{mg} / \mathrm{L})$

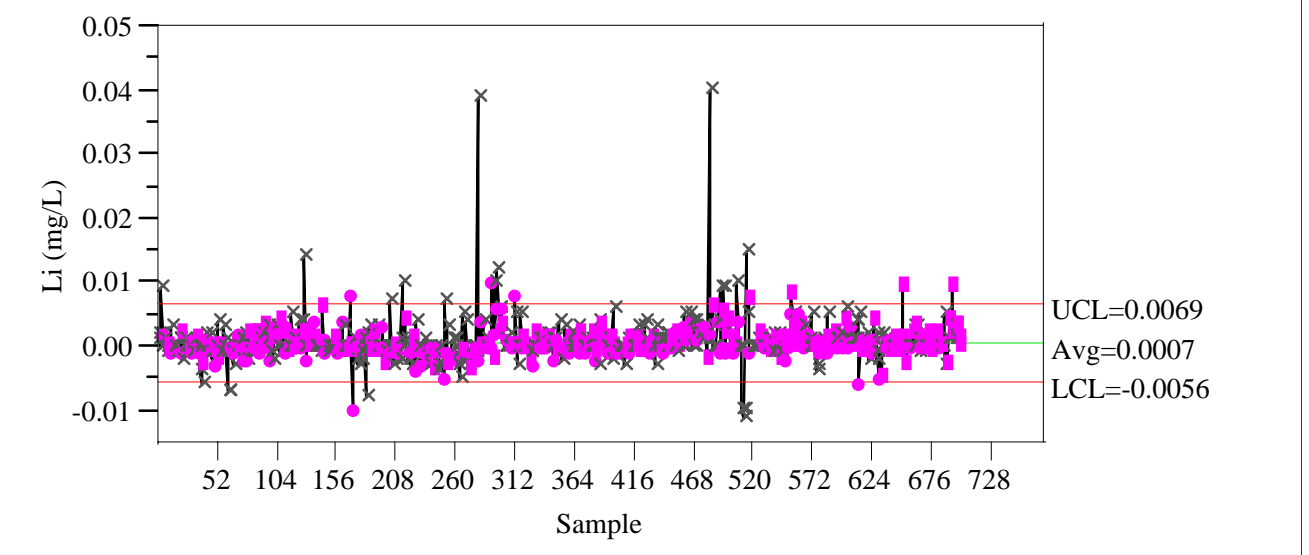

Individual Measurement of $\mathrm{Mg}(\mathrm{mg} / \mathrm{L})$

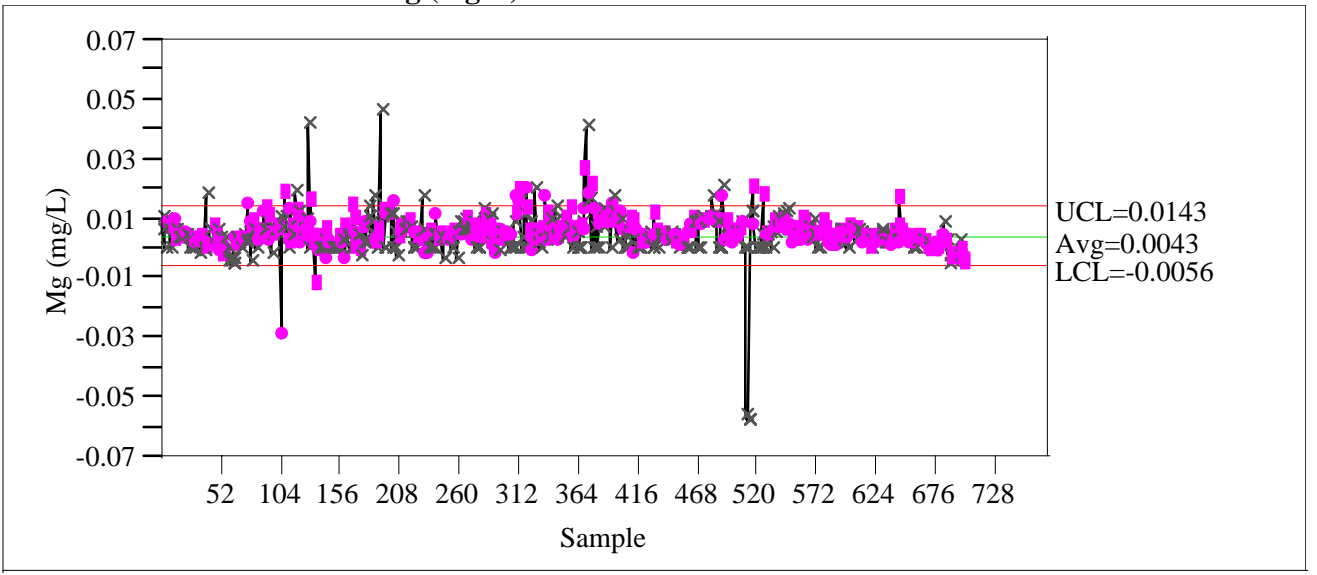


WSRC-TR-2004-00576

\section{Exhibit A11. MA Standards in Analytical Sequence}

\section{Revision 0}

Individual Measurement of Mn (mg/L)

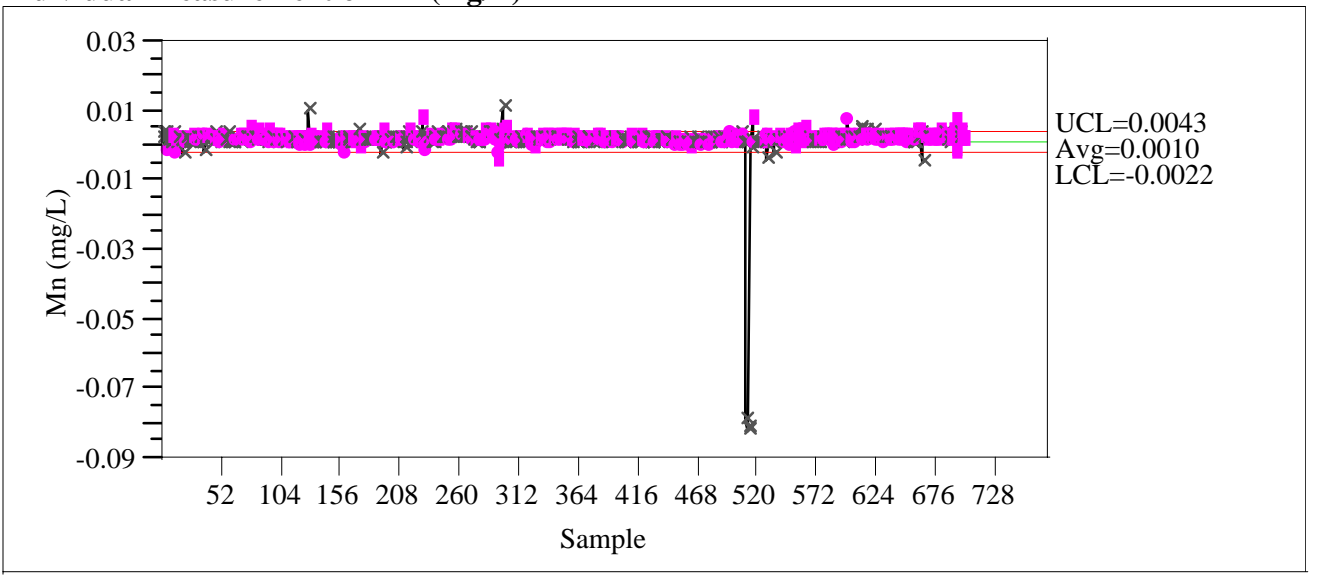

Individual Measurement of $\mathrm{Na}$ (mg/L)

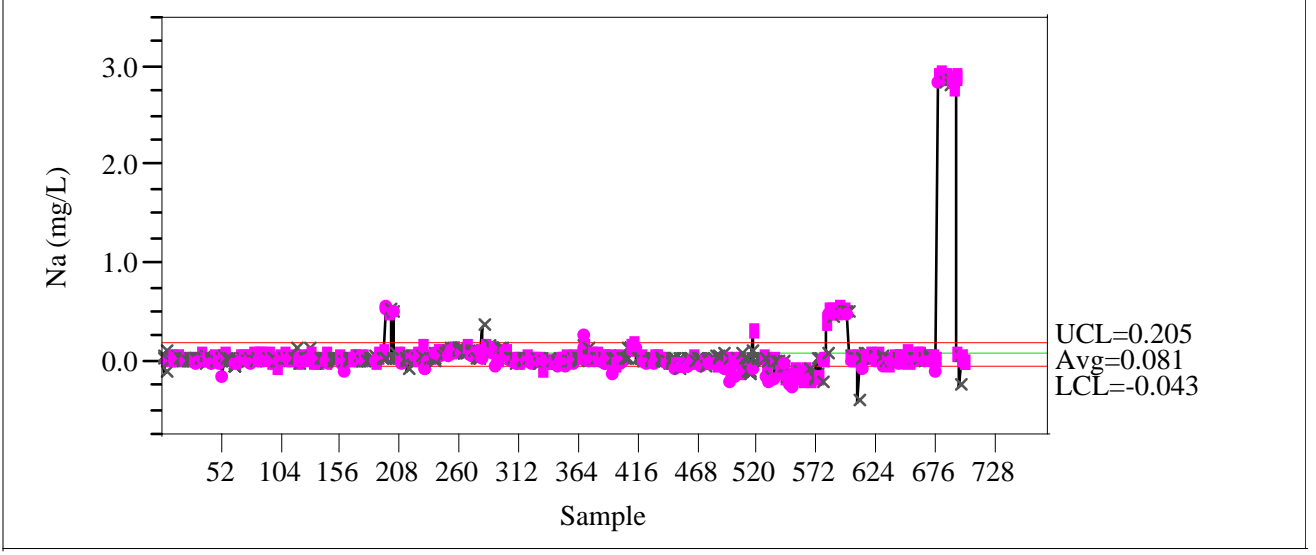

Individual Measurement of $\mathrm{Ni}(\mathrm{mg} / \mathrm{L})$

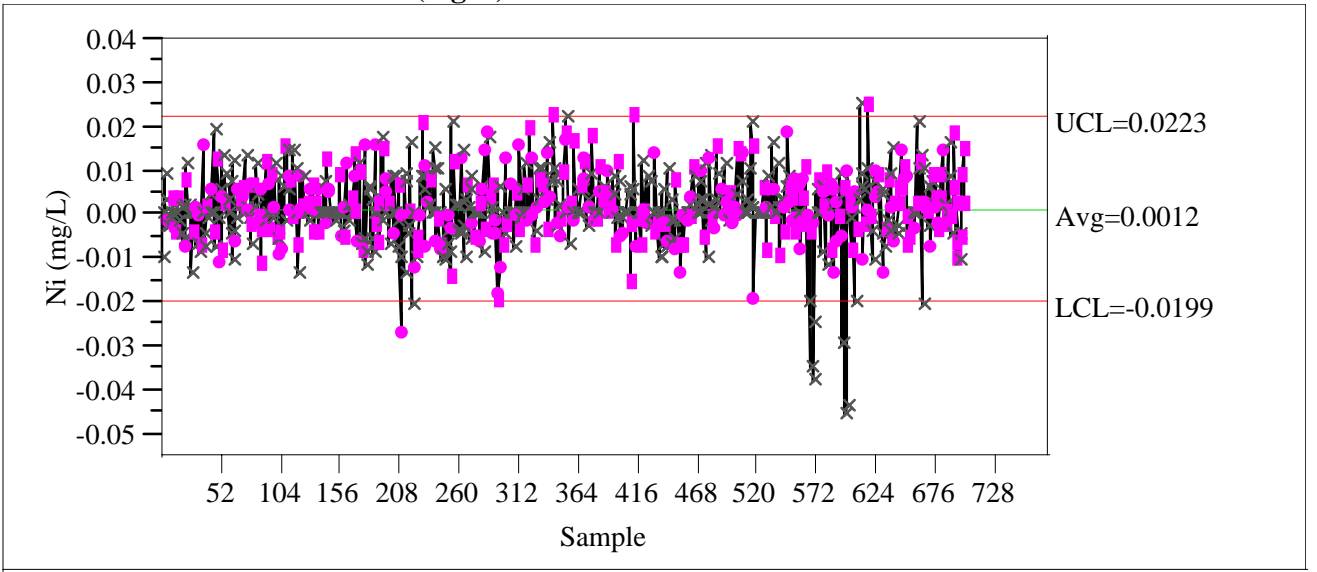




\section{Exhibit A11. MA Standards in Analytical Sequence}

Individual Measurement of Si (mg/L)

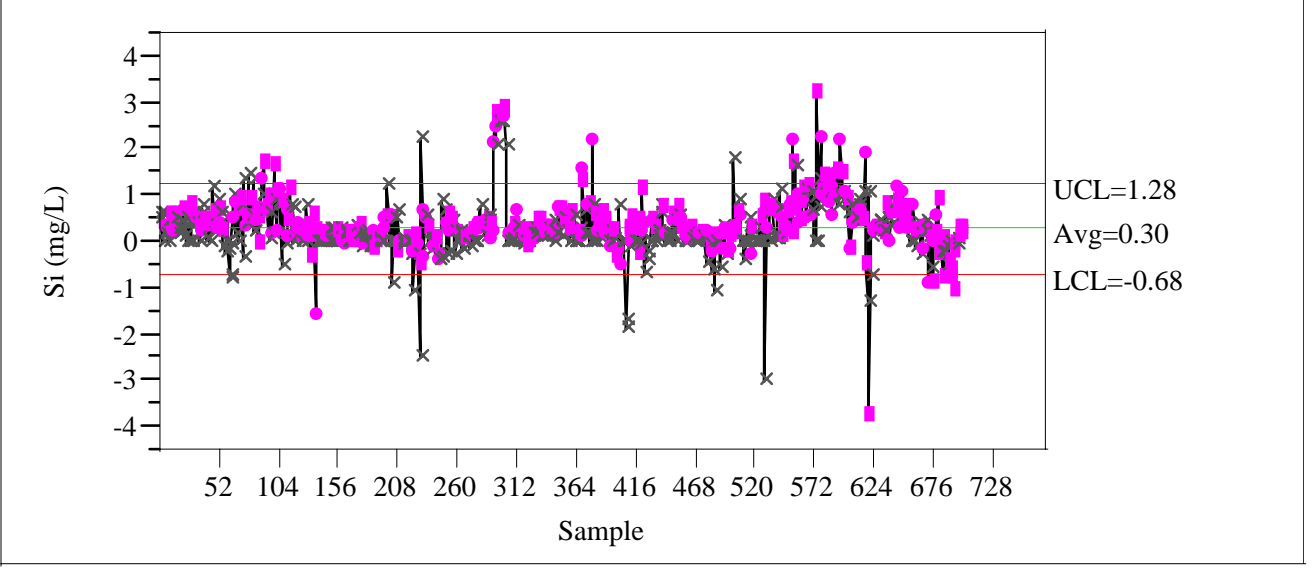

Individual Measurement of Ti (mg/L)

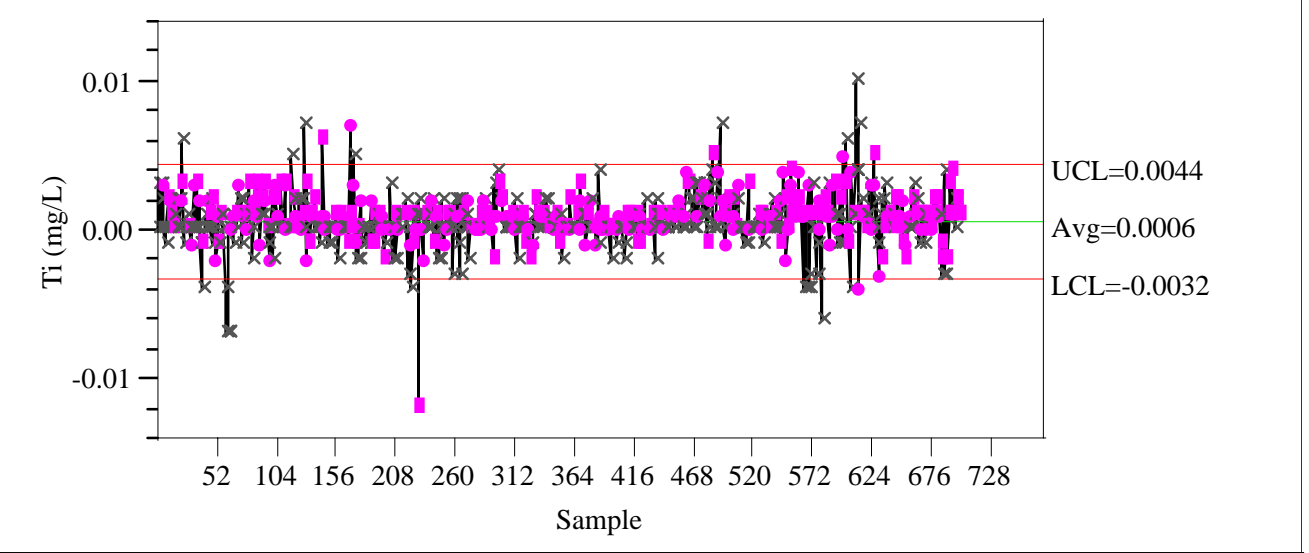

Individual Measurement of U (mg/L)

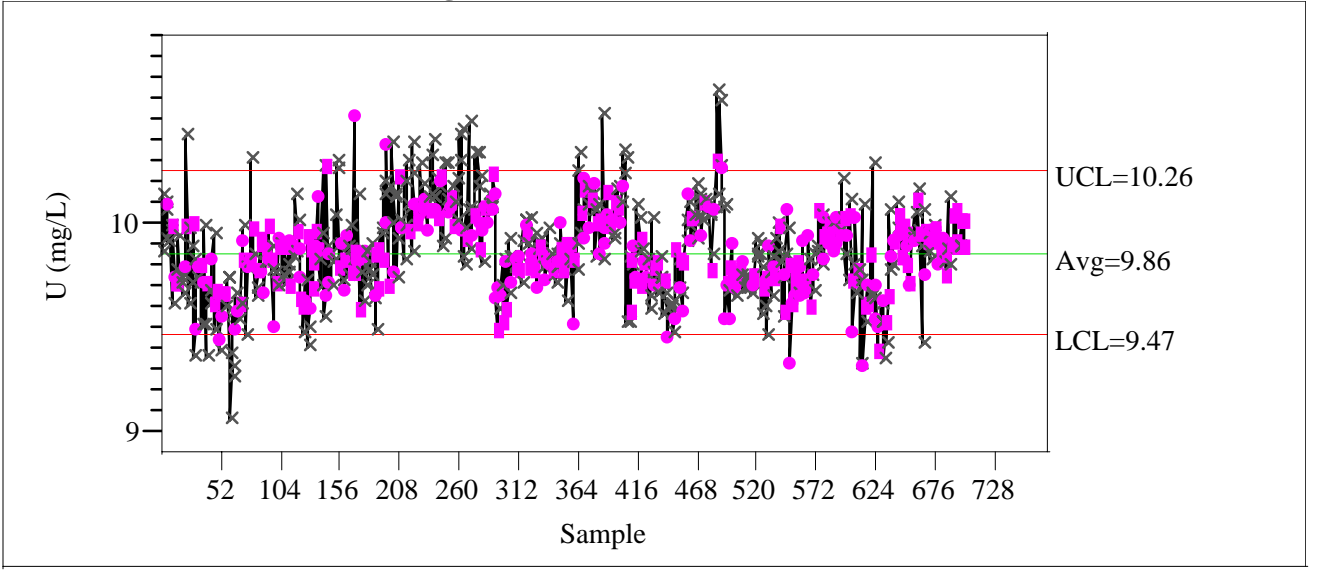


WSRC-TR-2004-00576

\section{Exhibit A11. MA Standards in Analytical Sequence}

\section{Revision 0}

STCd=SM38

Control Chart

Individual Measurement of $\mathrm{Al}$ (mg/L)

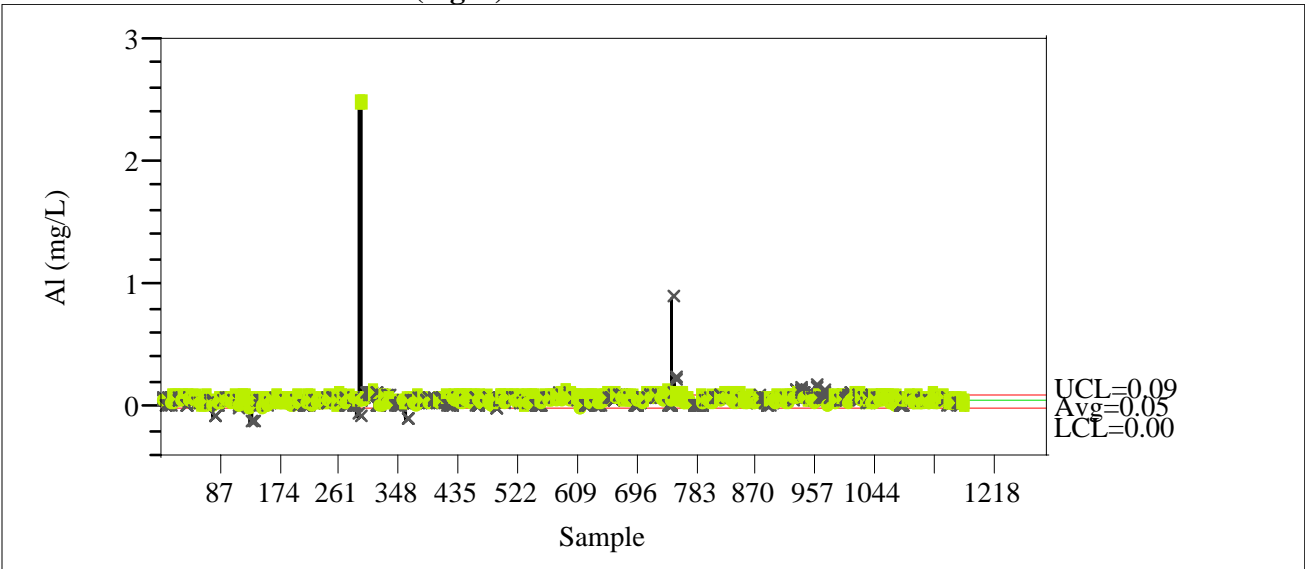

Individual Measurement of Ca (mg/L)

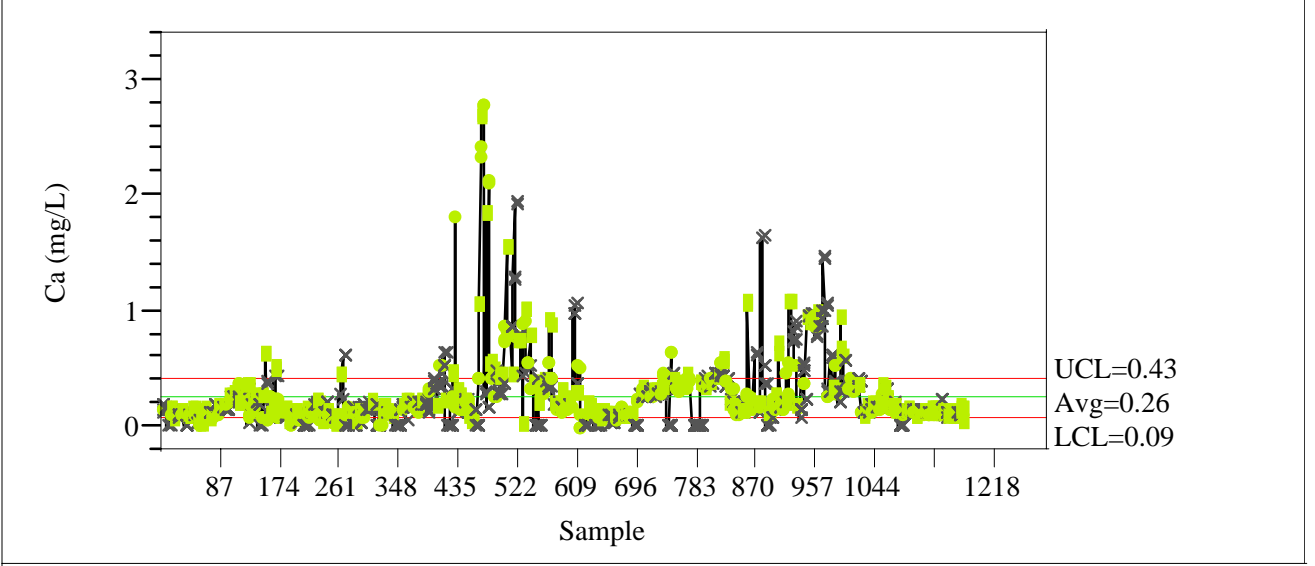

Individual Measurement of $\mathrm{Cr}$ (mg/L)

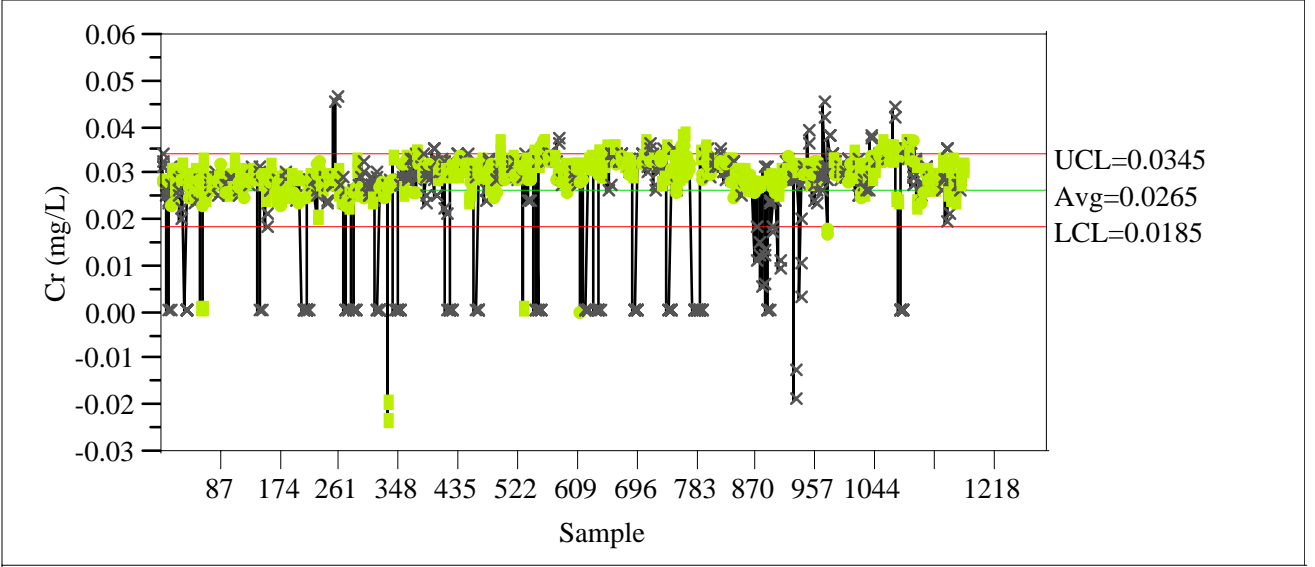


WSRC-TR-2004-00576

Exhibit A11. MA Standards in Analytical Sequence

Revision 0

Individual Measurement of $\mathrm{Cu}(\mathrm{mg} / \mathrm{L})$

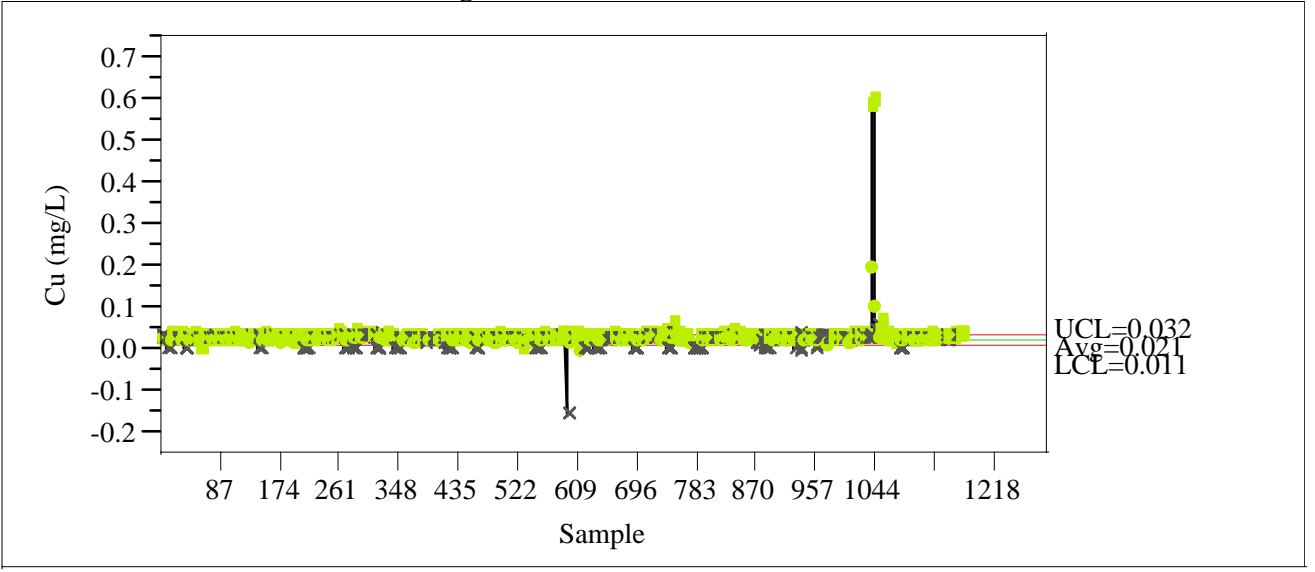

Individual Measurement of Fe (mg/L)

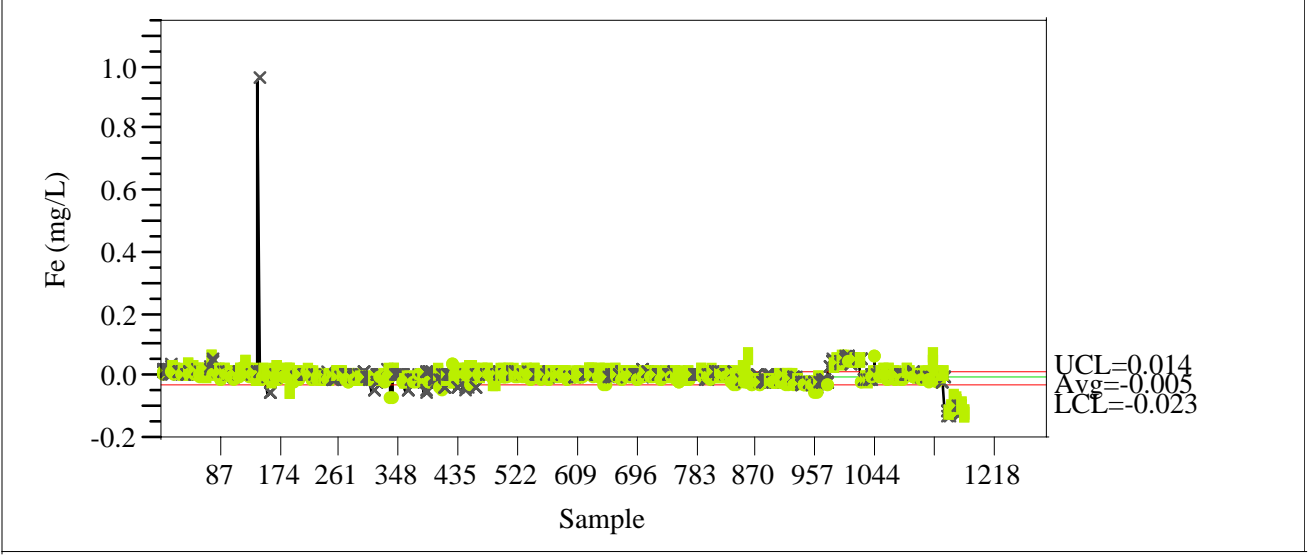

Individual Measurement of K (mg/L)

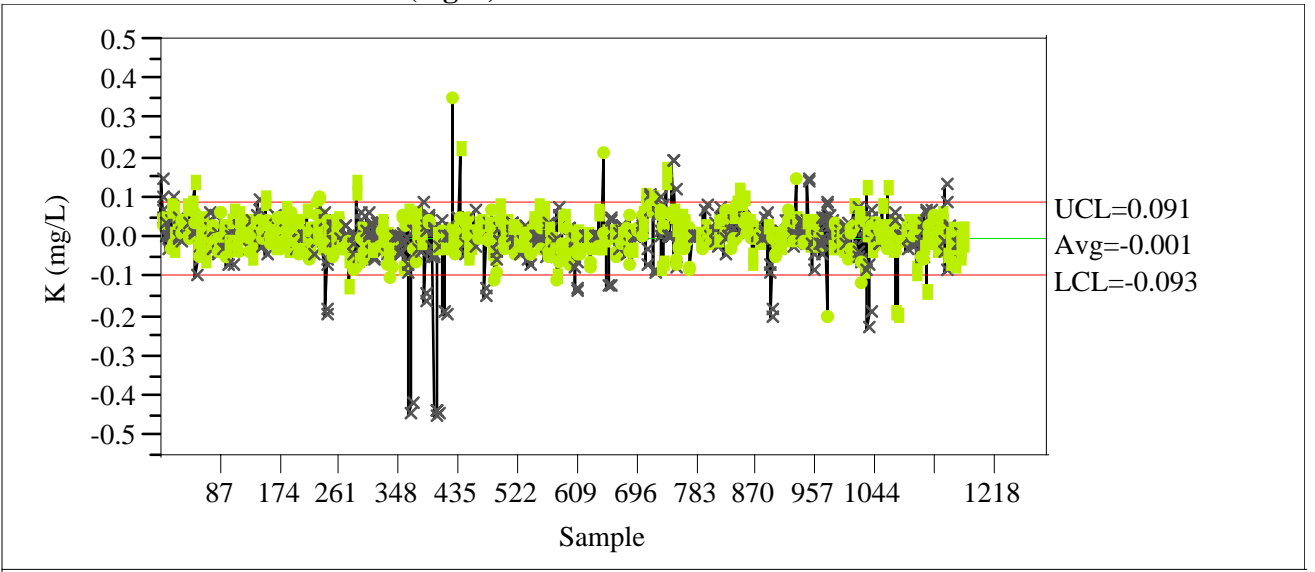


WSRC-TR-2004-00576

\section{Exhibit A11. MA Standards in Analytical Sequence}

\section{Revision 0}

Individual Measurement of $\mathrm{Li}(\mathrm{mg} / \mathrm{L})$

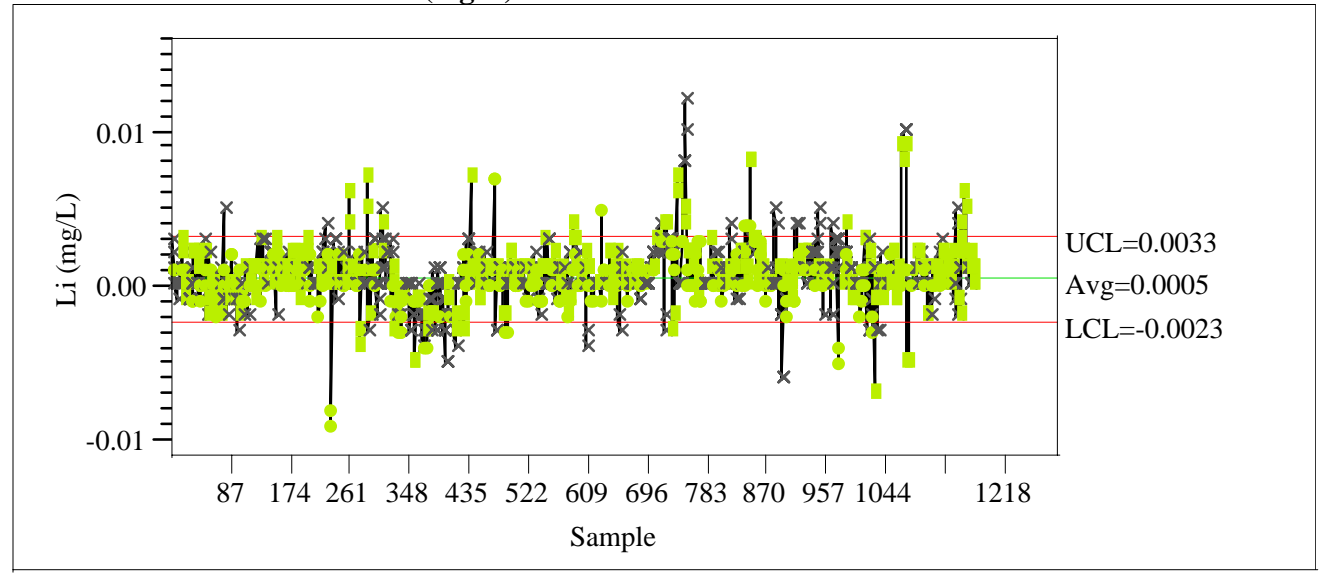

Individual Measurement of $\mathrm{Mg}(\mathrm{mg} / \mathrm{L})$

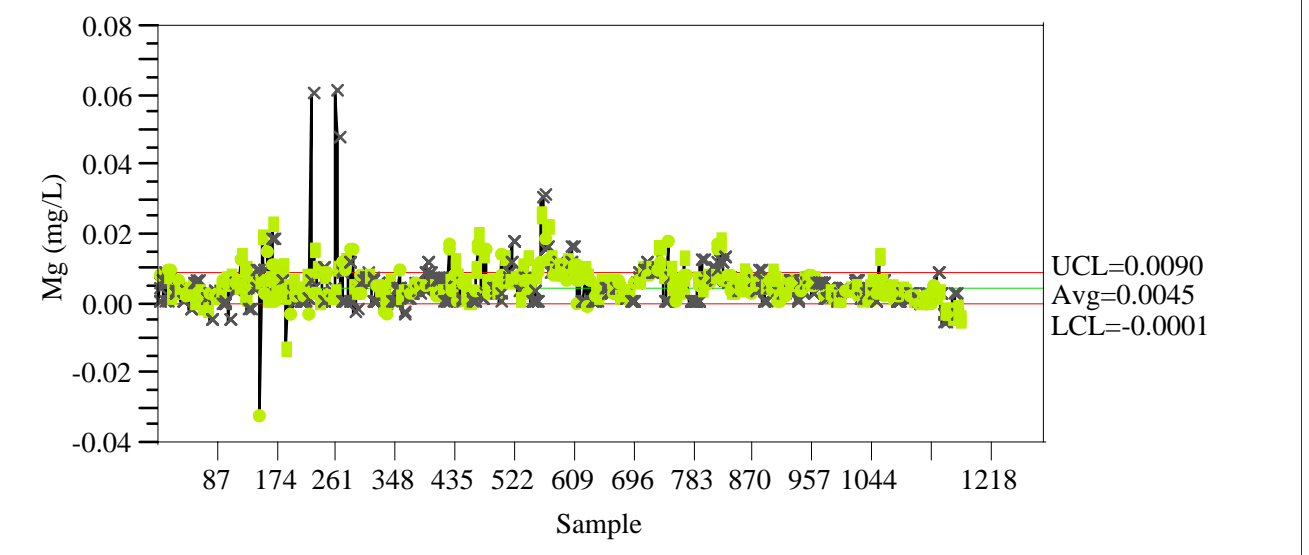

Individual Measurement of Mn (mg/L)

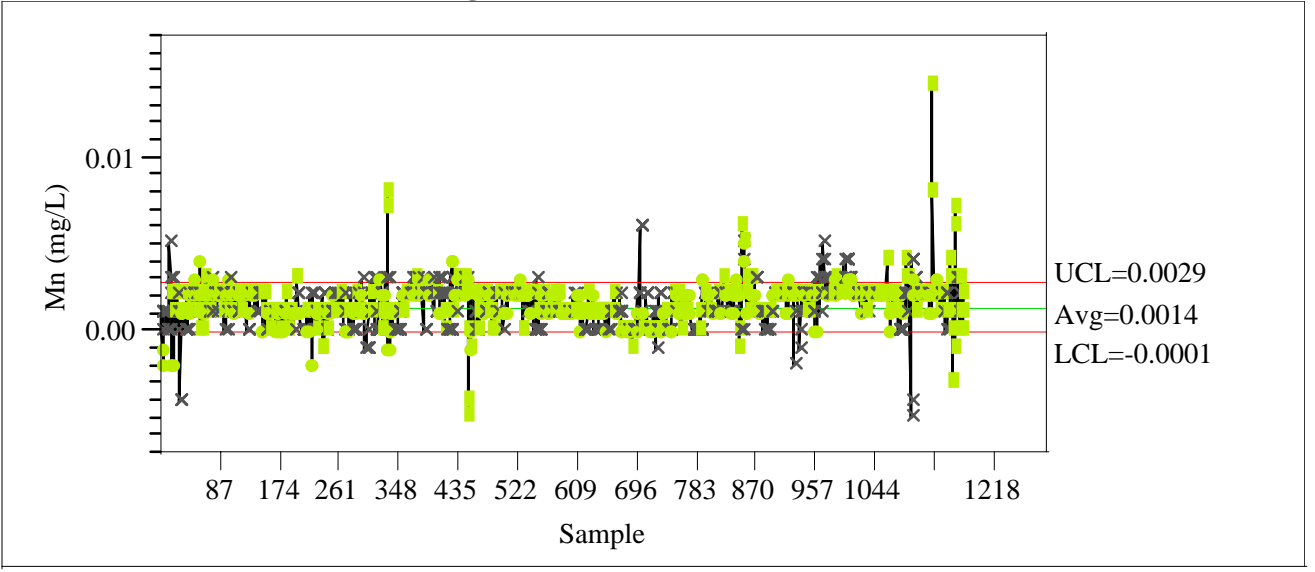


WSRC-TR-2004-00576

\section{Exhibit A11. MA Standards in Analytical Sequence}

\section{Revision 0}

Individual Measurement of $\mathrm{Na}(\mathrm{mg} / \mathrm{L})$

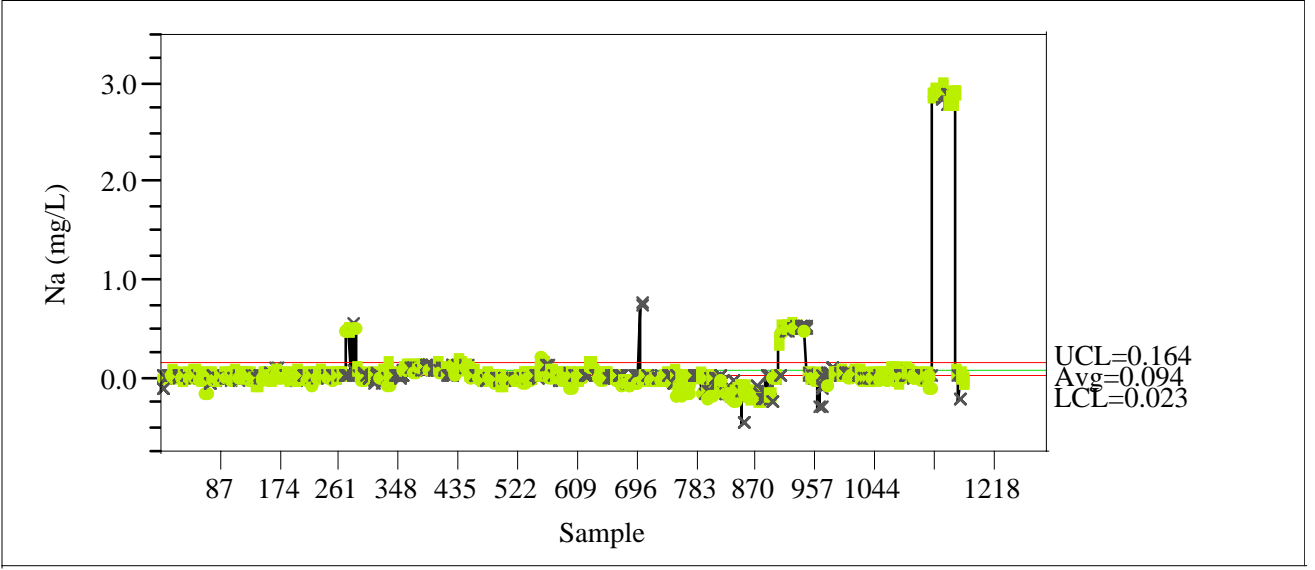

Individual Measurement of $\mathrm{Ni}(\mathrm{mg} / \mathrm{L})$

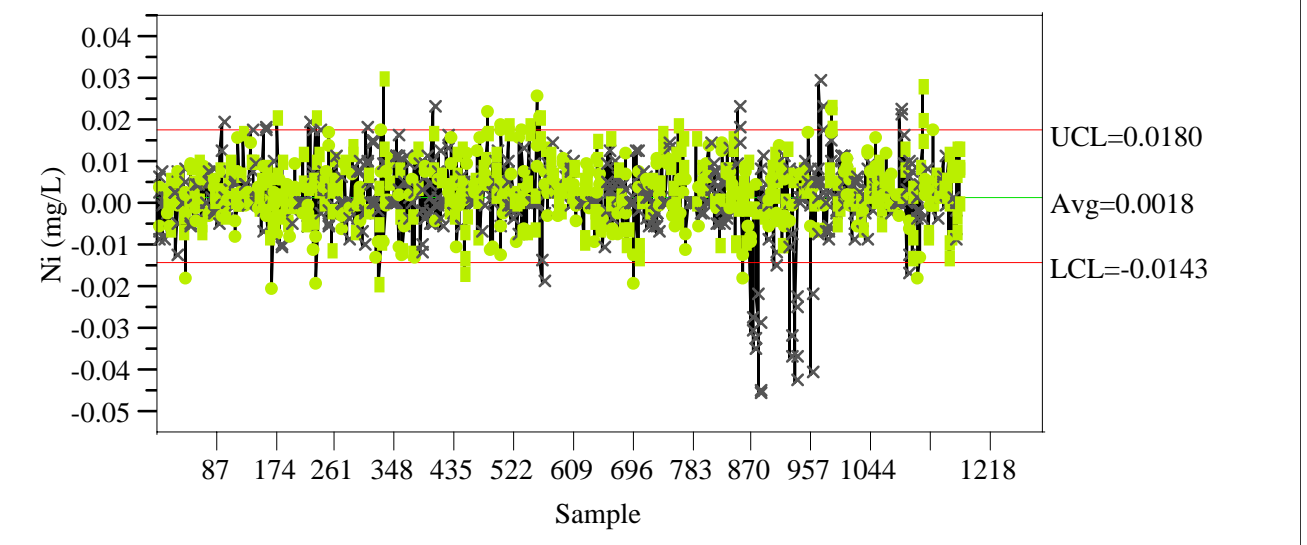

Individual Measurement of Si (mg/L)

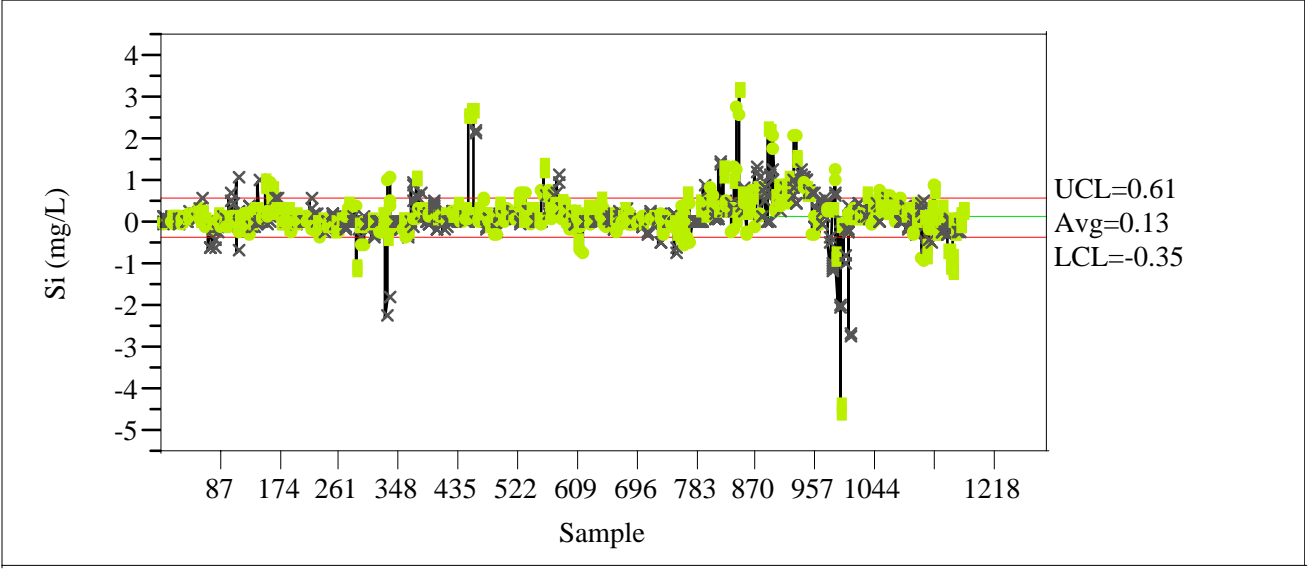


WSRC-TR-2004-00576

\section{Exhibit A11. MA Standards in Analytical Sequence}

Revision 0

Individual Measurement of Ti (mg/L)

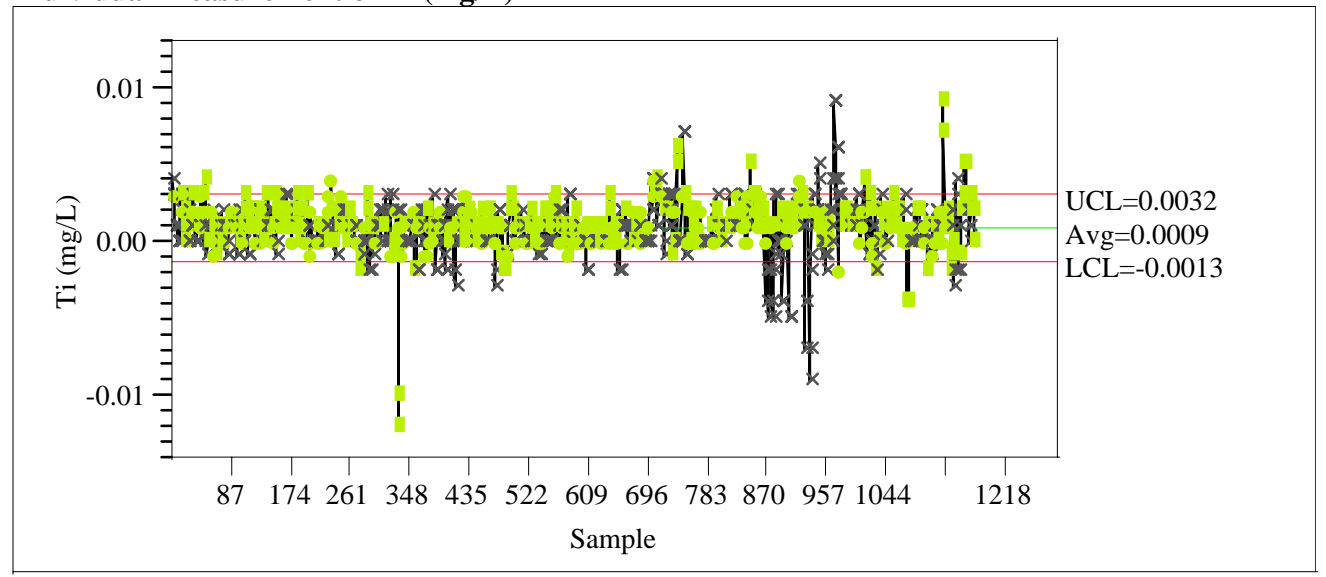

Individual Measurement of U (mg/L)

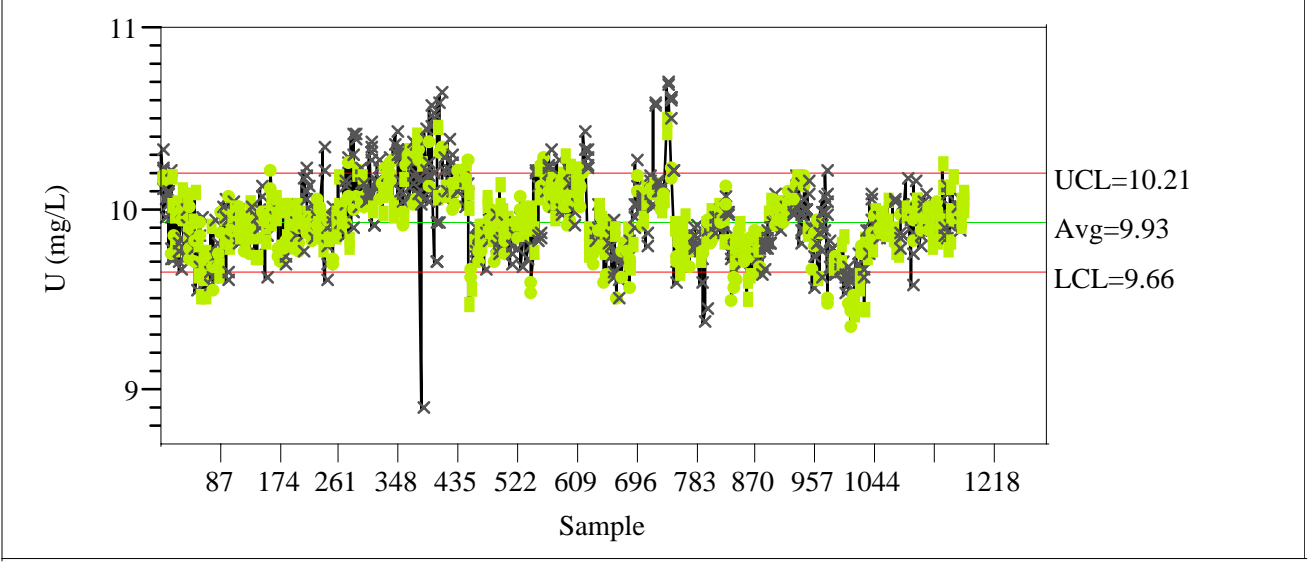


WSRC-TR-2004-00576

Revision 0

Exhibit A12. Histograms and Descriptive Statistics for Mixed Acid Measurements of Calibration and Bench Standards

STCd=SM32

Distributions

Al (mg/L)

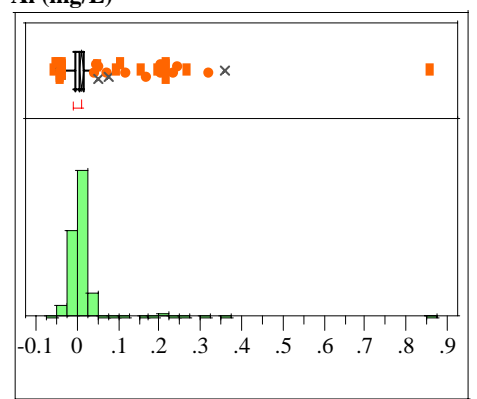

Quantiles

$100.0 \%$ maximum

$99.5 \%$

$97.5 \%$

$90.0 \%$

$75.0 \%$

$50.0 \%$

$25.0 \%$

$10.0 \%$

$2.5 \%$

$0.5 \%$

$0.0 \%$

Moments

Mean

Std Dev

Std Err Mean

upper 95\% Mean

lower 95\% Mean

$\mathrm{N}$

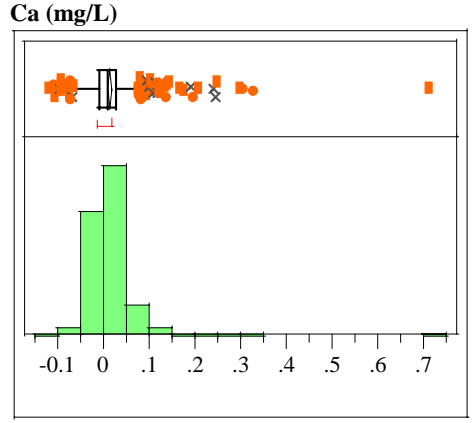

Quantiles

$\begin{array}{llr}100.0 \% & \text { maximum } & 0.7120 \\ 99.5 \% & & 0.3037 \\ 97.5 \% & & 0.1278 \\ 90.0 \% & & 0.0600 \\ 75.0 \% & \text { quartile } & 0.0250 \\ 50.0 \% & \text { median } & 0.0070 \\ 25.0 \% & \text { quartile } & -0.0090 \\ 10.0 \% & & -0.0230 \\ 2.5 \% & & -0.0636 \\ 0.5 \% & & -0.1050 \\ 0.0 \% & \text { minimum } & -0.1190 \\ \text { Moments } & & \\ & & 0.0141092 \\ \text { Mean } & & 0.0526992 \\ \text { Std Dev } & & 0.0020106 \\ \text { Std Err Mean } & & 0.0180568 \\ \text { upper 95\% Mean } & 0.0101615 \\ \text { lower 95\% Mean } & 687 \\ \text { N } & \end{array}$

$-0.0338$

$-0.0452$

minimum

$-0.0560$
Cr (mg/L)

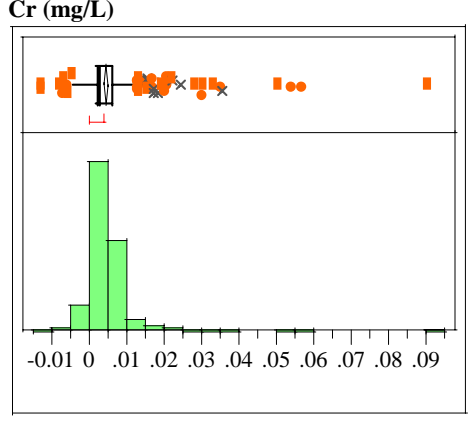

Quantiles

$100.0 \%$ maximum 0.0900

$99.5 \% \quad 0.0522$

$97.5 \%$

$90.0 \%$

$75.0 \%$

$50.0 \%$

$25.0 \%$

$10.0 \%$

$2.5 \%$

$0.5 \%$

$0.0 \%$ minimum

quartile

median

quartile

Moments

Mean

Std Dev

Std Err Mean

upper 95\% Mean

lower 95\% Mean

N

Cu (mg/L)

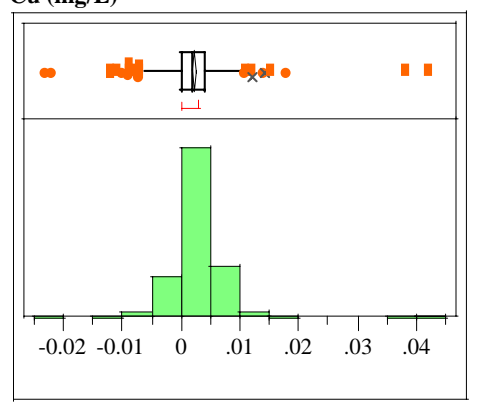

Quantiles

$100.0 \%$

$99.5 \%$

$97.5 \%$

$90.0 \%$

$75.0 \%$

$50.0 \%$

$25.0 \%$

$10.0 \%$

$2.5 \%$

$0.5 \%$

$0.0 \%$

Moments

minimum

Mean

Std Dev

Std Err Mean

upper 95\% Mean

lower 95\% Mean

$\mathrm{N}$
Fe (mg/L)

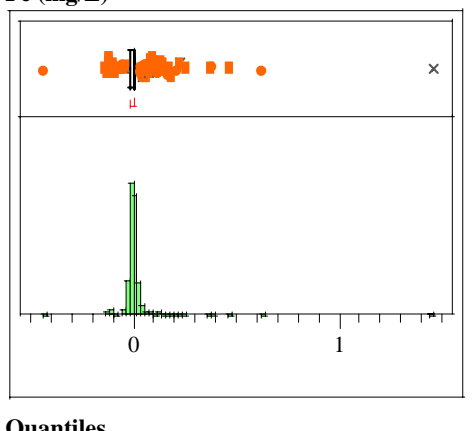

Quantiles

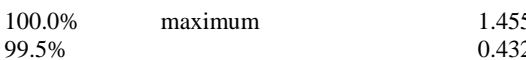

$97.5 \% \quad 0.123$

$90.0 \% \quad 0.032$

$\begin{array}{ll}75.0 \% & \text { quartile } \\ 50.0 \% & 0.010\end{array}$

$50.0 \%$ median 0.000

$25.0 \%$ quartile $\quad-0.011$

$10.0 \% \quad-0.024$

$2.5 \%-0.102$

$0.5 \% \quad-0.128$

$0.0 \% \quad$ minimum $\quad-0.430$

Moments

Mean

Std Dev

Std Err Mean

upper 95\% Mean

lower $95 \%$ Mean

0.0777535

0.0029665

0.0119409

0.0002575

0.0049728

0.0039617

687

K (mg/L)

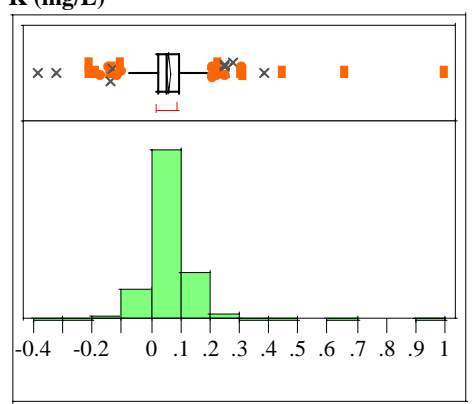

Quantiles

$100.0 \%$ maximum 0.9970

$99.5 \% \quad 0.4187$

$\begin{array}{ll}99.5 \% & 0.4187 \\ 97.5 \% & 0.2118\end{array}$

$75.0 \%$ quartile $\quad 0.0930$

$50.0 \%$ median 0.0540

$25.0 \%$ quartile 0.0220

$10.0 \%$

$2.5 \%-0.0632$

$\begin{array}{ll}2.5 \% & -0.2137 \\ 0.0 \% & -0.3900\end{array}$

$0.0 \%$ minimum -0.3900

Moments

0.0602824

Mean

Std Dev

Std Err Mean

upper $95 \%$ Mean

lower 95\% Mean

$\mathrm{N}$

0.082613

0.0031519

0.0664709

0.0540939

687 
WSRC-TR-2004-00576

Revision 0

\section{Exhibit A12. Histograms and Descriptive Statistics for Mixed Acid Measurements of} Calibration and Bench Standards

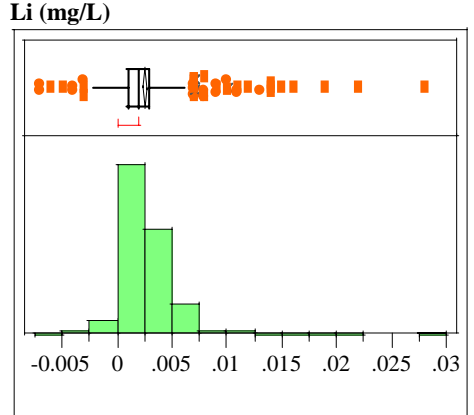

Quantiles

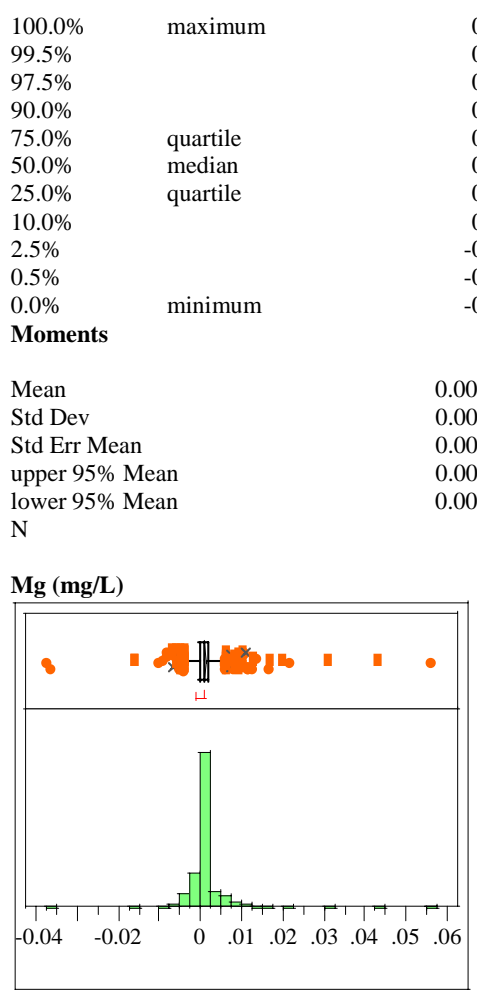

Quantiles

$100.0 \% \quad$ maximum

$99.5 \%$

$97.5 \%$

$90.0 \%$

$75.0 \%$

$50.0 \%$

$25.0 \%$

$10.0 \%$

$2.5 \%$

$0.5 \%$

$0.0 \%$
Moments

minimum

Mean

Std Dev

Std Err Mean

upper 95\% Mean

lower 95\% Mea

$\mathrm{N}$
Mn (mg/L)

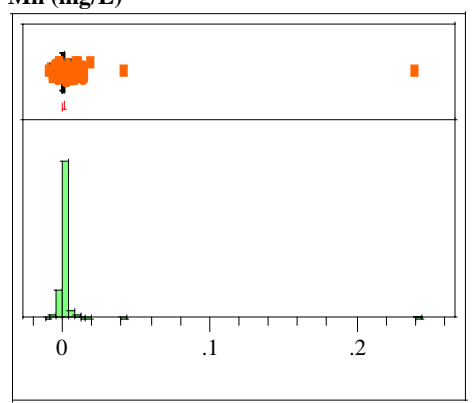

Quantiles

$100.0 \%$

$99.5 \%$

$97.5 \%$

$90.0 \%$

$75.0 \%$

$50.0 \%$

$25.0 \%$

$10.0 \%$

$2.5 \%$

$0.5 \%$

$0.0 \%$

Moments

maximum

Mean

Std Dev

Std Err Mean

upper 95\% Mean

lower 95\% Mean

$\mathrm{N}$

Na (mg/L)

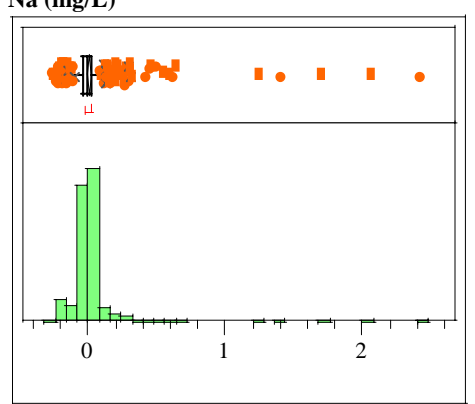

Quantiles

$100.0 \%$ maximum

$99.5 \%$

$97.5 \%$

$90.0 \%$

$75.0 \%$

$50.0 \%$

$25.0 \%$

$10.0 \%$

$2.5 \%$

$0.5 \%$

$0.0 \%$ minimum

Moments

quartile

median

Mean

Std Dev

Std Err Mean

upper 95\% Mean

lower 95\% Mean

$\mathrm{N}$
Ni (mg/L)

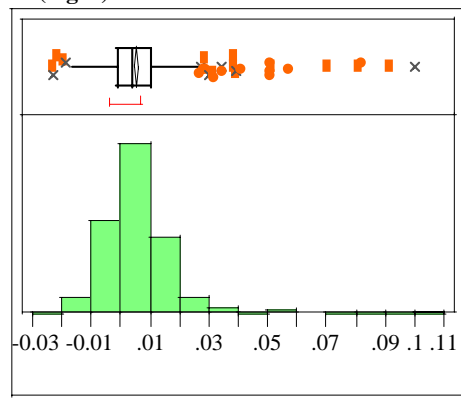

Quantiles

$100.0 \%$

maximum

0.1000

$99.5 \%$

$97.5 \%$

$90.0 \%$

$75.0 \%$

$50.0 \%$

$25.0 \%$

$10.0 \%$

$2.5 \%$

$0.5 \%$

$0.0 \%$

Moments

quartile

median

quartile

$-0.0090$

Mean

Std Dev

Std Err Mean

upper $95 \%$ Mean

lower 95\% Mean

0.000365

0.0018404

0.0004071

687

$\mathrm{N}$

Si (mg/L)

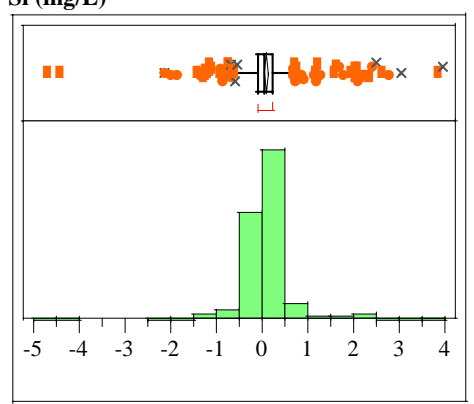

Quantiles

$100.0 \%$ maximum 3.917

$99.5 \% \quad 2.938$

$97.5 \%$

$75.0 \%$

$50.0 \%$

$25.0 \%$

$10.0 \%$

$2.5 \%$

$0.5 \%$

$0.0 \%$

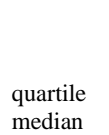

median

quartile

0.0004685

0.0062096

0.0043697

687

Moments

0.0117118

Mean

Std Dev

Std Err Mean

upper 95\% Mean

lower 95\% Mean

$\mathrm{N}$ 
WSRC-TR-2004-00576

Revision 0

Exhibit A12. Histograms and Descriptive Statistics for Mixed Acid Measurements of Calibration and Bench Standards

Ti (mg/L)

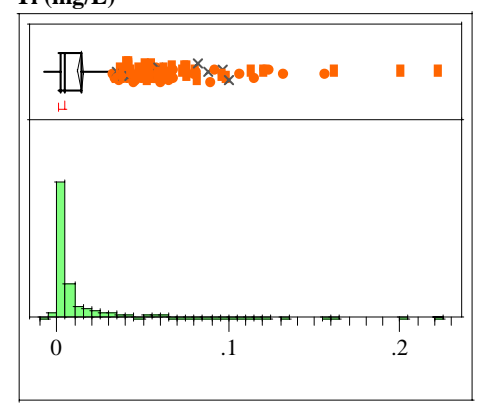

Quantiles

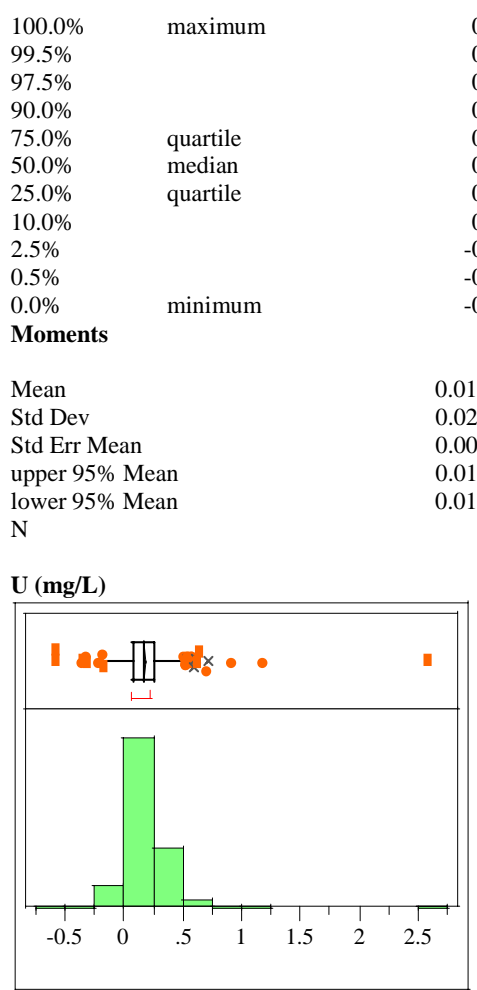

Quantiles

$100.0 \%$

maximum

$99.5 \%$

$97.5 \%$

$90.0 \%$

$75.0 \%$

$50.0 \%$

$25.0 \%$

$10.0 \%$

$2.5 \%$

$0.5 \%$

$0.0 \%$

Moments

Mean

Std Dev

Std Err Mean

upper 95\% Mean

lower 95\% Mean

$\mathrm{N}$

quartile
median

quartile

minimum
Zr (mg/L)

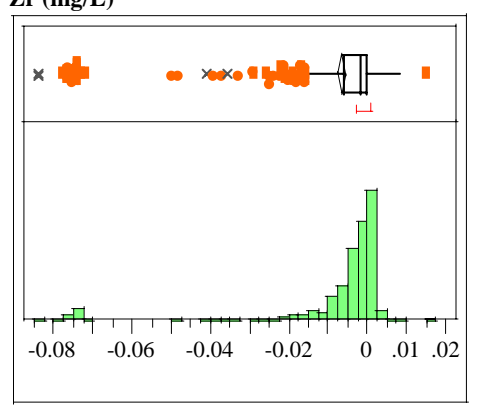

Quantiles

$99.5 \%$

$97.5 \%$

$90.0 \%$

$75.0 \%$

$50.0 \%$

$25.0 \%$

$10.0 \%$

$2.5 \%$

$0.5 \%$

$0.0 \%$ minimum

Moments

quartile

median

quartile

Mean

Std Dev

Std Err Mean

upper 95\% Mean

lower 95\% Mean

$\mathrm{N}$

STCd=SM33

Distributions

Al (mg/L)

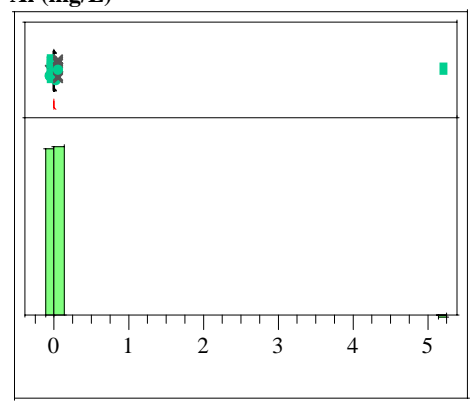

Quantiles

$100.0 \%$ maximum

$99.5 \%$

$97.5 \%$

$90.0 \%$

$75.0 \%$

$50.0 \%$

$25.0 \%$

$10.0 \%$

$2.5 \%$

$0.5 \%$

$0.0 \%$

$-0.583$

0.1764978

0.1865608

0.0071177

0.190473

0.1625226

quartile

median

quartile

minimum
Moments

Mean

Std Dev

Std Err Mean

upper 95\% Mean

lower 95\% Mean

$\mathrm{N}$

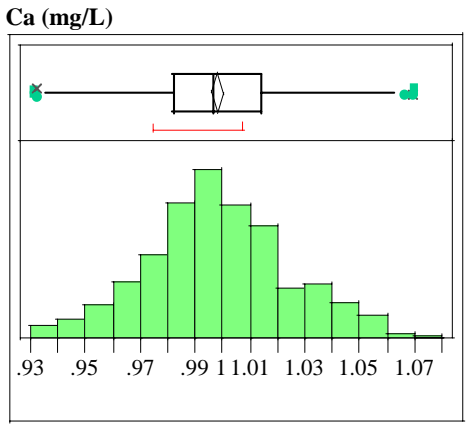

Quantiles

$100.0 \%$ maximum $\quad 1.0700$

$99.5 \% \quad 1.0695$

$97.5 \% \quad 1.0523$

1.0341

$\begin{array}{ll}75.0 \% & \text { quartile } \\ 50.0 \% & 1.0140\end{array}$

$50.0 \%$ median 0.9970

$25.0 \%$ quartile 0.9820

0.9639

$2.5 \% \quad 0.9430$

$0.5 \% \quad 0.9320$

$0.0 \%$ minimum 0.9310

Moments

0.9983071

Mean

Std Dev

Std Err Mean

upper 95\% Mean

lower 95\% Mean 0.9960062

$-0.005428$

687

Cr (mg/L)

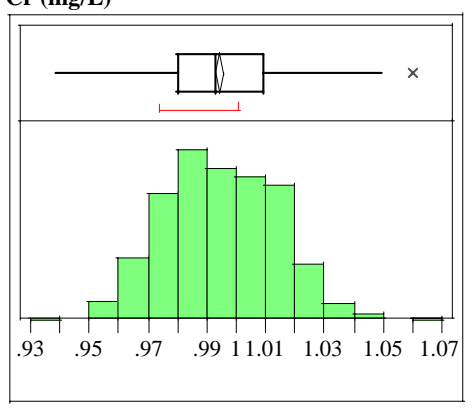

Quantiles

$100.0 \%$

$99.5 \%$

$97.5 \%$

$90.0 \%$

$75.0 \%$

$50.0 \%$

$25.0 \%$

$10.0 \%$

$2.5 \%$

$0.5 \%$

$0.0 \%$

maximum

1.0600

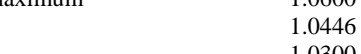

quartile

median

quartile

Moments

minimum

1.0190

1.0090

0.9930

0.9800

0.9710

0.9607

0.9511

Mean

Std Dev

Std Err Mean

upper 95\% Mean

lower 95\% Mean

$\mathrm{N}$

0.994374

0.0188004

0.0008341

0.9960128

0.9927352 
WSRC-TR-2004-00576

Revision 0

\section{Exhibit A12. Histograms and Descriptive Statistics for Mixed Acid Measurements of} Calibration and Bench Standards

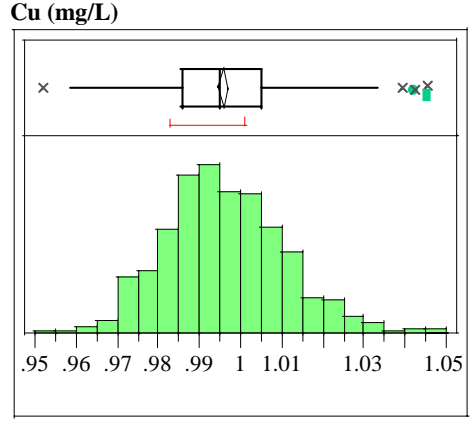

Quantiles

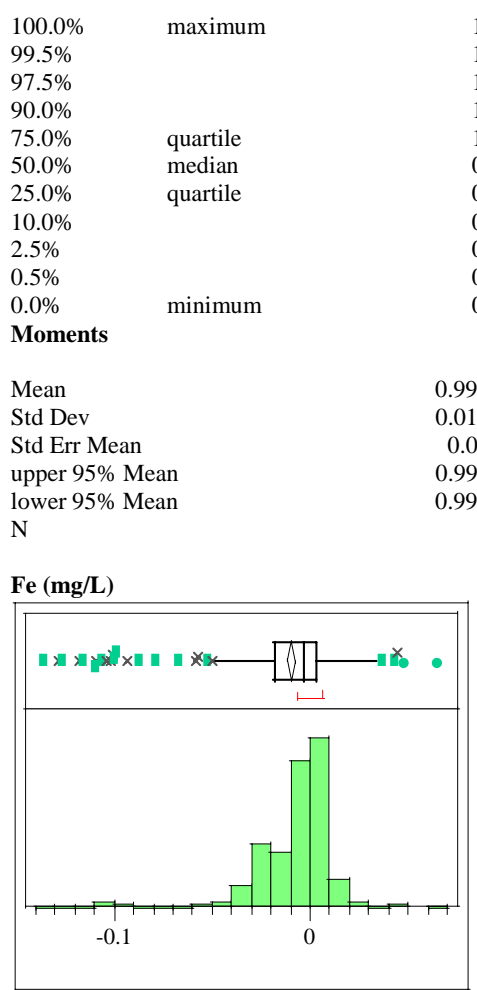

Quantiles

$100.0 \%$

$99.5 \%$

$97.5 \%$

$90.0 \%$

$75.0 \%$

$50.0 \%$

$25.0 \%$

$10.0 \%$

$2.5 \%$

$0.5 \%$

$0.0 \%$
Moments

maximum

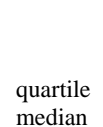

median

quartile

minimum

Mean

Std Dev

Std Err Mean

upper 95\% Mean

lower 95\% Mean

$\mathrm{N}$
K (mg/L)

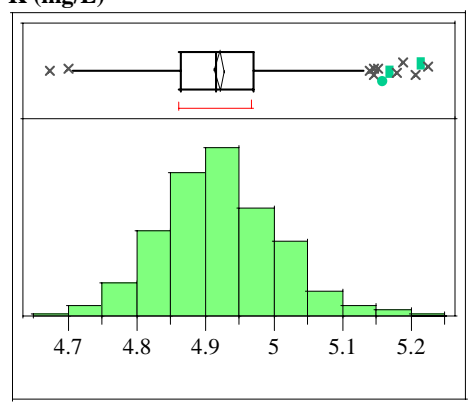

Quantiles

$100.0 \%$

$99.5 \%$

$97.5 \%$

$90.0 \%$

$75.0 \%$

$50.0 \%$

$25.0 \%$

$10.0 \%$

$2.5 \%$

$0.5 \%$

$0.0 \%$

Moments

maximum

Mean

Std Dev

Std Err Mean

upper 95\% Mean

lower 95\% Mean

$\mathrm{N}$

Li (mg/L)

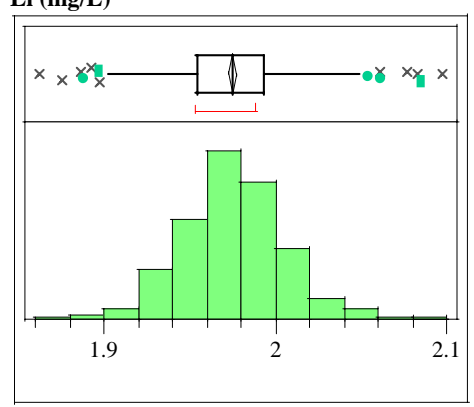

Quantiles

$100.0 \% \quad$ maximum

$99.5 \%$

$97.5 \%$

$90.0 \%$

$75.0 \%$

$50.0 \%$

$25.0 \%$

$10.0 \%$

$2.5 \%$

$0.5 \%$

$0.0 \%$ minimum

Moments

quartile

median
quartile

Mean

Std Dev

Std Err Mean

upper 95\% Mean

lower 95\% Mean

$\mathrm{N}$
$\operatorname{Mg}(\mathrm{mg} / \mathrm{L})$

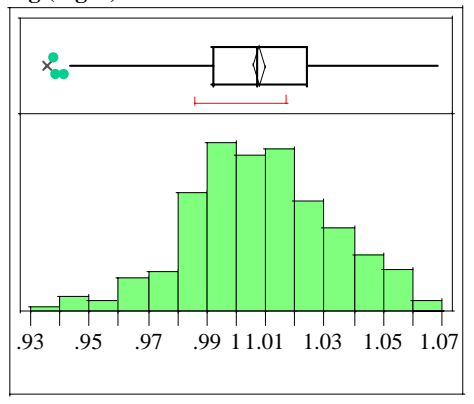

Quantiles

$100.0 \%$

$99.5 \%$

$97.5 \%$

$90.0 \%$

$75.0 \%$

$50.0 \%$

$25.0 \%$

$10.0 \%$

$2.5 \%$

$0.5 \%$

$0.0 \%$

Moments

maximum

1.0680

Mean

Std Dev

Std Err Mean

upper 95\% Mean

lower 95\% Mean

0.0039207

4.929506

4.9141003

$\mathrm{N}$

quartile

median

quartile

minimum

1.0573

1.0573

1.0401

1.0240

1.0070

0.9920

0.9779

0.9567

0.9385

0.9350

Mn (mg/L)

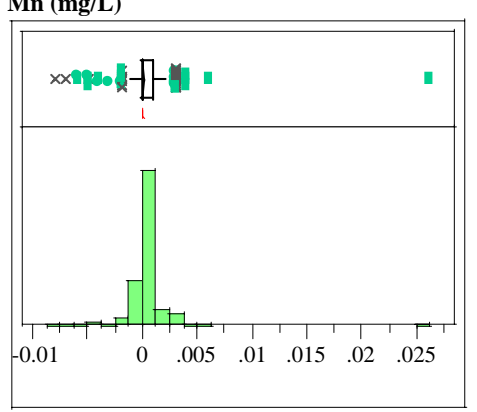

Quantiles

$100.0 \%$

maximum

1.0074862 0.0248284 0.0011016 1.0096505 1.005322 508

0.0233795

0.0010373

$-0.007163$

508

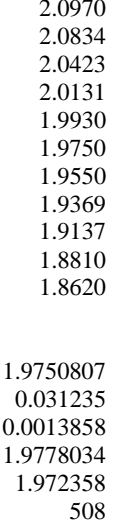

$99.5 \%$

$97.5 \%$

$90.0 \%$

$75.0 \%$

$50.0 \%$

$25.0 \%$

$10.0 \%$

$2.5 \%$

$0.5 \%$

$0.0 \%$

Moments

Mean

Std Dev

Std Err Mean

upper 95\% Mean

lower 95\% Mean

$\mathrm{N}$
0.0260 0.0049 0.0030 0.0020 0.0010 0.0000 0.0000 $-0.0010$ $-0.0020$ $-0.0065$ $-0.0080$ 0.0000795 0.0002822 $-0.00003$ 
WSRC-TR-2004-00576

Revision 0

\section{Exhibit A12. Histograms and Descriptive Statistics for Mixed Acid Measurements of} Calibration and Bench Standards

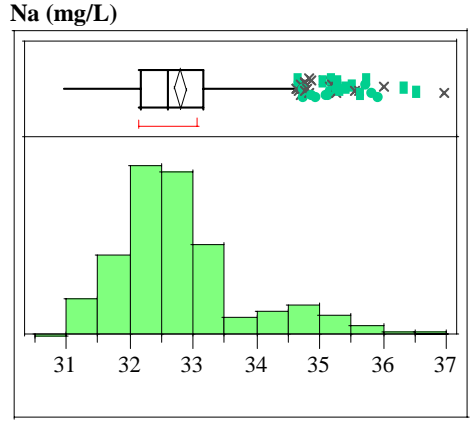

Quantiles

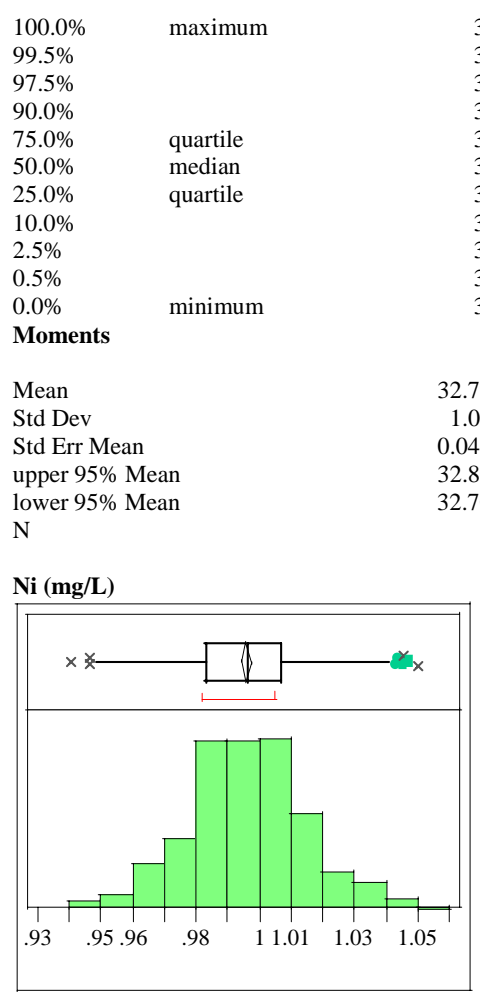

Quantiles

$100.0 \% \quad$ maximum

$99.5 \%$

$97.5 \%$

$90.0 \%$

$75.0 \%$

$50.0 \%$

$25.0 \%$

$10.0 \%$

$2.5 \%$

$0.5 \%$

$0.0 \% \quad$ minimum

Moments

Mean

Std Dev

Std Err Mean

upper 95\% Mean

lower 95\% Mean

$\mathrm{N}$
Si (mg/L)

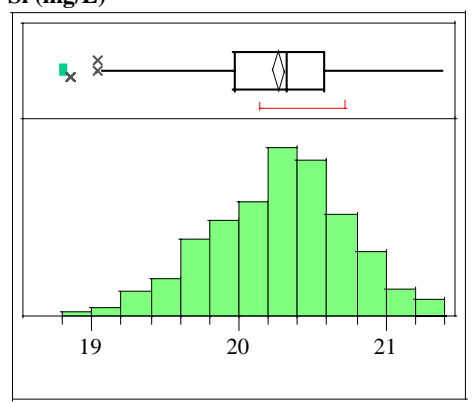

Quantiles

$100.0 \%$

$99.5 \%$

$97.5 \%$

$90.0 \%$

$75.0 \%$

$50.0 \%$

$25.0 \%$

$10.0 \%$

$2.5 \%$

$0.5 \%$

$0.0 \%$

Moments

maximum

Mean

Std Dev

Std Err Mean

upper 95\% Mean

lower 95\% Mean

$\mathrm{N}$

Ti (mg/L)

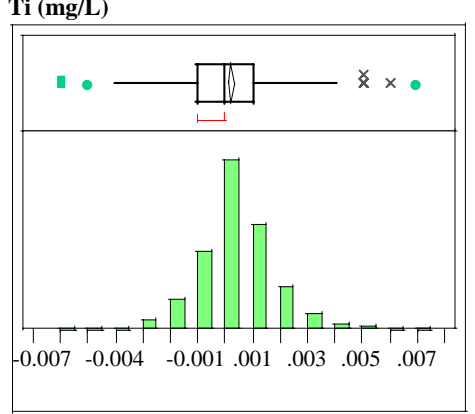

Quantiles

$100.0 \%$ maximum

$99.5 \%$

$97.5 \%$

$90.0 \%$

$75.0 \%$

$50.0 \%$

$25.0 \%$

$10.0 \%$

$2.5 \%$

$0.5 \%$

$0.0 \%$ minimum

Moments

quartile

median

Mean

Std Dev

Std Err Mean

upper 95\% Mean

lower 95\% Mean

$\mathrm{N}$
U (mg/L)

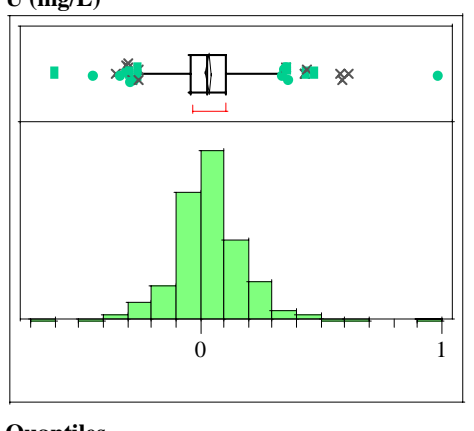

Quantiles

$100.0 \%$

$99.5 \%$

$97.5 \%$

$90.0 \%$

$75.0 \%$

$50.0 \%$

$25.0 \%$

$10.0 \%$

$2.5 \%$

$0.5 \%$

$0.0 \%$

Moments

maximum

0.9900

Mean

Std Dev

Std Err Mean

upper $95 \%$ Mean

lower 95\% Mean

0.0211122

20.310071

20.227114

$\mathrm{N}$

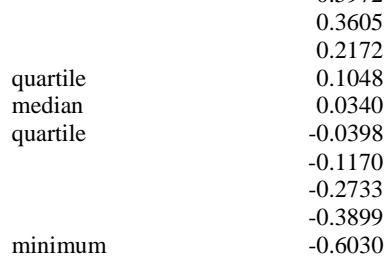

Zr (mg/L)

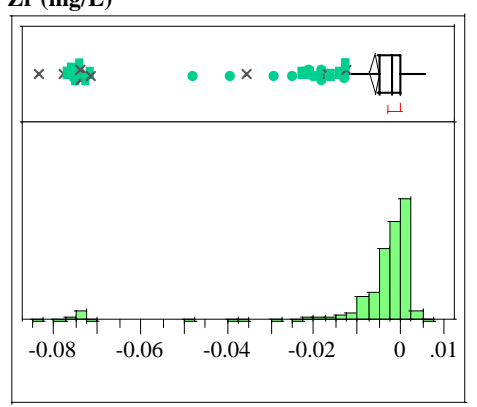

Quantiles

$100.0 \%$ maximum 0.0050

$99.5 \% \quad 0.0040$

$90.0 \%$

$75.0 \%$

$50.0 \%$

$25.0 \%$

$10.0 \%$

$2.5 \%$

$0.5 \%$

$0.0 \%$

quartile

median

quartile

0.0382205

0.1485597

0.05117

0.0252709

508

$-0.0045$

minimum

$-0.0120$

$-0.0743$

$-0.0775$

$-0.0840$

Moments

Mean

Std Dev

Std Err Mean

upper 95\% Mean

lower 95\% Mean

0.0014531

0.000359

$\mathrm{N}$ 
WSRC-TR-2004-00576

Revision 0

\section{Exhibit A12. Histograms and Descriptive Statistics for Mixed Acid Measurements of} Calibration and Bench Standards

STCd=SM34

Distributions

Al (mg/L)

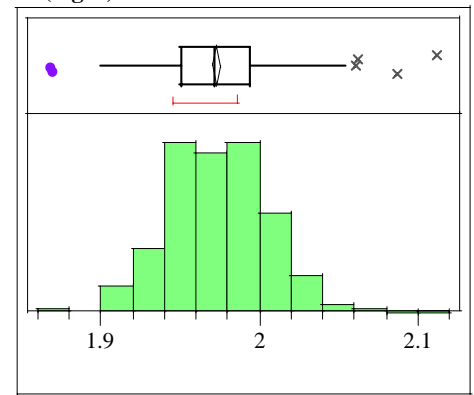

Quantiles

$100.0 \% \quad$ maximum

$99.5 \%$

$97.5 \%$

$90.0 \%$

$75.0 \%$

$50.0 \%$

$25.0 \%$

$10.0 \%$

$2.5 \%$

$0.5 \%$

$0.0 \%$

Moments

Mean

Std Dev

Std Err Mean

upper 95\% Mean

lower 95\% Mean

$\mathrm{N}$

Ca (mg/L)

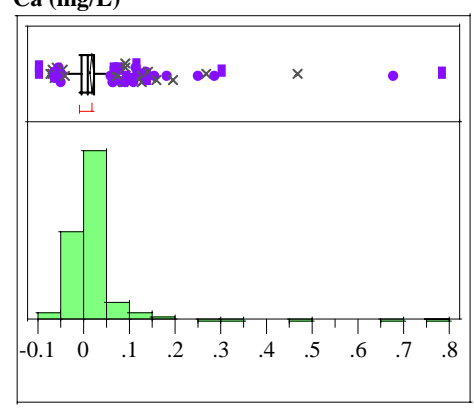

Quantiles

$\begin{array}{ll}100.0 \% & \text { maximum } \\ 99.5 \% & \\ 97.5 \% & \\ 90.0 \% & \\ 75.0 \% & \text { quartile } \\ 50.0 \% & \text { median } \\ 25.0 \% & \text { quartile } \\ 10.0 \% & \\ 2.5 \% & \\ 0.5 \% & \\ 0.0 \% & \text { minimum } \\ \text { Moments } & \\ & \\ \text { Mean } & \\ \text { Std Dev } & \\ \text { Std Err Mean } & \\ \text { upper 95\% Mean } \\ \text { lower 95\% Mean } \\ \text { N }\end{array}$

$\mathrm{N}$

1.9360

minimum

1.9761822

$-0.0970$

0.0028657

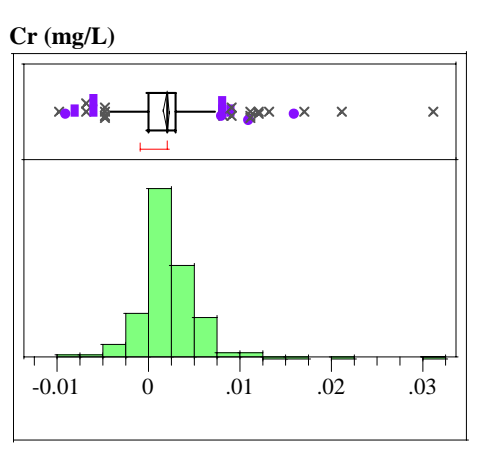

Quantiles

$100.0 \%$ maximum 0.0310

$99.5 \% \quad 0.0188$

$97.5 \%$

$90.0 \%$

$75.0 \%$

$50.0 \%$

$25.0 \%$

$10.0 \%$

$2.5 \%$

$0.5 \%$

$0.0 \%$

Moments

quartile

median

quartile

1.8870

1.8690

1.9734244

0.0316693

0.0014037

1.9706666

509

Mean

Std Dev

Std Err Mean

upper 95\% Mean

lower 95\% Mean

$\mathrm{N}$

Cu (mg/L)

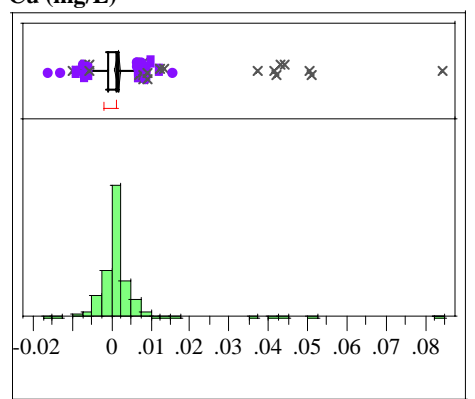

Quantiles

$100.0 \%$

$99.5 \%$

$97.5 \%$

$90.0 \%$

$75.0 \%$

$50.0 \%$

$25.0 \%$

$10.0 \%$

$2.5 \%$

$0.5 \%$

$0.0 \%$

Moments

maximum

quartile

median

quartile

$-0.0190$

0.0179764

0.064652

0.0236064

0.0123464
Mean

Std Dev

Std Err Mean

upper 95\% Mean

lower 95\% Mean

$\mathrm{N}$
0.000312

509
Fe (mg/L)

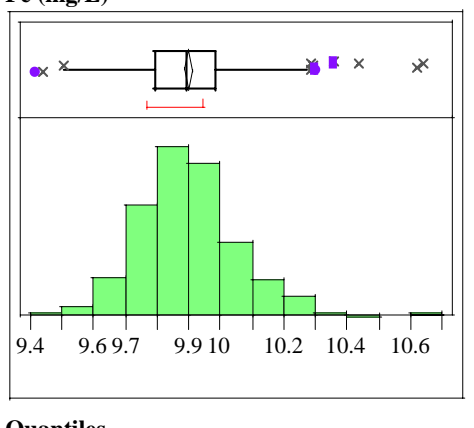

Quantiles

maximum

10.640

$99.5 \%$

$97.5 \%$

$90.0 \%$

$75.0 \%$

$50.0 \%$

$25.0 \%$

$10.0 \%$

$2.5 \%$

$0.5 \%$

$0.0 \%$

Moments

quartile

median

quartile

$-0.0010$

-0.0040
-0.0084

$-0.0100$

Mean

Std Dev

Std Err Mean

upper 95\% Mean

lower 95\% Mean

0.0034051

0.0001509

0.0022926

0.0016996

509

K (mg/L)

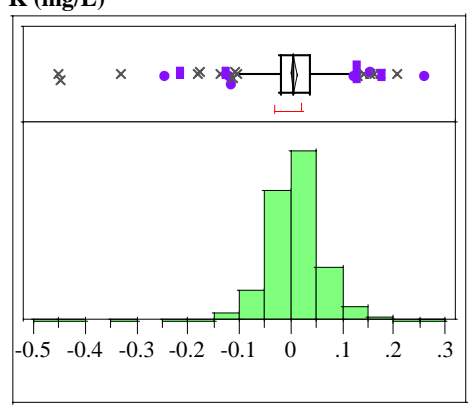

Quantiles

$100.0 \%$

maximum

0.2600

$99.5 \%$

$97.5 \%$

$90.0 \%$

$75.0 \%$

$50.0 \%$

$25.0 \%$

$10.0 \%$

$2.5 \%$

$0.5 \%$

$0.0 \%$

minimum

$-0.0030$

$-0.0060$

Moments

quartile

median

quartile

Mean

Std Dev

Std Err Mean

upper 95\% Mean

lower 95\% Mean

$\mathrm{N}$
10.517

10.249

10.108

9.986

9.796

9.714

9.615

9.474

9.418

9.9013301

0.1585996

0.0070298

9.9151411

9.887519

509 
WSRC-TR-2004-00576

Revision 0

\section{Exhibit A12. Histograms and Descriptive Statistics for Mixed Acid Measurements of} Calibration and Bench Standards

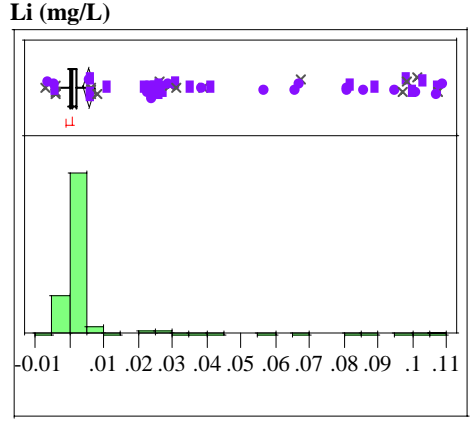

Quantiles

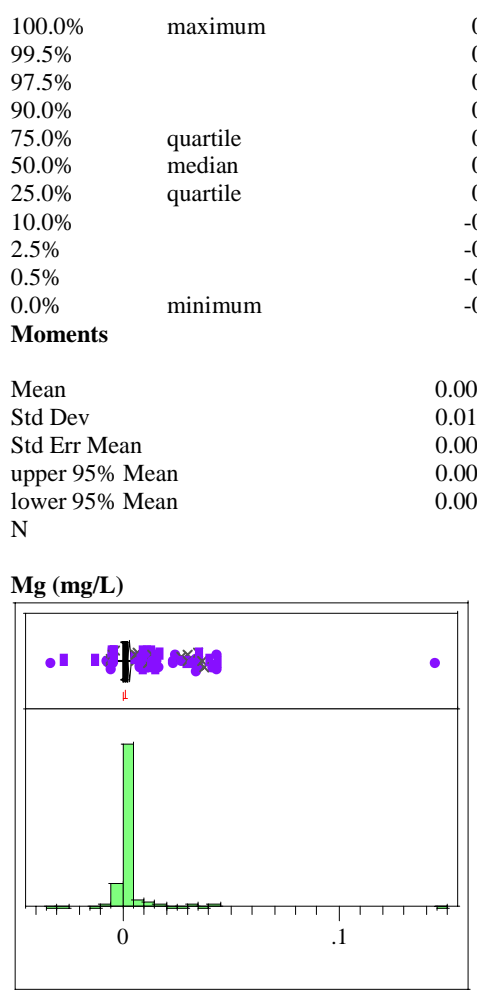

Quantiles

$100.0 \%$

$99.5 \%$

$97.5 \%$

$90.0 \%$

$75.0 \%$

$50.0 \%$

$25.0 \%$

$10.0 \%$

$2.5 \%$

$0.5 \%$

$0.0 \%$

minimum

Mean

Std Dev

Std Err Mean

upper $95 \%$ Mean

lower 95\% Mean

$\mathrm{N}$
Mn (mg/L)

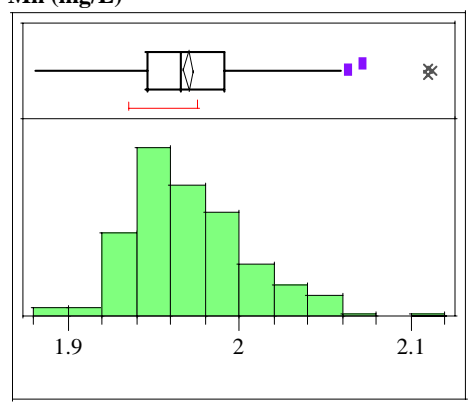

Quantiles

$\begin{array}{ll}100.0 \% & \text { maximum } \\ 99.5 \% & \\ 97.5 \% & \\ 90.0 \% & \\ 75.0 \% & \text { quartile } \\ 50.0 \% & \text { median } \\ 25.0 \% & \text { quartile } \\ 10.0 \% & \\ 2.5 \% & \\ 0.5 \% & \\ 0.0 \% & \text { minimum } \\ \text { Moments } & \\ & \\ \text { Mean } & \\ \text { Std Dev } & \\ \text { Std Err Mean } & \\ \text { upper 95\% Mean } \\ \text { lower 95\% Mean } \\ \text { N }\end{array}$

Na (mg/L)

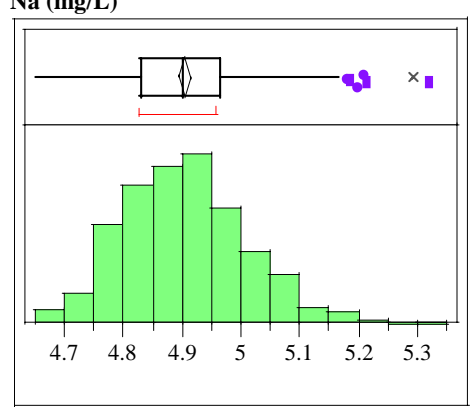

Quantiles

$100.0 \%$ maximum

$99.5 \%$

$97.5 \%$

$90.0 \%$

$75.0 \%$

$50.0 \%$

$25.0 \%$

$10.0 \%$

$2.5 \%$

$0.5 \%$

$0.0 \%$ minimum

Moments

quartile

median

Mean

Std Dev

Std Err Mean

upper 95\% Mean

lower 95\% Mean

$\mathrm{N}$

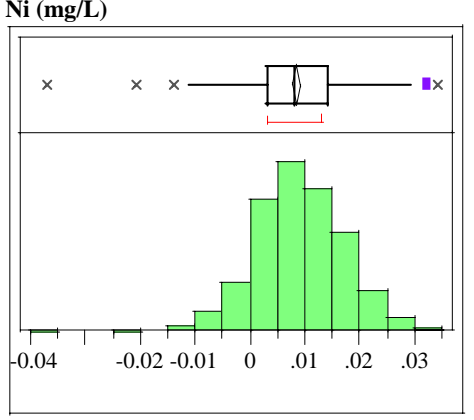

Quantiles

$100.0 \%$

$99.5 \%$

$97.5 \%$

$90.0 \%$

$75.0 \%$

$50.0 \%$

$25.0 \%$

$10.0 \%$

$2.5 \%$

$0.5 \%$

$0.0 \%$

Moments

maximum

0.0340

0.0303

0.0243

0.0243
0.0190

0.0140

0.0080

0.0030

0.0030
-0.0010

$-0.0080$

$-0.0171$

Mean

Std Dev

Std Err Mean

upper 95\% Mean

lower 95\% Mean

quartile

quartile

minimum

$-0.0370$

0.0015615

1.9738105

509

$\mathrm{N}$

0.008556

0.0082909

0.0003675

0.009278

0.007834

Si (mg/L)

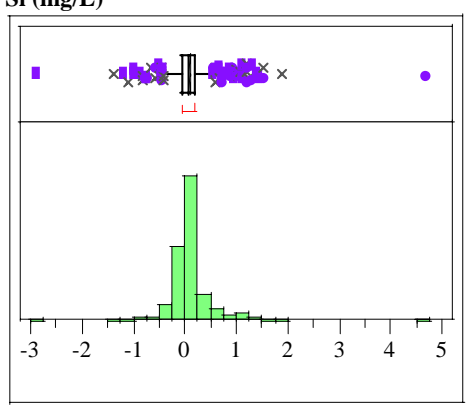

Quantiles

$100.0 \%$ maximum 4.701

$99.5 \% \quad 1.701$

$97.5 \%$

$75.0 \%$

$50.0 \%$

$25.0 \%$

$10.0 \%$

$2.5 \%$

$0.5 \%$

$0.0 \%$

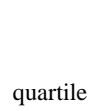

1.701
1.178

0.454

0.188

0.188

$-0.050$

$-0.205$

$-0.738$

$-1.291$

Moments

minimum

$-2.901$

Mean

Std Dev

Std Err Mean

upper 95\% Mean

lower 95\% Mean

$\mathrm{N}$ 
WSRC-TR-2004-00576

Revision 0

Exhibit A12. Histograms and Descriptive Statistics for Mixed Acid Measurements of Calibration and Bench Standards

Ti (mg/L)

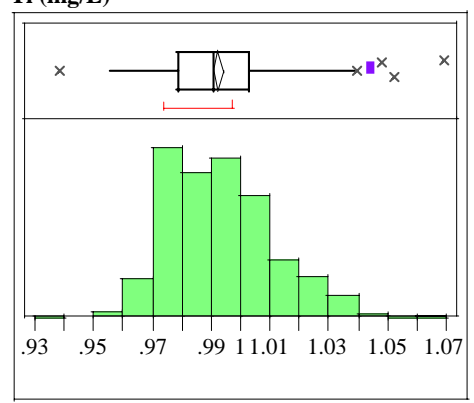

Quantiles

$99.5 \%$

$97.5 \%$

$90.0 \%$

$75.0 \%$

$50.0 \%$

$25.0 \%$

$10.0 \%$

$2.5 \%$

$0.5 \%$

$0.0 \%$

Moments

Mean

Std Dev

Std Err Mean

upper 95\% Mean

lower 95\% Mean

$\mathrm{N}$

U (mg/L)

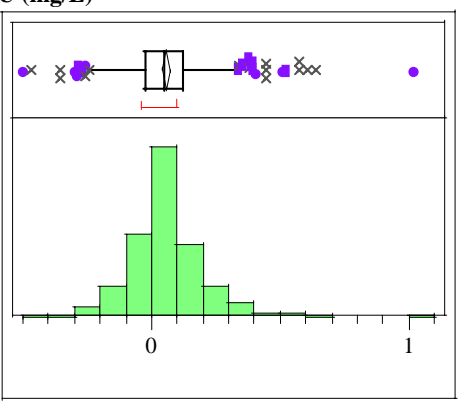

Quantiles

$100.0 \% \quad$ maximum

$99.5 \%$

$97.5 \%$

$90.0 \%$

$75.0 \%$

$50.0 \%$

$25.0 \%$

$10.0 \%$

$2.5 \%$

$0.5 \%$

$0.0 \%$

Moments

Mean

Std Dev

Std Err Mean

upper 95\% Mean

lower 95\% Mean

$\mathrm{N}$
$\operatorname{Zr}(\mathrm{mg} / \mathrm{L})$

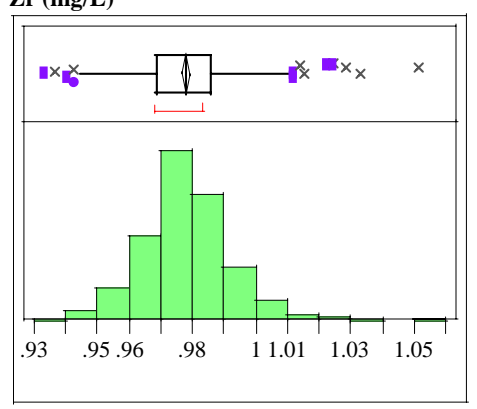

Quantiles

$100.0 \%$

$99.5 \%$

$97.5 \%$

$90.0 \%$

$75.0 \%$

$50.0 \%$

$25.0 \%$

$10.0 \%$

$2.5 \%$

$0.5 \%$

$0.0 \%$

Moments

maximum

Mean

Std Dev

Std Err Mean

upper 95\% Mean

lower 95\% Mean

$\mathrm{N}$

STCd=SM35

Distributions

Al (mg/L)

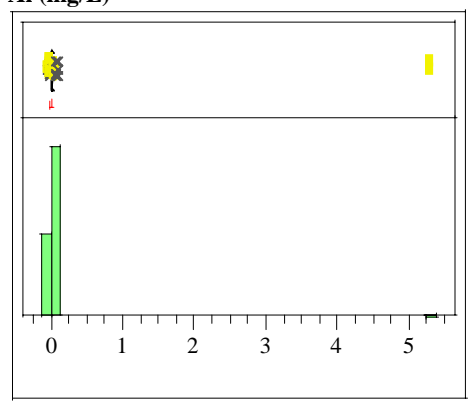

Quantiles

quartile

median

quartile

minimum

(1)

upper 95\% Mean

lower 95\% Mean

$\mathrm{N}$
$99.5 \%$

$97.5 \%$

$90.0 \%$

$75.0 \%$

$50.0 \%$

$25.0 \%$

$10.0 \%$

$2.5 \%$

$0.5 \%$

$0.0 \%$

Moments

Mean

Std Dev

Std Err Mean

maximum

quartile

median

quartile

minimum

0.147824

0.0707195

0.044974
Ca (mg/L)

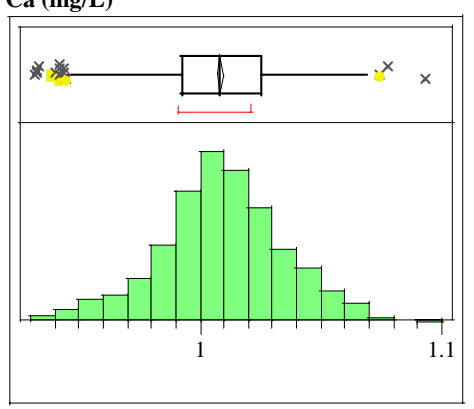

Quantiles

$100.0 \% \quad$ maximum $\quad 1.0930$

$99.5 \% \quad 1.0675$

$\begin{array}{ll}97.5 \% & 1.0590\end{array}$

$90.0 \% \quad 1.0410$

$\begin{array}{lll}75.0 \% & \text { quartile } & 1.0250\end{array}$

$50.0 \%$ median 1.0080

$25.0 \%$ quartile 0.9930

$10.0 \% \quad 0.9770$

$2.5 \% \quad 0.9550$

$0.5 \% \quad 0.9390$

$0.0 \%$ minimum 0.9310

Moments

0.977725

0.0144137

0.0006389

0.9789801

0.9764698

Mean

Std Dev

Std Err Mean

upper 95\% Mean

lower 95\% Mean

1.0084511

0.0252653

0.0007604

1.0099431

1.0069591

$\operatorname{Cr}(\mathrm{mg} / \mathrm{L})$

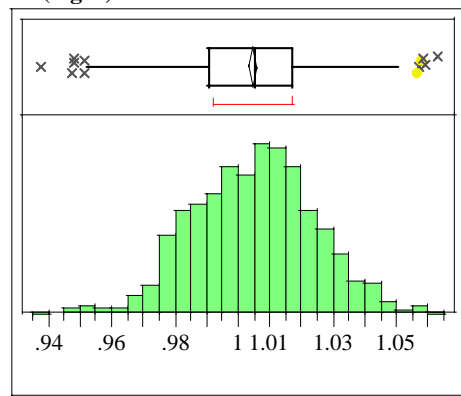

Quantiles

$100.0 \%$

$99.5 \%$

$97.5 \%$

$90.0 \%$

$75.0 \%$

$50.0 \%$

$25.0 \%$

$10.0 \%$

$2.5 \%$

$0.5 \%$

$0.0 \%$

maximum

1.0630

1.0630
-1.0420

quartile

median

quartile

$-0.011$

$-0.062$

Moments

minimum

1.0290

1.0290
1.0170

1.0050

0.9910

0.9800

0.9680

0.9510

Mean

Std Dev

Std Err Mean

upper 95\% Mean

0.2252538

0.0067793

0.0270211

0.0004173

$\mathrm{N}$
1.004529

0.0190072

0.000572

1.0056514

1.0034066 
WSRC-TR-2004-00576

Revision 0

\section{Exhibit A12. Histograms and Descriptive Statistics for Mixed Acid Measurements of} Calibration and Bench Standards

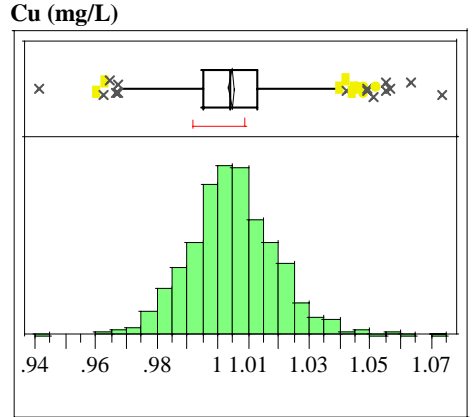

Quantiles

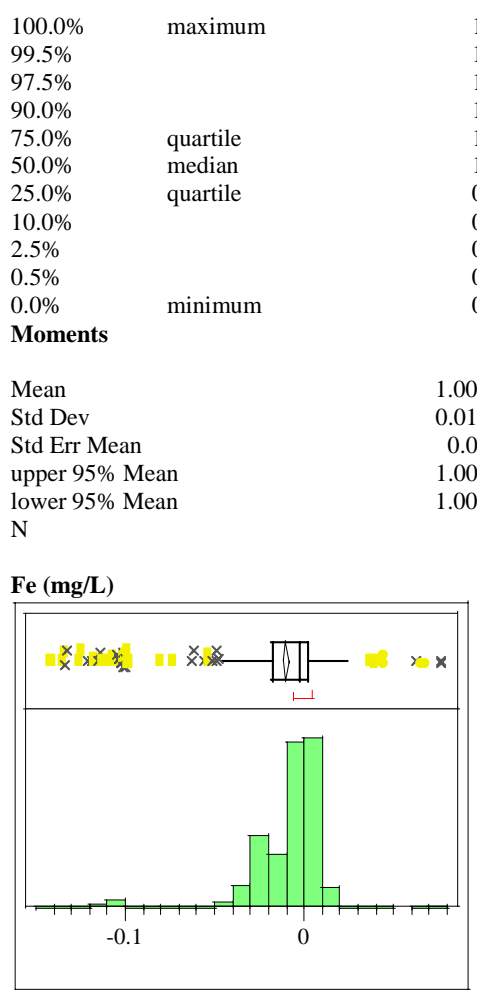

Quantiles

$100.0 \% \quad$ maximum

$99.5 \%$

$97.5 \%$

$90.0 \%$

$75.0 \%$

$50.0 \%$

$25.0 \%$

$10.0 \%$

$2.5 \%$

$0.5 \%$

$0.0 \%$

Moments

minimum

Mean

Std Dev

Std Err Mean

upper 95\% Mean

lower 95\% Mean

$\mathrm{N}$
K (mg/L)

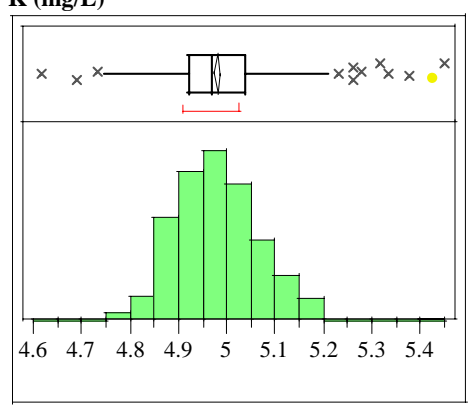

Quantiles

$100.0 \%$

$99.5 \%$

$97.5 \%$

$90.0 \%$

$75.0 \%$

$50.0 \%$

$25.0 \%$

$10.0 \%$

$2.5 \%$

$0.5 \%$

$0.0 \%$

Moments

maximum

Mean

Std Dev

Std Err Mean

upper 95\% Mean

lower 95\% Mean

$\mathrm{N}$

Li (mg/L)

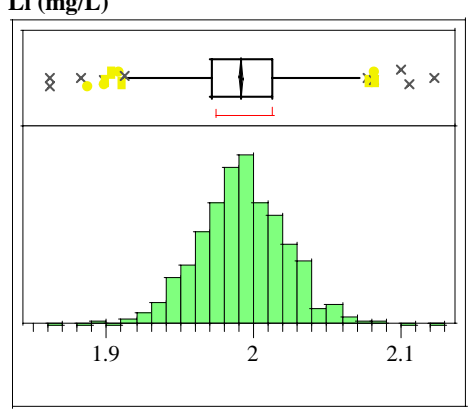

Quantiles

$100.0 \%$ maximum

$99.5 \%$

$97.5 \%$

$90.0 \%$

$75.0 \%$

$50.0 \%$

$25.0 \%$

$10.0 \%$

$2.5 \%$

$0.5 \%$

$0.0 \%$ minimum

Moments

quartile

median

quartile

minimum

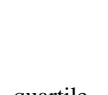

quartile

quartile

Mean

Std Dev

Std Err Mean

upper 95\% Mean

lower 95\% Mean

$\mathrm{N}$
$\operatorname{Mg}(\mathrm{mg} / \mathrm{L})$

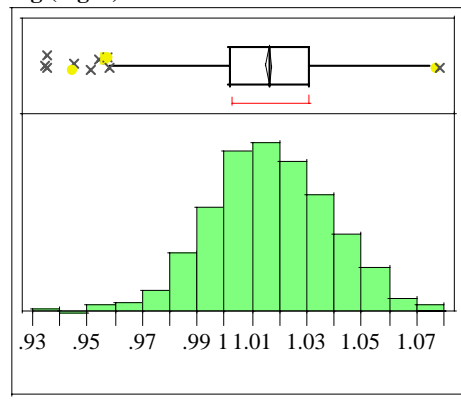

Quantiles

$100.0 \%$

$99.5 \%$

$97.5 \%$

$90.0 \%$

$75.0 \%$

$50.0 \%$

$25.0 \%$

$10.0 \%$

$2.5 \%$

$\begin{array}{llll}4.7683 & 0.5 \% & & 0.9481 \\ 4.6130 & 0.0 \% & \text { minimum } & 0.9340\end{array}$

$\begin{array}{llll}4.7683 & 0.5 \% & & 0.9481 \\ 4.6130 & 0.0 \% & \text { minimum } & 0.9340\end{array}$

Moments

maximum

1.0780

1.0720

1.0590

1.0590
1.0440

quartile

median

quartile

1.0020

0.9880

0.9730

$\begin{array}{llll}4.7683 & 0.5 \% & & 0.9481 \\ 4.6130 & 0.0 \% & \text { minimum } & 0.9340\end{array}$

Mean

Std Dev

Std Err Mean

upper 95\% Mean

lower 95\% Mean

0.0906175

0.0027273

4.9860958

4.9753933

1104

$\mathrm{N}$

0.0006693

1.0174438

1.0148171

1104

Mn (mg/L)

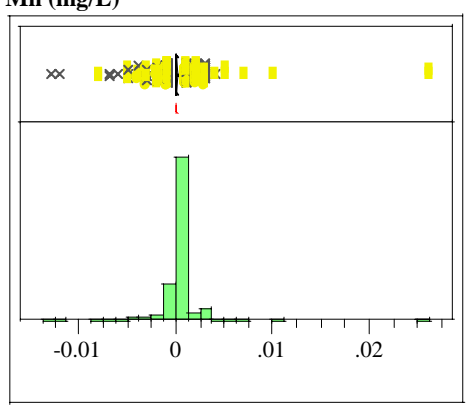

Quantiles

$100.0 \%$

maximum

0.0260

$99.5 \%$

$97.5 \%$

$90.0 \%$

$75.0 \%$

$50.0 \%$

$25.0 \%$

$10.0 \%$

$2.5 \%$

$0.5 \%$

$0.0 \%$

quartile

median

quartile

Moments

minimum

0.0030

0.0010

0.0000
0.0000

0.0000
0.0000

$-0.0010$

$-0.0030$

$-0.0070$

Mean

Std Dev

Std Err Mean

upper 95\% Mean

lower 95\% Mean

$\mathrm{N}$

0.000067

0.0017877

0.0000538

0.0001726

$-0.000039$

1104 
WSRC-TR-2004-00576

Revision 0

Exhibit A12. Histograms and Descriptive Statistics for Mixed Acid Measurements of Calibration and Bench Standards

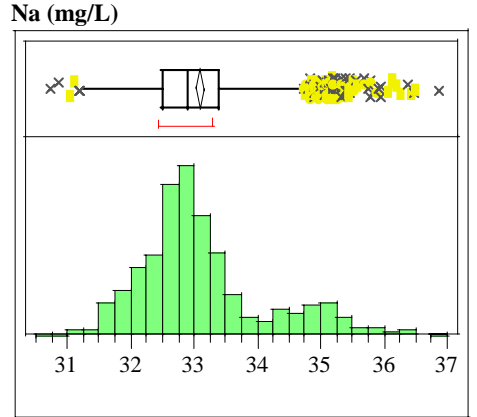

Quantiles

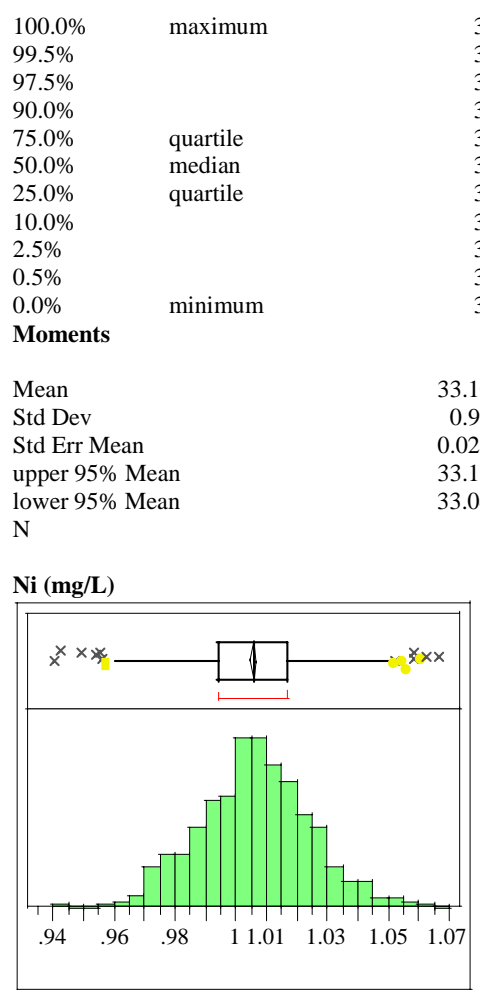

Quantiles

$100.0 \% \quad$ maximum

$99.5 \%$

$97.5 \%$

$90.0 \%$

$75.0 \%$

$50.0 \%$

$25.0 \%$

$10.0 \%$

$2.5 \%$

$0.5 \%$

$0.0 \%$ minimum

Moments

Mean

Std Dev

Std Err Mean

upper 95\% Mean

lower 95\% Mean

$\mathrm{N}$
Si (mg/L)

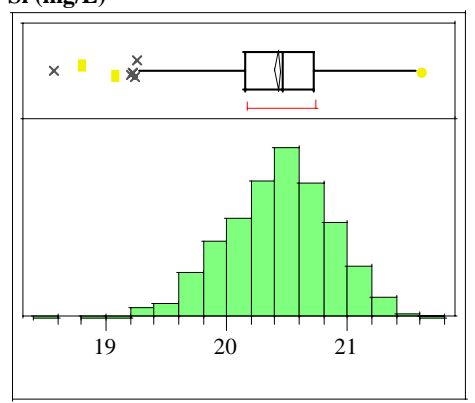

Quantiles

$100.0 \%$

$99.5 \%$

$97.5 \%$

$90.0 \%$

$75.0 \%$

$50.0 \%$

$25.0 \%$

$10.0 \%$

$2.5 \%$

$0.5 \%$

$0.0 \%$

Moments

maximum

Mean

Std Dev

Std Err Mean

upper 95\% Mean

lower 95\% Mean

$\mathrm{N}$

Ti (mg/L)

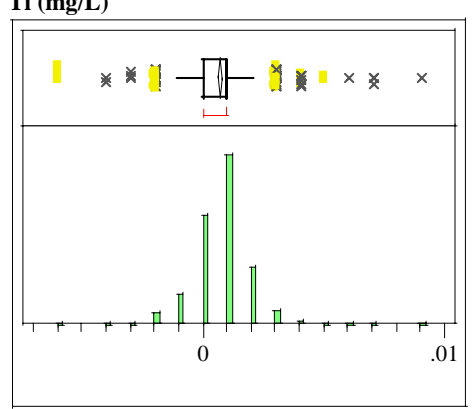

Quantiles

$100.0 \%$ maximum

$99.5 \%$

$97.5 \%$

$90.0 \%$

$75.0 \%$

$50.0 \%$

$25.0 \%$

$10.0 \%$

$2.5 \%$

$0.5 \%$

$0.0 \%$ minimum

Moments

quartile

median

quartile

Mean

Std Dev

Std Err Mean

upper 95\% Mean

lower 95\% Mean

$\mathrm{N}$
U (mg/L)

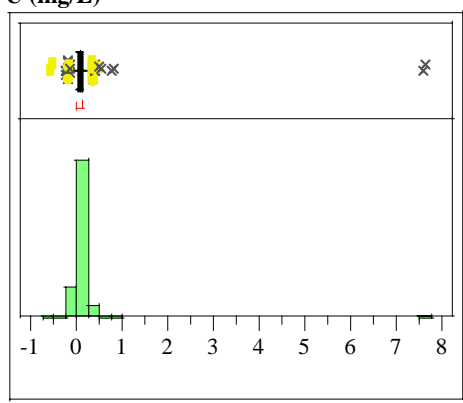

Quantiles

$100.0 \%$

maximum

7.594

$99.5 \%$

$97.5 \%$

$90.0 \%$

$75.0 \%$

$50.0 \%$

$25.0 \%$

$10.0 \%$

$2.5 \%$

$0.5 \%$

$0.0 \%$

Moments

\section{quartile}

median

quartile

Mean

Std Dev

Std Err Mean

upper 95\% Mean

lower 95\% Mean

0.0130055

20.450942

20.399906

1104

$\mathrm{N}$

Zr (mg/L)

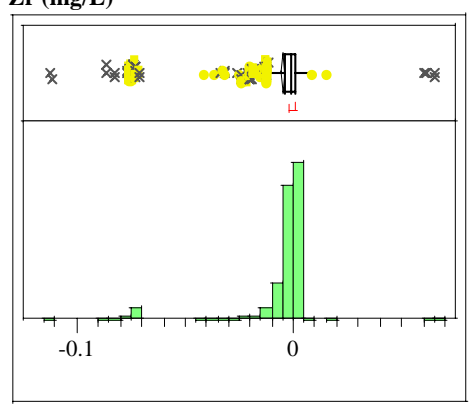

Quantiles

$100.0 \% \quad$ maximum $\quad 0.0650$

$99.5 \%$

$97.5 \%$

$90.0 \%$

$75.0 \%$

$50.0 \%$

$25.0 \%$

$10.0 \%$

$2.5 \%$

$0.5 \%$

$0.0 \%$

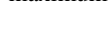

quartile

median

quartile

$-0.0010$

-0.0020
-0.0030

$-0.0060$

Moments

minimum

0.1080634

0.3372052

0.0101487

0.1279763

0.0881505

1104

\section{Mean}

Std Dev

Std Err Mean

upper 95\% Mean

lower 95\% Mean

$\mathrm{N}$ 0.0123
0.0030 0.0010

0.0010 $-0.0010$ $-0.0040$

$-0.0105$ $-0.0740$ $-0.0830$ $-0.1130$ 0.0163872 0.0004932 $-0.00419$ $-0.006125$ 1104 
WSRC-TR-2004-00576

Revision 0

\section{Exhibit A12. Histograms and Descriptive Statistics for Mixed Acid Measurements of} Calibration and Bench Standards

STCd=SM36

Distributions

Al (mg/L)

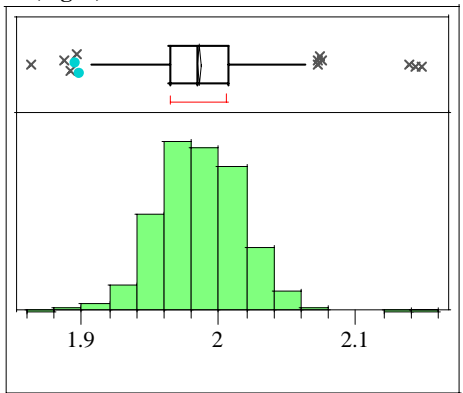

Quantiles

$100.0 \% \quad$ maximum

$99.5 \%$

$97.5 \%$

$90.0 \%$

$75.0 \%$

$50.0 \%$

$25.0 \%$

$10.0 \%$

$2.5 \%$

$0.5 \%$

$0.0 \%$

Moments

Mean

Std Dev

Std Err Mean

upper 95\% Mean

lower $95 \%$ Mean

$\mathrm{N}$

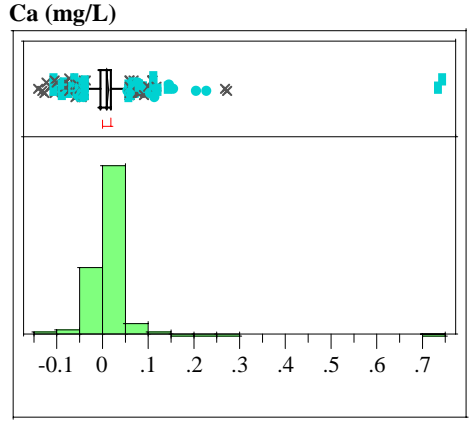

Quantiles

$\begin{array}{lcr}100.0 \% & \text { maximum } & 0.7410 \\ 99.5 \% & & 0.2191 \\ 97.5 \% & & 0.0810 \\ 90.0 \% & & 0.0370 \\ 75.0 \% & \text { quartile } & 0.0200 \\ 50.0 \% & \text { median } & 0.0090 \\ 25.0 \% & \text { quartile } & -0.0030 \\ 10.0 \% & & -0.0170 \\ 2.5 \% & & -0.0580 \\ 0.5 \% & & -0.1130 \\ 0.0 \% & -0.1410 \\ \text { Moments } & & \\ & & \\ \text { Mean } & & 0.0105228 \\ \text { Std Dev } & & 0.0451304 \\ \text { Std Err Mean } & & 0.001362 \\ \text { upper 95\% Mean } & 0.0131951 \\ \text { lower 95\% Mean } & 0.0078504 \\ \text { N } & \end{array}$

1.9490

1.8965

minimum

1.8620

1.9861302

0.0310151

0.000936

1.9879668

1.9842937

1098
Cr (mg/L)

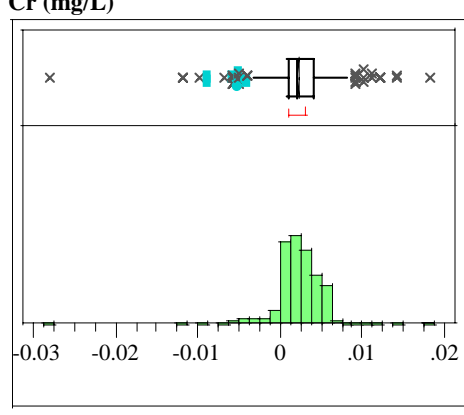

Quantiles

$100.0 \%$ maximum 0.0180

$99.5 \% \quad 0.0120$

$97.5 \%$

$90.0 \%$

$75.0 \%$

$50.0 \%$

$25.0 \%$

$10.0 \%$

$2.5 \%$

$0.5 \%$

$0.0 \% \quad$ minimum

Moments

quartile

median

quartile

Mean

Std Dev

Std Err Mean

upper 95\% Mean

lower 95\% Mean

$\mathrm{N}$

Cu (mg/L)

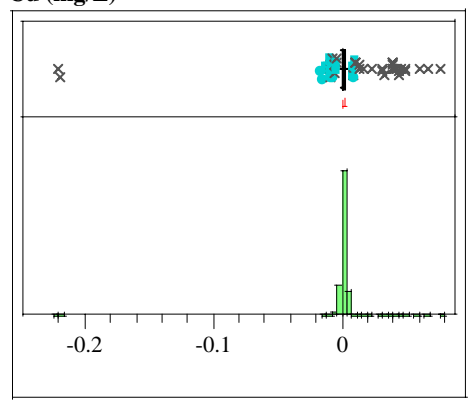

Quantiles

$100.0 \%$

$99.5 \%$

$97.5 \%$

$90.0 \%$

$75.0 \%$

$50.0 \%$

$25.0 \%$

$10.0 \%$

$2.5 \%$

$0.5 \%$

$0.0 \%$

Moments

minimum

Mean

Std Dev

Std Err Mean

upper 95\% Mean

lower 95\% Mean

$\mathrm{N}$
Fe (mg/L)

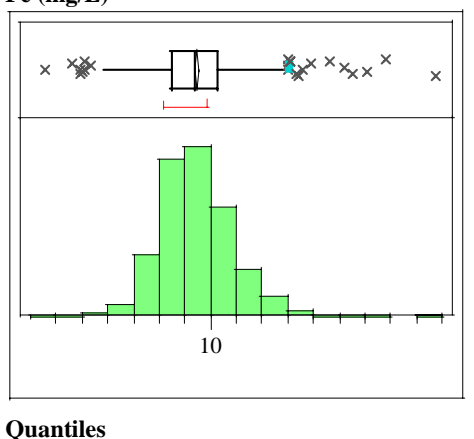

Quantiles

maximum

10.875

$99.5 \% \quad 10.488$

$97.5 \% \quad 10.255$

$75.0 \%$

quartile

median

$25.0 \%$

$10.0 \%$

$2.5 \%$

$0.5 \%$

$0.0 \%$

Moments

quartile

10.132
10.026

9.936

9.847

9.780

9.686

9.505

9.352

Mean

Std Dev

Std Err Mean

upper $95 \%$ Mean

lower 95\% Mean

minimum

9.9434681

0.1471541

0.0044409

9.9521817

0.0000825

0.0024142

0.0020904

1098

K (mg/L)

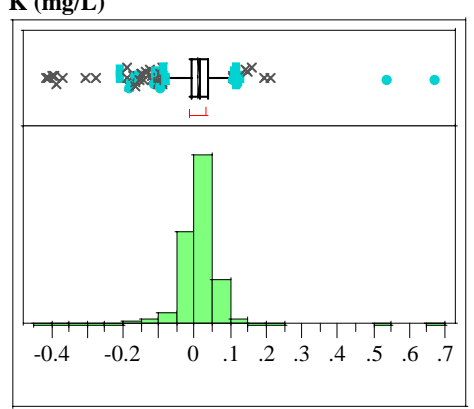

Quantiles

$100.0 \%$

maximum

0.6730

$99.5 \%$

$97.5 \%$

$90.0 \%$

$75.0 \%$

$50.0 \%$

$25.0 \%$

$10.0 \%$

$2.5 \%$

$0.5 \%$

$0.0 \%$

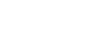

0.1526

0.0945

0.0610

0.0360

0.0360
0.0130

0.0130
-0.0100

$-0.0331$

-0.1261
-0.3801

Moments

minimum

$-0.4200$

Mean

Std Dev

Std Err Mean

upper 95\% Mean

lower 95\% Mean

$\begin{array}{rlr}0.000853 & \mathrm{~N} & 1098\end{array}$

0.0096667

0.0613561

0.0018516

0.0132998

0.0060335 
WSRC-TR-2004-00576

Revision 0

\section{Exhibit A12. Histograms and Descriptive Statistics for Mixed Acid Measurements of} Calibration and Bench Standards

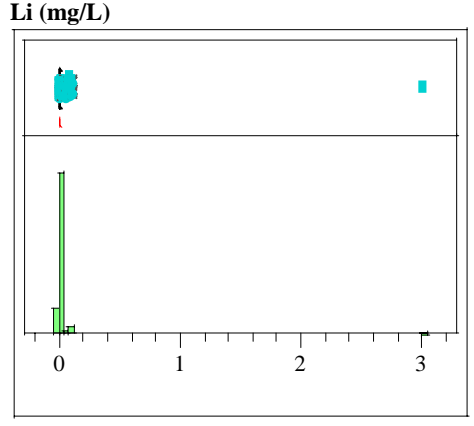

Quantiles

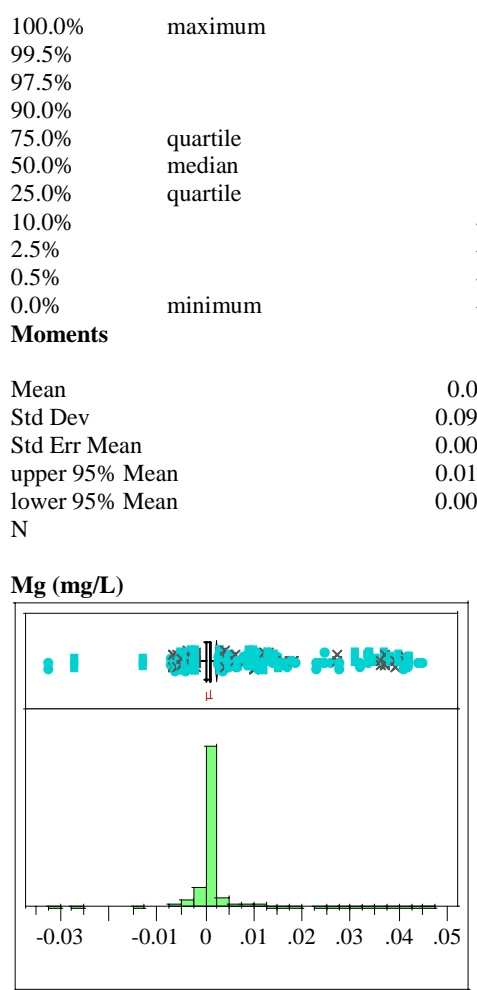

Quantiles

$100.0 \% \quad$ maximum

$99.5 \%$

$97.5 \%$

$90.0 \%$

$75.0 \%$

$50.0 \%$

$25.0 \%$

$10.0 \%$

$2.5 \%$

$0.5 \%$

$0.0 \%$
Moments

minimum

Mean

Std Dev

Std Err Mean

upper 95\% Mean

lower 95\% Mean

$\mathrm{N}$
Mn (mg/L)

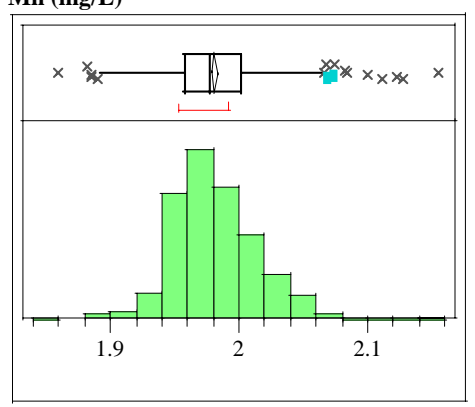

Quantiles

$100.0 \%$

$99.5 \%$

$97.5 \%$

$90.0 \%$

$75.0 \%$

$50.0 \%$

$25.0 \%$

$10.0 \%$

$2.5 \%$

$0.5 \%$

$0.0 \%$

Moments

maximum

Mean

Std Dev

Std Err Mean

upper 95\% Mean

lower 95\% Mean

$\mathrm{N}$

Na (mg/L)

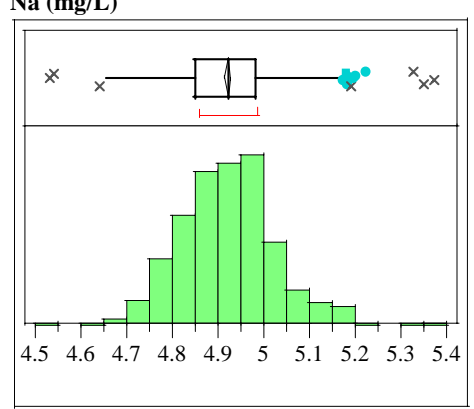

Quantiles

$100.0 \%$ maximum

$99.5 \%$

$97.5 \%$

$90.0 \%$

$75.0 \%$

$50.0 \%$

$25.0 \%$

$10.0 \%$

$2.5 \%$

$0.5 \%$

$0.0 \%$ minimum

Moments

quartile

median

quartile

Mean

Std Dev

Std Err Mean

upper 95\% Mean

lower 95\% Mean

$\mathrm{N}$
Ni (mg/L)

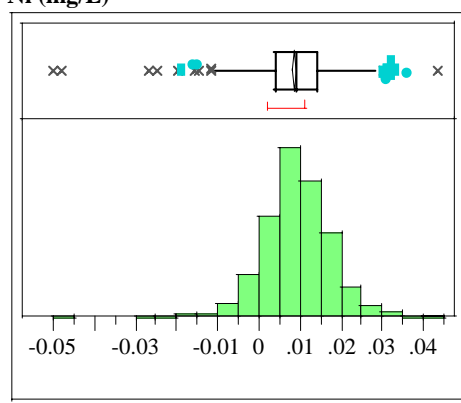

Quantiles

$100.0 \%$

maximum

0.0430

$99.5 \%$

$97.5 \%$

$90.0 \%$

$75.0 \%$

$50.0 \%$

$25.0 \%$

$10.0 \%$

$2.5 \%$

$0.5 \%$

$0.0 \%$

Moments

quartile

median

quartile

Mean

Std Dev

Std Err Mean

upper $95 \%$ Mean

lower 95\% Mean

0.0010008

1.9832953

1.9793678

1098

$\mathrm{N}$

Si (mg/L)

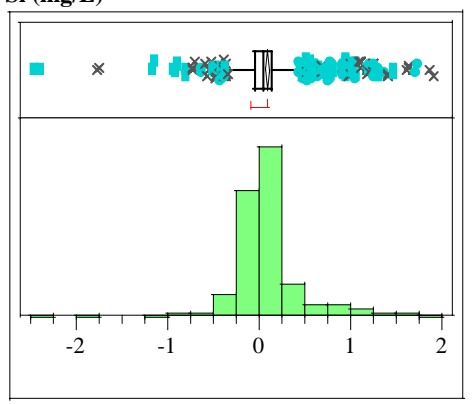

Quantiles

$100.0 \%$ maximum 1.888

$\begin{array}{ll}99.5 \% & 1.651\end{array}$

$97.5 \%$

$75.0 \%$

$50.0 \%$

$25.0 \%$

$10.0 \%$

$2.5 \%$

$0.5 \%$

$0.0 \%$

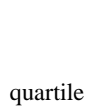

quartile
median

quartile

0.00025

0.0091808

0.0081998

1098

Moments

4.9208015

Mean

Std Dev

Std Err Mean

upper 95\% Mean

0.0030975

4.9268791

lower 95\% Mean

1098 
WSRC-TR-2004-00576

Revision 0

Exhibit A12. Histograms and Descriptive Statistics for Mixed Acid Measurements of Calibration and Bench Standards

Ti (mg/L)

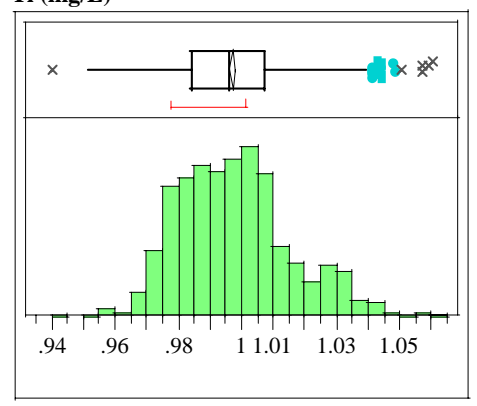

Quantiles

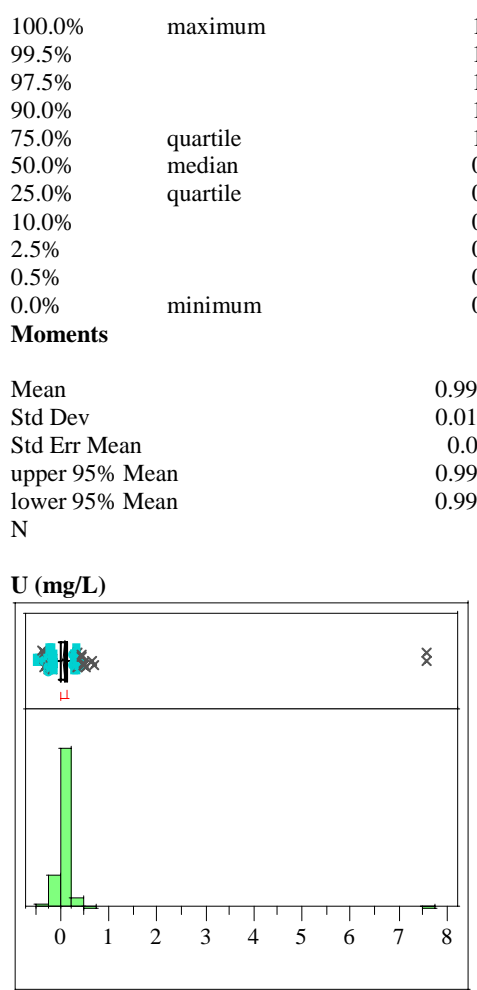

Quantiles

$100.0 \%$

$99.5 \%$

$97.5 \%$

$90.0 \%$

$75.0 \%$

$50.0 \%$

$25.0 \%$

$10.0 \%$

$2.5 \%$

$0.5 \%$

$0.0 \%$

Moments

Mean

Std Dev

Std Err Mean

upper 95\% Mean

lower 95\% Mean

$\mathrm{N}$
Zr (mg/L)

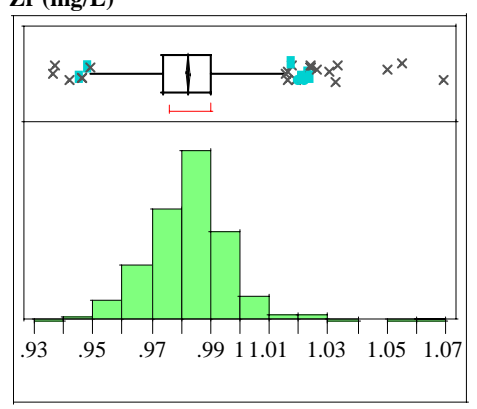

Quantiles

$100.0 \%$

$99.5 \%$

$97.5 \%$

$90.0 \%$

$75.0 \%$

$50.0 \%$

$25.0 \%$

$10.0 \%$

$2.5 \%$

$0.5 \%$

$0.0 \%$ minimum

Moments

maximum

quartile

median

quartile

Mean

Std Dev

Std Err Mean

upper 95\% Mean

lower 95\% Mean

$\mathrm{N}$

STCd=SM37

Distributions

Al (mg/L)

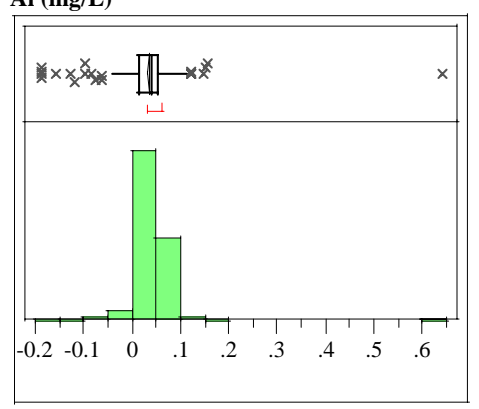

Quantiles

$100.0 \%$ maximum

$99.5 \%$

$97.5 \%$

$90.0 \%$

$75.0 \%$

$50.0 \%$

$25.0 \%$

$10.0 \%$

$2.5 \%$

$0.5 \%$

$0.0 \%$

0.0924772

0.3378057

0.0101945

0.1124801

0.0724743

1098
Moments

Mean

Std Dev

Std Err Mean

upper 95\% Mean

lower 95\% Mean

$\mathrm{N}$ quartile

median

quartile

minimum

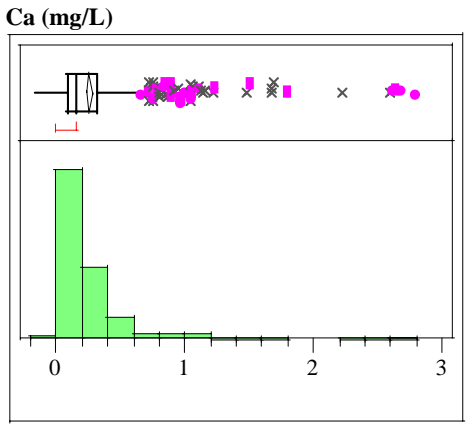

Quantiles

$100.0 \%$ maximum 2.796

$99.5 \% \quad 2.627$

$\begin{array}{ll}97.5 \% & 1.114\end{array}$

0.514

$\begin{array}{ll}75.0 \% & \text { quartile } \\ 50.0 \% & 0.320\end{array}$

$50.0 \%$ median 0.160

$25.0 \%$ quartile 0.091

$10.0 \% \quad 0.000$

$2.5 \% \quad 0.000$

$0.5 \% \quad-0.026$

$0.0 \%$ minimum -0.156

Moments

0.9822222

0.0137874

0.0004161

0.9830386

0.9814058

Mean

Std Dev

Std Err Mean

upper 95\% Mean

lower 95\% Mean

0.256359

0.3390257

0.0127957

0.2814815

Cr (mg/L)

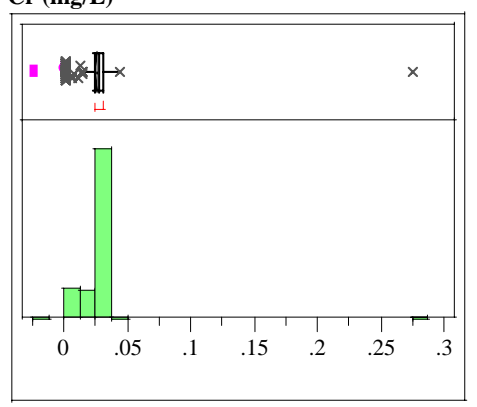

Quantiles

$100.0 \%$

$99.5 \%$

maximum

0.2750

$97.5 \%$

$90.0 \%$

$75.0 \%$

$50.0 \%$

$25.0 \%$

$10.0 \%$

$2.5 \%$

$0.5 \%$

$0.0 \%$

Moments

minimum

quartile

median

quartile

Mean

Std Dev

Std Err Mean

upper 95\% Mean

0.0420383

0.0015866

0.0378145
0.0315843

$\mathrm{N}$ 
WSRC-TR-2004-00576

Revision 0

\section{Exhibit A12. Histograms and Descriptive Statistics for Mixed Acid Measurements of} Calibration and Bench Standards

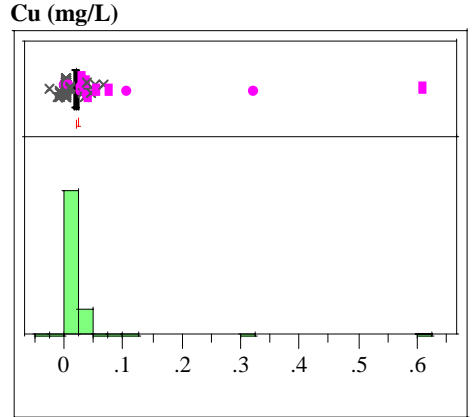

Quantiles

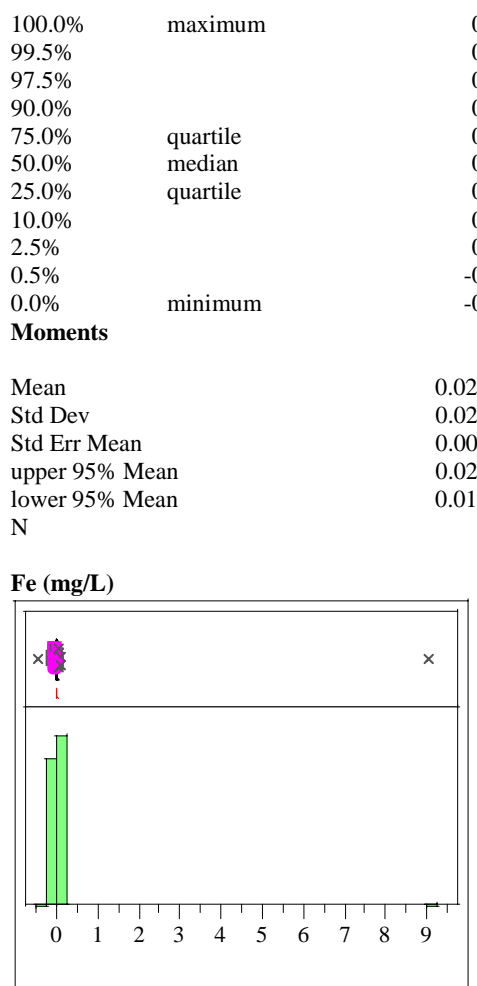

Quantiles

$100.0 \%$

$99.5 \%$

$97.5 \%$

$90.0 \%$

$75.0 \%$

$50.0 \%$

$25.0 \%$

$10.0 \%$

$2.5 \%$

$0.5 \%$

$0.0 \%$

minimum

Mean

Std Dev

Std Err Mean

upper $95 \%$ Mean

lower 95\% Mean

$\mathrm{N}$
K (mg/L)

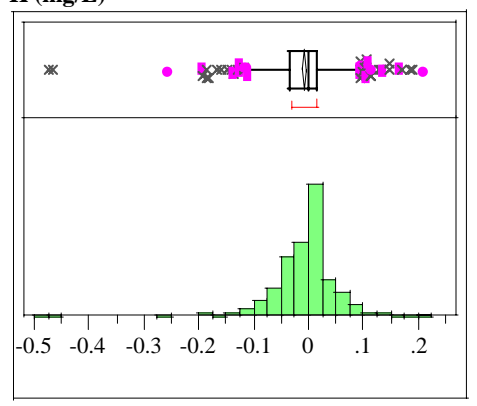

Quantiles

$\begin{array}{ll}100.0 \% & \text { maximum } \\ 99.5 \% & \\ 97.5 \% & \\ 90.0 \% & \\ 75.0 \% & \text { quartile } \\ 50.0 \% & \text { median } \\ 25.0 \% & \text { quartile } \\ 10.0 \% & \\ 2.5 \% & \\ 0.5 \% & \\ 0.0 \% & \text { minimum } \\ \text { Moments } & \\ & \\ \text { Mean } & \\ \text { Std Dev } & \\ \text { Std Err Mean } & \\ \text { upper 95\% Mean } \\ \text { lower 95\% Mean } \\ \text { N }\end{array}$

Li (mg/L)

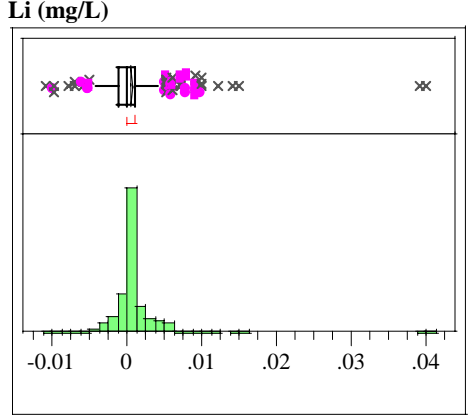

Quantiles

$100.0 \%$ maximum

$99.5 \%$

$97.5 \%$

$90.0 \%$

$75.0 \%$

$50.0 \%$

$25.0 \%$

$10.0 \%$

$2.5 \%$

$0.5 \%$

$0.0 \%$ minimum

Moments

quartile

median

quartile

Mean

Std Dev

Std Err Mean

upper 95\% Mean

lower 95\% Mean

$\mathrm{N}$
Mg (mg/L)

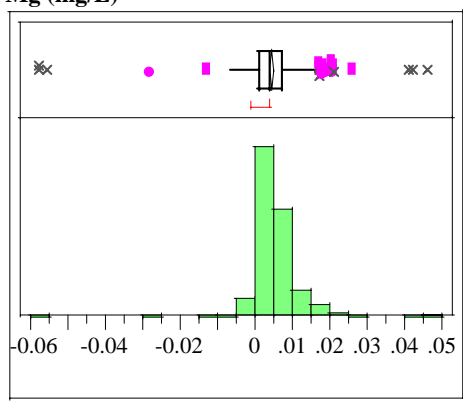

Quantiles

$100.0 \%$

maximum

0.0460

$99.5 \%$

$97.5 \%$

$90.0 \%$

$75.0 \%$

$50.0 \%$

$25.0 \%$

$10.0 \%$

$2.5 \%$

$0.5 \%$

$0.0 \%$

Moments

quartile

median

quartile

$-0.0657$

$-0.2251$

$-0.4760$

Mean

Std Dev

Std Err Mean

upper 95\% Mean

lower 95\% Mean

$-0.003566$

$-0.01201$

$\mathrm{N}$

minimum

0.0174

0.0100

0.0070

0.0040

0.0010

0.0000

$-0.0040$

$-0.0416$

$-0.0580$

Mn (mg/L)

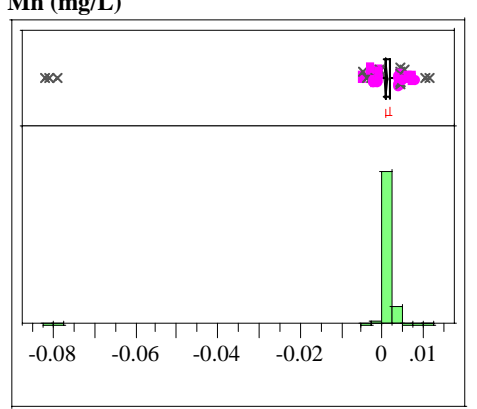

Quantiles

$100.0 \%$ maximum 0.0110

$99.5 \% \quad 0.0075$

$97.5 \%$

$75.0 \%$

$50.0 \%$

$25.0 \%$

$10.0 \%$

$2.5 \%$

$0.5 \%$

$0.0 \%$

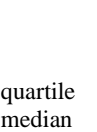

median

quartile

0.0043091

0.0068012

0.0002567

0.0048131

702

Moments

minimum

0.0040

0.0030

0.0020

0.0020
0.0010

0.0010

0.0000

$-0.0010$

$-0.0409$

$-0.0820$

\section{Mean}

Std Dev

Std Err Mean

upper $95 \%$ Mean

lower 95\% Mean

$\mathrm{N}$
702 
WSRC-TR-2004-00576

Revision 0

\section{Exhibit A12. Histograms and Descriptive Statistics for Mixed Acid Measurements of} Calibration and Bench Standards

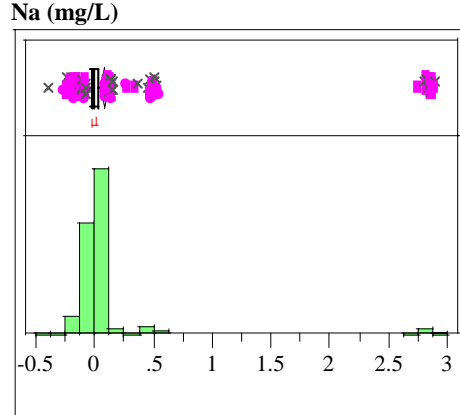

Quantiles

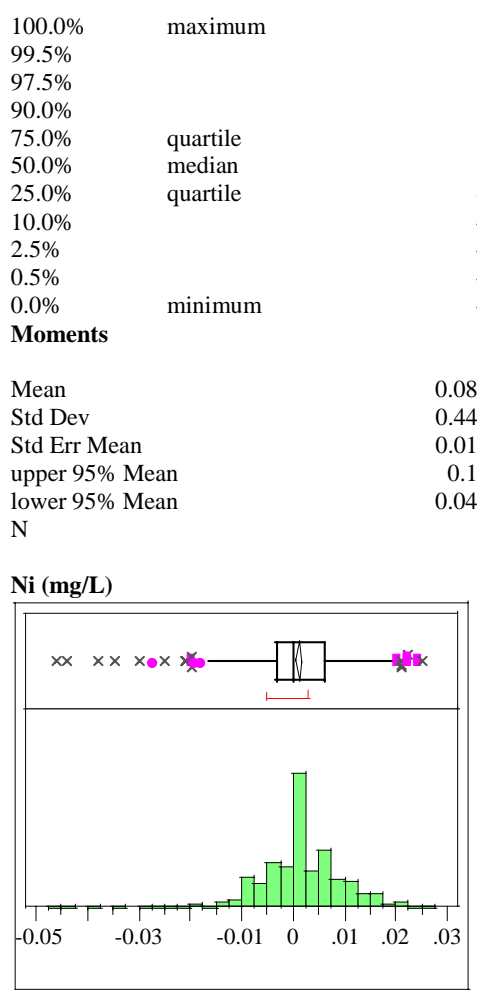

Quantiles

$100.0 \%$

$99.5 \%$

$97.5 \%$

$90.0 \%$

$75.0 \%$

$50.0 \%$

$25.0 \%$

$10.0 \%$

$2.5 \%$

$0.5 \%$

$0.0 \%$
Moments

maximum

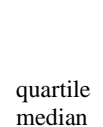

median

quartile

minimum

Mean

Std Dev

Std Err Mean

upper 95\% Mean

lower 95\% Mean

$\mathrm{N}$
Si (mg/L)

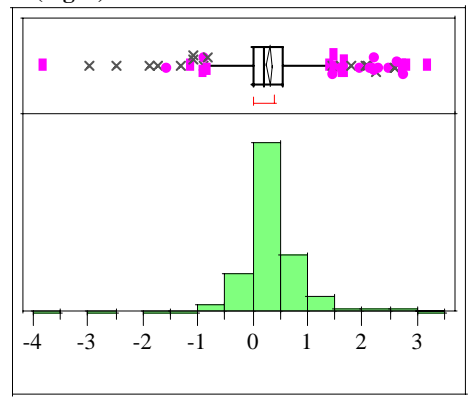

Quantiles

$100.0 \%$

$99.5 \%$

$97.5 \%$

$90.0 \%$

$75.0 \%$

$50.0 \%$

$25.0 \%$

$10.0 \%$

$2.5 \%$

$0.5 \%$

$0.0 \%$

Moments

maximum

Mean

Std Dev

Std Err Mean

upper 95\% Mean

lower 95\% Mean

$\mathrm{N}$

Ti (mg/L)

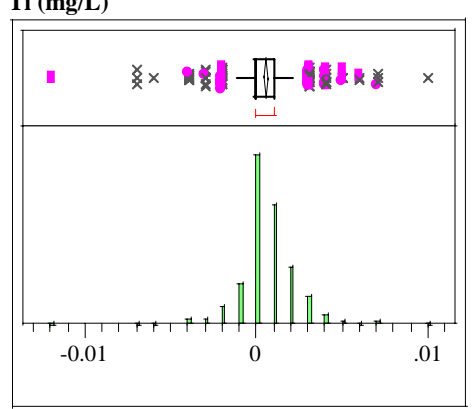

Quantiles

$100.0 \%$ maximum

$99.5 \%$

$97.5 \%$

$90.0 \%$

$75.0 \%$

$50.0 \%$

$25.0 \%$

$10.0 \%$

$2.5 \%$

$0.5 \%$

$0.0 \%$ minimum

Moments

quartile

median

Mean

Std Dev

Std Err Mean

upper 95\% Mean

lower 95\% Mean

$\mathrm{N}$
U (mg/L)

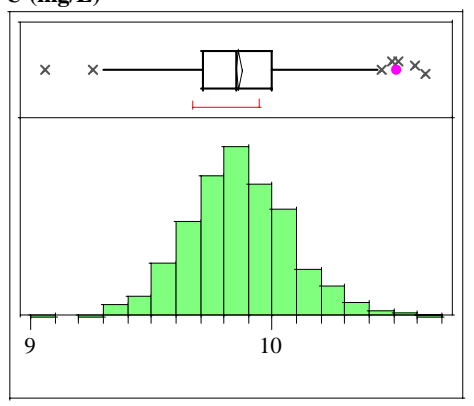

Quantiles

$100.0 \%$

maximum

10.629

$99.5 \%$

$97.5 \%$

$90.0 \%$

$75.0 \%$

$50.0 \%$

$25.0 \%$

$10.0 \%$

$2.5 \%$

$0.5 \%$

$0.0 \%$

Moments

quartile

median

quartile

Mean

Std Dev

Std Err Mean

upper 95\% Mean

lower 95\% Mean

0.3470193

702

$\mathrm{N}$

Zr (mg/L)

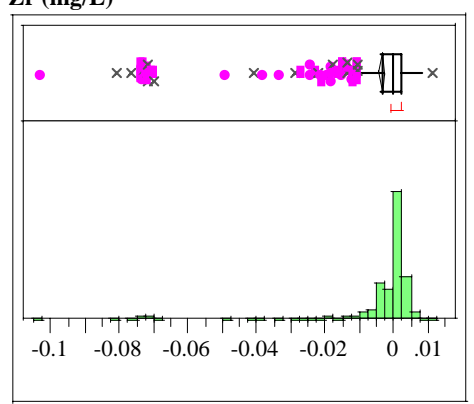

Quantiles

$100.0 \%$ maximum 0.0110

$99.5 \% \quad 0.0070$

$90.0 \%$

$75.0 \%$

$50.0 \%$

$25.0 \%$

$10.0 \%$

$2.5 \%$

$0.5 \%$

$0.0 \%$

minimum

9.8617365

0.215953

0.0081506

9.877739

9.8457339

702

Moments

quartile

median

quartile

minimum

0.0050

0.0030

0.0020

0.0020
0.0000

0.0000
-0.0030

$-0.0080$

$-0.0714$

$-0.0755$

$-0.1030$

\section{Mean}

Std Dev

Std Err Mean

upper 95\% Mean

lower 95\% Mean

$\mathrm{N}$
$-0.003417$

0.0137093

0.0005174

$-0.002401$

$-0.004433$ 
WSRC-TR-2004-00576

Revision 0

Exhibit A12. Histograms and Descriptive Statistics for Mixed Acid Measurements of Calibration and Bench Standards

STCd=SM38

Distributions

Al (mg/L)

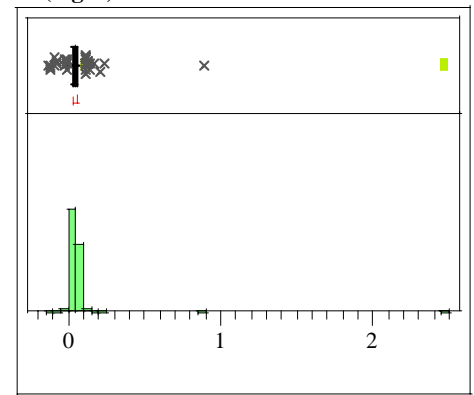

Quantiles

$100.0 \%$ maximum

$99.5 \%$

$97.5 \%$

$90.0 \%$

$75.0 \%$

$50.0 \%$

$25.0 \%$

$10.0 \%$

$2.5 \%$

$0.5 \%$

$0.0 \%$

Moments

Mean

Std Dev

Std Err Mean

upper 95\% Mean

lower $95 \%$ Mean

$\mathrm{N}$

Ca (mg/L)

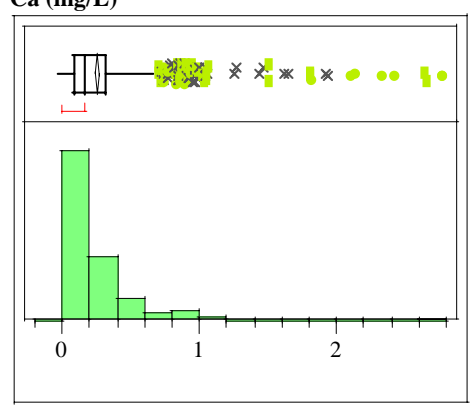

Quantiles

$\begin{array}{ll}100.0 \% & \text { maximum } \\ 99.5 \% & \\ 97.5 \% & \\ 90.0 \% & \\ 75.0 \% & \text { quartile } \\ 50.0 \% & \text { median } \\ 25.0 \% & \text { quartile } \\ 10.0 \% & \\ 2.5 \% & \\ 0.5 \% & \\ 0.0 \% & \text { minimum } \\ \text { Moments } & \\ & \\ \text { Mean } & \\ \text { Std Dev } & \\ \text { Std Err Mean } & \\ \text { upper 95\% Mean } \\ \text { lower 95\% Mean } \\ \text { N }\end{array}$

$\mathrm{N}$
Cr (mg/L)

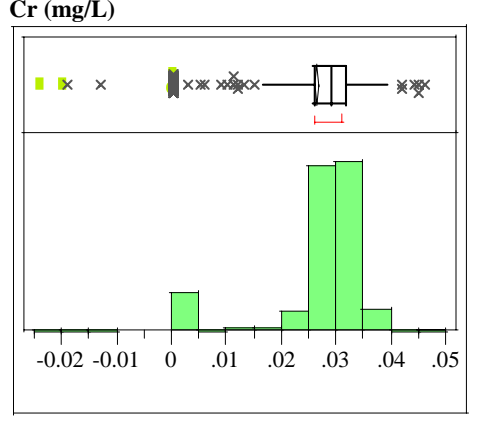

Quantiles

$100.0 \%$ maximum 0.0460

$99.5 \%$

$97.5 \%$

$90.0 \%$

$75.0 \%$

$50.0 \%$

$25.0 \%$

$10.0 \%$

$2.5 \%$

$0.5 \%$

$0.0 \%$

Moments

quartile

median

quartile

minimum

Mean

Std Dev

Std Err Mean

upper 95\% Mean

lower 95\% Mean

N

Cu (mg/L)

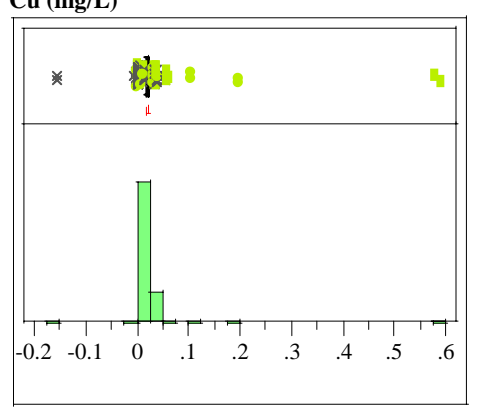

Quantiles

$100.0 \%$

$99.5 \%$

$97.5 \%$

$90.0 \%$

$75.0 \%$

$50.0 \%$

$25.0 \%$

$10.0 \%$

$2.5 \%$

$0.5 \%$

$0.0 \%$

Moments

minimum

Mean

Std Dev

Std Err Mean

upper 95\% Mean

lower 95\% Mean

$\mathrm{N}$

median
Fe (mg/L)

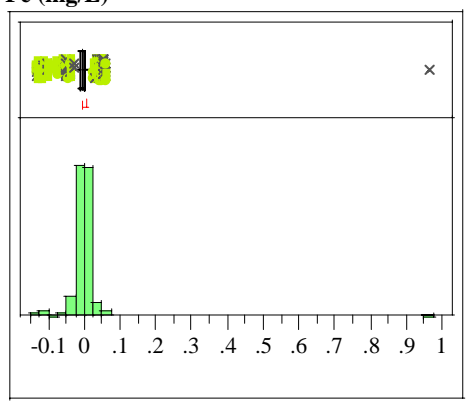

Quantiles

maximum

0.9620

$99.5 \%$

$97.5 \%$

$90.0 \%$

$75.0 \%$

$50.0 \%$

$25.0 \%$

$10.0 \%$

$2.5 \%$

$0.5 \%$

$0.0 \%$

Moments

quartile

median

quartile

0.0117

0.0000

$-0.0240$

Mean

Std Dev

Std Err Mean

upper 95\% Mean

lower 95\% Mean

$\mathrm{N}$

0.0270635

0.0259637

1176

K (mg/L)

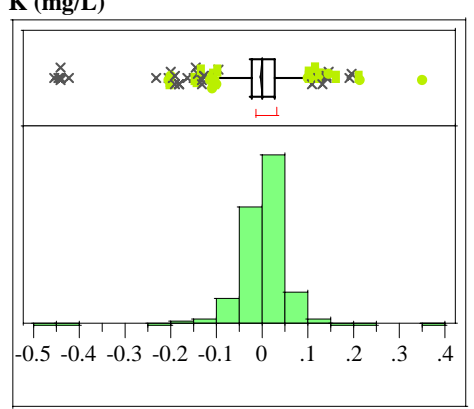

Quantiles

$100.0 \%$

$99.5 \%$

$97.5 \%$

$90.0 \%$

$75.0 \%$

$50.0 \%$

$25.0 \%$

$10.0 \%$

$2.5 \%$

$0.5 \%$

$0.0 \%$

Moments

maximum

0.3520

0.1642

0.0920

0.0523

0.0270

0.0270
0.0000

quartile

median

quartile

$-0.0510$

$-0.1208$

$-0.4261$

$-0.0042$

$-0.1590$

Mean

Std Dev

Std Err Mean

upper 95\% Mean

lower 95\% Mean

minimum

$-0.4580$

0.0213801

0.0007824

0.0229151

0.0198451

$\mathrm{N}$

$-0.001117$

0.0578298

0.0016864

0.0021913

$-0.004426$

1176 
WSRC-TR-2004-00576

Revision 0

\section{Exhibit A12. Histograms and Descriptive Statistics for Mixed Acid Measurements of} Calibration and Bench Standards

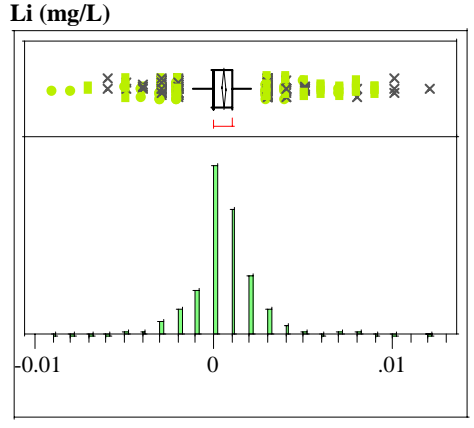

Quantiles

$\begin{array}{ll}100.0 \% & \text { maximum } \\ 99.5 \% & \\ 97.5 \% & \\ 90.0 \% & \\ 75.0 \% & \text { quartile } \\ 50.0 \% & \text { median } \\ 25.0 \% & \text { quartile } \\ 10.0 \% & \\ 2.5 \% & \\ 0.5 \% & \\ 0.0 \% & \text { minimum } \\ \text { Moments } & \\ & \\ \text { Mean } & \\ \text { Std Dev } & \\ \text { Std Err Mean } & \\ \text { upper 95\% Mean } \\ \text { lower 95\% Mean } \\ \text { N }\end{array}$

N

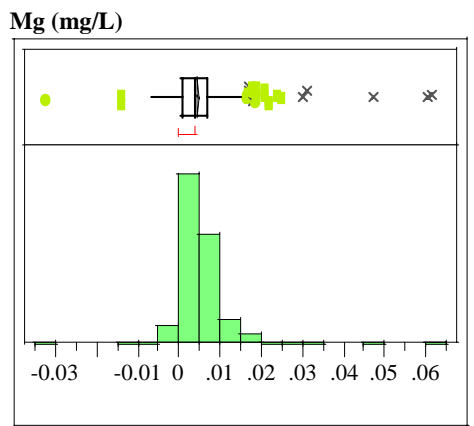

Quantiles

$100.0 \%$

maximum

$99.5 \%$

$97.5 \%$

$90.0 \%$

$75.0 \%$

$50.0 \%$

$25.0 \%$

$10.0 \%$

$2.5 \%$

$0.5 \%$

$0.0 \%$
Moments

minimum

Mean

Std Dev

Std Err Mean

upper 95\% Mean

lower 95\% Mea

$\mathrm{N}$
Mn (mg/L)

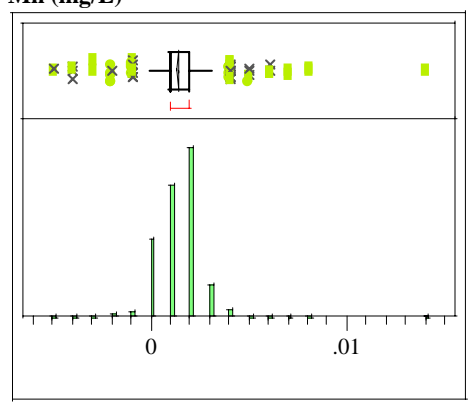

Quantiles

$\begin{array}{rll}0.0120 & 100.0 \% & \text { maximum } \\ 0.0090 & 99.5 \% & \\ 0.0046 & 97.5 \% & \\ 0.0020 & 90.0 \% & \\ 0.0010 & 75.0 \% & \text { quartile } \\ 0.0000 & 50.0 \% & \text { median } \\ 0.0000 & 25.0 \% & \text { quartile } \\ -0.0013 & 10.0 \% & \\ -0.0030 & 2.5 \% & \\ -0.0051 & 0.5 \% & \\ -0.0090 & 0.0 \% & \text { minimum } \\ & \text { Moments } & \\ & & \\ 0.000523 & \text { Mean } & \\ 0.0018981 & \text { Std Dev } & \\ 0.0000554 & \text { Std Err Mean } & \\ 0.0006316 & \text { upper 95\% Mean } \\ 0.0004144 & \text { lower 95\% Mean } \\ 1176 & \text { N }\end{array}$

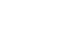

Na (mg/L)

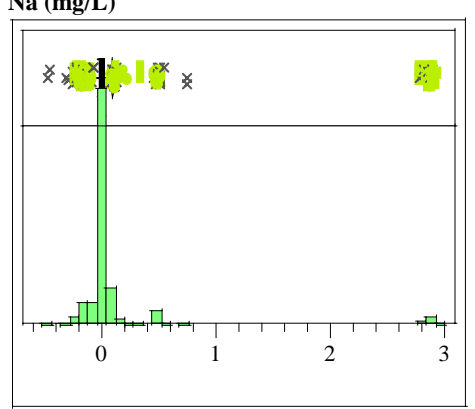

Quantiles

$100.0 \%$ maximum

$99.5 \%$

$97.5 \%$

$90.0 \%$

$75.0 \%$

$50.0 \%$

$25.0 \%$

$10.0 \%$

$2.5 \%$

$0.5 \%$

$0.0 \%$ minimum

Moments

quartile

median

quartile

$-0.0040$

$-0.0320$

0.0044702

0.0054961

0.0001603

0.0047847

0.0041558

1176

\section{Mean}

Std Dev

Std Err Mean

upper 95\% Mean

lower 95\% Mean

$\mathrm{N}$
0.0000364

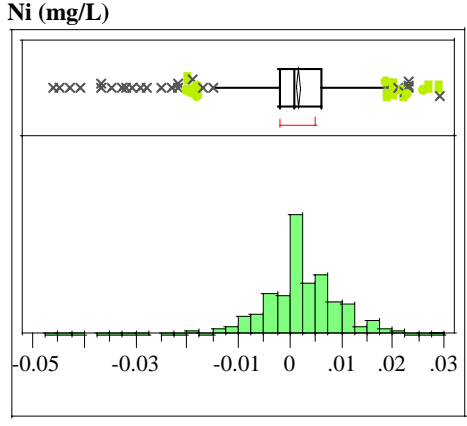

Quantiles

$100.0 \%$

maximum

0.0290

$99.5 \%$

$97.5 \%$

$90.0 \%$

$75.0 \%$

$50.0 \%$

$25.0 \%$

$10.0 \%$

$2.5 \%$

$0.5 \%$

$0.0 \%$

Moments

quartile

median

quartile

-0.0040
-0.0050

Mean

Std Dev

Std Err Mean

upper 95\% Mean

lower 95\% Mean

0.001471

0.0013283

1176

$\mathrm{N}$

Si (mg/L)

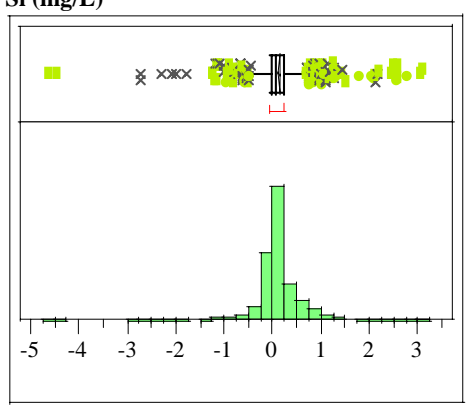

Quantiles

$100.0 \%$ maximum 3.072

$99.5 \% \quad 2.574$

$90.0 \%$

$75.0 \%$

$75.0 \%$
$50.0 \%$

$25.0 \%$

$10.0 \%$

$2.5 \%$

$0.5 \%$

$0.0 \%$

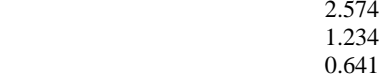

quartile

median

quartile

0.258

$-0.037$

$-0.220$

$-0.800$

$-2.139$

Moments

minimum

$-4.630$

Mean

Std Dev

Std Err Mean

upper 95\% Mean

lower 95\% Mean

$\mathrm{N}$

0.1311216

0.5247348

0.0153016

0.1611431

0.1011001

1176 
WSRC-TR-2004-00576

Revision 0

\section{Exhibit A12. Histograms and Descriptive Statistics for Mixed Acid Measurements of} Calibration and Bench Standards

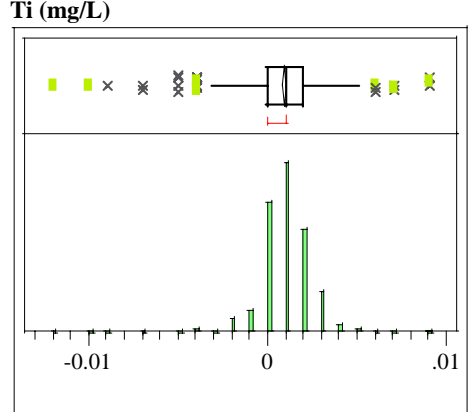

Quantiles

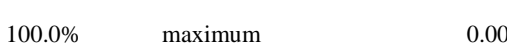

$99.5 \%$

$97.5 \%$

$90.0 \%$

$75.0 \%$

$50.0 \%$

$25.0 \%$

$10.0 \%$

$2.5 \%$

$0.5 \%$

$0.0 \%$

Moments

Mean

Std Dev

Std Err Mean

upper 95\% Mean

lower 95\% Mea

N

\section{U (mg/L)}

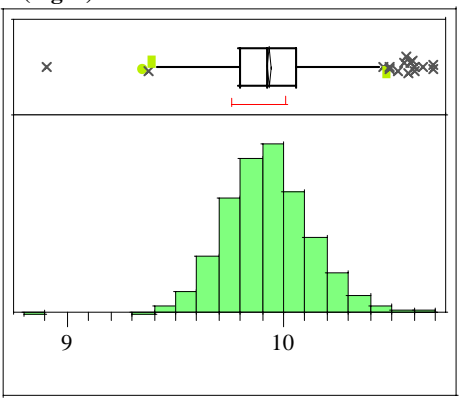

Quantiles

$\begin{array}{lcr}100.0 \% & \text { maximum } & 10.686 \\ 99.5 \% & & 10.588 \\ 97.5 \% & 10.354 \\ 90.0 \% & & 10.188 \\ 75.0 \% & \text { quartile } & 10.056 \\ 50.0 \% & \text { median } & 9.925 \\ 25.0 \% & \text { quartile } & 9.798 \\ 10.0 \% & & 9.689 \\ 2.5 \% & & 9.539 \\ 0.5 \% & & 9.436 \\ 0.0 \% & \text { minimum } & 8.898 \\ \text { Moments } & & \\ & & \\ \text { Mean } & & 9.9324371 \\ \text { Std Dev } & & 0.202447 \\ \text { Std Err Mean } & & 0.0059035 \\ \text { upper 95\% Mean } & 9.9440196 \\ \text { lower 95\% Mean } & 9.9208545 \\ \text { N }\end{array}$

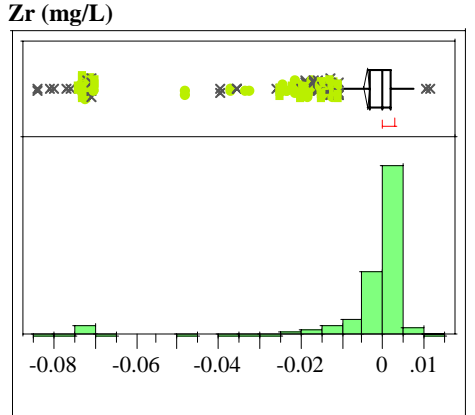

Quantiles

$\begin{array}{llr}100.0 \% & \text { maximum } & 0.0110 \\ 99.5 \% & & 0.0060 \\ 97.5 \% & & 0.0050 \\ 90.0 \% & & 0.0030 \\ 75.0 \% & \text { quartile } & 0.0020 \\ 50.0 \% & \text { median } & 0.0000 \\ 25.0 \% & \text { quartile } & -0.0030 \\ 10.0 \% & & -0.0110 \\ 2.5 \% & & -0.0720 \\ 0.5 \% & & -0.0761 \\ 0.0 \% & \text { minimum } & -0.0840 \\ \text { Moments } & & \\ & & -0.003895 \\ \text { Mean } & & 0.0149417 \\ \text { Std Dev } & & 0.0004357 \\ \text { Std Err Mean } & & -0.00304 \\ \text { upper 95\% Mean } & -0.004749 \\ \text { lower 95\% Mean } & 1176 \\ \text { N } & \end{array}$

$\mathrm{N}$

1176 
WSRC-TR-2004-00576

Revision 0

Exhibit A13. Components of Variance for the Mixed Acid Measurements of Calibration and Bench Standards

STCd=SM32

Least Squares Fit

Response Al (mg/L)

Summary of Fit

$\begin{array}{lr}\text { RSquare } & 0.731448 \\ \text { RSquare Adj } & 0.459397 \\ \text { Root Mean Square Error } & 0.037056 \\ \text { Mean of Response } & 0.010732 \\ \text { Observations (or Sum Wgts) } & 619\end{array}$

Analysis of Variance

$\begin{array}{lrrrr}\text { Source } & \text { DF } & \text { Sum of Squares } & \text { Mean Square } & \text { F Ratio } \\ \text { Model } & 311 & 1.1482092 & 0.003692 & 2.6886 \\ \text { Error } & 307 & 0.4215662 & 0.001373 & \text { Prob }>\text { F } \\ \text { C. Total } & 618 & 1.5697755 & & <.0001\end{array}$

Variance Component Estimates

\begin{tabular}{lrr} 
Component & Var Comp Est & Percent of Total \\
Tank \& Batch\&Random & 0.001169 & 45.980 \\
Residual & 0.001373 & 54.020 \\
\hline
\end{tabular}

Total $0.002542 \quad 100.000$

These estimates based on equating Mean Squares to Expected Value.

Response Ca (mg/L)

Summary of Fit

RSquare 0.645226

RSquare Adj $\quad 0.285831$

Root Mean Square Error $\quad 0.043983$

Mean of Response

Observations (or Sum Wgts) 619

Analysis of Variance

Source DF Sum of Squares Mean Square F Ratio

$\begin{array}{llrrr}\text { Model } & 311 & 1.0800944 & 0.003473 & 1.7953\end{array}$

$\begin{array}{lllll}\text { Error } & 307 & 0.5938827 & 0.001934 & \text { Prob }>\text { F }\end{array}$

$\begin{array}{llll}\text { C. Total } & 618 & 1.6739771 & <.0001\end{array}$

Variance Component Estimates

$\begin{array}{lrr}\text { Component } & \text { Var Comp Est } & \text { Percent of Total } \\ \text { Tank \& Batch\&Random } & 0.000775 & 28.616 \\ \text { Residual } & 0.001934 & 71.384 \\ \text { Total } & 0.00271 & 100.000\end{array}$

These estimates based on equating Mean Squares to Expected Value.

Response Cr (mg/L)

Summary of Fit

$\begin{array}{lr}\text { RSquare } & 0.637097 \\ \text { RSquare Adj } & 0.269467 \\ \text { Root Mean Square Error } & 0.005754 \\ \text { Mean of Response } & 0.004292 \\ \text { Observations (or Sum Wgts) } & 619\end{array}$

Observations (or Sum Wgts) $\quad 619$

Analysis of Variance

Source DF Sum of Squares Mean Square F Ratio

$\begin{array}{lllll}\text { Model } & 311 & 0.01784132 & 0.000057 & 1.7330\end{array}$

$\begin{array}{lllll}\text { Error } & 307 & 0.01016275 & 0.000033 & \text { Prob }>\text { F }\end{array}$

$\begin{array}{llll}\text { C. Total } & 618 & 0.02800407 & <.0001\end{array}$

Variance Component Estimates

$\begin{array}{lrr}\text { Component } & \text { Var Comp Est } & \text { Percent of Total } \\ \text { Tank \& Batch\&Random } & 0.000012 & 26.979 \\ \text { Residual } & 0.000033 & 73.021 \\ \text { Total } & 0.000045 & 100.000\end{array}$

These estimates based on equating Mean Squares to Expected Value.
Response Cu (mg/L)

Summary of Fit

RSquare

0.819704

RSquare Adj $\quad 0.637059$

Root Mean Square Error $\quad 0.002582$

Mean of Response 0.00209

Observations (or Sum Wgts) 619

Analysis of Variance

Source DF Sum of Squares Mean Square F Ratio

$\begin{array}{lllll}\text { Model } & 311 & 0.00930768 & 0.000030 & 4.4880\end{array}$

$\begin{array}{lllll}\text { Error } & 307 & 0.00204725 & 0.000007 & \text { Prob }>\text { F }\end{array}$

$\begin{array}{lllr}\text { C. Total } & 618 & 0.01135493 & 0.000007 \\ \end{array}$

Variance Component Estimates

Component Var Comp Est Percent of Total

Tank \& Batch\&Random $\quad 0.000012$

Residual $\quad 0.000007 \quad 36.256$

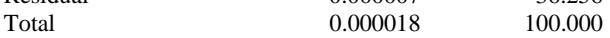

These estimates based on equating Mean Squares to Expected Value.

Response Fe (mg/L)

Summary of Fit

RSquare

RSquare Adj

0.6433

0.281953

0.047725

Mean of Response $\quad 0.004237$

Observations (or Sum Wgts) $\quad 619$

Analysis of Variance

Source DF Sum of Squares Mean Square F Ratio

$\begin{array}{lllll}\text { Model } & 311 & 1.2610783 & 0.004055 & 1.7803\end{array}$

$\begin{array}{lllll}\text { Error } & 307 & 0.6992477 & 0.002278 & \text { Prob }>\text { F }\end{array}$

$\begin{array}{llll}\text { C. Total } & 618 & 1.9603261 & <.0001\end{array}$

Variance Component Estimates

Component Var Comp Est Percent of Total

$\begin{array}{llr}\text { Tank \& Batch\&Random } & 0.000896 & 28.228\end{array}$

Residual $\quad 0.002278 \quad 71.772$

Total $0.003174 \quad 100.000$

These estimates based on equating Mean Squares to Expected Value.

Response K (mg/L)

Summary of Fit

RSquare

0.749507

RSquare Adj $\quad 0.49575$

Root Mean Square Error $\quad 0.055593$

Mean of Response

0.059318

Observations (or Sum Wgts)

619

Analysis of Variance

Source DF Sum of Squares Mean Square F Ratio

$\begin{array}{lllll}\text { Model } & 311 & 2.8389248 & 0.009128 & 2.9536\end{array}$

$\begin{array}{lllll}\text { Error } & 307 & 0.9487995 & 0.003091 & \text { Prob }>\text { F }\end{array}$

$\begin{array}{llll}\text { C. Total } 618 & 3.7877243 & <.0001\end{array}$

Variance Component Estimates

Component Var Comp Est Percent of Total

Tank \& Batch\&Random $\quad 0.003043$

Residual $\quad 0.003091 \quad 50.384$

$\begin{array}{lrr}\text { Total } & 0.006134 & 100.000\end{array}$

These estimates based on equating Mean Squares to Expected Value. 
WSRC-TR-2004-00576

Revision 0

Exhibit A13. Components of Variance for the Mixed Acid Measurements of Calibration and Bench Standards

Response Li (mg/L)

Summary of Fit

$\begin{array}{lr}\text { RSquare } & 0.815283 \\ \text { RSquare Adj } & 0.628159 \\ \text { Root Mean Square Error } & 0.001742 \\ \text { Mean of Response } & 0.00253 \\ \text { Observations (or Sum Wgts) } & 619\end{array}$

Analysis of Variance

Source DF Sum of Squares Mean Square F Ratio

$\begin{array}{lllll}\text { Model } & 311 & 0.00411245 & 0.000013 & 4.3569\end{array}$

$\begin{array}{lllll}\text { Error } & 307 & 0.00093175 & 0.000003 & \text { Prob }>\text { F }\end{array}$

$\begin{array}{llll}\text { C. Total } & 618 & 0.00504420 & <.0001\end{array}$

Variance Component Estimates

\begin{tabular}{lrr} 
Component & Var Comp Est & Percent of Total \\
Tank \& Batch\&Random & 0.000005 & 62.854 \\
Residual & 0.000003 & 37.146 \\
\hline
\end{tabular}

Total $0.000008 \quad 100.000$

These estimates based on equating Mean Squares to Expected Value.

Response Mg (mg/L)

Summary of Fit

RSquare $\quad 0.710526$

RSquare Adj $\quad 0.41728$

Root Mean Square Error $\quad 0.00383$

Mean of Response

Observations (or Sum Wgts) 619

Analysis of Variance

Source DF Sum of Squares Mean Square F Ratio

$\begin{array}{lrrrr}\text { Model } & 311 & 0.01105217 & 0.000036 & 2.4230\end{array}$

$\begin{array}{lllll}\text { Error } & 307 & 0.00450275 & 0.000015 & \text { Prob }>\text { F }\end{array}$

C. Total $618 \quad 0.01555492$

$<.0001$

Variance Component Estimates

$\begin{array}{lrr}\text { Component } & \text { Var Comp Est } & \text { Percent of Total } \\ \text { Tank \& Batch\&Random } & 0.000011 & 41.768 \\ \text { Residual } & 0.000015 & 58.232 \\ \text { Total } & 0.000025 & 100.000\end{array}$

Total $0.000025 \quad 100.000$

These estimates based on equating Mean Squares to Expected Value.

Response Mn (mg/L)

Summary of Fit

RSquare

0.514042

RSquare Adj

0.021752

Root Mean Square Error $\quad 0.00994$

Mean of Response

0.001163

Observations (or Sum Wgts) 619

Analysis of Variance

Source DF Sum of Squares Mean Square F Ratio

$\begin{array}{lrrrr}\text { Model } & 311 & 0.03208677 & 0.000103 & 1.0442\end{array}$

$\begin{array}{lllll}\text { Error } & 307 & 0.03033375 & 0.000099 & \text { Prob }>\text { F }\end{array}$

$\begin{array}{llll}\text { C. Total } & 618 & 0.06242052 & 0.3522\end{array}$

Variance Component Estimates

$\begin{array}{lrr}\text { Component } & \text { Var Comp Est } & \text { Percent of Total } \\ \text { Tank \& Batch\&Random } & 0.000002 & 2.179 \\ \text { Residual } & 0.000099 & 97.821 \\ \text { Total } & 0.000101 & 100.000\end{array}$

Total $0.000101 \quad 100.000$

These estimates based on equating Mean Squares to Expected Value.

\begin{tabular}{|c|c|c|c|c|c|}
\hline \multicolumn{6}{|c|}{$\begin{array}{l}\text { Response Na }(\mathrm{mg} / \mathrm{L}) \\
\text { Summary of Fit }\end{array}$} \\
\hline \multicolumn{3}{|l|}{ RSquare } & \multicolumn{3}{|c|}{0.612569} \\
\hline \multicolumn{3}{|c|}{ RSquare Adj } & \multicolumn{3}{|c|}{0.220091} \\
\hline \multicolumn{3}{|c|}{ Root Mean Square Error } & \multicolumn{3}{|c|}{0.169108} \\
\hline \multicolumn{3}{|c|}{ Mean of Response } & \multicolumn{3}{|c|}{0.013015} \\
\hline \multicolumn{5}{|c|}{ Observations (or Sum Wgts) } & \\
\hline \multicolumn{6}{|c|}{ Analysis of Variance } \\
\hline Source & DF & Sum of Squ & & Mean Square & F Ratio \\
\hline Model & 311 & 13.88 & & 0.044634 & 1.5608 \\
\hline Error & 307 & 8.77 & 484 & 0.028598 & Prob $>$ F \\
\hline C. Total & 618 & 22.66 & 791 & & $<.0001$ \\
\hline
\end{tabular}

Variance Component Estimates

\begin{tabular}{lrr} 
Component & Var Comp Est & Percent of Total \\
Tank \& Batch\&Random & 0.008084 & 22.037 \\
Residual & 0.028598 & 77.963 \\
\hline
\end{tabular}

0.036681

These estimates based on equating Mean Squares to Expected Value.

Response Ni (mg/L)

Summary of Fit

RSquare

0.700966

RSquare Adj $\quad 0.398036$

Root Mean Square Error $\quad 0.009129$

Mean of Response 0.005105

Observations (or Sum Wgts) 619

Analysis of Variance

Source DF Sum of Squares Mean Square F Ratio

$\begin{array}{lrrrr}\text { Model } & 311 & 0.05996917 & 0.000193 & 2.3140\end{array}$

$\begin{array}{lllll}\text { Error } & 307 & 0.02558300 & 0.000083 & \text { Prob }>\text { F }\end{array}$

$\begin{array}{llll}\text { C. Total } 618 & 0.08555217 & <.0001\end{array}$

Variance Component Estimates

$\begin{array}{lrr}\text { Component } & \text { Var Comp Est } & \text { Percent of Total } \\ \text { Tank \& Batch\&Random } & 0.000055 & 39.843 \\ \text { Residual } & 0.000083 & 60.157 \\ \text { Total } & 0.000139 & 100.000\end{array}$

$\begin{array}{lcc}\text { Total } & 0.000139 & 100.000 \\ \text { These estimates based on equating Mean Squares to Expected Value. }\end{array}$

Response Si (mg/L)

Summary of Fit

RSquare

0.901673

RSquare Adj 0.802064

Root Mean Square Error $\quad 0.252245$

Mean of Response 0.088454

Observations (or Sum Wgts) 619

Analysis of Variance

Source DF Sum of Squares Mean Square F Ratio

$\begin{array}{lllll}\text { Model } & 311 & 179.12582 & 0.575967 & 9.0522\end{array}$

$\begin{array}{llrrr}\text { Error } & 307 & 19.53365 & 0.063628 & \text { Prob }>\text { F }\end{array}$

$\begin{array}{llll}\text { C. Total } & 618 & 198.65948 & <.0001\end{array}$

Variance Component Estimates

$\begin{array}{lrr}\text { Component } & \text { Var Comp Est } & \text { Percent of Total } \\ \text { Tank \& Batch\&Random } & 0.258251 & 80.232 \\ \text { Residual } & 0.063628 & 19.768 \\ \text { Total } & 0.321878 & 100.000 \\ \text { These estimates based on equating Mean Squares to Expected Value. }\end{array}$


WSRC-TR-2004-00576

Revision 0

Exhibit A13. Components of Variance for the Mixed Acid Measurements of Calibration and Bench Standards

Response Ti (mg/L)

Summary of Fit

$\begin{array}{lr}\text { RSquare } & 0.832348 \\ \text { RSquare Adj } & 0.662511 \\ \text { Root Mean Square Error } & 0.014828 \\ \text { Mean of Response } & 0.013963 \\ \text { Observations (or Sum Wgts) } & 619\end{array}$

Analysis of Variance

Source DF Sum of Squares Mean Square F Ratio

$\begin{array}{lllll}\text { Model } & 311 & 0.33511490 & 0.001078 & 4.9009\end{array}$

$\begin{array}{lllll}\text { Error } & 307 & 0.06749925 & 0.000220 & \text { Prob }>\text { F }\end{array}$

$\begin{array}{llll}\text { C. Total } & 618 & 0.40261415 & <.0001\end{array}$

Variance Component Estimates

$\begin{array}{lrr}\text { Component } & \text { Var Comp Est } & \text { Percent of Total } \\ \text { Tank \& Batch\&Random } & 0.000432 & 66.288 \\ \text { Residual } & 0.00022 & 33.712 \\ \text { Total } & 0.000652 & 100.000\end{array}$

Total $0.000652 \quad 100.000$

These estimates based on equating Mean Squares to Expected Value.

Response U (mg/L)

Summary of Fit

$\begin{array}{lr}\text { RSquare } & 0.810443 \\ \text { RSquare Adj } & 0.618417 \\ \text { Root Mean Square Error } & 0.115758 \\ \text { Mean of Response } & 0.171911 \\ \text { Observations (or Sum Wgts) } & 619\end{array}$

Analysis of Variance

$\begin{array}{lrrrr}\text { Source } & \text { DF } & \text { Sum of Squares } & \text { Mean Square } & \text { F Ratio } \\ \text { Model } & 311 & 17.588249 & 0.056554 & 4.2205 \\ \text { Error } & 307 & 4.113757 & 0.013400 & \text { Prob > F } \\ \text { C. Total } & 618 & 21.702006 & & <.0001\end{array}$

Variance Component Estimates

$\begin{array}{lrr}\text { Component } & \text { Var Comp Est } & \text { Percent of Total } \\ \text { Tank \& Batch\&Random } & 0.021752 & 61.880 \\ \text { Residual } & 0.0134 & 38.120 \\ \text { Total } & 0.035152 & 100.000\end{array}$

These estimates based on equating Mean Squares to Expected Value.

Response Zr (mg/L)

Summary of Fit

RSquare $\quad 0.99585$

RSquare Adj $\quad 0.991646$

Root Mean Square Error $\quad 0.001429$

Mean of Response

Observations (or Sum Wgts) 619

Analysis of Variance

Source DF Sum of Squares Mean Square F Ratio

$\begin{array}{lrrrr}\text { Model } & 311 & 0.15045249 & 0.000484 & 236.8699\end{array}$

$\begin{array}{lllll}\text { Error } & 307 & 0.00062700 & 0.000002 & \text { Prob }>\text { F }\end{array}$

$\begin{array}{llll}\text { C. Total } & 618 & 0.15107949 & <.0001\end{array}$

Variance Component Estimates

$\begin{array}{lrr}\text { Component } & \text { Var Comp Est } & \text { Percent of Total } \\ \text { Tank \& Batch\&Random } & 0.000243 & 99.166 \\ \text { Residual } & 0.000002 & 0.834 \\ \text { Total } & 0.000245 & 100.000\end{array}$

These estimates based on equating Mean Squares to Expected Value.

\author{
STCd=SM35 \\ Least Squares Fit \\ Response Al (mg/L) \\ Summary of Fit \\ RSquare \\ 0.999908 \\ RSquare Adj \\ 0.999821 \\ Root Mean Square Error $\quad 0.003954$ \\ Mean of Response $\quad 0.021078$ \\ Observations (or Sum Wgts) $\quad 640$
}

Analysis of Variance

Source DF Sum of Squares Mean Square F Ratio

$\begin{array}{llrrr}\text { Model } & 311 & 55.759775 & 0.179292 & 11468.9\end{array}$

$\begin{array}{lllll}\text { Error } & 328 & 0.005128 & 0.000016 & \text { Prob > F }\end{array}$

$\begin{array}{llll}\text { C. Total } & 639 & 55.764902 & 0.0000\end{array}$

Variance Component Estimates

Component Var Comp Est Percent of Total

$\begin{array}{lrr}\text { Tank \& Batch\&Random } & 0.087404 & 99.982\end{array}$

Residual $\quad 0.000016 \quad 0.018$

Total

These estimates based on equating Mean Squares to Expected Value.

Response Ca (mg/L)

Summary of Fit

RSquare

0.931703

RSquare Adj $\quad 0.866946$

Root Mean Square Error $\quad 0.008818$

Mean of Response $\quad 1.01023$

Observations (or Sum Wgts) 640

Analysis of Variance

Source DF Sum of Squares Mean Square F Ratio

$\begin{array}{lrrrr}\text { Model } & 311 & 0.34794390 & 0.001119 & 14.3877\end{array}$

$\begin{array}{lllll}\text { Error } & 328 & 0.02550533 & 0.000078 & \text { Prob }>\text { F }\end{array}$

$\begin{array}{llll}\text { C. Total } & 639 & 0.37344924 & <.0001\end{array}$

Variance Component Estimates

Component Var Comp Est Percent of Total

Tank \& Batch\&Random $\quad 0.000508 \quad 86.715$

$\begin{array}{lll}\text { Residual } & 0.000078 & 13.285\end{array}$

Total $\quad 0.000585 \quad 100.000$

These estimates based on equating Mean Squares to Expected Value.

Response Cr (mg/L)

Summary of Fit

RSquare

0.958953

RSquare Adj

0.920033

Root Mean Square Error $\quad 0.004855$

Mean of Response 1.006403

Observations (or Sum Wgts) 640

Analysis of Variance

Source DF Sum of Squares Mean Square F Ratio

$\begin{array}{lllll}\text { Model } & 311 & 0.18060916 & 0.000581 & 24.6392\end{array}$

$\begin{array}{lllll}\text { Error } & 328 & 0.00773083 & 0.000024 & \text { Prob }>\text { F }\end{array}$

$\begin{array}{llll}\text { C. Total } & 639 & 0.18833999 & <.0001\end{array}$

Variance Component Estimates

$\begin{array}{lrr}\text { Component } & \text { Var Comp Est } & \text { Percent of Total } \\ \text { Tank \& Batch\&Random } & 0.000272 & 92.016 \\ \text { Residual } & 0.000024 & 7.984 \\ \text { Total } & 0.000295 & 100.000 \\ \text { These estimates based on equating Mean Squares to Expected Value. }\end{array}$


WSRC-TR-2004-00576

Revision 0

Exhibit A13. Components of Variance for the Mixed Acid Measurements of Calibration and Bench Standards

Response Cu (mg/L)

Summary of Fit

$\begin{array}{lr}\text { RSquare } & 0.945467 \\ \text { RSquare Adj } & 0.893761 \\ \text { Root Mean Square Error } & 0.004551 \\ \text { Mean of Response } & 1.005462 \\ \text { Observations (or Sum Wgts) } & 640\end{array}$

Analysis of Variance

Source DF Sum of Squares Mean Square F Ratio

$\begin{array}{lllll}\text { Model } & 311 & 0.11775902 & 0.000379 & 18.2854\end{array}$

$\begin{array}{lllll}\text { Error } & 328 & 0.00679208 & 0.000021 & \text { Prob }>\text { F }\end{array}$

$\begin{array}{llll}\text { C. Total } & 639 & 0.12455110 & <.0001\end{array}$

Variance Component Estimates

$\begin{array}{lrr}\text { Component } & \text { Var Comp Est } & \text { Percent of Total } \\ \text { Tank \& Batch\&Random } & 0.000175 & 89.392 \\ \text { Residual } & 0.000021 & 10.608 \\ \text { Total } & 0.000195 & 100.000\end{array}$

Total $0.000195 \quad 100.000$

These estimates based on equating Mean Squares to Expected Value.

Response Fe (mg/L)

Summary of Fit

RSquare

RSquare Adj 0.988586

Root Mean Square Error $\quad 0.002517$

Mean of Response $\quad-0.0085$

Observations (or Sum Wgts) 640

Analysis of Variance

$\begin{array}{lrrrr}\text { Source } & \text { DF } & \text { Sum of Squares } & \text { Mean Square } & \text { F Ratio } \\ \text { Model } & 311 & 0.35271724 & 0.001134 & 178.9525 \\ \text { Error } & 328 & 0.00207875 & 0.000006 & \text { Prob }>\text { F } \\ \text { C. Total } & 639 & 0.35479599 & & <.0001\end{array}$

Variance Component Estimates

Component Var Comp Est Percent of Total

$\begin{array}{lrr}\text { Tank \& Batch\&Random } & 0.00055 & 98.861\end{array}$

Residual $\quad 0.000006 \quad 1.139$

Total $0.000556 \quad 100.000$

These estimates based on equating Mean Squares to Expected Value.

Response K (mg/L)

Summary of Fit

RSquare $\quad 0.91076$

RSquare Adj $\quad 0.826149$

Root Mean Square Error $\quad 0.035144$

Mean of Response

Observations (or Sum Wgts) 640

Analysis of Variance

Source DF Sum of Squares Mean Square F Ratio

$\begin{array}{lllll}\text { Model } & 311 & 4.1346358 & 0.013295 & 10.7639\end{array}$

$\begin{array}{lllll}\text { Error } & 328 & 0.4051186 & 0.001235 & \text { Prob }>\text { F }\end{array}$

$\begin{array}{llll}\text { C. Total } 639<.0001 & 4.5397543\end{array}$

Variance Component Estimates

$\begin{array}{lrr}\text { Component } & \text { Var Comp Est } & \text { Percent of Total } \\ \text { Tank \& Batch\&Random } & 0.005879 & 82.640 \\ \text { Residual } & 0.001235 & 17.360 \\ \text { Total } & 0.007115 & 100.000\end{array}$

These estimates based on equating Mean Squares to Expected Value.

$\begin{array}{lr}\begin{array}{l}\text { Response Li (mg/L) } \\ \text { Summary of Fit }\end{array} \\ \text { RSquare } & 0.958425 \\ \text { RSquare Adj } & 0.919004 \\ \text { Root Mean Square Error } & 0.008964 \\ \text { Mean of Response } & 1.993216 \\ \text { Observations (or Sum Wgts) } & 640\end{array}$

Analysis of Variance

Source DF Sum of Squares Mean Square F Ratio

$\begin{array}{lllll}\text { Model } & 311 & 0.60755916 & 0.001954 & 24.3129\end{array}$

$\begin{array}{lllll}\text { Error } & 328 & 0.02635508 & 0.000080 & \text { Prob }>\text { F }\end{array}$

$\begin{array}{lllr}\text { C. Total } & 639 & 0.63391424 & <.0001\end{array}$

Variance Component Estimates

Component Var Comp Est Percent of Total

Tank \& Batch\&Random $\quad 0.000913 \quad 91.913$

Residual $\quad 0.00008 \quad 8.087$

$\begin{array}{lll}\text { Total } & 0.000994 & 100.000\end{array}$

These estimates based on equating Mean Squares to Expected Value.

Response Mg (mg/L)

Summary of Fit

$\begin{array}{lr}\text { RSquare } & 0.930673 \\ \text { RSquare Adj } & 0.864939 \\ \text { Root Mean Square Error } & 0.007845 \\ \text { Mean of Response } & 1.017252 \\ \text { Observations (or Sum Wgts) } & 640\end{array}$

Observations (or Sum Wgts)

Analysis of Variance

Source DF Sum of Squares Mean Square F Ratio

$\begin{array}{lllll}\text { Model } & 311 & 0.27096583 & 0.000871 & 14.1581\end{array}$

$\begin{array}{lllll}\text { Error } & 328 & 0.02018467 & 0.000062 & \text { Prob }>\text { F }\end{array}$

$\begin{array}{llll}\text { C. Total } 639<0.29115050 & <.0001\end{array}$

Variance Component Estimates

Component Var Comp Est Percent of Total

$\begin{array}{lrr}\text { Tank \& Batch\&Random } & 0.000395 & 86.514\end{array}$

Residual $\quad 0.000062 \quad 13.486$

Total $0.000456 \quad 100.000$

These estimates based on equating Mean Squares to Expected Value.

Response Mn (mg/L)

Summary of Fit

RSquare

0.98071

RSquare Adj $\quad 0.96242$

Root Mean Square Error $\quad 0.000382$

Mean of Response $\quad 0.000142$

Observations (or Sum Wgts) $\quad 640$

Analysis of Variance

$\begin{array}{lrrrr}\text { Source } & \text { DF } & \text { Sum of Squares } & \text { Mean Square } & \text { F Ratio } \\ \text { Model } & 311 & 0.00243614 & 0.0000078 & 53.6204 \\ \text { Error } & 328 & 0.00004792 & 1.4609 \mathrm{e}-7 & \text { Prob }>\text { F } \\ \text { C. Total } & 639 & 0.00248406 & & <.0001\end{array}$

C. Total $639 \quad 0.00248406$

Variance Component Estimates

$\begin{array}{lrr}\text { Component } & \text { Var Comp Est } & \text { Percent of Total } \\ \text { Tank \& Batch\&Random } & 0.000004 & 96.248 \\ \text { Residual } & 1.461 \mathrm{e}-7 & 3.752 \\ \text { Total } & 0.000004 & 100.000\end{array}$

These estimates based on equating Mean Squares to Expected Value. 
WSRC-TR-2004-00576

Revision 0

Exhibit A13. Components of Variance for the Mixed Acid Measurements of Calibration and Bench Standards

Response Na (mg/L)

Summary of Fit

$\begin{array}{lr}\text { RSquare } & 0.987919 \\ \text { RSquare Adj } & 0.976463 \\ \text { Root Mean Square Error } & 0.14046 \\ \text { Mean of Response } & 33.05044 \\ \text { Observations (or Sum Wgts) } & 640\end{array}$

Analysis of Variance

Source DF Sum of Squares Mean Square F Ratio

$\begin{array}{lllll}\text { Model } & 311 & 529.15574 & 1.70147 & 86.2419\end{array}$

$\begin{array}{llrr}\text { Error } & 328 & 6.47111 & 0.01973 \quad \text { Prob }>\text { F }\end{array}$

$\begin{array}{llll}\text { C. Total } & 639 & 535.62685 & <.0001\end{array}$

Variance Component Estimates

$\begin{array}{lrr}\text { Component } & \text { Var Comp Est } & \text { Percent of Total } \\ \text { Tank \& Batch\&Random } & 0.819909 & 97.650 \\ \text { Residual } & 0.019729 & 2.350 \\ \text { Total } & 0.839638 & 100.000\end{array}$

Total $\quad 0.839638 \quad 100.000$

These estimates based on equating Mean Squares to Expected Value.

Response Ni (mg/L)

Summary of Fit

RSquare

RSquare Adj

0.837895

O $\quad 0.009906$

Mean of Response 1.007205

Observations (or Sum Wgts) 640

Analysis of Variance

$\begin{array}{lrrrr}\text { Source } & \text { DF } & \text { Sum of Squares } & \text { Mean Square } & \text { F Ratio } \\ \text { Model } & 311 & 0.16635085 & 0.000535 & 5.4514 \\ \text { Error } & 328 & 0.03218333 & 0.000098 & \text { Prob > F }\end{array}$

C. Total $639 \quad 0.19853419$

$<.0001$

Variance Component Estimates

Component Var Comp Est Percent of Total

$\begin{array}{lrr}\text { Tank \& Batch\&Random } & 0.000213 & 68.456\end{array}$

Residual $\quad 0.000098 \quad 31.544$

Total $0.000311 \quad 100.000$

These estimates based on equating Mean Squares to Expected Value.

Response Si (mg/L)

Summary of Fit

RSquare $\quad 0.918035$

RSquare Adj $\quad 0.840319$

Root Mean Square Error $\quad 0.164221$

Mean of Response 20.4581

Observations (or Sum Wgts) 640

Analysis of Variance

Source DF Sum of Squares Mean Square F Ratio

$\begin{array}{lrrrr}\text { Model } & 311 & 99.07467 & 0.318568 & 11.8126\end{array}$

$\begin{array}{lllll}\text { Error } & 328 & 8.84567 & 0.026969 & \text { Prob }>\text { F }\end{array}$

C. Total 639

Variance Component Estimates

$\begin{array}{lrr}\text { Component } & \text { Var Comp Est } & \text { Percent of Total } \\ \text { Tank \& Batch\&Random } & 0.142166 & 84.055 \\ \text { Residual } & 0.026969 & 15.945 \\ \text { Total } & 0.169134 & 100.000\end{array}$

Total $0.169134 \quad 100.000$

These estimates based on equating Mean Squares to Expected Value.

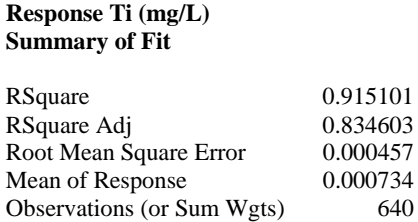

Analysis of Variance

Source DF Sum of Squares Mean Square F Ratio

$\begin{array}{lllll}\text { Model } & 311 & 0.00073834 & 0.0000024 & 11.3679\end{array}$

$\begin{array}{lllll}\text { Error } & 328 & 0.00006850 & 2.0884 \mathrm{e}-7 & \text { Prob }>\text { F }\end{array}$

$\begin{array}{llll}\text { C. Total } 639<0.00080684 & <.0001\end{array}$

Variance Component Estimates

Component Var Comp Est Percent of Total

Tank \& Batch\&Random $\quad 0.000001 \quad 83.484$

Residual 2.088e-7 16.516

Total $\quad 0.000001 \quad 100.000$

These estimates based on equating Mean Squares to Expected Value.

Response U (mg/L)

Summary of Fit

$\begin{array}{lr}\text { RSquare } & 0.94539 \\ \text { RSquare Adj } & 0.89361 \\ \text { Root Mean Square Error } & 0.034337 \\ \text { Mean of Response } & 0.100041 \\ \text { Observations (or Sum Wgts) } & 640\end{array}$

Analysis of Variance

Source DF Sum of Squares Mean Square F Ratio

$\begin{array}{lllll}\text { Model } & 311 & 6.6947554 & 0.021527 & 18.2579\end{array}$

$\begin{array}{lllll}\text { Error } & 328 & 0.3867196 & 0.001179 & \text { Prob }>\text { F }\end{array}$

$\begin{array}{llll}\text { C. Total } 639 & 7.0814749 & <.0001\end{array}$

Variance Component Estimates

Component Var Comp Est Percent of Total

$\begin{array}{lrr}\text { Tank \& Batch\&Random } & 0.00992 & 89.377\end{array}$

$\begin{array}{lll}\text { Residual } & 0.001179 & 10.623\end{array}$

Total $0.011099 \quad 100.000$

These estimates based on equating Mean Squares to Expected Value.

Response Zr (mg/L)

Summary of Fit

RSquare 0.998234

RSquare Adj $\quad 0.99656$

Root Mean Square Error $\quad 0.000885$

Mean of Response

Observations (or Sum Wgts) $\quad 640$

Analysis of Variance

Source DF Sum of Squares Mean Square F Ratio

Model $\quad 311 \quad 0.14539981 \quad 0.000468 \quad 596.2970$

$\begin{array}{lllll}\text { Error } & 328 & 0.00025717 & 0.000001 & \text { Prob }>\text { F }\end{array}$

$\begin{array}{llll}\text { C. Total } & 639 & 0.14565698 & 0.0000\end{array}$ 
WSRC-TR-2004-00576

Revision 0

Exhibit A13. Components of Variance for the Mixed Acid Measurements of Calibration and Bench Standards

STCd=SM36

Least Squares Fit

Response Al (mg/L)

Summary of Fit

RSquare Adj 0.917723

Root Mean Square Error $\quad 0.008164$

Mean of Response 1.985738

Observations (or Sum Wgts) 642

Analysis of Variance

$\begin{array}{lrrrr}\text { Source } & \text { DF } & \text { Sum of Squares } & \text { Mean Square } & \text { F Ratio } \\ \text { Model } & 312 & 0.49727254 & 0.001594 & 23.9159 \\ \text { Error } & 329 & 0.02192550 & 0.000067 & \text { Prob }>\text { F } \\ \text { C. Total } & 641 & 0.51919804 & & <.0001\end{array}$

C. Total $641 \quad 0.51919804$

Variance Component Estimates

\begin{tabular}{lrr} 
Component & Var Comp Est & Percent of Total \\
Tank \& Batch\&Random & 0.000745 & 91.785 \\
Residual & 0.000067 & 8.215 \\
\hline
\end{tabular}

Total $0.000811 \quad 100.000$

These estimates based on equating Mean Squares to Expected Value.

Response Ca (mg/L)

Summary of Fit

$\begin{array}{lr}\text { RSquare } & 0.98817 \\ \text { RSquare Adj } & 0.976952 \\ \text { Root Mean Square Error } & 0.007646 \\ \text { Mean of Response } & 0.014014 \\ \text { Observations (or Sum Wgts) } & 642\end{array}$

Analysis of Variance

Source DF Sum of Squares Mean Square F Ratio

$\begin{array}{lllll}\text { Model } & 312 & 1.6065306 & 0.005149 & 88.0846\end{array}$

$\begin{array}{lllll}\text { Error } & 329 & 0.0192322 & 0.000058 & \text { Prob }>\text { F }\end{array}$

$\begin{array}{llll}\text { C. Total } & 641 & 1.6257629 & <.0001\end{array}$

Variance Component Estimates

$\begin{array}{lrr}\text { Component } & \text { Var Comp Est } & \text { Percent of Total } \\ \text { Tank \& Batch\&Random } & 0.002482 & 97.699 \\ \text { Residual } & 0.000058 & 2.301 \\ \text { Total } & 0.002541 & 100.000\end{array}$

These estimates based on equating Mean Squares to Expected Value.

Response Cr (mg/L)

Summary of Fit

RSquare Adj

0.945494

Root Mean Square Error $\quad 0.000691$

0.893804

Mean of Response 0.002301

Observations (or Sum Wgts)

642

Analysis of Variance

Source DF Sum of Squares Mean Square F Ratio

$\begin{array}{lllll}\text { Model } & 312 & 0.00272773 & 0.0000087 & 18.2916\end{array}$

$\begin{array}{lllll}\text { Error } & 329 & 0.00015725 & 4.7796 \mathrm{e}-7 & \text { Prob }>\text { F }\end{array}$

$\begin{array}{llll}\text { C. Total } 641 \quad 0.00288498 & <.0001\end{array}$

Variance Component Estimates

$\begin{array}{lrr}\text { Component } & \text { Var Comp Est } & \text { Percent of Total } \\ \text { Tank \& Batch\&Random } & 0.000004 & 89.397 \\ \text { Residual } & 4.78 \mathrm{e}-7 & 10.603 \\ \text { Total } & 0.000005 & 100.000\end{array}$

Total $0.000005 \quad 100.000$

These estimates based on equating Mean Squares to Expected Value.

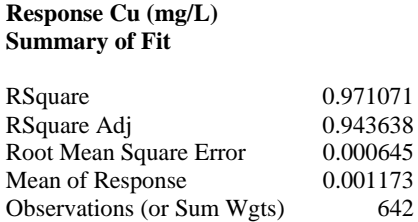

Analysis of Variance

Source DF Sum of Squares Mean Square F Ratio

$\begin{array}{lllll}\text { Model } & 312 & 0.00459881 & 0.000015 & 35.3970\end{array}$

$\begin{array}{lllll}\text { Error } & 329 & 0.00013700 & 0.000000 & \text { Prob }>\text { F }\end{array}$

$\begin{array}{llll}\text { C. Total } 641<0.00473581 & <.0001\end{array}$

Variance Component Estimates

Component Var Comp Est Percent of Total

Tank \& Batch\&Random $\quad 0.000007 \quad 94.373$

Residual $\quad 4.164 \mathrm{e}-7 \quad 5.627$

$\begin{array}{lll}\text { Total } & 0.000007 & 100.000\end{array}$

These estimates based on equating Mean Squares to Expected Value.

Response Fe (mg/L)

Summary of Fit

$\begin{array}{lr}\text { RSquare } & 0.945694 \\ \text { RSquare Adj } & 0.894194 \\ \text { Root Mean Square Error } & 0.040845 \\ \text { Mean of Response } & 9.939983 \\ \text { Observations (or Sum Wgts) } & 642\end{array}$

Observations (or Sum Wgts)

Analysis of Variance

$\begin{array}{lrrrr}\text { Source } & \text { DF } & \text { Sum of Squares } & \text { Mean Square } & \text { F Ratio } \\ \text { Model } & 312 & 9.558196 & 0.030635 & 18.3629 \\ \text { Error } & 329 & 0.548877 & 0.001668 & \text { Prob }>\text { F } \\ \text { C. Total } & 641 & 10.107073 & & <.0001\end{array}$

Variance Component Estimates

Component Var Comp Est Percent of Total

$\begin{array}{llr}\text { Tank \& Batch\&Random } & 0.014124 & 89.436\end{array}$

Residual $\quad 0.001668 \quad 10.564$

Total $0.015792 \quad 100.000$

These estimates based on equating Mean Squares to Expected Value.

Response K (mg/L)

Summary of Fit

RSquare $\quad 0.808647$

RSquare Adj $\quad 0.627181$

Root Mean Square Error $\quad 0.032376$

Mean of Response $\quad 0.014528$

Observations (or Sum Wgts) 642

$\begin{array}{lrrrr}\text { Analysis of Variance } & & & \\ \text { Source } & \text { DF } & \text { Sum of Squares } & \text { Mean Square } & \text { F Ratio } \\ \text { Model } & 312 & 1.4573787 & 0.004671 & 4.4562 \\ \text { Error } & 329 & 0.3448652 & 0.001048 & \text { Prob }>\text { F } \\ \text { C. Total } & 641 & 1.8022440 & & <.0001\end{array}$

Variance Component Estimates

Component Var Comp Est Percent of Total

Tank \& Batch\&Random $\quad 0.001766$

$\begin{array}{lll}\text { Residual } & 0.001048 & 37.242\end{array}$

Total $0.002815 \quad 100.000$

These estimates based on equating Mean Squares to Expected Value. 
WSRC-TR-2004-00576

Revision 0

Exhibit A13. Components of Variance for the Mixed Acid Measurements of Calibration and Bench Standards

Response Li (mg/L)

Summary of Fit

$\begin{array}{lr}\text { RSquare } & 0.51178 \\ \text { RSquare Adj } & 0.048788 \\ \text { Root Mean Square Error } & 0.117031 \\ \text { Mean of Response } & 0.011338 \\ \text { Observations (or Sum Wgts) } & 642\end{array}$

Analysis of Variance

Source DF Sum of Squares Mean Square F Ratio

$\begin{array}{lllll}\text { Model } & 312 & 4.7235519 & 0.015140 & 1.1054\end{array}$

$\begin{array}{lllll}\text { Error } & 329 & 4.5060957 & 0.013696 & \text { Prob }>\text { F }\end{array}$

$\begin{array}{llll}\text { C. Total } & 641 & 9.2296477 & 0.1849\end{array}$

Variance Component Estimates

$\begin{array}{lrr}\text { Component } & \text { Var Comp Est } & \text { Percent of Total } \\ \text { Tank \& Batch\&Random } & 0.000704 & 4.887 \\ \text { Residual } & 0.013696 & 95.113 \\ \text { Total } & 0.0144 & 100.000\end{array}$

These estimates based on equating Mean Squares to Expected Value.

Response Mg (mg/L)

Summary of Fit

RSquare

0.99744

RSquare Adj

0.995012

Root Mean Square Error $\quad 0.000597$

Mean of Response

0.002645

Observations (or Sum Wgts) 642

Analysis of Variance

Source DF Sum of Squares Mean Square F Ratio

$\begin{array}{lrrrr}\text { Model } & 312 & 0.04568378 & 0.000146 & 410.8568\end{array}$

$\begin{array}{lllll}\text { Error } & 329 & 0.00011725 & 0.000000 & \text { Prob }>\text { F }\end{array}$

C. Total $641 \quad 0.04580103$

0.0000

\author{
Response Na (mg/L) \\ Summary of Fit \\ 0.975938 \\ RSquare Adj $\quad 0.95312$ \\ Root Mean Square Error $\quad 0.020942$ \\ Mean of Response 4.92141 \\ Observations (or Sum Wgts) 642 \\ Analysis of Variance

$\begin{array}{lrrrr}\text { Source } & \text { DF } & \text { Sum of Squares } & \text { Mean Square } & \text { F Ratio } \\ \text { Model } & 312 & 5.8521605 & 0.018757 & 42.7698 \\ \text { Error } & 329 & 0.1442848 & 0.000439 & \text { Prob }>\text { F } \\ \text { C. Total } & 641 & 5.9964453 & & <.0001\end{array}$

Variance Component Estimates

$\begin{array}{lrr}\text { Component } & \text { Var Comp Est } & \text { Percent of Total } \\ \text { Tank \& Batch\&Random } & 0.008932 & 95.320 \\ \text { Residual } & 0.000439 & 4.680 \\ \text { Total } & 0.00937 & 100.000\end{array}$

These estimates based on equating Mean Squares to Expected Value.

Response Ni (mg/L)

Summary of Fit

RSquar

0.781709

RSquare Adj $\quad 0.574697$

Root Mean Square Error $\quad 0.004956$

Mean of Response $\quad 0.009037$

Observations (or Sum Wgts) 642

Analysis of Variance

Source DF Sum of Squares Mean Square F Ratio

$\begin{array}{lrrrr}\text { Model } & 312 & 0.02894285 & 0.000093 & 3.7762\end{array}$

$\begin{array}{lllll}\text { Error } & 329 & 0.00808225 & 0.000025 & \text { Prob }>\text { F }\end{array}$

$\begin{array}{llll}\text { C. Total } & 641 & 0.03702510 & <.0001\end{array}$

Variance Component Estimates

$\begin{array}{lrr}\text { Component } & \text { Var Comp Est } & \text { Percent of Total } \\ \text { Tank \& Batch\&Random } & 0.000033 & 57.512 \\ \text { Residual } & 0.000025 & 42.488 \\ \text { Total } & 0.000058 & 100.000 \\ \text { These estimates based on equating Mean Squares to Expected Value. }\end{array}$

These estimates based on equating Mean Squares to Expected Value.

Response Si (mg/L)

Summary of Fit

Summary of Fit

RSquare $\quad 0.942504$

RSquare Adj $\quad 0.887978$

Root Mean Square Error $\quad 0.126195$

Mean of Response $\quad 0.105315$

Observations (or Sum Wgts) $\quad 642$

$\begin{array}{lrrrr}\text { Analysis of Variance } & & & \\ \text { Source } & \text { DF } & \text { Sum of Squares } & \text { Mean Square } & \text { F Ratio } \\ \text { Model } & 312 & 85.886059 & 0.275276 & 17.2856 \\ \text { Error } & 329 & 5.239385 & 0.015925 & \text { Prob }>\text { F } \\ \text { C. Total } & 641 & 91.125444 & & <.0001\end{array}$

Variance Component Estimates

$\begin{array}{lrr}\text { Component } & \text { Var Comp Est } & \text { Percent of Total } \\ \text { Tank \& Batch\&Random } & 0.126453 & 88.815 \\ \text { Residual } & 0.015925 & 11.185 \\ \text { Total } & 0.142378 & 100.000 \\ \text { These estimates based on equating Mean Squares to Expected Value. }\end{array}$

Variance Component Estimates

$\begin{array}{lrr}\text { Component } & \text { Var Comp Est } & \text { Percent of Total } \\ \text { Tank \& Batch\&Random } & 0.000831 & 93.499 \\ \text { Residual } & 0.000058 & 6.501 \\ \text { Total } & 0.000888 & 100.000\end{array}$

These estimates based on equating Mean Squares to Expected Value.
These estimates based on equating Mean Squares to Expected Value. 
WSRC-TR-2004-00576

Revision 0

Exhibit A13. Components of Variance for the Mixed Acid Measurements of Calibration and Bench Standards

Response Ti (mg/L)

Summary of Fit

$\begin{array}{lr}\text { RSquare } & 0.977565 \\ \text { RSquare Adj } & 0.95629 \\ \text { Root Mean Square Error } & 0.003696 \\ \text { Mean of Response } & 0.997285 \\ \text { Observations (or Sum Wgts) } & 642\end{array}$

Analysis of Variance

Source DF Sum of Squares Mean Square F Ratio

$\begin{array}{lllll}\text { Model } & 312 & 0.19585209 & 0.000628 & 45.9477\end{array}$

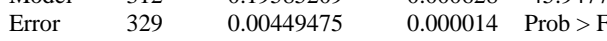

$\begin{array}{llll}\text { C. Total } & 641 & 0.20034684 & <.0001\end{array}$

Variance Component Estimates

$\begin{array}{lrr}\text { Component } & \text { Var Comp Est } & \text { Percent of Total } \\ \text { Tank \& Batch\&Random } & 0.000299 & 95.636 \\ \text { Residual } & 0.000014 & 4.364 \\ \text { Total } & 0.000313 & 100.000\end{array}$

These estimates based on equating Mean Squares to Expected Value.

Response U (mg/L)

Summary of Fit

\begin{tabular}{|c|c|c|c|c|}
\hline \multicolumn{3}{|l|}{ RSquare } & \multicolumn{2}{|c|}{0.964136} \\
\hline \multicolumn{3}{|c|}{ RSquare Adj } & \multicolumn{2}{|c|}{0.930125} \\
\hline \multicolumn{3}{|c|}{ Root Mean Square Error } & \multicolumn{2}{|c|}{0.028213} \\
\hline \multicolumn{3}{|c|}{ Mean of Response } & \multicolumn{2}{|c|}{0.080436} \\
\hline \multicolumn{3}{|c|}{ Observations (or Sum Wgts) } & \multicolumn{2}{|l|}{642} \\
\hline \multicolumn{5}{|c|}{ Analysis of Variance } \\
\hline Source & DF & Sum of Squares & Mean Square & F Ratio \\
\hline Model & 312 & 7.0399106 & 0.022564 & 28.3477 \\
\hline Error & 329 & 0.2618732 & 0.000796 & Prob $>$ F \\
\hline C. Total & 641 & 7.3017839 & & $<.0001$ \\
\hline
\end{tabular}

Variance Component Estimates

$\begin{array}{lrr}\text { Component } & \text { Var Comp Est } & \text { Percent of Total } \\ \text { Tank \& Batch\&Random } & 0.010613 & 93.024 \\ \text { Residual } & 0.000796 & 6.976 \\ \text { Total } & 0.011409 & 100.000\end{array}$

Total $0.011409 \quad 100.000$

These estimates based on equating Mean Squares to Expected Value.

Response Zr (mg/L)

Summary of Fit

\begin{tabular}{|c|c|c|c|c|c|}
\hline \multicolumn{3}{|l|}{ RSquare } & \multicolumn{3}{|c|}{0.947128} \\
\hline \multicolumn{3}{|c|}{ RSquare Adj } & \multicolumn{3}{|c|}{0.896987} \\
\hline \multicolumn{3}{|c|}{ Root Mean Square Error } & \multicolumn{3}{|c|}{0.003751} \\
\hline \multicolumn{3}{|c|}{ Mean of Response } & \multicolumn{3}{|c|}{0.981547} \\
\hline \multicolumn{3}{|c|}{ Observations (or Sum Wgts) } & \multicolumn{3}{|c|}{642} \\
\hline \multicolumn{6}{|c|}{ Analysis of Variance } \\
\hline Source & $\mathrm{DF}$ & Sum of Squ & & Mean Square & F Ratio \\
\hline Model & 312 & 0.08291 & 260 & 0.000266 & 18.8895 \\
\hline Error & 329 & 0.00462 & 350 & 0.000014 & Prob $>\mathrm{F}$ \\
\hline C. Total & 641 & 0.08754 & 110 & & $<.0001$ \\
\hline
\end{tabular}

Variance Component Estimates

$\begin{array}{lrr}\text { Component } & \text { Var Comp Est } & \text { Percent of Total } \\ \text { Tank \& Batch\&Random } & 0.000123 & 89.715 \\ \text { Residual } & 0.000014 & 10.285 \\ \text { Total } & 0.000137 & 100.000\end{array}$

These estimates based on equating Mean Squares to Expected Value.

\author{
STCd=SM38 \\ Least Squares Fit \\ Response Al (mg/L) \\ Summary of Fit \\ RSquare \\ 0.999352 \\ RSquare Adj \\ 0.998742 \\ Root Mean Square Error $\quad 0.004835$ \\ Mean of Response $\quad 0.053603$ \\ Observations (or Sum Wgts) $\quad 640$ \\ Analysis of Variance \\ Source DF Sum of Squares Mean Square F Ratio \\ $\begin{array}{lrrrr}\text { Model } & 310 & 11.867425 & 0.038282 & 1637.441\end{array}$ \\ $\begin{array}{lllll}\text { Error } & 329 & 0.007692 & 0.000023 & \text { Prob }>\text { F }\end{array}$ \\ $\begin{array}{llll}\text { C. Total } & 639 & 11.875117 & 0.0000\end{array}$
}

Variance Component Estimates

$\begin{array}{lrr}\text { Component } & \text { Var Comp Est } & \text { Percent of Total } \\ \text { Tank \& Batch\&Random } & 0.018593 & 99.874 \\ \text { Residual } & 0.000023 & 0.126 \\ \text { Total } & 0.018616 & 100.000\end{array}$

These estimates based on equating Mean Squares to Expected Value.

Response Ca (mg/L)

Summary of Fit

RSquare Adj

Root Mean Square Error $\quad 0.068089$

Mean of Response 0.276666

Observations (or Sum Wgts) 640

Analysis of Variance

Source DF Sum of Squares Mean Square F Ratio

$\begin{array}{lllll}\text { Model } & 310 & 76.638089 & 0.247220 & 53.3247\end{array}$

$\begin{array}{lllll}\text { Error } & 329 & 1.525283 & 0.004636 & \text { Prob }>\text { F }\end{array}$

$\begin{array}{llll}\text { C. Total } & 639 & 78.163372 & <.004636\end{array}$

Variance Component Estimates

$\begin{array}{lrr}\text { Component } & \text { Var Comp Est } & \text { Percent of Total } \\ \text { Tank \& Batch\&Random } & 0.117891 & 96.216 \\ \text { Residual } & 0.004636 & 3.784 \\ \text { Total } & 0.122527 & 100.000\end{array}$

These estimates based on equating Mean Squares to Expected Value.

Response Cr (mg/L)

Summary of Fit

0.934417

RSquare Adj $\quad 0.872622$

Root Mean Square Error $\quad 0.001823$

Mean of Response $\quad 0.029059$

Observations (or Sum Wgts) 640

Analysis of Variance

Source DF Sum of Squares Mean Square F Ratio

$\begin{array}{lllll}\text { Model } & 310 & 0.01557649 & 0.000050 & 15.1211\end{array}$

$\begin{array}{lllll}\text { Error } & 329 & 0.00109325 & 0.000003 & \text { Prob }>\text { F }\end{array}$

$\begin{array}{llll}\text { C. Total } 639<0001 & 0.01666974\end{array}$

Variance Component Estimates

$\begin{array}{lrr}\text { Component } & \text { Var Comp Est } & \text { Percent of Total } \\ \text { Tank \& Batch\&Random } & 0.000023 & 87.282 \\ \text { Residual } & 0.000003 & 12.718 \\ \text { Total } & 0.000026 & 100.000 \\ \text { These estimates based on equating Mean Squares to Expected Value. }\end{array}$


WSRC-TR-2004-00576

Revision 0

Exhibit A13. Components of Variance for the Mixed Acid Measurements of Calibration and Bench Standards

Response Cu (mg/L)

Summary of Fit

$\begin{array}{lr}\text { RSquare } & 0.998625 \\ \text { RSquare Adj } & 0.99733 \\ \text { Root Mean Square Error } & 0.001727 \\ \text { Mean of Response } & 0.024969 \\ \text { Observations (or Sum Wgts) } & 640\end{array}$

Analysis of Variance

Source DF Sum of Squares Mean Square F Ratio

$\begin{array}{lllll}\text { Model } & 310 & 0.71264238 & 0.002299 & 770.9689\end{array}$

$\begin{array}{lllll}\text { Error } & 329 & 0.00098100 & 0.000003 & \text { Prob }>\text { F }\end{array}$

C. Total $639 \quad 0.71362337$

0.0000

Variance Component Estimates

$\begin{array}{lrr}\text { Component } & \text { Var Comp Est } & \text { Percent of Total } \\ \text { Tank \& Batch\&Random } & 0.001116 & 99.733 \\ \text { Residual } & 0.000003 & 0.267 \\ \text { Total } & 0.001119 & 100.000\end{array}$

These estimates based on equating Mean Squares to Expected Value.

Response Fe (mg/L)

Summary of Fit

RSquare $\quad 0.983698$

RSquare Adj $\quad 0.968338$

Root Mean Square Error $\quad 0.004469$

Mean of Response $\quad-0.00586$

Observations (or Sum Wgts) 640

Analysis of Variance

Source DF Sum of Squares Mean Square F Ratio

$\begin{array}{llrrr}\text { Model } & 310 & 0.39653137 & 0.001279 & 64.0418\end{array}$

$\begin{array}{lllll}\text { Error } & 329 & 0.00657125 & 0.000020 & \text { Prob }>\text { F }\end{array}$

C. Total $639-0.40310262$

Variance Component Estimates

$\begin{array}{lrr}\text { Component } & \text { Var Comp Est } & \text { Percent of Total } \\ \text { Tank \& Batch\&Random } & 0.000612 & 96.839 \\ \text { Residual } & 0.00002 & 3.161 \\ \text { Total } & 0.000632 & 100.000\end{array}$

Total $0.000632 \quad 100.000$

These estimates based on equating Mean Squares to Expected Value.

Response K (mg/L)

Summary of Fi

RSquare

0.843468

RSquare Adj $\quad 0.695977$

Root Mean Square Error $\quad 0.026611$

Mean of Response $\quad 0.003142$

Observations (or Sum Wgts) 640

Analysis of Variance

$\begin{array}{lrrrr}\text { Source } & \text { DF } & \text { Sum of Squares } & \text { Mean Square } & \text { F Ratio } \\ \text { Model } & 310 & 1.2554488 & 0.004050 & 5.7187 \\ \text { Error } & 329 & 0.2329873 & 0.000708 & \text { Prob }>\text { F } \\ \text { C. Total } & 639 & 1.4884361 & & <.0001\end{array}$

C. Total $639 \quad 1.4884361$

$<.0001$

Variance Component Estimates

$\begin{array}{lrr}\text { Component } & \text { Var Comp Est } & \text { Percent of Total } \\ \text { Tank \& Batch\&Random } & 0.001624 & 69.635 \\ \text { Residual } & 0.000708 & 30.365 \\ \text { Total } & 0.002332 & 100.000\end{array}$

Total $0.002332 \quad 100.000$

These estimates based on equating Mean Squares to Expected Value.

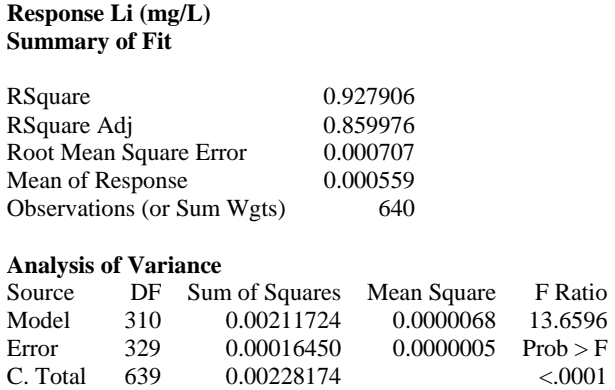

Variance Component Estimates

\begin{tabular}{lrr} 
Component & Var Comp Est & Percent of Total \\
Tank \& Batch\&Random & 0.000003 & 86.019 \\
Residual & $5 \mathrm{e}-7$ & 13.981 \\
\hline
\end{tabular}

These estimates based on equating Mean Squares to Expected Value.

Response Mg (mg/L)

Summary of Fit

RSquare

0.986396

RSquare Adj

0.973578

Root Mean Square Error $\quad 0.000848$

Mean of Response 0.004812

Observations (or Sum Wgts) 640

Analysis of Variance

Source DF Sum of Squares Mean Square F Ratio

$\begin{array}{lrrrr}\text { Model } & 310 & 0.01716675 & 0.000055 & 76.9542\end{array}$

$\begin{array}{lllll}\text { Error } & 329 & 0.00023675 & 0.000001 & \text { Prob }>\text { F }\end{array}$

$\begin{array}{llll}\text { C. Total } 639 & 0.01740350 & <.0001\end{array}$

Variance Component Estimates

$\begin{array}{lrr}\text { Component } & \text { Var Comp Est } & \text { Percent of Total } \\ \text { Tank \& Batch\&Random } & 0.000027 & 97.362 \\ \text { Residual } & 7.196 \mathrm{e}-7 & 2.638 \\ \text { Total } & 0.000027 & 100.000 \\ \text { These estimates based on equating Mean Squares to Expected Value. }\end{array}$

Response Mn (mg/L)

Summary of Fit

RSquare

0.935927

RSquare Ad

0.875554

Root Mean Square Error $\quad 0.00045$

Mean of Response $\quad 0.001481$

Observations (or Sum Wgts) $\quad 640$

$\begin{array}{lrrrr}\text { Analysis of Variance } & & & \\ \text { Source } & \text { DF } & \text { Sum of Squares } & \text { Mean Square } & \text { F Ratio } \\ \text { Model } & 310 & 0.00097502 & 0.0000031 & 15.5024 \\ \text { Error } & 329 & 0.00006675 & 2.0289 e-7 & \text { Prob }>\text { F } \\ \text { C. Total } & 639 & 0.00104178 & & <.0001\end{array}$

Variance Component Estimates

$\begin{array}{lrc}\text { Variance Component Estimates } & \\ \text { Component } & \text { Var Comp Est } & \text { Percent of Total } \\ \text { Tank \& Batch\&Random } & 0.000001 & 87.574 \\ \text { Residual } & 2.029 \mathrm{e}-7 & 12.426 \\ \text { Total } & 0.000002 & 100.000 \\ \text { These estimates based on equating Mean Squares to Expected Value. }\end{array}$


WSRC-TR-2004-00576

Revision 0

Exhibit A13. Components of Variance for the Mixed Acid Measurements of Calibration and Bench Standards

Response Na (mg/L)

Summary of Fit

$\begin{array}{lr}\text { RSquare } & 0.999931 \\ \text { RSquare Adj } & 0.999867 \\ \text { Root Mean Square Error } & 0.00642 \\ \text { Mean of Response } & 0.11727 \\ \text { Observations (or Sum Wgts) } & 640\end{array}$

Analysis of Variance

Source DF Sum of Squares Mean Square F Ratio

$\begin{array}{lllll}\text { Model } & 310 & 197.61906 & 0.637481 & 15464.34\end{array}$

$\begin{array}{llrrr}\text { Error } & 329 & 0.01356 & 0.000041 & \text { Prob }>\text { F }\end{array}$

$\begin{array}{llll}\text { C. Total } & 639 & 197.63262 & 0.0000\end{array}$

Variance Component Estimates

\begin{tabular}{lrr} 
Component & Var Comp Est & Percent of Total \\
Tank \& Batch\&Random & 0.309782 & 99.987 \\
Residual & 0.000041 & 0.013 \\
\hline
\end{tabular}

Total $0.309824 \quad 100.000$

These estimates based on equating Mean Squares to Expected Value.

Response Ni (mg/L)

Summary of Fit

RSquare $\quad 0.801291$

RSquare Adj $\quad 0.614058$

$\begin{array}{lr}\text { Root Mean Square Error } & 0.004689 \\ \text { Mean of Response } & 0.00248\end{array}$

Observations (or Sum Wgts) 640

Analysis of Variance

Source DF Sum of Squares Mean Square F Ratio

$\begin{array}{llrrr}\text { Model } & 310 & 0.02916999 & 0.000094 & 4.2796\end{array}$

$\begin{array}{lllll}\text { Error } & 329 & 0.00723375 & 0.000022 & \text { Prob }>\text { F }\end{array}$

C. Total $639 \quad 0.03640374$

$<.0001$

Variance Component Estimates

$\begin{array}{lrr}\text { Component } & \text { Var Comp Est } & \text { Percent of Total } \\ \text { Tank \& Batch\&Random } & 0.000035 & 61.447 \\ \text { Residual } & 0.000022 & 38.553 \\ \text { Total } & 0.000057 & 100.000\end{array}$

Total $0.000057 \quad 100.000$

These estimates based on equating Mean Squares to Expected Value.

Response Si (mg/L)

Summary of Fit

RSquare

0.898259

RSquare Adj $\quad 0.802393$

Root Mean Square Error $\quad 0.256714$

Mean of Response 0.171572

Observations (or Sum Wgts) 640

Analysis of Variance

$\begin{array}{lrrrr}\text { Source } & \text { DF } & \text { Sum of Squares } & \text { Mean Square } & \text { F Ratio } \\ \text { Model } & 310 & 191.42465 & 0.617499 & 9.3700 \\ \text { Error } & 329 & 21.68175 & 0.065902 & \text { Prob }>\text { F } \\ \text { C. Total } & 639 & 213.10640 & & <.0001\end{array}$

C. Total $639 \quad 213.10640$

$<.0001$

Variance Component Estimates

$\begin{array}{lrr}\text { Component } & \text { Var Comp Est } & \text { Percent of Total } \\ \text { Tank \& Batch\&Random } & 0.268065 & 80.267 \\ \text { Residual } & 0.065902 & 19.733 \\ \text { Total } & 0.333967 & 100.000\end{array}$

Total $0.333967 \quad 100.000$

These estimates based on equating Mean Squares to Expected Value.

\begin{tabular}{|c|c|c|c|c|c|}
\hline \multicolumn{5}{|c|}{ Response Ti (mg/L) } & Summary of Fit \\
\hline \multicolumn{3}{|l|}{ RSquare } & \multicolumn{3}{|c|}{0.938576} \\
\hline \multicolumn{3}{|c|}{ RSquare Adj } & \multicolumn{3}{|c|}{0.880699} \\
\hline \multicolumn{3}{|c|}{ Root Mean Square Error } & \multicolumn{3}{|c|}{0.000491} \\
\hline \multicolumn{3}{|c|}{ Mean of Response } & 0.0 & 01117 & \\
\hline \multicolumn{3}{|c|}{ Observations (or Sum Wgts) } & & 640 & \\
\hline \multicolumn{6}{|c|}{ Analysis of Variance } \\
\hline Source & $\mathrm{DF}$ & Sum of Squ & & Mean Square & F Ratio \\
\hline Model & 310 & 0.00121 & & 0.0000039 & 16.2168 \\
\hline Error & 329 & 0.00007 & & $2.4088 \mathrm{e}-7$ & Prob $>$ F \\
\hline C. Total & 639 & $0.0012 \mathrm{C}$ & & & $<.0001$ \\
\hline
\end{tabular}

$\begin{array}{lrr}\text { Variance Component Estimates } & \\ \text { Component } & \text { Var Comp Est } & \text { Percent of Total } \\ \text { Tank \& Batch\&Random } & 0.000002 & 88.088 \\ \text { Residual } & 2.409 \mathrm{e}-7 & 11.912 \\ \text { Total } & 0.000002 & 100.000\end{array}$

These estimates based on equating Mean Squares to Expected Value.

Response U (mg/L)

Summary of Fit

RSquare $\quad 0.924231$

RSquare Adj 0.852838

Root Mean Square Error $\quad 0.068853$

Mean of Response 9.913252

Observations (or Sum Wgts) 640

Analysis of Variance

Source DF Sum of Squares Mean Square F Ratio

$\begin{array}{lrrrr}\text { Model } & 310 & 19.025153 & 0.061371 & 12.9457\end{array}$

$\begin{array}{lllll}\text { Error } & 329 & 1.559687 & 0.004741 & \text { Prob }>\text { F }\end{array}$

$\begin{array}{llll}\text { C. Total } & 639 & 20.584840 & <.0001\end{array}$

Variance Component Estimates

$\begin{array}{lrr}\text { Component } & \text { Var Comp Est } & \text { Percent of Total } \\ \text { Tank \& Batch\&Random } & 0.027521 & 85.306 \\ \text { Residual } & 0.004741 & 14.694 \\ \text { Total } & 0.032262 & 100.000\end{array}$

$\begin{array}{ccc}\text { Total } & 0.032262 & 100.000 \\ \text { These estimates based on equating Mean Squares to Expected Value. }\end{array}$

Response Zr (mg/L)

Summary of Fit

RSquare

0.997853

RSquare Adj

0.99583

Root Mean Square Error $\quad 0.00099$

Mean of Response $\quad-0.00467$

Observations (or Sum Wgts) $\quad 640$

Analysis of Variance

Source DF Sum of Squares Mean Square F Ratio

$\begin{array}{lrrrr}\text { Model } & 310 & 0.14999034 & 0.000484 & 493.2093\end{array}$

$\begin{array}{lllll}\text { Error } & 329 & 0.00032275 & 0.000001 & \text { Prob }>\text { F }\end{array}$

$\begin{array}{llll}\text { C. Total } & 639 & 0.15031309 & 0.0000\end{array}$

Variance Component Estimates

$\begin{array}{lrr}\text { Variance Component Estimates } & \\ \text { Component } & \text { Var Comp Est } & \text { Percent of Total } \\ \text { Tank \& Batch\&Random } & 0.000235 & 99.584 \\ \text { Residual } & 9.81 \mathrm{e}-7 & 0.416 \\ \text { Total } & 0.000236 & 100.000 \\ \text { These estimates based on equating Mean Squares to Expected Value. }\end{array}$


WSRC-TR-2004-00576

Revision 0

\section{Exhibit A14. Paired Comparisons of SM35 versus SM33 for Mixed Acid Data}
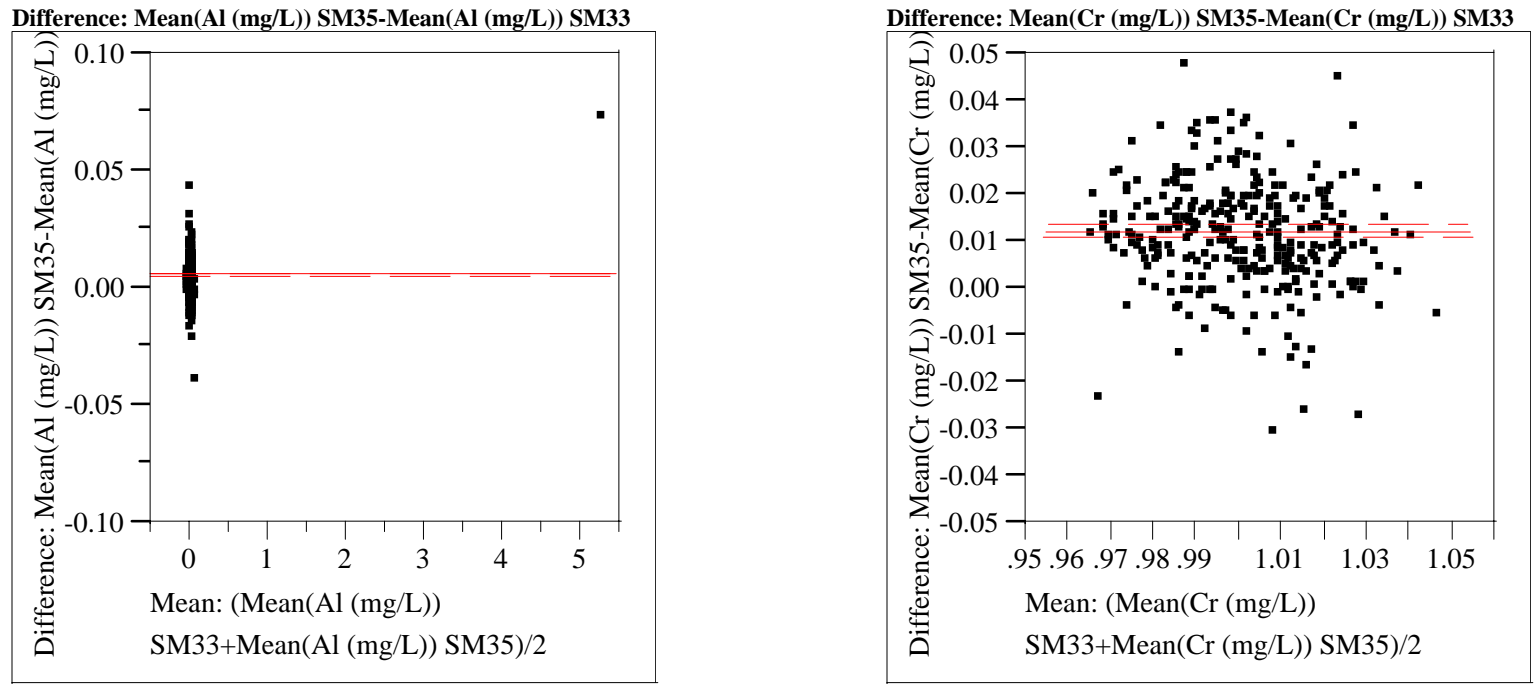

$\begin{array}{lrlr}\text { Mean(Al (mg/L)) SM35 } & 0.02175 & \text { t-Ratio } & 10.66921 \\ \text { Mean(Al (mg/L)) SM33 } & 0.01616 & \text { DF } & 304 \\ \text { Mean Difference } & 0.00559 & \text { Prob }>|t| & <.0001 \\ \text { Std Error } & 0.00052 & \text { Prob }>t & <.0001 \\ \text { Upper95\% } & 0.00662 & \text { Prob }<\mathrm{t} & 1.0000 \\ \text { Lower95\% } & 0.00456 & & \\ \text { N } & 305 & & \\ \text { Correlation } & 0.99962 & & \end{array}$

Difference: Mean(Ca (mg/L)) SM35-Mean(Ca (mg/L)) SM33

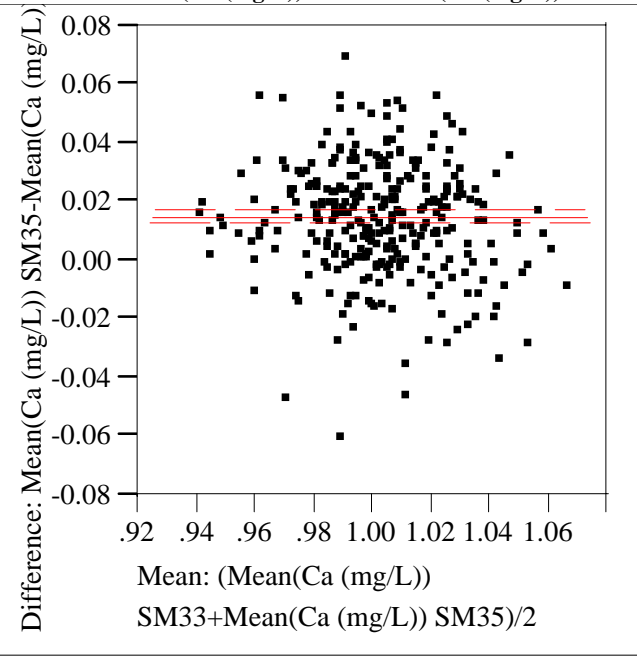

$\begin{array}{lrlr}\text { Mean(Ca (mg/L)) SM35 } & 1.01034 & \text { t-Ratio } & 13.64267 \\ \text { Mean(Ca (mg/L)) SM33 } & 0.99531 & \text { DF } & 304 \\ \text { Mean Difference } & 0.01503 & \text { Prob }>|t| & <.0001 \\ \text { Std Error } & 0.0011 & \text { Prob }>t & <.0001 \\ \text { Upper95\% } & 0.0172 & \text { Prob }<t & 1.0000 \\ \text { Lower95\% } & 0.01286 & & \\ \text { N } & 305 & & \\ \text { Correlation } & 0.69877 & & \end{array}$

$\begin{array}{lrlr}\text { Mean(Cr (mg/L)) SM35 } & 1.00657 & \text { t-Ratio } & 18.35433 \\ \text { Mean(Cr (mg/L)) SM33 } & 0.99444 & \text { DF } & 304 \\ \text { Mean Difference } & 0.01213 & \text { Prob }>|t| & <.0001 \\ \text { Std Error } & 0.00066 & \text { Prob }>t & <.0001 \\ \text { Upper95\% } & 0.01344 & \text { Prob }<\mathrm{t} & 1.0000 \\ \text { Lower95\% } & 0.01083 & & \\ \text { N } & 305 & & \\ \text { Correlation } & 0.79083 & & \end{array}$

Difference: Mean(Cu (mg/L)) SM35-Mean(Cu (mg/L)) SM33

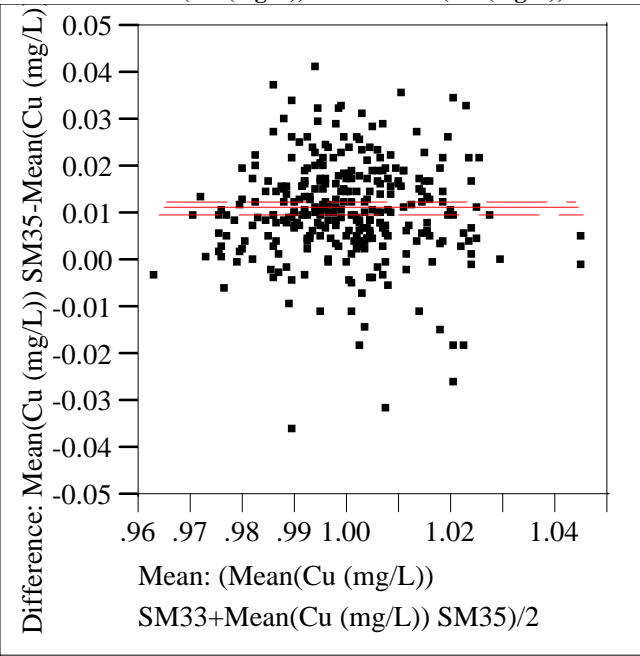

$\begin{array}{lrlr}\text { Mean }(\mathrm{Cu}(\mathrm{mg} / \mathrm{L})) \text { SM35 } & 1.00538 & \mathrm{t}-\text { Ratio } & 18.38029 \\ \text { Mean(Cu (mg/L)) SM33 } & 0.99421 & \text { DF } & 304 \\ \text { Mean Difference } & 0.01117 & \text { Prob }>|\mathrm{t}| & <.0001 \\ \text { Std Error } & 0.00061 & \text { Prob }>\mathrm{t} & <.0001 \\ \text { Upper95\% } & 0.01237 & \text { Prob }<\mathrm{t} & 1.0000 \\ \text { Lower95\% } & 0.00997 & & \\ \mathrm{~N} & 305 & & \\ \text { Correlation } & 0.70216 & & \end{array}$


WSRC-TR-2004-00576

Revision 0

\section{Exhibit A14. Paired Comparisons of SM35 versus SM33 for Mixed Acid Data}
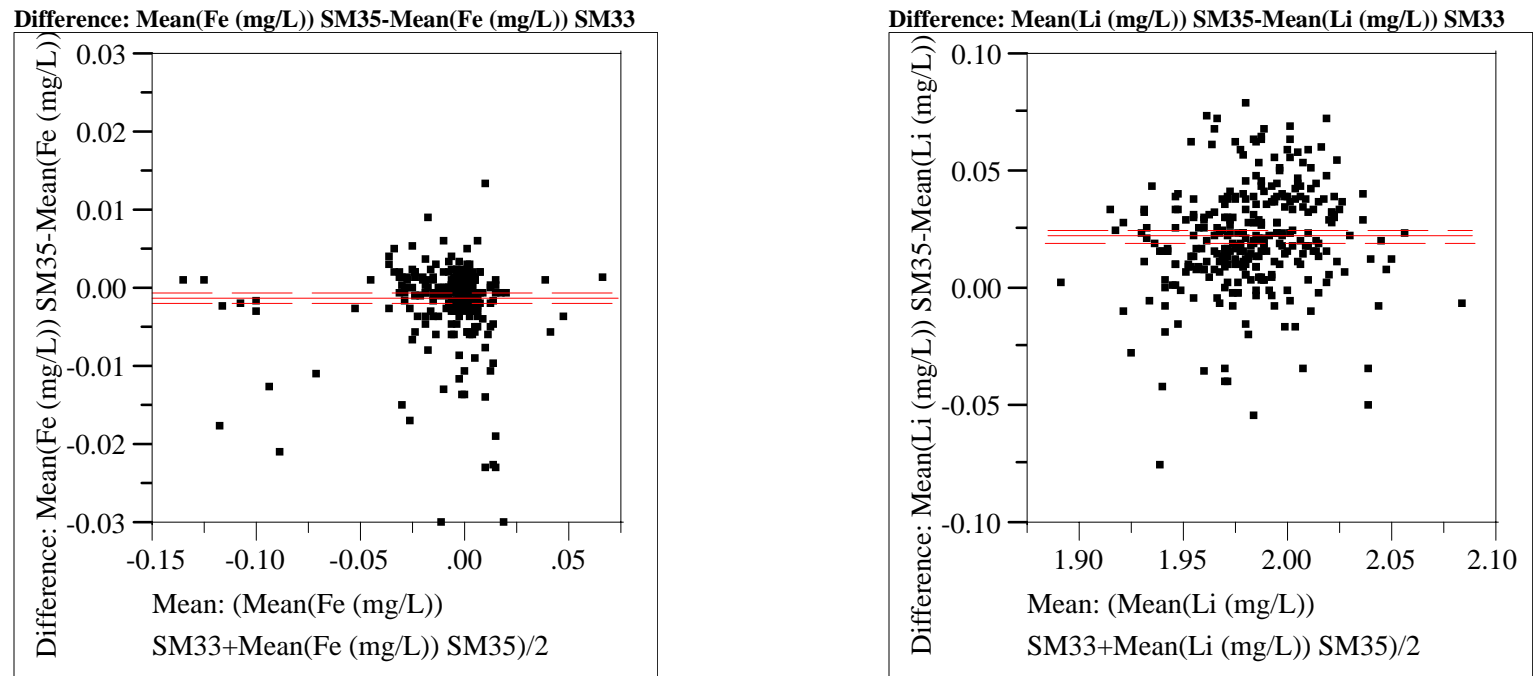

$\begin{array}{lrlr}\text { Mean(Fe (mg/L)) SM35 } & -0.0081 & \mathrm{t}-\text { Ratio } & -3.89603 \\ \text { Mean(Fe (mg/L)) SM33 } & -0.007 & \text { DF } & 304 \\ \text { Mean Difference } & -0.0011 & \text { Prob }>|t| & 0.0001 \\ \text { Std Error } & 0.00029 & \text { Prob }>\text { t } & 0.9999 \\ \text { Upper95\% } & -0.0006 & \text { Prob }<\mathrm{t} & <.0001 \\ \text { Lower95\% } & -0.0017 & & \\ \text { N } & 305 & & \\ \text { Correlation } & 0.97592 & & \end{array}$

Difference: Mean(K (mg/L)) SM35-Mean(K (mg/L)) SM33

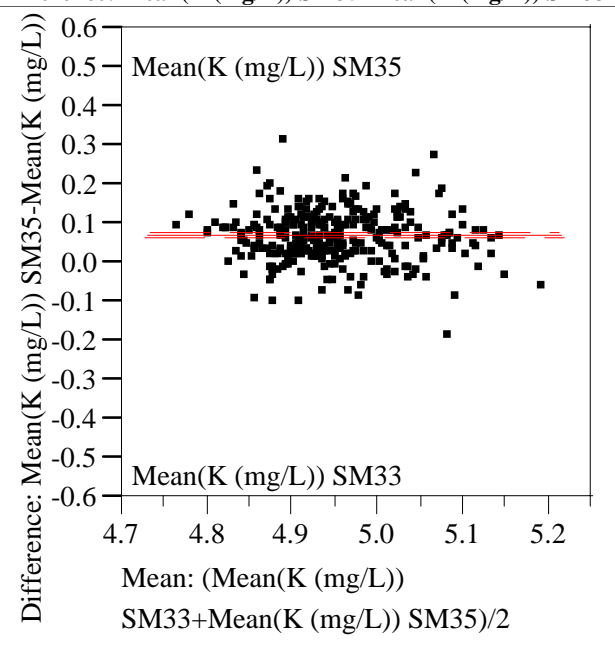

$\begin{array}{lrlr}\text { Mean }(\mathrm{K}(\mathrm{mg} / \mathrm{L})) \text { SM35 } & 4.98468 & \text { t-Ratio } & 19.43239 \\ \text { Mean(K (mg/L)) SM33 } & 4.91212 & \text { DF } & 304 \\ \text { Mean Difference } & 0.07256 & \text { Prob }>|t| & <.0001 \\ \text { Std Error } & 0.00373 & \text { Prob }>t & <.0001 \\ \text { Upper95\% } & 0.07991 & \text { Prob }<\mathrm{t} & 1.0000 \\ \text { Lower95\% } & 0.06521 & & \\ \text { N } & 305 & & \\ \text { Correlation } & 0.68301 & & \end{array}$

$\begin{array}{lrlr}\text { Mean(Li (mg/L)) SM35 } & 1.99297 & \text { t-Ratio } & 17.36545 \\ \text { Mean(Li (mg/L)) SM33 } & 1.9706 & \text { DF } & 304 \\ \text { Mean Difference } & 0.02237 & \text { Prob }>|t| & <.0001 \\ \text { Std Error } & 0.00129 & \text { Prob }>t & <.0001 \\ \text { Upper95\% } & 0.02491 & \text { Prob }<\mathrm{t} & 1.0000 \\ \text { Lower95\% } & 0.01984 & & \\ \text { N } & 305 & & \\ \text { Correlation } & 0.71247 & & \end{array}$

Difference: Mean(Mg (mg/L)) SM35-Mean(Mg (mg/L)) SM33

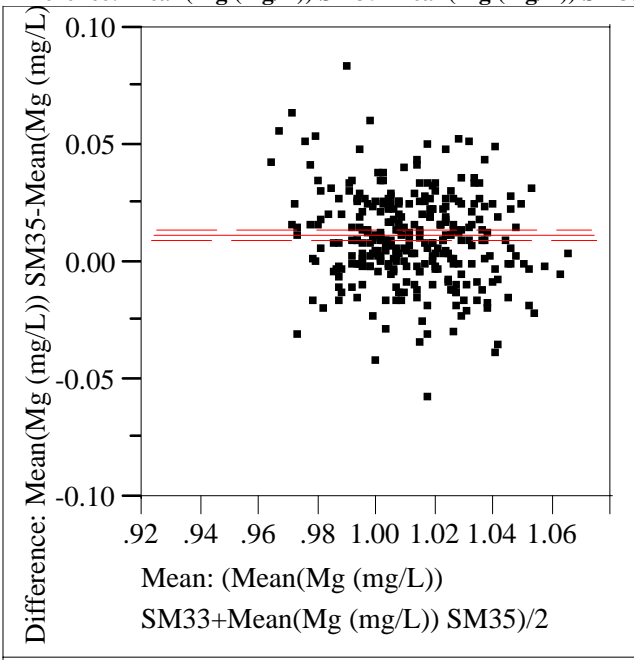

$\begin{array}{lrlr}\text { Mean }(\mathrm{Mg}(\mathrm{mg} / \mathrm{L})) \text { SM35 } & 1.01765 & \mathrm{t}-\text { Ratio } & 10.22383 \\ \text { Mean(Mg (mg/L)) SM33 } & 1.00642 & \mathrm{DF} & 304 \\ \text { Mean Difference } & 0.01123 & \text { Prob }>|\mathrm{t}| & <.0001 \\ \text { Std Error } & 0.0011 & \text { Prob }>\mathrm{t} & <.0001 \\ \text { Upper95\% } & 0.01339 & \text { Prob }<\mathrm{t} & 1.0000 \\ \text { Lower95\% } & 0.00907 & & \\ \mathrm{~N} & 305 & & \\ \text { Correlation } & 0.60749 & & \end{array}$


WSRC-TR-2004-00576

Revision 0

\section{Exhibit A14. Paired Comparisons of SM35 versus SM33 for Mixed Acid Data}
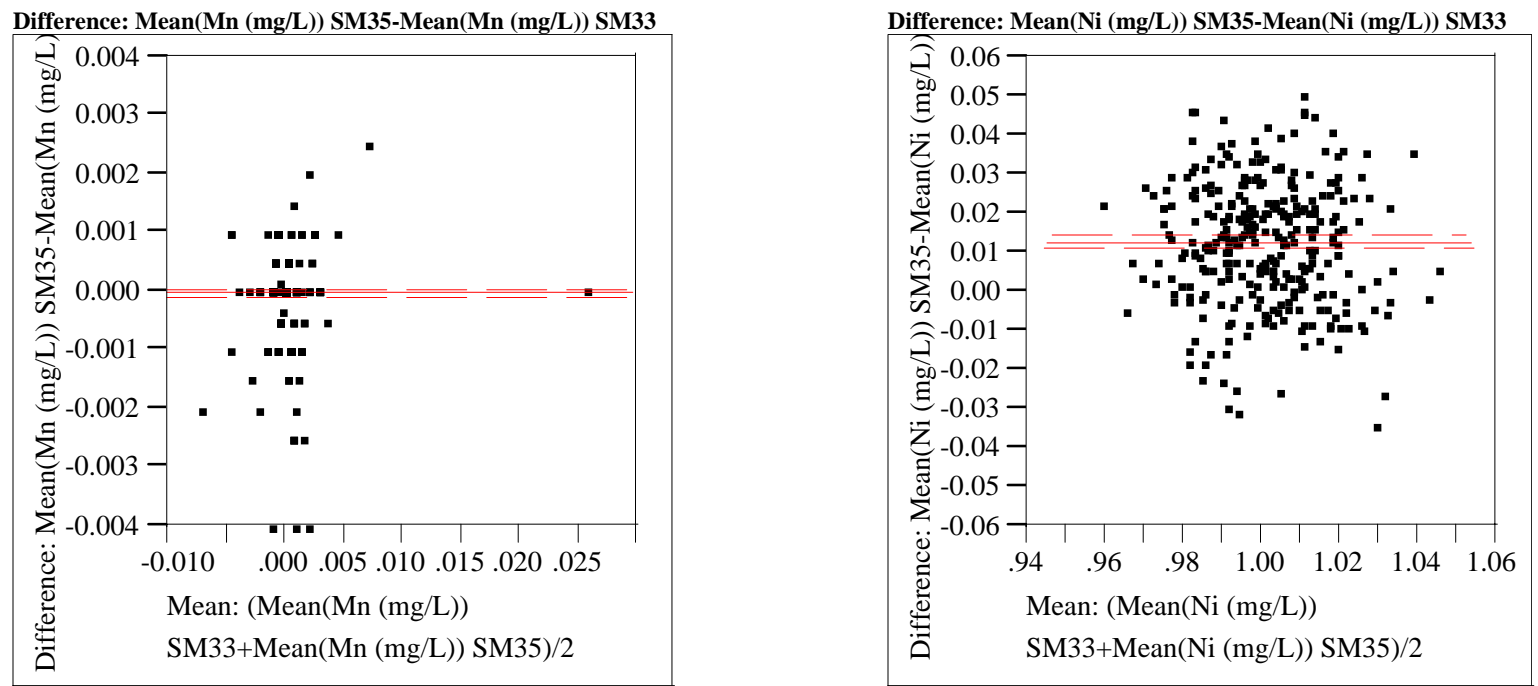

$\begin{array}{lrlr}\text { Mean(Mn (mg/L)) SM35 } & 0.00012 & \text { t-Ratio } & -0.97016 \\ \text { Mean(Mn (mg/L)) SM33 } & 0.00016 & \text { DF } & 304 \\ \text { Mean Difference } & -4.2 \mathrm{e}-5 & \text { Prob }>|\mathrm{t}| & 0.3327 \\ \text { Std Error } & 0.00004 & \text { Prob }>\mathrm{t} & 0.8336 \\ \text { Upper95\% } & 0.00004 & \text { Prob }<\mathrm{t} & 0.1664 \\ \text { Lower95\% } & -0.0001 & & \\ \text { N } & 305 & & \\ \text { Correlation } & 0.92751 & & \end{array}$

Difference: Mean(Na (mg/L)) SM35-Mean(Na (mg/L)) SM33

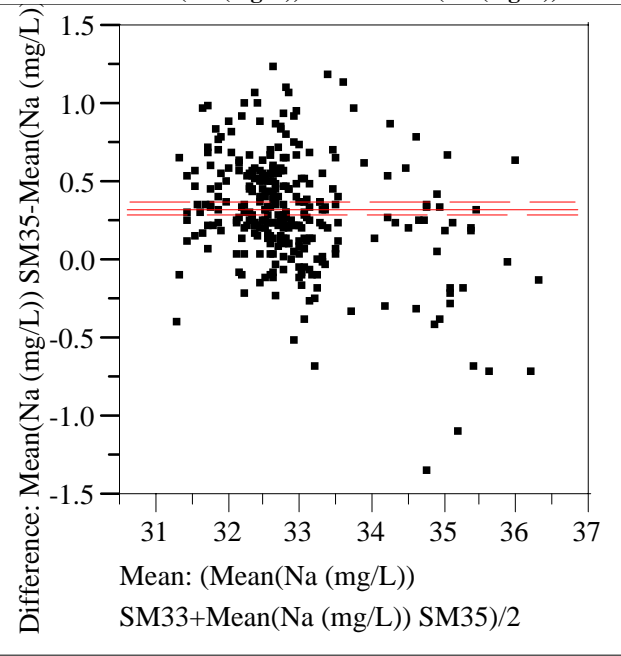

$\begin{array}{lrlr}\text { Mean(Na (mg/L)) SM35 } & 33.0419 & \text { t-Ratio } & 16.24694 \\ \text { Mean(Na (mg/L)) SM33 } & 32.7116 & \text { DF } & 304 \\ \text { Mean Difference } & 0.33031 & \text { Prob }>|t| & <.0001 \\ \text { Std Error } & 0.02033 & \text { Prob }>t & <.0001 \\ \text { Upper95\% } & 0.37031 & \text { Prob }<t & 1.0000 \\ \text { Lower95\% } & 0.2903 & & \\ \text { N } & 305 & & \\ \text { Correlation } & 0.93697 & & \end{array}$

Mean(Ni (mg/L)) SM35 $1.00718 \quad$ t-Ratio 13.89571 Mean(Ni (mg/L)) SM33 $0.99469 \quad$ DF 304 Mean Difference $\quad 0.01249 \quad$ Prob $>|t| \quad<.0001$ $\begin{array}{llll}\text { Std Error } & 0.0009 & \text { Prob }>t & <.0001\end{array}$ Upper95\% $\quad 0.01426 \quad$ Prob $<\mathrm{t} \quad 1.0000$ $\mathrm{N}$ Correlation $\quad 0.56414$

Difference: Mean(Si (mg/L)) SM35-Mean(Si (mg/L)) SM33

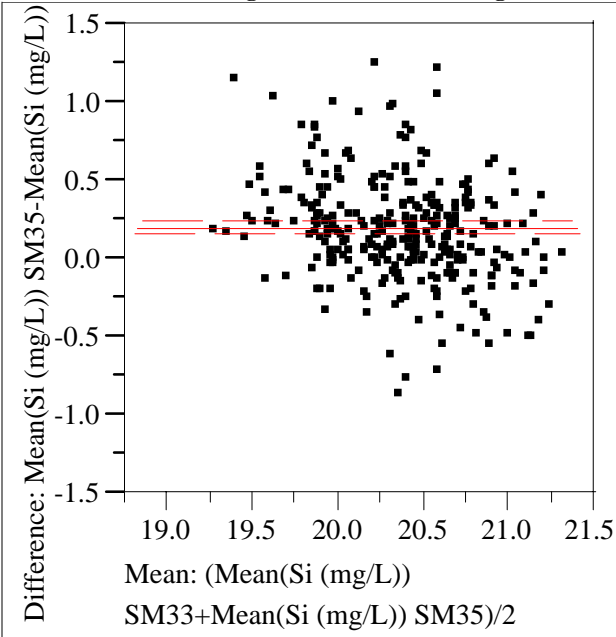

$\begin{array}{lrlr}\text { Mean(Si (mg/L)) SM35 } & 20.4596 & \text { t-Ratio } & 10.5016 \\ \text { Mean(Si (mg/L)) SM33 } & 20.2611 & \text { DF } & 304 \\ \text { Mean Difference } & 0.19853 & \text { Prob }>|t| & <.0001 \\ \text { Std Error } & 0.01891 & \text { Prob }>t & <.0001 \\ \text { Upper95\% } & 0.23574 & \text { Prob }<\mathrm{t} & 1.0000 \\ \text { Lower95\% } & 0.16133 & & \\ \text { N } & 305 & & \\ \text { Correlation } & 0.73459 & & \end{array}$


WSRC-TR-2004-00576

Revision 0

Exhibit A14. Paired Comparisons of SM35 versus SM33 for Mixed Acid Data
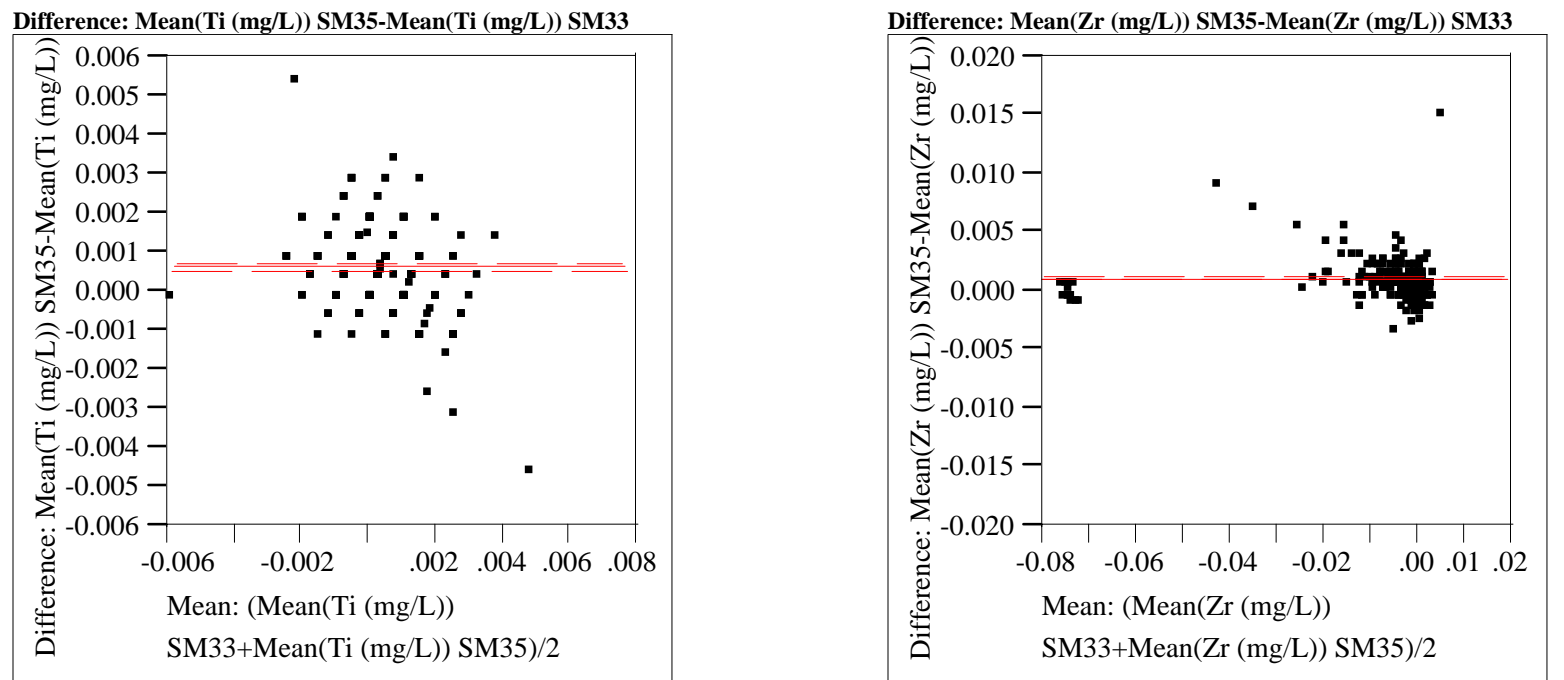

$\begin{array}{lrlr}\text { Mean(Ti (mg/L)) SM35 } & 0.00072 & \mathrm{t}-\text { Ratio } & 10.42806 \\ \text { Mean(Ti (mg/L)) SM33 } & 0.00011 & \text { DF } & 304 \\ \text { Mean Difference } & 0.00061 & \text { Prob }>|t| & <.0001 \\ \text { Std Error } & 0.00006 & \text { Prob }>\mathrm{t} & <.0001 \\ \text { Upper95\% } & 0.00072 & \text { Prob }<\mathrm{t} & 1.0000 \\ \text { Lower95\% } & 0.00049 & & \\ \text { N } & 305 & & \\ \text { Correlation } & 0.68648 & & \end{array}$

$\begin{array}{lrlr}\text { Mean }(\mathrm{Zr}(\mathrm{mg} / \mathrm{L})) \text { SM35 } & -0.0058 & \mathrm{t}-\text { Ratio } & 12.28788 \\ \text { Mean }(\mathrm{Zr}(\mathrm{mg} / \mathrm{L})) \text { SM33 } & -0.0069 & \text { DF } & 304 \\ \text { Mean Difference } & 0.00111 & \text { Prob }>|\mathrm{t}| & <.0001 \\ \text { Std Error } & 0.00009 & \text { Prob }>\mathrm{t} & <.0001 \\ \text { Upper95\% } & 0.00128 & \text { Prob }<\mathrm{t} & 1.0000 \\ \text { Lower95\% } & 0.00093 & & \\ \text { N } & 305 & & \\ \text { Correlation } & 0.99485 & & \end{array}$

Difference: Mean(U (mg/L)) SM35-Mean(U (mg/L)) SM33

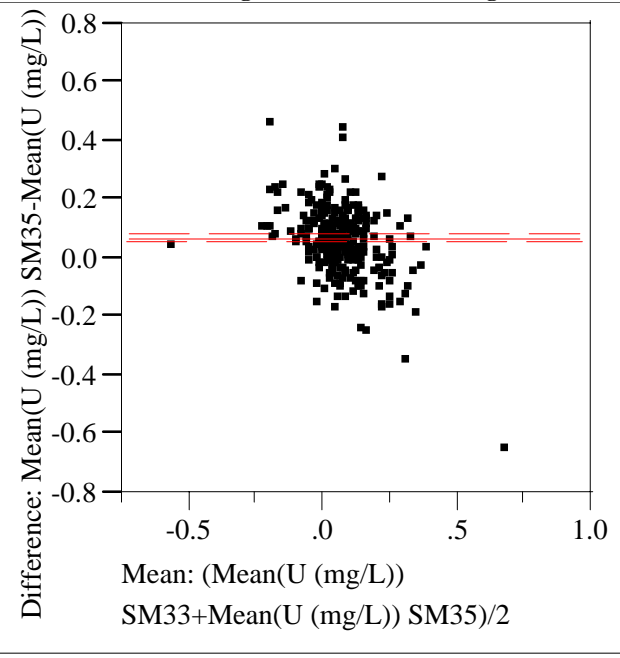

$\begin{array}{lrlr}\text { Mean(U (mg/L)) SM35 } & 0.09804 & \text { t-Ratio } & 10.59003 \\ \text { Mean(U (mg/L)) SM33 } & 0.02976 & \text { DF } & 304 \\ \text { Mean Difference } & 0.06828 & \text { Prob }>|t| & <.0001 \\ \text { Std Error } & 0.00645 & \text { Prob }>\text { t } & <.0001 \\ \text { Upper95\% } & 0.08097 & \text { Prob }<\mathrm{t} & 1.0000 \\ \text { Lower95\% } & 0.05559 & & \\ \text { N } & 305 & & \\ \text { Correlation } & 0.6441 & & \end{array}$


WSRC-TR-2004-00576

Revision 0

\section{Exhibit A15. Paired Comparisons of SM36 versus SM34 for Mixed Acid Data}

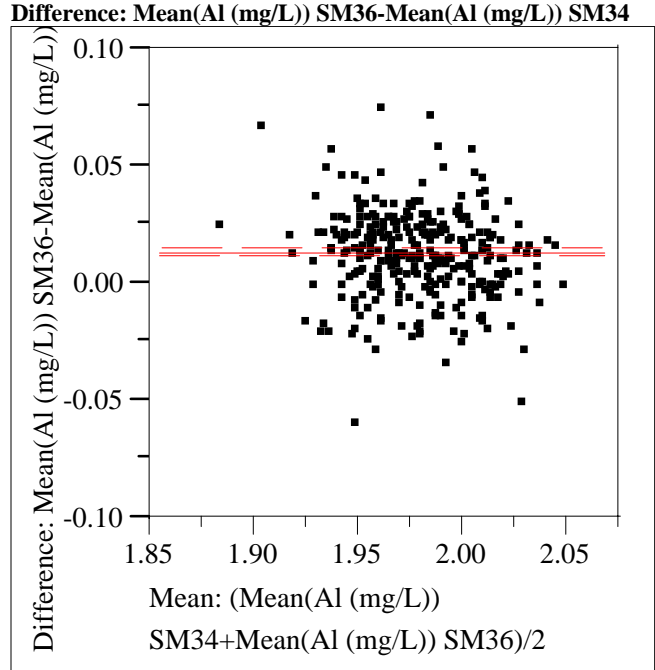

$\begin{array}{lrlr}\text { Mean(Al (mg/L)) SM36 } & 1.98574 & \text { t-Ratio } & 12.68897 \\ \text { Mean(Al (mg/L)) SM34 } & 1.97255 & \text { DF } & 312 \\ \text { Mean Difference } & 0.01319 & \text { Prob }>|t| & <.0001 \\ \text { Std Error } & 0.00104 & \text { Prob }>t & <.0001 \\ \text { Upper95\% } & 0.01524 & \text { Prob }<\mathrm{t} & 1.0000 \\ \text { Lower95\% } & 0.01115 & & \\ \text { N } & 313 & & \\ \text { Correlation } & 0.8036 & & \end{array}$

Difference: Mean(Ca (mg/L)) SM36-Mean(Ca (mg/L)) SM34

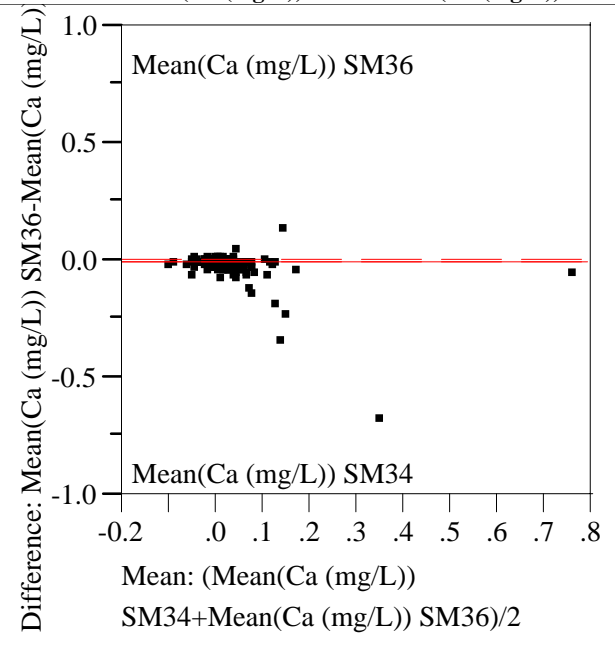

$\begin{array}{lrlr}\text { Mean(Ca (mg/L)) SM36 } & 0.01416 & \text { t-Ratio } & -1.65222 \\ \text { Mean(Ca (mg/L)) SM34 } & 0.01868 & \text { DF } & 312 \\ \text { Mean Difference } & -0.0045 & \text { Prob }>|t| & 0.0995 \\ \text { Std Error } & 0.00274 & \text { Prob }>\text { t } & 0.9503 \\ \text { Upper95\% } & 0.00086 & \text { Prob }<\mathrm{t} & 0.0497 \\ \text { Lower95\% } & -0.0099 & & \\ \text { N } & 313 & & \\ \text { Correlation } & 0.72302 & & \end{array}$

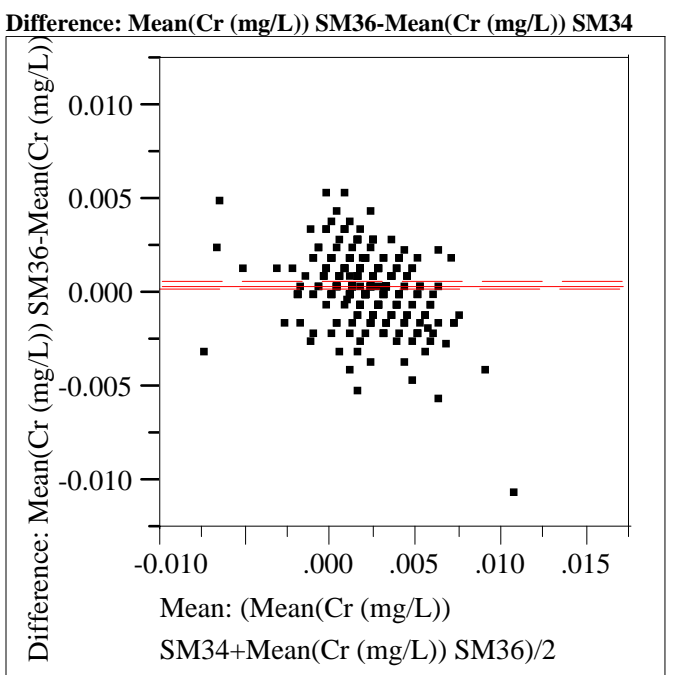

$\begin{array}{lrlr}\text { Mean(Cr (mg/L)) SM36 } & 0.00229 & \text { t-Ratio } & 3.805284 \\ \text { Mean(Cr (mg/L)) SM34 } & 0.00191 & \text { DF } & 312 \\ \text { Mean Difference } & 0.00038 & \text { Prob }>|t| & 0.0002 \\ \text { Std Error } & 0.0001 & \text { Prob }>t & <.0001 \\ \text { Upper95\% } & 0.00058 & \text { Prob }<\mathrm{t} & 0.9999 \\ \text { Lower95\% } & 0.00019 & & \\ \text { N } & 313 & & \\ \text { Correlation } & 0.74744 & & \end{array}$

Difference: Mean(Cu (mg/L)) SM36-Mean(Cu (mg/L)) SM34

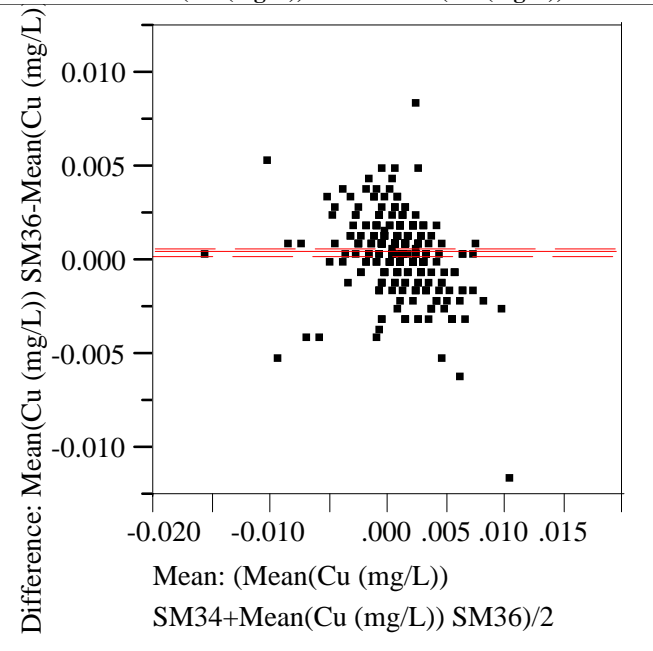

$\begin{array}{lrlr}\text { Mean }(\mathrm{Cu}(\mathrm{mg} / \mathrm{L})) \text { SM36 } & 0.00115 & \mathrm{t}-\text { Ratio } & 4.304008 \\ \text { Mean(Cu (mg/L)) SM34 } & 0.00068 & \text { DF } & 312 \\ \text { Mean Difference } & 0.00047 & \text { Prob }>|\mathrm{t}| & <.0001 \\ \text { Std Error } & 0.00011 & \text { Prob }>\mathrm{t} & <.0001 \\ \text { Upper95\% } & 0.00069 & \text { Prob }<\mathrm{t} & 1.0000 \\ \text { Lower95\% } & 0.00026 & & \\ \mathrm{~N} & 313 & & \\ \text { Correlation } & 0.80579 & & \end{array}$


WSRC-TR-2004-00576

Revision 0

\section{Exhibit A15. Paired Comparisons of SM36 versus SM34 for Mixed Acid Data}
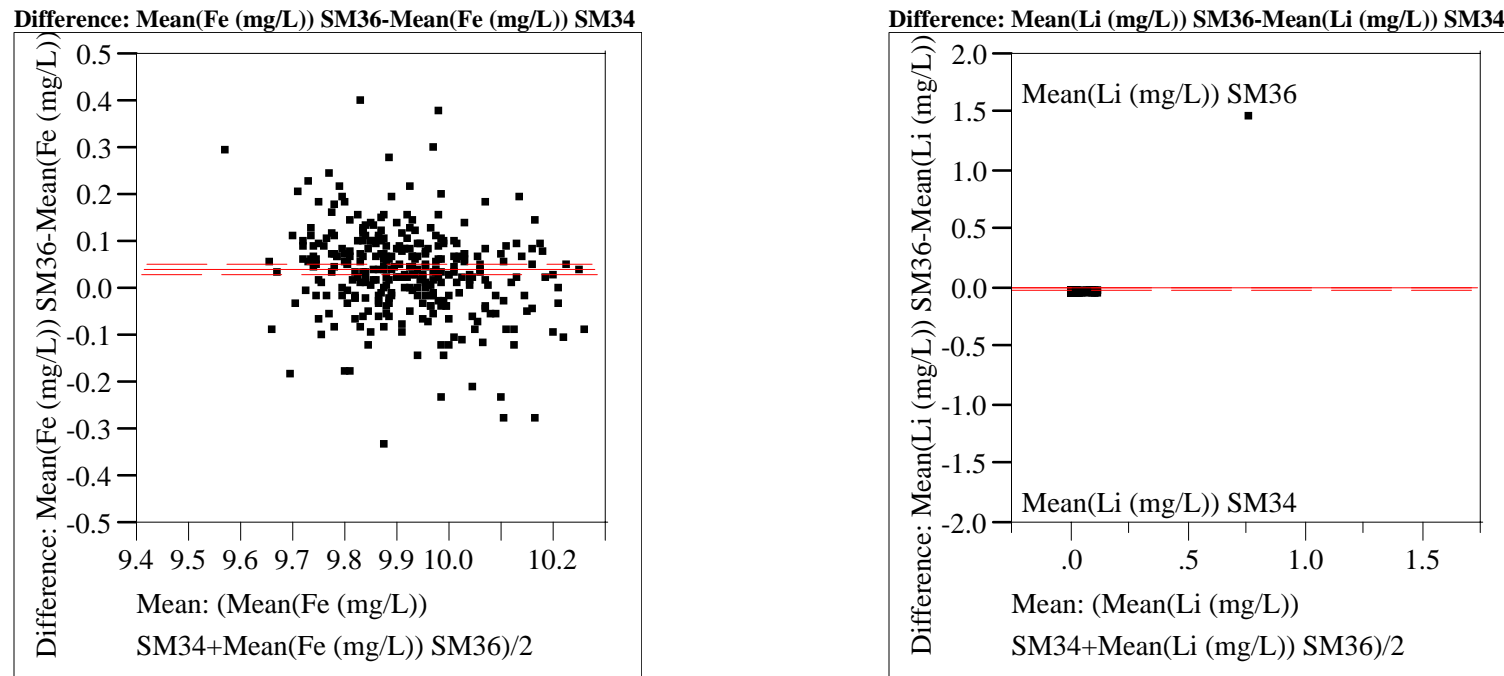

$\begin{array}{lrlr}\text { Mean(Fe (mg/L)) SM36 } & 9.93903 & \text { t-Ratio } & 7.955643 \\ \text { Mean(Fe (mg/L)) SM34 } & 9.89681 & \text { DF } & 312 \\ \text { Mean Difference } & 0.04222 & \text { Prob }>|t| & <.0001 \\ \text { Std Error } & 0.00531 & \text { Prob }>t & <.0001 \\ \text { Upper95\% } & 0.05266 & \text { Prob }<\mathrm{t} & 1.0000 \\ \text { Lower95\% } & 0.03178 & & \\ \text { N } & 313 & & \\ \text { Correlation } & 0.75927 & & \end{array}$

Difference: Mean(K (mg/L)) SM36-Mean(K (mg/L)) SM34

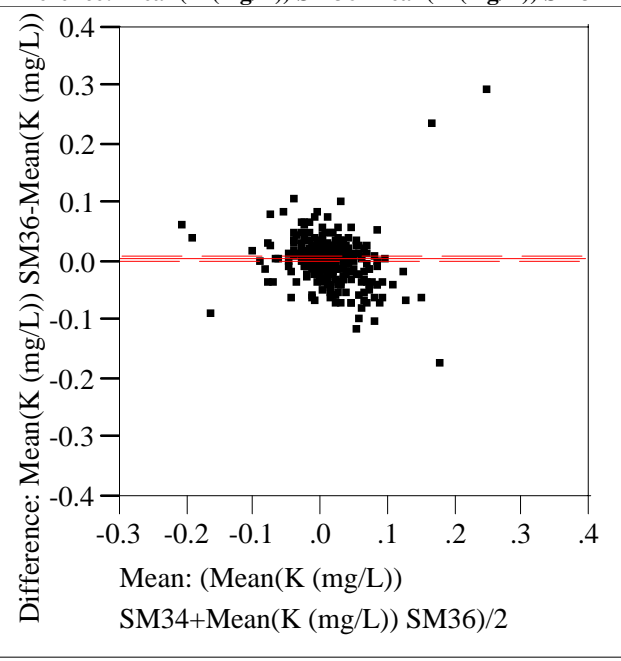

$\begin{array}{lrlr}\text { Mean }(\mathrm{K}(\mathrm{mg} / \mathrm{L})) \text { SM36 } & 0.01434 & \mathrm{t}-\text { Ratio } & 2.943505 \\ \text { Mean(K (mg/L)) SM34 } & 0.00746 & \text { DF } & 312 \\ \text { Mean Difference } & 0.00688 & \text { Prob }>|\mathrm{t}| & 0.0035 \\ \text { Std Error } & 0.00234 & \text { Prob }>\mathrm{t} & 0.0017 \\ \text { Upper95\% } & 0.01147 & \text { Prob }<\mathrm{t} & 0.9983 \\ \text { Lower95\% } & 0.00228 & & \\ \text { N } & 313 & & \\ \text { Correlation } & 0.66582 & & \end{array}$

$\begin{array}{lrlr}\text { Mean(Li (mg/L)) SM36 } & 0.01161 & \text { t-Ratio } & 0.988498 \\ \text { Mean(Li (mg/L)) SM34 } & 0.00687 & \text { DF } & 312 \\ \text { Mean Difference } & 0.00474 & \text { Prob }>|t| & 0.3237 \\ \text { Std Error } & 0.0048 & \text { Prob }>\text { t } & 0.1618 \\ \text { Upper95\% } & 0.01418 & \text { Prob }<\mathrm{t} & 0.8382 \\ \text { Lower95\% } & -0.0047 & & \\ \text { N } & 313 & & \\ \text { Correlation } & 0.22067 & & \end{array}$

Difference: Mean(Mg (mg/L)) SM36-Mean(Mg (mg/L)) SM34

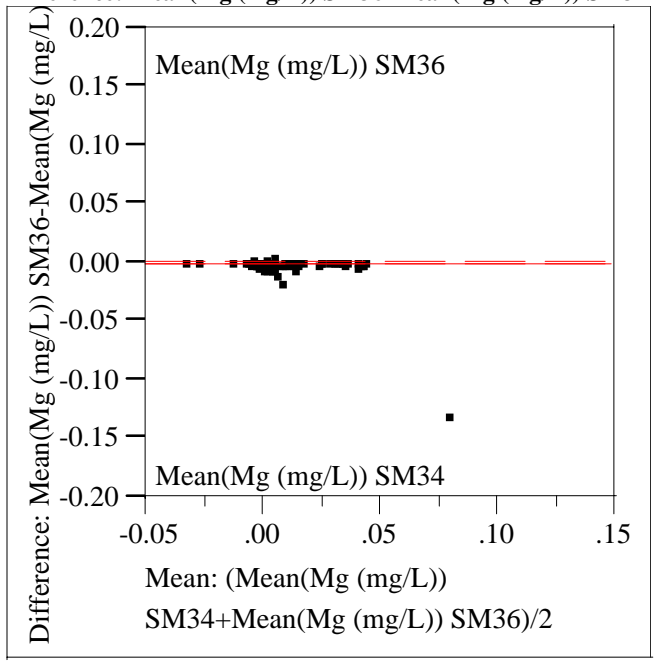

$\begin{array}{lrlr}\text { Mean }(\mathrm{Mg}(\mathrm{mg} / \mathrm{L})) \text { SM36 } & 0.00268 & \mathrm{t}-\text { Ratio } & -1.80112 \\ \text { Mean(Mg (mg/L)) SM34 } & 0.00345 & \text { DF } & 312 \\ \text { Mean Difference } & -0.0008 & \text { Prob }>|\mathrm{t}| & 0.0726 \\ \text { Std Error } & 0.00043 & \text { Prob }>\mathrm{t} & 0.9637 \\ \text { Upper95\% } & 0.00007 & \text { Prob }<\mathrm{t} & 0.0363 \\ \text { Lower95\% } & -0.0016 & & \\ \text { N } & 313 & & \\ \text { Correlation } & 0.77139 & & \end{array}$


WSRC-TR-2004-00576

Revision 0

\section{Exhibit A15. Paired Comparisons of SM36 versus SM34 for Mixed Acid Data}
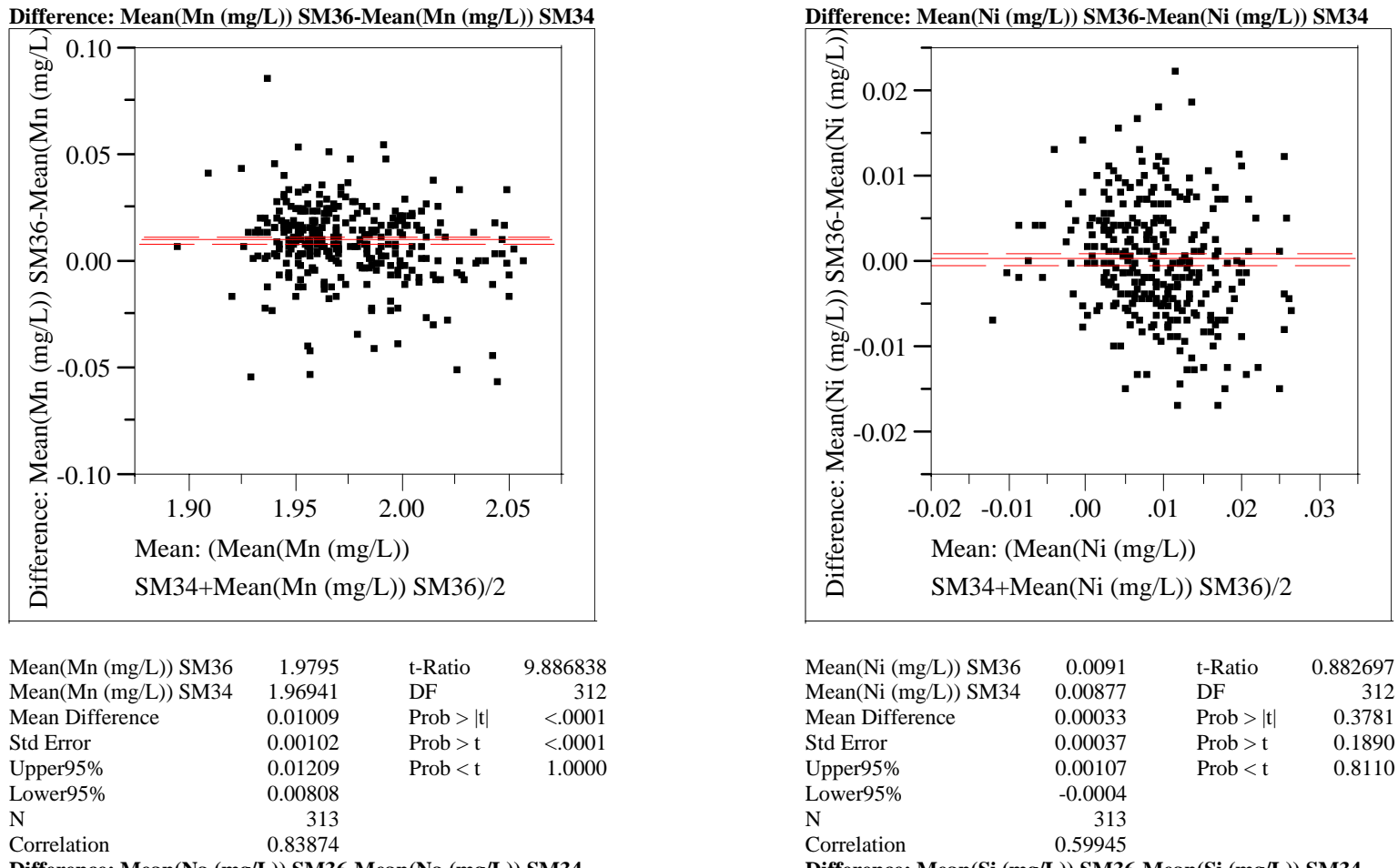

Difference: Mean(Na (mg/L)) SM36-Mean(Na (mg/L)) SM34

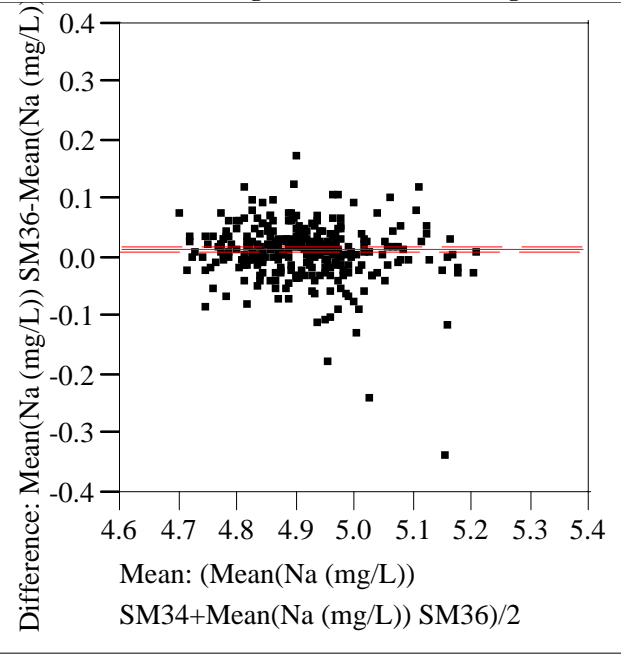

$\begin{array}{lrlr}\text { Mean(Na (mg/L)) SM36 } & 4.92084 & \text { t-Ratio } & 5.361701 \\ \text { Mean(Na (mg/L)) SM34 } & 4.90595 & \text { DF } & 312 \\ \text { Mean Difference } & 0.01488 & \text { Prob }>|t| & <.0001 \\ \text { Std Error } & 0.00278 & \text { Prob }>t & <.0001 \\ \text { Upper95\% } & 0.02035 & \text { Prob }<\mathrm{t} & 1.0000 \\ \text { Lower95\% } & 0.00942 & & \\ \text { N } & 313 & & \\ \text { Correlation } & 0.88203 & & \end{array}$

$\begin{array}{lrlr}\text { Mean(Ni (mg/L)) SM36 } & 0.0091 & \text { t-Ratio } & 0.882697 \\ \text { Mean(Ni (mg/L)) SM34 } & 0.00877 & \text { DF } & 312 \\ \text { Mean Difference } & 0.00033 & \text { Prob > }|\mathrm{t}| & 0.3781 \\ \text { Std Error } & 0.00037 & \text { Prob }>\mathrm{t} & 0.1890 \\ \text { Upper95\% } & 0.00107 & \text { Prob }<\mathrm{t} & 0.8110 \\ \text { Lower95\% } & -0.0004 & & \\ \text { N } & 313 & & \\ \text { Correlation } & 0.59945 & & \\ \text { Difference: } & & \end{array}$

Difference: Mean(Si (mg/L)) SM36-Mean(Si (mg/L)) SM34

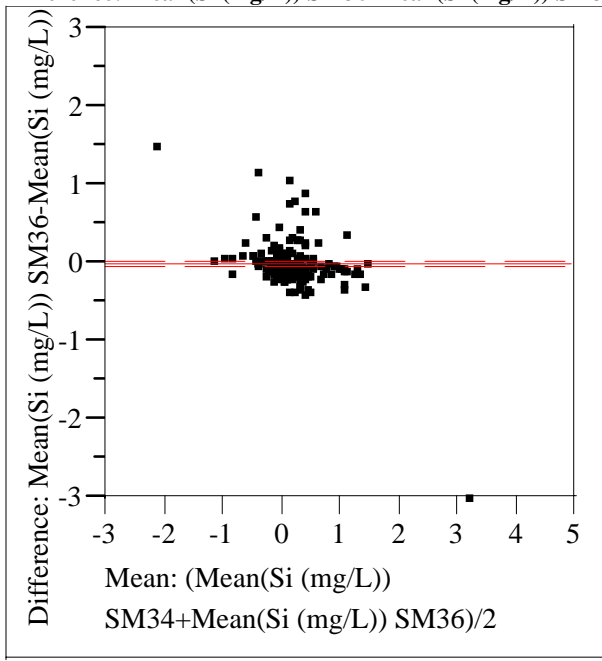

$\begin{array}{lrlr}\text { Mean(Si (mg/L)) SM36 } & 0.1131 & \text { t-Ratio } & -0.77095 \\ \text { Mean(Si (mg/L)) SM34 } & 0.12468 & \text { DF } & 312 \\ \text { Mean Difference } & -0.0116 & \text { Prob }>|t| & 0.4413 \\ \text { Std Error } & 0.01503 & \text { Prob }>\text { t } & 0.7793 \\ \text { Upper95\% } & 0.01798 & \text { Prob }<\mathrm{t} & 0.2207 \\ \text { Lower95\% } & -0.0411 & & \\ \text { N } & 313 & & \\ \text { Correlation } & 0.8324 & & \end{array}$


WSRC-TR-2004-00576

Revision 0

Exhibit A15. Paired Comparisons of SM36 versus SM34 for Mixed Acid Data
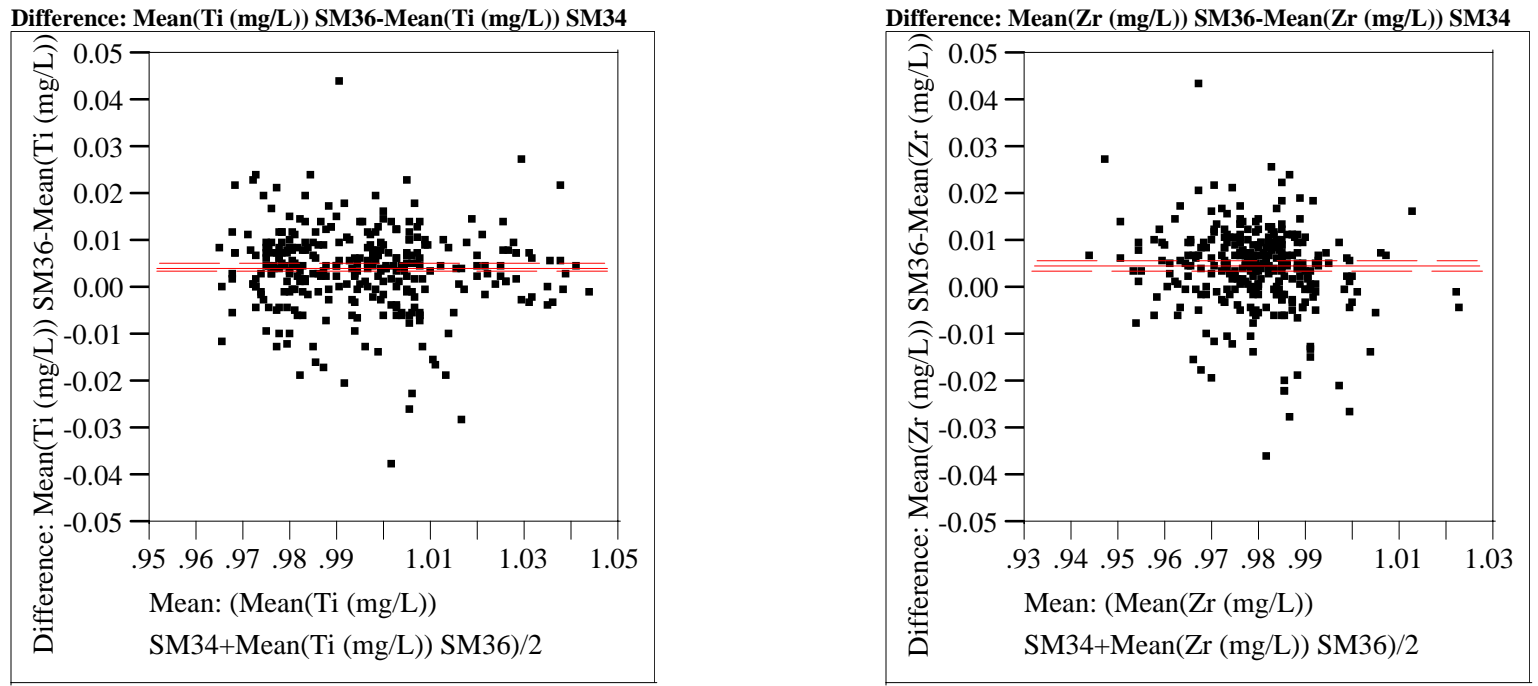

$\begin{array}{lrlr}\text { Mean(Ti (mg/L)) SM36 } & 0.99744 & \text { t-Ratio } & 8.82996 \\ \text { Mean(Ti (mg/L)) SM34 } & 0.993 & \text { DF } & 312 \\ \text { Mean Difference } & 0.00444 & \text { Prob }>|t| & <.0001 \\ \text { Std Error } & 0.0005 & \text { Prob }>\text { t } & <.0001 \\ \text { Upper95\% } & 0.00543 & \text { Prob }<\mathrm{t} & 1.0000 \\ \text { Lower95\% } & 0.00345 & & \\ \text { N } & 313 & & \\ \text { Correlation } & 0.87666 & & \end{array}$

$\begin{array}{lrlr}\text { Mean(Zr (mg/L)) SM36 } & 0.98149 & \text { t-Ratio } & 9.288836 \\ \text { Mean(Zr (mg/L)) SM34 } & 0.97686 & \text { DF } & 312 \\ \text { Mean Difference } & 0.00463 & \text { Prob }>|t| & <.0001 \\ \text { Std Error } & 0.0005 & \text { Prob }>\text { t } & <.0001 \\ \text { Upper95\% } & 0.00561 & \text { Prob }<\mathrm{t} & 1.0000 \\ \text { Lower95\% } & 0.00365 & & \\ \text { N } & 313 & & \\ \text { Correlation } & 0.73919 & & \end{array}$

Difference: Mean(U (mg/L)) SM36-Mean(U (mg/L)) SM34

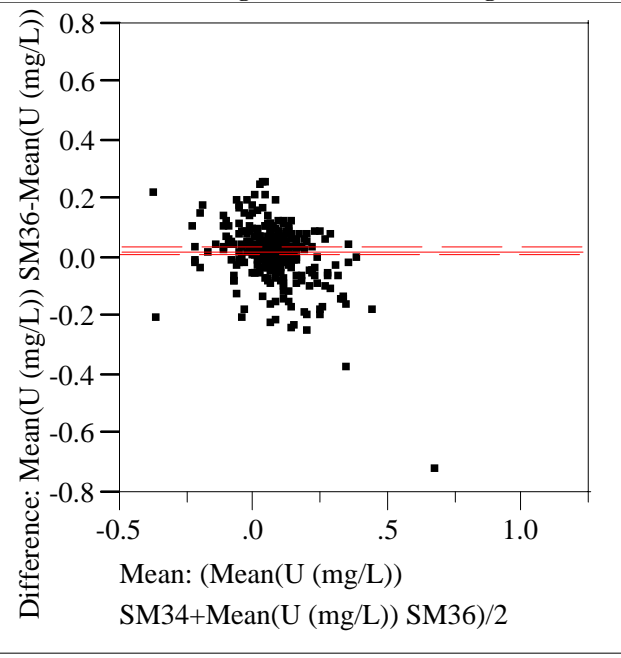

$\begin{array}{lrlr}\text { Mean(U (mg/L)) SM36 } & 0.08016 & \text { t-Ratio } & 4.406798 \\ \text { Mean(U (mg/L)) SM34 } & 0.0554 & \text { DF } & 312 \\ \text { Mean Difference } & 0.02476 & \text { Prob }>|t| & <.0001 \\ \text { Std Error } & 0.00562 & \text { Prob }>t & <.0001 \\ \text { Upper95\% } & 0.03582 & \text { Prob }<\mathrm{t} & 1.0000 \\ \text { Lower95\% } & 0.01371 & & \\ \text { N } & 313 & & \\ \text { Correlation } & 0.7284 & & \end{array}$


WSRC-TR-2004-00576

Revision 0

Exhibit A16. Paired Comparisons of SM38 versus SM37 for Mixed Acid Data

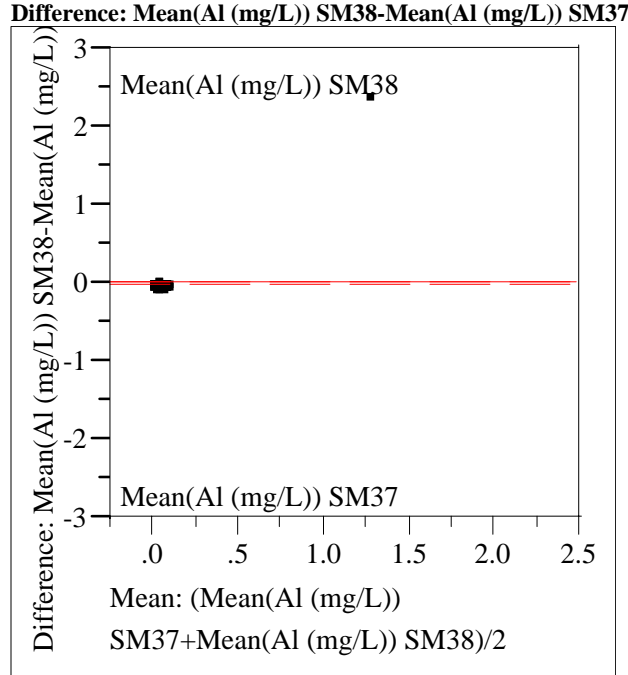

Mean(Al (mg/L)) SM38 $0.05396 \quad$ t-Ratio 1.230675

Mean(Al (mg/L)) SM37 0.04436

Mean Difference $\quad 0.0096$

Std Error

Upper95\%

Lower95\%

$\mathrm{N}$

Correlation

Difference: Mean(Ca (mg/L)) SM38-Mean(Ca (mg/L)) SM37

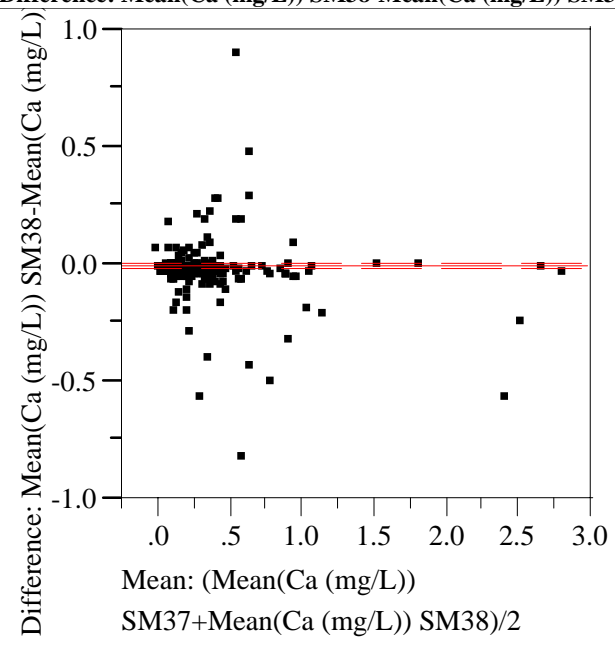

Mean(Ca (mg/L)) SM38

Mean $(\mathrm{Ca}(\mathrm{mg} / \mathrm{L}))$ SM37 0.28758

Mean Difference

Std Error

Upper95\%

Lower95\%

$\mathrm{N}$

Correlation
t-Ratio $\quad-1.38752$

DF 308

Prob $>|t| \quad 0.1663$

Prob $>\mathrm{t} \quad 0.9169$

Prob $<\mathrm{t} \quad 0.0831$
Difference: Mean(Cr (mg/L)) SM38-Mean(Cr (mg/L)) SM37

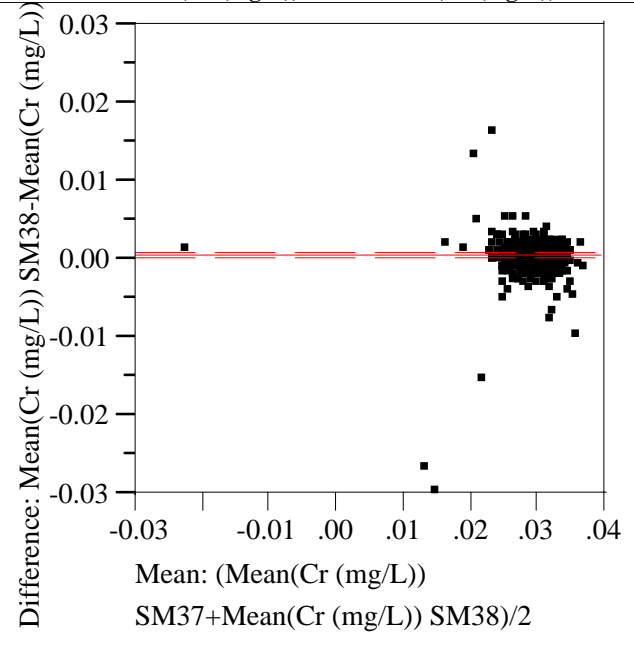

$\begin{array}{lrlr}\text { Mean }(\mathrm{Cr}(\mathrm{mg} / \mathrm{L})) \text { SM38 } & 0.02906 & \text { t-Ratio } & 2.724395 \\ \text { Mean(Cr (mg/L)) SM37 } & 0.02855 & \text { DF } & 308 \\ \text { Mean Difference } & 0.00051 & \text { Prob > tt } & 0.0068 \\ \text { Std Error } & 0.00019 & \text { Prob }>\text { t } & 0.0034 \\ \text { Upper95\% } & 0.00087 & \text { Prob }<\mathrm{t} & 0.9966 \\ \text { Lower95\% } & 0.00014 & & \\ \text { N } & 309 & & \\ \text { Correlation } & 0.77076 & & \\ \text { Difference: Mean(Cu (mg/L)) SM38-Mean(Cu (mg/L)) SM37 }\end{array}$

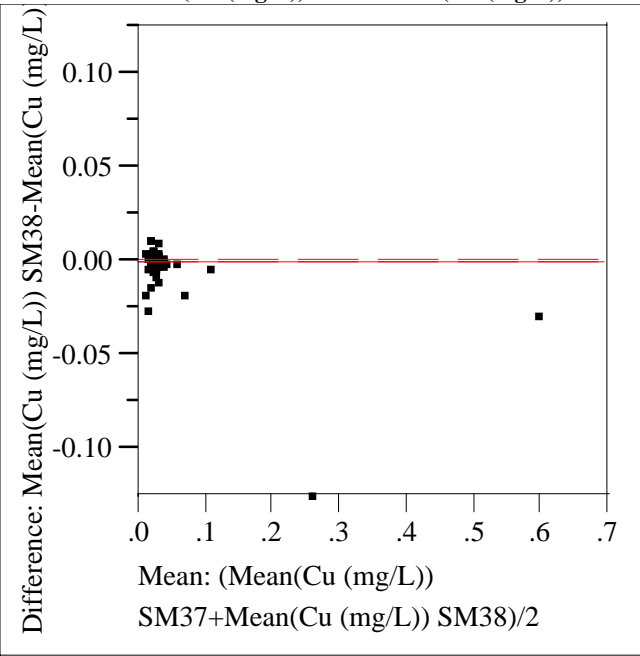

Mean(Cu (mg/L)) SM38 Mean(Cu (mg/L)) SM37 Mean Difference

Std Error

Upper95\%

Lower95\%

$\mathrm{N}$

Correlation
t-Ratio $\quad-0.01795$

DF 308

Prob $<\mathrm{t} \quad 0.4928$
Prob $>|\mathrm{t}| \quad 0.9857$

Prob $>\mathrm{t} \quad 0.5072$ 
WSRC-TR-2004-00576

Revision 0

Exhibit A16. Paired Comparisons of SM38 versus SM37 for Mixed Acid Data
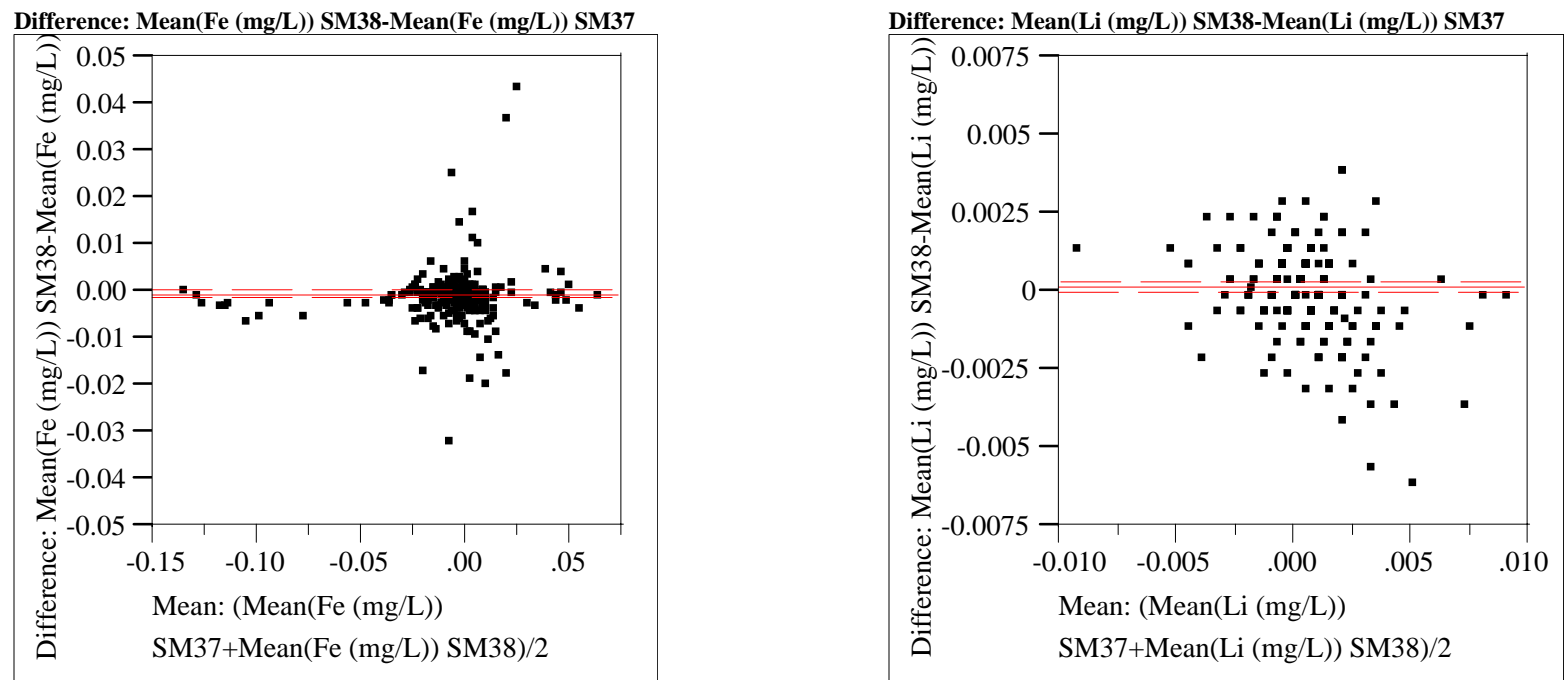

$\begin{array}{lrlr}\text { Mean(Fe (mg/L)) SM38 } & -0.0061 & \text { t-Ratio } & -1.8934 \\ \text { Mean(Fe (mg/L)) SM37 } & -0.0055 & \text { DF } & 308 \\ \text { Mean Difference } & -0.0006 & \text { Prob }>|t| & 0.0592 \\ \text { Std Error } & 0.00031 & \text { Prob }>\text { t } & 0.9704 \\ \text { Upper95\% } & 0.00002 & \text { Prob }<\mathrm{t} & 0.0296 \\ \text { Lower95\% } & -0.0012 & & \\ \text { N } & 309 & & \\ \text { Correlation } & 0.9754 & & \end{array}$

Difference: Mean(K (mg/L)) SM38-Mean(K (mg/L)) SM37

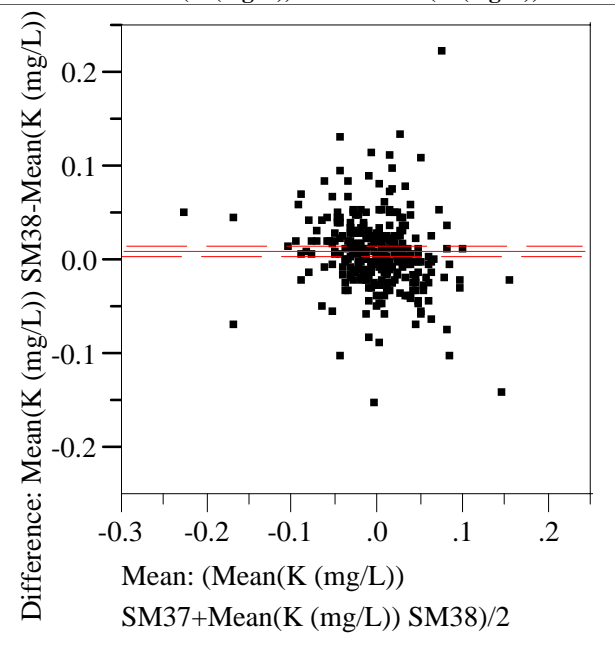

$\begin{array}{lrlr}\text { Mean(K (mg/L)) SM38 } & 0.00275 & \text { t-Ratio } & 4.424413 \\ \text { Mean(K (mg/L)) SM37 } & -0.0072 & \text { DF } & 308 \\ \text { Mean Difference } & 0.00998 & \text { Prob }>|t| & <.0001 \\ \text { Std Error } & 0.00226 & \text { Prob }>t & <.0001 \\ \text { Upper95\% } & 0.01442 & \text { Prob }<\mathrm{t} & 1.0000 \\ \text { Lower95\% } & 0.00554 & & \\ \text { N } & 309 & & \\ \text { Correlation } & 0.66467 & & \end{array}$

$\begin{array}{lrlr}\text { Mean(Li (mg/L)) SM38 } & 0.00055 & \text { t-Ratio } & 1.650136 \\ \text { Mean(Li (mg/L)) SM37 } & 0.00042 & \text { DF } & 308 \\ \text { Mean Difference } & 0.00013 & \text { Prob }>|t| & 0.0999 \\ \text { Std Error } & 0.00008 & \text { Prob }>\text { t } & 0.0500 \\ \text { Upper95\% } & 0.00028 & \text { Prob }<\mathrm{t} & 0.9500 \\ \text { Lower95\% } & -2.5 \mathrm{e}-5 & & \\ \mathrm{~N} & 309 & & \\ \text { Correlation } & 0.77398 & & \end{array}$

Difference: Mean(Mg (mg/L)) SM38-Mean(Mg (mg/L)) SM37

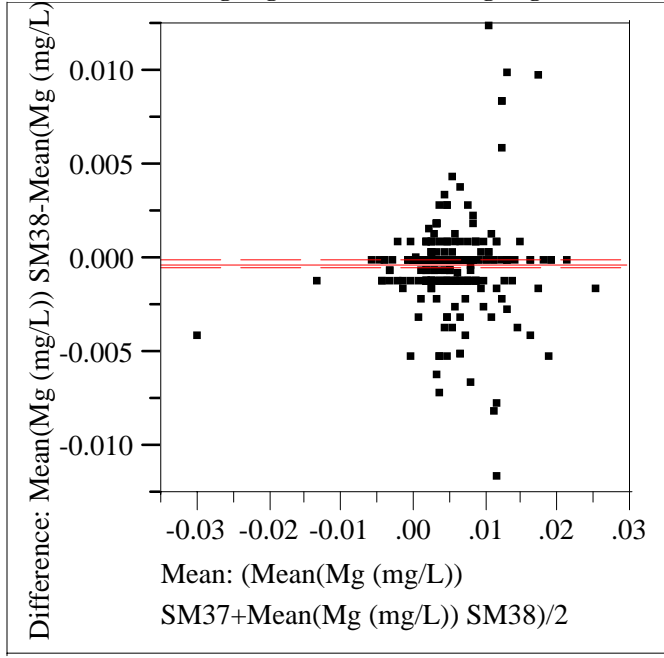

$\begin{array}{lrlr}\text { Mean }(\mathrm{Mg}(\mathrm{mg} / \mathrm{L})) \text { SM38 } & 0.00485 & \mathrm{t}-\text { Ratio } & -2.45937 \\ \text { Mean(Mg (mg/L)) SM37 } & 0.00516 & \text { DF } & 308 \\ \text { Mean Difference } & -0.0003 & \text { Prob }>|\mathrm{t}| & 0.0145 \\ \text { Std Error } & 0.00012 & \text { Prob }>\mathrm{t} & 0.9928 \\ \text { Upper95\% } & -6.1 \mathrm{e}-5 & \text { Prob }<\mathrm{t} & 0.0072 \\ \text { Lower95\% } & -0.0005 & & \\ \text { N } & 309 & & \\ \text { Correlation } & 0.91143 & & \end{array}$


WSRC-TR-2004-00576

Revision 0

\section{Exhibit A16. Paired Comparisons of SM38 versus SM37 for Mixed Acid Data}
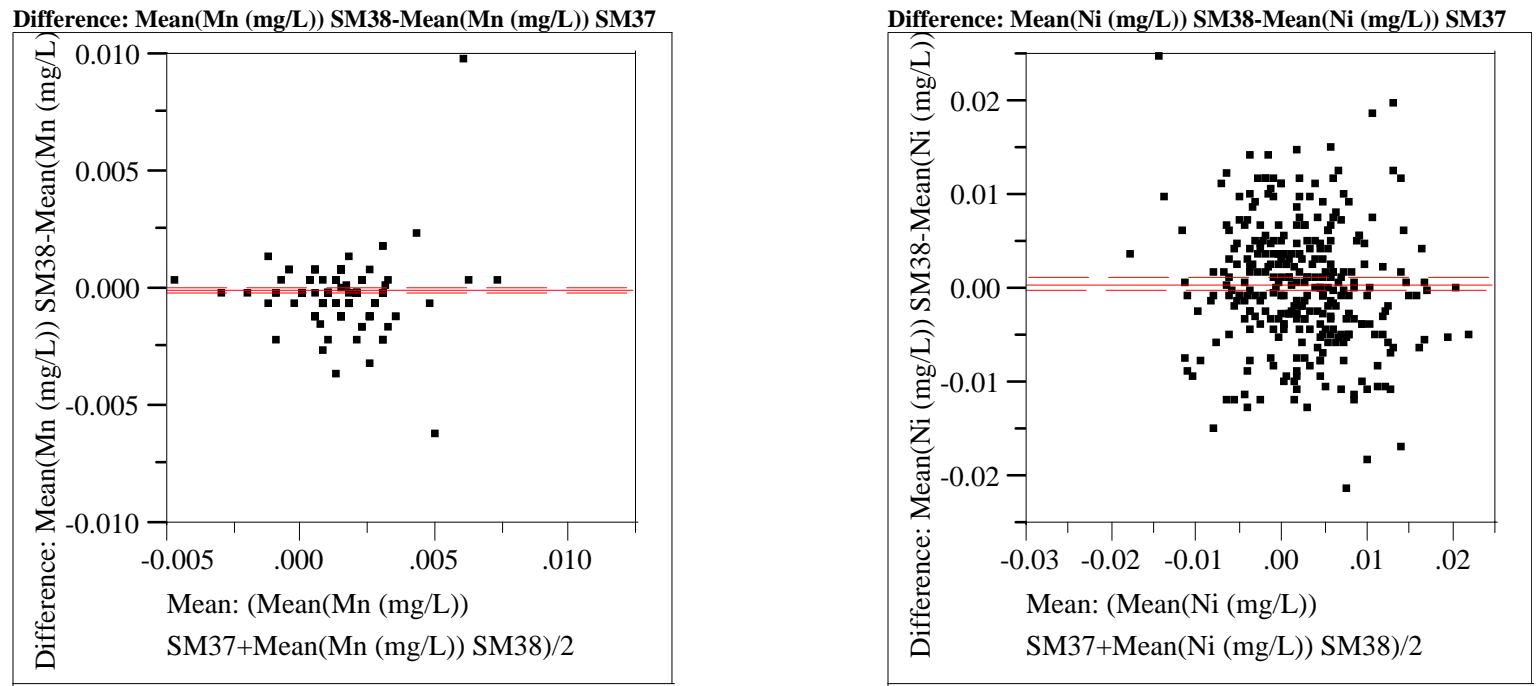

$\begin{array}{lrlr}\text { Mean(Mn (mg/L)) SM38 } & 0.00147 & \text { t-Ratio } & -1.31912 \\ \text { Mean(Mn (mg/L)) SM37 } & 0.00155 & \text { DF } & 308 \\ \text { Mean Difference } & -7 \mathrm{e}-5 & \text { Prob }>|t| & 0.1881 \\ \text { Std Error } & 0.00005 & \text { Prob }>\mathrm{t} & 0.9059 \\ \text { Upper95\% } & 0.00003 & \text { Prob }<\mathrm{t} & 0.0941 \\ \text { Lower95\% } & -0.0002 & & \\ \text { N } & 309 & & \\ \text { Correlation } & 0.71016 & & \end{array}$

Difference: Mean(Na (mg/L)) SM38-Mean(Na (mg/L)) SM37

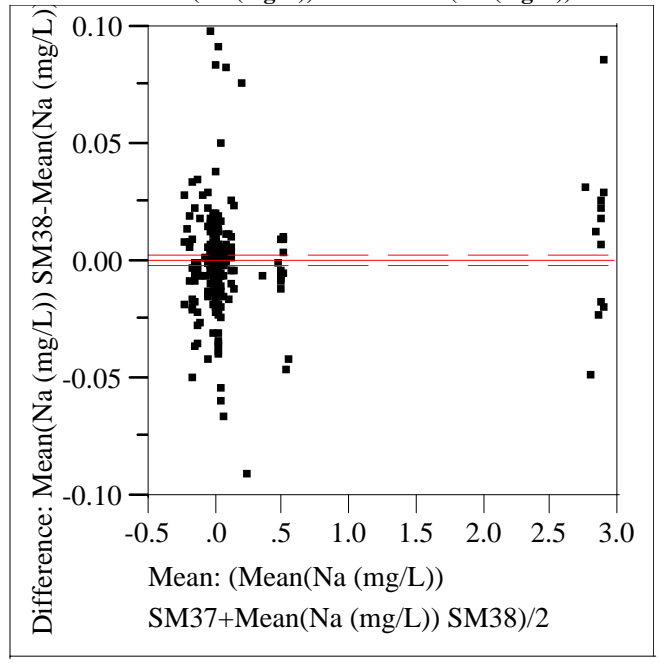

$\begin{array}{lrlr}\text { Mean(Na (mg/L)) SM38 } & 0.11885 & \text { t-Ratio } & 0.472686 \\ \text { Mean(Na (mg/L)) SM37 } & 0.1183 & \text { DF } & 308 \\ \text { Mean Difference } & 0.00054 & \text { Prob }>|t| & 0.6368 \\ \text { Std Error } & 0.00115 & \text { Prob }>t & 0.3184 \\ \text { Upper95\% } & 0.0028 & \text { Prob }<\mathrm{t} & 0.6816 \\ \text { Lower95\% } & -0.0017 & & \\ \text { N } & 309 & & \\ \text { Correlation } & 0.99937 & & \end{array}$

$\begin{array}{lrlr}\text { Mean(Ni (mg/L)) SM38 } & 0.00251 & \text { t-Ratio } & 1.404357 \\ \text { Mean(Ni (mg/L)) SM37 } & 0.00198 & \text { DF } & 308 \\ \text { Mean Difference } & 0.00053 & \text { Prob }>|t| & 0.1612 \\ \text { Std Error } & 0.00038 & \text { Prob }>\mathrm{t} & 0.0806 \\ \text { Upper95\% } & 0.00128 & \text { Prob }<\mathrm{t} & 0.9194 \\ \text { Lower95\% } & -0.0002 & & \\ \mathrm{~N} & 309 & & \\ \text { Correlation } & 0.57971 & & \end{array}$

Difference: Mean(Si (mg/L)) SM38-Mean(Si (mg/L)) SM37

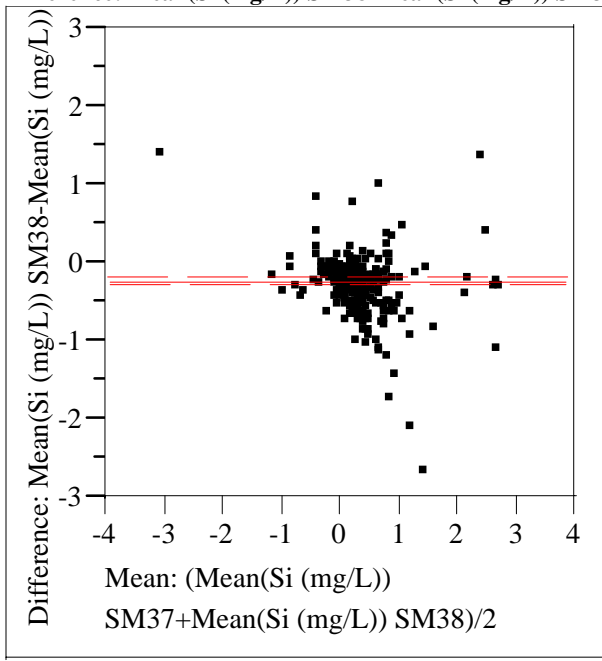

$\begin{array}{lrlr}\text { Mean(Si (mg/L)) SM38 } & 0.18767 & \mathrm{t}-\text { Ratio } & -11.1414 \\ \text { Mean(Si (mg/L)) SM37 } & 0.42607 & \text { DF } & 308 \\ \text { Mean Difference } & -0.2384 & \text { Prob }>|t| & <.0001 \\ \text { Std Error } & 0.0214 & \text { Prob }>\mathrm{t} & 1.0000 \\ \text { Upper95\% } & -0.1963 & \text { Prob }<\mathrm{t} & <.0001 \\ \text { Lower95\% } & -0.2805 & & \\ \text { N } & 309 & & \\ \text { Correlation } & 0.79916 & & \end{array}$


WSRC-TR-2004-00576

Revision 0

\section{Exhibit A16. Paired Comparisons of SM38 versus SM37 for Mixed Acid Data}
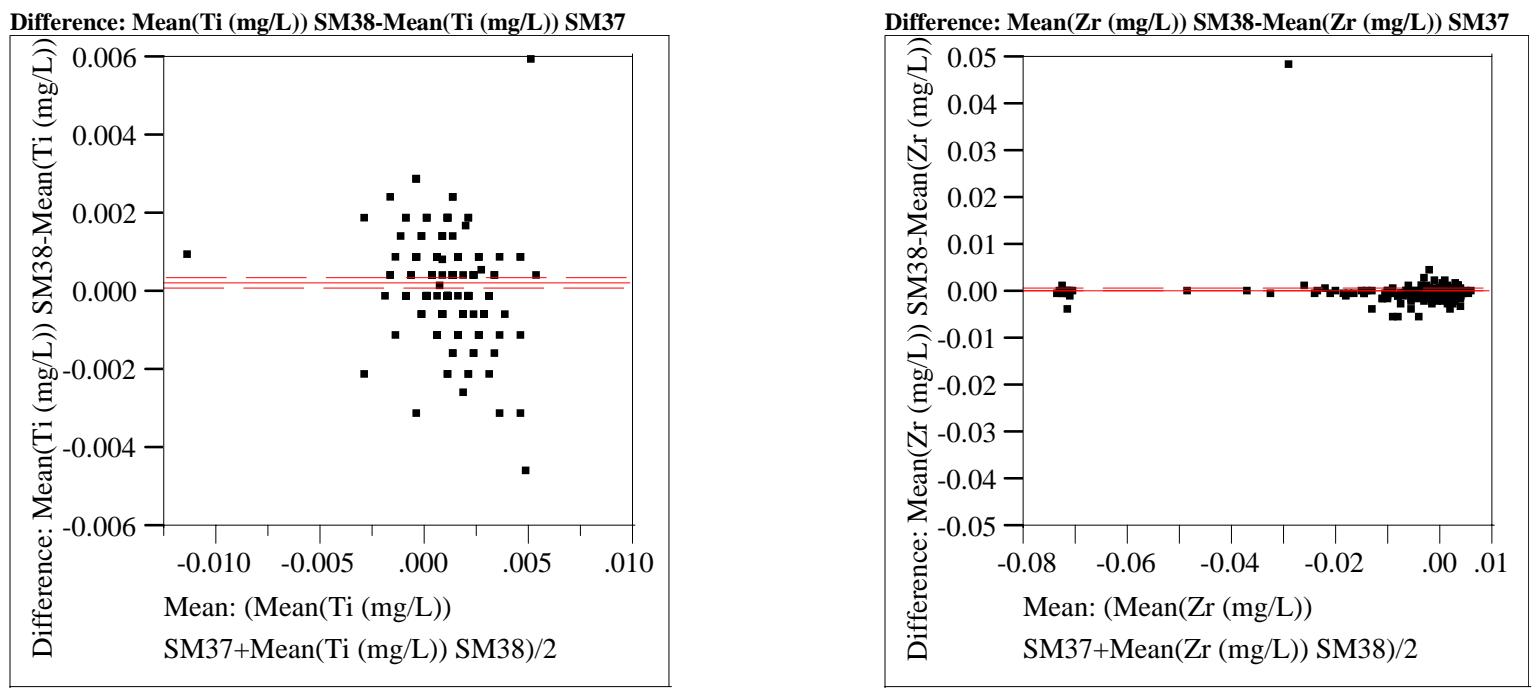

$\begin{array}{lrlr}\text { Mean(Ti (mg/L)) SM38 } & 0.00111 & \text { t-Ratio } & 4.147508 \\ \text { Mean(Ti (mg/L)) SM37 } & 0.00086 & \text { DF } & 308 \\ \text { Mean Difference } & 0.00025 & \text { Prob }>|t| & <.0001 \\ \text { Std Error } & 0.00006 & \text { Prob }>t & <.0001 \\ \text { Upper95\% } & 0.00036 & \text { Prob }<t & 1.0000 \\ \text { Lower95\% } & 0.00013 & & \\ \text { N } & 309 & & \\ \text { Correlation } & 0.76455 & & \end{array}$

$\begin{array}{lrlr}\text { Mean }(\mathrm{Zr}(\mathrm{mg} / \mathrm{L})) \text { SM38 } & -0.0049 & \mathrm{t}-\text { Ratio } & 2.110308 \\ \text { Mean }(\mathrm{Zr}(\mathrm{mg} / \mathrm{L})) \text { SM37 } & -0.0052 & \text { DF } & 308 \\ \text { Mean Difference } & 0.00036 & \text { Prob }>|\mathrm{t}| & 0.0356 \\ \text { Std Error } & 0.00017 & \text { Prob }>\mathrm{t} & 0.0178 \\ \text { Upper95\% } & 0.0007 & \text { Prob }<\mathrm{t} & 0.9822 \\ \text { Lower95\% } & 0.00002 & & \\ \text { N } & 309 & & \\ \text { Correlation } & 0.9819 & & \end{array}$

Difference: Mean(U (mg/L)) SM38-Mean(U (mg/L)) SM37

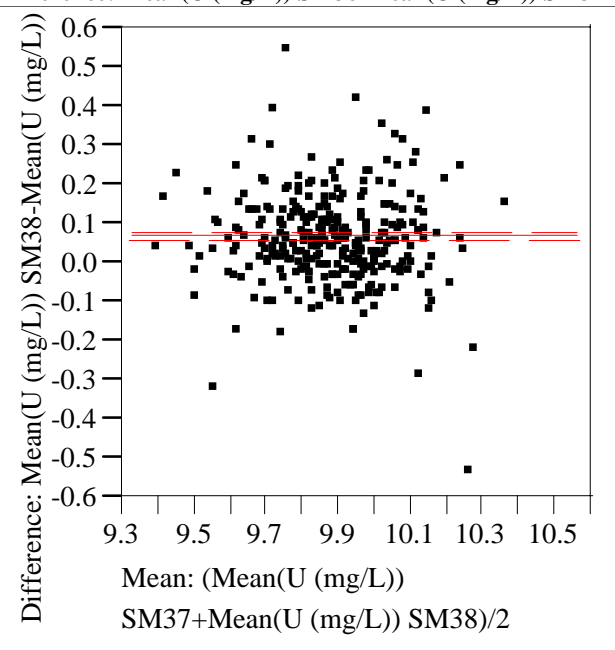

$\begin{array}{lrlr}\text { Mean(U (mg/L)) SM38 } & 9.91223 & \text { t-Ratio } & 10.20373 \\ \text { Mean(U (mg/L)) SM37 } & 9.84519 & \text { DF } & 308 \\ \text { Mean Difference } & 0.06705 & \text { Prob }>|t| & <.0001 \\ \text { Std Error } & 0.00657 & \text { Prob }>t & <.0001 \\ \text { Upper95\% } & 0.07997 & \text { Prob }<\mathrm{t} & 1.0000 \\ \text { Lower95\% } & 0.05412 & & \\ \text { N } & 309 & & \\ \text { Correlation } & 0.78196 & & \end{array}$




\section{Exhibit A17. Time Series Plots of ARG-1 Measurements for Mixed Acid Data}

Control Chart

Individual Measurement of $\mathrm{Al}(\mathrm{wt} \%)$

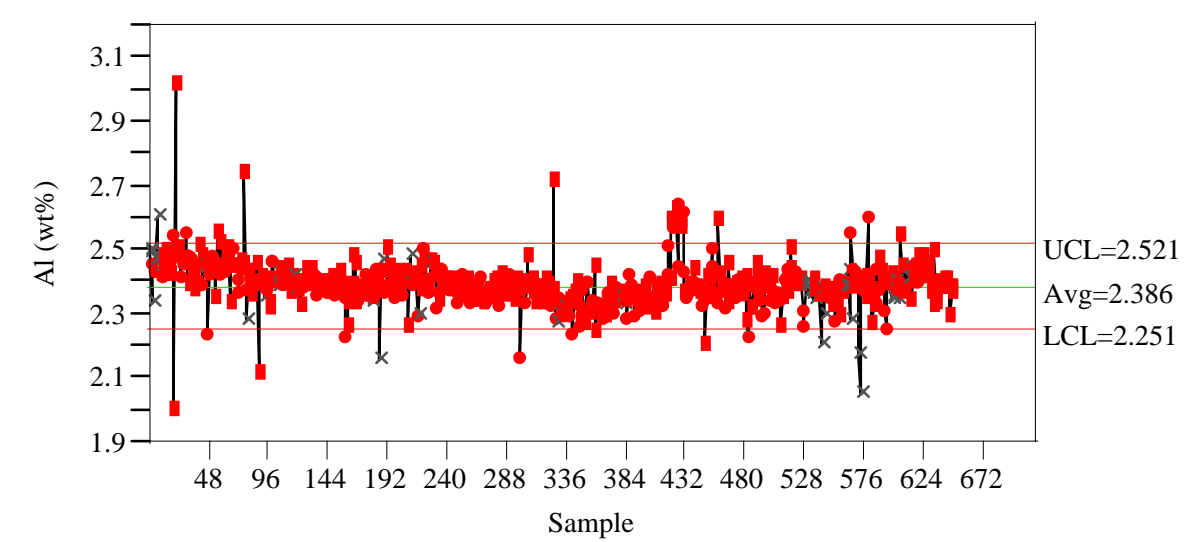

Individual Measurement of Ca (wt \%)

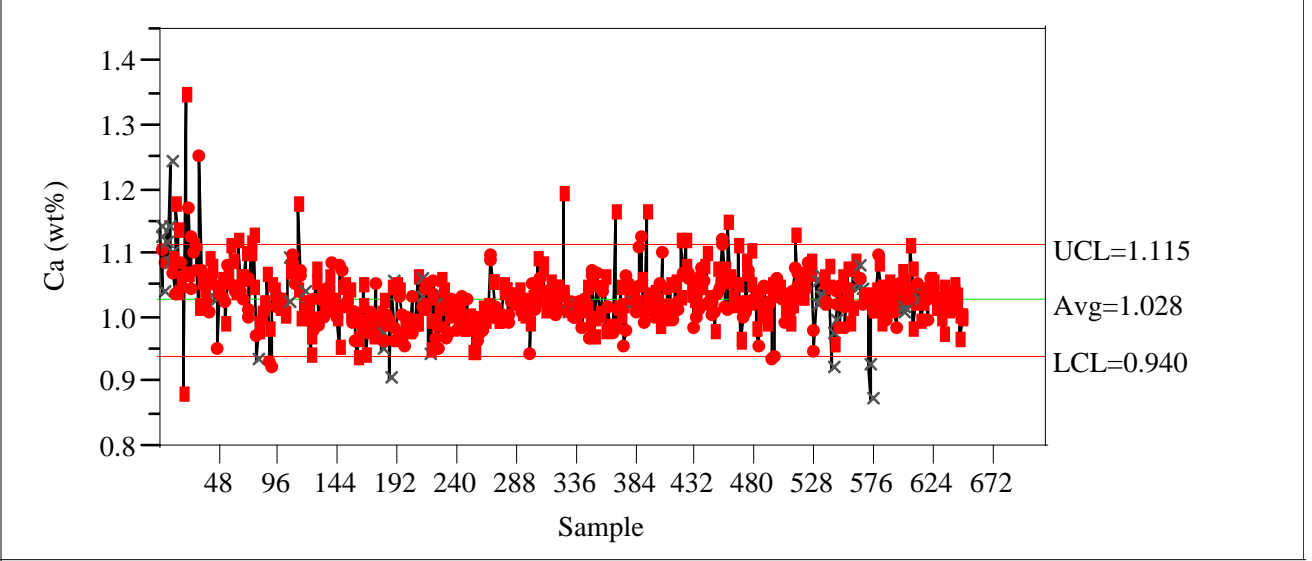

Individual Measurement of $\mathrm{Cr}(\mathrm{wt} \%)$

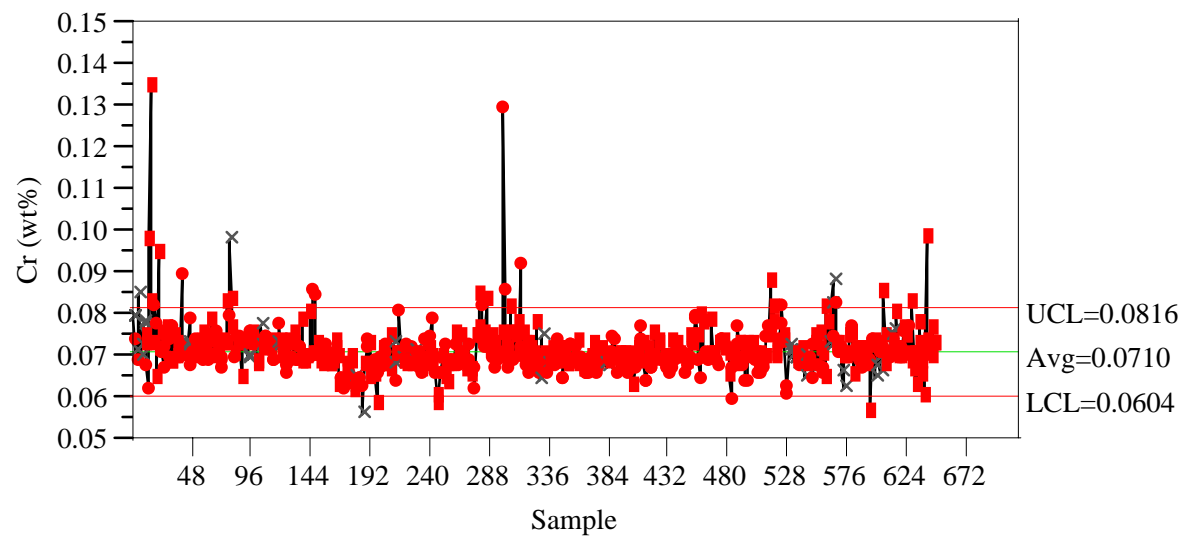




\section{Exhibit A17. Time Series Plots of ARG-1 Measurements for Mixed Acid Data}

Individual Measurement of $\mathrm{Cu}(\mathrm{wt} \%)$

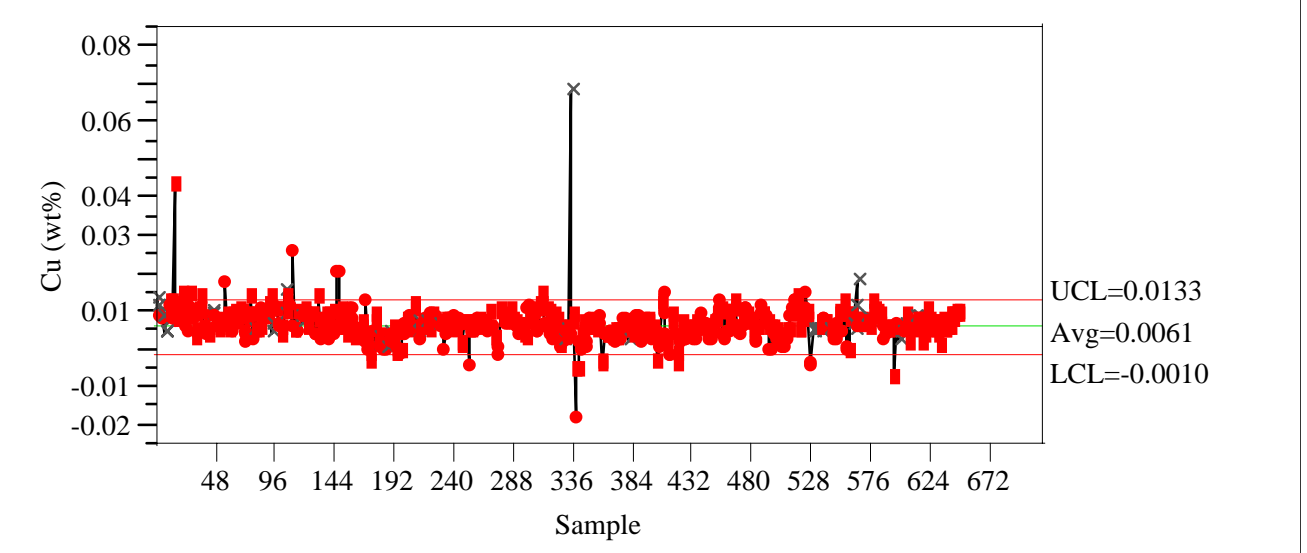

Individual Measurement of Fe (wt \%)

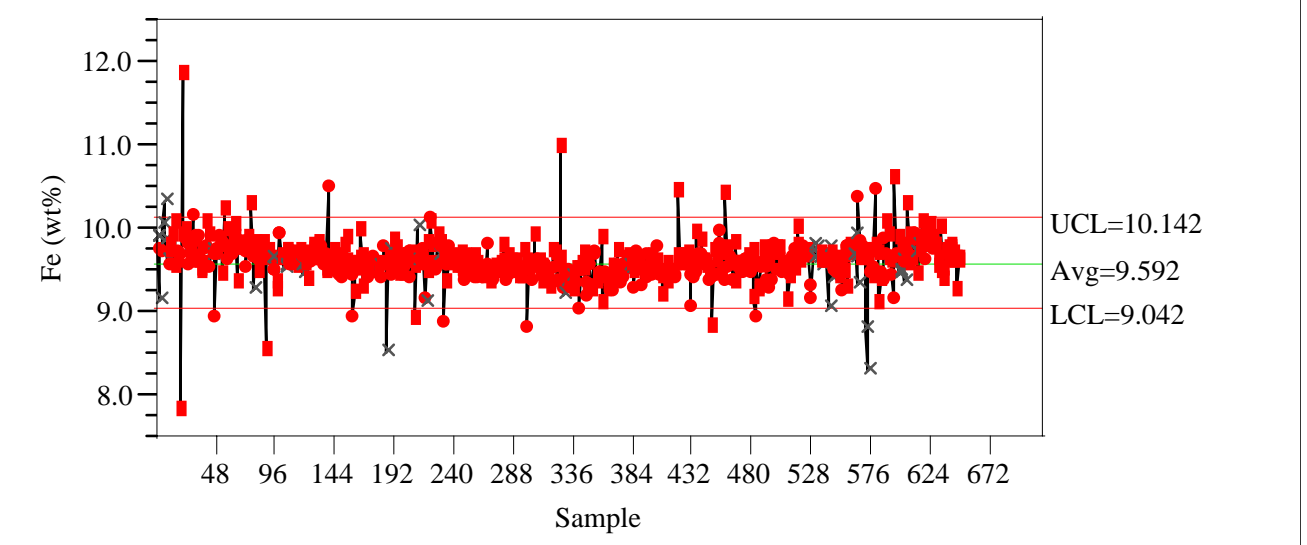

Individual Measurement of K (wt \%)

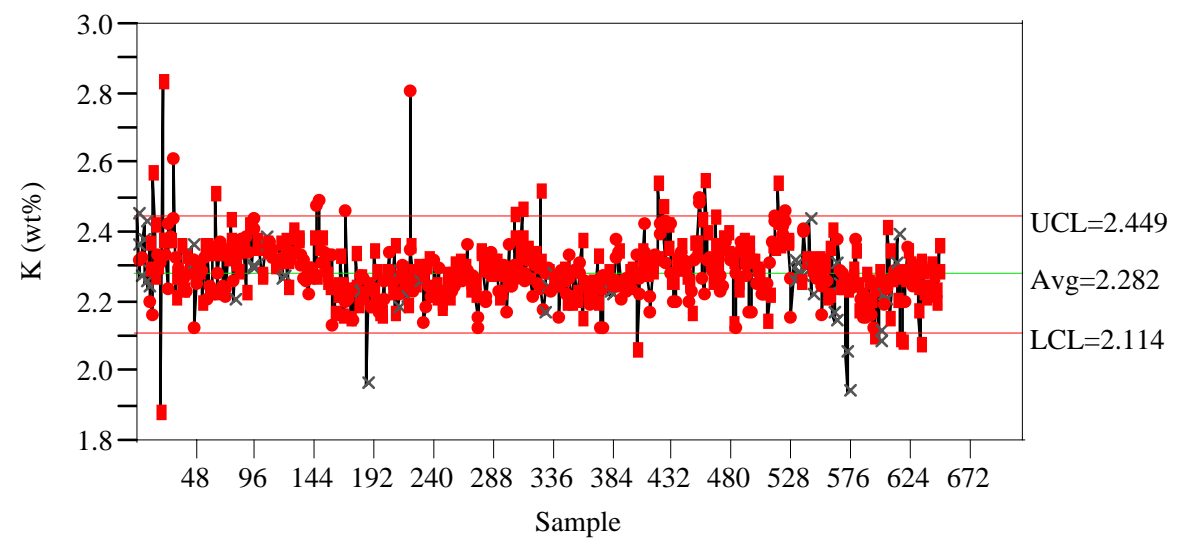




\section{Exhibit A17. Time Series Plots of ARG-1 Measurements for Mixed Acid Data}

Individual Measurement of Li (wt \%)

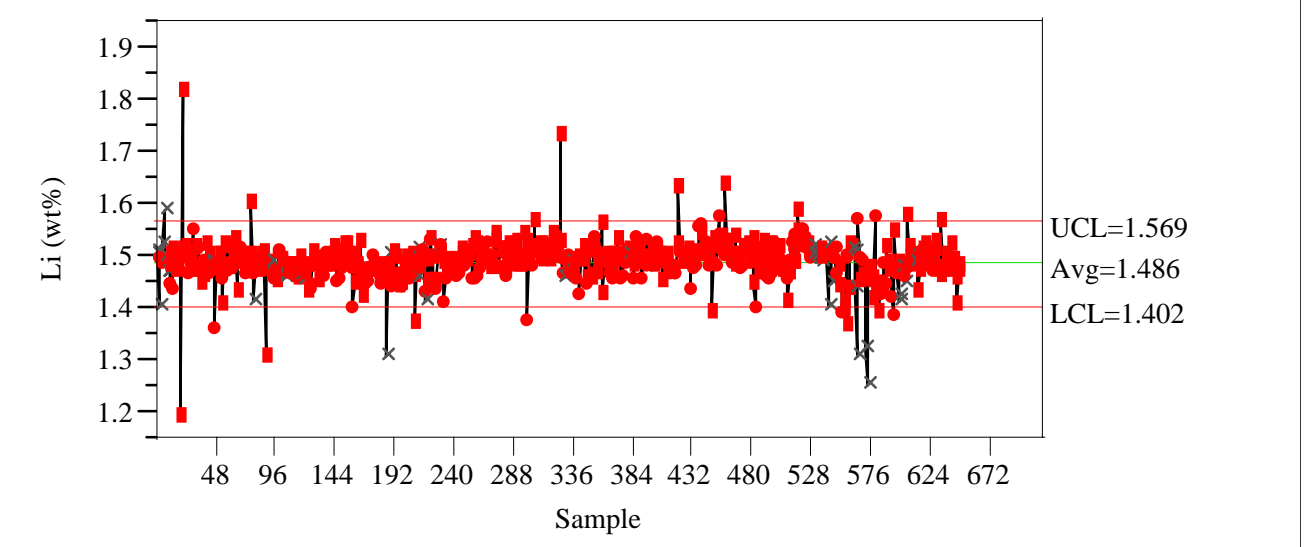

Individual Measurement of $\mathrm{Mg}(\mathrm{wt} \%)$

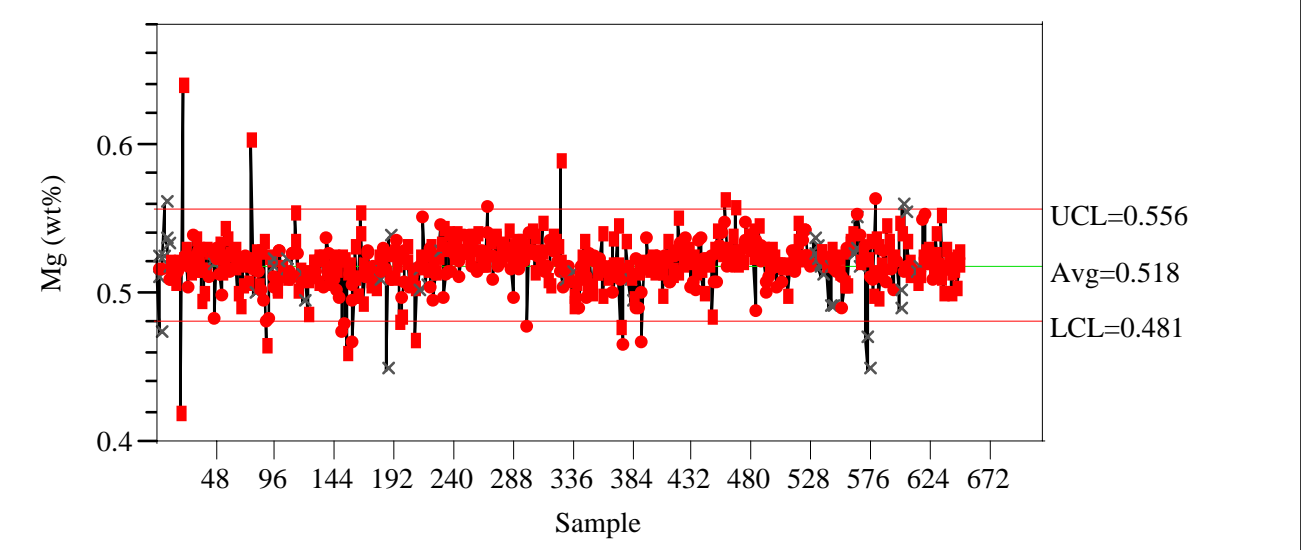

Individual Measurement of Mn (wt\%)

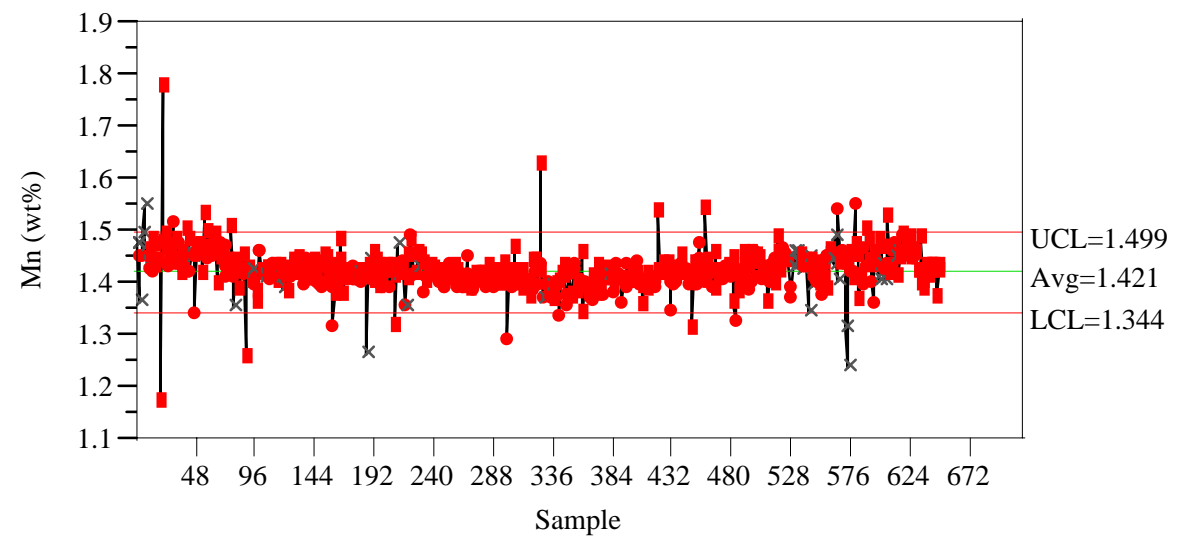


WSRC-TR-2004-00576

Revision 0

\section{Exhibit A17. Time Series Plots of ARG-1 Measurements for Mixed Acid Data}

Individual Measurement of $\mathrm{Na}(\mathrm{wt} \%)$

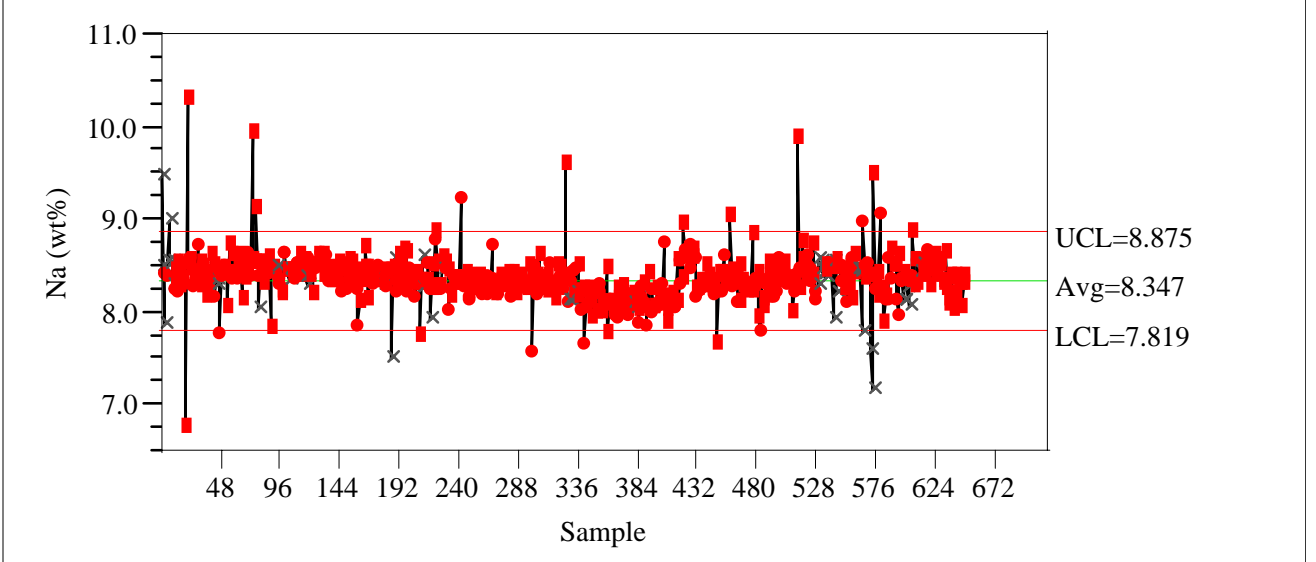

Individual Measurement of $\mathrm{Ni}(\mathrm{wt} \%)$

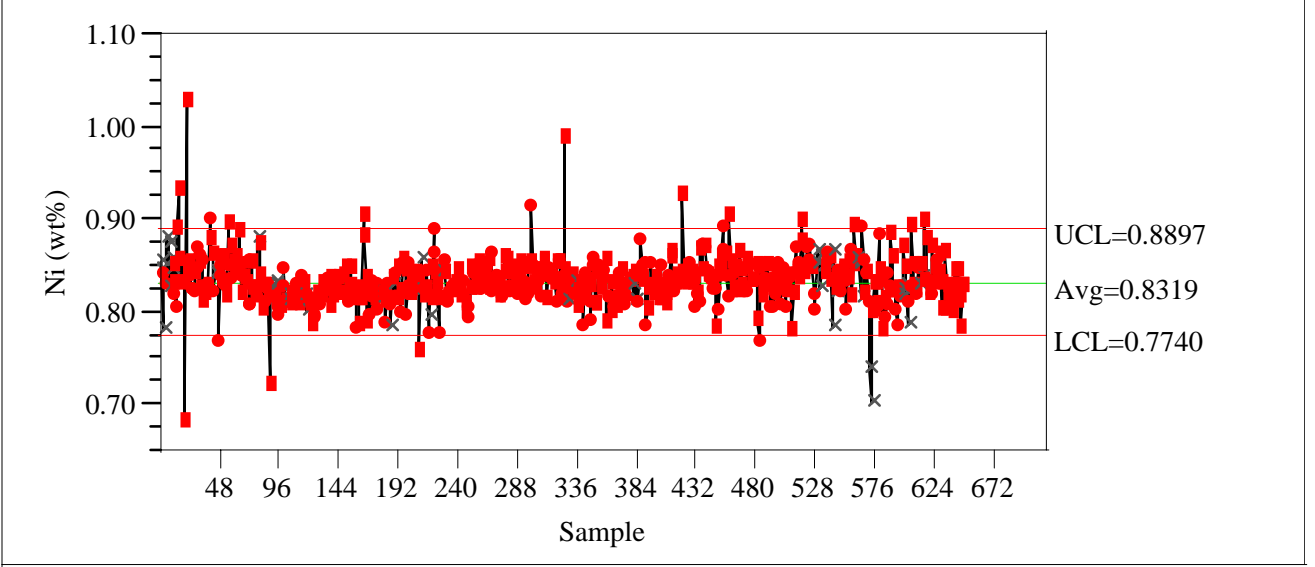

Individual Measurement of Si (wt\%)

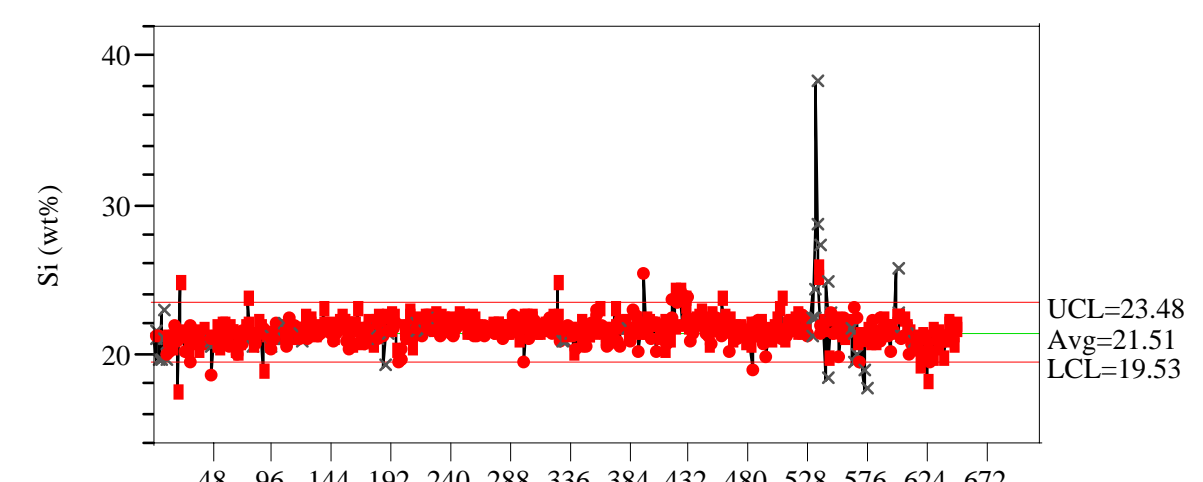

$\begin{array}{llllllllllllll}48 & 96 & 144 & 192 & 240 & 288 & 336 & 384 & 432 & 480 & 528 & 576 & 624 & 672\end{array}$

Sample 


\section{Exhibit A17. Time Series Plots of ARG-1 Measurements for Mixed Acid Data}

Individual Measurement of Ti (wt\%)

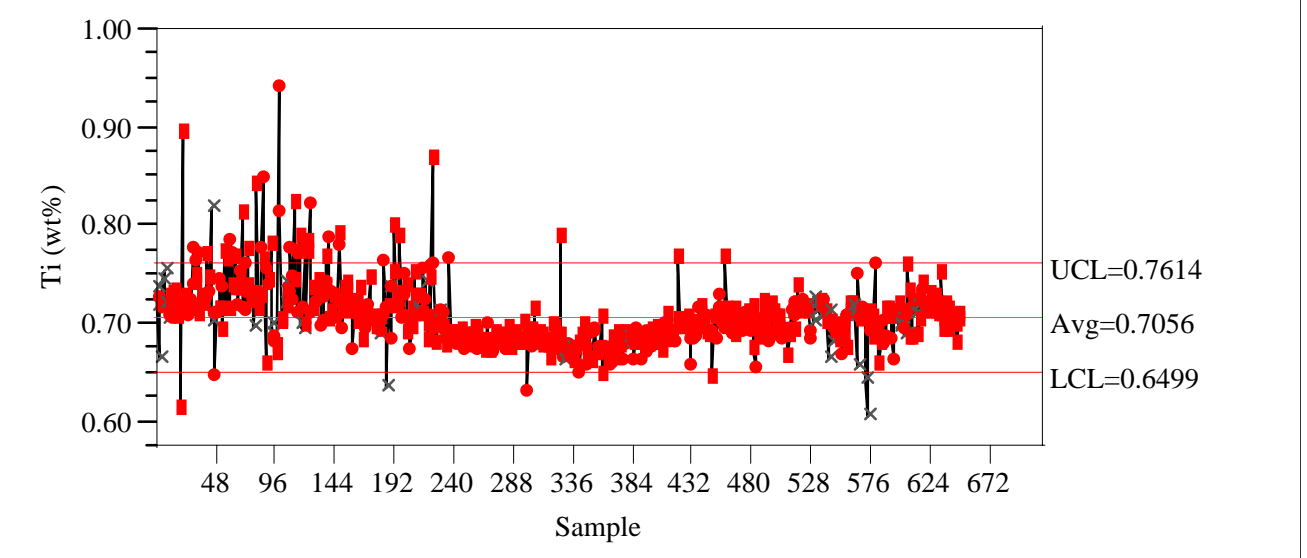

Individual Measurement of U (wt\%)

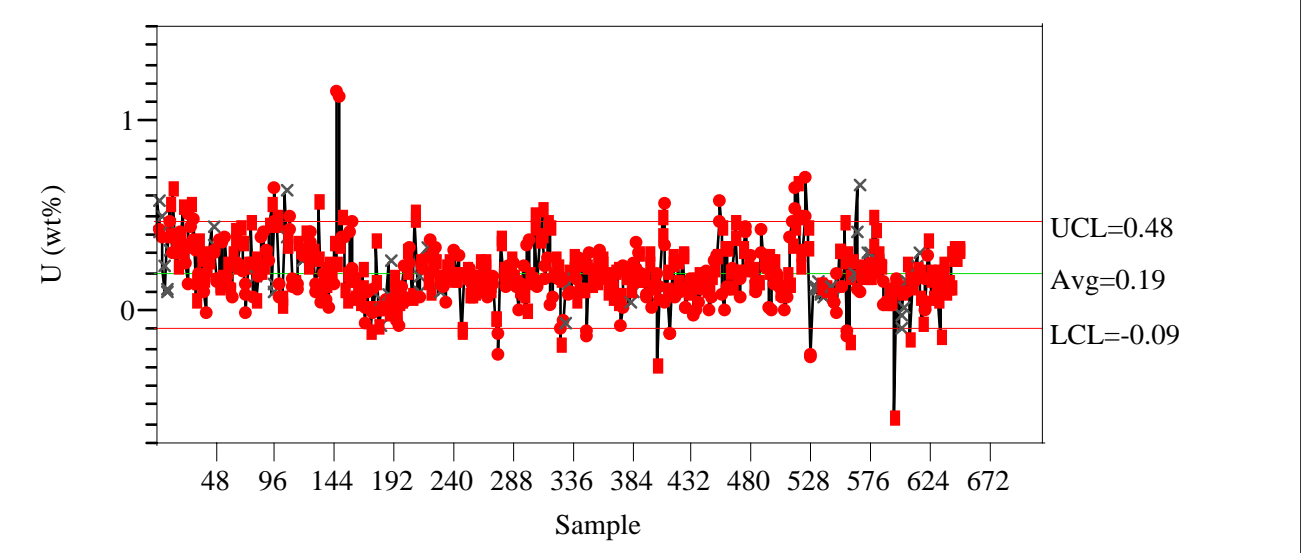

Individual Measurement of $\mathrm{Zr}$ (wt\%)

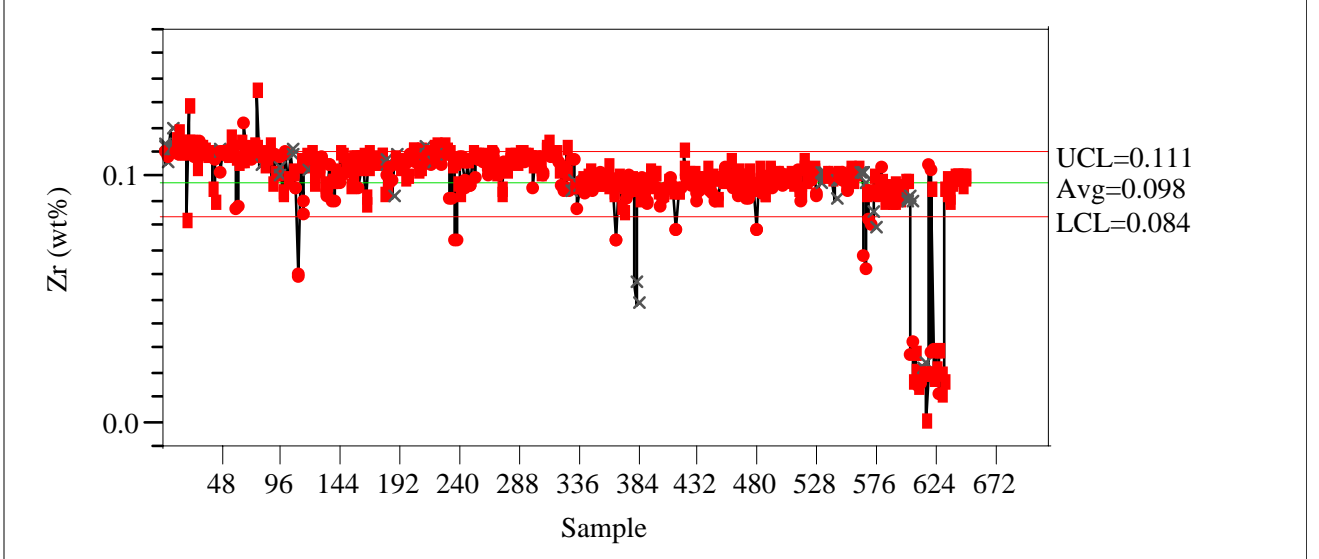


WSRC-TR-2004-00576

Revision 0

\section{Exhibit A18. Histograms and Descriptive Statistics for All ARG-1 Measurements for Mixed Acid Data}

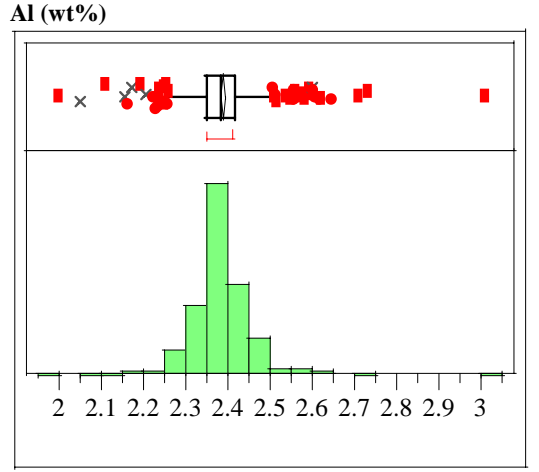

Quantiles

$100.0 \%$ maximum 3.0070

$99.5 \%$

$97.5 \%$

$90.0 \%$

$75.0 \%$

$50.0 \%$
$25.0 \%$

$10.0 \%$

$2.5 \%$

$0.5 \%$

$0.0 \%$

Mean

Std Dev

Std Err Mean

upper $95 \%$ Mean

lower 95\% Mean

$\mathrm{N}$

Ca (wt\%)

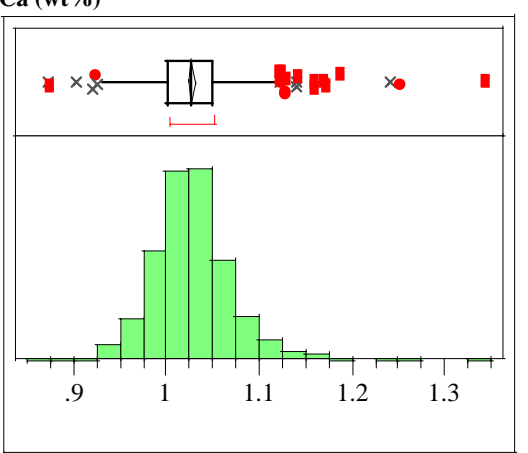

Quantiles

$100.0 \%$ maximum 1.3440

$99.5 \%$

$97.5 \%$

$90.0 \%$

$75.0 \%$

$50.0 \%$

$25.0 \%$

$2.5 \%$

$0.5 \%$

$0.0 \%$
Moment

Mean

Std Dev

Std Err Mean

upper 95\% Mean 1.0309853

lower 95\% Mean 1.0240409

$\mathrm{N}$
Cr (wt\%)

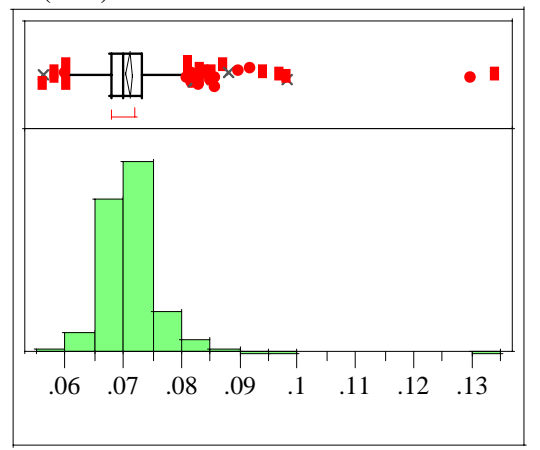

Quantiles

$100.0 \%$ maximum 0.13400

$99.5 \%$

$97.5 \%$

$90.0 \%$

$75.0 \%$

$50.0 \%$

$25.0 \%$

$10.0 \%$

$2.5 \%$

$0.5 \%$

$0.0 \%$

Mean

Std Dev

Std Err Mean

upper 95\% Mean

lower 95\% Mean

$\mathrm{N}$

Cu (wt\%)

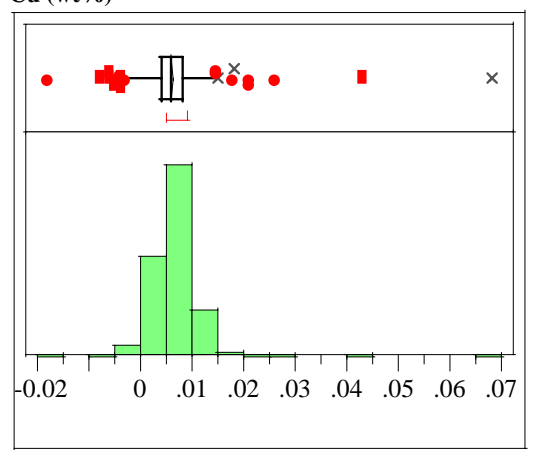

Quantiles

$100.0 \%$ maximum 0.0680

$99.5 \%$

$97.5 \%$

$90.0 \%$

$75.0 \%$

$50.0 \%$

$25.0 \%$

$10.0 \%$

$2.5 \%$

$0.5 \%$

Momen

Mean

Std Dev

Std Err Mean

upper 95\% Mean

lower 95\% Mean

$\mathrm{N}$
Fe (wt \%)

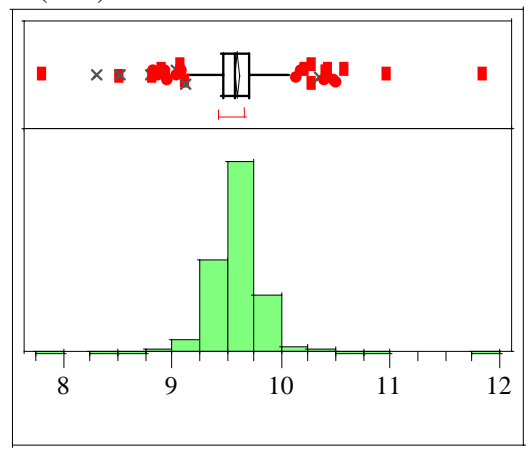

Quantiles

$100.0 \%$ maximum 11.843

$99.5 \%$

$97.5 \%$

$90.0 \%$

$75.0 \%$ quartile $\quad 9.710$

$50.0 \%$ median $\quad 9.582$

$25.0 \%$ quartile $\quad 9.475$

$10.0 \% \quad 9.354$

$2.5 \% \quad 9.078$

$0.5 \% \quad 8.515$

$0.0 \%$ minimum $\quad 7.806$

Moments

Mean $\quad 9.5919815$

Std Dev $\quad 0.2628951$

Std Err Mean $\quad 0.0103195$

upper 95\% Mean 9.6122453

lower 95\% Mean 9.5717177

$\mathrm{N}$

K (wt \%)

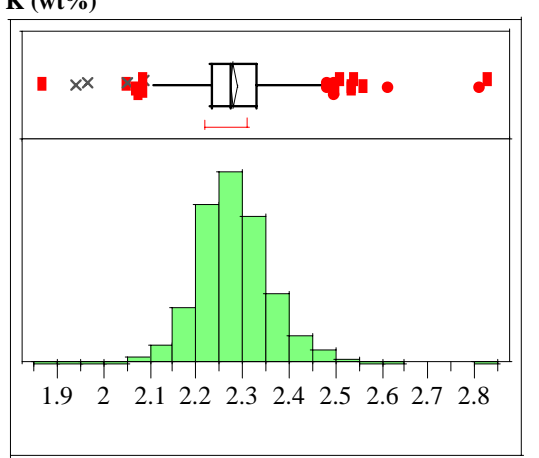

Quantiles

$100.0 \%$ maximum 2.8260

$99.5 \% \quad 2.6015$

$\begin{array}{ll}97.5 \% & 2.4605\end{array}$

$90.0 \% \quad 2.3760$

$75.0 \%$ quartile $\quad 2.3290$

$50.0 \%$ median 2.2760

$25.0 \%$ quartile 2.2330

$10.0 \% \quad 2.1900$

$2.5 \% \quad 2.1290$

$0.5 \% \quad 1.9835$

$0.0 \%$ minimum $\quad 1.8680$

Moments

Mean

2.2818166

Std Dev

0.0865792

Std Err Mean $\quad 0.0033985$

upper 95\% Mean 2.2884901

lower 95\% Mean 2.2751432

$\mathrm{N}$

649 
WSRC-TR-2004-00576

Revision 0

\section{Exhibit A18. Histograms and Descriptive Statistics for All ARG-1 Measurements for Mixed Acid Data}

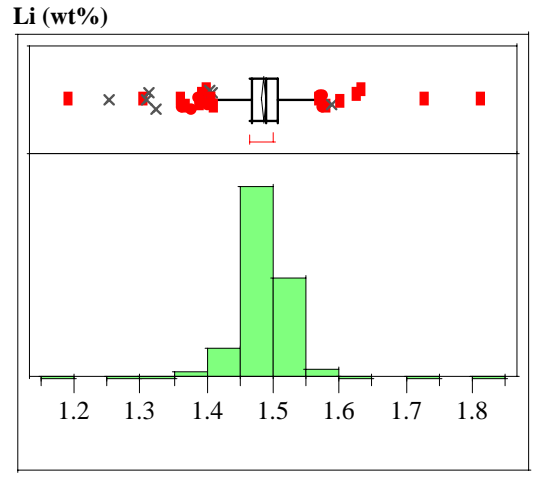

Quantiles

$100.0 \%$ maximum 1.8120

$99.5 \%$

$97.5 \%$

$90.0 \%$

$75.0 \%$

$50.0 \%$
$25.0 \%$

$10.0 \%$

$2.5 \%$

$0.5 \%$

$0.0 \%$

Mean

Std Dev

Std Err Mean

upper $95 \%$ Mean

$\mathrm{N}$

Mg (wt\%)

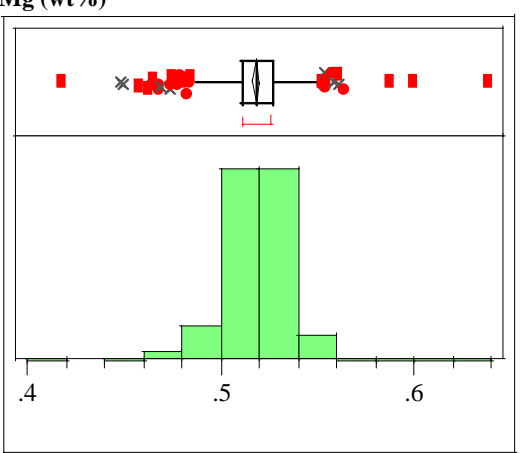

Quantiles

$100.0 \%$ maximum 0.63800

$99.5 \%$

$97.5 \%$

$90.0 \%$

$75.0 \%$

$50.0 \%$

$25.0 \%$

$2.5 \%$

$0.5 \%$

$0.0 \%$
Moment

Mean

Std Dev

Std Err Mean

upper 95\% Mean

lower 95\% Mean

$\mathrm{N}$
Mn (wt\%)

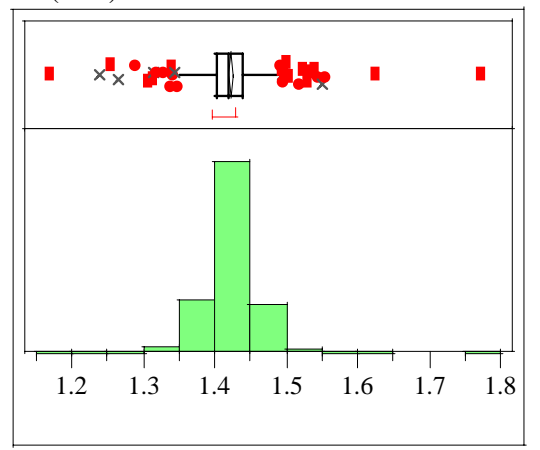

Quantiles

$100.0 \%$ maximum 1.7720

$99.5 \%$

$97.5 \%$

$90.0 \%$

$75.0 \%$

$50.0 \%$

$25.0 \%$

$10.0 \%$

$2.5 \%$

$0.5 \%$

$0.0 \%$

Mean

Std Dev

Std Err Mean

upper 95\% Mean

lower 95\% Mean

$\mathrm{N}$

Na (wt\%)

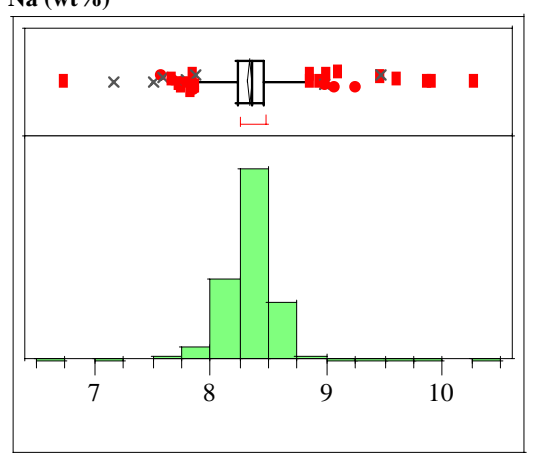

Quantiles

$100.0 \%$ maximum 10.271

$99.5 \%$

$97.5 \%$

$90.0 \%$

$75.0 \%$

$50.0 \%$

$25.0 \%$

$10.0 \%$

$2.5 \%$

$0.5 \%$

Momen

Mean

Std Dev

Std Err Mean

upper 95\% Mean

lower 95\% Mean

$\mathrm{N}$

4183736

649
Ni (wt \%)

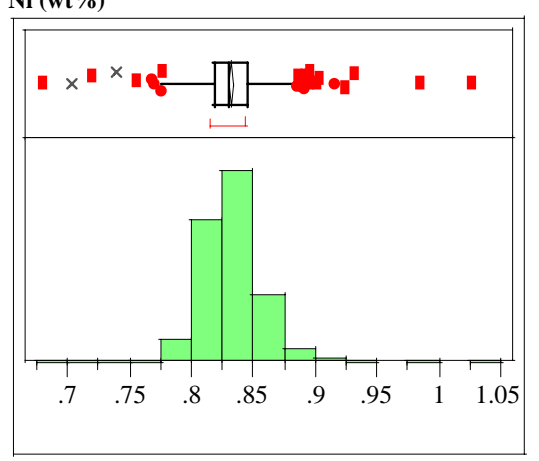

Quantiles

$100.0 \%$ maximum 1.0260

$99.5 \%$

$97.5 \%$

$90.0 \%$

$75.0 \%$

$50.0 \%$ median $\quad 0.8300$

$25.0 \%$ quartile 0.8180

$10.0 \% \quad 0.8060$

$2.5 \% \quad 0.7835$

$\begin{array}{lll}0.5 \% & & 0.7248 \\ 0.0 \% & \text { minimum } & 0.6790\end{array}$

Moments

Mean

0.8318706

Std Dev

Std Err Mean

upper 95\% Mean

lower 95\% Mean

0.0263452

0.0010341

$\mathrm{N}$

649

Si (wt \%)

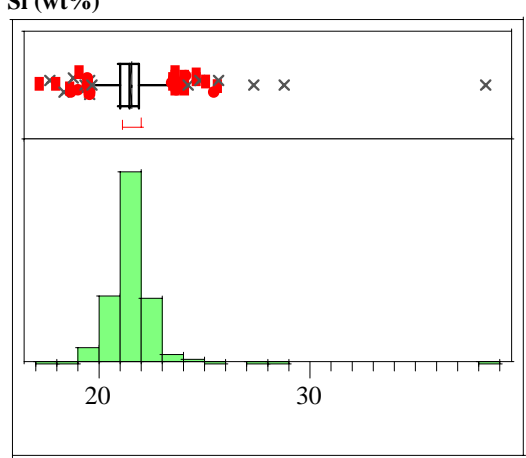

Quantiles

$100.0 \%$ maximum 38.227

$99.5 \%$

$97.5 \%$

$90.0 \%$

$75.0 \%$

$50.0 \%$

$25.0 \%$

$10.0 \%$

$2.5 \%$

$0.5 \%$

$0.0 \%$

Moments

Mean
Std Dev

Std Err Mean

upper 95\% Mean

lower 95\% Mean

$\mathrm{N}$

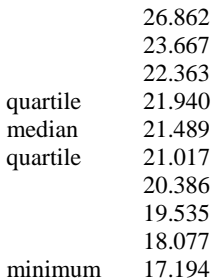

$\begin{array}{ll} & 18.077 \\ \end{array}$

21.506364 1.1897877

0.0467033

21.598072

21.414656 
WSRC-TR-2004-00576

Revision 0

\section{Exhibit A18. Histograms and Descriptive Statistics for All ARG-1 Measurements for Mixed Acid Data}

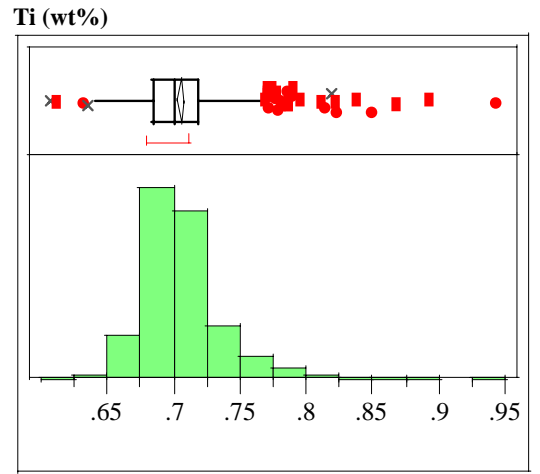

\begin{tabular}{lrr}
\multicolumn{2}{l}{ Quantiles } & \\
$900.0 \%$ & maximum & 0.94300 \\
$99.5 \%$ & & 0.86275 \\
$97.5 \%$ & & 0.78600 \\
$90.0 \%$ & & 0.74200 \\
$75.0 \%$ & quartile & 0.71800 \\
$50.0 \%$ & median & 0.70100 \\
$25.0 \%$ & quartile & 0.68500 \\
$10.0 \%$ & & 0.67500 \\
$2.5 \%$ & & 0.65925 \\
$0.5 \%$ & 0.63350 \\
$0.0 \% \quad$ minimum & 0.60600 \\
Moments & \\
Mean & 0.7056055 \\
Std Dev & 0.033036 \\
Std Err Mean & 0.0012968 \\
upper 95\% Mean & 0.7081519 \\
lower 95\% Mean & 0.7030592 \\
N & 649
\end{tabular}

U (wt\%)

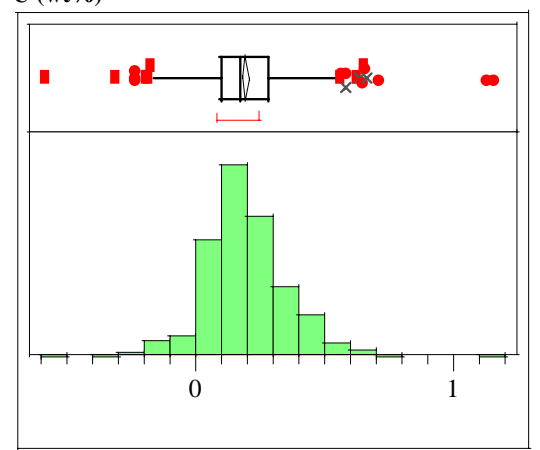

Quantiles

$100.0 \%$ maximum 1.158

$99.5 \% \quad 0.700$

$97.5 \% \quad 0.540$

$90.0 \% \quad 0.402$

$75.0 \%$ quartile $\quad 0.280$

$50.0 \%$ median $\quad 0.177$

$25.0 \%$ quartile $\quad 0.099$

$10.0 \% \quad 0.023$

$2.5 \% \quad-0.115$

$\begin{array}{lll}0.5 \% & & -0.234 \\ 0.0 \% & \text { minimum } & -0.585\end{array}$

Moments

Mean

0.1941279

Std Dev

0.1622673

Std Err Mean $\quad 0.0063695$

upper 95\% Mean 0.2066353

lower 95\% Mean 0.1816204

$\mathrm{N}$

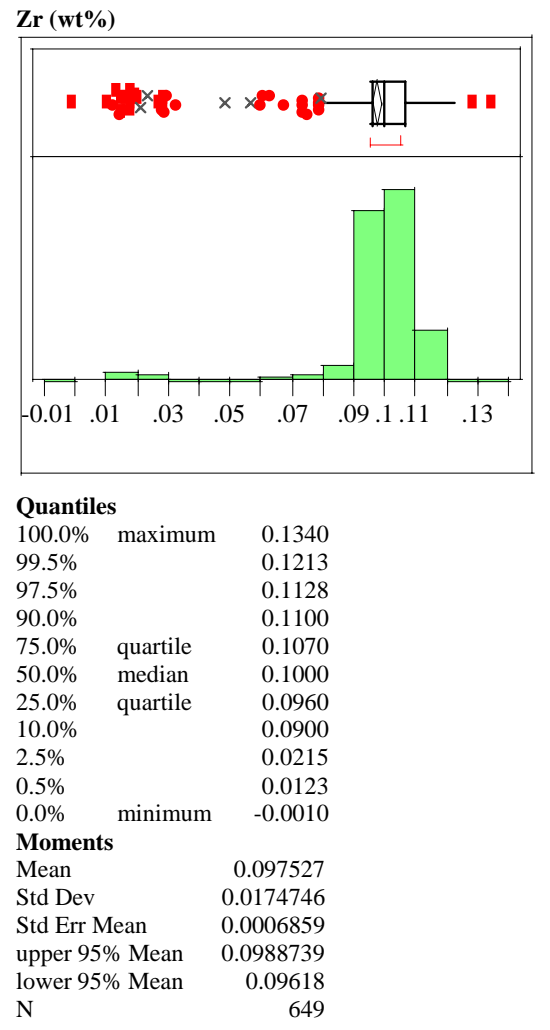


WSRC-TR-2004-00576

Revision 0

Exhibit A19. Histograms and Descriptive Statistics for ARG-1 Measurements for Mixed Acid Data Associated with SME and MFT Batches

Al (wt\%)

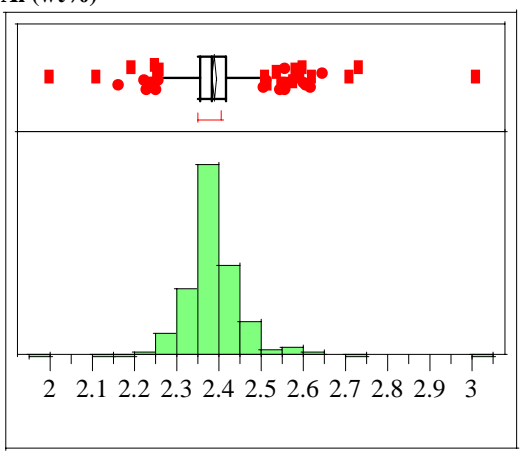

Quantiles

$100.0 \%$ maximum 3.0070

$99.5 \%$

$97.5 \%$

$90.0 \%$

$75.0 \%$

$50.0 \%$
$25.0 \%$

$10.0 \%$

$2.5 \%$

$0.5 \%$

$0.0 \%$

Mean

Std Dev

Std Err Mean

upper $95 \%$ Mean

$\mathrm{N}$

Ca (wt\%)

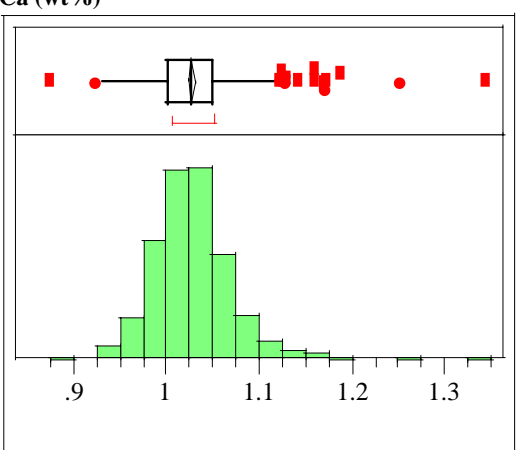

Quantiles

$100.0 \%$ maximum 1.3440

$99.5 \% \quad 1.1886$

$97.5 \%$

$90.0 \%$

$75.0 \%$

$50.0 \%$

$25.0 \%$

$10.0 \%$

$2.5 \%$

$0.5 \%$

$0.0 \%$

Mean

Std Dev

Std Err Mean

upper 95\% Mean

lower 95\% Mean

$\mathrm{N}$
Cr (wt\%)

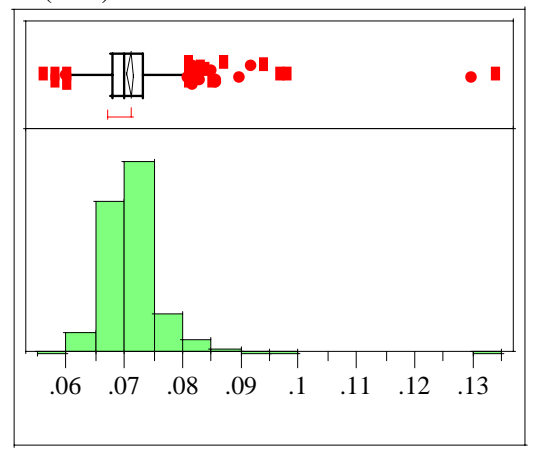

Quantiles

$100.0 \%$ maximum 0.13400

$99.5 \%$

$97.5 \%$

$90.0 \%$

$75.0 \%$

$50.0 \%$

$25.0 \%$

$10.0 \%$

$2.5 \%$

$0.5 \%$

$0.0 \%$

Moments

Mean

Std Dev

Std Err Mean

upper $95 \%$ Mean

lower 95\% Mean

$\mathrm{N}$

Cu (wt\%)

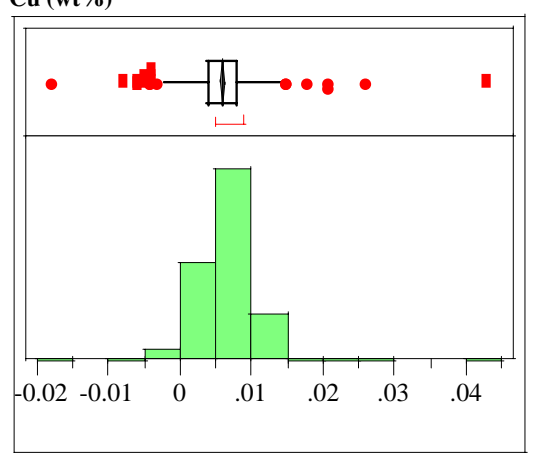

Quantiles

$100.0 \%$ maximum 0.0430

$99.5 \%$

$97.5 \%$

$90.0 \%$

$75.0 \%$ quartile

$50.0 \%$

$25.0 \%$

$10.0 \%$

$2.5 \%$

$0.5 \%$

Momen

Mean

Std Dev

Std Err Mean

upper 95\% Mean

lower 95\% Mean

$\mathrm{N}$
Fe (wt \%)

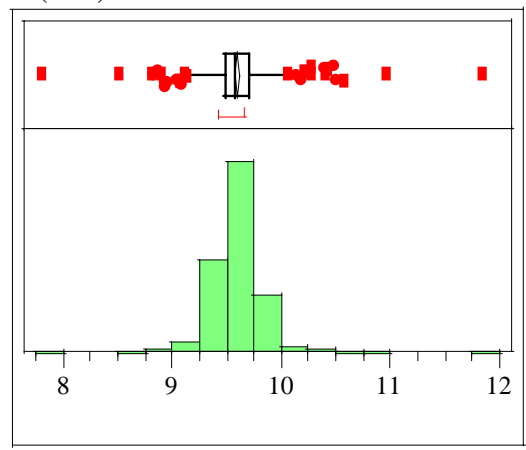

Quantiles

$100.0 \%$ maximum 11.843

$99.5 \%$

$97.5 \%$

$90.0 \%$

$75.0 \%$ quartile $\quad 9.710$

$50.0 \%$ median $\quad 9.582$

$25.0 \%$ quartile $\quad 9.479$

$10.0 \% \quad 9.372$

$2.5 \% \quad 9.126$

$\begin{array}{lll}0.5 \% & & 8.807 \\ 0.0 \% & \text { minimum } & 7.806\end{array}$

Moments

Mean $\quad 9.5967018$

Std Dev $\quad 0.2540405$

Std Err Mean $\quad 0.0103972$

upper 95\% Mean 9.6171214

lower 95\% Mean 9.5762823

$\mathrm{N}$

K (wt \%)

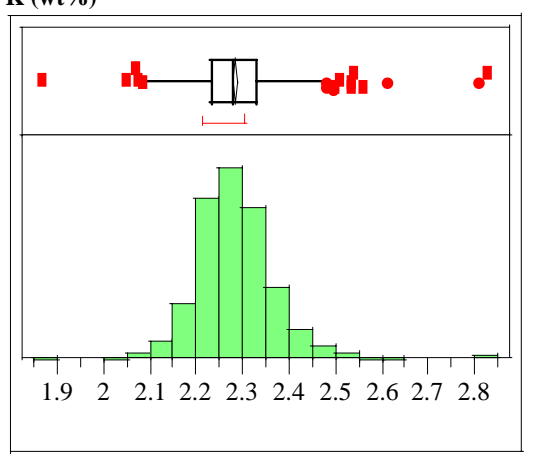

Quantiles

$100.0 \%$ maximum 2.8260

$99.5 \% \quad 2.6180$

$\begin{array}{ll}97.5 \% & 2.4642\end{array}$

$90.0 \% \quad 2.3760$

$75.0 \%$ quartile 2.3315

$50.0 \%$ median 2.2790

$25.0 \%$ quartile 2.2330

$10.0 \% \quad 2.1930$

$2.5 \% \quad 2.1348$

$\begin{array}{ll}0.5 \% & 2.0698\end{array}$

$0.0 \%$ minimum $\quad 1.8680$

Moments

Mean

Std Dev

2.2843719

Std Err Mean $\quad 0.0034688$

upper 95\% Mean $\quad 2.2911843$

lower 95\% Mean 2.2775594

$\mathrm{N}$

597 
WSRC-TR-2004-00576

Revision 0

Exhibit A19. Histograms and Descriptive Statistics for ARG-1 Measurements for Mixed Acid Data Associated with SME and MFT Batches

Li (wt\%)

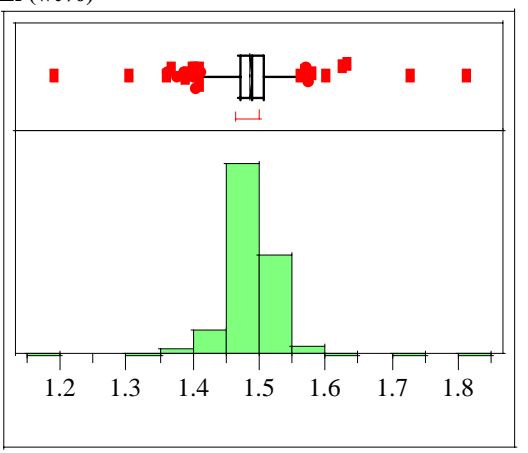

Quantiles

$100.0 \%$ maximum 1.8120

$99.5 \%$

$97.5 \%$

$90.0 \%$

$75.0 \%$

$50.0 \%$
$25.0 \%$

$10.0 \%$

$2.5 \%$

$0.5 \%$

Moments

Mean

Std Dev

Std Err Mean

upper $95 \%$ Mean

lower 95\% Mean 1.4844646

$\mathrm{N}$

$\operatorname{Mg}(w t \%)$

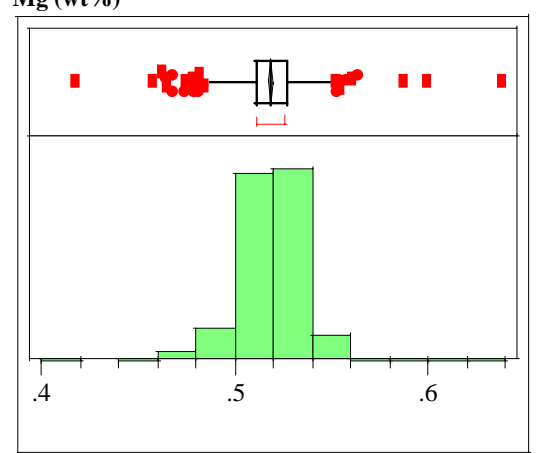

Quantiles

$100.0 \%$ maximum 0.63800

$99.5 \%$

$97.5 \%$

$90.0 \%$

$75.0 \%$

$50.0 \%$

$25.0 \%$

$10.0 \%$

$2.5 \%$

$0.5 \%$

Moment

Mean

Std Dev

Std Err Mean

upper 95\% Mean

lower 95\% Mean

$\mathrm{N}$
Mn (wt\%)

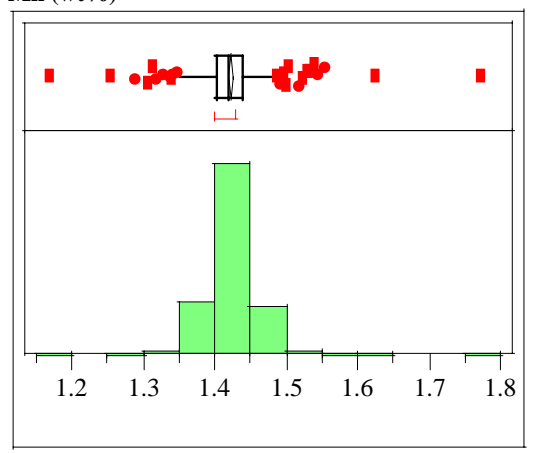

Quantiles

$100.0 \%$ maximum 1.7720

$99.5 \%$

$97.5 \%$

$90.0 \%$

$75.0 \%$

$50.0 \%$

$25.0 \%$

$10.0 \%$

$2.5 \%$

$0.5 \%$

$0.0 \%$

Mean

Std Dev

Std Err Mean

upper 95\% Mean

lower 95\% Mean

$\mathrm{N}$

Na (wt\%)

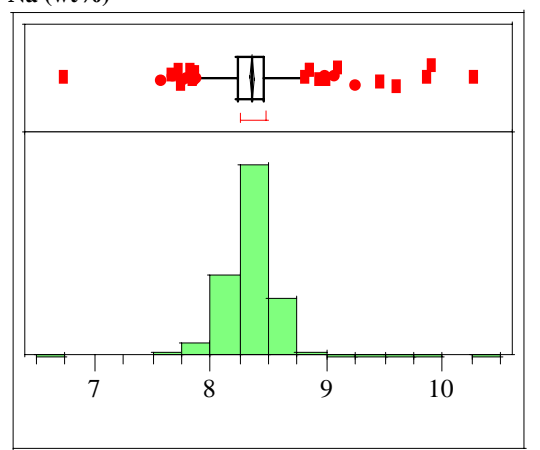

Quantiles

$100.0 \%$ maximum 10.271

$99.5 \%$

$97.5 \%$

$90.0 \%$

$75.0 \%$

$50.0 \%$

$25.0 \%$

$10.0 \%$

$2.5 \%$

$0.5 \%$

Momen

Mean

Std Dev

Std Err Mean

upper 95\% Mean

lower 95\% Mean

$\mathrm{N}$
Ni (wt\%)

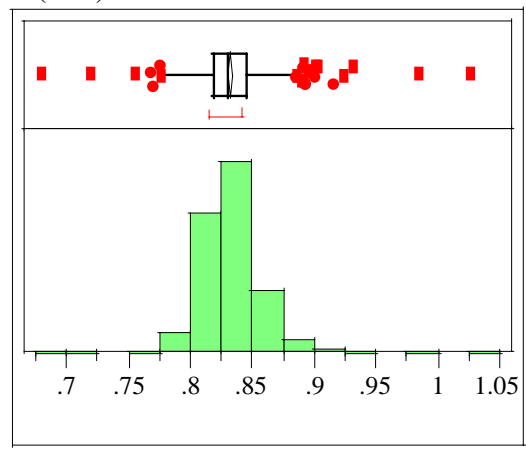

Quantiles

$100.0 \%$ maximum 1.0260

$99.5 \%$

$97.5 \%$

$90.0 \%$

$75.0 \%$

$50.0 \%$ median $\quad 0.8310$

$25.0 \%$ quartile 0.8180

$10.0 \% \quad 0.8060$

$2.5 \% \quad 0.7860$

$0.5 \% \quad 0.7556$

$0.0 \%$ minimum 0.6790

Moments

Mean 0.832206

Std Dev $\quad 0.0257768$

Std Err Mean $\quad 0.001055$

upper 95\% Mean 0.8342779

lower 95\% Mean $\quad 0.8301341$

$\mathrm{N}$

Si (wt \%)

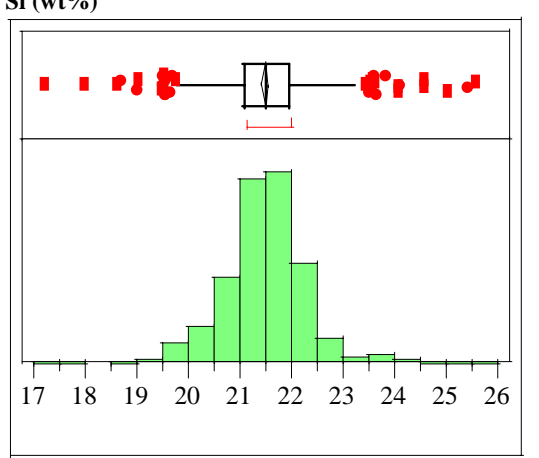

Quantiles

$100.0 \%$ maximum 25.573

$99.5 \% \quad 25.017$

$\begin{array}{ll}97.5 \% & 23.532\end{array}$

$90.0 \% \quad 22.350$

$75.0 \%$ quartile $\quad 21.940$

$50.0 \%$ median 21.506

$25.0 \%$ quartile 21.070

$10.0 \% \quad 20.510$

$2.5 \% \quad 19.652$

$0.5 \% \quad 18.622$

$0.0 \%$ minimum 17.194

Moments

Mean

21.488307

Std Dev

0.8630585

Std Err Mean $\quad 0.0353226$

upper 95\% Mean 21.557678

lower 95\% Mean 21.418935

$\mathrm{N}$ 
WSRC-TR-2004-00576

Revision 0

Exhibit A19. Histograms and Descriptive Statistics for ARG-1 Measurements for Mixed Acid Data Associated with SME and MFT Batches

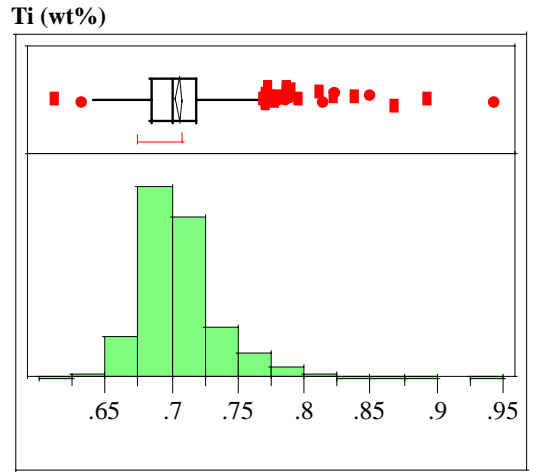

Quantiles

$100.0 \%$ maximum 0.94300

$99.5 \%$

$97.5 \%$

$90.0 \%$

$75.0 \%$

$25.0 \%$

$10.0 \%$

$2.5 \%$

$0.5 \%$

$0.0 \%$

Mean

Std Dev

Std Err Mean

upper 95\% Mean

$\mathrm{N}$

U (wt \%)

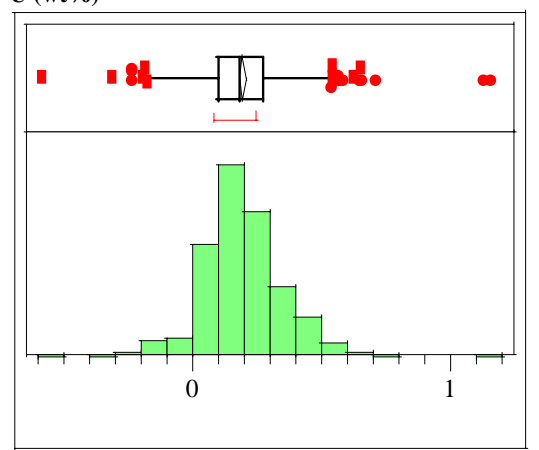

Quantiles

$100.0 \%$ maximum 1.158

$99.5 \%$

$97.5 \%$

$90.0 \%$

$75.0 \%$ quartile

$50.0 \%$ median

$25.0 \%$ quartile

$10.0 \%$

$2.5 \%$

$0.5 \%$

$0.0 \%$

Moments

Mean

Std Dev

Std Err Mean

upper 95\% Mean

lower 95\% Mean

$\mathrm{N}$

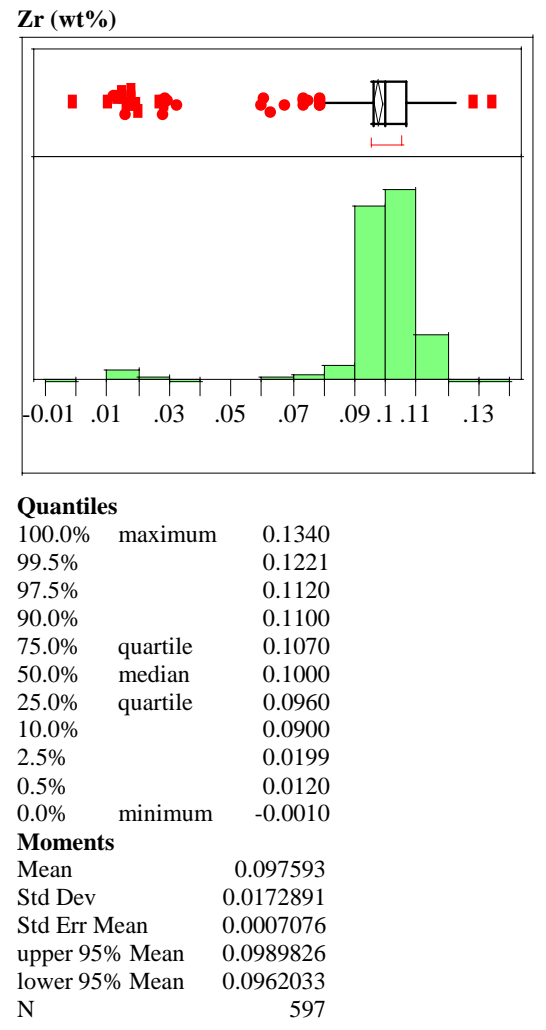

Zr (wt\%)

\begin{tabular}{lrr}
\multicolumn{2}{l}{ Quantiles } \\
$100.0 \%$ & maximum & 0.1340 \\
$99.5 \%$ & & 0.1221 \\
$97.5 \%$ & & 0.1120 \\
$90.0 \%$ & & 0.1100 \\
$75.0 \% \quad$ quartile & 0.1070 \\
$50.0 \% \quad$ median & 0.1000 \\
$25.0 \% \quad$ quartile & 0.0960 \\
$10.0 \%$ & & 0.0900 \\
$2.5 \%$ & & 0.0199 \\
$0.5 \%$ & 0.0120 \\
$0.0 \%$ & -0.0010 \\
Moments & \\
Mean & 0.097593 \\
Std Dev & 0.0172891 \\
Std Err Mean & 0.0007076 \\
upper 95\% Mean & 0.0989826 \\
lower 95\% Mean r & 0.0962033 \\
N & 597
\end{tabular}


WSRC-TR-2004-00576

Revision 0

\section{Exhibit A20. Variance Components for ARG-1 Measurements for Mixed Acid Data Associated with SME and MFT Batches}

Response Al (wt\%)

Summary of Fit

$\begin{array}{lr}\text { RSquare } & 0.61327 \\ \text { RSquare Adj } & 0.227911 \\ \text { Root Mean Square Error } & 0.061605 \\ \text { Mean of Response } & 2.387021 \\ \text { Observations (or Sum Wgts) } & 564\end{array}$

Analysis of Variance

Source DF Sum of Squares Mean Square F Ratio

$\begin{array}{lllll}\text { Model } & 281 & 1.6971587 & 0.006040 & 1.5914\end{array}$

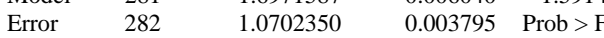

C. Total $563 \quad 2.7673937$

$<.0001$

Variance Component Estimates

$\begin{array}{lrr}\text { Component } & \text { Var Comp Est } & \text { Percent of Total } \\ \text { Tank\&Batch\&Random } & 0.001122 & 22.822 \\ \text { Residual } & 0.003795 & 77.178 \\ \text { Total } & 0.004917 & 100.000\end{array}$

Total $\quad 0.004917 \quad 100.000$

These estimates based on equating Mean Squares to Expected Value.

Response Ca (wt\%)

Summary of Fit

RSquare

RSquare Adj 0.449615

Root Mean Square Error $\quad 0.031993$

Mean of Response 1.027449

Observations (or Sum Wgts) 564

Analysis of Variance

$\begin{array}{lrrrr}\text { Source } & \text { DF } & \text { Sum of Squares } & \text { Mean Square } & \text { F Ratio } \\ \text { Model } & 281 & 0.7583890 & 0.002699 & 2.6367 \\ \text { Error } & 282 & 0.2886485 & 0.001024 & \text { Prob }>\text { F } \\ \text { C. Total } & 563 & 1.0470375 & & <.0001\end{array}$

Variance Component Estimates

$\begin{array}{lrr}\text { Component } & \text { Var Comp Est } & \text { Percent of Total } \\ \text { Tank\&Batch\&Random } & 0.000838 & 45.006 \\ \text { Residual } & 0.001024 & 54.994 \\ \text { Total } & 0.001861 & 100.000\end{array}$

These estimates based on equating Mean Squares to Expected Value.

Response Cr (wt\%)

Summary of Fit

$\begin{array}{lr}\text { RSquare } & 0.761515 \\ \text { RSquare Adj } & 0.523876 \\ \text { Root Mean Square Error } & 0.00409 \\ \text { Mean of Response } & 0.071009 \\ \text { Observations (or Sum Wgts) } & 564\end{array}$

Analysis of Variance

$\begin{array}{lrrrr}\text { Source } & \text { DF } & \text { Sum of Squares } & \text { Mean Square } & \text { F Ratio } \\ \text { Model } & 281 & 0.01506046 & 0.000054 & 3.2045 \\ \text { Error } & 282 & 0.00471650 & 0.000017 & \text { Prob }>\text { F } \\ \text { C. Total } & 563 & 0.01977696 & & <.0001\end{array}$

Variance Component Estimates

$\begin{array}{lrr}\text { Component } & \text { Var Comp Est } & \text { Percent of Total } \\ \text { Tank\&Batch\&Random } & 0.000018 & 52.432 \\ \text { Residual } & 0.000017 & 47.568 \\ \text { Total } & 0.000035 & 100.000\end{array}$

Total $0.000035 \quad 100.000$

These estimates based on equating Mean Squares to Expected Value.

\begin{tabular}{|c|c|c|c|c|}
\hline \multicolumn{5}{|c|}{$\begin{array}{l}\text { Response Cu (wt\%) } \\
\text { Summary of Fit }\end{array}$} \\
\hline \multicolumn{3}{|l|}{ RSquare } & \multicolumn{2}{|c|}{0.845709} \\
\hline \multicolumn{3}{|c|}{ RSquare Adj } & \multicolumn{2}{|c|}{0.691965} \\
\hline \multicolumn{3}{|c|}{ Root Mean Square Error } & \multicolumn{2}{|c|}{0.002141} \\
\hline \multicolumn{3}{|c|}{ Mean of Response } & \multicolumn{2}{|c|}{0.006117} \\
\hline \multicolumn{3}{|c|}{ Observations (or Sum Wgts) } & 564 & \\
\hline \multicolumn{5}{|c|}{ Analysis of Variance } \\
\hline Source & $\mathrm{DF}$ & Sum of Squares & Mean Square & F Ratio \\
\hline Model & 281 & 0.00708728 & 0.000025 & 5.5008 \\
\hline Error & 282 & 0.00129300 & 0.000005 & Prob $>$ F \\
\hline C. Total & 563 & 0.00838028 & & $<.0001$ \\
\hline
\end{tabular}

Variance Component Estimates

Component Var Comp Est Percent of Total

Tank\&Batch\&Random $\quad 0.00001 \quad 69.234$

Residual $\quad 0.000005 \quad 30.766$

Total $\quad 0.000015 \quad 100.000$

These estimates based on equating Mean Squares to Expected Value.

Response Fe (wt\%)

Summary of Fit

\begin{tabular}{|c|c|c|c|c|}
\hline \multicolumn{3}{|l|}{ RSquare } & \multicolumn{2}{|c|}{.515347} \\
\hline \multicolumn{3}{|c|}{ RSquare Adj } & \multicolumn{2}{|c|}{0.032412} \\
\hline \multicolumn{3}{|c|}{ Root Mean Square Error } & \multicolumn{2}{|c|}{0.24159} \\
\hline \multicolumn{3}{|c|}{ Mean of Response } & \multicolumn{2}{|c|}{9.590246} \\
\hline \multicolumn{3}{|c|}{ Observations (or Sum Wgts) } & \multicolumn{2}{|l|}{564} \\
\hline \multicolumn{5}{|c|}{ Analysis of Variance } \\
\hline Source & DF & Sum of Square & Mean Square & F Ratio \\
\hline Model & 281 & 17.501453 & 0.062283 & 1.0671 \\
\hline Error & 282 & 16.45908 & 0.058366 & Prob $>$ F \\
\hline C. Total & 563 & 33.960535 & & 0.2931 \\
\hline
\end{tabular}

Variance Component Estimates

$\begin{array}{lrr}\text { Component } & \text { Var Comp Est } & \text { Percent of Total } \\ \text { Tank\&Batch\&Random } & 0.001959 & 3.247 \\ \text { Residual } & 0.058366 & 96.753 \\ \text { Total } & 0.060324 & 100.000\end{array}$

These estimates based on equating Mean Squares to Expected Value.

Response K (wt\%)

Summary of Fit

$\begin{array}{lr}\text { RSquare } & 0.712031 \\ \text { RSquare Adj } & 0.425082 \\ \text { Root Mean Square Error } & 0.064217 \\ \text { Mean of Response } & 2.284351 \\ \text { Observations (or Sum Wgts) } & 564\end{array}$

Analysis of Variance

$\begin{array}{lrrrr}\text { Source } & \text { DF } & \text { Sum of Squares } & \text { Mean Square } & \text { F Ratio } \\ \text { Model } & 281 & 2.8754175 & 0.010233 & 2.4814 \\ \text { Error } & 282 & 1.1629170 & 0.004124 & \text { Prob }>\text { F } \\ \text { C. Total } & 563 & 4.0383345 & & <.0001\end{array}$

Variance Component Estimates

$\begin{array}{lrc}\text { Component } & \text { Var Comp Est } & \text { Percent of Total } \\ \text { Tank\&Batch\&Random } & 0.003054 & 42.552 \\ \text { Residual } & 0.004124 & 57.448 \\ \text { Total } & 0.007178 & 100.000 \\ \text { These estimates based on equating Mean Squares to Expected Value. }\end{array}$


WSRC-TR-2004-00576

Revision 0

\section{Exhibit A20. Variance Components for ARG-1 Measurements for Mixed Acid Data Associated with SME and MFT Batches}

Response Li (wt\%)

Summary of Fit

$\begin{array}{lr}\text { RSquare } & 0.53644 \\ \text { RSquare Adj } & 0.074524 \\ \text { Root Mean Square Error } & 0.03665 \\ \text { Mean of Response } & 1.486697 \\ \text { Observations (or Sum Wgts) } & 564\end{array}$

Analysis of Variance

Source DF Sum of Squares Mean Square F Ratio

$\begin{array}{lllll}\text { Model } & 281 & 0.43833865 & 0.001560 & 1.1613\end{array}$

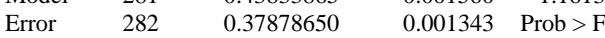

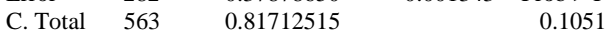

$\begin{array}{lrr}\text { Variance Component Estimates } & \\ \text { Component } & \text { Var Comp Est } & \text { Percent of Total } \\ \text { Tank\&Batch\&Random } & 0.000108 & 7.465 \\ \text { Residual } & 0.001343 & 92.535 \\ \text { Total } & 0.001452 & 100.000\end{array}$

These estimates based on equating Mean Squares to Expected Value.

Response Mg (wt \%)

Summary of Fi

RSquare $\quad 0.601894$

RSquare Adj 0.205199

Root Mean Square Error $\quad 0.014507$

Mean of Response

Observations (or Sum Wgts) 564

Analysis of Variance

Source DF Sum of Squares Mean Square F Ratio

$\begin{array}{lllll}\text { Model } & 281 & 0.08973001 & 0.000319 & 1.5173\end{array}$

$\begin{array}{lllll}\text { Error } & 282 & 0.05934950 & 0.000210 & \text { Prob }>\text { F }\end{array}$

$\begin{array}{lll}\text { C. Total } 563 & 0.14907951 & 0.0002\end{array}$

Variance Component Estimates

$\begin{array}{lrr}\text { Component } & \text { Var Comp Est } & \text { Percent of Total } \\ \text { Tank\&Batch\&Random } & 0.000054 & 20.549 \\ \text { Residual } & 0.00021 & 79.451 \\ \text { Total } & 0.000265 & 100.000\end{array}$

$\begin{array}{lcc}\text { Total } & 0.000265 & 100.000 \\ \text { These estimates based on equating Mean Squares to Expected Value. }\end{array}$

Response Mn (wt\%)

Summary of Fit

RSquare

0.539631

RSquare Adj $\quad 0.080895$

Root Mean Square Error $\quad 0.035375$

Mean of Response $\quad 1.421126$

Observations (or Sum Wgts) 564

Analysis of Variance

$\begin{array}{lrrrr}\text { Source } & \text { DF } & \text { Sum of Squares } & \text { Mean Square } & \text { F Ratio } \\ \text { Model } & 281 & 0.41364256 & 0.001472 & 1.1763 \\ \text { Error } & 282 & 0.35288550 & 0.001251 & \text { Prob }>\text { F } \\ \text { C. Total } & 563 & 0.76652806 & & 0.0868\end{array}$

$\begin{array}{llll}\text { C. Total } & 563 & 0.76652806 & 0.001251-\text { Prob }>\text { F }\end{array}$

Variance Component Estimates

$\begin{array}{lrc}\text { Variance Component Estimates } & \\ \text { Component } & \text { Var Comp Est } & \text { Percent of Total } \\ \text { Tank\&Batch\&Random } & 0.00011 & 8.103 \\ \text { Residual } & 0.001251 & 91.897 \\ \text { Total } & 0.001362 & 100.000 \\ \text { These estimates based on equating Mean Squares to Expected Value. }\end{array}$

\begin{tabular}{|c|c|c|c|c|}
\hline \multicolumn{5}{|c|}{$\begin{array}{l}\text { Response Na (wt\%) } \\
\text { Summary of Fit }\end{array}$} \\
\hline \multicolumn{3}{|l|}{ RSquare } & \multicolumn{2}{|c|}{0.531534} \\
\hline \multicolumn{3}{|c|}{ RSquare Adj } & \multicolumn{2}{|c|}{0.06473} \\
\hline \multicolumn{3}{|c|}{ Root Mean Square Error } & \multicolumn{2}{|c|}{0.237979} \\
\hline \multicolumn{3}{|c|}{ Mean of Response } & \multicolumn{2}{|c|}{8.349112} \\
\hline \multicolumn{3}{|c|}{ Observations (or Sum Wgts) } & \multicolumn{2}{|c|}{564} \\
\hline \multicolumn{5}{|c|}{ Analysis of Variance } \\
\hline Source & DF & Sum of Squares & Mean Square & F Ratio \\
\hline Model & 281 & 18.120888 & 0.064487 & 1.1387 \\
\hline Error & 282 & 15.970784 & 0.056634 & Prob $>$ F \\
\hline C. Total & 563 & 34.091672 & & 0.1383 \\
\hline
\end{tabular}

$\begin{array}{lrr}\text { Variance Component Estimates } & \\ \text { Component } & \text { Var Comp Est } & \text { Percent of Total } \\ \text { Tank\&Batch\&Random } & 0.003927 & 6.484 \\ \text { Residual } & 0.056634 & 93.516 \\ \text { Total } & 0.060561 & 100.000\end{array}$

These estimates based on equating Mean Squares to Expected Value.

Response Ni (wt \%)

Summary of Fit

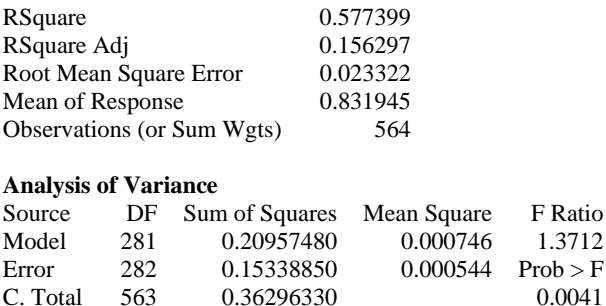

$\begin{array}{lrc}\text { Variance Component Estimates } & \\ \text { Component } & \text { Var Comp Est } & \text { Percent of Total } \\ \text { Tank\&Batch\&Random } & 0.000101 & 15.653 \\ \text { Residual } & 0.000544 & 84.347 \\ \text { Total } & 0.000645 & 100.000 \\ \text { These estimates based on equating Mean Squares to Expected Value. }\end{array}$

Response Si (wt\%)

Summary of Fit

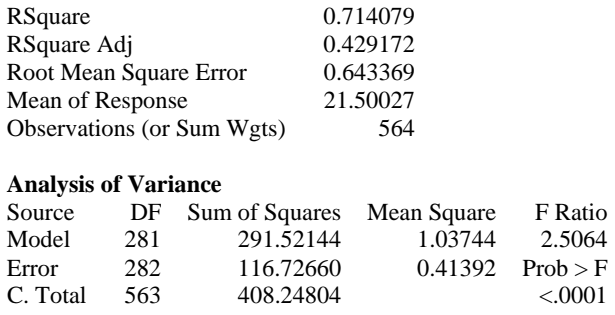

Variance Component Estimates

$\begin{array}{lrc}\text { Variance Component Estimates } & \\ \text { Component } & \text { Var Comp Est } & \text { Percent of Total } \\ \text { Tank\&Batch\&Random } & 0.311759 & 42.961 \\ \text { Residual } & 0.413924 & 57.039 \\ \text { Total } & 0.725683 & 100.000 \\ \text { These estimates based on equating Mean Squares to Expected Value. }\end{array}$


WSRC-TR-2004-00576

Revision 0

\section{Exhibit A20. Variance Components for ARG-1 Measurements for Mixed Acid Data Associated with SME and MFT Batches}

\begin{tabular}{|c|c|c|c|c|c|}
\hline \multicolumn{5}{|c|}{ Response Ti (wt\%) } & Summary of Fit \\
\hline \multicolumn{3}{|l|}{ RSquare } & \multicolumn{3}{|c|}{0.755915} \\
\hline \multicolumn{3}{|c|}{ RSquare Adj } & \multicolumn{3}{|c|}{0.512695} \\
\hline \multicolumn{3}{|c|}{ Root Mean Square Error } & \multicolumn{3}{|c|}{0.023315} \\
\hline \multicolumn{3}{|c|}{ Mean of Response } & 0.7 & 05715 & \\
\hline \multicolumn{5}{|c|}{ Observations (or Sum Wgts) } & \\
\hline \multicolumn{6}{|c|}{ Analysis of Variance } \\
\hline Source & DF & Sum of Squ & ares & Mean Square & F Ratio \\
\hline Model & 281 & 0.47475 & 454 & 0.001690 & 3.1079 \\
\hline Error & 282 & 0.15329 & 850 & 0.000544 & Prob $>$ F \\
\hline C. Total & 563 & 0.62805 & 304 & & $<.0001$ \\
\hline
\end{tabular}

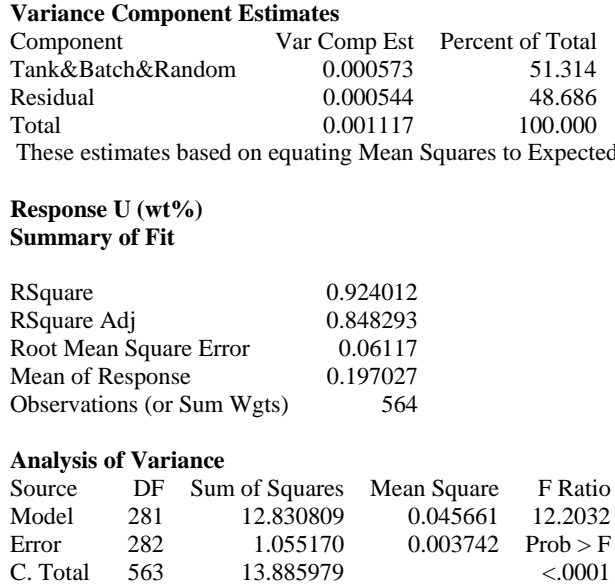

$\begin{array}{lrr}\text { Variance Component Estimates } & \\ \text { Component } & \text { Var Comp Est } & \text { Percent of Total } \\ \text { Tank\&Batch\&Random } & 0.02096 & 84.852 \\ \text { Residual } & 0.003742 & 15.148 \\ \text { Total } & 0.024701 & 100.000\end{array}$

These estimates based on equating Mean Squares to Expected Value.

Response Zr (wt\%)

Summary of Fit

\begin{tabular}{|c|c|c|c|c|}
\hline \multicolumn{3}{|l|}{ RSquare } & \multicolumn{2}{|c|}{981072} \\
\hline \multicolumn{3}{|c|}{ RSquare Adj } & \multicolumn{2}{|c|}{0.962211} \\
\hline \multicolumn{3}{|c|}{ Root Mean Square Error } & \multicolumn{2}{|c|}{0.003198} \\
\hline \multicolumn{3}{|c|}{ Mean of Response } & \multicolumn{2}{|c|}{0.098002} \\
\hline \multicolumn{3}{|c|}{ Observations (or Sum Wgts) } & \multicolumn{2}{|l|}{564} \\
\hline \multicolumn{5}{|c|}{ Analysis of Variance } \\
\hline Source & DF & Sum of Squares & Mean Square & F Ratio \\
\hline Model & 281 & 0.14950650 & 0.000532 & 52.0154 \\
\hline Error & 282 & 0.00288450 & 0.000010 & Prob $>$ F \\
\hline C. Total & 563 & 0.15239100 & & $<.0001$ \\
\hline
\end{tabular}

$\begin{array}{lrr}\text { Variance Component Estimates } & \\ \text { Component } & \text { Var Comp Est } & \text { Percent of Total } \\ \text { Tank\&Batch\&Random } & 0.000261 & 96.228 \\ \text { Residual } & 0.00001 & 3.772 \\ \text { Total } & 0.000271 & 100.000\end{array}$

Total $0.000271 \quad 100.000$

These estimates based on equating Mean Squares to Expected Value. 
WSRC-TR-2004-00576

Revision 0

\section{Exhibit A21. Fusion Preparation Standards in Analytical Sequence}

STCd=SM51

Control Chart

Individual Measurement of AL (mg/L)

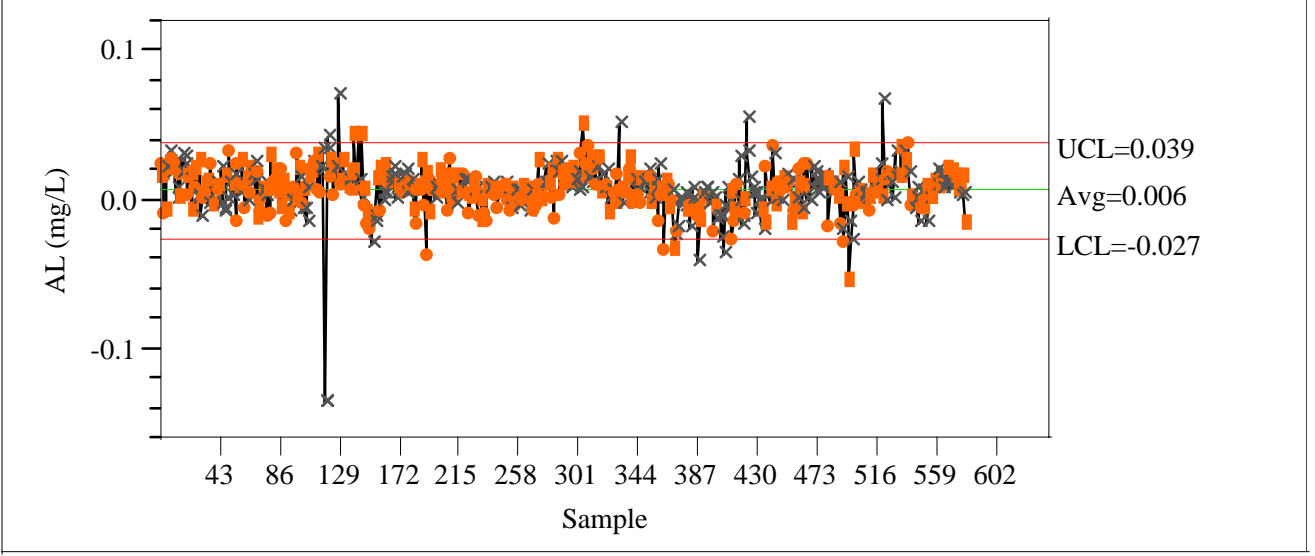

Individual Measurement of B (mg/L)

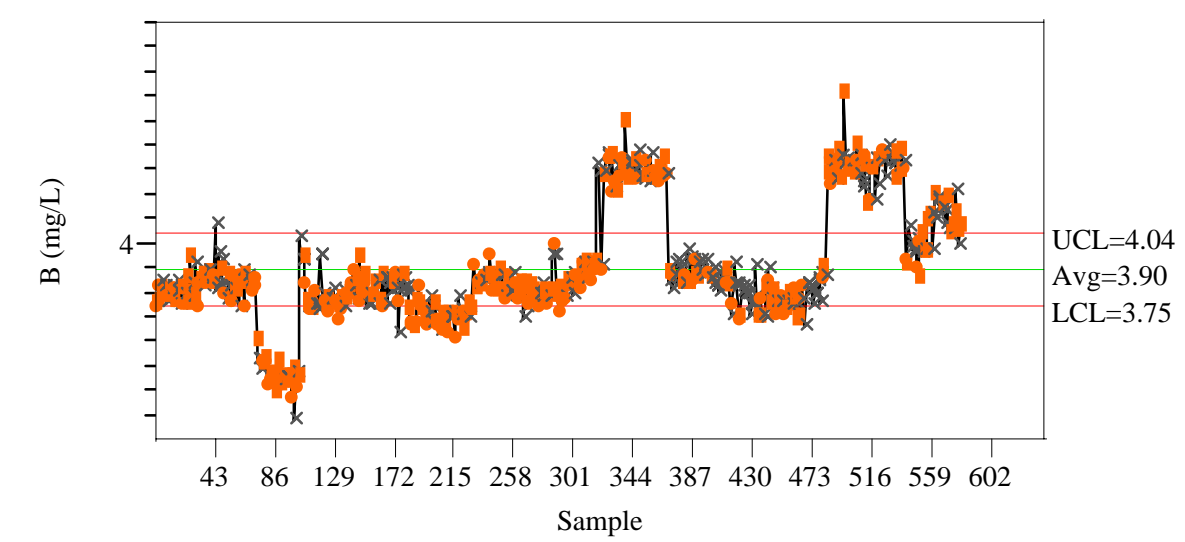

Individual Measurement of Ca (mg/L)

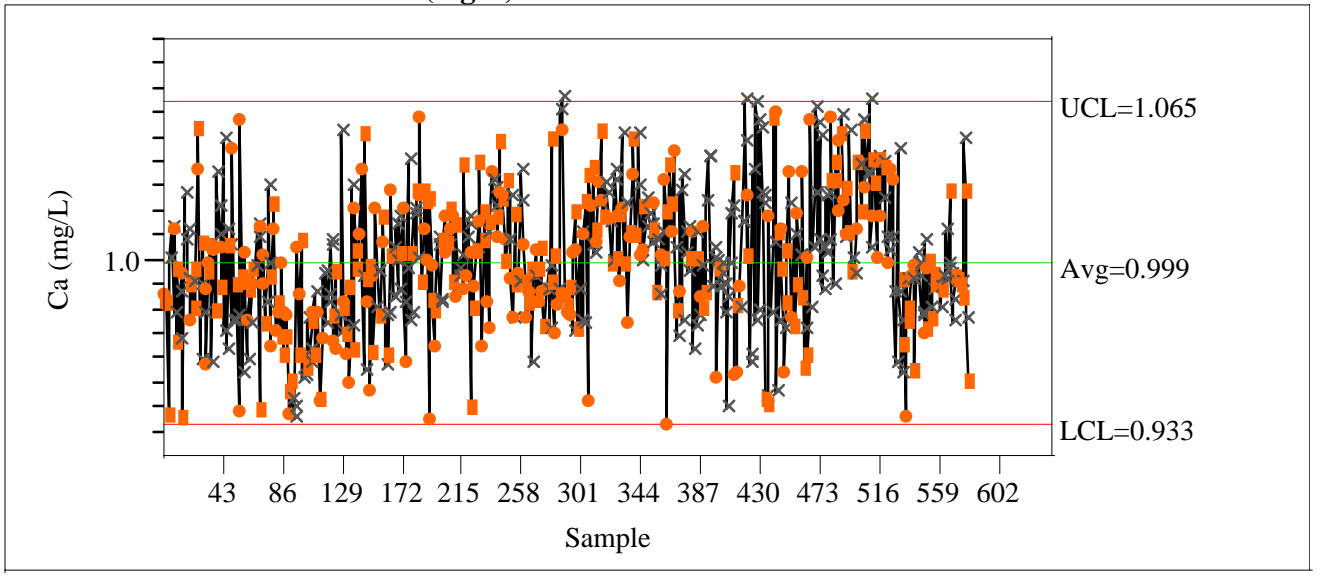


WSRC-TR-2004-00576

Revision 0

Exhibit A21. Fusion Preparation Standards in Analytical Sequence

Individual Measurement of $\mathrm{Cr}$ (mg/L)

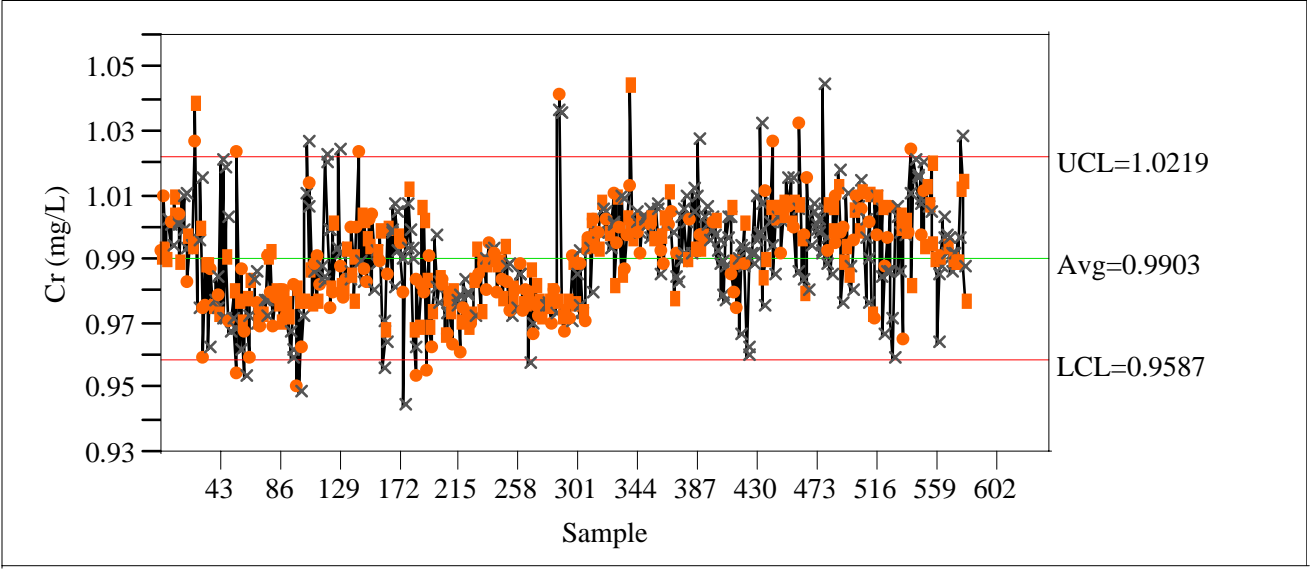

Individual Measurement of $\mathrm{Cu}(\mathrm{mg} / \mathrm{L})$

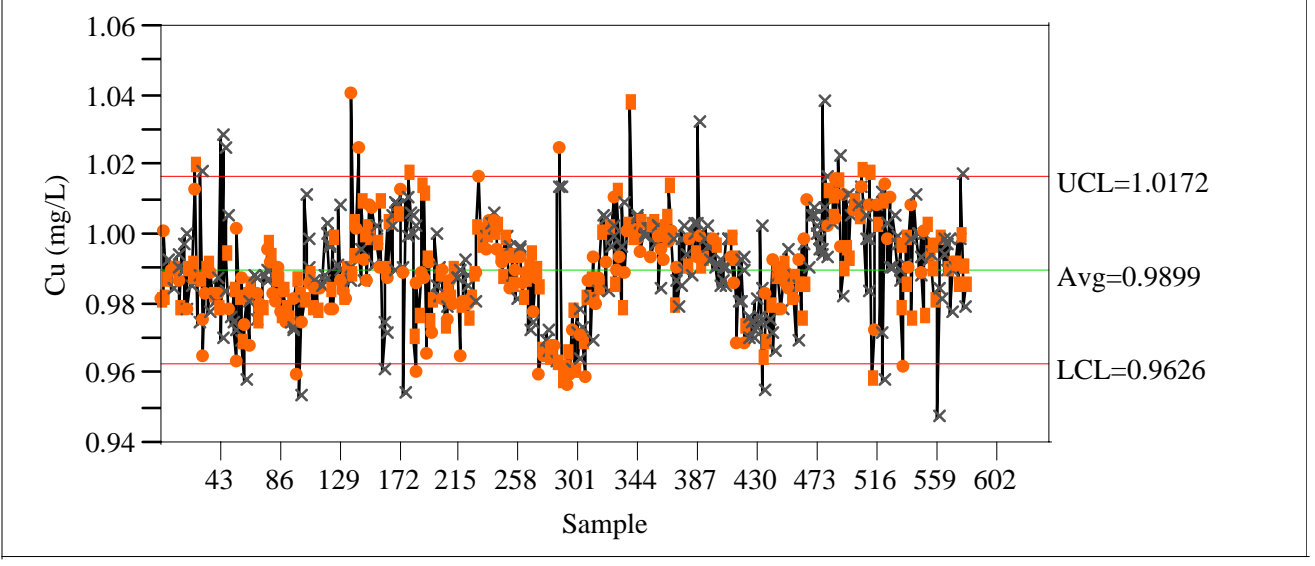

Individual Measurement of Fe (mg/L)

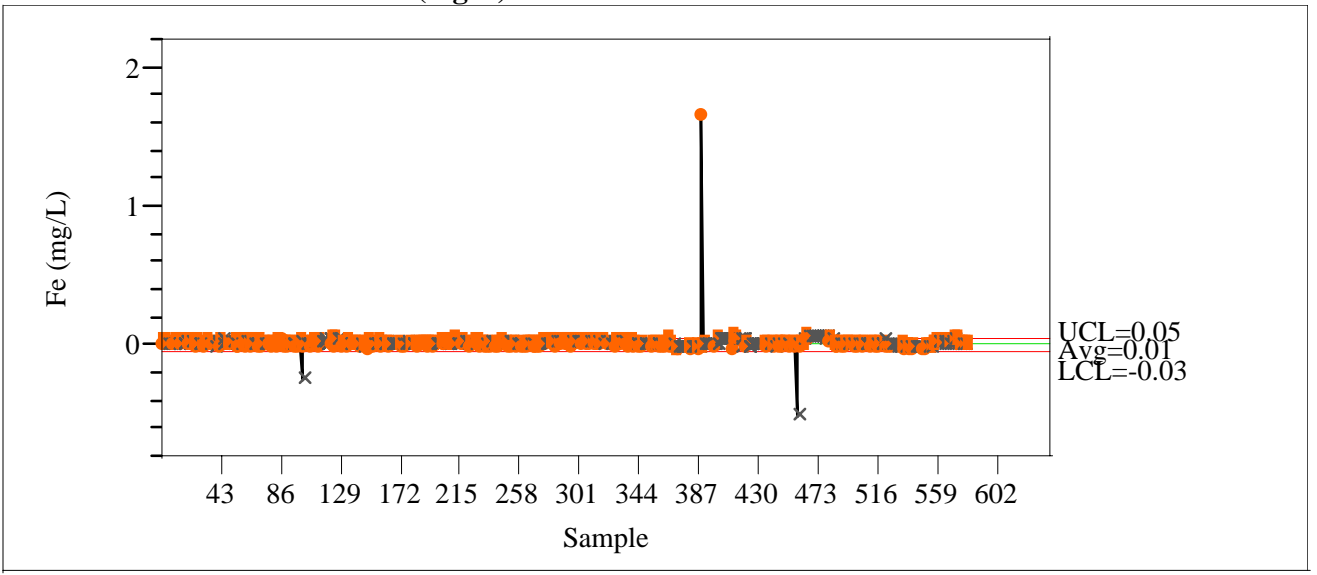


Exhibit A21. Fusion Preparation Standards in Analytical Sequence

Individual Measurement of K (mg/L)

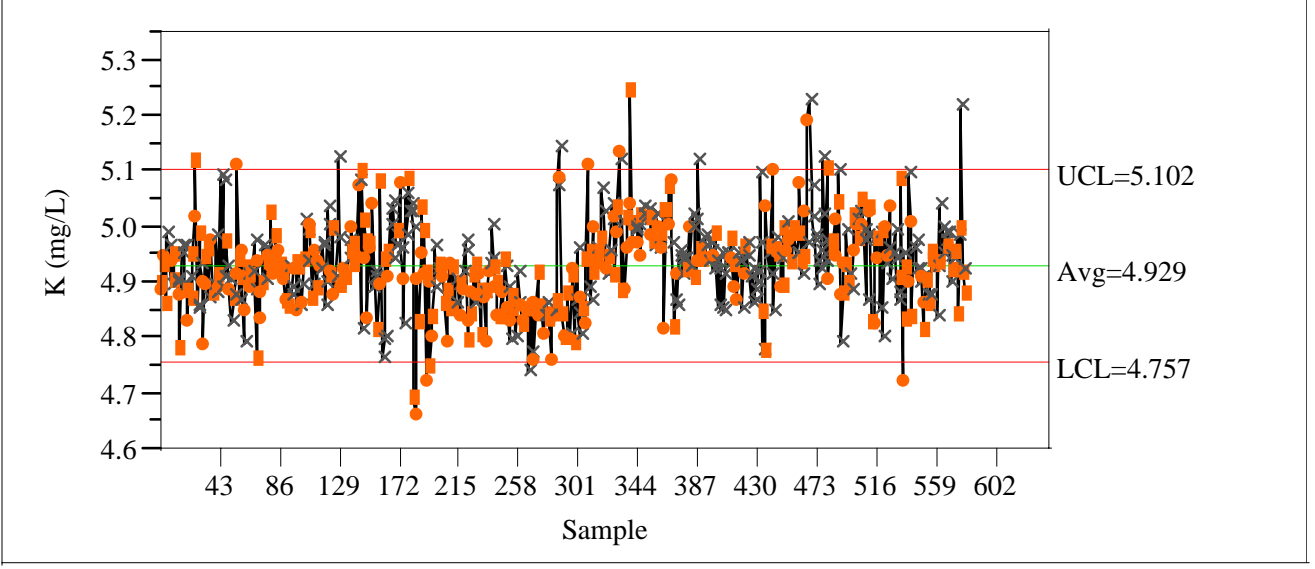

Individual Measurement of $\mathrm{Li}(\mathrm{mg} / \mathrm{L})$

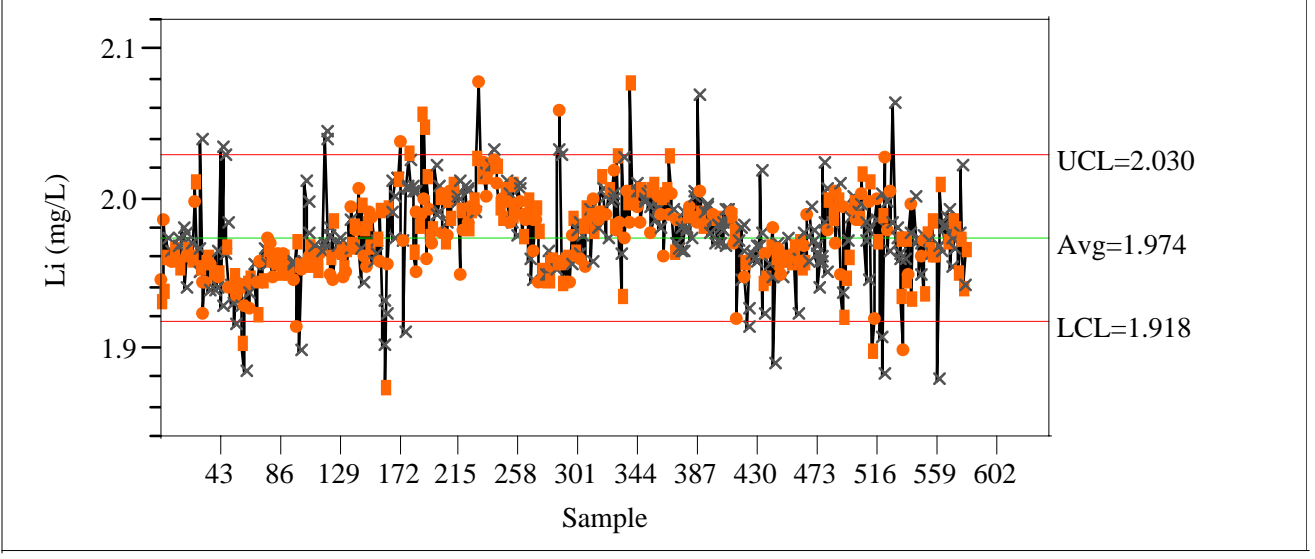

Individual Measurement of $\mathrm{Mg}$ (mg/L)

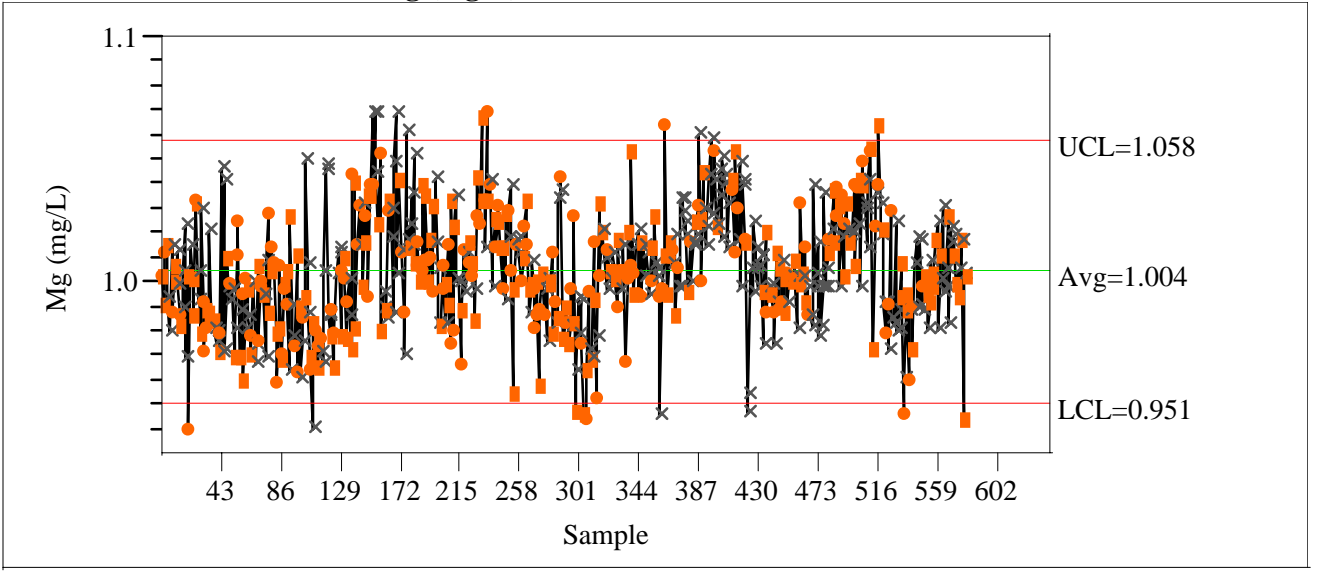


WSRC-TR-2004-00576

Revision 0

Exhibit A21. Fusion Preparation Standards in Analytical Sequence

Individual Measurement of Mn (mg/L)

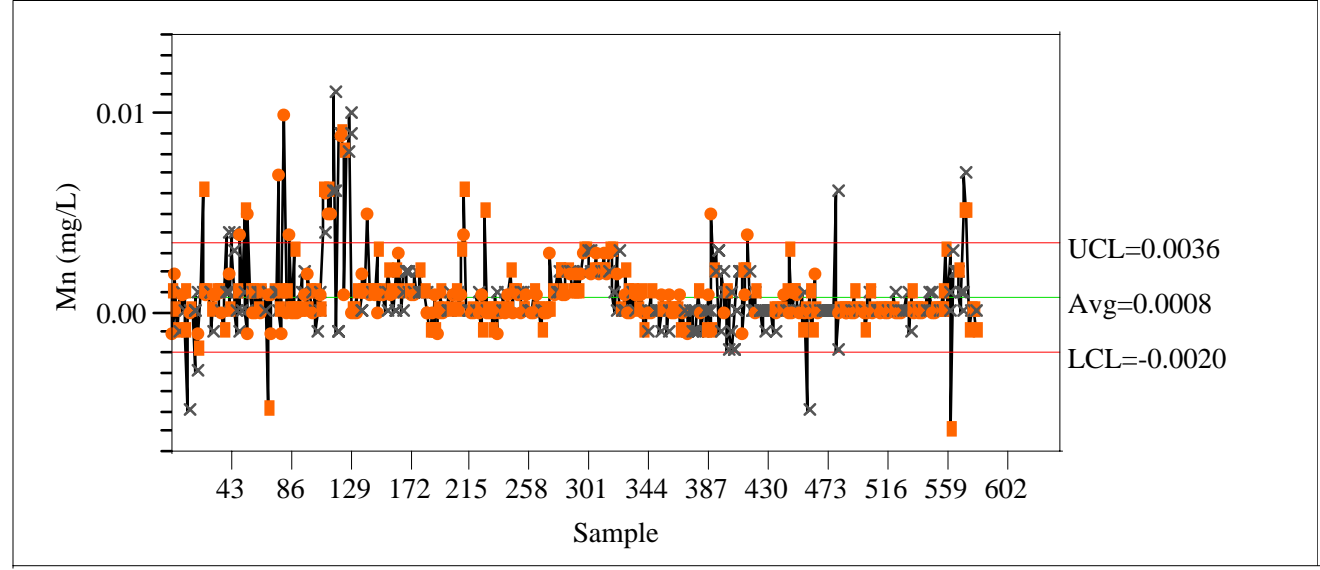

Individual Measurement of $\mathrm{Ni}(\mathrm{mg} / \mathrm{L})$

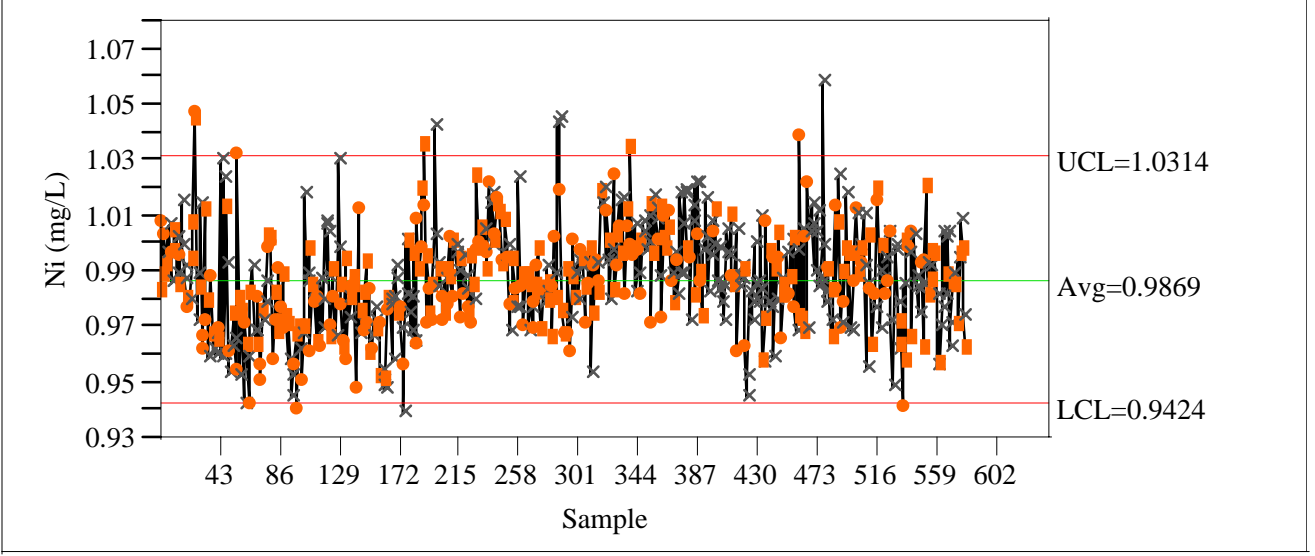

Individual Measurement of Si (mg/L)

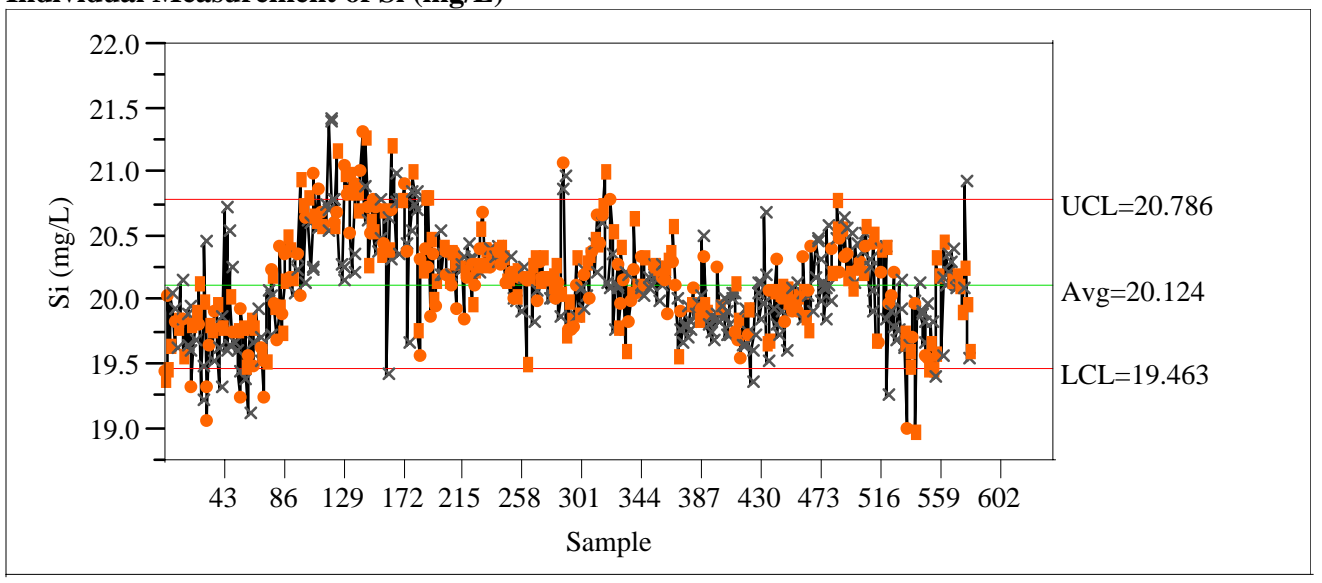


WSRC-TR-2004-00576

Revision 0

Exhibit A21. Fusion Preparation Standards in Analytical Sequence

Individual Measurement of Ti (mg/L)

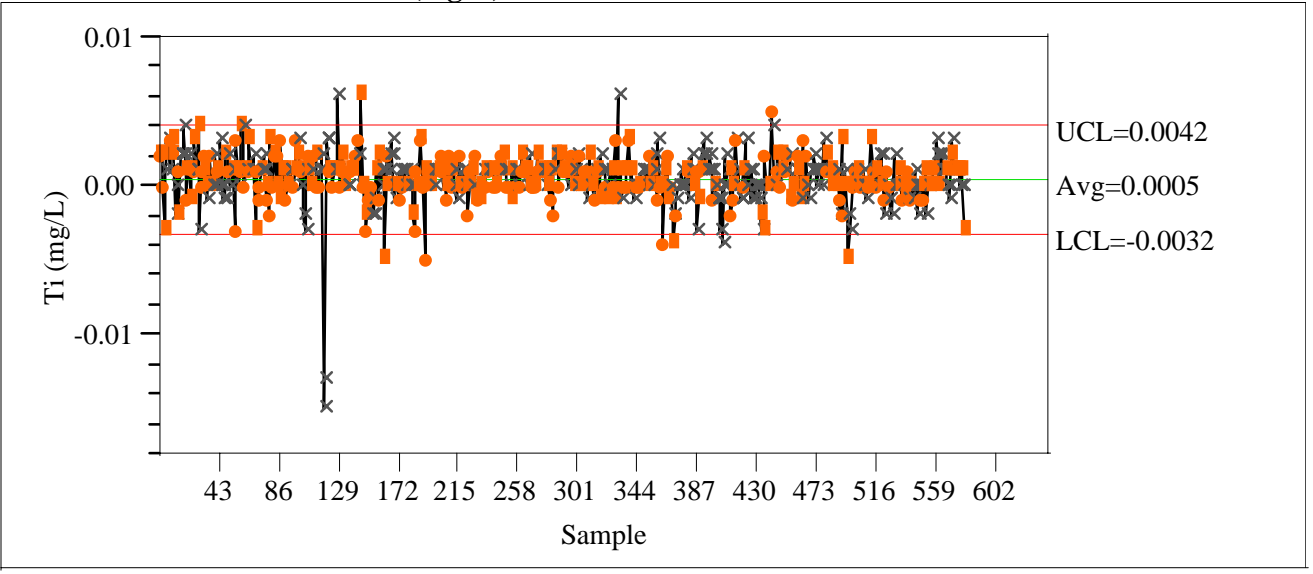

STCd=SM52

Control Chart

Individual Measurement of AL (mg/L)

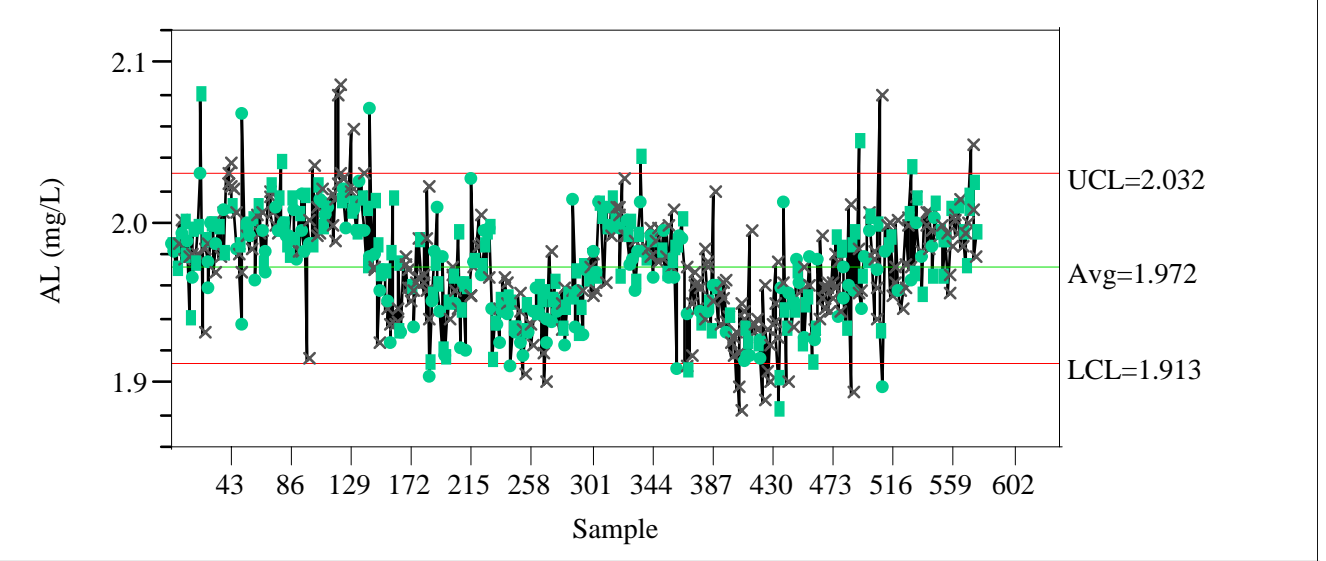

Individual Measurement of B (mg/L)

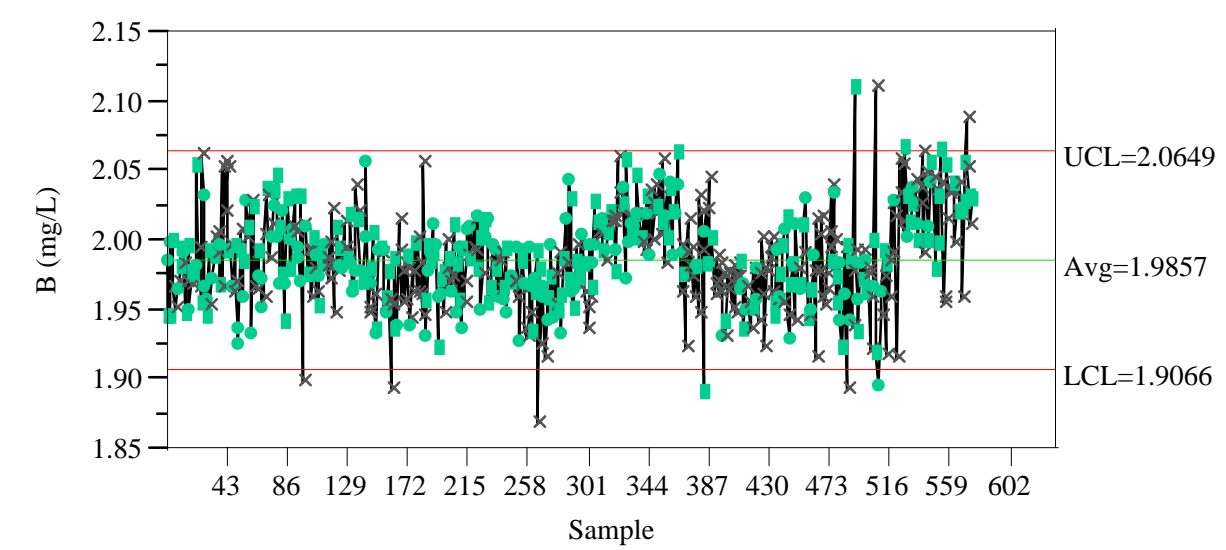


WSRC-TR-2004-00576

Revision 0

\section{Exhibit A21. Fusion Preparation Standards in Analytical Sequence}

Individual Measurement of Ca (mg/L)

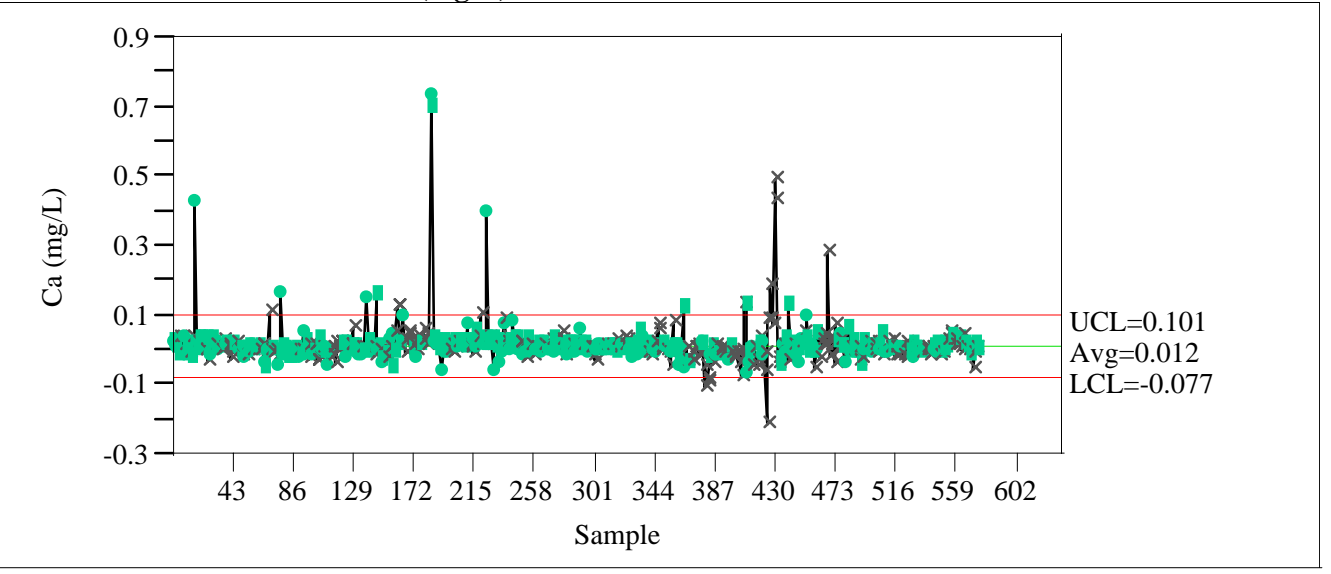

Individual Measurement of $\mathrm{Cr}$ (mg/L)

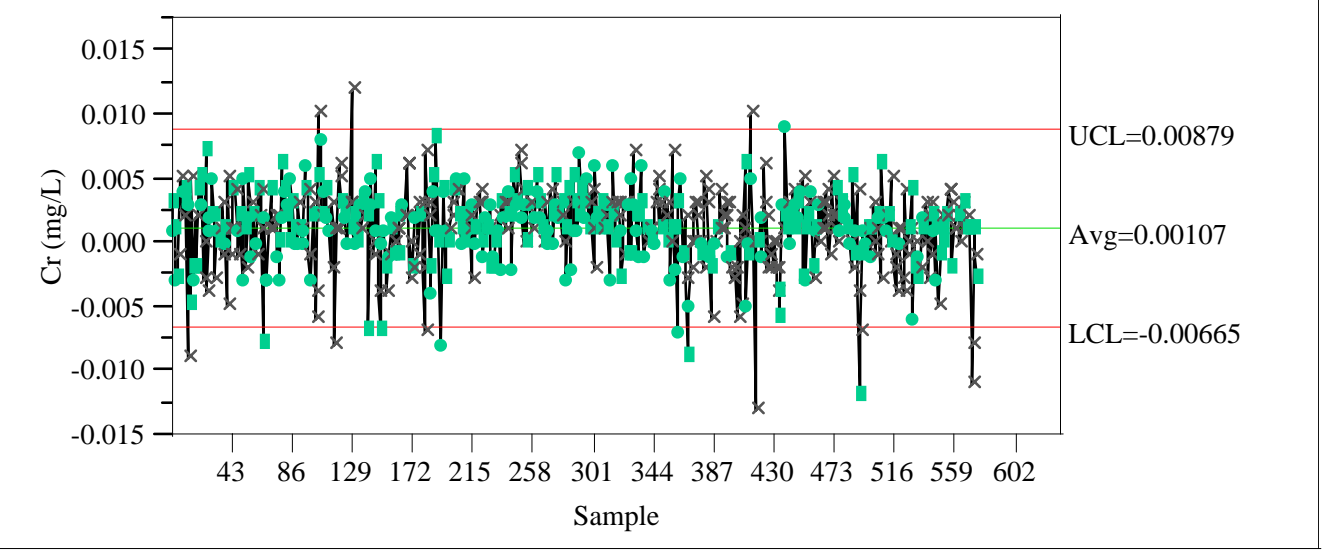

Individual Measurement of $\mathrm{Cu}(\mathrm{mg} / \mathrm{L})$

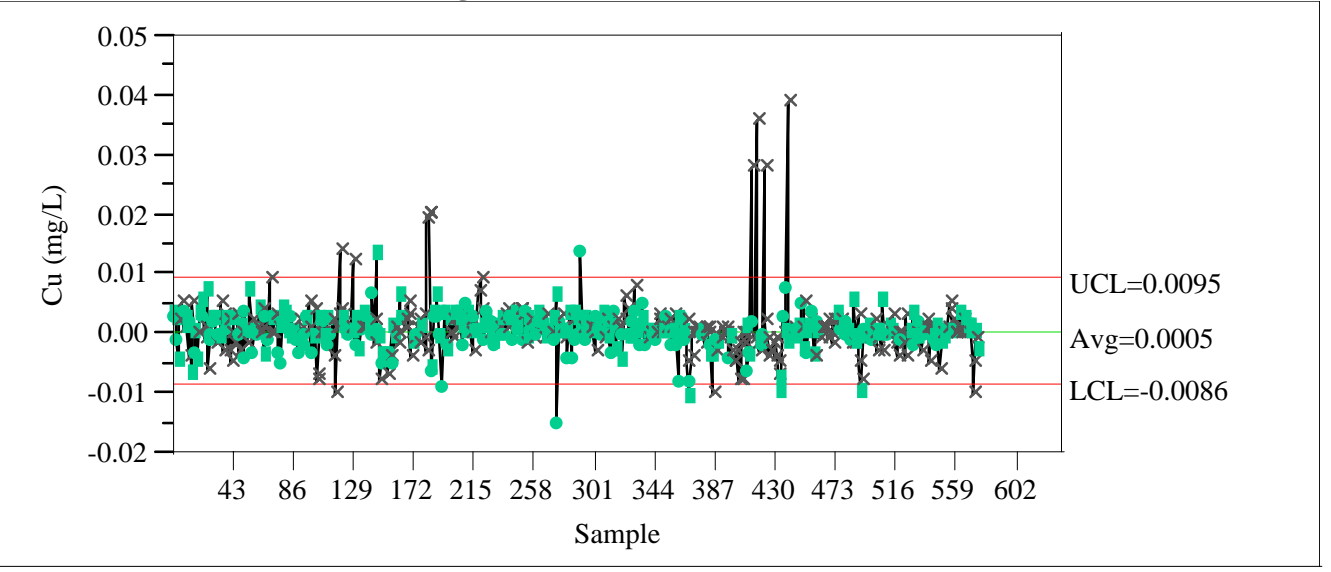


Exhibit A21. Fusion Preparation Standards in Analytical Sequence

Individual Measurement of Fe (mg/L)

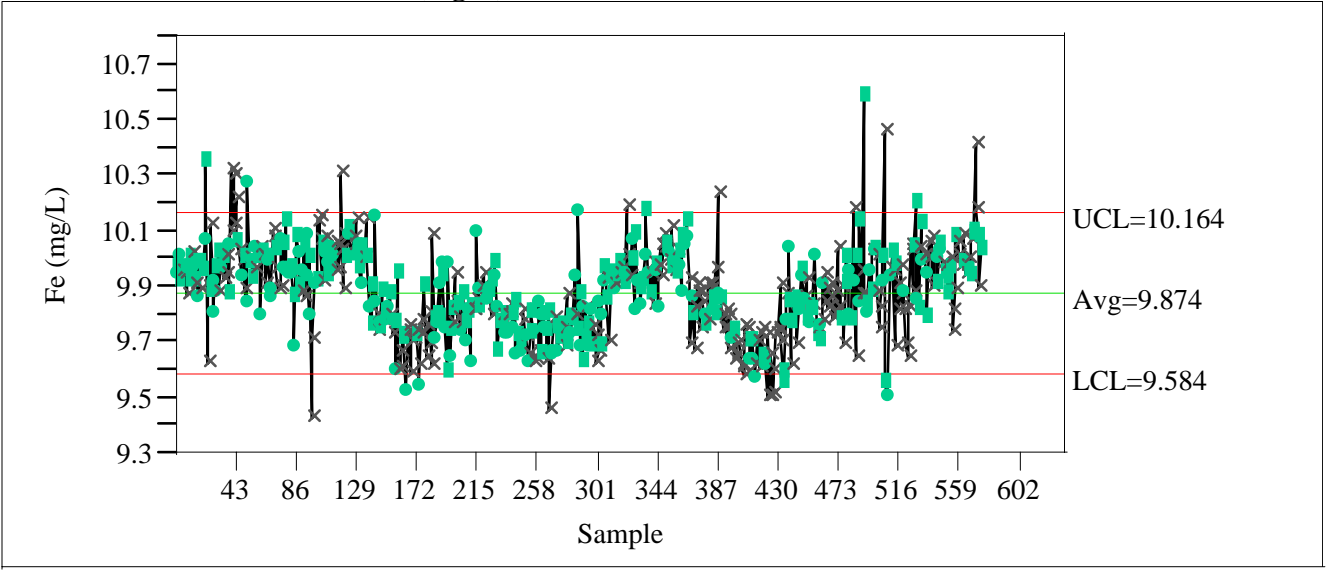

Individual Measurement of K (mg/L)

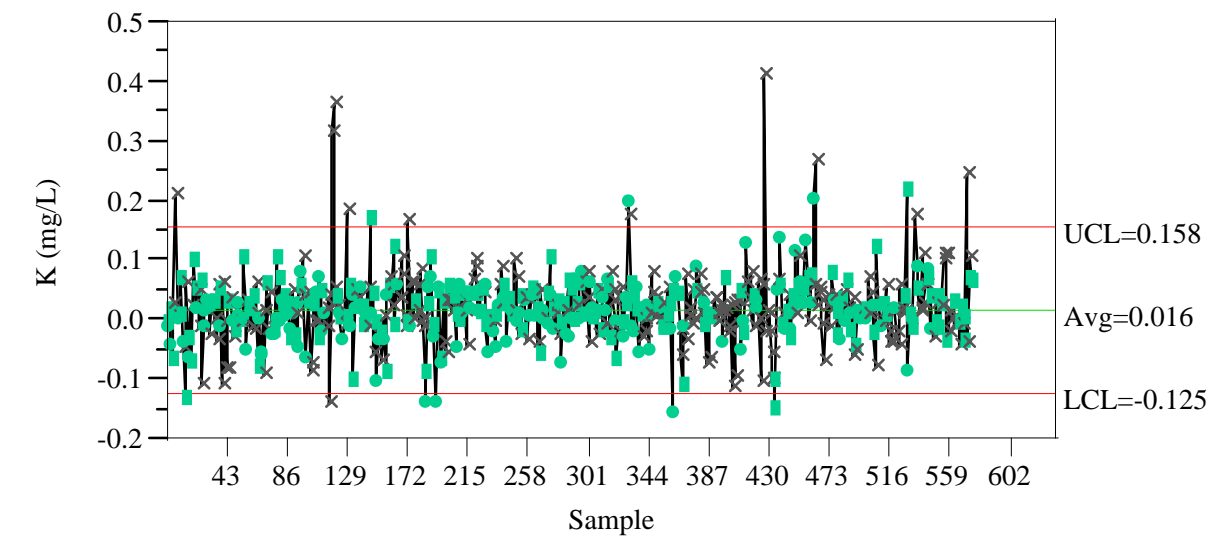

Individual Measurement of $\mathrm{Li}(\mathrm{mg} / \mathrm{L}$ )

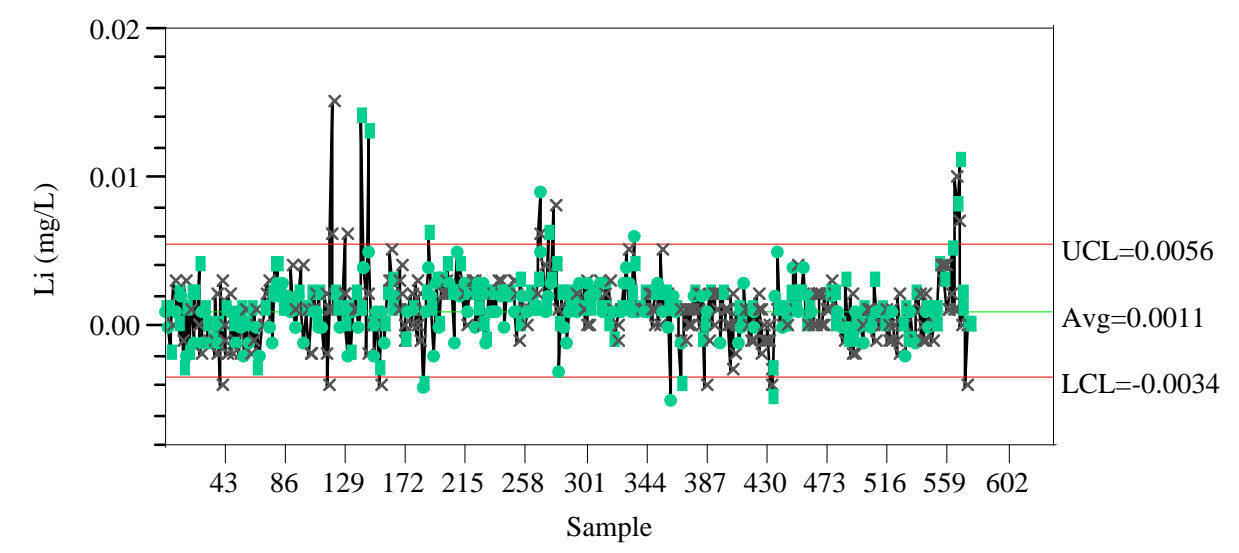


WSRC-TR-2004-00576

Revision 0

Exhibit A21. Fusion Preparation Standards in Analytical Sequence

Individual Measurement of $\mathrm{Mg}$ (mg/L)

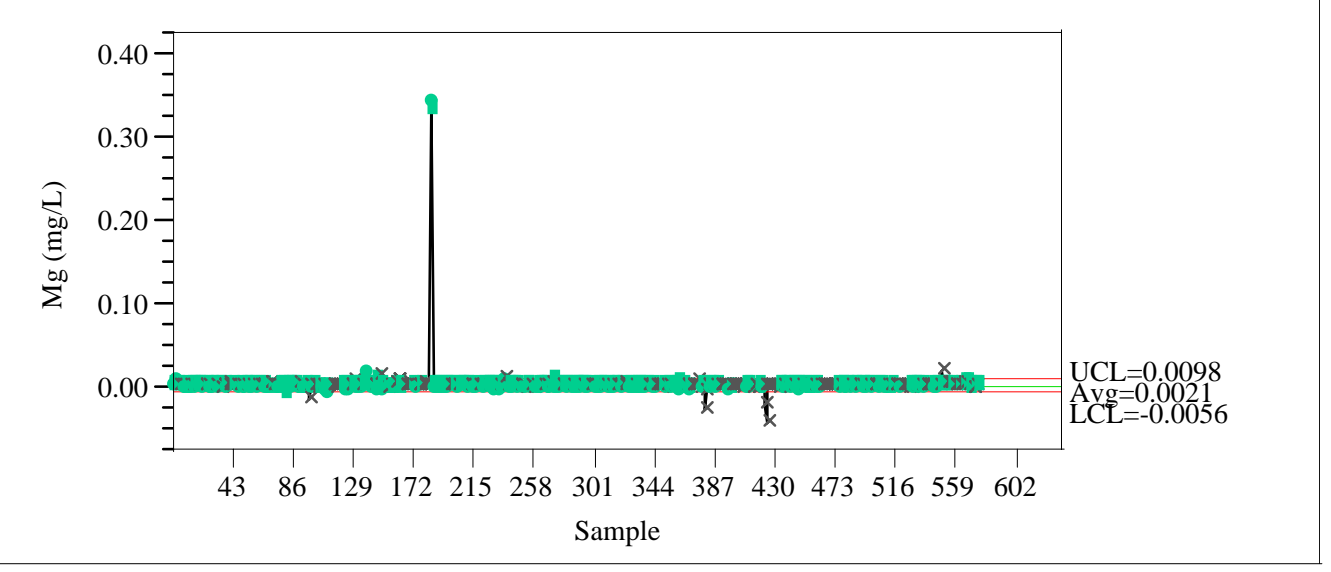

Individual Measurement of Mn (mg/L)

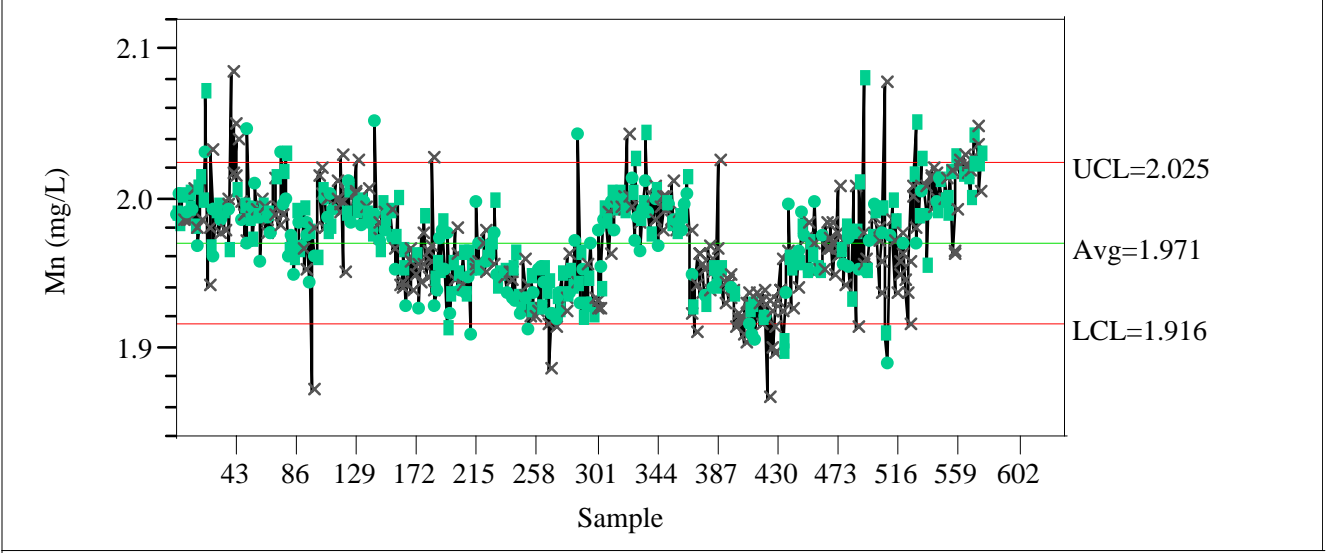

Individual Measurement of $\mathrm{Ni}$ (mg/L)

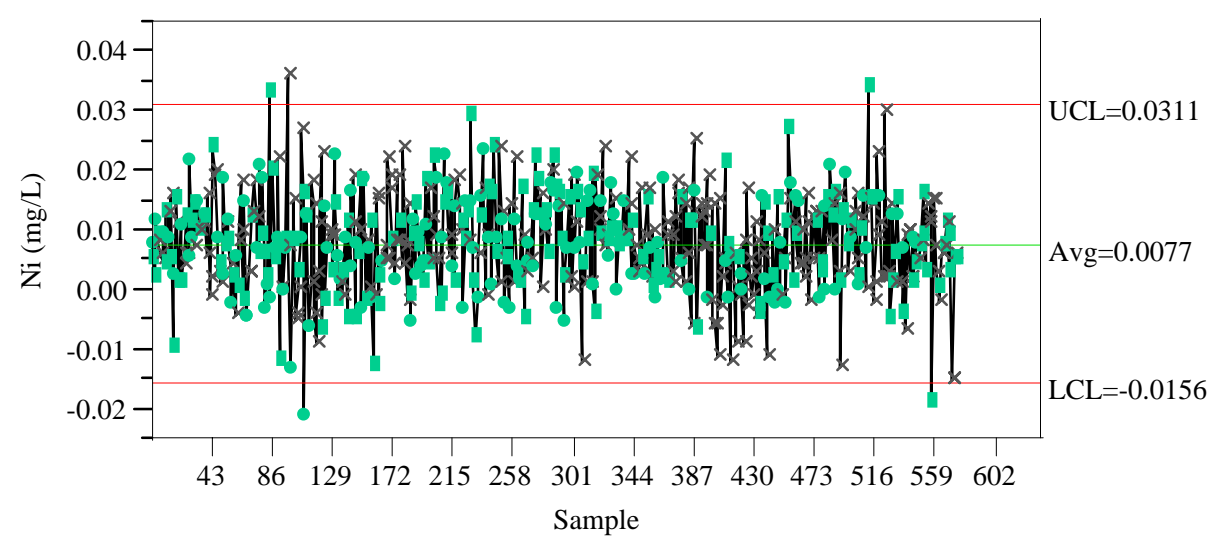


WSRC-TR-2004-00576

Revision 0

Exhibit A21. Fusion Preparation Standards in Analytical Sequence

Individual Measurement of Si (mg/L)

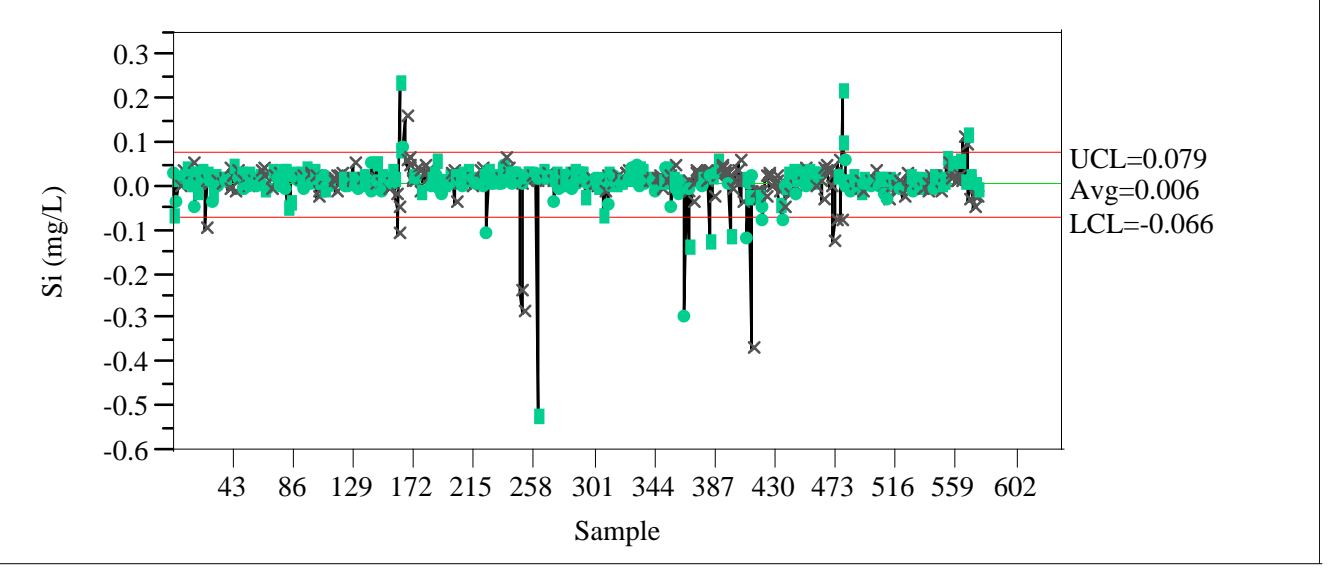

Individual Measurement of $\mathrm{Ti}$ (mg/L)

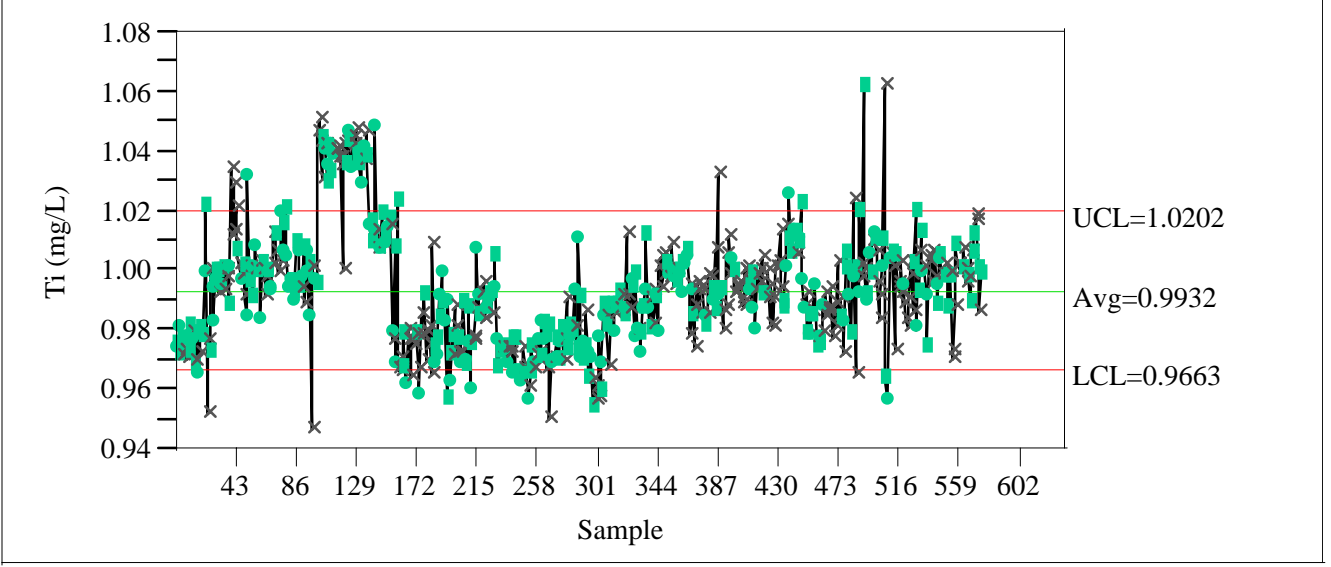

STCd=SM53

Control Chart

Individual Measurement of AL (mg/L)

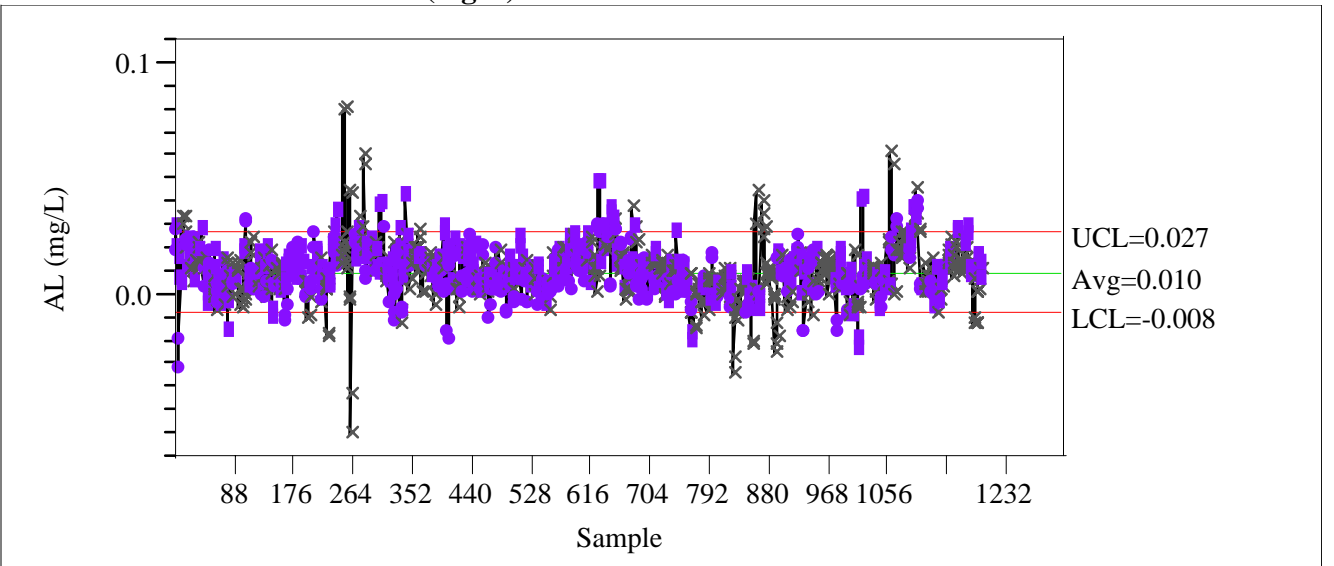


Exhibit A21. Fusion Preparation Standards in Analytical Sequence

Individual Measurement of B (mg/L)

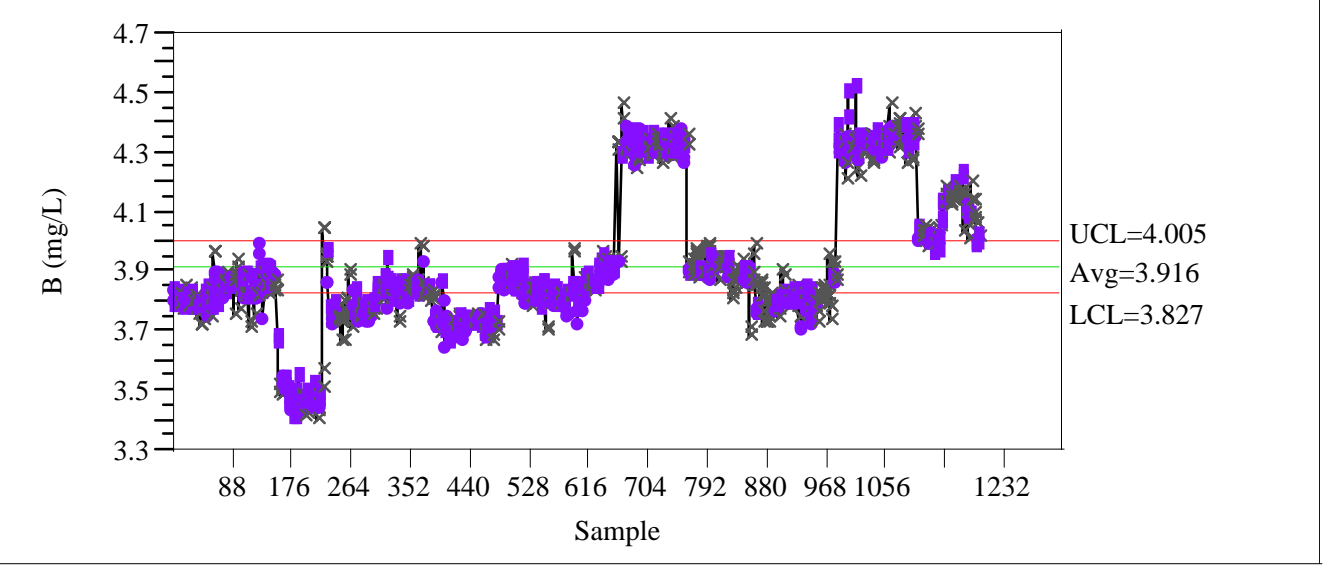

Individual Measurement of Ca (mg/L)

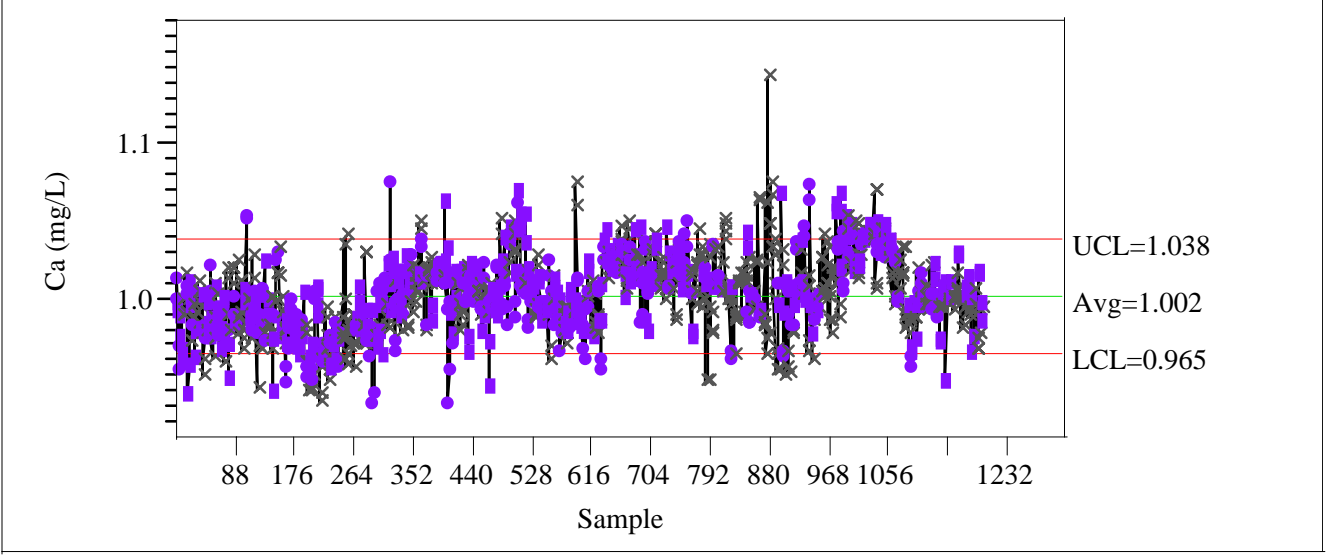

Individual Measurement of $\mathrm{Cr}(\mathrm{mg} / \mathrm{L})$

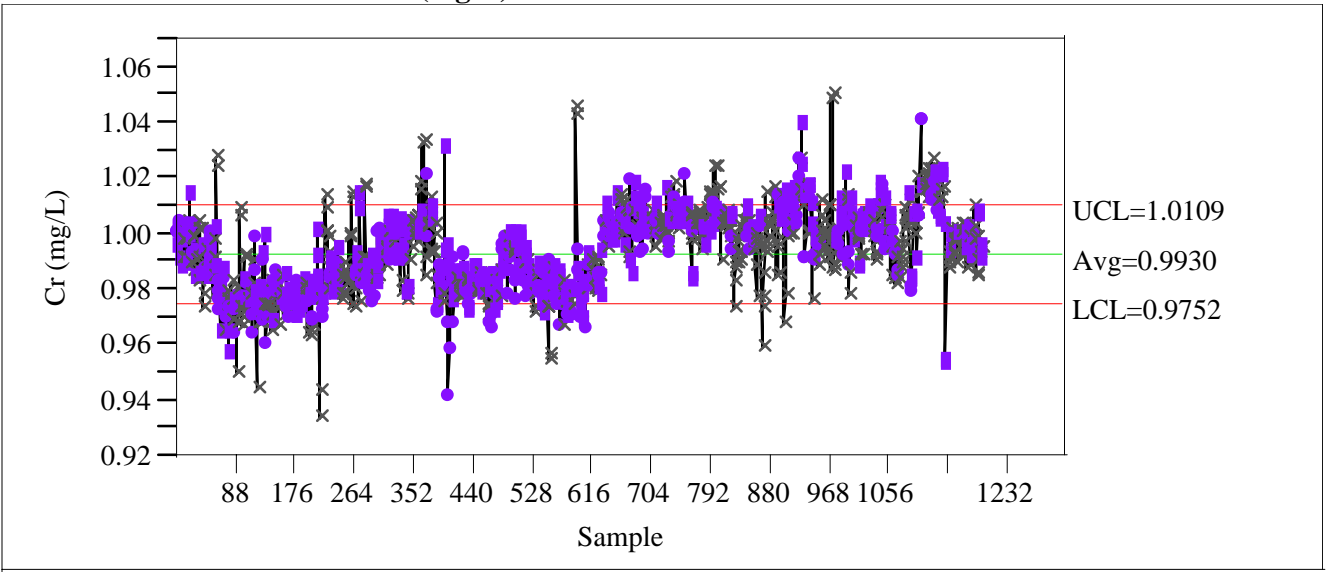


WSRC-TR-2004-00576

Revision 0

Exhibit A21. Fusion Preparation Standards in Analytical Sequence

Individual Measurement of $\mathrm{Cu}(\mathrm{mg} / \mathrm{L})$

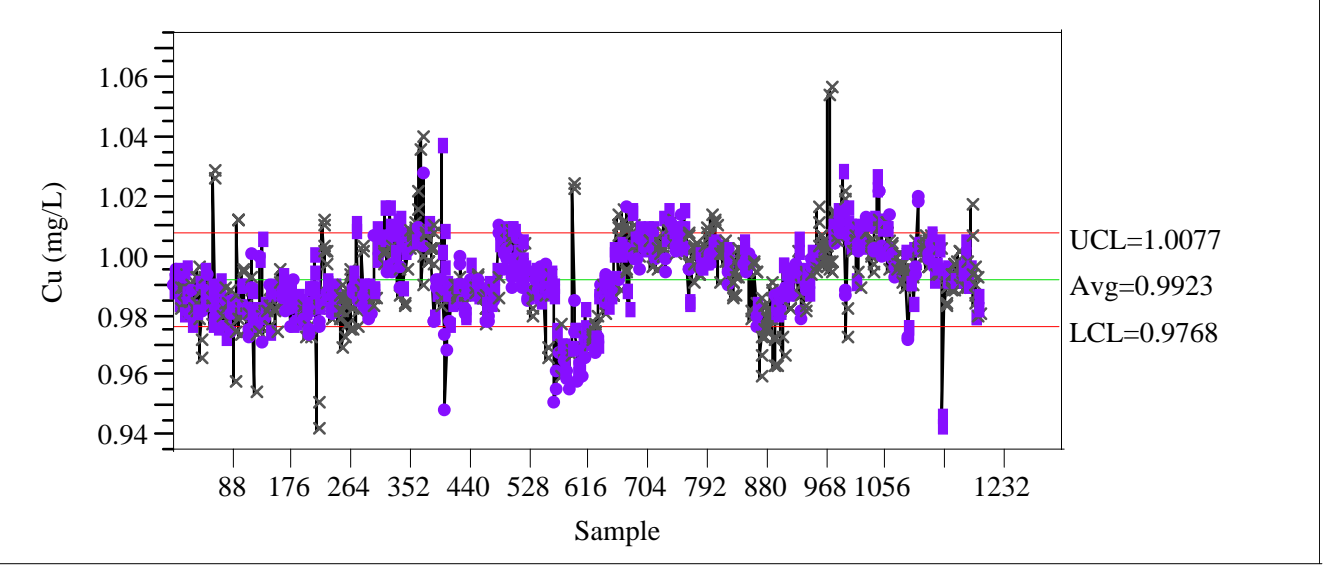

Individual Measurement of Fe (mg/L)

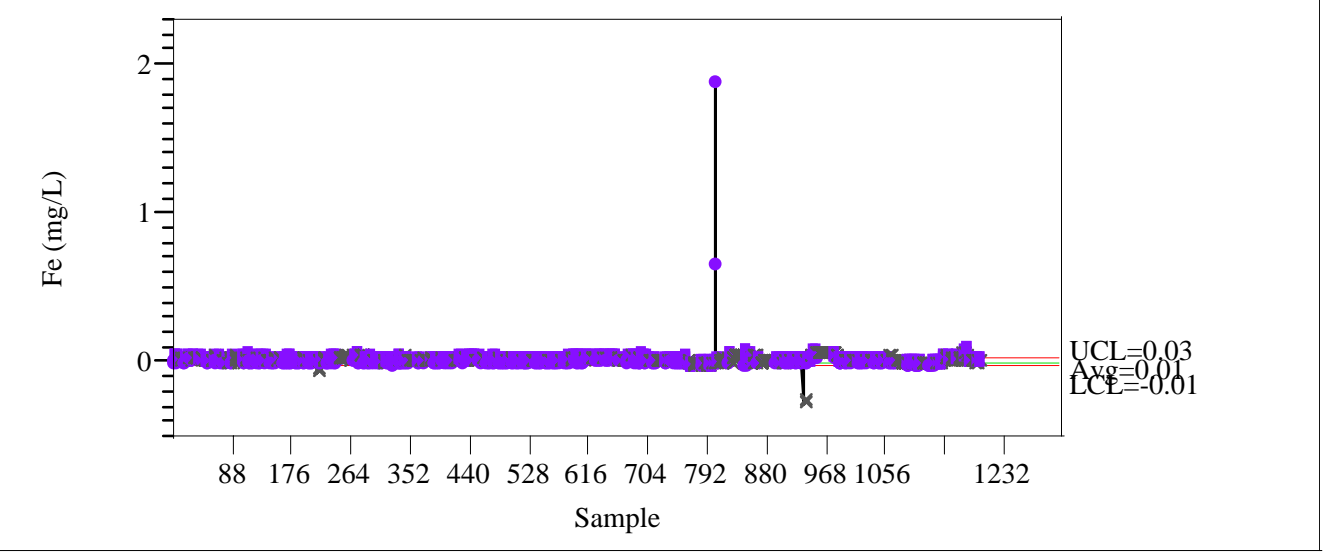

Individual Measurement of K (mg/L)

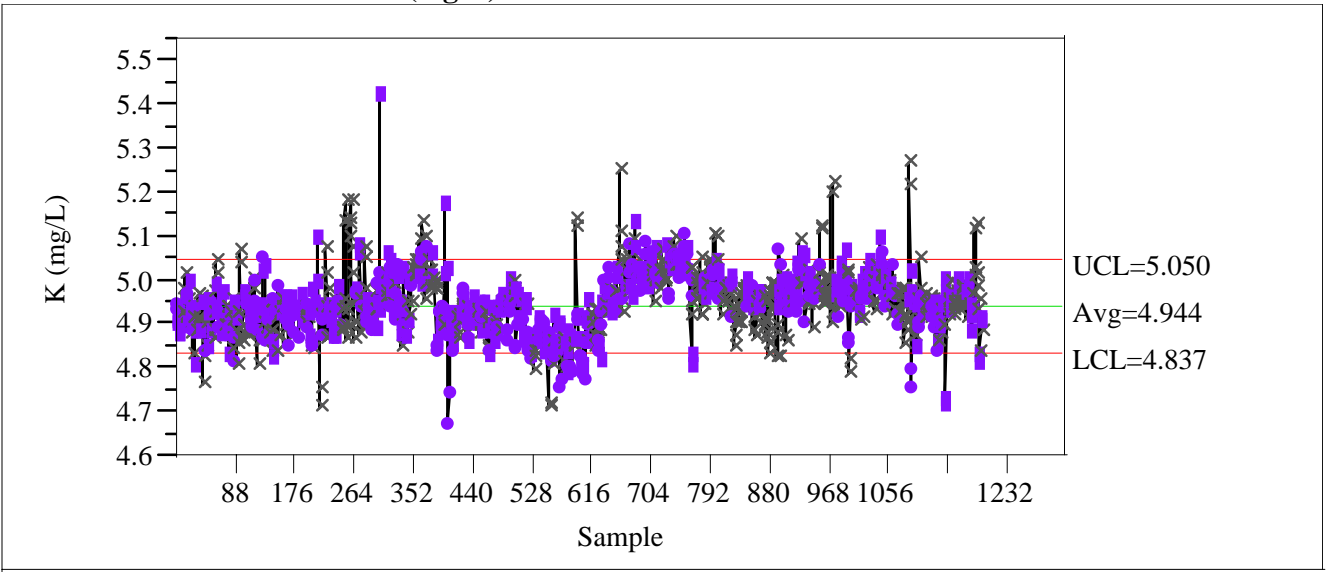


Exhibit A21. Fusion Preparation Standards in Analytical Sequence

Individual Measurement of Li (mg/L)

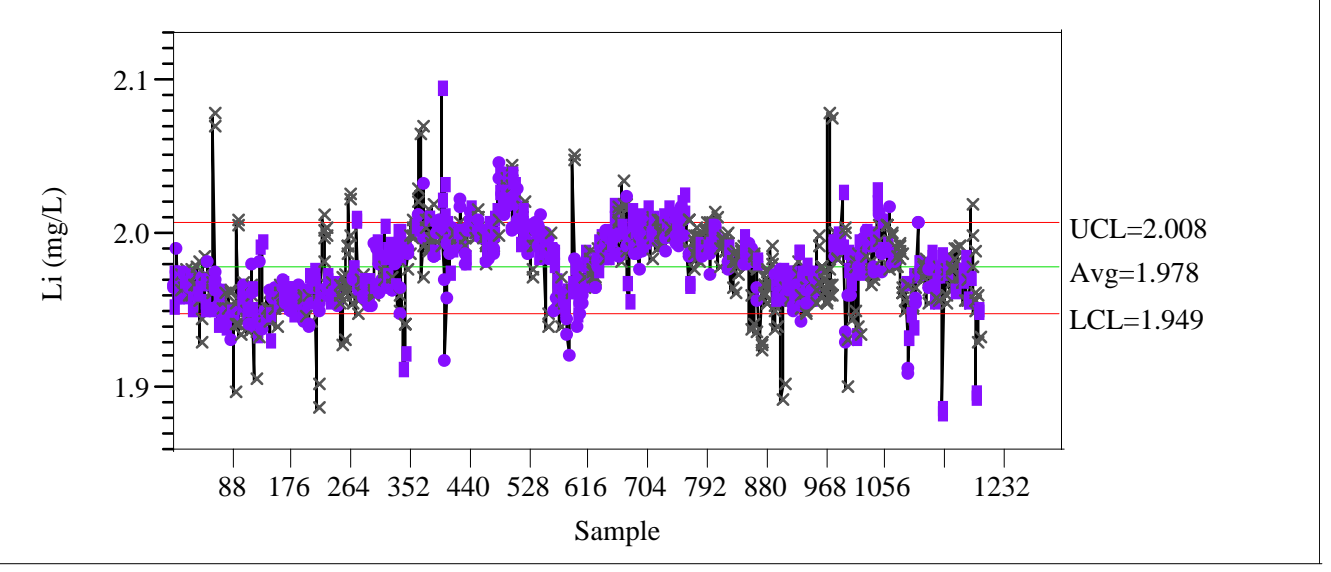

Individual Measurement of Mg (mg/L)

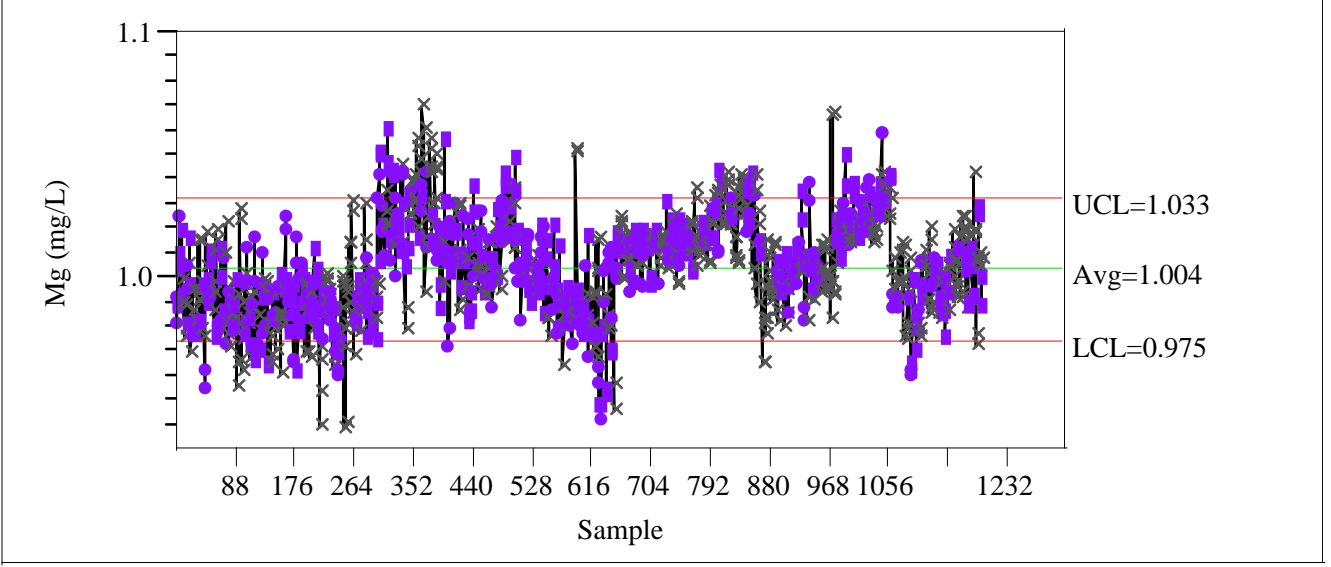

Individual Measurement of Mn (mg/L)

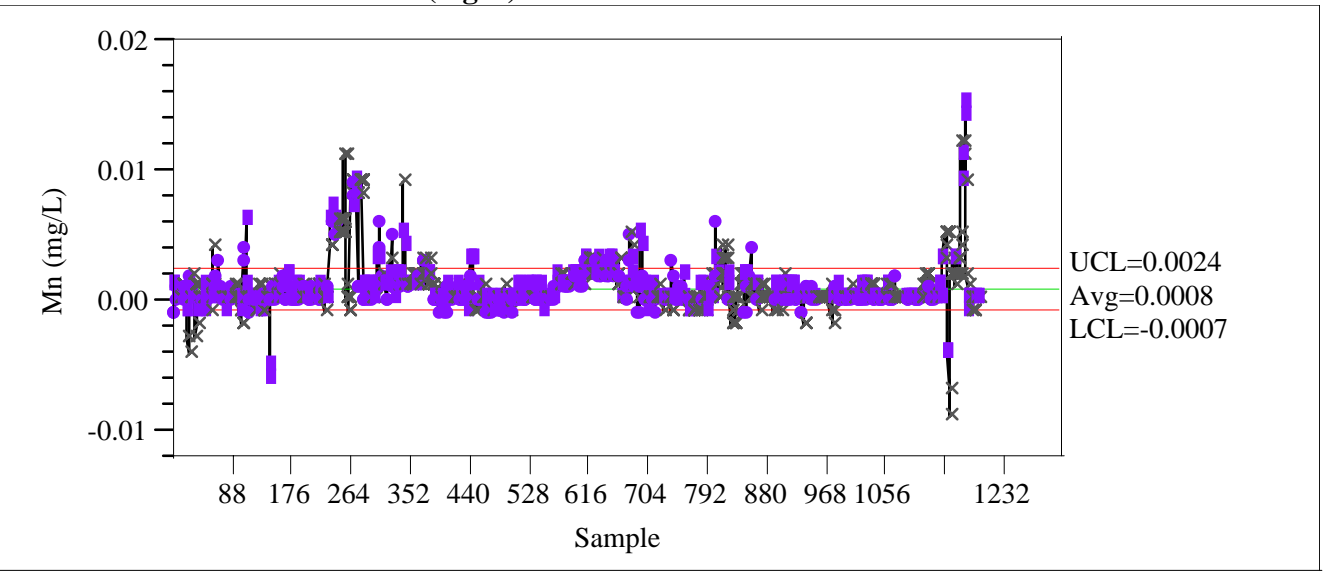


WSRC-TR-2004-00576

Revision 0

Exhibit A21. Fusion Preparation Standards in Analytical Sequence

Individual Measurement of Ni (mg/L)

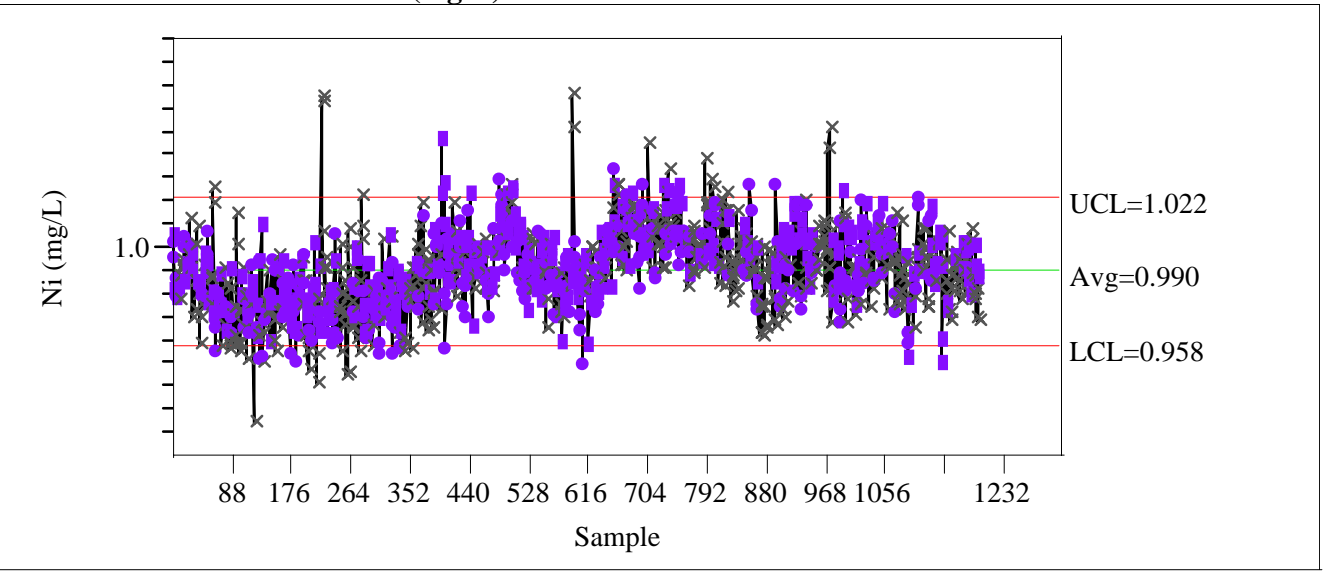

Individual Measurement of Si (mg/L)

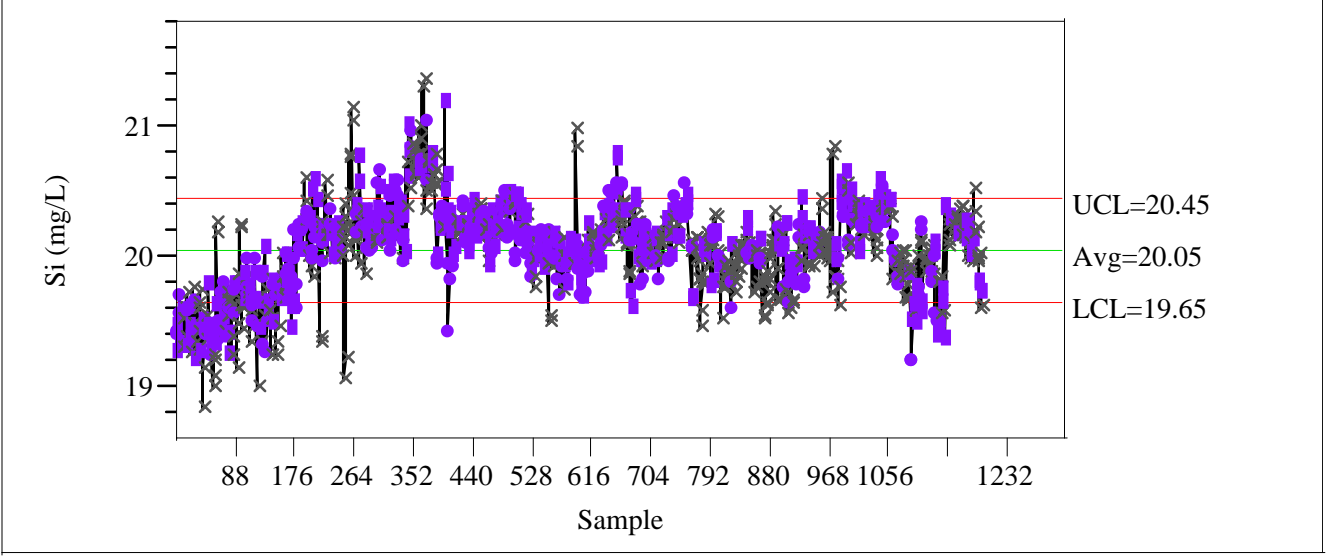

Individual Measurement of $\mathrm{Ti}$ (mg/L)

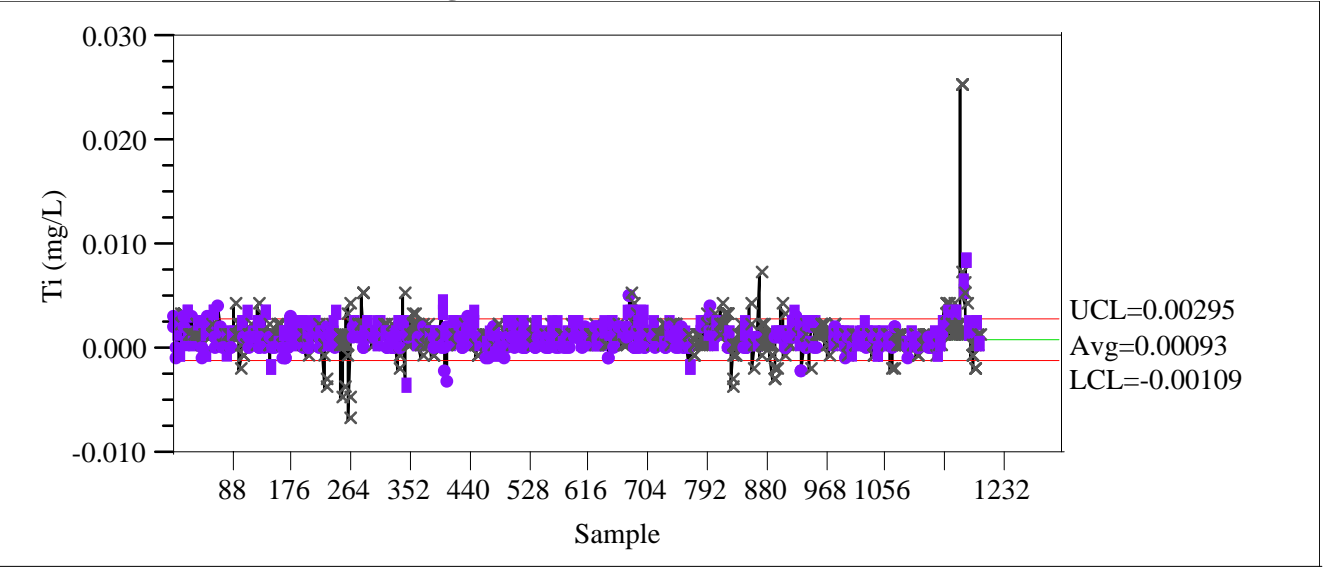


WSRC-TR-2004-00576

Revision 0

\section{Exhibit A21. Fusion Preparation Standards in Analytical Sequence}

\section{STCd=SM54}

Control Chart

Individual Measurement of AL (mg/L)

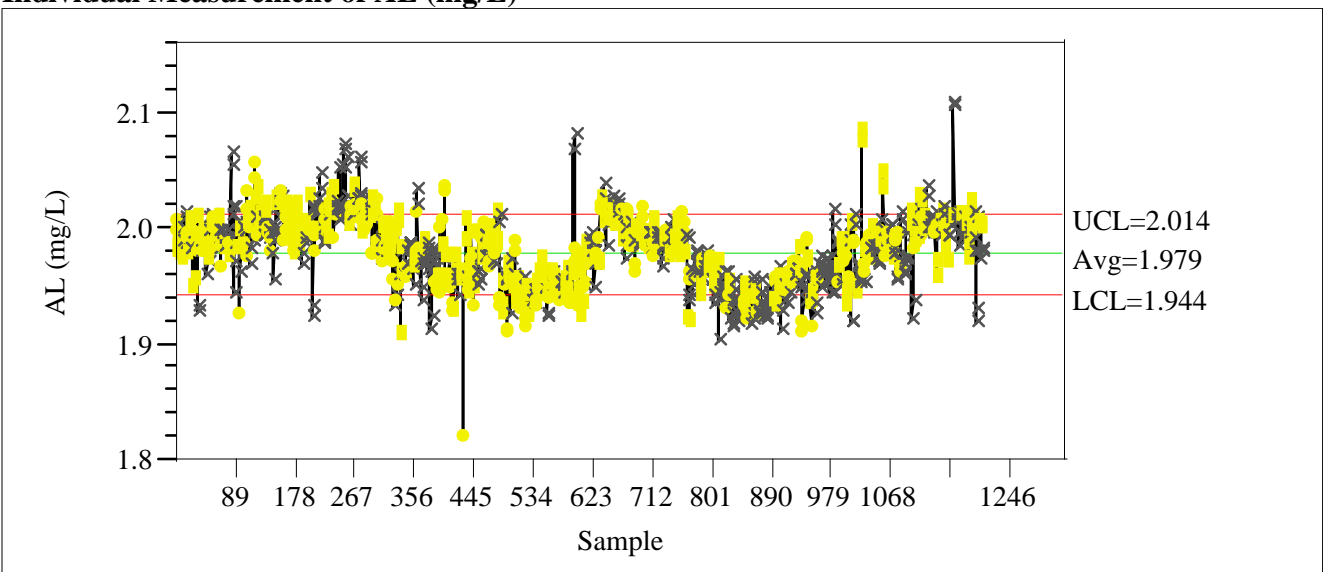

Individual Measurement of B (mg/L)

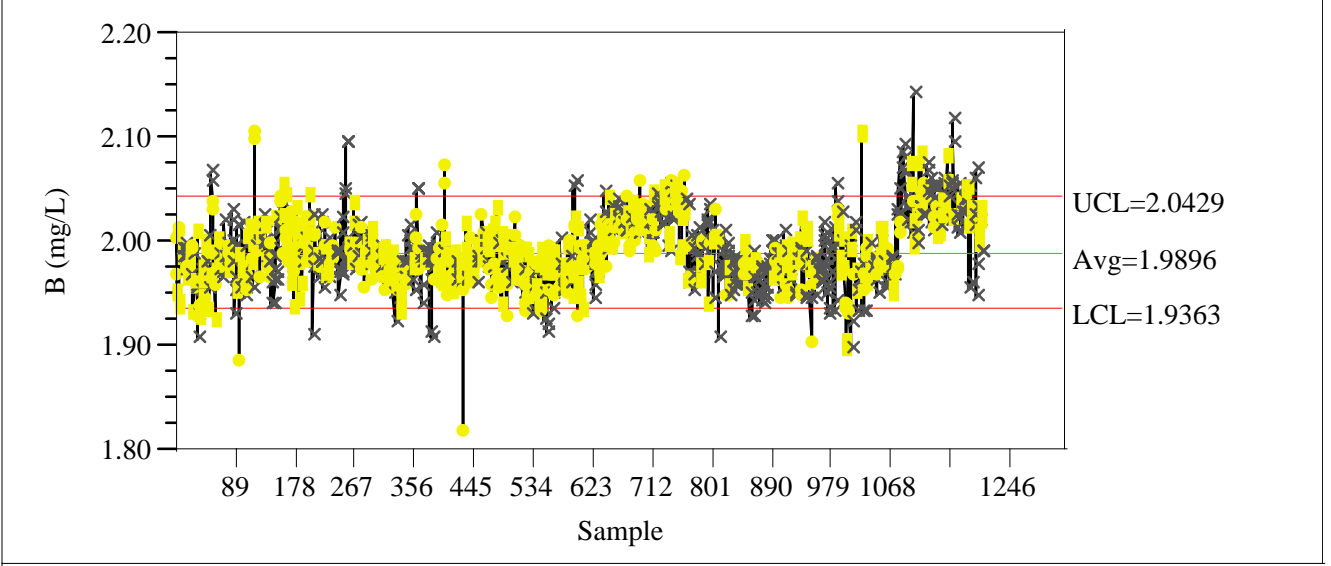

Individual Measurement of Ca (mg/L)

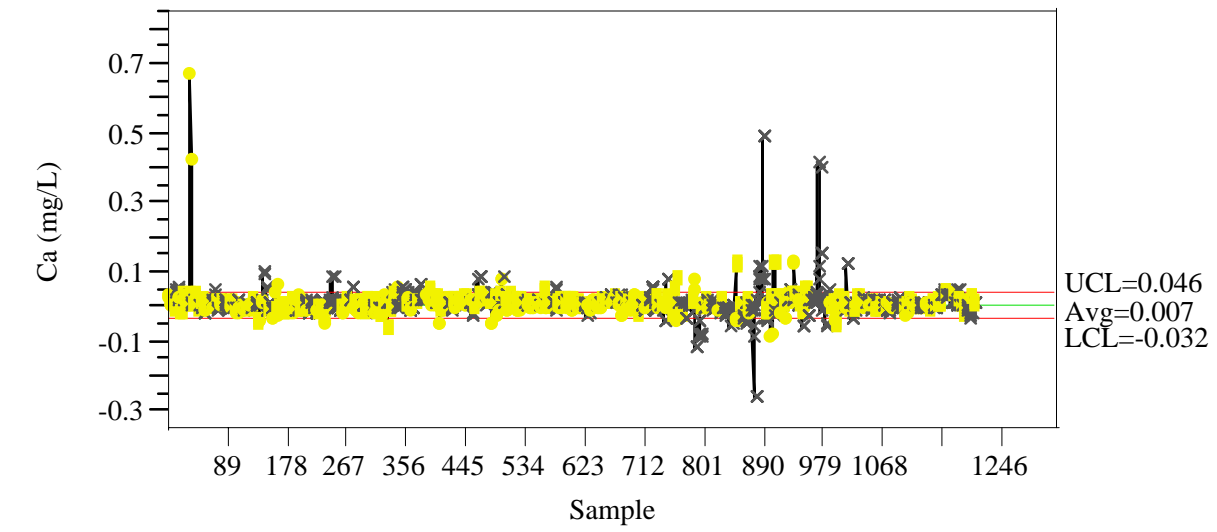


WSRC-TR-2004-00576

Revision 0

\section{Exhibit A21. Fusion Preparation Standards in Analytical Sequence}

Individual Measurement of $\mathrm{Cr}$ (mg/L)

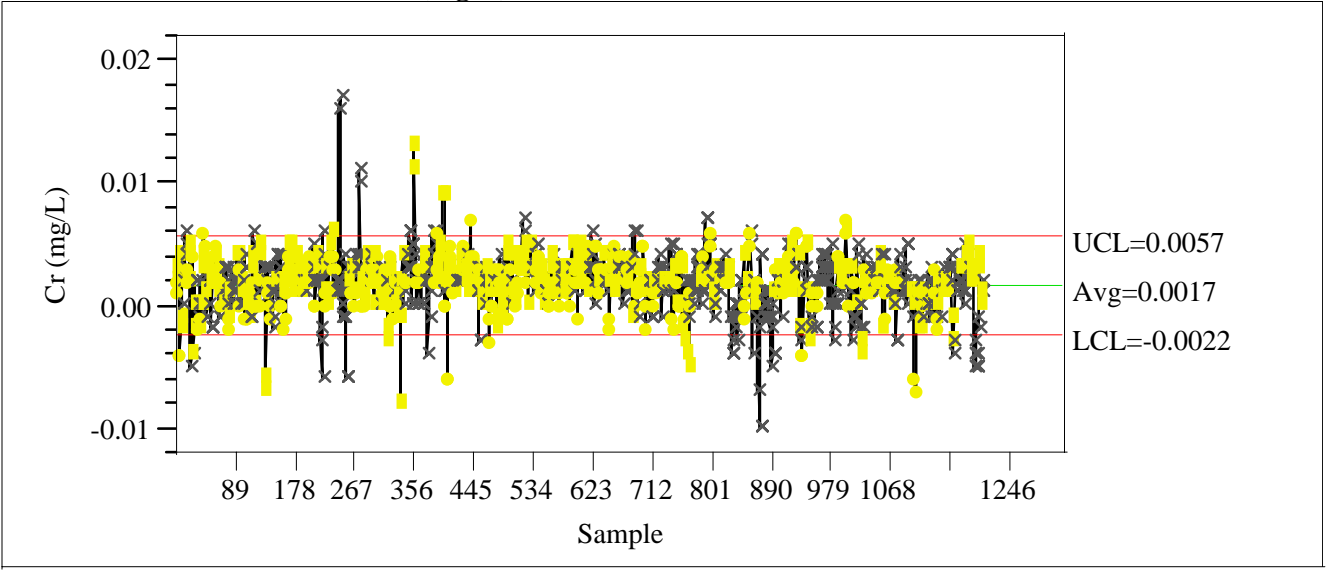

Individual Measurement of $\mathrm{Cu}$ (mg/L)

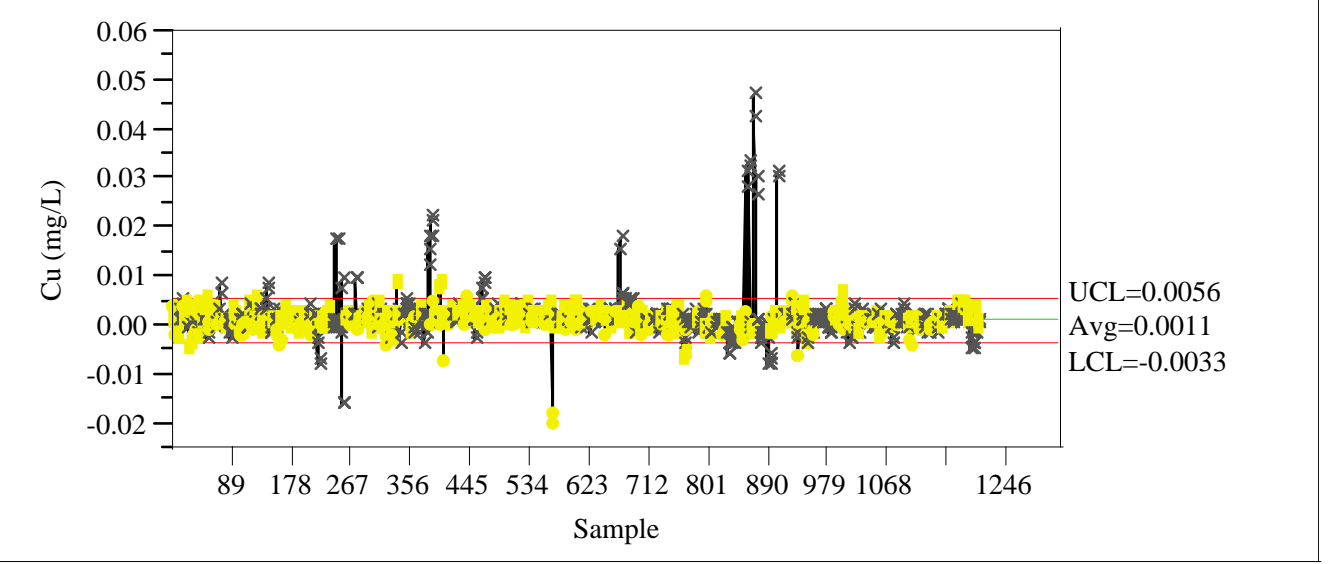

Individual Measurement of Fe (mg/L)

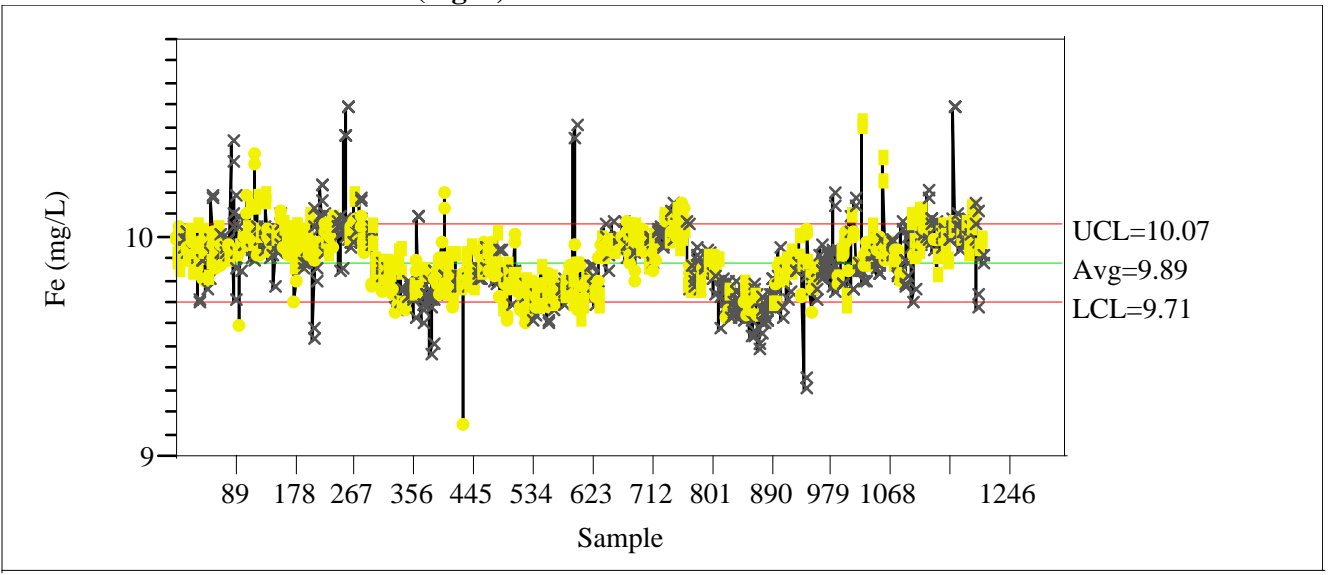


WSRC-TR-2004-00576

Revision 0

\section{Exhibit A21. Fusion Preparation Standards in Analytical Sequence}

Individual Measurement of K (mg/L)

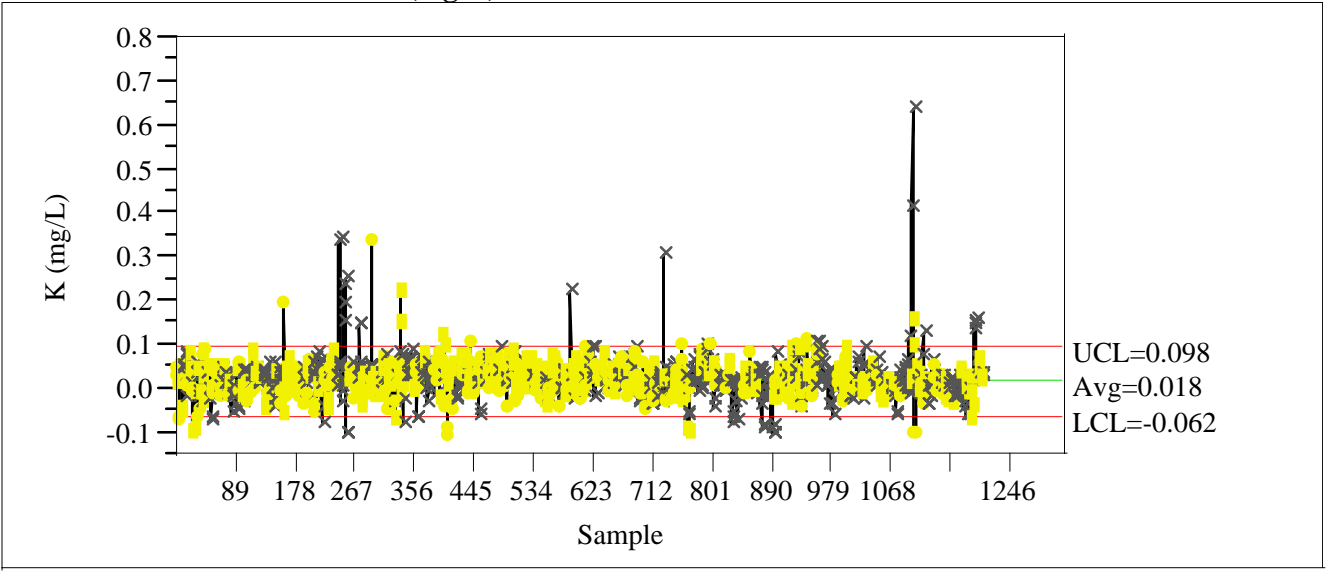

Individual Measurement of $\mathrm{Li}(\mathrm{mg} / \mathrm{L})$

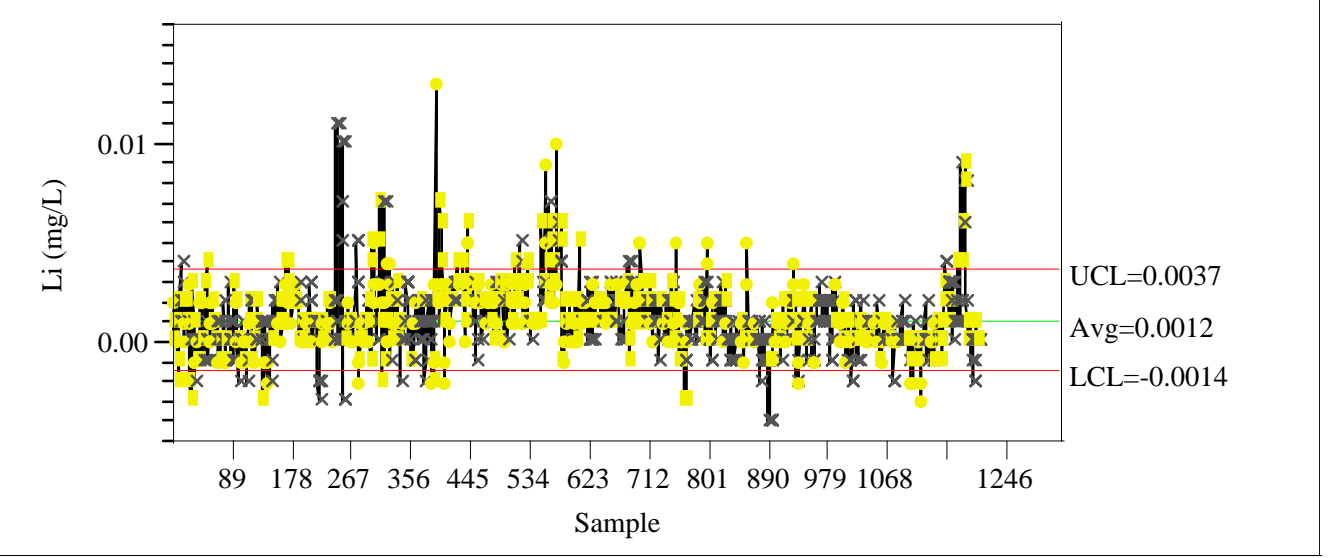

Individual Measurement of $\mathrm{Mg}(\mathrm{mg} / \mathrm{L})$

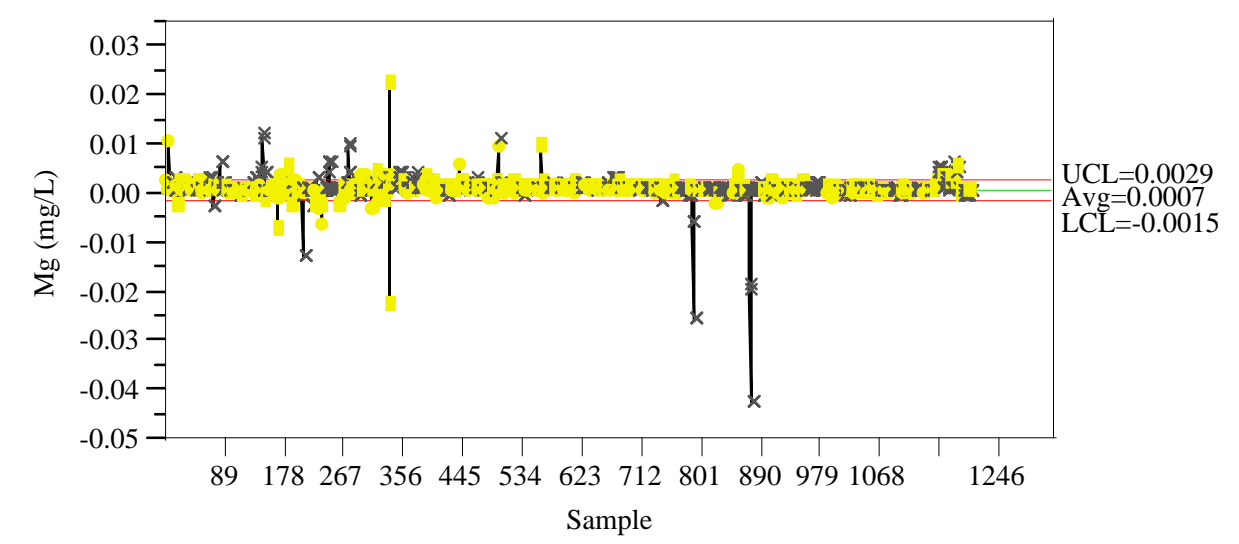


WSRC-TR-2004-00576

Revision 0

\section{Exhibit A21. Fusion Preparation Standards in Analytical Sequence}

Individual Measurement of Mn (mg/L)

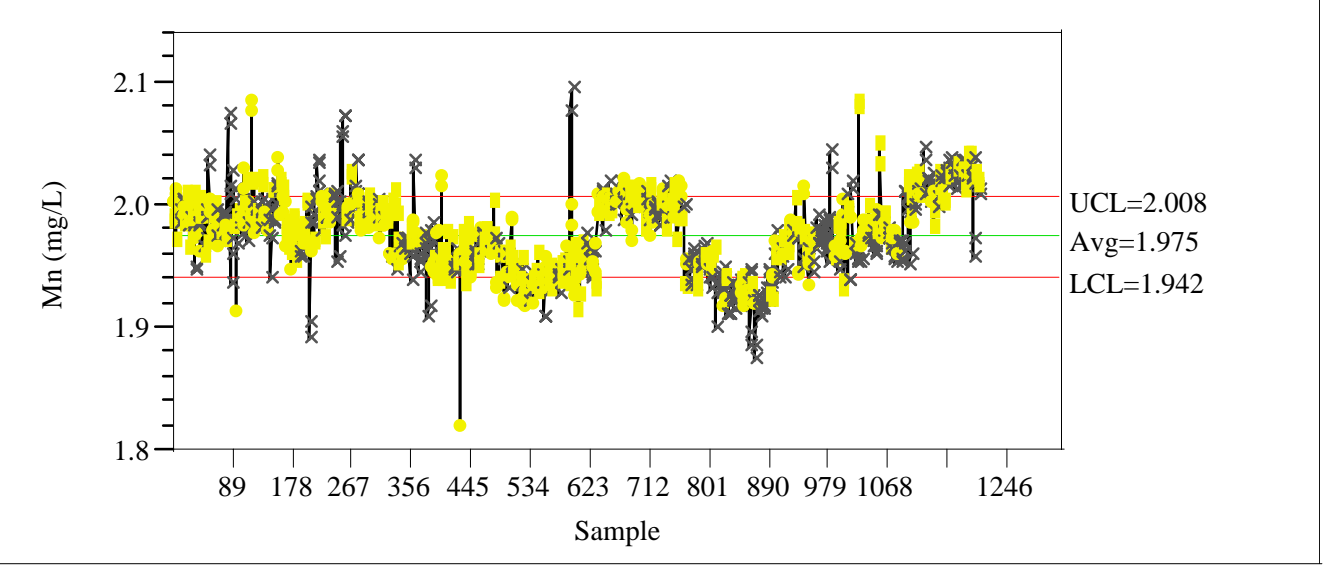

Individual Measurement of $\mathrm{Ni}(\mathrm{mg} / \mathrm{L})$

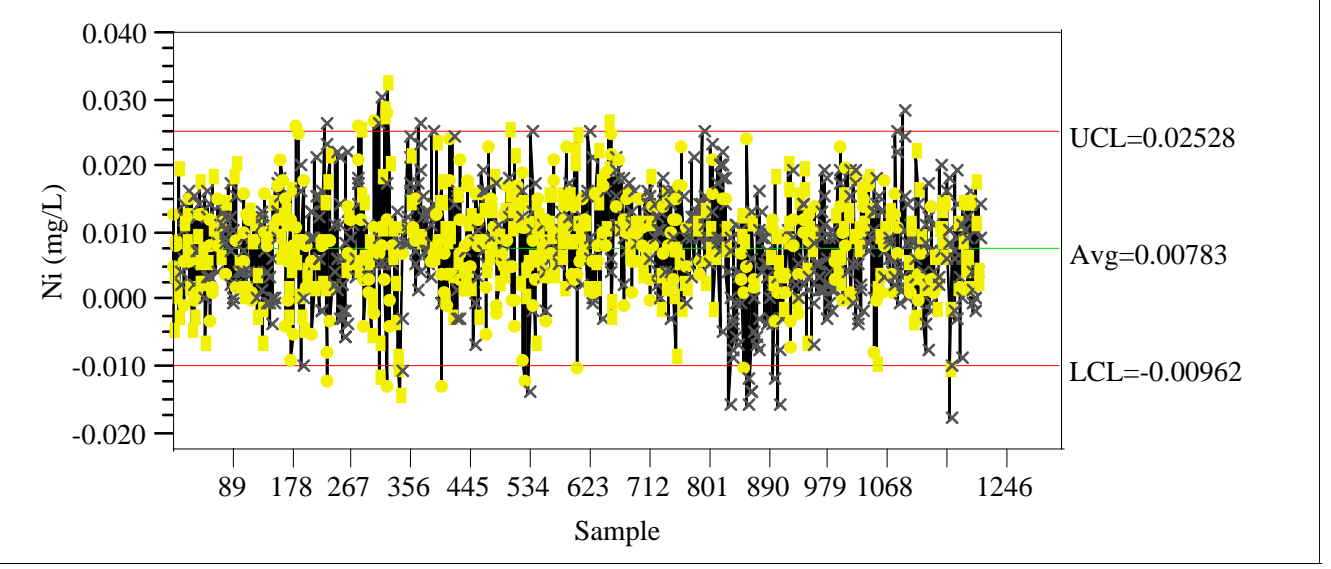

Individual Measurement of Si (mg/L)

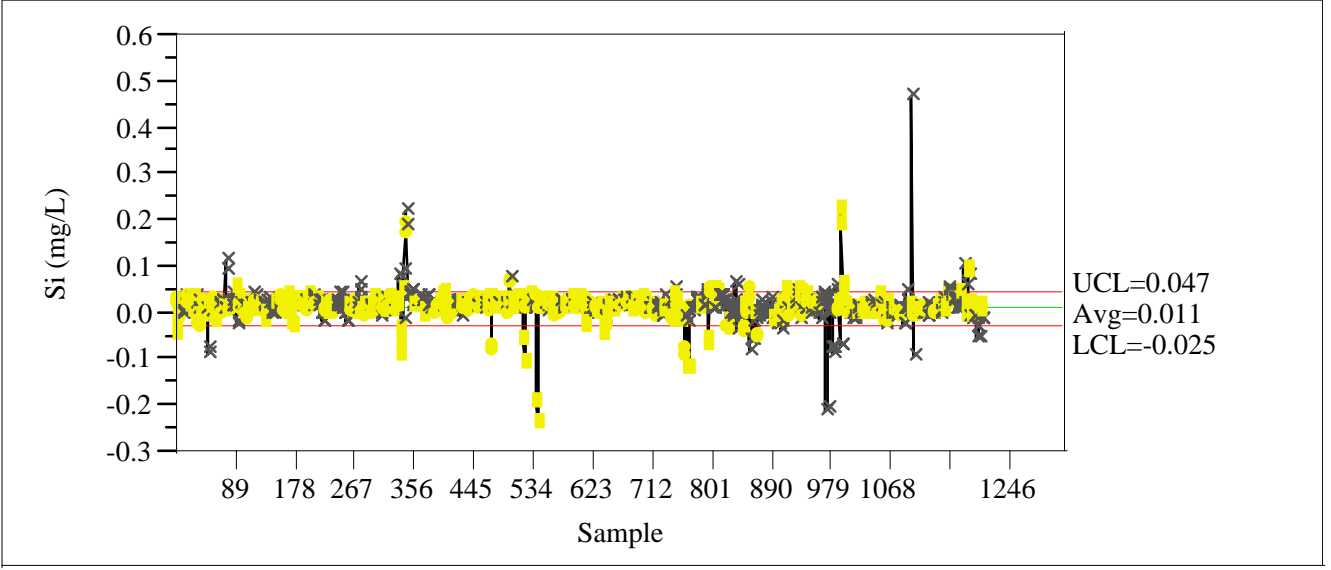


WSRC-TR-2004-00576

Revision 0

\section{Exhibit A21. Fusion Preparation Standards in Analytical Sequence}

Individual Measurement of $\mathrm{Ti}(\mathrm{mg} / \mathrm{L})$

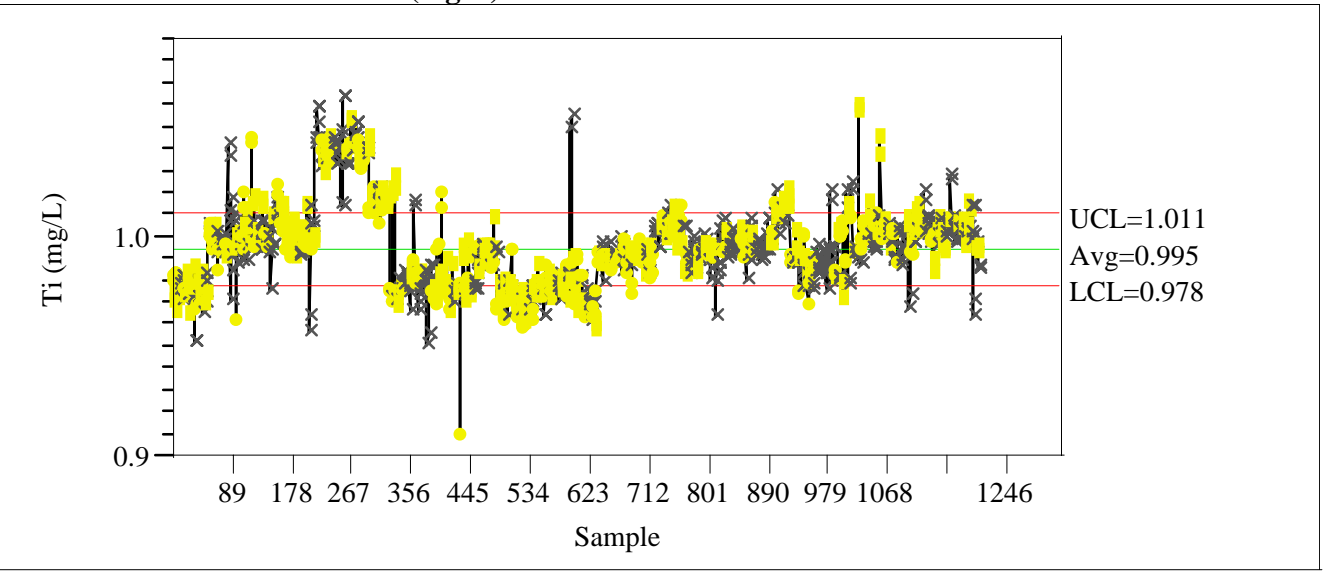

STCd=SM55

Control Chart

Individual Measurement of AL (mg/L)

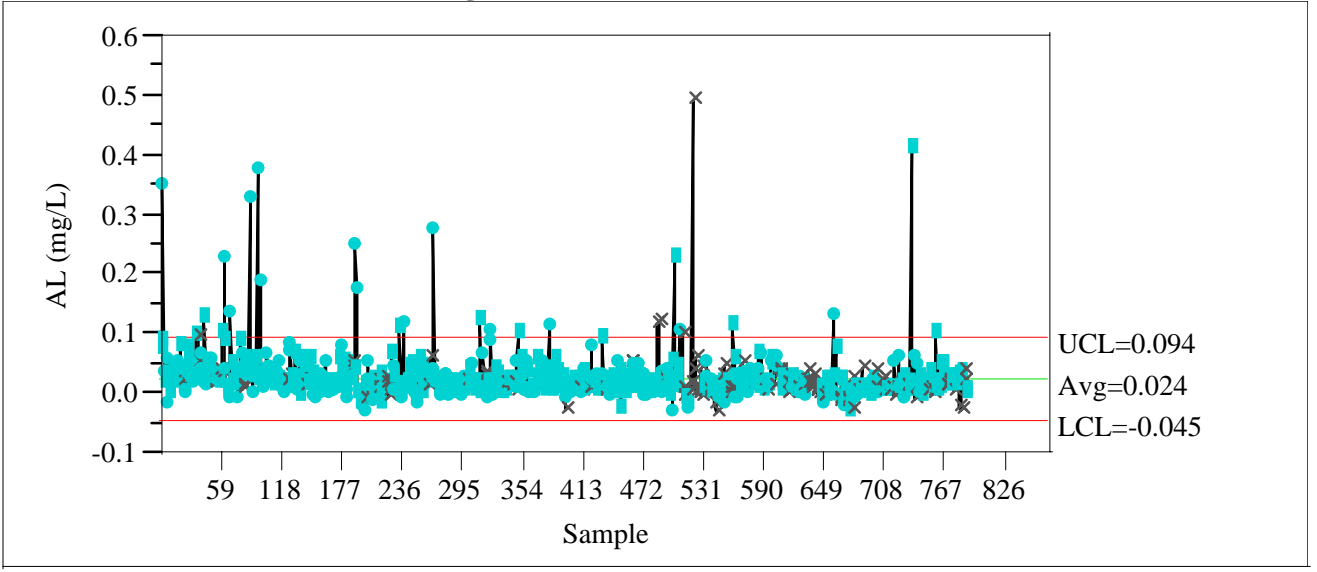

Individual Measurement of B (mg/L)

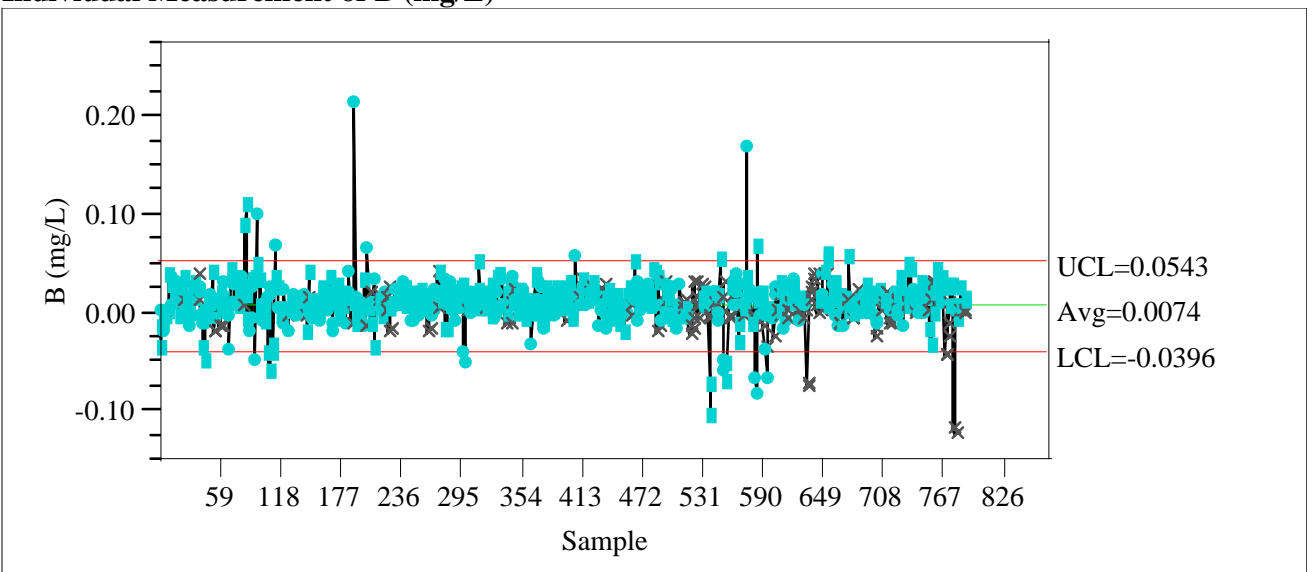


WSRC-TR-2004-00576

Revision 0

\section{Exhibit A21. Fusion Preparation Standards in Analytical Sequence}

Individual Measurement of Ca (mg/L)

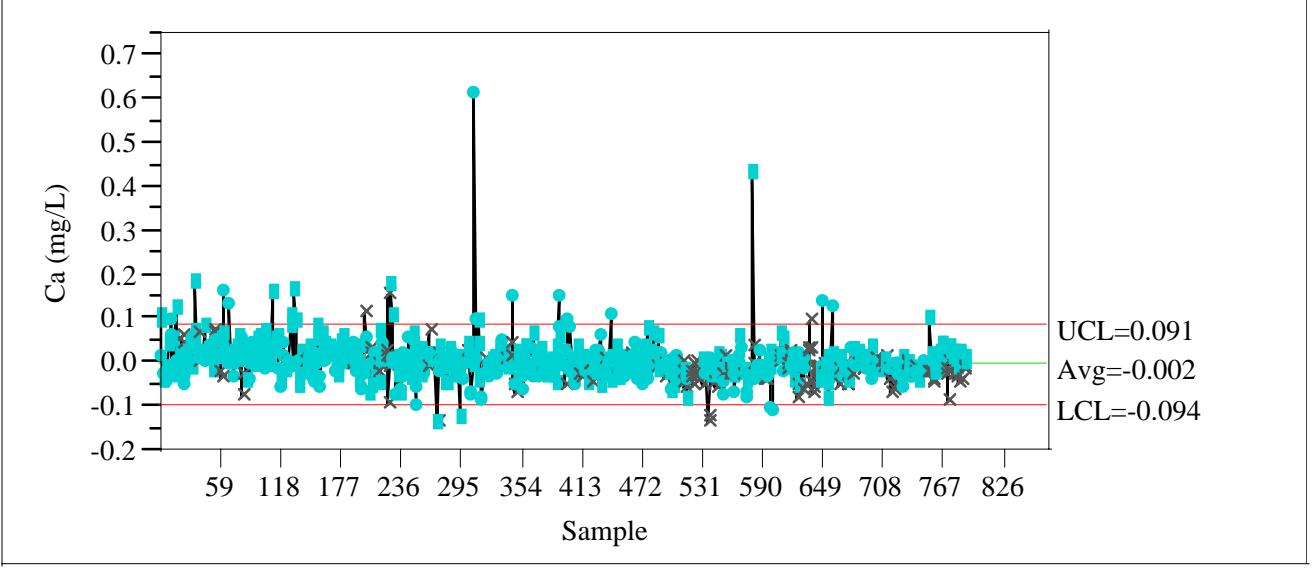

Individual Measurement of $\mathrm{Cr}$ (mg/L)

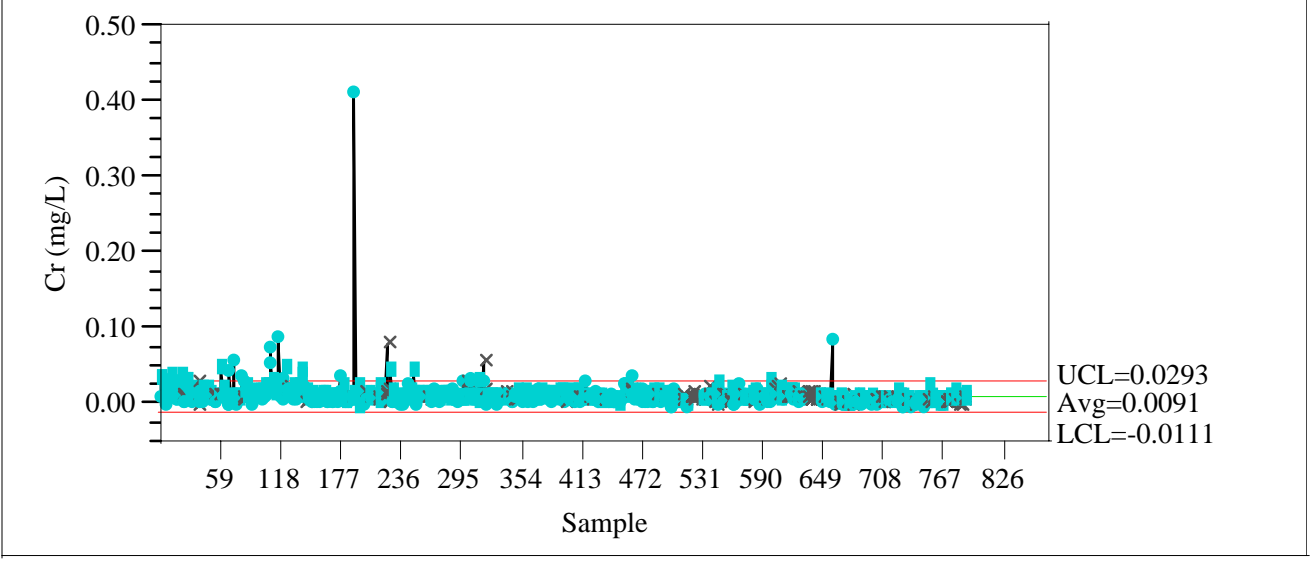

Individual Measurement of $\mathrm{Cu}(\mathrm{mg} / \mathrm{L})$

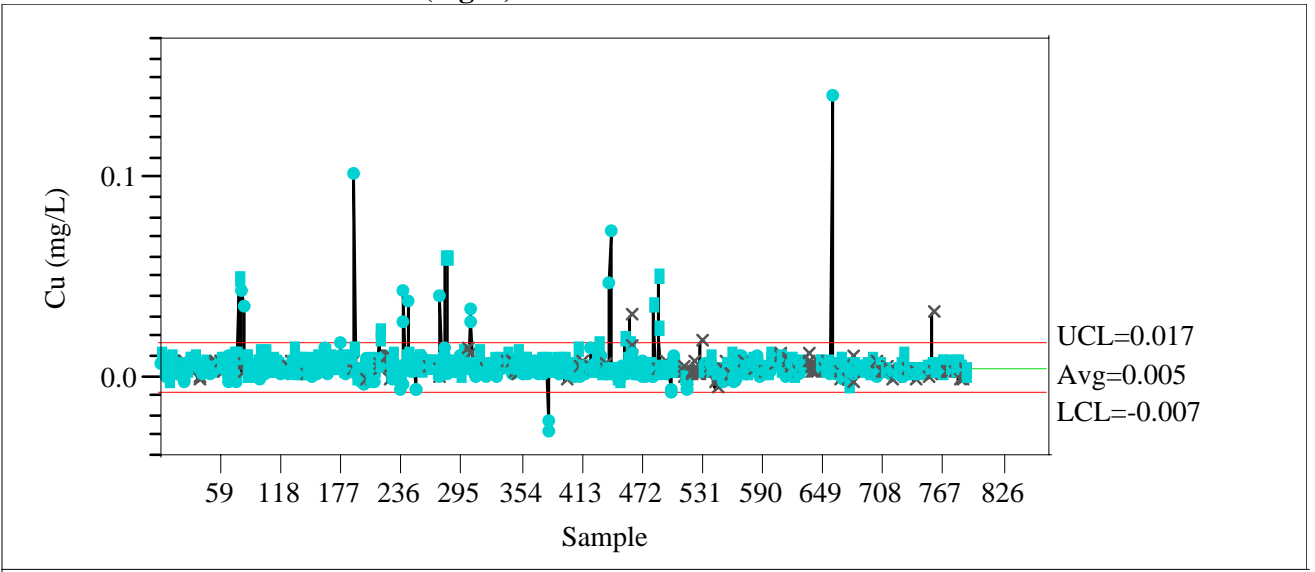


WSRC-TR-2004-00576

Revision 0

\section{Exhibit A21. Fusion Preparation Standards in Analytical Sequence}

Individual Measurement of Fe (mg/L)

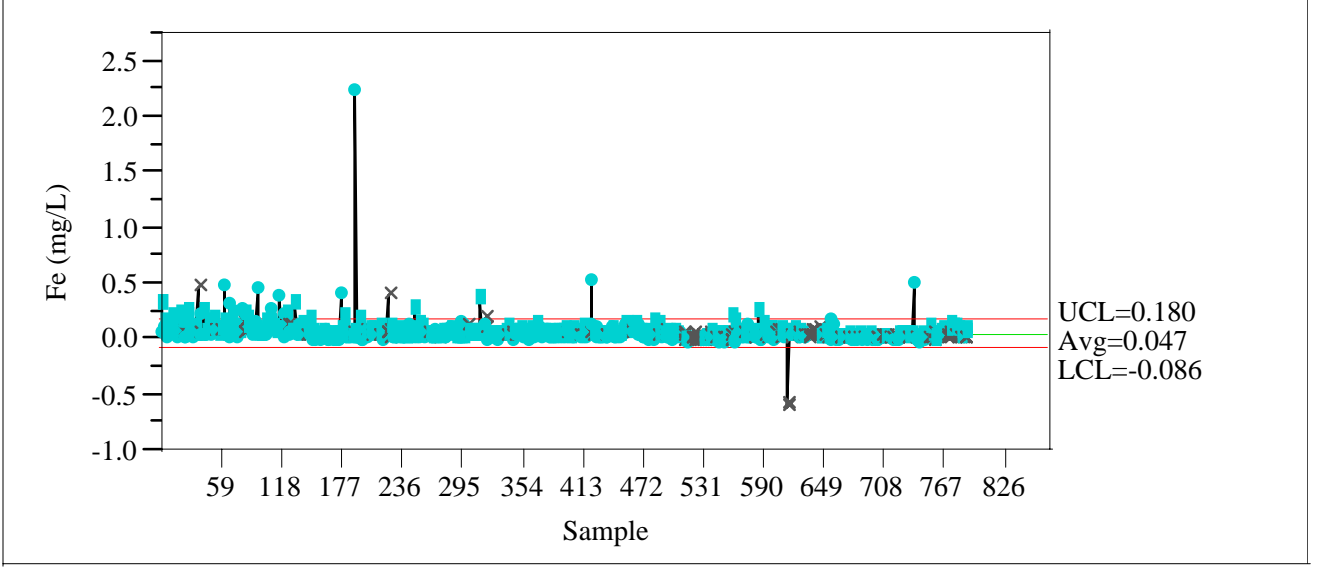

Individual Measurement of K (mg/L)

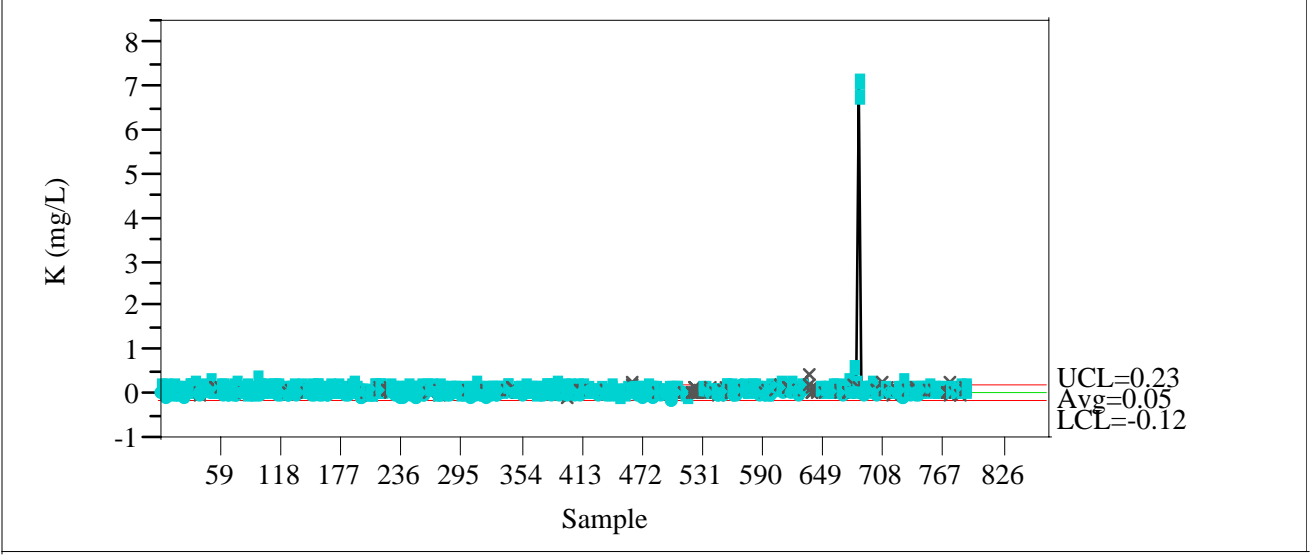

Individual Measurement of $\mathrm{Li}(\mathrm{mg} / \mathrm{L})$

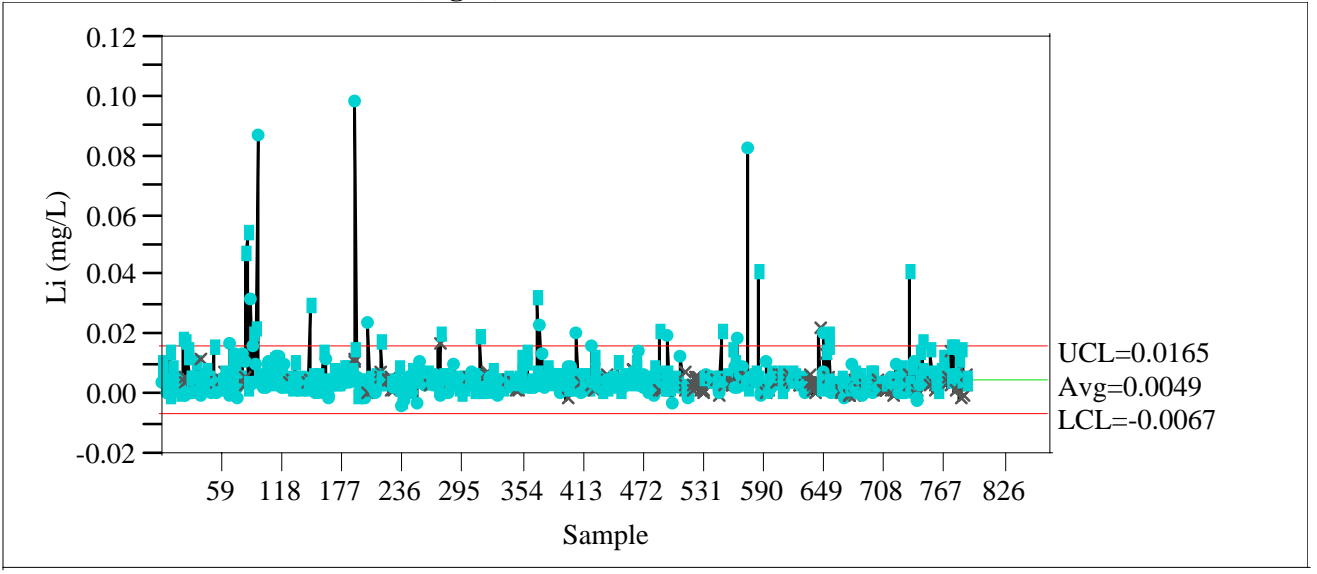


WSRC-TR-2004-00576

Revision 0

\section{Exhibit A21. Fusion Preparation Standards in Analytical Sequence}

Individual Measurement of $\mathrm{Mg}(\mathrm{mg} / \mathrm{L})$

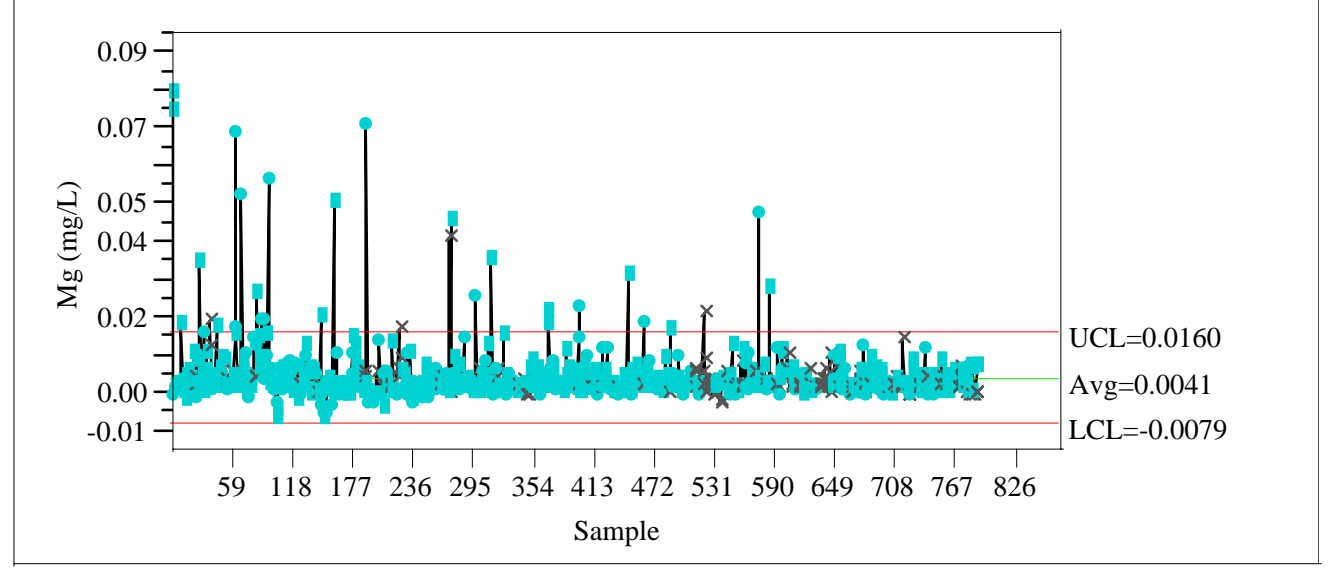

Individual Measurement of Mn (mg/L)

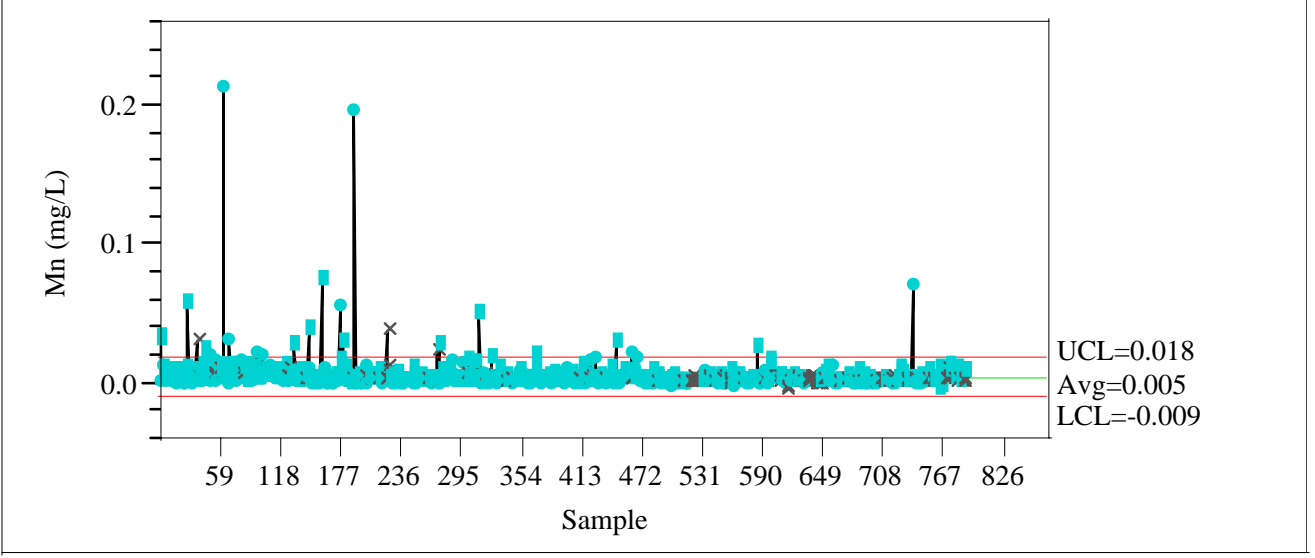

Individual Measurement of $\mathrm{Ni}(\mathrm{mg} / \mathrm{L})$

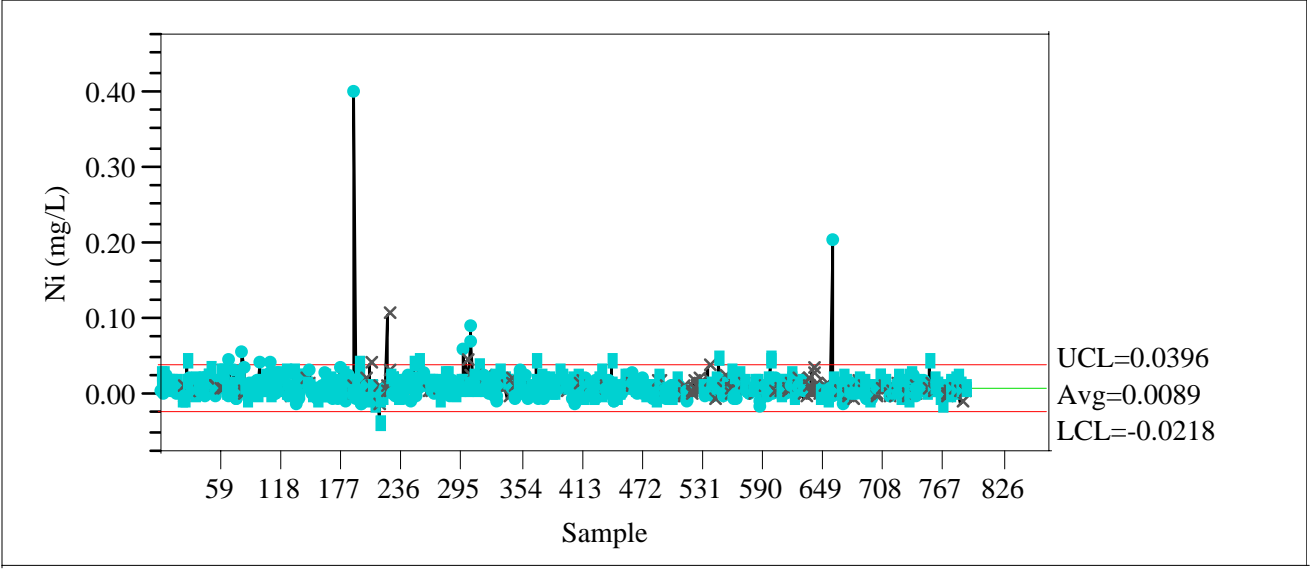


WSRC-TR-2004-00576

Revision 0

\section{Exhibit A21. Fusion Preparation Standards in Analytical Sequence}

Individual Measurement of Si (mg/L)

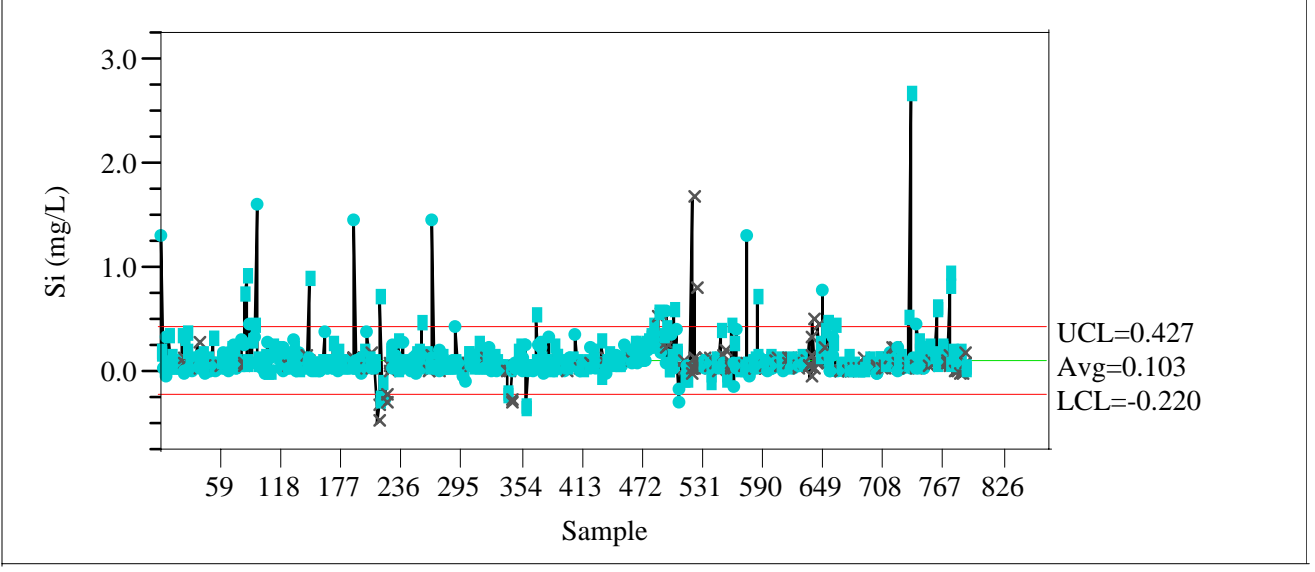

Individual Measurement of Ti (mg/L)

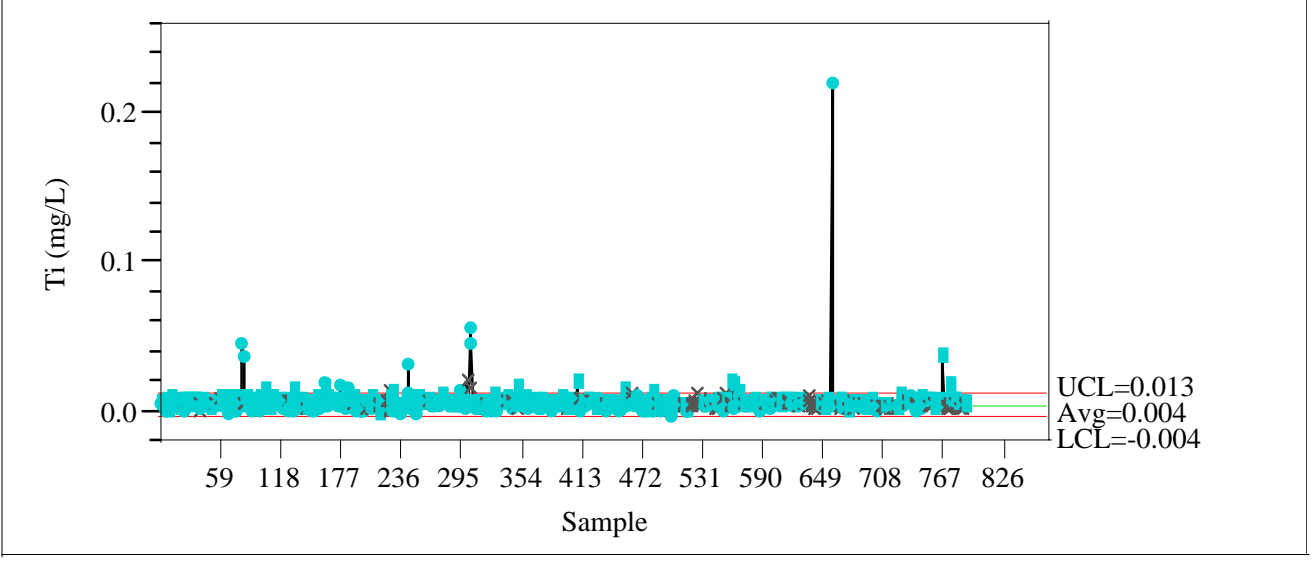


WSRC-TR-2004-00576

Revision 0

\section{Exhibit A22. Histograms and Descriptive Statistics for Fusion Measurements of Calibration and Bench Standards}

STCd=SM51

Distributions

AL (mg/L)

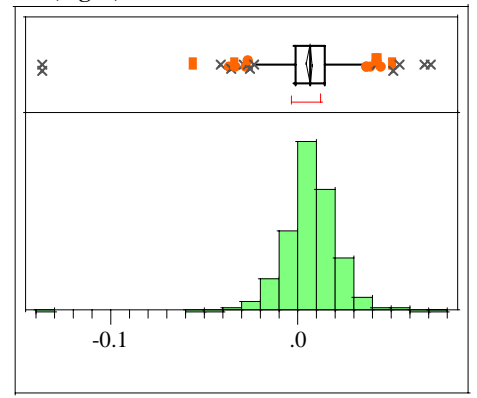

Quantiles

$100.0 \%$ maximum 0.0700

$99.5 \%$

$97.5 \%$

$90.0 \%$

$75.0 \%$

$50.0 \%$

$25.0 \%$

$10.0 \%$

$2.5 \%$

$0.5 \%$

$0.0 \%$

Moments

Mean

Std Dev

Std Err Mean

upper 95\% Mean

lower 95\% Mean

$\mathrm{N}$

B (mg/L)

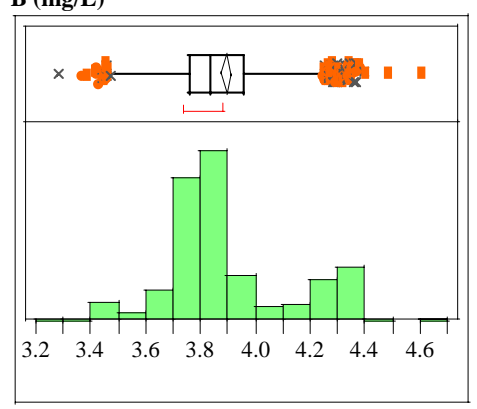

Quantiles

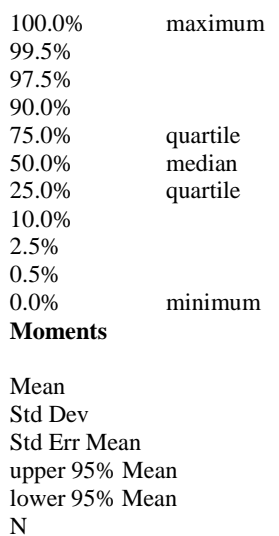

$\mathrm{N}$
Ca (mg/L)

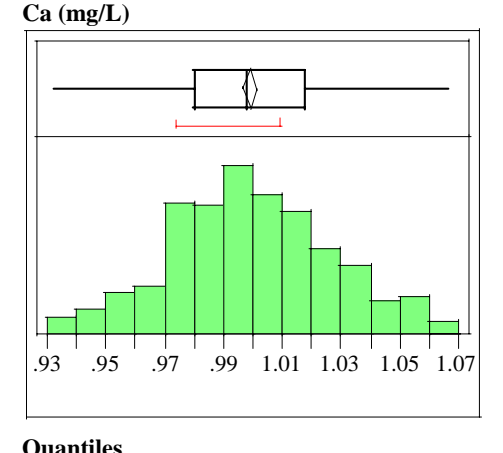

Quantiles

$100.0 \%$ maximum 1.0660

$99.5 \% \quad 1.0650$

$97.5 \%$

$90.0 \%$

$75.0 \%$

$50.0 \%$

$25.0 \%$

$10.0 \%$

$2.5 \%$

$0.5 \%$

$0.0 \% \quad$ minimum

Moments

Mean

Std Dev

Std Err Mean

upper 95\% Mean

lower 95\% Mean

$\mathrm{N}$

Cr (mg/L)

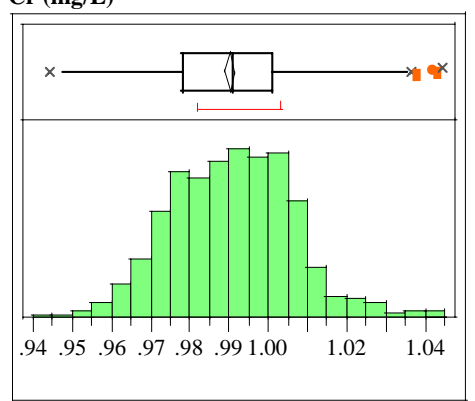

Quantiles

$100.0 \%$

$99.5 \%$

$97.5 \%$

$90.0 \%$

$75.0 \%$

$50.0 \%$

$25.0 \%$

$10.0 \%$

$2.5 \%$

$0.5 \%$

$0.0 \%$

Moments

minimum

Mean

Std Dev

Std Err Mean

upper 95\% Mean

lower 95\% Mean

$\mathrm{N}$

1.0012568 0.9967432

580

median

quartile

0.9710
0.9605

0.9507

0.9440
Cu (mg/L)

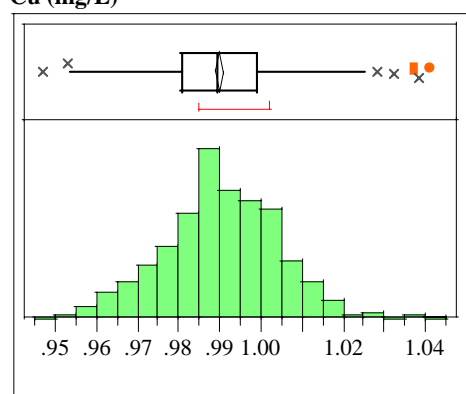

Quantiles

$100.0 \%$

$99.5 \%$

$97.5 \%$

$90.0 \%$

$75.0 \%$

$50.0 \%$

$25.0 \%$

$10.0 \%$

$2.5 \%$

$0.5 \%$

$0.0 \%$

Moments

maximum

1.0410

1.0371

1.0170

1.0170
1.0080

0.9990

0.9895

0.9810

0.9720

0.9605

0.9539

0.9470

Mean

Std Dev

Std Err Mean

upper 95\% Mean

lower 95\% Mean

minimum

0.9898672

0.0141485

0.0005875

0.9910211

0.9887134

Fe (mg/L)

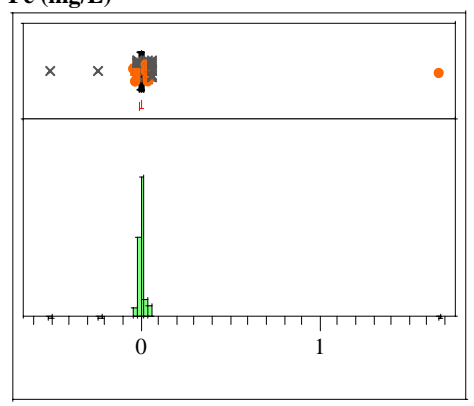

Quantiles

$100.0 \%$ maximum 1.673

$99.5 \% \quad 0.056$

$\begin{array}{ll}99.5 \% & 0.056 \\ 97.5 \% & 0.046\end{array}$

$90.0 \%$

$75.0 \%$ quartile

$50.0 \%$ median

$25.0 \%$ quartile

$10.0 \%$

$2.5 \%$

$0.5 \%$

$0.0 \%$

Moments

minimum

0.026

0.010

Mean

Std Dev

Std Err Mean

upper 95\% Mean

lower 95\% Mean

$\mathrm{N}$ 
WSRC-TR-2004-00576

Revision 0

\section{Exhibit A22. Histograms and Descriptive Statistics for Fusion Measurements of Calibration and Bench Standards}

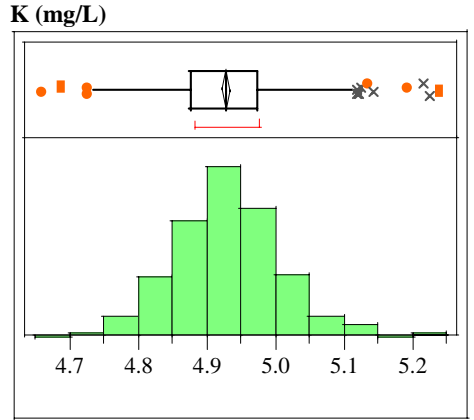

Quantiles

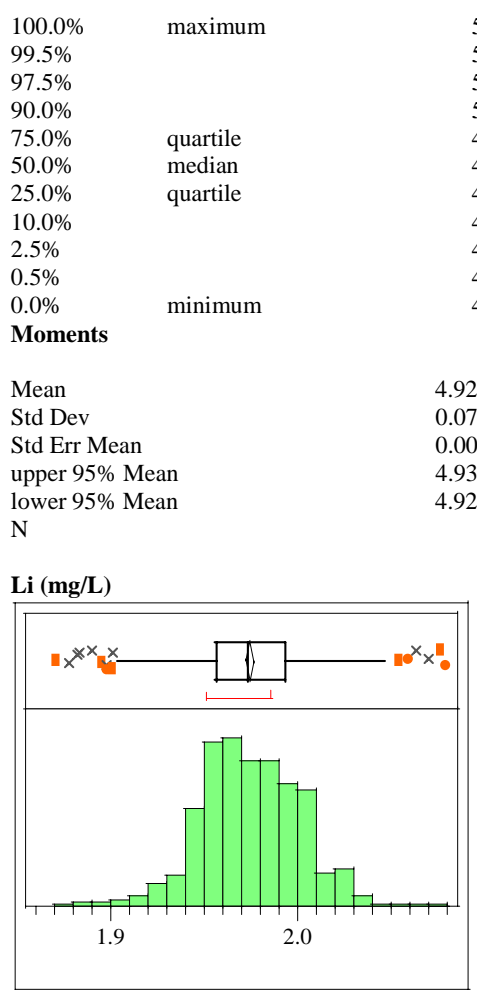

Quantiles

$100.0 \%$

$99.5 \%$

$97.5 \%$

$90.0 \%$

$75.0 \%$

$50.0 \%$

$25.0 \%$

$10.0 \%$

$2.5 \%$

$0.5 \%$

$0.0 \%$

minimum

Mean

Std Dev

Std Err Mean

upper $95 \%$ Mean

lower 95\% Mean

$\mathrm{N}$
$\operatorname{Mg}(\mathbf{m g} / \mathrm{L})$

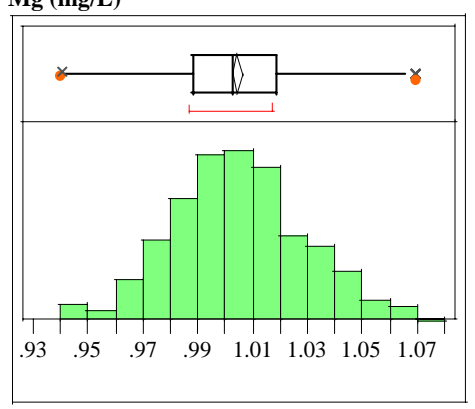

Quantiles

$\begin{array}{ll}100.0 \% & \text { maximum } \\ 99.5 \% & \\ 97.5 \% & \\ 90.0 \% & \\ 75.0 \% & \text { quartile } \\ 50.0 \% & \text { median } \\ 25.0 \% & \text { quartile } \\ 10.0 \% & \\ 2.5 \% & \\ 0.5 \% & \\ 0.0 \% & \text { minimum } \\ \text { Moments } & \\ & \\ \text { Mean } & \\ \text { Std Dev } & \\ \text { Std Err Mean } & \\ \text { upper 95\% Mean } \\ \text { lower 95\% Mean } \\ \text { N }\end{array}$

Mn (mg/L)

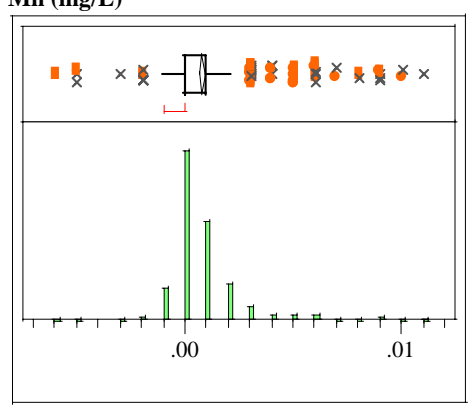

Quantiles

$100.0 \% \quad$ maximum

$99.5 \%$

$97.5 \%$

$90.0 \%$

$75.0 \%$

$50.0 \%$

$25.0 \%$

$10.0 \%$

$2.5 \%$

$0.5 \%$

$0.0 \% \quad$ minimum

Moments

quartile

median

quartile

1.9140
1.8815

1.8700

1.9743517

0.0288364

0.0011974

1.9767034

1.972

580
Mean

Std Dev

Std Err Mean

upper 95\% Mean

$\mathrm{N}$

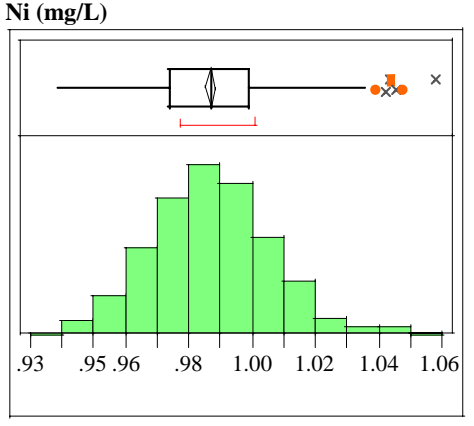

Quantiles

$\begin{array}{lcr}100.0 \% & \text { maximum } & 1.0580 \\ 99.5 \% & & 1.0453 \\ 97.5 \% & & 1.0235 \\ 90.0 \% & & 1.0110 \\ 75.0 \% & \text { quartile } & 0.9990 \\ 50.0 \% & \text { median } & 0.9870 \\ 25.0 \% & \text { quartile } & 0.9740 \\ 10.0 \% & & 0.9620 \\ 2.5 \% & & 0.9510 \\ 0.5 \% & & 0.9419 \\ 0.0 \% & \text { minimum } & 0.9390 \\ \text { Moments } & & \\ & & 0.9868741 \\ \text { Mean } & & 0.0188345 \\ \text { Std Dev } & & 0.0007821 \\ \text { Std Err Mean } & & 0.9884102 \\ \text { upper 95\% Mean } & 0.9853381 \\ \text { lower 95\% Mean } & 580 \\ \text { N } & \end{array}$

Si (mg/L)

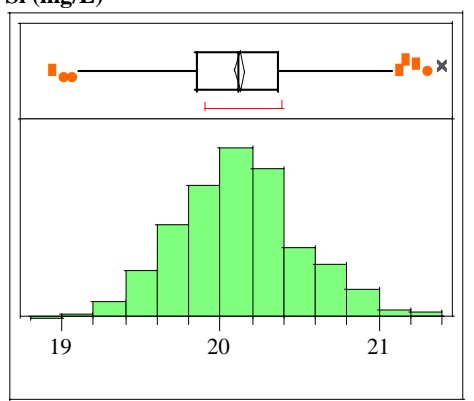

Quantiles

$100.0 \% \quad$ maximum 21.392

$99.5 \% \quad 21.325$

$90.0 \%$

$75.0 \%$

$50.0 \%$

$25.0 \%$

$10.0 \%$

$2.5 \%$

$0.5 \%$

$0.0 \%$

\section{quartile}

20.949

20.679

20.365

0.0000
0.0000

$-0.0009$

-0.0010
-0.0050

$-0.0060$

Moments

minimum

19.852

19.378

19.061

18.934

0.0008121

Mean

Std Dev

Std Err Mean

upper 95\% Mean

lower 95\% Mean

$\mathrm{N}$

20.124321

0.3956577

0.0164288

20.156588

20.092053 
WSRC-TR-2004-00576

Revision 0

\section{Exhibit A22. Histograms and Descriptive Statistics for Fusion Measurements of Calibration and Bench Standards}

Ti (mg/L)

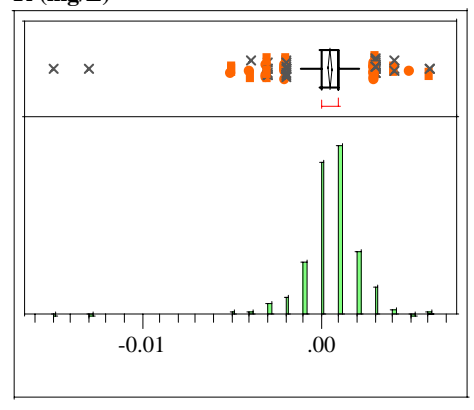

Quantiles

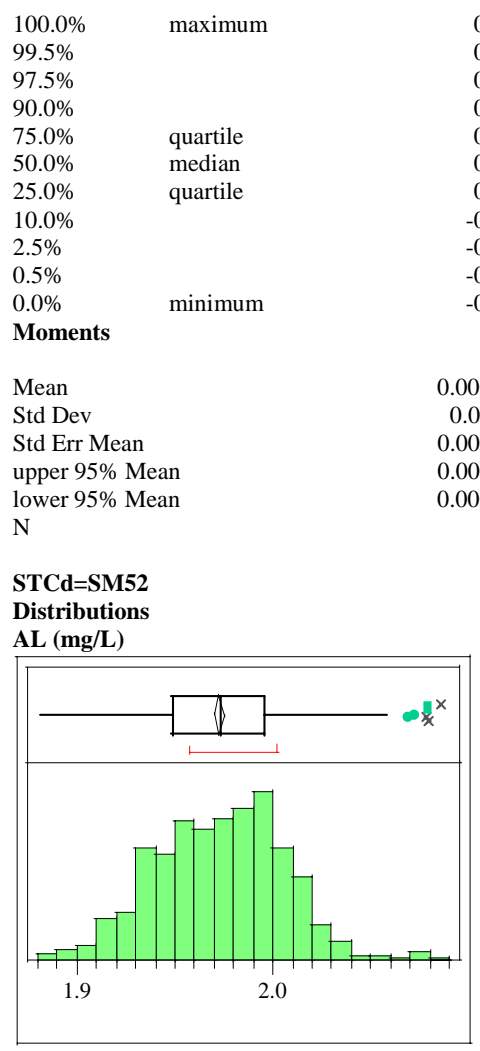

Quantiles

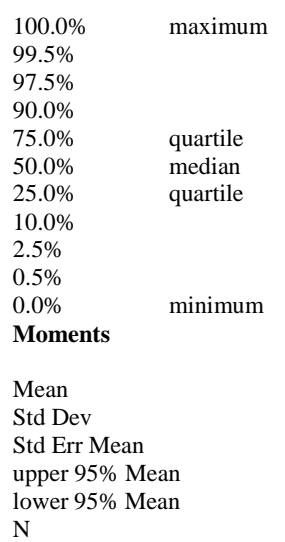

$\mathrm{N}$

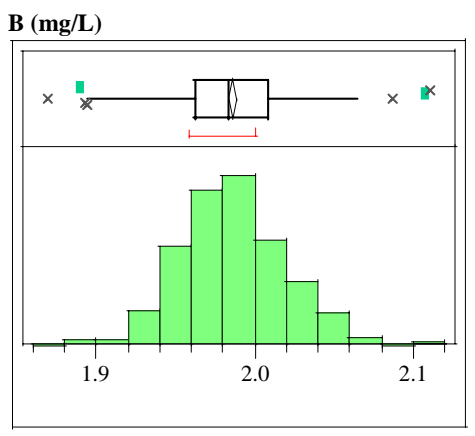

Quantiles

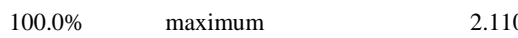

$99.5 \%$

$99.5 \%$

$97.5 \%$

$90.0 \%$

$75.0 \%$

$50.0 \%$

$25.0 \%$

$10.0 \%$

$2.5 \%$

$0.5 \%$

$0.0 \%$

quartile

median
quartile

Moments

Mean

Std Dev

Std Err Mean

upper 95\% Mean

lower 95\% Mean

$\mathrm{N}$

Ca (mg/L)

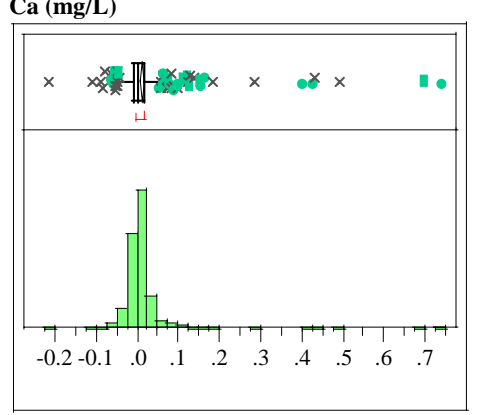

Quantiles

$100.0 \%$

$99.5 \%$

$97.5 \%$

$90.0 \%$

$75.0 \%$

$50.0 \%$

$25.0 \%$

$10.0 \%$

$2.5 \%$

$0.5 \%$

$0.0 \%$

Moments

minimum

Mean

Std Dev

Std Err Mean

upper 95\% Mean

lower 95\% Mean

$\mathrm{N}$

0.0119653

0.0650883

0.002712
Cr (mg/L)

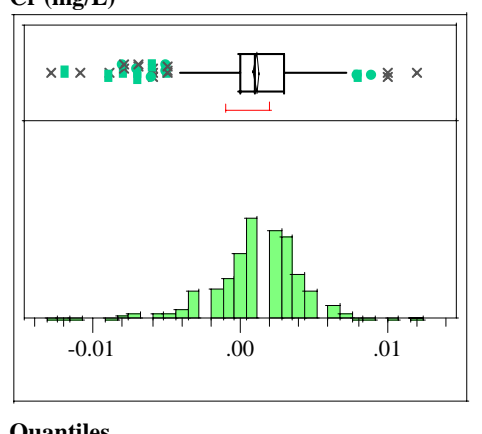

Quantiles

maximum

0.0120

$99.5 \%$

$97.5 \%$

$90.0 \%$

$75.0 \%$

$50.0 \%$

$25.0 \%$

$10.0 \%$

$2.5 \%$

$0.5 \%$

$0.0 \%$

Moments

quartile

median

quartile

Mean

Std Dev

Std Err Mean

upper 95\% Mean

lower 95\% Mean

0.0014125

1.9885174

1.9829687

N

Cu (mg/L)

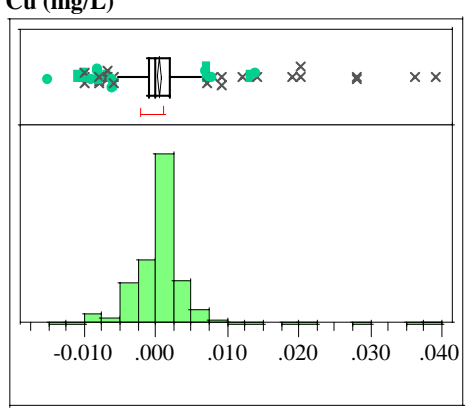

Quantiles

$100.0 \%$

maximum

0.0390

$99.5 \%$

$97.5 \%$

$90.0 \%$

$75.0 \%$

$50.0 \%$

$25.0 \%$

$10.0 \%$

$2.5 \%$

$0.5 \%$

$0.0 \%$

minimum

0.0060

0.0040
0.0030

0.0010

0.0000

0.0000
-0.0030

$-0.0066$

$-0.0111$

$-0.0130$

0.0010677

0.0030355

0.0001265

0.0013161

0.0008193

576

quartile

median

quartile

Moments

Std Dev

Std Err Mean

upper 95\% Mean

lower 95\% Mean

$0.0066386 \mathrm{~N}$ 
WSRC-TR-2004-00576

Revision 0

\section{Exhibit A22. Histograms and Descriptive Statistics for Fusion Measurements of Calibration and Bench Standards}

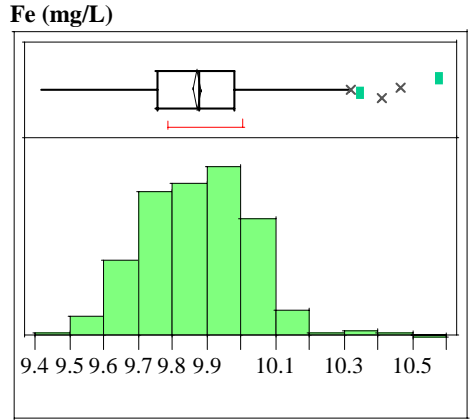

Quantiles

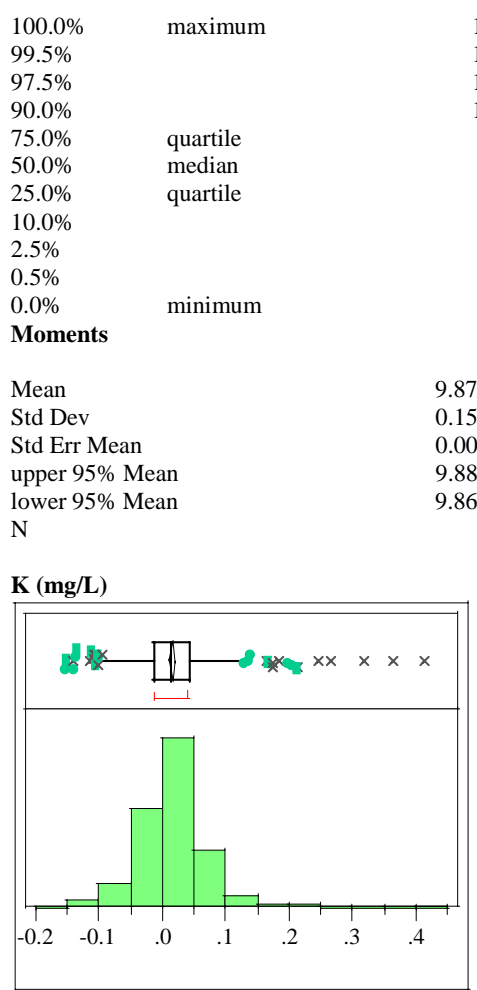

Quantiles

$100.0 \% \quad$ maximum

$99.5 \%$

$97.5 \%$

$90.0 \%$

$75.0 \%$

$50.0 \%$

$25.0 \%$

$10.0 \%$

$2.5 \%$

$0.5 \%$

$0.0 \%$

Mean

Std Dev

Std Err Mean

upper 95\% Mean

lower 95\% Mean

$\mathrm{N}$
Li (mg/L)

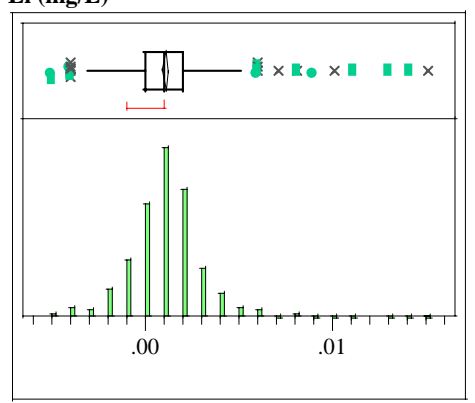

Quantiles

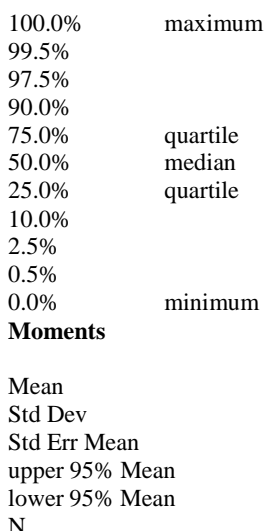

$\mathrm{N}$

$\operatorname{Mg}(\mathrm{mg} / \mathrm{L})$

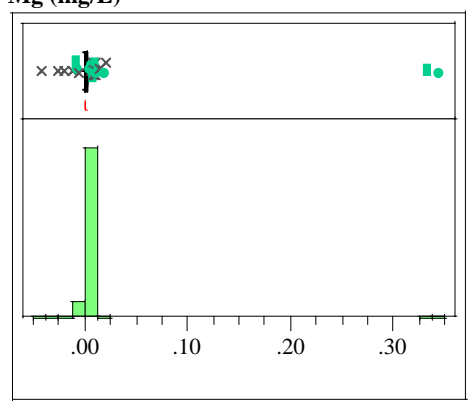

Quantiles

$100.0 \%$ maximum

$99.5 \%$

$97.5 \%$

$90.0 \%$

$75.0 \%$

$50.0 \%$

$25.0 \%$

$10.0 \%$

$2.5 \%$

$0.5 \%$

$0.0 \%$ minimum

Moments

quartile

median
quartile

Mean

Std Dev

Std Err Mean

upper 95\% Mean

lower 95\% Mean

$\mathrm{N}$
Mn (mg/L)

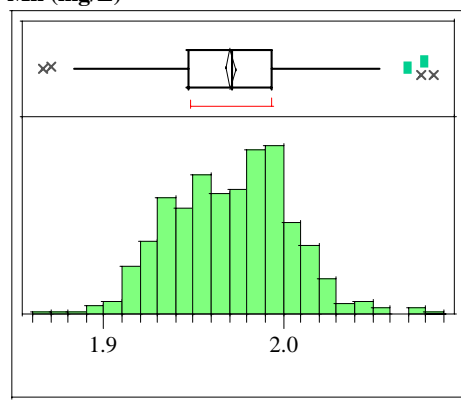

Quantiles

$100.0 \%$

$99.5 \%$

$97.5 \%$

$90.0 \%$

$75.0 \%$

$50.0 \%$

$25.0 \%$

$10.0 \%$

$2.5 \%$

$0.5 \%$

$0.0 \%$

Moments

maximum

2.0840

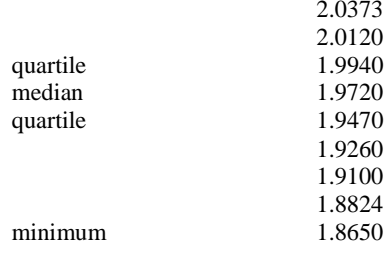

Ni (mg/L)

0.3440

0.0560

0.0070

0.0030

0.0020

0.0010

0.0000

0.0000

$-0.0020$

$-0.0208$

$-0.0430$

0.0024364

0.0212541

576
Mean

Std Dev

Std Err Mean

upper 95\% Mean

lower 95\% Mean

$\mathrm{N}$

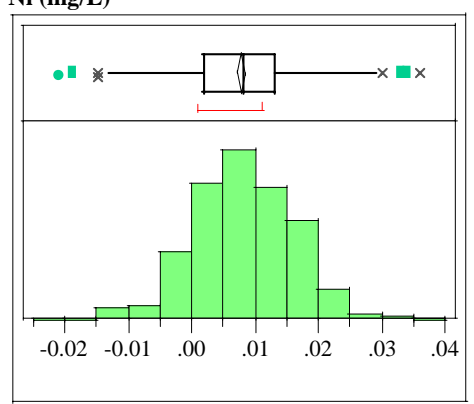

Quantiles

$100.0 \%$

$99.5 \%$

$97.5 \%$

$90.0 \%$

$75.0 \%$

$50.0 \%$

$25.0 \%$

$10.0 \%$

$2.5 \%$

$0.5 \%$

$0.0 \%$

maximum

1.9705347

0.0335045

0.001396

1.9732766

1.9677928

576

\section{Moments}

0.0021111

0.0201373

0.0008391

0.0037591

Std Dev

Std Err Mean

upper 95\% Mean

lower 95\% Mean

$576 \mathrm{~N}$
0.0360 0.0331 0.0236 0.0180 0.0130 0.0080 0.0020 $-0.0020$ $-0.0090$ $-0.0155$ $-0.0210$ 0.0083909 0.0070639 
WSRC-TR-2004-00576

Revision 0

\section{Exhibit A22. Histograms and Descriptive Statistics for Fusion Measurements of Calibration and Bench Standards}

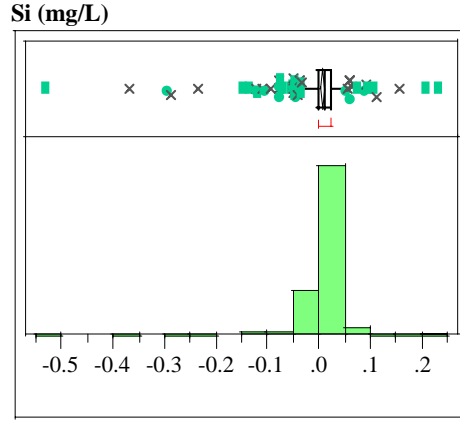

Quantiles

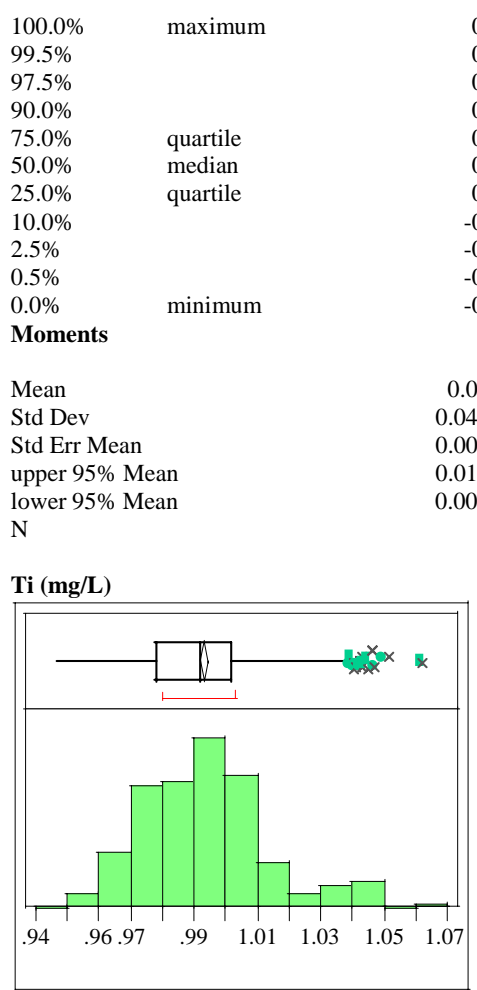

Quantiles

$100.0 \% \quad$ maximum

$99.5 \%$

$97.5 \%$

$90.0 \%$

$75.0 \%$

$50.0 \%$

$25.0 \%$

$10.0 \%$

$2.5 \%$

$0.5 \%$

$0.0 \%$

Moments

Mean

Std Dev

Std Err Mean

upper 95\% Mean

lower 95\% Mean

$\mathrm{N}$
STCd=SM53

Distributions

AL (mg/L)

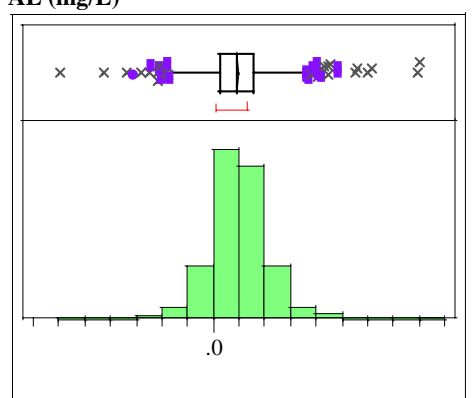

Quantiles

$100.0 \%$ maximum 0.0800

0.9932135

0.0196068

0.0008169

0.9948181

0.991609
$99.5 \%$

$97.5 \%$

$90.0 \%$

$75.0 \%$

$50.0 \%$

$25.0 \%$

$10.0 \%$

$2.5 \%$

$0.5 \%$

$0.0 \%$

Moments

Mean

Std Dev

Std Err Mean

upper 95\% Mean

lower 95\% Mean

$\mathrm{N}$

B (mg/L)

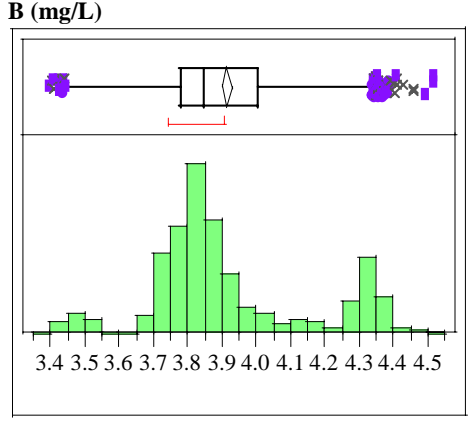

Quantiles

$100.0 \%$

$99.5 \%$

$97.5 \%$

$90.0 \%$

$75.0 \%$

$50.0 \%$

$25.0 \%$

$10.0 \%$

$2.5 \%$

$0.5 \%$

$0.0 \%$

quartile

median

quartile

minimum

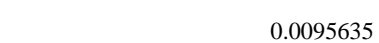

0.0095635 0.0116537

0.000337

0.0102247

0.0089024 1196

Moments

Mean

Std Dev

Std Err Mean

upper 95\% Mean

lower 95\% Mean

$\mathrm{N}$

minimum

3.4220

0.2339384

0.0067645

3.9293076

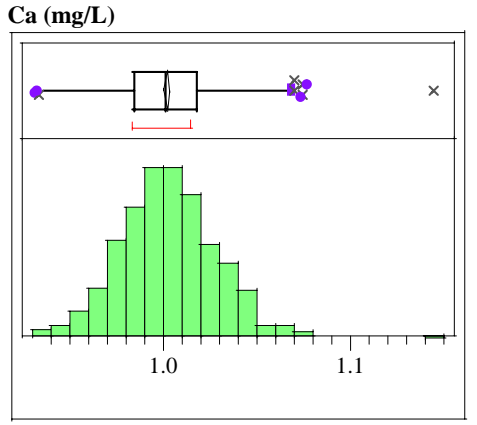

Quantiles

$100.0 \%$ maximum $\quad 1.1440$

$99.5 \%-1.0701$

$\begin{array}{ll}97.5 \% & 1.0510\end{array}$

1.0350

$75.0 \%$ quartile 1.0180

$50.0 \%$ median 1.0010

$25.0 \%$ quartile 0.9850

0.9710

$2.5 \% \quad 0.9539$

$0.5 \% \quad 0.9380$

$0.0 \%$ minimum 0.9320

Moments

Mean

Std Err Mean

lower 95\% Mean 1.0004747

1196

$\operatorname{Cr}(\mathrm{mg} / \mathrm{L})$

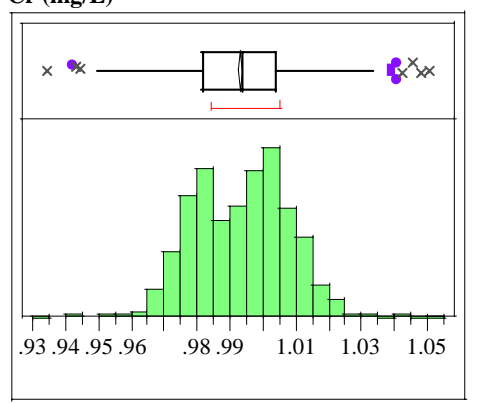

Quantiles

$100.0 \%$

$99.5 \%$

$97.5 \%$

$90.0 \%$

Moments

maximum

1.0500
$75.0 \%$

$50.0 \%$

$25.0 \%$

$10.0 \%$

$2.5 \%$

$0.5 \%$

$0.0 \%$

Mean

Std Dev

Std Err Mean

upper 95\% Mean

$\mathrm{N}$

quartile

median

quartile

minimum

.9670

0.9520

0.9340

0.9930376

0.0146903

0.0004248

0.993871

0.9922042

1196 
WSRC-TR-2004-00576

Revision 0

\section{Exhibit A22. Histograms and Descriptive Statistics for Fusion Measurements of Calibration and Bench Standards}

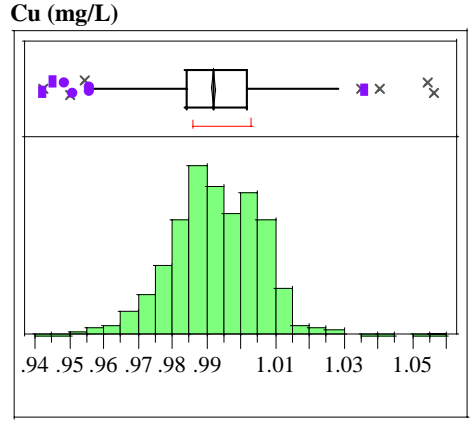

Quantiles

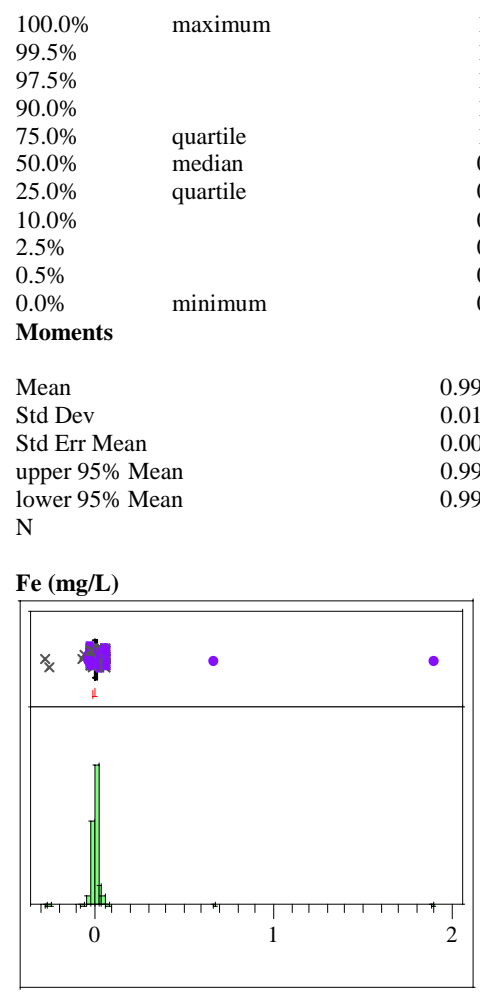

Quantiles

$100.0 \%$

$99.5 \%$

$97.5 \%$

$90.0 \%$

$75.0 \%$

$50.0 \%$

$25.0 \%$

$10.0 \%$

$2.5 \%$

$0.5 \%$

$0.0 \%$

minimum

Mean

Std Dev

Std Err Mean

upper $95 \%$ Mean

lower 95\% Mean

$\mathrm{N}$
K (mg/L)

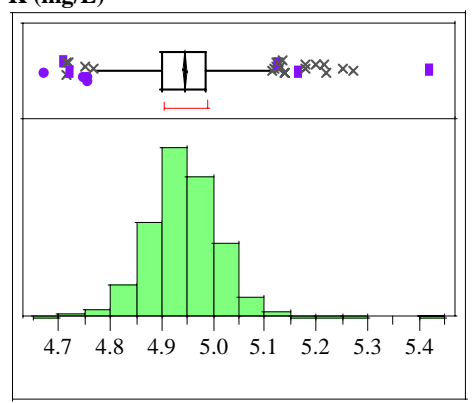

Quantiles

$\begin{array}{ll}100.0 \% & \text { maximum } \\ 99.5 \% & \\ 97.5 \% & \\ 90.0 \% & \\ 75.0 \% & \text { quartile } \\ 50.0 \% & \text { median } \\ 25.0 \% & \text { quartile } \\ 10.0 \% & \\ 2.5 \% & \\ 0.5 \% & \\ 0.0 \% & \text { minimum } \\ \text { Moments } & \\ & \\ \text { Mean } & \\ \text { Std Dev } & \\ \text { Std Err Mean } & \\ \text { upper 95\% Mean } \\ \text { lower 95\% Mean } \\ \text { N }\end{array}$

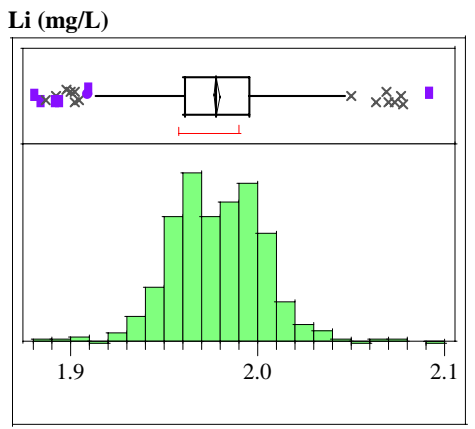

Quantiles

$100.0 \%$ maximum

$99.5 \%$

$97.5 \%$

$90.0 \%$

$75.0 \%$

$50.0 \%$

$25.0 \%$

$10.0 \%$

$2.5 \%$

$0.5 \%$

$0.0 \%$ minimum

Moments

quartile

median

quartile

Mean

Std Dev

Std Err Mean

upper 95\% Mean

lower 95\% Mean

$\mathrm{N}$
$\operatorname{Mg}(\mathrm{mg} / \mathrm{L})$

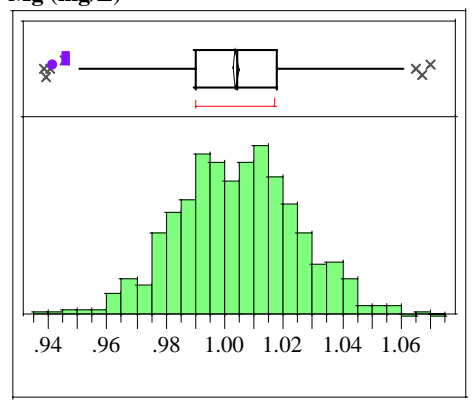

Quantiles

$100.0 \%$

$99.5 \%$

$97.5 \%$

$90.0 \%$

$75.0 \%$

$50.0 \%$

$25.0 \%$

$10.0 \%$

$2.5 \%$

$0.5 \%$

$0.0 \%$

Moments

maximum

1.0700

1.0590

1.0420
1.0300

1.0180

1.0040

0.9900

0.9780

0.9649

0.9460

0.9380

Mean

Std Dev

Std Err Mean

upper 95\% Mean

lower 95\% Mean

minimum

1.0037065

0.0203674

0.0005889
1.004862

1196

$\mathrm{N}$

1.0025511

1196

Mn (mg/L)

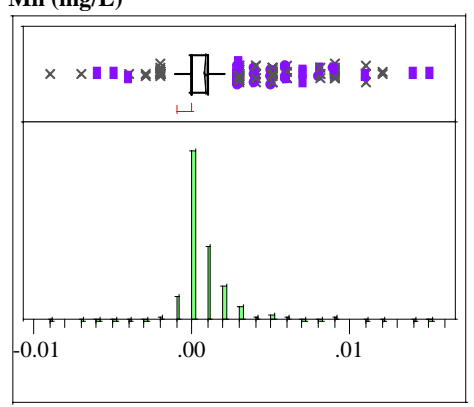

Quantiles

$100.0 \%$ maximum 0.0150

$99.5 \% \quad 0.0110$

$97.5 \%$

$75.0 \%$

$50.0 \%$

$25.0 \%$

$10.0 \%$

$2.5 \%$

$0.5 \%$

$0.0 \%$

quartile

median

quartile

1.9497

1.8940

1.8810

Moments

minimum

0.0070

0.0030

0.0010
0.0000

0.0000
0.0000

0.0000

$-0.0010$

$-0.0040$

$-0.0090$

Mean

Std Dev

Std Err Mean

upper $95 \%$ Mean

lower 95\% Mean

$\mathrm{N}$

0.0008403

0.0019744

0.0000571

0.0009523

0.0007283

1196 
WSRC-TR-2004-00576

Revision 0

\section{Exhibit A22. Histograms and Descriptive Statistics for Fusion Measurements of Calibration and Bench Standards}

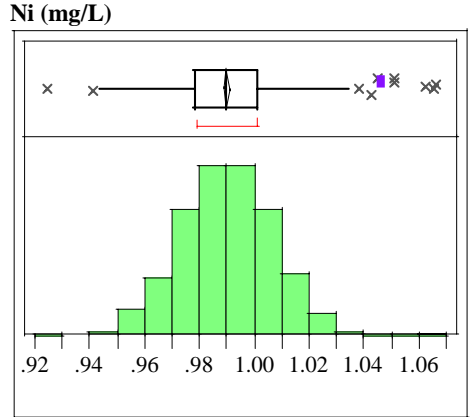

Quantiles

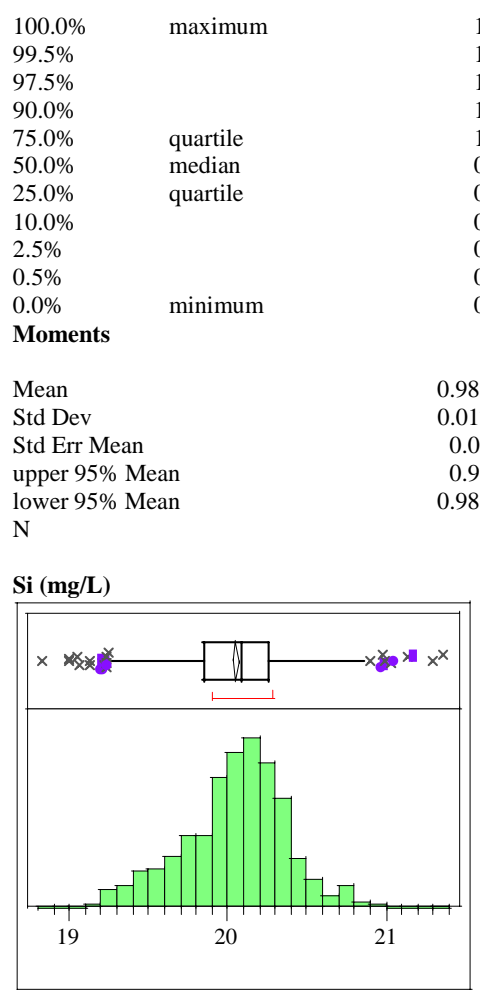

Quantiles

$100.0 \% \quad$ maximum

$99.5 \%$

$97.5 \%$

$90.0 \%$

$75.0 \%$

$50.0 \%$

$25.0 \%$

$10.0 \%$

$2.5 \%$

$0.5 \%$

$0.0 \%$

Moments

Mean

Std Dev

Std Err Mean

upper $95 \%$ Mean

lower 95\% Mean

$\mathrm{N}$
Ti (mg/L)

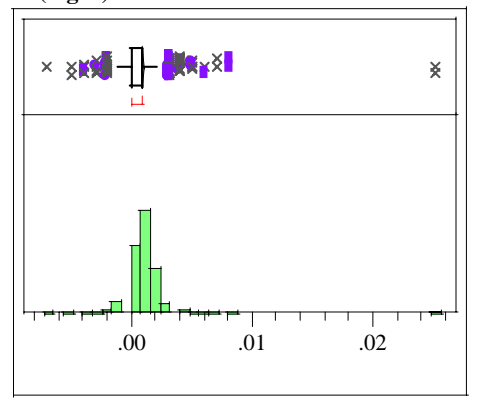

Quantiles

$\begin{array}{ll}100.0 \% & \text { maximum } \\ 99.5 \% & \\ 97.5 \% & \\ 90.0 \% & \\ 75.0 \% & \text { quartile } \\ 50.0 \% & \text { median } \\ 25.0 \% & \text { quartile } \\ 10.0 \% & \\ 2.5 \% & \\ 0.5 \% & \\ 0.0 \% & \text { minimum } \\ \text { Moments } & \\ & \\ \text { Mean } & \\ \text { Std Dev } & \\ \text { Std Err Mean } & \\ \text { upper 95\% Mean } \\ \text { lower 95\% Mean } \\ \text { N } & \\ & \\ \text { STCd=SM54 } & \\ \text { Distributions } & \end{array}$

AL (mg/L)

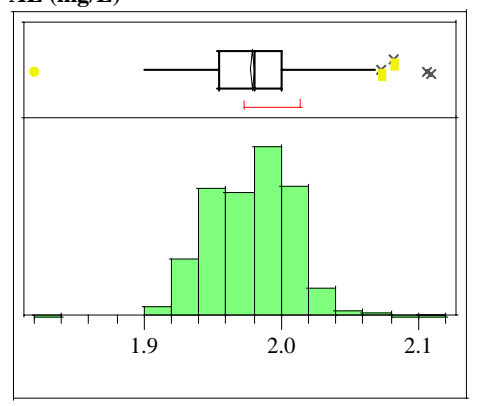

Quantiles

$100.0 \% \quad$ maximum

$99.5 \%$

$97.5 \%$

$90.0 \%$

$75.0 \%$

$50.0 \%$

$25.0 \%$

$10.0 \%$

$2.5 \%$

$0.5 \%$

$0.0 \%$

20.049995

0.3401347

0.0098352

20.069291

20.030699

1196
Moments

Mean

Std Dev

Std Err Mean

upper 95\% Mean

lower 95\% Mean

$\mathrm{N}$ minimum

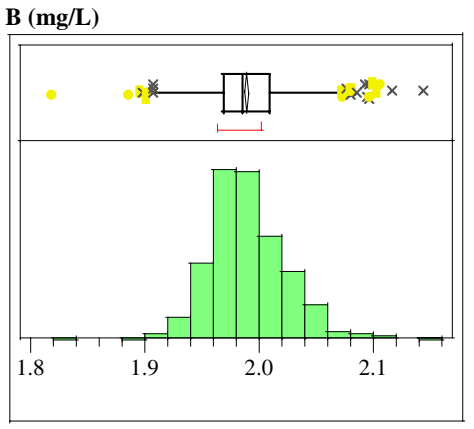

Quantiles

$100.0 \%$ maximum 2.1420

$99.5 \% \quad 2.0979$

$\begin{array}{ll}97.5 \% & 2.0578\end{array}$

2.0340

$\begin{array}{ll}75.0 \% & \text { quartile }\end{array}$

$50.0 \%$ median 1.9855

$\begin{array}{ll}25.0 \% & \text { quartile } \\ 10.0 \% & 1.9690\end{array}$

1.9520

$2.5 \% \quad 1.9310$

$0.5 \% \quad 1.9031$

$0.0 \%$ minimum 1.8200

Moments

1.9895705

Mean

$\begin{array}{ll}\text { Std Dev } & 0.0332189 \\ \text { Std Err Mean } & 0.0009566\end{array}$

Std Err Mean

upper 95\% Mean

lower 95\% Mean 1.9876938

$\mathrm{N}$

Ca (mg/L)

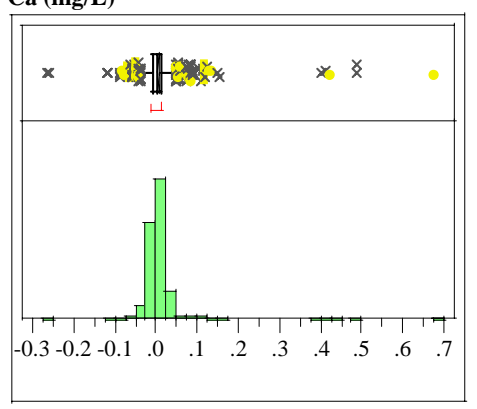

Quantiles

$100.0 \%$

$99.5 \%$

maximum

0.6770

$97.5 \%$

$90.0 \%$

$75.0 \%$

$50.0 \%$

$25.0 \%$

$10.0 \%$

$2.5 \%$

$0.5 \%$

$0.0 \%$

1.9110

Moments

minimum

quartile

median

quartile

Mean

1.9786725

0.030148

0.0008681

Std Dev

Std Err Mean

upper 95\% Mean

1.9803757

1.9769693

lower 95\% Mean

$\mathrm{N}$

0.0442153

0.0012732

0.0091447

0.0041488

1206 
WSRC-TR-2004-00576

Revision 0

\section{Exhibit A22. Histograms and Descriptive Statistics for Fusion Measurements of Calibration and Bench Standards}

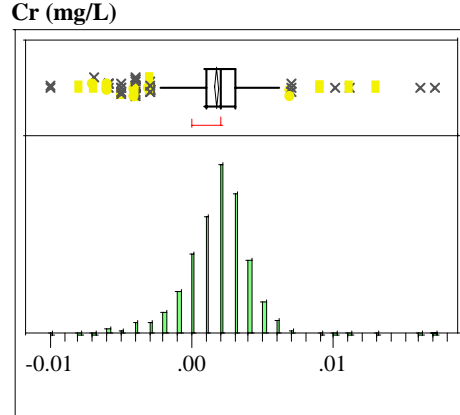

Quantiles

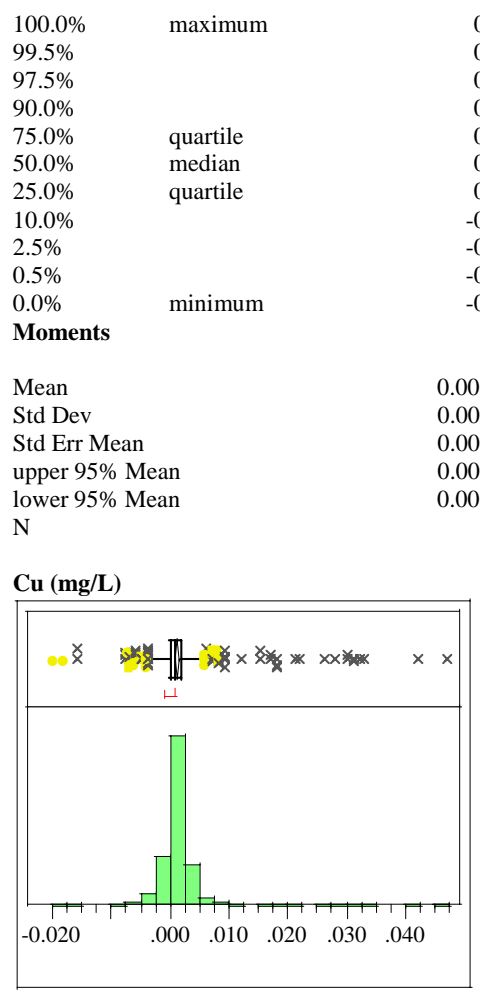

Quantiles

$100.0 \%$ maximum

$99.5 \%$

$97.5 \%$

$90.0 \%$

$75.0 \%$

$50.0 \%$

$25.0 \%$

$10.0 \%$

$2.5 \%$

$0.5 \%$

$0.0 \%$

minimum

Mean

Std Dev

Std Err Mean

upper 95\% Mean

lower 95\% Mean

$\mathrm{N}$
Fe (mg/L)

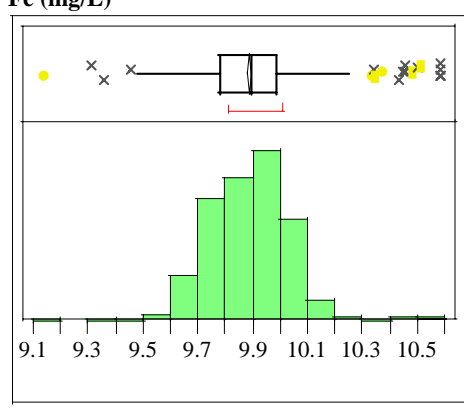

Quantiles

$100.0 \%$

$99.5 \%$

$97.5 \%$

$90.0 \%$

$75.0 \%$

$50.0 \%$

$25.0 \%$

$10.0 \%$

$2.5 \%$

$0.5 \%$

$0.0 \%$

Moments

maximum

Mean

Std Dev

Std Err Mean

upper 95\% Mean

lower 95\% Mean

$\mathrm{N}$

K (mg/L)

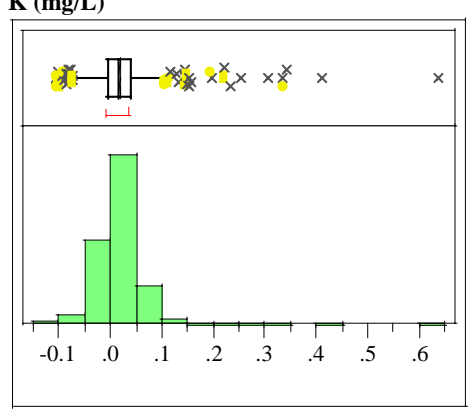

Quantiles

$100.0 \%$ maximum

$99.5 \%$

$97.5 \%$

$90.0 \%$

$75.0 \%$

$50.0 \%$

$25.0 \%$

$10.0 \%$

$2.5 \%$

$0.5 \%$

$0.0 \%$ minimum

Moments

quartile

median

quartile

$-0.0040$

$-0.0200$

0.0011368

0.0041057

0.0001182

0.0013688

0.0009049

1206
Mean

Std Dev

Std Err Mean

upper 95\% Mean

lower 95\% Mea

$\mathrm{N}$
Li (mg/L)

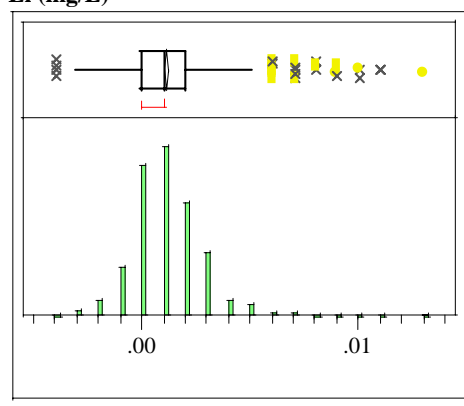

Quantiles

$99.5 \%$

$97.5 \%$

$90.0 \%$

$75.0 \%$

$50.0 \%$

$25.0 \%$

$10.0 \%$

$2.5 \%$

$0.5 \%$

$0.0 \%$

Moments

maximum

0.0130

Mean

Std Dev

Std Err Mean

upper $95 \%$ Mean

lower 95\% Mean

0.0043094

9.8971405

9.8802309

$\mathrm{N}$

quartile

median

quartile

minimum

0.0050

0.0030

0.0020

0.0010

0.0000

$-0.0010$

$-0.0020$

$-0.0030$

$-0.0040$

$\operatorname{Mg}(\mathrm{mg} / \mathrm{L})$

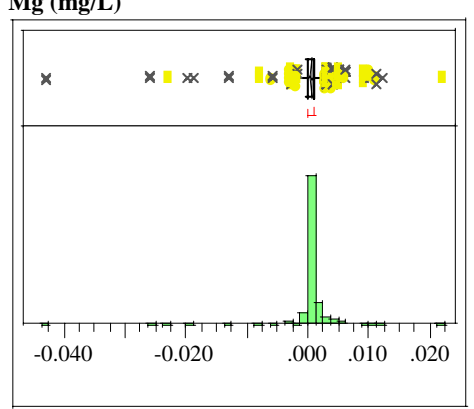

Quantiles

$100.0 \%$

maximum

0.0011609

0.0017904

0.0000516

0.001262

0.0010597

1206

$\begin{array}{llr}100.0 \% & \text { maximum } & 0.0220 \\ 99.5 \% & & 0.0110 \\ 97.5 \% & & 0.0050 \\ 90.0 \% & & 0.0020 \\ 75.0 \% & \text { quartile } & 0.0010 \\ 50.0 \% & \text { median } & 0.0010 \\ 25.0 \% & \text { quartile } & 0.0000 \\ 10.0 \% & & 0.0000 \\ 2.5 \% & & -0.0030 \\ 0.5 \% & & -0.0200 \\ 0.0 \% & -0.0430 \\ \text { Moments } & & \\ & & \\ \text { Mean } & & 0.0006766 \\ \text { Std Dev } & & 0.0029765 \\ \text { Std Err Mean } & & 0.0000857 \\ \text { upper 95\% Mean } & 0.0008448 \\ \text { lower 95\% Mean } & 0.0005085\end{array}$

1206 
WSRC-TR-2004-00576

Revision 0

\section{Exhibit A22. Histograms and Descriptive Statistics for Fusion Measurements of Calibration and Bench Standards}

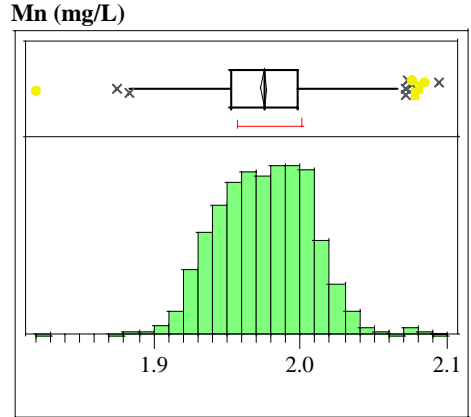

Quantiles

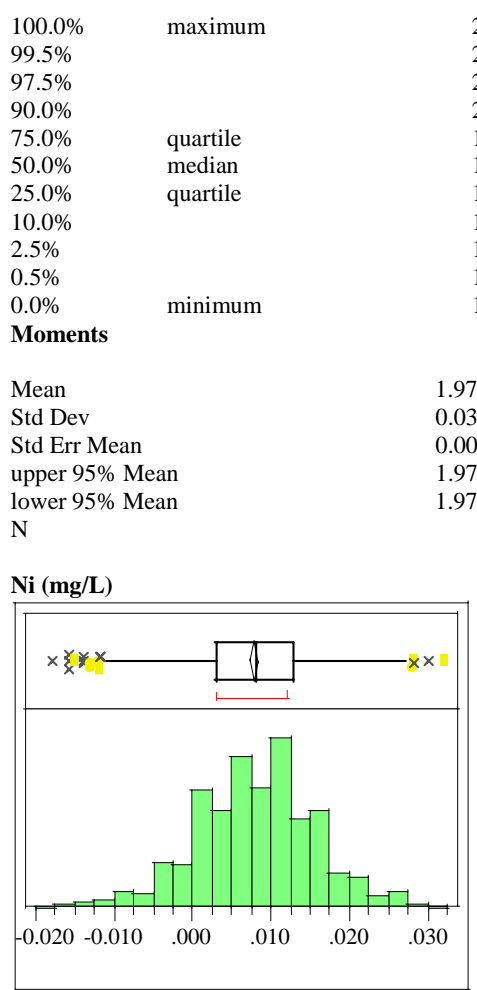

Quantiles

$100.0 \%$

$99.5 \%$

$97.5 \%$

$90.0 \%$

$75.0 \%$

$50.0 \%$

$25.0 \%$

$2.5 \%$

$0.5 \%$

$0.0 \%$

minimum

Mean

Std Dev

Std Err Mean

upper $95 \%$ Mean

lower 95\% Mean

$\mathrm{N}$
Si (mg/L)

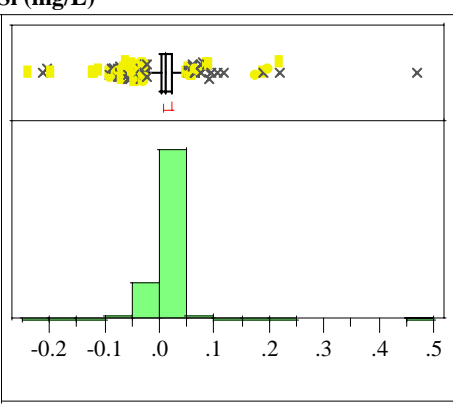

Quantiles

$\begin{array}{ll}100.0 \% & \text { maximum } \\ 99.5 \% & \\ 97.5 \% & \\ 90.0 \% & \\ 75.0 \% & \text { quartile } \\ 50.0 \% & \text { median } \\ 25.0 \% & \text { quartile } \\ 10.0 \% & \\ 2.5 \% & \\ 0.5 \% & \\ 0.0 \% & \text { minimum } \\ \text { Moments } & \\ & \\ \text { Mean } & \\ \text { Std Dev } & \\ \text { Std Err Mean } & \\ \text { upper 95\% Mean } \\ \text { lower 95\% Mean } \\ \text { N }\end{array}$

Ti (mg/L)

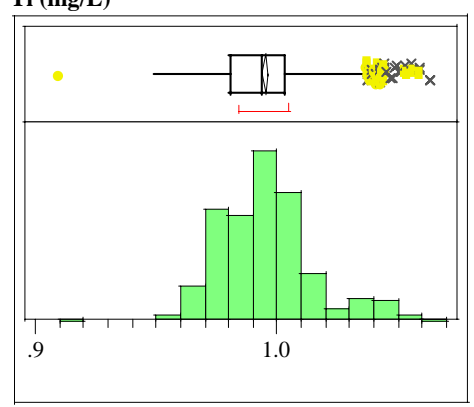

Quantiles

$100.0 \% \quad$ maximum

$99.5 \%$

$97.5 \%$

$90.0 \%$

$75.0 \%$

$50.0 \%$

$25.0 \%$

$10.0 \%$

$2.5 \%$

$0.5 \%$

$0.0 \% \quad$ minimum

Moments

Mean

Std Dev

Std Err Mean

upper 95\% Mean

lower 95\% Mean

$\mathrm{N}$
STCd=SM55

Distributions

AL (mg/L)

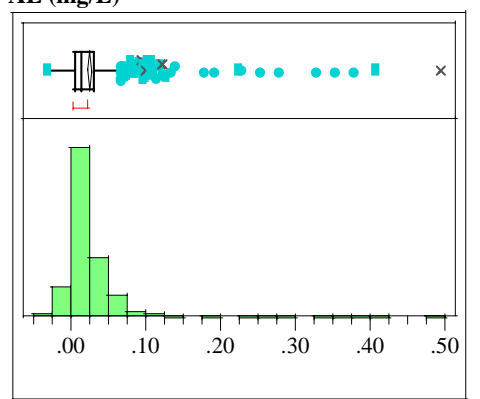

Quantiles

$100.0 \% \quad$ maximum 0.4930

$99.5 \% \quad 0.3561$

$97.5 \%$

$90.0 \%$

$75.0 \%$ quartile

$50.0 \%$ median

$25.0 \%$ quartile

$10.0 \%$

$2.5 \%$

$0.5 \%$

$0.0 \%$

0.0114088

0.0316349

0.0009109

0.013196

0.0096216

1206

Mean

Std Err Mea

upper 95\% Mean

lower 95\% Mean

$\mathrm{N}$

0.1121

0.0540

0.0302

0.0150

0.0060

$-0.0010$

$-0.0150$

$-0.0290$

$-0.0310$

minimum

0.0242329

0.0423149

0.0015055

0.0271882

0.0212777

790

B (mg/L)

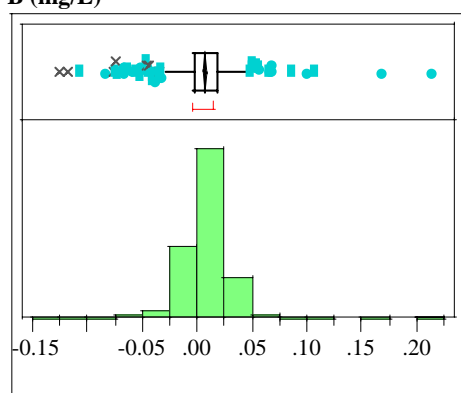

Quantiles

$100.0 \%$ maximum $\quad 0.2140$

$99.5 \%-0.1003$

$97.5 \%$

$90.0 \%$

$75.0 \%$

$50.0 \%$

$25.0 \%$

$10.0 \%$

$2.5 \%$

$0.5 \%$

$0.0 \%$

quartile

median

quartile

0.9560

Moments

minimum

0.0412

0.0290

0.0180

0.0080

$-0.0020$

$-0.0130$

$-0.0444$

$-0.0841$

$-0.1260$

Mean

Std Dev

Std Err Mean

upper 95\% Mean

lower 95\% Mean

$\mathrm{N}$

0.0073949

0.0230525

0.0008202

0.0090049

0.005785 
WSRC-TR-2004-00576

Revision 0

\section{Exhibit A22. Histograms and Descriptive Statistics for Fusion Measurements of Calibration and Bench Standards}

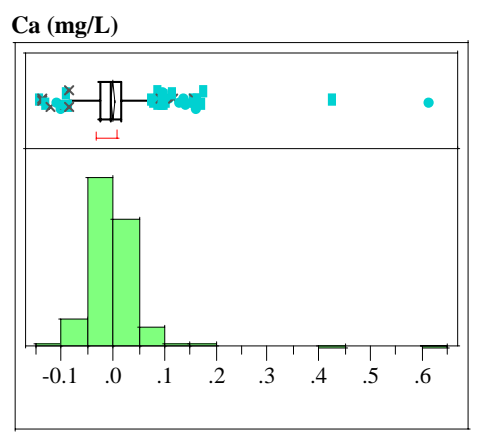

Quantiles

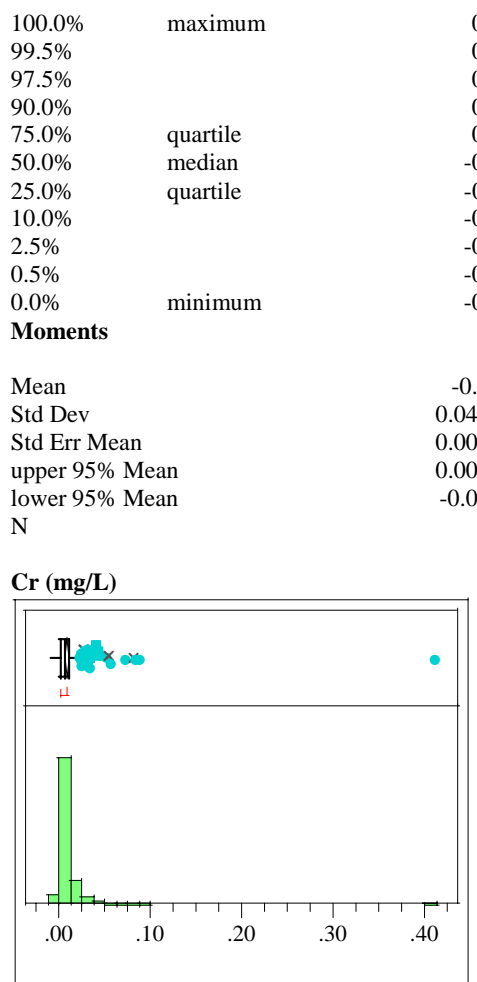

Quantiles

$100.0 \% \quad$ maximum

$99.5 \%$

$97.5 \%$

$90.0 \%$

$75.0 \%$

$50.0 \%$

$25.0 \%$

$10.0 \%$

$2.5 \%$

$0.5 \%$

$0.0 \%$

Moments

minimum

Mean

Std Dev

Std Err Mean

upper 95\% Mean

lower $95 \%$ Mean

$\mathrm{N}$
Cu (mg/L)

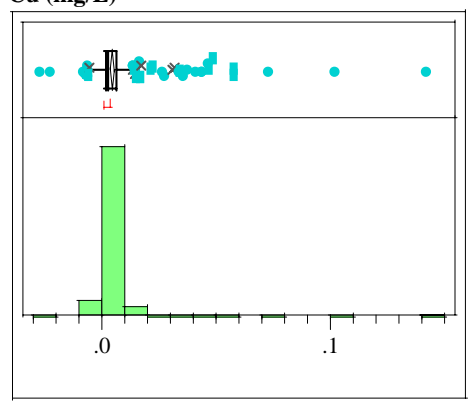

Quantiles

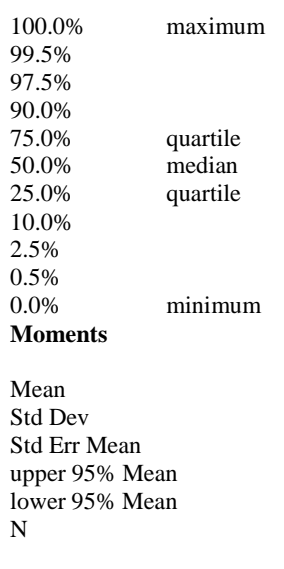

Fe (mg/L)

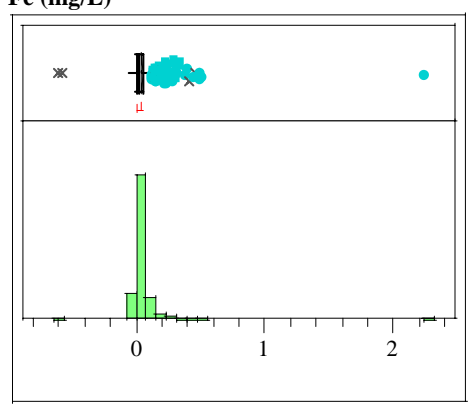

Quantiles

$100.0 \% \quad$ maximum

$99.5 \%$

$97.5 \%$

$90.0 \%$

$75.0 \%$

$50.0 \%$

$25.0 \%$

$10.0 \%$

$2.5 \%$

$0.5 \%$

$0.0 \%$ minimum

Moments

Mean

Std Dev

Std Err Mean

upper 95\% Mean

lower 95\% Mean

$\mathrm{N}$
K (mg/L)

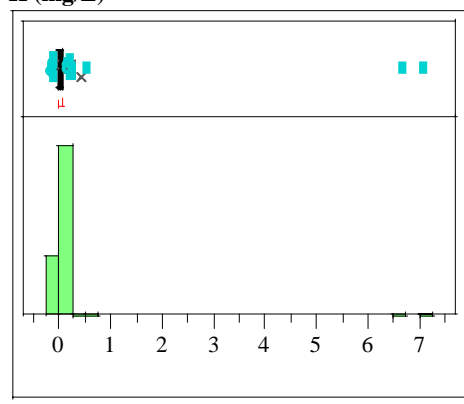

Quantiles

$100.0 \% \quad$ maximum $\quad 7.072$

$99.5 \% \quad 0.397$

$97.5 \%-0.163$

$90.0 \% \quad 0.098$

$\begin{array}{ll}75.0 \% & \text { quartile } \\ 50.0 \% & 0.062\end{array}$

$50.0 \%$ median 0.031

$25.0 \%$ quartile -0.001

$10.0 \% \quad-0.033$

$2.5 \%-0.072$

$0.5 \% \quad-0.122$

$0.0 \% \quad$ minimum $\quad-0.165$

Moments

Mean

Std Dev

Std Err Mean

upper $95 \%$ Mean

lower $95 \%$ Mean

0.0092372

0.0003286

0.0052122

0.003922

$\mathrm{N}$

0.3490953

0.0124203

0.0749047

0.0261434

790

Li (mg/L)

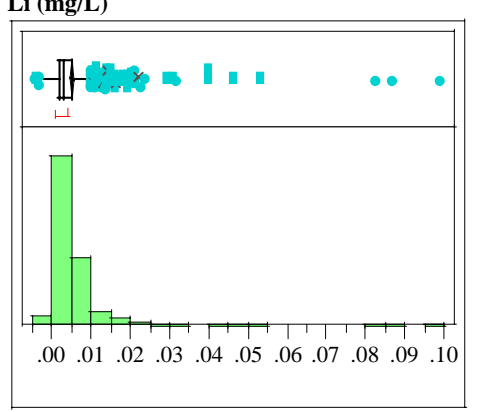

Quantiles

$100.0 \%$ maximum 0.0990

$99.5 \% \quad 0.0543$

$90.0 \%$

$75.0 \%$ quartile

$50.0 \%$ median

$25.0 \%$ quartile

$10.0 \%$

$2.5 \%$

$0.5 \%$

$0.0 \%$

Moments

minimum

0.0192

0.0090

0.0050
0.0030

0.0020

0.0010

$-0.0010$

$-0.0030$

$-0.0040$

Mean

Std Dev

Std Err Mean

upper 95\% Mean

lower 95\% Mean

$\mathrm{N}$
0.0048785

0.0073499

0.0002615

0.0053918

0.0043652 
WSRC-TR-2004-00576

Revision 0

\section{Exhibit A22. Histograms and Descriptive Statistics for Fusion Measurements of Calibration and Bench Standards}

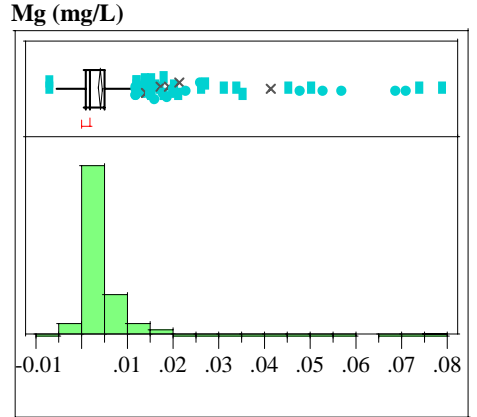

Quantiles

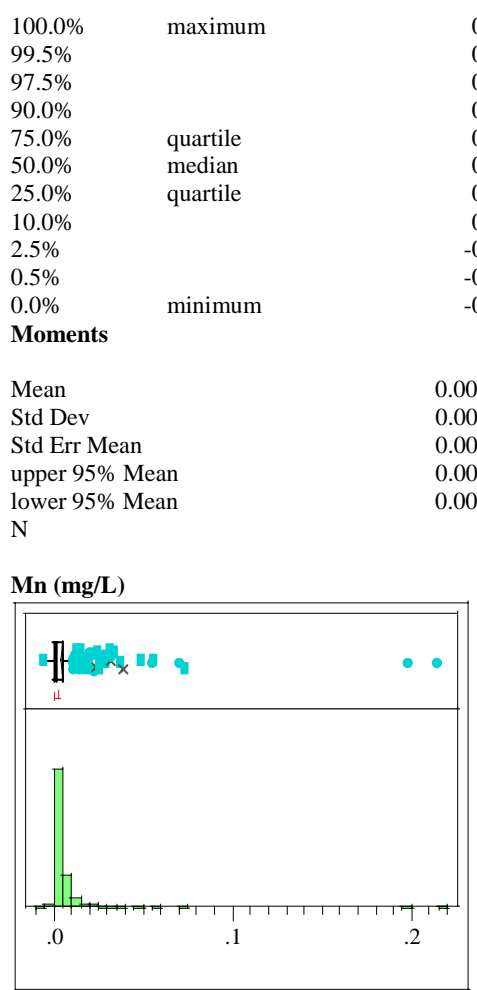

Quantiles

$100.0 \%$

$99.5 \%$

$97.5 \%$

$90.0 \%$

$75.0 \%$

$50.0 \%$

$25.0 \%$

$10.0 \%$

$2.5 \%$

$0.5 \%$

$0.0 \%$
Moments

Mean

Std Dev

Std Err Mean

upper $95 \%$ Mean

lower $95 \%$ Mean

$\mathrm{N}$
Ni (mg/L)

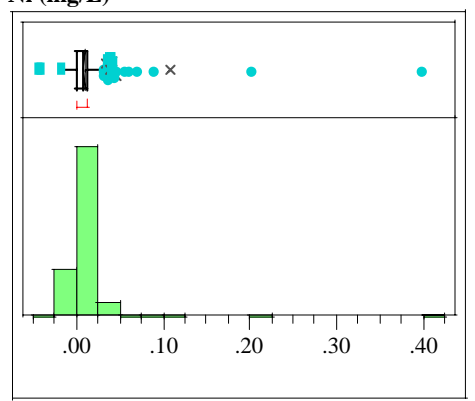

Quantiles

$\begin{array}{ll}100.0 \% & \text { maximum } \\ 99.5 \% & \\ 97.5 \% & \\ 90.0 \% & \\ 75.0 \% & \text { quartile } \\ 50.0 \% & \text { median } \\ 25.0 \% & \text { quartile } \\ 10.0 \% & \\ 2.5 \% & \\ 0.5 \% & \\ 0.0 \% & \text { minimum } \\ \text { Moments } & \\ & \\ \text { Mean } & \\ \text { Std Dev } & \\ \text { Std Err Mean } & \\ \text { upper 95\% Mean } \\ \text { lower 95\% Mean } \\ \text { N }\end{array}$

Si (mg/L)

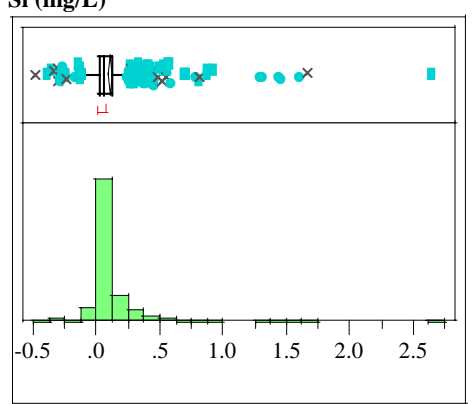

Quantiles

$100.0 \%$

$99.5 \%$

$97.5 \%$

$90.0 \%$

$75.0 \%$

$50.0 \%$

$25.0 \%$

$10.0 \%$

$2.5 \%$

$0.5 \%$

$0.0 \%$

Moments

0.0196088

0.0006976

0.010296

0.0075571

\begin{tabular}{l}
7951 \\
\hline
\end{tabular}
Ti (mg/L)

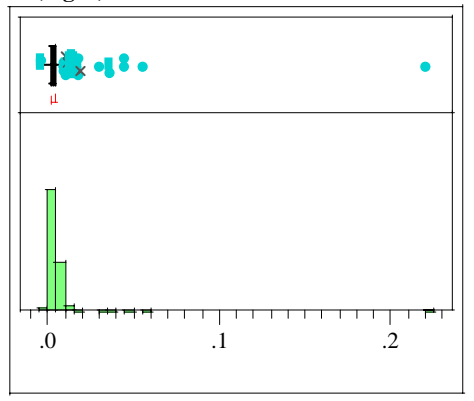

Quantiles

$100.0 \%$

maximum

0.2210

$99.5 \%$

$97.5 \%$

$90.0 \%$

$75.0 \%$

$50.0 \%$

$25.0 \%$

$10.0 \%$

$2.5 \%$

$0.5 \%$

$0.0 \%$

Moments

quartile

median

quartile

Mean

Std Dev

Std Err Mean

upper 95\% Mean

lower 95\% Mean

$\mathrm{N}$
0.0450

0.0140

0.0070

0.0050

0.0030

0.0020

0.0010

0.0000

$-0.0020$

$-0.0040$

0.0044203

0.0088443

0.0003147

0.0050379

0.0038026

\section{Mean}

Std Dev

Std Err Mean

upper 95\% Mean

lower 95\% Mean

$\mathrm{N}$ maximum

$\begin{array}{lr}\text { maximum } & 2.637 \\ & 1.471 \\ & 0.560 \\ \text { quartile } & 0.261 \\ \text { median } & 0.116 \\ \text { quartile } & 0.056 \\ & 0.026 \\ & 0.002 \\ \text { minimum } & -0.126 \\ & -0.340 \\ & -0.485\end{array}$

0.1032595

0.2061269

0.0073337

0.1176553

0.0888637 
WSRC-TR-2004-00576

Revision 0

Exhibit A23. Components of Variance for Fusion Measurements of Calibration and Bench Standard Measurements Reported in a Process Batch

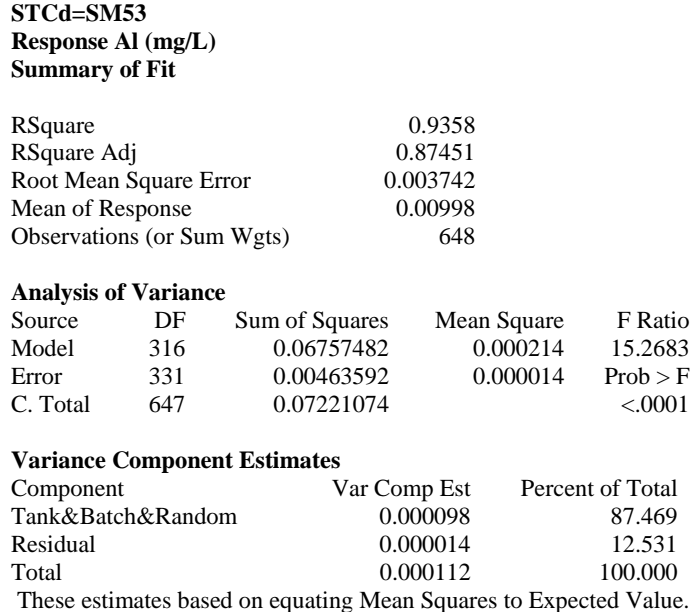

Response B (mg/L)

Summary of Fi

RSquare $\quad 0.989335$

RSquare Adj 0.979152

Root Mean Square Error $\quad 0.034664$

Mean of Response 3.902182

Observations (or Sum Wgts) 648

Analysis of Variance

$\begin{array}{lrrrr}\text { Source } & \text { DF } & \text { Sum of Squares } & \text { Mean Square } & \text { F Ratio } \\ \text { Model } & 316 & 36.892338 & 0.116748 & 97.1636 \\ \text { Error } & 331 & 0.397716 & 0.001202 & \text { Prob }>\text { F } \\ \text { C. Total } & 647 & 37.290055 & & <.0001\end{array}$

Variance Component Estimates

$\begin{array}{lrr}\text { Component } & \text { Var Comp Est } & \text { Percent of Total } \\ \text { Tank\&Batch\&Random } & 0.056529 & 97.919 \\ \text { Residual } & 0.001202 & 2.081 \\ \text { Total } & 0.057731 & 100.000\end{array}$

These estimates based on equating Mean Squares to Expected Value.

Response Ca (mg/L)

Summary of Fit

RSquare 0.939638

RSquare Adj $\quad 0.882012$

Root Mean Square Error $\quad 0.008566$

Mean of Response 1.001775

Observations (or Sum Wgts) 648

Analysis of Variance

$\begin{array}{lrrrr}\text { Source } & \text { DF } & \text { Sum of Squares } & \text { Mean Square } & \text { F Ratio } \\ \text { Model } & 316 & 0.37810969 & 0.001197 & 16.3058 \\ \text { Error } & 331 & 0.02428942 & 0.000073 & \text { Prob }>\text { F } \\ \text { C. Total } & 647 & 0.40239910 & & <.0001\end{array}$

Variance Component Estimates

$\begin{array}{lrr}\text { Component } & \text { Var Comp Est } & \text { Percent of Total } \\ \text { Tank\&Batch\&Random } & 0.000549 & 88.219 \\ \text { Residual } & 0.000073 & 11.781 \\ \text { Total } & 0.000623 & 100.000 \\ \text { These estimates based on equating }\end{array}$

These estimates based on equating Mean Squares to Expected Value.

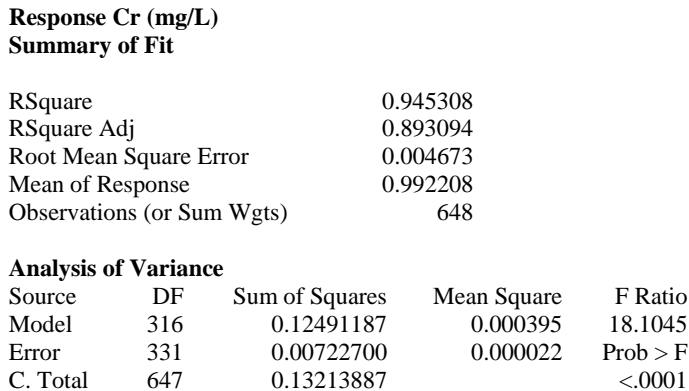

Variance Component Estimates

$\begin{array}{lrr}\text { Component } & \text { Var Comp Est } & \text { Percent of Total } \\ \text { Tank\&Batch\&Random } & 0.000183 & 89.325 \\ \text { Residual } & 0.000022 & 10.675 \\ \text { Total } & 0.000205 & 100.000\end{array}$

These estimates based on equating Mean Squares to Expected Value.

\begin{tabular}{|c|c|c|c|c|}
\hline \multicolumn{4}{|c|}{ Response Cu (mg/L) } & Summary of Fit \\
\hline \multicolumn{3}{|l|}{ RSquare } & \multicolumn{2}{|c|}{0.949232} \\
\hline \multicolumn{3}{|c|}{ RSquare Adj } & \multicolumn{2}{|c|}{0.900765} \\
\hline \multicolumn{3}{|c|}{ Root Mean Square Error } & \multicolumn{2}{|c|}{0.004072} \\
\hline \multicolumn{3}{|c|}{ Mean of Response } & \multicolumn{2}{|c|}{0.991847} \\
\hline \multicolumn{3}{|c|}{ Observations (or Sum Wgts) } & 648 & \\
\hline \multicolumn{5}{|c|}{ Analysis of Variance } \\
\hline Source & DF & Sum of Squares & Mean Square & F Ratio \\
\hline Model & 316 & 0.10259671 & 0.000325 & 19.5851 \\
\hline Error & 331 & 0.00548717 & 0.000017 & Prob $>$ F \\
\hline C. Total & 647 & 0.10808387 & & $<.0001$ \\
\hline
\end{tabular}

Response Fe (mg/L)

Summary of Fit

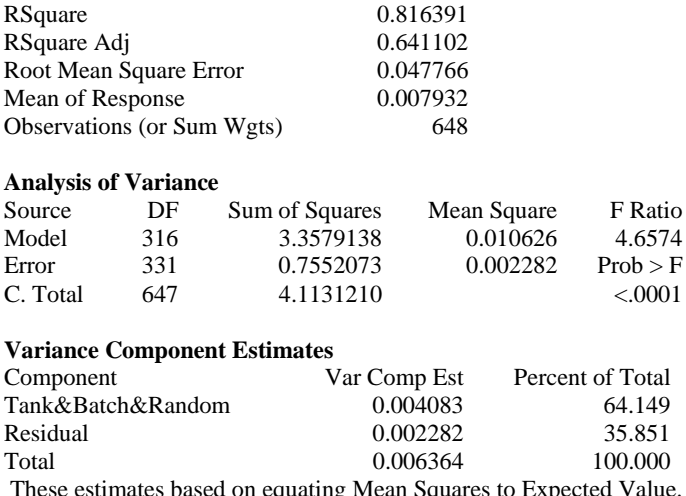


WSRC-TR-2004-00576

Revision 0

Exhibit A23. Components of Variance for Fusion Measurements of Calibration and Bench Standard Measurements Reported in a Process Batch

Response K (mg/L)

Summary of Fit

$\begin{array}{lr}\text { RSquare } & 0.87852 \\ \text { RSquare Adj } & 0.762546 \\ \text { Root Mean Square Error } & 0.033723 \\ \text { Mean of Response } & 4.938444 \\ \text { Observations (or Sum Wgts) } & 648\end{array}$

Analysis of Variance

$\begin{array}{lrrrr}\text { Source } & \text { DF } & \text { Sum of Squares } & \text { Mean Square } & \text { F Ratio } \\ \text { Model } & 316 & 2.7222093 & 0.008615 & 7.5751 \\ \text { Error } & 331 & 0.3764207 & 0.001137 & \text { Prob }>\text { F } \\ \text { C. Total } & 647 & 3.0986300 & & <.0001\end{array}$

Variance Component Estimates

$\begin{array}{lrr}\text { Component } & \text { Var Comp Est } & \text { Percent of Total } \\ \text { Tank\&Batch\&Random } & 0.003658 & 76.285 \\ \text { Residual } & 0.001137 & 23.715 \\ \text { Total } & 0.004795 & 100.000\end{array}$

$\begin{array}{lcr}\text { Total } & 0.004795 & 100.000 \\ \text { These estimates based on equating Mean Squares to Expected Value. }\end{array}$

Response Li (mg/L)

Summary of Fit

RSquare Adj

0.938513

Root Mean Square Error $\quad 0.008523$

Mean of Response 1.979003

Observations (or Sum Wgts) 648

Analysis of Variance

$\begin{array}{lrrrr}\text { Source } & \text { DF } & \text { Sum of Squares } & \text { Mean Square } & \text { F Ratio } \\ \text { Model } & 316 & 0.36702433 & 0.001161 & 15.9882 \\ \text { Error } & 331 & 0.02404567 & 0.000073 & \text { Prob }>\text { F } \\ \text { C. Total } & 647 & 0.39106999 & & <.0001\end{array}$

$\begin{array}{lll}\text { C. Total } & 647 & 0.39106999\end{array}$

Variance Component Estimates

$\begin{array}{lrr}\text { Component } & \text { Var Comp Est } & \text { Percent of Total } \\ \text { Tank\&Batch\&Random } & 0.000533 & 87.999 \\ \text { Residual } & 0.000073 & 12.001 \\ \text { Total } & 0.000605 & 100.000\end{array}$

$\begin{array}{lcr}\text { Total } & 0.000605 & 100.000 \\ \text { These estimates based on equating } & \text { Mean Squares to Expected Value. }\end{array}$

Response Mg (mg/L)

Summary of Fit

RSquare

0.92405

RSquare Adj

0.851541

Root Mean Square Erro

0.007659

Mean of Response

1.003215

Observations (or Sum Wgts)

Analysis of Variance

$\begin{array}{lrrrr}\text { Source } & \text { DF } & \text { Sum of Squares } & \text { Mean Square } & \text { F Ratio } \\ \text { Model } & 316 & 0.23620293 & 0.000747 & 12.7440 \\ \text { Error } & 331 & 0.01941425 & 0.000059 & \text { Prob }>\text { F } \\ \text { C. Total } & 647 & 0.25561718 & & <.0001\end{array}$

Variance Component Estimates

$\begin{array}{lrr}\text { Component } & \text { Var Comp Est } & \text { Percent of Total } \\ \text { Tank\&Batch\&Random } & 0.000337 & 85.175 \\ \text { Residual } & 0.000059 & 14.825 \\ \text { Total } & 0.000396 & 100.000\end{array}$

These estimates based on equating Mean Squares to Expected Value.
Response Mn (mg/L)

Summary of Fit

RSquare

0.948209

RSquare Adj 0.898766

Root Mean Square Error $\quad 0.000566$

Mean of Response $\quad 0.000799$

Observations (or Sum Wgts)

648

Analysis of Variance

$\begin{array}{lrrrr}\text { Source } & \text { DF } & \text { Sum of Squares } & \text { Mean Square } & \text { F Ratio } \\ \text { Model } & 316 & 0.00194375 & 0.0000062 & 19.1776 \\ \text { Error } & 331 & 0.00010617 & 3.2075 \mathrm{e}-7 & \text { Prob }>\text { F } \\ \text { C. Total } & 647 & 0.00204992 & & <.0001\end{array}$

Variance Component Estimates

Component Var

$0.000003 \quad 89.892$

Residual $\quad 3.207 \mathrm{e}-7 \quad 10.108$

$\begin{array}{lr}\text { Total } & 0.000003 \\ \text { These } & 100.000\end{array}$

These estimates based on equating Mean Squares to Expected Value.

Response Ni (mg/L)

Summary of Fit

$\begin{array}{lr}\text { RSquare } & 0.837913 \\ \text { RSquare Adj } & 0.683172 \\ \text { Root Mean Square Error } & 0.009166 \\ \text { Mean of Response } & 0.990213 \\ \text { Observations (or Sum Wgts) } & 648\end{array}$

\begin{tabular}{|c|c|c|c|c|}
\hline \multicolumn{5}{|c|}{ Analysis of Variance } \\
\hline Source & $\mathrm{DF}$ & Sum of Squares & Mean Square & F Rat \\
\hline Model & 316 & 0.14374619 & 0.000455 & 5.41 \\
\hline Error & 331 & 0.02780642 & 0.000084 & Prob > \\
\hline C. Total & 647 & 0.17155261 & & $<.00$ \\
\hline
\end{tabular}

Variance Component Estimates

$\begin{array}{lrr}\text { Component } & \text { Var Comp Est } & \text { Percent of Total } \\ \text { Tank\&Batch\&Random } & 0.000181 & 68.354 \\ \text { Residual } & 0.000084 & 31.646 \\ \text { Total } & 0.000265 & 100.000\end{array}$

Total $0.000265 \quad 100.000$

These estimates based on equating Mean Squares to Expected Value.

Response Si (mg/L)

Summary of Fit

$\begin{array}{lr}\text { RSquare } & 0.944727 \\ \text { RSquare Adj } & 0.891959 \\ \text { Root Mean Square Error } & 0.107271 \\ \text { Mean of Response } & 20.06408 \\ \text { Observations (or Sum Wgts) } & 648\end{array}$

\begin{tabular}{|c|c|c|c|c|}
\hline \multicolumn{5}{|c|}{ Analysis of Variance } \\
\hline Source & DF & Sum of Squares & Mean Square & F Rat \\
\hline Model & 316 & 65.100755 & 0.206015 & 17.90 \\
\hline Error & 331 & 3.808832 & 0.011507 & Prob $>$ \\
\hline C. Total & 647 & 68.909587 & & $<.000$ \\
\hline
\end{tabular}

Variance Component Estimates

$\begin{array}{lrr}\text { Component } & \text { Var Comp Est } & \text { Percent of Total } \\ \text { Tank\&Batch\&Random } & 0.09516 & 89.212 \\ \text { Residual } & 0.011507 & 10.788 \\ \text { Total } & 0.106667 & 100.000\end{array}$

$\begin{array}{lcr}\text { Total } & 0.106667 & 100.000 \\ \text { These estimates based on equating Mean Squares to Expected Value. }\end{array}$ 
WSRC-TR-2004-00576

Revision 0

Exhibit A23. Components of Variance for Fusion Measurements of Calibration and Bench Standard Measurements Reported in a Process Batch

Response Ti (mg/L)

Summary of Fit

$\begin{array}{lr}\text { RSquare } & 0.896927 \\ \text { RSquare Adj } & 0.798525 \\ \text { Root Mean Square Error } & 0.000504 \\ \text { Mean of Response } & 0.000934 \\ \text { Observations (or Sum Wgts) } & 648\end{array}$

Analysis of Variance

$\begin{array}{lrrrr}\text { Source } & \text { DF } & \text { Sum of Squares } & \text { Mean Square } & \text { F Ratio } \\ \text { Model } & 316 & 0.00073023 & 0.0000023 & 9.1149 \\ \text { Error } & 331 & 0.00008392 & 2.5352 \mathrm{e}-7 & \text { Prob }>\text { F } \\ \text { C. Total } & 647 & 0.00081415 & & <.0001\end{array}$

$\begin{array}{lll}\text { C. Total } & 647 & 0.00081415\end{array}$

Variance Component Estimates

$\begin{array}{lrr}\text { Component } & \text { Var Comp Est } & \text { Percent of Total } \\ \text { Tank\&Batch\&Random } & 0.000001 & 79.880 \\ \text { Residual } & 2.535 \mathrm{e}-7 & 20.120 \\ \text { Total } & 0.000001 & 100.000\end{array}$

Total $0.000001 \quad 100.000$

These estimates based on equating Mean Squares to Expected Value.

STCd=SM54

Response Al (mg/L)

Summary of Fit

RSquare Adj

0.940636

0.883618

Root Mean Square Error $\quad 0.009705$

Mean of Response 1.979769

Observations (or Sum Wgts) 646

Analysis of Variance

$\begin{array}{lrrrr}\text { Source } & \text { DF } & \text { Sum of Squares } & \text { Mean Square } & \text { F Ratio } \\ \text { Model } & 316 & 0.49102763 & 0.001554 & 16.4971 \\ \text { Error } & 329 & 0.03098900 & 0.000094 & \text { Prob }>\text { F } \\ \text { C. Total } & 645 & 0.52201663 & & <.0001\end{array}$

C. Total $645 \quad 0.5220166$

Variance Component Estimates

$\begin{array}{lrr}\text { Component } & \text { Var Comp Est } & \text { Percent of Total } \\ \text { Tank\&Batch\&Random } & 0.000716 & 88.379 \\ \text { Residual } & 0.000094 & 11.621 \\ \text { Total } & 0.000811 & 100.000\end{array}$

$\begin{array}{lcr}\text { Total } & 0.000811 & 100.000 \\ \text { These estimates based on equating Mean Squares to Expected Value. }\end{array}$

Response B (mg/L)

Summary of Fit

Mean of Response 1.988483

Observations (or Sum Wgts) 646

Analysis of Variance

$\begin{array}{lrrrr}\text { Source } & \text { DF } & \text { Sum of Squares } & \text { Mean Square } & \text { F Ratio } \\ \text { Model } & 316 & 0.57341431 & 0.001815 & 8.4338 \\ \text { Error } & 329 & 0.07078700 & 0.000215 & \text { Prob }>\text { F } \\ \text { C. Total } & 645 & 0.64420131 & & <.0001\end{array}$

Variance Component Estimates

$\begin{array}{lrr}\text { Component } & \text { Var Comp Est } & \text { Percent of Total } \\ \text { Tank\&Batch\&Random } & 0.000785 & 78.486 \\ \text { Residual } & 0.000215 & 21.514 \\ \text { Total } & 0.001 & 100.000\end{array}$

These estimates based on equating Mean Squares to Expected Value.
Response Ca (mg/L)

Summary of Fit

0.961124

RSquare Adj

0.923785

Root Mean Square Error $\quad 0.010556$

Mean of Response $\quad 0.005833$

Observations (or Sum Wgts) 646

Analysis of Variance

$\begin{array}{lrrrr}\text { Source } & \text { DF } & \text { Sum of Squares } & \text { Mean Square } & \text { F Ratio } \\ \text { Model } & 316 & 0.90633044 & 0.002868 & 25.7400 \\ \text { Error } & 329 & 0.03665950 & 0.000111 & \text { Prob }>\text { F } \\ \text { C. Total } & 645 & 0.94298994 & & <.0001\end{array}$

Variance Component Estimates

$\begin{array}{lrr}\text { Component } & \text { Var Comp Est } & \text { Percent of Total } \\ \text { Tank\&Batch\&Random } & 0.001353 & 92.390 \\ \text { Residual } & 0.000111 & 7.610 \\ \text { Total } & 0.001464 & 100.000\end{array}$

$\begin{array}{lcr}\text { Total } & 0.001464 & 100.000 \\ \text { These estimates based on equating Mean Squares to Expected Value. }\end{array}$

Response Cr (mg/L)

Summary of Fit

RSquare

0.89941

RSquare Adj

0.802794

Root Mean Square Error $\quad 0.000971$

Mean of Response 0.001819

Observations (or Sum Wgts) 646

\begin{tabular}{|c|c|c|c|c|}
\hline \multicolumn{5}{|c|}{ Analysis of Variance } \\
\hline Source & DF & Sum of Squares & Mean Square & F Ratio \\
\hline Model & 316 & 0.00277181 & 0.0000088 & 9.3092 \\
\hline Error & 329 & 0.00031000 & $9.4225 \mathrm{e}-7$ & Prob $>$ F \\
\hline C. Total & 645 & 0.00308181 & & $<.0001$ \\
\hline
\end{tabular}

Variance Component Estimates

$\begin{array}{lrr}\text { Component } & \text { Var Comp Est } & \text { Percent of Total } \\ \text { Tank\&Batch\&Random } & 0.000004 & 80.306 \\ \text { Residual } & 9.422 \mathrm{e}-7 & 19.694 \\ \text { Total } & 0.000005 & 100.000\end{array}$

Total $0.000005 \quad 100.000$

These estimates based on equating Mean Squares to Expected Value.

Response Cu (mg/L)

Summary of Fit

RSquare

0.939669

RSquare Adj

0.881722

Root Mean Square Error $\quad 0.000771$

Mean of Response 0.000836

Observations (or Sum Wgts) 646

\begin{tabular}{|c|c|c|c|c|}
\hline \multicolumn{5}{|c|}{ Analysis of Variance } \\
\hline Source & $\mathrm{DF}$ & Sum of Squares & Mean Square & F Ratio \\
\hline Model & 316 & 0.00304886 & 0.0000096 & 16.2160 \\
\hline Error & 329 & 0.00019575 & 5.9498e-7 & Prob $>$ F \\
\hline C. Total & 645 & 0.00324461 & & $<.0001$ \\
\hline
\end{tabular}

Variance Component Estimates

$\begin{array}{lrr}\text { Component } & \text { Var Comp Est } & \text { Percent of Total } \\ \text { Tank\&Batch\&Random } & 0.000004 & 88.190 \\ \text { Residual } & 5.95 \mathrm{e}-7 & 11.810 \\ \text { Total } & 0.000005 & 100.000\end{array}$

Total $0.000005 \quad 100.000$

These estimates based on equating Mean Squares to Expected Value. 
WSRC-TR-2004-00576

Revision 0

Exhibit A23. Components of Variance for Fusion Measurements of Calibration and Bench Standard Measurements Reported in a Process Batch

Response Fe (mg/L)

Summary of Fit

$\begin{array}{lr}\text { RSquare } & 0.932824 \\ \text { RSquare Adj } & 0.868302 \\ \text { Root Mean Square Error } & 0.047873 \\ \text { Mean of Response } & 9.894831 \\ \text { Observations (or Sum Wgts) } & 646\end{array}$

Analysis of Variance

$\begin{array}{lrrrr}\text { Source } & \text { DF } & \text { Sum of Squares } & \text { Mean Square } & \text { F Ratio } \\ \text { Model } & 316 & 10.470202 & 0.033134 & 14.4575 \\ \text { Error } & 329 & 0.753997 & 0.002292 & \text { Prob }>\text { F } \\ \text { C. Total } & 645 & 11.224199 & & <.0001\end{array}$

Variance Component Estimates

$\begin{array}{lrr}\text { Component } & \text { Var Comp Est } & \text { Percent of Total } \\ \text { Tank\&Batch\&Random } & 0.015135 & 86.849 \\ \text { Residual } & 0.002292 & 13.151 \\ \text { Total } & 0.017427 & 100.000\end{array}$

$\begin{array}{lcr}\text { Total } & 0.017427 & 100.000 \\ \text { These estimates based on equating Mean Squares to Expected Value. }\end{array}$

Response K (mg/L)

Summary of Fit

RSquare Adj

0.813318

Root Mean Square Error $\quad 0.02398$

Mean of Response $\quad 0.015715$

Observations (or Sum Wgts) 646

Analysis of Variance

$\begin{array}{lrrrr}\text { Source } & \text { DF } & \text { Sum of Squares } & \text { Mean Square } & \text { F Ratio } \\ \text { Model } & 316 & 0.8242128 & 0.002608 & 4.5359 \\ \text { Error } & 329 & 0.1891828 & 0.000575 & \text { Prob }>\text { F } \\ \text { C. Total } & 645 & 1.0133956 & & <.0001\end{array}$

Variance Component Estimates

$\begin{array}{lrr}\text { Component } & \text { Var Comp Est } & \text { Percent of Total } \\ \text { Tank\&Batch\&Random } & 0.000998 & 63.440 \\ \text { Residual } & 0.000575 & 36.560 \\ \text { Total } & 0.001573 & 100.000\end{array}$

$\begin{array}{lcr}\text { Total } & 0.001573 & 100.000 \\ \text { These estimates based on equating Mean Squares to Expected Value. }\end{array}$

Response Li (mg/L)

Summary of Fit

0.765499

Root Mean Square Error $\quad 0.000848$

Mean of Response $\quad 0.001291$

Observations (or Sum Wgts) 646

Analysis of Variance

$\begin{array}{lrrrr}\text { Source } & \text { DF } & \text { Sum of Squares } & \text { Mean Square } & \text { F Ratio } \\ \text { Model } & 316 & 0.00174254 & 0.0000055 & 7.6630 \\ \text { Error } & 329 & 0.00023675 & 7.196 \mathrm{e}-7 & \text { Prob }>\text { F } \\ \text { C. Total } & 645 & 0.00197929 & & <.0001\end{array}$

Variance Component Estimates

$\begin{array}{lrr}\text { Component } & \text { Var Comp Est } & \text { Percent of Total } \\ \text { Tank\&Batch\&Random } & 0.000002 & 76.580 \\ \text { Residual } & 7.196 \mathrm{e}-7 & 23.420 \\ \text { Total } & 0.000003 & 100.000\end{array}$

These estimates based on equating Mean Squares to Expected Value.
Response Mg (mg/L)

Summary of Fit

RSquare Adj 0.226437

Root Mean Square Error $\quad 0.001799$

Mean of Response 0.000785

Observations (or Sum Wgts) 646

Analysis of Variance

$\begin{array}{lrrrr}\text { Source } & \text { DF } & \text { Sum of Squares } & \text { Mean Square } & \text { F Ratio } \\ \text { Model } & 316 & 0.00163409 & 0.0000052 & 1.5975 \\ \text { Error } & 329 & 0.00106500 & 0.0000032 & \text { Prob }>\text { F } \\ \text { C. Total } & 645 & 0.00269909 & & <.0001\end{array}$

Variance Component Estimates

$\begin{array}{lrr}\text { Component } & \text { Var Comp Est } & \text { Percent of Total } \\ \text { Tank\&Batch\&Random } & 9.491 \mathrm{e}-7 & 22.673 \\ \text { Residual } & 0.000003 & 77.327 \\ \text { Total } & 0.000004 & 100.000\end{array}$

These estimates based on equating Mean Squares to Expected Value.

Response Mn (mg/L)

Summary of Fit

0.950951

RSquare Adj 0.90384

Root Mean Square Error $\quad 0.009028$

Mean of Response $\quad 1.976125$

Observations (or Sum Wgts) 646

\begin{tabular}{|c|c|c|c|c|}
\hline \multicolumn{5}{|c|}{ Analysis of Variance } \\
\hline Source & DF & Sum of Squares & Mean Square & F Ratio \\
\hline Model & 316 & 0.51983434 & 0.001645 & 20.1854 \\
\hline Error & 329 & 0.02681250 & 0.000081 & Prob $>$ F \\
\hline C. Total & 645 & 0.54664684 & & $<.0001$ \\
\hline
\end{tabular}

Variance Component Estimates

$\begin{array}{lrr}\text { Component } & \text { Var Comp Est } & \text { Percent of Total } \\ \text { Tank\&Batch\&Random } & 0.000767 & 90.398 \\ \text { Residual } & 0.000081 & 9.602 \\ \text { Total } & 0.000849 & 100.000\end{array}$

Total $0.000849 \quad 100.000$

These estimates based on equating Mean Squares to Expected Value.

Response Ni (mg/L)

Summary of Fit

0.794376

RSquare Adj

0.596877

Root Mean Square Error $\quad 0.004717$

Mean of Response 0.007923

Observations (or Sum Wgts) 646

\begin{tabular}{|c|c|c|c|c|}
\hline \multicolumn{5}{|c|}{ Analysis of Variance } \\
\hline Source & $\mathrm{DF}$ & Sum of Squares & Mean Square & F Ratio \\
\hline Model & 316 & 0.02827513 & 0.000089 & 4.0222 \\
\hline Error & 329 & 0.00731900 & 0.000022 & Prob $>$ F \\
\hline C. Total & 645 & 0.03559413 & & $<.0001$ \\
\hline
\end{tabular}

Variance Component Estimates

$\begin{array}{lrr}\text { Component } & \text { Var Comp Est } & \text { Percent of Total } \\ \text { Tank\&Batch\&Random } & 0.000033 & 59.728 \\ \text { Residual } & 0.000022 & 40.272 \\ \text { Total } & 0.000055 & 100.000\end{array}$

$\begin{array}{lcr}\text { Total } & 0.000055 & 100.000 \\ \text { These estimates based on equating Mean Squares to Expected Value. }\end{array}$ 
WSRC-TR-2004-00576

Revision 0

Exhibit A23. Components of Variance for Fusion Measurements of Calibration and Bench Standard Measurements Reported in a Process Batch

Response Si (mg/L)

Summary of Fit

$\begin{array}{lr}\text { RSquare } & 0.956326 \\ \text { RSquare Adj } & 0.914378 \\ \text { Root Mean Square Error } & 0.008383 \\ \text { Mean of Response } & 0.010935 \\ \text { Observations (or Sum Wgts) } & 646\end{array}$

Analysis of Variance

$\begin{array}{lrrrr}\text { Source } & \text { DF } & \text { Sum of Squares } & \text { Mean Square } & \text { F Ratio } \\ \text { Model } & 316 & 0.50627452 & 0.001602 & 22.7978 \\ \text { Error } & 329 & 0.02312075 & 0.000070 & \text { Prob }>\text { F } \\ \text { C. Total } & 645 & 0.52939527 & & <.0001\end{array}$

Variance Component Estimates

$\begin{array}{lrr}\text { Component } & \text { Var Comp Est } & \text { Percent of Total } \\ \text { Tank\&Batch\&Random } & 0.000752 & 91.451 \\ \text { Residual } & 0.00007 & 8.549 \\ \text { Total } & 0.000822 & 100.000\end{array}$

$\begin{array}{lcr}\text { Total } & 0.000822 & 100.000 \\ \text { These estimates based on equating Mean Squares to Expected Value. }\end{array}$

Response Ti (mg/L)

Summary of Fit

0.975601

RSquare Adj $\quad 0.952165$

Root Mean Square Error $\quad 0.00417$

Mean of Response 0.994164

Observations (or Sum Wgts) 646

Analysis of Variance

$\begin{array}{lrrrr}\text { Source } & \text { DF } & \text { Sum of Squares } & \text { Mean Square } & \text { F Ratio } \\ \text { Model } & 316 & 0.22875161 & 0.000724 & 41.6295 \\ \text { Error } & 329 & 0.00572100 & 0.000017 & \text { Prob }>\text { F } \\ \text { C. Total } & 645 & 0.23447261 & & <.0001\end{array}$

$<.0001$

Variance Component Estimates

$\begin{array}{lrr}\text { Component } & \text { Var Comp Est } & \text { Percent of Total } \\ \text { Tank\&Batch\&Random } & 0.000347 & 95.224 \\ \text { Residual } & 0.000017 & 4.776 \\ \text { Total } & 0.000364 & 100.000\end{array}$

$\begin{array}{lcr}\text { Total } & 0.000364 & 100.000 \\ \text { These estimates based on equating } & \text { Mean Squares to Expected Value. }\end{array}$

STCd=SM55

Response Al (mg/L)

Summary of Fit

Root Mean Square Error $\quad 0.037528$

Mean of Response 0.025602

Observations (or Sum Wgts) 635

Analysis of Variance

$\begin{array}{lrrrr}\text { Source } & \text { DF } & \text { Sum of Squares } & \text { Mean Square } & \text { F Ratio } \\ \text { Model } & 314 & 0.6501650 & 0.002071 & 1.4702 \\ \text { Error } & 320 & 0.4506832 & 0.001408 & \text { Prob }>\text { F } \\ \text { C. Total } & 634 & 1.1008482 & & 0.0003\end{array}$

Variance Component Estimates

$\begin{array}{lrr}\text { Component } & \text { Var Comp Est } & \text { Percent of Total } \\ \text { Tank\&Batch\&Random } & 0.000329 & 18.914 \\ \text { Residual } & 0.001408 & 81.086 \\ \text { Total } & 0.001737 & 100.000\end{array}$

These estimates based on equating Mean Squares to Expected Value.
Response B (mg/L)

Summary of Fit

RSquare Adj $\quad 0.472025$

Root Mean Square Error $\quad 0.01645$

Mean of Response 0.009044

Observations (or Sum Wgts) 635

Analysis of Variance

$\begin{array}{lrrrr}\text { Source } & \text { DF } & \text { Sum of Squares } & \text { Mean Square } & \text { F Ratio } \\ \text { Model } & 314 & 0.23834577 & 0.000759 & 2.8051 \\ \text { Error } & 320 & 0.08659100 & 0.000271 & \text { Prob }>\text { F } \\ \text { C. Total } & 634 & 0.32493677 & & <.0001\end{array}$

Variance Component Estimates

$\begin{array}{lrr}\text { Component } & \text { Var Comp Est } & \text { Percent of Total } \\ \text { Tank\&Batch\&Random } & 0.000242 & 47.244 \\ \text { Residual } & 0.000271 & 52.756 \\ \text { Total } & 0.000513 & 100.000\end{array}$

Total $0.000513 \quad 100.000$

These estimates based on equating Mean Squares to Expected Value.

Response Ca (mg/L)

Summary of Fit

$\begin{array}{lr}\text { RSquare } & 0.691343 \\ \text { RSquare Adj } & 0.388474 \\ \text { Root Mean Square Error } & 0.038398 \\ \text { Mean of Response } & 0.001937 \\ \text { Observations (or Sum Wgts) } & 635\end{array}$

\begin{tabular}{|c|c|c|c|c|}
\hline \multicolumn{5}{|c|}{ Analysis of Variance } \\
\hline Source & DF & Sum of Squares & Mean Square & F Rat \\
\hline Model & 314 & 1.0568048 & 0.003366 & 2.28 \\
\hline Error & 320 & 0.4718207 & 0.001474 & Prob > \\
\hline C. Total & 634 & 1.5286255 & & $<.00$ \\
\hline
\end{tabular}

Variance Component Estimates

$\begin{array}{lrr}\text { Component } & \text { Var Comp Est } & \text { Percent of Total } \\ \text { Tank\&Batch\&Random } & 0.000938 & 38.887 \\ \text { Residual } & 0.001474 & 61.113 \\ \text { Total } & 0.002413 & 100.000\end{array}$

Total $0.002413 \quad 100.000$

These estimates based on equating Mean Squares to Expected Value.

Response Cr (mg/L)

Summary of Fit

RSquare

0.5618

RSquare Adj

0.131816

Root Mean Square Error $\quad 0.017543$

Mean of Response 0.009624

Observations (or Sum Wgts) 635

\begin{tabular}{lcrrr}
\multicolumn{2}{l}{ Analysis of Variance } & & & \\
Source & DF & Sum of Squares & Mean Square & F Ratio \\
Model & 314 & 0.12626738 & 0.000402 & 1.3066 \\
Error & 320 & 0.09848767 & 0.000308 & Prob > F \\
C. Total & 634 & 0.22475505 & & 0.0087
\end{tabular}

Variance Component Estimates

$\begin{array}{lrr}\text { Component } & \text { Var Comp Est } & \text { Percent of Total } \\ \text { Tank\&Batch\&Random } & 0.000047 & 13.201 \\ \text { Residual } & 0.000308 & 86.799 \\ \text { Total } & 0.000355 & 100.000\end{array}$

These estimates based on equating Mean Squares to Expected Value. 
WSRC-TR-2004-00576

Revision 0

Exhibit A23. Components of Variance for Fusion Measurements of Calibration and Bench Standard Measurements Reported in a Process Batch

Response Cu (mg/L)

Summary of Fit

$\begin{array}{lr}\text { RSquare } & 0.691523 \\ \text { RSquare Adj } & 0.388831 \\ \text { Root Mean Square Error } & 0.007824 \\ \text { Mean of Response } & 0.004891 \\ \text { Observations (or Sum Wgts) } & 635\end{array}$

Analysis of Variance

$\begin{array}{lrrrr}\text { Source } & \text { DF } & \text { Sum of Squares } & \text { Mean Square } & \text { F Ratio } \\ \text { Model } & 314 & 0.04391000 & 0.000140 & 2.2846 \\ \text { Error } & 320 & 0.01958750 & 0.000061 & \text { Prob }>\text { F } \\ \text { C. Total } & 634 & 0.06349750 & & <.0001\end{array}$

Variance Component Estimates

$\begin{array}{lrr}\text { Component } & \text { Var Comp Est } & \text { Percent of Total } \\ \text { Tank\&Batch\&Random } & 0.000039 & 38.923 \\ \text { Residual } & 0.000061 & 61.077 \\ \text { Total } & 0.0001 & 100.000 \\ \text { These estimates based on equating Mean Squares to Expected Value. }\end{array}$

Response Fe (mg/L)

Summary of Fit

0.559378

RSquare Adj 0.127017

Root Mean Square Error $\quad 0.103396$

Mean of Response 0.05398

Observations (or Sum Wgts) 635

Analysis of Variance

$\begin{array}{lrrrr}\text { Source } & \text { DF } & \text { Sum of Squares } & \text { Mean Square } & \text { F Ratio } \\ \text { Model } & 314 & 4.3430983 & 0.013832 & 1.2938 \\ \text { Error } & 320 & 3.4210624 & 0.010691 & \text { Prob }>\text { F } \\ \text { C. Total } & 634 & 7.7641607 & & 0.0110\end{array}$

Variance Component Estimates

$\begin{array}{lrr}\text { Component } & \text { Var Comp Est } & \text { Percent of Total } \\ \text { Tank\&Batch\&Random } & 0.001558 & 12.720 \\ \text { Residual } & 0.010691 & 87.280 \\ \text { Total } & 0.012249 & 100.000\end{array}$

$\begin{array}{lcr}\text { Total } & 0.012249 & 100.000 \\ \text { These estimates based on equating } & \text { Mean Squares to Expected Value. }\end{array}$

Response K (mg/L)

Summary of Fit

Root Mean Square Error $\quad 0.043083$

Mean of Response 0.054534

Observations (or Sum Wgts) 635

Analysis of Variance

$\begin{array}{lrrrr}\text { Source } & \text { DF } & \text { Sum of Squares } & \text { Mean Square } & \text { F Ratio } \\ \text { Model } & 314 & 94.890086 & 0.302198 & 162.8094 \\ \text { Error } & 320 & 0.593966 & 0.001856 & \text { Prob }>\text { F } \\ \text { C. Total } & 634 & 95.484052 & & <.0001\end{array}$

Variance Component Estimates

$\begin{array}{lrr}\text { Component } & \text { Var Comp Est } & \text { Percent of Total } \\ \text { Tank\&Batch\&Random } & 0.148998 & 98.770 \\ \text { Residual } & 0.001856 & 1.230 \\ \text { Total } & 0.150854 & 100.000\end{array}$

These estimates based on equating Mean Squares to Expected Value.
Response Li (mg/L)

Summary of Fit

0.584718

RSquare Adj $\quad 0.177223$

Root Mean Square Error $\quad 0.007257$

Mean of Response $\quad 0.005272$

Observations (or Sum Wgts) 635

Analysis of Variance

$\begin{array}{lrrrr}\text { Source } & \text { DF } & \text { Sum of Squares } & \text { Mean Square } & \text { F Ratio } \\ \text { Model } & 314 & 0.02372895 & 0.000076 & 1.4349 \\ \text { Error } & 320 & 0.01685292 & 0.000053 & \text { Prob }>\text { F } \\ \text { C. Total } & 634 & 0.04058187 & & 0.0007\end{array}$

Variance Component Estimates

$\begin{array}{lrr}\text { Component } & \text { Var Comp Est } & \text { Percent of Total } \\ \text { Tank\&Batch\&Random } & 0.000011 & 17.747 \\ \text { Residual } & 0.000053 & 82.253 \\ \text { Total } & 0.000064 & 100.000\end{array}$

Total $0.000064 \quad 100.000$

These estimates based on equating Mean Squares to Expected Value.

Response Mg (mg/L)

Summary of Fit

$\begin{array}{lr}\text { RSquare } & 0.698651 \\ \text { RSquare Adj } & 0.402952 \\ \text { Root Mean Square Error } & 0.006475 \\ \text { Mean of Response } & 0.004339 \\ \text { Observations (or Sum Wgts) } & 635\end{array}$

\begin{tabular}{|c|c|c|c|c|}
\hline \multicolumn{5}{|c|}{ Analysis of Variance } \\
\hline Source & $\mathrm{DF}$ & Sum of Squares & Mean Square & F Rat \\
\hline Model & 314 & 0.03110129 & 0.000099 & 2.36 \\
\hline Error & 320 & 0.01341492 & 0.000042 & Prob $>$ \\
\hline C. Total & 634 & 0.04451620 & & $<.00$ \\
\hline
\end{tabular}

Variance Component Estimates

$\begin{array}{lrr}\text { Component } & \text { Var Comp Est } & \text { Percent of Total } \\ \text { Tank\&Batch\&Random } & 0.000028 & 40.335 \\ \text { Residual } & 0.000042 & 59.665 \\ \text { Total } & 0.00007 & 100.000\end{array}$

$\begin{array}{lcr}\text { Total } & 0.00007 & 100.000 \\ \text { These estimates based on equating Mean Squares to Expected Value. }\end{array}$

Response Mn (mg/L)

Summary of Fit

RSquare

0.534931

RSquare Adj

Root Mean Square Error $\quad 0.012836$

Mean of Response 0.005301

Observations (or Sum Wgts) 635

\begin{tabular}{|c|c|c|c|c|}
\hline \multicolumn{5}{|c|}{ Analysis of Variance } \\
\hline Source & DF & Sum of Squares & Mean Square & F Rat \\
\hline Model & 314 & 0.06064805 & 0.000193 & 1.172 \\
\hline Error & 320 & 0.05272750 & 0.000165 & Prob $>$ \\
\hline C. Total & 634 & 0.11337555 & & 0.0788 \\
\hline
\end{tabular}

Variance Component Estimates

$\begin{array}{lrr}\text { Component } & \text { Var Comp Est } & \text { Percent of Total } \\ \text { Tank\&Batch\&Random } & 0.000014 & 7.870 \\ \text { Residual } & 0.000165 & 92.130 \\ \text { Total } & 0.000179 & 100.000\end{array}$

$\begin{array}{lcr}\text { Total } & 0.000179 & 100.000 \\ \text { These estimates based on equating Mean Squares to Expected Value. }\end{array}$ 
WSRC-TR-2004-00576

Revision 0

Exhibit A23. Components of Variance for Fusion Measurements of Calibration and Bench Standard Measurements Reported in a Process Batch

\begin{tabular}{|c|c|c|c|c|c|}
\hline \multicolumn{6}{|c|}{$\begin{array}{l}\text { Response Ni (mg/L) } \\
\text { Summary of Fit }\end{array}$} \\
\hline \multicolumn{3}{|l|}{ RSquare } & \multicolumn{3}{|c|}{0.559588} \\
\hline \multicolumn{3}{|c|}{ RSquare Adj } & \multicolumn{3}{|c|}{0.127434} \\
\hline \multicolumn{3}{|c|}{ Root Mean Square Error } & 0.019659 & & \\
\hline \multicolumn{3}{|c|}{ Mean of Response } & 0923 & & \\
\hline \multicolumn{3}{|c|}{ Observations (or Sum Wgts) } & \multicolumn{3}{|l|}{635} \\
\hline \multicolumn{6}{|c|}{ Analysis of Variance } \\
\hline Source & DF & Sum of Squares & \multicolumn{2}{|c|}{ Mean Square } & F Ratio \\
\hline Model & 314 & 0.15713476 & \multicolumn{2}{|c|}{0.000500} & 1.2949 \\
\hline Error & 320 & 0.12366967 & \multicolumn{2}{|c|}{0.000386} & Prob $>$ F \\
\hline C. Total & 634 & 0.28080443 & & & 0.0108 \\
\hline \multicolumn{6}{|c|}{ Variance Component Estimates } \\
\hline \multicolumn{3}{|c|}{ Component } & \multicolumn{3}{|c|}{ Percent of Total } \\
\hline \multicolumn{2}{|c|}{ Tank\&Batch\&Random } & \multicolumn{2}{|c|}{0.000057} & & 12.762 \\
\hline \multicolumn{2}{|l|}{ Residual } & \multicolumn{2}{|c|}{0.000386} & & 87.238 \\
\hline \multicolumn{2}{|l|}{ Total } & \multicolumn{3}{|c|}{0.000443} & 100.000 \\
\hline
\end{tabular}

Response Si (mg/L)

Summary of Fit

0.55291

RSquare Adj 0.114203

Root Mean Square Error $\quad 0.197776$

Mean of Response 0.111131

Observations (or Sum Wgts) 635

\begin{tabular}{|c|c|c|c|c|}
\hline \multicolumn{5}{|c|}{ Analysis of Variance } \\
\hline Source & DF & Sum of Squares & Mean Square & F Ratio \\
\hline Model & 314 & 15.479474 & 0.049298 & 1.2603 \\
\hline Error & 320 & 12.516892 & 0.039115 & Prob $>$ F \\
\hline C. Total & 634 & 27.996366 & & 0.0199 \\
\hline
\end{tabular}

Variance Component Estimates

$\begin{array}{lrr}\text { Component } & \text { Var Comp Est } & \text { Percent of Total } \\ \text { Tank\&Batch\&Random } & 0.005051 & 11.437 \\ \text { Residual } & 0.039115 & 88.563 \\ \text { Total } & 0.044167 & 100.000\end{array}$

$\begin{array}{lcr}\text { Total } & 0.044167 & 100.000 \\ \text { These estimates based on equating Mean Squares to Expected Value. }\end{array}$

Response Ti (mg/L)

Summary of Fit

$\begin{array}{lr}\text { RSquare } & 0.573782 \\ \text { RSquare Adj } & 0.155556 \\ \text { Root Mean Square Error } & 0.008967 \\ \text { Mean of Response } & 0.004639 \\ \text { Observations (or Sum Wgts) } & 635\end{array}$

\begin{tabular}{|c|c|c|c|c|}
\hline \multicolumn{5}{|c|}{ Analysis of Variance } \\
\hline Source & DF & Sum of Squares & Mean Square & F Ratio \\
\hline Model & 314 & 0.03463717 & 0.000110 & 1.3719 \\
\hline Error & 320 & 0.02572925 & 0.000080 & Prob $>$ F \\
\hline C. Total & 634 & 0.06036642 & & 0.0025 \\
\hline
\end{tabular}

Variance Component Estimates

$\begin{array}{lrr}\text { Component } & \text { Var Comp Est } & \text { Percent of Total } \\ \text { Tank\&Batch\&Random } & 0.000015 & 15.578 \\ \text { Residual } & 0.00008 & 84.422 \\ \text { Total } & 0.000095 & 100.000\end{array}$

$\begin{array}{lcc}\text { Total } & 0.000095 & 100.000 \\ \text { These estimates based on equating Mean Squares to Expected Value. }\end{array}$ 
WSRC-TR-2004-00576

Revision 0

Exhibit A24. Paired Comparisons of SM53 vs SM51 for Fusion Measurements
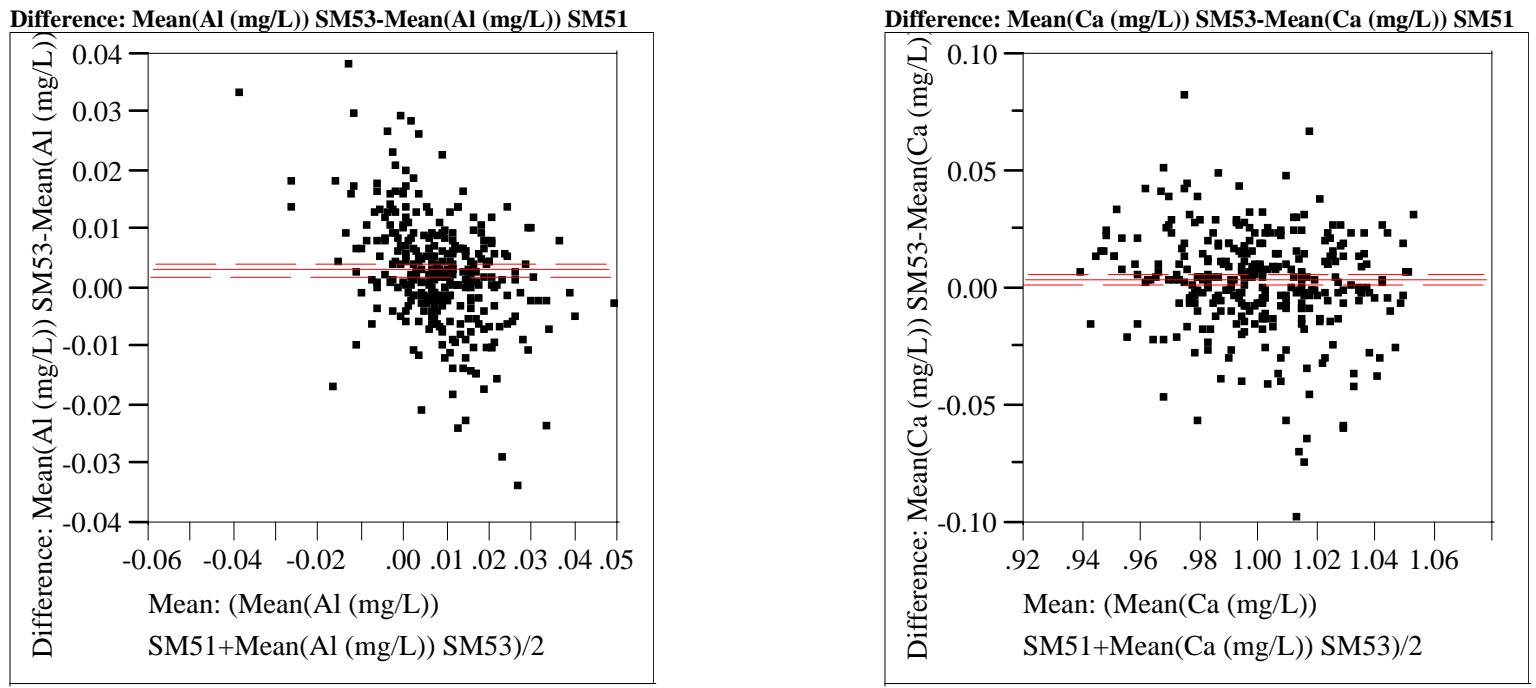

$\begin{array}{lrlr}\text { Mean(Al (mg/L)) SM53 } & 0.00986 & \text { t-Ratio } & 6.00785 \\ \text { Mean(Al (mg/L)) SM51 } & 0.00668 & \text { DF } & 316 \\ \text { Mean Difference } & 0.00318 & \text { Prob }>|t| & <.0001 \\ \text { Std Error } & 0.00053 & \text { Prob }>t & <.0001 \\ \text { Upper95\% } & 0.00422 & \text { Prob }<\mathrm{t} & 1.0000 \\ \text { Lower95\% } & 0.00214 & & \\ \text { N } & 317 & & \\ \text { Correlation } & 0.7309 & & \end{array}$

$\begin{array}{lrlr}\text { Mean(Ca (mg/L)) SM53 } & 1.00173 & \text { t-Ratio } & 3.068298 \\ \text { Mean(Ca (mg/L)) SM51 } & 0.99797 & \text { DF } & 316 \\ \text { Mean Difference } & 0.00376 & \text { Prob }>|t| & 0.0023 \\ \text { Std Error } & 0.00122 & \text { Prob }>\text { t } & 0.0012 \\ \text { Upper95\% } & 0.00616 & \text { Prob }<\mathrm{t} & 0.9988 \\ \text { Lower95\% } & 0.00135 & & \\ \text { N } & 317 & & \\ \text { Correlation } & 0.65821 & & \end{array}$

Difference: Mean(B (mg/L)) SM53-Mean(B (mg/L)) SM51

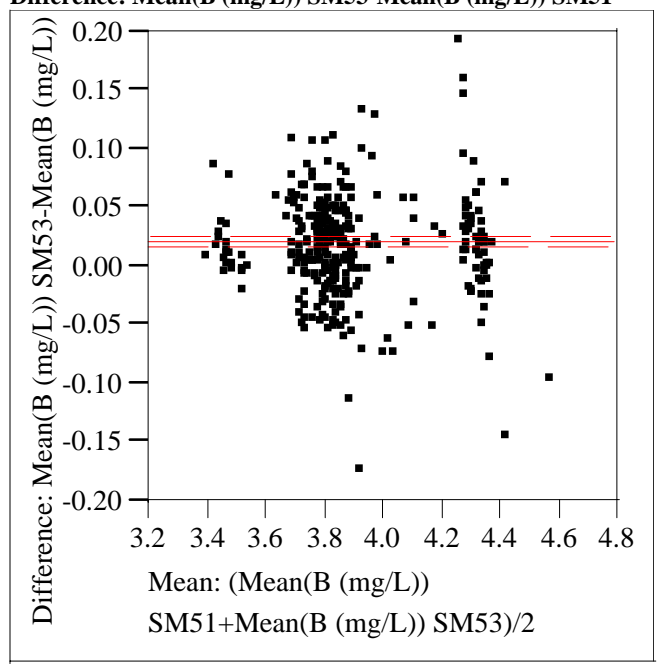

Difference: Mean(Cr (mg/L)) SM53-Mean(Cr (mg/L)) SM51

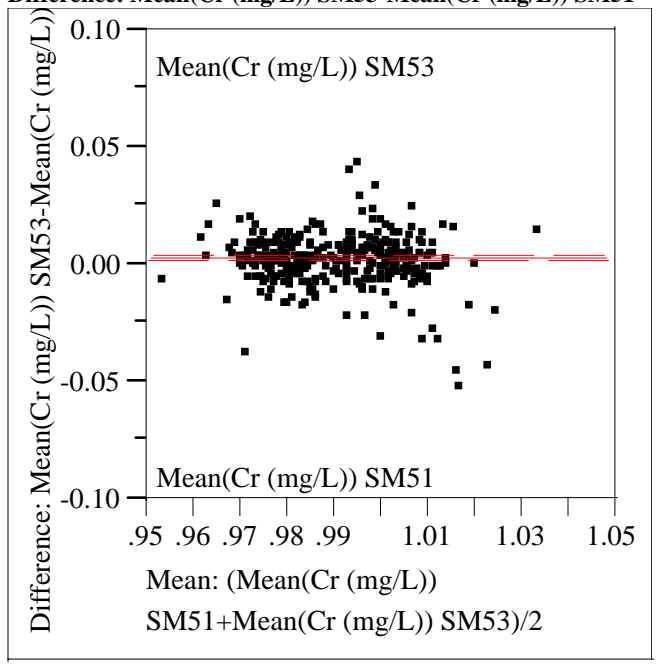

$\begin{array}{lrlr}\text { Mean(B (mg/L)) SM53 } & 3.90127 & \mathrm{t}-\text { Ratio } & 8.461604 \\ \text { Mean(B (mg/L)) SM51 } & 3.88062 & \text { DF } & 316 \\ \text { Mean Difference } & 0.02066 & \text { Prob }>|\mathrm{t}| & <.0001 \\ \text { Std Error } & 0.00244 & \text { Prob }>\mathrm{t} & <.0001 \\ \text { Upper95\% } & 0.02546 & \text { Prob }<\mathrm{t} & 1.0000 \\ \text { Lower95\% } & 0.01585 & & \\ \mathrm{~N} & 317 & & \\ \text { Correlation } & 0.98346 & & \end{array}$

$\begin{array}{lrlr}\text { Mean(Cr (mg/L)) SM53 } & 0.99211 & \text { t-Ratio } & 4.714181 \\ \text { Mean(Cr (mg/L)) SM51 } & 0.98913 & \text { DF } & 316 \\ \text { Mean Difference } & 0.00297 & \text { Prob }>|t| & <.0001 \\ \text { Std Error } & 0.00063 & \text { Prob }>t & <.0001 \\ \text { Upper95\% } & 0.00421 & \text { Prob }<\mathrm{t} & 1.0000 \\ \text { Lower95\% } & 0.00173 & & \\ \text { N } & 317 & & \\ \text { Correlation } & 0.70515 & & \end{array}$


WSRC-TR-2004-00576

Revision 0

\section{Exhibit A24. Paired Comparisons of SM53 vs SM51 for Fusion Measurements}

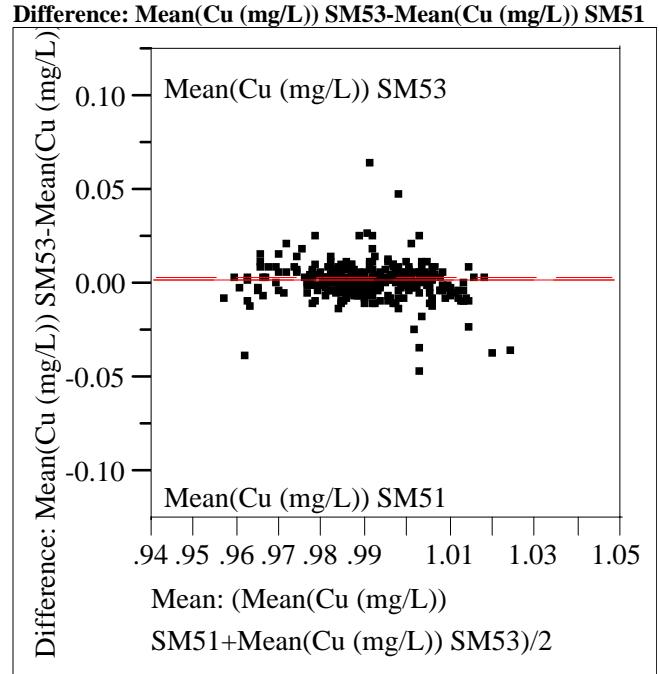

$\begin{array}{lrlr}\text { Mean(Cu (mg/L)) SM53 } & 0.99183 & \text { t-Ratio } & 4.740339 \\ \text { Mean(Cu (mg/L)) SM51 } & 0.98919 & \text { DF } & 316 \\ \text { Mean Difference } & 0.00263 & \text { Prob }>|t| & <.0001 \\ \text { Std Error } & 0.00056 & \text { Prob }>t & <.0001 \\ \text { Upper95\% } & 0.00373 & \text { Prob }<\mathrm{t} & 1.0000 \\ \text { Lower95\% } & 0.00154 & & \\ \text { N } & 317 & & \\ \text { Correlation } & 0.72872 & & \end{array}$

Difference: Mean(Fe (mg/L)) SM53-Mean(Fe (mg/L)) SM51

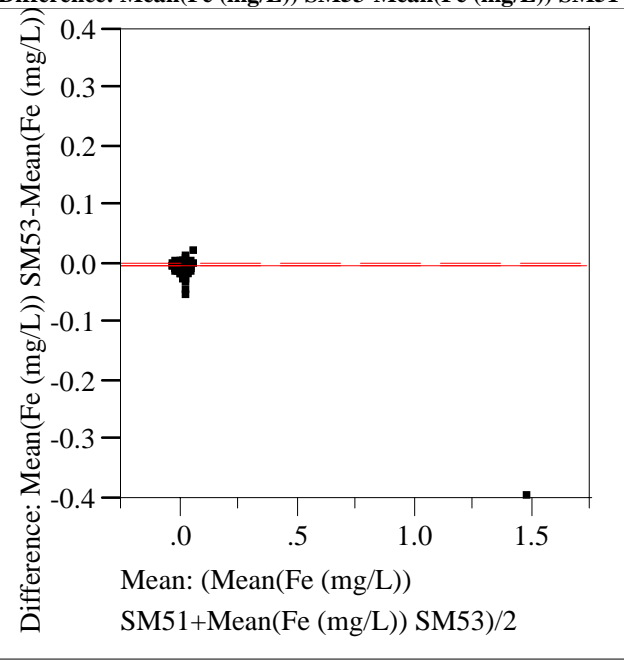

Mean(Fe (mg/L)) SM53

Mean(Fe (mg/L)) SM51 0.00999

Mean Difference

Std Error

Upper95\%

Lower95\%

Correlation
t-Ratio $\quad-1.5284$

Prob $>|t| \quad 0.1274$

Prob $>\mathrm{t} \quad 0.9363$

Prob $<\mathrm{t} \quad 0.0637$
DF $\quad 316$
Difference: Mean(K (mg/L)) SM53-Mean(K (mg/L)) SM51

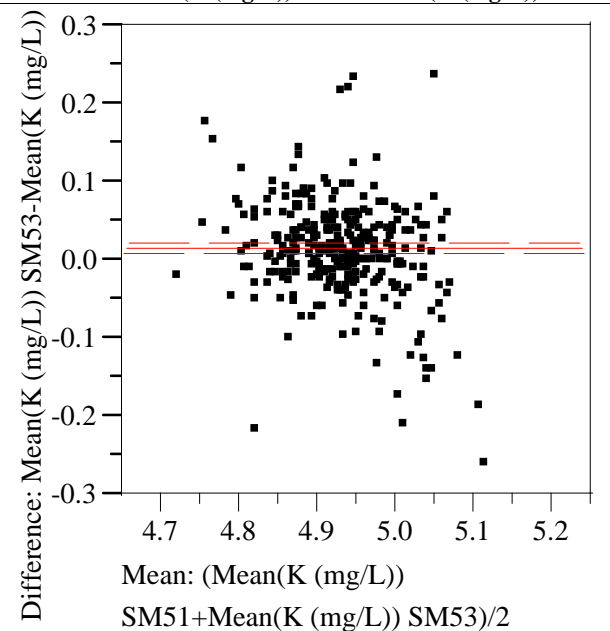

$\begin{array}{lrlr}\text { Mean(K (mg/L)) SM53 } & 4.9381 & \text { t-Ratio } & 4.652804 \\ \text { Mean(K (mg/L)) SM51 } & 4.92172 & \text { DF } & 316 \\ \text { Mean Difference } & 0.01638 & \text { Prob }>|t| & <.0001 \\ \text { Std Error } & 0.00352 & \text { Prob }>t & <.0001 \\ \text { Upper95\% } & 0.02331 & \text { Prob }<\mathrm{t} & 1.0000 \\ \text { Lower95\% } & 0.00945 & & \\ \text { N } & 317 & & \\ \text { Correlation } & 0.64919 & & \end{array}$

Difference: Mean(Li (mg/L)) SM53-Mean(Li (mg/L)) SM51

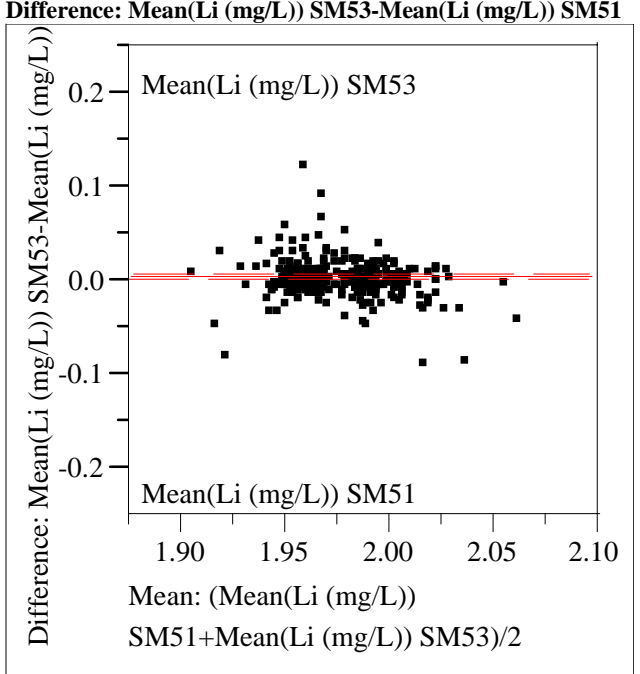

Mean(Li (mg/L)) SM53

Mean(Li (mg/L)) SM51 1.97428

Mean Difference $\quad 0.00491$

Std Error

Upper95\%

Lower95\%

$\mathrm{N}$

Correlation 
WSRC-TR-2004-00576

Revision 0

Exhibit A24. Paired Comparisons of SM53 vs SM51 for Fusion Measurements
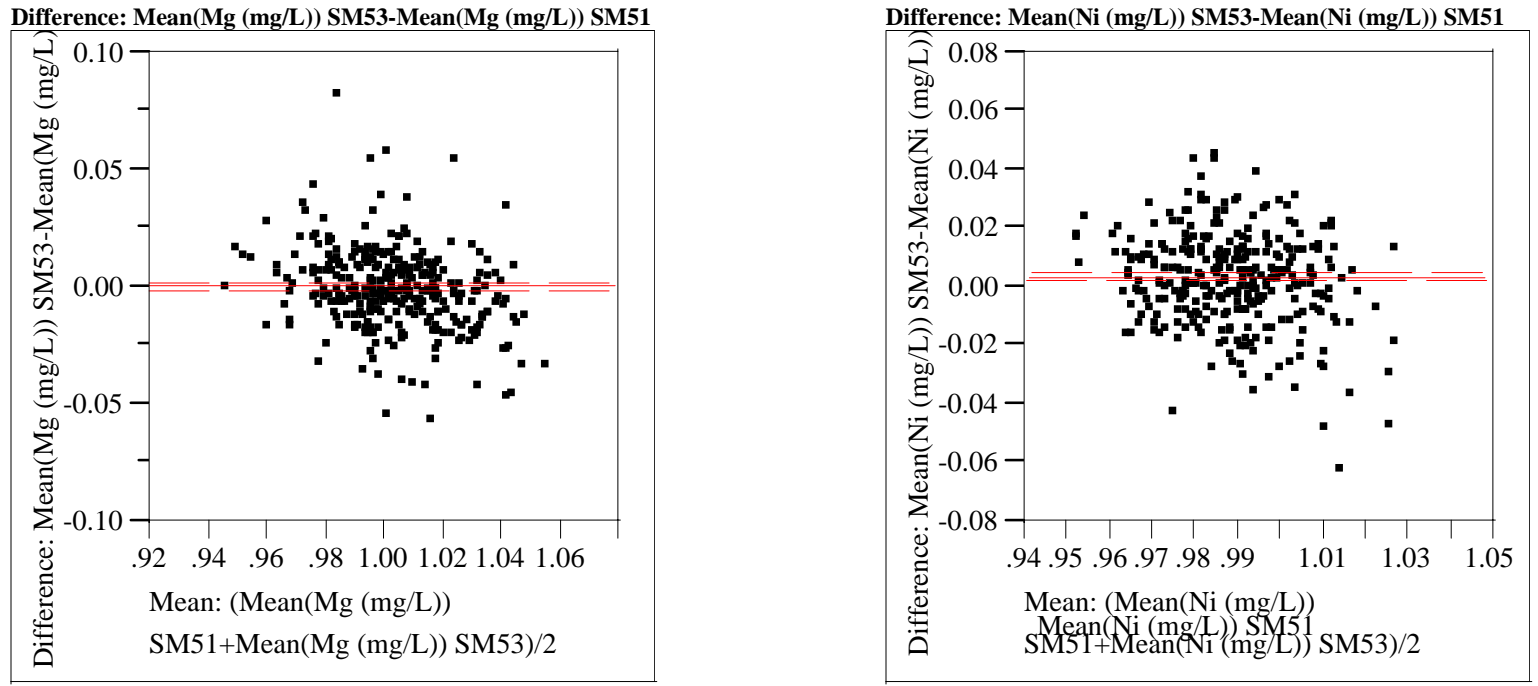

$\begin{array}{lrlr}\text { Mean }(\mathrm{Mg}(\mathrm{mg} / \mathrm{L})) \text { SM53 } & 1.00324 & \mathrm{t}-\text { Ratio } & 0.046533 \\ \text { Mean(Mg (mg/L)) SM51 } & 1.0032 & \text { DF } & 316 \\ \text { Mean Difference } & 0.00004 & \text { Prob }>|\mathrm{t}| & 0.9629 \\ \text { Std Error } & 0.00097 & \text { Prob }>\mathrm{t} & 0.4815 \\ \text { Upper95\% } & 0.00195 & \text { Prob }<\mathrm{t} & 0.5185 \\ \text { Lower95\% } & -0.0019 & & \\ \text { N } & 317 & & \\ \text { Correlation } & 0.70106 & & \\ \end{array}$

Difference: Mean(Mn (mg/L)) SM53-Mean(Mn (mg/L)) SM51

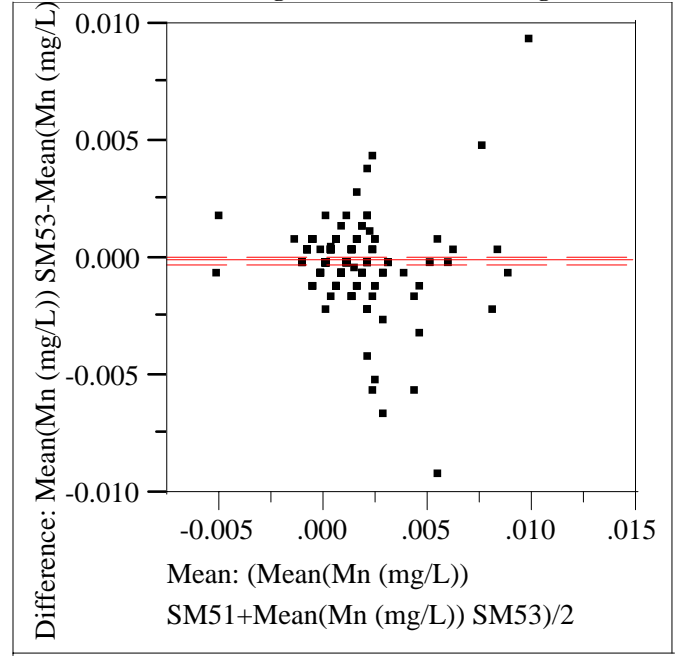

$\begin{array}{lrlr}\text { Mean(Ni (mg/L)) SM53 } & 0.99014 & \text { t-Ratio } & 3.977338 \\ \text { Mean(Ni (mg/L)) SM51 } & 0.98661 & \text { DF } & 316 \\ \text { Mean Difference } & 0.00353 & \text { Prob }>|t| & <.0001 \\ \text { Std Error } & 0.00089 & \text { Prob }>t & <.0001 \\ \text { Upper95\% } & 0.00528 & \text { Prob }<\mathrm{t} & 1.0000 \\ \text { Lower95\% } & 0.00179 & & \\ \text { N } & 317 & & \\ \text { Correlation } & 0.54352 & & \end{array}$

Difference: Mean(Si (mg/L)) SM53-Mean(Si (mg/L)) SM51

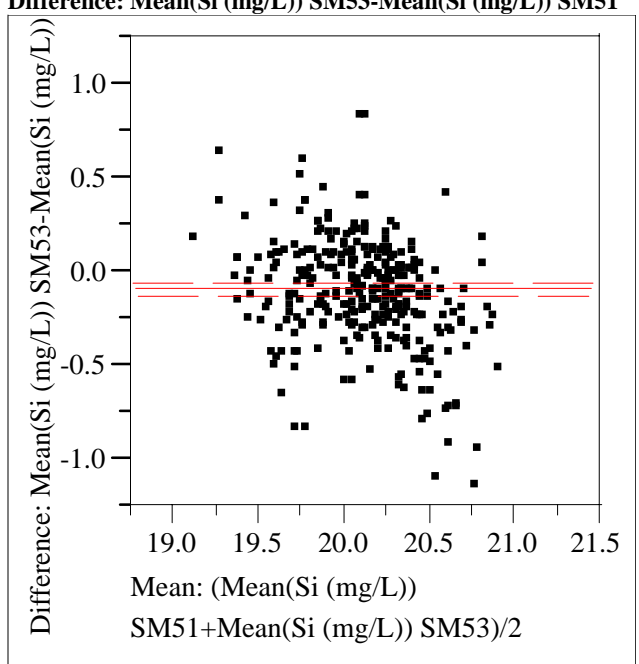

$\begin{array}{lrlr}\text { Mean(Mn (mg/L)) SM53 } & 0.0008 & \mathrm{t}-\text { Ratio } & -1.24501 \\ \text { Mean(Mn (mg/L)) SM51 } & 0.00089 & \text { DF } & 316 \\ \text { Mean Difference } & -9.3 \mathrm{e}-5 & \text { Prob }>|\mathrm{t}| & 0.2141 \\ \text { Std Error } & 0.00007 & \text { Prob }>\mathrm{t} & 0.8930 \\ \text { Upper95\% } & 0.00005 & \text { Prob }<\mathrm{t} & 0.1070 \\ \text { Lower95\% } & -0.0002 & & \\ \text { N } & 317 & & \\ \text { Correlation } & 0.71957 & & \end{array}$

$\begin{array}{lrlr}\text { Mean(Si (mg/L)) SM53 } & 20.063 & \text { t-Ratio } & -5.9821 \\ \text { Mean(Si (mg/L)) SM51 } & 20.1574 & \text { DF } & 316 \\ \text { Mean Difference } & -0.0944 & \text { Prob }>|t| & <.0001 \\ \text { Std Error } & 0.01578 & \text { Prob }>\text { t } & 1.0000 \\ \text { Upper95\% } & -0.0634 & \text { Prob }<\text { t } & <.0001 \\ \text { Lower95\% } & -0.1254 & & \\ \text { N } & 317 & & \\ \text { Correlation } & 0.71814 & & \end{array}$


WSRC-TR-2004-00576

Revision 0

Exhibit A24. Paired Comparisons of SM53 vs SM51 for Fusion Measurements

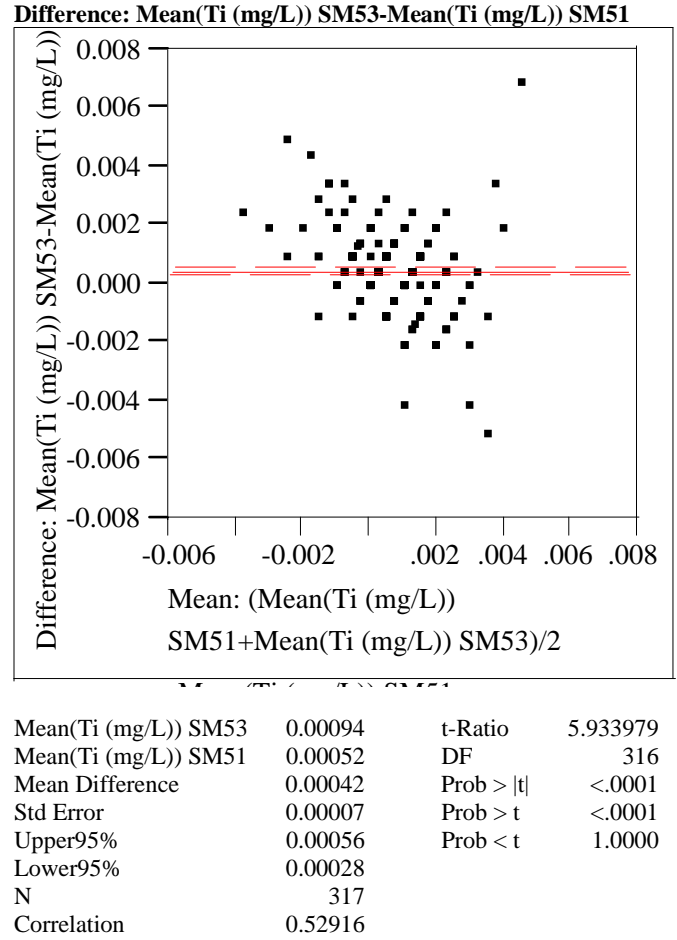


WSRC-TR-2004-00576

Revision 0

\section{Exhibit A25. Paired Comparisons of SM54 vs SM52 for Fusion Measurements}

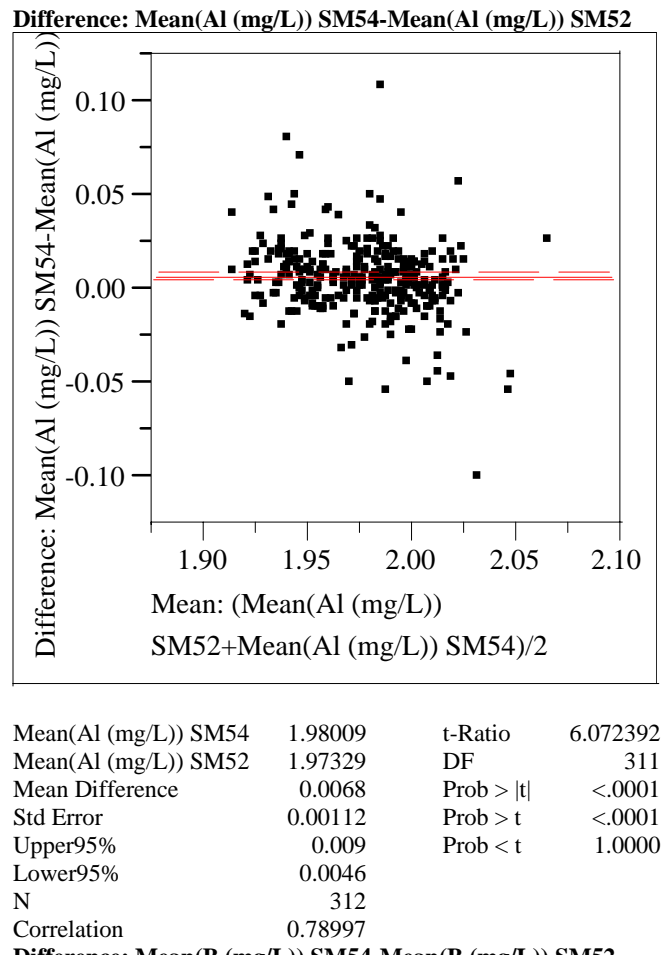

Difference: Mean(B (mg/L)) SM54-Mean(B (mg/L)) SM52

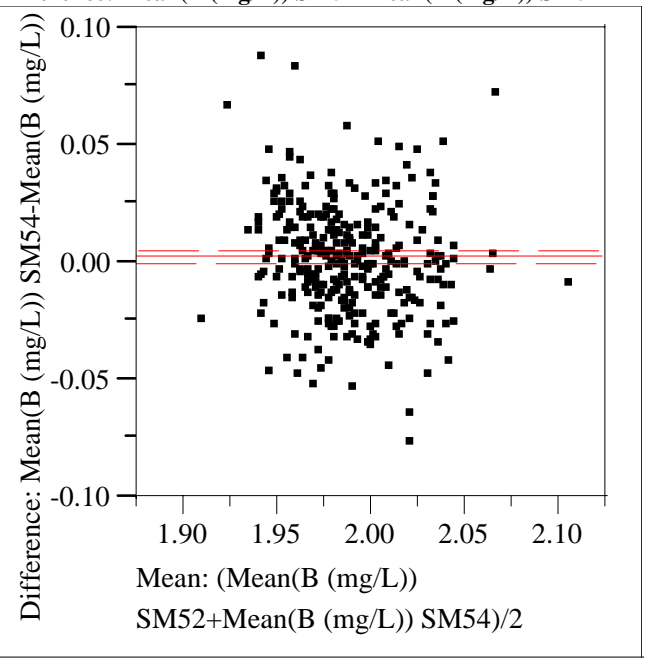

$\begin{array}{lrlr}\text { Mean(B (mg/L)) SM54 } & 1.98862 & \text { t-Ratio } & 1.762773 \\ \text { Mean(B (mg/L)) SM52 } & 1.98628 & \text { DF } & 311 \\ \text { Mean Difference } & 0.00234 & \text { Prob }>|t| & 0.0789 \\ \text { Std Error } & 0.00133 & \text { Prob }>\mathrm{t} & 0.0395 \\ \text { Upper95\% } & 0.00496 & \text { Prob }<\mathrm{t} & 0.9605 \\ \text { Lower95\% } & -0.0003 & & \\ \text { N } & 312 & & \\ \text { Correlation } & 0.71103 & & \end{array}$

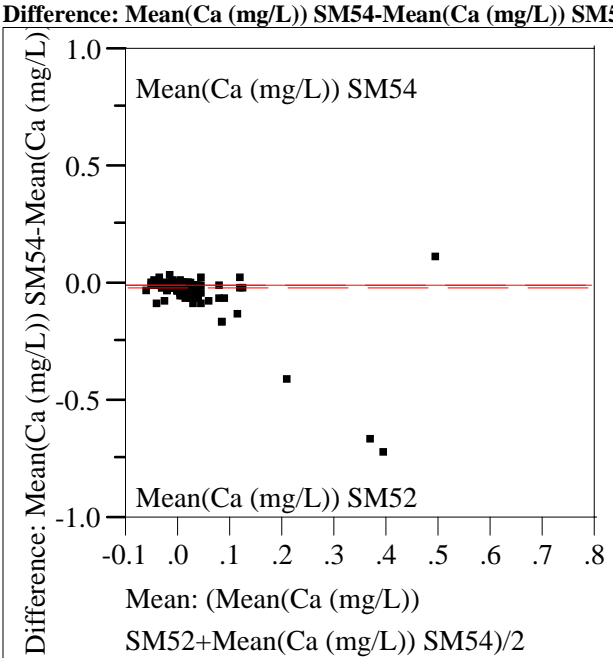

$\begin{array}{lrlr}\text { Mean(Ca (mg/L)) SM54 } & 0.00571 & \text { t-Ratio } & -2.50136 \\ \text { Mean(Ca (mg/L)) SM52 } & 0.01448 & \text { DF } & 311 \\ \text { Mean Difference } & -0.0088 & \text { Prob }>|t| & 0.0129 \\ \text { Std Error } & 0.00351 & \text { Prob }>\mathrm{t} & 0.9936 \\ \text { Upper95\% } & -0.0019 & \text { Prob }<\mathrm{t} & 0.0064 \\ \text { Lower95\% } & -0.0157 & & \\ \text { N } & 312 & & \\ \text { Correlation } & 0.50328 & & \end{array}$

Difference: Mean(Cr (mg/L)) SM54-Mean(Cr (mg/L)) SM52

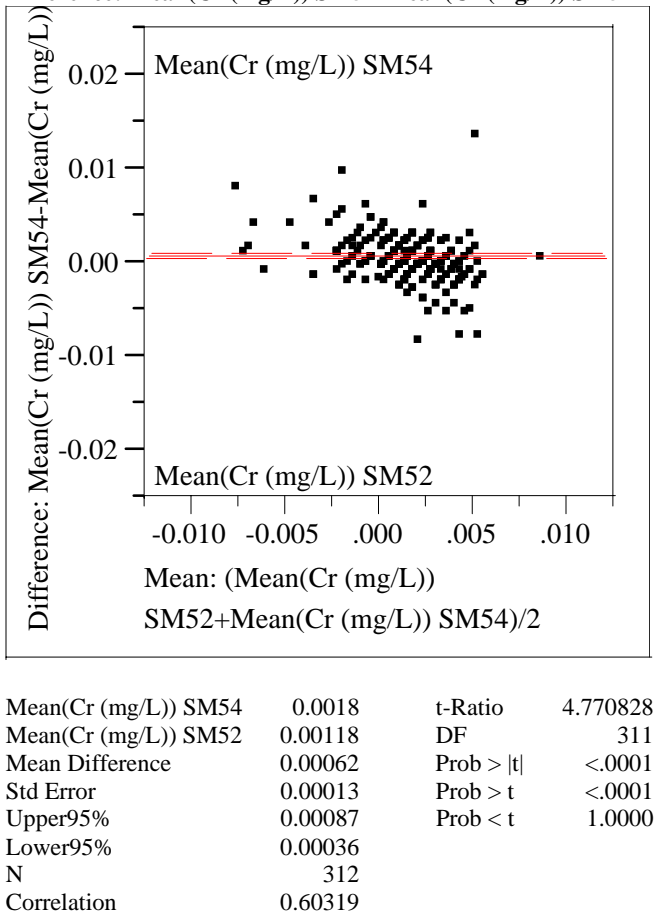


WSRC-TR-2004-00576

Revision 0

\section{Exhibit A25. Paired Comparisons of SM54 vs SM52 for Fusion Measurements}
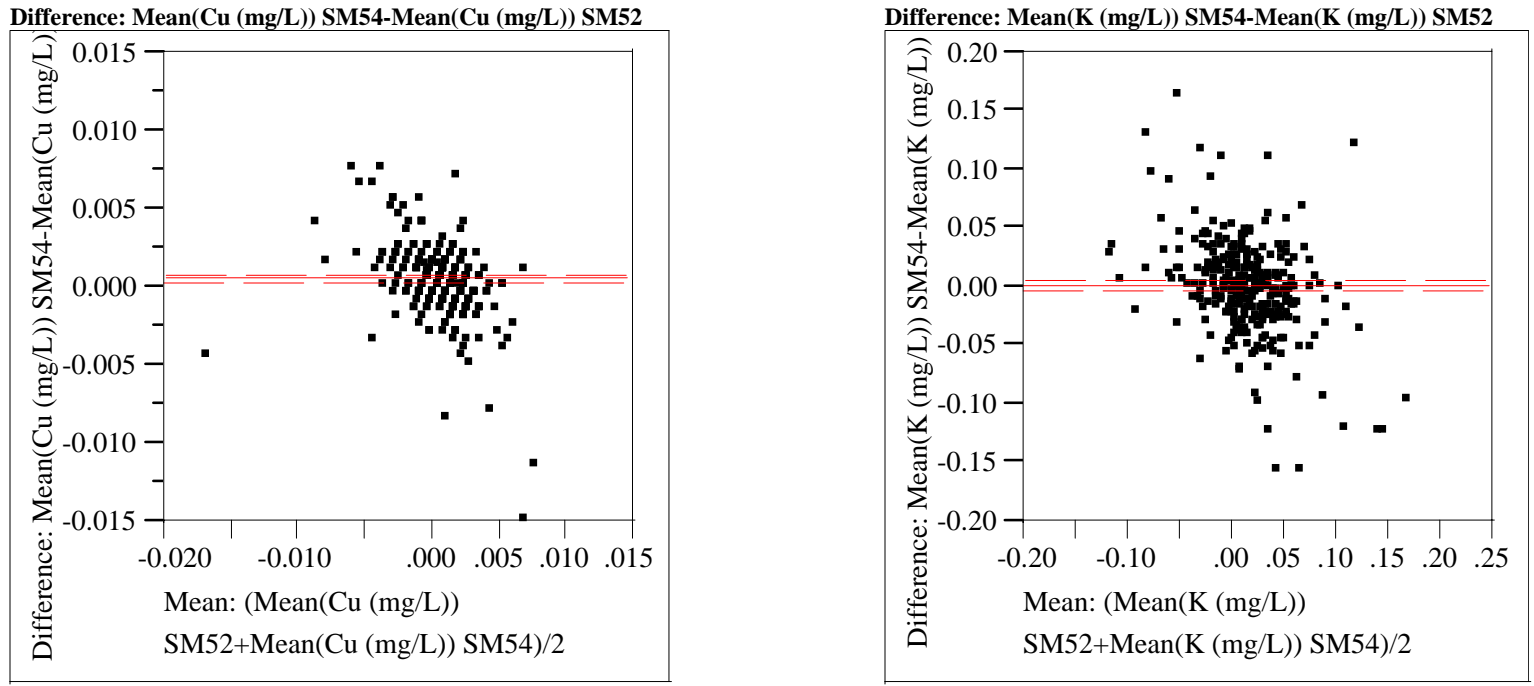

$\begin{array}{lrlr}\text { Mean }(\mathrm{Cu}(\mathrm{mg} / \mathrm{L})) \text { SM54 } & 0.0008 & \mathrm{t}-\text { Ratio } & 4.231774 \\ \text { Mean }(\mathrm{Cu}(\mathrm{mg} / \mathrm{L})) \text { SM52 } & 0.00025 & \text { DF } & 311 \\ \text { Mean Difference } & 0.00056 & \text { Prob }>|\mathrm{t}| & <.0001 \\ \text { Std Error } & 0.00013 & \text { Prob }>\mathrm{t} & <.0001 \\ \text { Upper95\% } & 0.00081 & \text { Prob }<\mathrm{t} & 1.0000 \\ \text { Lower95\% } & 0.0003 & & \\ \text { N } & 312 & & \\ \text { Correlation } & 0.65834 & & \end{array}$

Difference: Mean(Fe (mg/L)) SM54-Mean(Fe (mg/L)) SM52

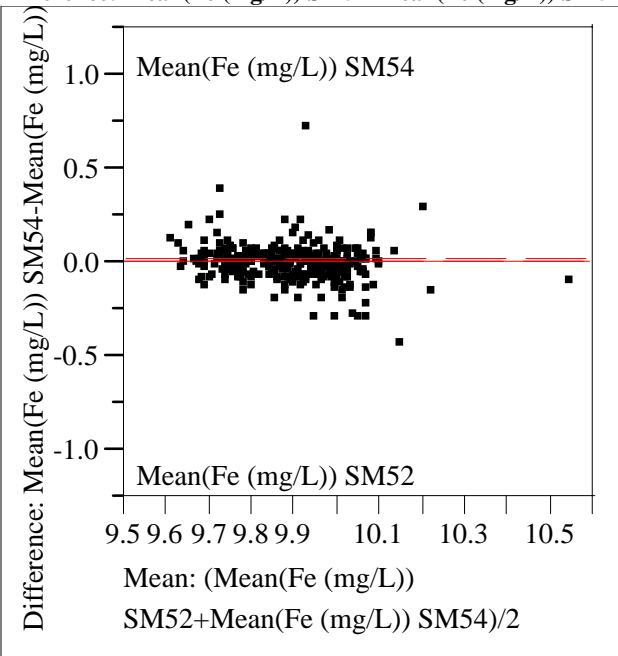

$\begin{array}{lrlr}\text { Mean(K (mg/L)) SM54 } & 0.01486 & \text { t-Ratio } & 0.670486 \\ \text { Mean(K (mg/L)) SM52 } & 0.01335 & \text { DF } & 311 \\ \text { Mean Difference } & 0.00151 & \text { Prob }>|t| & 0.5030 \\ \text { Std Error } & 0.00226 & \text { Prob }>t & 0.2515 \\ \text { Upper95\% } & 0.00596 & \text { Prob }<\mathrm{t} & 0.7485 \\ \text { Lower95\% } & -0.0029 & & \\ \text { N } & 312 & & \\ \text { Correlation } & 0.61011 & & \end{array}$

Difference: Mean(Li (mg/L)) SM54-Mean(Li (mg/L)) SM52

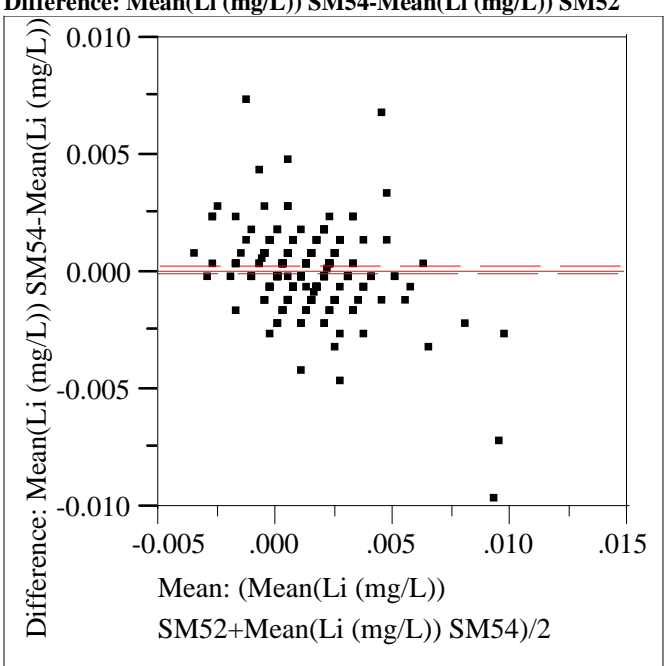

$\begin{array}{lrlr}\text { Mean(Fe (mg/L)) SM54 } & 9.89664 & \text { t-Ratio } & 2.241425 \\ \text { Mean(Fe (mg/L)) SM52 } & 9.88419 & \text { DF } & 311 \\ \text { Mean Difference } & 0.01245 & \text { Prob }>|t| & 0.0257 \\ \text { Std Error } & 0.00556 & \text { Prob }>\text { t } & 0.0129 \\ \text { Upper95\% } & 0.02339 & \text { Prob }<\mathrm{t} & 0.9871 \\ \text { Lower95\% } & 0.00152 & & \\ \text { N } & 312 & & \\ \text { Correlation } & 0.74436 & & \end{array}$

$\begin{array}{lrlr}\text { Mean(Li (mg/L)) SM54 } & 0.00129 & \text { t-Ratio } & 0.856487 \\ \text { Mean(Li (mg/L)) SM52 } & 0.00121 & \text { DF } & 311 \\ \text { Mean Difference } & 0.00007 & \text { Prob }>|t| & 0.3924 \\ \text { Std Error } & 0.00009 & \text { Prob }>t & 0.1962 \\ \text { Upper95\% } & 0.00024 & \text { Prob }<\mathrm{t} & 0.8038 \\ \text { Lower95\% } & -9.5 \mathrm{e}-5 & & \\ \text { N } & 312 & & \\ \text { Correlation } & 0.70833 & & \end{array}$


WSRC-TR-2004-00576

Revision 0

\section{Exhibit A25. Paired Comparisons of SM54 vs SM52 for Fusion Measurements}
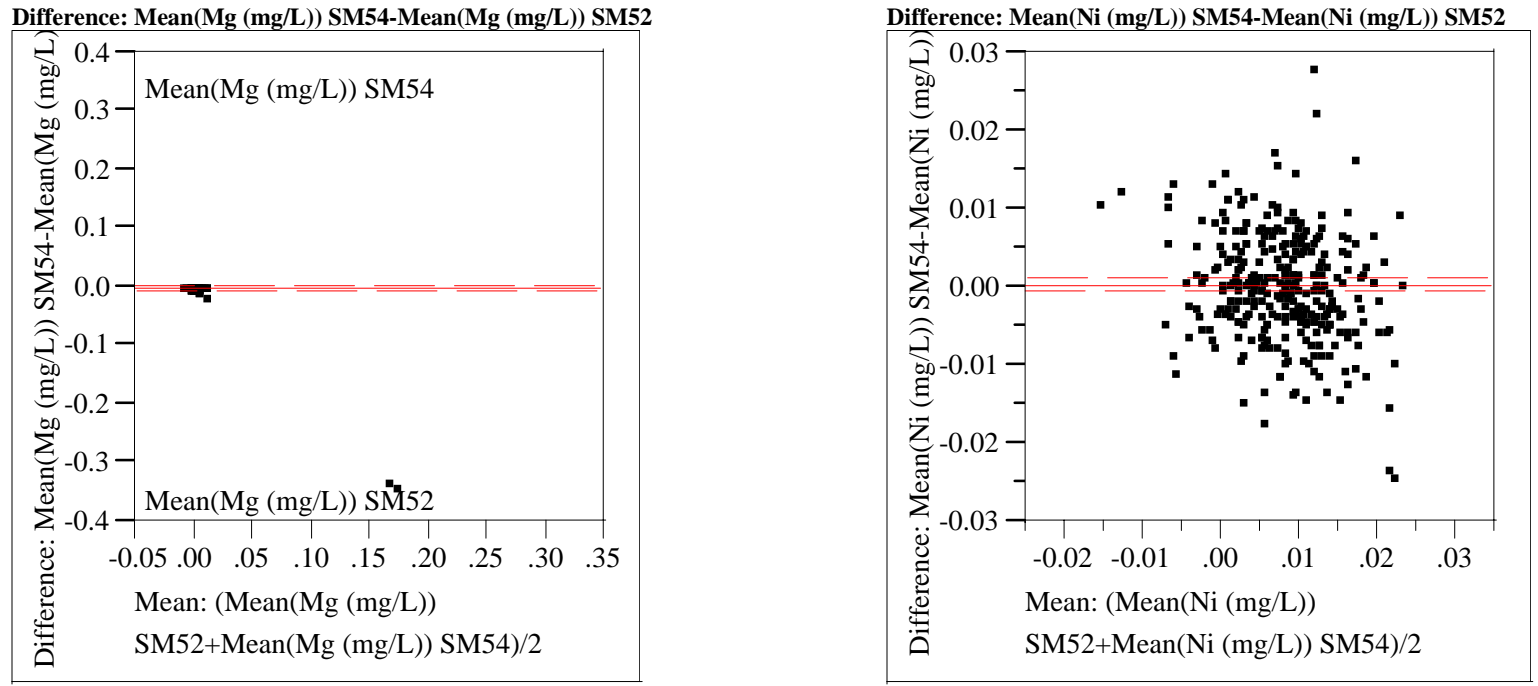

$\begin{array}{lrlr}\text { Mean(Mg (mg/L)) SM54 } & 0.00078 & \text { t-Ratio } & -1.6184 \\ \text { Mean(Mg (mg/L)) SM52 } & 0.00324 & \text { DF } & 311 \\ \text { Mean Difference } & -0.0025 & \text { Prob }>|t| & 0.1066 \\ \text { Std Error } & 0.00152 & \text { Prob }>t & 0.9467 \\ \text { Upper95\% } & 0.00053 & \text { Prob }<\mathrm{t} & 0.0533 \\ \text { Lower95\% } & -0.0055 & & \\ \text { N } & 312 & & \\ \text { Correlation } & 0.14638 & & \end{array}$

Difference: Mean(Mn (mg/L)) SM54-Mean(Mn (mg/L)) SM52

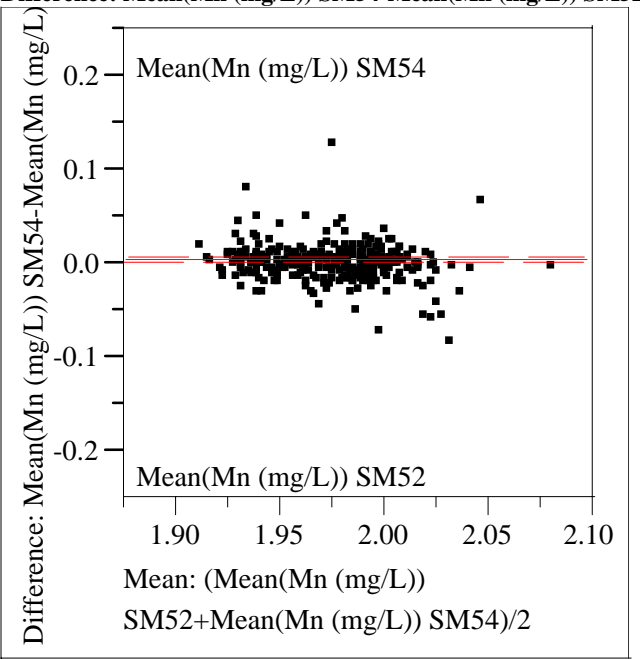

$\begin{array}{lrlr}\text { Mean(Mn (mg/L)) SM54 } & 1.97641 & \text { t-Ratio } & 3.806688 \\ \text { Mean(Mn (mg/L)) SM52 } & 1.97222 & \text { DF } & 311 \\ \text { Mean Difference } & 0.00419 & \text { Prob }>|t| & 0.0002 \\ \text { Std Error } & 0.0011 & \text { Prob }>t & <.0001 \\ \text { Upper95\% } & 0.00635 & \text { Prob }<\mathrm{t} & 0.9999 \\ \text { Lower95\% } & 0.00202 & & \\ \text { N } & 312 & & \\ \text { Correlation } & 0.79156 & & \end{array}$

$\begin{array}{lrlr}\text { Mean(Ni (mg/L)) SM54 } & 0.008 & \text { t-Ratio } & 0.648544 \\ \text { Mean(Ni (mg/L)) SM52 } & 0.00774 & \text { DF } & 311 \\ \text { Mean Difference } & 0.00025 & \text { Prob }>|t| & 0.5171 \\ \text { Std Error } & 0.00039 & \text { Prob }>\mathrm{t} & 0.2586 \\ \text { Upper95\% } & 0.00103 & \text { Prob }<\mathrm{t} & 0.7414 \\ \text { Lower95\% } & -0.0005 & & \\ \text { N } & 312 & & \\ \text { Correlation } & 0.56474 & & \end{array}$

Difference: Mean(Si (mg/L)) SM54-Mean(Si (mg/L)) SM52

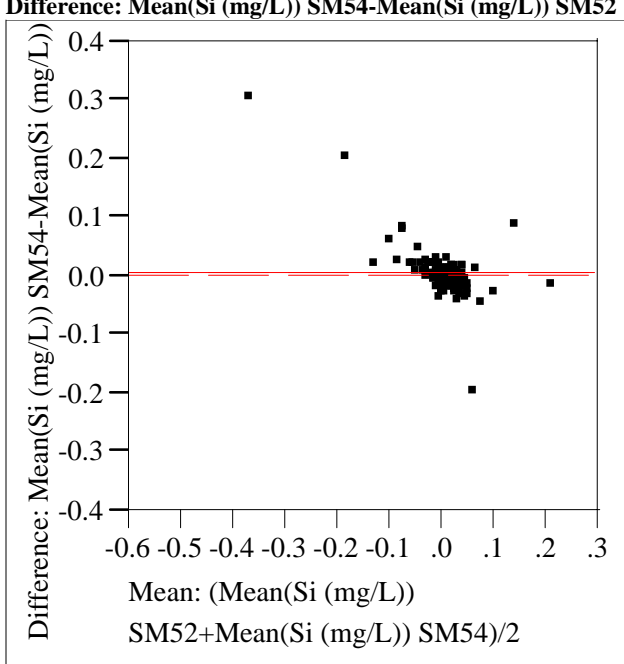

$\begin{array}{lrlr}\text { Mean(Si (mg/L)) SM54 } & 0.01117 & \text { t-Ratio } & 3.17119 \\ \text { Mean(Si (mg/L)) SM52 } & 0.00613 & \text { DF } & 311 \\ \text { Mean Difference } & 0.00504 & \text { Prob }>|t| & 0.0017 \\ \text { Std Error } & 0.00159 & \text { Prob }>\text { t } & 0.0008 \\ \text { Upper95\% } & 0.00817 & \text { Prob }<\mathrm{t} & 0.9992 \\ \text { Lower95\% } & 0.00191 & & \\ \text { N } & 312 & & \\ \text { Correlation } & 0.8293 & & \end{array}$


WSRC-TR-2004-00576

Revision 0

Exhibit A25. Paired Comparisons of SM54 vs SM52 for Fusion Measurements

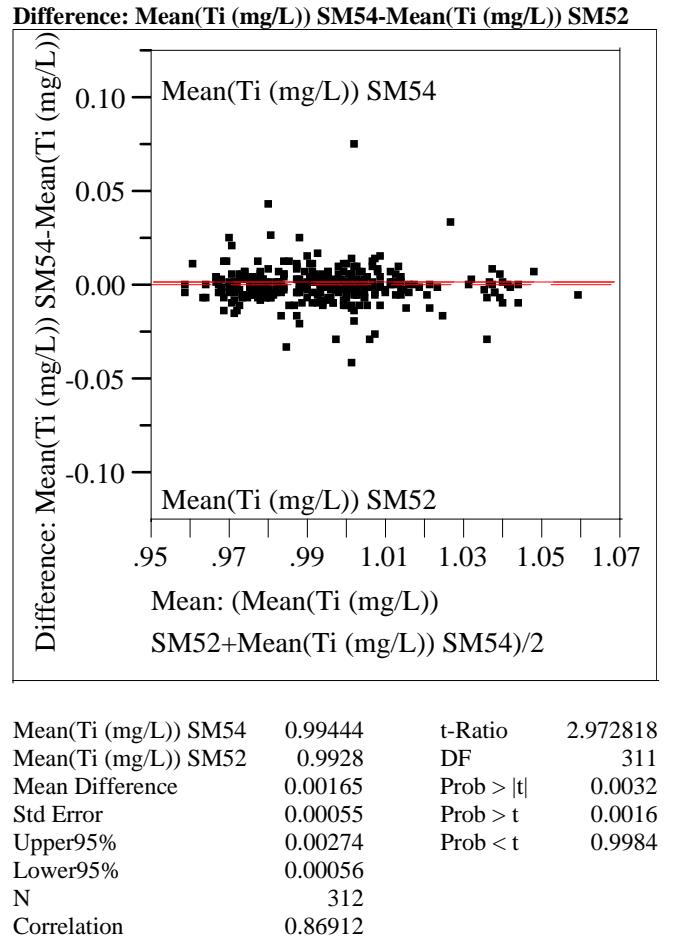


Exhibit A26. Time Series Plot (Control Charts) for ARG-1 Measurements Using Fusion Prep
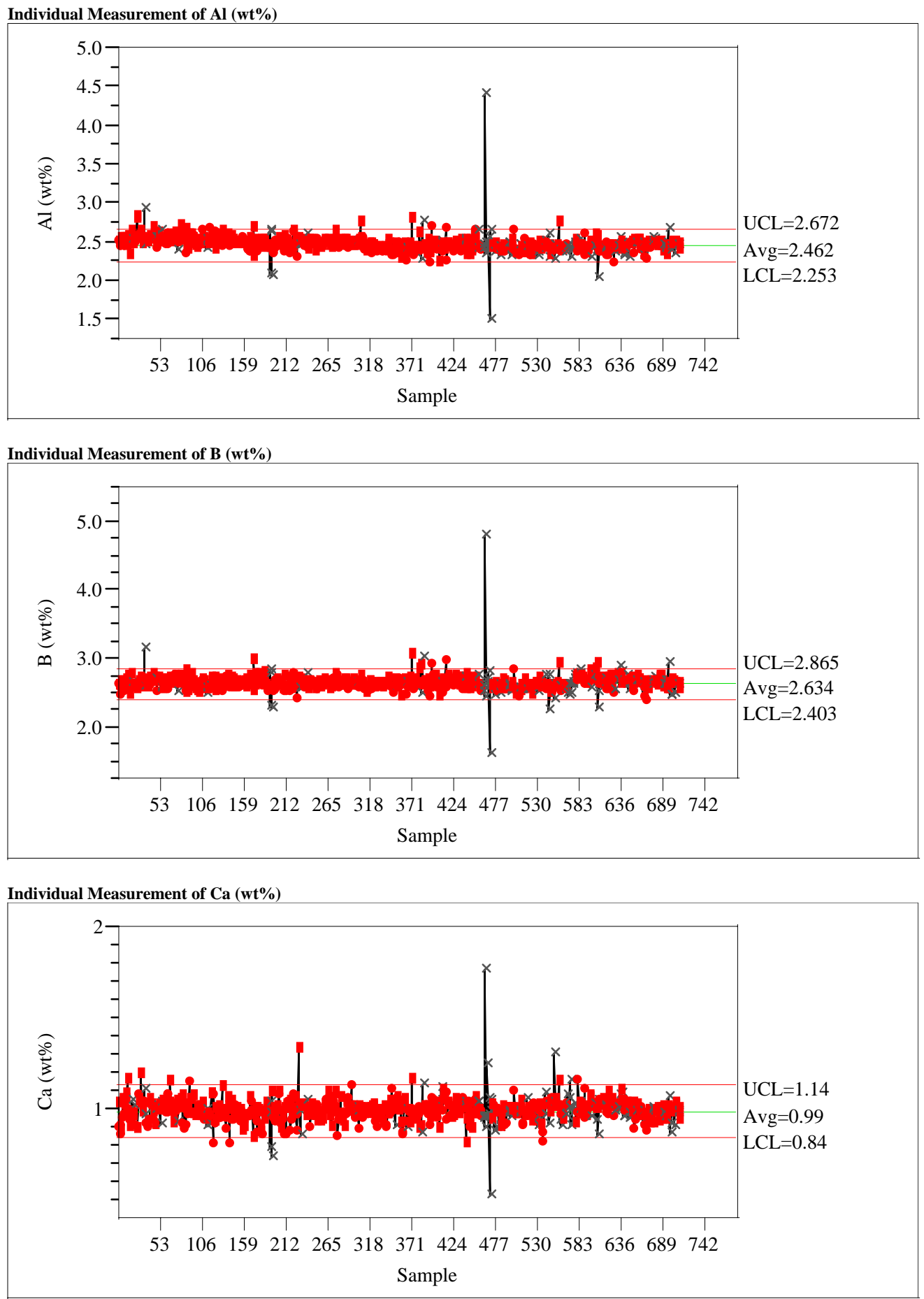
WSRC-TR-2004-00576

Revision 0

Exhibit A26. Time Series Plot (Control Charts) for ARG-1 Measurements Using Fusion Prep

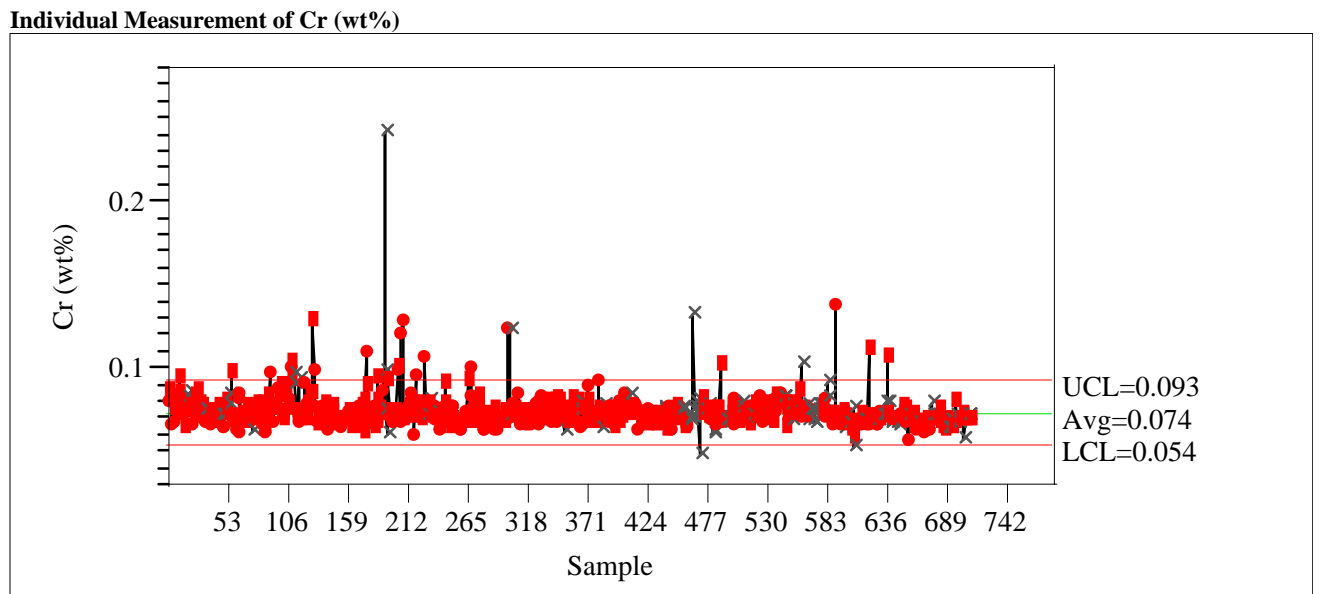

Individual Measurement of $\mathrm{Cu}(\mathrm{wt} \%)$

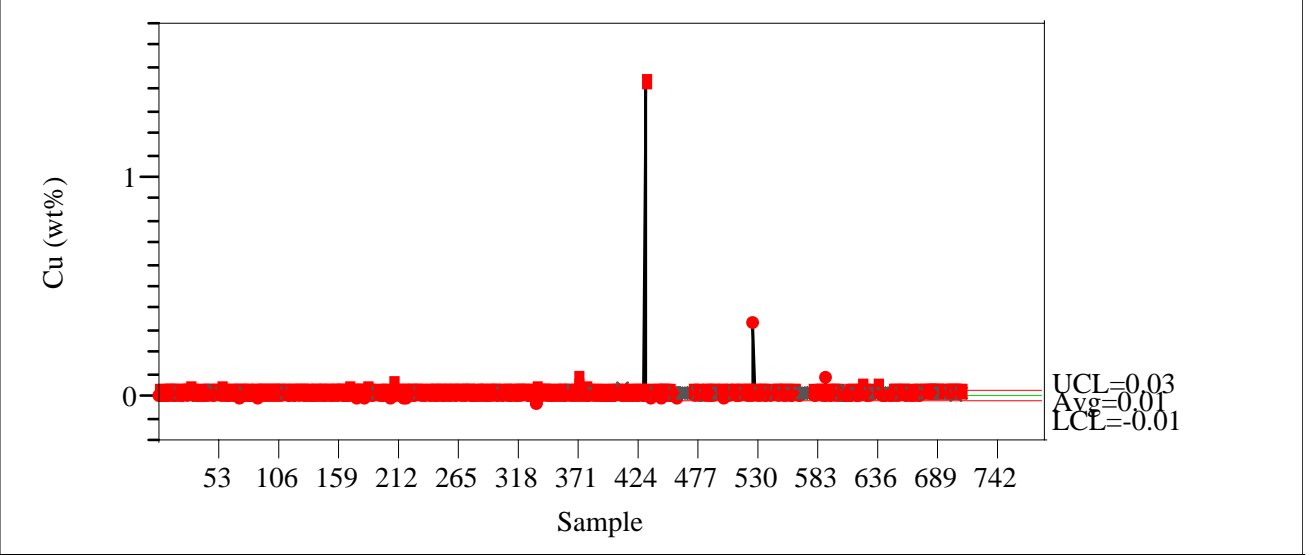

Individual Measurement of Fe (wt \%)

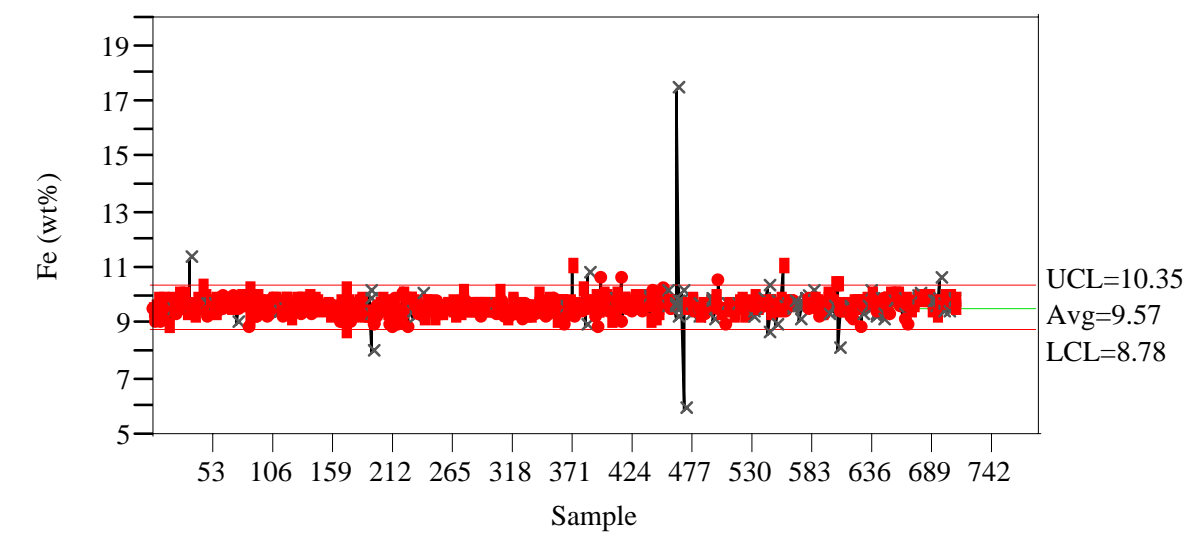


WSRC-TR-2004-00576

Revision 0

Exhibit A26. Time Series Plot (Control Charts) for ARG-1 Measurements Using Fusion Prep

Individual Measurement of $\mathrm{K}(\mathrm{wt} \%)$

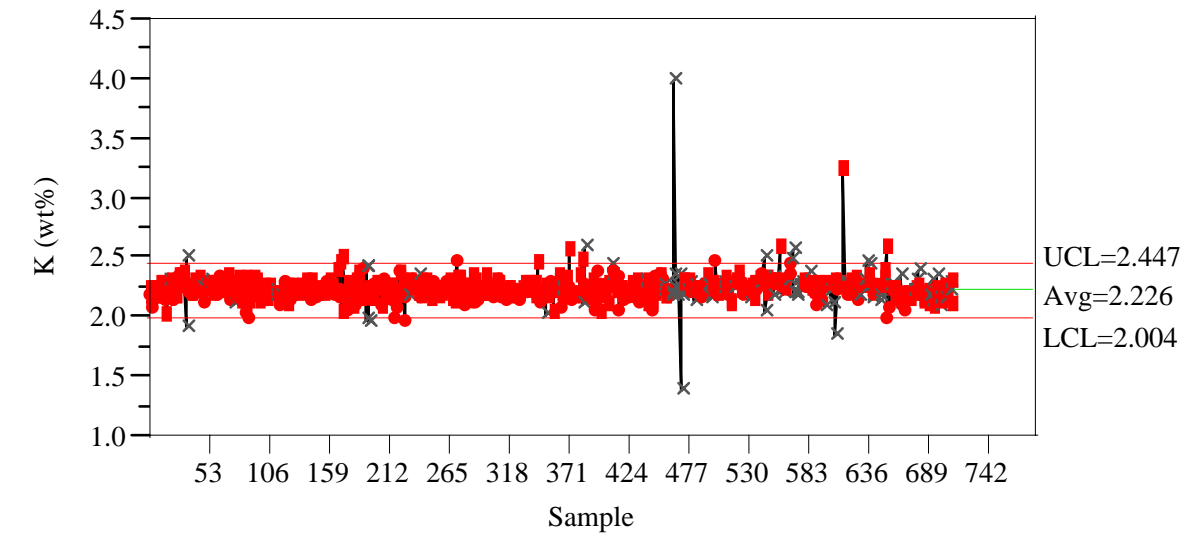

Individual Measurement of $\mathrm{Li}(\mathrm{wt} \%)$

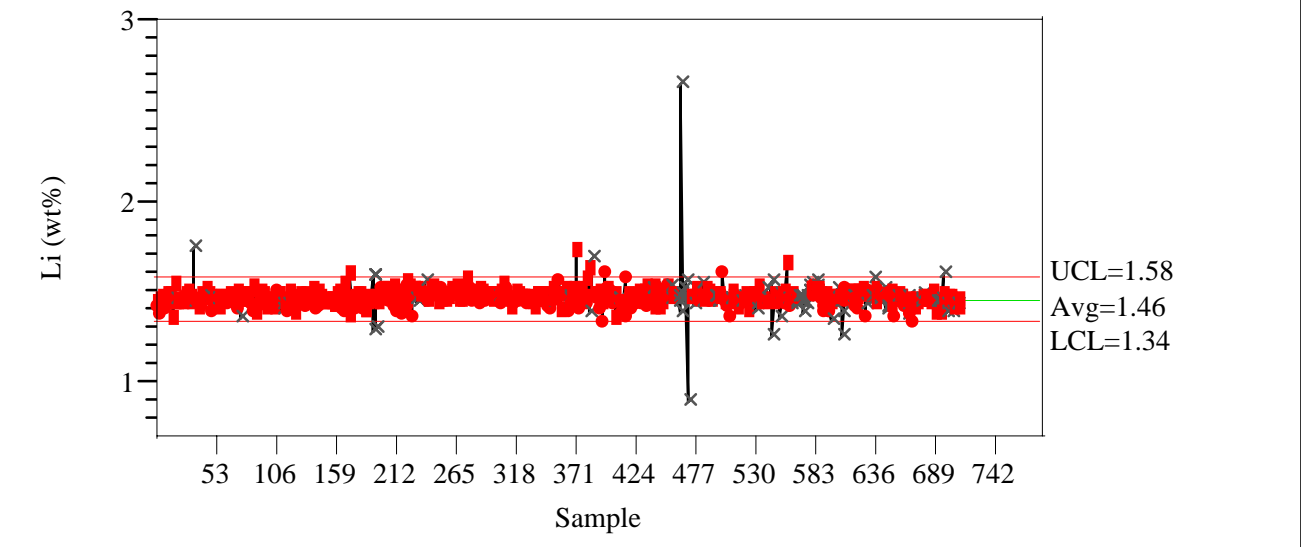

Individual Measurement of Mg (wt\%)

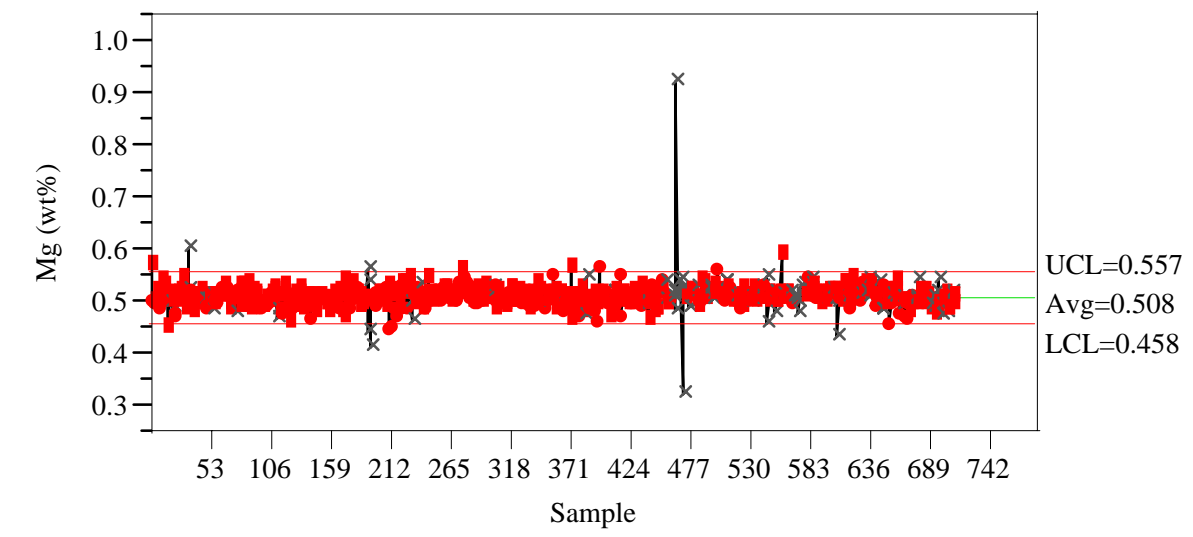


WSRC-TR-2004-00576

Revision 0

\section{Exhibit A26. Time Series Plot (Control Charts) for ARG-1 Measurements Using Fusion Prep}
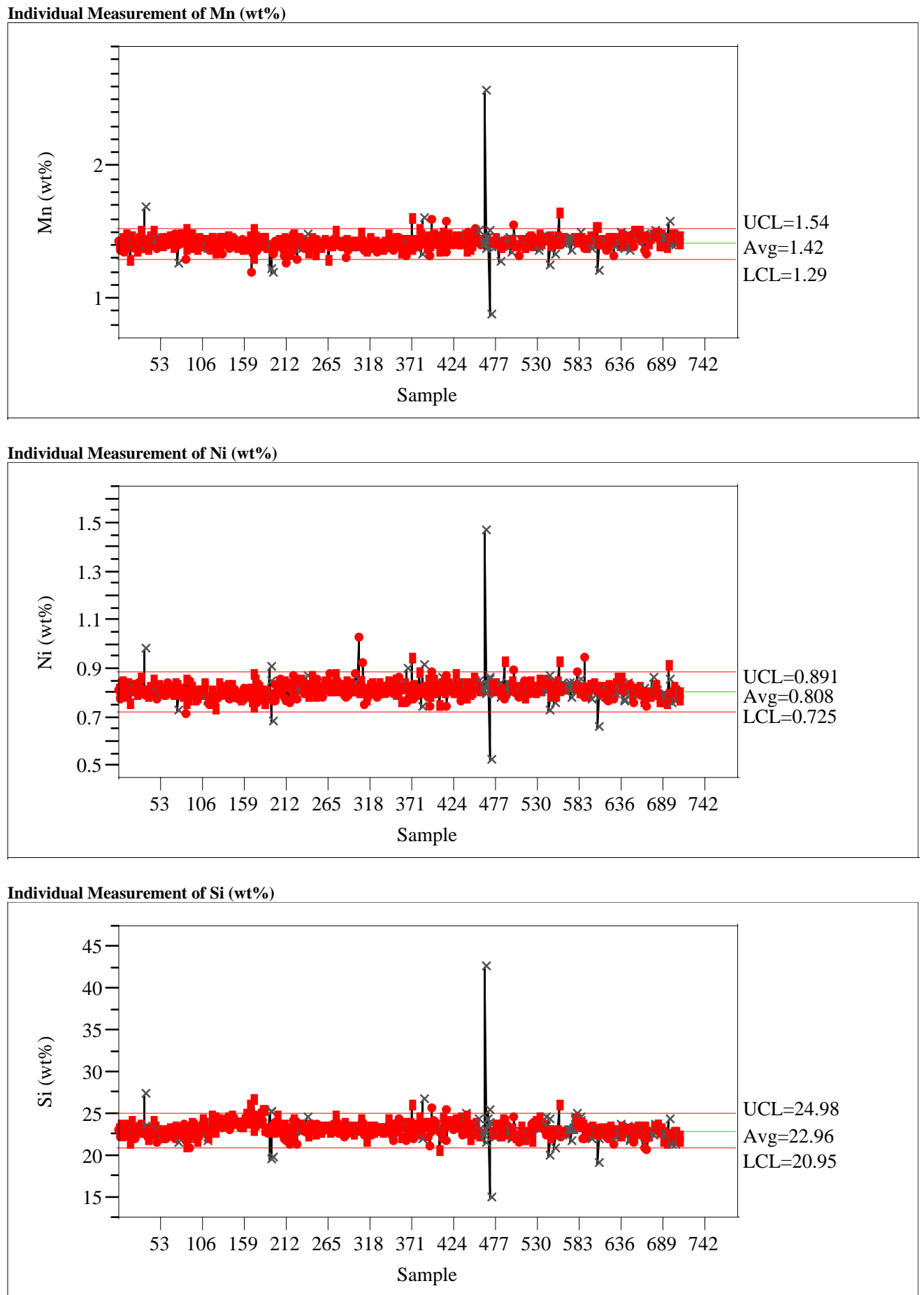
WSRC-TR-2004-00576

Revision 0

Exhibit A26. Time Series Plot (Control Charts) for ARG-1 Measurements Using Fusion Prep

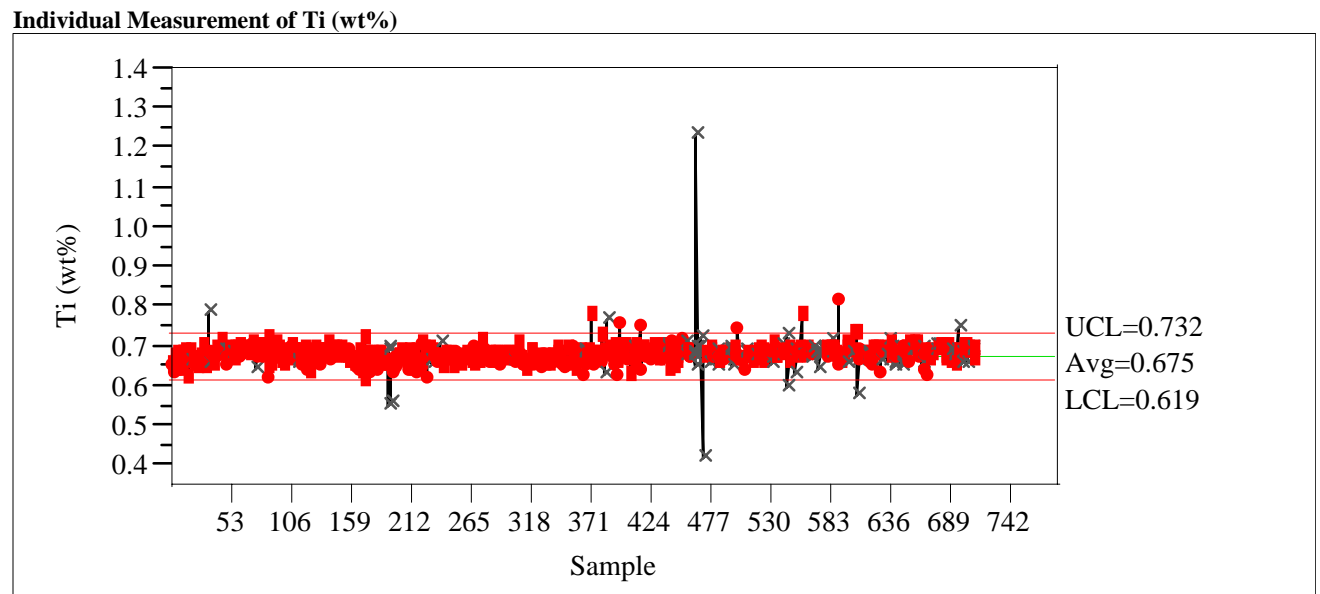


WSRC-TR-2004-00576

Revision 0

\section{Exhibit A27. Histograms and Descriptive Statistics for the ARG-1 Measurements Using Fusion Prep}

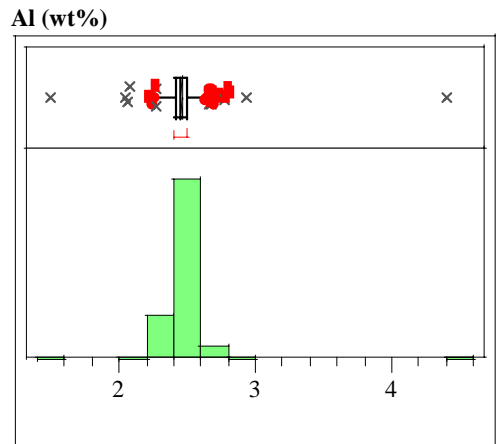

\section{Quantiles}

$100.0 \%$ maximum 4.4000

$99.5 \% \quad 2.8021$

$97.5 \%$

$90.0 \% \quad 2.5570$

$75.0 \%$ quartile 2.5040

$50.0 \%$ median 2.4540

$25.0 \%$ quartile $\quad 2.4130$

$10.0 \%$

$\begin{array}{ll}1.5 \% & 2.3017\end{array}$

$0.5 \% \quad 2.0665$

$0.0 \%$ minimum 1.4940

Moments

Mean $\quad 2.462316$

Std Dev $\quad 0.1180327$

Std Err Mean $\quad 0.0044235$

upper 95\% Mean 2.4710006

lower 95\% Mean 2.4536314

$\mathrm{N}$

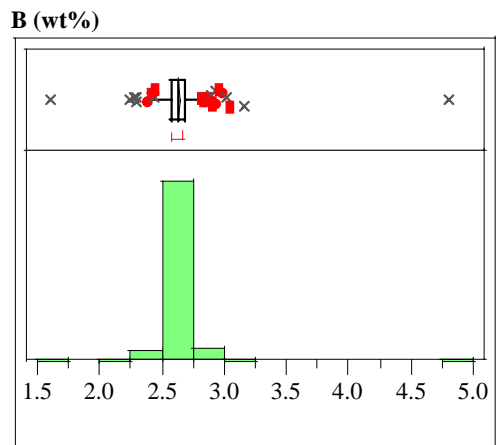

\section{Quantiles}

$100.0 \%$ maximum 4.8030

$99.5 \% \quad 3.030$

$97.5 \% \quad 2.8157$

$90.0 \% \quad 2.7260$

$75.0 \%$ quartile 2.6790

$50.0 \%$ median 2.6295

$25.0 \%$ quartile 2.5850

$10.0 \% \quad 2.5400$

$2.5 \% \quad 2.4588$

$0.5 \% \quad 2.2743$

$0.0 \%$ minimum $\quad 1.6000$

Moments

Mean 2.6339129

Std Dev $\quad 0.1249286$

Std Err Mean $\quad 0.0046819$

upper 95\% Mean 2.6431049

lower 95\% Mean $\quad 2.6247209$

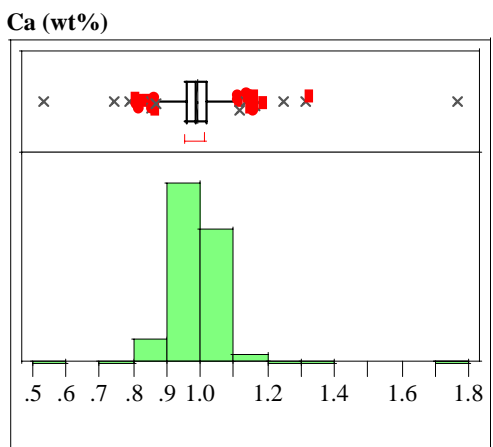

\section{Quantiles}

$100.0 \%$ maximum 1.7660

$99.5 \% \quad 1.2750$

$97.5 \% \quad 1.1120$

$90.0 \%$

$75.0 \%$

$50.0 \%$

$25.0 \%$

$10.0 \%$

$2.5 \%$

$0.5 \%$

minimum 0.5300

Moments

Mean

Std Dev

Std Err Mea

upper $95 \%$ Mean

lowe

$\mathrm{N}$

Cr (wt\%)

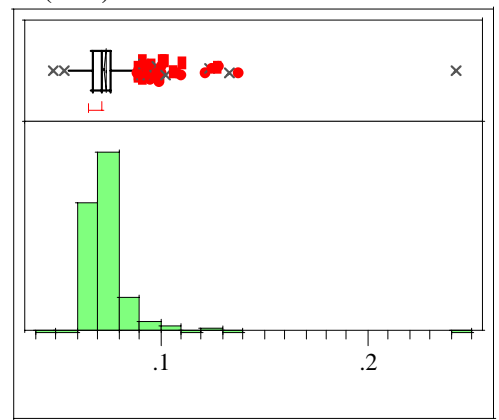

Quantiles

$100.0 \%$ maximum 0.24200

$99.5 \%$

$97.5 \%$

$90.0 \%$

$75.0 \%$

$50.0 \%$

$25.0 \%$

$10.0 \%$

$2.5 \%$

$0.5 \%$

Momen

Mean

Std Dev

upper $95 \%$ Mean

lower 95\% Mean

$\mathrm{N}$

0.0112944

0.0004233
0.0744307

0.0727687
Cu (wt\%)

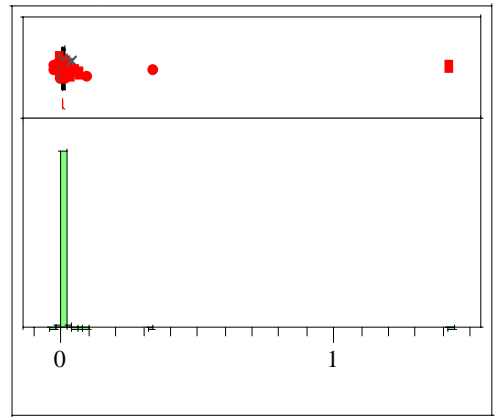

Quantiles

$100.0 \%$ maximum 1.424

$99.5 \%$

$97.5 \%$

$90.0 \%$

$75.0 \%$ quartile

$50.0 \%$ median

$25.0 \%$

$10.0 \%$

$2.5 \%$

$0.5 \%$

Moments

Mome

Std Dev

Std Err Mean

upper 95\% Mean

lower 95\% Mean

$\mathrm{N}$

Fe (wt \%)

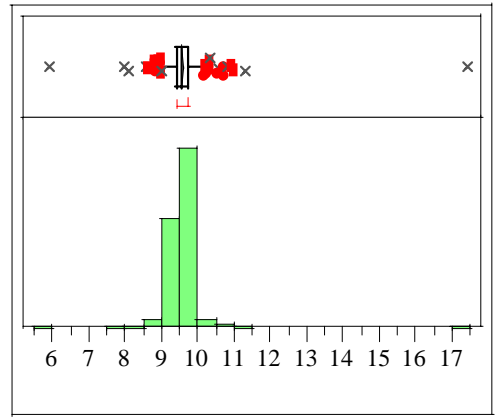

Quantiles

$100.0 \%$ maximum 17.414

$99.5 \% \quad 10.968$

$97.5 \% \quad 10.121$

$90.0 \%$

$75.0 \%$ quartile $\quad 9.717$

$50.0 \%$ median 9.566

$25.0 \%$ quartile $\quad 9.419$

$10.0 \% \quad 9.233$

$2.5 \% \quad 8.969$

$0.5 \% \quad 8.351$

$0.0 \%$ minimum 5.892

Moments

Mean

9.5666138

Std Dev

Std Err Mean

0.4359804

upper 95\% Mean 9.5986923

lower 95\% Mean 9.5345352

$\mathrm{N}$

712 
WSRC-TR-2004-00576

Revision 0

\section{Exhibit A27. Histograms and Descriptive Statistics for the ARG-1 Measurements Using Fusion Prep}

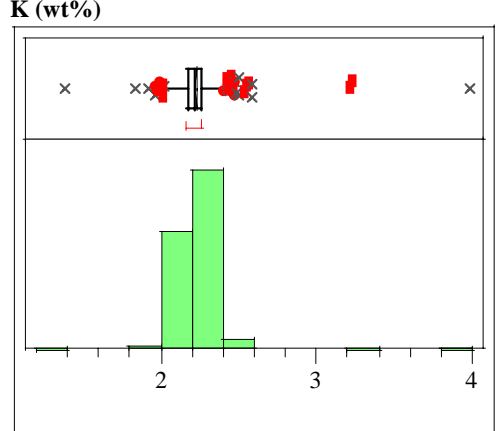

\section{Quantiles}

$100.0 \%$ maximum 3.9830

$99.5 \%$

$97.5 \%$

$90.0 \%$

$75.0 \%$

$50.0 \%$

$25.0 \%$

$10.0 \%$

$2.5 \%$

$0.5 \%$

$0.0 \%$
Moments

Mean

Std Dev

Std Err Mean

upper 95\% Mean

low

$\mathrm{N}$

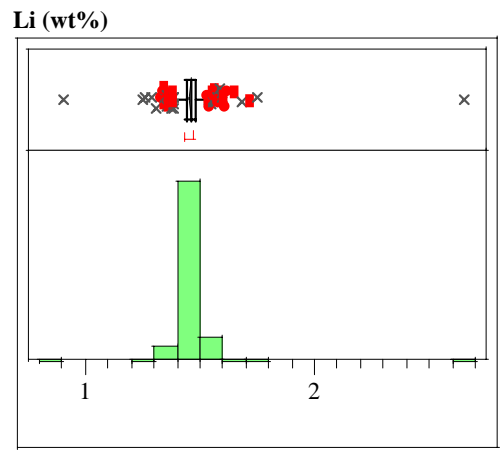

\section{Quantiles}

$100.0 \%$ maximum 2.6500

$99.5 \%$

$97.5 \%$

$90.0 \%$

$75.0 \%$

$50.0 \%$

$25.0 \%$

$10.0 \%$

$2.5 \%$

$0.5 \%$

Moment

Mean

Std Dev

Std Err Mean

upper 95\% Mean

lower 95\% Mean
Mg (wt\%)

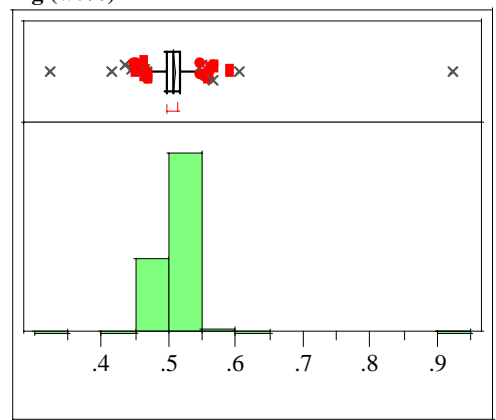

Quantiles

$100.0 \%$ maximum 0.92200

$99.5 \%$

$97.5 \%$

$90.0 \%$

$75.0 \%$

$50.0 \%$

$25.0 \%$

$10.0 \%$

$2.5 \%$

$0.5 \%$

$0.0 \%$ minimum

Moments

Mean

Std Dev

Std Err Mean

upper 95\% Mean

lower $95 \%$ Mea

$\mathrm{N}$

Mn (wt\%)

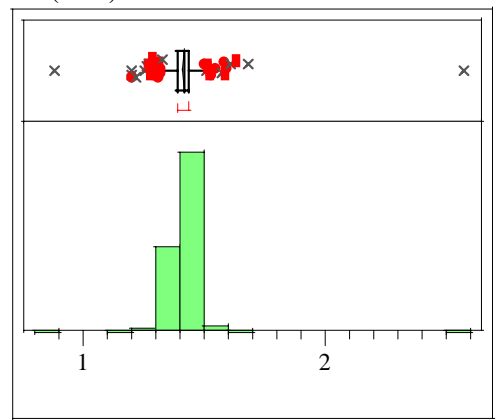

Quantiles

$100.0 \%$ maximum 2.5640

$99.5 \%$

$97.5 \%$

$90.0 \%$

$75.0 \%$

$50.0 \%$

$25.0 \%$

$10.0 \%$

$2.5 \%$

$0.5 \%$

$0.0 \%$

Moments

Mean

Std Dev

upper 95\% Mean

lower 95\% Mean

$\mathrm{N}$
Ni (wt\%)

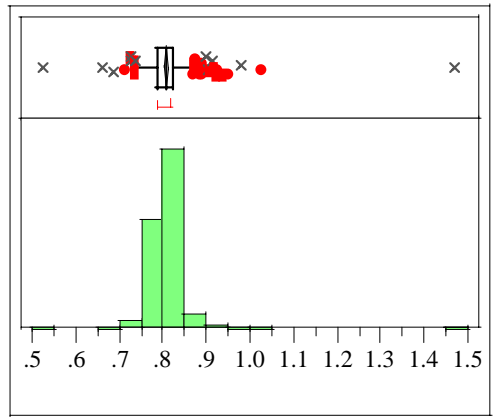

Quantiles

$100.0 \%$ maximum 1.4680

$99.5 \%$

$97.5 \%$

$90.0 \%$

$75.0 \%$

$50.0 \%$

$25.0 \%$

$10.0 \%$

$2.5 \%$

$0.5 \%$

Moment

Mean

Std Dev

upper 95\% Mean

Si (wt\%)

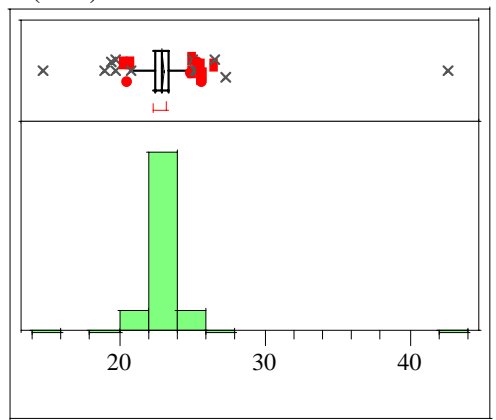

Quantiles

$100.0 \%$ maximum 42.493

$99.5 \% \quad 26.508$

$97.5 \% \quad 24.743$

$90.0 \% \quad 23.980$

$75.0 \%$ quartile 23.426

$50.0 \%$ median 22.907

$25.0 \%$ quartile 22.455

$10.0 \% \quad 22.002$

$2.5 \% \quad 21.265$

$0.5 \% \quad 19.546$

$0.0 \%$ minimum 14.693

Moments

Mean

Std Dev $\quad 1.1730263$

Std Err Mean $\quad 0.043961$

upper 95\% Mean 23.051285

lower 95\% Mean $\quad 22.878667$

$\mathrm{N}$ 
WSRC-TR-2004-00576

Revision 0

Exhibit A27. Histograms and Descriptive Statistics for the ARG-1 Measurements Using Fusion Prep

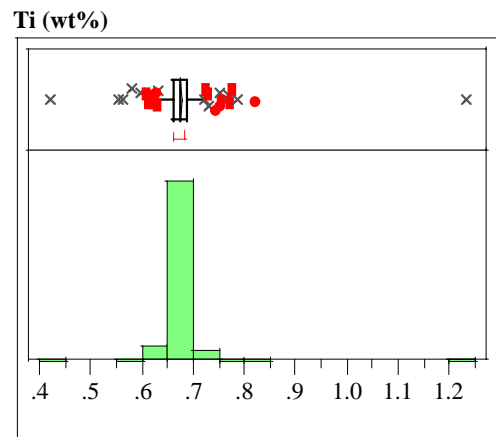

Quantiles

$100.0 \%$ maximum 1.2320

$99.5 \% \quad 0.7793$

$97.5 \% \quad 0.7162$

$90.0 \%-0.6950$

$75.0 \%$ quartile $\quad 0.6860$

$50.0 \%$ median 0.6760

$25.0 \%$ quartile $\quad 0.6640$

$10.0 \%$

$2.5 \% \quad 0.6328$

$0.5 \% \quad 0.5692$

$0.0 \% \quad$ minimum $\quad 0.4190$

Moments

Mean 0.6751798

Std Dev $\quad 0.0316071$

Std Err Mean $\quad 0.0011845$

upper 95\% Mean 0.6775054

lower 95\% Mean 0.6728542

$\mathrm{N}$ 
WSRC-TR-2004-00576

Revision 0

\section{Exhibit A28. Histograms and Descriptive Statistics for the ARG-1 Measurements from SME and MFT Batches for Fusion Prep}

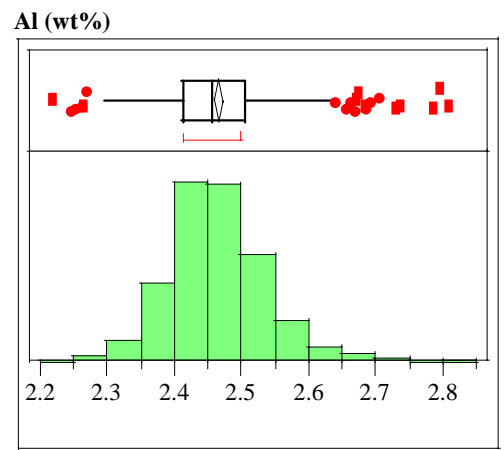

\section{Quantiles}

$100.0 \%$ maximum 2.8100

$99.5 \% \quad 2.7852$

$97.5 \% \quad 2.6423$

$90.0 \% \quad 2.5553$

$75.0 \%$ quartile 2.5048

$50.0 \%$ median 2.4560

$25.0 \%$ quartile $\quad 2.4150$

$10.0 \% \quad 2.3807$

$2.5 \% \quad 2.3168$

$0.5 \% \quad 2.2539$

$0.0 \%$ minimum 2.2190

Moments

Mean $\quad 2.464797$

Std Dev $\quad 0.0772526$

Std Err Mean $\quad 0.0031644$

upper 95\% Mean 2.4710117

lower 95\% Mean 2.4585823

$\mathrm{N}$

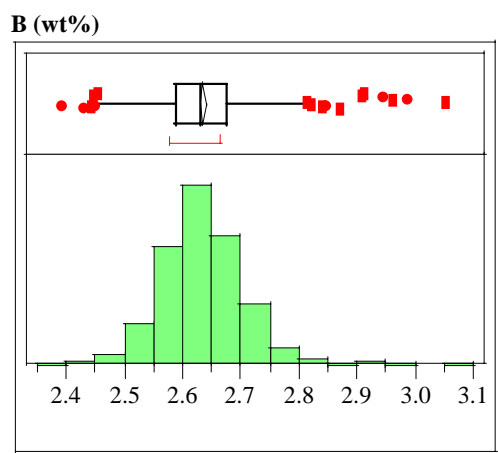

Quantiles

$100.0 \%$ maximum 3.0510

$99.5 \% \quad 2.9624$

$97.5 \% \quad 2.7864$

$90.0 \% \quad 2.7196$

$75.0 \%$ quartile 2.6770

$50.0 \%$ median 2.6315

$25.0 \%$ quartile 2.5880

$10.0 \% \quad 2.5497$

$2.5 \% \quad 2.4949$

$0.5 \% \quad 2.4409$

$0.0 \% \quad$ minimum 2.3940

Moments

Mean

Std Dev $\quad 0.0745982$

Std Err Mean $\quad 0.0030557$

upper 95\% Mean 2.6408066

lower 95\% Mean 2.6288042

$\mathrm{N}$

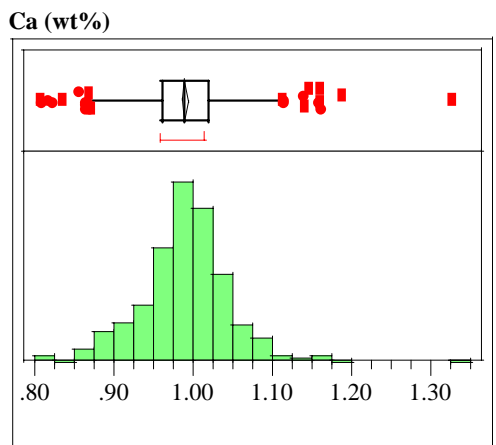

\section{Quantiles}

$100.0 \%$ maximum 1.3260

$99.5 \%$

$97.5 \%$

$90.0 \%$

$75.0 \%$

$50.0 \%$

$25.0 \%$

$10.0 \%$

$2.5 \%$

$0.5 \%$

Moments

Mean

Std Dev

Std Err Mean

upper 95\% Mean

lower 95\% Mea

$\mathrm{N}$

Cr (wt\%)

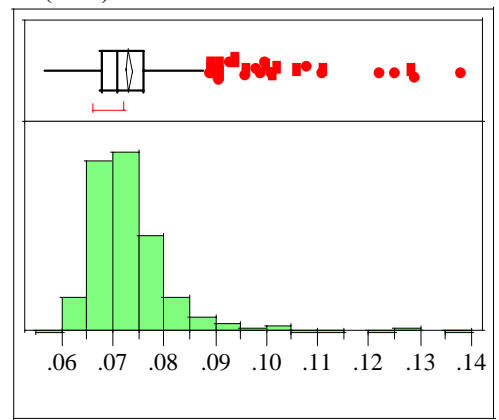

Quantiles

$100.0 \%$ maximum 0.13800

$99.5 \%$

$97.5 \%$

$90.0 \%$

$75.0 \%$

$50.0 \%$

$25.0 \%$

$10.0 \%$

$2.5 \%$

$0.5 \%$

$0.0 \%$

Mome

Std Dev

Std Err Mean

upper 95\% Mean

lower 95\% Mean

$\mathrm{N}$
Cu (wt\%)

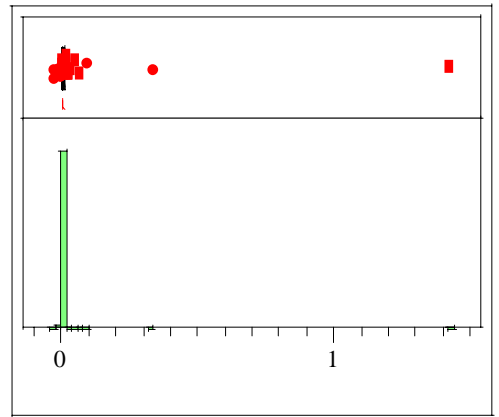

Quantiles

$100.0 \%$ maximum 1.424

$99.5 \%$

$97.5 \%$

$75.0 \%$

median

$10.0 \%$

$2.5 \%$

$0.5 \%$

$0.0 \%$
Mome

Momen

Std Dev

Std Err Mean

upper 95\% Mean

lower 95\% Mean

$\mathrm{N}$

Fe (wt \%)

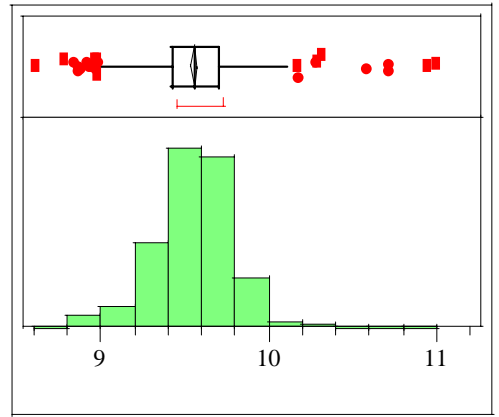

Quantiles

$100.0 \%$ maximum 10.999

$99.5 \%$

$97.5 \%$

$90.0 \%$

$75.0 \%$

$25.0 \%$

$10.0 \%$

$2.5 \%$

$0.5 \%$

8.847

Moments

Mean

Std Dev

Std Err Mean
0.010146 0.0599259

0.0024547

0.0149668

596 
WSRC-TR-2004-00576

Revision 0

\section{Exhibit A28. Histograms and Descriptive Statistics for the ARG-1 Measurements from SME and MFT Batches for Fusion Prep}

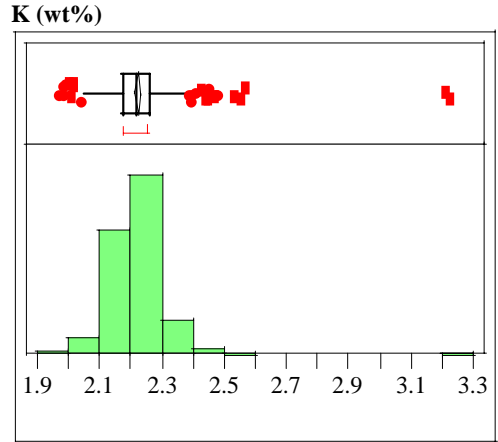

\section{Quantiles}

$100.0 \%$ maximum 3.2270

$99.5 \% \quad 2.5747$

$97.5 \% \quad 2.3961$

$90.0 \% \quad 2.3073$

$75.0 \%$ quartile 2.2600

$50.0 \%$ median 2.2185

$25.0 \%$ quartile $\quad 2.1750$

$10.0 \%$

$\begin{array}{ll}1.5 \% & 2.0629\end{array}$

$0.5 \% \quad 1.9900$

$0.0 \%$ minimum $\quad 1.9720$

Moments

Mean $\quad 2.2221342$

Std Dev $\quad 0.0954932$

Std Err Mean $\quad 0.0039116$

upper 95\% Mean $\quad 2.2298164$

lower 95\% Mean 2.2144521

$\mathrm{N}$

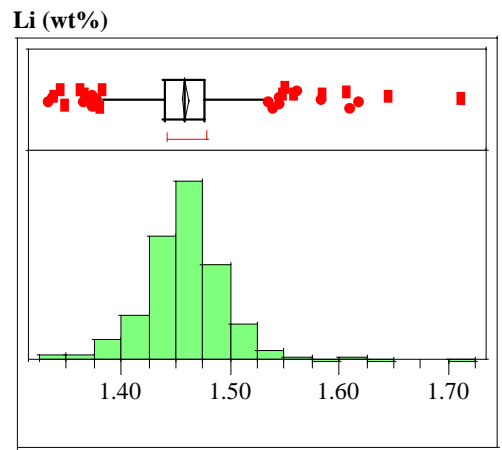

Quantiles

$100.0 \%$ maximum 1.7120

$99.5 \%$

$97.5 \%$

$90.0 \%$

$75.0 \%$

$50.0 \%$

$25.0 \%$

$10.0 \%$

$2.5 \%$

$0.5 \%$

Moment

Momen

Std Dev

Std Err Mean

upper 95\% Mean 1.4621838

lower 95\% Mean 1.455974

$\mathrm{N}$
Mg (wt\%)

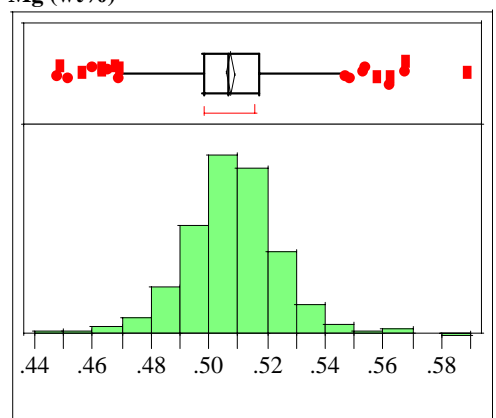

\section{Quantiles}

$100.0 \%$ maximum 0.58900

$99.5 \%$

$97.5 \%$

$90.0 \%$

$75.0 \%$

$50.0 \%$

$25.0 \%$

$10.0 \%$

$2.5 \%$

$0.5 \%$

Moments

Mome

Std Dev

Std Err Mean

upper 95\% Mean

lower 95\% Mean

$\mathrm{N}$

Mn (wt\%)

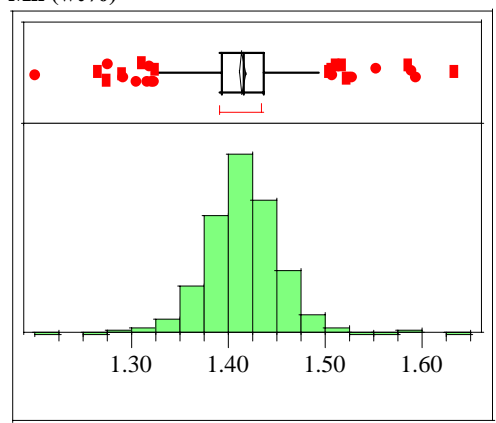

Quantiles

$100.0 \%$ maximum 1.6320

$99.5 \%$

$97.5 \%$

$90.0 \%$

$75.0 \%$

$50.0 \%$

$25.0 \%$

$10.0 \%$

$2.5 \%$

$0.5 \%$

$0.0 \%$

Mome

Std Dev

Std Err Mean

upper 95\% Mean

lower 95\% Mean
$\mathrm{Ni}(\mathrm{wt} \%)$

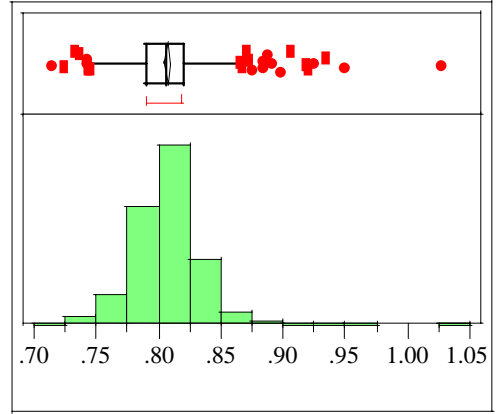

Quantiles

$100.0 \%$ maximum 1.0280

$99.5 \%$

$97.5 \%$

$90.0 \%$

$75.0 \%$

$50.0 \%$

$25.0 \%$

$10.0 \%$

$2.5 \%$

$0.5 \%$

Moment

Mome

Std Dev

Std Err Mean

upper 95\% Mean

lower 95\% Mean

$\mathrm{N}$

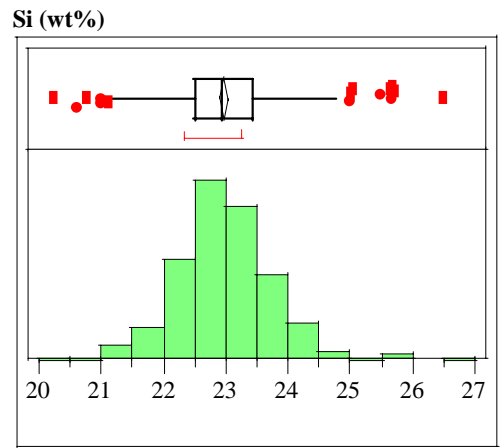

Quantiles

$100.0 \%$ maximum 26.500

$99.5 \%$

$97.5 \%$

$75.0 \%$

$50.0 \%$

$25.0 \%$

$10.0 \%$

$2.5 \%$

$0.5 \%$

Moments

Mean

Std Dev

Std Err Mean

upper 95\% Mean

lower 95\% Mean
0.807094

0.0011992

0.8094492

8047387

596

24.516

23.902

quartile $\quad 23.439$

artile $\quad 22.512$

22.089

20.752

20.218

22.977993

0.7698535

0.0315344

23.039926

22.916061 
WSRC-TR-2004-00576

Revision 0

Exhibit A28. Histograms and Descriptive Statistics for the ARG-1 Measurements from SME and MFT Batches for Fusion Prep

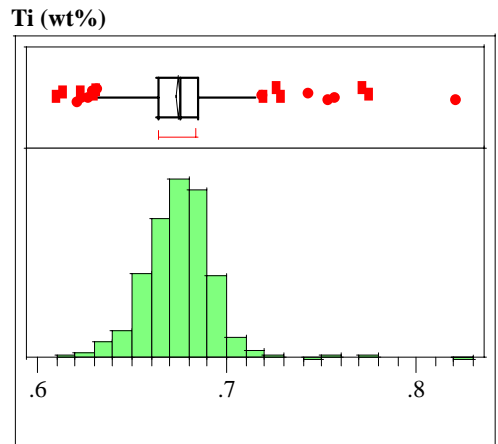

Quantiles

$100.0 \%$ maximum 0.82100

$\begin{array}{ll}99.5 \% & 0.77106\end{array}$

$97.5 \% \quad 0.70907$

$90.0 \%$

$75.0 \%$ quartile $\quad 0.68500$

$50.0 \%$ median 0.67500

$25.0 \%$ quartile $\quad 0.66400$

$10.0 \% \quad 0.65270$

$2.5 \% \quad 0.63492$

$0.5 \% \quad 0.62088$

$0.0 \%$ minimum $\quad 0.61000$

Moments

Mean $\quad 0.6744916$

Std Dev $\quad 0.0194301$

Std Err Mean $\quad 0.0007959$

upper 95\% Mean $\quad 0.6760547$

lower 95\% Mean 0.6729285

$\mathrm{N}$ 
WSRC-TR-2004-00576

Revision 0

\section{Exhibit A29. Components of Variance for the ARG-1 Measurements from SME and MFT Batches for Fusion Prep}

Response Al (wt\%)

Summary of Fit

RSquare

RSquare Adj

0.286528

0.06554

Mean of Response 2.46686

Observations (or Sum Wgts) 536

Analysis of Variance

$\begin{array}{lrrrr}\text { Source } & \text { DF } & \text { Sum of Squares } & \text { Mean Square } & \text { F Ratio } \\ \text { Model } & 267 & 2.0697860 & 0.007752 & 1.8047 \\ \text { Error } & 268 & 1.1511825 & 0.004295 & \text { Prob }>\text { F } \\ \text { C. Total } & 535 & 3.2209685 & & <.0001\end{array}$

Variance Component Estimates

$\begin{array}{lrr}\text { Component } & \text { Var Comp Est } & \text { Percent of Total } \\ \text { Tank\&Batch\&Random } & 0.001728 & 28.691 \\ \text { Residual } & 0.004295 & 71.309 \\ \text { Total } & 0.006024 & 100.000\end{array}$

These estimates based on equating Mean Squares to Expected Value.

Response B (wt\%)

Summary of Fit

$\begin{array}{lr}\text { RSquare } & 0.566521 \\ \text { RSquare Adj } & 0.13466 \\ \text { Root Mean Square Error } & 0.068939 \\ \text { Mean of Response } & 2.635424 \\ \text { Observations (or Sum Wgts) } & 536\end{array}$

Analysis of Variance

$\begin{array}{lrrrr}\text { Source } & \text { DF } & \text { Sum of Squares } & \text { Mean Square } & \text { F Ratio } \\ \text { Model } & 267 & 1.6646354 & 0.006235 & 1.3118 \\ \text { Error } & 268 & 1.2737095 & 0.004753 & \text { Prob }>\text { F } \\ \text { C. Total } & 535 & 2.9383449 & & 0.0134\end{array}$

$\begin{array}{llll}\text { C. Total } & 535 & 2.9383449 & 0.0134\end{array}$

$\begin{array}{lrr}\text { Variance Component Estimates } & \\ \text { Component } & \text { Var Comp Est } & \text { Percent of Total } \\ \text { Tank\&Batch\&Random } & 0.000741 & 13.488 \\ \text { Residual } & 0.004753 & 86.512 \\ \text { Total } & 0.005494 & 100.000\end{array}$

These estimates based on equating Mean Squares to Expected Value.

Response Ca (wt \%)

Summary of Fit

\begin{tabular}{|c|c|c|c|c|}
\hline \multicolumn{3}{|l|}{ RSquare } & \multicolumn{2}{|c|}{0.695905} \\
\hline \multicolumn{3}{|c|}{ RSquare Adj } & \multicolumn{2}{|c|}{0.392945} \\
\hline \multicolumn{3}{|c|}{ Root Mean Square Error } & \multicolumn{2}{|c|}{0.04269} \\
\hline \multicolumn{3}{|c|}{ Mean of Response } & \multicolumn{2}{|c|}{0.990711} \\
\hline \multicolumn{3}{|c|}{ Observations (or Sum Wgts) } & \multicolumn{2}{|l|}{536} \\
\hline \multicolumn{5}{|c|}{ Analysis of Variance } \\
\hline Source & DF & Sum of Squares & Mean Square & F Ratio \\
\hline Model & 267 & 1.1176987 & 0.004186 & 2.2970 \\
\hline Error & 268 & 0.4884095 & 0.001822 & Prob $>$ F \\
\hline C. Total & 535 & 1.6061082 & & $<.0001$ \\
\hline
\end{tabular}

Variance Component Estimates

$\begin{array}{lrr}\text { Component } & \text { Var Comp Est } & \text { Percent of Total } \\ \text { Tank\&Batch\&Random } & 0.001182 & 39.339 \\ \text { Residual } & 0.001822 & 60.661 \\ \text { Total } & 0.003004 & 100.000\end{array}$

Total $0.003004 \quad 100.000$

These estimates based on equating Mean Squares to Expected Value.
Response Cr (wt\%)

Summary of Fit

\begin{tabular}{|c|c|c|c|c|c|}
\hline \multicolumn{3}{|l|}{ RSquare } & \multicolumn{3}{|c|}{0.678199} \\
\hline \multicolumn{3}{|c|}{ RSquare Adj } & \multicolumn{3}{|c|}{0.357598} \\
\hline \multicolumn{3}{|c|}{ Root Mean Square Error } & \multicolumn{3}{|c|}{0.00708} \\
\hline \multicolumn{3}{|c|}{ Mean of Response } & \multicolumn{3}{|c|}{0.073149} \\
\hline \multicolumn{3}{|c|}{ Observations (or Sum Wgts) } & \multicolumn{3}{|c|}{536} \\
\hline \multicolumn{6}{|c|}{ Analysis of Variance } \\
\hline Source & DF & Sum of Squ & & Mean Square & F Rati \\
\hline Model & 267 & 0.02830 & & 0.000106 & 2.1154 \\
\hline Error & 268 & 0.01343 & & 0.000050 & Prob $>$ F \\
\hline C. Total & 535 & 0.04174 & 006 & & $<.0001$ \\
\hline
\end{tabular}

Variance Component Estimates

$\begin{array}{lrr}\text { Component } & \text { Var Comp Est } & \text { Percent of Total } \\ \text { Tank\&Batch\&Random } & 0.000028 & 35.803 \\ \text { Residual } & 0.00005 & 64.197 \\ \text { Total } & 0.000078 & 100.000\end{array}$

These estimates based on equating Mean Squares to Expected Value.

Response Cu (wt \%)

Summary of Fit

0.499352

RSquare Adj $\quad 0.000571$

Root Mean Square Error $\quad 0.063157$

Mean of Response $\quad 0.010494$

Observations (or Sum Wgts) 536

Analysis of Variance

$\begin{array}{lrrrr}\text { Source } & \text { DF } & \text { Sum of Squares } & \text { Mean Square } & \text { F Ratio } \\ \text { Model } & 267 & 1.0662265 & 0.003993 & 1.0011 \\ \text { Error } & 268 & 1.0689955 & 0.003989 & \text { Prob }>\text { F } \\ \text { C. Total } & 535 & 2.1352220 & & 0.4962\end{array}$

Variance Component Estimates

$\begin{array}{lrr}\text { Component } & \text { Var Comp Est } & \text { Percent of Total } \\ \text { Tank\&Batch\&Random } & 0.000002 & 0.057 \\ \text { Residual } & 0.003989 & 99.943 \\ \text { Total } & 0.003991 & 100.000\end{array}$

These estimates based on equating Mean Squares to Expected Value.

Response Fe (wt\%)

Summary of Fit

RSquare

0.540001

RSquare Adj

0.081718

Root Mean Square Error $\quad 0.24665$

Mean of Response 9.563257

Observations (or Sum Wgts) 536

Analysis of Variance

Source DF Sum of Squares Mean Square F Ratio

$\begin{array}{lrrrr}\text { Model } & 267 & 19.139734 & 0.071684 & 1.1783\end{array}$

$\begin{array}{lllll}\text { Error } & 268 & 16.304158 & 0.060836 & \text { Prob }>\text { F }\end{array}$

$\begin{array}{llll}\text { C. Total } & 535 & 35.443892 & 0.0901\end{array}$ 
WSRC-TR-2004-00576

Revision 0

\section{Exhibit A29. Components of Variance for the ARG-1 Measurements from SME and MFT Batches for Fusion Prep}

Response K (wt\%)

Summary of Fit

RSquare

RSquare Adj

0.061952

Mean of Response 2.223088

Observations (or Sum Wgts) $\quad 536$

Analysis of Variance

$\begin{array}{lrrrr}\text { Source } & \text { DF } & \text { Sum of Squares } & \text { Mean Square } & \text { F Ratio } \\ \text { Model } & 267 & 4.0747884 & 0.015261 & 3.9763 \\ \text { Error } & 268 & 1.0286005 & 0.003838 & \text { Prob }>\text { F } \\ \text { C. Total } & 535 & 5.1033889 & & <.0001\end{array}$

Variance Component Estimates

$\begin{array}{lrr}\text { Component } & \text { Var Comp Est } & \text { Percent of Total } \\ \text { Tank\&Batch\&Random } & 0.005712 & 59.810 \\ \text { Residual } & 0.003838 & 40.190 \\ \text { Total } & 0.00955 & 100.000\end{array}$

These estimates based on equating Mean Squares to Expected Value.

Response Li (wt \%)

Summary of Fit

RSquare Adj

0.55432

Root Mean Square Error $\quad 0.036186$

Mean of Response $\quad 1.46064$

Observations (or Sum Wgts) 536

Analysis of Variance

Source DF Sum of Squares Mean Square F Ratio

Model $\quad 267 \quad 0.43646601 \quad 0.001635 \quad 1.2484$

$\begin{array}{lllll}\text { Error } & 268 & 0.35092350 & 0.001309 & \text { Prob }>\text { F }\end{array}$

$\begin{array}{llll}\text { C. Total } & 535 & 0.78738951 & 0.0351\end{array}$

Variance Component Estimates

$\begin{array}{lrr}\text { Component } & \text { Var Comp Est } & \text { Percent of Total } \\ \text { Tank\&Batch\&Random } & 0.000163 & 11.049 \\ \text { Residual } & 0.001309 & 88.951 \\ \text { Total } & 0.001472 & 100.000\end{array}$

Total

These estimates based on equating Mean Squares to Expected Value.

Response Mg (wt \%)

Summary of Fi

\begin{tabular}{|c|c|c|c|c|}
\hline \multicolumn{3}{|l|}{ RSquare } & \multicolumn{2}{|c|}{0.617697} \\
\hline \multicolumn{3}{|c|}{ RSquare Adj } & \multicolumn{2}{|c|}{0.23682} \\
\hline \multicolumn{3}{|c|}{ Root Mean Square Error } & \multicolumn{2}{|c|}{0.014229} \\
\hline \multicolumn{3}{|c|}{ Mean of Response } & \multicolumn{2}{|c|}{0.50778} \\
\hline \multicolumn{3}{|c|}{ Observations (or Sum Wgts) } & \multicolumn{2}{|l|}{536} \\
\hline \multicolumn{5}{|c|}{ Analysis of Variance } \\
\hline Source & DF & Sum of Squares & Mean Square & F Ratio \\
\hline Model & 267 & 0.08766602 & 0.000328 & 1.6218 \\
\hline Error & 268 & 0.05425800 & 0.000202 & Prob $>$ F \\
\hline C. Total & 535 & 0.14192402 & & $<.0001$ \\
\hline
\end{tabular}

Variance Component Estimates

$\begin{array}{lrr}\text { Component } & \text { Var Comp Est } & \text { Percent of Total } \\ \text { Tank\&Batch\&Random } & 0.000063 & 23.716 \\ \text { Residual } & 0.000202 & 76.284 \\ \text { Total } & 0.000265 & 100.000\end{array}$

Total $0.000265 \quad 100.000$

These estimates based on equating Mean Squares to Expected Value.
Response Mn (wt\%)

Summary of Fit

RSquare

0.550444

RSquare Adj

0.102565

Root Mean Square Error $\quad 0.03863$

Mean of Response 1.414802

Observations (or Sum Wgts) 536

Analysis of Variance

$\begin{array}{lrrrr}\text { Source } & \text { DF } & \text { Sum of Squares } & \text { Mean Square } & \text { F Ratio } \\ \text { Model } & 267 & 0.48968204 & 0.001834 & 1.2290 \\ \text { Error } & 268 & 0.39993100 & 0.001492 & \text { Prob }>\text { F } \\ \text { C. Total } & 535 & 0.88961304 & & 0.0462\end{array}$

Variance Component Estimates

$\begin{array}{lrr}\text { Component } & \text { Var Comp Est } & \text { Percent of Total } \\ \text { Tank\&Batch\&Random } & 0.000171 & 10.274 \\ \text { Residual } & 0.001492 & 89.726 \\ \text { Total } & 0.001663 & 100.000\end{array}$

These estimates based on equating Mean Squares to Expected Value.

Response Ni (wt \%)

Summary of Fit

0.548413

RSquare Adj

0.098511

Root Mean Square Error $\quad 0.027995$

Mean of Response 0.807976

Observations (or Sum Wgts) 536

Analysis of Variance

Source DF Sum of Squares Mean Square F Ratio

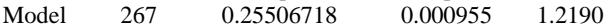

$\begin{array}{lllll}\text { Error } & 268 & 0.21003350 & 0.000784 & \text { Prob }>\text { F }\end{array}$

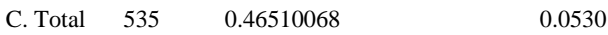

Variance Component Estimates

$\begin{array}{lrr}\text { Component } & \text { Var Comp Est } & \text { Percent of Total } \\ \text { Tank\&Batch\&Random } & 0.000086 & 9.868 \\ \text { Residual } & 0.000784 & 90.132 \\ \text { Total } & 0.00087 & 100.000\end{array}$

These estimates based on equating Mean Squares to Expected Value.

Response Si (wt\%)

Summary of Fit

RSquare Adj

0.702951

Square Error

22.98275

Observations (or Sum Wgts) 536

Analysis of Variance

Source DF Sum of Squares Mean Square F Ratio

$\begin{array}{llrrr}\text { Model } & 267 & 221.33015 & 0.828952 & 2.3753\end{array}$

$\begin{array}{llrrr}\text { Error } & 268 & 93.52862 & 0.348987 & \text { Prob }>\text { F }\end{array}$

$\begin{array}{llll}\text { C. Total } 535 & 314.85877 & <0001\end{array}$ 
WSRC-TR-2004-00576

Revision 0

\section{Exhibit A29. Components of Variance for the ARG-1 Measurements from SME and MFT Batches for Fusion Prep}

\begin{tabular}{|c|c|c|c|c|}
\hline \multicolumn{5}{|c|}{ Response Ti (wt\%) } \\
\hline \multicolumn{3}{|l|}{ RSquare } & \multicolumn{2}{|c|}{0.535873} \\
\hline \multicolumn{3}{|c|}{ RSquare Adj } & \multicolumn{2}{|c|}{0.073478} \\
\hline \multicolumn{3}{|c|}{ Root Mean Square Error } & \multicolumn{2}{|c|}{0.018632} \\
\hline \multicolumn{3}{|c|}{ Mean of Response } & \multicolumn{2}{|c|}{0.674841} \\
\hline \multicolumn{3}{|c|}{ Observations (or Sum Wgts) } & \multicolumn{2}{|l|}{536} \\
\hline \multicolumn{5}{|c|}{ Analysis of Variance } \\
\hline Source & DF & Sum of Squares & Mean Square & F Ratio \\
\hline Model & 267 & 0.10742302 & 0.000402 & 1.1589 \\
\hline Error & 268 & 0.09304050 & 0.000347 & Prob $>$ F \\
\hline C. Total & 535 & 0.20046352 & & 0.1142 \\
\hline
\end{tabular}

Variance Component Estimates

Var Comp Est Percent of Total

Tank\&Batch\&Random $\quad 0.000028$

$\begin{array}{lrr}\text { Residual } & 0.000347 & 92.639\end{array}$

Total $0.000375 \quad 100.000$

These estimates based on equating Mean Squares to Expected Value. 


\section{Exhibit A30. Time Series Plots as Control Charts for the ARG-1 Sum of Oxides in Analytical Sequence by Preparation Method}

Method=Cold Chem

Control Chart

Individual Measurement of Sum of Oxides (as available)

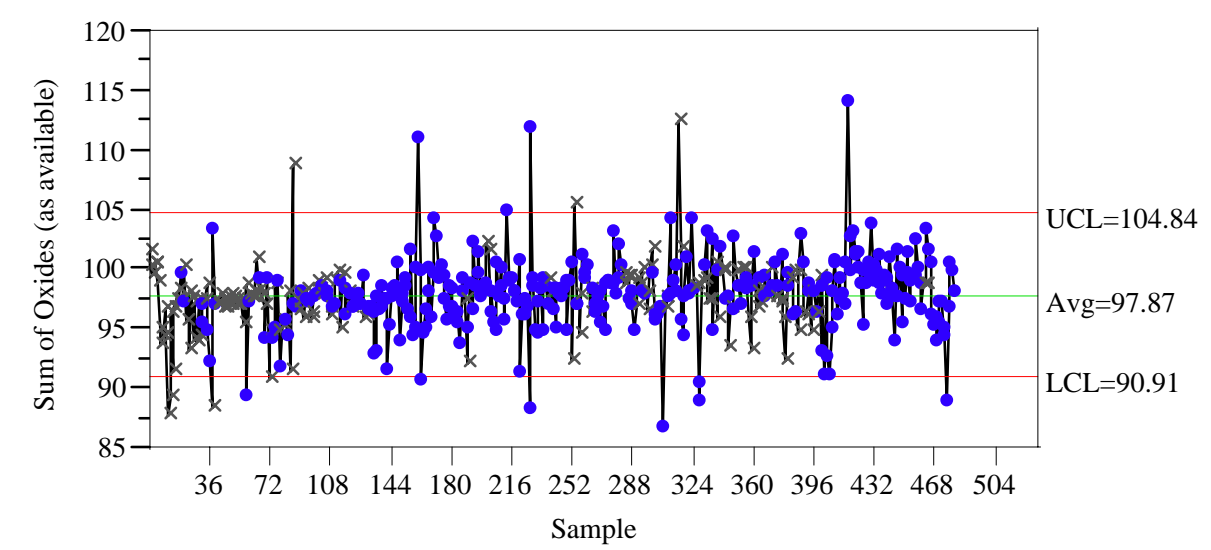

Method=Fusion

Control Chart

Individual Measurement of Sum of Oxides (as available)

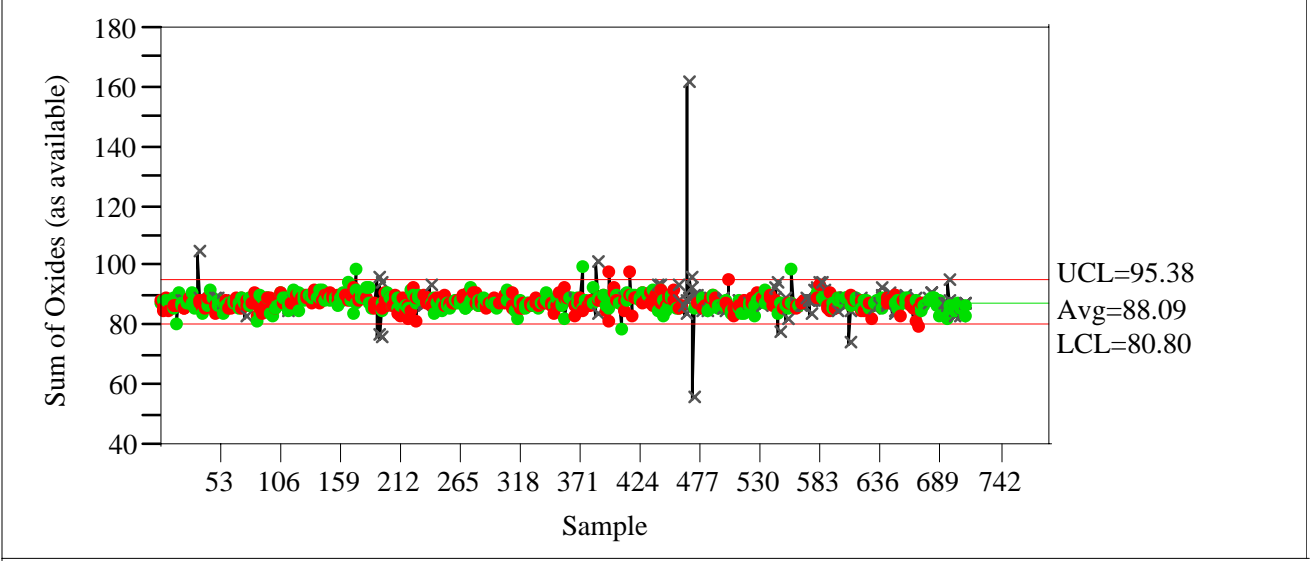

Method=Mixed Acid

Control Chart

Individual Measurement of Sum of Oxides (as available)

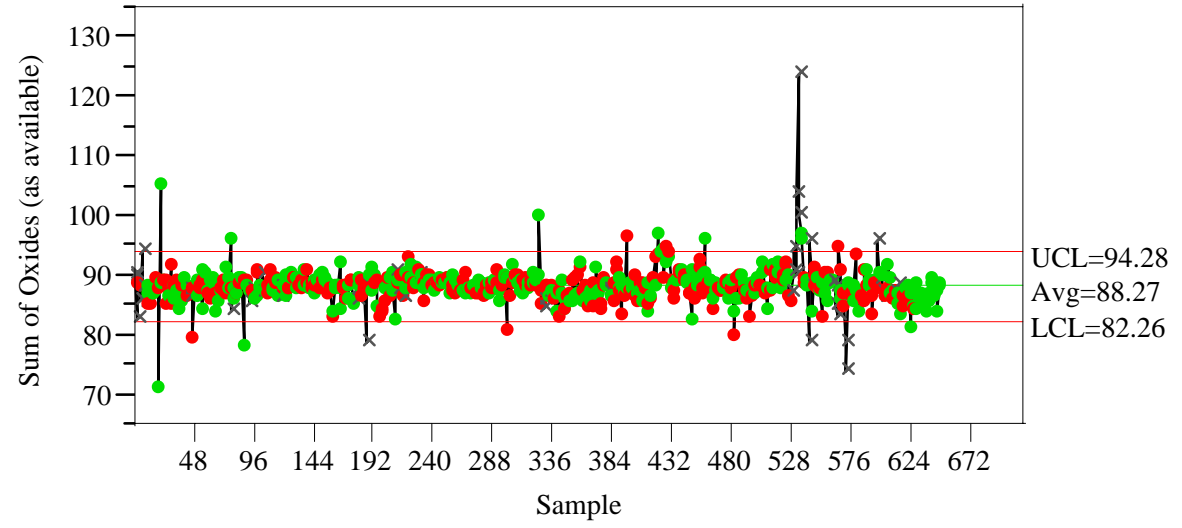




\section{Exhibit A31. Time Series Plots as Control Charts for the ARG-1 Sum of Oxides in Analytical Sequence by Preparation Method with Process Batch Information}

\section{Method=Cold Chem}

Control Chart

Individual Measurement of Sum of Oxides (as available)

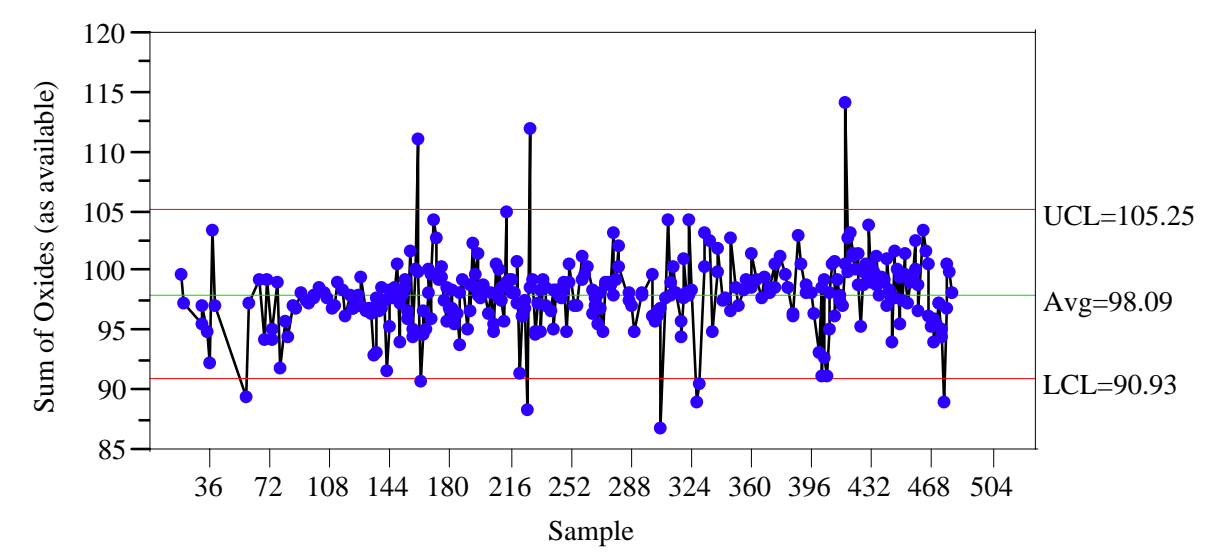

Method=Fusion

Control Chart

Individual Measurement of Sum of Oxides (as available)

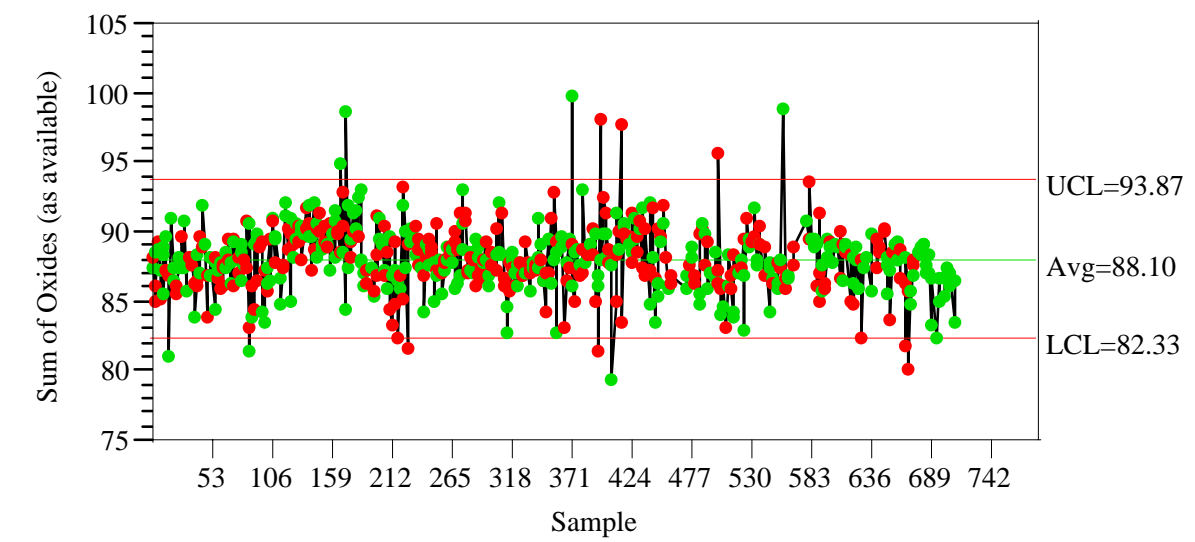

Method=Mixed Acid

Control Chart

Individual Measurement of Sum of Oxides (as available)

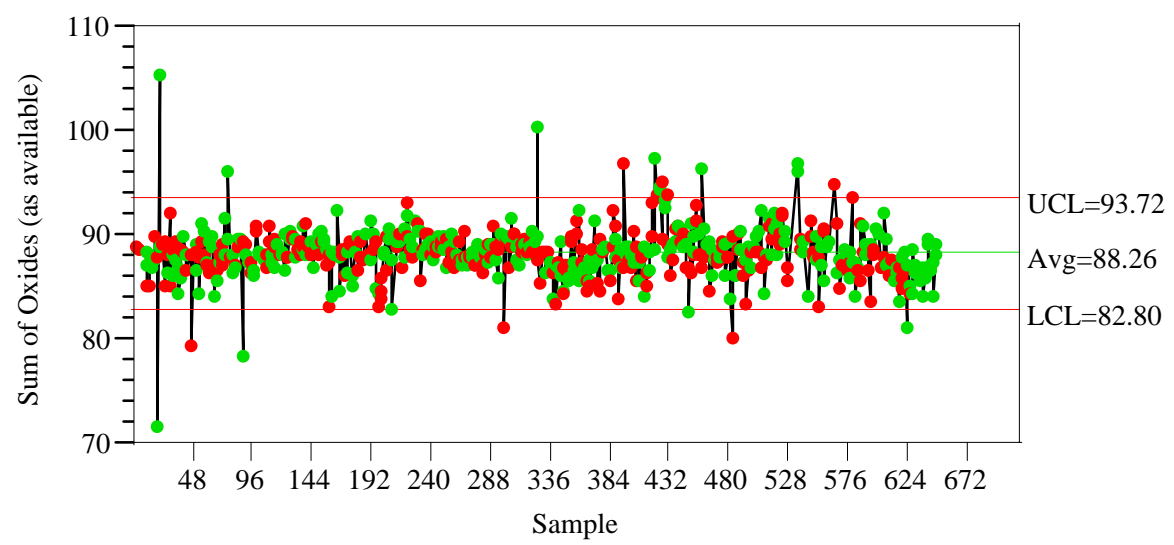


WSRC-TR-2004-00576

Revision 0

Exhibit A32. Histograms and Descriptive Statistics for the ARG-1 Sum of Oxides in Analytical Sequence by Preparation Method with Process Batch Information

Descriptor=Cold Chem-SRAT-With Batch

Sum of Oxides (as available)

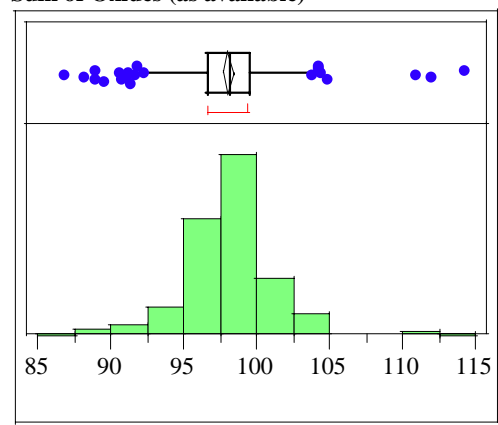

Quantiles

$100.0 \% \quad$ maximum $\quad 114.27$

$99.5 \%$

$97.5 \%$

$90.0 \%$

$75.0 \%$

$50.0 \%$

$25.0 \%$

$10.0 \%$

$2.5 \%$

$0.5 \%$

$0.0 \%$

Moments

Mean

Std Dev

Std Err Mean

upper 95\% Mean

lower 95\% Mean

$\mathrm{N}$

Descriptor=Fusion-MFT-With Batch Sum of Oxides (as available)

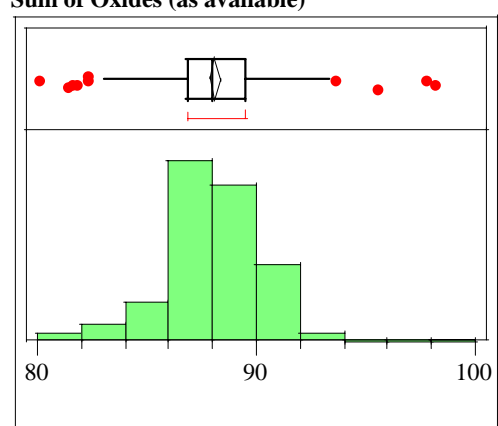

Quantiles

$100.0 \%$

$99.5 \%$

maximum

98.236

$99.5 \%$
$97.5 \%$

$90.0 \%$

$75.0 \%$

$50.0 \%$

$25.0 \%$

$10.0 \%$

$2.5 \%$

$0.5 \%$

$0.0 \%$

Moments

Mean

Std Dev

Std Err Mean upper 95\% Mean

lower 95\% Mean

$\mathrm{N}$

98.089771

3.0383525

98.42237

97.757172

323
Descriptor=Fusion-SME-With Batch

Sum of Oxides (as available)

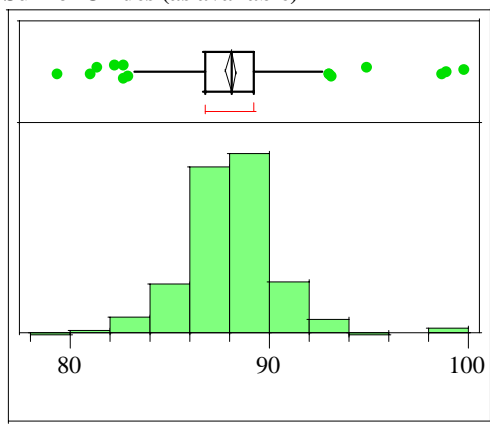

Quantiles

$100.0 \%$

$99.5 \%$

$97.5 \%$

$90.0 \%$

$75.0 \%$

$50.0 \%$

$25.0 \%$

$10.0 \%$

$2.5 \%$

$0.5 \%$

$0.0 \%$

Moments

Mean

Std Dev

Std Err Mean

maximum

99.873

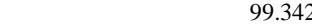

92.612

89.256

median 88.097

quartile $\quad 86.813$

85.429
83.333

80.372

minimum $\quad 79.346$

lower 95\% Mean

88.08721

2.4180965

0.1356001

88.354

87.82042

$\mathrm{N}$

Descriptor=Mixed Acid-MFT-With Batch Sum of Oxides (as available)

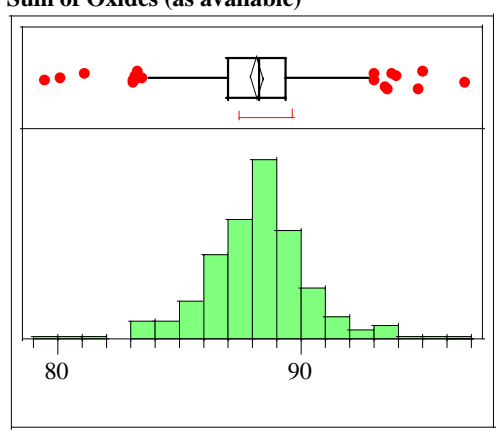

Quantiles

$100.0 \%$ maximum 96.755

$99.5 \%$

$97.5 \%$

$90.0 \%$

$75.0 \%$

$50.0 \%$

$25.0 \%$

$10.0 \%$

$2.5 \%$

$0.5 \%$

$0.0 \%$

Moments

Mean

Std Dev

Std Err Mean

upper 95\% Mean

lower 95\% Mean

$\mathrm{N}$

$\begin{array}{lr}\text { maximum } & 96.755 \\ & 96.094 \\ & 93.513 \\ & 90.830 \\ \text { quartile } & 89.330 \\ \text { median } & 88.251 \\ \text { quartile } & 87.009 \\ & 85.545 \\ & 83.273 \\ \text { minimum } & 79.743 \\ & 79.494 \\ & \\ & 88.20173 \\ & 2.2359544 \\ & 0.1341036 \\ & 88.465722 \\ & 87.937738 \\ \text { an } & 278\end{array}$


WSRC-TR-2004-00576

Revision 0

Exhibit A32. Histograms and Descriptive Statistics for the ARG-1 Sum of Oxides in Analytical Sequence by Preparation Method with Process Batch Information

Descriptor=Mixed Acid-SME-With Batch

Sum of Oxides (as available)

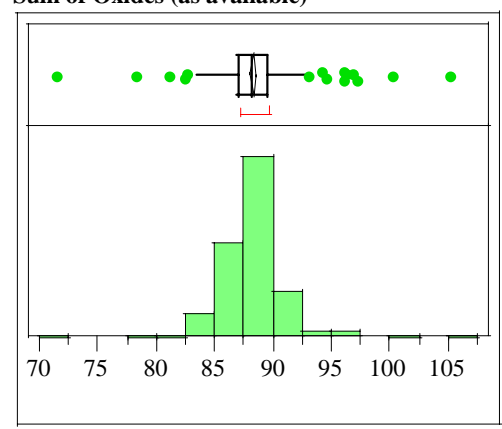

Quantiles

$100.0 \%$ maximum $\quad 105.31$

$99.5 \%$

$97.5 \% \quad 94.69$

$90.0 \% \quad 90.70$

$75.0 \% \quad$ quartile $\quad 89.47$

$50.0 \%$ median 88.25

$25.0 \%$ quartile 86.98

$10.0 \%$

$2.5 \%$

$0.5 \% \quad 75.63$

$0.0 \%$ minimum 71.55

Moments

Mean

Std Dev

Std Err Mean

88.303786

2.7229765

upper $95 \%$ Mean $\quad 88.603739$

lower 95\% Mean $\quad 88.003833$

$\mathrm{N}$

319 